Title Learning to Teach English in Hong Kong: Effects of the Changeover in Sovereignty

Name Alan Urmston

This is a digitised version of a dissertation submitted to the University of Bedfordshire.

It is available to view only.

This item is subject to copyright. 


\title{
LEARNING TO TEACH ENGLISH IN HONG KONG: EFFECTS OF THE CHANGEOVER IN SOVEREIGNTY
}

\begin{abstract}
Alan Urmston
A thesis submitted to the University of Luton, in partial fulfilment of the requirements for the degree of Doctor of Philosophy
\end{abstract}

July 2003

VOLUME 1

\section{THESIS}

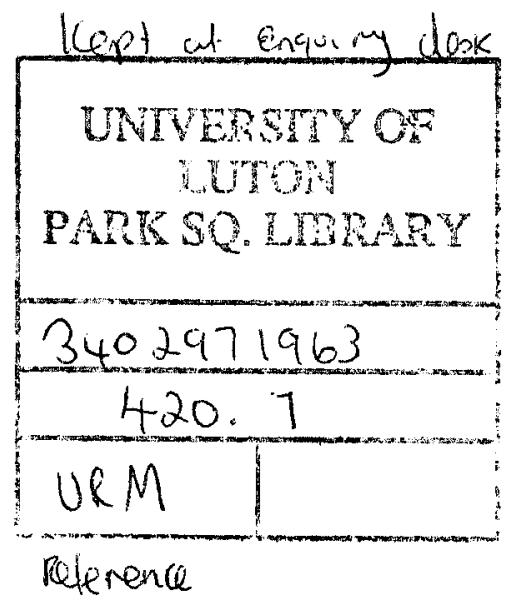




\begin{abstract}
Learning to Teach English in Hong Kong: Effects of the Changeover in Sovereignty
\end{abstract}

Alan Urmston

University of Luton, 2003

\title{
Director of Studies: Professor Martha C. Pennington
}

Teachers undergo changes in their beliefs, knowledge and practices on an individual level as they learn how to teach. If society undergoes significant change, as Hong Kong did during the transition from British to Chinese rule in 1997 , then social groups within society such as teachers are likely to react to change in different and complex ways. The purpose of this investigation is to examine the changes experienced by teachers of English in Hong Kong, with a focus on teachers who received their teacher preparation at one Hong Kong institution during the final years leading up to the transition. The educational, linguistic, social and political context of Hong Kong is first described through a study of the research literature and a number of theories and models of change are presented through which the findings of the investigation are analysed. The main sources of data for the investigation consist of questionnaire responses, interview transcriptions and lesson observation reports of trainee English teachers during and after graduation from a BA course in TESL at a Hong Kong university. The main conclusions of the investigation are: (i) Educational issues and particularly those affecting ELT became more high-profile and politicised in the lead up to and after the changeover. (ii) English teachers in Hong Kong experience conflict 
between their desired approaches and the realities and constraints of the Hong Kong teaching context. These constraints provide a common justification for lack of innovative behaviour and make it possible for teachers to put off being innovative in the classroom indefinitely. (iii) At the same time, English teachers in Hong Kong are becoming more empowered within the educational system in reaction to challenges to their competency and as they have realised that they can affect educational policy through individual and collective action. The findings suggest that colonial discourses as documented by Pennycook (1998) of English language teaching still persist in Hong Kong, as they have been shown to do in other post-colonial societies, and Hong Kong is undergoing a post-handover period of change as it struggles to synthesise the educational legacies of the colonial period with new initiatives adopted to address Hong Kong's changing educational and social needs. The results of the research are developed into an original model of the factors impacting English language education in Hong Kong. The generic model is then elaborated in two versions, one of which applies before the changeover and the other after it. 


\section{ACKNOWLEDGEMENTS}

I would like to thank my Director of Studies, Professor Martha C. Pennington of the University of Luton for her invaluable guidance, inspiration, patience and mentorship throughout this investigation.

I would also like to thank my External Supervisor, Dr Graham Lock of the City University of Hong Kong for his valuable input on thesis drafts; and my Second Internal Supervisor, Dr Helen Spencer-Oatey of the University of Luton for critical comment at an early stage of the research.

Furthermore, I would like to acknowledge the help given to me by the following: The students of and graduates from the BATESL course and the staff of the English Department of the City University of Hong Kong; the many English language practitioners who gave up their valuable time to be interviewed for the investigation; Dr Francis Yue of the City University of Hong Kong and Dr Bart Yeung of the Hong Kong Polytechnic University for their advice on statistical

procedures; Professor Jack C. Richards for advice and inspiration; and all my superiors, colleagues and students in the institutions that I have worked in over the past six years.

Last but not least, I owe an extreme debt of gratitude to my wife, Kathy, for her patience and unwavering support throughout the last six years. 


\section{DECLARATION}

I declare that this thesis is my own unaided work. It is being submitted for the degree of Doctor of Philosophy at the University of Luton. It has not been submitted before for any degree or examination in any other University.

Alan Urmston

July 2003 


\section{CONTENTS (This volume)}

\author{
Abstract \\ Acknowledgements \\ List of figures
}

i

iii

viii

2.2.2 English language learning and teaching in Hong Kong schools

2.2.3 The training of teachers of English in Hong Kong 2-9

2.3 The language situation in Hong Kong (1994-1999) 2-10

2.4 Hong Kong society during the period leading up to and after $\quad 2-16$ the changeover in sovereignty (1994-1999)

2.4.1 Social structure and social change 2-17

2.4.2 Hong Kong's political culture 2-29

2.4.3 Diffusion of innovations 2-31

$2-75$

$2-75$

$2-78$

2.5.3 Previous studies of pre-service teachers directly 2-90 related to this research

2.6 A framework for analysing change in the social system of 2-92

Hong Kong English language teachers

2.6.1 Educational change 2-92

2.6.2 Framework for analysing teacher change 2-94

$2-99$

\subsection{The investigation}


3.1 Background 3-1

3.2 Survey of the issues pertaining to English language teaching 3-3 as reported in the English language newspapers in 1997

3.2.1 Methodology 3-3

3.2.2 Findings 3-4

3.2.3 Discussion 3-13

3.2.4 Concluding remarks on newspaper survey 3-14

3.3 Interview survey of people connected with the teaching of 3-16 English in Hong Kong on the issues affecting English language teaching in the run up to and after the changeover in sovereignty

3.3.1 Methodology 3-17

3.3.2 Findings 3-21

3.3.3 Discussion 3-49

3.4 Conclusions $3-60$

CHAPTER 4 REFLECTIONS ON TEACHING OF TRAINEE 4-1 TEACHERS STUDYING ON A BATESL COURSE IN HONG KONG (1994 AND 1997)

4.1 Background 4-1

4.2 Methodology 4-3

4.2.1 Respondents 4-3

4.2.2 Survey instrument $4-4$

4.2.3 Procedures $4-7$

4.3 Results $4-9$

4.3.1 Language use 4-10

4.3.2 Instructional planning and decision-making 4-12

4.3.3 Teaching approach 4-14

4.3.4 Professional relationships and responsibilities 4-16

4.3.5 Perceptions and values 4-21

4.4 Discussion 4-25

4.4.1 Similarities across the three sets of responses (survey $\quad 4-26$ groups)

4.4.2 Differences across the three response groups 4-53

4.4.3 Teaching philosophies 4-83

4.4.4 The 1997 BATESL graduates' views on the potential 4-85 effects of the changeover in sovereignty on the teaching of English in Hong Kong

4.5 Conclusions

4.5.1 Common characteristics of trainee English teachers in 4-94 Hong Kong 
4.5.2 Evidence of change in cohort 2 over period of

BATESL course

4.5.3 Evidence of differences between Cohort 1 and

Cohort 2

4.5.4 Evidence of changes in the BATESL course during 4-99 the period 1994-1997

4.5.5 Evidence of changes in the teaching culture in Hong Kong during period 1994-1997

CHAPTER 5 A POST-1997 PICTURE OF GRADUATES IN HONG KONG SCHOOLS

5.1 Background

5.2 Methodology

5.2.1 Subjects and procedures

5.3 Discussion of findings

5.3.1 Interviews with 1997 BATESL graduates one year

5.3.2 Lesson observations of 1997 BATESL graduates two 5-11 years after graduation

5.3.3 Lesson observations of 1994 BATESL graduates five 5-37 years after graduation

5.3.4 Reflections of BATESL course administrators/ teachers in 1999 (eight years after the launch of the course)

5.4 Conclusions

5.4.1 Language use in English lessons

5.4.2 The situation and status of English teachers in Hong

5.4.3 The potential of teachers for adopting innovative teaching approaches

CHAPTER 6 GENERAL DISCUSSION AND CONCLUSIONS 6-1

6.1 Summary of findings 6-1

6.2 Areas of change 6-5

6.2.1 Society 6-5

6.2.2 Educational system 6-14

6.2.3 Language (medium of instruction) 6-24

6.2.4 Teachers 6-31

6.3 A model for change in English language education at a time of $\quad 6-38$ political change

6.4 Generalisations and projections 6-51

6.5 Possible areas of future study 6-57 
6.6 Concluding reflections $6-60$

References $\quad$ R-1

\section{APPENDICES (Second volume)}

Contents

i

List of tables

Appendix A: Chapter $3 \quad$ A-1

A.1 List of interviewees $\quad$ A-1

A.2 Outline of research sent to interviewees in 1997 A-4

A.3 List of questions sent to interviewees in 1997 A-6

A.4 Full list of comments from interviews 1997 A-7

A.5 Interview transcripts from interviews in $1997 \quad$ A-29

A.6 List of questions sent to interviewees in 1999 A-206

A.7 Full list of comments from interviews in $1999 \quad$ A-207

A.8 Interview transcripts from interviews in 1999 A-226

A.9 Tables of results of interview study A-364

Appendix B: Chapter $4 \quad$ B-1

B.1 Transcriptions of interviews with BATESL graduates in 1994 B-1

B.2 Questionnaire administered to BATESL graduates in 1997 B-92

B.3 Transcriptions of interviews with BATESL graduates in 1997 B-111

B.4 Statistical results of questionnaire surveys $\quad$ B-203

B.5 BATESL students' philosophies of teaching B-244

Appendix C: Chapter $5 \quad$ C-1

C.1 Transcriptions of interviews with BATESL graduates in 1998 C-1

C.2 Main points to emerge during interviews with BATESL $\quad \mathrm{C}-47$ graduates in 1998

C.3 Lesson observation reports of 1997 and 1994 BATESL C-54 graduates

C.4 Transcriptions of interviews with BATESL course leaders in C-93 1999

C.5 Main points to emerge from interviews with BATESL course C-104 leaders in 1999 


\section{Figures}

2.1 An s-shaped diffusion curve 2-43

2.2 The diffusion curve for an unsuccessful diffusion $\quad 2-43$

2.3 The diffusion curve for a diffusion which takes a long time to $\quad 2-44$ take off

2.4 The diffusion curve for a diffusion which takes a long time to $\quad 2-45$ take off before discontinuance begins

2.5 The diffusion curve for a diffusion which takes a long time to $\quad 2-45$ take off before plummeting

2.6 Henrichsen's Hybrid Model of diffusion of innovations in 2-57 educational reform

2.7 The formation of new teaching frameworks 2-65

2.8 Centra \& Potter's model of school and teacher effects on 2-102 student learning outcomes

2.9 Pennington's model of the relationship of innovations to 2-103 outcomes in education

6.1 Model of the English language education system in Hong 6-39 Kong

6.2 Revised model of the English language education system in $\quad 6-42$ Hong Kong

6.3 Complete model of English language education system in $\quad 6-44$ Hong Kong

6.4 Model of English language education system in Hong Kong $\quad 6-47$ prior to the changeover in sovereignty

6.5 Model of English language education system in Hong Kong 6-50 after the changeover in sovereignty 


\section{INTRODUCTION}

\subsection{General Introduction}

This introductory chapter first briefly describes the concepts of continuity and change and how they relate to societies and social systems before going on to look at issues of post-colonialism and the special character of Hong Kong. Then the thesis is introduced by outlining the main methodologies used and the research questions that it attempts to answer. Finally, a brief overview of each chapter is given followed by a brief summary of the major conclusions reached.

\subsubsection{Continuity and Change}

Continuity of a system occurs when there is a self-regulatory process or systemic ability to cope with events or behaviours which deviate from the norm and this ability to cope through self-regulatory mechanisms becomes the norm for the system. The system is not destroyed (by the deviant) because it has the ability to incorporate that which is seemingly different - or even to prevent it through certain practices (Kegley \& Wittkopf, 1997). In other words, continuity is maintained in a system when there is familiar or expected change which is cyclical, such as the cycle of the seasons, or of life and death and new growth and new life once again (Dellios, 2001; Pennington, 2002). Change can occur at a subsystem level resulting in changes in relationships between sub-systems and these changes will normally occur in order to maintain systemic continuity.

Change can take different forms and can be considered either stable evolutionary (or progressive), cyclical or retrogressive - or it can be unstable revolutionary, accelerating, 'flip' or even catastrophic (Dellios, 2001; Pennington, 2002). Evolutionary change is that likely to occur in situations where the change 
is over a long period and is from a relatively simple to a more complex form and could be classed as development. An example of evolutionary change could be an education system that develops over time to cope with changes in society. The education system in Hong Kong, the focus of this thesis, developed after the end of World War II and the resumption of British colonial rule to cope with the rapidly increasing population caused by mass migration from Mainland China. ${ }^{1}$ Sweeting (1998) describes how provision was first made for primary education, followed by secondary and then tertiary over a period of forty years. As such the education system evolved due to a series of pre-planned measures and a number of extenuating factors. Cyclical change can occur within a situation of continuity, as mentioned, and is characterised by a return to a familiar position. Education in most societies follows a school year, either from September through to July (as is the case in Hong Kong) or from January to December (as in Australia) and is a cycle of events which is repeated year after year whilst education progresses. Change does occur within the cycle, but ultimately the same position is reached at the end and then a new year begins again. Another form of relatively stable change is retrogressive change, which is the opposite of evolutionary change and is characterised by a development from a more to a less complex system. Such a retrogression may occur under a 'return to basics' movement such as using simpler forms of transport like the bicycle for environmental reasons, or in the case of English language teaching, a return to a more form-focused approach when it is seen that a communicative approach is not working.

What might be considered as more immediate, dramatic or unstable change can occur in a number of forms. Revolutionary change can be thought of as the replacement of one system, policy or condition by another that differs from it in fundamental ways. Revolution in Marxist terms refers to the overthrow of a ruling social class by another social class. In 1997, there was a change of sovereignty when Hong Kong changed from a British dependent territory to a special administrative region of China. ${ }^{2}$ In Hong Kong's case, the British colonial system was replaced by what China's paramount leader, Deng Xiaoping termed the 'one country [China], two systems [Chinese socialism and Hong Kong's free-market 
capitalism]'. The case of Hong Kong was less one of revolutionary change since it was not disruptive of the system and involved a considerable amount of planned continuity. Another more immediate or unstable form of change is accelerating change, which occurs when a series of changes occurs progressively such that the process of changes increases or accelerates. The series of uprisings and revolutions that occurred in 1989/90 in Eastern Europe and led to the fall of communism in the countries of the so-called Eastern or Soviet Bloc were said to have been set into motion by Mikhail Gorbachev in the USSR, resulting in a process of rapidly accelerating change that swept communist regimes from power across the continent (Simpson, 1990). A change process that results in change in two directions has been termed flip or flip-flop. Change in one direction is reversed and a change back to a former condition results. Such a situation can occur in democratic politics (such as in the UK) when a particular political party is in power for one term and enacts policies consistent with its ideologies, and is then replaced by the opposition party at a general election which brings with it its own policies. Then the first party may resume power again at the following election and the process is reversed. Finally, the most extreme form of change can be termed catastrophic change, which may be the result of war or natural disaster. Each of these forms of change may occur at different times in a society or may occur simultaneously.

The change processes described briefly above can occur as the result of deliberate action on the part of an individual or group of individuals or they can be said to be resultant of other changes or to be of unknown origin. Frequently, it is difficult to determine which and so a more realistic description is of a change process beginning at a point along a continuum between deliberate or planned action at one end and independent or accidental action at the other end. Most change processes will occur over a length of the continuum, i.e., they will be in part as a result of deliberate action and in part of incidental or associated action. The ways in which these different change patterns combine will define the nature of the change. Whilst the study of the nature of different changes is interesting in itself, it is through an understanding of change and continuity in a society that the 
structure of that society and its potential for further change can be determined. A detailed discussion of change patterns and how they can be applied to English language teaching is the focus of Chapter 2. It is the purpose of this thesis to examine the changes that occurred in society and in education in Hong Kong that affected English language education there prior to, after and as a result of the changeover in sovereignty. ${ }^{3}$

\subsubsection{Postcolonialism}

Each of the change processes (except for catastrophic change - although there were some people who did not rule this out as a possibility) may have been in operation in Hong Kong during the second half of the final decade of the twentieth century, as political factors converged on the Territory in the run up to the handover. As Hong Kong was the last significant colony of Great Britain to lose that status, the world watched with fascination as the Prince of Wales and Chinese President Jiang Zemin officiated at the handover ceremony on 30 June 1997 (Hong Kong Government Information Service, 1998). Like other former British colonies in Asia, such as India, Malaysia and Singapore, Hong Kong was left with a number of legacies which would help to shape its future, at least in the short term. One of the most important of these legacies was the English language. Much has been written about how these and other postcolonial societies have dealt with the English language (e.g., Kachru on English in India ${ }^{4}$ and Pennycook on Hong Kong ${ }^{5}$ ) and the insights of Pennycook in particular are sourced in relation to the concepts of language policy and linguistic imperialism in postcolonial societies (Chapter 2). Crucially, it is Pennycook's (1998) recognition that language policy within a society is linked to the socio-political structure of society and Rassool's (1998) linking of language policy to socio-political change that reflect key themes in this thesis. In the postcolonial context, societies such as Hong Kong are left with the remnants of the departing colonial power and can take ownership of them and adapt them to meet their own needs or can reject them in favour of their own ways of doing things. Postcolonialism is, according to 
Pennycook (1999) 'a taking over of and reuse of language, culture and knowledge' (p. 11). In this sense, postcolonialism can be described as a form of recycling. How the recycling is done and what is recycled depends on sociopolitical and socio-cultural factors within the postcolonial state, as well as the extent of the lingering influence of the departed colonist.

\subsubsection{The Special Character of Hong Kong}

A major difference between Hong Kong and most postcolonial societies is that Hong Kong did not gain independence in 1997; rather, there occurred a process of decolonisation followed by recolonisation (Law, 1997) as the Territory's new colonial master became the PRC. However, under Hong Kong's post-handover constitution, the Basic Law (Hong Kong SAR Government, 1990) the SAR was promised its autonomy from the Mainland for a period of fifty years. This meant that in theory after the handover Hong Kong was in a position to decide for itself what aspects of British colonialism it would recycle. The English language and the provisions for the teaching and learning of it have received great attention in Hong Kong, no more so than in 1997 (Chapter 3). Prior to the handover, attention was focused on English as the language of the ruling power (and the dominant language of government, the law, international trade and higher education) and the belief that an education through English (as the medium of instruction) would provide people with greater access to resources (Pennycook, 1999). Given the character of Hong Kong, which has been described by Pennington (1998a) as a 'unique blend of East and West, of international and regional characteristics' ( $p$. 24), the importance of English as the conduit for that internationalism has always been strong. Yet as the handover approached, the use of English as the medium of learning in a society where the vast majority of the population speak Cantonese as their first language and do not have access to English in their home environment began to be questioned. At the same time, the impending reunification with the Mainland brought with it increased expressions of patriotism and affinity for 
things Chinese, including language, and the language debate became one of the central issues surrounding the transition of political power.

The British left in place a system of education whereby the majority of secondary schools were in name English-medium. However, the élitist nature of the education system meant that a number of highly prestigious and well-established schools did maintain a high level of English medium; whilst in the majority it was a reality of English heavily supported by Cantonese. Within schools, English was (and still is) taught as a core subject (along with Chinese and mathematics) under the influence of methods adapted from the British and other Western education systems infused with locally developed and maintained methods of language teaching. (A full description and evaluation of the English teaching situation prior to the handover is given in Chapter 2). The colonial nature of the education system and in particular its language policies has been held responsible by Pennycook (1998) for the inappropriacy of the education system for the majority of the population, who do not have access to the cultural capital that facilitates access to elite schools where an effective English medium of instruction is maintained (see section 2.3) and are not adequately served either by a system which uses a mixture of English and Chinese as the teaching media. Pennycook (1998) discusses the legacy of colonial discourses attached to English in Hong Kong as being (at least partly) responsible for the problems in the education system and the continued attachment of these discourses to English in the postcolonial society as resulting in change being difficult to enact. Such discourses as English being the route to economic success and of Chinese people only wanting English for its capital value, Chinese learners being more suited to rote learning, native-speaking English teachers being superior to non-native teachers, and an English-medium education being superior to mother-tongue education, are enduring and (according to Pennycook) inhibit the development of education in post-colonial societies such as Hong Kong.

For education to develop in tune with the changing needs of society after the changeover in sovereignty, the government will need to play an active role and 
will need to be seen to be doing so by the public. It is likely that greater demands will be made on the government to address educational issues, particularly those seen as legacies of the colonial administration and it can be expected that education will become more politicised as the public come to regard Hong Kong as being run by Hong Kong people and as such being more publicly accountable than the colonial government was. Ironically, the democratic political reforms brought in by the colonial government towards the end of their period of rule may well result in greater involvement in political affairs by the public and educational issues will be high on the agenda as they affect all members of society. Some members of society will be more involved in education than others, of course, especially teachers, and how they react to the changing political and social atmosphere will be crucial to the development of education. Teachers in particular can affect education greatly through both individual and collective action. Individual action in the classroom means the extent to which they adopt or do not adopt new initiatives and reforms introduced by education authorities. Collective action may involve political protest or even resistance to policy. Either way, teachers are at the forefront of any educational change mechanism and it is important to consider their reactions and behaviour.

What is important also is to consider is the ways that education in Hong Kong is structured, i.e., the roles that the different groups or organisations, or what might be termed sub-systems play, and the relationships between these sub-systems in providing education for the population. Given the complexity of these roles and relationships and their dependence on the social structure of society, it seems inevitable that the changeover in sovereignty would affect this social structure and hence would affect education. The added complexities connected with the provision for English language in a predominantly Chinese society under Western and Eastern influences, may make it even more difficult to detect and describe the patterns of change and to evaluate the resulting educational structure. However, when doing so, a greater understanding of the ways that changing societies change their provisions for language education should be achieved. 


\subsection{Introduction to Thesis}

\subsubsection{Purpose, Nature and Scope of the Investigation}

The research described in this thesis is an investigation into the teaching and learning of English as a second language in Hong Kong during the time of the changeover in sovereignty from British to Chinese rule which took place in July 1997. The investigation takes as its focus a study of the opinions, orientations, teaching approaches and practices of a cohort of students on the Bachelor of Arts in Teaching English as a Second Language (BATESL) course at the City University of Hong Kong over a five-year period as they passed from schoolleavers entering the course in September 1994, to graduates from it in 1997 and practising teachers of English in Hong Kong secondary schools in 1998/99. The research evolved from the researcher's previous studies of the orientations to teaching of students on the BATESL course (Pennington \& Urmston, 1998; Urmston, 1994; Urmston, Pennington \& Brock, 1996) and from the work that the researcher did with the BATESL students on a funded research project (led by Professor Martha C. Pennington and Professor Jack C. Richards) on which the present researcher served as research assistant (Pennington \& Richards, 1993; Pennington, Richards, Urmston, \& Lee, 1996; Richards \& Pennington, 1995) ${ }^{6}$

In addition to the studies of the BATESL student teachers, the context of the education system in Hong Kong and the teaching of English there is examined in terms of the historical background of English use in society, English teaching and English teacher preparation. Consideration is also made of the social structure of Hong Kong society and its political culture at the time of the handover. A framework is then developed based the Innovation-Diffusion model of change, especially as applied to educational contexts by Rogers, Pennington, Henrichsen and others; the ethno-cognitive model of language teachers' decision-making of Woods; and prior applications and development of these models in the context of English teaching in Hong Kong, particularly in the work of Pennington and others at the City University of Hong Kong. The framework incorporates as well 
considerations of the history and politics of English language teaching in a colonial and postcolonial context. This framework provides a theoretical foundation with which to analyse the changes taking place in English language teaching and in English language teachers in Hong Kong during this significant period in its history.

The purpose of the study is to develop an understanding of the change processes at work at the time of the changeover in sovereignty and how they impacted on education and English language teaching. From this understanding a model of educational change is developed to represent the situation in Hong Kong both prior to and following this major socio-political change. This model will serve as an update of the English language education situation in the SAR as well as a representation of systemic change in postcolonial societies.

\subsubsection{Research Questions}

The research question guiding the investigation is the following:

What are the effects in terms of teacher change at an individual level and educational change at a system level of the changeover in sovereignty in Hong Kong occurring in July 1997 ?

The focus of this question is on the teaching of English in a former British colony as it is transferred back to local rule. The assumption is that there are many issues and complexities concerning the educational status of the international and formerly colonial language in a period of increased attention to local identity, heightened regionalism, retrenchment from western ideas, and conservatism. It is further assumed that the answer to this question can extend the largely Western literature on educational change and enrich the database as well as the social theory of educational change by attention to (1) cognitive theory of teacher change and (2) the politics of English language around the world. 
In answering this question, it is necessary to answer a number of subsidiary questions:

(i) Is the situation vis-à-vis English teaching and learning in Hong Kong continuing in the direction predicted by published studies in the 1980s and early 1990s? Is it continuing to evolve towards 'Western' philosophies and methodologies (involving communicative language teaching, process writing, and other interpretive, learner-centered educational modes), or is it becoming more localised, regionalised, conservative, and less 'Western' and interpretive in orientation?

(ii) What were the key issues connected to English language teaching as reported in the local English language newspapers during the period immediately before and immediately after the changeover, and was there any change in emphasis on the different issues over this period?

(iii) How were the changing needs and perceptions of English teaching in Hong Kong addressed by prominent English language educators and representatives of the organisations charged with English language teaching provision before and after the changeover?

(iv) What were the opinions of teachers-in-training due to enter the teaching profession in 1997 as regards teaching English in Hong Kong, and how had these opinions changed during the run-up to the changeover, since they began their teacher training course in 1994 ?

(v) What evidence was there of changes to the beliefs, assumptions, knowledge and practices of novice teachers of English in Hong Kong as a result of their teaching experience post-graduation and of the changeover?

(vi) What insights are gained from the Hong Kong case for the theory and practice of English language teaching, and educational and social change?

(vii) How did the changeover in sovereignty affect the main languages used in Hong Kong, i.e., Cantonese, English and Putonghua? 


\subsubsection{Methodologies}

In order to answer these questions, a variety of research procedures were employed. The chapters of the thesis reporting on the empirical studies that comprise the investigation are Chapters 3,4 and 5 and detailed descriptions of the methodologies used can be found in each chapter. The following is a summary of the methods used in order of the relative weight of each and their contributions to the investigation as a whole.

\section{Questionnaires}

A questionnaire, developed for use in the First Year Teacher Project, was modified and administered to a cohort of students entering the BATESL course at the City University of Hong Kong in September 1994. The questionnaire, with additional questions concerning the changeover in sovereignty, was administered to the same cohort of BATESL students as they were about to graduate from the course in May 1997. The responses from the two administrations were compared to determine changes in teaching orientations of the cohort over the three-year period. In addition, the responses from an earlier administration of the questionnaire, which was done by the cohort of BATESL students who graduated in June 1994 and that formed the basis for the earlier studies (Pennington \& Urmston, 1998; Urmston, 1994; Urmston, Pennington \& Brock, 1996), served as a third source of data and enabled comparisons to be made across cohorts and across time periods. Comparisons were carried out quantitatively (using descriptive and non-parametric statistics) and qualitatively (using respondents' written comments to add perspective to the quantitative data). (Chapter 4) 
A large number of interviews were carried out throughout the investigation and contribute to each of the chapters of findings.

Firstly, to help to qualify the questionnaire responses of the BATESL students, follow-up interviews were held with sample groups of the 1997 and 1994 graduating students a few days following each administration of the questionnaire. In the interviews, each student was asked to elaborate on his/her responses so as to garner a more global perspective of each cohort's orientations and to ensure that the items on the questionnaire itself were correctly interpreted. (Chapter 4)

Secondly, interviews were carried out with a small sample (five) of the 1997 BATESL graduates in June 1998 to ascertain if their orientations had changed since graduation. In these interviews (carried out in two groups of two and three graduates) the graduates were given summarised results from the 1997 questionnaire administration and asked to comment on them. (Chapter 5)

In another strand of the investigation, interviews were carried out with a number of English language practitioners to glean their views on the major issues affecting ELT. Two rounds of interviews were carried out, in May-August 1997 and June-October 1999. In both rounds of interviews there were twenty-one practitioners. Nine of the practitioners took part in both rounds of interviews. The interviewees were sent a list of questions to consider prior to the interviews and these questions formed the basis for the interviews. (Chapter 3)

All interviews were audio recorded, transcribed and the transcriptions analysed for content using the constant comparative method (Strauss, 1987). 


\section{Lesson Observations}

Lesson observations were carried out of five English teachers in secondary schools in May/June 1999. Three members of the 1997 BATESL graduating cohort and two members of the 1994 graduating cohort were observed twice. Each observation was followed by a teacher-researcher conference in which the teacher was asked to reflect on the lesson just taught and on their general teaching situation. The reports of the observations were written up and the information from them used as the basis of a discussion of the teaching practices of BATESL graduates in post-handover Hong Kong. (Chapter 5)

\section{Newspaper Survey}

A record was taken of the issues related to English language education that appeared in the English language newspapers (i.e., in reports, editorials, columns or letters) on each Monday during 1997. In addition, a database of all news items from the English press from 1997 onwards was collected. (Chapter 3)

\subsubsection{Overview of Thesis}

This chapter has served as the introduction to the thesis. In the next chapter, Chapter 2, the thesis is framed though a review of the literature and a discussion of the context of English language education in Hong Kong at the time of the changeover in sovereignty. In so doing, research question (i) will be addressed. Theories of change in sociological and educational contexts are discussed and applied to the Hong Kong case. The empirical studies that constitute the investigation are foregrounded through a review of current thinking on English language teaching and teacher education. Finally, a framework for teacher and educational change is developed and models of educational change considered in 
anticipation of the development of a new model based on the findings from the investigation to be presented in Chapter 6.

Chapter 3 presents the context of English language education in Hong Kong at the time of the handover and the period immediately after it through a review of the dominant issues at large at that time and attempts to answer research questions (ii) and (iii). This is done through a review of the English language newspapers during 1997 and an examination of the opinions of English language practitioners in 1997 and 1999.

Chapter 4 describes in depth the questionnaire and interview surveys of the students on the BATESL course carried out between 1994 and 1997. Through these surveys, answers to research question (iv) are sought and English language teaching in Hong Kong is characterised from the perspective of students (as they were at the beginning of the BATESL course) and teachers (as they were at the end of it).

Chapter 5 serves as a post-handover view of the English language teaching context in Hong Kong though the views of BATESL graduates, the teaching practices of novice and more experienced English teachers, and the perspectives of teacher educators. This chapter addresses research question (v).

Chapter 6 concludes the investigation by summarising the main findings and synthesising these with the other studies reviewed in Chapter 2 towards the development of a new model of change in the educational context as it applies to English language education in Hong Kong. Research questions (vi) and (vii) are addressed in this final chapter.

In Chapter 6, the main points to emerge from the investigation are presented and discussed. These include major themes of conflict in English language education, including conflict in teachers, in schools and in the education system that provides English language learning and teaching. In addition, there emerges evidence of 
changing structures of the sub-systems and changing relationships between the sub-systems that comprise the education system and changing degrees of influence or power of certain sub-systems or possibly agents within them. The extent to which the English language education system represents the wider society is discussed in relation to issues of political change and postcolonialism. The thesis is concluded with descriptions of the ways that societies such as Hong Kong are seen to deal with such change. 


\section{Notes}

${ }^{1}$ In this thesis, the People's Republic of China will be referred to variously as Mainland China, the Mainland, the PRC or China.

${ }^{2}$ From 20 January 1841 until 30 June 1997, Hong Kong was a colony and then a dependent territory of Great Britain. On 1 July 1997, it became the Hong Kong Special Administrative Region of the People's Republic of China (HKSAR). In this thesis, Hong Kong will be referred to either by its pre-1997 abridged title, the Territory, its post-1997 abbreviation, the $S A R$, or simply by name, whichever is appropriate.

${ }^{3}$ Throughout this thesis, Hong Kong's reversion to Chinese rule will be referred to either as the changeover in sovereignty, the handover or the transition.

${ }^{4}$ See for example Kachru, B. (1983). The Indianisation of English: The English language in India: New Delhi and New York, Oxford University Press.

5 See for example Pennycook, A. (1998). English and the discourses of colonialism. London: Routledge

${ }^{6}$ The project followed five graduates from the BATESL course in their first year in the classroom in 1994-95 and will henceforth be referred to as the First Year Teacher Project. 


\section{FRAMING THE INVESTIGATION}

\subsection{Background}

At the time of the changeover in sovereignty, Hong Kong had been in a pattern of change that had been ongoing since the end of the Second World War. After the Japanese occupation, Hong Kong reverted back to being a British colony and the economy began to recover. The massive outflow of population that had occurred during the war reversed and the population grew dramatically, from an estimated 600,000 in 1945 to about 1.9 million in 1949 and about 2.5 million in 1955 (Sweeting, 1998). The vast majority of these new immigrants came from over the border in China and consisted of mainly young people, resulting in a 'baby boom' in the 1950 s and ' 60 s and a ready supply of labour. In addition, the flight of factory owners and other entrepreneurs from Shanghai and other Mainland cities as a result of the Chinese Revolution led to Hong Kong becoming a major manufacturing centre (Sweeting, 1998). The population increased again during the 1960 s, due to the influx of people fleeing the Cultural Revolution in China, to about 4.3 million in 1974 and 5.8 million in 1992, whilst the economy diversified into financial and commercial sectors as the Mainland took over as the manufacturing centre (Sweeting, 1998). These major changes in population and demography resulted in concurrent changes in the provision of education as the primary, secondary and then tertiary sectors were expanded to cope with the increasing population and the changing needs of society.

After the signing of the Joint Declaration between Britain and China in 1984, thereby ensuring that Hong Kong would be returned to Chinese sovereignty on 1 July 1997, the relative stability of society was disturbed and the Territory began to experience a heightened period of sociological change. The added factors of a 
downturn in the economy caused by external factors in the 1990s and political 'events' such as the Tiananmen Square 'incident' in 1989 had significant effects on the sociological framework of society. At the same time, perceived needs to reform the education sector in response to changes in society and in popular educational thinking led to major changes in this sector also.

In this chapter, theories of sociological, political and educational change are described and discussed with a view to developing a theoretical framework which can be used to put into perspective the changes in education which occurred during the period leading up to and at the time of the handover, specifically as they impinged on English language and the teaching and learning of it. The first two sections look at the educational and language contexts of Hong Kong to provide background for the discussions of change and the empirical studies to follow in subsequent chapters. In addition, this and the following chapters will address research question (i):

Is the situation vis-à-vis English teaching and learning in Hong Kong continuing in the direction predicted by published studies in the 1980s and early 1990s? Is it continuing to evolve towards 'Western' philosophies and methodologies (involving communicative language teaching, process writing, and other interpretive, learner-centered educational modes), or is it becoming more localised, regionalised, conservative, and less 'Western' and interpretive in orientation? 


\subsection{The Educational Situation in Hong Kong (1994-1999)}

\subsubsection{The Hong Kong Educational Context}

The educational context in Hong Kong has been described by Pennington (1995c) as one in which teachers:

generally work under conditions of low autonomy, with little influence over strategic decisions, few opportunities for collaboration with colleagues and indeed little emphasis on collegiality; minimal positive feedback or work incentives such as promotions or societal recognition; and generally poor resources in the way of an orderly environment, administrative support, adequate physical conditions, instructional resources, and reasonable workloads. (p. 708)

Hong Kong teachers generally have to cope with large classes and a lack of encouragement to experiment with innovative teaching methods (Pennington, 1995c). They teach under an extremely heavy schedule and paper-marking load, and they must cover a required syllabus densely packed with linguistic content knowledge and with numerous assignments expected to be completed each term. Hong Kong is regarded as having an educational culture which is transmissive, teacher-centered, and examination-based (Pennington, 1995c; Young \& Lee, 1987); and traditional, Chinese principles of control and respect for authority still dominate (Pennington \& Cheung, 1995; So, 1992). The result is that teachers suffer from low work satisfaction and commitment and indulge in minimal innovative behavior (Pennington, 1996).

The transmission-interpretation dichotomy of teaching, which was first developed by Barnes and Shemilt (1974) in their study of teachers' approaches to the teaching of writing, can be applied to the culture of language teaching in a community such as Hong Kong that exemplifies a tension between traditional grammar-based and modern communication-based approaches. The beliefs about knowledge and the approach to teaching and learning of teachers who fall into these two categories were summarised by Barnes and Shemilt as follows: 
(1) believes knowledge to exist in the form of public disciplines which include content and criteria of performance;

(1) believes knowledge to exist in the knower's ability to organize thought and action:

(2) values the learners' performances insofar as they conform to the criteria of the discipline;

(3) perceives the teacher's task to be the evaluation and correction of the learner's performance, according to criteria of which he is the guardian;

(2) values the learner's commitment to interpreting reality, so that criteria rise as much from the learner as from the teacher;

(3) perceives the teacher's task to be the setting up of a dialogue in which the learner can reshape his knowledge through interaction with others;

(4) perceives the learner as an uninformed acolyte for whom access to knowledge will be difficult since he must qualify himself through tests of appropriate performance.

(4) perceives the learner as already possessing systematic and relevant knowledge, and the means of reshaping that knowledge.

(Barnes and Shemilt, 1974, p. 166)

The educational culture in Hong Kong is and has been predominantly based on transmissive beliefs (Pennington, 1995c; Pennington \& Cheung, 1995; So, 1992; Young \& Lee, 1987). The nature and history of Hong Kong point to it being 'an intensely competitive, materialistic, results-oriented society, where personal status is to a great extent measured by the wealth derived from a successful career in government or business' (Evans, 1996, p. 36). Thus, the achievement of high grades in public examinations is paramount. This examination pressure has resulted in a strong orientation to the sorts of discrete-item knowledge of language - primarily grammar and vocabulary - which is tested in traditional normreferenced, standardised tests.

Whether this educational culture is changing is a matter for debate. Pennington et al. (1996), in their examination of English teachers' beliefs, values and teaching 
practices with regard to the process approach to writing in the Asia-Pacific region, found that in Hong Kong there is a significant gap between teachers' view of their ideal and actual teaching situations. The results indicated that teachers of English in Hong Kong were becoming more inclined towards a process approach, indicating a more interpretive or liberal ideology, a finding supported by Lockhart (1996) in another study of Hong Kong English teachers. However, Pennington et al. (1996) found that these changes in belief were not reflected in the teachers' actual teaching practices, which still tended to be transmissive and conservative. The findings of a project which studied the changes in teaching of a group of firstyear English teachers in 1994/5 (Pennington \& Richards, 1997; Pennington, Richards, Urmston, \& Lee, 1996) support this finding and suggest that teachers have a mixture of transmissive and interpretive teaching philosophies when they start out, which soon become highly transmissive to meet the realities of their teaching context. The study also found conflict between teachers' ideal and actual practice, as they continued to express their desire for a less conservative and constrained teaching culture that would allow them the space to innovate in their classrooms, such as by applying principles of communicative and learner-centered language teaching.

\subsubsection{English Language Learning and Teaching in Hong Kong Schools}

The history of English language teaching in Hong Kong schools has been described by So (1992), drawing on terminology from chaos theory, as one of 'bifurcation' into English-medium or Chinese-medium. So (1992) attributes this bifurcation to:

three sets of historical circumstances ... the cultural backwardness and foreign encroachment of Modern China, as well as her effort to strengthen herself; ... the colonization of Hong Kong, and the needs of the local colonial administration to cultivate a class of bilingual brokers to mediate between itself and the governed; and ... the Chinese Nationalist Party's political platforms on modem education and the Overseas Chinese. (p. 71) 
Before the Second World War, English-medium secondary schools dominated as the colonial government strove to cultivate a 'Westernized and English-speaking elite among the local population' (So, 1992, p. 71). This emphasis on the teaching of English did not, however, seem to produce the high standards that the government was looking for, as British education inspector Burney concluded when he visited the colony in the 1930s. Half a century later in 1982, exemplifying the continuing discourses associated with language in the Territory, the Llewellan Report again remarked that standards in both Chinese and English had to be improved and recommended a bilingual programme by the end of Form 3 (Grade/Year 9), with increasing emphasis on English in the upper forms in preparation for higher education (Lord, 1987).

Yet as Morris (1995) points out 'As secondary schooling is now effectively provided for all pupils (and has been since 1979), its curriculum has become increasingly inappropriate for the academically less able and those who are going directly from school into the labour market' (p. 125). While expectations as to the standard of English that pupils should aspire to have risen, the numbers and abilities of pupils have changed substantially over the last few decades, resulting in the popular opinion, often voiced in letters to the editor in local newspapers, that standards of English are falling. Consequently, efforts have been made to improve the situation over the last decade and a half through recommendations made in the Hong Kong Education Commission Reports 1, 2, 4, 5 and 6 (Hong Kong Education Commission, 1984, 1986, 1990, 1992, 1996) ${ }^{1}$ and carried out by the Education Department. These include:

- The employment of expatriate, native-speaking teachers (NETs) who, it is hoped, will bring with them modern, innovative language teaching methodologies. $^{2}$

- The move towards a fully-qualified local English teacher workforce through the phasing out of non-graduate teaching posts and the introduction of the Language Proficiency Assessment for Teachers (LPAT). ${ }^{3}$ 
- Changes in the curriculum of English by the Education Department's Curriculum Development Council to reflect modern developments in TESL, such as communicative language teaching and task-based and target-oriented teaching. ${ }^{4}$

- Provisions for more teachers with the aim of reducing class sizes, particularly for students with learning difficulties. ${ }^{5}$

Added to the concerns over falling English standards has been the view that standards of Chinese (spoken Cantonese and written Modern Standard Chinese) are also falling, with increasing calls for Putonghua (or Mandarin Chinese) to be introduced as a school subject in the run-up to the changeover in sovereignty. In respect of the perceived drop in the standards of Chinese amongst school students, there have been calls from teachers and government officials for an increase in the amount of Chinese language education (Johnson, 1998). The Education Commission in its Report No. 6 recommended an increased focus on the teaching of Chinese, including more provision for the teaching of Putonghua (Hong Kong Education Commission, 1995).

During the late 1990s, while parents and students pushed to maintain an English language teaching culture, many in education advocated mother tongue (Chinese) instruction (Evans et al., 1998; Tung et al., 1997), a position which was consistent with the return to Chinese rule. According to Tung (1990), prior to 1984 and the release of Education Commission Report No.1 (Hong Kong Education Commission, 1984), the Education Department had adopted a laissez faire policy towards the medium of instruction, allowing schools to teach through whatever medium they chose. This resulted in the majority of secondary schools using English as it was considered to be the language of status with the desired linguistic capital as the language of higher education and of intermational commerce (So, 1992). For this reason, schools would attract the best students as well as winning approval from parents by being English medium (Tung et al., 1997). However, after 1984, the Education Department made concerted efforts to encourage schools to adopt Chinese as the medium of instruction (Johnson, 1998; 
Tung, 1990). This culminated in the issuing of the Arrangements for Firm Guidance on Secondary Schools ' Medium of Instruction consultation document in March 1997, in which clear instructions were set out for the adoption by the majority of schools of Chinese-medium instruction starting from September 1998 (Hong Kong Education Department, 1997).

The Education Department decided to act in order to arrest what they considered to be the increasing and educationally inefficient use of mixed-code in schools (Hong Kong Education Commission, 1990). The English language education system was and is changing and evolving towards a greater acceptance of Chinese in English-medium schools (Boyle, 1997a). While a reality of bilingual teaching obtains in some subjects, including English (Johnson, 1998; Pennington, 1995c; Pennington et al., 1996; Pennington \& Richards, 1997; So, 1992; Tung, 1990), in maths and science, teaching has for some time been essentially in Cantonese, with many technical terms from English mixed in (Johnson, 1991). In addition, textbooks and other teaching materials and, most importantly for most students, examinations, ${ }^{6}$ are still generally in English. This conflict of usage and importance between the two languages is a familiar scenario in Hong Kong schools. Research has shown that students in Hong Kong have for a long time appreciated the instrumental value of English to their futures (Axler et al., 1998; Pennington \& Yue, 1994; Pierson et al., 1980). Prior to the handover there may have existed feelings of resentment towards the English language as a symbol of colonial domination (Pennington, 1996) or simply as a medium through which they were forced to learn (Pennycook, 1998). Since the handover, with the colonial power departed, and even before the handover (Pennington \& Yue, 1994) those feelings may have dissipated to be replaced by an ambivalence as regards any sociopolitical connotations the language may have once held (Axler et al., 1998; Li, 1999). 


\subsubsection{The Training of Teachers of English in Hong Kong}

Bickley (1987) reviewed the development of English language teaching and English language teacher education in Hong Kong, concluding that 'general teacher training is not sufficient for English language teachers and ... some kind of initial specific training, reinforced by further training at a later stage, is essential' (p. 187). In the past, a reasonable proficiency in the language was considered a sufficient qualification for teaching English, as specialist training in English language teaching was only available in the teacher training colleges for full-time students of the Certificate in Education course or for graduates on the full-time Diploma in Education course at the University of Hong Kong. ${ }^{7}$ In the last few years, this situation has changed noticeably. Expansions in the tertiary education sector as a result of substantial government investment have led to an increase in the provision of English language teacher training courses.

There would appear to be a need for this increased provision for professional training of English language teachers; in 1993, of the cohort of 3700 English teachers, it was found that only 14.2 per cent were both subject and professionally trained (Falvey \& Coniam, 1997). The instigation of the LPAT scheme for teachers of English (and of Putonghua) is evidence of the government's determination to be seen to be paying attention to the quality of language teaching. Added to this is the move towards an all-graduate teaching profession (as announced by Hong Kong's head of state, the Chief Executive, Tung Cheehwa in his annual Policy Address in November 1998) within which people will not be able to teach in schools whilst holding a Certificate of Education only, and so the post of Certificated Master/Mistresses (CM) will gradually be phased out. 


\subsection{The Language Situation in Hong Kong (1994-1999)}

Education, like every aspect of life in the SAR, has been shaped by the frequently conflicting influences of Britain and the People's Republic of China (PRC), and the issue of language use and language teaching has been pivotal. The vast majority of the population (approximately 95 per cent) is Chinese and speaks Cantonese, the linguistic variety of the southern Chinese province of Guangdong, as a first language $(\mathrm{Li}, 1999)$. English has been considered the prestige or status language and has been used in government, the law courts, and as the main means of communication in international trade and commerce, upon which Hong Kong has built its prosperity (Fu, 1987; Lord, 1987). It has also generally been considered a language of wider access and upward mobility (So, 1992).

An argument can be made that English language teaching has served the purposes of British colonialism in Hong Kong. Not only have institutions such as the British Council thrived there, but government policy served to create a huge market for teachers, textbooks, examinations and other features of language learning (Pennycook, 1994). This situation has continued up to the present day. However, after the signing of the Joint Declaration in 1984, which paved the way for the reunification of Hong Kong with the PRC, it became clear that Cantonese was growing in importance (Pierson, 1998). In addition, Putonghua, the spoken form of Chinese officially sanctioned by the PRC, and its written equivalent, Modern Standard Chinese, which functions as the standard written form of all varieties of Chinese, also began to increase in prominence ( $\mathrm{Fu}, 1987$; Lord, 1987).

Although Chinese has been an official language in Hong Kong since 1974, it had until recently lagged behind English, particularly in education and in written correspondence within government and commercial organisations (Pierson, 1998). The ambiguous nature of the term 'Chinese' and the linguistic mix of the local population has contributed to this situation. Although most Chinese people in Hong Kong speak Cantonese, the term 'Chinese' could also refer to Putonghua, other dialects or varieties of spoken Chinese, and Standard Written Chinese 
(SWC). In fact, in terms of status, the linguistic variety commonly referred to as Chinese is generally considered to be based on Putonghua and SWC. BrucheSchulz (1997) makes the point that:

Although Cantonese is used at home as well in government, legal, church, and cultural, especially entertainment-related domains as the medium of oral communication, the common perception of Cantonese is one of not being the 'real' Chinese. (p. 296)

Cantonese is the spoken lingua-franca of the population in Hong Kong and since the transition to Chinese rule it has become the language used in the Legislative Council and in most meetings in government; is growing in use in the law courts (dependant upon the choice of the judge or magistrate involved); and in the media, i.e., radio (Pennington, Lau \& Chan, 1998), film and television (Li, 1999). Cantonese is not, however, accepted as a written form in legal, governmental or educational domains, although there is evidence of growing usage of written forms of Cantonese in popular newspapers and magazines (Bruche-Schulz, 1997; $\mathrm{Li}, 1998$ ). What remains to be seen is whether written forms of Cantonese will eventually become acceptable in the 'high' functions mentioned above (Luke, 1998).

Under British rule, English was thought of as the status language. However, as China has become more 'open' with regard to economic and political links with Hong Kong, Chinese, that is, Putonghua and Standard Written Chinese, have increased in importance for government functions, as Hong Kong government officials now have to deal regularly with their counterparts from Beijing. Added to this has been the increase in what Pierson calls 'Cantonese ethnicity', which has led to an increase in the status of Cantonese (Pennington and Balla, 1998; Pierson, 1998). Consequently, it can be said that the language balance in Hong Kong has shifted, with 'Chinese' rising in status, possibly with English falling (Flowerdew \& Scollon, 1997; Kwo, 1987; Pennington, 1994b; Pennington \& Yue, 1994). A quote from a local English language newspaper discussing a proposal to increase the amount of Chinese-language programming on local English-language television highlighted this shift in importance: 
There has recently been in Hong Kong society an increase in the importance and popularity of Chinese language, a reduced emphasis on English and an attendant shrinking of the advertising market for English-language media.

(South China Morning Post, 16 October 1998a)

Just what will the future status of English be in postcolonial Hong Kong? Comparisons with Hong Kong's South-East Asian neighbour Singapore are inevitable. Singapore gained independence from Britain in 1959 and English has retained a high status there as it is seen as a neutral form of access to science and technology. However, during the early period of its post-colonial development, the Singaporean government considered that English could be a possible vehicle for decadent Western values, so Putonghua was pushed in a desire to promulgate Confucian ideals of 'loyalty, conformity and family values' (Pennycook, 1994, p. 251). In addition, Putonghua was considered to be the desired form of 'high' Chinese that the ethnic Chinese people in Singapore should adopt as lingua franca, at the expense of the native varieties used such as Teochew (Chiuchow), Cantonese and Hokkien (Fukien) (Tan, 1997). The result has been that English and Putonghua dominate public life, including education, even though both Malay and Tamil are also designated 'official' languages. Although Hong Kong has not gained independence, and its linguistic mix is rather different from that of its South-East Asian neighbour, comparisons can be drawn in terms of language hegemony. It seems likely that in Hong Kong, as in Singapore, albeit for different reasons, Putonghua will grow in importance, as the language of recolonisation, ${ }^{8}$ to be added to English, the language of colonisation (Law, 1997). For a long time, China could be said to have been in a 'muted' condition and Britain 'dominant' as influences on Hong Kong (and hence its language); since the handover, there has been a 'flip' in which 'muted' and 'dominant' influences have been reversed (Pennington, personal communication). ${ }^{9}$

Pennycook (1998), in his discussion of colonial discourses, makes strong arguments for the continuing effects of colonialism on language policy: 
Although it cannot be said to have been government policy to promote English as widely as possible in Hong Kong and thus that the government has immediate responsibility for the current problems in the education system, ${ }^{10}$ it nevertheless seems to be the case that the sources of the problem can be found in the constant micropolitics of colonial governance, in the support for an elitist school system and an English-language university, in the refusal to acknowledge degrees from Chinese universities, in the constant use of English-speaking advisors and 'experts'. Coupled with the development of a highly egalitarian society with English as the decisive distributor of possible social and economic advancement, the apparently laissez-faire policies of the colonial decision-makers in Hong Kong can be seen to have far more responsibility for the state of affairs than they admit. The dismantling of some of these colonial structures is underway, and yet the continuing reproduction of colonial discourses through English suggests these changes may not effect much change. (p. 200)

From the observations of Pennycook (1998), it can be seen that language policy is inextricably linked to social policy, to the socio-political structure of society and to socio-political change within society (Rassool, 1998). The effects of colonialism on language policy, language use and language rights in Hong Kong are tied in with the notions of linguistic imperialism (Phillipson, 1992, 1998) and English as the global language of power (Fairclough, 1989), and with the notion of linguistic capital, developed from Bourdieu's (1997) ideas on cultural capital (Morrison \& Lui, 2000).

Linguistic imperialism, or the use of language for economic and political domination (Morrison \& Lui, 2000), is a theory that has been applied to the Hong Kong case, at least the colonial Hong Kong case. As Pennycook (1998) points out (above), there is little evidence to suggest that the colonial government purposely used English to dominate, although through its policies as regards government, education and the law, it could be argued that this was the result. Legislators were forced to debate in what for most of them was their second language. Children were forced to learn in schools in what for most of them could be said to be a foreign language. And people unfortunate enough to find themselves up before a court of law had to sit through the proceedings conducted in a language of which 
at best they might have had a rudimentary knowledge and so had to rely on translations and the potential problems associated with them.

English has long been promoted in Hong Kong as the international language of business, which is crucial to Hong Kong in its role as an erstwhile entrepôt and future 'bridgehead for international consortiums to do business with mainland China' (Li, 1999, p. 88). In addition, English has been and still is regarded as being of high instrumental value to the citizenry (Axler et al., 1998; Pennington, 1994b; Pennington \& Yue, 1994) and the means by which Hong Kong people can move up the social ladder (Li, 1999; Pennington \& Yue, 1994). However, as Boyle (1997b) remarks, 'English, under the innocuous guise of a helpful language for business and travel [became] a potent weapon for cultural and economic domination' (p. 169). Whether the Chinese people of Hong Kong have always wanted English as Boyle (1997b) puts it, or they have been pushed into the situation whereby they have had no choice but to pursue and live with it is open for debate.

Whilst linguistic imperialism can go some way towards describing the language situation in Hong Kong pre-handover, it is the issues of language rights and linguistic capital that have been achieving more attention from observers in the field. Bourdieu (1997), in his discussion of the forms of capital which enable people to 'appropriate social energy' (p. 46) or gain life advantages over others, argues that in educational terms some groups in society possess cultural capital in the form of attitudes to schooling, social advantage, parental support and linguistic facility and these advantages enable them to achieve greater educational success than others in less advantaged situations. In this way, social stratification occurs and is promulgated (Morrison \& Lui, 2000) as those children with the cultural capital, frequently linked to economic and social capital, will take the most advantage of education and pass on the advantage to their offspring. Conversely, linguistic capital, or fluency in and comfort with a high-status, world-wide language such as English, is held by those who have the cultural, social and political capital. In Hong Kong, the English language has been considered a 
desirable and achievable form of capital with the result being that parents have endeavoured to send their children to English-medium schools in the belief that they will acquire the linguistic capital necessary to accumulate other forms of capital for the family.

Lin (1997) poses the question as to whether there are equal opportunities of access to English for the children of disadvantaged monolingual (Chinese) social groups who have little English linguistic and cultural capital compared with the children of bilingual, middle and professional classes, who have more English resources. In other words, there are issues of language rights to be addressed. Lin (1997) goes on to say:

A schooling and education funding system founded on the principle of ignoring the children's varying amounts of initial linguistic and cultural capital contributes to the reproduction of existing social stratification and the lack of social mobility of children from disadvantaged groups. (p. 25)

Lin's (1997) point of view is that Hong Kong people have been victims of the colonial government's policies leading to symbolic domination of English over Chinese. Countering this argument is Boyle (1997b), who describes Hong Kong people as pragmatic enough to decide for themselves the value of English for social and economic advancement (summarised by $\mathrm{Li}, 1999$ ). The medium of instruction issue has been central to the debate over language rights and linguistic capital in Hong Kong. Prior to the handover, the majority of schools offered a nominally English-medium education and parents were relatively free to choose which school to send their children to in the (sometimes mistaken) belief that through immersion they would emerge from their schooling with sufficient English capital to make a decent life for themselves. Since the changeover in sovereignty and the change in the medium of instruction of most secondary schools from English to Chinese, there has been, according to Lin (1997), an increase in social division as an English-medium education and hence access to the desired linguistic capital is now even farther from the reach of the socially disadvantaged in society. In opposition to this view is that held by Benson (1997) 
and others that the move to Chinese-medium schooling for the Cantonesespeaking majority means that their language rights are being upheld in allowing them to be educated through their mother tongue.

Like the methods employed to teach languages, the roles, functions and relative positions of the languages in society are susceptible to the forces of change and the diffusion of innovations perspective (to be discussed in later sections). As such, the analysis of the changes in these areas of language and education will contribute to and complement the understanding of the societal changes underway during the period. The next section looks more widely at how changes in society, particularly as a result of or at the time of the changeover in sovereignty may have affected the teaching of language in Hong Kong.

\subsection{Hong Kong Society during the Period Leading up to and after the Changeover in Sovereignty (1994-1999)}

The following section aims to describe the social structure of Hong Kong with reference to established constructs and theories in social science and then considers the impact that the changeover might have had on the SAR in terms of social, political and educational change. Against this background, attempts are made to describe patterns in the progression of reforms in education during the period encapsulating the changeover. It is shown how educational reforms, which can be characterised as innovations, have come under the influence of numerous factors determining the relative speed and success of their implementation (or non-implementation). Innovation processes are described in terms of general patterns of change, both regular and irregular, which can elucidate the specific changes documented in English language education which occurred during this period and subsequent to it. Given that change is a natural and regular feature of any social system or society, the changeover can be viewed as an added factor in the change processes which were already occurring in Hong Kong during the changeover period. The impact of this significant new factor is considered on the 
patterns of change. These change patterns are then used to describe the changes in the education system with a view to gaining a deeper understanding of how societies in transition accommodate and react to change, with particular reference to educational and linguistic change.

\subsubsection{Social Structure and Social Change}

As background for a discussion of how, and possibly why, Hong Kong society may have changed as an effect of the handover of the colony back to China, it is useful to look at theories of how societies are structured and how they behave, and then to consider how these theories, or certain features of them, might be applied to the case of Hong Kong. The field of social change is a vast one and it is not the intention here to review all theories, from either an historical or epistemological point of view. Rather, a number of theories and perspectives are considered to provide a broad framework within which the social changes affecting one society, Hong Kong, in one period, the mid to late 1990s, can be discussed.

Firstly, a number of descriptions of society are based on the inequalities between individuals and groups of individuals within society, what is termed social stratification. One definition of social stratification is given by Ho (1994) as:

... the persistent existence of social groups whereby one group is ranked above the other group, usually in terms of the amount of material resources and or immaterial resources it possesses. (p. 2.8)

As Ho (1994) remarks, few, if any, societies can truly be termed egalitarian, in which people enjoy equal status, wealth and power, and the relationships between the different social strata are an important determinant of how a society functions and how it changes.

As reviewed by Harper (1993), there are three major contemporary theories or perspectives of social change, each of which is based on an assumption of social 
stratification, namely: structural functionalist theory, conflict theory and interpretive theory. Each of these theories will be briefly reviewed in turn, and each considered in terms of their applicability to the Hong Kong context.

\subsubsection{Structural Functionalist Theory}

Structural functionalism, as developed by sociologists such as Durkheim (e.g., Durkheim, 1947) and Parsons (e.g., Parsons, 1951), holds that society exists in a state of equilibrium as a result of social systems acting to perpetuate the survival and prosperity of the whole system (Harper, 1993; Viswanath \& Demers, 1999). These social systems, such as government, education, religion, the economy and the family, and the interrelations among them, make up the structure of society. As defined by Vago (1996), 'structure generally refers to a set of relatively stable and patterned relationships of social units' (p. 55). Functions are the necessary activities and operations or 'the dynamic processes that make societies work' (Vago, 1996, p. 55). These functions can be either mandated, intended (de jure) functions, or they can be latent or unintended (de facto) functions. As an example, the intended function (de jure) of making the majority of secondary schools in Hong Kong adopt the first language of the vast majority of the population, Cantonese, as the teaching medium was to facilitate ease of understanding and expression of ideas in the local student population, as opposed to the difficulties they were perceived to have in trying to learn through the second language, English. An unintended (de facto) function of this policy has been to polarise schools into the more desirable - from the point of view of parents - minority of English-medium schools and the majority Chinese-medium, due to the perceived socio-economic benefits of an English-medium education. This is an example of a function causing 'strains' in the structure of society and resulting in adjustments being made to maintain equilibrium or stability. Both the original source of strain as well as the adjustments made to counter the strain are typically effected by government in the form of structural innovations, of which more will be discussed later. 
The structure or patterns of interaction between social systems in a given society are heavily influenced by the common value system or culture of that society. Within the common value system, individuals have certain role expectations, the conformity to which is regarded as a moral obligation, assuming that the ultimate goal is the stability of the particular social system to which they belong and the society more generally (Parsons, 1951). What Parsons terms the common value patterns which an individual adopts and which guide his or her actions are generally learned or acquired in order to survive within the society and avoid negative sanctions. Such sanctions could be in the form of state-administered punishment or perhaps expulsion from the social system or group to which the individual belongs. Individuals who perform well within the common value system of the society will tend to be successful and so will rise to positions of influence and be rewarded accordingly. People are encouraged to work hard to increase their social position and individual resources (Harper, 1993). As a result, society consists of individuals with clearly defined role expectations based on their abilities to perform their allotted functions. That society is stratified is considered a natural and desirable phenomenon that maintains the status quo and the continued prosperity and well-being of the whole.

Aspects of the structural functionalist theory are relevant to the Hong Kong situation. Firstly, functionalism stresses the importance of stability within a common value system. Hong Kong in common with other East Asian societies such as China, Korea, Japan and Vietnam could be said to have a common value system in its own version of Confucianism, which stresses the importance in guiding behaviour of set roles and relationships, particularly familial and filial. Within this philosophy, society is seen as one big family in which social and political relationships are modelled on familial relationships (Huque et al., 1997). Stability is seen as a desirable state of affairs, although Hong Kong people see it as the ultimate responsibility of the state to maintain this stability, rather than of individuals in the society (Huque et al., 1997; Wilding \& Mok, 1997). Most importantly, Hong Kong society is stratified and this is an accepted state of affairs. There is undoubted support for equality of opportunity at the expense of 
equality of outcomes (Huque et al., 1997; Wong \& Lui, 2000), meaning that people generally feel that it is the natural order of things for there to be disparities in wealth and status, so long as everyone has the opportunity to increase their socio-economic status through their own and/or their family members' efforts and/or good fortune. Functionalist thinking deems it the responsibility of the populace to work hard for the good of society. However, this does not seem to apply to the Hong Kong situation as the people there tend to display characteristics more attuned to individualism and familism (Huque et al., 1997; Wong \& Lui, 2000).

When considering the extent to which structural functionalism can be applied to the Hong Kong case, it is necessary to examine how the theory deals with social change. As mentioned above, its focus on structure and stability means that any changes introduced, such as in educational policy, are carried out in response to perceived strains in the structure of social systems in the attempt to maintain the status quo. Thus, according to Parsons (1951), social change is resisted by individuals who have vested interests, that is, their 'interest in maintaining the gratifications involved in an established system of role-expectations [which includes] conformity with institutionalized expectations, of the effectively neutral and often the moral type [and] also includes the interest in the relational rewards of love, approval and esteem' (p. 492). Parsons describes society as a boundarymaintaining system within which changes do occur in a persistent state of reequilibration.

Because of the phenomenon of vested interests ... we may assume that the introduction of the change in the relevant part of the system imposes strains in the actors in those other parts on which the change impinges. The reactions to these strains constitute the tendencies to re-equilibration of the system, that is, to the elimination of the change and the restoration of the state of the system before its introduction. But these changes may be 'coped with' so that the change becomes consolidated and perhaps extended. But unless the system is loosely integrated, this consolidation will mean that the other parts of the system than the original area of change have also been changed, so that what is 
eventually reached is a new state of the system as a whole. (Parsons, 1951, p. 496)

The structural-functional approach, then, regards the process of social change as a (slightly) shifting equilibrium point and as such does not take into account the dynamics of the change process, nor the possibility of challenges to equilibrium and disruption of the status quo. It does not consider the ways in which reforms in the system can drive major societal change through class division or changes in relationships between social systems (Pun, 1996). Further, the relationships between social systems are not explained and so the potential for investigation of changes in such relationships is absent (Harper, 1993). Therefore, whilst the ideas of social stratification $(\approx$ the structure of the society) supported by a common value system ( $\approx$ the functional core of the society) can usefully be incorporated into a model of Hong Kong society, further descriptive-theoretical strands are needed to account for the complex changes in relationships which occur between different social groups and their respective social systems as a result of strains that might be caused by the imposition of innovations and by conflicts between social groups and systems such as those of education authorities and teachers.

In relation to education in Hong Kong, structural functionalism would see the education system as a closed, boundary-maintaining system under Parsons' conception in which efforts are made to overcome strains in the system through reform and the introduction of government-directed edicts. The focus on stability of structural-functionalism would appear to apply to education in the same way that it can be applied to society in general, as education could be said to be a microcosm of society, or at least one sub-system of the larger social system. Yet within education there are separate systems according to children's age and relevant curriculum: pre-primary, primary, secondary and tertiary. There are also separate systems according to the school funding base: government, governmentaided and private schools. Furthermore, there are separate systems depending on the medium of instruction. This all adds up to a complex system of interrelated and overlapping sub-systems which the government, through its agencies, tries to keep stable, whilst at the same time responding to endogenous changes impacting 
from other systems outside of education, such as the economy, the citizenry and the polity. The imposition of educational reforms in Hong Kong, particularly as they relate to language, such as the change in the medium of instruction, revision of the curriculum to take into account new educational and linguistic theories, and the upgrading of teaching standards, have caused friction between the sub-groups in education and between the education system and the other systems in society. Here again, functionalism does not account for such changes in relationships, which are crucial in determining whether changes imposed proceed as planned. The theory also does not explain the natural evolution which can be ascribed to curricular development and teacher change (Fullan, 1991; Woods, 1996). In addition to the theory of stability, a theory that takes into account changes in relationships between systems is required.

\subsubsection{Conflict Theory}

As with structural functionalism, conflict theory attempts to explain how inequalities or stratification in society lead to change within it. However, whilst functionalism is basically a theory of systemic order or stability, in which the sources of change are unclear or unaccounted for, in conflict theory, which is based on Marxist thought, strains in society are considered to be inevitable and inherent. In a conflict theory view, societal strains are based on the efforts of individuals and groups within the society to control scarce resources or the means of producing them (Harper, 1993). In essence, conflict theory is based on the struggle between socio-economic classes to control the means of production, but is also applicable to societal conflicts of different natures. Harper (1993), summarising the views of the conflict theorist Ralph Dahrendorf (Dahrendorf, 1959) gives the following characterisation of the dynamic of societal conflicts and resolution of those conflicts over time:

... conflict may or may not result in change, and may result in change of different types. Conflict can produce stability as an ongoing stalemate, in which there are no winners between groups 
that are bound in conflict relationships. It can result in the defeat of established powers or of insurgent groups. It can result in total or partial system change regarding the distribution of rights, resources, and authority ... [and] ... each new 'resolution' of the problem of rights and authority creates new categories of 'those in charge' and 'those who are not,' and the new categories are likely to become new cleavages along which conflict will emerge. (p. 100)

In other words, conflict leads to disruption or destruction of the old order and creation of a new order, which can be as liable to conflicts as before.

There is no doubt that societal conflicts have existed and continue to exist in Hong Kong. During the period leading up to the changeover in sovereignty, there were major conflicts created as the Chinese and British governments each sought to protect their own interests (Pun, 1996). The democratic reforms introduced by the last colonial Governor, Christopher Patten, which allowed for more members of the Legislative Council to be directly elected, greatly upset the PRC government, resulting in a 'war of words' between the Governor and the Mainland officials responsible for Hong Kong and between Patten and the pro-China press (Flowerdew, 2001). The situation also led to a virtual stalemate between the colonial government and the Mainland as the latter refused to negotiate on key areas of policy, preferring a 'stand firm and wait it out' approach. Patten himself in reference to the Joint Liaison Group, ${ }^{11}$ described the mood at that time in vivid terms:

Hardly a week went by without British and Hong Kong negotiators ... heading for the trenches in Hong Kong's Kennedy Road, where the talks with China usually took place. The front would move a few inches this way and a few inches that. None of our negotiators actually died on the barbed wire, but several must have come close to passing away from sheer boredom. (Patten, 1999, p. 79)

As a result of the democratic reforms, in 1995 elected democrats and populists held a slight majority in the legislature, which was perennially at odds with the pro-business government (Lau, 2001). ${ }^{12}$ As a result, the atmosphere in which educational reforms were introduced was one of conflict in the political arena. 
There was likely to be opposition to any innovations proposed by the government or any of its agencies as, one might say, a natural consequence of democratic politics.

Major conflicts existed as well in language (English vs. Chinese) and education (parents vs. authorities, teachers trying to implement the examination system vs. communicative goals, etc.). In terms of language in Hong Kong, the conflict between English, the language of the colonial power, and Chinese (in generic terms) the language of the majority of the population, has been a highly visible one. In education, conflicts have existed between parents and the authorities responsible for education, between teachers and the authorities, and within teaching itself. An example of such conflict is that between trying to adhere to the requirements of the examination system and trying to accede to the communicative goals of the teaching syllabus or the communicative and other requirements of the students. Further discussion of these conflicts will occur in later sections throughout the thesis.

However, to attempt to analyse the changes occurring in education at that time on the basis of conflict theory alone would be too simplistic. We next look at how the nature of conflicts both between and within groups in society can be interpreted in terms of the cultural makeup of that society and how such an approach can help to further understand Hong Kong society and its reactions to the changes taking place during the political transition.

\subsubsection{Interpretive Theory}

A third group of theories of social construction or change have been termed social-psychological theories (Vago, 1996). These theories deal with the ways that individuals shape societies through the 'psychological determinants that drive people to act, to push forward, to invent, to discover, create, acquire, build, or expand' (Vago, 1996, p. 62). Probably the most influential proponent of this 
approach was Max Weber (e.g. Weber, 1958). Harper (1993) uses the term Interpretive Theory as a collective term for these theories based on Weber's insights, i.e.:

... a 'full' sociological understanding [of social action and social change] would focus not only on the overt behaviour and events, but also on how they are interpreted, defined and shaped by the cultural meanings that people give to them. Sociology is therefore the 'interpretive understanding' of social action. (p. 104)

Weber's description of capitalist society dominated by the work ethic arose from his observations of the relationship between Protestantism, which encouraged hard work and frugality, and industrial development (Harper, 1993). This influence of a belief system on the social structure of society seems to more closely represent the situation in Hong Kong than does conflict theory, considered as focusing on class conflict, as class differentiation is less distinct in the community than it was in the past. The expansion of the middle class, which includes increasing numbers of the more highly paid working class, means that there is now greater intra-class fragmentation in Hong Kong society (Ho, 1994), and as a result class boundaries are not as clear as they were before the rapid growth in the economy of the 1970s and 1980s (Grant, 2001).

In fact, class is not the only (or necessarily the best) way of describing and differentiating Hong Kong people; culture and beliefs play an equally important role (Hung, 2001; Huque et al., 1997). The Chinese people of Hong Kong have been characterised as tending to be home-centred and less inclined to collective action, such as trade unionism, unless it serves their personal or familial interests. This reluctance to engage in activities that might involve conflict - which has been less true of the Mainland Chinese, at least in the past - indicates that they tend to favour stability over change. This is not to say that the Hong Kong Chinese are not prepared to engage in collective protest action, particularly against the government. Individuals do form themselves into political organisations to protect their own interests against persecution by the state. Examples of such groupings, which tend to cut across class and status boundaries 
and to be based on common beliefs or ideals, are (i) the pro-democracy movement, begun in the aftermath of the Tiananmen Square incident in Beijing in June 1989 (Butenhoff, 1999); and, in terms of education, (ii) the Professional Teachers' Union, which led protests by teachers against the proposed Language Proficiency Assessment or 'benchmarking' of primary and secondary school English teachers (LPAT) in May and June 2000 (South China Morning Post, 28 May 2000, 11 June 2000).

The Weberian perspective of social stratification is more complex and involves a greater degree of diffusion and fragmentation of individual groups within society than either the structural functionalist or conflict perspectives. Harper (1993) describes a social-psychological theory based on Weber's perspective as well as a number of other theories which focuses on 'the way actors define their social situations and the effect of these definitions on ensuing action and interaction' ( $p$. 104). In Harper's concept of Interpretive Theory, social structure exists within the framework of accepted outlooks, meanings and definitions, or in other words, the common value system or culture (or functions) of the society. Within this functional or cultural framework exist the ever-changing social groups and structures based on class, ethnicity, occupation, religion and other social structures, attributes and practices. In the characterisation of Harper (1993), change occurs when individual groups or structures of groups are successful in reconstructing social reality by deliberate action. They do this by interpreting and then articulating what is unsatisfactory in the existing situation, what a better situation might be and what action should be taken to achieve it. Claims-making agents, as they are called, must use their power to overcome challenge by other claims-makers and must challenge those groups held responsible for the problem. Successful claims-making involves having access to the structures and facilities that control the definition of social reality, which Harper (1993) identifies as the mass media and the judicial system. Through the media, claims are disseminated; and then the judicial system, generally the government, has the job of promulgating laws and policies to transform social reality. If the government does 
not like the attempts to alter society, it may act to regulate the behaviour of the claims-making agents.

Relating interpretive theory to Hong Kong, the education system, which is in part a facet of the government but in part also a separate system, can be seen as one of the structures and facilities that control the definition of social reality. Within the education system are examples of prominent claims-making agents. Representing teachers are Szeto Wah and Cheung Man-kwong. Szeto Wah was previously President of the Professional Teachers' Union and also represented the education sector in Hong Kong's governmental legislature, the Legislative Council. He now serves as an elected member of the Legislative Council. Cheung Man-kwong succeeded Szeto Wah as leader of the PTU and likewise now represents the education sector in the Legislative Council; he is also a member of the influential Education Commission. Antony Leung Kam-chung served as Chairman of the Education Commission from 1998 to 2001, when he was appointed (or one could say, promoted) to the position of Financial Secretary (in effect the third-highest position in the SAR government after the Chief Executive and the Chief Secretary). His place was taken by Rosanna Wong Yick-ming, former Director of Housing. Within the government itself, the most influential agent is probably Fanny Law Fan Chiu-fun, formerly Director of Education, with responsibility to supervise how schools are run and to ensure that education policy is implemented (Pun, 1996), from 1998 to 2000. In 2001, she became Secretary of the Education and Manpower Bureau, which is responsible for formulating and reviewing all education and training policies. Another regularly-heard voice is that of Tsang Yok-sing, an outspoken school headmaster and legislator, who is also leader of the political party, The Democratic Alliance for the Betterment of Hong Kong, a pro-PRC group. Finally, there are prominent academics such as Cheng Kai-ming and Amy Tsui Bik-mei from the University of Hong Kong, whose views on education and English language are well-respected and influential within the community. 
The link between the English language and the common value system or culture of the society is not so clear-cut. Being a former British colony, English has been and will remain a significant part of the social fabric in Hong Kong. In terms of English being a part of the structure of society, there are different attitudes towards and demands for English from different groups. Such groups include the commercial sector, the academic community and the general public. Within each of these groups there are sub-groups which also differ in their affinity for English and depend to a large extent on factors such as their need (extrinsic) or desire (intrinsic) for English and their social class or the amount of cultural capital they posses which would put them in the position of being able to take advantage of provisions to supply English. Examples of such a provision are the Englishmedium schools, both local and international, in which places are much soughtafter (and in the case of the international schools, beyond the means of most Hong Kong people). As for education more generally, within the structure that is the English language in Hong Kong, claims-making agents have emerged who have been influential in terms of the changing roles, functions and status of English. Given the high prominence that English has had within the education sector, the majority of those agents mentioned in the previous paragraph are those that have had the greatest influence on English.

Claims-making agents do not necessarily have to be so high-profile. A group, such as parents, teachers or students, or individual representatives of them, can make claims on government or other decision-making groups providing they are vocal enough. However, high-profile individuals tend to attract attention, from the media and through them from the public and can use their influence to effect or (sometimes) to oppose reforms in education, leading to the situation whereby opposing forces serve to slow down or even stop the processes of educational change, as heterogeneity within the society impairs or even obstructs the diffusion of innovations (Rogers, 1995). As a foundation for a deeper discussion of societal change in terms of innovation theory in a later section of this thesis, the ideas on social structure and social change that have already been described can be 
elaborated by looking at the political culture within Hong Kong and the part it has played in the changes that have occurred during the transitional period.

\subsubsection{Hong Kong's Political Culture}

In order to examine how Hong Kong's population has attempted to implement and then either accepted or rejected educational change and innovation, it is necessary to look at the culture within which the changes have been introduced, that is, the political culture, which can be thought of as a bridge between social culture (or culture itself) and political structure (Chodak, 1973). According to Chodak (ibid.), a society's political culture can be evaluated in terms of:

Its ability to change ... Its capacity for absorption of innovations both from other political cultures and from other spheres of social activity. (p. 242)

The political structure in Hong Kong, as mentioned, did undergo changes during the final years of British rule as the colonial power attempted to leave in place a degree of self-determination by promoting democratic reforms. How much these changes affected the political culture is unclear, however, given the fact that, as Chodak (1973) has remarked:

The character of the political system and regimes change much faster and more drastically than political cultures ... The continuity of political cultures also reflects the persistence of the national psychological traits commonly regarded as national character. ( $p$. 244)

To paraphrase Chodak, structure changes faster than (underlying) function. It is possible that the underlying functions or political culture in Hong Kong remained fairly stable even during this period of dramatic political change. What needs to be examined is the true nature of this political culture, whether it did or has changed as a result of the handover and what effects that has had on its capacity for absorption of innovations. 
The vast majority of the population of Hong Kong are of ethnic Chinese origin and according to Huque et al. (1997) Confucianism is at the core of the 'national character' that exists there. The prominent Hong Kong sociologist, Lau Siu-kai, used the term utilitarianistic familism to define the approach to life adopted by the people of Hong Kong (Flowerdew, 2001; Ho, 1994: Huque et al., 1997, Lau, 1982; Lau \& Kuan, 1988) in that they put family concerns above those of society as a whole, indicating a lack of interest or apathy towards political issues, unless they impinge directly on them or their family. Lau characterises Hong Kong society as being a 'minimally integrated socio-political system' in which social groups, including the family and the polity, exist in near isolation. As a result of this isolation, a status quo exists in which social groups go about their own business and conflicts with other groups are rare, resulting in a desired (from the government's point of view) condition of stability (Lau, 1982: Wong \& Lui, 2000b). Chodak (1973) described this more broadly as an Asian characteristic, to be parochial and communalistic (family or tribe) rather than individualistic or collectivistic (whole society or state).

Countering this view is the opinion that the family has become less significant as the basis of society as a result of the rapid growth in the economy leading to greater individualism and less reliance on the family group (Ho, 1994; Huque et al., 1997). However, as the economy began to suffer periods of recession in the late 1980 s, particularly as a result of the weakness in the international stock market and the aftermath of the Tiananmen Square crackdown in 1989, people began to see limits to their opportunities for upward mobility, a growing inequality in income distribution (Ho, 1994) and uncertainty over the stability of the Territory under Chinese rule (Cheung, 2001). Increasing numbers of the middle class in a position to do so migrated to other countries to secure a foreign passport, ${ }^{13}$ later returning to Hong Kong to take part in the economic boom of the early 1990s (Cheung, 2001); the rest of the population began making more claims on the government, as its responsibility to intervene in the functions of society in order to safeguard the general well-being of populace became more overt (Ho, 1994). The people may also have become more 'democratised' under the 
influence of the Tiananmen Square incident and the strive towards democracy in government by the colonial ruler in the person of the last Governor, Chris Patten (Flowerdew, 1998). Patten was a politician by nature, in contrast to his predecessor, Lord Wilson, a career diplomat, sinologist and old-style colonial governor whose style of governorship tended towards maintenance of the status quo, especially with regard to relations with China (Flowerdew, 1998). Under Patten, it seems that the political culture in Hong Kong changed during the period prior to the handover, partially in response to the changing political structure and partially due to economic factors imposed by forces external to Hong Kong that impacted on the Territory's internal affairs. The change can be summarised as a transition from a situation of stability brought about by colonial rule and acceptance of the status quo by the populace, to less stability caused by people's concerns about the future under Chinese rule, their greater expectations of and demands on the government, as well as higher levels of mobility of the population. $^{14}$

Returning to Chodak's (1973) view that a society's political culture can be measured in terms of its ability to absorb innovations, changes in the political culture should logically lead to changes in the ways in which the society deals with change itself. Of particular interest to the present study is change which is generated from within society, even though it may have its origins in another political culture, and the ways in which it is accepted, rejected or ignored altogether. The next section looks at innovations and some different theories as to how they diffuse throughout a social system within a political system and culture.

\subsubsection{Diffusion of Innovations}

There is a large and diverse literature on the absorption and diffusion of innovations in a number of disciplines. Rogers (1995) identified a total of 3,890 publications in the field of diffusion research in 1994. From this survey, ten major diffusion research traditions were detected in terms of their percentage share of 
the total number of publications. The ten were: anthropology, early sociology, rural sociology, education, public health and medical sociology, communication, marketing and management, geography, general sociology and general economics. Whilst Rogers (1995) documents the growth of diffusion research in all of these (and other) areas, since the mid-1990s, two areas which have seen a particular increase in research activity have been communication and general economics the former due to rapid developments in electronic communications and information systems (McMaster, 2001) and the latter as it is 'mainly concerned with the economic analyses of technological innovations' (Rogers, 1995, p. 88), and as such is related to the former. The field of education, although important in terms of the number of studies completed, has been criticised for not contributing enough to a theoretical understanding of diffusion processes (Rogers, 1995). The work of Cooper (1989), Fullan (1991, 1993), Hargreaves (1999), Henrichsen (1989), Kennedy (1988), Markee (2001), Miles (1964), Nicholls (1983), Pennington (1995c, 1996, 1997b, 2001) and others is evidence to the contrary. It is hoped that this thesis will also make a contribution to this growing field.

The work of Everett Rogers in Diffusion of Innovations (Rogers, 1962, 1995) is considered seminal in the innovation-diffusion area (Mitsufuji, 2001) and is particularly important for an understanding of the social mechanisms of change. Rogers (1995) describes diffusion as:

a kind of social change, defined as the process by which alteration occurs in the structure and function of a social system. When new ideas are invented, diffused and are adopted or rejected, leading to certain consequences, social change occurs. (p. 6)

More precisely, Rogers (ibid.) defines diffusion as 'the process by which an innovation is communicated through certain channels over time among the members of a social system' [italics in original] (p. 10). These dimensions of change can be applied to the Hong Kong context of English language teaching.

An innovation, according to Rogers (1995) is 'an idea, practice, or object that is perceived as new by an individual or other unit of adoption' (p. 11). Innovation, in 
terms of English language education in Hong Kong, can be said to include more interpretive teaching methodologies, such as communicative language teaching (Evans, 1996; Young \& Lee, 1987), process approaches to writing (Pennington, 1995c) and a task-based or target-oriented curriculum (Hau et al., 1996; Morris, 1995). In terms of educational change impinging on English language education, it could also include the push towards mother-tongue education (Tung et al., 1997), as in a sense the 'return' to Chinese is an innovation in the majority of Hong Kong schools, where English is still the preferred medium. This is in spite of recent efforts by the Education Department to designate more schools as Chinese medium. These innovations, all of which have been imposed 'top-down', have been or are being pushed onto teachers and students in Hong Kong by the government through the Education Department and its Curriculum Development Council, resulting in potential resistance to them, particularly from teachers (Arias, 1994; Woods, 1996). In addition, Hong Kong teachers have been characterised as being typically resistant to change, due in part to the cautious, authority-upholding nature of Chinese people (Pennington \& Cheung, 1995) and the constraining factors within the curriculum and within schools (Pennington, 1995c; Tsui, 1996).

Rogers (1995) defines communication in terms of the diffusion of innovations as 'the information exchange through which one individual communicates a new idea to one or several others' (p. 18). This communication flow between individuals is essential if innovations are to be adopted. The nature of the relationships between the individuals will determine how easily information and hence innovation is spread. The transfer of information occurs most frequently between individuals who are alike, who share common beliefs, education and social status. Such individuals are described as being homophilous. Homophily encourages information flow as it is more comfortable to communicate with peers who share beliefs and a common language. Communication with those from a different social grouping results in heterophilous interpersonal diffusion networks, which are more difficult and require more effort. Rogers (1995) describes how homophily can sometimes be a barrier to diffusion as innovations are generally 
introduced by individuals of higher status and a high degree of homophily would mean that these individuals would only interact with each other, the result being that an innovation would not 'trickle down to non-elites' (p. 288). On the other hand, heterophilous network links can sometimes connect cliques of socially dissimilar groups, which can serve to carry information about innovations. The relationships between social groups within English language education in Hong Kong will be examined in section 2.5 .

Such links or communication channels can be either mass media channels such as television, newspapers, journals, and possibly the Internet, or interpersonal channels, where two or more people communicate face-to-face. The available channels through which innovations in education can be transmitted in Hong Kong are many. There are both Chinese and English-medium television and radio channels, as well as local Chinese and English language newspapers (although, understandably, in both media the Chinese-language dominates). There is ready access to international mass media and educational journals. There are institutes of tertiary education, which offer courses in English language education designed and delivered by internationally recognised authorities in the field. In addition, there are frequent international conferences and open lectures and seminars given by world-renowned scholars. In effect, there is no shortage of means through which innovations in English language education might be communicated.

The time factor in Rogers' (1995) model relates to the time it takes for an innovation to be adopted. Rogers describes the process of innovation as consisting of a number of different stages before an innovation is fully adopted. He also develops categories for the 'innovativeness' of an individual, and describes the rate of adoption of an innovation as occurring within a social system. The time period relevant to the present study is that surrounding the transfer of sovereignty, beginning in 1994 and continuing on to 1999, but also stretching back to the mid1980s, just after it became certain that Hong Kong would revert to Chinese rule. 
Finally, the social system, according to Rogers (1995), is 'a set of interrelated units that are engaged in joint problem solving to accomplish a common goal' ( $p$. 24). Rogers describes how the structure of a social system affects the diffusion process. Within any social system, there exist patterns of relationships between individuals, based on perceived rank or position, or shared beliefs or cultures. These role relationships will affect the communication flow in a system and hence facilitate or impede the diffusion of innovations. Every social system also has its norms or established conventions and patterns of behaviour, which can serve as barriers to change (Rogers, 1995). Within the social system that is Hong Kong, or more specifically that is the culture of teachers of English in Hong Kong, the norm is one of cautious attraction to innovations.

Another feature of social systems are people Rogers calls opinion leaders and change agents. Opinion leaders are individuals who are able to exert influence over other individuals with regard to the adoption of new ideas. According to Rogers, 'Opinion leadership is earned and maintained by the individual's technical competence, social accessibility, and conformity to the systems' norms' (p. 27). While opinion leaders exert their influence from within the social system, change agents influence the diffusion of innovations from outside the social system and usually posses technical expertise and the status that goes with it. Such change agents typically work for a change agency.

The social system also affects the diffusion of new ideas by the types of innovation decisions that are made. Innovations can be adopted (or rejected) by individuals within the system, or they can be adopted (or rejected) by the whole system. In the latter case, adoption or rejection can be by collective or authority decision (Rogers, 1995). The idea of enforced or top-down change can again be highlighted here. Decisions made at government level, such as the implementation of the Target Oriented Curriculum, of Chinese-medium education and of the Language Proficiency Assessment for Teachers, are examples of these kinds of collective, authority-decisions. When innovations are imposed top-down, it takes time and energy on the part of the change agents and others in the system for the 
innovation to work its way through the entire system. The social systems within which English language education could be said to exist in Hong Kong include the society as a whole and then within this all-encompassing system, separate but interconnected sub-systems such as government departments, tertiary institutions, schools, teachers, parents and students. Ideally, each of these sub-systems adopts an innovation leading to adoption by the larger system of which it is a part. At the same time, different rates, degrees and types of adoption may occur in each subsystem and thus affect the quality and scale of adoption in the system or society overall.

The consequences of innovations 'are the changes that occur to an individual or to a social system as a result of the adoption or rejection of an innovation' (Rogers, 1995, p. 30). These consequences are defined by Rogers (1995) as desirable/undesirable, direct/indirect and anticipated/unanticipated. The desired outcomes are often not those that in fact occur, and changes may occur which are both unanticipated and undesirable, including indirect changes occurring as a result of an innovation. The change in the medium of instruction in Hong Kong schools from English to Chinese is intended to have an effect of enabling the majority of students to gain more from their learning. However, it has been pointed out here that a possible undesirable outcome could be a reduced status of Chinese-medium schools, which will encourage the best students to seek Englishmedium schools, either within the local or international school systems or overseas, thereby reducing the overall standard of students in the Chinese-medium schools. An indirect outcome of this could be a fall in the perceived importance of English in Hong Kong.

In the remainder of this section, the diffusion-of-innovations (DOI) concepts developed by Rogers will be further applied to the context of education in Hong Kong during the period of the changeover, taking into consideration the theories of societal development discussed above as well as the changing socio-political culture at that time. 
The value of the diffusion of innovations approach to an understanding of changes in language teaching has been stated by Markee (2001):

[T] his perspective provides a unified framework for conceptualizing both the development and evaluation of innovations in language teaching. (p. 118)

Markee (2001) (following Cooper, 1989) adopts a componential approach to examining how DOI approaches influence language teaching by positing the question, 'Who adopts what, where, when, why and how?' [italics in original] (p.118). These are essentially the questions asked by Rogers (1995), and it seems logical to follow the same approach here, bringing in where appropriate, theories and ideas on DOI as applied to language teaching from other sources and then applying them to the Hong Kong context.

\subsubsection{Who is Involved in the Innovation-Diffusion Process?}

In terms of the adoption of a language teaching innovation, it is inevitably the teacher and by design the student who becomes the adopter or end user of an innovation. Without the full participation in the process of diffusion by the teacher, no educational innovation can be considered to have been adopted (Fullan, 1991) and the core of this investigation will look at the teacher as adopter. This is not to say that they are the only groups of individuals involved in the process. Rogers (1995) identifies two types of individuals, adopters and change agents. Adopters vary in their innovativeness or the rate at which they adopt and include in their midst, opinion leaders, who can greatly influence the rate at which diffusions are adopted either positively or, in a more conservative social group or society, negatively ( $\mathrm{Li}, 2001)$. Opinion leaders will reflect the nature of the system to which they belong, such that in a more progressive society, they will be the ones pushing for adoption. In a more conservative system, they will reflect this characteristic by tending to resist anything new. The Hong Kong education system, perhaps unlike the more innovative commercial sector, has been described 
as conservative and tending to resist change. In that context, the opinion leaders will tend to be people within schools such as principals, panel chairs ${ }^{15}$ and experienced teachers, as well as respected educationalists. (The opinions of such a group on educational issues affecting English language education during the period just following the changeover are discussed in Chapter 3).

Change agents may use opinion leaders to influence adopters to either adopt or resist adoption of an innovation (Rogers, 1995). Change agents have also been described as entrepreneurs (Markee, 2001) or, as referred to earlier in terms of societal development theory, claims-making agents (Harper, 1993). A change agent normally represents a change-making agency, which might include the government, or one of its 'agencies' such as (in the case of Hong Kong) the Education Commission or the Education and Manpower Bureau, a teachereducation institution such as the Hong Kong Institute of Education, or a group of adopters such as the Professional Teachers' Union. These people can exert great influence and a look at the recent history of education in Hong Kong has shown this.

\section{What is Meant by 'Adoption'?}

According to Rogers (1995), adoption is 'a decision to make full use of an innovation as the best course of action available' (p. 21). In addition, an innovation can be rejected or even discontinued, which means it is rejected after first having been adopted. Adoption may also be thought of in terms of levels of implementation (Markee, 2001), i.e., measures of the depth to which changes have occurred. In some situations, adoption may not take place in a linear fashion, in that the sequential processes described by Rogers (1995) of (1) knowledge, (2) persuasion, (3) decision, (4) implementation and (5) confirmation of an innovation may well suffer regression at any stage, particularly at the implementation stage, due to conflicting information or other factors (as discussed in the forthcoming pages). An educational innovation, such as a change in the 
medium of instruction or the implementation of a new mode of teaching, may or may not go through all the above stages before it is either adopted, partially implemented or rejected.

The change in the medium of instruction in Hong Kong schools from predominantly English to predominantly Chinese illustrates the stages of the innovation-adoption process. From 1984 onwards, the government expressed the desire to increase the emphasis in schools on teaching in Chinese. Through the Education Commission and its reports 1, 2 and 4 (Hong Kong Education Commission, 1984; 1986; 1990) knowledge concerning the benefits of a Chinese medium of instruction (CMI) was dissipated to the public. However, the move proved unpopular with parents, who preferred their children to study in English as they considered that it offered them the best hope of a bright future. Consequently, the persuasion stage took some time. It was not until 1995, when Education Commission Report No. 6 was published (Hong Kong Education Commission, 1995) that clear guidelines for the implementation of the MOI policy were introduced and the decision made to go ahead with it in 1998. It was decided that from the start of the school year in September 1998, about 70 per cent of secondary schools would be required to teach through the medium of Chinese (Johnson, 1998; Poon, 2000; Tung, 1990; Tung et al., 1997). The evidence appears to demonstrate that the decision reached the last stage in the adoption process, confirmation, although there have been reports of Chinese-medium schools using English as the medium for higher forms (South China Morning Post, 29 September 2001). ${ }^{16}$ Therefore, although the normal evolutionary process of innovation has been followed, the adoption process is a good deal more complicated than this brief explanation allows and there is much that has to be considered in order to fully understand the reasons why the process takes so long and varies so much in form. 
Innovation, in language teaching contexts, has been defined by Markee (2001) as:

... proposals for qualitative change in pedagogical materials, approaches, and values that are perceived as new by individuals who comprise a formal (language) education system. (p. 120)

The change is described as 'qualitative' given the difficulty in actually measuring the degree of change involved, or whether an innovation creates 'fundamental' change (Markee, 2001). While it might be possible to ask individual teachers whether or not they have confirmed their adoption of an innovation, such as switching to the use of the mother tongue in teaching, this is not the same as determining to what degree that change can be differentiated from the language use the teacher adopted previously. Even when changes occur in the materials used, approaches adopted and values of the individual teacher, the extent of the changes, their precise nature, and their precise outcome in the classroom, are difficult to measure.

Similarly, changes can only be perceived as new by each individual, as the idea may not be new as such, but may be new to the individual concerned (Pennington, 1996). Markee (2001) gives the example of the Natural Approach to language teaching being considered new in the early 1980s and still viewed in this light by teachers who are introduced to it today. In Hong Kong, the approach known as Communicative Language Teaching (CLT) has been pushed as an innovation in Hong Kong since the early 1980s (Curriculum Development Committee, 1983; Curriculum Development Council, 1999) but is still considered 'new' by many within the language education system. This may be as a result of the lack of success in implementing it caused by the reluctance of teachers to stray from the transmissive, examination-focused approach which pervades the entire system (Evans, 1996, 1997; Pennington, 1995c; Pennington \& Richards, 1997; Richards, Tung \& Ng, 1991; Young \& Lee, 1987). Anything different from this norm or 
tradition is seen as new, even though it might have been around, albeit in the background, for some time.

The factors within language education that are liable to change have been termed materials, approaches and values by Markee (2001). These terms are not altogether clearly defined, and therefore the categories used by Pennington (1996) when developing teaching frameworks to model change might be more applicable. Instead of materials, approaches and values, Pennington (1996), following Richards and Rodgers (1982, 1986, 2001), refers to design, procedure and approach. These factors are summarised below:

Design: $\quad$ The teacher's teaching objectives, syllabus, planned teaching and learning activities and instructional materials.

Procedure: The actions performed by the teacher in interacting with students within the teaching context.

Approach: The theoretical framework of beliefs and values which influences the teacher's thoughts and actions.

All three of these components are affected to varying degrees when an innovation is adopted and the changing relationships between them are used by Pennington (1996) to describe different patterns of teacher change. They could also explain how certain innovations are diffused and why they are either adopted or not adopted on a more systemic level, which will be discussed in the forthcoming sections.

\subsubsection{Where does the Innovation-Diffusion Process Take Place?}

The question of 'where' an innovation-diffusion process takes place has more to do with the socio-cultural context than the geographical location (Markee, 2001). 
However, this study is concerned specifically with educational change in one location, Hong Kong, which did and does have particular socio-cultural and sociopolitical factors which have impinged and do impinge directly and indirectly on the change process. Markee (2001) identifies the following factors which affect the diffusion process: cultural, ideological, historical, political, economic, administrative, institutional and sociolinguistic. Pennington (1996) puts the factors into a hierarchy and refers to the context frames within which an educational innovation process takes place of classroom, within school, within society, within culture, which is similar to those of Kennedy (1988), i.e., classroom, institution, education system, administrative system, political system and finally, again, culture. All of these factors, as discussed earlier, were affected to some degree by the changeover in sovereignty, particularly the 'middle' context frames of society (Pennington, 1996) or political system (Kennedy, 1988). Changes in these middle context frames work inwards (or downwards) on those below and are influenced by the overarching frame of culture from above, which creates changes in the contexts within which the diffusions of the innovations ongoing at that time are occurring. It is anticipated that an analysis of these changes can help provide explanations of the patterns of adoption, non-adoption and partial adoption that characterise the changeover period, specifically in terms of English language education.

\subsubsection{When are Innovations Adopted?}

The innovation-diffusion process takes place over a certain time period, which ranges from the introduction of the innovation to the point at which there is no further increase in the numbers of adopters or it is rejected. The process can be represented by a graph of the percentage of the population who adopt the innovation against time (Rogers, 1995, p. 106): 


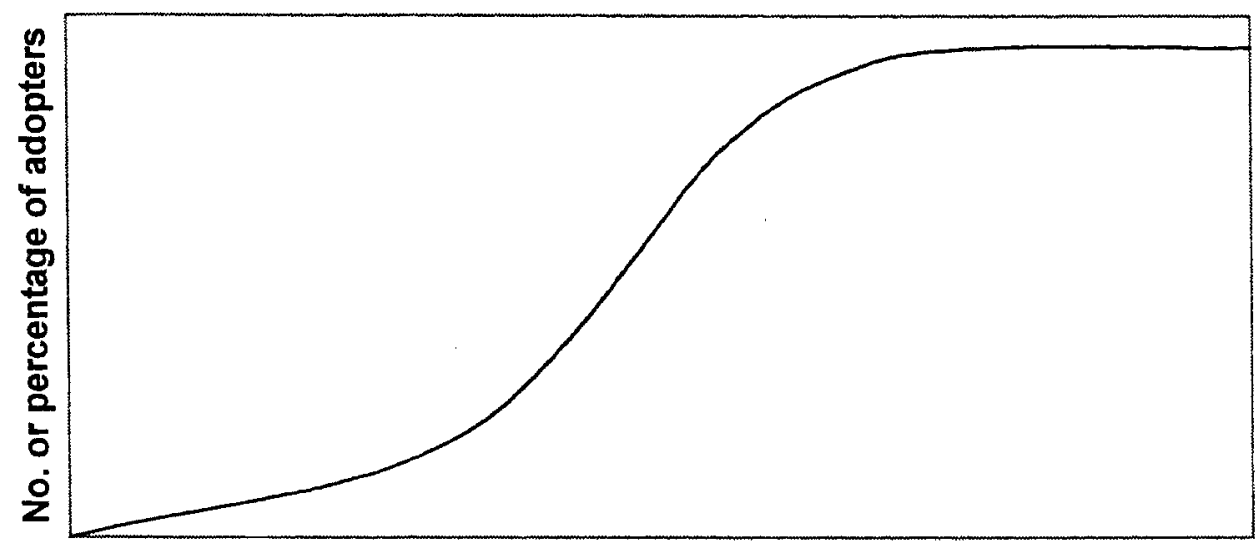

Time

Figure 2.1: An s-shaped diffusion curve

The diffusion-adoption curve has a characteristic shape, showing that adoption at first occurs slowly and then reaches a critical mass when approximately 25 per cent of potential adopters have adopted, and then the process takes off. Finally, diffusion slows down and plateaus as the majority who are likely to adopt, have already done so. The exact shape of the curve, i.e., its steepness and the height it reaches before levelling off, reflects the rate of adoption of an innovation. Figure 2.2 shows the curve which might represent an unsuccessful innovation:

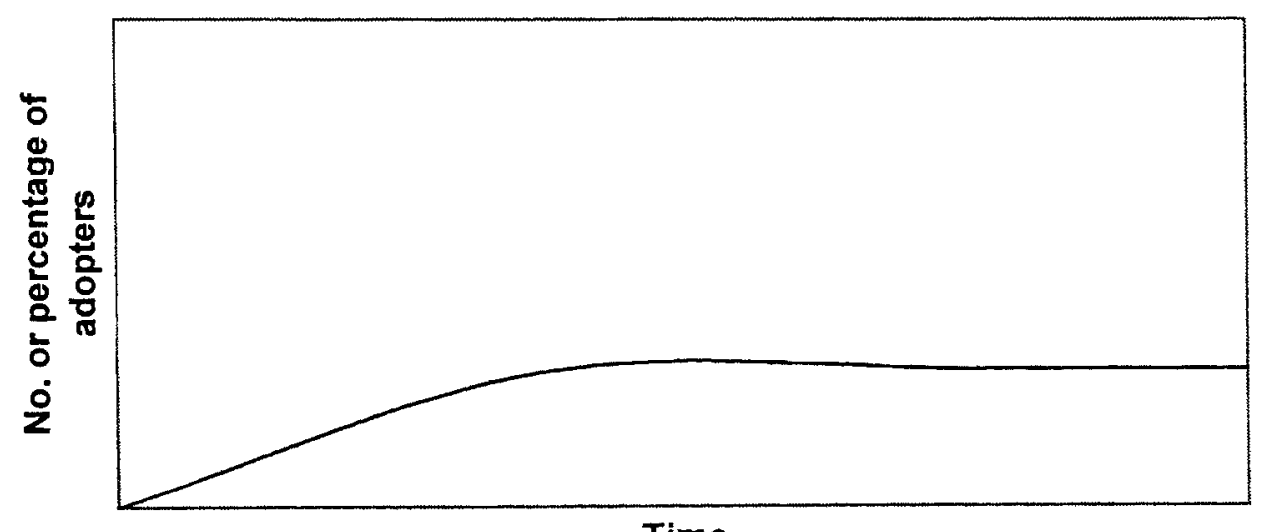

Time

Figure 2.2: The diffusion curve for an unsuccessful diffusion 
In this case, adoption has plateaued relatively early in the process with no sign of increased adoption (Rogers, 1995); in other words, the diffusion has failed to take off.

A third possibility is that an innovation could have been ongoing for a considerable period of time and have existed 'under the surface' - or in a 'muted' guise, in the description of Pennington $(1996,2002)$ - before it appears to suddenly take off (Figure 2.3):

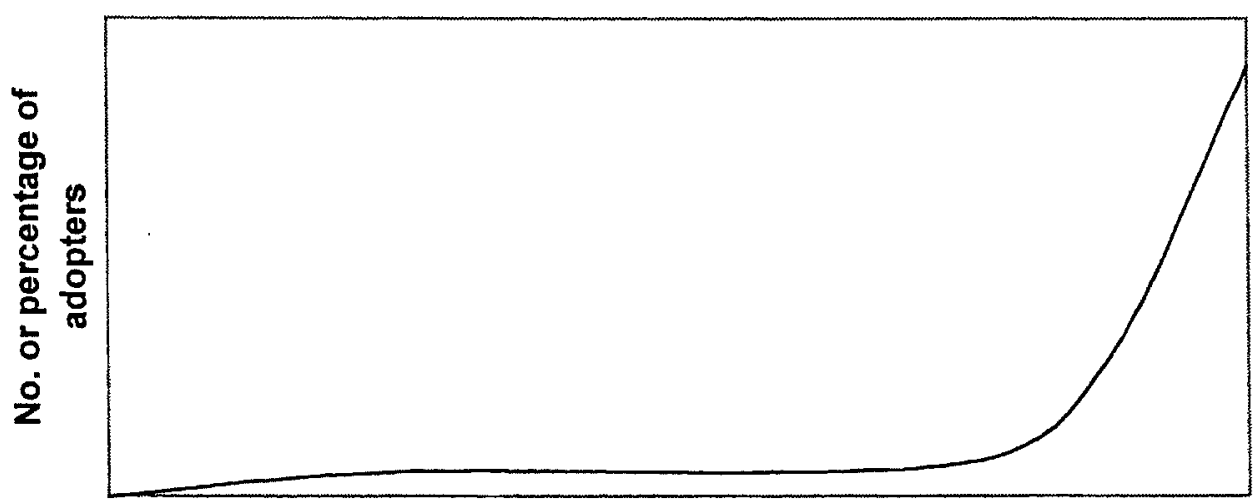

Time

Figure 2.3: The diffusion curve for a diffusion which takes a long time to take off

Such a situation as that illustrated by Figure 2.3 could be as a result of certain factors - political, sociological or administrative - that have led to an innovation either being given a 'push' through communication channels by change agents or perhaps as a reaction by adopters to some other 'event' which might or might not be directly connected to the innovation but has an effect on it. An example of this could be the medium of instruction issue in schools in Hong Kong discussed earlier. There was a long period during the 1980s and 1990s when schools were reluctant to change from the nominally English medium that they were using, to becoming officially Chinese medium. It took intervention from the government in order for the majority of schools to adopt the change from September 1998. Given that some schools have reportedly been switching back from Chinese to English medium to some degree, there may be some regression or discontinuance (Rogers, 
1995 ) evident which would cause a dropping off of the curve as indicated in Figure 2.4:

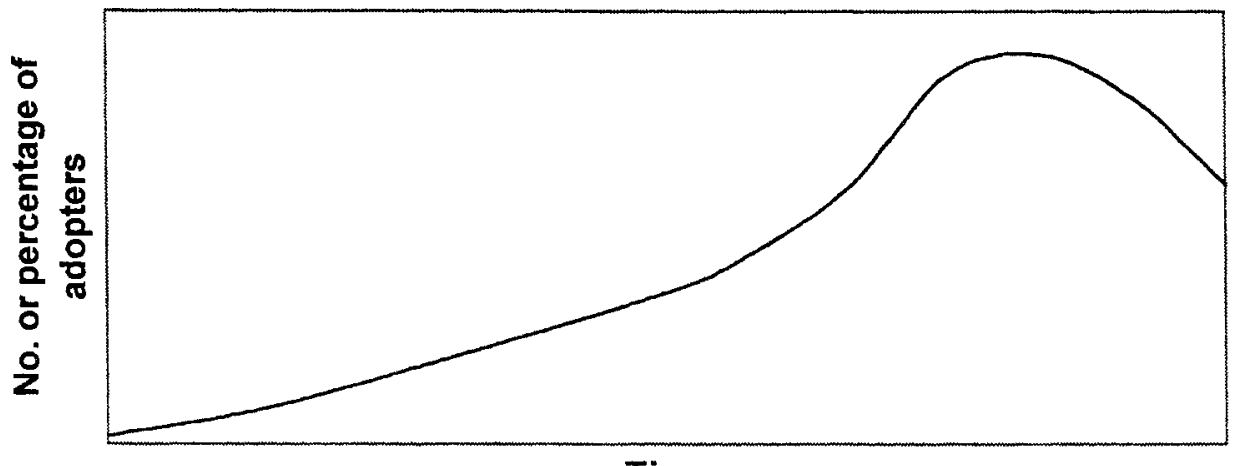

Time

Figure 2.4: The diffusion curve for a diffusion which takes a long time to take off before discontinuance begins

Plummeting, in contrast to a dropping off, would be a sharp fall in use, i.e., a reversion to the status quo prior to the innovation (Figure 2.5).

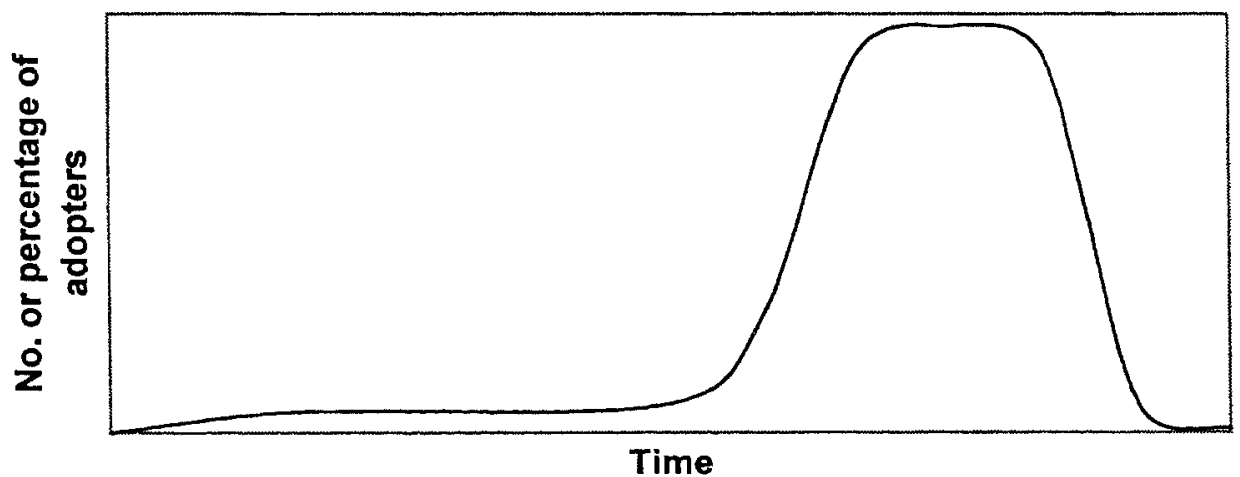

Figure 2.5: The diffusion curve for a diffusion which takes a long time to take off before plummeting

In the Hong Kong case, this could be exemplified by a rapid change to Chinese medium which survives only a short period before a rapid return to mixed-mode teaching. 
The diffusion curve can illustrate the rate of adoption of an innovation in a social system. The rate of adoption itself depends on a number of factors:

Variables Determining the Rate of Adoption (from Rogers, 1995, p. 207)

I. Communication Channels (e.g., mass media or interpersonal)

II. Nature of the Social System (e.g., its norms, degree of network interconnectedness etc.)

III. Extent of Change Agents' Promotion Efforts

IV. Perceived Attributes of Innovations

1. Relative advantage

2. Compatibility

3. Complexity

4. Trialability

5. Observability

V. Types of Innovation Decision

1. Optional

2. Collective

3. Authority

The above variables are those which Rogers (1995) puts forward as determining the rate of adoption of innovations. The first three of these variables, communication channels, the nature of the social system and the efforts of change agents, have already been discussed; the types of innovation decision will be examined shortly in terms of how innovations diffuse (section 2.4.3.7). In the next section, the specific attributes of the innovation itself, together with those of the innovation resource and user systems will be described in an attempt to determine why some innovations diffuse better than others.

\subsubsection{6}

Why are Innovations Adopted?

According to Rogers (1995), 49 to 87 per cent of the variance in the rate of adoption is explained by the following five attributes of the innovation: relative advantage, compatibility, complexity, trialability and observability; and these 
factors certainly go a long way towards determining why an innovation is adopted at all. The differing attributes of the innovation and of the adopters are key reasons why adoption occurs (Markee, 2001). Henrichsen (1989) ${ }^{17}$ puts forward a range of factors that hinder or facilitate change, specifically in respect of English language teaching:

\begin{tabular}{|c|c|c|c|}
\hline $\begin{array}{l}\text { Within the } \\
\text { innovation itself }\end{array}$ & $\begin{array}{l}\text { Within the } \\
\text { resource } \\
\text { system }\end{array}$ & $\begin{array}{l}\text { Within the intended- } \\
\text { user system }\end{array}$ & Inter-element: \\
\hline Originality & Capacity & Geographic location & Compatibility \\
\hline Complexity & Structure & Centralisation of power & Linkage \\
\hline Explicitness & Openness & administration & Reward \\
\hline Relative & Harmony & Size of adopting unit & Proximity \\
\hline advantage & & Communication structure & Synergism \\
\hline Observability & & Group orientation and & \\
\hline Flexibility/ & & tolerance of deviancy & \\
\hline adaptability & & Openness & \\
\hline Practicality & & Teacher factors & \\
\hline Trialability & & Learner factors & \\
\hline Status & & Capacities & \\
\hline Primacy & & Educational philosophy & \\
\hline Form & & Examinations & \\
\hline
\end{tabular}

Henrichsen (1989) includes factors inherent in the innovation itself, those in the system from which the innovation originates, those in the system into which the innovation is diffused and what he calls 'inter-elemental' factors, which exist between rather than within the different stages of the diffusion process. These factors will later be applied to the Hong Kong case to illustrate both their significance in the diffusion process generally and the nature of the changes taking place there at that time.

\section{Characteristics of the Innovation}

Originality. The degree to which an innovation is seen as being 'original' can have a significant effect on its potential to be adopted. The innovation may be 
invented locally with no prior model or prototype on which to base it, adapted from another innovation that may have been partially successful or borrowed from another innovation without very much change (Henrichsen, 1989). The extent to which any change is seen as something 'foreign' being foisted onto the local system, particularly by a colonial power, could lead to dissension by potential adopters and questions of compatibility caused by differences in culture.

The complexity of an innovation, taken to mean how difficult an innovation is to understand and use (Rogers, 1995), can be broken down into different levels, such as technical, organisational and intellectual complexity, as well as its radicalness (Henrichsen, 1989). For example, the resistance to the use of IT by some teachers could partly be explained by its perceived complexity. If teachers feel that they do not understand how to use the new technology or how to exploit it for use in their teaching, then they will avoid using it at all (Pennington, 2001). Related to this is the amount of explicitness inherent in the innovation. If the goals and rationale of the innovation are not clear then it becomes more difficult to understand and to implement.

If teachers are to adopt an educational innovation, they must be able to see its relative advantage, in other words, it must be seen to be 'better' than the idea it supersedes (Rogers, 1995). Allied to the relative advantage of an innovation is its observability. If the benefits are visible to potential adopters then they are more likely to accept and use the innovation (Henrichsen, 1989). The benefits of new curricula or approaches to teaching are not often (immediately) visible, unless, as in the case of the use of IT in schools, students can be seen to be improving their IT skills. Even then, the pedagogical benefits may not become apparent until later, if at all, or until the adopter has reached a stage in the diffusion process where $\mathrm{s} / \mathrm{he}$ is able to adapt or reconstruct the innovation to suit her/his own needs and teaching situation (Pennington, 1994a; 1995a; 1995c; 2001). This adaptability/ flexibility is another factor included in Henrichsen's framework. 
Practicality is another factor that applies particularly to educational innovations. If teachers do not know how a new idea, approach or system can be implemented in the classroom, then they will not have the motivation to try it out. There needs to be practical support, in terms of materials and assistance from colleagues, school and administration. If teachers are able to experiment with an innovation on a relatively small scale, then there is a chance that they will adopt it as a whole. In other words, if the innovation is divisible and has trialability, it is more likely to be adopted in stages. If the innovation is trialable, then adopters may be persuaded to try it rather than waiting until the beneficial effects of adoption become more observable (Henrichsen, 1989). If an innovation has status it can also be particularly appealing. In Hong Kong, as the English language has always been associated with status, any innovation which is seen to be promoting English has that associated status.

The importance of timing of an innovation cannot be overlooked; in other words, it should have primacy. People will tend to stick with whatever innovation they adopted first and this may form a barrier to new innovations (Henrichsen, 1989). The final characteristic of the innovation listed by Henrichsen (1989) is form. In terms of education it means that innovations that are formalised, in particular those that result in published texts have a better chance of being adopted than those that consist of abstract philosophies or approaches. Textbooks and materials are observable, concrete and practical ways of gaining information about teaching.

\section{Characteristics of the Resource System}

Henrichsen (1989) describes four characteristics of the resource system that promote an innovation. They are capacity, structure, openness and harmony. Firstly, the system must have the capacity to control the resources necessary, including the relevant communication channels, to ensure that the innovation is given the freedom from restriction and the support needed for it to be diffused successfully. Educational innovations in Hong Kong are normally developed and 
implemented by the government in the form of the Education and Manpower Bureau (EMB) via its agents, the Education Department (ED), which includes the Curriculum Development Council (CDC), and the Examinations Authority (HKEA). In addition, textbook publishers play a key role (and have a vested interest) in determining how subjects are presented (Morris, 1995). These public bodies and private concerns possess the power and the resources to implement change. They also have a high degree of structure, which is important in terms of division and co-ordination of effort (Henrichsen, 1989). The primary concern of these groups when influencing curriculum decisions is described by Morris (1995, p. 103) as follows:

\begin{tabular}{|l|l|}
\hline Group & \multicolumn{1}{|c|}{ Primary concern } \\
\hline EMB & $\begin{array}{l}\text { To be able to demonstrate that the government has a } \\
\text { policy designed to address all foreseeable educational } \\
\text { problems and to be able to control any part of the system } \\
\text { which might threaten its status. }\end{array}$ \\
\hline ED & $\begin{array}{l}\text { To implement policy, minimise any possible conflicts or } \\
\text { problems and ensure a smooth-running bureaucracy. }\end{array}$ \\
\hline HKEA & $\begin{array}{l}\text { To provide a fair and objective system of public } \\
\text { assessment. }\end{array}$ \\
\hline Textbook publishers & To maximise sales and profits. \\
\hline
\end{tabular}

However, (as will be discussed further below) these groups sometimes find that they have conflicting interests and often act to protect them, resulting in failure to implement initiatives as they have been intended.

Openness is a characteristic perhaps not so immediately applicable, particularly to the Hong Kong government, if one defines openness, as Henrichsen (1989) does, as being willing to listen and be influenced by user needs as well as being open to 
new developments in technology. Morris (1995) described the Hong Kong colonial government's approach to education in the 1990s as follows:

In Hong Kong, the government is ... in a very difficult position. It wants to be seen to be responsive to social expectations and demands, but it also wants to make sure that policies do not emerge which do not support or which threaten its status. Consequently it has developed a fairly complex system for consulting and obtaining advice from the public. Examples of consultative committees in the area of the curriculum are the Curriculum Development Council and Subject Committees, the HKEA Committees, the Education Commission and the Board of Education. It also uses a range of techniques to control and influence these bodies. These include: setting up a number of committees to study the same topic, careful control of the agenda, careful selection of committee members, ignoring recommendations which are not consistent with the government's view or implementing policies in a way which is contrary to that recommended. (p. 100)

After the changeover in sovereignty, the structure described by Morris did not change. The same bodies were responsible for formulating educational policy as before and they appeared to be doing it in much the same way. The government has to be seen to be open to the needs of the population, but the population's needs and aspirations vary widely, as has been described. Students, teachers, parents, principals, school governors, teacher trainers and others in the field all have different perspectives on the ways that education should be organised (Evans et al., 1998) and how and when the innovations should be introduced. Governments often try to compromise and choose 'the path of least resistance' when deciding education policy, seeking, as Morris (1995) says, to preserve their status and, in some cases, social order.

The final characteristic of the resource system of an innovation put forward by Henrichsen (1989) is harmony between the different people and groups within the system responsible for generating the innovation. Poor social relations between these elements can seriously undermine efforts to have an innovation adopted. In education in Hong Kong, the groups responsible for changes in curriculum - i.e., the government agencies, textbook publishers as well as local 
and overseas consultants connected with tertiary institutions - need to maintain good relations in order to facilitate educational change or reform. Unfortunately, this has not always been the case and disagreements between groups have resulted in the failure of educational reforms. Morris (1995) gives the example of the Expatriate English Teachers Scheme, in which native English speaking teachers were recruited into Hong Kong secondary schools to help improve English standards during the 1990s. The Education and Manpower Bureau wanted the scheme, as it was highly visible and reflected to the public that something was being done to tackle the perceived problem of falling standards of English amongst school students, and was able to get it introduced through the Education Commission and its Report No. 3 (Hong Kong Education Commission, 1988). However, the Education Department, which did not favour the initiative, perhaps due to its expense and implications vis-à-vis local teachers (the expatriate teachers were paid housing and other allowances that local teachers were not entitled to) managed to 'implement [this policy] in ways that did not maximize [its] chances of being successful in the long term' (Morris, 1995, p. 104). Thus, an innovation can be undermined for various reasons even by stakeholders who are closely associated with it.

\section{Characteristics of the Intended-User System}

The characteristics of the system through which an educational innovation is to be diffused and by which it is to be adopted are many and diverse and include physical (geographical location and size and the capacities of the adopting units to support an innovation), structural (centralisation of power and administration, communication structure and examinations), cultural (group orientation and tolerance of deviancy, openness, and educational philosophy) and characteristics of the groups of individuals within the system (teacher and leamer factors) (Henrichsen, 1989). The user system of education in Hong Kong is as varied and complicated as one might expect considering the political, economic and social changes that were affecting it during the period leading up to and prior to the 
changeover in sovereignty and considering its long-term political, social and linguistic history (Pennington, 1998a, b). The nature of Hong Kong and its education system are discussed at length in other sections of this review.

\section{Inter-Elemental Factors}

The factors that exist between the elements of the diffusion process have been termed by Henrichsen (1989) compatibility, linkage, reward, proximity and synergism. Compatibility needs to be considered between both the innovation and its intended users and between the resource system and the intended-user system. If the user and the innovation itself are not compatible, i.e., it is not consistent with their existing values and past experiences, then a great deal of

effort has to be put in to overcome the differences. The degree of compatibility between the resource system and the intended users affects the diffusion process greatly and is related to Rogers' (1995) conceptions of relationships between and within social systems during the diffusion process, which can be either homophilous or heterophilous. Individuals within homophilous social systems tend to share common beliefs, values, education, social status and other characteristics. In such homophilous groups, ease of communication and role expectation will foster diffusion of innovations throughout the social system. Likewise, if the resource and user systems are homophilous with respect to each other, more successful adoption will result. The resource system is that responsible for originating the innovation and the user system is that of the potential users of the innovation and through which the innovation is diffused. Heterophily of systems will result in difficulties in the process of diffusion, which may even be insurmountable. The nature of social systems within English language education and how the relative degree of homophily affects change, particularly in teachers, are discussed in section 2.5.

The linkages between the resource system and the user system refer to the amount of contact between the two and is of obvious importance to furthering diffusion of 
innovations. In language education, for instance, support networks such as professional teacher organisations, tertiary institutions, educational agencies and professional journals are all important in maintaining contacts between the parties involved in educational reform (Henrichsen, 1989). In Hong Kong, as described by Morris (1995), the Education Department and the Examinations Authority through their various subject committees, the membership of which is purposely representative of education officials, university faculty, school principals and teachers, controls education and any reforms to it. In addition, public consultation is sought when new initiatives such as the medium of instruction or curriculum reform are proposed by posting consultation documents on the Internet or making them available through other channels (see Hong Kong Education Department, 1997a, for example) and by holding public forums in which members of the public as well as teachers and other stakeholders are invited to quiz representatives of the resource system.

That reward serves as a motivator for adoption of innovation may seem to be an obvious point to make. The rewards can be in numerous forms including profitability, recognition by colleagues, personal satisfaction and feedback from colleagues (Henrichsen, 1989). Although there is little obvious scope for profitability in state-run education, there is such scope in the private sector, especially through private tuition schools and centres. Such enterprises have proved successful in Hong Kong, where good performance in examinations is highly valued (South China Morning Post, 25 August, 1997). Unfortunately, as Henrichsen (1989) points out, "it is not uncommon for innovation decisions to be negatively reinforced' (p. 93). Most often teachers have no choice but to adopt a new curriculum or accept new teaching mandates. Both positive rewards and negative incentives can lead to an increased rate of diffusion and a greater likelihood of adoption (Rogers, 1995).

Proximity literally means how close the resource and user systems are in time, space and context (Henrichsen, 1989). The closer together the two systems are in these senses, the more likely they are to be familiar with and similar to each other, 
thus enhancing the probability and the process of diffusion. If a resource system with its own characteristics, values and ideologies is distant - where 'distant' is taken not only in the geographical sense - from the user system, such as in the case of a colonial power overseeing a colonised territory, diffusion will necessarily be more difficult than if the resource and user systems share the same 'local' environment.

The final inter-elemental factor identified by Henrichsen (1989) is synergism, or working together, i.e., 'the number, variety, frequency and persistence of forces that can be mobilised to produce a knowledge utilization effect' (p. 94). It perhaps takes the other linkage factors one step further by describing the unseen forces that come into play when individuals and groups come into contact with some kind of common interest or goal in mind.

It is now clear that to determine why an innovation is adopted (or not) involves consideration of a large number of factors. A number of models or theories of change based on the principles of diffusion of innovations have been developed which attempt in different ways to explain how innovations are diffused. Three such models are presented here and relevant features of these models will later be applied to the Hong Kong situation (see Chapter 6) towards the goal of explaining the effects that the changing political system had on the educational innovations that were in the process of being diffused at the time of the handover.

\subsubsection{How are Innovations Adopted?}

To examine how innovations are diffused and subsequently adopted or rejected, three different models, which have each been developed to describe change in educational contexts, will be discussed. The first is Henrichsen's (1989) Hybrid Model, developed to describe the implementation of a new approach to the teaching of English in Japan. The second, by Pennington (2001), is a description of innovation-adoption cycles as applied to the adoption of Information 
Technology by teachers of English. The third, also by Pennington (1995c, 1996, $1997 \mathrm{~b}$ ) is an analysis of teaching frameworks and how they evolve to incorporate innovations.

\section{The Hybrid Model}

According to Henrichsen (1989), for a framework of innovation-diffusion to be comprehensive enough to be applied in all situations, the following criteria must be met: coherent framework, abstractness, completeness, relevance to directed change and cross-cultural applicability. Taking into consideration the work in sociology on diffusion of Everett Rogers and colleagues, ${ }^{18}$ as well as that in education of Jack Richards and others, ${ }^{19}$ Henrichsen developed what he termed 'The Hybrid Model' (being a hybrid of the models developed by these scholars and others) as a way to analyse the diffusion of English language teaching reform in Japan in the late 1950s and early 1960s. The Hybrid Model consists of three main phases: antecedents, process and consequences; the components of each can be seen in Figure 2.6. 


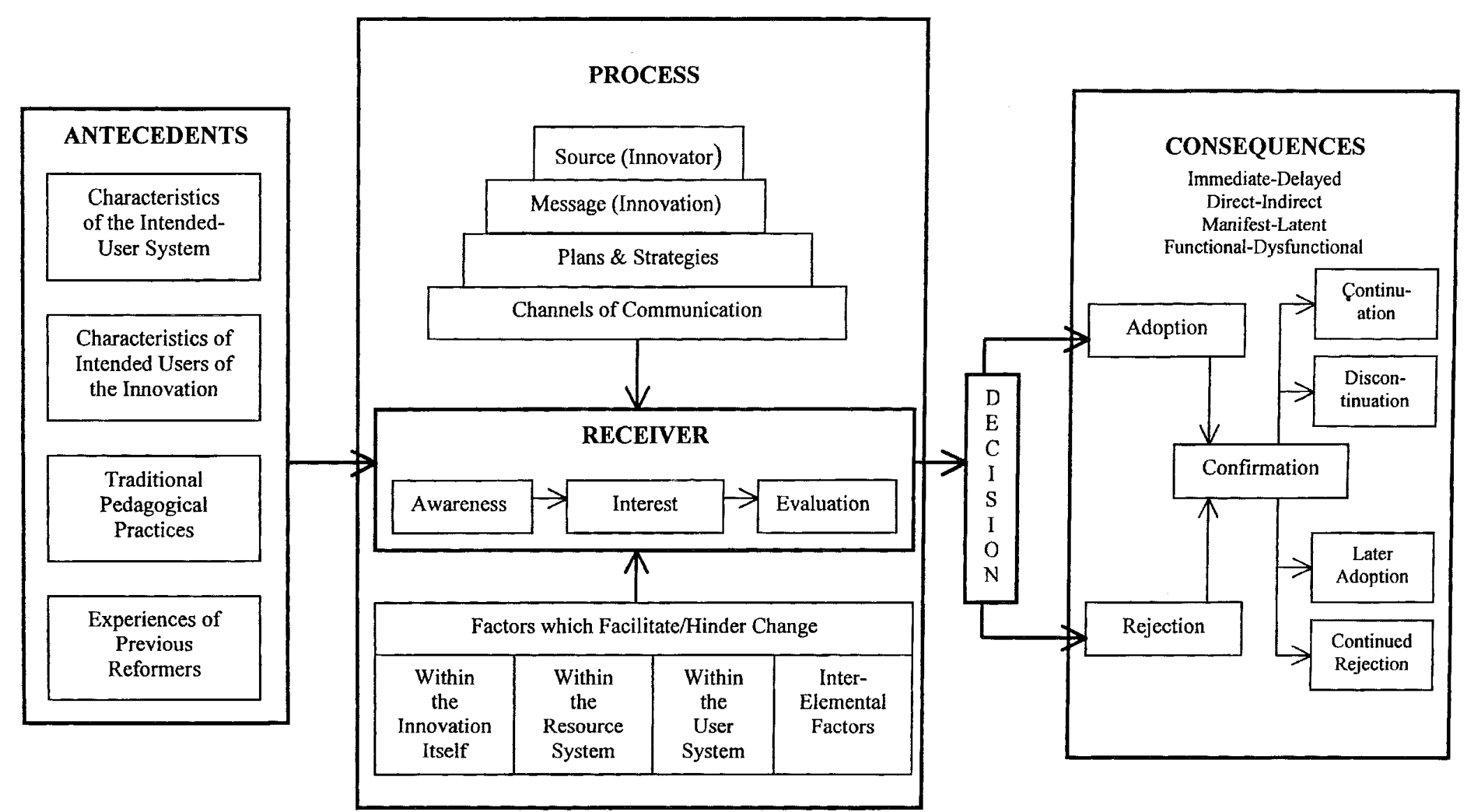

Figure 2.6: Henrichsen's Hybrid Model of Diffusion of Innovations in Educational Reform (Henrichsen, 1989, p. 80) 
The nature of the process of diffusion can be seen as a number of factors impacting on the receiver or potential adopter, who goes through the stages of awareness, interest and evaluation before making a decision whether to adopt or reject. After that is confirmation, which can lead to any of four consequences, continuation or discontinuation if adoption has occurred, or later adoption or continued rejection if the decision was at first to reject the innovation. Henrichsen (1989), following the work of Rogers and others, describes four different types of decisions:

- Optional decisions - the adopter makes an individual decision

- Collective decisions - groups of adopters make decision by consensus

- Authority decisions - made on behalf of adopters by superordinate individual(s)

- Contingent decisions - depend on prior decisions

The type of decision taken will be dependent on the nature of the innovation and of the user system and it is likely that there will be more than one type of decision involved in each adoption case. To illustrate the ways that different types of decision are involved in the diffusion of an innovation, the five main reform initiatives to affect English language teaching in Hong Kong during the 1990s and early 2000 s are discussed.

Although communicative language teaching $(\mathrm{CLT})^{20}$ was officially proposed as the means by which English should be taught in secondary schools in 1983 (Curriculum Development Committee, 1983), and was thus an authority decision, it has generally been accepted that it has had little impact on Hong Kong classrooms (Evans, 1996). Traditions of transmissive, examination-oriented teaching have persisted such that the teacher, even if s/he wishes to adopt the CLT approach, is constrained by the system and is only able to use communicative activities on a piecemeal basis (Evans, 1996; Pennington, 1995c; Pennington \& Richards, 1997; Pennington, Richards, Urmston \& Lee, 1996; Pennington \& Urmston, 1998; Young \& Lee, 1987). A central fact is the contingent nature of the 
decision to adopt CLT, i.e., its limited adoption as a result of the preponderance and domination of traditional teaching modes. ${ }^{21}$ In spite of this authority decision, collective decisions were made by groups or 'panels' of teachers within individual schools as to whether or not they would adopt CLT. Traditionally, teachers in Hong Kong schools are provided with a syllabus and a book list and are under some pressure to adhere to it. This to a great extent determines their approach to teaching, although some have limited flexibility to adopt their own teaching approach, as long as the items in the syllabus or units in the textbook are covered. Consequently, optional decisions have also played a role in the adoption or lack of adoption of CLT in Hong Kong classrooms. Given that CLT has had little impact, it appears that the authority decision was overridden by contingent, collective and optional decisions in a different direction.

The change in the medium of instruction in Hong Kong schools from predominantly nominally English to Chinese (Cantonese) came about after a long period in which the actual language being used in classrooms had been shifting from English to Chinese (Johnson, 1998; Johnson \& Lee, 1987; Li, 1999; Pennington, 1995a; Walters \& Balla, 1998). Yet when the government imposed its Firm Guidance and those schools which could not demonstrate that the majority of their students would benefit from an education through the medium of English were directed to use Chinese, beginning from Form 1 in September 1998 (Hong Kong Education Department, 1997b), there was much resistance from schools and from parents (Hong Kong Standard, 2 December, 1997; South China Morning Post, 15 December, 1997). The innovation decision, therefore, was made on behalf of schools by the government and as such was an authority decision, although it could also be said to have been contingent as a consequence of the gradual change in the language of instruction already occurring in schools. Collective decisions by parents, school governors and principals had an effect on schools deciding whether to apply to be English medium or not, or whether to appeal against the decision laid down by government, and teachers, specifically English teachers in Chinese-medium schools, decided for themselves (optional) the relative amounts of English and Cantonese that they use during their lessons 
(Pennington, 1995b; 1998). Thus, while the de facto movement was towards an increasing amount of Cantonese in the classroom, the de jure decision to impose Chinese medium on a wide scale was resisted at a collective and an individual level.

The decision to implement the Target Oriented Curriculum (TOC) was made by government once the consultants had developed the materials and guidelines for implementation, and it was launched into all schools from 1995 (authority decision). ${ }^{22}$ Once schools were faced with having to use the TOC, it became a collective decision on the part of teachers, panel chairs and school principals as to the degree to which the 'new' curriculum would be followed, in opposition to the more traditional approaches that schools and teachers were used to (contingent decision). That the initiative has failed to become adopted, at least on a systemwide basis, can be attributed to teachers' (optional) decisions to reject it due to its more troublesome aspects (excessive administration, incoherence and lack of relation to previous initiatives) (Adamson et al., 2000).

The decision to run the Native English Teachers (NET) scheme $e^{23}$ came again from government (authority decision), despite resistance from local English teachers (collective decision), who resented the NET teachers' extra benefits, as well as the implied suggestion that they themselves were not up to the task of teaching English to Hong Kong students (South China Morning Post, 20 October, 1997). The scheme was (or rather, is) a continuation or a revival of the Expatriate English Teachers Scheme (contingent decision) and was introduced to counter fears expressed by the public concerning reduced exposure to English of students in Chinese-medium schools. It was also optional as school principals and panel chairs could decide how much and how to use them - either as a speaking coach or as a teacher with a full range of teaching duties to perform.

The fifth educational reform affecting English language education in Hong Kong, the Language Proficiency Assessment for Teachers (LPAT) initiative, ${ }^{24}$ arose 
out of recommendations made by the Education Commission in its Report No. 6 (Hong Kong Education Commission, 1995), that:

\begin{abstract}
... minimum language proficiency standards should be specified for all new teachers to ensure that they can teach competently through the chosen medium of instruction; and that all new Chinese or English language teachers should, as from a certain cutoff date, be required (a) to have a high level of academic attainment in Chinese or English, and (b) to have completed satisfactorily professional training in the teaching of Chinese or English as a subject. (p. viii)
\end{abstract}

From 1995 until the initiative was finally launched in March 2001 (authority decision) with the first administration of the Language Proficiency Assessment for Teachers (LPAT) in English and Putonghua, there was much debate as to whether or not there should be a requirement, not only for new teachers but for all teachers of English and Putonghua, to reach the benchmark level. ${ }^{25}$ Teachers, represented by the Professional Teachers Union, strongly objected to having to face the pressure of a test of their language proficiency or having to attend courses to prove that they had the proficiency to teach the language effectively (an opposing collective decision). As the cut-off date has been set at 2006, teachers still have the option (optional decision) to adopt this innovation up until that date. In fact, indications are that many will adopt a wait-and-see approach, thinking that the government may go back on its commitment and decide to relax the conditions of the assessment or even abandon it altogether. If this were to be the case, collective action by teachers will have played a large part in the ultimate fate of the initiative and may well reverse the authority decision. It was contingent on the previous poor standard of English teachers, e.g., based on decisions to allow people to teach English who did not have degrees in teaching English.

Henrichsen's Hybrid Model takes into account the great number of factors involved in the diffusion process and allows a systematic analysis of change leading to explanations of why innovations are adopted or not. It looks at change as a linear process with a clear beginning, middle and end. An alternative 
approach put forward by Pennington (2001) looks at innovation adoption in terms of cycles.

\section{Innovation-Adoption Cycles}

Pennington (2001), acknowledging the influence of Rogers (1995), describes innovation adoption in terms of three cycles:

- Phase 1 Cycle - Expansion of old forms (Continuity Cycle)

- Phase 2 Cycle - Expansion of innovation (Creativity Cycle)

- Phase 3 Cycle - Transformation of forms (Discontinuity Cycle)

In the first phase, the innovation is described as 'a new form added to an existing context' (p. 12), which is why it is called the Continuity Cycle. The new innovation is regarded as an additional resource rather than a replacement for an existing one and must be accommodated into existing knowledge structures. For this reason, users may neglect or fail to notice the true nature of the innovation, tending to concentrate on what is familiar about it. They do not yet have the experiential framework to incorporate it.

In the second phase, the Creativity Cycle, the innovation is expanded and developed as it becomes naturalised, in the sense that users become familiar with it and adapt it to fit the needs of the user system. As the process continues, the innovation may become 'completely incorporated into the context of adoption and uses up its innovative potential, thus leaving a "slot" for a new innovation to take centre stage' (Pennington, 2001, p. 20). In other words, the innovation is no longer new and has been absorbed by the system into which it was diffused.

Following on from the Creativity Cycle, an additional phase is possible. That is the Discontinuity Cycle, so called because there occurs a discontinuity or break away of the innovation from its original context and new forms and functions 
develop. These may be hybrid forms which become markedly different from the original form of the innovation. In this case, there needs to be an assimilation of the new form or function into the newly created context, which may have any of a series of outcomes (Pennington, 2001):

(i) There may be rejection of the innovation as people become aware of what has been lost or given up by (formerly) adopting the innovation.

(ii) People may be led to recycle to an older view as the innovation might be seen as too idealistic. The desire will be to revert back to something more tangible and workable.

(iii) Existing forms may be replaced completely by the innovation.

(iv) The innovation may be replaced by another form, either old or new.

(v) There may occur a reconstruction of the innovation and/or its context to take account of new features.

The above possible outcomes can lead into new cycles as the context of the user system changes under the influence of successive innovations. The notion of change being cyclical is expressed here by Pennington rather than the more linear model of Henrichsen. Pennington's insights can be extended to the present case by noting that such cyclical change can occur at a sub-system level within a systemic condition of stability or continuity. A changing context as a result of changing political and social structures will certainly influence the ways, including the changing rates of diffusion and the changing directions of diffusion that the innovation-adoption process proceeds. By looking at patterns of diffusion of reforms and relating them to the education system in Hong Kong, it will be possible to qualify the effects that the changeover in sovereignty had on this sector of society. Before doing that, a more detailed examination of possible change structures is needed. 
Pennington (1996) looked at the ways that language teachers change in response to innovations through evolutions or modifications of the frameworks according to which they think and act in teaching contexts. These frameworks are conceived in cognitive and behavioural terms as consisting of three dimensions of teaching, which Richards and Rodgers $(1982,1986)$ had characterised as approach, design and procedure (see 2.4.3.3), the components of what they referred to as 'teaching method'. In Pennington's view, such methods can be described as a conceptual hierarchy of approach (one's overall philosophy of teaching), dominating design (one's curriculum, whether implicit or explicit), dominating procedures (one's teaching behaviours), i.e.,

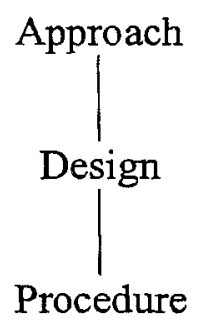

Teachers' frameworks, in Pennington's (1996) view, can change at any of these levels as a result of a new type of approach, design or procedure becoming attached to the framework from outside and modifying it. As Pennington (1996) explains:

When a teacher's teaching framework is modified in response to new input from a different teaching framework at the level of approach, design and/or procedure, it can be said to incorporate some aspect of that framework by any of the methods of learning ... i.e., accretion/augmentation, accommodation/subsumption, assimilation/evolution, or construction/revolution. In so doing the teacher's primary framework for teaching is enriched by a secondary framework. (p. 98)

This secondary framework can be described as a new cognitive-behavioural structure added to the teacher's primary framework, which may over time begin to 
infiltrate the old framework. Figure 2.7 illustrates four possible outcomes based on changes in teaching approach:

\begin{tabular}{|c|c|c|c|c|}
\hline \multirow[b]{3}{*}{ 1-A A2 } & \multicolumn{4}{|c|}{ A3 } \\
\hline & & \multicolumn{2}{|c|}{11} & \multirow{3}{*}{$\mathbf{A} 3-\mathrm{A} 1--\mathrm{A} 2$} \\
\hline & $\mathrm{A} 1--\mathbf{A}^{\prime}--\mathrm{A} 2$ & A1 & A2 & \\
\hline & 11 & 1 & 1 & \\
\hline $\mathrm{D} 2$ & D2 & D1 & D2 & D3 D1 - D2 \\
\hline 1 & 1 & 1 & 1 & 1 \\
\hline P2 & P1 & P1 & P2 & P3 P1 -- P2 \\
\hline ugmented & (ii) Assimilated & (iii) $\mathrm{H}$ & & (iv) Mutated \\
\hline pproach & Approach & & & $\begin{array}{l}\text { Method } \\
\text { (Approach- } \\
\text { based) }\end{array}$ \\
\hline
\end{tabular}

Figure 2.7: The formation of new teaching frameworks (Pennington, 1996, p. 100)

$$
\text { (Features: } \mathrm{A}=\text { Approach, } \mathrm{D}=\text { Design, } \mathrm{P}=\text { Procedure) }
$$

$\underline{\text { Key }}$

$\mathrm{A} 1, \mathrm{D} 1, \mathrm{P} 1=$ Old framework

$\mathrm{A} 2, \mathrm{D} 2, \mathrm{P} 2=$ New framework

$\mathrm{A}^{\prime}, \mathrm{D}^{\prime}, \mathrm{P}^{\prime}=$ Assimilations of features of two frameworks, $\mathrm{A} 1$ and $\mathrm{A} 2, \mathrm{D} 1$ and $\mathrm{D} 2$, $\mathrm{P} 1$ and $\mathrm{P} 2$

$\mathrm{A} 3, \mathrm{D} 3, \mathrm{P} 3=$ Hybrids or mutations of two frameworks, $\mathrm{A} 1$ and A2, D1 and D2, P1 and P2

Boldface $=$ Dominant or primary framework

- $\quad=$ Connection within a framework

$=$ Connection to a mutated or subordinate structure

From Figure 2.7, the following possible outcomes are illustrated:

(i) The old framework may be 'shadowed' by a new framework through the addition of a new feature at the approach (belief), design (curriculum) or procedure (behaviour) level (Figure 2.7 (i) shows the example of an augmented approach, i.e., where a teacher's belief system or teaching philosophy has been expanded by some new views (beliefs or values) based on knowledge of a new method or teaching innovation). Eventually, the influence of the additional feature may grow to cause changes at the other levels resulting in a gradual change in structure. 
(ii) The original framework may become substantially changed under the influence of new frameworks through assimilation of new features. A new framework evolves as a combination of old and new structures. In the example, a teacher's adoption of new beliefs results in a new set of beliefs or philosophy of teaching (approach) bridging between the (still) separate curriculum and procedures of the teacher's primary framework and the new framework.

(iii) New hybrid frameworks may develop in which two original frameworks become equally dominant and result in a higher-level synthesis that allows them to be joined conceptually - e.g., by a generalisation at the level of philosophy or approach.

(iv) A completely new framework may result from contact with another framework which is a mutation of the old but with none of its original features. In this case, a whole new method results - in the figure illustrated - from a new belief system growing out of two frameworks, both of which have become backgrounded in comparison to the dominant framework.

The structures shown and described above are a way of looking at how individual teachers respond to innovations at the conceptual level and how innovations can lead to changes in conceptual frameworks, which are necessary if the innovation is to be adopted totally or in part. Non-adoption of an innovation would mean that information about the innovation had failed to get through the teacher's cognitive (or cognitive-effective, Pennington, 1994) filter, i.e., it had been rejected at some point as being too incompatible with the teacher's existing framework at any of the levels of approach, design or procedure and hence there had been no augmentation or assimilation of new features of method, no hybridisation and no mutation of new frameworks. For there to be no change in any of the features of the framework would seem to be unlikely, though Pennington $(1996,1997 \mathrm{~b})$ says it does happen. The question is to what degree change in one or more of the elements of the framework would percolate up or down to influence the others (Pennington, 1996), to the extent that the framework changes to accommodate the innovation at the approach, design and/or procedural level. 
Pennington (1997b) put these models of change into the context of language teaching in Hong Kong by looking at teachers' responses to communicative language teaching and the process approach to writing. The responses are categorised as augmentation, integration, reconstruction, replacement, crisscrossing, dual teaching, double-voicing, regression to context and oppositional stance. The last of the responses occurs when an innovation is rejected.

Augmentation can occur when a 'new' technique is added to a teacher's repertoire at the procedural level, such that the approach or design of their teaching does not change; it is more likely that activities or materials that are familiar to the teacher are used in a different way in an attempt to adopt a new method. Pennington (1997b) found that English teachers in Hong Kong are apt to use communicative activities on a piecemeal basis when they have the time and in order to add variety to their teaching. This sort of low-level, procedurally augmented system, is a common outcome in terms of educational reform in Hong Kong as changing the underlying approach or curriculum is difficult given the constraints present, such as set materials, an outcomes-driven philosophy and curriculum, large class sizes (Pennington, 1995c; Young \& Lee, 1987) and lack of opportunity and motivation to use the target language outside the classroom $(\mathrm{Li}$, 1999).

Integration, (combining assimilation and accommodation) involves a more substantive adoption of an aspect of another framework or method to the extent that some feature or features of the new method become(s) integrated into the preexisting framework. It is a stage further than augmentation, when assimilation or accommodation at the approach, design or procedural level occurs and the teacher 'blends' the new aspect into his/her existing framework. In this sense there is a greater degree of adoption and the new feature is beginning to influence the status quo.. An innovation or reform measure can become integrated into the teaching system if it is partially adopted and is an appropriate adaptation to context (Pennington, 1997b). 
Reconstruction relates to the kind of situation illustrated in Figure 2.7 (iii) or (iv) in which connections are made between existing approaches and new ones resulting in a mutation, a new synthesis, or a hybridisation of the two. If a teacher is introduced to a new framework, whether or not it is compatible with the teacher's existing framework, some change is likely to occur. As Pennington (1997b) explains:

... the very attempt to establish a link between two teaching approaches is likely to inspire a creative change process. Thus some teachers may react to a new approach by creatively developing some kind of syntheses of it with their old approach. In some cases, teachers may reconceptualise their teaching in light of the new teaching approach, creating an innovative hybrid of old and new approaches. (p. 17)

In the same way, an innovation introduced into a system may cause reconstruction to occur such that the innovation causes change within the system as it is partially or completely adopted. The old system and the new innovation combine but this time systemic change occurs. The introduction of an innovation into a system could be said to set off a 'chain reaction' or 'domino effect' in that change of the system begins to occur and subsequent innovations are diffused through a system which is now in a state of evolution. The culture of change has the potential to engender more change, making adoption of new innovations more likely.

Replacement occurs when a teacher replaces his/her teaching framework with a totally new one. This is not a common outcome but is possible. A teacher might decide that the approach or method s/he has been using is not proving effective in the context in which s/he teaches and therefore is prepared to abandon it altogether and try something new. Alternatively, the teacher may gain new insights into teaching from professional development through reading, attending conferences or talking with colleagues that stimulate her/him to make a change. Another possibility is that there may be an event that changes the teacher's whole outlook on teaching or maybe on life in general, something which acts as a strong catalyst to change. It may be that the potential or desire for change was present before, but constraints in the system kept it backgrounded. An event, be it 
personal, institutional or societal, could trigger the release of the shackles of constraint resulting in a radical change. That the changeover of sovereignty of Hong Kong could act as such a catalyst to change is one premise that this thesis is based on. Looking at how reforms are adopted (or not adopted) at a social-system level can indicate the extent to which the reforms were adopted by teachers and other individuals in the system. Similarly, examination of the adoption patterns of individuals in the system can facilitate an understanding of the diffusion throughout the whole system.

Pennington (1997b) describes criss-crossing as occurring when the classroom design and procedures that a teacher deploys are not linked to the approach that they characterise. Commonly in Hong Kong, teachers find themselves switching between communicative designs and procedures within a structural or functional approach and vice-versa as a result of the constraints under which they teach. Morris (1995) lists the constraints that affect the classroom pedagogy employed by teachers in Hong Kong as: textbooks, resource constraints, classroom control, examinations, subject cultures, school cultures, teacher isolation and career considerations. Because of these factors, teachers are unable to adopt, or lack sufficient pedagogical content knowledge to adopt a consistent approach, design or procedure in their teaching. As Pennington (1997b) remarks, the result is often that the teacher focuses on procedural concerns alone without having a clear sense of the approach or design they are using.

Dual teaching is another possible outcome when a teacher wants to marry one approach to another, but the two approaches are not compatible. Pennington (1997b) gives the example of a teacher who decided to abandon her product approach to the teaching of writing in favour of a more communicative, process approach. After some initial success with the new approach, the teacher found that it became unworkable in her teaching context and decided to revert back to the product approach. Then, as the reasons for her change in approaches resurfaced, she tried to marry the two approaches together. This also proved problematic as the combination of the two approaches was not satisfactory due to their 
incompatibility (Tsui, 1996). The attempted joining together of two incompatible approaches, or 'flip-flop' between them, cannot produce an integrated or new hybridised teaching method under these circumstances. As a pattern of change, this 'flip-flopping' describes what happens when a new educational reform is introduced which is seen as revolutionary and the solution to existing problems (such as TOC) but is so different from what already exists that for a time the new approach and the older ones sit uneasily together, whilst the education system has to flip between old and new in terms of resource allocation, teacher retraining, new textbooks and changed examination syllabuses. Such flip-flopping pendulum swings are characteristic of educational systems caught up in the polarising tendencies of political rhetoric (Pennington, 2002).

When teachers display double-voicing, they support a new approach in principle but fail to adopt it in practice, either due to perceived constraints preventing them from adopting it in their teaching context, or they feel that it cannot be applied in their culture. Support is often voiced for innovations in education without the intention of practitioners ever to implement them. This can be seen as an indication of a schism between teachers' ideal and actual views of teaching (Pennington et al., 1996).

When teaching constraints, such as those existing in Hong Kong (Morris, 1995), prove to be too much of a barrier to the adoption of a certain teaching approach, then there is likely to occur a regression to context on the part of the teacher. Pennington and Richards (1997) report a study of five graduates from the BATESL course at the City University of Hong Kong in their first year in secondary school classrooms (1994-1995). ${ }^{26}$ Instead of using the communicative language model they had been introduced to during their training, constraints resulted in those five teachers reverting to the use of a transmissional, productoriented model. The constraints were: heavy workload; the need to cover the set syllabus; lack of time; pressure to achieve good examination results; students' low motivation and ability; large class size; and logistical constraints resulting in complaints from other teachers should students make too much noise when 
involved in 'communicative' activities. Their teaching context became overwhelming for them: with their limited experience and low professional standing, they could not overcome its constraints (Pennington, 1997b; Pennington \& Richards, 1997; Pennington, Richards, Urmston \& Lee, 1996).

Finally, Pennington (1997b) postulates that in Hong Kong since the changeover of sovereignty, there has developed a reaction to what are seen as 'Western' innovations in education and in language teaching in particular as a result of a localisation of the culture in the transition to Chinese rule. Innovations that are seen as 'foreign', particularly British, in origin are deemed to be inappropriate to and incompatible with the Hong Kong Chinese way of doing things. Pennington calls this pattern oppositional stance. In this connection, Sweeting (1997) has observed:

There are some signs ... that individual groups connected with education policy are attempting at least to be seen to be considering carefully the further convergence of Hong Kong's education system with that of China and continuing ostensible divergence of Hong Kong's education system with that of the U.K. (p. 36)

'Imported' methods such as communicative language teaching (CLT) have been blamed for the fall in language standards and calls have been made for a return to a more grammar or form-focused approach so that Hong Kong can again produce university graduates who can use English accurately (South China Morning Post, 26 January 1999).

The link between English language teaching (ELT) and political and cultural influence, particularly as it applies to colonial and post-colonial cases, has been discussed by Pennycook (1994), who has examined the spread of English throughout the world and its reciprocal relationship with English teaching practices. From a historical perspective, Pennycook (1994) attributes the promotion of English, in part, to institutions such as the British Council, which has used ELT as a means to foster social development in developing countries around the world. In this respect, ELT practices are seen as a method of 
promoting British culture overseas. The British Council, prominent in ELT in Hong Kong as in many other Anglicised societies, has been charged by Pennycook as being expansionist in its push for English as a world language and for British culture, which, according to Pennycook (1994) have been for 'clear commercial and political reasons' (p. 150). In addition, not only has the promotion of English been part of the 'mission' of the British Council (and other organisations such as the $\mathrm{BBC}$ in Britain, and the Peace Corps and the Ford Foundation in the USA) so too has the promotion of particular approaches to English language teaching (Pennycook, 1994). In the case of the British Council, it was the communicative approach that was, and still is, seen as being the definitive way to learn and teach a language. This approach, and others that are said to be linked to Anglo centric or Western-based cultural and political ideologies, have come into conflict with local cultural practices in the many corners of the world in which they have been promulgated (Evans, 1997; Parakrama, 1995; Pennycook, 1994; Strevens, 1987).

In Hong Kong, ELT approaches, such as the communicative approach and others predating it such as audiolingualism, were seen as being 'modern', 'scientific' or 'innovative' at the time of their introduction and were embraced by the local educational system as the best way to teach English. That they might not be appropriate in the local culture was a possibility that was generally ignored. Taking the communicative approach as an example, the dependence of the approach on students' active participation and willingness to 'take risks' by making mistakes, so long as communicative functions are achieved, clearly does not match Chinese characteristics of conservatism and humility. Similarly, the monolingual approach to ELT of using only English in the classroom was at the core of Western ELT theory (Pennycook, 1994) but rather unrealistic in many non-English-speaking societies such as Hong Kong for the vast majority of its inhabitants. The discussion of the political influences on the adoption of innovations in language teaching needs to take into account the history of ELT and how it has been driven by Western and, in the case of Hong Kong, specifically British interests. At the time of the changeover in sovereignty, 
reactions to such interests began to surface and the rejection which Pennington (1997b) refers to as an oppositional stance are perhaps not surprising.

It has been suggested that the postcolonial government in Hong Kong, as part of a concerted effort to build up a cultural identity in the local population, pushed through the policy of using Chinese as the medium of instruction in schools in 1998, consistent with the long-standing recommendations of the Education Commission $(\mathrm{Ng}, 2000)$. In this case, the opposition was not to an innovation but to the English language, an important cultural symbol of the former colonial power. However, whilst moving towards majority Chinese-medium of instruction, the government did not lose sight of the importance of English as linguistic capital leading to both cultural and economic capital, as it maintained English-medium status in the most prestigious schools (Morrison \& Lui, 2000). This form of elitism is characteristic of colonialism, only in post-handover Hong Kong, the PRC had become the colonial power.

The purpose of the previous discussion of societal change and how it could be applied to the situation in Hong Kong during the period straddling the changeover in sovereignty has been to develop an understanding of the context in which English language teaching and the training of new English language teachers was being carried out at that time. The discussion can help to tease out patterns that can help to contextualise the research findings of this project and put them into a perspective from which conclusions can be drawn that can be applied to other, similar contexts. In this way, the present study may provide useful lessons for other second language contexts, while offering some insights at a more general level about the nature and spread of or resistance to innovations.

This section has examined the nature of Hong Kong society in terms of theories of social and political change, leaning heavily on diffusion of innovation theory. In order to attempt to put the effects of the changeover of sovereignty on education into a form through which observed changes in policy and practice as well as the attitudes, beliefs and approaches of teachers, specifically, language teachers, can 
be understood, it is necessary to look at the possible patterns of change. These patterns are those that have emerged from the previous discussion, i.e.:

(i) Resistance to anything new or non-traditional The oppositional stance in which anything regarded as contrary to prescribed and accepted norms is rejected as being unsuitable or inappropriate.

(ii) Regression to the past The recycling or reversion to an older view when an innovation is seen as not having the desired effect.

(iii) Retreat to a more conservative pattern When an innovation or change is deemed too idealistic or progressive and a retreat to a more conservative approach seems called for.

(iv) No change No reforms are introduced, or they are introduced but not implemented, and the status quo is maintained.

(v) Accelerated change Change already in the works occurs at an increasing rate as time progresses. Change begets more change and leads to a context within which change becomes both highly desirable and inevitable in the same or a new direction.

(vi) Major innovation A major change occurs in a relatively short time span which has a profound influence on the system.

(vii) Disorderly pattern of change and non-change occurring simultaneously A scenario in which changes are occurring on one level or in one sector while other levels or sectors remain unchanged or resistant to change.

(viii) A series of innovations in succession Such innovations may be linked together or have interactive effects between them. 
(ix) A switching or 'flip-flop' between patterns at different times As change patterns develop on the back of innovations, there can be a switching backwards and forwards between patterns under the influence of different innovations originating from different resource systems, each with different characteristics.

(x) More than one cycle of flipping to the opposite extreme and back within the time frame under study Similar to the situation above but with a clear polarisation of the system such that there is a complete switch between, for example, total acceptance of change and total rejection of it and then back again to total acceptance.

These patterns of change provide a means by which the educational changes affecting English language teaching and teachers in Hong Kong at the time of the transition of sovereignty can be characterised. Evidence of change in teachers and, through analysis and extrapolation, changes in the nature of, provisions for and approaches to English language teaching can then be placed into context, enabling inferences to be made as to the effects caused by the transition.

The next section will look in more detail at the teacher as the central player in the education system, examining his/her beliefs, approaches and practices and how these might be open to change in a changing environment.

\subsection{Teacher Change and Development}

\subsubsection{Pre-Service Teachers' Concepts of Method}

There is a growing school of thought that the prior experiences as a student which pre-service teachers bring to the classroom are the major factor shaping their teaching method. Teachers are coming to be recognised as individuals who enter teacher education programmes with prior experiences, personal values, and beliefs 
that inform their knowledge about teaching and shape what they do in their classrooms' (Freeman \& Johnson, 1998, p. 401). Golombek (1998) found that language teachers' 'personal practical knowledge' serves as a framework through which they make sense of their classrooms:

it filters experience so that teachers reconstruct it and respond to the exigencies of a teaching situation ... it informs practice by giving physical form to practice (p. 259).

In other words, it is a teacher's beliefs, assumptions and knowledge which determine how s/he will interpret events related to teaching, in both planning and implementation, and therefore the teaching decisions that are made and the changes that result (Aitken \& Mildon, 1992; Arias, 1994; Woods, 1996). Littlewood (1999) calls the knowledge and experience base of teachers, 'classroom schemata', and describes it thus:

These classroom schemata provide teachers with assumptions about how people learn, conceptions of what it means to be a 'learner' and a 'teacher', ways of deciding what to do in routine situations and predicting what the effects of these actions will be ... (p. 4)

Littlewood (1999) goes on to describe the teacher's 'mental map' of classroom reality, which is shaped by a number of sources, such as personal values and intuition, cultural and educational tradition, pre-service and in-service courses, ongoing experience and encounters. This mental map can be compared to the teaching framework concept developed by Pennington (1996) and discussed earlier (see 2.4.3.7) in relation to reactions to innovations, i.e.:

A teaching framework is a schema for thinking about and acting in learning contexts. It includes a personalized or subjective theory of the nature of language learning and language teaching as well as proceduralized routines for behavior and interpretation of classroom events. (p. 90)

Teachers' reactions to change depend on how changes are accommodated into their teaching framework, and they develop this framework in response to new 
experiences and information on a day-to-day basis in their teaching context at the levels of approach (philosophy or beliefs), design (curriculum or planning) and procedure (teaching behaviour).

Looking at the development of teachers' experiences, Calderhead and Robson (1991) followed twelve student primary school teachers through the first year of a BEd programme in the UK. They found that the student teachers held particular images of teaching mainly derived from their experiences as pupils, which were found to be highly influential in the interpretation of their course and of their classroom practice. Clift (1991) carried out a case study of the first two years of a teacher's experience of learning to teach secondary school English. Clift formulated three conclusions about her subject's knowledge development, which she identified as consisting of ten knowledge schemes that the subject drew upon as she worked through the various incidents that occurred during her teaching;

(a) Multiple schemata are called upon almost simultaneously when an English teacher begins interacting with students in classrooms; (b) these schemata are not equally well developed, and the gaps in knowledge become apparent when teachers are required to integrate across schemes as they put knowledge into practice, and (c) teacher preparation curricula are not designed to foster knowledge integration across schemes. (p. 364)

The schemes that Clift identified can be related to Pennington's (1996) teaching frameworks and also to the beliefs, assumptions and knowledge (BAK) that teachers use to interpret their teaching experiences conceptualised by Woods (1996). In terms of teacher preparation, both Calderhead and Robson (1991) and Clift (1991) found gaps between student teachers' existing knowledge and what they were 'taught' in their teacher training programmes. They also both maintained that there is a need for such programmes to better integrate the two, to foster the growth of student teachers' knowledge of teaching so that they will be able to adapt to a variety of teaching contexts.

If it is the assumption that pre-service teachers bring their prior knowledge and beliefs to the classroom, then the period in which they spend training to be a 
teacher should play a major part in shaping this knowledge and these beliefs. However, it is also felt that pre-service teachers' beliefs are relatively resistant to change (Farrell, 1999; Freeman, 1991). With this thought in mind, one may consider the question of how resistant to change the beliefs, assumptions and knowledge of a group of student teachers on a pre-service degree programme in TESL are likely to be as they progress through the course from being initially

school-leavers, to eventually serving teachers. ${ }^{27}$ Pre-service teachers are likely to have a number of concerns: about their own progress as students, about their survival as teachers, about their performance as teachers and about having a positive effect on students (Pigge \& Marso, 1997). These concerns may manifest themselves in this order as the teacher passes from being a student teacher to novice and then to experienced teacher (Fuller, 1969) or there may be elements of all of them present to varying degrees as the teacher progresses through his/her period of training.

\subsubsection{The Orientations to Teaching of Pre-Service Teachers}

The orientations to teaching of novice English teachers in Hong Kong secondary schools were divided into five areas by Pennington and Richards (1993) as the basis for the project 'Learning to teach English in Hong Kong: The first year in the classroom', i.e.,

- Language Use

- Lesson Planning and Decision-Making

- Teaching Approach

- Professional Relationships and Responsibilities

- Perceptions and Values

Taking each area in turn, it becomes clear that they encompass the major issues affecting teachers, whether novice or expert. 


\section{$\underline{\text { Language Use }}$}

Since the vast majority of teachers of English in Hong Kong are non-native speakers (NNS) of the language, their language use, particularly in the classroom but also outside of it, is of obvious importance. In a society as linguistically complex as Hong Kong (see section 2.3) the ways in which teachers make use of both the target language, English, and the vernacular language of the vast majority of both students and teachers, Cantonese, takes on added importance. As Pennington (1995a) remarks:

In Hong Kong, the teacher's habitual teaching pattern and the students' low language proficiency pressure towards use of the mother tongue when lesson material presented in English is not understood by the students. (p. 11)

Prior to 1997, the majority of secondary schools were 'Anglo-Chinese', in which the medium of instruction was purported to be English. In reality, the mother tongue, Cantonese was used in the classroom for various purposes (Johnson \& Lee, 1987; Li, 1999; Pennington, 1994a, b; 1995a, b; 1996; 1997a). Richards (1998, p. 7) lists some of the typical functions or speech acts that a second language teacher has to perform within the classroom:

- requesting, ordering and giving rules

- establishing attention

- questioning

- repeating and reporting what has been said

- giving instructions

- giving and refusing permission

- warning and giving advice

- giving reasons and explaining

Whilst beginning teachers of ESL in English-medium schools might be expected to start off by attempting to use only English in the classroom, there is evidence which suggests that they may soon resort to compensatory code-switching into Cantonese as a reaction to students' and teachers' needs as well as time restrictions (Pennington, 1995a). Furthermore, as the teacher learns to cope with 
the particular educational and institutional culture in which s/he teaches, compensatory code-switching may give way to more strategic code-switching, leading to a pattern of bilingual classroom language use that is both predictable and difficult to reverse (Pennington, 1995a). In a study of the language use of the cohort of student teachers who graduated from the BATESL at the City University of Hong Kong in 1994, Pennington (1994a, 1997a) found the following motives for both compensatory and strategic use of the mother tongue in English lessons:

\section{Compensatory Use of Mother Tongue}

General necessity or expediency

Teacher's need

Students' level

Students' communication difficulties

Difficult content/lexis

\section{Strategic Use of Mother Tongue}

Discipline

Explanation

Clarification

Efficiency

Classroom management

Reduction of psychological distance between teacher and students

Creation of a sense of belonging or safety

Nature of content

Subject-appropriate use of English

Maximum exposure to English

Research in other countries has shown that in bilingual classrooms, the pattern is for the second language to be used for whole-class instruction, i.e., the provision of 'content', and the first language for individual teacher-student interaction (Pennington, 1995a). This is a pattern also shown to exist in Hong Kong classrooms (Johnson \& Lee, 1987; Lin, 1990). In addition, strategic switching into the first language has been shown to be for reasons of giving instructions, maintaining discipline and explicating ideas (Johnson \& Lee, 1987) and for being more friendly towards the students (Lin, 1990).

Evidence exists which shows that progressively less English and more Cantonese is being used in classrooms in Hong Kong (Johnson, 1991), although it has also been shown that English teachers have been less 'guilty' of this and have been more resistant to the use of Cantonese and mixed-code in lessons (Johnson, 1998) 
than teachers of other subjects. In fact, research has indicated an acceptance of the use of Cantonese and mixed code in teaching by undergraduate students on a BA course in TESL compared with more experienced English teachers, who were more in favour of using the second language wherever possible (Pennington \& Balla, 1998). The section on language use in the present study will attempt to determine changes in the student teachers' attitudes towards the use of English and Cantonese to see whether they fit the patterns described or whether in fact, despite the disapproval of code-mixing in classrooms by the Hong Kong Education Department (Johnson, 1998; Li, 1998; 1999) and their subsequent moves to eradicate, or at least minimise, it (Hong Kong Education Commission, 1995), teachers are becoming more accepting of the need to utilise the mother tongue to make for more effective teaching of ESL.

\section{Lesson Planning and Decision-Making}

According to Yost et al (2000):

Teachers making decisions are basing their decision making on rational choices after carefully weighing and analyzing alternative courses of action. (p. 40)

Whilst this may be true for experienced teachers, who have knowledge of what the various 'alternative courses of action' may entail, can the same be said to be true for novice teachers? Whilst experienced or 'expert' teachers might be described as 'improvisational performers' (Galton, 2000), the novice has to rely to a greater extent on what $\mathrm{s}$ /he has planned in advance. As Woods (1989) points out, what a teacher does in the classroom is based on advance planning and decisions made while a lesson is in progress. Novice teachers may receive instruction in lesson planning during their course of training, but there are many things that can happen during a lesson that can force the teacher to change what s/he had originally planned to do (Woods, 1989). Furthermore, there is evidence to suggest that pre-service teachers are unable to integrate and apply to their 
teaching the pedagogical information they learn during their training unless they have sufficient guidance (Richards, 1998; Yost et al., 2000). In other words, they may have learned how to plan lessons but once in the classroom, carrying out those plans may prove to be more difficult than they might have anticipated, just as the NNS English teacher has to compensate and make use of the students' (and their own) first language when s/he finds that $s /$ he is not able to perform the necessary communicative functions in English alone.

Such compensatory code-switching is a form of behaviour that the teacher adopts within the classroom at a local level in order to solve problems in the short term (Pennington, 1995a); it is a form of on-the-spot decision-making or improvisation. As teachers become more experienced they learn how to respond to the performance of their students by drawing on their 'repertoire of teaching routines' (Richards, 1998, p. 114). The teacher makes many such interactive decisions in the course of a lesson. Richards (1998), in a study of in-service English teachers on an MATESL programme in Hong Kong, found that the teachers' interactive decisions could be classified into four categories: timing, affective factors, pedagogical factors and language factors. When comparing the more experienced teachers with their less experienced classmates, the latter were found to be more concerned with the timing of lessons, being less able to carry out their plans within the time available to them. In contrast, the more experienced teachers were better able to make on-the-spot decisions to modify their lesson plans for both affective and pedagogical reasons. Richards (1998) concludes that student teachers can learn to recognise what the explicit difficulties or challenges of a lesson might be and what adaptations might be needed by developing their pedagogical reasoning skills through discussion and collaborative planning activities.

If what novice teachers believe about teaching is based mainly on their experience as students, it is this experience that most probably reflects their decisions and actions and drives their major decisions and the practices (including their language use) which they adopt in the classroom (Stuart \& Thurlow, 2000). From 
this point of view, it is important for teacher educators and teacher training course developers to know how much influence their course has on teachers' decisionmaking and how flexible or improvisational they are prepared or able to be in their teaching. Given the constraints that teachers, especially new teachers, are under, i.e., inexperience, the need to be seen to be competent by their supervisor and the nature of the teaching norms, will the limited experience they have during practice teaching change their attitudes towards planning and decision-making or will they retain the beliefs they had as school-leavers? This study will attempt to find this out, and in the process, look at how responsive and improvisational teachers of English in Hong Kong actually are. The need to be flexible and adaptable with regard to lesson planning, language use and teaching approach are central dimensions of teaching (Richards, 1998, pp. 65-85) within the micro environment of the classroom. That this micro environment exists within the wider environments described in earlier sections such as institution, educational system and society means that being flexible in reaction to change in these environments is even more of a requirement of the teacher than might normally be considered.

\section{Teaching Approach}

It seems that most student teachers believe that no amount of teaching methodology taught in an education college can match what they learn from experience as teachers (and students) themselves and in real teaching situations (Ethell \& McMeniman, 2000; Freeman, 1991). This suggests that the reality of the classroom situation will have more effect on their approach to teaching than will the philosophies, methods and techniques to which they are exposed during their course of training. As former students of the secondary school system, they are well aware of the teaching environment that they will have to teach in, and hence the approaches that they believe are likely to be effective in it. The question to be answered is whether their training can in any way change the fundamental beliefs that they have regarding what approaches would be both successful and practical. 
Practicality has been shown to be perhaps the major concern of pre-service teachers and this generally involves the two key factors of maintaining discipline and motivating students (Stuart \& Thurlow, 2000). In order to accomplish the first, direct instruction is most often used as it ensures teacher control (Galton, 2000). For the second, novices are 'inclined to believe that there exists somewhere a set of guidelines, or even tips, which will enable them to survive as teachers no matter what the context of their teaching' (Galton, 2000, p. 5). So while they may not feel that they can adopt a particular teaching philosophy, such as communicative, interpretational or innovative approaches, they will look for specific lesson plans or activities that they think will motivate their students whilst at the same time maintaining discipline. In the study of five of the graduates from the first cohort of the BATESL course at the City University of Hong Kong in their first year as teachers, Pennington \& Richards (1997) found that the teachers did indeed diverge significantly from the communicative, process-oriented principles and practices emphasised on the course once they were in the classroom. In terms of changes to teachers' frameworks of teaching, the teachers underwent a regression to context in that they were oriented to the transmissional, product approaches of the Hong Kong educational context and the need to cover the syllabus they were given to teach whilst maintaining discipline in what were often difficult teaching conditions.

Pennington \& Richards (1997) felt that there were a number of reasons why these novice teachers, fresh from their course of training, diverged from what they had been taught. Firstly, the different cultures and backgrounds of their instructors on the BATESL course (expatriates with little experience of the Hong Kong teaching culture as well as Hong Kong Chinese with both overseas and local experience) led to inconsistencies in teaching philosophy and a lack of as strong an emphasis on communicative approaches as the course developers would have envisaged. Secondly, the teachers' prior experiences as students within the educational system, as already discussed, proved more resistant to change than had been hoped. Thirdly, the influences on the teachers during their practice teaching, i.e., supervising teachers, panel chairs (heads of department) and other colleagues 
served to reinforce the teaching norms and discourage innovative, 'alternative' approaches as being too risky and diverting attention away from the goal of preparing the students for examinations. Fourthly, the overwhelming constraints of the system, such as heavy workload, large class size and students' low English proficiency and motivation meant that, given their inexperience and lack of confidence, they were unable and unwilling to step out from the safety of what they were expected to do. Finally, their nearness in age to their students meant that they could in some ways sympathise with them in terms of the difficulties they were facing in learning the language, resulting in use of the mother tongue for support and in conflicts in their perceptions as to their roles as teachers and near-peers.

The section on teaching approach in the present study will try to find out whether the cohort which began their BATESL course just as those studied above were finishing theirs, changed their approaches to the teaching of English during the period of the course. A close examination of the changes undergone by the student teachers will allow for a deeper investigation of the influence that the course had and that similar courses have on potential teachers of English. It will also be interesting to see how the added factors of the great changes in the socio-political makeup of society impinged on them in comparison with their predecessors.

\section{Professional Relationships and Responsibilities}

The perceptions that student teachers bring to their practice teaching about teachers' relationships and responsibilities are often in conflict with the reality of the school and the pedagogical knowledge they acquire during their pre-service training. This means that student teachers need to overcome the 'culture shock' that they may experience while they attempt to construct their own teaching identity (Fairbanks et al., 2000). To help them to do this, it is important for them to develop relationships with other teachers so that they can see themselves as a member of staff. Unfortunately, what Hargreaves (1999) refers to as 'The Age of 
the Autonomous Professional' is still to a large extent the case in Hong Kong and most teachers teach 'in a box' (Hargreaves, 1999, p. 69, see also Bullough, 1989; Huling-Austin, 1992), meaning that they seldom share teaching ideas or discuss classroom management techniques. This can make it very difficult for practising teachers to learn the skills they need. Such teacher isolation can be explained partially by the fact that they often are unable to find the time to meet to discuss teaching matters. What time there is outside of the classroom is generally taken up with other duties, and 'teaching includes not only planning and presenting lessons but a host of additional tasks that impinge upon the daily routine: paperwork, after school meetings, bus duty, and parent conferences' (Fairbanks et al., 2000, p. 105). This is especially the case in Hong Kong schools as a full teaching load, large classes and the subsequent large piles of marking add to the large variety of other school activities they are normally expected to carry out (Pennington, $1995 \mathrm{c})$. It is not surprising that meeting with colleagues to discuss teaching ideas or strategies is considered a luxury. Meetings that do take place are normally confined to the beginning and end of the school year and are restricted to matters concerning syllabus, textbooks and assessment.

On a more informal level, teachers may discuss individual students or the relative merits of certain textbooks but they seldom talk about their teaching. There are two possible reasons for this. Firstly, the asking of advice from a colleague could be seen as a sign of weakness and an admission of failure on the part of the teacher concerned (Hargreaves, 1999; Huling-Austin, 1992). This attitude is prevalent in Hong Kong where people are especially concerned to avoid losing face in front of others. In addition, it is a characteristic of Hong Kong people, in the Confucian tradition, to maintain a conservative and humble image and so to avoid situations in which one might be accused of 'showing off'. A teacher wishing to share a successful teaching approach or strategy might be seen to be seeking self-glorification, which is frowned upon. Instead, teachers are expected to follow the rules and not disturb the status quo. 
Coming back to the roles and responsibilities that most teachers have to perform in the modern school situation, the question needs to be asked whether practising teachers should have to take up such additional tasks and whether it will have a positive or negative effect on their development as teachers if they do. HulingAustin (1992) thinks, for example, that beginning teachers should not be expected to take part in extra-curricular activities as this can interfere with the planning they need to do to ensure that their lessons run smoothly. On the other hand, as Fairbanks et al (2000) put it, 'novices learn through legitimate peripheral participation in the activities of the profession ... and engage in the actual work of the profession and have opportunities to learn its discourse, values, and skills' ( $p$. 104).

In addition, novice teachers need to pay attention to their own professional development if they are to pursue a career in teaching (Pennington, 1989). Research has found that pre-service teachers seldom participate in action research projects (Yost et al., 2000) and may feel that they do not have the time to attend seminars or workshops outside of their course of study or even read research publications. Once they project themselves into a future as a full-time teacher, they may well feel that they will have even less time and opportunity for this kind of professional development. The findings from the section on professional relationships and responsibilities should give some indication of the relative importance of these complicated facets of teaching within the Hong Kong education community.

\section{Perceptions and Values}

The final section of the questionnaire study deals with the pre-service teachers' perceptions and values. Pre-service teachers tend to be 'reluctant to challenge the status quo. Having been in classrooms for many years, they have internalised through an apprenticeship of observation [italics in original], many of the values, practices and beliefs of their teachers' (Stuart \& Thurlow, 2000, p. 114) through 
which they can end up believing that teaching is simply a matter of liking children and transmitting knowledge (Huling-Austin, 1992; Stuart \& Thurlow, 2000; Yost et al., 2000). It is these firmly established beliefs that form the basis for their actions and can be very resistant to the efforts of teacher educators to impress on them new and innovative philosophies and methods of teaching. Instead, they tend to stick to traditional notions of schools and schooling (Farrell, 1999; Yost et al., 2000). Consequently, if teacher educators wish to instil in their student teachers the capacity to embrace new ideas and approaches, they need to provide opportunities for them to measure how their beliefs compare with the philosophy of the teacher education programme (Yost et al., 2000) in which they are enrolled.

The BATESL course at the City University of Hong Kong was set up with two basic aims:

Graduates of the BATESL program are ... expected to be comfortable teaching according to the principles of communicative language teaching, while at the same time being able to adapt their teaching to student expectations and to the type of class they are teaching. They are also expected to be able to interpret what they have learned flexibly based on their own judgement. (Pennington, Richards, Urmston \& Lee, 1996, p. 19)

The opportunity for the student teachers to consider and formulate new beliefs will most likely come during or as a result of internment, during which they observe and assist classroom teachers, and practice teaching. Although these periods are relatively short (approximately three weeks each), the impact that they have on the student teachers should be discernible by monitoring changes in their responses to questions which are aimed at testing their fundamental beliefs and values.

According to Richards (1998):

[T] eachers posses rational orientations toward teaching as well as personal beliefs about what constitutes good teaching, and these lead them to try to create specific conditions in their classrooms. These conditions reflect the teachers' view of the role of the 
teacher and of the learners, their beliefs about the kind of classroom climate they think best supports learning, what they believe constitutes good methodology, and the quality of classroom interaction and language use they seek to achieve. (p. 58)

Richards (1996b, 1998) used the term maxims to describe the 'rules' or 'principles' that guide teachers in the actions they take within the classroom and these are based on teachers' individual philosophies of teaching, personal beliefs and value systems, and are shaped by their experiences in classrooms (as students and teachers) and from their teacher training. However, the degree to which teachers are able to employ their teaching maxims has been found to be dependent on the constraints of a prescriptive syllabus, lack of resources, and student motivation and proficiency. The first-year teacher study of Pennington and Richards (1997) confirmed that this was the case in Hong Kong schools.

Teacher belief systems also greatly influence novice teachers' practices and often serve as 'a lens through which they view both the content of the teacher development program and their language teaching experiences' (Richards, 1998, p. 71). Novice teachers are likely to accept certain aspects of their training if it matches their own beliefs or values while rejecting others which do not. In addition, different novice teachers will interpret different models of teaching in different ways and then try to implement them based on their own developing teaching maxims within the constraints of the education system in which they find themselves. It is expected that not all of the undergraduates of the BATESL course will accept the communicative, interpretational philosophy that is the foundation of the course and certainly, as has been found, not all of them will be able to implement it once they have the chance to do so in the classroom. 


\subsubsection{Previous Studies of Pre-Service Teachers Directly Related to this Research}

According to a group of related studies (Pennington \& Richards, 1997; Pennington, Richards, Urmston, \& Lee, 1996; Richards \& Pennington, 1995; Urmston, 1994; Urmston, Pennington \& Brock, 1996) the surveyed first-year BATESL cohort (those who began their studies in September 1994) differed from the third-year cohort (those who finished their studies in May 1994) in a number of ways.

In Urmston (1994), it was found that the third-year cohort, who were the first graduates of the course, had been influenced by the course, by their practice teaching, and by their own experience as students in Hong Kong secondary schools. Whilst the group clearly favoured the interpretive, student-centered approach advocated by the BATESL course at the level of approach, they were aware of the constraints that are placed on teachers within the Hong Kong teaching context at the level of design and procedure, such as large class sizes, heavy workloads resulting in lack of time for adequate planning, and a lack of cooperation among colleagues. They were keen to try out innovative approaches during their practice teaching (especially bearing in mind that they were being assessed on their performance), but were doubtful as to whether they would adopt such approaches if they were to teach full-time after graduation. When considering their futures, many of the group were unsure about their intentions to teach after graduation, citing worries about their own career goals, possible discipline problems and teachers' lack of status in Hong Kong. It was concluded that the BATESL course had not had the influence on the students that might have been hoped for, and instead of producing graduates ready to enter the teaching profession as qualified and confident novice teachers of English, most of the group were undecided as to their intentions to teach and somewhat disillusioned with the BATESL course and teaching in general. 
The studies by Urmston, Pennington and Brock (1996) and Pennington and Urmston (1998) compared this third-year group with the first-year group who are the subjects of this study. It was found that in comparison, the first-year group were more idealistic and less cynical than the third-year group. The first-year group showed that they had been influenced by their years as students in Hong Kong, and perhaps not surprisingly, were more enthusiastic about the course they were about to embark on and the third-year group were about to complete. There was an obvious naiveté shown by the first-year group as regards aspects of teaching that they had had no experience of, such as the duties and responsibilities of teachers, the relationships between teaching colleagues and the constraining effects of such factors as class size and examination requirements. On the other hand, there was evidence of an ability to see teaching in a wider or more general educational or societal context.

The purpose of the present investigation is to take the previous studies outlined above one step further by determining the orientations to teaching of the group of BATESL students who began their course in 1994 at the point when they were about to graduate in the summer of 1997 . Then it will be possible to assess any changes that may have taken place in their orientations over the three years of the course and determine whether such changes are the result of the influence of the course itself or other, 'external' factors within the Hong Kong teaching context, or a combination of the two. The changes undergone by this one group at such a crucial time in the history of the Territory will serve as a window on the changes in progress at the time in the wider context of education in society.

The study of the BATESL students will hope to further the growing body of knowledge concerning the relationships between student teachers' belief systems and their practices as well as looking into how these belief systems are affected by their training and the educational environment at large in society at the time. In the next section, conceptions of teaching and teacher change are examined and a framework developed through which any changes evidenced by the study can be discussed and which will in turn be modified to take account of the changes. 


\subsection{A Framework for Analysing Change in the Social System of Hong Kong English Language Teachers}

In order to allow for a closer examination of the changes undergone by the group of trainee English teachers and to relate those changes to societal change, it is necessary to develop a framework which takes into account different approaches to teaching, change and teacher change.

\subsubsection{Educational Change}

Woods (1996) developed a model of language teachers' decision-making processes in order to study the planning and decision-making of eight teachers of ESL in tertiary institutions in Canada. Using as a basis concepts of cognitive science, Woods developed a model which included three main aspects of the teaching process: the events which make up the teaching, the planning processes of the teachers, and the interpretive processes of the teachers' (p. 184). Woods developed his initial model by considering the different levels involved when decisions are made throughout a course of teaching, rather than in isolated incidents in lessons. This model is hierarchical and includes the factors of implementation (or events), planning and assessment (interpretation). Woods sees this model as being three dimensional, with the planning and assessment aspects feeding both 'backwards' and 'forwards' in a dynamic way as the course progresses and changes are made to the course to incorporate these new insights or new courses are developed as 'offshoots' or alternatives to the original course. The biggest variable in this model is the teacher. Her/his beliefs, assumptions and knowledge (for which Woods uses the acronym BAK) determine how s/he will interpret events related to teaching, in both planning and implementation, and therefore the teaching decisions that are made and the changes that result (Woods, 1996). 
Within this model, Woods examines two aspects of language teaching, "teacher change and curricular evolution' (p. 251). As teachers change, so the ways that they teach a given curriculum will change as well. Furthermore, as different teachers change and teach the same curriculum in different ways, feeding back their ideas to other teachers working in the same context, so the curriculum will evolve even if the curricular guidelines do not change (Woods, 1996). In other words, curriculum development is a natural evolutionary process in which the teacher, by implementing the curriculum, undergoes change and then by providing input into the curriculum through his/her interpretation of it, facilitates curricular change.

For such a two-way change process, communication channels must remain open in both directions. Moves to create changes from a top-down perspective are unidirectional and create blocks in the channels of communication and are frequently unsuccessful for this reason. In Hong Kong, the Curriculum Development Council (CDC) produces the curricula to be followed in schools. In reality however, 'what goes on in classrooms is often not consistent with the intentions of the official or planned curriculum' (Morris, 1995, p. 107). In terms of what is taught in Hong Kong classrooms and how it is taught, the major influences have been the examination syllabuses and the available textbooks rather than the curriculum provided by the CDC (Morris, 1995). When these have changed, so has teaching changed accordingly, and a process of evolution has taken place.

Fullan (1991) describes educational change in terms of classroom innovation as being multidimensional, with at least three components necessary for any new program or policy to be successfully implemented:

(1) the possible use of new or revised materials (direct instructional resources such as curriculum materials or technologies), (2) the possible use of new teaching approaches (i.e., new teaching strategies or activities), and (3) the possible alteration of beliefs (e.g., pedagogical assumptions and theories underlying particular new policies or programs). (p. 37) 
Fullan's dimensions of educational change, mirroring Richards \& Rodgers (1982, 1986) and Pennington's (1996) components of teaching frameworks, i.e., design, procedure and approach (see 1.3.3), complement Woods' (1996) notions of teacher change and curricular evolution in that all of the dimensions are necessary to achieve an educational goal or set of goals (Fullan, 1991). As far as Fullan is concerned, change must 'occur in practice along the three dimensions in order for it to have a chance of affecting the outcome' (p. 37). In other words, change can only occur as a result of teachers using new materials or technologies, developing new strategies or activities to go with the new materials or technologies and having their beliefs altered in the process, either as a first step or as a last step. Once their beliefs have been altered, then they can embrace change and feed their ideas back into further development of new materials, technologies and activities. In other words, changes in personal teaching beliefs feed forward into changes in curriculum, and changes in curriculum and procedures 'percolate up' to changes in beliefs. In this way, a change in one aspect of a teaching framework can radiate out to other parts. Hence, without the complete involvement of teachers, educational innovation is bound to fail (Fullan, 1991).

\subsubsection{Framework for Analysing Teacher Change}

As has been discussed, in Hong Kong there is 'a strong orientation to tradition, authority, and order which fosters a reluctance to challenge the status quo, a low tolerance for uncertainty and instability, and a cultural expectation of tight control by the teacher over class activities and students' behaviour' (Pennington \& Cheung, 1995, p. 19). On the other hand, Moser (1985) describes the peoples who populate Hong Kong and the Guangdong region of China bordering Hong Kong as 'clannish, diligent in pursuit of their own advantage or that of their own family, eager to embrace new ideas or novel things, [and] often impulsive' (cited in Pierson, 1998, p. 94). Consequently, as we have seen, the climate for change does exist in Hong Kong, provided that the people involved, and in educational terms that means the teachers, can see the benefit that they and their students are likely 
to receive. As Pennington and Cheung (1995) discovered in their investigation of Hong Kong English teachers' adoption of process writing:

... our initial findings suggest that others would like to see changes come about in the Hong Kong educational context that would make it more feasible to try out and to adapt, in a form modified or 'reinvented' to suit the conditions of the local context, interpretationbased educational innovations such as process writing. (p. 33)

Just how much are teachers involved in the process of educational change in Hong Kong? They are the ones who are charged with implementing changes in the classroom, but are all three of Fullan's dimensions present at the time of implementation of any educational initiative or innovation? There may be new materials or technologies due to curriculum reform (for example, new syllabuses for the main public examinations, HKCEE (at Form 5) and Advanced Level (at Form 7), and there may even be new teaching approaches advocated to go with them; but changing teachers' beliefs, or BAK (beliefs, assumptions and knowledge) as Woods (1996) labelled them, is a much more lengthy process. It may be that teachers as a group are resistant to change, or it may be that they are prepared to endorse change provided that it satisfies a number of criteria, as suggested by favorable answers to the following questions posed by Fullan (1991):

1) Does the change potentially address a need? Will students be interested? Will they learn? Is there evidence that the change works?

2) How clear is the change in terms of what the teacher will have to do?

3) How will it affect the teacher personally in terms of time, energy, new skill, sense of excitement and competence and interference with existing priorities?

4) How rewarding will the experience be in terms of interaction with peers or others?

(p. 128)

Consequently, any analysis of change in an educational context should consider the teacher as the pivotal 'actor' in the change process. The Hong Kong teacher, as previously noted, generally works under conditions which would be considered 
less than ideal from the point of view of being able to 'experiment' with educational innovation - heavy workloads, large classes and an examinationdriven curriculum are the norm. How much will teachers be consulted when educational change is mooted? Will teachers' working conditions be considered? Will teachers be made the focus of changes or merely the implementers?

In addition to teachers, the social systems or sub-systems that are involved in English language education include government departments, tertiary institutions, schools, parents and students. Looking at these sub-systems individually, it may be possible to gauge their potential for change based on the nature of the relationships between the individuals within the sub-system, or on the dynamics of the sub-system itself. Each sub-system would, to a varying degree, have the potential to change by adopting an innovation (Rogers, 1995) or through evolution (Woods, 1996), or to resist change in that sub-system. One would expect that the degree to which a sub-system is likely to change would depend on how cohesive it is. A sub-system that is very tightly knit or cohesive would be less likely to change than one which is not cohesive. It could be that within a sub-system such as parents, in which, in the Hong Kong case, almost all of the individuals are of the same race and cultural background and have similar beliefs concerning the education of their children, the relationships would be homophilous. They would form a cohesive social system that would be less open to change as they would be isolated from the innovators (the Education Department, academics etc.), who would form a separate social system. In a similar way, Hong Kong teachers, the majority of whom, like the parents (and many of them are parents), are ethnic Chinese, middle class and of a certain educational level, would be considered a homogenous and cohesive group. On the other hand, relationships within a subsystem such as English teachers, in which there might be both local Chinese and expatriates, who might be either locally trained or trained overseas, would be more heterophilous and the group less cohesive and thus more open to influence from outside the social system and therefore more open to change. With the growth in the number of Native English Teachers or NETs, the internal dynamic of this social system is changing and becoming more susceptible to change. 
Given that social systems are to some extent homogenous or heterogeneous, it is possible that external factors could influence their members' reactions to change. A sub-system which is initially quite cohesive could become more open to change as a result of being 'agitated' from outside. Such agitation could come through new training that promotes a particular philosophy or set of principles, such as a BA course for teaching English. Should the political climate be conducive to change, such as it was during the build-up to and the aftermath of the transfer of sovereignty, then a previously tightly knit sub-system could become more disparate as individuals within the group are influenced by external events and their beliefs or attitudes changed. This could lead to the group becoming more open to change, as opinion leaders would become more visible and open to influence from forces outside the group. Alternatively, a sub-system might react to external pressures or enforced changes by drawing together and becoming more cohesive, thereby rendering the system and its individuals more resistant to change as opinion leaders would be less visible.

An alternative or perhaps complementary view is that a sub-system could be agitated from the inside by change agents. In the case of language teachers, such agents could be newcomers, such as the NETs, who bring with them from outside the system unfamiliar, innovative approaches, which the existing members of the system will either accept, reject or ignore. Either way, changes will result in relationships within the sub-system leading to the potential for greater heterophily and hence possible greater acceptance of change. Another source of agitation is from existing members of the system who have learned of new methods and approaches from training courses or even from overseas and attempt to bring them into the system.

The issue of whether innovations that are considered to be of Western origin will be readily adopted in Hong Kong has become more acute due to the transfer of sovereignty. The political climate has been such that some effects will have been felt by all of the sub-systems involved in English language education. Given the various potential reactions to change of different types of social system (as 
discussed above), it can be postulated that some systems will have become more open to change or evolution, whilst some will have become less open to being affected by the changes occurring at the time of the handover. Through an analysis of the nature of each sub-system, it should be possible to assess the cohesiveness of each and thence its potential reaction to enforced change or adoption of innovation, and in which directions evolution or internally generated change might progress.

As Hong Kong passes from British to Chinese rule, it would seem logical that Chinese would increase in importance and that there would be a push for Chinesemedium education (Bray, 1997). In addition, an increasing sense of Chinese identity in the population (Pennington \& Yue, 1994; Pierson, 1998) might offer resistance to any innovations deemed as Western in origin as well as to the teaching of English in general, as a symbol of colonial rule or Western influence, in what Pennington (1997b) termed an oppositional stance (see 2.4.3.7). As previously suggested, there was resistance to the implementation of communicative language teaching as it was seen to be in conflict with the traditional transmissional methodologies practised in Hong Kong schools. However, this innovation has been adopted by other social systems, such as the tertiary institutions and teacher training bodies. The BA in TESL at the City University of Hong Kong, which was at the time the only degree course in the community in the teaching of English as a second language, was designed with this philosophy of teaching firmly in mind (Pennington and Richards, 1997). Whilst on the one hand acting as change agents in their own right within the Hong Kong educational context, these institutions can also be regarded as opinion leaders by virtue of their status as employing/containing 'experts' in the field.

According to Woods (1996), '[t]he likelihood of an individual adopting a particular action will depend on his beliefs and perceptions and not just on the recommendation of a professional' (p. 72). Within the Hong Kong education system, which has already been described as examination oriented - or what might be termed 'ends-driven' or 'outcomes-driven' - there clearly exist 
individual practitioners who are open to change and are ready to adopt innovations (Pennington \& Cheung, 1995). Such practitioners could be said to be 'input-driven' or 'process-driven', in the sense that they see teaching and learning as more of a personal, creative and developmental enterprise. Clearly, a conflict exists between these teachers and the education system as a whole. A major question to be answered is whether this conflict between teachers who are interpretational in their beliefs, and a school system which is basically transmissive, is causing change to occur from within the educational system, or whether the changes being imposed from outside are in response to these internal changes.

\subsection{The Investigation}

This chapter has discussed the educational and sociological structure of the territory of Hong Kong, once a colony of Great Britain and since 1 July 1997, a Special Administrative Region of the People's Republic of China. The purpose has been to set a context so that it might be possible to relate any significant changes in the beliefs, approaches and practices of a group of trainee English teachers to the many changes in education, particularly English language education, that have been documented as occurring there over the past decade. By looking at the relationships between the changes at the levels of teacher, education system, political system and social system, it might be possible to gain insights into the ways in which change itself in one or more social sub-systems engenders change in those sub-systems related to it/them.

This issue of English language education could be said to be at the very core of the changes that have manifested themselves and affected life in the Territory during the latter stages of the 20th century, not only for educational, but also for political, social and economic reasons. Within this social system, teachers form a highly significant social group or sub-group. Their reactions to the changes in education and society and the ways in which they and the sub-systems around 
them, such as language teacher education, language policy and language education policy, changed during the key period leading up to and during this crucial time in Hong Kong's history will provide a fascinating view of how social systems react to such changes.

The purpose of this thesis is to track the changes undergone in terms of beliefs, assumptions and knowledge (Woods, 1996) or design, approach and procedure (Pennington, 1996) by a group of trainee English teachers from the point at which they enrol in a course of training, through that training and into schools as teachers. Further dimensions are added to the study by the fact that the period during which these teachers learned the skills and knowledge they need to function in the educational environment in which they find themselves corresponded with potentially great political and social changes. By considering all aspects of influence on this group and utilising theories of innovation and change, the changes that are identified and confirmed will be interpreted by the patterns of change developed earlier. In addition, the framework for change based on the notions of the interaction of elements of social systems will be refined through the examination of the study findings in relation to the societal and educational changes discussed in this chapter, building to a model of educational change that can represent the changing relationships that effect English language education in Hong Kong.

Henrichsen (1989) gives the following description of what a model of educational change should comprise:

A model ... is more than a mere list of suggestions. A model shows the structure of the thing it represents. It provides a cohesive framework that recognises that the various individual factors do not exist only as independent elements. A model shows how these factors affect each other, indicating their interrelationships and the direction(s) of influence. An adequate model also shows process. Many of the elements involved in a reform campaign are observable and can be listed, but the change process itself and many of the forces that affect it are intangible and cannot be directly observed. For this reason, the framework of a model must 
depict not only the forces that affect the change process but also the process itself. (p. 67)

Henrichsen's (1989) own model of diffusion of educational innovations, the Hybrid Model, has already been discussed and is reproduced in Figure 2.6. Another model, which can serve as an example of relationships between the various elements in an educational system is Centra and Potter's (1980) model of school and teacher effects on student learning (reproduced from Pennington, 1995c) (Figure 2.8).

Centra and Potter's model is a useful indicator of the relationships between educational systems, but it does not indicate the relative importance of the various factors impacting educational outcomes (Pennington, 1995c). Neither does it show the contexts or domains within which the processes take place. A model which does take into account the contexts within which educational activities take place, is Pennington's (1995c) model of the relationship of innovations to outcomes (Figure 2.9). ${ }^{28}$ In this model, the process of innovation affecting outcomes is shown to take place within the context frames of the classroom, within the school, within the society within the culture 


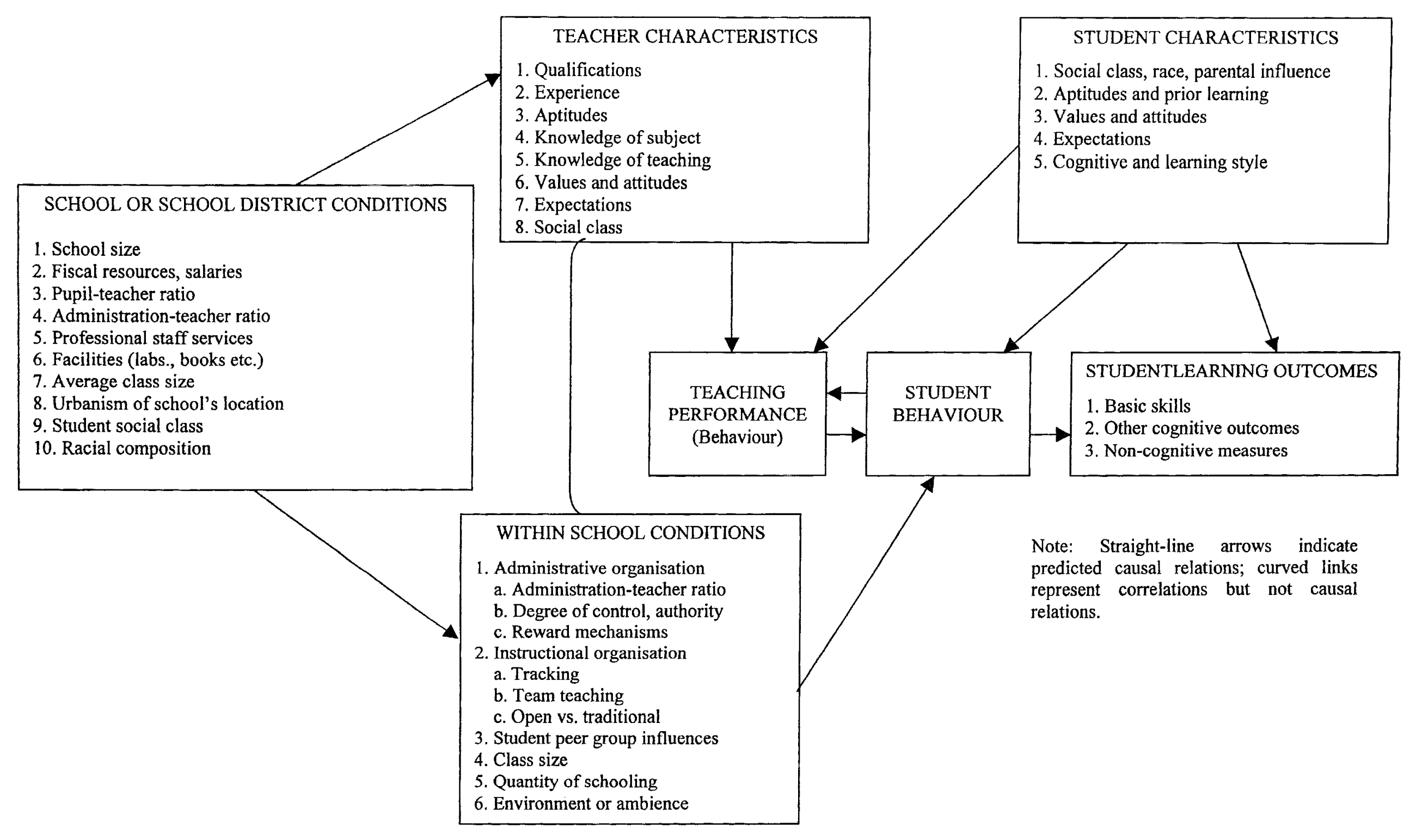

Figure 2.8: Centra \& Potter's Model of School and Teacher Effects on Student Learning Outcomes (Reproduced from Pennington, 1995c, p. 34) 
CONTEXT FRAMES

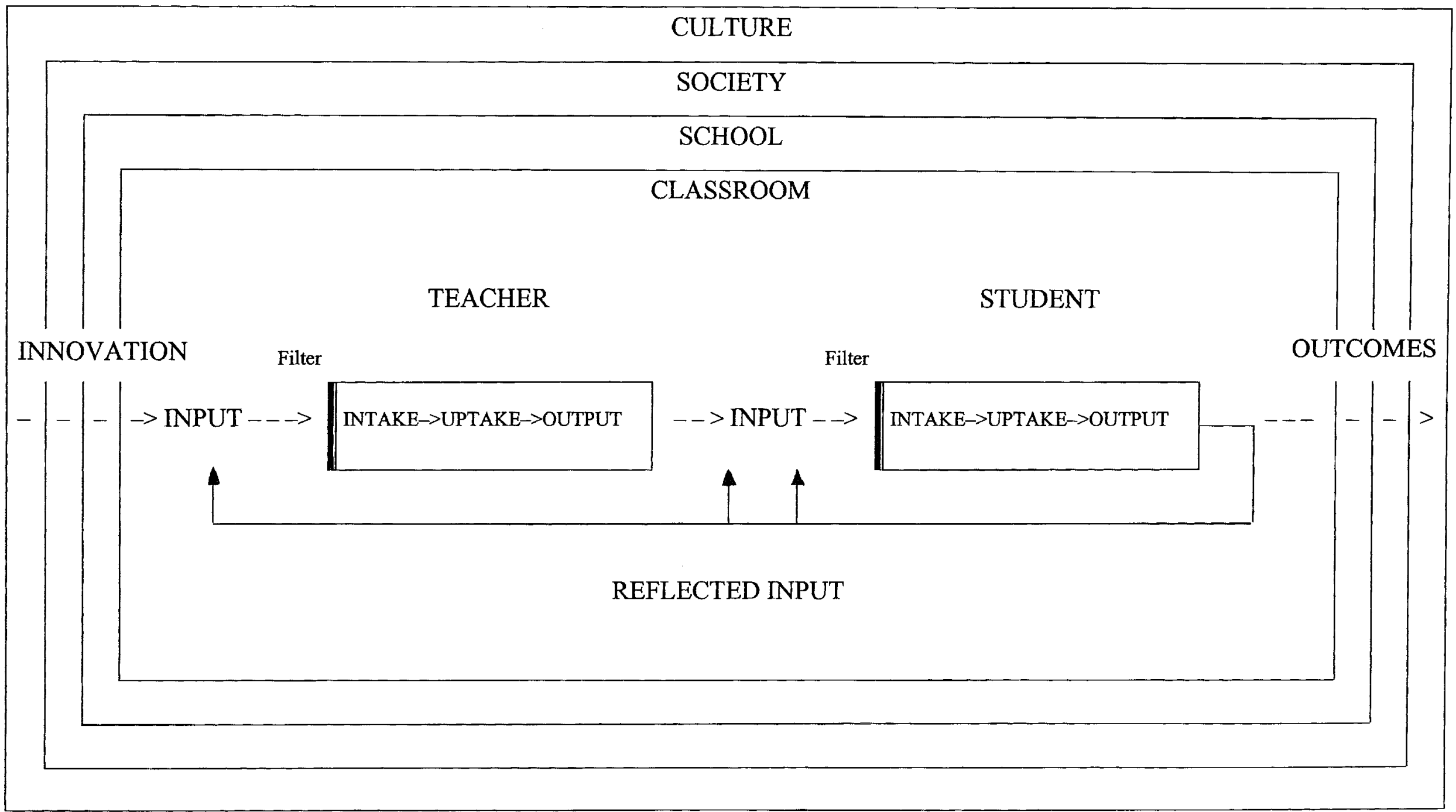

Figure 2.9 Pennington's Model of the Relationship of Innovations to Outcomes in Education (Pennington, 1995c, p. 75)

$$
2-103
$$


A model of educational change as it impacted the teacher of English in Hong Kong schools during the period of the changeover of sovereignty should show contexts, relationships (both causal and non-causal), and the relative importance and influence that separate components or systems have on each other. This thesis will take into consideration the different components, contexts and relationships gleaned from the prior literature reviewed in this chapter together with the findings from the studies outlined in Chapters 3-5 towards a coherent updating of the situation in Hong Kong that includes an original model of this complex context. 


\section{Notes}

${ }^{1}$ The Education Commission is a powerful body of politicians, educationalists and business leaders appointed by the Chief Executive (prior to the handover, the Governor) which advises the government on the development of education and educational policy (Hong Kong Government Information Services, 1998).

2 The NET scheme, as it is known, will be discussed in further detail in subsequent sections.

${ }^{3}$ The LPAT has been introduced by the government so as to ensure a minimum level of language competency of teachers of English and Putonghua and will be further discussed in later sections.

${ }^{4}$ To this end, the Target Oriented Curriculum (TOC) was developed, originally under the name, Targets and Target Related Assessment (TTRA), in response to recommendations made in Education Commission Report No. 4 (Hong Kong Education Commission, 1990) concerning the need for an assessment system that assessed the strengths and weaknesses of individual students against agreed targets (Morris, 1995). The initiative was developed by a team from the Hong Kong Institute of Language in Education under the leadership of Dr. John Clark.

${ }^{5}$ In Hong Kong, education policy is formulated by the government in the form of the Education and Manpower Bureau (EMB). Policy is then carried out by the education agencies, the Education Department (ED) and the Examinations Authority (HKEA). It is the Education Department that is responsible for developing curricula under its Curriculum Development Council (CDC) and managing schools. The HKEA is responsible for development and administration of public examinations.

${ }^{6}$ Most public examinations are now administered in both English and Chinese, although the English versions remain the most popular (Pennington, 1996).

7 In Hong Kong, teachers are employed in government-aided schools (the majority) as either Graduate Master/Mistress (GM) or Certificated Master/Mistress (CM). Government schools employ university graduates as Assistant Education Officer, which is the equivalent of the GM. GM's receive a higher salary than CM's and are on a higher point on the salary scale if they have a teaching qualification. Private schools are free to employ whomever they wish. GM English teachers do not necessarily have to be English graduates.

${ }^{8}$ Law (1997) refers to the 'institutionalization of national sovereignty among the subject people by the incoming sovereign power', that is the PRC, as recolonisation.

${ }^{9}$ See Pennington (1996) for discussion of 'muted' and 'dominant' influences in this sense, which she derived from Muted Group Theory by Edwin and Shirley Ardener (Ardener, 1975).

${ }^{10}$ The 'problems' Pennycook was referring to were those of inequality, elitism, discrimination, inadequate planning and emphasis on quantity rather than quality.

${ }^{11}$ The Joint Liaison Group, consisting of representatives from Britain, Hong Kong and China, was set up at China's insistence to deal with issues regarding the transition of sovereignty and met intermittently in the years leading up to the changeover. 
${ }^{12}$ The Hong Kong government was and is still executive-led. Whilst the bureaux and offices are staffed by career civil servants, decisions on policy are made by the Chief Executive (under British rule, the Governor) under advice from the Executive Council, a body made up mainly of representatives from the business field all personally selected by the Chief Executive.

${ }_{13}$ In the early 1980 s, approximately 20,000 Hong Kong citizens per year emigrated. This figure rose to 66,000 in 1992 and 62,000 in 1994 (Hong Kong Government Information Service, 1996).

${ }^{14}$ In what was seen as the first major post-handover test of its strength and autonomy from the Mainland, in June 1999, the SAR government overturned a decision made in the Court of Final Appeal (Hong Kong's highest legal establishment) on whether children of Hong Kong residents born on the Mainland had right of abode in Hong Kong. This was done after the government asked Beijing to step in for a 're-interpretation' of the Basic Law (South China Morning Post, 11 January, 2002). The case was seen by many to be indicative of the lack of independence held by Hong Kong from Beijing since the handover, even if it did serve to show that Hong Kong people are prepared to stand up to China on a point of principle, a feature which met the approval of former Governor, Chris Patten (Patten, 1999).

${ }^{15}$ In Hong Kong schools, the teachers assigned to teach a particular subject are known as the 'panel' of teachers and as such the 'panel chairperson' or simply 'panel chair' is the teacher-in-charge of that subject.

${ }^{16}$ Despite acceptance of CMI in schools, EMI is still considered to be most appropriate for higher forms in preparation for tertiary study, which mostly remains EMI.

${ }^{17}$ Henrichsen (1989) describes the campaign conducted by the Rockefeller-funded English Language Exploratory Committee (ELEC) to revolutionise English language teaching practices in Japan in the 1950s and 1960s via Charles Fries' Oral Approach. Henrichsen developed a hybrid diffusion-of-innovations model, representing both the diffusion/implementation process and the multitude of factors which affect it, and used this model to try to determine why the campaign had failed. He argued that creating change across cultural boundaries requires both a careful analysis of the target setting, one which takes into account the potential barriers to change, and an appropriate implementation strategy to overcome those barriers.

${ }^{18}$ Rogers \& Kincaid (1981); Rogers \& Shoemaker (1971).

${ }^{19}$ Richards (1983; 1985), Gee (1984)

${ }^{20}$ Communicative Language Teaching emerged in Britain and North America due to dissatisfaction with the Oral-Structural and Situational Approach, which had been the adopted approach to language teaching in these areas and also in Hong Kong in the post-war years (Richards \& Rodgers, 2001).

${ }^{21}$ When the Institute of Language in Education (one of the original five institutes or colleges which amalgamated to form the Hong Kong Institute of Education) promoted CLT, it can be seen as a different kind of contingency in that the whole field of English language teaching (including tertiary scholars and outside consultants) was pushing for it. The decision by Hong Kong teachers to resist it could have been as a reaction against this 'outside' influence. 
22 Although not explicitly stated, the TOC is clearly based on the UK National Curriculum introduced in 1988 , with which it shares many characteristics. It was felt that the emphasis on guiding students to acquire achievable and practical skills was as applicable to Hong Kong as it was to the UK (Hau et al., 1996), although it could be argued that the colonial government of Hong Kong at the time tried to push an approach which had high face validity due to positive results in its early stages in an altogether different educational context.

${ }^{23}$ The Expatriate English Teachers Scheme (EETS), the forerunner of the Native English Teachers scheme, placed expatriate native-speaker teachers of English into Hong Kong secondary schools. The scheme was initially organised with the help of the British Council and teachers were recruited from the UK Since its revival as the Native English Teachers scheme, it has been expanded into all secondary and primary schools and the Education Department has broadened its catchment to include Australia, New Zealand, the USA, Canada, South Africa and Singapore. The idea of having native-speaker teachers is based on the concept of language assistants, commonly used as support for the teaching of French and other languages as foreign languages in schools in the UK (Boyle, 1997).

24 The consultation exercise which led to the establishment of the Language Proficiency Assessment for Teachers initiative took into account similar standardsetting initiatives of ESL teachers, particularly in the countries mentioned (Falvey \& Coniam, 1997).

${ }^{25}$ The so-called 'benchmark' level is set at Level 3 on a 5-point scale across the four skills that are assessed by the Hong Kong Examinations Authority of reading, writing, listening and speaking; plus a classroom language assessment which is carried out by the Education Department. For more details of how the benchmark level was arrived at see Coniam \& Falvey (1996) and Falvey \& Coniam (1997).

26 This project, 'Learning to teach English in Hong Kong: The first year in the classroom', was supported by a Competitive Earmarked Research Grant from the Hong Kong Research Grants Committee. The author worked as a Senior Research Assistant on the project.

${ }^{27}$ In fact, the trainee teachers of this study, enrolled in a Bachelor's degree course in Teaching English as a Second Language, although able to teach in schools upon graduation, would be required to complete a Certificate of Education in addition to their degree to be considered 'fully qualified' teachers. They would normally complete this extra course work on a one-year part-time Cert. Ed. course whilst they were teaching full-time.

28 Slight modifications have been made to Pennington's model for ease of reproduction. The modifications do not affect the features of the model itself. 


\section{COMMUNITY VIEWS: THE ISSUES RELATED TO ENGLISH LANGUAGE TEACHING IN HONG KONG IN THE POST-HANDOVER PERIOD}

\subsection{Background}

In 1997, the major issue affecting English language teaching in Hong Kong was the change in the medium of instruction (MOI) in secondary schools from predominantly English medium (EMI) to mother tongue or Chinese medium (CMI). This had resulted from the objection by education authorities to the use of a 'mixed code' of English interspersed with Cantonese in teaching (Boyle, 1997; Li, 1998) as had been described in Education Commission Reports Nos. 4 and 6 (Hong Kong Education Commission; 1990, 1995). Consequently, measures to force schools to revert to Chinese medium, unless they could pass certain criteria which would show that the majority of their students would benefit from an English-medium education, were outlined in the government consultative paper, Arrangements for Firm Guidance on Medium of Instruction in Secondary Schools (Hong Kong Education Department, 1997a) and resulted in approximately 25 per cent of secondary schools retaining English medium status and the rest being designated Chinese medium from September 1998 (Hong Kong Education Department, 1997b).

Another initiative which proved to be both prominent and controversial was the introduction of Benchmark language levels for teachers, since re-named the Language Proficiency Assessment for Teachers (LPAT) (Coniam \& Falvey, 1999; Falvey \& Coniam, 1997). Since the announcement of the scheme, by which all teachers of English and Putonghua will be required to reach certain Benchmarks 
of language proficiency by 2005 in order to continue teaching, objections have been voiced in increasingly vigorous terms by language teachers and teacher representatives in primary and secondary schools.

Furthermore, in January 1999 the Education Department, through the Education Commission, launched what it has come to refer to as its Review of the Education System, with the consultation document Education Blueprint for the 21st Century Review of Academic System: Aims of Education (Hong Kong Education Commission, 1999). Although this document did no more than state a number of aims for reforming the education system, it did indicate the government's intention to be seen to be paying attention to the need to reform the education system. The LPAT is one initiative that has been introduced as well as an updated curriculum for secondary schools based on the Target Oriented Curriculum, the increase of provision for use of Information Technology in schools and the teaching and learning of Putonghua.

In this chapter, the issues that pertained to the teaching of English in Hong Kong at the time of the changeover in sovereignty in 1997 and in the subsequent two years will be investigated from two perspectives: That of the general public through the news media, and that of the English language education system through English language teaching practitioners. There are two parts to this investigation: The first looks into the coverage given to the issues of English language teaching (ELT) by the English language newspapers in Hong Kong during the watershed year of 1997; the second examines the views on the key issues of a representative sample of expert ELT practitioners through two rounds of interviews, in 1997 and two years later in 1999. The intention of these studies is to build a picture of the state of the education system at the time of the handover as well as gain some perspective on directions of change in the system after the handover, such that these insights can form the basis of the studies of teachers to come and of the models of change to be developed from all the studies. 


\subsection{Survey of the Issues Pertaining to English Language Teaching as Reported in the English Language Newspapers in 1997}

At the time of the study, in 1997, there were two English language newspapers in Hong Kong, The South China Morning Post (SCMP) ${ }^{1}$ and The Hong Kong Standard (HKS). ${ }^{2}$ Both were daily papers with a Sunday edition. It was considered that by recording the coverage of issues related to English language teaching over the twelve months straddling the changeover in sovereignty, i.e., the whole of 1997, it would be possible to gauge the relative importance of individual issues. Furthermore, it would be possible to see how such issues moved into (and out of) public attention over the course of the year.

The possible issues that could emerge were anticipated to include: language use, language planning and policy, the teaching of language, language teacher issues, and larger education issues affecting language teaching. In addition, it was anticipated that certain organisations, such as the Education Department, would feature regularly in the press and so these would be included in the survey also.

\subsubsection{Methodology}

Both the South China Morning Post and the Hong Kong Standard contain(ed) news sections (local, China and international), comment sections and editorial pages including letters to the editor. In addition, there are occasional special report sections when local or international events warrant them. In order to gain a representative measure of the frequency of reporting of the issues connected to ELT, relevant news items, articles, editorials and letters published in the two newspapers on one day each week (Monday) were collected. Consequently, from Monday 6 January until Monday 29 December 1997, all relevant items were collected. These items were then analysed and individual issues or organisations 
were identified and quantified, and the date on which they were reported was recorded.

Although it was anticipated that there would be Mondays on which no items concerning ELT would appear in the newspapers, and there would be other days of the week on which there would be substantial numbers of items, within the scope of the study it was felt that the news on Monday would be a fair representation of key information of interest to the community. The number of times each issue or organisation was mentioned within each individual edition of either newspaper was not recorded, rather, it was simply considered to have been in the news on any one Monday. Additionally, in order to provide a context to the survey, a database of news articles, editorials and letters published in the newspapers on the other six days of each week was built up throughout the year. Using this database, it was possible to identify which issues or organisations were being highlighted by the media and thus of concern to people in Hong Kong. This database makes it possible to identify what might be termed 'key dates' on which events occurred or announcements were made which impacted directly on ELT in Hong Kong.

\subsubsection{Findings}

\subsubsection{Identified Issues and Organisations}

A summary of the issues and organisations related to English language teaching in Hong Kong which were in the news reported on in either of the two English language newspapers on at least two Mondays in 1997 is shown in Table 3.1 (All tables can be found in Appendix A). The issues and organisations are listed in descending order in terms of the number of days on which each was reported.

In all, 32 different issues or organisations relevant to ELT were identified in the English language newspapers on at least two Mondays in 1997. The most 
common was, unsurprisingly, the Education Department (on a total of 17 Mondays, or approximately 33 per cent of the total of 52). The Department was in the news consistently throughout the year. The most common issue was that of the medium of instruction in schools (MOI) (15 days / 29 per cent), followed by the more general one of language standards (14 days / 27 per cent).

Pattern of Items in the News Throughout the Year of the Changeover

In order to gain an insight into the reasons behind the occurrence of certain issues at certain times in 1997, the following 'key dates' relating to language teaching have been identified:

14th March

Announcement made by Secretary for Education and Manpower, Joseph Wong Wing-ping of planned pilot exercise to establish English language proficiency standards for teachers. It was announced that from 1999, new English teachers would have to meet a certain language 'Benchmark' in order to be eligible to teach in schools. In addition, all serving English teachers would be required to meet the Benchmark after a certain period of time and would be given training to help them reach the standard. There was support for the initiative from government officials but opposition from teachers' unions (SCMP, 15 March 1997).

27th March Education Department issues Consultation Document, Arrangements for Firm Guidance on Secondary Schools' Medium of Instruction (Hong Kong Education Department, 1997a). The measures propose that from 1998, schools would need to teach in Chinese from Form 1 unless they could prove that they could teach effectively through English. 
1st July

11 th July

7th August

Ist September

25th September

7th October

8th October
Sovereignty of Hong Kong handed over by Britain to the People's Republic of China.

Results of Advanced Level examinations announced, showing that 12,000 candidates reached the university entrance grade in English, with 14,500 places available.

Results of Certificate of Education examinations announced, showing a slight improvement in results over the previous year and an increase in the number of candidates choosing to do exams in Chinese rather than English.

Schools re-open after summer break.

Education Department issues Medium of Instruction Guidance for Secondary Schools (Hong Kong Education Department, 1997b). The guidelines follow the proposals made in March. It is anticipated that a large number of schools will apply for exemption.

Education Commission issues Education Commission Report No.7, 'Quality School Education' (Hong Kong Education Commission, 1997). The report focuses on ways to improve school management and performance by offering cash incentives to schools.

Chief Executive, Tung Chee-hwa delivers his first policy address, in which it is announced that 700 new native Englishspeaking teachers will be employed to teach in secondary schools from September 1998 (HKS, 10 October 1997). 
Education Department announces that 100 out of a total of 124 schools which had applied to retain English as the medium of instruction would be allowed to do so and all other schools would be expected to teach through the medium of Chinese (HKS, 2 December 1997)

A detailed breakdown of the findings is shown in Tables 3.2a (January-June) and $3.2 \mathrm{~b}$ (July-December), in which the Monday on which each issue or organisation was a news item is indicated. The figures indicate on a general level the periods in the year in which there was a high degree of interest shown in issues related to education and language teaching. The clustering of points indicates when certain issues were in the news.

Clearly, the periods of March-April, August-October and December were the times when there was a lot of attention paid to ELT-related issues/organisations. This pattern can be explained by referring to the 'key dates' outlined above. In addition, it is also possible to explain the occurrence of individual issues/organisations at certain times and over the whole year.

The medium of instruction and language standards issues were in the news particularly during March and April. This corresponds with the issuing by the Education Department of the consultation document on the MOI in schools (Hong Kong Education Department, 1997a), which raised public awareness of ELT issues and caused much comment in the press. Indeed, a wide range of different sectors of society voiced their opinions on the MOI issue, including educators, politicians, union leaders and parents; and the whole issue of standards of English was discussed during this period. Furthermore, with the publication of the Medium of Instruction Guidance for Secondary Schools (Hong Kong Education Department, 1997b) in September, these issues also had a high profile later in the year. This also corresponded with the beginning of the school year. Then in December, the government announced which schools would be allowed to use English as the MOI. 
There was frequent reporting of the influence, concern and interests of parents during 1997, related mainly to the MOI issue (11 days / 21 per cent). It was reported that parents were extremely anxious for their children to be given the opportunity to learn through the medium of English as they considered it a prerequisite for a successful career to be fluent in the language and they assumed that learning through English was the most effective way for them to reach such a level of fluency (SCMP, 8 December 1997). In fact, during December, when the Education Department announced which schools could teach through English, many parents of children enrolled at schools which were set to become Chinesemedium expressed anger and bitterness (SCMP, 15 December 1997). In addition, it was reported that many parents suffered from 'exam stress' due to the Hong Kong Advanced Level and Certificate of Education examinations, the results of which were announced in July and August respectively (HKS, 18 August 1997). In fact, examinations was the issue that was reported on the next most frequently ( 9 days / 17 per cent), peaking in popularity in August and September.

The Professional Teachers' Union (PTU) was also in the news on 9 out of 52 Mondays, mainly during the period from August to October. The major contexts in which the PTU was mentioned were the Benchmarking of language teachers (HKS, 17 March 1997); the MOI issue (HKS, 29 September 1997) and the recruitment of Native English Teachers (NETs) announced by the Chief Executive in his policy address (HKS, 13 October 1997; SCMP, 20 October 1997). It was the last of these issues that raised the profile of the PTU the most. There were frequent objections by the PTU to the proposed conditions of service to be offered to the NETs (housing and re-location allowances among others) that their members (local teachers) did not receive. The NETs issue occurred three times, in October and December. Furthermore, the issue of the Benchmarking of language teachers made the news three times, the first in March after the announcement by the government of the initiative. In fact, this was a major issue that received fairly widespread coverage in the newspapers, just not particularly on Mondays. The initiative was intended as a means of raising the language standards of teachers of Inglish (and Putonghua) by requiring them to either pass a series of tests or take a 
designated course with in-built assessment. There was initially some confusion as to the nature of the tests that teachers would need to take and which teachers would need to take them. It was in fact an issue that was set to increase greatly in profile as the PTU, who were strongly against it, became more heavily involved in the debate over the issue.

Indications that the transfer of sovereignty to Chinese rule was imminent were provided by the occurrence of a number of related factors: Putonghua ( 9 days / 17 per cent), new immigrants from China ( 9 days $/ 17$ per cent), civic education ( 6 days / 12 per cent) and political interference by China ( 3 days / 6 per cent). These were amongst the very few issues to find news space during the period straddling the handover (June-July), when other issues were more prominent. In fact, it could be argued that many of the other factors identified, such as the status of English (7 days / 13 per cent), the colonial or British influence on education, language and the law ( 3 days / 6 per cent), simplified Chinese characters ( 3 days / 6 per cent) and even the use of mixed-code in schools ( 3 days / 6 per cent) were in some way connected to the changeover, or at least were newsworthy because of it.

Organisations in the news other than the Education Department and the PTU, were the Education Commission ( 6 days / 12 per cent); the Education Policy Concern Organisation ( 4 days $/ 8$ per cent); the English Schools Foundation ( 3 days $/ 6$ per cent); and the Examinations Authority ( 2 days $/ 4$ per cent). In October, the Education Commission issued its 7th report, concerned with the quality of education (Hong Kong Education Commission, 1997); the major issue of the year, the MOI, was the direct result of recommendations made in Education Commission Report No. 6 (Hong Kong Education Commission, 1995). The Education Policy Concern Organisation was mainly mentioned in relation to the provision for immigrant children from Mainland China and the MOI issue. The English Schools Foundation, the semi-independent, semi-government-funded body which provides the bulk of the international schooling in Hong Kong, was quoted in relation to its increasing role as the main provider of a true Englishmedium education. The Examinations Authority, besides being charged with the 
development and administration of public examinations, also came into the news in relation to the language Benchmarking of teachers initiative, as it (the Authority) was expected to be given the job of implementing the assessment part of the initiative.

Teaching issues addressed were the question of school textbooks ( 5 days / 10 per cent); resources for schools ( 4 days $/ 8$ per cent) and changes in the curriculum (2 days / 4 per cent). It was felt that school textbooks would need to be updated to take account of the changes that were taking place as a result of the handover (SCMP, 7 April 1997); in addition, should schools change over to Chinesemedium, they would need to have textbooks in Chinese, rather than in English (HKS, 29 September 1997). The call for more resources for schools was made particularly for those schools which would have to accept new immigrant children from Mainland China (SCMP, 25 August 1997).

Issues directly connected to teachers included standards of teachers ( 7 days / 13 per cent), and other teachers' issues ( 5 days / 10 per cent). It was generally expressed that there was a need to improve the standard of teachers, particularly the language standard of English and Chinese teachers (SCMP, 10 March 1997), and ungrade their qualifications (HKS, 6 October 1997), including a call by the Chief Executive for all teachers to hold a minimum qualification of a bachelor's degree. While the teachers themselves, as represented by the PTU, appeared to object to the language Benchmarking initiative, it was clear that both the press and the public were supportive of it. Other teachers' issues to emerge concerned teacher stress and the setting up of a self-governing body for teachers (HKS, 3 March 1997).

Finally, there were a number of other issues identified in the English language press as connected with language: bilingualism ( 4 days / 8 per cent), language and the law ( 3 days $/ 6$ per cent) and language learning ( 3 days $/ 6$ per cent). In addition, the main issues identified as affecting tertiary education were the language qualifications for university entry ( 3 days $/ 6$ per cent) and the prospect 
of four-year university degrees ( 3 days / 6 per cent), as opposed to the current three years. Additionally, in late August, much attention was paid in the press to tutorial schools at the time of the publication of the Hong Kong Advanced Level examination results. In some cases it was reported that students preferred these schools, with their 'guaranteed results' and examination tips and techniques, to their secondary schools, despite the fact that some of them were found to have been operating illegally (SCMP, 25 August 1998).

\subsubsection{Summary of Issues and Organisations Reported on in English Language Newspapers Pre and Post-Changeover in 1997}

To summarise, the issues/organisations which proved to be high profile before and after the changeover in sovereignty are shown in Table 3.3 in order of the number of Mondays on which they were reported.

Language standards, the medium of instruction and the Education Department featured prominently before and after the changeover, while examinations and the Professional Teachers Union came more into prominence after the handover for the reasons previously mentioned. There was also, in total, more attention paid to issues affecting English language education after than before the changeover.

\subsubsection{Breakdown of Pattern of Issues}

In order to give a useful picture of the kinds of issues impacting education in Hong Kong in 1997, the above findings have been divided into four subcategories of issues: Language Issues, Organisations and Groups, Educational Issues and Socio-Political Issues. 


\section{$\underline{\text { Language Issues }}$}

Table 3.4 shows the issues related to language that were covered in the English language newspapers on Mondays in 1997, both before and after the changeover.

Language issues comprised the biggest proportion of the total issues/organisations identified, approximately 38 per cent, showing how important such issues were at that time and probably still are. However, there seemed to be slightly more attention paid to this area before the changeover than after it, with the exception of the medium of instruction, as more attention was paid to examinations, educational policy and teacher issues than language itself.

\section{Educational Issues}

The next most covered issues were those relating to education (23 per cent) as shown in Table 3.5. Examinations dominated this category, especially in the second half of the year, when the results of the school examinations were announced. This was followed by standards of teachers, which was closely related to the Benchmarking of language teachers initiative. The initiative was first announced in March but came more into the public eye in the latter part of the year as speculation began to grow about its implementation.

\section{Socio-Political Issues}

Finally, issues that could be described as socio-political and affecting English language education made up the remaining 4 per cent (Table 3.6). Although there was a great deal of news coverage of handover-related issues concerning the potential political makeup of the SAR post-handover, the prospects for political and social freedoms and the effects the handover might have on the economy and other social institutions, only those two shown related specifically to language education during the time frame of the survey. 


\subsubsection{Discussion}

According to Morris (1995), writing before the changeover, the factors that might affect the school curriculum after the changeover would be politics, the economy, and social factors, and it was assumed that these would be the factors affecting language use in general in Hong Kong as well. The findings of this survey indicate that while politics did play some part, with the impending and then the actual transition of sovereignty increasing the attention paid to China and things Chinese (Putonghua, new immigrants, civic education, political interference and simplified characters), it was the medium of instruction issue that dominated news coverage. It could be argued that as the MOI issue was first mooted in Education Commission Report No.4 (Hong Kong Education Commission, 1990), it was not connected to the changeover and the planned implementation of majority Chinese MOI in 1998/99 was not a decision influenced by political factors. Yet as has been pointed out, the timing of the announcement of it and its subsequent implementation did coincide with the transition and with the myriad of other changes occurring at the time. In a similar way, the planned introduction of Benchmark language levels for language teachers had been in development since before the handover, although the implementation of this initiative did not occur until 2001.

There was no evidence of a decrease in the value placed on English and proficiency in English by businesses, students and their parents. Parents in particular felt that it was vital for their children to have the advantage of an education in English in order to be competitive in the labour market upon graduation (Balla \& Pennington, 1996; Evans et al., 1998; Tung et al., 1997). Whilst it was clear that Putonghua was beginning to become more influential, by far the most dominant language was still Cantonese; and with the switch of the majority of schools to Chinese-medium, as well as the increasing use of the language in government, it was set to continue to be so. In addition, English clearly still held onto its place as the second language, due mainly to its position as the language of international trade (Lord, 1987), and to the preponderance of 
English-speakers as well as the tradition of British-style English-based law in the courts.

Social factors possibly relevant to English language teaching (referred to by Morris, 1995) could have been the changing needs of the population. There was a general perception that the education system was failing a great many students, and the change to Chinese-medium of instruction was designed to help address the problem. The estimated increase in the population of Hong Kong due to the influx of immigrants from Mainland China was anticipated to increase the pressure on schools. More schools would need to be built and more resources given to schools to help them cope with the influx.

\subsubsection{Concluding Remarks on Newspaper Survey}

This review of the issues related to English language teaching covered by the two English language newspapers in Hong Kong during the landmark year of 1997 has attempted to address research question (i), i.e.:

What were the key issues connected to English language teaching as reported in the local English language newspapers during the period immediately before and immediately after the changeover, and was there any change in emphasis on the different issues over this period?

The review revealed that govemment-initiated factors dominated over factors initiated by teachers, students or the general public. Furthermore, there was a noticeable influence on matters caused by the transfer of sovereignty and the increasing presence of the People's Republic of China and its government in Hong Kong affairs. 
The dominant issue was the medium of instruction in schools. Although this had been an ongoing issue for some years, it had mainly been confined to the educational sector. With the issuing of the Firm Guidance policy and the subsequent widespread news coverage of it, the issue was thrust into the public's attention. There had been concern expressed about falling standards of language (most especially English) for some time in the media prior to 1997 and announcements by the government that most schools would actually be forced to comply with the Chinese-as-medium policy caused controversy on two fronts. Firstly, it was seen by large sections of the media, and by the public, as an 'admission of defeat' in the battle to maintain (or even raise) standards of English amongst students. As the majority could not function adequately and could not learn English sufficiently well, then better let them use their mother tongue. Secondly, the mandatory nature of the policy appeared by many to contradict Hong Kong's tradition of laissez faire government and coming at a time when the Territory was preparing for the changeover, and there was already a politicallycharged atmosphere, may explain why the issue raised such attention.

Connected with these key issues of medium of instruction and language standards were the groups of stakeholders involved, the Education Department (ED), the Professional Teachers Union (PTU) and parents. As two more issues came into focus that were specifically related to English standards, the Benchmarking of teachers initiative and the NET scheme, there developed a greater politicisation of what had initially been essentially language issues. Consequently, the three bodies increased in profile during the year; the number of occasions on which they were in the news (or at least the number of Mondays) in the second half of the year increased when compared to the first half. With the raised profile of the government (represented by ED), teachers (represented by the PTU) and parents, the focus of reporting seemed to move from the issues of standards of English and how perceived dropping of these was affecting society, to the relative influence, rights and interests of the stakeholder groups. So whilst language standards were seen to be of high importance to the community, there was evidence perhaps of 
the growing influence of different social groups on the issues of concern to society during this year of great socio-political change.

This survey of the issues which were in the 'public eye' in 1997 has served as a foretaste of the more in-depth analysis of the issues pertinent to ELT as perceived by the people involved, the practitioners charged with the teaching of English, the preparation of English teachers, the administration of the education and examination systems and the development of curricula. In the second part of this chapter, issues of educational change in a changing society are revealed by examining the teaching and learning of the English language in Hong Kong through the perceptions of individual teachers, teacher educators, administrators and the community generally in the run up to and two years after the changeover in sovereignty.

\subsection{Interview Survey of People Connected with the Teaching of English in Hong Kong on the Issues Affecting English Language Teaching in the Run Up to and After the Changeover in Sovereignty}

This survey was begun in the summer of 1997 and set out to answer research question (iii), i.e.:

How were the changing needs and perceptions of English teaching in Hong Kong addressed by prominent English language educators and representatives of the organisations charged with English language teaching provision before and after the changeover?

In addition, it was the purpose to determine the most prominent issues to emerge in 1997 and 1999, and whether there would there be any noticeable shift in importance of certain issues that could form the foundations for the models (to be 
developed in Chapter 6) of the English language education system before and after the changeover.

In what follows, the content of two rounds of interviews is discussed. Certain key issues to emerge from the interviews are identified and the various aspects of those key issues are discussed, comparing the attention paid to them in the two rounds. As a further analysis of the interview data, comparisons are made of the responses of those interviewees that took part in both rounds of interviews. Finally, conclusions are drawn as to the possible shift in importance of the major factors affecting English language teaching in Hong Kong at the time of and just after the changeover in sovereignty with a view to determining whether the changeover itself had any bearing on these changes.

\subsubsection{Methodology}

\subsubsection{1}

\section{Subjects}

In order to attempt to answer the above questions, it was decided that two rounds of interviews would be conducted with a wide variety of people connected to the teaching of English in Hong Kong. The interviewees were chosen on the basis of being in one of the following categories:

- publicly recognised authorities on language teaching issues in the Hong Kong context

- people in positions of authority within teacher-training and language departments in tertiary institutes

- people in positions of authority within the Education Department and the Examinations Authority

- people in positions of authority within secondary schools

- teachers of English in secondary schools 
Some of the subjects (about 20 per cent) were known personally by the researcher, while the others were approached independently (based on their specific reputation and/or key position) and asked to participate in the survey. ${ }^{3}$ The composition of the group of interviewees is shown in Table 3.7.

It was felt that while there should be a balance between the number of teachers, teacher trainers and other practitioners, it was also important to obtain the views of people who, via their involvement in the process of educational decisionmaking and high public profile, had taken leading roles in language education in Hong Kong. It was also felt that there should be as many representatives as possible from the different facets of English language education, that is, teachers, teacher educators, and people involved in the administration of educational policy. As much as possible the same interviewees who took part in 1997 were asked to participate again in 1999. However, for various reasons, not all of the original group were able to do so (some had retired and left Hong Kong, some had changed positions and felt they were no longer qualified to comment on the issues tabled and others just did not wish to take part). Consequently, other suitably qualified practitioners were approached to participate, with a view to maintaining the overall composition of the group as much as possible. In total, nine of the original group of interviewees also took part in the second round of interviews. It was felt that to maintain a representative group of practitioners was the most important consideration.

\subsubsection{2 $\quad$ Procedures}

The first round of interviews was held during the period May-August 1997 at the time when Hong Kong was changing from a British Dependent Territory to a Special Administrative Region of the PRC. At this time, the interviewees (21 in 19 interviews) ${ }^{4}$ were asked for their predictions of the effects of the changeover on English language education in Hong Kong. All of the interviews took place at the interviewees' place of work and beforehand each of the interviewees was sent 
a copy of an outline of the survey detailing the background, procedures and expectations of the research. ${ }^{5}$ In addition, they were sent a copy of the questions which would be asked in the interviews, relating to the issues affecting English language teaching in Hong Kong secondary schools and the changeover in sovereignty. ${ }^{6}$

The second round of interviews was carried out from June-October 1999. This time, 18 people were interviewed. In addition, copies of the invitation letter and questions were sent out to school principals in a number of schools. This mailedout survey resulted in one response each from a principal and a panel chair, both of whom responded anonymously in writing. A further respondent, an official of the government's Education and Manpower Bureau, also preferred to give a written response. This brought the total number of respondents to 21 , the same number as for the first administration. The questions of the second administration of the survey differed in some ways from those of the first administration as some were no longer relevant. In addition, an attempt was made in the 1999 survey to move away from an exclusive focus on the changeover, so that other issues of importance to language and education for the future might be discussed. ${ }^{7}$

Most of the questions refer to possible changes affecting English language teaching in secondary schools after the changeover. While these questions formed the basis of the interviews and approximately eighty per cent of the responses given, the interviewees were encouraged to talk freely on any issues which arose and which it was felt were relevant to the study.

The interviews lasted for generally just under an hour and were audio-taped, with the tape recordings transcribed for content. (Copies of the transcriptions and written responses can be found in Appendix A.) Each transcription was reviewed to identify issues raised that were related to English language education in Hong Kong. When a respondent addressed an issue of relevance to this study, each separate point raised was counted as one instance. For example, when discussing the Benchmarking of language teachers issue, one interviewee said: 
[They have been talking about some form of incentive for people who, let's say, pass with a distinction or whatever, do very well in their thesis or whatever assessment method they're going to use.] But I think they have to think seriously about this now and II was told more schools are now going to get expat teachers, they will have less of a problem with the language and so on.]

Two distinct points are identified here (shown in parentheses), the first categorised under 'Incentives for those who reach the Benchmark' on the Benchmarking issue, and the second coming under Teachers' issues and categorised as 'Native English Teachers'. From this procedure, a total of 493 points was found in 1997 from a total of 153 pages of interview transcripts, as compared to 1999 when the interviews totalled 365 points in 126 pages.

The collated comments or statements made by the group as a whole were then analysed using the constant-comparative method, in which categories are identified within the data and then as new categories are identified they are continually compared with the existing categories so as to develop ever more encompassing core categories until all categories are saturated (Strauss, 1987). ${ }^{8}$ In the following section, the core categories are listed under headings that reflect their nature and it has been possible to group the headings into major areas of conecrn to English language education. This process was carried out for both rounds of interviews and the findings are compared to gain an understanding of the variance in the perceived areas of coneern over this crucial two-year period. 


\subsubsection{Findings}

\subsubsection{Findings in full}

The full lists of comments made by the interviewees during the two round of interviews can be found in Appendix A.

\subsubsection{Overview of Main Issues Addressed in the Two Interview Surveys}

In the following section, comparisons are made of the main points raised under the headings of the major issues identified for the two rounds of interviews.

The major issues addressed by the interviewees are listed in Table 3.8 in terms of the total number of interviews in which they were discussed. In 1997, significant points were made in all interviews on the medium of instruction and Benchmarking issues, with teachers, proportion of the timetable, teaching methodology and curriculum and the English language also raising significant comment in most interviews. In 1999, the MOI was the only issue that all interviewees said something noteworthy about, whilst teachers, methodology and curriculum and the English language also featured strongly in respondents' comments. Although questions were asked about certain issues, such as education policy (in 1997) and students (in 1999) many of the interviewees had few comments, if any, on these subjects.

In Table 3.9, the major issues discussed by the interviewees are presented again, this time in descending order of the total number of comments given. Table 3.9 shows a similar breakdown of the issues discussed as that of Table 3.8. In other words, of the total of identified comments made in 1997, 31 per cent concerned some aspect of the medium of instruetion issue. Clearly, as was found in the survey of the issues covered in the newspapers in 1997, it was this issue that dominated discussion, in both 1997 and two years later in 1999. Teachers' issues also showed up strongly in both rounds of interviews, which is to be expected 
given the strong focus on teachers of the questions and the fact that most of the interviewees were teachers or teacher educators, or possibly had been teachers earlier in their careers. Other points to note are that the Benchmarking of language teachers did not feature so strongly in 1999. Yet, although it was not addressed directly in the questions, it still commanded 5 per cent of the comments made. At the time of the second round of interviews, this initiative, although at that time in development, had perhaps lost some of the impact that it had had when first discussed in 1997. The other major issue to receive attention was the English language itself, which increased slightly in terms of percentage of comments made over the two rounds.

\subsubsection{Detailed Findings for Each Issue}

In the following pages, the core categories identified above are broken down into sub-categories, representing aspects or subtopics of the individual issues identified. Where appropriate, quotations are included from the interviews in order to illustrate the findings.

\section{The Medium of Instruction Issue}

Several aspeets of the medium of instruction (MOI) issue, which dominated the thoughts of educationalists during this period, were discussed during the two rounds of interviews.

The decision to adopt (hinese as the medium of instruction (CMI) in most schools was believed by many respondents in 1997 to be a political decision and the reason it was being done at this time (and not before) was thought to be that it would be easier for the post-handover government of the SAR to implement a Chinese language policy than it would have been for the British colonial government: 
... the very fact that the Firm Guidance was planned in 1998 and not 1996 is because I think they would see much less opposition after the handover than before the handover. (University Educator, 1997)

In the past they would probably not have been able to get away with enforcing something like this, but they now obviously feel that they can ... hecause it's just one of the many changes taking place, this has otviously proved to be the best time for the ED to try and do this. (Secondary School English Panel Chair, 1997)

A Chinese MOI was perhaps being left to the SAR as a kind of legacy, or, as another interviewee put it, the colonial government was afraid that the SAR government would try to implement $\mathrm{CMI}$ in all schools, and so was attempting to pre-empt such a move:

There might be this worn that the Chinese government, or the SAR government, which will be under Chinese influence anyway will say change the MOI in all schools into Chinese, so they can pre'mpt that by doing this. (University Educator, 1997)

A further focus of attention of the respondents in 1997 was the influence that the various stakeholders had on the decision to implement majority CMI. Many respondents felt that the government had stopped short of a fully Chinese-medium secondary sehool sector for fear of the backlash from parents (as well as students and teachers), who wanted English-medium education for its educational and cultural capital:

Thy [the government] have got it in the neck from the parents, they will get it in the neck from the parents, the pressure will be right on the schools to cominue to pretend to be English-medium. (University fducator, 1997) 
Parents were really anxious about getting their kids into Englishmedium classes, so that's the situation now, that parents prefer English-medium. And I suppose that market forces would force the principals to go for English medium. (Education Officer, 1997)

Attention had switched more to problems with the change to CMI in 1999. It was felt by respondents that teachers would have to learn the Chinese translations of terms that they previously expressed in English, even when they had been speaking in Cantonese to their students (i.e., through code-mixing of technical or academic terms). There was concern that teachers would be forced to teach in Chinese but that textbooks would still be mainly in English. It was also felt that schools forced to change to CMI would experience a drop in the standard of the intake of their students and standards of English would fall because students would no longer have the exposure to English outside of the English class. Other issues that it was felt needed to be addressed were the possibility of different training for English teachers teaching in EMI or CMI schools, and even different examinations for students from either kind of school. It was clear that the interviewees felt that as a result of the implementation of CMI, English standards would fall and consequently extra resources and new methods would need to be put into the teaching of English in these schools:

We have introduced a lot of measures to try to make up the exposure, to enrich the language environment in those schools which are now using Chinese as the medium of instruction. (Education Officer, 1999)

In 1999, the respondents indicated that measures were being taken to arrest the possible decline in English standards in CMI schools in the shape of more resources such as employing more Native English Teachers (two for every CMI school and one for every EMI school), encouraging English learning activities through schemes such as the Quality Education Fund and provision for language leaming facilities such as language labs and multi-media rooms: 
My personal opinion is that it [the implementation of CMI] has made school authorities more aware of the fact that they need to put more resources into teaching English language and it has also coincided with some IT resources being given to schools and ... they are hiring native speakers ... as well. (University English Teacher, 1999)

The issue of the MOI in the tertiary institutes, which had been raised by a number of participants in 1997, remained a prominent issue in 1999. Questions were raised as to how students from CMI schools would be able to cope with university study through the medium of English. One suggestion which they reported was to have a stream within CMI schools for students with the potential to enter university. Although there were a few participants who intimated that it might be feasible for some universities to adopt CMI, or for most of the students in the universities to be taught through the medium of Chinese, it was felt more widely that the universities must maintain English as the MOI in order to remain internationally recognised.

The practitioners interviewed in 1999 felt that it would take some time before the effects of the change to CMI could be assessed and before people would get used to the idea. They pointed out that eventually, high-ranking government officials would be graduates from CMI schools, instead of the situation at present where almost all come from Anglo-Chinese EMI schools, which might affect the relative prominence of English. However, local businesses as well as the tertiary institutes would still regard English as the status language.

\section{Language Benchmarking ${ }^{9}$}

The other major government-backed initiative to be announced just prior to the changeover was the Benchmarking of language teachers, in which teachers of English would be required to reach a certain level of proficiency. The details of 
the implementation of the initiative were still at the planning stage in 1997 and generated a lot of conjecture among the interviewees. ${ }^{10}$ Some felt that if the Benchmark level was set too high, then this would result in a shortage of teachers. It was also felt that it should not be set too low just to fill posts. Others were concerned about how the results of the Benchmarking might be interpreted, and it was felt that they did not know enough about it yet:

... what are you going to accept as a Benchmark? What does a certain Benchmark mean?

There may be a different Benchmark for primary teachers than for secondary ...

I think there probably will also be a distinction between teachers who teach through the medium of English in other subjects and teachers who teach English, there will be a different requirement.

(Education Officers, 1997)

Table 3.11 shows that the issue of language Benchmarking was less prominent for the respondents in 1999, when the participants were not asked about it directly, but were still concerned about the details of its implementation. Initial reactions in this group had been against the Benchmarking; participants felt that some teachers might deliberately fail it so as not to have to teach English. Respondents also felt that it was unnecessary to Benchmark teachers who had already qualified as English teachers and that teachers would consider it an insult to their integrity. The biggest worry seemed to be that the Benchmarking would put added pressure on teachers who were already overloaded.

... some teachers oppose it [the Benchmark initiative] because they think it's an interference in their professional independence. Once they've gone through their education system and they've been 
certified as good enough to teach, then they shouldn't have to prove themselves. (Education Officer, 1999)

This anxiety or apprehension would be felt most acutely by experienced teachers, who would maintain that they had already qualified and were now having their professionalism called into question. Consequently, some participants called for the Benchmarking to be for new teachers only, at least at first.

On the other hand, there were several participants who felt that if the Benchmarking were done in a positive manner, it could be beneficial to language teachers. It was felt that the emphasis must be on the training of teachers and on helping them to improve their standards:

I feel now that there's a distinct feeling amongst the English teachers that they need to keep up with the latest of the English teaching methodologies or the resources and also to make sure their own English keeps up with the times. This Benchmarking thing has put a lot of pressure on them [teachers]. Again, it's good and bad ... good in the sense that this will give an incentive to teachers to improve their teaching ... On the other hand, the anxiety may make people less generally motivated ... with the changes come all the uncertainties and apprehension. (University Educator, 1999)

It was hoped that this would help to increase the professionalism and the status of language teachers and hence their morale. On a positive note, interviewees suggested that incentives could be offered to teachers who reached the Benchmark. 


\section{$\underline{\text { Teachers }}$}

The next most popular subject for the interviewees to comment on in 1997 was teachers, and the standard of English teachers featured prominently (Table 3.12). Many felt that teachers in schools were either not sufficiently qualified to teach English or not of the required standard, and those entering the profession were not as good as might be hoped for. There was a call from respondents to step up entry requirements for teachers as it was felt that often people went into teaching, particularly during periods of economic recession, only because the salary and conditions were attractive. The major problem for teachers was considered to be that of language ability. It was considered vital by respondents that English teachers have a higher standard of English than their students in order to be able to teach them effectively:

... if you expect students to be able to speak and write good English, teachers should be able to teach good English. The truth is there are some teachers, even in our school, who are teaching English, whose English is really not as good as we expect. (Secondary School English Panel Chair, 1997)

It was felt by some that the morale of English teachers was relatively low and that they lacked motivation. Possible reasons given were that teachers were often forced into teaching English, especially at lower school level (Forms 1-3). A number of concerns of teachers came out during the interviews: that teachers were overworked, that they were being treated unfairly, that the teaching environment in schools was not conducive to high standards, and that they were worried about self-censorship after the changeover. The Native English Teachers (NET) scheme was also prominent, being seen to some extent as a controversial issue in 1997 due to the effects that the scheme had on local teachers' status and morale.

In 1999 there was still a great deal of concern over teachers' standards, morale and status. The issue of English teachers' language proficiency was linked directly 
to the Benchmarking initiative and it was felt in 1999 that the initiative was a positive move in this direction. By 1999, the NETs were being seen in a more positive light as more of a useful resource in the drive towards higher standards of teaching and proficiency of both students and local teachers.

The teachers I've talked to see them as a resource. I think those [local teachers] who are threatened, those who are on the defensive will feel threatened and defensive about anything. Those who feel reasonably confident about themselves as language speakers just take it on board. These people [the NETs] are well-qualified, some of them are superb, they really are a good bunch and some of them are doing superb work and ... once they get over their first contract then they get trusted. (University Educator, 1999)

... for many students who are in Chinese-medium schools now they have more opportunities to enjoy the benefits from this scheme because more resources have been allocated to them and nowadays because of the NET scheme, Native English Teachers scheme, they have ... more schools can be in touch with foreigners ... so students in Chinese-medium schools can have better advantage in receiving English education. (Secondary School English Teacher, 1999)

\section{English as Proportion of Timetable}

Table 3.13 shows that in 1997 there was conjecture over the amount of time that would be given to the teaching of English after the changeover. When asked about this, participants felt that the amount of English on the timetable in CMI schools would be increased in order to compensate for the loss of English as the MOI. In contrast, a small number felt that English might be reduced on the timetable as a result of an increase in the amount of Putonghua. ${ }^{11}$ It was thought that in order to 
reassure parents that English was not going to be neglected if the school changed from EMI to CMI, principals may decide to increase the emphasis on English teaching and increasing the number of lessons might be one way of doing this. However, the view was expressed that school timetables are already full, and with new subjects such as Information Technology, Civic Affairs and Putonghua being introduced, any changes in the timetable would have to be managed very carefully:

... for our school, the school is taking our English lesson to make way for Putonghua, so even though you may be using EMI, you have so many subjects struggling for time. I don't see any positive change in the timetable for teaching English. But for the Chinesemedium school, if I planned the curriculum for the school, if they have other subjects in Chinese, I think they can spend less time for the individual subjects and they should give more support and emphasis on English. (Secondary School English Panel Chair, 1997)

This issue was not raised in 1999, when participants were not asked to comment on it directly.

\section{Teaching Methodology and Curriculum Issues}

Turning to issues of what is taught and what teaching methodology is used, there were rather varied ideas expressed (Table 3.14). In terms of content, points raised before the handover included a potential change in the cultural content of lessons away from a predominantly British approach, more emphasis on oral instruction, a call for students to be taught more functional English, and even an opinion that students need to be taught patriotism. Of those participants who expressed a view that there might be a change in teaching methodology, it was thought that there could be a reaction from teachers against communicative language teaching: 
I do hope that we don't have a reaction against communicative methodology because that's a hangover from imperial times. I do hope we don't go back to practice and manipulation. Teachers might want to do it because the easy way out for teachers is exercises, exercise manipulation, lexical/grammatical workover, no work at the macro level and no work at the oral level. (University Educator, 1997)

There was some support from respondents for the learning of grammar and also for the use of extra-curricular activities for the teaching of English. There was also some mention of existing problems with teaching methodology, mainly to criticise traditional methods, and it was felt that the curriculum needed to be revised. It was recognised that this was the purpose of the Target Oriented Curriculum (TOC).

In 1999, there was more discussion of the curriculum and examination syllabuses and the TOC had gained greater attention in the interviewed group.

And in fact the exam syllabus, the last change in 1996 was in $C E^{12}$ and I suppose to a lesser extent to changes that were made to the oral in $U E{ }^{13}$ well they were, I mean the changes in the $C E$ were a pre-emptive strike designed to anticipate the introduction of TOC and therefore make it unnecessary to have to change our syllabus, so it was made more task-based ... (Education Officer, 1999)

It seems to me that there hasn't been any fundamental change in the classroom because of TOC. The textbooks have TOC on the cover and claim to be using TOC methods, the teachers and the schools claim to have been using it, but the substance of the lesson, what is happening in the lesson, what the students go through doesn't seem to have gone through the expected changes. This 
worries me, but I guess it will take time for it to be implemented properly. (University Educator, 1999)

\section{Focus on English}

At the time of the changeover in 1997, some respondents had felt that the status of English would decrease and this would result in a fall in the status of English teachers. It was even suggested by one interviewee that the name of the course of study English Literature, be changed so as to disassociate it from England, indicating a possible linguistic backlash against the former colonial power. ${ }^{14}$ This was partly attributed to a possible increase in the importance of Chinese (Putonghua), which would push English down from first to second foreign language (assuming that it is a foreign and not a second language at present). Some participants felt that English was not a second language in Hong Kong at all. Two participants even thought that English could become the common language between speakers of Cantonese and Putonghua.

There was less evidence of this two years after the handover in 1999, as the majority of interviewees were convinced of the continuing importance of English. Interviewees felt that English was becoming even more established as an international language and Hong Kong needed to keep up with this trend. Also, there was a feeling that the English being spoken in Hong Kong was becoming more internationalised and less British. With more Hong Kong people emigrating to North America than to Britain, the spread of American culture, and the importance of mastering American-produced Information Technology, it was remarked that American English was perhaps more relevant to Hong Kong than British English:

Probably ... the emphasis now is even greater as we don't have the stigma of English being a colonial thing now, we recognise the fact that English is a world language, an international language, and in 
order for Hong Kong to continue its present status, English plays a very important part. (Education Officer, 1999)

Student teachers should know American English well because the English used in computer programmes is Americanised and most of the well-established business corporations are US-based. In order to help our future generations to maintain Hong Kong as a major financial and commercial centre, American English has to be taught. (Secondary School English Panel Chair, 1999)

\section{Putonghua}

Following on from a focus on the English language, the next most common issue to be raised in 1997 was that of the variety of Chinese adopted by the PRC as the national language, i.e., Putonghua (or Mainland standard spoken Mandarin) (Table 3.16). There was some discussion from respondents as to whether or not Putonghua would be adopted as the MOI in schools. At the very least, it was thought that it could become the medium through which the Chinese language would be taught. It was felt by respondents that Putonghua would inevitably become more prominent in Hong Kong society than it had previously been, to the extent even of replacing English as the status language:

Give it another ten years, fifteen years and it may be Cantonese that's being threatened as the medium of instruction and Putonghua will be the move, that will be where the pressure is coming from. (University Educator, 1997)

Yes, yes, I think Putonghua is replacing English as a status language for the Chinese community in Hong Kong in the near future. (Teacher Educator, 1997) 
Views in the group of interviewees had changed somewhat in 1999 as the push for Putonghua as an MOI failed to materialise. There was still debate as to how Putonghua would fit into the school system: Would it be offered as a compulsory or an optional subject? Would learning Putonghua be an extra-curricular activity? Furthermore, would there be enough teachers capable of teaching Putonghua? These were the questions being asked by respondents in 1999 .

\section{References to Changeover}

Table 3.17 shows which aspects of the changeover in sovereignty were discussed in both years. In 1997, most of the people who expressed a view on the changeover felt that the event itself would not bring about any changes. They felt that there would be no effect on government policy towards education and also no effect on the situation, status or morale of English teachers. In their view, any changes that might occur would not be as a result of the changeover and would be more likely to be due to advances in technology in such areas as computers and information technology:

I think as far as education is concerned, apart from a few history texts which seem to have come up as an issue, the Basic Law is absolutely clear that all policy decisions are the business of the SAR government and nobody else's and I don't think there is any reason why the changeover, the One Country Two Systems, why the changeover should have any impact at all. (University Educator, 1997)

Two years later, more people in the interviewed group actually felt that the changeover had had an effect. It was thought by a few participants that people in Hong Kong had begun to question the role of English in what had become a fully Chinese society. Two participants even felt that some students were using the changeover as an excuse for not trying as hard in English as they might have done 
before. However, the majority feeling was that any changes in education were a result of ongoing curriculum development or had arisen from initiatives under development from long before the changeover. There was a noticeable reluctance to attribute any changes to the handover itself:

I think it's just coincidental because ... any changes to be made to the curriculum or the syllabuses takes a long period of time and the thought probably began when the committee members saw the need for revision rather than really because of the changeover. Yes there are changes but they are not because of the changeover. (Education Officer, 1999)

\section{English Teaching in China}

The view was quite strongly expressed that learning English is a very popular activity in Mainland China (Table 3.18). In 1997, some participants surmised that the Mainland Chinese did not have the 'colonial hang-ups' that affected the people in Hong Kong and that consequently there was not the same British 'cultural baggage' associated with the language:

I'll tell you one more thing ... people in China have no hang-ups about learning English, except the usual ones, but they're the hang-ups that a German or a Russian might have. (University Educator, 1997)

It was thought that although the teaching methodology as regards English in China was still rather traditional and based on grammar-translation, new, more communicative methods were being introduced there.

Two years later and the feeling was still that English learning was popular in China. In fact, there were some who felt that Hong Kong could be left behind in 
terms of English proficiency, especially that of its graduates:

... there are a few who are now saying that Hong Kong cannot keep up with its English standard or in terms of producing people who can speak fluent English and very soon we are going to lose out because major cities in Mainland China are producing people of high quality, including their command of English and obviously this sense of crisis is coming along and coming along very strongly among the business sector. Parents will get the message as well. (Education Officer, 1999)

\section{Focus on Students}

Table 3.19 shows the points made about students in the two rounds of interviews. There was some criticism of Hong Kong students from the 1997 participants, who felt that among other things they had a short attention span, were unmotivated and actually turning away from English:

... there's a culture within the schools which frowns on people being fluent in English. (University Educator, 1997)

There was also evidence provided by respondents in 1997 that students no longer considered English as the key to a successful future:

Now when we ask the students to improve their English, some of them will say, oh Chinese is okay. Even if I fail in English, I can still get a good job in Mainland China ... they will think that if their Chinese is alright, if their other subjects or their ability to work is alright, they don't worry if they really fail in English. (Secondary School Principal, 1997) 
In 1999, there was some evidence that people had higher expectations of students as they were not only expected to master English, Chinese and other content subjects, but also to embrace new technologies, particularly information technologies. There was also some discussion as to whether students and young people generally were coming more to terms with Chinese culture after the handover:

... the children that I see, many of them are very bright, they are very quick, they can learn new things, they can solve problems. Now their language standard isn't very good and you frown on what they say and how they write letters and reports and you worry about that. (University Educator, 1999)

That somehow international contact and being able to cope with that I think is important and is complementary to the soft communication skills because that's something Hong Kong students could have if they were willing to go for it. With some of them ... I think there is an ambivalence generally about whether to throw in their lot entirely with Chinese culture. (University Educator, 1999)

\section{$\underline{\text { Education Policy }}$}

In terms of education policy (Table 3.20), the participants in 1997 felt that any changes could result from other changes already implemented or from demands within society:

I think that government policy may change, not really initiated by the government, but in response to the changes in demand and the atmosphere in society ... there may be some sort of change of the attitude from the students, and so I don't know how the government 
will respond to this or the government will think that Chinese is more important than English. (Secondary School Principal, 1997)

The view was expressed that the colonial government may have wanted to be seen to be supporting Chinese values by implementing the Firm Guidance and promoting mother-tongue instruction. Another view was that China would not want any major changes to be made to the education system prior to the handover. According to some interviewees, if there were to be changes, these should come in the form of extra resources to improve the conditions within schools and so attract better people to be teachers. There was also a call for the universities to revert to four-year programmes instead of the three-year, British-style bachelor's degrees offered at that time.

In 1999, the main focus of discussion was the educational reforms brought in by the government and whether they would be successful or not. There was criticism of the government as being too ambitious in trying to implement too many reforms at the same time without a clear focus or direction:

So what is going to happen is you start making changes on all fronts and then you begin to lose what is the main focus, what are the major issues you must tackle. And I think this is some kind of handover, post-colonial syndrome, which is very much in line with what the Chief Executive is doing, because the Chief Executive is saying, let's take a look at everything we're doing, so civil service, you have to reform, the medical welfare, you have to reform and you're beginning to have reforms everywhere. (University Educator, 1999)

People thought that changes were necessary but that teachers were rather overwhelmed by the number of them. Some respondents felt that the Chairman of the Education Commission, Antony Leung Kam-chung was tending to act more as 
a politician or businessman than as someone with the future success of the education system at heart.

\section{Focus on Schools}

It was felt by the respondents in 1997 that schools have a responsibility to parents and in fact very often base their policy on what parents want:

So I'm afraid what schools do is largely what is convenient for the school and what is convenient for the teachers and suits the purposes of the parents, without thinking about it too much. (University Educator, 1997)

It was added that more local parents were sending their children to private schools, often international schools, where they could get an education through the medium of English and where the facilities and environment were usually better than in government or government-aided schools. As Table 3.21 shows, there was little comment on schools as such in 1999.

\section{New Immigrants from China}

At the time of the 1997 administration, there was little doubt among the participants that there would be more students coming to Hong Kong as immigrants from Mainland China, and consequently the schools would have to absorb them. This would mean either extra classes being formed or an increase in the sizes of the current classes. It would be most likely that extra classes would be needed, especially for English, as these immigrant children could be expected to be at least one or two years behind their peers of the same age in terms of English proficiency. This could cause problems for schools, and in fact it was reported that 
some schools were rejecting new immigrants because they simply did not have the capacity to absorb them:

I even promised her [a new immigrant student from Mainland China applying for a place in a Hong Kong secondary school] that if she did the test well, I might get another extra place in one of the classes. She told me that she heard all the statements from the schools she went, they all speak to her, well no place. I have no place here but if you are doing well and you are eager to learn, I might give you a place. It all depends on how you did in the test. They have to have a place to learn, right? And the time is short, you know? It is late August. I don't know, but people are rejecting new immigrants all over the place. (Secondary School Principal, 1997)

The issue of educational provision for Mainland immigrants was not discussed in 1999 (Table 3.22), although the issue of immigrants from China coming to Hong Kong to live was very high profile due to the case of the children of Hong Kong residents being refused the right of abode in 1999 (reported in Chapter 2).

\section{Reference to Hong Kong People}

From Table 3.23 it can be seen that in 1997 there was some discussion during the interviews about the people of Hong Kong looking upon the acquisition of English from a very pragmatic point of view, seeing English as a means to get a better job or to gain promotion. It was felt by respondents in the first interviews that Hong Kong people had little interest in learning the language for enjoyment or intrinsic interest or simply to improve themselves, unless they could see some (mainly financial) benefit in it. In the view of the respondents, this could serve as a legitimate motivation to learn the language, although it often meant that learners did not appreciate more innovative or unorthodox methods of learning. It was felt 
that people preferred a more functional approach, as they could see how it would improve their English in the ways they understood or thought they needed:

We all know that Hong Kong people are very pragmatic, if this is what they need to do, they'll probably do it if it's going to lead to a better job or promotion. (University Educator, 1997)

It was also felt by respondents in 1997 that Hong Kong people no longer, if they ever, resented English as the language of the (former) colonial ruler. In their view, Hong Kong people saw it as a means to an end, and as such it was rather apolitical:

... people of my generation will always appreciate the fact that whatever we say about English, colonial power, that sort of thing, then it will just represent a way of opening up a horizon for us. It does allow us to know a lot of things if I were only Chinese speaking, I would never know. (University Educator, 1997)

The views of Hong Kong people as mercantile with respect to learning English, identified as an aspect of colonial discourse by Pennycook (1998), is here expressed and endorsed by Hong Kong people themselves. This is evidence that such discourses are present in local people educated under the colonial system.

In 1999 there was much discussion in the interview response about the public's views on education. As a result of the many reforms taking place and how they had been brought into the public arena by the media, it was felt by the respondents that the public were no longer prepared to accept the education system as it was and were taking a much more pro-active role:

... but what I think the effect that it [the reforms in education] has on teachers and the community as whole is that I think for the first time they are involving parents, they're involving business people 
and teachers and the response from the people outside education is very enthusiastic because they get to air their views. (University Educator, 1999)

Here there is evidence of the increasing politicisation of education and language issues in society.

\section{$\underline{\text { Reference to Chinese }}$}

Lastly, it was felt by the participants in both administrations that standards of Chinese would become a big issue and that inevitably, like Putonghua, the simplified Chinese characters that are used in Mainland China would have to be taught in Hong Kong (Table 3.24).

\subsubsection{Case Studies of Interviewees Present in Both Rounds}

Having looked at the main issues discussed by each group of interviewees as a whole in the two rounds of interviews, to gain a clearer picture of which issues were felt to be of most importance at each time, the next section will summarise the responses of those nine interviewees that were present in both rounds. ${ }^{15}$

\section{Interviewee I}

University Educator

Before the changeover, Subject I clearly supported the switch to Chinese medium of schools in Hong Kong. In fact, I advocated all schools changing, not just the majority, and warned that there was a danger of EMI schools becoming even more élitist than they already were. I also felt that there would be more resources put into English teaching in CMI schools. In 1999, I showed concern that standards of 
English in these schools would decline unless those resources were put in place. I also spoke of the worries in EMI schools that their standards were being closely monitored and that in general the change in MOI had focused much attention on language issues.

In 1997 this subject spoke of the need for better qualified English teachers, which is a theme picked up on and discussed more in 1999. I felt that there were many teachers who were teaching in schools but who should not have been because they were not qualified to do so and/or did not have adequate English proficiency. I also expressed the view that the Benchmarking initiative had the support of the public who were aware of the deficiencies in the standards of English teachers. ${ }^{16}$

Subject I was reluctant to assign any changes in language teaching or in education generally to the handover. I felt that there had been normal processes of curriculum reform which accounted for the changes being witnessed and in fact that new initiatives were being fuelled more on the basis of research than as a result of popular trends. I did feel that students' attitudes had changed, particularly tertiary students, as they were likely to place more importance on the learning of Putonghua than of English and to see English as merely a means to progress to tertiary education.

\section{Interviewee II}

University Educator

Interviewee II believed that the government decided to implement the change in the MOI after the changeover as there would be potentially less opposition to it. II felt that this change had caused a lot of controversy and had had a significant effect on education, such as fostering more interaction in content lessons, although II thought that it had had no real effect on English language teaching. In fact, this subject believed that the government had no clear focus as to the direction in 
which their educational reforms were going; rather, they were making changes on all fronts, perhaps as a result of what II called 'post-colonial syndrome'.

The interviewee was critical of the NET scheme, saying that it has not been properly thought out and had resulted in a situation of low morale in schools. II also felt that the Benchmarking initiative would have a positive impact on teachers as it would add to their prestige.

As regards the English language, before the changeover, this interviewee felt that the status of English would remain high, as businesses and educators were anxious for it to do so. After the changeover, II felt that this had become more manifest as concern over English standards in the workplace had been raised. In addition, the interviewee remarked on the increased involvement of parents in education issues, expressing the view that since the handover the public had developed a rather negative view of the education system in Hong Kong.

\section{Interviewee III}

University Educator

When discussing the change in the MOI, the interviewee was conservative about possible implications of the change before the handover, expressing the view that it would take some time before any changes were noticeable. After the handover, III spoke more about how the change had been accepted by some parents and students but had caused a lot of reaction and anxiety. III felt that the change had caused divisions in education and that the knock-on effect to tertiary education was significant, with university teachers making more use of Chinese in their otherwise English-medium teaching, and university students finding it increasingly difficult to cope with an English-medium learning environment.

The interviewee spoke after the handover about the need for teachers to develop themselves professionally if they were to 'survive' in the 'new' teaching 
environment. As with the previous interviewees, III had in 1997 been more concerned with the details of the implementations of the Benchmarking, whereas in 1999 III looked more at the implications of it and the pressure that it was putting on teachers. In addition, III felt that the changes in education were putting more pressure on students, in the sense that more was expected of them than had ever been in the past. Not only were they expected to master content and language, but nowadays they also had to develop new skills, particularly in IT.

\section{Interviewee IV}

English Teacher in Secondary School (1997) and University (1999)

This subject had been an English teacher in an EMI school at the time of the first interview then had moved on to higher education by the time of the second. IV spoke quite negatively about the change in the MOI, regarding it as threatening the provision for English teaching and potentially English standards. IV was also quite concerned about the lack of qualifications and low morale of English teachers, at the time of the handover. IV felt that teachers of English in Hong Kong are either too over-worked to be innovative or are not committed enough to the profession. In 1999, IV seemed appreciative of the government's efforts, through the Benchmarking, to raise the standards of English teachers.

The subject showed concern also at the limitations of the Hong Kong English syllabus, accusing it of being too localised and functional. IV would have preferred to see students given a wider, more international perspective on language, possibly through the appreciation of literature. The subject made comparisons with Singapore, where standards of English amongst students are, according to IV, much higher and students have a more cosmopolitan outlook. IV felt the situation in Hong Kong had not changed since the handover as students attitudes to learning were basically the same. However, IV thought that some students who resented learning English would regard Putonghua as more relevant to their future needs, whilst there were others who were motivated to take it upon 
themselves to improve their English language skills if the provision for it were not adequate.

\section{Interviewee $\mathrm{V}$}

University Educator

This interviewee expressed the view, both before and after the handover, that the change in the MOI would be socially divisive. Interviewee $\mathrm{V}$ foresaw an increased élitism as places in EMI schools would be most sought-after and the teachers there would receive a boost to their morale, as opposed to those in the majority of CMI schools, who he felt had become demoralised since the change. Yet $\mathrm{V}$ also felt that the washback effect of having an English-medium tertiary education system meant that provision for English would remain strong in both EMI and CMI schools. V was critical of the NET scheme, thinking that the NETs were not being used effectively in schools and were a further contributor to the low morale of local English teachers, as was the Benchmarking. Although this interviewee supported the initiative, $\mathrm{V}$ felt that teachers should be rewarded, possibly with a salary increment, when they had reached the Benchmark level.

As regards English in society, this interviewee felt that people were now using the language more than they had done previously, particularly people in service industries, and proficiency in the language had been a necessary accoutrement for an educated Hong Kong person. $\mathrm{V}$ thought that Putonghua was becoming more important but not at the expense of English.

\section{Interviewee VI}

Secondary School English Teacher

In 1997, this interviewee believed that the change in MOI had been made for political rather than educational reasons, that the government wanted to be seen to 
be moving away from an English-dominated educational system to one which was more Chinese-focused. The interviewee expressed concern that this would result in lower standards of English amongst students and within society. VI felt that more resources would need to be put into English teaching if schools were to change to $\mathrm{CMI}$ in order to maintain the provision for English instruction. Two years later VI was able to report that resources had been made available but that there was now some pressure on English teachers, both in CMI schools, as students there would no longer be getting exposure to English during content lessons, and in EMI schools, who were anxious to maintain their status.

This teacher was rather more positive about the NET scheme than other interviewees had been. VI felt that although the scheme had had difficulty at first in recruiting teachers, the presence of these teachers in schools had been very beneficial as they had brought in new methods and approaches in addition to exposing the students to a new culture. From VI's experience, students actually felt more comfortable speaking to NETs in English than they did to local teachers, with whom they shared the first language.

In general, the interviewee felt that there had been no change in the importance attached to English by students and the public at large after the handover when compared to the situation before. VI did feel also that there would be a greater attention paid to IT in schools in the future as well as possibly new approaches to learning such as self-access.

\section{Interviewees VII, VIII and IX}

Government Education Officers ${ }^{17}$

The interviewees spoke at length about the change in the MOI, particularly about the effects that it would have on teachers and students. Before the handover, they expressed support for the change to CMI, but felt there was concern that teachers would find the change difficult, as both English and content teachers would need 
to adapt their teaching styles significantly. This point was also made after the changeover. As a result of the change, they felt that students would regard English as being less useful than before, at least in the short term. Within the schools, they thought that the use of mixed code would continue in CMI schools, despite the government's stated intention to eliminate it. On the other hand, the interviewees felt that students in Form 1 in EMI schools were at that time, with the change in its first year, getting a 'purer' form of English in their lessons as there was pressure on these schools to stick to the medium that they had been designated to use.

The interviewees had not said much of note about teachers in their first interview. In 1999, they expressed the view that teachers do not like change and that the new initiatives introduced since the handover had affected their morale, particularly the Benchmarking. Before the handover, these interviewees had spoken about the ways that the Benchmarking might be implemented, such as who would need to sit for the assessment. In 1999, their focus had shifted somewhat to the wider implications of the Benchmarking such as its effects on teachers and teacher education.

Before the changeover there was also little discussion of possible changes in teaching methods or the curriculum, but two years later there was some discussion of the change in the English teaching syllabus for schools, which had just been released. The interviewees felt that the syllabus had changed as there was a need to bring it up to date with modern thinking and pedagogy. There was some discussion of innovations such as the Target Oriented Curriculum, IT and schoolbased assessment, all of which were becoming more important.

\section{Summary of the Patterns in the Case Studies}

The above interviewees, those that took part in the study in both 1997 and 1999, showed some differences in their opinions on the major issues but were also in 
agreement to a large extent. There was a discernible bias towards their own teaching situation evident, with the teacher educators (Interviewees A, B and E) showing concern for teacher issues and educational issues in general, the educational officers more concerned with curriculum and assessment issues, and the teachers focusing on school-based issues affecting teachers and students.

\subsubsection{Discussion}

In the last part of the Findings section, the issues have been grouped from the interview data to show the general pattern of change in the whole social system of education in Hong Kong from the perspective of those participating in those changes at the time of the great political event of the handover.

The issue categories identified from the interview data were:

- Administrative and Institutional

- Behavioural

- Educational

- Intercultural

- Language

- Professional

- Socio-Political

In the following section, each of these issue categories is presented as a tabulation of comments made by the interviewees during the two rounds of interviews. The findings are then related to those reviewed previously. A summary of the number of comments made on each of these issues in the two rounds of interviews is shown in Table 3.25. There was very little difference in the attention paid to these issues across the two rounds of interviews. In fact, the two types of issues most commonly discussed, language issues and professional issues, made up identical proportions of the total comments made on all issues. 
The most important of these issues in terms of the number of comments made was that relating generally to language.

\section{Language Issues}

A breakdown of the comments in both rounds of interviews can be seen in Table 3.26. The predominant comment to be made concerning language issues prior to the changeover in sovereignty was expressions of support for the change to CMI in schools ( 9 per cent of total comments made). Although many may have voiced dissatisfaction with the way that the government went about enforcing this, there was clear support for the principle of teaching students through their first language. Although the point should be made that there were also comments made against CMI (5 per cent of total comments). The fact that there were no comments made either way after the changeover shows that the situation had changed to one of acceptance of the reality of the situation rather than debate over its merits. Prior to the implementation of the change in MOI, there was naturally much discussion amongst ELT professionals about whether the change would benefit students in terms of their English language proficiency. The high profile of the change, as evidenced by the amount of coverage given to it in the press as well as the attention it was given at the time in academic circles in conference presentations and publications, showed that it was a key issue that was demonstrative of the changes occurring at the time of the handover, despite the fact that, as remarked upon by many interviewees, the process leading to the change had been underway for some years previously. Debate on language issues in Hong Kong has always centred around the uses, proficiency, status and value attached to the languages of the home (Cantonese) and of power (English). The MOI issue epitomised these facets and hence generated much comment. Once the decision had been made and implemented, people accepted it and attention moved more towards dealing with it. In other words, there were signs that the innovation was causing reconstruction within the system, as school administrators, teachers and students were either changing their own practices to deal with it or were avoiding having to change and were working around it in some way. In either case, change in the system was 
resulting and the potential for more change was enhanced. Evidence of how teachers were coming to terms with the change will be presented in Chapter 5 .

There was confirmation that the status of English in Hong Kong would remain high, with the number of comments making this point more than doubling in percentage terms in 1999 as compared to 1997 . The potential threat to the status of English from the transition to Chinese rule did not seem to materialise, if indeed people might have thought it would. As discussed in previous sections, the number of new innovations designed to maintain or improve English standards not only in schools but in society generally shows at least that the government wished to be seen to be addressing the issue of falling English language standards. Yet, standards and status are two different issues and whilst the importance of having at least a minimum accepted standard of English in order to fulfil one's role in society to a satisfactory degree remained and remains significant, the attachment of English to colonial rule clearly dissipated. The view was expressed by interviewees in 1999, and also in 1997 but less so, that English is seen by the majority of the population as a functional tool to enable them to achieve a better position in life. Questions about the role of English became more prevalent after the changeover, as did discussion of the involvement of parents and the public generally in educational matters affecting English language education. The innovations or initiatives referred to, particularly the change in MOI and language

Benchmarking, generated much controversy and raised interest in such matters, where previously there had been little. Undoubtedly, the changing language situation and the efforts of administrators and educators to address those issues seen to be in need of addressing, brought issues of language into more into general public discourse than they had perhaps ever been before.

\section{Professional Issues}

Table 3.27 shows the comments made on what might be termed 'Professional Issues' in the two rounds of interviews. The dominant issue for teachers of 
English at this time was the language Benchmarking. In 1997 views expressed showed concern for the morale of teachers in the face of pressure caused by the imminent 'threat' of having to take an assessment to continue teaching. As the details of this assessment had not been worked out at this stage, conjecture was rife and the tendency was to lean towards a negative view of the effects that the Benchmarking would have on local English teachers. Yet there was concern expressed as to the standard of local English teachers; and although there were voices speaking out against the initiative, there were also those who believed that as a way of raising standards and increasing professionalism, it should be supported.

The same issues were being discussed two years later, but views were more focused. Almost one-third of the comments made on these issues were concerned with needs for and potential changes in teacher training. Whilst interviewees did not generally feel that the changeover had had any effect on teacher training, or would do in the future, there was a consensus that English teacher preparation was changing, but as a consequence more of changes in the education system and the needs of society. The change in the MOI in schools would have a washback effect on teacher training as decisions would need to be made as to whether content teachers should be taught how to teach through Cantonese, or through English, as they would previously have done. The Hong Kong Institute of Education, the SAR's major teacher-preparation institution, has for many years run its courses through two streams, Chinese and English; the relative weighting put on each stream would have to be addressed in the light of the potential future needs of the graduates. In addition, the Benchmarking highlighted concerns for teachers' language proficiency; more attention would need to be paid to this in their preparation. As an example, the BATESL course at the City University of Hong Kong, which had previously devoted approximately one-third of its first year to improving students' English, would, in future, provide more language support for those students deemed to require it, throughout the three-year course. ${ }^{18}$ 
The motivation and morale of teachers was also a major point of discussion in 1999. It was felt that the pace of reform in education brought in by the government was having a damaging effect on teachers, who were expected to have to adapt to changes in curriculum (TOC), teaching medium, school structure (the employment of NETs, change in the admission procedures for secondary school students, change in banding etc.), whilst at the same being time put under the pressure of possibly losing their job if they did not reach the Benchmark in language proficiency. All of these changes were impacting on teachers directly and they were being given no extra 'space', in terms of a lighter teaching load, to take the changes on board and react to them in the ways that the initiators of the changes might have hoped that they would. The demonstrations by teachers against the Benchmarking are perhaps an indication of the frustration felt by teachers, particularly English teachers.

\section{$\underline{\text { Administrative and Institutional Issues }}$}

Table 3.28 shows a breakdown of the comments made on issues that were considered to be either administrative or institutional. Again, it was the MOI and Benchmarking issues which seemed to dominate. As a result of the change in the medium of instruction, there were questions asked before the changeover about how flexible the measures would be in terms of whether the Firm Guidance, which decreed that all schools designated CMI would be required to teach all subjects other than English though the medium of Chinese from Form 1 from September 1998, would be flexible to some degree. It was felt that there would have to be flexibility since tertiary education, external examinations and textbooks would all still be geared towards English-medium. There was also conjecture on whether the amount of English being taught in schools, particularly CMI schools, would change to compensate for the loss of English input through content subjects. Most interviewees felt it unlikely that there would be any change since the timetable was already full and the introduction of 'new' subjects such as IT and Putonghua would further increase pressure on the more 'traditional' subjects 
such as History and Geography, not to mention the core subjects of English, Chinese and Mathematics. As new initiatives begin to diffuse throughout a system, change results in reorientation of priorities regarding what is important to teach, and this effect can therefore be expected in the present case. More than one interviewee remarked on the fact that education in Hong Kong was becoming more functional or even vocational, with the emphasis moving towards skills development and problem solving and away from the accumulation of knowledge and development of social and ethical values that had traditionally been the strengths of Chinese education.

In 1999, the focus had shifted more to a consideration of the effects of the changes underway, such as the effect of the change in MOI on schools. There was noticeable discussion of examination syllabuses in 1999, as this was a topic on which the interviewees were asked to comment, and most people felt that the changes in the syllabuses were being made as a natural result of changes in other parts of the education system. In other words, innovations in one social subsystem were impacting other, interconnected systems, as one might expect. Questions concerning the implementation of the Benchmarking initiative surfaced here rather than being seen as affecting teachers professionally. At the time of the second round of interviews, as previously discussed, the initiative was in the piloting stage and had been rather removed from the public or the eye of the teaching profession. Yet concerns remained as to how it would be implemented and who would be affected by it; and these concerns were set to continue through to the first live administration in March 2001.

The other major administrative issue raised at this time, and this could as well come under educational issues, was the effective deployment of NETs. There was the concern that decisions were being made as to how to use NETs from administrative rather than pedagogical or educational standpoints. These decisions were in most cases made by the individual school principal, resulting in some NETs being used as 'oral coaches', providing speaking practice for every student (other than examination classes perhaps), whilst others were 'thrown in at the 
deep end' and told to take on a full timetable of duties alongside their local colleagues, a task for which many were not adequately prepared.

\section{$\underline{\text { Socio-political Issues }}$}

Comments on the issues identified as being socio-political in nature are shown in Table 3.29. Prior to the changeover in sovereignty, the change in MOI was discussed in relation to the extent to which the decision to finally implement it, after many years of consideration, was, in fact, political. Amongst the views expressed were that the decision to go ahead with the change at that time was made because the then colonial government felt that the change would be more acceptable to the public after the handover, when Chinese identity would be at a peak. Another view was that as so many other changes were occurring at the time, this change in the education system would not stand out as being such a major upheaval. In fact, it did stand out and a great deal of attention was paid to the change in MOI, particularly through the news media. Whilst educators may have agreed with the switch to majority CMI, many did not agree with the way that the government decided to carry out this change (the Firm Guidance). It was felt by some that the policy had been heavy-handed, that schools had not been given enough notice, or that the decision as to which schools would be allowed to continue as EMI were inconsistent and non-transparent. The charge was made that the change would be socially divisive, creating more, rather than less, élitism within the education system. Furthermore, there would be linguistic hegemony as government would be seen to be supporting Chinese whilst the business sector were still strongly supportive of English for its commercial and international value. The public too would still favour an English-medium education, with those

in the position to do so seeking either to send their children overseas or to withdraw them from the local system and move them into the international school system, which continues to prosper. 
With regard to the changeover and its possible effects, interviewees were conservative in their opinions as to whether it would have any effect on English language education. Those changes or reforms in process or being considered were thought to have been undertaken for educational rather than political reasons. Interviewees did not foresee the political change impacting directly on education. Changes were predicted but they would be due to the reforms themselves. In 1999, there was more discussion of change in a general sense, with references made to the reforms in education and the effects that they were having. Interviewees recognised that the government was intent on enacting change in the education system, and people began to consider the possibility that the changeover had acted in some way as a catalyst to change. Changes attributed directly to the changeover were in the area of language and people's attitudes towards English and Chinese. There was a belief amongst some that there had been an increase in Chinese ethnocentrism and as a consequence, Chinese ethnolinguistic identity and vitality. It was foreseen that there would be conflict between this rise in attention to Chinese and the renewed efforts on the parts of the private and public sectors to maintain a high level of proficiency and attention to English.

Another noticeable difference in the focus of discussions was the increase in the references to the views of parents and the public. As noted earlier, education issues came much more into the public eye during this period, with the public beginning to take on a more participatory role in educational discourse. In addition to such stakeholders as teachers, students, tertiary institutes and school administrators, by 1999, the general public had become another social group which had to be considered if innovations in education were to be successfully diffused. At this stage, it is logical to say that the public consists of people who are either students, or parents of students, or, if they do not come into the previous two categories, they are people who were once students themselves. It might be possible to define 'parents' as a group by considering them as those parents who take an active role in education by joining parent-teacher associations, becoming involved in their children's school activities such as open days, or simply taking an active interest in their children's learning by communicating with teachers and 
school authorities and following educational issues in the media. The general public, on the other hand, can be considered as the section of the populations who are not directly involved in education through employment or voluntary participation. The greater involvement of the public in education has manifested itself through an increase in the numbers of letters to the editor of newspapers, of attendance at public forums on educational reforms, and through the fact that news items on educational matters have appeared with seemingly greater frequency than in the past. It is assumed that newspapers print coverage of news affecting education as this is what their readership wants.

The increased involvement of the public in educational affairs could be a reflection of the greater empowerment of the middle class, which had become enlarged due mainly to the economic boom in Hong Kong of the 1980s and early 1990s. As more and more people became financially secure; with jobs, housing (either privately owned or government-provided) and investments, they perhaps began to feel that they had choices (in terms of the education that they desired for their children) and that their were ways of making claims on the government, as evidenced by the demonstrations against the suppression of students in Beijing in 1989. In general, the public began to have more participation in public affairs and the democratic reforms in government promulgated by the British colonial administration in the early 1990 s tried to capitalise on this added participation, or were perhaps a manifestation of it. The result has been that there has been an increase in what might be termed 'grassroots forces' impacting on the ideology of Hong Kong affairs. The progress of prospective reforms occurred increasingly in the public arena during the post-handover period, possibly contrary to fears before 1997 of stricter government control and less accountability.

\section{$\underline{\text { Educational Issues }}$}

The comments related to pedagogical or educational issues are shown in Table 3.30. Of the issue categories from the interview data, this was the only one in 
which there were more comments in the second round of interviews than in the first. The comments before the handover tended to be concerned with the inadequacies of the language teaching methodology adopted in Hong Kong classrooms. It was felt that the typical, transmissive approach used to teach English would prevail after the changeover since the teaching approaches of teachers in Hong Kong schools tended to be determined more by contextual or situational factors relative to the school in which they taught and the political change would have minimal effect on this. Rather, changes in the MOI and in the curriculum, as a result of TOC, would have more of an effect. It was suggested that as a result of the change to CMI of most schools, more extra-curricular activities would be employed as a way of increasing or at least maintaining students' exposure to English.

The comments made after the changeover reflected the effects on teaching and methodology of the changes implemented on the system, i.e., the switch to CMI, the TOC and the increase in attention to IT in teaching. With CMI had come an increase in resources for English teaching as well as a significant increase in interaction between students and teachers in content lessons. Changes in the curriculum had come about as a result of, according to some, changes in society, as well as modern thinking on language teaching. There was to be a greater focus on skills development and the task-based, Target Oriented Curriculum was set to change teaching approaches. The need for change in examination syllabuses was also mentioned but, as stated by the education officers (Interviewees VII, VIII and IX), there was no immediate plan to change the examination syllabuses, a process which was said to be long and difficult.

\section{Behavioural Issues}

Table 3.31 shows what have been termed behavioural issues, in that they relate to the characteristics of individual groups, such as students and teachers. Before the handover, there was a certain amount of criticism of students, including 
statements that they have a short attention span, that they are reluctant to speak English in class and that they are not motivated to improve their English. In addition to these criticisms were observations about the culture of students, such that demonstrations of ability in English are frowned upon by peers. There was less criticism and more sympathy with students from the interviewees in the second round of interviews. People felt that more was expected of them than had been in the past and that they were coming to terms with IT although their communication skills were declining. Certainly more is expected of students as the timetable becomes fuller and competition for places at tertiary institutions and in the workforce becomes fiercer. The interviewees may have recognised that students had to cope with the education reforms just as much as teachers and hence a softening of their attitudes towards them.

There was reference to the pragmatism of Hong Kong people with regard to educational matters, meaning that they saw the intrinsic value of a good standard of English for their children and were prepared to take action in order to achieve it. Teachers practices were also mentioned, in the second round of interviews, with respect to there being gaps in teachers' beliefs and practices.

\section{Intercultural Issues}

There were a small number of comments that could be classified as intercultural, and these are shown in Table 3.32. These comments were concerned with the possible differences between Chinese and Western culture that might impact upon English language education. It might be expected that cultural differences would be an issue in schools where NETs and local English teachers have to work together, yet there was little evidence from the interviews that this was a major issue in 1997 or in 1999. Differences in teaching approaches and beliefs are to be expected and there has been evidence since the interviews were carried out that it has been proving difficult for some NETs to become accustomed to the culture within schools. The restraints to teaching that Hong Kong teachers take for 
granted and that have been discussed in earlier sections have proved to be exasperating and frustrating for teachers whose experiences have often come in very different contexts. ${ }^{19}$

The other inter-cultural issue touched upon was the implications to Chinese of studying the language of the former colonial ruler. This was mentioned only indirectly, as a comment that people in Mainland China do not have any cultural hang-ups about learning English. Relating this to the Hong Kong case is more problematic and further study would need to be carried out to confirm whether the utilitarianism or pragmatism attributed to Hong Kong in regard to language learning would tend to overshadow any cultural or linguistic bias that might remain as a legacy of colonial rule.

\subsection{Conclusions}

The issue that dominated policy and discourse on English language education at the time of the changeover in sovereignty and the two years following it was that of the choice of medium of instruction within secondary schools. Whilst there was some suspicion, particularly amongst teachers, that the decision to announce the plan just prior to the changeover was based on political motivations, those with a knowledge of the background to the initiative felt that there was no connection between this change and any political event. Although educators were in favour of the initiative, they were aware that it would be opposed by parents and students, who have for so long associated an English-medium education with the élite of Hong Kong and had aspired towards achieving the symbolic capital that control of the English language brings.

There were problems envisaged with the implementation of CMI in erstwhile EMI schools, mainly due to the fact that teachers would have to adapt to a system whereby they would need to teach in their mother tongue, something they were not used to (an anomaly that possibly is characteristic not only of Hong Kong but 
also of other former colonial societies such as India and Vietnam). There was a lot of attention paid to the language ability of teachers, both those who actually teach language and those who teach other subjects. As the 'front line' of language teaching, language teachers, and English teachers in particular, attracted sympathy for their predicament, and suggestions were plentiful as to how they might be helped. In-service training, more resources and the employment of more native speakers were mentioned, although this latter initiative had been the cause of some anxiety amongst local English teachers who saw their jobs under threat.

That the government was taking seriously the reported lack of proficiency of English language teachers and teachers of other subjects in English-medium schools, was indicated by their announcement of the Benchmarking initiative. There was confusion over the exact details of the initiative, which had not been made clear, and there was what might at that stage be considered a 'knee-jerk' reaction from educators - that it was a threat to teachers' integrity or that it was unnecessary. Whilst it had been accepted that teachers needed help in the form of extra training and were not generally teaching at a high enough standard, there was notable opposition to the idea of teachers having to go through an assessment process, with the accompanying pressures and stresses which that entails.

Of all the relevant social groups involved in English language education, it is the teachers who emerged as the central focus of attention in the interviews. The educational reforms that were in the process of diffusion through the education system at the time required the teacher to be an active adopter of those reforms and without the participation of the teacher, these reforms were unlikely to succeed. In fact, it is very difficult to define what 'succeed' means in the case of educational reforms since it is only in the classroom that one can measure whether or not the intended reform, innovation, initiative or scheme is being practised as intended by the resource system that introduced it. Evidence has been found that measures were being taken to ensure that the MOI policy was being carried out as intended, through close monitoring of the language being used for instructional purposes. Similarly, school principals and panel chairs were responsible for 
ensuring that the new, target-oriented curriculum was being implemented by teachers. However, ultimately, it is the ways that teachers adapt their teaching frameworks to take new methods and approaches on board that shapes what goes on in classrooms. The evidence from this chapter suggests that teachers of English in Hong Kong were under great pressure trying to cope with the changes they were having to face and this pressure tended to result in less than ideal conditions for the 'successful' adoption of the changes themselves. It can therefore be predicted that they may not in fact have been implementing the new initiatives as fully or as successfully as they might have under other circumstances (see Chapter 5 for further discussion).

Turning to the question of language itself, prior to the changeover, it was thought that English would either maintain its importance as Hong Kong developed further as an international financial and distribution centre, or it would start to lose out to the principal language of Mainland China, Mandarin Chinese (Putonghua, the spoken Mandarin standard or Standard Written Chinese). Certainly the MOI issue made people think about whether there was a need for the majority of the population to be proficient in English, or whether being mainly proficient in Chinese was adequate for the majority of the population. On the other hand, would there become a need for the more highly educated members of the population to not only be bilingual in Cantonese and English, but trilingual in Cantonese, English and Putonghua as described by So (1992)? It would seem that the three languages (Cantonese, English and Putonghua) had come to represent the tripartite nature of the Special Administrative Region: Cantonese representing Hong Kong's uniqueness of character; English its history as a colonial outpost and its increasing internationalisation; and Putonghua its future as part of 'the motherland' of China. After the changeover, the anticipated surge in attention paid to Putonghua did not seem to materialise; instead a renewed focus on English as the socially and economically valuable language occurred as the SAR government was keen to be seen not to be losing the 'advantage' that it had, as a former British colony, in terms of high levels of proficiency in English. 
The handover was held up as a potential cause of change in the ways that English is taught, although other factors such as the MOI or the Target Oriented Curriculum were thought likely to have more of an effect, as was the introduction into schools of new technology such as computer multimedia. In general, there was a reluctance to speculate on any potential changes due to the handover itself, evidence of a desire to downplay the significance of the handover and in the conservatism prevalent in Hong Kong society and education. Teachers in particular are wont to complain about conditions, standards of students and lack of support, but are suspicious of any changes implemented by the government. They feel that any changes will result in more work for them, and in fact seldom look upon change of any kind as being positive. There was a lot of reference, especially from the teachers, to the increasing influx of children from Mainland China. The teachers seemed to regard these children as a 'problem' that schools, and ultimately they as teachers, would need to deal with. These responses are consistent with what might be termed a 'culture of inertia' or even resistance to change in Hong Kong teachers. The possible reasons for this inertia have already been discussed to some extent in previous sections. In later sections, empirical evidence is presented with a view to determining the changes in beliefs and practices of teachers of English during this time.

The participants in the interview study described in the present chapter, all experts in the field of English language teaching in Hong Kong, had much to say about the 'state of the art' and where it might be leading during this crucial time. There were opinions on many issues and many of the opinions conflicting; but an impression that surfaced from many of the interviews was an enthusiasm for and commitment to English as a subject of study. Despite some pessimism, and the inertia or resistance to change mentioned previously, there was no doubt that the majority of practitioners felt that they were doing something worthwhile in promoting English and were doing their best to cope with the myriad of changes going on around them. 


\section{Notes}

${ }^{1}$ Given the frequency of reference to the two newspapers, the abbreviations, SCMP and HKS are used in place of the full names in this chapter.

${ }^{2}$ The Hong Kong Standard ceased publication in 2001, to be replaced by the $I$ Mail, which in turn was relaunched later that year as a business and sportsoriented paper. The Standard had always lagged behind its competitor, the South China Morning Post in terms of circulation and had for many years ceased to be profitable. It was able to stay in circulation only because of the support of its parent company, Sing Tao, one of the leading publishers of Chinese newspapers and magazines in the Territory. In an effort to revitalise the Standards' fortunes, it was reoriented towards a more defined market.

${ }^{3}$ For a detailed list of all interviewees, see Appendix A.1.

${ }^{4}$ Eighteen of the interviewees were interviewed individually and the remaining three were interviewed together.

${ }^{5}$ For a copy of this outline, see Appendix A.2.

${ }^{6}$ For a copy of the question list sent to the first administration, see Appendix A.3.

${ }^{7}$ For a copy of the question list sent to the second administration, see Appendix A.6.

${ }^{8}$ Although the main topics of discussion were introduced to the interviewees, they were free to talk for as long as they wanted and to make as many points as they wanted on each topic. For this reason, it is considered that the constant comparative method is still valid for analysis of the interview data.

${ }^{9}$ The Benchmarking was, as has previously been discussed, officially named the Language Proficiency Assessment for Teachers upon its first implementation in 2001. The term 'Benchmarking' will be used here as that was the term familiar to participants at the time of the interviews.

${ }^{10}$ For a detailed report on the background to the Benchmarking initiative as well as its planned implementation, see Falvey \& Coniam (1997).

${ }^{11}$ In general, schools in Hong Kong devote about 20 per cent of the timetable to each of the core subjects of Chinese, English and Mathematics. The proportion devoted to each subject is at the discretion of the school and the principal.

${ }^{12}$ Hong Kong Certificate of Education, the Form 5 (Grade 11) examination.

${ }_{14}^{13}$ Advanced Level Use of English, the Form 7 (Grade 13) examination.

${ }^{14}$ This, in fact, has occurred, with the change in the official name given to the subject of English Literature in school examinations and syllabuses to Literature in English in 2002.

${ }^{15}$ The nine interviewees are designated by upper case Roman numerals. The transcripts of their interviews can be found in Appendix A.

${ }^{16}$ This subject was one of the team of consultants engaged by the government to develop the Benchmarking or Language Proficiency Assessment for Teachers initiative.

${ }^{17}$ The views of the three officers who took part in the interviews in 1997 and 1999 are considered together as they tended to speak from the same perspective with little difference in their opinions on the major themes of the interviews.

${ }^{18}$ Information provided from one of the interviewees in the second round of interviews, who was at the time Acting Head of the Department of English (since renamed English and Communication) at the City University of Hong Kong. 
${ }^{19}$ The pages of the Hong Kong English language newspaper, the South China Morning Post, have frequently contained articles by and letters from NETs and local teachers discussing the problems faced by NETs in adapting to the Hong Kong education system. In particular, see Bowman (2000) and Bunce (1999, 2001). 


\section{REFLECTIONS ON TEACHING OF TRAINEE TEACHERS STUDYING ON A BATESL COURSE IN HONG KONG (1994 AND 1997)}

\subsection{Background}

This chapter presents the findings from a series of questionnaire studies of teachers learning to teach English in Hong Kong secondary schools who were enrolled in the BA course in Teaching English as a Second Language (BATESL) at the City University of Hong Kong during the period leading up to the changeover in sovereignty in July 1997. The purpose of the studies was to determine the extent to which the teaching method, comprising approach, design and procedure (Richards \& Rodgers, 1982, 1986, 2001), of pre-service English teachers changed between leaving secondary school and entering the teaching profession.

A number of previous studies form the background to the findings presented here. The first of these studies was the author's MA thesis (Urmston, 1994) in which a questionnaire and follow-up interviews were used to explore the attitudes to teaching of the third-year cohort of students on the BATESL course who completed their course in May 1994. In Urmston (1994), it was found that this cohort of graduates ${ }^{1}$ had been influenced by the BATESL course, by their practice teaching, and by their own experience as students in Hong Kong secondary schools. Whilst the cohort clearly favoured the interpretive, student-centered approach advocated by the course, they were aware of the constraints that are placed on teachers within the Hong Kong teaching context. They were keen to try out innovative approaches during their practice teaching (especially bearing in mind that they were being assessed on their performance), but were doubtful that 
they would adopt such approaches were they to teach full-time after graduation. When considering their future, many of the cohort were unsure about their commitment to teaching, citing worries about their own career goals, having to deal with student discipline problems and the lack of status of teachers. It was concluded that the BATESL course had not had the influence on the students that might have been hoped for, and instead of producing graduates ready to enter the teaching profession as qualified and confident novice teachers of English, most of the cohort were undecided as to their intentions to teach and somewhat disillusioned with the BATESL course and teaching in general.

The studies presented in this chapter bring the previous studies together by investigating the orientations to teaching of the cohort of BATESL students who began their course in 1994 at the point when they were about to graduate in the summer of 1997. Comparisons are made of the teaching orientation of this cohort of trainee teachers at the time of graduation with their own teaching orientation three years earlier at the start of their course in 1994, and with that of the BATESL cohort who had been about to graduate in 1994 (i.e., when the focal cohort were just starting their course). Through this three-way comparison, it is possible to identify the common elements of teaching orientation across the different administrations of the questionnaire as well as differences among them. From this comparison it may be possible to gain a view of both continuity and change over the three years (1994-1997) leading up to the handover related to the BATESL course, its students, and the larger context of English language teacher education and English language teaching in Hong Kong. 


\subsection{Methodology}

\subsubsection{Respondents}

The focal group of interest in the present study is the BATESL cohort who started their course in 1994 and graduated just before the handover, in June 1997. This cohort, which was surveyed at the beginning and end of their course, in September 1994 and June 1997, is also compared to the first cohort of the BATESL course the group that began their studies in 1991 - at the point of their graduation in 1994. For statistical reasons, the different administrations of the questionnaire are analysed as independent comparisons, so that the focal cohort forms two groups for purposes of analysis (see section 4.2 .3 below). The comparison is thus made across the following three groups:

\section{Cohort 1 Cohort $2 a \quad$ Cohort $2 b$}

$\begin{array}{lccc}\text { Year graduated from BATESL } & 1994 & 1997 & 1997 \\ \text { Year completed questionnaire } & 1994 & 1994 & 1997 \\ \text { Statistical Group Label } & 94(3) & 94(1) & 97(3) \\ \text { Number of respondents } & 48 & 40 & 30^{2} \\ \text { Percentage of class } & 80 & 100 & 75 \\ \text { Ethnicity } & \text { Chinese }^{3} & \text { Chinese } & \text { Chinese } \\ \text { Native language } & \text { Cantonese }^{4} & \text { Cantonese } & \text { Cantonese }\end{array}$

The statistical groups are designated according to the year in which they were administered the questionnaire and the year of study on the BATESL course i.e., first or third and final year as, 94(3), 94(1) and 97(3). As cohorts, the groups are numbered (1), (2a) and (2b), respectively, for ease of identification of comments and of interview excerpts attributed to members of each of the groups.

The respondents were Hong Kong Chinese who spoke Cantonese as their native or first language and English as a second language. ${ }^{5}$ Most had no previous 
teaching experience before joining the course.

Follow-up interviews were carried out with six members of the First Cohort designated $A(94), B(94), C(94) \ldots F(94)$ - and with eight members of the Second Cohort - N(97), $\mathrm{O}(97), \mathrm{P}(97)$... U(97). In both cases, respondents were chosen to be interviewed who intended to teach English after graduating and appeared (from the questionnaire and/or the author's knowledge of them ${ }^{6}$ ) to have opinions which were considered to be interesting, insightful and representative of the variation in their group as a whole. The purpose of these interviews was to ask the graduates to expand upon the responses they gave on the questionnaire so as to gain additional perspectives on their opinions with reference to their course and future teaching. ${ }^{7}$ All interviews were tape-recorded and transcribed. Transcriptions of the interviews can be found in Appendix B.

\subsubsection{Survey Instrument}

The survey instrument was a questionnaire originally developed on the basis of objectives laid out by Pennington and Richards (1993) for their first-year teacher project, on which the present researcher was employed as a Research Assistant and had a central role in developing the questionnaire adapted for the current study (see Urmston, 1994 for earlier version and report of prior work). The first-year teacher project aimed:

To describe and map changes experienced by beginning teachers, both as individuals and as a group, in five aspects of teaching:

1) Language use (Cantonese, English and mixed Cantonese-English)

2) Instructional planning and decision-making

3) Teaching approaches

4) Professional relationships and responsibilities

5) Teaching-related perceptions and values (Pennington \& Richards 1993, p. 2) ${ }^{8}$

Prior to the construction of the questionnaire, the course coordinator of the BATESL course was interviewed to obtain input in terms of possible questions to include on 
the questionnaire and the likely reactions of the students to the research.

The first section of the questionnaire, Language Use, was designed to look at three things: First, the respondents' opinions of their own standards of English, written and spoken, both before they began the BATESL course and at the time of completing the questionnaire (if different); second, the respondents' views on language use in English lessons; and finally, the respondents' use of English and Cantonese in their practice teaching.

The second section of the questionnaire, Instructional Planning and Decision Making, examines the respondents' views on the importance of lesson planning and the factors that influenced the planning of their own lessons during their practice teaching.

The section Teaching Approach, aims to elicit the opinions of the respondents as to the pragmatics of teaching. Questions concentrate on their beliefs about lesson content and teaching strategies, and how they approached their teaching during their practice teaching.

In the fourth section, Professional Relationships and Responsibilities, questions are asked to determine the respondents' feelings about teaching, about themselves as teachers, and about what they expected to experience as first-year teachers should they decide to go into teaching.

In the section titled Perceptions and Values, the beliefs of the respondents as teachers are examined. The questions anticipate that their experiences as student teachers and their position as prospective teachers should have instilled in them definite opinions and views about teachers and teaching.

In addition to the five sections described above, a sixth section, General Reflections on the BATESL Course, was added to the questionnaire administered to the graduating students so that the respondents could communicate their feelings about 
the course, and the course writers could get some feedback towards future improvements to meet the needs of Hong Kong students and future English teachers. In addition, for the third administration in 1997, items were added to the original questionnaire used in the earlier studies to garner the respondents' opinions as regards their expectations for changes in the above five areas in the years ahead, with particular reference to the changeover in sovereignty of Hong Kong. It was made clear to them that they did not need to write their names on the questionnaire, so as to encourage freedom of expression, but that if they were willing to be interviewed then they should put their name on the form.

The questionnaire was written with the bilingual respondents in mind. It was considered important that the respondents not find any parts of the questionnaire ambiguous. Being students of TESL in Hong Kong, they should be familiar with terms such as 'code-mixing', but other pedagogical terms were kept to a minimum. It was not intended that the questionnaire should be in any way a test of the respondents' knowledge of TESL jargon. Some items required a certain format, but it was also felt that there should be a variety of item types, so as to maintain the interest of the respondents. All methods of questioning have their advantages and disadvantages; it was felt that by varying the question types, the disadvantages arising from any one question type could be minimised. The same basic questionnaire was used in all three administrations, although minor changes in tense or aspect were made to some items for reasons of coherence. A copy of the questionnaire as used in the final administration can be found in Appendix B.

Item types used in the questionnaire are: frequency scales; Likert scales (a type of Trating scale); items with a choice of responses to be circled; items requiring responses to be ranked in order of influence or importance; items requiring factors to be numbered in terms of importance; and 'open-response' items requiring respondents to respond in their own words. In items in which respondents were asked to provide an assessment of their practices or opinions, a five-point Likert scale was used so as to allow a wider range of response. In those scaled items asking for respondents' agreement to certain statements about teaching and learning, four 
options, i.e., 'Absolutely agree', 'Quite agree', 'Quite disagree' and 'Absolutely disagree', were used to avoid compounding problems of central-tendency or indecision. With these items, and with others where it was felt that the respondents might want to add further information, space was given for them to add comments.

\subsubsection{Procedures}

The questionnaire administrations all took place at the City University of Hong Kong: in June 1994 - 94(3), September 1994 - 94(1) and June 1997 - 97(3). Each of the administrations was carried out during a scheduled class period under the supervision of the researcher or, in the case of the 94(3) group, the researcher's MA thesis supervising professor. Once the group responses to the items on the questionnaire for the three administrations had been tallied, statistical analysis of the data was carried out to determine any significant differences. ${ }^{9}$ Unless stated, differences were considered significant at the 5 per cent confidence level. As the data consisted of rankings (ordinal data) and various types of classifications (nominal data), non-parametric tests were chosen to compare the responses across administrations. Although the 94(1) group and the 97(3) group were part of the same cohort, it was not possible to compare responses across individuals, as the survey did not require respondents to identify themselves and the majority of the respondents completed their questionnaires anonymously; and 10 people ( 25 per cent) did not complete the second administration. For this reason, it was necessary to consider the three sets of responses in the separate administrations of the questionnaire as three independent groups for purposes of statistical analysis. The analysis was done as follows using SPSS 10.0 for Windows statistical analysis software:

(i) Those items that used a Likert or other rating scale - including yes/no/somewhat (scaled as yes/somewhat/no) - were analysed descriptively by calculating the means and standard deviations for each administration. Kruskal-Wallis $H$ test (non-parametric test for 3 
independent groups) was carried out to determine any significant differences in the responses of the three groups. This procedure computes the average rank for each item of the scores in each group based on the total ranking of all scores in the three groups. Where significant differences were detected, post-hoc tests - Mann-Whitney $U$ test (nonparametric test for two independent groups) - were carried out for each pair of administrations to see which groups differed from each other. In order to adjust approximately for the 'repeated test' type of error - i.e., the problem of the increased probability of finding significance due to repeating tests - as there were three pair-wise post-hoc comparisons the significance level was adjusted to 1.67 per cent (i.e., 5/3).

(ii) For trichotomous nominal response, i.e., where respondents must select one of three non-scalar choices, chi-square test on frequency per response ( $3 \times 2$ table) was used to determine significant differences between groups. There was one item of this type, involving comparison of responses of the 94(3) and 97(3) groups only.

(iii) For items which asked the respondents to circle all those factors which apply to a certain situation, i.e., one subject may select all, some or nothing on the list, the mean order of ranking for the three administrations was compared as a descriptive measure. Then, each response choice on the list was analysed as a separate item to which each respondent responded either 'yes' or 'no' and chi-square computed for all the respondents in the three groups ( $2 \times 3$ table). Where significant differences between the three groups were found, post-hoc comparison was carried out ( $2 \times 2$ table) to find significant pairs. All chi-square tests were carried out using cross tabulations and applying Yates' continuity correction to $2 \times 2$ tables to improve the approximation to the chi-square distribution when frequency values were small. In addition, in situations where one or more cells in a 2 x 3 table had a frequency value of zero, chi-square was not carried out as 
the computed value could not be considered reliable. ${ }^{10}$ In such cases, comparisons could only be made informally.

(iv) For items which required the respondents to rank a number of factors, the mean order of ranking for the three different administrations was compared and the bivariate correlation coefficient for non-parametric groups - Spearman's rho - was calculated to determine if the mean rank order was significantly similar. Each factor on the list was then compared for the three groups using Kruskal-Wallis test and post-hoc (MannWhitney) tests carried out where significant difference was found in order to determine which groups differed, again, at the 1.67 per cent confidence level to adjust for repeated-test type of statistical error.

For all items, comparisons are made across all three groups, i.e., 94(1) vs. 94(3) vs. 97(3). Then the pairs of groups in three matched pairs, i.e., 94(1) vs. 97(3), 94(3) vs. 97(3) and 94(1) vs. 94(3) are compared. In this way it is possible to pinpoint those significant differences which might be attributable to differences in the two cohorts, the BATESL course, other factors within the Hong Kong context, or combinations of these.

The qualitative data from the administrations, i.e., the respondents' comments and philosophies of teaching, are referred to in the discussion of the findings and are used to add perspective to the quantitative data. In addition, where appropriate, excerpts from the follow-up interviews with the selected respondents, which were analysed for content, are used to illustrate the questionnaire data.

\subsection{Results}

In this section, the results from the three administrations of the questionnaire are presented. Reference is made to the tabulated results of the statistical analysis of the 
questionnaire data to be found in Appendix B. Discussion of the results is in a separate section following.

\subsubsection{Language Use}

Table 4.1 shows respondents' self-perceived ability in written English at the time of the administration of the questionnaire. Kruskal-Wallis $H$ Test for independent groups found a significant difference between the three groups, $\mathrm{K}-\mathrm{W}=13.65, p=$ .001 . Post-hoc analysis using Mann-Whitney $U$ Test showed that Cohort 2 at the beginning of their studies (the 94(1) group) considered their written English to be significantly worse than did both the graduating groups, $M-W=12.69, p=.000$ and $6.37, p=.012$. There was no significant difference in this perception between the two Year 3 groups $(\mathrm{K}-\mathrm{W}=0.39, p=.53)$.

In terms of spoken English (Table 4.2), Kruskal-Wallis again uncovered a significant difference between the three groups, $\mathrm{K}-\mathrm{W}=19.69, p=.000$. Post-hoc MannWhitney testing found significant differences between the first-year group (94(1)) and both of the third-year groups: $94(1)$ v 97(3), $M-W=17.73, p=.000$ and 94(1) v 94(3), $M-W=8.30, p=.004$; but no difference between the two graduating groups, 94(3) v 97(3), $\mathrm{M}-\mathrm{W}=4.50, p=.034$.

The next issue addressed was the extent of code-mixing with friends (Table 4.3), Kruskal-Wallis revealed that the three groups differed significantly in this respect, $\mathrm{K}-\mathrm{W}=14.74, p=.001$, Post-hoc Mann-Whitney tests showed that the 94(1) group reported code-mixing significantly less than both the $94(3)$ group, $\mathrm{M}-\mathrm{W}=13.46, p=$ .000 and the $97(3)$ group, $\mathrm{M}-\mathrm{W}=6.32, p=.012$. No significant difference was found between the two Year 3 groups (M-W $=1.52, p=.22)$.

A similar pattern emerged for the respondents' self-reported use of code-switching with friends (Table 4.4). A significant difference was found for the three groups by the Kruskal-Wallis procedure, $\mathrm{K}-\mathrm{W}=8.08, p=.018$. The post-hoc Mann-Whitney 
test revealed that there was a significant difference only between the two groups surveyed in $1994(94(1)$ and 94(3)) $\mathrm{M}-\mathrm{W}=8.26, p=.004$, with the graduating (94(3)) group reporting that they code-switched significantly more than the first-year group. The other two comparisons uncovered no significant differences (94(3) v 97(3), $\mathrm{M}-\mathrm{W}=0.98, p=.32 ; 94(1)$ v 97(3), $\mathrm{M}-\mathrm{W}=2.23, p=.13$ ).

Regarding classroom language use, a significant difference was indicated by Kruskal-Wallis for the three groups in the level of agreement on use of English only in English lessons (Table 4.5), $\mathrm{K}-\mathrm{W}=10.85, p=.004$. The post-hoc Mann-Whitney tests showed that the 97(3) group held significantly stronger views against the use of only English than did the 94(1) group, $\mathrm{M}-\mathrm{W}=9.58, p=.002$, and the 94(3) group, $\mathrm{M}-\mathrm{W}=6.82, p=.009$. The two groups surveyed in 1994 did not differ significantly in this respect $(\mathrm{M}-\mathrm{W}=0.48, p=.49)$.

The three groups did not differ significantly in their opinion as to whether mixed code could be used in English lessons (Table 4.6). Kruskal-Wallis test resulted in $\mathrm{K}$ $\mathrm{W}=1.23, p=.54$. All three groups tended towards slight disagreement, having means between 2 and 3 on a frequency scale where $1=$ 'Absolutely agree' and $4=$ 'Absolutely disagree'.

The two Year 3 groups, 94(3) and 97(3) stated their level of use of English only in their classes during practice teaching (Table 4.7). The responses for both groups were compared using Mann-Whitney $U$ Test for two independent groups and no significant difference was found, $\mathrm{M}-\mathrm{W}=1.19, p=.28$, with both groups having means between 1 and 2 on a frequency scale where $1=$ 'Always' and $4=$ 'Never'. The difference between the Year 3 groups' reported change in use of English only during practice teaching (Table 4.8) was not significant based on the Mann-Whitney result of $2.72, p=.099$. Both groups felt that there had been a slight decrease in their use of English during the period.

Finally in the Language Use section, the respondents stated the situations in which they would or might use Cantonese during English lessons (Table 4.9). The 
responses were tabulated into two columns for each group representing the numbers of respondents selecting each option ('Yes') and the remainder of the group who were assumed to have not selected that situation ('No'). This arrangement resulted in a $2 \times 3$ table, for which chi-square test was carried out to determine whether there were any significant differences between the responses of the three groups for each situation or item. Most of the items did not yield significant differences: 'To be friendly with students' $\left(\chi^{2}=0.70, p=.71\right)$, 'To present lesson content' $\left(\chi^{2}=2.19, p=.34\right)$, 'To explain lesson content' $\left(\chi^{2}=3.40\right.$, $p=.18)$, and 'None' $\left(\chi^{2}=1.27, p=.53\right) .{ }^{11}$ One item showed significant difference - 'To establish discipline': $\chi^{2}=16.60, p=.000$. Post-hoc chi-square test across three pairs of groups showed that significant differences existed between the 94(1) group and both the 94(3) group, $\chi^{2}=14.90, p=.000$ and the 97(3) group, $\chi^{2}=$ $4.41, p=.036$. The difference between the two graduating groups was not significant $\left(\chi^{2}=1.53, p=.21\right)$.

\subsubsection{Instructional Planning and Decision-making}

Table 4.10 shows the respondents' level of agreement on it being impossible to have a successful lesson that has not been well-planned in advance. Kruskal-Wallis test for the three groups revealed no significant difference between the mean rankings of the groups $(\mathrm{K}-\mathrm{W}=0.59, p=.74)$, with all showing moderate agreement.

The findings for respondents' views on whether teachers should write detailed lesson plans for all their lessons are shown in Table 4.11. The Kruskal-Wallis test indicated that there were significant differences in the groups' responses, $\mathrm{K}-\mathrm{W}=21.94, p=$ .000 . The two-way comparisons of the 94(1) group with each of the graduating groups were significant: 94(1) v 94(3), M-W = 20.11, $p=.000 ; 94(1) \vee 97(3), M-W$ $=9.37, p=.002$. The two graduating groups, 94(3) $\mathrm{v} 97(3)$, did not differ significantly from each other $(\mathrm{M}-\mathrm{W}=1.68, p=.19)$.

There were no significant differences across groups on their views on the degree to 
which lessons should be planned (Table 4.12), $\mathrm{K}-\mathrm{W}=4.96, p=.084$. The response in all cases was in the direction of less detailed, more flexible planning.

The two Year 3 groups were asked to rank the factors which influenced their lesson planning during their practice teaching (Table 4.13). The factors of 'Syllabus' and 'Regular teacher's instructions' were ranked highest by both groups. There was no significant difference (at the $p<.05$ level) in the mean rankings of any of the factors for these two graduating groups according to the Mann-Whitney $U$ test: 'Syllabus' $(\mathrm{M}-\mathrm{W}=0.00, p=.99)$, 'Regular teacher's instructions' $(\mathrm{M}-\mathrm{W}=0.26, p=.61)$, 'Textbook teachers' instructions' (M-W $=0.38, p=.54)$, 'Own ideas' $(\mathrm{M}-\mathrm{W}=1.06$, $p=.30)$, 'Perceived needs of students' ( $\mathrm{M}-\mathrm{W}=1.50, p=.22)$, 'BATESL course guidelines' $(\mathrm{M}-\mathrm{W}=3.72, p=.054)$. The bivariate correlation coefficient for ranked data, Spearman's rho, calculated for the mean rank order of the factors for the two groups was 0.77 , but this correlation coefficient did not achieve significance $(p=$ $.072)$.

A similar result was obtained from the two Year 3 groups' rankings of factors affecting the decisions that they made during their practice teaching (Table 4.14). According to the Mann-Whitney test, the mean rankings of factors did not differ significantly (at the $\mathrm{p}<.05$ level) between groups: 'Students' needs' $(\mathrm{M}-\mathrm{W}=1.18, p$ $=.28)$, 'Regular teacher's input' ( $\mathrm{M}-\mathrm{W}=1.27, p=.26)$, 'Fellow student teachers' advice' $(\mathrm{M}-\mathrm{W}=1.89, p=.17)$, 'Supervisor's advice' $(\mathrm{M}-\mathrm{W}=3.09, p=.08)$. Although the mean rank order of factors for both groups was similar, the Spearman's rho correlation coefficient of 0.80 was not significant $(p=.20)$ due to the small number of factors (4).

Lastly, there was no significant difference between the three groups on whether the teacher should be more responsive to student needs than the set syllabus, all three groups indicating agreement (Table 4.15). The Kruskal-Wallis test gave the result, $\mathrm{K}-\mathrm{W}=4.21, p=.12$. 


\subsubsection{Teaching Approach}

The first item under Teaching Approach concerned respondents' agreement as to whether examination requirements should determine what is taught in English lessons (Table 4.16). All three groups showed moderate agreement to this statement, with the 97(3) group showing stronger agreement than the other two groups. Kruskal-Wallis $H$ test revealed significant difference in the three-group comparison, $\mathrm{K}-\mathrm{W}=9.08, p=.011$. Pair-wise comparisons between groups using Mann-Whitney $U$ test indicated that only the 94(3) and 97(3) groups differed significantly in their mean responses, $\mathrm{M}-\mathrm{W}=7.30, p=.007$. (Non-significant differences - 94.(1) v 94(3), $\mathrm{M}-\mathrm{W}=2.87, p=.090 ; 94(1) \vee 97(3), \mathrm{M}-\mathrm{W}=3.48, p .062)$.

Table 4.17 presents respondents' opinions on whether students learn better though interaction with each other or through teacher-instruction. Descriptive statistics revealed that the $97(3)$ group were more strongly in favour of student-student interaction than the other two groups. The Kruskal-Wallis test showed there to be significant difference in the groups on this issue, $\mathrm{K}-\mathrm{W}=7.93, p=.019$. Post-hoc Mann-Whitney test indicated that the only significant pair-wise difference in response was between the two graduating groups, $94(3)$ and $97(3)(\mathrm{M}-\mathrm{W}=8.19, p=$ $.004)$, whilst the 94(1) $\vee 94(3)(\mathrm{M}-\mathrm{W}=1.18, p=.28)$ and $94(1) \vee 97(3)(\mathrm{M}-\mathrm{W}=$ $3.48, p=.062$ ) were not significant. In addition, these two groups were asked whether they thought that their students had learned English better through interaction or instruction during practice teaching (Table 4.18). The respondents answered on a trichotomous nominal response scale of 'Through interaction', 'Through instruction' and 'No difference'. Chi-square test of the $3 \times 2$ table showed no significant difference between the groups, $\chi^{2}=3.95, p=.14$.

The three groups were asked for their opinions on whether a class should be strictly controlled and all lesson content directed by the teacher (Table 4.19). All three group means indicated that they moderately disagreed with this premise, with no. significant difference in the groups' orientations according to the Kruskal-Wallis test, $\mathrm{K}-\mathrm{W}=5.67, p=.059$. Similarly, the groups did not differ significantly in their 
level of agreement as to whether a teacher should be prepared to experiment with teaching materials and strategies, all groups showing strong agreement (Table 4.20), $\mathrm{K}-\mathrm{W}=0.098, p=.95$. The groups also did not differ significantly in their assessment of their own teaching style (Table 4.21), as the mean response in each group fell between learner-centered and teacher-centered orientations, $\mathrm{K}-\mathrm{W}=2.40, p=.30$.

Table 4.22 shows the respondents' rankings of the factors that they considered to have the most effect on their teaching of English. The mean rankings for the three groups were compared for all factors by the Kruskal-Wallis test. Seven of the factors showed no significant difference in the groups' mean ranking of perceptions of their relative importance: 'Examination requirements' $(\mathrm{K}-\mathrm{W}=0.22, p=.89)$, 'Noise in classroom' $(\mathrm{K}-\mathrm{W}=0.64, p=.73)$, 'Personality of teacher' $(\mathrm{K}-\mathrm{W}=0.89, p=.87)$, 'Availability/quality of teaching aids' $(\mathrm{K}-\mathrm{W}=1.25, p=.54)$, 'Availability/quality of teaching materials' $(\mathrm{K}-\mathrm{W}=1.71, p=.42)$, 'School policy' $(\mathrm{K}-\mathrm{W}=1.77, p=.41)$, 'Motivation of students' $(\mathrm{K}-\mathrm{W}=5.71, p=.057)$. Of these factors, 'Motivation of students' was ranked highly by all three groups, i.e., as a key factor affecting their teaching.

The remaining factors included in the response list for this item were significantly different in their position in the ranking of factors in the three-group comparison according to the Kruskal-Wallis test. 'Teacher training' proved to be highly significant in the three-way group comparison, $\mathrm{K}-\mathrm{W}=18.74, p=.000$. Two pairwise post-hoc comparisons were significant, those of each of the graduating teacher groups compared to the beginning teacher group, who had a very high ranking of this factor: $94(1) \vee 94(3), \mathrm{M}-\mathrm{W}=16.57, p=.000$ and $94(1) \vee 97(3), \mathrm{M}-\mathrm{W}=10.34, p$ $=.001$. The two graduating groups did not differ significantly from each other $(\mathrm{M}-\mathrm{W}$ $=0.41, p=.52$ ).

'Classroom size' yielded a Kruskal-Wallis value of $\mathrm{K}-\mathrm{W}=16.12, p=.000$, with the 94(1) group differing significantly from both of the third-year groups in ranking this factor lower than they did: $M-W=13.09, p=.000(94(1) \vee 94(3))$ and $M-W=8.37$, 
$p=.004(94(1) \vee 97(3))$. The comparison between the third-year groups was not significant $(\mathrm{M}-\mathrm{W}=3.69, p=.055)$.

'English proficiency of students' was ranked highly by all groups, especially by the 94(3) group, with significant difference found in the Kruskal-Wallis comparison, K$\mathrm{W}=9.93, p=.007$. The post-hoc tests revealed significant difference only between the two 1994 groups, $\mathrm{M}-\mathrm{W}=9.70, p=.002$ but no significant difference between the $94(1)$ and $97(3)$ groups $(\mathrm{M}-\mathrm{W}=1.31, p=.25)$ or between the two graduating groups $(\mathrm{M}-\mathrm{W}=3.01, p=.083)$.

For 'Class size' (K-W $=9.07, p=.011)$, post-hoc Mann-Whitney tests showed the 94(1) v 94(3) comparison to be the only one with a significant difference, $M-W=$ 7.94, $p=.005$; the 94(1) group ranked the factor of class size as least important. The other two comparisons were not significant $-94(3) \mathrm{v} 97(3), \mathrm{M}-\mathrm{W}=0.24, p=.62$; 94(1) $\vee 97(3), \mathrm{M}-\mathrm{W}=5.35, p=.021$.

Finally, 'Current trends in ESL teaching' also showed significant difference, $\mathrm{K}-\mathrm{W}=$ $8.89, p=.012$, with the Mann-Whitney test indicating that the two groups surveyed in 1994 differed significantly, $\mathrm{M}-\mathrm{W}=8.86, p=.003$; in 1994, the first-year group considered this factor to be more important than did the third-year group. The other two comparisons were not significant: 94(3) v 97(3), M-W $=1.51, p=.22$ and 94(1) v 97(3), $\mathrm{M}-\mathrm{W}=2.10, p=.15$.

\subsubsection{Professional Relationships and Responsibilities}

The first item in this section concerned the respondents' level of confidence in their ability to become a successful teacher (Table 4.23). All three groups indicated moderate confidence and Kruskal-Wallis test showed there to be no significant difference, $\mathrm{K}-\mathrm{W}=1.03, p=.60$. 
Groups differed in their opinions on the status of teachers (Table 4.24), with the three-way Kruskal-Wallis significant, $\mathrm{K}-\mathrm{W}=8.38, p=.015$. Post-hoc MannWhitney tests uncovered a significant difference between the 94(1) and 94(3) groups, $\mathrm{M}-\mathrm{W}=7.98, p=.005$ as the 1994 entrants raked this factor significantly higher than the 1994 graduates did. The other two comparisons (94(1) v 97(3), M-W $=0.32, p=.57$; and 94(3) $\vee 97(3), \mathrm{M}-\mathrm{W}=3.34, p=.068)$ were not significant.

The respondents' ranking of their own best attributes as a teacher resulted in only one factor showing significant difference in the three groups as a result of KruskalWallis test (Table 4.25). This factor was 'Careful planner', $\mathrm{K}-\mathrm{W}=7.51, p=.023$. Post-hoc Mann-Whitney tests showed that the two Year 3 groups differed significantly in their assessment of this factor, $\mathrm{M}-\mathrm{W}=6.74, p=.009$, with $94(1) \mathrm{v}$ 97(3) $(\mathrm{M}-\mathrm{W}=1.82, p=.18)$ and $94(1) \vee 94(3)(\mathrm{M}-\mathrm{W}=2.57, p=.11)$ proving not significant. The factors which did not demonstrate significant difference were: 'Good relationships with colleagues' (K-W $=0.47, p=.79)$, 'Strict disciplinarian' $(\mathrm{K}-\mathrm{W}=1.06, p=.59)$, 'Innovative in teaching ideas' $(\mathrm{K}-\mathrm{W}=1.68, p=.43)$, 'Empathy with students' ( $\mathrm{K}-\mathrm{W}=1.78, p .41)$, 'Aware of latest trends in TESL' (K$\mathrm{W}=2.20, p=.33)$, 'Good command of language' $(\mathrm{K}-\mathrm{W}=2.91, p=.23)$, and 'Good communication skills' $(\mathrm{K}-\mathrm{W}=2.99, p=.22)$. The first two of these factors were ranked lowest by all three groups and the last two were ranked highest by all three groups. Spearman's rho test of correlation across the entire ranking for best attributes as a teacher revealed that all groups correlated highly and significantly: 94(1) and $97(3), \mathrm{r}=0.98, p=.000 ; 94(1)$ and $94(3), \mathrm{r}=0.98, p=.000 ;$ and 94(3) and 97(3), $\mathrm{r}=$ $0.95, p=.000$.

The next item asked for the respondents' views on whether they were considering teaching as a long-term career (Table 4.26). The Kruskal-Wallis test indicated significant difference in the three-group comparison, $\mathrm{K}-\mathrm{W}=8.33, p=.016$. In Mann-Whitney tests for paired groups, only the two third-year groups differed significantly, $\mathrm{M}-\mathrm{W}=6.13, p=.013$, with the group that graduated in 1997 being more positive about teaching than the group that graduated in 1994. The 94(1) $v$ 
97(3) $(\mathrm{M}-\mathrm{W}=0.22, p=.64)$ and 94(1) $\vee 94(3)(\mathrm{M}-\mathrm{W}=5.53, p=.019)$ group comparisons were non-significant.

Respondents were asked to select from a list those people who they felt would be helpful to them in their first year as a teacher (Table 4.27). Chi-square test on a $2 \times 3$ table was carried out to determine if any of the groups differed from the others in their pattern of choice across each selection. As the selection 'Principal' contained one cell with zero, its chi-square value could not be considered reliable and was not computed. Only the selection 'Panel chair' proved to be significantly different in the comparison of the three groups $\left(\chi^{2}=6.30, p=.043\right)$. Post-hoc tests showed that the 94(1) and 97(3) groups differed significantly $\left(\chi^{2}=4.05 p=.044\right)$, whilst the 94(1) and 94(3) groups $\left(\chi^{2}=0.51, p=.47\right)$ and the two graduating groups $\left(\chi^{2}=1.33, p=\right.$ .25) did not differ significantly. The remaining selections - 'Teacher-training supervisor' $\left(\chi^{2}=.21, p=.90\right)$, 'Senior teachers' $\left(\chi^{2}=1.50, p=.47\right)$, 'More experienced teachers' $\left(\chi^{2}=1.71, p=.43\right)$ and 'Fellow first-year teachers' $\left(\chi^{2}=2.59\right.$, $p=.27$ ) - showed no significant differences. Only 'More experienced teachers' was chosen by more than 50 per cent of the members of each group as likely to be helpful to them in their first year of teaching.

The next item considered the respondents' expectations of the duties or activities that they expected to have to carry out as first-year teachers (Table 4.28). Each activity/duty on the list was analysed for the three groups by means of chi-square $2 \mathrm{x}$ 3 table. A number of activities/duties did not differ significantly across the three groups: 'Extra-curricular activities' $\left(\chi^{2}=0.099, p=.95\right)$, 'Religious activities' $\left(\chi^{2}=\right.$ $0.48, p=.78)$, 'Be involved in English club' $\left(\chi^{2}=1.11, p=.57\right)$, 'Counsel students' $\left(\chi^{2}=2.10, p=.35\right)$, 'Train students for speech festivals etc.' $\left(\chi^{2}=3.58, p=.17\right)$, and 'Prepare lessons' $\left(\chi^{2}=5.62, p=.060\right)$. The activities/duties that did show significant difference across the three groups were then tested for differences between pairs of groups using $2 \times 2$ chi-square. The results were:

'Administer examinations': $\chi^{2}=21.20, p=.000$; post-hoc 94(1) v 94(3), $\chi^{2}=16.26$, $p=.000 ; 94(1) \vee 97(3), \chi^{2}=10.82, p=.001 ; 94(3) \vee 97(3), \chi^{2}=0.00, p=.98 n s$. 
'Attend meetings with principal/panel chair': $\chi^{2}=18.51, p=.000$; post-hoc $94(1) \mathrm{v}$ 97(3), $\chi^{2}=12.31, p=.000 ; 94(1) \vee 94(3), \chi^{2}=9.60 p=.002 ; 94(3) \vee 97(3), \chi^{2}=$ $0.56, p=.45 n s$.

'Be a form teacher': $\chi^{2}=17.32, p=.000$; post-hoc 94(1) v 94(3), $\chi^{2}=11.21, p=$ $.001 ; 94(1) \vee 97(3), \chi^{2}=10.33, p=.001 ; 94(3) \vee 97(3), \chi^{2}=0.01, p=.92 n s$.

'Meet parents': $\chi^{2}=11.63, p=.003$; post-hoc 94(1) v 97(3), $\chi^{2}=8.61, p=.003$; $94(1) \vee 94(3), \chi^{2}=5.55, p=.019 ; 94(3) \vee 97(3), \chi^{2}=0.55, p=.49 n s$.

'Prepare examinations': $\chi^{2}=10.07, p=.007$; post-hoc 94(1) $\vee 94(3), \chi^{2}=6.04, p=$ $.014 ; 94(1) \vee 97(3), \chi^{2}=4.74, p=.030 ; 94(3) \vee 97(3), \chi^{2}=0.00, p=1.00 n s$.

'Mark papers': $\chi^{2}=10.01, p=.007$; post-hoc 94(1) $\vee 97(3), \chi^{2}=6.28, p=.012$; $94(1) \vee 94(3), \chi^{2}=3.95, p=.047 ; 94(3) \vee 97(3), \chi^{2}=0.49, p=.48 n s$.

'Attend courses': $\chi^{2}=7.31, p=.026$; post-hoc 94(1) v 94(3), $\chi^{2}=5.00, p=.025$; $94(1) \vee 97(3), \chi^{2}=0.00, p=1.00 n s ; 94(3) \vee 97(3), \chi^{2}=3.59, p=.058 n s$.

'Mark examinations': $\chi^{2}=7.22, p=.027$; post-hoc 94(3) v 97(3), $\chi^{2}=0.025, p=.88$ $n s ; 94(1) \vee 94(3), \chi^{2}=3.21, p=.073 n s ; 94(1) \vee 97(3), \chi^{2}=3.64, p=.056 n s$.

'Attend meetings with colleagues': $\chi^{2}=6.69, p=.035$; post-hoc $94(1) \mathrm{v} 97(3), \chi^{2}=$ $5.35, p=.021 ; 94(1) \vee 94(3), \chi^{2}=0.29, p=.59 n s ; 94(3) \vee 97(3), \chi^{2}=3.06, p=.08$ ns.

As can be seen in these figures, the two graduating groups did not differ on any of these items, and any significant differences were between beginning and graduating groups.

Following up from the last item, the respondents were given the same list of 
activities/duties and asked to select those which they expected would take up the most of their time during their first year in the classroom (Table 4.29). After the three-group chi-square analysis, only one of the activities/duties, 'Marking papers', showed significant difference in the three-group comparison, $\chi^{2}=20.27, p=.000$. Post-hoc chi-square for paired groups showed that the 94(1) and 97(3) groups differed significantly, $\chi^{2}=15.40, p=.000$, as did the 94(1) and 94(3) groups, $\chi^{2}=$ $12.82, p=.000$. The two Year 3 groups did not differ significantly from each other $\left(\chi^{2}=0.31, p=.57\right)$. The selections 'Train students for speech festivals', 'Religious activities', 'Meet parents' and 'Administer examinations' had cells which contained values of zero and so chi-square was not carried out. The items which did not differ significantly in the three-group comparison were: 'Attend courses' $\left(\chi^{2}=0.09, p=\right.$ 95), 'Extra-curricular activities' $\left(\chi^{2}=0.21, p=.90\right)$, 'Attend meetings with principal/panel chair' $\left(\chi^{2}=0.83, p=.66\right)$, 'Be a form teacher' $\left(\chi^{2}=1.09, p=.58\right)$, 'Prepare lessons' $\left(\chi^{2}=1.38, p=.50\right)$, 'Be involved in English Club etc.' $\left(\chi^{2}=1.90\right.$, $p=.39)$, 'Attend meetings with colleagues' $\left(\chi^{2}=2.26, p=.32\right)$, 'Counsel students' $\left(\chi^{2}=3.03, p=.22\right)$, 'Meet parents' $\left(\chi^{2}=3.31, p=.19\right)$, 'Train students for speech festivals etc.' $\left(\chi^{2}=3.31, p=.19\right)$, 'Mark examinations' $\left(\chi^{2}=3.46, p=.18\right)$, 'Administer examinations' $\left(\chi^{2}=3.78, p=.15\right)$, 'Religious activities' $\left(\chi^{2}=3.97, p=\right.$ $.14)$, 'Prepare examinations' $\left(\chi^{2}=5.60, p=.060\right)$.

The respondents' views on the extent to which they might work closely with other teachers differed significantly (Table 4.30) according to the Kruskal-Wallis test for three groups, $\mathrm{K}-\mathrm{W}=20.00, p=.000$. Post-hoc tests for paired groups using the Mann-Whitney test indicated that the 94(1) and 94(3) groups differed significantly $(\mathrm{M}-\mathrm{W}=17.65, p=.000)$, as did the $94(3)$ and $97(3)$ groups $(\mathrm{M}-\mathrm{W}=10.21, p=$ .001). The first cohort of teachers on the BATESL (94(3) group) were less positive in this respect than the later cohort at the beginning (1994) and end (1997) of the course. The focus cohort did not differ significantly on this point over the three years of the course $(\mathrm{M}-\mathrm{W}=0.58, p=.44)$

The next item required the respondents to rank a number of factors according to how 
helpful they thought each would be in their professional development (Table 4.31). Kruskal-Wallis uncovered no significant difference in groups' mean rankings of all but one of the factors: 'Getting feedback from the panel chair' $(\mathrm{K}-\mathrm{W}=0.09, p=$ $.95)$, 'Learning from other teachers' ( $\mathrm{K}-\mathrm{W}=0.15, p=.93$ ), 'Designing new materials' ( $\mathrm{K}-\mathrm{W}=2.05, p=.36)$, 'Finding new materials' ( $\mathrm{K}-\mathrm{W}=4.75, p=.09)$, and 'Reading articles' $(\mathrm{K}-\mathrm{W}=5.93, p=.052)$. One factor, 'Attending conferences' $(\mathrm{K}-\mathrm{W}=6.10, p=.047)$, showed a significant difference in the three-way comparison but not in the pair-wise post-hoc Mann-Whitney $U$ tests carried out at the $p<.0167$ level, which were non-significant for each pairing of groups $-94(3) \vee 97(3), \mathrm{M}-\mathrm{W}=$ $0.045, p=.83 ; 94(1) \vee 94(3), \mathrm{M}-\mathrm{W}=4.20, p=.04 ; 94(1) \vee 97(3), \mathrm{M}-\mathrm{W}=4.9, p=$ .027. The bivariate correlation coefficient for ranked data, Spearman's rho, was calculated for the three pairs of groups, yielding coefficients of $0.26, p=.62(94(1) \mathrm{v}$

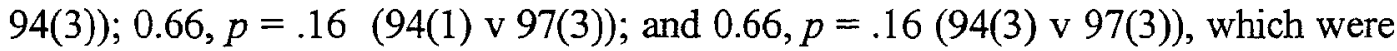
not significant.

\subsubsection{Perceptions and Values}

The first item in the final section of the questionnaire asked the respondents whether or not they had a definite philosophy of teaching (Table 4.32). A Kruskal-Wallis test for the three groups yielded a significant value, $\mathrm{K}-\mathrm{W}=8.60, p=.014$. According to post-hoc Mann-Whitney tests, two pairs of groups differed significantly, 94(3) v 97(3) $(\mathrm{M}-\mathrm{W}=7.15, p=.008)$ and 94(1) $\vee 97(3)(\mathrm{M}-\mathrm{W}=6.26, p=.012)$, with the 97(3) group being more definite about their philosophy. The two 1994 groups did not differ significantly from each other on this item (M-W $=0.06, p=.81)$.

Based on the previous item, those respondents who did not have a definite philosophy of teaching were assumed to have factors that they were unsure about and they were asked to select from a list those that most concerned them (Table 4.33). Each factor was treated as a $2 \times 3$ table for three groups and a chi-square test was carried out for each one that satisfied the conditions described in 4.2.3. The following factors were analysed for three groups by chi-square and showed no 
significant difference: 'Working conditions' $\left(\chi^{2}=0.31, p=.86\right)$, 'Professional responsibilities' $\left(\chi^{2}=0.43, p=.81\right)$, 'Ideas on teaching methods' $\left(\chi^{2}=1.07, p=\right.$ $.59)$, Ways to handle discipline' $\left(\chi^{2}=1.81, p=.40\right)$, 'Ideas on teaching content' $\left(\chi^{2}\right.$ $=2.14, p=.34)$, and 'Personal career goals' $\left(\chi^{2}=4.01, p=.13\right)$. The factor 'Professional status' proved significant in the three-group chi-square analysis $\left(\chi^{2}=\right.$ $10.32, p=.006)$. Post-hoc tests showed significant difference between the response of the focal cohort in their initial (1994) versus their final (1997) questionnaire results, $\chi^{2}=7.80, p=.005$, with the other comparisons not significant (94(1) v94(3), $\chi^{2}=1.26, p=.26$; 94(3) $\left.\mathrm{v} 97(3), \chi^{2}=2.79, p=.095\right)$. Similarly, the factor of 'Professional relationships' was significant in the three-group comparison $\left(\chi^{2}=6.95\right.$, $p=.031)$ and post-hoc chi-square revealed significant pair-wise difference in the focal cohort 94(1) $\vee 97(3)$ comparison $\left(\chi^{2}=4.47, p=.035\right)$. Again the other comparisons were not significant (94(1) v 94(3), $\chi^{2}=0.10, p=.75 ; 94(3) \vee 97(3), \chi^{2}$ $=2.79, p=.095)$. For the factors 'Relationships with students' and 'Salary', at least one cell in the $2 \times 3$ table had a value of zero and so chi-square could not be considered reliable and was not calculated.

As a parallel item to that asked earlier (Table 4.22) concerning the respondents' views on the factors that affected their teaching, they were next asked for the factors that affected what goes on in the classroom in Hong Kong schools generally (Table 4.34). Of the thirteen factors listed, four did not differ significantly by group Kruskal-Wallis test: 'Efficiency of English department' ( $\mathrm{K}-\mathrm{W}=0.58, p=.75$ ), 'Backup of colleagues' (K-W =1.84, $p=.40)$, Design of classroom' (K-W = 2.14, $p$ $=.34$ ), and 'Businesses or employers' $(\mathrm{K}-\mathrm{W}=2.64, p=.27)$. The other factors, which did prove significant, were further tested for significant difference between pairs using Mann-Whitney test. The results were:

'Hong Kong Education Department': $\mathrm{K}-\mathrm{W}=26.27, p=.000$; post hoc 94(3) v 97(3), $\mathrm{M}-\mathrm{W}=21.93, p=.000 ; 94(1) \vee 94(3), \mathrm{M}-\mathrm{W}=12.10, p=.001 ; 94(1) \vee 97(3), \mathrm{M}-\mathrm{W}$ $=6.71, p=.010$. 
'Hong Kong Examinations Authority': K-W $=17.18, p=.000$; post hoc $94(3) \mathrm{v}$ 97(3), $\mathrm{M}-\mathrm{W}=16.83, p=.000 ; 94(1) \vee 94(3), \mathrm{M}-\mathrm{W}=5.97, p=.015 ; 94(1) \vee 97(3)$, $\mathrm{M}-\mathrm{W}=2.78, p=.095 n s$.

'Hong Kong government': $\mathrm{K}-\mathrm{W}=16.58 p=.000$; post hoc 94(3) v 97(3), $\mathrm{M}-\mathrm{W}=$ $14.79, p=.000 ; 94(1) \vee 94(3), \mathrm{M}-\mathrm{W}=7.58, p=.005 ; 94(1) \vee 97(3), \mathrm{M}-\mathrm{W}=2.32, p$ $=.13 \mathrm{~ns}$.

Competence of teacher': $\mathrm{K}-\mathrm{W}=16.23, p=.000$; post hoc 94(1) $\vee 97(3), \mathrm{M}-\mathrm{W}=$ $15.29, p=.000 ; 94(3) \vee 97(3), \mathrm{M}-\mathrm{W}=4.15, p=.042 n s ; 94(1) \vee 94(3), \mathrm{M}-\mathrm{W}=5.74$, $p=.017 n s$.

'Language ability of students': $\mathrm{K}-\mathrm{W}=16.01, p=.000$; post hoc 94(1) $\mathrm{v} 97(3)$, $11.93, p=.001 ; 94(1) \vee 94(3), \mathrm{M}-\mathrm{W}=11.31, p=.001 ; 94(3) \vee 97(3) ; \mathrm{M}-\mathrm{W}=0.28, p$ $=.60 \mathrm{~ns}$.

'School policy': $\mathrm{K}-\mathrm{W}=12.05, p=.002$; post hoc 94(3) $\vee 97(3), \mathrm{M}-\mathrm{W}=12.92, p=$ $.000 ; 94(1) \vee 97(3), \mathrm{M}-\mathrm{W}=2.74, p=.098 n s ; 94(1) \vee 94(3), \mathrm{M}-\mathrm{W}=3.02, p=.08 n s$.

'Class size': $\mathrm{K}-\mathrm{W}=9.19, p=.010$; post hoc 94(1) v 97(3), $\mathrm{M}-\mathrm{W}=7.92, p=.005$; 94(3) v 97(3), $\mathrm{M}-\mathrm{W}=6.22, p=.013 ; 94(1) \mathrm{v} 94(3), \mathrm{M}-\mathrm{W}=0.38, p=.54 \mathrm{~ns}$.

'Location of school': $\mathrm{K}-\mathrm{W}=7.36, p=.025$; post hoc 94(1) $\vee 97(3), \mathrm{M}-\mathrm{W}=7.33, p=$ $.007 ; 94(1) \vee 94(3), \mathrm{M}-\mathrm{W}=1.12, p=.29 n s ; 94(3) \vee 97(3), \mathrm{M}-\mathrm{W}=2.80, p .09 \mathrm{~ns}$.

'Motivation of students': $\mathrm{K}-\mathrm{W}=6.04, p=.049$; post hoc $94(3) \vee 97(3), \mathrm{M}-\mathrm{W}=6.25$, $p=.012 ; 94(1) \vee 94(3), \mathrm{M}-\mathrm{W}=0.49, p=.48 n s ; 94(1) \vee 97(3), \mathrm{M}-\mathrm{W}=3.04, p=.08$ $n s$.

Despite the recorded significant differences between the groups on these items, the bivariate correlation, Spearman's rho, yielded a significant degree of correlation in the overall pattern of response between the two graduating groups $(\mathrm{r}=0.84, p=$ $.000)$, the two 1994 groups $(\mathrm{r}=0.87, p=.000)$ and between the earlier and later 
responses of the focal group that began their studies in 1994 and graduated in 1997 (r $=0.64, p=.019$ ).

Table 4.35 shows the respondents' opinions on the relative importance of the teaching of the different language skills. The Kruskal-Wallis test found no significant differences in the groups' rankings of the skills: 'Speaking' $(\mathrm{K}-\mathrm{W}=$ $0.00, p=.99)$, 'Listening' ( $\mathrm{K}-\mathrm{W}=0.91, p=.63)$, 'Reading' $(\mathrm{K}-\mathrm{W}=1.54, p=.46)$, 'Writing' $(\mathrm{K}-\mathrm{W}=3.87, p=.14)$, 'Grammar' $(\mathrm{K}-\mathrm{W}=5.52, p=.063)$, 'Pronunciation' $(\mathrm{K}-\mathrm{W}=5.96, p=.051)$. The two groups surveyed in 1994 ranked 'Speaking' first, while the 1997 graduates ranked this skill second behind 'Grammar'. Although the differences across the individual skills were not significant, the bivariate correlation coefficient, Spearman's rho, was not significant for the three pair-wise comparisons of the overall rank ordering of the skills by each group (94(1) $\vee 97(3), \mathrm{r}=-0.31, p=.54 ; 94(3) \vee 97(3), \mathrm{r}=0.31, p=.54 ; 94(1) \mathrm{v}$ $94(3), \mathrm{r}=0.26, p=.63)$.

In the next item, the respondents were asked to choose from a list of possible assertions governing the roles that a teacher should play in regard to his/her relationships with his/her students (Table 4.36). Of the eight roles/relationships, two were found to mark the groups as differing significantly. The chi-square test of a $2 \mathrm{x}$ 3 table was carried out on each role/relationship for the three groups and the following were not significant: 'The teacher should become friends with the students' $\left(\chi^{2}=1.43, p=.49\right)$, 'The teacher should be prepared to devote as much time as is necessary to helping his/her students' $\left(\chi^{2}=4.10, p=.13\right)$, 'The teacher should allow students to decide the direction that their learning should take' $\left(\chi^{2}=\right.$ 4.63, $p=.099)$, 'The teacher should act like a parent' $\left(\chi^{2}=4.87, p=.088\right)$, and 'The teacher should be a transmitter of knowledge' $\left(\chi^{2}=5.66, p=.059\right)$. The assertion 'The teacher should be in total control at all times' contained one cell with a value of zero and so the chi-square was not computed. The remaining two assertions, which were found to differ significantly in terms of the three-way group comparison, were subjected to post-hoc testing using $2 \times 2$ chi-square. The first was 'The teacher should be a facilitator of learning', $\chi^{2}=13.02, p=.001$; post-hoc $94(1) \mathrm{v}$ 
97(3), $\chi^{2}=9.50, p=.002 ; 94(3) \vee 97(3), \chi^{2}=3.88, p=.049 ; 94(1) \vee 94(3), \chi^{2}=$ $2.04, p=.15 n s$. The second was 'The teacher should refrain from punishing the students', $\chi^{2}=8.60, p=.014$; post-hoc 94(1) v 97(3), $\chi^{2}=7.13, p=.008 ; 94(3) \mathrm{v}$ $97(3), \chi^{2}=0.88, p=.35 n s$ and 94(1) $\vee 94(3), \chi^{2}=3.06, \mathrm{p}=.08 \mathrm{~ns}$.

The last item of the questionnaire asked the respondents to assess their strengths and weaknesses as teachers (Table 4.37). The Kruskal-Wallis test for three groups on each factor revealed no significant differences: 'Teaching grammar' $(\mathrm{K}-\mathrm{W}=$ $0.03, p=.98$ ), 'Relationships with colleagues' ( $\mathrm{K}-\mathrm{W}=0.12, p=.94$ ), 'Organisation' $(\mathrm{K}-\mathrm{W}=0.34, p=.84)$, 'Relationships with students' $(\mathrm{K}-\mathrm{W}=0.36$, $p=.83$ ), 'Planning' $(\mathrm{K}-\mathrm{W}=0.43, p=.81)$, 'Motivating students' $(\mathrm{K}-\mathrm{W}=0.47, p=$ .79), 'Innovation' ( $\mathrm{K}-\mathrm{W}=0.60, p=.74)$, 'Communication skills' $(\mathrm{K}-\mathrm{W}=3.18, p$ $=.20)$, 'Discipline' (K-W = 1.06, $p=.59$ ), 'Teaching writing' ( $\mathrm{K}-\mathrm{W}=1.31, p=$ $.52)$, 'Teaching speaking' $(\mathrm{K}-\mathrm{W}=1.86, p=.39)$, 'Voice' $(\mathrm{K}-\mathrm{W}=2.29, p=.32)$, 'Strength of personality' ( $\mathrm{K}-\mathrm{W}=2.36, p=.21)$, 'Teaching listening' $(\mathrm{K}-\mathrm{W}=2.96$, $p=.23$ ), 'Teaching pronunciation' ( $\mathrm{K}-\mathrm{W}=3.05 p=.22$ ), 'Language ability' ( $\mathrm{K}-\mathrm{W}$ $=3.84, p=.15)$, 'Teaching reading' $(\mathrm{K}-\mathrm{W}=4.17, p=.12)$. The factor 'Relationships with students' was ranked highest by all three groups, whilst 'Discipline' was ranked lowest by all three groups. Spearman's tho demonstrated significant correlations between the 94(3) and 97(3) groups ( $\mathrm{r}=0.73, p=.001)$, the 94(1) and 94(3) groups $(\mathrm{r}=0.61, p=.009)$, and between the 94(1) and 97(3) groups $(\mathrm{r}=0.54, p=.026)$.

\subsection{Discussion}

In this section, the findings from the three administrations of the questionnaire are discussed. The intention is to illustrate similarities and differences in the responses and hence ascertain characteristics of the three groups. The findings documented above show that the groups held similar views on most aspects of teaching and the similarities in responses outweighed the differences. Therefore, the discussion will 
first focus on these similarities as a base of common culture of teaching that can put the differences between the groups into perspective.

The main focus of the discussion of differences is the comparison between the respondents who began the BATESL course in 1994 and who graduated in 1997, as it is the intention to examine the possible effects of the run-up to 1997 on these trainee teachers. As a secondary focus, the comparison between the 1997 graduates and the 1994 graduates will provide an indication of changing priorities for teachers of English who were ready to begin teaching in the year of the handover as contrasted with three years earlier. Finally, the comparison between the two groups studied in 1994, one the 1994 entrants and the other the graduates of that year, will provide additional information regarding differences in the characteristics of the two cohorts. Where appropriate, comments made by the respondents on the questionnaires, and/or points raised during the follow-up interviews, will be added to show either differences or similarities in the groups. ${ }^{12}$

\subsubsection{Similarities Across the Three Sets of Responses (Survey Groups)}

The purpose of this section is to look at those items which, by their lack of difference, reveal characteristics of the three sets of responses which can be attributed to the educational culture in Hong Kong and the influence of the BATESL course.

\subsubsection{Language Use}

All three response groups showed moderate disagreement (on average) to the use of mixed code English and Cantonese by teachers in English lessons (Table 4.6). ${ }^{13}$ The popular notion that English should be taught through English would appear to have affected the subjects in this regard. Yet their responses were very much hedged and through their comments, which were consistent, they expressed the need on occasion 
to 'resort' to the mother tongue when necessary. The following comments were made by subjects from each survey group who all 'quite disagreed' with the use of mixed code in English lessons:

(1) In my opinion, only some difficult and abstract ideas can be presented in a mixed code Cantonese-English.

(1) Most of the time use English, switch to Chinese only when necessary.

(1) Depends on the levels of the students.

(1) Try to use English as much as possible.

(2a) It largely depends on the ability of the pupils and the topic.

(2a) Cantonese should be used only in some occasions or cases, but not always.

(2a) Only fail to explain in simple English, Cantonese can be used.

(2a) I think some Cantonese can be used, but not so much. Students may be too depend on Cantonese.

(2a) We should try to let the students to use English as much as possible.

(2b) This may cause bad effects to Ss', either English or Chinese.

(2b) It will lead to interlingual errors.

Subject $\mathrm{P}(97)$ added:

(2b) It would be better if you could use English only, but just like I have said, in certain situations, you have to cope with the difficulty, you have to teach the syllabus, you have to follow the instructions of the panel chair, for example, so you have to use Cantonese sometimes. But I believe ... using English is good, rather than using mixed code.

As the overall pattern of response and the gist of these comments suggests, there was a reluctant acceptance of the use of mixed code when necessary, although respondents in all groups believed that maximum exposure to English was the ideal. The conflict between teachers' preferred approach and the realities of the teaching 
situation was apparent here. Teachers felt that they had no choice but to make use of the mixed-code style of lesson presentation as the pressures of the teaching load restricted the time that they would need to facilitate their lessons only in English. As belief that this mixed-code approach was detrimental to students' leaming of both English and Chinese grew, the decision was made to switch to Chinese as the medium of instruction in the majority of schools. As discussed in Chapters 2 and 3, the intention to adopt Chinese as the medium of instruction had been in the pipeline for some time, but it was decided to announce the change in 1997 and to implement it in 1998. The government may have felt that the process of change underway in 1997 would mask any controversy caused by the change in the MOI, but as described in Chapter 3, this was not to be the case.

When asked about their use of English as the exclusive medium of instruction in their practice teaching, the two graduating groups had a similar response, suggesting a high level of use of English (Table 4.7), though with a slight decline in that level over the practice teaching period (Table 4.8). An indication of the typical situation faced by these students getting ready to teach in Hong Kong was given by Q(97):

(2b) Yes, but I made some concessions during my practice teaching because all my students are grade [Band] 5 students, so in other words that means I have to extend my proportion of using Cantonese in my lessons. I made some concessions, but I still insist for most of the time I must give them English versions at first and try to explain to them in some simple English first and if they still can't understand it then I code-switch to Cantonese.

The sense of the student teachers wishing to maintain a high level of English use came out of these results and comments but with the added caveat of 'resorting' to the use of Cantonese when necessary. That the patterns of classroom language use of the BATESL graduates from the two cohorts was similar in 1994 and 1997 indicates no lessening in the resolve of trainee English teachers to maintain English use in English lessons as the handover approached. Rather, there could have been a strengthening of the resolve to counter the increased expectation of use of Cantonese 
within the education system more generally by maintaining English as the dominant language in English lessons and in English language learning contexts. However, it should be pointed out that the student teachers' practice teaching situation may well have been unrepresentative of the situation in classrooms generally, and the fact that their use of Cantonese began to increase indicates a reality of bilingual classroom language use, even in English lessons.

Respondents throughout the survey agreed that it was necessary to use Cantonese for some teaching functions (Table 4.9). A large majority of the respondents in each group were against the use of Cantonese to present lesson content, while there were mixed responses in each group as regards whether they would use Cantonese to explain content or to be friendly with students. Most of the respondents in each of the three groups stated that there were no situations in which they would not use Cantonese in English lessons (1997 graduates - 93 per cent, 1994 entrants - 88 per cent, 1994 graduates - 94 per cent). This pattern of expectation of use of Cantonese in English lessons in Hong Kong is consistent with the findings of other studies of Hong Kong English teachers' classroom language use in which the propagation of content is performed in the target language but teacher-student interaction takes place in the first language (Johnson \& Lee, 1987; Lin, 1990; Pennington, 1995a, b). Nearly all the respondents, being familiar with Hong Kong classrooms, recognised the prevalence of Cantonese use, even in English lessons. There was

significant difference only on the question of using the mother tongue for establishing discipline, an issue which will be discussed in the later sections that deal with differences in survey response.

\subsubsection{Instructional Planning and Decision-Making}

The respondents throughout the survey were in agreement that it is impossible to have a successful lesson that has not been well planned in advance (Table 4.10). These future Hong Kong English teachers were in favour of planning to achieve successful lessons but were of the opinion that it very much depends on teachers' 
experience and ability to incorporate flexibility in their teaching. Some of the comments from the three groups were:

(1) It depends on one's experience in teaching.

(1) For speaking lessons, spontaneity is very impossible. Too many unpredictable things can happen in the course of the lesson. Flexibility and sensitivity of the teacher is more important.

(1) But I think experience can help teachers to overcome the problem of not enough preparation.

(2a) If the tutor is very professional and experienced, it is $O K$. But if he or she is a green hand, it is a must to have well preparation before lessons.

(2a) Though well-planning is important, stimulation, interaction and brainstorming ideas are also important in a lesson.

(2b) It depends on the experience of the teachers.

(2b) Maybe, it's possible for experienced Ts!!

(2b) Especially for new teachers. They may not have enough experience to predict students' responses and reactions.

Subject $\mathrm{P}(97)$ explained how experienced teachers might not need to devote so much time to planning lessons:

(2b) I mean, if the teacher is very experienced, then maybe she don't need to spend so much time on planning the lesson in advance and she can just teach based on the pace of the lesson and based on the ability of the students.

Subject $R(97)$, in contrast, focused on the need of inexperienced teachers to plan well:

(2b) It is especially true for us, for inexperienced young teachers, because it 
gives us confidence when we have something in hand. If we don't have something in mind, something planned, when we go into the classroom, we would feel less confident. We need to have something planned in mind before we go into the classroom.

However, from Table 4.11, it was found that both Year 3 groups disagreed that teachers should write detailed lesson plans for every lesson. Whilst feeling that as inexperienced teachers themselves, they would need to plan their lessons, they were also aware of the constraints that prevent teachers from doing so. Respondents across the surveyed groups of BATESL students agreed on the extent of detail required for lesson plans (Table 4.12), with a moderate degree of flexibility the preference. Subject $R(97)$ commented on the need to be flexible to cover all possible eventualities:

(2a) You need to have that sort of detailed element because you may forget before teaching that lesson, and you need to have flexibility because there are so many unexpected classroom happenings and you need to have that sort of flexibility to change.

This view was shared by Subject O(97):

(2a) To me the time constraint is the main concern, so we can't write detailed lesson plans. So maybe loosely detailed, that will do, and flexibility is always very important. We should bear in mind that the lesson plan can be changed all the time, it depends on the situations you are in, especially in the classroom, there are so many students with different expectations.

In 1994, Subject B(94) reflected on the changes to the planning she had made during her practice teaching:

(1) Because sometimes I can't catch up with my plan, so I have to change it, cut it short, but sometimes maybe they need more examples or sometimes I feel 
that the students need another activity other than the activity that I've planned, then I will change it.

In 1997, Subject N(97) explained that in her case she often changed her lesson plan because her students were more responsive than she had anticipated:

(2b) Actually, I'm quite aggressive. Usually I plan more than I can teach, and because the students are very responsive, and I had a lot of interactive activities with the students, so usually this kind of activities took much much more time than I planned, so I had to change a lot.

For Subject $\mathrm{P}(97)$, it was quite the opposite:

(2b) Because I set quite a lot of activities and they don't know how to respond always because they used to be taught by a very experienced teacher, but she always use Cantonese and she always focus on the textbook page by page and then do all the comprehension exercises and go over all the grammatical points, but she won't do any activities. What we've learned here is designing some activities for your students and they can use English in the context, instead of just focus on the grammatical points, just learning the rules, and so I find it difficult to organise the activity and how to explain to the students, it's a great problem.

The respondents commented on the constraints that prevent teachers from either adhering to a detailed plan or even having the time to write one, a feature found in other studies of teaching in Hong Kong (Pennington \& Cheung, 1995; Pennington, Richards, Urmston \& Lee, 1996; Richards et al., 1991). The concern with such constraints (heavy workload, large classes, limited resources etc.) may reflect (or may have caused) a lack of commitment to teaching on behalf of some of the respondents (Pennington et al., 1996). Of greatest concern to the trainee teachers was the issue of timing, either not having sufficient time to plan lessons in detail, or not being able to accurately judge the pace of lessons and make decisions on how to 
cope when students either make slower or faster than anticipated progress. Similar findings for inexperienced teachers have been made by Richards (1998) in a study of experienced and novice teachers of English in Hong Kong. The evidence from the questionnaire and interview surveys of this investigation suggests that the BATESL student teachers possessed similar approaches to planning as novice teachers in other teaching contexts. Similar to Bullough's (1989) first-year junior high school teacher, the respondents' thinking seemed to focus more on activities and timing in the early stages of their training, then begin to move more towards student abilities and interests as they began to think more about their teaching approaches and, like $\mathrm{P}(97)$, compare themselves with the cooperating teachers they were working alongside during their practice teaching.

In terms of the factors upon which the Year 3 subjects planned their lessons during practice, there were some differences, but they were not significant (Table 4.13). Having to follow the syllabus and the instructions given to them by the regular teacher with whom they were working (cooperating teacher) proved to be the norm for the teachers in both graduating groups. Subject T(97) reflected this:

(2b) Not much freedom because I have to finish the exam syllabus because the past teaching period is just before the examination, so the time was quite tight. So I had to finish what the teacher assigned me, the school teacher.

Lack of time was a factor that was mentioned by both graduating groups. Subject $\mathrm{O}(97)$ felt this was a major problem:

(2b) Yes, it's too short so I can't put all my ideas and for example my first lesson of précis writing is not that good, maybe I don't have a chance to correct it because the practice is too short.

The two Year 3 groups also agreed on the factors affecting their decision-making during their practice teaching (Table 4.14), which were the cooperating teacher's 
input, students' needs and their supervisor's advice. Subject E(94) stressed the importance of the supervisor:

(1) That's kind of silly because I was being assessed in practice teaching. If I want to score high I have to follow what he says, so that's what I meant by 'Supervisor's advice'. But I don't take that as assessment guidelines because he was a nice guy, my supervisor and he was really good in teaching us. He give us some good advice, so what the hell, let's take it.

Subject T(97) agreed:

(2b) Yes, because she [their supervisor] assessed me. If I don't follow her instructions, maybe she would reduce my marks. Generally speaking there's not much freedom during practice teaching, I have to obey the instructions of the cooperating teacher and the tutor of the university.

The importance of the supervisor related by the respondents above is in terms of his/her role as the assessor of their performance as well as their advisor. The Year 3 respondents were still having to think as students while at the same time being expected to think and act as teachers.

There was also agreement among respondent groups that teachers should be more responsive to student needs than the set syllabus (Table 4.15). A selection of comments on this issue reveals that the majority had a strong student or learnerorientation, although there were some in the Year 3 groups who expressed the view that the reality of teaching in Hong Kong is one of syllabus and examination-focus:

(1) Sometimes students need more than what are stated in the syllabus. If we follow strictly the syllabus, we'll neglect some of the students' need and this in turn will affect their motivation to learn.

(1) The aim of teaching is to enable students to learn so the consideration of student needs is very important. 
(1) Students in Hong Kong are very exam-oriented; most of them just want their teachers to stick to the syllabus.

(2a) Students' response is very important. Teachers should be flexible rather than strictly follow the set syllabus.

(2a) Different students have difficulties on different aspects. Teacher should adjust any things that suit the students.

(2a) The most important thing for the teacher to do is to raise the interests of the students.

(2a) Different students have different needs, so, teacher should be more responsive to their needs than the set syllabus.

(2b) Students will enjoy more if they've learnt something they WANT to learn.

(2b) Ts have to adjust the syllabus to match Ss' needs.

(2b) I agree with you, but teachers may face pressure from schools, parents or students if the teaching schedule is far behind the normal one which is based on the examination syllabus.

The idea of the examination-oriented syllabus was reflected by Subject U(97):

(2b) For the junior form students, yes, it's possible, but for the senior form students, their needs are according to the syllabus. When you are responsible to their needs, actually you are responsible to the syllabus. Unlike the senior form students, the junior form students may want to have some interesting lessons so that they can pay attention, so I think to make them more like your lesson, you should be more responsible to their needs instead. But not for senior form students.

As with teachers' planning, the strong focus on constraints in teaching came out strongly from the respondents' comments with the recognition of the influence on teaching played by examinations (both internal and external) and the syllabuses that are designed to ensure that students are prepared for the examinations. 
On the other hand, Subject N(97) felt that for weaker students, it is essential to cater to their needs:

(2b) I also believe that if the students' level is not up to the syllabus level, they will understand nothing. You just stand in front of them and talk and talk, and they understand nothing. It's no use. So I think teachers should try to adjust the syllabus, or should not just blindly follow the syllabus. They should think about the students' needs.

Whilst there was clear evidence of awareness of the teaching norms and constraints of Hong Kong, there was also an entrenched belief in the importance of addressing students' needs. This sense of conflict is a recurring theme and one which could be said to challenge teachers' framework of beliefs and practices on an almost daily basis.

\subsubsection{Teaching Approach}

There was moderate agreement across the survey groups that a class should not be strictly controlled by and all lesson content directed by the teacher (Table 4.19). Comments from the three survey groups indicate that they were against strict control but with some provisos to maintain discipline:

(1) It should be flexible, but it is important not to let students control the lesson. It's dangerous!

(1) The students have their own responsibility to control themselves and they should have rights to decide lesson content.

(1) Very often, it is the teacher who understand what the students need. However, I think teacher should also give chances for the students to voice out their own needs and opinions. 
(2a) Spoon-filling is not a good way. Students should be taught in an innovative and creative way.

(2a) Teacher should guide the students but not control them. Students will perform better if they have chance to commit in choosing and designing the lesson content.

(2a) Students should have enough interaction with both teacher and their classmate in order to keep the language learning lively.

(2a) The active involvement of the students is vital for the possibility of success for lessons. Too disciplined class and teacher-directed lessons are not healthy for teaching language.

(2b) It depends upon the levels of students.

(2b) Need room for Ss to experiment with the language.

(2b) Students can be guided to explicit the language items themselves.

(2b) Teacher-student interaction is recommended by my viewpoints.

(2b) Lessons should be student-centered.

(2b) Students may feel bored.

(2b) It's because of the class size you know!

Subject R(97) did not like the word 'control' and felt that teachers should form good relationships with their students based on respect rather than control:

(2b) If you take care of student needs, then teacher cannot control all the lesson content, and a class should not be strictly controlled by the teacher because you are just teaching students, you are not having a parent-son relationship. Don't control them. If you want to have good classroom management, maybe you can control them in a mild way. For me control is not a good word.

However, Subject $P(97)$ felt that students needed to have their lessons tightly structured or they would not feel comfortable: 
(2b) For some reason, like if the lesson is not controlled by the teacher, then the students will feel loose and they don't know what to do. Based on my own experience when I was a Form 4 student, the teacher won't plan anything for us to do during the English lessons and that's what I feel. I feel loose and I don't know what to do and I'm worried about my proficiency and I'm worried about my performance.

The term 'strictly controlled' seemed to cause concern for many of the respondents as the idea of strict control was not consistent in their thinking with a studentcentered approach to teaching. Some of the respondents looked at the question from the teachers' point of view, as wishing to form good working relationships with students based on mutual trust and respect. Others, like $\mathrm{P}(97)$, looked at this issue from the a students' point of view, expressing the view that students need the sense of security that a 'controlled' lesson can bring. As the overall response of the trainee teachers was against strict control, the indication is that they had not yet begun to think about classroom management issues. On the other hand, it could be an indication of a change in teaching culture away from the traditionally strict (in Chinese societies) authoritarian teacher-student relationship.

The survey groups were in agreement that teachers should be prepared to experiment with teaching materials and strategies (Table 4.20). It would be expected that the interpretive, innovative philosophy of the BATESL course would have influenced the graduating teachers towards experimentation, but the Year 1 respondents agreed also, showing that they held such a view even before they began the course. They could possibly have been influenced by the induction that they would have received before beginning the course, or perhaps it was the fact that they held these beliefs in the first place that made them want to be English teachers. Typical comments from respondents in the three survey groups were:

(1) Teachers should be risk takers. Never try, never know the result.

(1) It can help the teacher to find out what kinds of teaching strategies interest the students most and the best way to help the students. 
(1) Yes, I agree this statement but there are too many constraints in the real situation.

(2a) To stick to the traditional method of teaching, especially language will burden students ability to learn.

(2a) Pupils are composed of different learning styles, which should be catered for by different teaching methods and materials.

(2a) So that he/she knows what best suits a certain type of students.

(2a) Students can supply some materials and teacher strategies. It is also a chance to learn.

(2b) Teachers need more support/input in terms of training.

(2b) As long as there is enough time.

The comments showed that they were in favour of experimentation for the benefit of their students, with the Year 3 respondents also aware of the constraints that often prevent teachers from experimenting. This was reflected in their comments concerning the experimentation they did during their practice teaching:

(1) As my cooperating teacher asked me to cover a lot of teaching materials during that period, I did not have much time to experiment with my own materials or even strategies.

(1) There is much to cover only from the course book itself. However, the way to deliver may be different from the suggested ones.

(2b) You must ensure your lesson flow is all right.

(2b) I tried what I've learned in this course when I taught.

(2b) But teaching syllabus and textbook had to follow the school policies ... school views English learning as a top priority.

(2b) Time constraints. 
Subject $O(97)$ felt that it was important for teachers to modify their teaching materials or strategies to match their students:

(2b) I think all those materials may not be suitable for your class, or the suggested teaching strategies in those teachers' textbooks, you may not use them in your class, your specific class, so we should always bear in mind that we shouldn't use those materials directly, just use them in your class, we should think about whether they are suitable or not, so we should experiment with those materials with the students first and you can confidently use them later on.

This view was shared by Subject Q(97), who also commented on the ESL materials produced by commercial publishers in Hong Kong:

(2b) I know that in Hong Kong so many publishers would like to provide some materials to teachers ... and some teachers, they just like to adopt directly from the publishers. They just adopt everything and they do not think that this is appropriate to our students or not ... I find that the materials are designed for those countries where English is the second language and the students have to use English in their daily life but in Hong Kong this isn't the case.

Thus, the teachers again showed their awareness of and concern about the teaching constraints that tend to inhibit experimentation and innovation in teaching, but the Year 3 respondents especially showed that they recognise the importance of developing specific designs and procedures appropriate to the students being taught, within an individual teaching approach or framework

All three survey groups hedged on their own teaching style, declaring themselves to be in the middle of the scale between 'mostly learner-centred' and 'mostly teachercentred' (Table 4.21). Given their inexperience, even after their practice teaching, which was only three weeks long on a reduced timetable, they may not have been 
too sure of their style. Comments from the Year 3 respondents indicated that they perceived their teaching style to be very much affected by constraints within the teaching context such as school, syllabus, proficiency and age of students. Subject $B(94)$ felt constrained by the scheme she had to teach to:

(1) Because I have some activities for them to practice in pairs or in groups but not very often because of the scheme, because of the lessons. For example composition, you can't ask them to do group work, they have to write individually. And also the comprehension is explaining the textbook, maybe the questions I will ask them to do in pairs or to do it at home, then I check the answers. And the listening, they will listen to the tape, there is no interaction. Only the oral lesson they will have the interaction.

Subject U(97) thought that a teacher's teaching style was influenced by the proficiency of the students that he or she taught:

(2b) Actually it depends on the banding of school and my students. If it's like my students in the practice teaching, I may give them more chance to be learner-centred because they can control themselves, they can help themselves or help the others. But if they are in like Band Four or Five schools, they need more help. So it depends, but to my own style, I really prefer teacher-centred. It will be more efficient, I still think that.

Subject R(97) felt that it depended on which form the students were in:

(2b) Because I was teaching young learners, I had to show them some authority. But I have no opportunity to teach upper form students, which I really want to teach. If I had that opportunity I think my approach would change to more like learner-centred. You need to bring out this problem. It seems to me that this BATESL course is prepare you for teaching only junior form students ... we have not much coverage on how to teach senior form students, we need that. 
There is clearly a conflict demonstrated between the subjects' desired teaching style or the approach that they would like to adopt, and that which they feel they are forced to adopt. As teacher-trainees or new teachers, it is likely that they would have felt that they needed to conform to the constraints present within their teaching situation. An alternative view is that they may have been unsure as to whether they should follow the approaches that they had learned in their course, what might be described as the norms of ESL teaching or 'Western' approaches, or they may have been more oriented towards what they saw as the norms of teaching in the Hong Kong context. Once they become more experienced and established, they may be able to develop their own approach and realise it more, or they may simply become increasingly absorbed into the 'resident' teaching culture in Hong Kong. The following chapter will look at how some of the BATESL students did change once they became more experienced teachers and whether they were able to develop more personalised teaching approaches.

English teaching by nature is open to Western influence, partly through the English language and partly because of innovative teaching approaches which have their origins in Western countries, particularly in Hong Kong's case, Britain (see Chapter 2). This influence was strong during the colonial period and remained so after the handover, as has been discussed in Chapter 2. Resistance to innovations that are seen as Western in origin is often manifested by teachers who feel that such 'foreign' approaches are not suitable for Hong Kong and resentment is directed to the government and education authorities for simply imposing unworkable approaches or initiatives instead of developing ones to meet the specific needs of Hong Kong.

The factors that respondents felt were influential in the teaching of English (Table 4.22) caused the groups to differ in some respects. These are discussed in the later sections. However, those factors that respondents agreed upon included examination requirements. This factor was ranked third most important (out of twelve) by the two graduating groups and fourth by the Year 1 group. The beginning student teachers recognised the importance of examinations in the education culture of Hong Kong 
and this feeling did not diminish after three years of the BATESL course. The high profile of the public examinations in Hong Kong has been demonstrated and described in Chapter 3. This importance showed no sign of diminishing after the changeover in sovereignty as qualifications were seen as a hedge against the uncertainty caused by the ongoing and anticipated changes in society.

\subsubsection{4}

\section{Professional Relationships and Responsibilities}

All survey groups showed a similar degree of confidence in their own ability to become a successful teacher (Table 4.23), with each group mean falling in the range between the descriptors 'Unsure' and 'Quite confident'. Whereas a lack of confidence on the part of the beginning students would be understandable, it might be expected that the graduating BATESL students would be more confident after three years of teacher training. Subject $O(97)$ mentioned relationships with students and colleagues as well as his English proficiency as factors affecting his confidence:

(2b) I really don't know how I can get along with my colleagues and my students, and which school I will be in. I think those factors may disrupt my confidence in being a successful teacher. My English proficiency is also another factor. I don't speak very fluently. I have no experience in using simple English to explain those English terms or something like that. I think what I lack is the confidence, I mean the experience.

Subject U(97) was quite confident in her own ability, but at the time was finding it difficult to secure a job:

(2b) Yes, if I can be employed. Yes, I really believe that. At least if I can be a good teacher, if I can have a good inter-relation with my students, I can be very instructive and informative to my students. But I have no chance to explain to any one of the principals. 
One problem faced by the BATESL graduates was a lack of recognition of their qualification by school principals, as the course was still relatively new, even in 1997, and did not have the face validity of more recognised teacher-training courses provided by the Hong Kong Institute of Education, the University of Hong Kong and the Chinese University of Hong Kong. This was despite the fact that at that time the aforementioned institutions were providing postgraduate diplomas and certificates of education rather than bachelor's degrees. Schools were more inclined to favour university graduates with degrees in English or related disciplines from the University of Hong Kong over the specially-trained graduates of the BATESL course, as the former possessed greater currency due to the perceived higher standards (of English) at this institution and its status as Hong Kong's premier university.

In general, the subjects' limited confidence suggests that they felt some unease about entering the English language teaching profession in Hong Kong in the period leading up to the handover. Views expressed by English language educators in Chapter 3 corroborate the impression that at that time, (and most likely even more since then - see Chapter 5), English teaching was seen as a less than attractive option for university graduates than medicine, law or the civil service. The uncertain future, as well as causing an increase in the influence of examinations on education in the post-handover period, may also have strengthened the desire of students to achieve the security of such professions, whilst teaching, with its related unknowns, was not as attractive an option.

All respondent groups considered good command of the language, good communication skills and innovativeness as their best attributes (Table 4.25). Subject B(94) made this comment:

(1) If you are a teacher, of course you must show good command of English otherwise the students have no confidence in you, they will tease you at the back. So I think the teacher himself or herself needs to be a very good communicator or a very good English so that they can give confidence to the 
students, so it's very important. And also, he or she must have some new ideas or teachings because traditional teaching is too boring. So he or she can use some new ideas to stimulate the students.

Being students on a BATESL course, they had confidence in their English language skills. They also felt that they had a lot of ideas as regards teaching and they were very aware of the importance of not being boring in their teaching. The Year 1 group also had a strong sense of being creative in the classroom, even though they had not yet had the benefit of the BATESL course and its stress on innovative and communicative approaches. Forming relationships with colleagues and discipline were ranked the weakest attributes by all groups. Subject $P(97)$ explained why she felt it difficult to form relationships with colleagues:

(2b) In a school there are many teachers and their age range from their twenties to their fifties. There may not be many fresh teachers in the school that you are teaching, and that's why I think it's quite difficult to form a good relationship with the other colleagues. And also they teach different subjects, and you can only communicate better with the English teacher, than other teachers. And if the English teachers in that school are in their fifties or in their forties, it's very difficult to communicate with them.

Subject $Q(97)$ even felt it unnecessary to form professional relationships with colleagues:

(2b) I think that teaching is just a matter between the students and the teachers, but not the relationship with your colleagues. Of course sometimes we need to cooperate about our teaching progress, to let them know about this, but that's it. If you have a good professional relationship with colleagues, it doesn't mean that it will help you to improve your students' standard or help you to solve those problems that you face in the classroom. So I think that's not important, but it's very important for your promotion. 
It seems that it is not part of the culture of teaching in Hong Kong to perceive oneself as part of a group or team of colleagues, as would be typical in other (Western) cultures. This could be related to the competitive culture of education in general in Hong Kong. The lack of attractiveness of English language teaching may have resulted in a corresponding lack of camaraderie amongst colleagues. Instead of teachers becoming more cohesive in the face of the increasing difficulties associated with the job, they became more isolated as the pressure not to be seen to be failing mounted amidst the uncertainty associated with the handover and the changes in education.

Despite this impression of an isolationist culture of teachers, of the possible sources of help to the new teachers in their first years in the classroom, the subjects were in agreement that only more experienced teachers could be relied upon to offer advice and assistance (Table 4.27). Subject Q(97) commented:

(2b) Because sometimes more experienced teachers can give you some direct information about the classes and about the level of students, and about the characters, so it save you a lot of time in dealing with the class.

Subject N(97) found the same thing during her practice teaching:

(2b) Yes, because they can give me practical advice, practical strategies to solve some problem. I talked to my supervisor sometimes, but my supervisor was too theoretical sometimes and she helped me to solve the problem. So what I need is more practical things, I don't have the time to think, to solve the theory, so I think most experienced teachers helped me a lot, because these teachers understand more about the students.

Such comments show that these future teachers had the same sort of practical orientation as has been observed in teachers in other countries (Galton, 2000; Stuart \& Thurlow, 2000). They also underscore findings highlighted elsewhere in this chapter involving a stress on immediate needs, problems, and time constraints. 
That these future teachers did not think that such people as panel chairs, principals or senior teachers would be of help perhaps demonstrates the perceived distance between themselves as novices and those that could be thought of as 'school management'. In addition, as Subject N(97) pointed out, they felt that even their supervisor might not be of practical help to them in their teaching situation, demonstrating the perceived gap between theory and practice that is typical of teachers' views (Ethell \& McMeniman, 2000; Freeman, 1991), though perhaps less expected from those taking coursework in TESL. In the case of those studying on the BATESL course in Hong Kong, it is well to remember that 'experienced teachers' refers exclusively to Cantonese-speaking teachers, whereas 'supervisors' may include university lecturers - particularly, those from other countries who are nonChinese and non-Cantonese-speaking - who have never taught in Hong Kong schools.

Consistent with previous findings, respondents cited learning from other teachers as the factor that would most help them to develop professionally (Table 4.31). Subject Q(97) acknowledged the importance of other teachers, but also mentioned the importance of teaching materials:

(2b) I just think that of course we can learn from other teachers, from other experts, but that's their own experience, that may not be appropriate to you. So I just think that finding new materials and designing new materials will be more important than this.

All recognised the importance of learning from other teachers, despite the earlier findings that they were concerned about their relationships with colleagues. They recognised the value of experience. 
The groups did not disagree significantly on the relative importance of the language skills of reading, writing, listening, speaking, grammar and pronunciation (Table 4.35). ${ }^{14}$ Subject $\mathrm{P}(97)$ explained why she thought listening and reading were relatively less important than writing and speaking:

(2b) For listening and reading, sometimes you can guess if you don't understand the vocabulary in the story, you can guess from the context, and also, if you don't understand what the speaker said, you can just guess while you are listening. But in writing you can't use that kind of strategy. You have to know everything before you write or before you speak. You have to know exactly the wordings or the grammar.

Whilst Subject Q(97) was expressive in his support for the teaching of grammar:

(2b) Yes, I just don't understand why some so-called experts proclaim that grammar is not so important. They tend to use some inductive, is that right? They ask the students to generate some grammar rules from the lessons, a deductive model? They proclaim there's no need to teach those grammar rules explicitly, we just allow the students to generalise. Unfortunately, some of the teachers, especially the English teachers complain about this because they find that the students are not able to generalise the grammar rules. Instead of this they fossilise the mistakes only to the confusion, so I think grammar lesson is a must, I must teach my students grammar, even though it's boring. Of course I will try to teach in an interesting way, but I must teach grammar to our students.

Whilst the two groups surveyed in 1994 on average rated speaking as the most important language skill, the 1997 graduates gave the highest ranking on average to grammar, evidence perhaps of more attention being paid to accuracy in order to try to arrest what had been seen to be falling standards of English. The increased 
importance attributed to grammar could be said to be against the theory of communicative language teaching but consistent with the rediscovery of the importance of grammar in the ESL field at that time (Celce-Mercia et al., 1997). It could even be seen as a rejection of the communicative orientation that was pushed in the 1980 s and early 1990 s.

The respondents differed somewhat on their perceptions of the roles they should play and the relationships they should have with students (Table 4.36). One interesting finding was the consistency with which the respondent groups agreed that a teacher should try to become friends with students. According to Subject T(97):

(2b) Yes, I think teachers should try to become friends with the students because if the teacher has a good relationship with the students, it may facilitate the teaching process, and the students may be more motivated.

In contrast, Subject $\mathrm{N}(97)$, one of the few in her group to disagree, said:

(2b) I'm not sure is it possible to become friends. Actually the relationship between friends is quite different from the relationship between teachers and students. Sometimes if you are too friendly, or the students regard you as a friend, he or she may not behave properly in class, or some other students may be jealous of that student or they don't like that. So it's quite difficult to be friends with the students.

Although Subject N(97) was in the minority, her comments would seem to represent the common-sense view of this issue. The fact that the majority of subjects in all three groups felt that this role/relationship was one they wished to adhere to $(97(3)-$ 77 per cent, 94(1) - 72 per cent, 94(3) - 65 per cent) indicates a high level of student-centeredness and a possible desire, not uncommon amongst teachers, especially young ones, to be liked. Alternatively, it may indicate a reaction against being a strict teacher, echoing earlier findings about strict control of lessons. A third possibility is that as English is (perceived as) a humanistic, student-centred 
profession, the concept of being friends with students complies with the respondents' desire to enter the profession. This desire to develop close relationships with students did not stretch to wishing to act like a parent, however, as the vast majority of respondents in all three groups did not consider this a suitable role for a teacher.

Emphasising the student-centeredness of the BATESL sample as a whole was the fact that respondents across the survey also agreed that the teacher should devote as much time as is necessary to helping his/her students. This finding indicates a teaching culture in which teaching is viewed as a social service geared to helping people, in this case, students, who are viewed by many teachers as being in need of help. There seems to be a strong orientation towards being protective of students and treating them (even older ones in senior secondary or tertiary education) as children. This 'mothering' role would tend to conflict with the professional image that a teacher is expected (by the majority in society) to perform and there was evidence from these surveys that teachers find the relationships that they have with their students to be one of their major concerns. However, there were mixed feelings within the survey groups as to whether the teacher should allow the students to decide the course that a lesson should take, echoing the earlier findings concerning the extent to which the teacher should control lessons. The conflict between a student-focused orientation and a fear of lack of control is evident here again.

There were differences in the perceptions of the survey groups in terms of the other roles/relationships stated and these are discussed in forthcoming sections.

When asked to consider a number of factors and rank them as either a strength or a weakness, there was almost total consistency in the groups' choices (Table 4.37). The biggest strength was listed as their relationships with students, consistent with the learner-centeredness described earlier, and their greatest weakness was discipline. Subject $\mathrm{R}(97)$ explained why she rated discipline as not one of her strong points:

(2b) We could be cool to students in lessons but we don't want to do so. I could 
do something in discipline but I won't do so, I won't control students. So I would say I'm still strong in discipline but I don't control students in a very strict way. Everyone can be strong, but we just don't want to do so.

In other words, the desire to be sympathetic to students is strong and affects their approaches to discipline. This possibly supports other findings that show that teachers may feel that there is a contradiction between being strict with students and building good relationships with them.

\subsubsection{Summary of Similar Findings Across the Three Response Groups}

The impressions emerging from the general similarities in survey results of the culture of trainee teachers of English in Hong Kong is one of empathy with students combined with a developing awareness of the realities of teaching. As initiates on the BATESL course, the 94(1) survey group did not have the benefit of practice teaching experience but appeared to have an awareness of the teaching norms of the Hong Kong context through their experience as students. The overall response pattern suggests that all three groups experienced conflicts that can be summed up as a series of contrasts and hedges along the following lines:

- To make use of the target language as much as possible but use the first language if necessary for explanation, interaction with students and classroom management.

- To be innovative and experimental in teaching whilst conforming to the syllabus and examination-driven teaching approach prevalent in most schools.

- To be friendly with students and at the same time to be authoritative and avoid discipline problems.

The areas in which a common culture of English language teaching exists in Hong Kong can be summarised as language use, teaching approaches and relationships. Language use, as described in the previous chapters, concerns the ways in which 
English and Chinese are used within education and society. The relative positions of the two languages in society backwashes into schools and this affects how they are used and taught. With the expectation of increasing amounts of Chinese (Cantonese and Putonghua) within society after the handover, and the change in the MOI in schools, English teachers, the majority of whom would teach in CMI schools, were unsure as to what policy or practice they would be obliged to conform to. The expectation was for there to be either a clearer polarisation of: English medium in EMI schools and in English lessons in CMI schools; or, more use of Cantonese in English lessons in CMI schools. As regards the teaching culture at that time, it would appear that the common bilingual teaching mode of Cantonese-English was being maintained, but with some degree of uncertainty as to how this would change after the handover and the change in MOI.

Secondly, uncertainty over changing curricula and the increasing dominance of the examination culture put more pressure on teachers to avoid using innovative teaching approaches for fear of disadvantaging their students. Students were turning in ever-greater numbers to tuition schools, which they felt offered them the extra examination coaching that was their primary objective. Teachers in schools had the option of either competing against these tuition schools by providing the examination preparation demanded by students and their parents (particularly those who perhaps could not afford to pay for extra tuition), or adopting innovative teaching approaches that might be objected to by students, parents and even by school authorities.

Thirdly, relationships with colleagues were still apt to conform to previous descriptions of the lack of collegiality amongst teachers, with the uncertainties of the handover tending to make teachers even more isolationist. On the other hand, there was evidence of closer teacher-student relationships as teachers became more sympathetic with students and more aware of students' needs and problems in a changing society. 


\subsubsection{Differences Across the Three Response Groups}

In the following sections, the instances in which there were significant differences found between the survey groups' responses are discussed. Firstly, significant differences between only one of the pairs of groups will be discussed. Then, differences found between two pairs of groups will be examined. These pairs of significant differences will provide interesting points of discussion as through such comparisons, certain factors, such as the effects on the respondents of the BATESL course, may be highlighted. Finally, the differences which were found between all three pairs of survey groups will be presented.

\subsubsection{Differences in Response of Cohort 2 in 1994 and 1997, as Beginning and Graduating Group}

In this section, the significant differences in responses that were unique to the cohort surveyed at the beginning (94(1)) and at the end (97(3)) of their BATESL course are described. Differences in this cohort from the earlier to the later survey may reflect the effects of the BATESL course. They could also be a result of respondents' increased experience and knowledge, and/or changes in education in Hong Kong during the three-year period while they were studying on their degree course. From the statistical results (which are conservative), it can be assumed that the other pairs of groups, i.e., the two groups surveyed in 1994 and the two graduating groups (in 1994 and in 1997), did not differ on these items. The only statistically significant differences in Cohort 2 and no other group were in the two relatively abstract areas of Professional Relationships and Responsibilities and of Perceptions and Values.

\section{Professional Relationships and Responsibilities}

The first item on which the second surveyed cohort as beginning students and 
graduates were the only survey groups to differ significantly was on the degree of helpfulness of the panel chair (Table 4.27). ${ }^{15}$ The respondents may have been unsure as to what a panel chair is at the start of the course as only one out of forty felt that he/she would be helpful. After practice teaching, a number of the BATESL students must have had a positive experience with the panel chair(s) in their schools and so this influenced six out of thirty to state that in this light they now felt that the panel chair would be helpful. Subject U(97) felt that the role of the panel chair was crucial in the development of a novice teacher:

(2b) Yes, maybe I can get immediate help from the experienced teacher, but if the panel chair doesn't understand you or doesn't accept your weakness, I think it will be quite harsh. And I believe that the panel chair should be the most experienced one, so he or she can give me the most help.

A further example of the lack of awareness of the support needed by a novice teacher was that few of the second cohort respondents in 1994 anticipated having to meet with colleagues during their first year as a teacher, whereas most of the 1997 graduates expected to have to do this (Table 4.28).

\section{Perceptions and Values}

There were two areas of concern on which there was significant difference between the respondents in Cohort 2 in 1994 and 1997 (Table 4.33). The first was 'Professional relationships'. This was a source of concern to more of the respondents after completion of the BATESL course. It was perhaps not an area that the Year 1 student teachers would have had a chance to think much about, whereas this would have been more of an issue to those student teachers in Year 3 , as they prepared to enter the profession. It could indicate that some members of the 1997 graduating group had had negative experiences in getting on with other teachers during their practice teaching, possibly confirming the notion that teachers in schools were becoming less cohesive as the handover approached. 
Their feeling that they would need to work closely with other teachers in their first years of teaching did not change significantly (Table 4.30), indicating that they may have realised that this was inevitable but they still did not feel too confident about it.

Professional status was more of a concern to the 1997 graduates than it had been to this group when they started their course in 1994. The change from student to potential teacher would be expected to alter their perceptions to the extent that the status of teachers would become more important to them. This finding could also reflect a change in the image of teachers within society over the three-year period. In 1997, Subject $U(97)$ expressed her feelings about the changing status of teachers:

(2b) Yes, I think so, because when I was a student in senior form or junior form or even primary schools, I really respect my teacher very much. I think it's necessary to respect my teacher, but I can't find this respect from nowadays' students. They just think that teachers receive the salary from schools, and they paid for the school fee, and actually teachers are working for the salary and working for them. So teachers should be so good, only because of the salary, not because of the teacher.

Subject $T(97)$ saw the emergence of private tuition schools as threatening the status of teachers in government-funded schools:

(2b) Because of the culture of the society. Because some people may think that the customer is always right. This theory may be applied to schools, that means the students are always right, they complain everything. The teaching method is boring, they punish the students etc. And the emergence of some private remedial classes outside the school. Such kind of teachers in private institute may like a superstar because they provide many tips, many internal tips, maybe some secret tips about the examination, and they can predict the questions of the public examinations. The students may 
think such kind of teachers is like a superstar, is superior, because the teacher in ordinary secondary school cannot do this.

As mentioned previously, the status of teachers (of all subjects) was being undermined by the increasing popularity of tuition schools and by the increased focus on English teaching standards in respect of the perceived falling standards of English in society during the period (as reported in Chapter 3). A further indication of the changing image of teachers was provided by the second cohort's considerations of the factors affecting what goes on in the classroom (Table 4.34). The 1997 graduates differed from the respondents in this cohort at the beginning of their course in regard to their view of the value of the competence of the teacher. By the time of graduation, respondents in Cohort 2 rated this factor higher than they had at the beginning of their course. Subject $\mathrm{P}(97)$ explained why she considered the competence of the teacher to be the most important factor impacting what goes on in a class:

(2b) If the teacher is not confident, maybe he or she will have to use Cantonese all the time and he or she may make mistakes himself or herself, and it will affect what goes on in the classroom. Very often the performance of the students will be affected by the teacher. If the teacher is good, then the students are potentially good.

There was understandably more of a focus on the teacher as the dominating presence in the classroom from the graduating group, who would have been more conscious of the impact of the teacher in the classroom based on their recent teaching practice experience. There was also, as noted, an increased focus within English language education on the teacher as provider and on teaching standards and teacher morale in the face of substantial change.

An interesting finding was that the 1997 Year 3 group considered location of school to be a much more important influence on what goes on in classrooms after having done the course and having taught in schools themselves. This was not a 
significant factor in the comparison between the two 1994 survey groups (94(1) and 94(3)), indicating that it may have been due to changes taking place in the three years that the 1994 entrants spent doing the course (1994-1997). There may have developed an increased awareness of the social demographics of Hong Kong; that certain areas, such as Central and Causeway Bay on Hong Kong Island, or Kowloon Tong in Kowloon, contain the more prestigious schools, whereas the New Territories new towns, such as Tsuen Mun and Ma On Shan, tend to cater for children from working class families and suffer from low student motivation and discipline problems. In addition, there was an increased emphasis in 1997, compared to 1994, on the status of schools as it related to the medium of instruction issue, and prestigious schools became even more in demand as demarcation between EMI (high prestige) and CMI (lower prestige) began to take effect.

In terms of roles and relationships with students (Table 4.36), more members of the 1997 graduating group were inclined not to punish students than the group as course entrants in 1994. This could indicate a greater level of maturity and confidence in the Year 3 respondents as well as the influence on them of the BATESL course. The student-centrered nature of ESL teaching that they would have become familiar with would have led to them feeling that they should not need to punish students. The points made earlier concerning the culture amongst teachers of needing to be friendly with students is illustrated again as a rejection of the idea of punishing students. The novice teachers may have been adopting an attitude of positive thinking and (naively) hoping that they would not need to punish students. The reaction against strictness in teaching is illustrated again, possibly showing a trend towards more liberal (or 'Western') approaches to teaching and classroom management as coming from the BATESL course.

\subsubsection{2} Differences in Response of the 1994 and 1997 Graduating Groups

This section describes the items on which the survey groups which graduated in 
1994 and 1997 (i.e., 94(3) v 97(3)) differed significantly whilst the other two comparisons did not. Such differences can illustrate how the two cohorts, who had both completed the BATESL course, differed in composition, as well as indicating possible changes in the Hong Kong teaching culture in the run-up to 1997.

\section{Teaching Approach}

Firstly, there was evidence that the 1997 graduates were more examinationoriented than the 1994 graduates in that there was stronger agreement from the 1997 graduates that examinations should determine what is taught in English lessons (Table 4.16). This fact reinforces the profile of this group gained from other parts of the questionnaire that they were more conservative than the 1994 graduating group. Comments from the 1994 graduates show that whilst they accepted that examinations are important, they rather wished that they were not:

(1) I hope it's not the case but IT IS actually. Exam present a strong motive for students to learn a language.

(1) Backwash of exam not always positive. Very often it shadows/conceals the real needs of students.

(1) In reality, teachers should teach students all the things and knowledge that the students are required to learn for examination.

Comments from the 1997 graduates indicate a stronger sense of acceptance of examinations:

(2b) Because Ss must sit for public exams!

(2b) Ss are exam-oriented.

(2b) I do not want to agree so, but the reality is like this!

(2b) Some extra or common knowledge can also be one examination requirement.

(2b) For students who will sit for public examinations. 
(2b) I disagree with this statement. Unfortunately, this is a fact that we cannot resist such situation in Hong Kong.

In addition, in 1997, Subject $\mathrm{P}(97)$ spoke of the importance placed on examinations:

(2b) It may not be a good thing because if you really want to learn a language, I think your interest and the motivation is the most important thing. But for Hong Kong, the students have to sit for the public examination and the principal in schools will force the English teacher to follow the syllabus and how to train the students to be a good test-taker.

These comments are perhaps a further indication of an increased focus on examinations and qualifications within education and society at that time (1997). Further, the 1997 graduates felt more strongly that students learn English better through interaction with other students than through direct instruction from the teacher (Table 4.17), which may indicate a possible change in teaching culture in which group work, including discussion, is more of a focus of the official curriculum and the examination systems as a result of the introduction of group discussion and role play components into the public English examinations in 1995. In this sense, the influence of government and the provider of examinations, the Hong Kong Examinations Authority, increased.

\section{Professional Relationships and Responsibilities}

The student teachers graduating in 1997 were more convinced that they would make teaching their long-term career than those who graduated in 1994 (Table 4.26), indicating their greater commitment to teaching than the earlier group. A member of the first cohort, $\mathrm{D}(94)$ responded that he would 'definitely not' make teaching his long-term career and gave this comment:

(1) I think because now teaching do not have so-called development. It is a quite 
stable job, if you don't do anything, nobody can ask you to do or force you to do this, unless you are highly motivated enough to keep on searching for brighter prospect, to keep on going for master degree or such kind of things, nobody will force you to do. That means in such a circumstances I don't think I will carry on too long.

Subject E(94) also said 'definitely not' for the following reasons:

(1) Because I'm not that kind of stable person, I'm not the kind of person who will stick with one profession, unless you really like it. Of course there are girls, there are even guys that like teaching, they like it a lot, take it as a lifetime career, but not for me. If I teach probably first because there is no way out, second because I am good in it.

Although the 1997 graduates seemed more committed to making teaching their career than the 1994 graduates, ${ }^{16}$ there were still concerns expressed by members of the 1997 group about becoming teachers. Subject Q(97) felt the relationships he might enjoy with students in the future would affect his decision to teach:

(2b) Of course I can enjoy to teach my students if they can give me some positive rewards, not in money but in a sense of achievement or in a sense of friendship between us, that would be quite good. However, I cannot foresee that we can have such a good relationship in future, or at least it is not very easy for us to find it in local schools. So I don't want to be disappointed so I'm quite unsure this moment.

Another factor which might have affected the 1997 graduates was the possible difficulty that they might have anticipated in acquiring a teaching post at that time (a factor which is further discussed in Chapter 5). With the onset of the economic recession in Asia and the imminent changeover in sovereignty creating uncertainty in society, it was anticipated that teaching posts, seen as being stable and secure, would be at a premium and new graduates would find it more difficult 
to break into the profession. There were also concerns that the stability of the profession, traditionally one of its major attractions (when compared to business for example), would be affected by the uncertain times ahead.

\section{Perceptions and Values}

The 1997 graduates felt that motivation of students was a greater influence on the teaching of English than did the 1994 graduates (Table 4.34). The studentcentered orientation of the respondents of all groups has been discussed and this would seem to be a factor in this finding. However, this result could also be a reflection of the concern felt by the teachers in 1997 about student behaviour and motivation for learning English, particularly after the changeover in sovereignty. Evidence to emerge from the interview surveys reported in Chapter 3 suggests a trend of increasing sympathy for or solidarity with students by teachers as the handover approached and then beyond it. Possible reasons for this are the feeling that the uncertainty caused by the many educational and political reforms as well as the economic downturn may have caused teachers to feel that both they and the students were having to face the same uncertainties, and as adults the teachers should do all they can to help the students cope with these changes and uncertainties. There was also the fact that changing societal conditions would affect students' motivation for learning, through influence from parents, peers and the popular media, all of which showed signs of increasing in degree.

The 1997 Year 3 group also felt that school policy was more influential than did the 1994 graduating group - perhaps an indication of more autonomy of schools within the education system or a greater degree of politicisation of education compared to 3 years earlier. In addition, the 1997 group being relatively more conservative, may also have been more accepting of school policy.

Finally, the 1997 graduates were more oriented towards the teacher as a transmitter of knowledge than were the 1994 graduates (Table 4.36). In this case, 
the group graduating in 1997 seemed to be clearer about their role as a teacher being both a transmitter and a facilitator. This could be an indication of their greater commitment to teaching. Alternatively, it could indicate an essentially conservative acceptance of the view of the teacher as transmitter. The ESL field went through a period beginning in the 1960s and 1970s in which it was stressed that the teacher should facilitate rather than transmit knowledge, but by the 1990s this orientation had reversed such that the teacher as transmitter was becoming more acceptable, in part as a backlash against communicative language teaching and other 'Western' innovations (see for example Anderson, 1993; Evans, 1997; $\mathrm{Li}, 2001$ ) and in part based on positive findings about an explicit focus on form (see for example Celce-Murcia et al., 1997; Long, 1991; Williams, 1995).

\subsubsection{3} Differences in Response of the 1994 Beginning and Graduating

\section{Groups}

The cases where there were only significant differences between the two groups studied in 1994 show differences primarily between new teacher trainees and those about to enter the profession, with possible differences also revealed between the two cohorts.

\section{Language Use}

Firstly, there was a greater admitted use of code switching by the first cohort when graduating in 1994 than by the second cohort as entrants in the same year (Table 4.4). This suggests a likely effect of the BATESL course as learning through the English medium, including being required to discuss and interact at length in English with both native speakers of English and highly proficient Hong Kong Chinese English lecturers, will have not only raised their confidence in using the language but also led to them becoming familiar and comfortable with this kind of dual-code language use, which has been found to be common amongst 
university undergraduates in Hong Kong (Bacon-Shone \& Bolton, 1998; Pennington \& Balla, 1998).

\section{Teaching Approach}

Of the factors having the most effect on the trainee teachers' teaching, the two groups surveyed in 1994 differed on the importance placed on 'Class size', the 'English proficiency of students' and 'Current trends in ESL teaching' (Table 4.22). Having experienced teaching, the 1994 graduates would have had a better idea of how class size can influence teaching. Although the government has put the teacher to student ratio at approximately 19.5:1 (Hong Kong Government Information Service, 1998), classes of over 40 are the norm in secondary schools.

The 1994 BATESL entrants felt less strongly than the 1994 graduates that the English proficiency of students was an influential factor on the teaching of English. The course entrants may have been more tolerant of students with a lower proficiency. Alternatively, the graduating group had experience of students' standards of English in schools from the teacher's perspective and were perhaps in a better position to comment on its effect on their teaching. Furthermore, current trends in TESL was considered more important by the Year 1 group than by the Year 3 group, showing a tendency for more idealistic behaviour or possibly less cynicism by the beginning group. An alternative view is that Cohort 2 might have been, on average, more oriented towards professional development in respect of keeping up with developments in the field of English teaching than Cohort 1.

\section{Professional Relationships and Responsibilities}

The 1994 beginning group felt that the status of teachers was higher than did the 1994 graduating group (Table 4.24). The course entrants perhaps still held teachers in high regard, as they were at the time straight out of school. Contrast 
this with the graduating group, who, having been into schools during practice teaching and internship, regarded themselves as teachers and were more cynical about their status, associating with the perceived plight of teachers. Subject $C(94)$ commented:

(1) Sometimes I feel that the teachers are not very much respected in society in a way that they should be respected. People just know that teachers go to school every day and teach, they don't know much about the burden, the responsibility, the work-load of the teachers so much. Maybe they only know, 'Oh, being a teacher you have a lot of holidays'. They don't see being a teacher as an important and great job influencing their children.

The most obvious and expected reason to explain the contrast between the two groups surveyed in 1994 was the greater experience and acquired knowledge of teaching of the Year 3 group. This was exemplified by the higher expectation of having to attend courses in the Year 3 group (Table 4.28). This perhaps indicates that the more experienced and, because of this, more pragmatic student teachers felt that they would not have the time to attend courses, at least not in their first year. In 1994, with the changeover in sovereignty still three years into the future, there was no evidence to suggest that the prospects of it caused there to be differences in opinion or practice of these two groups. The next set of comparisons may indicate how the impending handover did have an effect as the 1997 graduates are compared with the two groups surveyed in 1994.

\subsubsection{Differences in Response of the 1997 Group with both 1994 Groups}

Differences in the response of the 1997 Year 3 group as contrasted with the two groups surveyed in 1994 can be taken to indicate changes due to factors occurring during the three-year period in the educational culture or the BATESL course. 


\section{Language Use}

The second cohort as graduates were less inclined towards English being the only language in English lessons as compared to both the same cohort as entrants and to the first cohort (Table 4.5). This result may indicate a change in the emphasis in English language education in Hong Kong away from English only in English lessons and more acceptance of the use of the L1 to facilitate learning with students of low English proficiency and motivation. There is an indication here of a trend within society (as the handover approached) towards increasing Chinese ethnocentrism in language terms such that the L1, Cantonese, was emerging from the shadow of English in educational terms (as well as in others such as government and business). In addition, the expansion of education, particularly the provision of more opportunities for students to continue into tertiary or further education, meant that more students with lower levels of English were staying in education and needed the support and facilitation of the mother tongue. Comments on this issue by members of the two groups surveyed in 1994 indicated limited acceptance of the use of Cantonese:

(1) If teachers can use English only in English lessons, that's perfect. But this doesn't appear in most of the English lessons in Hong Kong.

(1) Since it gives Ss more exposure to English. However, if necessary, some Chinese can be used, but not much.

(1) It depends on the level of the students. Full English is good to boost the students' research but it could be demotivating if the students can't manage.

(1) Student's 1st language may be used for clarification in some cases.

(2a) It will improve their speakers English.

(2a) For difficult ideas, teachers or students can use simple English to explain.

(2a) It is reasonable but it's not suitable for those junior students.

(2a) Give more chance for student to use English in their communication.

(2a) In this way, we can train ourselves to listen, speak and think in English. 
(2a) In some situations, if we don't know the special terms in English, we can express them in Chinese.

However, there were signs of greater acceptance of Chinese in 1997:

(2b) LI may be useful to deliver instructions.

(2b) If some words are very difficult to students, Cantonese can be the fastest way to explain and therefore teachers can save time for lesson.

(2b) Some students find difficult to understand.

(2b) When teacher explains grammar rules, s/he should use some Cantonese for the sake of comprehension.

This increased acceptance was summed up by Subject $N(97)$ :

(2b) I think if the students don't understand you in English, there's no point for you to still talk to them in English because education is to let them understand something.

The greater acceptance of Chinese by the 1997 graduates is consistent with the findings of studies of the changes in language use in Hong Kong during the 1990s as discussed in Chapter 2, that more Cantonese was being used in Englishmedium schools (Boyle, 1997) and in English teaching (e.g., Johnson, 1998; Pennington, 1995c; Pennington \& Richards, 1997; So, 1992). Furthermore, it was found that the status of Chinese was rising (e.g., Flowerdew \& Scollon, 1997; Pennington \& Yue, 1994) and as a result, English teachers were less likely to consider it wrong to use Cantonese when required to make teaching easier and more efficient. The coming of the change in the medium of instruction to mainly Chinese was possibly having a backwash effect in that prior to the change, teachers were using more Chinese and were more accepting of it. As was found in Chapter 3, the change in MOI was supported by teachers for educational reasons. The conflict as regards English teaching was expressed by the student teachers as being between providing students with maximum exposure to the English 
language, while at the same time ensuring that they understood what they were learning and what they were supposed to be doing in lessons. The evidence from these studies is that the bilingual Cantonese-English mode of teaching was set to continue with the relative proportions of Cantonese and English changing in favour of Cantonese. Whilst earlier discussion suggested a desire amongst English teachers to maintain English-only use in English lessons, the reality seemed to be of greater acceptance that Cantonese needs to be used. The second cohort, being typical of novice teachers in Hong Kong, reflected this reality.

\section{Perceptions and Values}

The second cohort as graduates in 1997 reported that they had more definite philosophies of teaching than did the other two survey groups, emphasising the higher level of commitment to teaching of the later cohort after having completed the course. Being more definite about their approach to teaching may also be a sign that the 1997 graduates had a greater sense of professionalism in that they had thought more deeply about teaching and what part they could play in it. Alternatively, improvements in the BATESL course over the three-year period (since its inception) could have resulted in the graduates in 1997 feeling more confident or better qualified to teach than the 1994 graduates had felt as the first cohort to take the course. The philosophy of Subject $O(97)$ focused on students' needs:

(2b) Increase the chance of language use among Ss.

Conscious about the Ss' attitudes to learning English.

Give Ss a reason to learn English.

Care about Ss including their personal life and if they are willing to share with me.

He commented: 
(2b) First of all they can equip themselves with these resources, I mean linguistic resources, speaking English, and they can obtain a better job. That's the first concern. Secondly, if they can read English they can explore themselves with different cultures, different kinds of knowledge by reading English magazines or different kinds of books in English. They can learn British or American or Australian culture by reading their literature. For example, when they are walking on the streets they encounter a foreigner, they are asking how to get to somewhere, they can respond to that. That's a kind of need, a reason to learn.

Subject $\mathrm{P}(97)$ 's philosophy showed her concern for teaching issues:

(2b) Teaching is not something that can be taught. I think you have to really teach a class in order to learn what is teaching and teaching can only be improved through teaching.

She commented on this philosophy as follows:

(2b) Even if somebody tells you, you can teach like this, you can teach like that, you may still fail, because your students are different from other students and you can't use others' philosophy and you can't use others' idea to teach your own students, and you have to for example, based on the proficiency of the students, based on the environment of the school or the policy made by the panel chair or the principal. All these things will affect your teaching. Even if you attend another course in teaching, you will make no change. You may still stay on the same situation.

The teaching philosophies of the 1997 graduates illustrated their orientation to teaching and teachers' issues. It may be speculated also that the changes in society occurring at that time served to focus the graduates' attention and make them more pragmatic as a reaction to the uncertainties that lay ahead. To cope with the anticipated changes, they would need to be clear about their personal and 
professional orientations and goals. (The teaching philosophies of those respondents who gave them in the three administrations of the questionnaire are summarised in a later section of this chapter.)

The issue of class size occurred again in terms of the factors affecting what happens in the classroom (Table 4.34). The 1997 graduates placed significantly more importance on this factor that the two groups surveyed in 1994, possibly indicating the increased attention given to this (and other) constraining factors in teaching. There was certainly attention paid to it in the press in 1997 (see Chapter 3). Subject $P(97)$ stressed the importance of class size:

(2b) With a very large class you can't always use activities because you can't manage and you can't check every student at a time.

Subject Q(97) agreed that class size was important but felt that if the students were motivated then successful learning could take place:

(2b) Of course class size may help us to reduce the work load but if the students have a strong motivation we can still manage to teach them in a good way.

The comments from $Q(97)$ and the other members of the second cohort characterise this group as more conservative and more realistic or pragmatic than the earlier cohort, who were comparatively idealistic. In an earlier study comparing the responses to the questionnaire of the 1994 graduates with the 1994 entrants, it was found that the graduating group expressed greater pragmatism towards education than did the entry group (Pennington \& Urmston, 1998). It is interesting to observe that three years after this comparison, the 1994 entrants, now having completed the course themselves, show these characteristics even more strongly than the first cohort did in 1994. While the effects of the BATESL course and the teaching experience that they gained whilst doing the course can account for this increased practicality, there are differences evident in the two cohorts. In 1994, it was found that the then graduates were to a certain extent 
cynical about their course and their future as teachers. This cynicism was not evident in the 1997 graduates, who were more positive and committed to teaching than the earlier group. Whereas the first cohort seemed to be negatively affected by the uncertainty ahead of them due to the handover, the second cohort seemed to be more determined to face the uncertainties in a positive and pragmatic way. By 1997, the future may have been a little easier to predict than three years previously and the uncertainties surrounding the handover might have decreased somewhat when compared with 1994. This may have induced less anxiety in the novice teachers about to enter the profession at that time, compared with their counterparts three years previously.

\subsubsection{Differences in Response of the Beginning Group from Both Graduating Groups}

Differences between the beginning group (94(1)) and both of the graduating groups (94(3) and 97(3)) are likely to reflect the results of increased knowledge and experience of teaching and/or of the specific effects of the BATESL course rather than changed circumstances in Hong Kong education. As would be expected, a number of factors emerged which highlighted the differences in experience between student teachers at the beginning of their training and those about to enter the profession as qualified teachers. ${ }^{17}$

\section{Language Use}

The respondents were asked to assess their own abilities in English at the times of completion of the questionnaire. In both written and spoken English, the 1994 entrants rated their proficiency lower than the two graduating groups (Tables 4.1 and 4.2). One would expect that three years of university instruction in English and through English would have improved the student teachers' English 
proficiency and hence their confidence in it. Subject $\mathrm{O}(97)$ commented on this point:

(2b) Yes sure, especially in speaking, because I have to interact with those foreigners [the course tutors], so now I have no fear in talking with you, like I am now using English. But in those days in secondary school, I am scared if I have to use English. I can't express fully what I want to say. So I can see that there's very much improvement, especially in spoken English.

Subject $\mathrm{P}(97)$, in contrast, felt that the BATESL course did not do enough to help them improve their English proficiency:

(2b) I think I haven't got enough training on the English proficiency and that's why I think I haven't got any improvement in written English ... because we just focus on how to teach English and how to teach the students and that's why I think we haven't got enough attention on that aspect.

There is evidence that Subject P's concerns were addressed and that measures have been introduced to improve the English proficiency of the BATESL students. ${ }^{18}$ In Hong Kong, more emphasis tends to be placed on spoken English ability than written, as it is through the spoken form that people interface with others. This has been especially true for teachers as being able to conduct lessons through the medium of English was the norm until the change in the medium of instruction in 1998. The issue of language competency of teachers, particularly of language teachers, is a major concern of education policy makers and has resulted in the Language Proficiency Assessment for Teachers (or Benchmarking) initiative mentioned previously.

Both Year 3 survey groups admitted to more code-mixing than the Year 1 group (Table 4.3). Code-mixing is very much a part of Hong Kong university students' language repertoire. They commonly intersperse their Cantonese with English terms related to university life, such as 'seminar', 'semester' or 'term paper', as 
well as subject-specific terms like (e.g., for students on a BATESL course) 'language acquisition' or 'communicative approach' (as e.g., Gibbons, 1987 reports for students in other fields at the University of Hong Kong). Subject $O(97)$ explained why he 'Quite often' had to code-mix:

(2b) If I want to say like, I have to give a presentation this afternoon, I will use 'presentation' still, because I can hardly find any equivalent in Cantonese which can suit the translation of the English words. That sense I can't find.

The 1997 graduates changed their attitude to the use of mixed-code English and Cantonese during the three years that they spent doing the BATESL course; like the graduates of the first cohort, they reported using English terms quite frequently when talking with friends. While the government (through the MOI policy) were endeavouring to reduce code-mixing in teaching, in private life it seemed set to continue to be used, particularly by university graduates. The approach of the handover may have had the effect of increasing Chinese ethnolinguistic vitality (Pierson, 1998) as discussed in Chapter 2, but the influence of English was still strong, through education, media and popular culture. It remained to be seen whether the increased use of Chinese as a medium of instruction would result in a lessening of the 'problem' described by $O(97)$, in which English terms were more familiar than equivalent Chinese terms. It might be expected that this would be the case as Chinese use increased.

The graduating student teachers were more prepared to use Cantonese in English lessons than the first-year student teachers were, but only to establish discipline (Table 4.9). This is most likely as a result of experience gained in practice teaching, resulting in greater pragmatism. A number of members of the first cohort commented on this:

(1) When the teacher wants to manage the class, he/she can use Cantonese, as it is much easier and direct. 
(1) Could use Cantonese to scold students or to repeat instructions for lower form students.

(1) Depends on the levels of the students. And also for classroom management, Cantonese may be better.

(1) I think it's better and effective in maintaining the classroom discipline.

(1) I think the mother tongue (other than Eng) can only be used in dealing with the discipline problem in class.

Of the second cohort, Subject $S(97)$ explained how she used Cantonese to deal with discipline problems during her practice teaching:

(2b) I always use Cantonese when I have to handle the discipline problems because I have to handle the discipline problems first, and if I still use English, they are out of control. So in that situation I have to use Cantonese or the students will say, 'I don't know what you are talking about!' Then I can't do anything.

The use of the first language for interacting with students, including for discipline, has been found to be a commonly used strategy by teachers generally in bilingual teaching situations (Guthrie \& Guthrie, 1987; Wong-Fillmore, 1980) and in Hong Kong (Johnson \& Lee, 1987; Pennington, 1995a, b) and the findings from the current surveys of trainee English teachers conform to that pattern. It might be expected that after three years on an English-medium course training to be an English teacher that the subjects would become more willing to use pure English in their teaching, and there was some evidence (reported earlier) that they would do in an ideal situation of highly motivated and proficient students. However, this is not the case in Hong Kong. There appears to be a strong culture of mixed language use in teaching and a strong acceptance of it (Johnson, 1998; Pennington, 1995c; Pennington \& Richards, 1997; Pennington et al., 1996; So, 1992; Tung, 1990), despite the previously mentioned efforts of the government to encourage monolingual teaching by pushing for clear distinctions between English and Chinese-medium schools. 


\section{Instructional Planning and Decision-Making}

The second cohort as entrants were more in favour of writing detailed lesson plans than the two survey groups of graduates (Table 4.11). The graduating student teachers had learned through experience that teachers do not have the time, or perhaps the desire, to write detailed lesson plans, a point raised earlier in the comparison of Cohort 2 as entrants and as graduates. In addition, the course may have influenced the graduating student teachers to be more flexible in their approach and they may have had more confidence to be able to go into a lesson without a detailed plan to guide them. Some members of Cohort 2 commented in 1994:

(2a) Let students know what they will learn beforehand, then student can have good preparation, too.

(2a) Student can prepare themselves before lesson with the help of detailed lesson plans.

(2a) Only by this, students can be well-prepared before the lesson.

(2a) Students can have the chance to prepare for some related materials.

(2a) Because it can use as a guideline for students.

(2a) Then teachers will have their progress to follow.

(2a) Easier to follow the course.

From their comments, it would appear that the 1994 entrants misconstrued the statement and understood it to mean that students would be given lesson plans before lessons to help them prepare.

In 1997, the student teachers better understood the nature of lesson planning; some typical comments were:

(2b) But, it's impossible in Hong Kong school context.

(2b) It is impossible owing to the heavy workload. Yet, a lesson framework or rough plan should be needed. 
(2b) A brief lesson plan is ok!

(2b) Teaching is a lively and vivid job, it should not be too rigid and some accidental things may happen.

(2b) A simple or short lesson plan can make a good lesson too!

(2b) It's too time-consuming to write DETAILED LESSON PLAN for every lesson, sometimes brief notes will do. Also, there might be some unexpected things come up which cannot be planned in advance.

(2b) Unexpected events may occur - only rough outline of lesson plan is ok.

(2b) It's just necessary for fresh $T s$.

(2b) You have no time. Be flexible!

These comments were expanded upon by Subject $\mathrm{P}(97)$ :

(2b) Yes, I think teachers have to plan the lesson in advance if they are not experienced, but as far as I know, many teachers won't plan for every lesson they teach. From my own experience, I've done a very detailed lesson plan because it's required by the department for that module. We have to plan the whole lesson, but when I just go out to teach I found it difficult to follow the lesson plan because students are not the kind of students that you have expected, you have to change the whole lesson plan sometimes.

Comments from members of Cohort 1 in 1994 echoed those of Cohort 2 in 1997:

(1) It is impossible to do so as teachers have already got lots of work to do. $A$ draft plan or rough note will do.

(1) A rough lesson plan that makes clear what the teacher should teach is needed but not a detailed one since it's time-consuming. The teacher won't have enough time to do so.

(1) Although it's a good idea, in practice, impossible! 
The responses of the three groups indicate the naivety of the beginning group and the greater pragmatism of the third-year subjects. In addition, whereas Cohort 1 seemed to focus more the difficulty of planning in detail due to time constraints, Cohort 2 as graduates were more concerned with the need for flexibility in planning rather than planning in too much detail prior to lessons. As was found by Richards (1998), when teachers become more experienced, they are better able to make decisions during lessons to deal with changing situations and to hold 'higher level' pedagogical lesson goals in mind, rather than be merely concerned with procedural matters such as getting the required exercises done. The trainee teachers may have been able to plan lessons during their practice teaching, as they would have had sufficient time to do so, and in fact would have been assessed by their course supervisors on their lesson plans. However, research has shown that novice teachers such as these have difficulty in carrying out lesson plans as they are not able to make the kinds of decisions necessary to cope with unexpected events in the classroom (Woods, 1989; Yost et al., 2000). From their comments, it would seem that the 1997 graduates understood this need for flexibility better than did the 1994 graduates, indicating that the later cohort had a better understanding of teaching and a more practical (and less negative) approach.

\section{Teaching Approach}

The graduating student teachers placed more importance on classroom size and less on teacher training as affecting their teaching of English than the beginning student teachers (Table 4.22). Experience in classrooms would have had an impact on their consideration of such factors as classroom size and class size. The course entrants also attributed significantly more importance to their teacher training than did both groups of graduates, which is understandable as they were just beginning their course and would have been more idealistic than the respondents in the graduating groups. The graduates may have seen the influence of their training as diminishing once they took up the role of full-time teacher in a school, which they regarded as distant from the confines of their course of training. It is perhaps 
surprising that student teachers who were about to graduate from a course they had been on for the previous three years should be so negative about the value of their course. There is no evidence from any of the other strands of the investigation that the BATESL course was unpopular or that the majority of the students on it did not enjoy the course, yet when asked to rank its potential influence on their teaching, they did not consider it as important as such factors as the proficiency and motivation of students, examination requirements and, as mentioned, class size. Such rejection of the value of training that is not practical or 'hands on' in nature has been commonly found in teacher trainees (Ethell \& Mc Meniman, 2000; Freeman, 1991) and the BATESL graduates conformed to that characterisation. The two graduating groups did not differ significantly in this regard possibly indicating that there had been no significant change in the nature of the BATESL course that would make the 1997 graduates appreciate its value any more than the earlier cohort did. Evidence of how much of an influence the BATESL course has on its graduates may emerge from the post-1997 studies described in Chapter 5.

\section{Professional Relationships and Responsibilities}

A number of items appeared to indicate the effects of the time that the graduating teachers spent in schools during their practice teaching and internship. In terms of their expectations of the duties or activities they would have to carry out as firstyear teachers, they became more aware of the need to be a form teacher, attend meetings with parents and superiors, mark papers and prepare and administer examinations (Table 4.28). In 1997, Subject Q(97) felt that being a form teacher would be very demanding for a first-year teacher:

(2b) I think be a form teacher is very demanding, especially for a fresh graduate, or for those novice in teaching language, because we have to do a lot with lesson plans, language problems, but rather than students' emotional feelings or something like that. 
Subject $\mathrm{T}(97)$ agreed:

(2b) Because a form teacher have to do many administration job of a class, such as the parents day. You meet forty parents and some of the parents are quite troublesome. If your class have many behaviour problem, you have many follow-up tasks.

Subject Q(97) anticipated having to spend a lot of time in meetings:

(2b) That's quite time-consuming. In the first few years of teaching career, I think we have to be familiar with the school environment so that we need to talk with other teachers and also with the principal and panel chair, and then since we haven't established our own teaching materials during the first few years so we need to spend a lot of time. After this first few years we just modify our teaching materials.

Both graduating groups were also more aware than the first-year student teachers of the time that would be taken up by marking of students' work (Table 4.29). Subject $R(97)$ had an interesting perspective on the question of time spent marking, feeling that it could be controlled, whereas dealing with students' problems would be more time-consuming:

(2b) I guess marking is just a question of habit, you can mark very fast, you can do it in an efficient way. But if you do some counselling, it really takes time and it depends on who you are talking to. Maybe some would take you more time, maybe some less, but I guess most probably this kind of job will take up the most time and you couldn't control. The marking, I suppose you could have some sort-of ways to control.

The pattern of a strong focus on the difficulties or constraints inherent in teaching in Hong Kong is continued in these comments by the graduating student teachers. 


\section{Perceptions and Values}

In terms of the factors affecting what goes on in the classroom, the 1994 and 1997 graduates felt that language ability of students was more influential on what happened in the classroom than did the 1994 entrants (Table 4.34). The new entrants to the course may have felt, more idealistically, that they would teach in the same way, regardless of the language ability of the students and partially rejected the notion of there being any kind of hierarchy of language learners. The Year 3 student teachers had perhaps come to realise that there is frequently a relationship between language ability and motivation to learn and also behaviour in the classroom. By focusing on the constraints of teaching, the Year 3 student teachers would tend to find it difficult to think about being innovative in their teaching and to teach effectively, rather than simply 'survive', in what might be termed the 'real world' of the Hong Kong classroom. Subject Q(97) felt that English proficiency of students was the factor which affected his teaching the most:

(2b) So English proficiency is very important for the students, because according to the ... level of students, we may adopt different levels. Of course, two-way communications between learner and teacher will be the best, however, if the student level are not able to cope with this kind of requirement, so we just change it to a teacher-dominated class.

Yet the graduating groups were more oriented towards being a facilitator of learning than were the first-year group (Table 4.36). While the graduating student teachers were concerned about the problems that they faced in teaching, they understood the need to help students to cope with these problems as best they could. The sympathy for or solidarity with the students that was shown in other parts of the surveys is again indicated here. Additionally, the graduates may have felt that they had the confidence to be able to take on this role after having done the course. 
The extra experience and training of the graduates was clear from their responses to the questionnaire items and from the comments that they made. Differences between the two cohorts will be indicated more clearly through the next comparison of differences in pairs.

\subsubsection{Differences in Response of Cohort 1 with Cohort 2 Both as Beginning and Graduating Groups}

Items on which the first cohort differed significantly from the second cohort as beginners (in 1994) and as graduates (in 1997) should indicate differences in the characteristics of the two cohorts.

\section{Professional Relationships and Responsibilities}

There is further evidence that the first cohort were less committed to teaching than the later cohort from the fact that they were less sure about working with other teachers (Table 4.30). Although as has been reported, all three of the groups said that relationships with colleagues were a major concern, which could be accounted for by the student teachers' inexperience and lack of confidence. The first cohort may have been more individualistic in terms of teaching and would be less comfortable working as part of a team than the later group. Alternatively, it may be a sign that they had not fully embraced the profession and felt that they would have difficulty in fitting in. Subject C(94) expressed this to some extent:

(1) It depends on different schools because I've gone to my secondary school where the English teachers meet once or twice a month and then they discuss about their students and also the methods they use. And they also take turns to look at each other's teaching. But in some other school they really seldom talk about their teaching, they just gossip about their students but not constructive. 
In contrast, Subject $\mathrm{R}(97)$ thought that she would need to get help from more experienced teachers:

(2b) Yes, especially as we are inexperienced. We need to ask very often. If we want to learn within a short time, we need to ask. We need to work closely with my neighbours.

A further possible explanation is that the 1994 graduates may have experienced a lack of 'collegiality' during their practice teaching, which would have affected their opinions on the value of working together with colleagues. There was no significant difference between the second cohort as beginners and as graduates, both responding that they would expect to work closely with other teachers. It is not clear whether any inference can be drawn as to possible changes in collegiality in schools over the period of the investigation.

\section{Perceptions and Values}

The cohort which graduated in 1994 felt less strongly that the Hong Kong Examinations Authority and the Hong Kong government influence what goes on in the classroom than did the second cohort both as entrants and as graduates. The later cohort may have felt this on graduation in 1997 due to the increased profile of education issues as a result of media coverage in the build-up to the changeover in sovereignty. However, that Cohort 2 as entrants in 1994 also regarded these organisations as more influential than did Cohort 1 in 1994 perhaps shows a wider appreciation of educational issues on the part of the later cohort and helps to confirm the proposition that the second cohort were more realistic or practical and the first cohort more idealistic in their orientations. The profile of these educational bodies and their effects on education increased during the period of the investigation, as has been found (Chapters 2 and 3) but whether this raised profile alone accounts for the opinions of the second cohort upon graduation, or whether they had a greater appreciation of the influence of educational authorities 
and issues is difficult to determine. However, in common with the interviewees of Chapter 3, there were frequent references to government, Examinations Authority and the Education Department, both before the handover and after it (see also Chapter 5). The Education Department generated similar differences in opinion amongst respondents; in fact all three survey groups differed in their views of its importance.

Differences in Response Amongst all Pairs of Survey Groups

As a final discussion of differences in responses, those areas in which there were significant differences of opinion between all three of the groups are presented.

\section{Perceptions and Values}

All three survey groups differed on only one issue, their assessment of the importance of the Hong Kong Education Department. The second cohort as graduates considered the Department to be more influential than did the same cohort as entrants. The first cohort as graduates rated its influence lowest of the three survey groups, providing further evidence of their greater idealism and lower practicality. The increasing profile of the Department during the 1990s may have caused this to a certain extent. In Subject P(97)'s opinion, it was policy laid down by school principals and the Education Department which counted most:

(2b) For many English teachers in Hong Kong, they are not English major students when they were university students, and they may come from other disciplines, and as the salary of a teacher is really good in Hong Kong they will join the education. But since they are not that keen on teaching they will not share their ideas with other teachers and they just do what they have to do, follow the instructions by the ED or the principal and just do what they have to do and then nothing more. 
Certainly the Education Department became much more high profile during the period leading up to the changeover in sovereignty and beyond as a result of the changes in education discussed in previous sections.

\subsubsection{Teaching Philosophies}

In this section, the self-penned philosophies of teaching of the respondents from the three questionnaire administrations are compared and contrasted in relation to the findings from the previous discussions. The philosophies are listed in Appendix B.

The respondents' philosophies are grouped into three main areas: those focused on students; those focused on teachers; and those focused on teaching. The focus on students showed a real desire on the part of the trainee teachers to be studentcentred and to help students in some way. In fact the word 'help' was used quite frequently ( 9 times by respondents in Cohort 1, 6 times by Cohort $2 \mathrm{a}$ and 6 times by Cohort $2 \mathrm{~b}$ ), echoing the earlier findings that students in Hong Kong are perceived as needing help and that there is a strong sense of the teacher as helper to students. It was the 1994 beginners who showed the greatest orientation towards helping students, with 69 per cent of their philosophies expressing this. This was slightly higher than the 61 per cent of the 1994 graduating group.

The main issue as regards teachers was that of the responsibility that a teacher has, especially to his or her students. Only 15 per cent of the philosophies of Cohort 2a reflect this notion, as contrasted with 25 per cent of the first Year 3 group and 30 per cent of the second Year 3 group. Being three years older and more experienced, and casting themselves in the role of teacher, the graduates were aware that teaching is a career in which one has to deal with children and with it comes a considerable amount of responsibility. The picture emerging from these philosophies is that the respondents felt that a teacher has a responsibility towards the students under their tutelage, rather than a responsibility towards society to produce educated and socially-aware young people. Of those philosophies indicating the responsibility 
of teachers, only one from Cohort 1 and one from Cohort 2 a made any reference to having a responsibility to society. The others were focused clearly on the responsibility to students, which overlaps with those philosophies that were definitely focused on students' needs. Another issue expressed regarding teachers was that of the teacher's competency in English. Two members of Cohort $2 b$ expressed concern about this. Earlier findings had suggested that this survey group were concerned about their English proficiency and that the course planners were in the process of implementing measures to address this.

Both Year 3 survey groups and the 1997 group in particular focused more on teaching and the need to be well prepared and to plan carefully so as to optimise student learning. It would seem that Cohort 2 were more professional in their orientation, again indicating a more committed orientation to teaching of this cohort at graduation than the earlier cohort. By 1997, the group graduating that year seemed to have shifted their focus away from issues concerned with students (which accounted for 39 per cent of all their philosophies in 1997) and more towards professional issues, like teachers' responsibilities (30 per cent) and teaching issues (17 per cent). The student teachers of this cohort may have been representative of a 'new type' of Hong Kong English teacher, more practical and aware of the teaching norms and having a determination to adapt to these norms in order to pursue a career in teaching so that they could help the students. On the other hand, there is the possibility that the second cohort represented better the profile of the Hong Kong English teacher and the first cohort were not representative, being the first entrants to what was then a new course. The impression given is that the people entering the BATESL course in 1994 were more focused on teaching than perhaps had been the case when the course was new and was seen as more of a means of acquiring a bachelor's degree in English as a stepping stone to potential careers in other fields requiring English language skills. The course had evolved to the extent that its graduates were more committed to becoming teachers and with the uncertainty surrounding the handover and the concurrent unfavourable economic climate, the need to obtain qualifications in order to target a career path became more pronounced. The changes in education, particularly those that affected English 
teachers like the change in the MOI and the LPAT, only served to focus trainee teachers on more pragmatic concerns such as getting a job, managing a class and relating to more experienced colleagues. In times of uncertainty, concerns naturally turn towards the practical and to equipping oneself with the best means to cope with whatever changes should occur. The ways in which the handover might change English teaching were expressed by the 1997 graduates and are discussed in the next section.

\subsubsection{The 1997 BATESL Graduates' Views on the Potential Effects of the Changeover in Sovereignty on the Teaching of English in Hong Kong}

The final section of the findings from the questionnaire and interview surveys summarises the views of the second cohort on the changeover in sovereignty of Hong Kong. In the questionnaire that they completed in June 1997, they were asked to comment on how they thought the changeover would affect English language teaching in Hong Kong under the five categories of the questionnaire, beginning with language use.

\section{Language Use}

The respondents felt that the changeover would affect the use of English in schools 'To some extent' (mean of 2.03, s.d. 0.79 ) on 4-point scale where $1=$ 'To a large extent' and 4 = 'To no extent') (Table 4.38). A representative selection of their comments on this issue is presented below:

(2b) The use of English in secondary schools will reduce to a significant level. For instance, the strong guidance for MOI may lead to a consequential loss of students' exposure towards English vocabulary!

(2b) Less and less English will be used in the lesson (esp. those beside English lang. lesson). It's less important. 
(2b) Mother tongue education will be more common which leads to less use in English. Putonghua will be widely spoken by teachers and students.

(2b) For those schools which have been streamed into the Chinese medium, of course they (Ts) will have to use Cantonese to teach the content areas. For English lessons, I think both 'English as a Medium of Instruction' and 'Chinese as a MOI' schools can still use English to teach English.

(2b) Students will depend more on teachers' Cantonese translation.

(2b) The use of English will be decreased in secondary schools after the changeover as the medium of instruction of the content subjects will be taught in Chinese or Cantonese. The use of Chinese will be emphasised and the language focus may move to Chinese.

(2b) Mandarin or mother-tongue education is more influential. More educators agree to change the mode of medium of instruction from English to Cantonese or Mandarin.

(2b) The importance of English is 'comparatively' lower due to the rising importance of Chinese and Putonghua.

(2b) I do not think that the changeover in sovereignty will result in changes in the use of English in secondary schools. However, I only concern that the changes will be led by the change of policy of medium of instruction.

It is clear that the majority of the respondents felt that the status or importance of English would decrease after the changeover at the expense of Chinese, both Cantonese and Putonghua. Many also mentioned the change in the medium of instruction for most schools from English to Chinese. Subject Q(97) made two points:

(2b) I think this question is two-fold. First of all, after the handover of Hong Kong, we must regard that some students will think that, oh, we are Chinese right now, so they have no need to think about the usage of English in their daily life, they have no need to use English. ... And the second influence is that the ED proclaims that all schools that do not achieve a certain level, that means the students' level is not so high enough 
to learn in English, they should use Chinese as the MOI in the classroom lesson.

Subject $U(97)$ also made the point that this change in the status or prestige of English would affect students' motivation:

(2b) I mean, when we were the British colony, when we use English, or when we have the ability to speak fluent English, the others will think you are well educated. Although that may be one of the motivation for students to learn, if they want to stick to a higher social class, later, after the changeover, to me I think it will not be emphasised as before. So students may think that Chinese or Putonghua is more important later, and they have lost their interest, the motivation, the initiation to learn English.

Subject N(97), however, felt that there would be little change:

(2b) Yes, very little, because the world status of English is still very solid and Hong Kong is an international city, so I think it didn't change much. Maybe a little bit, because we can see that the status of Chinese is a little bit uprising, it's growing become more and more important. The two languages are both official languages now, so maybe a little bit affected, but not much.

Prior to the handover, there was concern expressed by the 1997 BATESL graduates in the questionnaire and follow-up interview surveys that the status of English would fall as a result of the increased status of Chinese, which could mean Cantonese or Putonghua or both. In this regard perhaps, there was also the tendency to equate the change in the medium of instruction with the handover, as the move towards increasing amounts of Chinese in education corresponded with the impression that Chinese would rise in profile in society generally. It appeared to the respondents (and to others in education - see Chapter 3) that the change to CMI was a natural extension of the change in sovereignty. 


\section{Instructional Planning and Decision-Making}

The 1997 BATESL graduates felt that the changeover in sovereignty would not greatly affect their lesson planning and decision-making (mean of 3.10, s.d. 0.61 on 4-point scale where $1=$ 'To a large extent' and $4=$ 'To no extent') (Table 4.39). A few comments were added:

(2b) I may include more topics in my lessons so as to increase my students' exposure towards English.

(2b) Details not know yet.

(2b) Some meaning of difficult words may be explained in Cantonese.

(2b) If the English syllabus is not change, it will not affect my lesson planning.

Subject Q(97) felt that after the changeover, students would receive less exposure to English:

(2b) Yes, that's a compensation, because owing to the handover, the students' exposure to English may be limited in future, so I think I may need to include more topics.

While Subject S(97) thought that there would be more use of the first language, Cantonese:

(2b) I guess the school principal may allow us to use more Cantonese in our lessons ... especially for those Anglo-Chinese schools.

And Subject R(97) shared the non-committal view of many of the group:

(2b) I couldn't tell at that moment, I don't know. When you look at the ... Basic Law? There's not much guidelines about education. I don't know if there are any big changes after 1997, so I couldn't tell. 
Unlike for language use, there was little evidence of anticipated change in the areas of lesson planning or decision-making and any changes that did occur would be as a result of changes in language use or language policy which might impact on planning and decision-making.

\section{Teaching Approach}

The anticipated likelihood of change in the teaching approaches of these novice teachers was also relatively low (mean of 3.33, s.d. 0.79 on 4-point scale where $1=$ 'To a large extent' and $4=$ 'To no extent') (Table 4.40). Subject Q(97) felt that after the changeover, teachers would need to work harder as student motivation to learn English would fall:

(2b) In previous years the teacher may just adopt a passive role in teaching, they just think that my students they urge to learn it so I've no need to encourage you so you just learn it by yourself, we just provide some sorts of channels for you to learn English, that will be enough. Nowadays it's not the case, we need to encourage, we need to take an active role to encourage my students or our students to learn English.

Subject S(97) felt that English would remain important, and more English lessons would still be needed:

(2b) And I think English is still an important language in Hong Kong nowadays. Instead I think maybe more English lessons will be taught in schools. They will increase the number of English lessons ... because in some schools, there will be a clear cut between English-medium and Chinese-medium, so for those Chinese-medium schools, they may have to increase more English lessons to compensate ... they may want to increase the chances for the students to speak up in English. 
Subject Q(97)'s point seemed to be that student motivation to learn English was lower than it had been in the past and the indication was that it would become even lower, perhaps due to the handover or to other, unnamed factors. As a result of this reduced motivation, teachers would need to adopt a range of approaches to motivate students to learn English.

Subject T(97) agreed that English would remain important:

(2b) It's still the English lesson. It's important, as Hong Kong is still the international city. The government hasn't told us that there's no English lesson, the nature of English should be demoted. They haven't said that.

The main point as regards teaching approach seemed to be that there would still be a need to teach English, although the actual teaching method may need to change to account for the reduced attention paid by society to English learning and the possible reduced motivation of students to learn it.

\section{Professional Relationships and Responsibilities}

The respondents had some worries about their future as teachers after the changeover of sovereignty (mean of 2.83 , s.d. 0.97 on 4-point scale where $1=$ 'To a large extent' and $4=$ 'To no extent') (Table 4.41). Some of the comments were:

(2b) Difficult to find a job.

(2b) Difficult to adapt to the policy.

(2b) Hong Kong schools start having native speakers as English teachers.

(2b) The socio-political status of English may decrease as we are going to be a foreign language teacher.

(2b) Less English teachers will be employed. I am afraid of being unemployed.

(2b) Worrying about the language teaching policy in Hong Kong. 
A major concern seemed to be the prospect of fewer jobs for English teachers. Also mentioned was the possible lower status or prestige of English and changes in government policy that might impact upon English teaching. Subject T(97) mentioned the problem of new immigrants from China:

(2b) Yes, because most immigrants from Mainland China, they are unable to speak or write English, totally maybe illiterate in English. This creates some problems to the teachers.

The ambiguous nature of this comment is a point worth discussing. It was felt by some of the 1997 BATESL graduates, and by a number of the teachers interviewed for the surveys described in Chapter 3, that the changeover would result in increasing numbers of people, and hence students, entering Hong Kong from Mainland China. The impression was, as indicated by $\mathrm{T}(97)$ above, that such immigrants would be so lacking in basic English skills as to be described as 'illiterate'. There would seem to be little evidence to back up this assertion, and a common opinion voiced by educators in Chapter 3 is that standards of English proficiency of students in the Mainland have been rising rapidly in the past decade. That new immigrants should be seen as a 'problem' is perhaps symptomatic of the way that some Hong Kong people tend to look down on people from the Mainland and regard them as less educated and less sophisticated as a result of major differences in economic development and political ideology (Grant, 2001; Lau, 2000). It illustrates that one of the major worries of people generally in Hong Kong was that large numbers of new immigrants would be allowed to settle in Hong Kong, so creating pressure in the already stretched resources such as housing, employment and education.

A second point, which was also raised in Chapter 3, is that rather than see the influx of people with perceived language learning needs as an opportunity or a challenge, many Hong Kong English teachers took the negative view that the situation would create problems. Again, there is shown the tendency to focus on the constraints and stresses in teaching as teachers did not seem to have 
confidence in the educational authorities' ability to provide the educational infrastructure necessary to cope with the impending changes. Likewise, some did not seem to have sufficient confidence in themselves as being able to deal with these changes.

However, Subject Q(97) was confident about the future of English teachers:

(2b) Yes, because we need English so we need English teachers, and that's it, so I do not worry about my status because if I'm a qualified teacher, I can teach English. Of course I cannot compare with native speakers, or compare with those native speakers who teach English in Hong Kong. However, I have my advantages over them because I know Cantonese, I know the students' needs, so I do not worry about my status, or the threats by the ED's schemes about importing native speakers from other countries because we have our own advantages.

This view was shared by Subject N(97):

(2b) Because as far as I know the Chinese government become more and more concerned about the importance of English, so they need quite a lot of professionals to teach English in Mainland China, so I think the prospect's better.

This point of view is in contrast to the pessimistic view expressed by $T(97)$, as it saw the potential for more need for English teachers, but in Mainland China rather than in Hong Kong. The increased ties with the Mainland in the run up to the handover were set to increase once the transition of power occurred and the potential of Hong Kong teachers working over the border was a possibility that some teachers might have been considering. In essence, it showed that the Mainland was expected to become increasingly influential in Hong Kong affairs, both political and educational. 
The respondents felt that the changeover would have only limited effect on their professional relationships and responsibilities (mean of 2.57, s.d. 0.56 on 3-point scale of 1 = 'Yes', 2 = 'Somewhat' and 3 = 'No') (Table 4.42). Subject U(97) envisaged no change:

(2b) I still have to teach. I still have to teach English. I don't think the profession will be changed after the changeover of sovereignty.

Subject R(97) was non-committal:

(2b) I don't know. Maybe you ask me this question one year after the handover, two years after the handover, then I could have a better answer.

Again, the consensus of opinion was that the changeover would not have a great effect, thus echoing attitudes in the larger society and comments by some of the interviewees reported in Chapter 3. In other words, there was more evidence of a reluctance to attribute any changes that had occurred or might occur in the future to the handover itself. The desire for stability and fear of change inherent in Hong Kong people (see Chapter 2) could account for this reluctance. If one cannot predict what changes might occur, then it would seem logical that one would tend to take the position that no changes will occur, or that any changes that do occur are as a result of natural and predictable evolution. This seems a likely scenario for the novice teachers who did not have the benefit of experience or a sufficiently in-depth knowledge of educational issues to be able to comment with any confidence or authority. The respondents were perhaps unclear as to how the changeover in sovereignty might affect such specific areas of their teaching as planning, approaches and roles and responsibilities. The major area of possible change was in the relative status of English and Chinese, which as novice teachers they thought would affect them, but they were not clear as to exactly how they might be affected. 


\subsection{Conclusions}

In this final section, the major points of interest to arise from the surveys of the BATESL students will be reviewed with a view to identifying characteristics of the Hong Kong English teaching and English teacher education contexts during the period leading up to the changeover in sovereignty and to provide answers to research question (iv), i.e.:

What were the opinions of teachers-in-training due to enter the teaching profession in 1997 as regards teaching English in Hong Kong, and how had these opinions changed during the run-up to the changeover, since they began their teacher training course in 1994 ?

Firstly, areas of commonality will be discussed, followed by aspects of difference and change.

\subsubsection{Common characteristics of Trainee English Teachers in Hong Kong}

As mentioned previously, the culture of trainee teachers of English in Hong Kong revealed by these studies is one of empathy with students combined with a developing awareness of the realities of teaching. This combination of influences resulted in a series of conflicts within the trainee teachers between the approaches that they were learning and had learned from the BATESL course as well as their own intuitive beliefs about what constitutes good teaching, and the constraints present within the teaching context. The first of these conflicts concerned the use of the target language, English, in English teaching. The respondents believed in giving students maximum exposure to English but felt, and this was expressed most strongly by the Year 3 student teachers who had completed their practice teaching, that it is necessary to use the first language to facilitate teaching and to maintain student interest and discipline. Whilst teachers such as those surveyed seem prepared 
to use the first language, Cantonese, for lesson facilitation (giving instructions, explaining content, interacting with students) the Hong Kong government has taken measures to reduce the amount of mixed code being used in classrooms by forcing schools to become either English or Chinese-medium and to adhere to a policy of monolingual teaching (in English or in Chinese) wherever possible. The position of teachers appears to be that the choice of language within classrooms is determined largely by the language proficiency of the students and the need to cover a set syllabus of language items within a limited period of time.

A second conflict felt by the trainee teachers of this study can be expressed as being between the desire to be professional in practice, e.g., by devoting sufficient time to planning lessons, whilst being constrained from being so by the nature of the teaching situation. The consensus of opinion amongst the subjects was that inexperienced teachers need to plan their lessons with in-built flexibility to allow for unexpected situations, such as students being unresponsive or students making faster than anticipated progress. The conflict arises because teachers may not have the time to spend on lesson planning, particularly as so much of their time is taken up with the marking of assignments. It was felt that new teachers must struggle through their first few years until they become experienced enough to be able to rely on less planning.

Thirdly, the teachers felt conflict between the desire to be innovative and experimental in teaching whilst conforming to the syllabus and examinationdriven teaching approach prevalent in most schools. Again, the view was expressed by the trainees that once they became more experienced as teachers, they would feel less constrained by the syllabus or by having to teach examination techniques and they would feel more confident about challenging these constraints and developing their own approaches and styles. The examination culture within Hong Kong education came out strongly throughout the surveys and did not seem to diminish at all over the survey period, even in the face of perceived (by educationalists) alternative approaches to education in the form of studentcentered, target and task-based methods. 
Another conflict concerned the student teachers' perceptions of the relationships that they would have with colleagues once they began teaching. Although there was agreement that they would benefit most in terms of professional development from the help and guidance that they would be able to get from their more experienced colleagues, relationships with colleagues was frequently mentioned as a source of concern to them and one of their weakest attributes. It has been noted that Hong Kong teachers have in the past tended to be reluctant to share ideas or discuss classroom management techniques for fear of being seen as weak or as simply not having the time to meet due to their busy schedules (Pennington, 1995c; Young \& Lee, 1987). There could be said to be a sense of professional isolation amongst Hong Kong teachers in that they may develop relationships with colleagues on a personal level but the hierarchical nature of professional relationships often makes it difficult for those 'at the bottom', i.e., the new teachers, to feel that they can relate to more experienced colleagues on a professional level and consult them for help with their teaching. The approach of the changeover in sovereignty, and the attendant uncertainty associated with it and the educational changes being introduced at that time, may have exacerbated this situation by causing teachers to retreat within themselves. In reaction to the changes underway, established teachers may have felt the need to protect their positions, resulting in a retreat to a more conservative pattern (Pennington, 1997b), in which they reacted to the changes that they found threatening by retreating to familiar modes of working, including familiar relationships, making it more difficult for newcomers coming into the system at that time to establish themselves within the social group of colleagues. In addition, it was found that after the handover, as the economic recession began to take effect, novice teachers were finding it difficult to secure teaching positions as uncertainty over the future continued.

Lastly, the respondents consistently expressed the desire to be friendly with students, while at the same time recognising the need to be authoritative and avoid discipline problems. As the respondents were students themselves, many of them were able to feel solidarity with the school students that were expected to teach. Relationships 
with students was considered to be one of their major strengths and this studentoriented approach came out strongly in the philosophies of teaching that each respondent was invited to write. There was a strongly expressed desire to help students, as though they saw Hong Kong students as being vulnerable to some extent. In many cases, respondents seemed to feel that it was their duty as teachers to provide holistic help and support to students more in the form of a mentor or perhaps the traditional Confucian role of master and pupil. The conflict emerged when the student teachers considered the discipline problems they would be likely to face should they fail to be sufficiently authoritative to maintain control within the classroom and whether they could at the same time be sympathetic with and friendly to the students. It might have been the case that they would relate to teachers that they had had who had both maintained close relationships with their students and also been able to control classroom behaviour. Such are the skills of teaching that to a large extent come with experience. Whilst this may have been recognised by these trainee teachers, how they would be able to achieve such skills themselves was understandably a concern to them at this early stage in their careers. The uncertainty of the handover may have increased this concern, while at the same time they may have felt sympathetic to the students who were also having to adapt to the changes underway in education and in society.

\subsubsection{Evidence of Change in Cohort 2 Over Period of BATESL Course}

The cohort which began the course in 1994 and graduated from it in 1997 seemed to strengthen in their commitment to teaching over the period of the course. There was some evidence from the interview data that a number of them entered the course unsure as to whether they planned to go into teaching after they had finished the course. The whole experience seemed to have increased their motivation to become teachers. There was also evidence to suggest that they were less sure about their professional relationships and professional status at the end of the course than they had been at the beginning. Such issues may have been considered too distant for the respondents when they were at the beginning of the 
course but as they were about to consider a career in teaching such issues will have taken on a greater importance. In 1997, the novice teachers also had an increased awareness of the teaching situation in Hong Kong, which is not surprising given their greater experience of it as well as the raised profile of education issues in the media during this period.

\subsubsection{Evidence of Differences Between Cohort 1 and Cohort 2}

Cohort 2 appeared from the results of the surveys to have been more professional in their outlook and in their approach to teaching than the earlier cohort. They were more committed to teaching as a career and had a greater awareness of educational issues in society. (Although from Chapter 3, it is clear that educational issues were highly visible in 1997 as a result of the major changes being proposed and implemented.) Cohort 1, on the other hand, were more negative about the status of teachers and generally less positive about teaching. Cohort 1 , were also less inclined than the second cohort to cooperate with fellow teachers. It seems that by nature the 1997 graduating cohort held more positive views towards teaching. These findings seem to suggest that Cohort 2 was a more typical group of prospective teachers than the earlier cohort. As the BATESL course was new in 1991 when the first cohort began their course, many of them may have enrolled because it was the first of its kind in Hong Kong and it was a degree in English. Evidence from the interviews with the first cohort reported in this chapter, as well as with the course leader at the time (Chapter 5) corroborates this impression. More members of Cohort 2 intended to become teachers from the beginning of their studies. This would help explain their greater commitment to teaching and their greater appreciation of the practicalities of teaching. Yet another point to be considered is the raised profile of educational matters in society in the months before the handover in 1997 and following it though to 1999 and beyond. Teachers and student teachers and also the public in general became more aware of and more involved in educational matters, especially those pertaining to language teaching. Whilst there were arguments played out in public 
over the ideological issues associated with the medium of instruction, language rights, teaching standards and so on, there was also renewed focus on the practicalities of teaching, such as student motivation, classroom language use and class size. Consequently, the cohort that graduated in 1997 were reflecting an increased focus within the educational system on the practicalities of teaching in the midst of rapidly changing political, social and educational paradigms.

\subsubsection{Evidence of Changes in the BATESL Course During the Period 1994- 1997}

The 1997 graduates rated their competence in speaking higher than the 1994 graduates, which may have reflected an increased provision for improving the students' English language ability in the BATESL course, as reported by the course leader during the interview with him in 1999 (see Chapters 3 and 5). The results also suggest that there could have been an increased academic focus of the BATESL course which may have resulted in the greater professionalism of the second cohort, although there is no other evidence to support this change. Other than these two points, there did not appear to have been substantial change in the course itself.

\subsubsection{Evidence of Changes in the Teaching Culture in Hong Kong during period 1994-1997}

The results from the questionnaire and interview surveys suggest that a number of changes were taking place in teaching during the period leading up to the changeover in sovereignty. It seemed that the teaching culture, already regarded as being product-oriented or ends-driven was becoming even more examinationfocused. A possible effect of the uncertainty surrounding the changeover and the effects of the economic recession which began to affect the Territory in 1997 was that qualifications became even more important than they had been previously. 
There was also evidence of the examination syllabuses driving education with the introduction of group discussion to the two public English examinations resulting in more interactive learning with more group work and discussion.

There was evidence of an increasing politicisation of education as the government itself and its agencies, the Education Department and the Examinations Authority seemed to increase their already high profile throughout the period. The trainee teachers became aware of the important influence that these bodies have on teaching and the attention paid to them in the media during the period would have contributed to that raised awareness (Chapter 3). Other issues that seemed to gain in prominence were class size and the use of Cantonese in English lessons. The issue of class size (i.e., being on average larger than would be considered desirable) was thought to be more of an influence by the 1997 graduates than by the earlier cohort, which may have been an indication of the former's greater sense of the practicalities of teaching, or it may have become more important in relation to the prospect of there being increasing numbers of new immigrants coming from Mainland China after the handover. There is no evidence that class sizes increased in real terms during the period.

The second cohort also seemed to be more accepting than the first cohort of the use of Cantonese in English lessons, which may have been a reflection of the second cohort's greater pragmatism or could signify the situation in schools as they had experienced it during their practice teaching, i.e., there may have been more Cantonese used in 1997 than there had been in 1994. Johnson (1991) found Cantonese use to be increasing in teaching generally through the 1980 s, although less so in English teaching (Johnson, 1998). This resistance of English teachers to use the first language may have been slipping in anticipation of the change in the medium of instruction to mainly Chinese due to begin in 1998. There is evidence that suggests that after the change in MOI, Cantonese use in English lessons did become more acceptable (see Chapter 5). 
There also seemed to be more attention paid to teaching standards, and questions were raised about the competency and image of the classroom teacher. This had a significant effect on the status afforded to teachers by the public, students and teachers themselves. Challenges to teachers' competency were increasing, evidenced by the increased popularity of private tuition schools, which were seen by teachers as a threat to them in terms of their ability to produce examination results acceptable to parents and school governing bodies (Chapter 3). In addition, there was a feeling expressed by the teachers that students no longer respected their teachers as they had done in the past and seemed to expect the teacher to provide them with an education without the students having to do too much in return. In other words there appeared to be a changing power structure in that students were becoming more empowered, whilst teachers were losing ground. The stronger orientation of the 1997 BATESL graduates towards wanting to 'help' students and become their friends indicates a more subservient teacher role than the more authoritarian one that has generally been found to be the case in the past (Pennington \& Cheung, 1995; So, 1992).

The 1997 graduates' views on the possible effect of the changeover in sovereignty on English language education in Hong Kong showed them to be concerned mainly with the status of English and of English teachers. It was felt that there was a likelihood of an increase in the status of Chinese (both Cantonese and Putonghua) and that this might effect how much English was taught. Whilst they could not envisage the handover affecting teaching approaches or teachers' roles and responsibilities directly, it was felt that the question of the changing needs for language instruction would filter down and possibly affect teaching and teachers indirectly.

This chapter has continued the investigation into the English language teaching situation in Hong Kong in the period leading up to the handover by looking at it through the eyes of trainee teachers. Emerging from the studies described in this chapter and the insights gained from the previous chapters is a picture of a system undergoing change, whilst maintaining basic equilibrium and continuity. 
Inevitably the system goes on, as it must, but there is evidence of change occurring within the system and within the sub-system of teachers. The structure of the system on the eve of the changeover is becoming clearer and the model to be constructed of it will serve to show this structure in a simple form that can represent this complicated situation. To continue and complete the investigation, the next chapter will review the progress of some members of the first and second cohorts during the years following the changeover to determine whether the trends in education and teacher development evidenced in these surveys continued. Then the model of the system after the changeover can be constructed. 


\section{Notes}

${ }^{1}$ The group studied were the first cohort to graduate from the BATESL, which started in 1991.

${ }^{2}$ The cohort of students still consisted of 40 in 1997 but only 30 ( 75 per cent) took part in the questionnaire study at that time.

${ }^{3}$ With one exception - one member of this cohort was from Iran.

${ }^{4}$ Again with one exception - the Iranian's native language was Persian, but she was of native-speaker competence in English.

${ }^{5}$ All entrants to the course had to have reached a minimum of Grade D on the Advanced Level Use of English examination. Whilst all had this minimum standard, some were of higher proficiency than others.

${ }^{6}$ During the academic year 1994-95, the author worked as a tutor in the English Department at the City University on one of the courses taken by the then firstyear group. Over a period of about fourteen weeks, he was able to observe the students and got to know them informally.

${ }^{7}$ Five of those members of the 97(3) group that were interviewed after the questionnaire administration in 1997 were interviewed again one year later to ascertain any changes in their teaching approaches. The findings from these interviews as well as more detailed information about the respondents are presented in Chapter 5.

${ }^{8}$ For a full discussion of the five categories of the questionnaire, see Chapter 2 , section 2.5.2.

${ }^{9}$ These procedures were verified by Dr. Francis Yue, a statistician at City University of Hong Kong ( $\mathrm{PhD}$ Statistics, University of Sheffield) and Dr. Bart Yeung of the AMA Statistics Unit at the Hong Kong Polytechnic University.

${ }^{10}$ These are two conditions recommended in SPSS Base 10.0 Applications Guide (SPSS Inc., 1999) and in most reference books on statistical methods (e.g., Lehmen, 1995; Levin \& Fox, 1988).

${ }^{11}$ A statistically similar number of respondents in each group selected the latter option to signify that there were no situations in which they would or might use Cantonese during English lessons.

${ }^{12}$ These comments/points are identified by the following labels to avoid confusion:

(1) 94(3) group

(2a) $94(1)$ group

(2b) $97(3)$ group

13 Throughout this section and other sections dealing with language use, it was found that the respondents sometimes used the term 'Chinese' and sometimes 'Cantonese' to describe their use of the first language. In terms of English teaching, within these studies there was no reported use of either written Chinese or other spoken varieties of Chinese, therefore the term 'Chinese' should be taken to mean 'Cantonese'.

${ }^{14}$ The bivariate correlation coefficient, Spearman's rho gave a value of -0.31 for the comparison between the second cohort as BATESL graduates and as entrants, indicating that the pattern of response was opposite for the two groups. This in fact was the case, although the skill of 'Speaking' was ranked first in 1994 and second in 1997. The major change for the cohort was in the ranking of 
'Grammar', which changed from fifth in 1994 to first in 1997, although the change was not statistically significant.

${ }^{15}$ In schools in Hong Kong, the teachers of a certain subject are referred to as the panel and the teacher in charge of that subject is known as the panel chair.

${ }^{16}$ Personal conversation with the Course Leader of the BATESL in 1994 revealed that the cohort which graduated in 1994 had been relatively uncommitted to a career in teaching, but had enrolled on the course as it was one of the few BA courses in an English discipline available at that time.

${ }^{17}$ In fact, as mentioned previously, the graduates from the BATESL course were able to teach in Hong Kong schools, although strictly speaking they were required to take further, post-graduate training in education (at another institute) before they could be considered as fully qualified. This is a situation that has since been partially resolved at the City University of Hong Kong with the launch of their own Post-graduate Diploma course in English Language Education.

${ }^{18}$ Information on changes to BATESL course provided by former Acting Head of Department of English during second round of interviews with ELT practitioners in 1999 (see Chapter 3). 


\section{A POST-1997 PICTURE OF GRADUATES FROM THE BATESL COURSE AT THE CITY UNIVERSITY OF HONG KONG AS TEACHERS IN HONG KONG SCHOOLS}

\subsection{Background}

The previous chapter looked at the changes undergone during the period of their training by two cohorts of student teachers, who began the BATESL course at the City University of Hong Kong in 1991 and 1994 and graduated from it in the summers of 1994 and 1997 respectively. It also examined evidence of change in the BATESL course itself, either as a result of implemented development of the course by course planners, or natural evolution as a result of changes in the educational culture of Hong Kong. In the summer of 1997, the cohort of student teachers graduating at that time found themselves about to enter the workforce at a critical time in the Territory's history, as its sovereignty was being returned to China. For them, there were more immediate concerns than the changeover; they (those that had chosen to become teachers) were looking to enter the teaching profession in order to put into action what they had learned during the previous three years. The earlier cohort had by then been graduated from the course for three years and some of them had been teaching English in local schools during that time. This chapter looks at how a sample of these new teachers, separated by three years, progressed in the profession in the two-year period following the handover, i.e., from July 1997 to the summer of 1999. It is anticipated that it will be possible to determine from this examination whether there was any lasting influence of the BATESL course on its graduates. In addition, the nature of the approaches and practices of the teachers within the Hong Kong education system 
will highlight features of the system and the ways in which these features were changing during the period.

\subsection{Methodology}

The data for this chapter came from interviews and lesson observations as listed chronologically below:

Date

Data Collection

Summary of Process

Method

August 1998

Interview

Five of the 1997 BATESL

graduates were interviewed in 2 separate sessions, one year after graduating from the course.

May/June 1999

Lesson observation

Three of the 1997 BATESL graduates were observed (twice) in their teaching environments, two years after graduation.

June 1999

Lesson observation

Two of the 1994 BATESL graduates were observed in their teaching environments, five years after graduation and four years after being observed previously. 
September/

Interview

October 1999
Two members of staff responsible for the teaching and administration of the BATESL course were interviewed and asked for their reflections on the course and its graduates.

\subsubsection{Subjects and Procedures}

In August 1998, the eight members of the 1997 BATESL graduating group who had been interviewed as a follow-up to the questionnaire survey (Chapter 4) were approached to be interviewed again, one year after graduation, to ascertain their progress as teachers and their views on education and the BATESL course in retrospect. Five of them $-\mathrm{N}, \mathrm{O}, \mathrm{Q}, \mathrm{T}$ and $\mathrm{U}$ - agreed to be interviewed again. ${ }^{1}$ The remaining three were unavailable at that time. It was decided to interview them in small groups to try to stimulate informal and reflective discussion. Two interviews were carried out, the first with $Q(97)$ and $T(97)$ together and the second with $N(97), O(97)$ and $U(97)$ together. In the interviews, the graduates were presented with preliminary findings from the questionnaire study which they had taken part in the year before. They were then asked for their reactions to the findings and whether their views had changed since the time of the original survey. They were also asked for their reflections on the BATESL course, their practice teaching experience and their experience post-graduation. The interviews were transcribed and the transcriptions analysed qualitatively for key points by the constant comparative method, as for the interviews of Chapter 3 (see section 3.3.1.2). The transcriptions of the interviews can be found in Appendix C.

Approximately one year later, in May/June 1999, three of the 1997 graduates were observed whilst teaching in their schools. They were: $O(97)$ and $U(97)$ and another graduate from the same cohort who had not previously been interviewed and who shall be referred to as W(97). The other BATESL graduates interviewed 
in $1998(\mathrm{~N}(97), \mathrm{Q}(97)$ and $\mathrm{T}(97))$ were not teaching at that time for various reasons given in the brief profiles of the graduates in the Findings section. ${ }^{2}$ Each of the graduates was observed twice in his/her classroom. During the observations, a detailed record was taken of the teacher's teaching procedures, student behaviours, teacher-student interactions and teacher and student language use. Each observation was immediately followed by an individual interview to elicit the observed teachers' reflections on the lesson just taught and on their general teaching situation, and comments were added by the observer as to whether the teacher's reflections corresponded to or contradicted the observed behaviours or not. The lesson reports were then written up and can also be found in Appendix C.

During the same period, the five members of the 1994 BATESL graduating group who had taken part in the First Year Teacher Project in 1994-95 (Pennington, 1997b; Pennington \& Richards, 1997; Pennington, Richards, Urmston \& Lee, 1996) and who had been observed five times throughout that year by the present researcher (working as research assistant on the project) as well as by the coinvestigators of the project and another research assistant, were contacted to see if they would agree to be observed again for the present project. Two of the group did agree and they were visited in their schools and lesson observations carried out as for the 1997 graduates. These two teachers (designated as $\mathrm{G}(94)$ and $\mathrm{H}(94)$ ) were asked for their views on how their teaching had changed during the five-year period since they graduated from the course. Lesson reports for these graduates can also be found in Appendix C.

Finally, two of the participants in the second round of the interview survey conducted in 1999 (see Chapter 3), who were staff members of the English Department at the City University of Hong Kong and who were heavily involved in the teaching and administration of the BATESL course, also gave their views on the course and its graduates throughout the period of the research (1994-1999) as additional parts of their interviews. The transcriptions of these additional 
sections were analysed qualitatively for important points and are presented in Appendix C.

\subsection{Discussion of Findings}

The findings from the four distinct parts of the post-1997 study are documented in Appendix C. In the following sections, the findings are discussed.

\subsubsection{Interviews with 1997 BATESL Graduates One Year After Graduation}

In this section, the main points made by each of the five graduates in the interviews which took place in 1998 are summarised. The points were recorded as being made whilst the findings of the questionnaire studies carried out in 1994 and 1997 (as described in Chapter 4) were being discussed. The questionnaires were divided into the five categories of:

- Language use

- Instructional planning and decision-making

- Teaching approaches

- Professional relationships and responsibilities

- Teaching-related perceptions and values

This meant that on occasion, points were made in relation to issues other than those coming under the particular category being discussed. It was decided to retain the order of points raised so as to maintain a sense of the discourse of the interviews.

In addition, a sixth category was added to gather the graduates' general reflections on the BATESL course, education and the English language in Hong Kong, one year on from graduation: 
- Post-graduation reflections

At the time of the interviews, the graduates were in the following positions:

$\underline{N(97)}$ - Was working as a reporter on educational issues for one of Hong Kong's two English-language newspapers. Had not taught since graduation and was not planning to teach in the future.

$\underline{O(97)}$ - Had been employed as a research assistant in the English Department at the City University of Hong Kong since graduation. Planned to teach once his one-year contract was completed. Began working in a post-secondary institute in September 1998 and was observed by the researcher in May 1999.

Q(97) - Had taught as a supply (substitute) teacher in primary and secondary schools for two months after graduation. At the time of the interview was working as a junior manager in a government department. Had enrolled on a parttime masters degree programme to begin in September that year. Was unsure as to whether he would teach in the future.

$\underline{T(97)}$ - Was working in an educational company as a part-time marker as he had been unable to find a teaching job. Planned to teach in secondary school in the future if he could find a suitable job.

$\underline{U(97)}$ - Had been working in the hotel business since graduation. Would like to teach but had been unable to find a teaching position. Managed to secure a position for the following academic year and was observed by the researcher in May 1999. 


\section{Discussion of Findings}

As can be seen from the above descriptions, of the five graduates, one had taught in schools since graduation, three had worked within the field of education in some capacity and one had been working in another field. From this point of view, their responses to the questions asked can be regarded as coming from informed graduates of a BA programme in TESL.

In terms of language use, there were signs that those graduates who had not been in teaching had suffered a loss of exposure to English and so were concerned that their proficiency in the language had suffered. At the same time, their use of mixed code (Cantonese with English) showed signs of having increased as they conversed regularly with fellow university graduates or Chinese returnees from overseas, and this form of spoken discourse was said to be common amongst young adults in Hong Kong (Pennington \& Balla, 1998). As regards language use in the classroom, the graduates felt that it was being strongly guided by school policy, with the effects of the change to CMI being felt even at this early stage. In schools which were designated EMI, there was reported to be strict monitoring to ensure that only English was being used. In CMI schools, Cantonese was being used the majority of the time, even in English lessons. This meant that the only English that was used was for lesson material, such as reading aloud or quoting English, in a Cantonese utterance frame. The possible polarity in language use in English lessons that was discussed in Chapter 4 seemed to have partially occurred, but with English not being maintained as the main teaching language in English lessons in CMI schools. Schools, through their particular language policy, had become more influential. There was evidence that the graduates held differing views on code-switching. There was the view (expressed by two graduates) that they preferred not to code-switch themselves, but that it was acceptable in teaching if it aided instruction. On the other hand, two graduates reported codeswitching a great deal with friends and colleagues but were not so positive about using it with students, unless school policy dictated it. These mixed views on dual 
language use reflect the confusion within teaching as to what language should be used, what is acceptable and what is not acceptable, and what actually occurs.

Reflecting on their practice teaching during the BATESL course, there was agreement that the period was too short. However, most felt that it had been worthwhile and had given them the opportunity to try a few of the teaching practices that they had learned. There was some disagreement over whether the high degree of examination-orientation prevalent in Hong Kong education was attributable more to students or to teachers. The consensus was that society is achievement-oriented and this is reflected in schools, with teachers feeling that they had to 'teach to the test' and students only interested in learning in order to get higher grades. Both teachers and students were seen as being locked into a transmissive, ends-driven system of teaching and learning. The domination of examinations was felt in terms of teaching approach as the graduates had found that they could not adopt interactive or innovative approaches with higher form students who would expect examination practice, whilst on the other hand lower form students might not have the proficiency required to engage in communicative activities. Hence, the picture is of Hong Kong becoming an increasingly examination-oriented system in the post-handover period.

There was evidence of the remaining influence of the BATESL course on the graduates when describing their teaching style. $\mathrm{N}(97)$, showing some measure of confidence, felt that if she were a class teacher, she alone would determine her approach. U(97) agreed but felt that it was also important to take into account the attitude of students. Both of these graduates had not taught in schools since graduation, while Q(97), who had, still preferred a learner-centred approach but within the constraints of the teaching situation. $\mathrm{T}(97)$ held the view that approach was entirely dependent on the ability of students. Hence, whilst the influence of the BATESL course was strong and at least three of the graduates expressed confidence in their ability to put their training into action, they were also aware of the realities of the classroom and the need to modify the approaches they had learned. 
It was felt that the combined factors of the economic downturn, which began to affect Hong Kong in the latter part of 1997, as well as the government's efforts at raising standards through Benchmarking, would help to increase the status of teachers. $\mathrm{O}(97)$ felt that teaching would become a more desirable profession to enter as opportunities in business or other fields decreased as a result of the economic recession. This would result in more high-calibre people entering the teaching profession so raising its status. $\mathrm{N}(97)$ disagreed, feeling that many of the people going into teaching would only be doing so as a temporary measure until the economy picked up again and would not have the motivation and the commitment needed to succeed. In this way, the quality of teachers or the teaching profession would fall. The influence of the economy on educational matters appeared to be increasing after the handover. $N(97)$ also believed that as parents became more proactive and were no longer afraid to challenge the authority of teachers, then the status of the latter was likely to fall. The added factor of the increase in expatriate English teachers or NETs meant that local teachers of English were under some pressure to prove their own ability and emphasise their strengths, such as their better understanding of local students and their educational needs. The graduates stressed the importance of understanding the needs of students and forming good relationships with them, although it was clear that they were now able to differentiate between having a good teacher-student relationship and trying to become friends with them. U(97) in particular expressed the need to separate in-class and out-of-class patterns of behaviour such that relationships could be maintained in which each party knew what was expected of them in each situation. In such a case the teacher would have to adopt two different personas, one in-class and one out-of-class, which may not be realistic and may reflect $\mathrm{U}(97)$ 's relative lack of experience as a teacher.

There was very little mention of the changeover in sovereignty; the graduates did not consider that it had had any direct impact on education. Although the topic was raised in the context of whether the changeover had had any effect on language use or teaching approaches, the tendency of the graduates was to link the handover directly to the change in medium of instruction and associate the two, 
such that when they said 'after 1997' they were generally talking about the MOI policy, although this did not actually come into force until September 1998. It seemed that the assumption was that the handover had led to the increased emphasis on Chinese (including Putonghua), although none of the graduates were prepared to link the political change to the educational one directly. (The timing of the announcement of the change in MOI - just prior to the changeover - was seen by some as a gesture on behalf of the departing British colonial government in support of the Hong Kong Chinese (Pennington, 1998b)). Their impressions were that society and schools were on the one hand moving more towards Chinese while on the other were anxious to maintain English standards. The English that was being pursued was thought to be more functional or targeted English for specific purposes, rather than for more academic or aesthetic purposes (as noted also in Chapter 4). Linked to this was the acknowledgement of the importance of teaching grammar for English examination and workplace requirements (also found in Chapter 4) and support for the spoken communication skills (speaking and listening).

The graduates were positive about the BATESL course and most felt that they had learned valuable language and teaching skills, and that campus life had helped them increase their confidence in terms of personality and social relationships. Interestingly, there was greater evidence of the lasting influence of the BATESL in $\mathrm{N}(97)$, who had not taught since graduation but had been reporting on educational matters for the English language press, possibly because she had not been influenced by the real teaching context and had remained relatively idealistic in her views. These graduates believed that the modifications to their teaching philosophies had been caused by the constraints present in the schools in which they had taught during practice teaching or post-graduation. The tendency to blame the constraints in teaching for restricting teachers' innovativeness is a strong theme that has emerged from the studies of this investigation (Chapters 3 and 4). The inconsistencies in the responses of the graduates can perhaps be attributed to their differing experiences post-graduation. There was also the point made that the BATESL course needed better promotion as its graduates (including 
members of this group) were finding it hard to get jobs as school principals did not recognise the BATESL as a qualification to teach English (as discussed in Chapter 4). It is worth noting that four of the five graduates interviewed (10 per cent of the cohort) had not been able to find teaching jobs a year after graduation. The economic recession referred to earlier seemed to be affecting the teaching market to the extent that fresh graduates were having difficulty getting into the profession, with fewer teachers leaving their 'secure' positions to try careers in other fields and increasing numbers of teachers returning to the profession from other fields. In addition, amongst those Hong Kong people returning to the SAR after living overseas, were teachers eager to resume their careers. With the NET scheme in its early stages, local, inexperienced teachers such as the members of this group, were under some pressure in their pursuit of a career in English language teaching. This was a situation that had reportedly not been the case to the same degree for the graduates from the first cohort in $1994 .^{3}$

\subsubsection{Lesson Observations of 1997 BATESL Graduates Two Years After Graduation}

One year after the interviews with the 1997 graduates of the BATESL course, three of the group agreed to be observed in their teaching situations so as to determine whether or not they were putting into practice the ideas that they had espoused in the interviews and whether there had been any major changes in education in Hong Kong since then. Two of the graduates, $O(97)$ and U(97), were teaching in schools and agreed to be observed. A third, W(97), who had not taken part in the interviews, also agreed. Full reports on the observations can be found in Appendix C. In the main text here, each of the graduates is reported on in the form of a case study, consisting of the key features of the observations of the graduates, reports on what the graduates said about their teaching in the follow-up meetings with them after their lessons, and the observer's comments on the consistency between the graduates' observed behaviour and their self-reported practices. 
Firstly, background information regarding each graduate and their teaching situation is given.

\subsubsection{Observation Report on Graduate O(97)}

After graduation in 1997, O(97) worked in the English Department at the City University as a research assistant for one year. He had been working in his current school since September 1998. The school was privately run and offered courses mainly for students who wished to improve the HKCEE or A Level grades that they had gained in secondary school. They could also study vocational courses such as business, hotel management and design, as well as core A Level Use of English and Chinese Language and Culture (the language qualifications required for entry to higher education in Hong Kong).

Two separate lessons were observed on the same day in May 1999. The first was a Use of English group who were asked to brainstorm ideas for speaking practice on activities through which they planned to improve their English during the summer vacation. The second lesson involved the teacher, $\mathrm{O}(97)$, giving feedback to students on their written assignments.

\section{$\underline{\text { Language Use }}$}

Observed

$\mathrm{O}(97)$ used mainly English to present and explain lesson Practices content and to interact with the students. In the first lesson observed, he used mostly English for presenting lesson content and a mixture of Cantonese and English for explanation, clarification and interaction with students on a one-to-one and whole-class basis. As the lesson progressed, he used more and more Cantonese for all purposes, including presenting and explaining content. The students also used mainly English in 
their group discussions. After approximately three-quarters of the first lesson, there was a noticeable increase in the amount of Cantonese being used by $\mathrm{O}(97)$ for all purposes. In the second lesson, which was predominantly teacher-directed, mostly Cantonese was used by $\mathrm{O}(97)$ and the students, with occasional switches into English when beginning a new topic or item of instruction (e.g. how to write the 'Referees' section of a résumé).

Post-

$\mathrm{O}(97)$ reported that sometimes he conducted a whole lesson in

Observation Remarks

Observer's Comments English, sometimes all in Cantonese. He would introduce a new topic in English, as the students tended to pay attention to something new. For revision or review, he would use Cantonese. So long as the students had the chance to use English, both spoken and written, $\mathrm{O}(97)$ felt that the use of Cantonese was acceptable. $\mathrm{O}(97)$ would use Cantonese for giving instructions and for explaining vocabulary and grammatical structures. If the students were not on task then he would repeat the instructions in Cantonese, as they might not listen if he used English. As regards language use in the school, the principal encouraged teachers to use English but there was no formal policy. Most teachers of content subjects used Cantonese, whilst English teachers used a mixture of English and Cantonese.

$\mathrm{O}(97)$ 's pattern of language use in lessons generally corresponded to what he said he did. He exhibited a pattern of functional language use. However, although he said that he preferred to use either pure English or pure Cantonese in lessons, he was found to have used both languages together in code-mixing and code-switching patterns in the lessons observed, with the proportion of Cantonese increasing as the 
lessons progressed. He agreed that the presence of the observer may have encouraged him to use more English (at first) than he might normally do.

\section{Instructional Planning and Decision-making}

Observed In the first lesson, $\mathrm{O}(97)$ made use of the fact that it was the Practices end of the school year and the students would soon be breaking up for the summer vacation. To encourage them to work on their English, $O(97)$ had the students generate ideas for working on their English. This formed the basis of the lesson, with the students preparing and giving short presentations and then engaging in group discussion about their planned activities. The format of the student-student interaction was based on that of the speaking test of the external examination for which they were being prepared. $\mathrm{O}(97)$ used no prepared teaching materials. In the second lesson, $\mathrm{O}(97)$ made use of models of the writing that the students had done previously (application letters and résumés) to demonstrate key features of these types of documents to the students. These models appeared to have come from a textbook. The lesson did not appear to go as planned since there was a period of about ten minutes left at the end in which the students had nothing to do. The impression of the observer was that the lessons were loosely planned and that $O(97)$ made a number of decisions during both lessons, especially during the first lesson, to keep the lesson flowing.

Post- $\quad \mathrm{O}(97)$ said that he usually used his own materials but Observation sometimes consulted textbooks. The students' books were Remarks exam-practice books. He said that he did not have the time to 
prepare his own materials but planned to spend some part of the summer doing so. $\mathrm{O}(97)$ reported that the students were not in a mood for working as the summer vacation was approaching, so fewer of them were willing to present to the class, leaving time at the end that he hadn't planned for.

Observer's In the first lesson $\mathrm{O}(97)$ made use of the students' own ideas to Comments create the context for the lesson. In the second, he made use of published materials for demonstration. Neither lesson showed evidence of much pre-planning. In the first lesson, there was time left at the end because the speaking activity had not lasted as long as it should have done as those students who gave a presentation did so for less that the required time and many others did not speak at all.

\section{Teaching Approach}

Observed

Practices
In the first lesson, approximately 20 minutes (out of 1 hour and 40 minutes) consisted of student-student interaction, with the remainder of the time taken up with teacher-directed instruction, teacher-student interaction (teacher calling on students to answer questions) and individual student presentations to the class. The teacher used the students' own experiences as a stimulus to get them to engage in discussion. He encouraged the students to speak and used group activities as a way to foster communication between students, although the students seemed to use predominantly Cantonese to interact with each other. His use of the whiteboard to consolidate students' responses was effective. The second lesson consisted mainly of teacher-directed instruction with some student response to questions. $O(97)$ encouraged the students to 
generate ideas of their own and he consolidated these ideas by writing key points and terms on the whiteboard. The content of the lessons was mainly functional in Lesson 2.

Post$\mathrm{O}(97)$ reported using interactive activities, mainly group work, Observation for speaking and listening and to a certain extent, writing, but Remarks not for Use of English Paper C (reading comprehension and usage) or Paper E (practical skills). The content of instruction was described by $O(97)$ as examination-oriented since the students attended the school to improve their exam grades, or as he put it, 'To make them function in the examination.' According to $\mathrm{O}(97)$, the teachers in his school had the flexibility to teach what they wanted, so long as there were no complaints from the students.

Observer's $\quad \mathrm{O}(97)$ made use of interactive activities in the first lesson, Comments while the second (which was a follow-up to a previous lesson in which the students had done job interviews) was more directly functional. The students in the first observation seemed less motivated than those in the second.

\section{Professional Relationships and Responsibilities}

Observed $\quad \mathrm{O}(97)$ seemed to have good relationships with his students in Practices that they respected his authority and also seemed to like him as a teacher. They were attentive and responsive during the lessons observed.

Post- $\quad \mathrm{O}(97)$ described his relationships with his students as ' $\mathrm{OK}$ '. $\mathrm{He}$ Observation spent time with them outside of class and they would come to Remarks him to ask questions. His relationships with colleagues were 
described as 'Very good'. He reported sharing ideas with one colleague as they rarely had official meetings and tended to work by themselves. In terms of duties, $O(97)$ reported that he was an advisor for the students' union, had been the emcee of the students' fashion show and had helped to escort visitors from the UK to Macau.

Observer's $\quad O(97)$ appeared to be well-liked by students and colleagues. Comments His friendly, out-going nature made him popular with both and he had become integrated into the school. He mentioned that the teachers rarely had official meetings and so any cooperation with colleagues in terms of sharing teaching ideas or materials or information about students tended to be done on an informal basis.

\section{Perceptions and Values}

Observed During the first observed lesson, it was clear that $O(97)$ Practices became somewhat frustrated with the students as some of them were not working on the task that they had been given and were leaving the work to other members of their particular group. He expressed this frustration to the students. However, he did maintain an enthusiastic and positive manner throughout both lessons and this had a similarly positive effect on most of the students. He came across as genuinely concerned that the students should work to improve their English during the summer vacation.

Post- $\quad \mathrm{O}(97)$ felt that what he was doing, i.e., teaching English, was Observation valuable and that it was what he wanted to do. He planned to Remarks teach in the school for another year and then to look for 
another institution, perhaps a Technical Institute, in which to work. He found the work of teaching English in his school enjoyable but hard. He described himself as like an actor, trying to come up to the expectations of the students, that each lesson was like a performance and he had to maintain a level of performance otherwise he would lose the respect of the students. Sometimes he felt frustrated with students' attitudes if they were impolite or not interested and did not see the importance of being proficient in English. He said that he felt confident in his ability to talk about teaching.

Observer's As $\mathrm{O}(97)$ valued the work he was doing, the students Comments responded and the lessons were generally successful. That he felt frustrated as he claimed to be, either by the students' attitudes to learning or the content that he was having to teach, was not appreciably obvious, as he planned to stay at the school, at least for one more year. However, in the long term, he did not plan to stay at the school and so perhaps saw it as a 'stepping stone' to another stage in his career.

\section{Post-Graduation Reflections}

Teacher $O(97)$ said that he felt more confident in his ability to talk about teaching as a result of doing the BATESL course, although he had not had much opportunity to use the skills he had learned during the course. Yet the evidence from the observations was that he was employing the skills. He was using interactive activities in which the students had to make their own interpretations and develop their own ideas. He was, as he reported, free to adopt whatever teaching style he chose, as long as the students did not complain, and the evidence from the observations was that they seemed unlikely to do so as they seemed to enjoy the activities they were doing. (Although it should be noted that the lessons 
observed came at the end of the term when perhaps pressure of examinations was no longer a factor.) Consequently, there seemed to be a conflict within this teacher between what he felt he was able to do and what he was doing. Perhaps his expectations had been raised during the BATESL course to the extent that he would find any teaching situation to be restrictive. As he had spent the year postgraduation working in the English Department at the City University, he had been further removed from the realities of Hong Kong school classrooms.

\subsubsection{2}

\section{Observation Report on Graduate U(97)}

The teacher had spent her first year after graduation in 1997 working in another field (hotel and tourism) and so was in her first year in the school at the time of the observation. She would not be staying on at the school after her contract expired at the end of the current school year. The school was a Band Four/Five government-aided co-educational secondary school in a new town in the New Territories. In each cycle of six days students had nine English lessons plus one from the NET, which would be used for speaking practice.

Two classes were observed on the same day in May 1999. The first class observed was a Form 2 class of about forty students. In the lesson, $U(97)$ prepared the students to write a composition about pandas. In the second observation, U(97) administered an oral examination to a class of thirty Form 3 students. U(97) sat at the teacher's desk and the students came out in pairs to read a short dialogue that they had prepared in advance. 
Language Use

Observed The teacher used approximately 90 per cent Cantonese during Practices the lessons observed. Cantonese was used for asking questions, explaining vocabulary, interacting with students (formally and informally) and giving instructions. In the first observation, U(97) wrote guidelines and vocabulary on the blackboard in English to help the students to write their compositions. These would be explained in Cantonese. In the second lesson, although the students were reading dialogues in English, all teacher-student interaction was in Cantonese.

Post-

U(97) said that it was necessary for her to use mainly Observation Cantonese in order for the students to be able to understand Remarks what she was saying. She had begun the year by using English, but the students had objected to this and so she used Cantonese for all purposes and interspersed it with key phrases in English. Only oral exams and attainment tests were carried out in English. U(97) did not do oral work with her students (this was done by the NET), so Cantonese was used almost exclusively since the school was CMI and had been since the teacher started working there.

Observer's U(97)'s language use in the classroom was consistent with her Comments reported practice. Cantonese was used extensively to foster student understanding. English-medium instruction was adopted only by the NET. 
Instructional Planning and Decision-making

Observed In the first lesson, U(97) used a worksheet which gave a Practices suggested plan and guided questions for a composition. She had found the information herself from the Internet. The worksheet was used as the input and U(97) asked the students questions to generate sentences that they could use in their composition. In the second lesson, the students used a standardised dialogue that had been set for all students in Form 2 as an oral examination. She seemed to made a number of decisions during her teaching, such as to write the down on the blackboard all the sentences that the students would need to write their composition (in Lesson 1), and to play the role of second speaker in the dialogue for students without partners (in Lesson 2).

Post$U(97)$ reported that there were no teacher-designed activities

Observation and teachers had to follow the scheme of work and use the Remarks textbook. She said that she made use of the Internet to get information and sometimes used PowerPoint in teaching. She found that the students were interested in this as they liked hitech. U(97) would also make use of materials from seminars she had attended. $U(97)$ felt that the students needed information support to aid their writing and this is why she had decided to provide them with the information on pandas, rather than have them find it out by themselves.

Observer's There was some contradiction as $U(97)$ said that teachers did Comments not design their own materials, but in fact in the first lesson observed the worksheet had been (at least partly) written by her. Her reported use of materials from seminars also contradicted this point. U(97) showed that she was able to 
make on-the-spot decisions to facilitate the learning process and to make more long-term decisions such as changing her language use in the classroom as a result of the students' requests.

\section{Teaching Approach}

Observed In the first lesson observed, U(97) adopted a teacher-directed Practices approach by explaining what was written on the students' worksheet and writing the main points on the blackboard for the students to copy. She occasionally asked the students questions to generate sentences that they could use to write a composition. There was no student-student interaction or communicative activity. The second lesson consisted of students reading a dialogue in pairs in front of the teacher. $\mathrm{U}(97)$ gave little in the way of feedback on their performance as she was assessing them. She did try to encourage them.

Post- U(97) reported that she used interactive strategies with her Observation Form 1 class but not with Form 2 due to low student Remarks motivation. With this class she felt that she had to use a totally grammar-based approach, fully dominated by the teacher and conducted in Cantonese. The communicative approach was encouraged but students were unable to cope with it because of poor English proficiency.

Observer's As reported by U(97), with the first class observed, there was Comments no attempt made to introduce communicative strategies in the class. She considered the students' motivation and proficiency to be too low, although she did say after the lesson that there was a wide range of abilities in the class. The students 
appeared to be motivated and attentive and it might have been possible to introduce some form of interactive activity to get the students to try to acquire the information for themselves rather than having it provided to them.

\section{Professional Relationships and Responsibilities}

Observed $\quad \mathrm{U}(97)$ seemed to have a good relationship with the students in Practices the first class observed and was able to joke with them to a certain extent. They were also responsive to her, although there was some calling out by students (in Cantonese), which appeared to be to each other rather than to the teacher, that may have been off task and potentially disruptive. In the second lesson the students were performing a test and were generally respectful of the teacher, but she had to employ one student to observe the class and note those who were not on task preparing for their turn in presenting their dialogue.

PostU(97) reported that her relationships with students depended

Observation Remarks

Observer's

Comments on the class and the students' attitude and level of appreciation. She felt closest to her former Form 1 students and felt that students tend to cling to their Form 1 class teacher. Relationships with colleagues were described as 'good' and she was able to go to her panel chair for advice. Non-teaching responsibilities included serving as house master, extracurricular activities committee member and careers committee member.

It was clear that U(97) was not particularly happy at the school and felt that she was not able to teach in the way that she would have liked, i.e., using a communicative, activity-based 
approach. Her relationships with the students were not as she would have wished, which seemed to be because she felt that they were not motivated to learn. She felt that her beliefs had changed in that she used to believe that if she were close to her students, she could communicate with them easily and they would pay more attention to her lessons. She now felt that this was only applicable to senior form students. Her assumption, that learning attitude is (positively or negatively) related to academic achievement had been proven to be correct from her observations.

$\underline{\text { Perceptions and Values }}$

Observed U(97) maintained a patient and professional approach Practices throughout the lessons, despite the obvious low ability and motivation of the students.

Post$\mathrm{U}(97)$ felt that she was wasting her time as she really wanted to Observation teach the students and be a good teacher but many students Remarks were only interested in what was on the syllabus. She felt that she might have been better teaching higher forms. She also felt that the workload was too heavy for most teachers. She considered herself more open to innovations than some colleagues, not necessarily because of the BATESL, but because some teachers were fossilised in their methods. She felt that she would become more open to innovations as she became more experienced and she was eager to adopt new technologies in her teaching. As an innovator, she felt that it depended on the support she received from the E.D. and the school. She saw herself as being central to her colleagues in this respect as she was more willing to learn new things. 
Observer's $\mathrm{U}(97)$ placed a great deal of importance on being able to Comments communicate with her students, and this was a feature of her lessons, but she expressed the view that she could only really communicate and motivate senior form students. She saw herself as open to innovations and as an innovator herself, but again, the constraints of heavy workload, having to stick to the syllabus and the reluctance of other teachers to adopt new ideas meant that she was frustrated. She said that she was central to her colleagues as an innovator but it was not clear in what respect. She presumably felt that as she was possibly younger than they were and had done the BATESL course, she was more up-to-date in terms of contemporary approaches, designs and procedures. Whether or not she played an active role in disseminating or encouraging innovation was not clear.

\section{Post-graduation Reflections}

$U(97)$ appeared to be dissatisfied with her teaching situation. She felt frustrated that she was unable to use the methods and approaches that she had learned during the BATESL course due to students' lack of motivation and the restrictions placed upon teachers by the school. Consequently, she would be looking for a new job after her contract ended in August. She felt that some teachers were concerned about the Benchmarking of language teachers, as older teachers were often reluctant to take on new skills and new technology. She was looking forward to teaching students from Mainland China as they tended to be more diligent and motivated and their grounding in English tended to be better than that of Hong Kong students. 
W(97) had been teaching at the school as a supply (substitute) teacher from November 1998 and had been employed as a replacement for a NET who had not taken up employment at the school. She had spent one year in the UK studying for an MBA after graduating from the BATESL course in 1997. She intended to remain at the school on a permanent contract from September 1999.

The school was new, having been open only for three years. At the time of the observation, there were Form 1 and 2 classes and Form 4 and 5. The school was described by the teacher as a Band Three or Four Chinese-medium (CMI) secondary school in a new town in Kowloon.

In addition to their English class, the students were required to attend an English Enhancement Scheme that took place over three or four mornings during school holidays. In this scheme they took part in activities using only English. The scheme was described by W(97) as an Education Department initiative for CMI schools and for which the school received funding.

The first class observed was a 'split-class' group of fourteen Form 1 students. It was the policy of the school to divide Form 1 English classes into smaller groups for all but one class of the ten in the teaching cycle. W(97) described the students as being of quite high ability but weak in English. In the lesson observed, the class did work on asking and answering $w h$ questions.

In the second observation, three days later, the class was a complete class of about thirty-five students, some of them the same as those in the previous observation. W(97) explained that the students lacked interest in English and some were unwilling to participate in class activities. In the lesson observed, the students worked from the textbook on identifying parts of the body. They had already done some preliminary work on this in the previous lesson. 
Language Use

Observed W(97) used approximately 60 per cent English and 40 per cent Practices Cantonese during the lessons. Both languages were used for presentation and explanation of lesson content in a set pattern of bilingual language use. Normally, English was used first to present and explain content with repetition and/or follow-up in Cantonese Mainly Cantonese was used for giving instructions and for interacting with students. English was used for writing on the blackboard. The students used English when doing pair work activities, but switched to Cantonese when the teacher was not near them.

Post-

W(97) said that she used Cantonese to give instructions, for

Observation classroom control and to give the students a rationale for

Remarks lesson activities. She said she would not give them a translation of anything that they found difficult; instead she would give them clues to try to get them to think for themselves. In addition, she would never write Chinese on the blackboard, although she said that some English teachers did, and she told her students not to write Chinese in their books. This was due to her own experience as a learner, when having to avoid the use of Cantonese/Chinese helped to push her to learn English.

Observer's During the lesson observed, there was a problem of the Comments students slipping into Cantonese whenever the teacher was not standing next to them. This was probably inevitable as they were communicating with each other and their natural inclination was to use their L1. The fact that $W(97)$ used Cantonese for explanation (contradicting what she had said earlier) probably led to the students using it also. As the school 
was CMI, the students expected to be given instructions and explanations in Cantonese.

\section{Instructional Planning and Decision-making}

Observed In the first lesson, W(97) used two worksheets, the first Practices consisting of a list of twelve questions with blanks for the students to fill in the missing words to make up the questions. This first worksheet appeared to have been prepared by one of the teachers in the school. The second worksheet was a questionnaire copied from a textbook. Similarly, in the second lesson W(97) used a worksheet with a crossword puzzle whose clues were made up of pictures of parts of the body. This also seemed to have been copied from a textbook. Both lessons were well planned and well carried out. Examples of on-thespot decision-making were to spend extra time on the pronunciation of parts of the body with particularly difficult consonant sounds such as the sounds [t $\left.\int\right]$ in chin and $\left[\int\right]$ in moustache.

Post- $\quad$ W(97) reported that the teachers worked from a syllabus that Observation was prepared at the beginning of the year by the Form Remarks Coordinator. The students were taught the four language skills but there was a heavy bias towards the teaching of grammar. The materials used were a mixture of published materials (core textbook, listening book, reader) and teacher-produced materials. Each teacher in the year group was given responsibility for developing materials for different topics and then other teachers would share them. W(97) said that they were given a lot of flexibility as regards how they taught. She said that she decided to make the students write on the 
blackboard because they liked doing it and it stopped them from falling asleep.

Observer's $\quad \mathrm{W}(97)$ had chosen the teaching materials rather than designing Comments them herself, and they were clear and simple and served as the focus of the lesson. The lessons were quite well planned with clear learning objectives in mind. She was able to make decisions to maintain student interest and participation.

Teaching Approach

Observed W(97) used a combination of approaches in the lessons Practices observed. Both lessons involved teacher-student interaction as $\mathrm{W}(97)$ asked the students questions and wrote the responses on the blackboard. There was also a communicative pair activity in which the students worked in pairs to ask each other questions. In the second lesson, W(97) used the Total Physical Approach (TPR) by having the students follow her instructions to perform certain actions. The students were then told to do the activity in pairs. There was a follow up written activity for consolidation. Another approach that $\mathrm{W}(97)$ used was to choose students to write answers on the blackboard.

Post- W(97) said she was pleased that the students had learned the Observation question forms and could say them without having the Remarks worksheet, although she had found that they could not consolidate what they had learned. She felt that she would like to be more interactive (in English) with the students but their lack of understanding meant that she had to use Cantonese in order to get her message across and to save time in order to cover the syllabus. 
Observer's Although W(97) expressed the view that she had been unable Comments to use a communicative approach in her teaching due to the level of the students, the lessons observed proved that it is possible to be communicative with fairly low proficiency students. That the students found the lesson content interesting was quite obvious. They participated actively and seemed to enjoy speaking to each other and finding things out about each other. W(97) used a visual and physical approach in the second lesson to teach the students how to identify and pronounce the different parts of the body. As in the previous lesson, the students enjoyed coming up to the blackboard. This was evidenced by the number who were keen to come up. In addition, they enjoyed the physical actions of touching their (own) body parts as the teacher gave them instructions. The crossword puzzle was a nice way of consolidating what they had learned during the lesson.

\section{Professional Relationships and Responsibilities}

Observed

Practices

Post-

Observation

Remarks
W(97) seemed to have a good relationship with the students who were active and interested in the lessons. She communicated with them in English to facilitate the lesson content and in Cantonese to maintain solidarity with them. Her manner conveyed confidence and professionalism.

W(97) reported that her relationships with the students were 'good' with the lower form students, especially the girls, but not with the upper form students (Form 4). With colleagues, relationships were 'very good' and she could share both ideas and problems. She was a member of the extra-curricular activities committee, which organised English activities for the 
students during holidays. She felt that some teachers were not committed to teaching as a career and were always complaining about class size, workload, materials support and insufficient support and equipment for IT.

Observer's W(97) appeared to be satisfied with her work and was Comments developing good relationships with the students and with colleagues. She expressed the intention to seek a permanent contract to stay at the school.

\section{Perceptions and Values}

Observed W(97) showed great enthusiasm during the observed lessons Practices and this generated reciprocal enthusiasm in the students. Although the students were sometimes noisy and occasionally off task, she was able to refocus their attention through a skilful combination of firm control and friendly, light-hearted interaction and persuasion.

Post- $\quad$ W(97) felt that that she needed more patience and experience Observation in order to get more satisfaction from the job. She felt that she Remarks was more objective than she had been in the past in that she was better able to step back and judge what the students needed. She felt that there had been no real changes in her beliefs as teaching had been what she had expected. She was still learning to shorten the distance between theory and practice and what she should and should not do. 
Observer's This BATESL graduate had been teaching for about seven Comments months but showed a relatively mature and professional approach to the job. She seemed to have confidence in her own ability and a consistency of beliefs about teaching that belied her relative inexperience. She still seemed to hold to the philosophies of the BATESL (communicative, interpretive and innovative teaching) whilst learning to come to terms with her own particular teaching situation. Despite claiming that she was unable to adopt the kinds of teaching approaches that the BATESL course had instilled in her, the evidence was that she was using such approaches in her lessons (at least in those with the lower form students observed).

\section{Post-graduation Reflections}

W(97) felt that she could only adopt the strategies learned on the BATESL course, such as the Communicative Approach, for 10-20 per cent of the time due to the low ability level of the students. When asked what she thought she had gained from the BATESL that had helped her in her teaching, she said that the course, particularly the practice teaching and microteaching, had given her the confidence to speak in front of a class and the ability to present a lesson. She felt that the course could be improved by having videos of real lessons so that the BATESL students could get an impression of the behaviour of students in real classrooms as it was often hard for them to visualise the real situation

\subsubsection{Discussion of Findings}

Observing the 1997 graduates in their teaching situations revealed more detailed information regarding their practices and approaches than had been acquired from the interviews carried out in 1998. In terms of language use, there was evidence of 
strategic use of Cantonese in all three of the graduates. Given that two of the schools visited were CMI and the other had no clear language policy, it was not surprising that there was a heavy reliance on the use of the first language. Cantonese was used by all three teachers to aid facilitation of their lessons - to give instructions, for explanation and for classroom management (Richards, 1998). Two of the graduates felt that this was necessary to maintain students' attention, while the third $(W(97))$ reported that she actively tried to avoid explaining lesson content in Cantonese but in fact sometimes did so in response to students' apparent inability to understand (Pennington, 1995a). In the case of $\mathrm{U}(97)$, she said that she had initially used only English, but had gradually used more and more Cantonese in response to pressure from the students to do so. The teacher was forced to compromise her intended approach in order to meet the needs of her students and felt that she was not experienced enough or did not have enough authority in the school to insist on English-only. She may also have felt that she was not supported by a clear language policy within the school and was also concerned at the potential discipline problems that might arise if students were not engaged in lessons due to their inability to follow what was going on. This points to a rejection of English medium by students in CMI schools, even in English lessons. In the lessons observed, almost all oral communication was in Cantonese. $\mathrm{O}(97)$ was different again, despite reporting that he tried to avoid 'mixing' languages in class by using either only English or only Cantonese, in the observations he began by using almost exclusively English but gradually brought in more and more Cantonese. He later reported that the presence of the observer had encouraged him to use only English but as the lesson progressed he 'slipped back' into his more familiar pattern of classroom language use dominated (but not exclusively) by Cantonese.

Two of the graduates reported having a certain amount of flexibility to use their own teaching materials but having limited time to spend designing them. They clearly felt constrained by needing to follow a syllabus and use a course textbook. O(97) said that he was able to use his own ideas but as his classes were examination classes, he had limited scope to introduce anything innovative. He 
said that he did try to use group work wherever possible for speaking practice. In one of the observed lessons, he attempted to use group work so that the students could share ideas on language learning activities, although there did not appear to be much genuine communication occurring in the target language. All three teachers felt frustrated by the fact that they were constrained by the proficiency of the students they were teaching as well as by the need to cater to the students' product-oriented needs. As a result of this they reported having limited opportunities to adopt communicative or innovative practices. It would appear to be a common factor that teachers in Hong Kong schools, particularly new teachers, tend to overstate the impact of constraints within the system as preventing innovation and that interacting with the system is the norm in all teaching situations, especially in public education. Throughout the studies of this investigation, the constraints within the educational system have been cited as the main reason for the lack of successful diffusion and adoption of educational innovations (Chapters 3 and 4). It could be said that the constraints are the norms of the system and innovations will be diffused to a degree dependent on how they interact with these constraints. The tendency seems to be for new teachers to be overly idealistic in their expectations, which may result in some of them (such as some of the BATESL graduates) either not becoming teachers or leaving the profession when their expectations are not met. Differences in the three teachers' observed and reported approaches were generally attributed to the level and/or age of the students that they were teaching. There was also evidence of the graduates regarding themselves as innovators within their schools and amongst their colleagues, possibly as a result of their background of the BATESL and their relatively fresh take on teaching compared to their more experienced colleagues. This apparent innovativeness was not particularly evident during the lessons observed, although W(97) did make good use of a variety of techniques to generate interest in her students.

In general, the graduates felt that their relationships with their colleagues were good and they could rely on them for support and guidance. There was no confirmation of the findings from Chapter 4 concerning Hong Kong teachers' 
isolationism. These three novice teachers did not place much emphasis on such relationships and may have regarded them as 'good', within the norms of Hong Kong, or within their expectations of what they should be. (A better indication of teachers' professional relationships may be gained from the more experienced teachers reported on in the next section.) The teachers' relationships with the students were also described as good. Subjects U(97) and W(97) reported that they were able to forge better relationships with the lower form students than those of the higher forms. $\mathrm{O}(97)$ was teaching in a post-secondary school and so all his students were of Form 4 or above. All three seemed, from the evidence of the observations, to have developed good working relationships with their students, although it was difficult to judge whether these relationships extended to the extent where the students might approach the teacher outside of class for consultation or simply to be friendly. $\mathrm{O}(97)$ did report that he would meet with students outside of class. There appeared to be a difference here as $O(97)$ was able to form good relationships with older students where U(97) and W(97) found this more difficult, although $\mathrm{O}(97)$ was teaching only older students. Each of the graduates had been given a number of what might be termed 'low level' responsibilities and was involved in numerous extra-curricular activities, consistent with the government's policy of increased English language support through activities for students in CMI schools.

The graduates expressed mixed views concerning their teaching situation at the time of the observations. $O(97)$ and $W(97)$ seemed reasonably content with what they were doing, expressing some frustration with students' attitudes and lack of motivation, whilst at the same time feeling that they were doing a good job and were gaining valuable experience. Only U(97) was wholly negative in her appraisal of her situation. She felt that it would have been better for her to be teaching higher form students, as she would be better able to communicate with them, this despite the fact that she had earlier admitted to having better relations with the lower than the higher form students. She seemed to accept the fact that new teachers are generally given mostly lower form students to teach and the higher forms, which mainly focus on examination preparation, are taken by the 
more experienced teachers. She was also disappointed at having to use mostly Cantonese in her lessons, which contradicted her beliefs about teaching. U(97) and also $O(97)$, but to a lesser extent, expressed their frustration with having to 'teach to the syllabus', as though it were not possible to be innovative in terms of teaching approach within the constraints of having to cover language items set out on the syllabus. They seemed to have separated content or design from approach and procedure and not considered the fact that they could adopt their own teaching style whilst still covering the items on the syllabus. It could be that they were shifting blame to the syllabus for not teaching in the way that they would have liked, when it may have been more a question of what they perceived to be student expectations that prevented them from designing innovative activities or developing innovative practices. The repeated theme of regarding the syllabus as a barrier to the use of innovative approaches, rather than as the framework within which to use such approaches is evident here.

There were no references made to the changeover in sovereignty during the lesson observations or the interviews that followed them. It would appear that it was not considered to be a factor that had had any influence on the teachers' teaching situations or their teaching methods, consistent with the tendency to play down the effects of the changeover.

One of the most noticeable aspects of the observations was the relative inconsistency demonstrated by the teachers. This was manifested in two ways: there was inconsistency between their reported behaviour and their observed practices; there was also inconsistency in their beliefs. An example of the former was W(97), who felt that she was not able to adopt communicative practices, but in fact did so during her lessons. For the latter, an example was U(97), who felt that she would prefer to teach higher form students as she felt she would be able to communicate better with them, but also said that she had better relations with the lower form students. It could be that these novice teachers had not yet developed consistent teaching frameworks (Pennington, 1996) with which to put the various factors that affected their teaching into perspective. Alternatively, it 
could be indicative of a more widespread inconsistency between beliefs and practices and a lack of integration of perspectives (Calderhead \& Robson; Clift, 1991; Woods, 1996) present within the Hong Kong school teaching context, which would have been particularly acute during such a period of change as occurred at the time of the handover.

The beliefs and practices of two more experienced teachers, also graduates from the BATESL course, are examined in the next section.

\subsubsection{Lesson Observations of 1994 BATESL Graduates Five Years After Graduation}

In addition to the lesson observations of the three graduates from the 1997 cohort, two of the teachers who took part in the First Year Teacher Project described earlier (see Chapter 2) agreed to be observed again. These observations took place in May/June 1999, five years after they had graduated from the BATESL course (as members of the 1994 graduating cohort surveyed for Chapter 4) and four years after they had been observed for the above project. It was anticipated that these teachers, now experienced and established within the teaching community, would offer a valuable insight into:

- How graduates from a teacher preparation course have progressed as teachers after the initial first year in the classroom.

- What effects the teaching culture prevalent in Hong Kong durring that period might have had on these teachers.

- How their teaching methods (approach, design and procedure) differed from those of the 1997 graduates and from themselves as teachers four years earlier.

The two teachers observed are designated as $G(94)$, who was observed twice, and $\mathrm{H}(94),{ }^{4}$ who was observed once. ${ }^{5}$ As in the previous section, the observations and follow-up interviews with each teacher will be presented in the form of case 
studies. Each case study will begin with background information on the particular teacher, including a brief summary of their teaching from the 1994-95 First-Year Teacher Project and a description of their teaching situation at the time of the 1999 observations.

\subsubsection{Observation Report on Graduate $G(94)$}

The teacher had been teaching in the same school (a Band Two/Three government-aided co-educational secondary school on Hong Kong Island) since his graduation in 1994. During that time he had also completed his Certificate of Education (at the University of Hong Kong) and was in the second year of the MATESL course (at the City University of Hong Kong), both by part-time study.

In 1995, G(94)'s teaching approach had been described as being based on textbook exercises with contextualisation of the material. He had made attempts to include communicative activities in his lessons to maintain student interest but his main focus was on grammatical form. He had been prepared to use Cantonese in lessons if it enabled him to cover the lesson material more efficiently. He had been developing the persona of a strict teacher who demanded high standards from his students.

\section{Language Use}

Observed During the lessons observed, G(94) used English for all lesson Practices functions including presentation of content, explanation, giving instructions and interacting with students. On one occasion a student asked a question in Cantonese and $G(94)$ told the student to repeat it in English. 
Post-

Observation

Remarks

Observer's

Comments

$\underline{\text { Instructional Planning and Decision-Making }}$

Observed

Practices commitment to being EMI.
G(94) reported using only English in the classroom. Outside of the classroom he used Cantonese to interact with lower form and/or students whose English was weak. The school was EMI and so had not changed its teaching medium. If anything, G(94) had noticed more emphasis on English in terms of activities, notices etc., as if there had been a renewal of

English was the medium of instruction of instruction in the school and $\mathrm{G}(94)$ was careful to maintain that pattern of language use. The fact that a student did ask a question in Cantonese may indicate that if the observer had not been present, G(94) would have answered in Cantonese. Alternatively, the student may have become accustomed to interacting with $\mathrm{G}(94)$ in Cantonese outside of class.
The learning materials used in both lessons were from published texts. In the first lesson, $G(94)$ used a past examination paper to give the students practice in doing this kind of oral exam in preparation for the school exam, which would follow the same format. In the second lesson, $G(94)$ first gave the students a vocabulary dictation test and then the students worked from their course textbook. During the lessons G(94) made a number of decisions, such as choosing students to write on the blackboard and to demonstrate asking questions. He also appeared to vary the pace of the lessons if he felt that time needed to be spent on certain points. This resulted in them not completing the planned activities and $G(94)$ setting them for the students to do themselves. 
Post-

Observation

Remarks

Observer's

Comments

G(94) said that he used only published materials as he had no time to develop his own. He added that there was no official school policy on materials development but some teachers would do some on an ad-hoc basis. From time to time he would make use of aids such as language learning web sites so as to try to keep his teaching fresh and interesting for the students. He would also advise that the textbook be changed as often as possible.

Teaching Approach

Observed The two lessons observed were teacher-centered and lacking in Practices It was clear that $G(94)$ preferred to use published materials wherever possible and to focus on enabling his students to grasp the basics of English grammar and vocabulary as well as giving them the required exam practice. communicative activities. In Lesson 1, the focus was on preparation for the oral exam, rather than actually practising the spoken element of the exam itself, which would have given the students the opportunity to engage in interaction. $G(94)$ used the technique of having students formulate or answer questions in pairs, during which minimal communication seemed to be going on, and then writing these on the blackboard for comment. There was some teacher-student interaction as students asked the teacher to repeat things that he had said or called out answers to teacher questions.

Post- Teacher G(94) said that he would use interactive activities for Observation between 60 and 70 per cent of the time with less motivated Remarks students to try to maintain their attention. He felt that his 
teaching had become less communicative since graduation from the BATESL course and more grammar-based and examoriented. Students and parents expected uniformity and for students to be taught what they needed to succeed in exams. He did make use of some methods from the BATESL, such as 'P.P.P.' and 'warmers'. The main constraint was lack of time to prepare, but he used some of the things he had learned when he could. School policy was also against anything that strayed away from the syllabus. As far as developing the curriculum was concerned, the teachers would work together, although there had been no real changes in lower form or exam curricula. He felt that the curriculum was changing because of the changing needs of students and it was becoming more skills-based.

Observer's Comments
There was little evidence of interactive activities in the lessons observed, despite G(94)'s assertion, although he may have regarded the pair work that the students did as interactive. The decision not to practice the speaking activities in both lessons could have been due to a lack of time or perhaps G(94) was more concerned with form rather than function and felt it important for the students to have the background knowledge in how to formulate questions or construct the present perfect. There may have opportunities to do these activities in followup lessons but the indication was that this would not be the case. 


\section{Professional Relationships and Responsibilities}

Observed

The students showed respect for $\mathrm{G}(94)$ and there was no

Practices

evidence of indiscipline in his lessons. The students responded well and seemed to enjoy the lessons, particularly the more active parts such as writing on the blackboard.

Post-

G(94) reported having 'good' relationships with most of the

Observation students that he taught but some disagreements with his Form Remarks 6 class as form teacher. Previously he had been very strict but had since learned to lower his expectations and be more accepting of students' weaknesses. His relations with colleagues were described as 'very good', although there may sometimes have been differences of opinion. He felt that he did take a central role in his group of colleagues by making suggestions and having other teachers work with him. As he was still studying for his MA, he found that colleagues would come to him for ideas.

Since he had been promoted to Junior Panel Chair with responsibility for Forms 1-3, he found that whereas previously he had been able to take sides in a dispute, the increased responsibility meant that he now had to remain neutral. He said that less experienced and other colleagues sometimes sought his advice due to his position of authority. His other responsibilities included LAC Co-ordinator, responsible for Form 1 bridging programme; EMI co-ordinator, responsible for dealing with the Hong Kong Institute of Education as regards EMI, but had not been able to participate up to then; and Careers Master, which took up approximately 40 per cent of his working time. 
Observer's G(94) showed authority in his teaching and in his manner Comments around the school, but also came across as being very approachable for both students and colleagues. He had taken on a number of responsibilities and was also pursuing an MA in TESL (at the City University of Hong Kong) in his spare time. Despite this, he was able to maintain an image of professionalism and maturity.

\section{Perceptions and Values}

Observed

Practices

Post-

Observation

Remarks

Observer's

Comments
$\mathrm{G}(94)$ showed that he was concerned for the students' progress and that they should be serious about their learning. He also made it clear that he was determined to cover what was on the teaching/examination syllabus even if it meant leaving out more interactive activities.

He felt that he had become more flexible and he put more effort into teaching students who were motivated and would do only the bare minimum for those who were not. He had learned how to allocate his time and energy to the best effect.

In general, $G(94)$ seemed to have developed into a pragmatic Hong Kong English teacher who had learned how to allocate his rather limited time in order to fulfil his many duties and responsibilities. He felt that after completing his MA he would be able to devote more time to such things as materials preparation and lesson planning. He had found the balance between professional responsibilities and professional and personal development. 


\section{Post-graduation Reflections}

Teacher $\mathrm{G}(94)$ seemed, from the evidence of the observations, to be more at ease with himself and his teaching compared with four years previously, which is understandable. He was also authoritative and confident in the classroom. It would seem that he was starting to settle down into the role of teacher. He said that he was looking forward to finishing his MA, when he would be able to devote more time to his teaching and perhaps employ some of the methods and practices learned in the BATESL and incorporate more innovations into his teaching. $\mathrm{He}$ still seemed to believe that what he had learned in the BATESL was essentially worthwhile (more so than the PGCE) and that it had given him a solid background from which to develop his career. He was not worried about the language Benchmarking personally but felt that if teachers were qualified they should not have to take another test. It was not fair on the teachers.

\subsubsection{Observation Report on Graduate $\mathrm{H}(94)$}

$\mathrm{H}(94)$ had been teaching in the school (a Band Three/Four government-aided coeducational secondary school in a New Territories new town) for three years at the time of the observation. She had been teaching there since it opened. Previously she had worked for two years in another, similar but older school. The school, being newly built was well appointed, having OHP and TV/video in each classroom. In addition, due to funds gained through the government's Quality Education Fund (QEF), the school had a fully equipped multi-media language lab (MMLL) with space for up to forty students and a full-time technician. $\mathrm{H}(94)$ said that the MMLL was generally used for student self-access work and writing. She made use of the MMLL once or twice per month with the class and told students to use it for self-access during lunch times or after school. The school was CMI for Form 1-3 and had been since it opened. Form 4 was EMI as all the students had come from other schools, some EMI and some CMI, and the students were being prepared to take examinations in English. H(94) felt that English was given 
a lot of emphasis within the school. There were 2 NETs, a lower teacher-tostudent ratio for English (1:20 for lower forms and 1:30 for upper) than most other schools, and extra teachers to teach the best students.

During the First-year Teacher Project, G(94) had expressed the wish to adopt communicative practices in her teaching and had made attempts to do so. However, she had been restricted mainly by the lack of motivation and poor behaviour of her students. As a result, her lessons consisted mainly of sentencelevel exercises and revisions of homework assignments. She frequently made use of Cantonese in lessons, as the students would not use English. Frequently the lessons observed would end early as she had not been able to carry out her preplanned activities and had been able to improvise due to lack of experience and low student motivation. She had indicated that such problems were more pronounced with her Form 4 students (most of the observations were with the Form 4 students) than with her Form 1 students.

In the later observation, the evidence was that $\mathrm{G}(94)$ had matured as a teacher and had developed more consistent teaching behaviours and the problems that had affected her teaching in 1995 were much less pronounced. The following is a record of the observation of $G(94)$ that took place in the spring of 1999.

\section{Language Use}

Observed The majority of the lesson was carried out in English with Practices some explanations and instructions repeated in Cantonese. Teacher-student interaction was generally in Cantonese.

Post$H(94)$ said that she used Cantonese more with her Form 4 class

Observation (50-80 per cent) than with her Form 1 class (10-20 per cent). Remarks She used Cantonese to explain grammar, to explain difficult words and for discipline. She also said that she used Cantonese 
friendly interaction with the students to show them that English is important, although how this was achieved was not really explained.

Observer's Teacher $\mathrm{G}(94)$ seemed to have a regular pattern of language Comments use consisting of presentation of content in English followed by explanation in English and Cantonese and instructions given briefly in English before being clarified in Cantonese. It is considered likely that more Cantonese would have been used if the observer were not present, particularly for explanation and instruction. G(94)'s assertion of 10-20 per cent Cantonese would appear to be conservative. Her reported use of majority Cantonese with her Form 4 class contradicts what she had said earlier about the higher forms being EMI.

\section{Instructional Planning and Decision-Making}

Observed

Practices

Post-

Observation

Remarks
The lesson consisted of students working on grammatical exercises using computer programmes such as Tense Buster. ${ }^{6}$ $\mathrm{G}(94)$ had designed the exercises herself to suit what was on the teaching syllabus. The lesson appeared to have adhered to the prescribed plan and the teacher did not seem to make any major on-the-spot decisions other than to offer extra explanation, either in English or in Cantonese when necessary.

Teacher $\mathrm{G}(94)$ estimated that at the beginning of the term, maybe 70 per cent of the materials used were published and the rest were adapted. Later this would change to about 50-50. The materials would be supplemented with games and activities designed by her. There was no teacher-in-charge of materials 
for Form 1, but there was for Forms 2 and 3, so she had taken on the role of preparing materials for Form 1.

Observer's The lesson observed was well planned and the students Comments obviously enjoyed working on the computers. In such lessons there is a heavy reliance on the hardware and this did not cause any problems as there was a full-time technician in attendance. $\mathrm{G}(94)$ was able to control the content of the lesson by designing the exercises herself and using the exercise-choice function of the programmes to ensure that the exercises done by the students were of an appropriate level and conformed with their syllabus.

\section{Teaching Approach}

Observed The main approach used during the observed lesson was one of Practices teacher-directed instruction and student completion of exercises, which were done on computer as opposed to on paper. The use of the multi-media lab's integrated work station system enabled the teacher to monitor individual students from a central location and also for the students to score points for their team or group whenever they answered a question correctly. This served as a strong motivating factor in encouraging the students. $G(94)$ regularly gave out 'prizes' to the various group members as a motivating tool.

Post- $\quad$ Teacher G(94) said that she made a lot of use of group work, Observation including competitions between the groups and group Remarks discussion. She tried to make her lessons interactive and communicative but sometimes there was not the time to plan specific activities. She liked them to work in groups. The 
content of instruction consisted mainly of grammar and writing with a little reading, mainly exam-oriented.

She used more procedures that she had learned in the BATESL with the lower forms as she had more time and the help of colleagues, but with the senior forms she used a more formfocused approach. She was still aware of the methods learned in the course and felt that they made up the basis of her knowledge of teaching. She felt that she was not using so much because of lack of time and energy, but she felt that her present school was not as restrictive as her previous school.

Observer's Teacher G(94) used group competitions during the lesson Comments observed but there was no group work as such and students worked individually. Although the teacher did walk around and monitor the students briefly, the majority of the monitoring was done at a distance by using the multi-media system.

\section{Professional Relationships and Responsibilities}

Observed The students in the class observed responded positively to the Practices teacher and enjoyed the lesson. G(94) showed patience and professionalism as she kept the students on task and focused on the lesson content.

Post- $\quad G(94)$ thought that 80 per cent of the students considered her Observation strict. Outside class she was friendly with students. Although Remarks she tended to spend more time with the upper form students, she reported being was more friendly with the lower form students. Her relationships with colleagues were described as 'good to above average' with most colleagues but not with all. 
She felt that she was rather outspoken, which might have lead to some teachers not liking her. She thought that the computer teachers might have been jealous of her use of the MMLL, or Chinese teachers jealous of the special status of English, which afforded it more attention, in terms of access to the MMLL, the extra provisions allowed for it, such as NETs and extra resources for English-enhancement schemes and activities.

She was a member of the MMLL Team, responsible for developing materials for use in the MMLL and for helping other teachers to get the most out of the facility. She was Resource Manager (for English resources), Form 5 Coordinator: Academic Section (to overlook academic processes of the school), PTA (to hold meetings, design notice boards etc.), ECA and form teacher.

Observer's Teacher G(94) conducted the lesson in a lively and interactive Comments manner which the students responded positively to. She did give the impression of being outspoken in nature and it seemed likely that her description of her not totally positive relations with some colleagues was indeed the case. Being an active teacher, it was not surprising to find that she performed a number of different roles within the school and was involved in various extra-curricular activities.

\section{Perceptions and Values}

Observed $\mathrm{G}(94)$ showed that she was enthusiastic about teaching and Practices determined to make full use of new technology and innovative practices to make learning more interesting for the students. 
Post-

Observation

Remarks

Observer's

Comments
Teacher $\mathrm{G}(94)$ felt that she was using innovations, such as games, group work and process writing on the computer. She stated that she was the only teacher who used groups in her classes, which she felt could have been because most of her colleagues were not as experienced as she was and so were not as confident in using innovations. As she had become more experienced, she felt that she had become more open to innovations and had learned how to adapt to change. She felt that she was a central influence to her group of colleagues, especially in the development of materials and in spreading innovative practices. Through staff development, innovation was being fed into the curriculum, an example being the use of multi-media. If something was found to be good, then it was shared between colleagues and included in the curriculum.

She felt that her beliefs had not changed: she still believed in discipline before teaching, being prepared (in terms of materials, ideas and knowledge) and being talkative at the right time. In addition, she had come to believe that English should be creative and fun.

The teacher saw herself as an innovator due to her background in the BATESL, her knowledge of the use of new technologies and her five years of experience as a teacher. She displayed what might be termed as high confidence or, less positively, self-centeredness when stating that she was the only teacher to use groups in her classes. It was not clear how she knew this. She clearly had strong beliefs and her experience perhaps gave her the status in the school to be able to express those beliefs and hence influence other teachers and teaching policy within the school. 


\section{Post-graduation Reflections}

Teacher G(94) displayed an air of authority and confidence and clearly saw herself as one of the more experienced and senior members of the teaching staff. She seemed to have developed her own teaching style and philosophy and was happy with it. Although she was not happy with the status and situation of teachers in general in Hong Kong, she seemed satisfied in her own situation. She was adopting innovations in her teaching, within the constraints of the system, and saw herself as an innovator and participant in curricular evolution. In her view, the Benchmarking of language teachers would mean more work, as teachers would need to prepare for it. She felt that it was unfair to test teachers as they were already qualified and the Benchmarking could not reveal the whole picture of what it took to be an English teacher.

\subsubsection{Discussion of Findings}

The two teachers observed and interviewed had at the time of the observations been teaching for almost five years and could be considered to be experienced teachers. As reported earlier, these two BATESL graduates had taken part in the project which followed five of the 1994 graduating cohort from the BATESL course through their first year in the classroom. During that year (1994-95), they were observed a total of eleven times. They also took part in group reflection sessions and completed questionnaires similar to those taken by the two BATESL cohorts in 1994 and 1997 (of which they were members of the former - Chapter 4). ${ }^{7}$ To gain a measure of the development of these graduates as teachers, where appropriate, comparisons are made with the findings from the First Year Teacher Project.

The teachers seemed sure about their language use and the justifications for it. G(94) was quite firm about using only English in the classroom (the school was EMI) and this was consistent with what was observed. Outside of class he said that he would use either Cantonese or English depending on the competency of 
the student he was addressing. This had perhaps been a strengthening of the resolve to maintain an English-medium environment in the classroom to mirror that of the school. ${ }^{8}$ In 1995, he had made use of Cantonese in his lessons as reported by Pennington \& Richards (1997, p. 167):

Because he was so focused on covering the required material, [G(94)] regularly used Cantonese to facilitate the review of grammar rules or exercises previously introduced in English. He felt that use of Cantonese saved time and ensured understanding.

His position in 1999 seemed to have moved towards greater use of English wherever possible.

$\mathrm{H}$ (94) used Cantonese to varying degrees depending on which class she was teaching. She admitted to using more Cantonese with her Form 4 class than with her Form 1 class. This contradicted the school's policy, which was for Forms 1-3 to be CMI and Forms 4 and above EMI, which is also the policy for CMI schools in Hong Kong. It would appear that more use of Cantonese was becoming accepted for higher form students in order to help them towards success in the public examinations. She used the mother tongue for explanations and discipline. There would appear not to have been much change in the patterns of the teacher's language use as in 1995 she:

... made use of Cantonese to an even greater extent in her attempts to keep the lesson going and the students on target. In addition, she resorted to the mother tongue to establish rapport as well as discipline her students (Pennington \& Richards, 1997, p. 167).

At that time (1995), Pennington, Richards, Urmston \& Lee (1996) described $\mathrm{H}(94)$ as suffering from 'cognitive-affective dissonance', in that she was trying to maintain an English-only teaching environment but was unable to do so. It would seem that in 1999 she had adopted this dual-language teaching style and was more comfortable with it, in other words she had resolved the dissonance by accepting dual-code use and then making strategic provision for it in her teaching. The evidence from the one lesson observed in 1999 was that this teacher employed a 
more strategic use of Cantonese, for explanation and giving instructions, and used English as much as possible.

In terms of teaching materials, $\mathrm{G}(94)$ reported that he used only the published materials which the students were required to buy as he did not have time to prepare his own. Hence, the amount of lesson planning that he did was minimised and reduced to deciding which parts of the textbook to cover in each lesson and which parts to omit. There seemed not to have been a great deal of change from the situation in 1994/5. H(94), on the other hand, claimed that she used an equal amount of published and what she termed 'adapted' or teacher-designed materials. She had taken on the role of producing materials for the Form 1 classes and seemed happy to do this. In 1994-5, like G(94), she had relied almost entirely on the textbook, with occasional supplementary activities. It would appear that $H(94)$ had become more skilled at lesson preparation or had developed a teaching style within which she felt that the teaching materials she used, or at least a proportion of them, be designed specifically for the students she or other teachers in her year group were teaching. In their 1994-5 observations, these two teachers had demonstrated that the core of their decision-making in class amounted to ways to make the textbook more accessible for the students by personalising it or by using exemplification that the students could relate to. In this way they were attempting to maintain the students' attention by relating what they were learning to contexts that they were familiar with. In 1999, the teachers felt confident enough to employ more of the procedures and strategies that they had learned during the BATESL in order to make their lessons more interesting for their students.

Whilst $G(94)$ reported using interactive activities in his lessons, there was little evidence of him doing so in the observations and he felt that his teaching had actually become less communicative since graduating from the BATESL as a result of parental and school pressure for uniformity in terms of teaching practices and examination results. In this sense there had been a retreat to a more conservative pattern (Pennington, 1997b) of conformity with the norms of the teaching culture within the school. He blamed constraints of lack of time and 
restrictive school policy for his having to keep to the set syllabus but as was found with the less experienced teachers observed, tended to use the constraints as a kind of excuse for not being innovative. The classes observed proved to be generally teacher-centred with a limited amount of pair work and opportunities for students to speak in controlled exercises. As mentioned, in 1994-5 his teaching style or approach 'centred on textbook exercises but with personalisation and contextualisation of the material. ... [He was] concerned to keep the lessons interesting and gave students chances to work on their oral skills. He also made attempts to apply communicative techniques in some segments of some lessons' (Pennington, Richards, Urmston \& Lee, 1996, p. 82). Judging from the lessons observed in 1999, his teaching approach had not changed to any great degree, despite his greater experience and authority within the school..

Teacher $\mathrm{H}(94)$ tried to make her lessons as interactive and communicative as possible by involving the students in group work and considered herself somewhat of a leader in this respect among her colleagues. She felt as she had become more experienced as a teacher she had become more confident and prepared to innovate, or perhaps the BATESL course had given her the confidence, the skills and the interest to innovate. Her teaching approach, based on her background in the BATESL course, had not changed but she had become more knowledgeable about teaching and more competent in terms of developing and implementing different teaching procedures. The main contrast with her teaching in 1994-5 was that then, her attempts to introduce communicative procedures were generally unsuccessful as her teaching style involved informal interaction with the students which frequently led to disorder within the classroom and she was forced to revert to 'routine completion of exercises and checking of answers' (Pennington \& Richards, 1997, p. 161). By 1999, she had moved to a new school, which had allowed her a fresh start and she had developed a more authoritative manner so that she was less constrained by student behaviour. During the lesson observed, she stayed firmly on task and did not become distracted or allow the students to become distracted. Where necessary she 
scolded students if they were too slow in doing the exercises or if they showed from their behaviour that they had not been listening to instructions.

In 1994-5, G(94) had worked hard to establish himself as an authority figure by being strict with the students and trying to move quickly through the lesson material. By 1999 he had achieved a considerable amount of status within the school, having been promoted to a position of authority within the teaching panel, and was a well-established teacher that the students clearly respected. He was able to adopt a more relaxed yet focused style of teaching as the students knew what was expected of them and responded well to his teaching. He felt that he had relaxed his former strict persona as he had developed better understandings of students and had become more accepting of their weaknesses. At that time he was studying on the MATESL course at the City University of Hong Kong and he found that his colleagues would come to him for advice on teaching matters.

In 1994-5, H(94) had tried to achieve solidarity with her students by acting as their peer and engaging in light-hearted chat with them during lessons and had tried to make friends with them outside of class. This had resulted in the students losing respect for her and the discipline problems mentioned earlier. In 1999 she reported that the majority of her students considered her to be strict. She had clearly developed in this respect. Like G(94), she took on a number of roles and responsibilities, both academic and extra-curricular. Unlike $G(94)$, however, she did not feel that she had good relationships with all her colleagues. She felt that her outspoken nature may have upset some colleagues, as teachers in Hong Kong are generally considered to be conservative in nature (Pennington \& Cheung, 1995).

Teacher G(94), through his pursuit of further qualifications and his promotion within the school had shown himself to be a serious teacher who was dedicated to a career in teaching. He had remained in the same school and was clearly settled there and had built up very good relationships with students and colleagues. He was appreciative of the grounding that the BATESL course had given him and 
still felt that he would be able to employ some of the methods and approaches he had learned once he had completed his MA and had more time to spend on lesson preparation. (This expectation of there being a future time when innovations can be incorporated is a common feature to emerge from the studies of the investigation and will be returned to later in this chapter.) $\mathrm{H}(94)$ had overcome what had been a difficult first two years in the classroom and had developed into a confident and enthusiastic teacher. She could still be considered a more innovative and less conservative teacher than G(94) and she had also matured, particularly in terms of her relationships with students. Both teachers were comfortable within their teaching environments, although both were also still labouring under the constraints of the Hong Kong school system which restricted their efforts to employ the methods that they had learned during their training. Yet both agreed that the BATESL course had provided the grounding that they needed to survive as teachers.

During the lesson observations and follow-up interviews with the two 1994 BATESL graduates, no reference was made to changes in education that might be a result of the changeover in sovereignty. As an event or even as a cause of change, it had not seemed to have affected these two teachers. The major educational change that the 1997 graduates had associated with the changeover, the medium of instruction policy, had affected G(94) insofar as his school had had to fight to maintain EMI status and so there was a policy of strict adherence to EMI within lessons for fear of losing that status. In the case of $\mathrm{H}(94)$, her school was relatively new and had not changed its MOI policy after the handover.

\subsubsection{Reflections of BATESL Course Administrators/Teachers in 1999 (Eight Years After the Launch of the Course)}

In October 1999, during the second round of interviews with English language practitioners that help to frame this thesis and form the core of Chapter 3, two of the practitioners interviewed were long-serving staff members of the Department 
of English at the City University of Hong Kong who had had very close connections with the BATESL course and its students. One was the leader of the course at that time (Course Leader 1999), and the other had held that post in 1994 (Course Leader 1994), when the first cohort graduated. In addition to the questions asked of them for the interview survey (reported in chapter 3), they also agreed to speak specifically about the BATESL course and give their reflections on the following aspects of it during the period from the time of the graduation of the first cohort in 1994 up to the present:

- Changes in the structure or content of the course.

- Changes in the standards and characteristics of the students enrolled on the course.

- Changes in the ways that the course was viewed by the educational community in Hong Kong.

The main points to emerge from the interviews are listed in Appendix C. What follows is a discussion of the main points raised.

\section{Discussion of Findings}

Both course leaders expressed concern at the language proficiency levels of the BATESL course students with discussion about the entry requirement for the course. Course Leader 1994 was of the opinion that a grade C in Use of English should be the minimum qualification; Course Leader 1999, whilst agreeing that C had been the minimum acceptable level, pointed out that in recent intakes they had been forced to accept students with a grade D. In such cases, he believed that contingency measures needed to be taken to ensure that these students received the extra support in the language proficiency that they needed. To emphasise this point, Course Leader 1999 related his experience of teaching the 1999 graduates, who had been relatively weak on entry to the course but who had developed into what he described as 'fairly solid teachers'. The trend would appear to be of 
falling standards of intake with changes in the course implemented to cope with the situation.

There was concern expressed for the graduates from the course once they became teachers. Course Leader 1994 felt that the first year, or in some cases two years, was often a difficult time for the graduates (as had been the case for $\mathrm{H}(94)$ )). One reason given for this was that as new teachers they generally considered themselves powerless and unable to control their own teaching situation. Consequently it was felt that there should be more support given to the graduates in the form of outreach. One suggestion was to run the course on a part-time basis so that teachers would receive their training concurrently whilst they were teaching and could then get the support they needed to help them cope with the pressures they were facing in the classroom. Another possibility was to add a fourth year to the course, which would be in schools and the course would then become a Bachelor in Education. The recurring theme of practice teaching being too short was evident as both course leaders admitted that they would like to give the student teachers more exposure to real classrooms. This exposure would ideally be as practising teachers but could also be in the form of observation in schools and the use of video recordings of lessons. The view was expressed that such exposure was very important for new teachers, as there was such a wide variety of teaching situations in Hong Kong with schools and school authorities having a lot of autonomy. New teachers needed to get as wide an experience base as possible before they went out into schools (Fairbanks et al., 2000).

The point made by the 1997 graduates about the lack of recognition of the course by schools was repeated by the course leader in 1999. He expressed frustration at the lack of response from school administrators to his efforts to publicise and promote the course. Furthermore, this course leader, echoing earlier findings (Chapter 4), felt that English teaching had a low status in Hong Kong and so the course was generally not considered a popular option for school-leavers. 


\subsection{Conclusions}

The four studies described in this chapter serve to complement and extend the findings of the analyses of the English language teaching and teacher education situation as it existed in Hong Kong during the period of 1994-1999 described in the previous chapters and to provide answers for research question (v), i.e.,

What evidence was there of changes to the beliefs, assumptions, knowledge and practices of novice teachers of English in Hong Kong as a result of their teaching experience post-graduation and of the changeover?

Three key areas emerged from these studies which mirror the three areas of common culture of English language teaching in Hong Kong identified in Chapter 4 (see 4.4.1), i.e., language use in the classroom, the potential for adopting innovative approaches and the situation and status of English teachers and their relationships with colleagues and students. These three areas illustrate the educational culture prevalent at the time (in 1999) and provide insights into the ways that the needs for language and language teacher education were changing and had changed since the handover. These three areas are discussed below.

\subsubsection{Language Use in English Lessons}

The language used in the classroom by teachers (and students) came in for a great deal of attention during the period of 1997 to 1999 as government policy had dictated the language of instruction in schools from 1998 onwards. One picture emerging from the findings of the studies reported on in this chapter and throughout the investigation is that by 1999 the profile of language use in English classes was becoming more clearly defined and in schools given EMI status there was a clear edict to use only English in class. There was purported to be strict monitoring of this by school management. However, evidence suggests that this 
monitoring might not have been as strict as the schools would wish parents to believe. EMI status was much sought-after as it represented higher status and the ability to attract better students, who generally came from the middle and upper middle classes and possessed the cultural capital required to take advantage of the English-medium education that these schools provided (Bourdieu, 1997). As the majority of schools in Hong Kong are government-aided, receiving financial support from the government, they tend to rely for further funding on donations from parents and benefactors. By attracting better students, the schools are likely to attract more funding as the parents of such students will have the economic capital and the social energy that will motivate them to take an active role in their children's education and support the schools both financially and/or through participation in school events.

The evidence from the observations carried out in 1999 is that teachers were adhering to school policy on medium of instruction. Those teaching in EMI schools were careful to use only English for instructional purposes, although interaction with students outside of class and for what might be termed noninstructional purposes (giving notices, reminding students about appointments etc.) was often done in Cantonese. The situation within schools designated as Chinese medium appeared to be more complex. The CMI schools visited did not seem to have a clear and consistent policy regarding language use in English lessons. The teachers were encouraged to use as much English as possible but the use of Cantonese was considered acceptable if learning was facilitated. The result as observed in the small sample of classes visited was that content was presented in English with explanation, exemplification, instructions and interaction all taking place in Cantonese. In the view of the teachers, making use of the first language is the only way to maintain the interest and participation of students whose English language proficiency is often very low. In at least one case a teacher reported that her students had demanded that she use Cantonese as they could not understand her when she used English. There would appear to be a power struggle within the classroom between teachers who had been trained to believe that English should be taught through English, as evidenced in the 
questionnaire studies detailed in Chapter 4, and students who believed that the teacher was obliged to serve them. Confucian principles of total respect for and obedience of the teacher by the pupil, of the master by the apprentice, would appear to have waned somewhat in contemporary Hong Kong society, with pupils (like parents) becoming more empowered as a social group. However, it should be pointed out that the teachers who demonstrated this apparent power struggle were young and relatively inexperienced and as such less confident than their contemporaries who had been teaching for a few more years and who were more comfortable with their patterns of language use.

\subsubsection{The Situation and Status of English Teachers in Hong Kong}

The majority of English teachers in Hong Kong, like the majority of the population, are Chinese with a first language of Cantonese. In 1999, two years after the reversion of the Territory to Chinese rule, reform measures or innovations imposed on the English teaching community, as well as other endogenous and exogenous factors, were having significant effects on these 'local' English teachers. As noted in the previous chapters, evidence was emerging to suggest that stakeholder groups within education, such as parents and students, who had previously taken a less prominent position, were becoming more visible and challenging the authority of the teacher.

The continuing expressions of concern at the apparent fall in standards of English proficiency in society in general and in students in particular throughout the 1990s and the disclosure that only about 14 per cent of those teaching English in schools in Hong Kong were actually trained to do so (Falvey \& Coniam, 1997) led to the introduction of measures designed to try to rectify the situation. These included the LPAT or Benchmarking of teachers to ensure a minimum competency level for those not already graduate-trained and the expansion of the provision of native English-speaking teachers or NETs. Each of these initiatives did little to improve the situation of local teachers of English, already burdened with large classes, 
heavy workloads and low student motivation. The feedback from the teachers observed in this study indicates that they themselves were not feeling under excessive pressure as a result of these changes, although there was evidence that the morale of local English teachers was being affected by the actions taken by the government. At the same time, Hong Kong was still suffering from the economic downturn which had affected the majority of countries in East and South-East Asia and many teachers were, for perhaps the first time in their careers, worried about their job security. In addition, as we have found, those new to the profession were not finding it easy to secure positions. The additional threat of having to reach the minimum proficiency level or Benchmark, only increased the pressure on them. Expressions of this pressure were evident when large numbers of teachers took to the streets to demonstrate against the Benchmark initiative (South China Morning Post, 28 May 2000).

\subsubsection{The Potential of Teachers for Adopting Innovative Teaching Approaches}

The influence of parents and society on the approaches and procedures adopted by teachers was felt keenly by the teachers observed as the educational system remained examination-driven. The teachers regarded themselves as innovators within their schools, in comparison with colleagues who had been teaching for some years and who had developed set routines for teaching. The teachers felt that the BATESL course had given them the background in teaching that they needed and the predisposition to be innovative in their teaching. However, the teachers found that the culture in the schools in which they were teaching was frequently not conducive to innovation as pressure to conform to the syllabus was high. This was especially true for the higher forms, those of Form 4 and above, who were being prepared for external examinations. The less experienced teachers felt a great deal of frustration as they felt that it was only possible to use innovative procedures or techniques with the higher form students as they had the ability and maturity to take advantage of them. Although they generally had more freedom to 
teach the lower forms using their own methods, the students were usually not capable of performing the tasks that the teachers required of them.

The more experienced teachers seemed to have settled into a pattern of teaching with which they felt at ease and through which they were able to make use of new or innovative procedures at times and in situations that suited the teaching situation and culture they were in. Whilst they were faced with the same constraints as the younger teachers, they had come to accept the situation and to learn when it was suitable to innovate and when it was not. Both the experienced teachers visited still intended to make more use of the techniques they had learned in the BATESL course, some time in the future when they were not so busy, indicating that they could put off any change indefinitely by projecting it into the future (Pennington, 1998a). As they became more experienced and their authority within the school increased, they thought it likely that they would be able to experiment more in their teaching. It seems to be a characteristic of teachers in Hong Kong to put off doing something that is perceived to be difficult or even controversial and to look ahead to some time in the future when conditions will be better (Pennington, 1998a). This form of unreality seems to be a feature of Hong Kong people in general and is perhaps related to the characterisations discussed previously (Chapter 2) of people being conservative towards change and not wishing to act, even though they may see the need to. ${ }^{9}$ Hence, the reality might be that teachers would continually put off introducing innovative practices into their teaching whilst citing the constraints of the system as working against them and suppressing their educational ideals. This point highlights the need, expressed by the 1994 BATESL Course Leader, for more support for novice teachers in their first year or two in the classroom. The 1994 BATESL graduates observed had this support as they took part in the First Year Teacher Project and in return received a lot of feedback on their teaching and guidance through the first year. The 1997 graduates did not have such support and so were having to deal with the frustrations of being a teacher by themselves. 
The findings from this chapter indicate that two years after the original studies of English teaching in Hong Kong, the major trends determined at the time were continuing, in that language use was becoming more polarised as EMI schools demanded English use only in English lessons and CMI schools adopted a more laissez-faire approach to allowing the teacher to decide the proportion of English and Cantonese to be used. For teachers new to the profession, the familiar constraints of heavy workload, large class size and the need to adhere to a syllabus heavily geared towards achieving parity between classes and to preparing students for internal and external assessment had been supplemented by increasing pressure to perform from schools, parents and government. At this time, curricular reform measures such as the Target Oriented Curriculum had not had any impact on secondary schools, but the change in the medium of instruction, the expansion of the NET scheme and the prospect of language Benchmarking had. What was clear was that changes were in progress, but caught in the middle were the teachers, both experienced and novice, who were having to cope with limited support or sympathy. The message seemed to be that there was a need to address these teachers' concerns if the planned reforms were to succeed.

Changes since the handover were detected, as discussed, but also the system of ELT showed the continuity that it has been characterised as having. In order to gain a clearer understanding of the whole situation with regard to English language education in Hong Kong before and after the changeover in sovereignty, it is necessary to conclude all the studies of the investigation and synthesise the major findings: This will be done in the next chapter, which addresses the remaining research questions on its way to the construction of the models of ELT in Hong Kong pre and post-handover. 


\section{Notes}

${ }^{1}$ To better identify these subjects as graduates in 1997 from the BATESL course, they shall henceforth be referred to as Graduates $\mathrm{N}(97), \mathrm{O}(97), \mathrm{P}(97)$, etc.

${ }^{2}$ The lesson observations were intended to be a small-scale follow-up study to the earlier questionnaire and interview studies.

${ }^{3}$ It was reported by the BATESL Course Leader in 1999 that this was his impression, although he had no statistical evidence to prove his case as maintaining contact with graduates was difficult. It was hoped that since the advent of email, maintaining links with graduates would be easier.

${ }^{4}$ These two teachers were referred to as 'King Fai' and 'Wing Yee' respectively in the reports on the First Year Teacher Project (Pennington, Richards, Urmston \& Lee, 1996; Pennington \& Richards, 1997).

${ }^{5}$ Originally two observations were to be carried out but due to examinations, it was only possible to do one at that time.

${ }^{6}$ A commercially-made computer software package consisting of grammar and reading comprehension exercises that teachers can author to match their students' needs and syllabus requirements.

${ }^{7}$ See Pennington et al. (1996) and Pennington \& Richards (1997).

${ }^{8}$ Teacher G(94)'s school had been one of those that had originally been designated to become CMI but had successfully appealed the decision and won the right to remain EMI.

${ }^{9}$ This unreality is often demonstrated in student compositions and letters to the editor of local newspapers which, after addressing some social or environmental problem, will invariably finish with a phrase such as 'I hope that this problem can be solved in the future'. 


\section{GENERAL DISCUSSION AND CONCLUSIONS}

\subsection{Summary of Findings}

The developing pattern throughout this investigation into the teaching and learning of English in Hong Kong at the time of the changeover in sovereignty has been one of change and continuity. In this pattern of change within continuity, changes have been shown to have taken place in the degrees of influence and importance of components of the system which makes up English language education, whilst the people within that system have demonstrated continuity through their resistance to some changes and their partial or, less frequently, total adoption of others. These changes have been referred to as initiatives or innovations in this investigation and as documented they have met with varying degrees of success in terms of being adopted by the user system, which in most cases means the teachers.

The major innovations to have affected ELT and which have been discussed in this thesis - communicative language teaching (CLT), the change in the medium of instruction (MOI), the Target Oriented Curriculum (TOC), the Native English Teachers Scheme (NETS) and the Language Proficiency Assessment for Teachers (LPAT), were all 'remnants' (as described in Chapter 1) of British colonial rule. In other words, they were developed prior to the handover to be adopted after it in the sense that Hong Kong's post-handover education system inherited these innovations from the pre-handover system (with the exception of CLT, which had been introduced earlier and by the time of the handover was in a more advanced stage of diffusion - see later discussion). Although these innovations were introduced into the education system, and there is evidence of resultant change in the system because of them, there was also continuity in the system through its enduring characteristics and unchanged basic structure. From this perspective, it would be misleading to describe the pre-handover and post-handover states of the 
Hong Kong education system as though they were separate (unrelated and/or disconnected) systems. It is nevertheless possible to view the education system in terms of 'snapshots' before and after the handover such as are provided in this thesis and from these 'snapshots' to gain a perspective on effects connected to the transition. To build towards the construction of a model of the education system as related to English language teaching, the main findings from each chapter of the investigation are first summarised.

Chapter 2 reviewed the previous research into language use, language teaching, and language teacher education in Hong Kong, as well as discussing the social structure and political culture of the Territory and later Special Administrative Region. Theories of change, such as Rogers' Diffusion of Innovations model (Rogers, 1962, 1995), and the work influenced by it of Henrichsen (1989), Pennington (1995c, 1996, 1997b, 2001) and others, was discussed and applied to the Hong Kong English language educational context. Furthermore, the work in teacher education and teacher change, particularly of Richards (1990b, 1994, $1996 \mathrm{~b}, 1998)$ and Woods $(1989,1991,1996)$, was used to develop a framework through which changes apparent in teachers' beliefs, assumptions and knowledge (Woods, 1996) or approach, design and procedure (Richards \& Rodgers, 1982, $1986,2001)$ could be interpreted. Finally, the requirements for a model of change were put forward in anticipation of the development of such a model based on the findings from the investigation. What the chapter revealed about the nature of Hong Kong was that on the surface it is a society heavily influenced by Confucian principles of respect for authority, desire for stability and protection of familial interest, whilst also being adaptable to change on a pragmatic level for economic and social gain. These principles apply to education also as reflected in the reported respect for teachers from students and parents, the desire for English of the population for its economic and social benefits, and the conservatism or resistance to innovations by teachers. At a deeper level, there were indications of conflicts in the political, social and educational contexts. Within English language education, teachers were coming under increasing pressure to consolidate or improve their standards. At the same time, the current research showed that 
teachers are prepared to adopt innovations if they can see the benefit to their students and to themselves, but they feel in many cases that the changes being introduced are not appropriate for the Hong Kong context and would not be successful because they were being imposed on them (the teachers) rather than in consultation with them.

Chapter 3 examined the educational context of Hong Kong at the time of the handover and the period following it through a survey of the issues related to ELT reported on in the English language newspapers and through two rounds of interviews with educators and education officials in 1997 and again in 1999. The issue which dominated news coverage in 1997 and the thoughts of English language practitioners both before and after the handover was the change in the medium of instruction in secondary schools. Other important issues were those concerning teachers, language standards and teaching methodology and curriculum. As education was brought much more into the public arena by the media during this period, the public were taking a much more proactive role as a result of the handover or of the political reforms enacted by the last colonial governor, Chris Patten. It was felt by many of the practitioners interviewed that the government was intent on enacting change in the education system, and people began to consider the possibility that the handover had acted in some way as a catalyst to change. There was, however, a general tendency amongst people in education to downplay the significance of the handover. Lastly, the impression given by some of the teachers interviewed was of an increased solidarity with and even sympathy for students for having to be a part of the changes going on in education and society and to cope with them just as they (the teachers) had to do.

The tendency to sympathise with students is also a factor arising from the data presented in Chapter 4, which showed the student teachers on the BATESL course to be strongly student-centered. These novice teachers also, however, showed their inexperience through being uncertain as to how to be student-centered but also authoritative so as to avoid discipline problems. Conflicts were a theme of the findings of this chapter, as the BATESL students indicated how conflicts exist 
amongst teachers generally in terms of language use (pure English vs. use of Cantonese), teaching approach (innovation vs. transmissive teaching culture) and professional relationships (recognition of value of collegiality vs. lack of it in local teaching culture). In terms of education more generally, there emerged evidence of increasing acceptance of Cantonese use in English teaching, an increased examination focus in education to cope with changing conditions within society, increased pressure on teachers in terms of their professional standards, and a greater politicisation of education generally as major issues, particularly those affecting language teaching, were gaining greater attention from the public through the media and were having to be addressed by those in authority.

Chapter 5 looked at the English language education system post-handover by seeking the views of BATESL graduates and by observing representatives of two cohorts in their teaching environments. The theme of strong student-centeredness was continued in this chapter with evidence of a caregiver or mothering orientation in which students were regarded as being rather helpless and in need of teachers' great attention. The attitude of the teachers observed was one of wishing to do their best for their students even if in some cases it meant compromising their beliefs about what constitutes good teaching practices. This was exemplified by the apparent increase in the use of Cantonese in English teaching by the teachers over time, despite their own reported adherence wherever possible to English as the main classroom language. In addition, the tendency to look to a time in the future when they would be able to adopt the kinds of innovative teaching approaches and practices that they would like to was apparent, and this also applied to the 1994 BATESL graduates who had been teaching for five years. In other words, these Hong Kong English teachers blamed the constraints of the teaching system for preventing them from teaching in the ways that they thought they should, and forcing them to put off doing so to an unknown time in the future. On the face of it, they seemed to be thinking that the conditions or the norms of the teaching context would somehow change, or that they would somehow master them eventually and then be able to teach in a more innovative way. Yet, the graduates from the BATESL course did regard 
themselves as innovators in comparison with their colleagues, whom they felt may have not had the benefit of a specialised programme of training and may have become resistant to new approaches. It can thus be concluded that although the degree of innovation introduced into Hong Kong education by these graduates may not have been extensive, it may nevertheless have been real.

This chapter concludes the investigation of the changes undergone by the various systems, including the social systems and groups which constitute English language teaching in Hong Kong, during the period leading up to and prior to the changeover in sovereignty from British to Chinese rule in 1997. Through a synthesis of the key findings from the different studies detailed in Chapters 2 to 5 and summarised above, areas in which change has been detected are identified and discussed in relation to the models of change discussed and presented in Chapter 2. As an outgrowth of the present research, a new model is then proposed for educational change at a time of socio-political change.

\subsection{Areas of Change}

Four key areas of change have been identified for the Hong Kong context, and these are change with respect to society in general, the educational system, language and teachers.

\subsubsection{Society}

In this section and the one that follows (6.2.2) research question (vi) will be addressed, i.e.: ${ }^{1}$

What insights are gained from the Hong Kong case for the theory and practice of English language teaching, and for educational and social change? 
The theories of how societies are structured offer a useful perspective for viewing the findings of the investigation. Hong Kong is describable in structural functionalist terms relating to its Confucian value system. However, unlike its East Asian neighbours which share this value system, Hong Kong has been greatly influenced by Western principles of economics and government due to its status as a colony of Great Britain before 1 July 1997 and as a self-declared 'world city' since then. Moreover, Seagrave (1995) argued that the characteristic family-based commercial networks of the southern Chinese actually developed in resistance to the values of the Confucian élite imposed on them from the north. This interplay of influences has created a complex society of 'free-market capitalist Confucianism' within which the people display characteristics ranging from 'utilitarian familism' to 'egotistical individualism' (Lau \& Kuan, 1988; Wong \& Lui, 2000a), but with a social conscience as well, as proved when a million people took to the streets of Hong Kong to protest against the brutality of the crackdown on student demonstrators in Beijing in June 1989. That the transition of sovereignty, which was much-anticipated with considerable trepidation, passed off remarkably smoothly is perhaps an indication of the capacity for absorbing and dealing with change inherent in a society whose population is made up mostly of migrants or their descendants (Pennington, 1998a), political and economic, from Mainland China and elsewhere. The old cliché 'borrowed time, borrowed place', which used to refer to the fact that the Territory (as it was) would eventually be returned to the Mainland, still applies, as under the terms of the SAR's constitution, the Basic Law (Government of Hong Kong SAR, 1990), Hong Kong will only retain its independence from the Mainland under the so-called one country two systems policy for fifty years. So the people and society of Hong Kong are very familiar with change and very accustomed to dealing with it.

Conceptions of conflict theory can be applied to the Hong Kong case, as was done in Chapter 2, and the pattern to emerge from the data of all the studies of teachers confirms the conflicts previewed there. Conflicts evident in language use and in teaching have been described. In political terms, the conflicts between the British 
and Chinese sides prior to the handover were apparent right up to the handover itself. Under Governor Patten's political reforms, the most democratic elections in the Territory's history, were held in 1995, resulting in a Legislative Council dominated by Democrats, most of whom were vocal in their objections to China and critical of her human rights violations and lack of democracy (Flowerdew, 1998; Patten, 1999). China's response was to hand-pick its own Legislature, known as the Provisional Legislative Council, which replaced the serving Legislative Council after the handover and served until new elections took place in 1999. Britain had also expressed strong opposition to the way in which the Chief Executive, Tung Chee-hwa, was 'elected'. ${ }^{2}$ After the handover and the departure of the British, conflicts became more apparent within society as the government's perceived inability to cope with changing (and worsening) economic, social and environmental factors in the climate of economic recession resulted in more claims on the government and greater civil unrest. Examples of such unrest are the 'Right of Abode' issue (see 2.4.2) and the protest by an estimated 35,000 civil servants (the biggest protest since the handover) and their supporters in opposition to proposed cuts in their pay (South China Morning Post, 8 July 2002). The indications are that conflict is coming more to the surface in society after a relatively long period of stability. Conflicts resulting in civil unrest did occur throughout the twentieth century in Hong Kong, as described by Pennycook (1998) and Sweeting (1998), but since the riots of 1967, the only major disturbance to occur was the 1989 pro-democracy demonstrations, and these were directed at the Mainland authorities rather than at the Hong Kong government.

The increase in claims on the government as a result of conflicts within society can be described in terms of the third theory of social structure presented in Chapter 2, Interpretive Theory, and it is important to consider that theory in relation to the findings from the investigation. To recap, Interpretive Theory states that within the functional or cultural framework of society exist the ever-changing social groups and structures based on class, ethnicity, occupation, religion and other social structures, attributes and practices, and that change occurs when 
individual groups or structures of groups are successful in reconstructing social reality by deliberate action. Attempts by right-of-abode seekers or by civil servants to reconstruct social reality by drawing attention to their grievances through protest action shows how conflict can result in attempts to change social structure. The right of abode issue protests, as an example, led to the government asking Beijing to intervene, so contradicting its own autonomy from the Mainland. In education, claims-making agents such as teachers' representative Cheung Man-kwong, were able to gather sufficient support from teachers and coverage from the media to force the government to backtrack to some degree on the LPAT issue. The media, as a controller of social reality (Harper, 1993), continues to grow in influence as telecommunications technology develops and society becomes more affluent, resulting in greater numbers of the population having access to information. The government, ever more aware of the importance of its image and the potential for civil unrest generated by the media, has been shown to have acted to maintain stability in society whilst addressing social needs in the key areas or systems of education, housing, social services and the environment. In addition, the social groups or structures within each system (such as education) have made claims on each other and challenged each other such that the relationships between them are ever-changing, on a surface level, whilst the system as a whole interacts with other systems in society and a general condition of continuity is seen to be maintained at the deeper, structural level of society.

The diffusion of innovations perspective allows a conceptualisation of the nature of the change process and the reactions of society to them. Hong Kong, not unlike most societies, has been changing over the past decade in terms of its social, economic and political characteristics (Kuan \& Lau, 2000). The political stability that characterised the Territory under colonial rule as a result of the influence of Confucian culture on the local population combined with the lassez-faire approach to business of the government, which produced and maintained economic prosperity ( $\mathrm{Lau}, 2001)$ gave way to a period of change due to a number of factors, summarised by Cheung and Louie (2000) as the following: 
... the impact of rapid economic development and urbanization, social structural and demographic changes, the rise of an increasingly articulate and demanding middle class, and perhaps more crucially, the 1997 question which has triggered much political anxiety as well as demands for political participation. (p. 64)

In a period of potentially great socio-political change such as that which Hong Kong experienced in the late 1990s, it would be expected that stresses and strains would be produced in the social fabric of society. Innovations (as discussed in the educational context) were introduced to reduce those stresses and produce more integrative conditions within the social system (Harper, 1993). The political change of the transfer of sovereignty, within a society accustomed to stability, meant that there existed a politically charged atmosphere, such that politics could be said to have been driving every aspect of society more than it had been previously, at least since the social unrest of the late 1960s (Cheung \& Louie, 2000).

However, although some changes were apparent on a surface level, change at a deeper level by its very nature is more difficult to detect. Such a change in governance might (not unreasonably) be expected to be accompanied by catastrophic change such as war, as happened after the founding of the state of Israel in 1949 and which has characterised the political changes in the aftermath of the break-up of the former Yugoslavia. In addition, some of the innovations introduced into the system, particularly those introduced into the education system, could be said to have been in a direction different to what might have been predicted, i.e., there has been a focus on maintaining and improving English standards in society through serious policy measures, when the expectation might have been for a diminishing of attention to English and a greater push for Putonghua. In other words, there appeared to be a reluctance to embrace the changeover and instead to carry on as though it was 'business as usual' and hope for the best. Changes introduced were meant to be at the surface level and not intended to effect any deeper level changes. 
Educational reforms had been ongoing since the 1980s, but as Bray (1997) points out:

$\ldots$ as the 1990 s progressed the pace of reform began to quicken as decision-makers at all levels sought to make adjustments prior to what they perceived to be the deadline for the change of sovereignty. (p. 20)

In other words, changes which had been in the works might have been accelerated as decision-makers sought to implement changes before the handover due to uncertainty as to what would happen after it. There could be a number of reasons for this accentuated period of change:

- The resource systems responsible for producing the reforms did not wish to see the work that they had put into them being wasted should the reforms be shelved after the handover.

- Given the mood of change prevalent within society in general, decisionmakers may have thought that reforms introduced at this time would be met with relatively less resistance, as they would be one of many changes occurring at the time.

- Decision-makers may have considered certain reforms potentially more likely to be accepted due to their nature as being either pro-Chinese or even antiBritish or anti-Western and so riding on a perceived wave of patriotism for the Motherland. Such reforms as the change in the medium of instruction to Chinese would apply in this case.

An alternative view is that the changeover acted as a kind of watershed, in that decision-makers preferred to wait until after the changeover before implementing reforms due to uncertainty over the nature of the socio-political situation after the resumption of Chinese sovereignty. Innovations which had been pending, or which had been evolving under the surface of society, may have seemed to suddenly take off after the handover, as if they were new or recently-conceived, when in fact they could have been in development, waiting in the wings or kept on hold until the political atmosphere was considered to be more stable or at least 
more conducive to acceptance of the innovation. The Language Proficiency Assessment for Teachers is a possible example of this. The initiative was first brought to the public's attention in March 1997 (South China Morning Post, 15 March 1997) with the announcement by the government of the launching of a pilot scheme to determine benchmark language proficiency levels for language teachers. The pilot scheme was not actually put into operation until some two years later. With the subsequent demonstrations by teachers against it in 2000, it was not until March 2001 that the first administration of the assessment was conducted. It would appear that this particular innovation suddenly took off, whereas in fact it had been in development since 1995 after recommendations in Education Commission Report No. 6 (Hong Kong Education Commission, 1995) led to expert consultants being engaged to develop the assessment (Coniam, 1997; Coniam \& Falvey, 1996; Falvey \& Coniam, 1997). Although there is no clear indication that the changeover of sovereignty had any direct influence on the rate of progression of this innovation, what is clear is that the controversial nature of this particular reform measure led to a delay in its implementation, until perhaps a time when the socio-political as well as educational conditions were deemed more suitable. In such situations, innovations often fail to take off at all as delays in implementation allow for counter-arguments and counter-measures to evolve against them.

Referring back to Pennington's (1997b) conception of replacement in terms of teachers' frameworks of teaching (see Chapter 2), the possibility that the changeover acted as a catalyst to change by altering what might be termed the macro-context within which teaching exists, can be considered. As has been discussed, changes in education were ongoing during the period prior to the handover. For example, the medium of instruction debate had been running for several years, but implementation of change had been forestalled as the resource systems responsible for implementation had been unable to agree on the form or characteristics of the innovation, or had been afraid of the possible negative consequences of implementation. The great political change had been anticipated for a long period (since the signing of the Joint Declaration in 1984), but may not 
have driven change in itself until the actual handover was imminent. (Hong Kong people are often characterised as preferring to wait until the last minute before acting, a trait commonly applied by teachers to students.) When the event finally took place, the euphoria surrounding it may have caused there to be a renewed confidence in the potential successful adoption of any changes that might be introduced at that time. Alternatively, previously cautious implementers may have decided that the time was right to take the risk. Either way, the handover acted to change people's perceptions and look at change in a different way. There is some evidence from the investigation that this was a view shared by some educators and members of the public.

The impression gained from the surveys carried out for the investigation is of a desire amongst people to downplay the potential and actual impacts of the handover, as though change (and its corresponding uncertainty) was something to be feared. In the past, unless obvious strains occurred in the fabric of society and intervention was deemed to be necessary, the government was content to let business decide. As Chris Patten wrote about his time as Governor (1992-1997):

Though I was occasionally pressed to intervene in the economy to twiddle like a pseudo-Keynesian with the dials on the macroeconomic control panel, to establish a 'winner-backing' industrial policy, to do anything rather than just stand there - by and large there was a political culture that both understood and celebrated the case for government restraint, for keeping bureaucracy's hands off the management of enterprise. (Patten, 1999, p. 244)

While times were good and the economy was healthy, the government left it to the market to decide. There were measures taken to improve areas such as health care and safety and, as we have seen, education and training, but by and large these were non-controversial and what might be termed 'low-impact' in terms of the creation of conflict within and between the systems or structures involved.

However, the approaching handover and the Asian financial crisis, both of which occurred in 1997, created divisions in Hong Kong society that had not been 
experienced for decades. By the time that these two events occurred, the middle class had grown to become the dominant sector in society. Political uncertainty in the lead up to the handover resulted in many of this group seeking the insurance of an overseas passport, even emigration, whilst those who stayed suddenly found themselves either unable to afford the vastly inflated private housing, or, after the financial crash, holding mortgages on properties that had overnight lost perhaps forty percent of their value. This is in addition to the loss of savings suffered by many as a direct result of the fall in the stock market. This changed the political culture to the extent that people were no longer afraid to make claims on the government, society became more fragmented than it had been and strains were produced that required the government to take action. In August 1998, the government decided to intervene to prop up a falling Hang Seng Index by investing HK $\$ 100$ billion in what many observers felt was contrary to the very nature of Hong Kong society (Ho, 1998; South China Morning Post, 16 October 1998b). ${ }^{3}$

As Hong Kong people faced uncertainty, the value of a good education came more sharply into focus and in particular the added value of a good standard of English. Whilst the political change may have led to an increase in Chinese ethnocentrism and Cantonese ethnolinguistic vitality, the value of English did not seem to decrease. The push for Chinese-medium education, which for educational reasons was supported in the early stages of its development as a policy, became more socially divisive than had perhaps been anticipated as the public began to see their chances of a value-added education, with mastery of English at the core of it, slipping away. The government's reaction was to introduce innovations such as the NET scheme and the LPAT to assure the populace that measures were being taken to address the issue.

The relative success of the main educational innovations to be diffused during the period of the investigation will be discussed in the next section, which looks at the changes in the social system of education. 


\subsubsection{Educational System}

The social system that constitutes (public) education in Hong Kong has been shown to have been undergoing change to a significant extent during the period prior to and following the changeover in sovereignty in 1997. This section first describes the impact of four of the major innovations ${ }^{4}$ affecting English language teaching and learning to be introduced during this period, from the perspective of patterns of change synthesised with the findings from the investigation and the prior research reviewed in Chapter 2. The discussion then moves on to look at how the findings from the investigation fit into a possible model of the educational system with the aim of developing a new model which can represent the continuity and change in the English language education system during the period spanning the handover.

\subsubsection{Communicative Language Teaching}

Although communicative language teaching (CLT) has not been adopted by teachers in Hong Kong to any great degree (as discussed in Chapter 2), as a result of the introduction of the innovation, it could be said that language teachers began to question their methods and there was a renewed focus on the constraints that prevent them from adopting new ideas. This constraints-focused view came out very strongly from all the studies of teachers and could be seen as a kind of reconstruction (Pennington, 1997b) of teachers' perceptions of the teaching context. Due to the introduction of the innovation, the system undergoes change in reaction to the new structure and even though it may not be adopted, it has caused some teachers to alter their teaching frameworks. Teachers become more aware of constraints to teaching and will either adjust or restructure their framework to accommodate the innovation, or they will partially or totally reject it but still change their teaching framework in response to the constraints that previously they may have accepted as the norms of the teaching context but not have considered them changeable. The BATESL programme, which has been at the 
heart of the studies in this investigation and which takes as its underlying philosophy the communicative approach to language teaching and learning, as well as other new courses of teacher training or preparation, both reflected the change process underway in the system and helped to promulgate it (Pennington, Richards, Urmston \& Lee, 1996). There is little evidence to suggest that CLT has been adopted to any greater degree since the changeover than it had prior to it (Evans, 1996, 1997). Concerns over falling standards, particularly of students' writing, have resulted in the claims that CLT is not feasible or appropriate in the Hong Kong English classroom and the features of the innovation: its relative advantage, compatibility, complexity, trialability and observability (Rogers, 1995) and of the user system, particularly teacher and learner factors, educational philosophy and examinations (Henrichsen, 1989) have all worked against CLT becoming established in the Hong Kong educational context.

\subsubsection{The Target Oriented Curriculum}

Educators in Hong Kong have criticised the TOC as being pushed onto teachers without giving them adequate materials and training to implement it (Adamson et al., 2000), a finding supported by the interview surveys carried out for this investigation (Chapter 3). Carless (2001) states that in order for teachers to be able to adopt an innovation such as the TOC, support from the school principal and senior colleagues as well as advisory visits from inspectors, teacher trainers and others is needed. However, evidence from research done into the initiative shows that such support has not been incorporated adequately into it. In other words, it has suffered from a lack of practicality (Rogers, 1995). Teachers may have supported TOC from a pedagogical point of view and may have seen the relative advantage of it, but when faced with the task of putting it into action with inadequate resources to do so, the tendency was to revert to more traditional methods of teaching, even with the 'new' materials that were provided. The TOC has also suffered from a problem of primacy (Henrichsen, 1989). Before the initiative was launched officially in 1991, there had been a number of other 
initiatives promoted by the government to address the same kinds of problems that the TOC was meant to address; thus, teachers had become familiar with the ideas of the activity approach and task-based learning. However, when the new initiative came along, with no reference to those that had preceded it, it created confusion and uncertainty amongst teachers as they tried to relate it to the practices that they had been undertaking:

\begin{abstract}
A perception emerged that there was an absence of a clear longterm policy direction, a tendency to lurch from one fashionable (and imported) initiative to another, an innovation overload, that further ad hoc reforms could be anticipated and that no single reform initiative was supported for long enough to allow it to have an impact on classrooms. (Lo et al., 2000, p. 3)
\end{abstract}

In this case, as with others affecting education, teachers felt that there was a lack of coherence between previous innovations and the new one such that the adopters (teachers) did not know whether to abandon what they had adopted before or try to accommodate the new innovation into the framework that they had built up around the previous innovations. A natural result of this would seem to be that teachers will switch - or criss-cross (Pennington, 1997b) - between aspects of a new approach such as the TOC and an older, more familiar one such as the structural syllabus or even grammar-translation, instead of adopting the new approach or integrating it successfully into their teaching schema.

Native English Teachers Scheme

As evidenced by the studies of the Hong Kong English language education context in Chapter 3, there was some resistance to the Native English Teachers (NET) scheme by local teachers of English, but the impression gained from the teachers surveyed throughout the investigation was that the initiative had been relatively successful in achieving its objective of having at least one NET in every secondary school as an English language resource. The successful diffusion of the innovation may have been because it had not been contingent on the involvement 
or co-operation of local teachers, unlike other initiatives such as CLT, the change in the MOI and the TOC. It was reported by teachers and others closely associated with schools and teacher education that some schools in Hong Kong have experimented with employing native-speaking English teachers by hiring expatriate 'speaking coaches' to teach oral lessons (although these coaches often have no teaching qualifications, unlike the NETs, who must all be fully qualified). By hiring part-time expatriate teachers, schools have been able to determine how best they can utilise their (full-time) NET once s/he is appointed. In this sense, it could be said that the innovation has trialability and flexibility (Rogers, 1995). Thus, schools are able to trial the innovation of expatriate coaches which may therefore have a better chance of succeeding than some other innovations not allowing such trialling. Schools also have the flexibility to make use of their NET(s) as they wish, so enabling them to fulfil whatever role the school authority or principal sees as the greatest need. Other factors that may have accounted for the successful implementation of the scheme are the familiarity of the teacher with the Hong Kong context and the quality of the NET teacher. There was no evidence to suggest that the Education Department had reduced its standards of recruitment of NETs, which may have contributed to the degree of success of the scheme.

Evidence provided by the teachers surveyed suggested that some Native English Teachers have been integrated into the system in that they have been given a full range of teaching, administrative and extra-curricular duties in common with locally-trained (or non-native English-speaking) colleagues. In other schools, NETs have been allotted certain clearly defined roles, such as teaching speaking to every class in the school for one lesson each week. Such a situation could not be described as integration and is in fact a kind of segregation of the NETs and of the teaching of English overall. In this case, as with others, a 'top-down' policy is reinterpreted and redesigned when implemented, creating unexpected and possibly undesirable effects 'on the ground'. 
A perceived problem with using a NET in this way is that only a part of the full framework of skills that they have to offer is utilised and hence their impact on the school is one of accommodation (Pennington, 1997b) - of the NET to the school rather than vice-versa - rather than possible deeper influence, because the teaching approaches, designs and procedures they bring to the new context, typically communicative and interpretational, may not be considered applicable within the local context. Their local colleagues are given the 'more important' task of preparing the students for examinations. In some cases the NETs' methods may diffuse through the system to influence other teachers such that reconstruction or hybridisation (Pennington, 1997b) of familiar and new approaches does occur. However, such a process can only occur over the long term and it will only be possible to determine whether this is actually happening once the scheme has been in operation for a number of years. It is of course also possible that the impact of the NETs will be just what the system administrators would like it to be, that is, improvement in the level of English in graduates of Hong Kong schools without any other (deep) systemic change - i.e., if they succeed in keeping the NETs relatively separate from the rest of the educational system.

\subsubsection{4}

\section{Language Proficiency Assessment for Teachers}

Opposition to the Language Proficiency Assessment for Teachers, and this was expressed by teachers interviewed for the investigation, emerged to a large extent because of a lack of explicitness or uncertainty over the ways it was going to be carried out, which teachers would need to sit for the Assessment and what the consequences would be if they did not (Coniam \& Falvey, 1999; South China Morning Post, 28 May 2000, 11 June 2000). However, despite this opposition, research has shown (again, confirmed by the various studies involving teachers in this investigation) that the majority of teachers support it in principle (Coniam \& Falvey, 1999) as they can see its relative advantage (Henrichsen, 1989) in raising standards. The reasons for resistance against the LPAT appear to be due to other 
factors such as the government's use of the threat of expulsion from the profession (i.e., teachers in government or government-aided schools will not be allowed to teach English) should teachers not be able to achieve the prerequisite standard, either through the Assessment or by completing a training course, by the end of the 2005-06 school year. In this case, the government is hoping that the use of a negative incentive can lead to an increased rate of diffusion and a greater likelihood of adoption (Rogers, 1995).

\subsubsection{Evidence of Change in English Language Education}

The new initiatives introduced into language education in Hong Kong during the time frame of this research would tend to come into the category of having been adapted from initiatives in other countries, mainly (and unsurprisingly given Hong Kong's status as a British colony) the UK. Such initiatives as Communicative Language Teaching (UK and USA), the Target Oriented Curriculum (TOC) (UK), the Native English Teachers scheme (UK) and the Language Proficiency Assessment for Teachers (USA, Canada \& Australia) have been adapted extensively from initiatives already tried in other countries in an attempt to meet the particular demands of the Hong Kong system. Alternatively, it could be argued that the initiatives were adopted as they were what was being done elsewhere - and hence had 'international currency' or face-validity - and not enough consideration was given to whether they would work in Hong Kong and whether they would address Hong Kong's needs. It was clear from the surveys of teachers and tertiary level educators that there were concerns about the compatibility of these innovations with the local system due to differences in teaching culture between the originators of the innovation (the resource system) and the local system, and the impression that the initiatives have been forced onto the local education system without regard to their suitability for local schools, teachers and students. The fact that the majority of these innovations have been derived from British systems and were initiated during the colonial period, may have induced resentment among local educators and resulted in resistance to them, 
at least until the time when they might be more suitably adapted to local circumstances as an augmentation or integration with respect to local teaching methods.

As in most societies, the government has to be seen to be open to the needs of the population, but the population's needs and aspirations vary widely. Students, teachers, parents, principals, school governors, teacher trainers and others in the field all have different perspectives on the ways that education should be organised (Evans et al., 1998) and how and when the innovations should be introduced. Governments often try to compromise and choose 'the path of least resistance' when deciding education policy, seeking, as Morris (1995) says, to preserve their status as well as social order. The demonstrations by teachers in Hong Kong against the LPAT may have persuaded the government to relax the criteria for those teachers who could be exempted from the Assessment due to their existing qualifications (South China Morning Post, 11 June 2000). In other words, teachers, as well as being under increasing pressure as we have seen, were becoming more visible and pro-active as a social group, a point which will be taken up again later in this chapter

\subsubsection{Educational Issues Emerging from the Research Data}

From the studies that have comprised this investigation, a number of potentially important points have emerged which show that education has risen greatly in the public consciousness over the past decade. These points are listed and discussed below:

- Education is primarily vocational or skills-acquiring and is less concerned with the development of the social and/or ethical skills of the student.

Evidence suggests that education in Hong Kong is becoming more practical or vocational, with more attention being paid to training people for practical 
activities. Reflecting trends in other countries, notably the UK, there is an increased attention to Information Technology; increased popularity (from students) of vocational or applied programmes at tertiary institutes, and a loss in popularity of areas of study such as history and the arts, coupled with a reenhanced attention to improving language skills for work or study (i.e., other than for purposes such as travel or reading literature). In Hong Kong the emphasis on vocational study among many pursuing post-secondary education is related to an increase in the provision for tertiary study by greater numbers of the population. This strong emphasis on practicality is occurring despite the intentions laid out in the government's blueprint for educational reform, which states that education should 'enable everyone to develop to their full and individual potential in all areas covering ethics, intellect, physique, social skills and aesthetics' (Hong Kong Education Commission, 1999).

In the past, Hong Kong required a small number of highly educated people to fill management positions in the leading trading houses or the civil service, and large numbers of workers to supply the manufacturing firms with labour. As society's needs have changed, educational provisions have changed with them. Hong Kong is now a financial and distribution centre with manufacturing having moved to Mainland China and elsewhere, where the cost of labour is significantly lower. There is now a need for adaptable, multi-skilled personnel who are able to step into positions in the workplace with the basic skills required for the job and the aptitude to learn the skills that they do not already have. Language skills are rated high on the list of required skills, especially Putonghua - and increasingly other Chinese varieties such as Shanghainese - and (still) English.

- Increased examination-focus in education results from uncertainty in society in relation to the handover and/or the economic recession.

There is evidence of a re-strengthening of Hong Kong's already examinationfocused education system as uncertainty in society due to the economic recession, and also possibly due to the changing political situation, has resulted in an 
enhanced appreciation of the value of qualifications. The continuing importance of examinations was reflected in the washback effects that the changes in the English language public examination syllabuses had on the teaching of English. With the introduction of more interactive speaking components such as group discussion in the Hong Kong Certificate of Education English (Syllabus B) and Hong Kong AS Level Use of English examinations, teachers have reported using more interactive teaching activities in classrooms.

- The lack of intention to change the examination syllabuses results in a 'brick wall' in the innovation process.

Although there was change in the public examination syllabuses for English language during the period of the investigation, and in spite of the washback effect just mentioned above, it was admitted by Hong Kong Examinations Authority officers interviewed for the investigation (Chapter 3 ) that the process of change in these syllabuses is long and it is expected that they are only changed every ten years or so. This means that the consequent changes in teaching methods allied to the examinations do not change over long periods, creating a stagnation in the process of innovation.

Another reason for the slow pace of innovation diffusion in schools is that, as Morris (1995) explains, few schools in Hong Kong seem to have the internal climate and organisation which is conducive to the implementation of curricular innovations, i.e., an open, collaborative culture amongst teachers with support from panel chairs (heads of departments) and principals. In most schools, the principal acts as a virtual 'dictator' and teachers work in isolation. The influence of Confucian ideals of the need for stability - possibly coupled with the influence of conservative Western missionaries and other colonial educationalists as well on the development of Hong Kong education apply, and there exists a reluctance to accept new ideas for fear of disrupting the system and 'making trouble', or creating more work for oneself. Schools prefer to rely on the government (i.e., the 
Education Department) for guidance on curriculum matters rather than being autonomous and adapting to the needs of their students.

- Education has become increasingly politicised.

The attention paid to educational issues from the media has been explored in this investigation (see especially Chapter 3), and there can be little doubt that educational issues have increased in profile within the public domain to the extent that they have become political issues. The major educational innovation to affect Hong Kong during the period, the change in the medium of instruction, received much attention from the media as it encapsulated such a large number of subissues, such as freedom of choice, socio-economic and class differences, language rights and colonialism, which affected a large proportion of the population. As a result, greater interest in educational matters was generated than had been the case previously. The change in MOI was associated with the changeover in sovereignty by many of the educators interviewed (Chapter 3 ) and the BATESL students and graduates surveyed (Chapters 4 and 5), not in respect of causality, but rather as an indication that after the handover, this change would be the dominating educational issue. It seems there was an expectation amongst educators and even amongst members of the public that there would be major changes as a result of or at least at the time of the handover. A change away from English, the colonial language and towards Chinese, the 'home' language, is a change that people might have expected, even wanted. Although those within education were aware of the background to the innovation and realised that in fact its implementation was not related to the handover, the 'illusion' that it might have been so related was difficult to avoid.

The extensive media coverage of the educational innovations which came to public attention after 1999, such as the NET scheme and the LPAT, and which did not have such a direct impact on the general public, shows that educational issues, particularly those related to language teaching, had become high profile and newsworthy and have remained in the public's attention. The politicisation which 
accompanied the changeover and which had been growing as a result of the attempts at increased democratisation of government by the last Governor, Chris Patten, meant that politics could be said to have been driving most aspects of public affairs and that, as a result, education was thrust into the public and legislative domains as never before. At this time, the public became more proactive in educational affairs and there was an increase in claims made against education authorities, against schools and against teachers, who began to have their authority and competence challenged to an increasing degree.

\subsubsection{Language (Medium of Instruction)}

The following section addresses research question (vii), i.e.:

How did the changeover in sovereignty affect the main languages used in Hong Kong, i.e., Cantonese, English and Putonghua?

The language situation in Hong Kong has been discussed at length in this thesis and has been found to be both complicated and a reflection of the socio-cultural and socio-political make-up of the society. During the handover period of 1994 to 1999 that is the focus of this thesis, issues connected with language remained prominent, not only in educational discourse, but also increasingly in political and socio-political discourse. This was clear from the newspaper survey of issues and from the interviews with practitioners and teachers. The language issue that dominated was that of the change in the medium of instruction (MOI) from English to Chinese in the majority of secondary schools. Whilst this enforced change met with some opposition from school authorities and parents, the consensus from educators was that the change was necessary for educational reasons. A picture to emerge from the different sources of data was that at the time of the handover in 1997 , the political changes that were underway were seen by some as being responsible for all changes, including the potentially divisive change in the medium of instruction. The inseparability of language and culture 
meant that some educators found it difficult to believe that the push for Chinese medium of instruction (CMI) was not part of a bigger plan to increase the cultural capital of Chinese at the expense of English.

The group of educators interviewed for this investigation supported CMI, as they believed that the majority of students would inevitably learn more effectively through their mother tongue (Chapter 3). The view was even expressed that students might become more interested in learning English as a subject once the burden of studying other subjects through it was lifted. It was felt that previously, a true English-medium (EMI) system had not been implemented properly in most schools and therefore it would be better to adopt an effective CMI system than an ineffective EMI one. There was some criticism expressed at the time (in 1997) in the press and by interviewees of the way that the government had imposed the change in the MOI. Opponents believed that it was an unpopular move with students, parents and teachers; that it was inconsistent with the view expressed by the Education Department that all students learn better through their mother tongue (so why not make all schools CMI?); and that schools were not given enough time and resources to implement the change properly.

Critics of the change in MOI amongst the interviewees pointed out that the EMI schools would become even more élitist than they already were. These critics made the further point that the reduction in the quantity of EMI schools would lead to élitism within the CMI schools as the best of those students who were unable to enter the EMI schools or whose parents wished to have a Chinesemedium education would gravitate to a handful of schools, which eventually would develop a reputation as being 'the best' CMI schools. On the other hand, there was support for the initiative from the school principals interviewed, who recognised the educational and administrative advantages of a mother-tongue education system. Two years after the announcement of the change and one year after the implementation of it began, there was more discussion during the interviews of the effects of the change on teaching and the curriculum, and on 
education more generally, showing that by 1999 , considerations had shifted from political to educational and social.

According to the government, the intention of the MOI policy was to ensure a clearer demarcation between the languages used for teaching (Hong Kong Education Commission, 1990, 1995). Observers of education in Hong Kong pointed out that the result of the change would be that the majority of schools could continue doing what they had always done - teach in Chinese (Cantonese) while the élite schools, those which regarded themselves as truly English-medium, could re-emphasise and if necessary strengthen their commitment to English and strive to maintain their higher status within the community. It was suggested by some of the teachers who took part in this investigation (Chapters 3 and 5) that within schools designated as English-medium after the imposition of the new MOI policy, the use of English only within classrooms was being strictly enforced. However, the evidence from the observations of teachers contradicted this assertion, finding that in EMI schools the use of the mother tongue was becoming more widespread rather than less as teachers found it increasingly difficult to maintain students' interest and attention when using the second language for teaching purposes. The furore, partly generated by the news media, surrounding the 'awarding' of English-medium status to 114 schools in 1997 and the promises made to education authorities and parents that an English-only policy would be strictly enforced seemed to have been quickly forgotten as the realities of teaching took control. In principle there may have been an Englishonly policy, but in practice it was found and reported that Cantonese was still being used for many purposes, just as it has always been. In terms of the patterns of change outlined in Chapter 2, the situation could be described as a regression to the past, or a regression to baseline (Pennington, 1997b, 2002) as teachers returned to their old patterns of language use once the initial period of heightened public attention to the new requirement for use of English only in some specially designated schools had passed. 
The lesson observations carried out for the investigation showed that within (CMI-designated) schools, teachers of English, such as the graduates from the BATESL course, were making use of Cantonese to facilitate their teaching, often at the insistence of their students. English teachers reported being encouraged to use English as the teaching medium within English lessons, whilst being given a free hand to use Cantonese if required. The result of this ambiguity was that the teachers felt demoralised, as they were experiencing conflict between their belief in using the target language for communicative purposes, and the reality of having to rely on Cantonese to present the target language as isolated, de-contextualised forms. In cases such as those observed for this study, teachers might attempt to use English but then resort to using Cantonese when required, resulting in a disorderly pattern of change and non-change occurring simultaneously (Chapter 2) rather than a pattern of strategic bilingual language use. Previous research indicates that more experienced teachers, such as those that had been teaching before the change in the MOI, would be expected to have developed a pattern of strategic classroom language use that would be resistant to change, even if the policy of the school changed or if they moved between schools with different policies.

It was clear from the different sources of data of this study that the mother-tongue education issue is an example of double-voicing (Pennington, 1997b), in that the policy is supported in principle but not adopted in practice. Previous research has found that whilst educators, parents and students indicate that they support the principles of mother-tongue education in Hong Kong (Morrison \& Lui, 2000; Tung et al., 1997) given the choice, schools will opt for English-medium due to its perceived higher status and the fact that they will attract more able students (Morrison \& Lui, 2000; Ng, 2000). In addition, parents will do whatever it takes to get their children into English-medium schools, and students will regard themselves as inferior or 'second class' if they fail to get admitted to an Englishmedium secondary school ( $\mathrm{Li}, 1999)$. In this case there is evidence of a culture which prevents the support of the mother-tongue approach; that is the 'addedvalue culture', which places socio-economic success above intrinsic value of the 
mother tongue and views language (particularly English) as power (Fairclough, 1989). The conclusion to be drawn is that in an 'ideal world' the Hong Kong Chinese might prefer to use Chinese in written and spoken varieties for all modes of communication, but in the real world parents realise that English is a definite value-added factor for their children's future success. Their ideal is for 'perfect bilingualism' but they will settle for the practical solution of a 'split world' of private Cantonese and public English.

However, many observers of Hong Kong education have noted that the push for Chinese-medium education, in the context of the increased usage of Chinese in various functions of society, the increased ethnolinguistic vitality in Cantonese and the promotion of Putonghua, have resulted in an elevation in Chinese linguistic capital in Hong Kong in the past few years (Morrison \& Lui, 2000). The most highly valued symbolic capital in the educational domain and several other high-status public domains was always English under colonial rule, and the importance of English in the Hong Kong marketplace (linguistic and otherwise) still holds. The amount of attention given to the subject of English standards has not diminished, as new initiatives such as the Language Proficiency Assessment for Teachers, the Native English Teachers scheme and the Workplace English Campaign ${ }^{5}$ prove. However, it is clear from the findings of this investigation that the several forms of Chinese in Hong Kong - Cantonese, Putonghua, and the written forms of these - have all increased the value and supply of their own symbolic capital. The profile of Putonghua has certainly been raised, with the Language Proficiency Assessment for Teachers (Putonghua), the equivalent of the LPAT for English teachers, being developed as a means of ensuring the quality of Putonghua teaching in schools.

Given the possible changes in the balance of power of the main languages used in Hong Kong, it would follow that the priorities of the system (educational and/or social) might also be changing. If the value (symbolic or otherwise) of English is becoming reduced in the wake of the increased value (symbolic or otherwise) of Chinese, society's requirements for English may be changing. With the increased 
political ties with Mainland China since the changeover as well as the opening up of the Mainland commercially and the improved infrastructural links between Hong Kong and the Mainland, it follows that an increasing amount of Hong Kong's external dealings in terms of trade and politics are now with China, rather than with the wider international community. To facilitate these dealings, Chinese language skills (not only Cantonese and Standard Written Chinese but also spoken varieties such as Putonghua, Shanghainese, and other varieties) are needed, and the evidence from the interview studies in particular is that proficiency in these is becoming more highly valued. People still need English for its value in furthering their job or career prospects when interfacing with the international community; however, English is increasingly seen as just one of the desired languages and has perhaps even fallen behind the Chinese languages in relative importance or symbolic value in Hong Kong. On the other hand, as Hong Kong universities (other than the Chinese University of Hong Kong) still seek to maintain a strong presence of English and as the PRC seeks to increase the level of English among its university students, English still retains considerable value in Hong Kong as well as larger China of which Hong Kong is now a part.

Whilst there has undoubtedly been an increased focus on Chinese since the handover, the government of Hong Kong is aware of the need to be seen to be addressing concerns about falling standards of English. Inter-city rivalry with other major trading centres in the Asia-Pacific region such as Singapore and Shanghai features consistently in the discourse connected with standards of English, as these cities (and others such as Beijing and Guangzhou) are reputed to be gaining ground on (or even ahead of) Hong Kong in terms of the eagerness of the population to learn English. Consequently, there has been a renewed push to improve English standards as a key feature in maintaining Hong Kong's position as what the people believe is 'an international city'. New initiatives have been introduced in an attempt to raise standards of English of the general public to levels acceptable for the functions that they need to carry out in their own particular working situation. This pattern of change could be characterised as a switching or flip-flop (Pennington, 1997b) between attention to Chinese in the 
wake of the handover, followed by renewed attention to English as a result of commercial or economic factors, which is perhaps part of a longer-term tendency to switch periodically between an emphasis on English and Chinese in public discourse that represents the Hong Kong people's divided loyalties and ongoing linguistic dilemma (see Pennington's 1998a discussion of the 'psychological duality' of Hong Kong bilingualism).

\section{Synthesis of Findings Concerning Language}

The possible change in the balance of power of the languages of Hong Kong is a manifestation of the social changes underway during the handover period. Whilst the methods used to teach language must change with the changing requirements of society, they must also change to reflect the relative importance that society places on the language(s) in question. The call in Hong Kong for 'trilingualism in Cantonese, Putonghua and English and biliteracy in Chinese and English' sounds straightforward; however, as we have seen, the language situation in Hong Kong is complicated, given the multitude of historical, social, political and economic factors involved. To large numbers in the population, changes in the relative status of the languages in use in society represent changes in the structure of society itself, as with language comes capital, both real wealth and the symbolic capital associated with education and social status. Increased symbolic capital or economic value of Chinese might represent closer ties with the Mainland, or greater isolation from the international community, if it is associated with a fall in the knowledge of English within the community. Such changes, if imposed by policymakers, are subject to resistance from social groups who perceive that they have something to lose. Change agents will be called into action and their efforts will go a long way to determining the future pattern of language use in society. 


\subsubsection{Teachers}

As a social group, the teachers of English in Hong Kong have constituted the major thread of this investigation. A picture of teacher change during the period surrounding the changeover in sovereignty has emerged by focusing on a group of trainee English language teachers as they passed from students to student teachers and then to novice serving teachers. The picture has been enhanced by an examination of the issues that affected teachers during this period and the opinions and insights of experienced teachers, teacher educators and others in prominent positions in education in the SAR. The picture that emerges is one of both internal conflict within teachers themselves and conflict between teachers and other social groups within education. The result of these conflicts has been that the innovations imposed on Hong Kong teachers of English have in some cases met resistance (e.g., TOC, LPAT) and the diffusion of them has been negatively affected, while in other cases innovations have been relatively successfully diffused (e.g., MOI, NET scheme). Conflict between teachers and education authorities has resulted in resistance to innovations such as the LPAT. On the other hand, conflict within teachers who are unsure as to whether they support an innovation or not has meant that they have not formed a cohesive, resistive cohort in opposition to an innovation resulting in its relatively easier diffusion. Likewise, when some teachers support an innovation and some do not, opposition is fragmented and sporadic and not sufficiently strong to resist diffusion.

From this perspective, teachers as a group have become crucial in the diffusion process as they are the 'user group' (Henrichsen, 1989) and are the ones who are charged with the implementation of any new initiative 'on the ground'. As has been discussed, educational policies are imposed on the system in a top-down manner in Hong Kong and teachers are expected to carry them out under the auspices of their school principals and panel chairs and within their particular teaching contexts. Yet the image of the teacher as a powerless purveyor of education policy is not the one that has emerged from the investigation. Instead, it 
is evident that teachers (of English) have a greater degree of autonomy within their classrooms than perhaps they are prepared to admit. Whilst it is true that they have the constraining demands of school authorities, parents, students and examination-driven syllabuses to meet, their actual teaching methods are in most cases theirs to decide. To this extent, the teacher has become in reality more empowered such that the success or failure of any teaching innovation is dependent on them. The innovations that have featured throughout this investigation, in particular the TOC and the LPAT, show that unless the teachers are prepared to adopt the innovation, it is bound to fail. Hence, at a surface level teachers appear to be under pressure and to have suffered a reduction in their relative power within the educational system. At the same time, at a deeper level there is evidence of a greater empowerment as teachers become more pro-active and more aware of their individual and collective ability to determine educational outcomes.

The evidence suggests then that English teaching is becoming more politicised in Hong Kong. Teachers as a group or social system have come to realise that they can affect the progress of educational reform and the educational authorities have become more sensitive to their demands. Since the changeover in sovereignty, the Hong Kong government has become more conscious of its image and the need to be seen to be enacting policy that is in the public interest. The public and the business sector have demanded action to address a perceived fall in standards of English, so the government has taken steps towards this, with the inevitable result that teachers are held responsible and must then raise their professional standards. In addition, changes in the curriculum must also be enacted with the cooperation of teachers. It appears that teachers are no longer prepared to accept what is imposed on them and simply 'get on with it', instead, they realise that they have the power to influence policy in their or their students favour and are more prepared to do so. Whether this change in the political will or mobility of teachers is as a result of greater democratic motivation within society generally, perhaps as a result of pre-handover political reforms, or as a counter-reaction to perceived 
lack of democracy on the Mainland (a reaffirmation of the 'one country-two systems' scenario) is open to debate.

At the heart of the greater empowerment of teachers within the educational system are issues of conflict. Where there is conflict, change is likely to occur as different actors within the system strive to resolve the conflicts by enacting change measures. The areas of conflict felt by the teachers studied emerged from Chapter 4 and are supported by the findings from the other studies of the investigation as well as the prior research surveyed in Chapter 2. The conflicts are summarised below:

- Conflict between the desire to perform as a professional and the sense of having to conform to the constraints of the teaching situation in Hong Kong schools.

Teachers would like to spend time planning their lessons, developing teaching materials and pursuing professional development but given the heavy workload they do not have the 'space' which would allow them to do so. Consequently, they usually improvise and compromise their own teaching beliefs and standards.

- Conflict between the desire to be innovative and the constraints of the teaching culture.

The teaching culture in Hong Kong is product-oriented or ends-driven and teachers do not feel that being innovative in teaching, or adopting a more processoriented (interpretational) or means-oriented approach, is compatible with that culture.

- Conflict between the desire to develop professional relationships and the culture within schools which does not facilitate this. 
Teachers recognise the value of good professional relationships, especially to a teaching novice, but find the teaching culture to be isolationist. Even experienced teachers in many Hong Kong schools do not like to share teaching ideas or procedures or to give advice or assistance to less experienced colleagues for fear of losing credibility should their practices be judged inappropriate or their skills questioned. There is also little time, space, or encouragement from administrators for teachers to share ideas, thus little incentive to do so.

- Conflict with regard to relationships with students.

Teachers wish to be a friend and mentor to their students (in the form of a masterand-apprentice relationship) but are afraid of losing their authority and generating discipline problems.

These conflicts were felt by the novice teachers graduating from the BATESL course (Chapters 4 and 5) and could be seen as expressions of the uncertainty felt by young people about to enter the teaching profession. Yet the evidence suggests that the same conflicts are encountered not only by new teachers but also by more experienced teachers in Hong Kong (Chapter 5) as well as elsewhere (Bullough, 1989, Galton, 2000; Hargreaves, 1999; Huling-Austin, 1992; Pigge \& Marso, 1997). If experienced teachers do not exhibit uncertainty in the same way as novices do, then the reason may be that the experienced teachers have learned to accept the constraints inherent in the prevailing teaching situation. Their teaching frameworks will have incorporated these constraints and the teachers will have learned how to live with them and adapt their teaching practices to cope with them.

The issue of teachers' relationships with students featured prominently in the findings from the questionnaire and interview surveys (Chapter 4). The evidence from this investigation suggests that teachers were becoming more sympathetic towards students, more understanding of their problems, and that they were showing a tendency to view students in a more positive light than they had done 
previously, before the onset of the educational and social changes of the mid-tolate 1990s. Perhaps teachers had come to realise that it was not only they who were struggling to come to terms with the many changes taking place in education, but their students also had to cope with them. In other words, there was enhanced solidarity between teachers and students. Another possible factor is that the student-centered approaches espoused by teacher education programmes such as the BATESL course had begun to have an effect on teachers in changing the culture from one of respectful hierarchical distance between teacher and student to a more complex relationship in which the teacher at various times serves the role of instructor, mentor, parent, castigator, social worker, friend and perhaps other roles as well. In some cases there was evidence of a caregiver or mothering stance in which (secondary) students were regarded as children who needed to be nurtured and who could not be expected to make decisions for themselves. In other cases teachers bowed to student pressure to conform to their desires in terms of classroom activities or language use. In this sense, students were perhaps becoming more demanding, possibly due to the influence of what might be termed the gratification-on-demand culture, in which young people expect to be entertained in school in the same way that they are at home when they switch on the television (by popular local culture such as pop music and TV drama). Alternatively, the rise in the popularity of tuition schools offering instant examination success perhaps meant that some students expected the same instant success from their regular teachers. In this sense, the teachers in schools may have felt obliged to compete with tuition school teachers in order to prove their professional standing and credibility with their students. The result of these changing demands on teachers is that they have had to become more responsive or 'tuned in' to the students than they might have been in the past when teacherstudent roles and relationships were more clearly defined.

There was a strong feeling from the teachers studied that they were under more scrutiny than teachers had been previously as teaching standards began to be questioned, especially (English) language teaching standards, resulting in new initiatives such as the Language Proficiency Assessment for Teachers and the 
Native English Teachers scheme. Some of the teachers have felt insulted or even threatened by such initiatives, feeling that they were being blamed for the perceived decline in language standards. It might be postulated that this may have agitated the locally-trained teachers of English enough to become less cohesive as a social group, in which case they may have become more open to the diffusion of the reforms or innovations, or, more likely, to have closed ranks and become more cohesive and more resistant to the new initiatives. The sight of an estimated 6000 teachers demonstrating against the LPAT in 2000 shocked many Hong Kong people and showed that many teachers had become agitated enough to take collective action. As discussed earlier, Hong Kong had enjoyed a period of relative social stability coinciding with the economic prosperity of the $1970 \mathrm{~s}$ and 1980 s and such protests, especially from public servants, was still considered unusual. Opinion leaders such as Professional Teachers' Union President and Legislator, Cheung Man-kwong, played a major role in organising this action. Such opinion leaders and change agents (Rogers, 1995) are very influential in such a small (geographically) and closely-knit society, in which social groupings such as teachers tend to be cohesive by nature due to the homogeneity (in terms of ethnicity, language, educational background and social class) of the individuals.

In addition to individuals acting as change agents, institutions, departments within them and courses of instruction such as the BATESL and their instructors can perform the role of change agents by sending teachers out into the schools with innovative teaching methods that can then be diffused through the system. There was some evidence shown (Chapter 5 ) of the graduates from the BATESL course acting as disseminators of innovations within their schools. Given the reported finding that the majority of English teachers in Hong Kong are unqualified, those that are - even if they are fresh graduates - may in many ways be seen as 'experts' who can provide leadership in methodology. More experienced graduates of courses like the BATESL, such as the two studied in Chapter 5, can quickly rise to positions of influence within their teaching panels by virtue of their perceived expertise and advantage over their non-qualified colleagues. In this way, there is some indication of the potential influence of such courses of training 
on the teaching community. As came out of the interviews of ELT practitioners, the expansion of the numbers of Native English Teachers in schools (from initially just secondary schools to primary schools also) may also serve to foster change and innovation within teaching as these new teachers coming from outside the system bring new ideas and practices which may be diffused through the system and adopted by local teachers. However, the feeling expressed was that the extent to which the NETs become change agents depends on the degree to which they are integrated into the schools and the communities or social systems of teachers.

Prior to the changeover in sovereignty, the majority of teachers (and other educators) surveyed did not think that the handover would affect them directly, although they did think that changes within education that might be related to the handover would affect teachers. After the handover, the evidence was that the curricular and educational changes that were being diffused were affecting teachers, but whether the handover itself resulted in the replacement (Pennington, 1997b) of teachers' methods through the implementation of curricular reform at a systemic level or innovation on an individual teacher level is not clear. As mentioned earlier, teachers as a social group appeared to become more empowered after the handover as the educational reforms put them under increasing pressure and called their professionalism into question.

On the surface, the popular notion is of Hong Kong society being utilitarian and oriented towards stability (Lau, 1982: Wong \& Lui, 2000b), a notion refuted by Pennycook (1998) as exemplifying colonial discourse designed to serve the interests of the colonisers. During colonial rule, in order to preserve stability, the government was keen to avoid interfering in the structure of society, instead allowing it to develop such that the needs of the Crown and her subjects were served first and those of the general (mainly Chinese) population served next. Political and social unrest did occur, as we have seen, but during periods when the empowered social groups in society were not adversely affected by unfavourable conditions or agitated by other social groups, stability did ensue. In other words, 
the 'deeper structure' of society could be said to be of social groupings content to retain the status quo unless their position is threatened by worsening conditions or challenge from other groups. Within this deeper structure are social groups such as teachers who have seen their position being threatened by the imposition of incompatible reform measures and through challenge from other groups such as educational authorities, school administrators, the media, parents and students. Their responses, as described in this thesis, have manifested themselves as conflicts and have ranged from personal, psychological unease to organised, cooperative protest action. The surface structure shows teachers taking action due to the changing deeper structure of alterations in the 'balance of power' between social groups. The next section will attempt to illustrate the structure of the area of society related to English language education through the development of a model based on the findings from the investigation and from other research into the Hong Kong context.

\subsection{A Model for Change in English Language Education at a Time of Political Change}

Taking into account the findings from the different strands of this investigation and with reference to other models of educational change, it is the intention here to put forward a new model which might illustrate the various factors and relationships involved in the changes in English language education that occurred during the period in which one society, Hong Kong, changed politically - from its status as the last of Great Britain's colonies, to that of the next in a growing number of Special Administrative Regions of the Peoples' Republic of China.

The sub-systems that constitute the education system of Hong Kong are shown in Figure 6.1. 


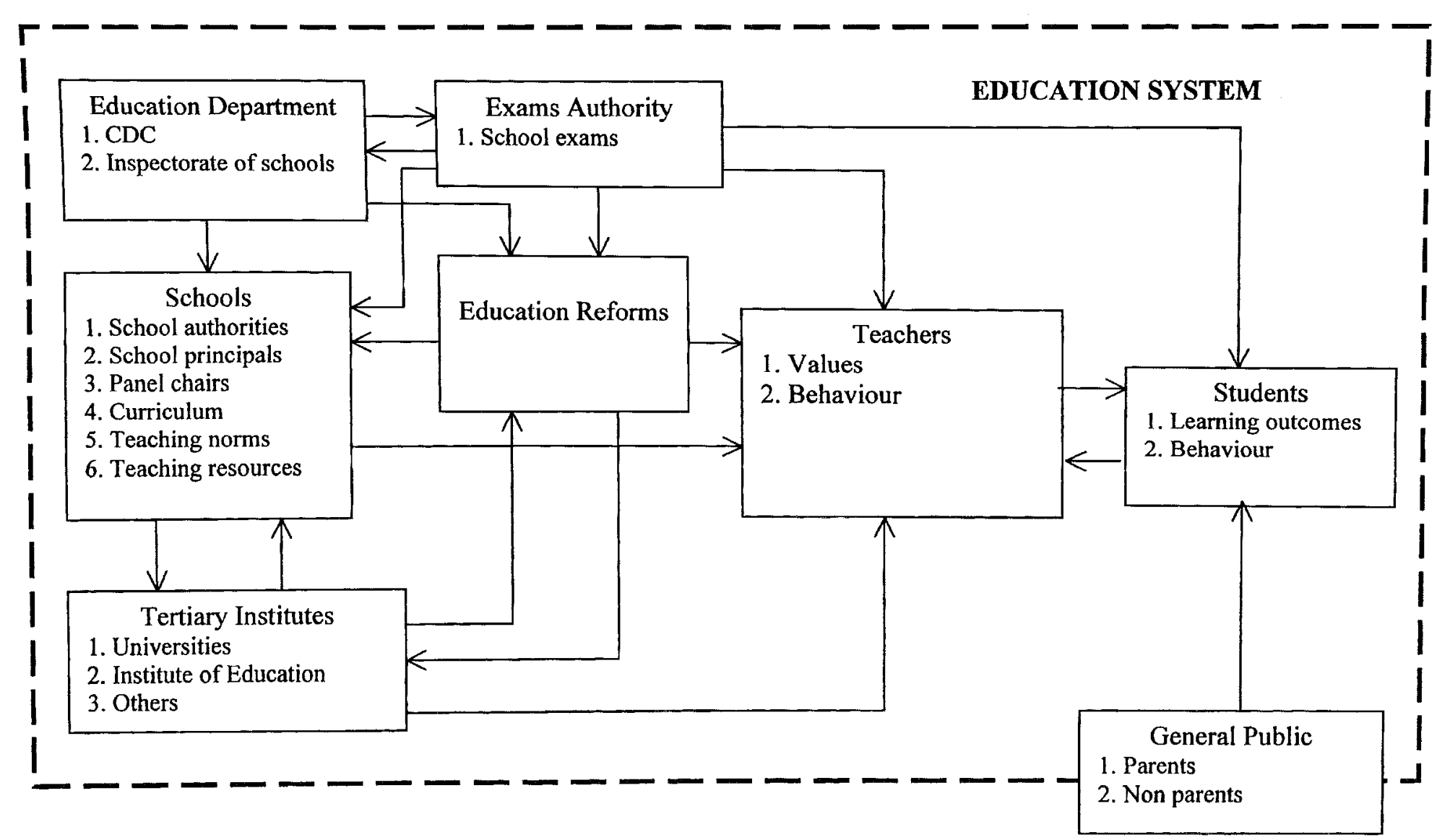

Figure 6.1: Model of the English language education system in Hong Kong 
The sub-systems which have been shown to constitute the English language education system in Hong Kong consist of the government's agents, the Education Department and the Examinations Authority; the institutions within which learning takes place, i.e., Schools and Tertiary Institutions and their administrators; the core groups involved in the practice of education, i.e., the Teachers and the Students; and as a separate sub-system, Educational Reforms or initiatives. Individual features of the Education Department, Schools, Tertiary Institutes, Teachers, Students and the General Public are also shown. ${ }^{6}$ In addition, the sub-system of the General Public is shown as occupying a position on the boundary of the Education System, reflecting that the public are both within education as parents or simply as concerned citizens, and outside of it in other sectors of society. The border of the Education System itself is shown as a broken line to indicate that it is not a closed system and is influenced by other systems in society such as business, health, transport and so on.

The causal relationships between the sub-systems are indicated by arrows showing the directions in which the relationships manifest themselves. There are two-way relationships of influence between the Education Department and the Examinations Authority, showing that these two organisations work together to set syllabuses and to regulate the flow of students through the Education System (or out of it) by means of public examinations. There is also a two-way relationship between Schools and Tertiary Institutes as schools are ever mindful of the requirements of the tertiary sector when preparing students for it, and the institutes are structured so as to provide a smooth path for students moving between the two sectors. The Tertiary Institutes are also involved in the development of Educational Reforms, through the expertise employed by the educational authorities from the institutes, and the reforms themselves will feed back influence on the tertiary sector though the changes in teacher education that are needed to follow the reforms implemented in schools. The final two-way relationship is shown as that between Teachers and Students. As the studies of teachers in this investigation have shown, teachers in Hong Kong are studentcentered and to varying extents their teaching is oriented by the students. 
One-way relationships shown in the model in Figure 6.1 suggest how some subsystems act in a top-down manner, such as the Education Department impacting on Schools and the Educational Reforms, the Schools and Tertiary Institutes impacting on Teachers, the Examinations Authority impacting on Teachers and Students, and the Reforms impacting on Schools and Teachers. Such one-way relationships show the lack of integration within certain parts of the system, particularly from the point of view of the Teachers, whose only direct causal influence is on Students. These teachers thus have no direct influence on Reforms or other components of the system directing their behaviour from the top-down, though they have considerable influence after the fact and on the ground, at the grassroots level. In fact, the teachers are the key influence on whether anything from the top-down ever gets into the system at more than a surface level, and in particular, in terms of what the outcomes will be in the students, and thus in the whole society eventually. The General Public are shown as influencing Students through the efforts of parents and possibly others such as other family members and friends.

There are sub-systems that exert influence on the education system from the outside. Most prominent is the government. The Hong Kong Government itself can be considered of consisting of three components: the Chief Executive (or prior to the changeover, the Governor), the Executive and Legislative Councils, and finally the Bureaux that deal with the day to day business of deciding policy. Of the bureaux, it is the Education and Manpower Bureau (EMB) that is charged with overseeing education in the Territory. In the model of the system influencing English language education, the EMB is represented as a separate sub-system to show the extent of its influence. In addition, as the government also influences education indirectly through means other than policy (i.e., through policy in other, related systems such as health, transport or security), a causal relationship is indicated (Figure 6.2). 


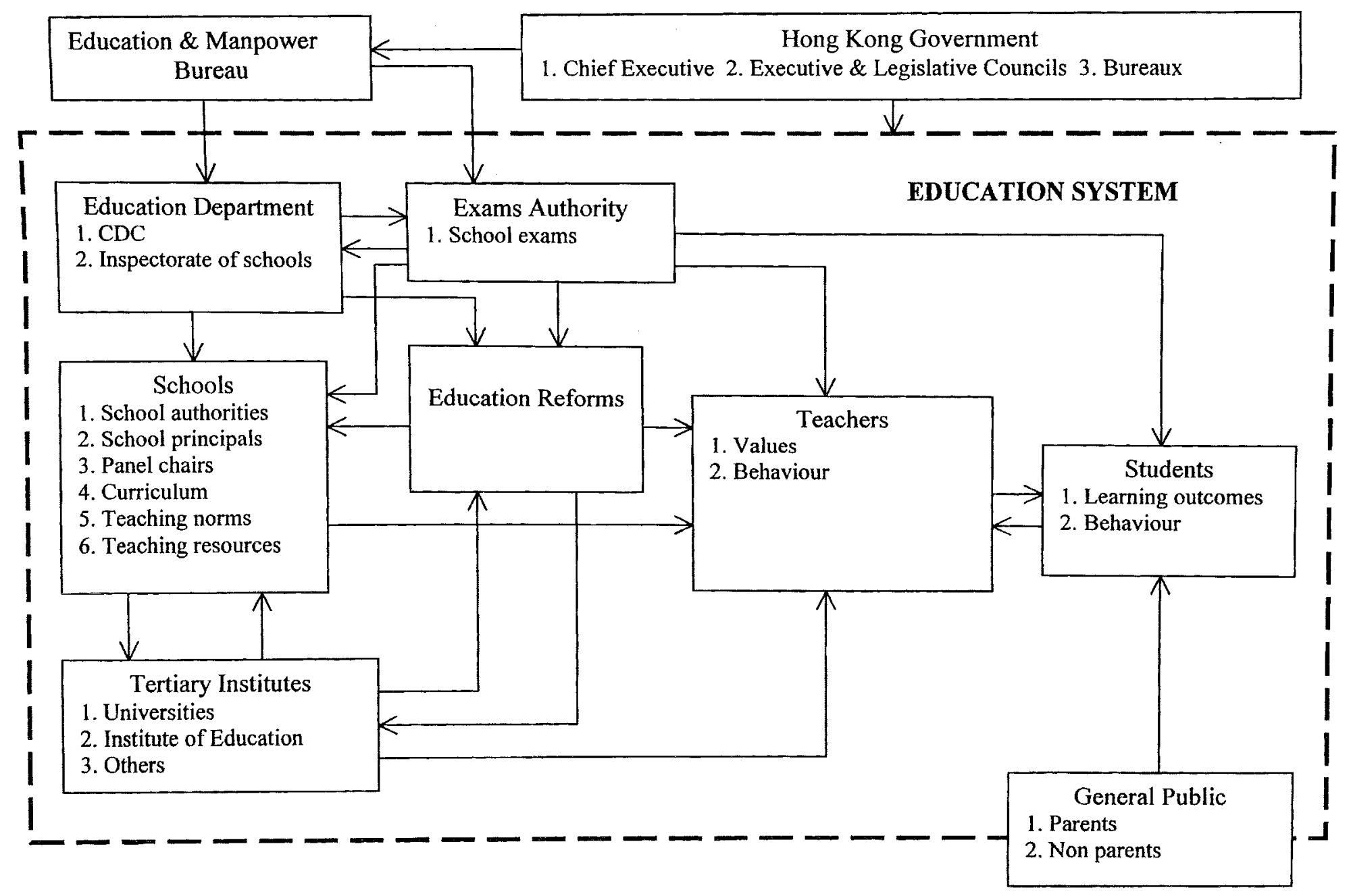

Figure 6.2: Revised model of the English language education system in Hong Kong 
In addition to the government, there are two other sub-systems that have been shown to have a major influence on English language education, the Media and the Business sector. The studies of the newspaper coverage of issues in 1997 indicated the extent to which the news media can influence education by helping to determine the social reality for the population and forcing government and other systems to take action. As is shown by the arrows in Figure 6.3, the Media influence education directly and is in turn influenced by it. The Media also have a strong influence on the public. The sub-system of Business can be used to represent employers generally and as such has a very marked influence on English language education. Employers need people who are proficient in English and hence their requirements greatly influence both policy and practice within the Education System. The non-causal link between the Media and Business indicates the fact that the media are privately owned and run concerns (and are in fact businesses themselves). Although there is undoubted political influence on Business and the Media, this influence is not clearly defined in terms of its effect on education and so no link will be drawn between Government and these components in the model.

Given the internal structure of the Education System and the external influences on it, it is necessary now to consider the context frames within which educational changes take place. Pennington's (1995c) outer context frames of 'Culture' and 'Society' (see Figure 2.9) can be combined and referred to as 'Societal Structure', in line with the characterisations of Hong Kong society and culture discussed in this thesis. Within this context frame can be placed the Education System and the bodies that influence it from within the Societal Structure but from outside the Education System itself.

The final influences that have been shown to have played a part in changes in English language education in Hong Kong are those outside of the Societal Structure, i.e., Great Britain and Mainland China. These two states are shown on either side of the outer context frame (Figure 6.3). 
HONG KONG SOCIETAL STRUCTURE

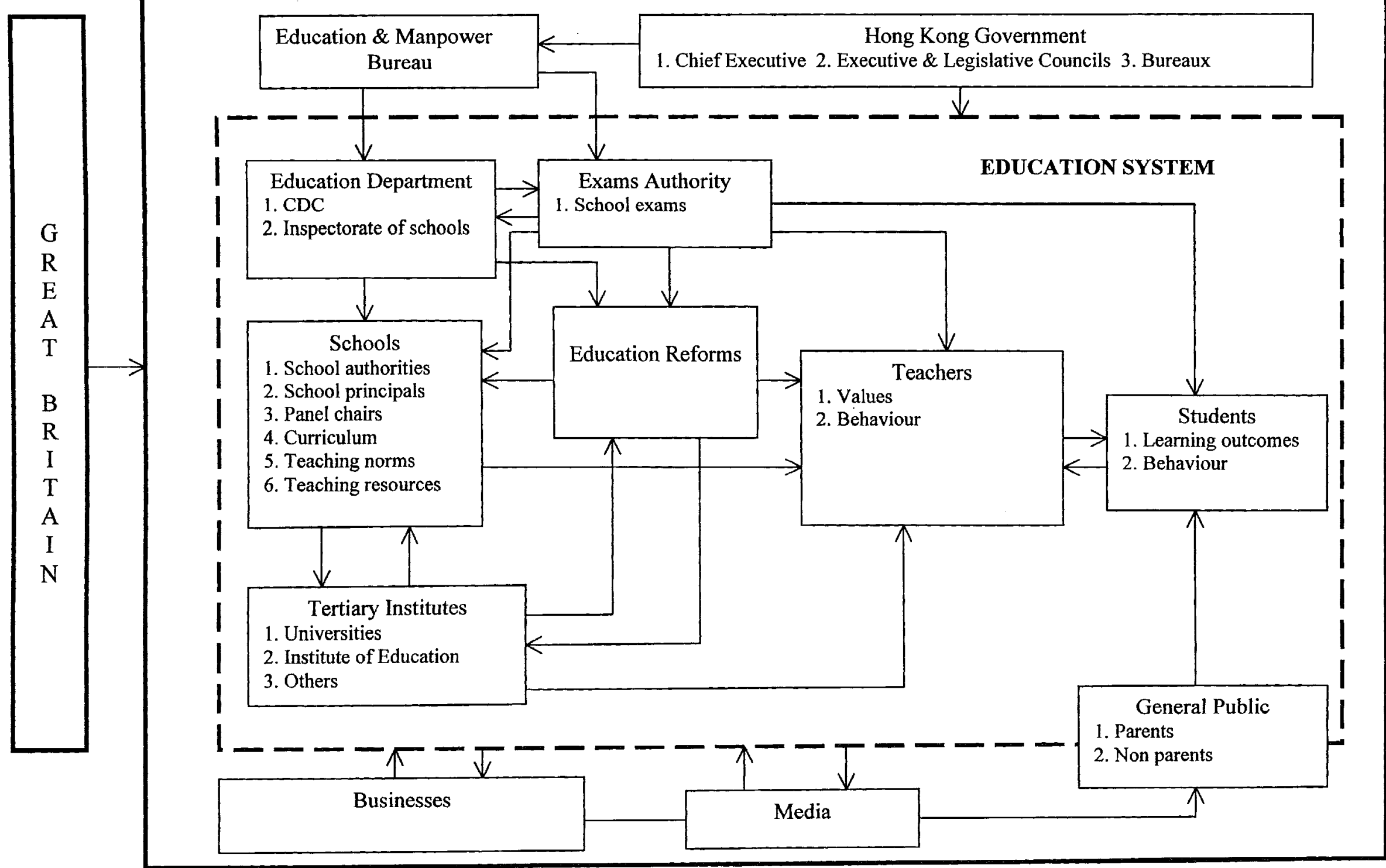

Figure 6.3: Complete model of English language education system in Hong Kong

$$
6-44
$$


Figure 6.3 shows the complete model of the English language education system in Hong Kong. The model is complete in that it shows the sub-systems within the system and those external to it and the causal relationships between them. However, the model is not fully representative of the situation for two reasons. Firstly, more detail needs to be added to the sub-systems that underwent change during the period of the investigation as described in this thesis. Secondly, the model does not show the degree of influence that any one sub-system has on another. Further, the model can only represent the situation at one given time. To indicate change in the situation, it will be necessary to modify the model based on observations and research data. In respect of the main research question guiding this investigation, to assess the effect of the changeover in sovereignty, two models can be produced, one before the handover and one after such that changes can be visualised.

Figure 6.4 shows the complete model for the period prior to the changeover in sovereignty. To illustrate the situation prior to the handover, the Educational Reforms in place or under development at that time have been itemised and they are communicative language teaching (CLT), the change in the medium of instruction (MOI) and the Target Oriented Curriculum (TOC). Further, in line with the analysis of teachers carried out in this investigation, the Teachers subsystem can be sub-divided into the five areas of the questionnaire studies (Chapter 4). The causal relationships between sub-systems are indicated by arrows showing the direction in which the influence occurs. The relative thickness of the arrows indicates the degree of influence that one system had on another during this time. It can be seen that prior to the handover, the government was exerting a relatively strong influence on the Education System, both directly and though policy from the Education and Manpower Bureau. The Education Department and the Examinations Authority were both influential in terms of being the resource systems (along with tertiary institutes) for the educational innovations or reforms that were in the process of being diffused at that time, but the latter less so as public assessment had not had a great influence on the reforms in question at that time. The influence of the Tertiary Institutes on the reforms was also relatively 
strong at this time due their role as developers of the reform initiatives. The Tertiary Institutes also exerted strong influence on Teachers through the preparation that they provided.

Given the examination culture present in Hong Kong schools, the Examinations Authority was a major factor affecting both Teachers and Students - the former through the influence that examinations had on the teaching approaches and designs of the teachers, and the latter as success in examinations was seen as the main motivation for schooling. Outside of the Educational System, significant roles were played by the Media and by the Business or commercial sectors. Lastly, the two nation states with the most interest in Hong Kong, Britain, the colonial ruler, and China, the future sovereign power, both exerted great influence as has been discussed in previous chapters. 


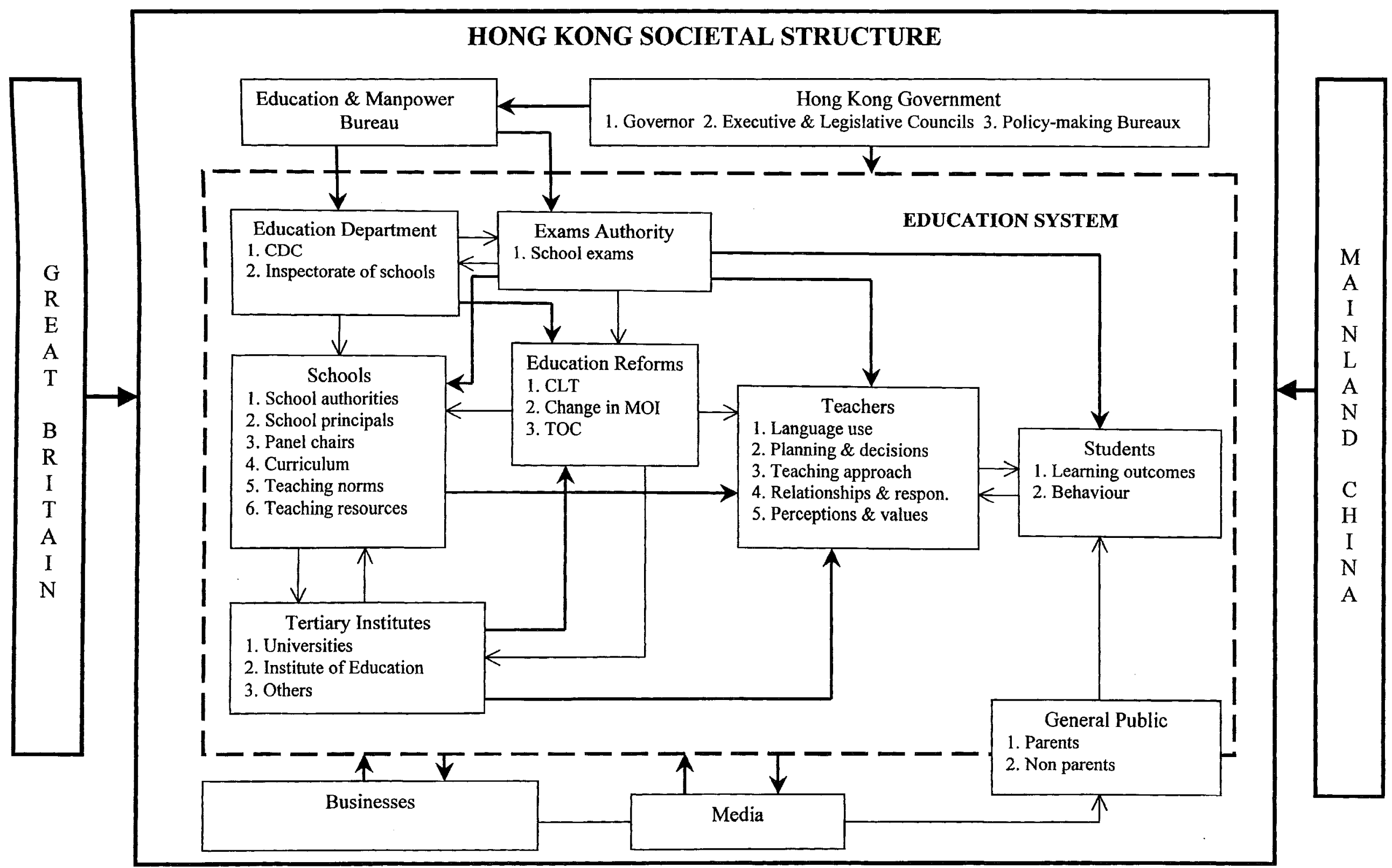

Figure 6.4: Model of English language education system in Hong Kong prior to the changeover in sovereignty

$$
6-47
$$


The model can be revised so that it applies to the period after the changeover in sovereignty, when Hong Kong had become an SAR of the People's Republic of China and more educational reforms began to be introduced (Figure 6.5). The following changes have been made in the model. Firstly, the items of Education Reform have been changed to indicate that after the handover the change in medium of instruction became the most significant reform measure and the NET scheme and the LPAT came into prominence. In connection with the latter, the LPAT has been added to the Education Department and the Examinations Authority as it is these two bodies who were charged with implementing the initiative. The changing degrees of influence are shown by changes in the thickness of the arrows connecting the various sub-systems.

The increase in the thickness of the arrows connecting the Hong Kong Government to both the Education and Manpower Bureau and the Education System shows that after the handover, the influence of the government on education increased as educational matters became more politicised. There was also an apparent increase in the extent to which Educational Reforms affected English language education, and these reforms seemed to have a greater impact on schools and on teachers. Particularly, the change in the MOI began to affect schools whilst the LPAT affected teachers. These changes are modelled as an increase in the thickness of the arrows from the Education Reforms to Schools and to Teachers. These reforms raised the profile and importance of the Education and Manpower Bureau and the Education Department and Examinations Authority, which is shown by the increase in thickness of the arrows joining the EMB to the other two bodies as well as those connecting these two bodies together. Further, the thickness of the arrows connecting the Education Department to Schools has been increased to signify the increased pressure put on schools by the ED in relation to reforms such as the MOI and TOC. The thickness of the arrows between the Examinations Authority and the Reforms and also between the latter and the Teachers have been increased to show how the Exams Authority increased its profile within the Education System (due to its involvement in the LPAT initiative). 
The arrows between the Teachers and Students sub-systems have both been increased in thickness to signify the apparent increased intensity in their relationships, with teachers seemingly more sympathetic to students' needs and students in turn more demanding of teachers. An arrow has been added from Teachers to Educational Reforms to indicate that teachers have begun to exert influence on the reforms through their adoption or rejection of them. The arrow added on the General Public component linking it to the Teachers sub-system indicates the increasing presence of the public in the education sector and the emergence of public pressure on teachers. An arrow has also been added from the General Public to Schools to signify the increasing tendency for parents to put pressure on schools to conform to their demands, e.g., for medium of instruction and even for parents to opt out of the public school sector and send their children to private (i.e., international) schools. Also, the increasing effects of the media on the public have been shown by a thickening of the arrow linking the two.

Finally, the influence of Great Britain had changed from a major causal factor, as indicated by a boldface arrow that impacts at the level of the whole society, to having a connection to that society (through the colonial legacy in the form of the English language, teaching methods and British institutions such as the British Council) without direct causal influence. Thus, what was a major influence on education in Hong Kong has diminished to a lingering connection that may diminish over time as other foreign powers, such as the USA, become more influential. In contrast, China has maintained its influence and will increase it as links in all areas between the Hong Kong SAR and the Mainland continue to grow. 


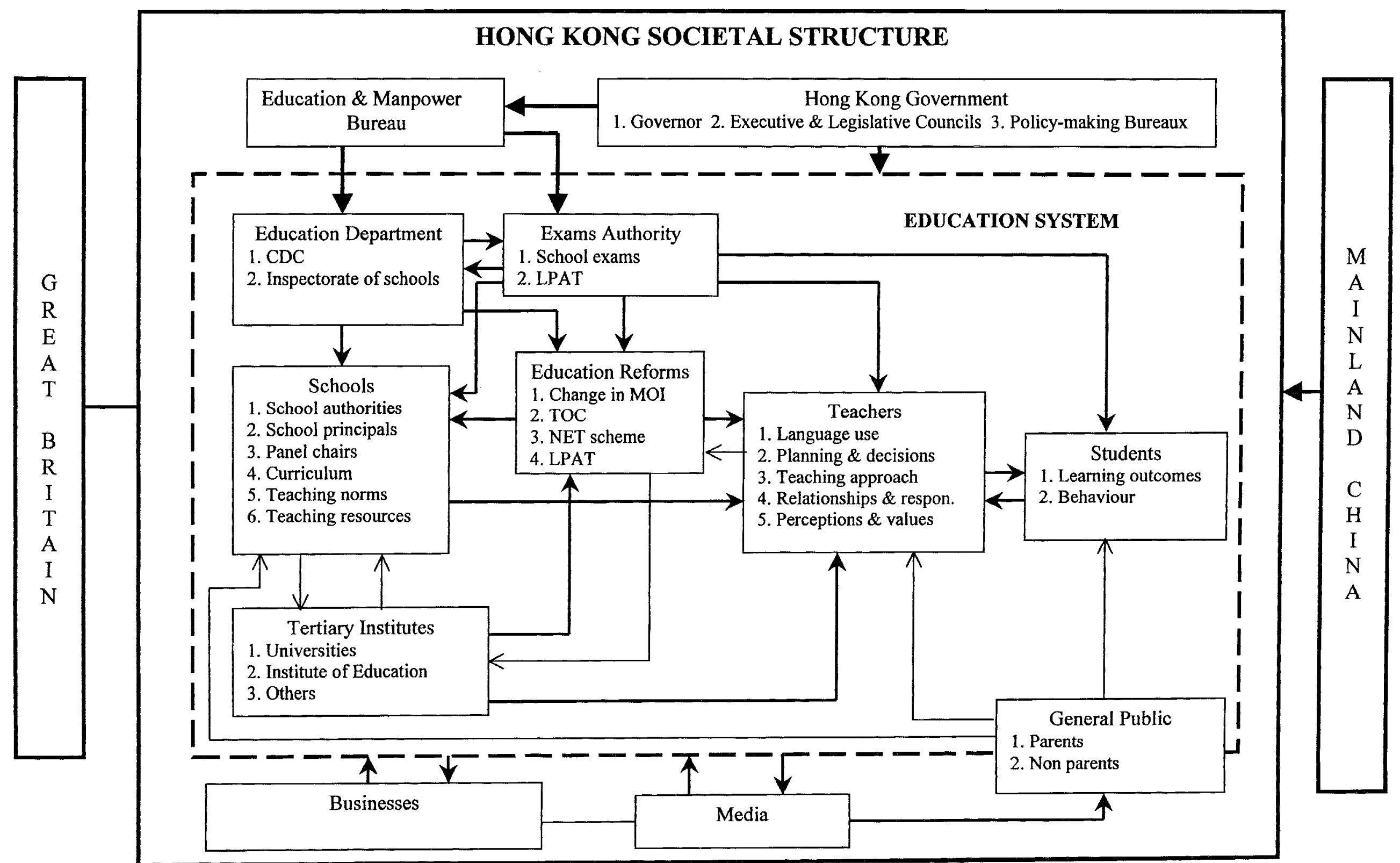

Figure 6.5: Model of English language education system in Hong Kong after the changeover in sovereignty 6-50 


\subsection{Generalisations and Projections}

The models of the English language education system of Hong Kong allow a visualisation of the interrelationships between the many different sub-systems involved in what is a complex social system within a complex society. As with any model, it only allows an indication of the factors involved and there are different interpretations that can be made of the evidence provided. However, it is possible to deduce trends and directions of potential change and to postulate as to how the situation might develop further into the future and how the picture to emerge might apply in other contexts.

The indications from the investigation are that educational matters in general and particularly those relating to language have risen in profile over the past decade and are set to continue doing so. Whilst Hong Kong is undoubtedly a society strongly geared towards commerce in its position as a distribution centre for Mainland China and other East Asian countries, it is not only issues relating to economics that shape the structure of society and characterise the ways in which changes occur. As the population generally has become more affluent and the middle class has grown, people have made more demands on the government for the basic services it is expected to provide: health care, public security, infrastructure and education amongst others. In education, it has been shown that parents have become more demanding of education resulting in increased pressure on schools and teachers to raise or at least maintain standards. As a result, the government is under pressure and has responded through the many new initiatives that have been introduced, whilst the private school sector continues to grow as parents grow dissatisfied with the public system and its seeming instability, as well as its lack of efficiency and public accountability.

Returning to the concepts of continuity and change introduced in Chapter 1 , it can be said that the case of English language education in Hong Kong represents continuity on the macro or societal scale incorporating change at the sub-system and inter-sub-system level. The individual sub-systems and the relations between 
them have changed and will continue to change into the future as has been discussed in this thesis, and the patterns of change are various and multi-layered. The education system and projecting it outward, Hong Kong society itself, can be characterised by a cyclical dynamic that incorporates both change and continuity. It is a picture of change in that innovations are developed, diffused, adopted or rejected; but it is also a picture of continuity, as the same underlying features remain dominant due to stable features of the social structure and the cultural makeup of people. Some of these enduring factors are: the value attached to a good education and gaining qualifications; the intrinsic value attached to proficiency in English; the natural conservatism which prevents people from public exhibition and so inhibits their ability to learn English through communicative and interactive approaches; and the orientation toward a hierarchical society in which power is concentrated at the top and school principals and managers are obeyed without contradiction.

In terms of cycles, the Hong Kong education system can be said to be in the first of Pennington's (2001) three cycles of innovation-adoption, i.e., the Continuity Cycle. Recalling the definition given in Chapter 2, i.e., the new innovation is regarded as an additional resource rather than a replacement for an existing one and must be accommodated into existing knowledge structures, in this case the new innovation could characterise any of the educational reforms introduced during the handover period or it could apply to the political and social structure in society post-handover. Furthermore (again recalling the definition from Chapter 2), users may neglect or fail to notice the true nature of the innovation, tending to concentrate on what is familiar about it. The sense here is of continuity at the deeper level whilst incorporating change at the surface level into existing structures. In the short period following the handover, Hong Kong was (understandably) still coming to terms with the many changes expected to take place, whether or not so many changes actually did take place. The changes in the education system coincided with the handover and teachers felt rather overwhelmed by the extent of the changes they were facing, resulting in the first stage of the cycle in which innovations are adopted as an additional feature to the 
existing system. The change in the medium of instruction to Chinese could be interpreted as a reversion to a situation in which English functioned as only a surface addition to education rather than a core component, as it was during the latter stages of colonial rule. Additionally, in the early stage of the adoption of Chinese medium of instruction, little change was noticeable as most teachers continued teaching in the same way that they had done before the change.

In time it would be expected that society and English language teaching would move into the second of the cycles, the Creativity Cycle, where the innovation is expanded and developed as it becomes naturalised, in the sense that users become familiar with it and adapt it to fit the needs of the user system. In this cycle, change begins to occur at a deeper level. Changes might occur in teachers' classroom language use, i.e., less (or more) code-mixing or code-switching - and there was evidence of this in the teacher studies. In the case of the NET scheme, for example, with time the NETs have become assimilated into the education system and their influence on teaching methods has grown. As the innovations have become more deeply diffused and have begun to impact more directly on teachers, the result has been more creative grass-roots political actions. In the case of the LPAT, once teachers became familiar with the initiative and with the government's determination to pursue it, they began to take action. Some have taken action against it and some have adopted it, whilst there is evidence also of the innovation itself being developed to meet the needs of the educational system. ${ }^{7}$

The third of the cycles of change described by Pennington (2001) (referring back to Chapter 2) is the Discontinuity Stage, wherein there occurs a discontinuity or break away of the innovation from its original context and new forms and functions develop. These may be hybrid forms which become markedly different from the original form of the innovation. Within the time frame of this investigation, this third cycle of change had yet to be reached in post-handover Hong Kong and the innovation-adoption process as regards English language education is still in progress. Yet it is useful to apply this conceptualisation to look at how the system might develop in the future. It seems likely that the 
innovations introduced prior to and during the handover period will reach some stage of discontinuity from their original forms. Firstly, there may be rejection of the innovation as people become aware of what has been lost or given up by (formerly) adopting the innovation. The relative failure of communicative language teaching to be adopted as a result of the perceived drop in English standards is an example of this form of discontinuity, as there has been a return to a more grammar-focused approach. In time, people in Hong Kong will look back at the pre-handover period and will compare present conditions with those found during colonial rule. They may compare them unfavourably, and call for a return to older, more proven methods, i.e., people may be led to recycle to an older view as the innovation might be seen as too idealistic. The desire will be to revert back to something more tangible and workable. Whilst some may view standards as slipping, it seems unlikely that the majority would wish for a return to prehandover methods. In addition to there being a will to innovate or 'move forward', the political undesirability of admitting that methods under colonial rule were better than those developed and implemented since the handover would prevent such a return or 'regression'. On the other hand, teachers who were learners of English and who may have trained to be a teacher before the handover, would have a natural disposition towards the methods which they were taught and which were used to teach them, and in this sense could be described as apolitical. The proclivity for adoption of innovations by teachers, as has been found, depends on many factors, not the least of which includes the nature of the innovation itself. Consequently, it may well be the case that teachers will revert to older, more workable methods, in opposition to imposed reform initiatives.

The third possibility is that existing forms may be replaced completely by the innovation. For this to occur, the innovation would need to be acceptable and adoptable by all major stakeholders, especially teachers. It is possible in some circumstances for this to occur, such as when there are major changes to the public examination syllabuses, which all schools, teachers and students must adhere to. The LPAT is an innovation that has received opposition from teachers and has been affected by that opposition, but the government's determination to 
proceed with it has resulted in greater attention to teachers' standards and qualifications. The indication is that the initiative will continue; however, if teachers do not attain the proficiency requirement by the cut-off date of September 2006, then it is unclear as to what action the government will take, especially if as a result there are not enough qualified or 'benchmarked' teachers to fill posts. As the deadline approaches, a power struggle between teachers and the government seems likely to ensue. Some teachers may leave it until the "last minute' to attempt to attain the proficiency requirement, whilst others may hold out to see what action the government may take.

Another possible future outcome is that the innovation may be replaced by another form, either old or new. It is quite feasible that the innovations that have been discussed will be replaced by other forms in the future. There may be greater emphasis on Putonghua, at the expense of English or of Cantonese. There may be significant changes in the curriculum or examinations to reflect changes in society, such as a shift away from traditional academic subjects towards practical and vocational skills or even a return to more artistic pursuits such as literature and music. There may be changes in the structure of education, with the traditional pyramidal pattern of primary, secondary and tertiary education, moving more towards an expansion of the higher levels so that increasing numbers of students can remain in education for as long as they wish. There may also be fundamental changes in the ways that education is provided, with greater use of information technology leading to more distance learning and less need for crowded classrooms and overworked teachers.

Finally, there may occur a reconstruction of the innovation and/or its context to take account of new features. Changing structures and relationships within society and the education system may result in changes in the innovations being diffused. The greater politicisation of education has meant that the contexts into which innovations are diffused are changing. As described, by setting a deadline for English teachers to reach the language proficiency requirement, the government has unwittingly empowered teachers. The teachers can now force the government 
into a reconstruction of the original form of its LPAT initiative, knowing that if there are not enough teachers who have achieved the requirement, then the government will be either forced to back down, or will have to introduce other innovations to deal with this particular strain in the system, such as reducing the amount of English taught in schools or employing more NETs.

The increased empowerment of teachers that has emerged as a feature of the discussions in this chapter would seem to contradict the commonly-held feeling of teachers themselves that they are dominated by circumstances. From the studies of teachers carried out, it is clear that they feel that they are actually powerless in that they are tied to a teaching syllabus and are forced to submit to the demands of policy makers, school administrators, parents and students. There has been a tendency for teachers to look upon their position from a defeatist standpoint, feeling that they are overworked and underappreciated - and this applies particularly to teachers of English in Hong Kong, who have been subject to the increased politicisation of their area of expertise. Yet there has also been an increased politicisation of society in general and this has resulted in government seemingly becoming more accountable to the public and more open to challenge from different groups within society, provided that these social groups are cohesive, have high-profile opinion leaders and have the support of significant numbers of the population at large. At present, Hong Kong society is in a posthandover period of transition as different social groups attempt to redefine their position in society or within their particular social system. Teachers are in that position too, and whether or not they will emerge as a more powerful social group, with greater autonomy, able to negotiate their own position rather than function at the behest of others, will depend on how changes in language, education, politics and culture lead to changes in the deeper structure of Hong Kong society.

Before concluding, the next section will describe the limitations of the investigation and outline areas of possible future research. 


\subsection{Possible Areas of Future Study}

This investigation into the effects of the changeover in sovereignty of Hong Kong on teachers and the teaching of English there has revealed possible patterns of change during the period prior to and immediately after the changeover that may be attributable in some part to the changeover but are also attributable to other, more complex factors inherent in society for both historical, political and cultural reasons. All research studies are to a certain extent limited in their scope and cannot claim to have explored all avenues of research into the area of study. Limitations of the present study can be viewed in a more positive and pragmatic light as providing directions for further research, as follows:

- A more extensive survey of the issues surrounding English language teaching in Hong Kong during the handover period could be carried out by including the coverage given to these issues by the Chinese press.

It is acknowledged that the Chinese newspapers in Hong Kong cover local issues in greater depth than the English newspapers and so would provide a valuable source of information in future studies to supplement that in English language newspapers.

- In addition to English language practitioners, officials and authorities, representatives from other relevant groups could also be interviewed for their perspectives on English language teaching.

In the present study, interviews were held with teachers, panel chairs, school principals, teacher educators, tertiary level English instructors, government education officials and prominent authorities in the field. In the second round of interviews, as many of the first group were sought out as possible, but in some cases it was not possible to interview them again and so other, equally qualified people were interviewed. Of the stakeholders involved in English language education, two groups not formally interviewed were parents and students. As 
many of the people interviewed were themselves parents of students within the system, it was felt that this group was represented. Similarly, most of the interviewees had been students within the system. Future studies of English teaching in Hong Kong might wish to consider including parents and students, and perhaps other designated groups such as business representatives as additional survey groups.

- Further numbers and groups of trainee teachers can be studied from the same and from different institutions.

The study of the trainee teachers encompassed two cohorts from the BATESL course at the City University of Hong Kong. In order to gain a larger picture of the culture of English teachers, further groups of trainee and graduate teachers from other institutions such as the Hong Kong Institute of Education or the University of Hong Kong, as well as additional groups in the future from City University, might be studied.

- Individual identification of questionnaire respondents such as the student teachers of the 1997 BATESL graduating cohort across two administrations of the same questionnaire might be considered to facilitate certain types of statistical analysis that are not possible when questionnaires are completed anonymously.

The student teachers in the present study were not required to identify themselves so as to encourage them to give their opinions freely. It might have been possible to incorporate some form of self-identification such that each individual's responses could have been compared across the two administrations, and this option can be considered in future research in which the same questionnaire is filled in by one group at two different times.

- A more extensive study of teachers' classroom behaviour can add to the findings of the present study. 
Further observations of graduates in their classrooms would help to gain (i) a clearer picture of the extent to which graduates from English teacher education courses implement what they are taught in those courses and in general (ii) a deeper understanding of teachers' classroom behaviour in relation to the specific teaching contexts that exist in Hong Kong schools.

To continue to build a picture of the nature of teaching English in Hong Kong and the effects on it of political and social change, it will be necessary to carry out more studies as the SAR develops its own, post-colonial characteristics. Further studies of the teaching approaches and practices of teachers are required, which may include those studied in the present investigation as well as others. Further analysis of the classroom behaviour and the orientations to teaching of the graduates from the BATESL course, both those of the 1994 cohort and the 1997 cohort, would help to clarify the impressions gained of their views and practices in the present study and to revise and update the model of educational change. In addition, parallel studies of trainee teachers from other courses and institutes could be carried out to ascertain differences in approaches to language teacher education in the SAR and whether individual courses have their own characteristics and whether the courses are developing or changing along similar lines to reflect changes in education. A third area that can benefit from further and ongoing investigation is the study of teacher's classroom practices. By comparing the methods of teachers from a wide variety of backgrounds, not only those from the BATESL and other courses of English teacher preparation, but also those with other backgrounds and experiences, including teachers of English from other parts of China, it will be possible to gain a more in-depth understanding of the nature of the teaching culture and how it is changing in relation to changes in society. 


\subsection{Concluding Reflections}

As a post-colonial society, Hong Kong is still in its infancy. Despite the independence promised to it by the PRC, it does not have that status in actuality, unlike its contemporaries in Asia such as India, Singapore, Malaysia and Burma/Myanmar (all former British colonies) and others such as Vietnam (French), Indonesia (Dutch) and the Philippines (USA). The government has to live with the legacies of the former colonists and marry them to what they see as the needs of the Hong Kong people, under the watchful eye of the Mainland. Discourses of colonialism, as described in detail relating to the SAR and to English and its teaching by Pennycook (1998), continue and are likely to continue for the foreseeable future, as educational policy has been shaped by these discourses and is now in the process of trying to emerge from their grip. The fact that the teaching and learning of English is so heavily imbued with these discourses means that we cannot expect any deep changes in the short term. Many teachers and their students will be integral parts of the change process as innovations are introduced with the intention of maintaining Hong Kong's position as a major world city and providing its subjects with an education that will equip them to meet the demands of its new role as a semi-autonomous region of one of the world's largest powers. The signs are that the role of the teacher is becoming more appreciated and that those charged with deciding educational and social policy are realising that they need to pay much more attention to the teachers than they have done in the past. 


\section{Notes}

1 The previous research questions are addressed in Chapter 2 (question (i)), Chapter 3 (questions (ii) and (iii)), Chapter 4 (question (iv)) and Chapter 5 (question (v)).

${ }^{2}$ Like the Provisional Legislative Council, the Chief Executive was 'elected' by a carefully chosen group of 400 prominent Hong Kong and Mainland citizens known as the 'Selection Committee'.

${ }^{3}$ Over a period of two weeks in August 1998, the government invested over $\$ 100$ billion $(\$ 100,000,000,000)$ in the Hong Kong stock market by buying up stock of major Hong Kong companies at prices of up to 20 per cent higher than their perceived value on the market, forcing speculators to sell their stock to the government and hence pushing up the Hang Seng Index.

${ }^{4}$ The fifth, the change in the medium of instruction, is discussed in 6.2.3.

5 Begun in 2000, under the Workplace English Campaign employees are encouraged (through sponsorship) to improve their English skills by taking courses leading to certain accredited examinations such as the UCLES Business English Certificate. Different grades of the approved examinations are assigned to different classes of employee, such that secretaries are required to reach a higher grade than clerks and so on (Hong Kong SAR Government, 2002).

${ }^{6}$ The individual features of the other systems will be added at the next stage in the development of the model as they relate more specifically to the situation at the time of the study.

${ }^{7}$ In the case of this particular initiative, the proficiency requirement for English teachers is set at a Level 3 on a 5-point scale, the so-called 'benchmark'. A development has been that schools now require teachers to have attained a Level 4 on the scale to be considered for promotion. It has even been reported that some schools will not hire English teachers unless they have a minimum of a Level 4 (or equivalent through other qualifications), so that they can boast to parents that their English teachers are of a high standard. This is an example of an innovation being developed to meet the demands of the system. 


\section{REFERENCES}

Adamson, B., \& Lai, W. A. (1997). Language and the curriculum in Hong Kong: Dilemmas of triglossia. In M. Bray \& W. O. Lee (Eds.), Education and political transition: Implications of Hong Kong's change of sovereignty (pp. 87-100). Hong Kong: Comparative Education Research Centre, University of Hong Kong.

Adamson, B., Kwan, T., \& Chan, K. K. (2000). Changing the curriculum. Hong Kong: Hong Kong University Press.

Aitken, J. L. \& Mildon, D. A. (1992). Teacher education and the developing teacher: The role of personal knowledge. In M. Fullan and A. Hargreaves (Eds.). Teacher development and educational change. London/Washington: The Falmer Press (pp.10-35).

Anderson, J, (1993). Is a communicative approach practical for teaching English in China? Pros and cons. System, 21, 471-480.

Ansaldo, U. (1995). Language diversity at sunset? Present and future of multilingualism in Hong Kong. Hong Kong Linguist, 15, 20-28.

Ardener, E. (1975). The 'problem' revisited. In S. Ardener (Ed.), Perceiving women (pp. 19-27). London: Malaby/Dent.

Arias, R. (1994). Towards a conceptualization of ESL teacher development: The interteaching development hypothesis. Unpublished manuscript. Teachers' College, Columbia University, New York.

Axler, M., Yang, A., \& Stevens, T. (1998). Current language attitudes of Hong Kong Chinese adolescents and young adults. In M. C. Pennington (Ed.), Language in Hong Kong at century's end (pp. 329-338). Hong Kong: Hong Kong University Press.

Bacon-Shone, J., \& Bolton, K. (1998). Charting multilingualism: Language censuses and language surveys in Hong Kong. In M. C. Pennington (Ed.), Language in Hong Kong at century's end (pp. 43-90). Hong Kong: Hong Kong University Press.

Bailey, K. D. (1987). Methods of social research. Third Edition. New York: Free Press.

Bailey, K. M. (1992). The processes of innovation in language teacher development: What, why and how teachers change. In J. Flowerdew, M. Brock \& S. Hsia (Eds.), Perspectives on second language teacher education. Hong Kong: Department of English, City Polytechnic of Hong Kong. 
Bailey, K. M., \& Nunan, D. (1996). Voices from the language classroom. Cambridge: Cambridge University Press.

Balla, J. \& Pennington, M.C. (1996). The perception of English-medium instruction by tertiary-level vocational students in Hong Kong. Education Research Journal, 24(1), 131-153.

Barnes, D., \& Schemilt, D (1974). Transmission and interpretation. Educational Review, 26, 213-228.

Barnes, P. (1987). The organisation of language teaching at tertiary Level. In R. Lord \& N. L. Cheng (Eds.), Language education in Hong Kong (pp. 141-154). Hong Kong: Chinese University Press.

Benson, P. (1997). Language rights and the medium of instruction issue in Hong Kong. Hong Kong Journal of Applied Linguistics, 2(2), 1-21.

Bickley, V. (1987). Developments in English language teacher education. In R. Lord \& N. L. Cheng (Eds.), Language education in Hong Kong (pp. 187-218). Hong Kong: Chinese University Press.

Boudon, R. (1986). Theories of social change: A critical appraisal. Cambridge: Polity.

Bourdieu, P. (1997). The forms of capital. In A. H. Halsey, H. Lauder, P. Brown, \& A. S. Wells (Eds.), Education: Culture, economy, society (pp. 46-58). New York: Oxford University Press.

Bowman, J. (2000). Our skills are squandered, say imported teachers. South China Morning Post, 13 August 2000.

Boyle, J. (1995). Parental influence on the language used in Hong Kong schools. Language Issues, 7(1), 21-24.

Boyle, J. (1997a). The use of mixed-code in Hong Kong English language teaching. System, 25(1), 83-89.

Boyle, J. (1997b). Imperialism and the English language in Hong Kong. Journal of Multilingual and Multicultural Development, 18(3), 169-181.

Bray, M. (1997). Education and colonial transition: The Hong Kong experience in comparative perspective. In M. Bray \& W. O. Lee (Eds.), Education and political transition: Implications of Hong Kong's change of sovereignty (pp. 11-24). Hong Kong: Comparative Education Research Centre, University of Hong Kong. 
Brindley, G., \& Hood, S. (1990). Curriculum innovation in adult ESL. In G. Brindley (Ed.). The second language curriculum in action. Sydney: National Centre for English Language Teaching Research, Macquarie University.

Bruche-Schulz, G. (1997). 'Fuzzy' Chinese: The status of Cantonese in Hong Kong. Journal of Pragmatics, 27, 295-314.

Bullough, R. V. (1989). First-year teacher: A case study. Teachers College Press.

Bullough, R. V., \& Baughman, K. (1993). Continuity and change in teacher development: First year teacher after five years. Journal of Teacher Education, 44(2), 86-95.

Bunce, P. (1999). The dreadful depths of dreary teaching. South China Morning Post, 27 October 1999.

Bunce, P. (2001). Stuck with six hundred names to put to a face. South China Morning Post, 7 April 2001.

Butenhoff, L. (1999). Social movements and political reform in Hong Kong. Westport, Connecticut: Praeger.

Calderhead, J. \& Robson, M. (1991). Images of teaching: Student teachers' early conceptions of classroom practice. Teaching and Teacher Education, 7(1), 1-8.

Carless, D. (2001). Curriculum implementation in Hong Kong. In D. R. Hall \& A. Hewings (Eds.), Innovation in English language teaching: A reader (pp. 263274). London \& New York: Routledge.

Celce-Mercia, M., Dornyei, Z, \& Thurrell, S. (1997). Direct approaches in L2 instruction: A turning point in communicative language teaching? TESOL Quarterly, 31(4), 141-152.

Carter, K. \& Gonzalez, L. (1993). Beginning teachers' knowledge of classroom events. Journal of Teacher Education, 44(3), 223-232.

Chan, A., Hoare, P., \& Johnson, R. K. (1997). English medium instruction in secondary 1 and 2 in Hong Kong schools: An evaluation of policy implementation. Hong Kong: Report of a Hong Kong Bank Language Development Fund project.

Cheng, M. S. (1997). Striving for higher goals in ELT in China. IATEFL Newsletter, April-May 1997.

Cheung, A. B. L. (2001). Seen, not heard. South China Morning Post. 5 September 2001. 
Cheung, A. B. L., \& Louie, K. S. (2000). Social conflicts: 1975-1986. In S. K. Lau (Ed.), Social development and political change in Hong Kong (pp. 63-114). Hong Kong: The Chinese University Press.

Cheung, M., \& Pennington, M. C. (1994). Cultivating product-oriented teachers to adopt process writing: An example of an innovation-related in-service programme. In D. Li, D. Mahoney, \& J. C. Richards (Eds.), Exploring second language teacher development (pp. 209-231). Department of English, City Polytechnic of Hong Kong.

Chodak, S. (1973). Societal development: Five approaches with conclusions from comparative analysis. New York: Oxford University Press.

Clift, R. (1991). Learning to teach English - maybe: A study of knowledge development. Journal of Teacher Education, 42, 357-372.

Coniam, D. (1997). Establishing minimum-standard qualifications in Hong Kong for English language teachers. In B. Asker (Ed.), Teaching language and culture: Building Hong Kong on education. Hong Kong: Longman

Coniam, D., \& Falvey, P. (1996). Setting language benchmarks for English language teachers in Hong Kong secondary schools. Hong Kong: Final report prepared for ACTEQ (The Advisory Committee on Teacher Education and Qualifications).

Coniam, D., \& Falvey, P. (1999). Setting standards for teachers of English in Hong Kong - The teachers' perspective. Curriculum Forum, 8(2), 1-27.

Cooper, R. L. (1989). Language planning and social change. Cambridge: Cambridge University Press.

Curriculum Development Committee. (1983). Syllabus for English (Forms 1-5). Hong Kong: Government Printer.

Curriculum Development Council. (1999). Syllabus for secondary schools: English Language (Secondary 1-5). Hong Kong: Government Printer.

Dahrendorf, R. (1959). Class and class conflict in industrial society. Stanford: Stanford University Press. Cited in Harper (1993).

Dellios, R. (2001). Continuity and change in international relations. No. IV of lecture series INTR13-306 \& INTR71/72-306 Prospects for International Relations. Department of International Relations, Bond University, Queensland, Australia. http://www.international-relations.com/ProspectsIV.htm. 
Dimmock, C. \& Walker, A. (1997). Hong Kong's change of sovereignty: School leader perceptions of the effects on educational policy and school administration. In M. Bray \& W. O. Lee (Eds.), Education and political transition: Implications of Hong Kong's change of sovereignty (pp. 131-156). Hong Kong: Comparative Education Research Centre, University of Hong Kong.

Durkheim, E. (1947). The division of labour in society. New York: Free Press.

Ellwein, M. C., Graue, M. E., \& Comfort, R. E. (1990). Talking about instruction: Student teachers' reflections on success and failure in the classroom. Journal of Teacher Education, 41, 3-14.

Ethell, R.G. \& McMeniman, M. M. (2000). Unlocking the knowledge in action of an expert practitioner. Journal of Teacher Education, 51(2), 87-101.

Evans, S. (1996). The context of English language education: The case of Hong Kong. RELC Journal, 27(2), 30-55.

Evans, S. (1997). Teacher and learner roles in the Hong Kong English classroom. Education Journal, 25(2), 43-61.

Evans, S., Jones, R., Rusmin, R. S., \& Cheung, O. L. (1998). Three languages: One future. In M. C. Pennington (Ed.), Language in Hong Kong at century's end (pp. 391-418). Hong Kong: Hong Kong University Press.

Faculty of Humanities and Social Sciences, (1992). Bachelor of Arts in Teaching English as a Second Language: Volume 1 (Course document). Hong Kong: City Polytechnic of Hong Kong.

Fairbanks, C.M., Freedman, D., \& Kahn, C. (2000). The role of effective mentors in learning to teach. Journal of Teacher Education, 51(2), 102-112.

Fairclough, N. (1989). Language and power. New York: Longman.

Falvey, P. \& Coniam, D. (1997). Introducing English language benchmarks for Hong Kong teachers: A preliminary overview. Curriculum Forum, 6(2), 16-35.

Farrell, T. (1999). The reflective assignment: Unlocking pre-service English teachers' beliefs on grammar teaching. RELC Journal, 30(2), 1-16.

Flowerdew, J. (1998). The final years of British Hong Kong: The discourse of colonial withdrawal. London and New York: Macmillan and St. Martin's Press.

Flowerdew, J. (2001). Public discourse in transitional Hong Kong. In P. T. Lee (Ed.), Hong Kong reintegrating with China: Political, cultural and social dimensions (pp. 39-58). Hong Kong: Hong Kong University Press. 
Flowerdew, J., \& Scollon, R. (1997). Public discourse in Hong Kong and the change of sovereignty. Journal of Pragmatics, 28, 417-426.

Freeman, D. (1989). Teacher training, development, and decision-making: A model of teaching and related strategies for language teacher education. TESOL Quarterly, 23(1), 27-46.

Freeman, D. (1991). 'Mistaken constructs': Re-examining the nature and assumptions of language teacher education. In J. E. Alatis (Ed.), Linguistics and language pedagogy: The state of the art. Georgetown University Round Table on Languages and Linguistics (pp. 25-39). Georgetown University Press.

Freeman, D. (1996). The 'unstudied problem'. Research on teacher learning in language teaching. In D. Freeman \& J. C. Richards (Eds.), Teacher learning in language teaching (pp. 351-378). Cambridge: Cambridge University Press.

Freeman, D. \& Johnson, K. E. (1998). Reconceptualizing the knowledge-base of language teacher education. TESOL Quarterly, 32(3), 397-417.

Freeman, D., \& Richards, J. C. (1993). Conceptions of teaching and education of second language teachers. TESOL Quarterly, 27(2), 192-216.

Fu, G. S., (1987). The Hong Kong bilingual. In R. Lord \& N. L. Cheng (Eds.), Language education in Hong Kong (pp. 27-50). Hong Kong: Chinese University Press.

Fullan, M. G. (1991). The new meaning of educational change. With S. Stiegelbauer. New York: Teachers' College Press.

Fullan, M. G. (1993). Change forces. London: The Falmer Press.

Fuller, E. F. (1969). Concerns of teachers: A developmental conceptualization. American Educational Research Journal, 6, 207-226.

Garner, J. (1999). Elite's imperfect values damaging society. Letter to the editor, South China Morning Post, 8 February.

Galton, M. (2000). Two decades of change in English teacher education: The consequences of neglecting educational theory. Asia-Pacific Journal of Teacher Education \& Development, 2(2), 1-10.

Gee, E. W. (1984). Applying effective instruction research findings in teacher education: Six influencing factors. Alexandria, Va.: ERIC document reproduction service, ED 246037.

Gibbons, J. (1987). Code mixing and code choice: A Hong Kong case study. Clevedon, England. Multilingual Matters. 
Glaser, B. \& Strauss, A. (1967). The discovery of grounded theory: Strategies for qualitative research. New York: Aldine.

Golombek, P. R. (1998). A study of language teachers' personal practical knowledge. TESOL Quarterly, 32(3), 447-464.

Good, T. L., \& Brophy, J. E. (1978). Looking in classrooms. New York: Harper and Row.

Grant, J.S. (2001). Cultural formation in post-war Hong Kong. In P. T. Lee (Ed.), Hong Kong reintegrating with China: political, cultural and social dimensions (pp. 159-180). Hong Kong: Hong Kong University Press.

Guthrie, L. F. , \& Guthrie, G. P. (1987). Teacher language use in a Chinese bilingual classroom. In S. R. Goldman \& H. T. Trueba (Eds.), Becoming literate in English as a second language (pp. 205-231). Norwood, NJ: Ablex.

Hargreaves, A. (1999). Reinventing professionalism: Teacher education and teacher development for a changing world. Asia-Pacific Journal of Teacher Education \& Development, 2(1), 65-74.

Harper, C. L. (1993). Exploring social change. Second Edition. Englewood Cliffs, N.J.: Prentice Hall.

Hau, K. T., Ip, M. H., \& Cheng, Z. (1996). Target Oriented Curriculum and interschool comparison. Education Journal, 24(2), 1-14.

Henrichsen, L. E. (1989). Diffusion of innovations in English language teaching: The ELEC effort in Japan, 1956-1968. New York: Greenwood Press.

Hirvela, A. \& Law, E. (1991). A survey of local English teachers' attitudes towards English and ELT. Institute of Language in Education Journal, 8, 25-38.

Ho, C. (1998). Intervention's bitter pill. South China Morning Post, 18 October, 1998.

Ho, J., Tang, E., \& Tam, P. (1993). Non-English major ESL teachers in Hong Kong: Problems in teaching and coping strategies. Research Report No. 34. Hong Kong: Department of English, City Polytechnic of Hong Kong.

Ho, K. (2000). Let's stand up for the benchmark tests, Hong Kong iMail. 9 June 2000 .

Ho, K. L. (1994). Hong Kong society. Volume 1. Hong Kong: Department of Applied Social Studies, Hong Kong Polytechnic. 
Hong Kong Education Commission (1984). Education Commission Report No. 1. Hong Kong: Government Printer.

Hong Kong Education Commission (1986). Education Commission Report No. 2. Hong Kong: Government Printer.

Hong Kong Education Commission (1988). Education Commission Report No. 3. Hong Kong: Government Printer.

Hong Kong Education Commission (1990). Education Commission Report No. 4. Hong Kong: Government Printer.

Hong Kong Education Commission (1992). Education Commission Report No. 5. Hong Kong: Government Printer.

Hong Kong Education Commission (1995). Education Commission Report No. 6. Hong Kong: Government Printer.

Hong Kong Education Commission (1997). Education Commission Report No. 7. Hong Kong: Government Printer.

Hong Kong Education Commission (1999). Education blueprint for the 21st century. Consultation Document. Hong Kong: Government Printer.

Hong Kong Education Department (1997a). Arrangements for firm guidance on secondary schools' medium of instruction. Consultation Document. Hong Kong: Government Printer.

Hong Kong Education Department (1997b). Medium of instruction guidance for secondary schools. Hong Kong: Government Printer.

Hong Kong Government Information Service (1996). Hong Kong 1996. Hong Kong: Government Printer.

Hong Kong Government Information Service (1998). Hong Kong - a new era: A review of 1997. Hong Kong: Government Printer.

Hong Kong SAR Government (1990). The Basic Law of the Hong Kong Special Administrative Region of the People's Republic of China. Hong Kong: Government Printer.

Hong Kong SAR Government (2002). The Workplace English Campaign. http://www.english.gov.hk.

Hong Kong Standard. Teach them well. 3 March, 1997.

Hong Kong Standard. No urgency to mind language. 17 March, 1997. 
Hong Kong Standard. More parents seeking help to overcome exams stress. 18 August, 1997.

Hong Kong Standard. Schools' shift to Chinese will take up to 15 years: union. 29 September, 1997.

Hong Kong Standard. Call for teachers to hold at least bachelor degree. 6 October, 1997.

Hong Kong Standard. Wanted: over 700 English teachers. 10 October, 1997.

Hong Kong Standard. Search for native English-speaking teachers 'discriminatory'. 13 October, 1997.

Hong Kong Standard. 100 schools get English go-ahead. 2 December, 1997.

Hong Kong Tourist Association (1996). Hong Kong Wondernet. http://www.hkta.org.

Huling-Austin, L. (1992). Research on learning to teach: Implications for teacher induction and mentoring programs. Journal of Teacher Education, 43, 173-180.

Hung, H. F. (2001). Identity contested: Rural ethnicity in the making of urban Hong Kong. In P. T. Lee (Ed.), Hong Kong reintegrating with China: Political, cultural and social dimensions (pp. 181-202). Hong Kong: Hong Kong University Press.

Huque, A. S., Tao, L. P. W., \& Wilding, P. (1997). Understanding Hong Kong. In P. Wilding, A. S. Huque \& L. P. W. Tao (Eds.), Social policy in Hong Kong (pp. 1-22). Cheltenham: Edward Elgar Publishing Ltd.

Johnson, K. E. (1992). Learning to teach: Instructional actions and decisions of preservice ESL teachers. TESOL Quarterly, 26(3), 507-534.

Johnson, K. E. (1995). Understanding communication in second language classrooms. Cambridge: Cambridge University Press.

Johnson, R. K., (1991). Teaching and learning in the English stream in secondary schools: Implications of ECR4. Institute of Language in Education Journal, 8, 10-24.

Johnson, R. K. (1998). Language and education in Hong Kong. In M. C. Pennington (Ed.), Language in Hong Kong at century's end (pp. 265-276). Hong Kong: Hong Kong University Press. 
Johnson, R. K., \& Lee, P. L. M. (1987). Modes of instruction: Teaching strategies and student responses. In R. Lord \& N. L. Cheng (Eds.), Language education in Hong Kong (pp. 99-122). Chinese University Press.

Kegley, C. W., \& Wittkopf, E. R. (1997). World Politics: Trend and Transformation. Sixth Edition. New York: St. Martin's Press.

Kennedy, C. (1988). Evaluation of the management of change in an ELT project. Applied Linguistics, 9, 329-342.

Kuan, H. C., \& Lau, S. K. (2000). Political attitudes in a changing context. In S. $\mathrm{K}$. Lau (Ed.), Social development and political change in Hong Kong (pp. 287308). Hong Kong: The Chinese University Press.

Kwo, O. W. Y. (1987). Language policies in Hong Kong secondary education: Alternative strategies. Chinese University of Hong Kong Education Journal, 15(1), 85-93.

Kwo, O. W. K. (1996). Learning to teach English in Hong Kong classrooms: Patterns of reflections. In D. Freeman \& J. C. Richards (Eds.), Teacher learning in language teaching (pp. 295-319). Cambridge: Cambridge University Press.

Lai, C. (1994). Communication failure in the language classroom: An exploration of causes. RELC Journal, 25(1), 99-129.

Lau, S. K. (1982). Society and politics in Hong Kong. Hong Kong: The Chinese University Press.

Lau, S. K. (2000). Hongkongese or Chinese :The problems of identity on the eve of resumption of Chinese sovereignty over Hong Kong. Social development and political change in Hong Kong (pp. 255-284). Hong Kong: Chinese University Press.

Lau, S. K. (2001). The Hong Kong Special Administrative Region Government in the new political environment. In P. T. Lee (Ed.), Hong Kong reintegrating with China: Political, cultural and social dimensions (pp. 59-77). Hong Kong: Hong Kong University Press.

Lau, S. K., \& Kuan, H. C. (1988). The ethos of the Hong Kong Chinese. Hong Kong: The Chinese University Press.

Law, W. W. (1997). The accommodation and resistance to the decolonisation, neocolonisation and recolonisation of higher education in Hong Kong. In M. Bray \& W. O. Lee (Eds.), Education and political transition: Implications of Hong Kong's change of sovereignty (pp. 41-63). Hong Kong: Comparative Education Research Centre, University of Hong Kong. 
Lehmen, R. S. (1995). Statistics in the behavioral sciences: A conceptual introduction. Pacific Grove, California: Brooks/Cole Publishing Company.

Levin, J. \& Fox, J. A. (1988). Elementary statistics in social research. Fourth Edition. New York: Harper and Row.

Li, D. (2001). Teachers' perceived difficulties in introducing the communicative approach in South Korea. In D. R. Hall \& A. Hewings (Eds.), Innovation in English language teaching: $A$ reader (pp. 149-167). London \& New York: Routledge.

Li, D. C. S. (1998). The plight of the purist. In M. C. Pennington (Ed.), Language in Hong Kong at century's end (pp. 161-190). Hong Kong: Hong Kong University Press.

Li, D. C. S. (1999). The functions and status of English in Hong Kong: A Post1997 update. English World-Wide, 20(1), 67-110.

Lin, A. M. Y. (1990). Teaching in two tongues: Language alternation in foreign language classrooms. Research Report No. 3, Department of English, City Polytechnic of Hong Kong.

Lin, A. M. Y. (1997a). Hong Kong children's' rights to a culturally compatible English education. Hong Kong Journal of Applied Linguistics, 2(2), 23-48.

Lin, A. M. Y. (1997b). Analysing the 'language problem' discourses in Hong Kong: How official, academic, and media discourses construct and perpetuate dominant models of language learning and education. Journal of Pragmatics, $28,427-440$.

Littlewood, W. (1999). Adapting classroom schemata. Hong Kong Journal of Applied Linguistics, 4(2), 1-15.

Lo, M. L., Adamson, B., \& Morris, P. (2000). Improving schools in Hong Kong Lessons from the past. Paper presented at $17^{\text {th }}$ Annual Conference of the Hong Kong Educational Research Association, University of Hong Kong, 18 November 2000.

Lockhart, C. (1996). Teachers' beliefs about writing in Hong Kong secondary schools. In Perspectives: Working papers of the Department of English, 8(2), 45-79. City University of Hong Kong

Long, M. H. (1991). Focus on form: A design feature in language teaching methodology. In K. de Bot, R. B. Ginsburg, \& C. Kramsch (Eds.), Foreign language research in cross-cultural perspectives (pp. 39-52). Amsterdam: John Benjamins. 
Lord, R. (1987). Language policy and planning in Hong Kong: Past, present and (especially) future. In R. Lord \& N. L. Cheng (Eds.), Language Education in Hong Kong (pp. 3-26). Chinese University Press.

Lucas, T., \& Katz, A. (1994). Reframing the debate: The roles of native languages in English-only programs for language minority students. TESOL Quarterly, 28, 537-561.

Luke, K. K. (1998). Why two languages might be better than one: Motivations of language mixing in Hong Kong. In M. C. Pennington (Ed.), Language in Hong Kong at century's end (pp. 145-160). Hong Kong: Hong Kong University Press.

Markee, N. (2001). The diffusion of innovation in language teaching. In D. R. Hall \& A. Hewings (Eds.), Innovation in English language teaching: A reader (pp. 118-126). London \& New York: Routledge.

Marks, R. (1990). Pedagogical content knowledge: From a mathematical case to a modified conception. Journal of Teacher Education, 41, 3-11.

McMaster, T. (2001). The illusion of diffusion in information systems research. In M. A. Ardis \& B. L. Marcolin (Eds.), Diffusing software product and process innovations (pp. 67-85). Norwell, Massachusetts: Kluwer Academic Publishers.

Miles, M. B. (1964). Innovation in education. New York: Teachers College Press.

Miles, M. B. \& Huberman, A. M. (1994). Qualitative data analysis. Second Edition. Thousand Oaks, London and New Delhi: Sage Publications.

Mitsufuji, T. (2001). A perspective of the innovation-diffusion process from the self-organizing system. In M. A. Ardis \& B. L. Marcolin (Eds.), Diffusing software product and process innovations (pp. 51-66). Norwell, Massachusetts: Kluwer Academic Publishers.

Morris, P. (1995). The Hong Kong school curriculum: Development, issues and policies. Hong Kong: Hong Kong University Press.

Morris, P., \& Chan, K. K. (1997). The Hong Kong school curriculum and the political transition: Politicisation, contextualisation and symbolic action. In $\mathrm{M}$. Bray \& W. O. Lee (Eds.), Education and political transition: Implications of Hong Kong's change of sovereignty (pp. 101-118). Comparative Education Research Centre, University of Hong Kong.

Morrison, K., \& Lui, I. (2000). Ideology, linguistic capital and the medium of instruction in Hong Kong. Journal of Multilingual and Multicultural Development, 21(6), 471-486. 
Moser, L. (1985). The Chinese mosaic: The people and provinces of China. London: Westview Press.

Ng, K. S. K. (2000). A multidimensional approach to promoting educational reform in Hong Kong: The case of mother tongue teaching. Paper presented at $17^{\text {th }}$ Annual Conference of the Hong Kong Educational Research Association, University of Hong Kong, 18 November 2000.

Nicholls, A. (1983). Managing educational change. London: George Allen \& Unwin.

Nunan, D. (1989a). Designing tasks for the communicative classroom. Cambridge: Cambridge University Press.

Nunan, D. (1989b). Understanding language classrooms: A guide for teacherinitiated action. Hemel Hempstead: Prentice Hall.

Nunan, D. (1991). Teachers' interactive decision-making processes. Research Report to the Adult Migrant English Program. National Centre for English Language Teaching and Research, Macquarie University.

Nunan, D. (1992a). Collaborative language learning and teaching. Cambridge: Cambridge University Press.

Nunan, D. (1992b). The teacher as decision-maker. In J. Flowerdew, M. Brock, \& S. Hsia (Eds.), Perspectives on second language teacher education (pp. 135165). Department of English, City University of Hong Kong.

Nunan, D., \& Lamb, C. (1996). The self-directed teacher. Cambridge: Cambridge University Press.

Parakrama, A. (1995). De-hegemonizing language standards: Learning from (post) colonial Englishes about 'English'. New York: St. Martin's Press.

Parsons, T. (1951). The social system. New York: Free Press.

Patten, C. (1999). East and West. London: Pan Books.

Pennington, M. C. (1989). Faculty development for language programs. In R. K. Johnson (Ed.), The second language curriculum (pp. 91-110). Cambridge University Press.

Pennington, M. C. (1994a). Projecting classroom language use in a group of bilingual graduates of a BATESL course. Perspectives (6)2, 93-110. Working Papers of the Department of English, City Polytechnic of Hong Kong. 
Pennington, M. C. (1994b). Forces shaping a dual code society: An interpretive review of the literature on language use and language attitudes in Hong Kong. Research Report No. 35, Department of English, City Polytechnic of Hong Kong.

Pennington, M. C. (1995a). Eight case studies of classroom discourse in the Hong Kong secondary English class. Research Report No. 42. Department of English, City University of Hong Kong.

Pennington, M. C. (1995b). Pattern and variation in use of two languages in the Hong Kong secondary English class. RELC Journal, 26(2), 80-105.

Pennington, M. C. (1995c). The teacher change cycle. TESOL Quarterly, 29(4), 705-732.

Pennington, M. C. (1996). Modeling teacher change: Relating input to output. Research Monograph No. 5. Hong Kong: Department of English, City University of Hong Kong.

Pennington, M. C. (1997a). Projecting classroom language use in a group of bilingual graduates of a BATESL course. Language, Culture and Curriculum, 10(3), 222-235.

Pennington, M. C. (1997b). Resistance and change: Hong Kong Teachers' reactions to "Western" innovations in language teaching. Invited lecture, University of Reading, 24 June 1997. Unpublished ms.

Pennington, M. C. (1998a). Perspectives on language use in Hong Kong at century's end. In M. C. Pennington (Ed.), Language in Hong Kong at century's end (pp. 3-40). Hong Kong: Hong Kong University Press.

Pennington, M. C. (1998b). The folly of language planning: A brief history of the English language in Hong Kong. English Today, 54 (April), 25-30.

Pennington, M. C. (2001). Innovation and synergy in IT and English language teaching. In B. Morrison, D. Gardner, K. Keobke \& M. Spratt (Eds.). ELT perspectives on IT \& multimedia. Plenary Address at the ITMELT 2001 Conference, Hong Kong Polytechnic University, June 2001.

Pennington, M. C. (2002). Bridging gaps: a dialectic perspective on teacher development. Plenary Address. International Association of Teachers of English as a Foreign Language (IATEFL). University of York, March 2002.

Pennington, M. C. \& Balla, J. (1998). Our future English teachers: Language use among graduate and undergraduate TESL students in Hong Kong. In M. C. Pennington (Ed.), Language in Hong Kong at century's end (pp. 243-264). Hong Kong: Hong Kong University Press. 
Pennington, M. C., \& Cheung, M. (1995). Factors shaping the introduction of process writing in Hong Kong secondary schools. Language, Culture and Curriculum, 8(1), 15-34.

Pennington, M. C., Costa, V., So, S., Li, J., Hirose, K., \& Niedzielski. K. (1996). A cross-country comparison of beliefs and practices of English composition teachers in the Asia-Pacific region. In G. Tinker Sachs, M. Brock \& R. Lo (Eds.), Directions in second language teacher education (pp. 147-162). Department of English, City University of Hong Kong.

Pennington, M. C., \& Richards, J. C. (1993). Learning to teach English in Hong Kong: The first year in the classroom. (Proposal for earmarked research grant to Hong Kong University and Polytechnic Grants Committee). Department of English, City Polytechnic of Hong Kong.

Pennington, M. C., \& Richards, J. C. (1997). Re-orienting the teaching universe: The experience of five first-year English teachers in Hong Kong. Language Teaching Research, 1(2), 149-178.

Pennington, M. C., Richards, J. C., Urmston, A. W., \& Lee, Y. P. (1996). Learning to teach English in Hong Kong: The first year in the classroom. Research Monograph No. 13, Department of English, City University of Hong Kong.

Pennington, M. C., \& Urmston, A. W. (1998). The teaching orientation of graduating students on a BATESL course in Hong Kong: A comparison with first-year students. Hong Kong Journal of Applied Linguistics, 3(2), 17-46.

Pennington, M. C., \& Yue, F. (1994). English and Chinese in Hong Kong: Pre1997 language attitudes. World Englishes, 13, 1-20.

Pennycook, A. (1994). The cultural politics of English as an international language. New York: Longman.

Pennycook, A. (1998). English and the discourses of colonialism. London: Routledge.

Phillipson, R. (1992). Linguistic imperialism. Oxford : Oxford University Press.

Phillipson, R. (1998). Globalizing English: Are linguistic human rights an alternative to linguistic imperialism? Language Sciences, 20(1), 101-112.

Pierson, H. D. (1998). Societal accommodation to English and Putonghua in Cantonese-speaking Hong Kong. In M. C. Pennington (Ed.), Language in Hong Kong at century's end (pp. 91-112). Hong Kong: Hong Kong University Press. 
Pierson, H. D., Fu, G. S., \& Lee, S. Y. (1980). An analysis of the relationship between language attitudes and English attainment of secondary students in Hong Kong. Language Learning, 30, 289-316.

Pigge, F. L., \& Marso, R. N. (1997). A seven-year longitudinal multi-factor assessment of teaching concerns development through preparation and early years of teaching. Teaching and Teacher Education, 12(2), 225-235.

Postiglione, G. A., \& Lee, W. O. (1997). Schooling in Hong Kong: Organization, teaching and social context. Hong Kong: Hong Kong University Press.

Poon, A. Y. K. (2000). Medium of instruction in Hong Kong: Policy and practice. Lanham, Maryland: University Press of America.

Pun, S. H. (1996). The educational policy development in Hong Kong: A study in the political economy of educational change. Unpublished Ph.D. dissertation, University of Hawaii. Ann Arbor: UMI Dissertation Services.

Rassool, N. (1998). Postmodernity, cultural pluralism and the nation-state: Problems of language rights, human rights, identity and power. Language Sciences, 20(1), 89-99.

Richards, J. C. (1983). The secret life of methods. Plenary address delivered at the annual meeting of Teachers of English to Speakers of Other Languages. Toronto, Canada, 19 March, 1983.

Richards, J. C. (1985). The context of language teaching. Cambridge: Cambridge University Press.

Richards, J. C. (1990a). Integrating theory and practice in second language teacher education. In J. E. Alatis (Ed.), Linguistics, language teaching and language acquisition: The interdependence of theory, practice and research. Georgetown University Round Table on Languages and Linguistics (pp. 219-227). Washington DC: Georgetown University Press.

Richards, J. C. (1990b). The dilemma of teacher education in second language teaching. In J. C. Richards \& D. Nunan (Eds.), Second language teacher education (pp. 3-15). Cambridge: Cambridge University Press.

Richards, J. C. (1991). Content knowledge and instructional practice in second language teacher education. In J. E. Alatis (Ed.), Linguistics and language pedagogy: The state of the art. Georgetown University Round Table on Languages and Linguistics (pp. 76-99). Washington DC: Georgetown University Press. 
Richards, J. C. (1994). The sources of language teachers' instructional decisions. Research Report No. 38. Hong Kong: Department of English, City Polytechnic of Hong Kong.

Richards, J. C. (1996a). The maxims of second language teaching. In G. Tinker Sachs, M. Brock \& R. Lo (Eds.), Directions in second language teacher education. Hong Kong: Department of English, City University of Hong Kong.

Richards, J. C. (1996b). Teachers' maxims in language teaching. TESOL Quarterly, 30(2), 281-296.

Richards, J. C. (1998). Beyond training: Perspectives on language teacher education. Cambridge: Cambridge University Press.

Richards, J. C. \& Ho, B. (1993). Reflective thinking through teacher joumal writing: Myths and realities. Prospect, 8(3), 8-24.

Richards, J. C., Ho, B., \& Gilbin, K. (1992). Learning how to teach: A study of EFL teachers in pre-service training. Research Report No. 19, Department of English, City Polytechnic of Hong Kong.

Richards, J. C., \& Pennington, M. C. (1995). The first year of teaching: Perspectives from second language classrooms. In J. C. Richards (Ed.), Research in second language teaching. New York: Cambridge University Press.

Richards, J. C., \& Rodgers, T. S. (1982). Method: Approach, design, procedure. TESOL Quarterly, 16, 153-168.

Richards, J. C., \& Rodgers, T. S. (1986). Approaches and methods in language teaching. New York: Cambridge University Press.

Richards, J. C., \& Rodgers, T. S. (2001). Approaches and methods in language teaching. Second Edition. New York: Cambridge University Press.

Richards, J. C., Tung, P. \& Ng, P. (1991). The culture of the English language teacher: A Hong Kong example. Research Report No. 6. Hong Kong: Department of English, City Polytechnic of Hong Kong.

Rogers, E. M. (1962). Diffusion of innovations. First Edition. New York: Free Press.

Rogers, E. M. (1995). Diffusion of innovations. Fourth Edition. New York: Free Press.

Rogers, E. M., \& Kincaid, D. L. (1981). Communication networks: Towards a new paradigm for research. New York: Free Press. 
Rogers, E. M., \& Shoemaker, F. F. (1971). Communication of innovations: A Cross-cultural approach. Second Edition. New York: Free Press.

Seagrave, S. (1993). Lords of the rim: The invisible empire of the overseas Chinese. New York: Putnam.

Shachar, H. (1997). Effects of a school change project on teachers' satisfaction with their work and their perceptions of teaching difficulties. Teaching and Teacher Education, 13(8), 799-813.

Simpson, J. (1990). Despatches from the barricades: An eye-witness account of the revolutions that shook the world 1989-90. London: Hutchinson.

So, D. (1987). Searching for a bilingual exit. In R. Lord \& H. N. L. Cheng (Eds.), Language education in Hong Kong (pp. 249-268). Hong Kong: Chinese University Press.

So, D. (1992). Language-based bifurcation of secondary education in Hong Kong: Past, present and future. In K. K. Luke (Ed.), Into the twenty-first century: Issues of language education in Hong Kong (pp. 69-95). Linguistic Society of Hong Kong.

South China Morning Post. Mixed-language teaching bad for students. 10 March, 1997.

South China Morning Post. Tests to raise school language standards. 15 March, 1997.

South China Morning Post. Publishers race to update textbooks. 7 April, 1997.

South China Morning Post. Deadline in school cash deal. 25 August, 1997.

South China Morning Post. Easier tests proposed as 40pc fail languages. 7 August, 1997

South China Morning Post. Lesson to be learned in college crackdown. 25 August, 1997.

South China Morning Post. College bans teacher criticism. 28 August, 1997

South China Morning Post. Teachers' $\$ 20,000$ flats perk 'illegal'. 20 October, 1997.

South China Morning Post. Parents are not ignorant or prejudiced. 8 December, 1997. 
South China Morning Post. Parents unite to fight for English-language teaching. 15 December, 1997.

South China Morning Post. TVB seeks more Chinese airtime. 16 October, 1998 a.

South China Morning Post. Intervention changes face of HK. 16 October 1998b.

South China Morning Post. Give priority to top students, says legislator. 26 January, 1999.

South China Morning Post. Education: the time is right for change. 4 February, 1999.

South China Morning Post. 2007 target on four-year degrees. 1 April, 2000.

South China Morning Post. Teachers vow to boycott test. 28 May, 2000.

South China Morning Post, 6,000 teachers march. 11 June, 2000.

South China Morning Post. Extension of language tests may be scrapped. 4 June, 2001.

South China Morning Post. Teachers flunk English test. 9 June, 2001.

South China Morning Post. 'Mother tongue' schools bow to English as best medium. 29 September, 2001.

South China Morning Post. The case of diminishing autonomy. 11 January 2002.

South China Morning Post. Thousands rally against pay cuts. 8 July 2002.

SPSS Inc. (1999). SPSS Base 10.0 User's Guide. Chicago: SPSS Inc.

Strauss, A. (1987). Qualitative Analysis for Social Scientists. Cambridge: Cambridge University Press.

Strevens, P. (1987). Cultural barriers to language learning. In L. E. Smith (Ed.), Discourse across cultures: Strategies in world Englishes (pp. 169-178). Hemel Hempstead: Prentice Hall.

Stuart, C. \& Thurlow, D. (2000). Making it their own: Preservice teachers' experiences, beliefs, and classroom practices. Journal of Teacher Education, 51(2), pp. 113-121. 
Sweeting, A. (1997). Education policy and the 1997 factor: The art of the possible interacting with the dismal science. In M. Bray \& W. O. Lee (Eds.), Education and political transition: Implications of Hong Kong's change of sovereignty (pp. 25-40). Comparative Education Research Centre, University of Hong Kong.

Sweeting, A. (1998). Education and development in Hong Kong. In P. Stimpson \& P. Morris (Eds.), Curriculum and assessment for Hong Kong: Two components, one system (pp. 5-50). Hong Kong: Open University of Hong Kong Press.

Tan, J. (1997). Education and colonial transition in Singapore and Hong Kong. In M. Bray \& W. O. Lee (Eds.), Education and political transition: Implications of Hong Kong's change of sovereignty (pp. 157-166). Hong Kong: Comparative Education Research Centre, University of Hong Kong.

T'sou, B. K. (1994). Language planning issues raised by English in Hong Kong: Pre- and post-1997. In T. Kandiah \& J. Kwan-Terry (Eds.), English and language planning: A Southeast Asian contribution (pp. 197-217). Singapore: Centre for Advanced Studies, National University of Singapore.

Tsui, A. B. M. (1996). Learning how to teach ESL writing. In D. Freeman \& J. C. Richards (Eds.), Teacher learning in language teaching (pp. 97-120). Cambridge: Cambridge University Press.

Tung, P. C. S. (1990). Why changing the medium of instruction in Hong Kong could be difficult. Journal of Multilingual and Multicultural Development, 11, 523-534.

Tung, P. C. S., Lam, R., \& Tsang, W. K. (1997). English as a medium of instruction in post 1997: What students, teachers and parents think. Journal of Pragmatics, 28, 441-459.

Urmston, A. W. (1994). An examination of the attitudes towards teaching of students in the final year of study in a BA level TESL course in Hong Kong. Unpublished MA dissertation. Department of English, City University of Hong Kong.

Urmston, A. W., Pennington, M. C., \& Brock, M. N. (1996). Attitudes towards teaching English of BATESL students: $A$ comparison of beginning and graduating cohorts. Research Monograph No. 9. Hong Kong: Department of English, City University of Hong Kong.

Vago, S. Social change. Third Edition. Upper Saddle River, New Jersey: Prentice Hall. 
Viswanath, K., \& Demers, D. (1999). Introduction. In K. Viswanath \& D. Demers (Eds.), Mass media, social control, and social change: A macrosocial perspective (pp.3-30). Ames, Iowa: Iowa State University Press.

Walters, S., \& Balla, J. (1998). Medium of instruction: Reality at one Hong Kong tertiary institution. In M. C. Pennington (Ed.), Language in Hong Kong at century's end (pp. 365-390). Hong Kong: Hong Kong University Press.

Watson-Gegeo, K. A. (1988). Ethnography in ESL: Defining the essentials. TESOL Quarterly, 22(4), 575-592.

Weber, M. (1958). The Protestant ethic and the spirit of capitalism. Translated by T. Parsons. New York: Scribner's.

Wilding, P., \& Mok, K. H. (1997). Conclusion: Coming to a judgement. In P. Wilding, A. S. Huque \& L. P. W. Tao (Eds.), Social policy in Hong Kong (pp. 145-165). Cheltenham: Edward Elgar Publishing Limited.

Williams, J. (1995). Focus on form in communicative language teaching: Research findings and the classroom teacher. TESOL Journal, 4, 12-16.

Wong, W. P., \& Lui, T. L. (2000a). Morality, class and the Hong Kong way of life. In S. K. Lau (Ed.), Social development and political change in Hong Kong (pp. 3-32). Hong Kong: The Chinese University Press.

Wong, W. P., \& Lui, T. L. (2000b). From one brand of politics to one brand of political culture. In S. K. Lau (Ed.), Social development and political change in Hong Kong (pp. 309-354). Hong Kong: The Chinese University Press.

Wong-Fillmore, L. (1980). Learning a second language: Chinese children in the American classroom. In J. E. Alatis (Ed.), Current issues in bilingual education. Georgetown University Roundtable on Languages and Linguistics (pp. 309-325). Georgetown University Press.

Woods, D. (1989). Studying ESL teacher's decision-making: Rationale, methodological issues and initial results. Carelton Papers in Applied Language Studies, VI, 107-123.

Woods, D. (1991). Teacher's interpretations of second language teaching curricula. RELC Journal, 22(2), 1-18.

Woods, D. (1996). Teacher cognition in language teaching. Cambridge: Cambridge University Press.

Yost, D.S., Sentner, S.M., \& Forlenza-Bailey, A. (2000). An examination of the construct of critical reflection: Implications for teacher education programming in the $21^{\text {st }}$ century. Journal of Teacher Education, 51(1), 39-49. 
Young, R., \& Lee, S. (1987). EFL curriculum innovation and teachers' attitudes. In R. Lord \& N. L. Cheng (Eds.), Language education in Hong Kong (pp. 8398). Hong Kong: Chinese University Press. 


\title{
LEARNING TO TEACH ENGLISH IN HONG KONG: EFFECTS OF THE CHANGEOVER IN SOVEREIGNTY
}

\begin{abstract}
Alan Urmston
A thesis submitted to the University of Luton, in partial fulfilment of the requirements for the degree of Doctor of Philosophy
\end{abstract}

July 2003

VOLUME 2

APPENDICES

lept at Enquy beje

$$
\begin{aligned}
& 3402972145 \\
& 420 \cdot 7 \\
& \text { URm } \\
& \text { Reference }
\end{aligned}
$$




\section{CONTENTS}

APPENDLX A: Chapter 3

A.1 List of interviewees

A.2 Outline of research sent to interviewees in 1997

A.3 List of questions sent to interviewees in 1997

A.4 Full list of comments from interviews 1997

A.5 Interview transcripts from interviews in 1997

A.6 List of questions sent to interviewees in 1999

A.7 Full list of comments from interviews in 1999

A.8 Interview transcripts from interviews in 1999

A.9 Tables of results of interview study

\section{APPENDIX B: Chapter 4}

B-1

B.1 Transcriptions of interviews with BATESL graduates in 1994

$\mathrm{B}-1$

B.2 Questionnaire administered to BATESL graduates in 1997

B-92

B.3 Transcriptions of interviews with BATESL graduates in 1997

B.4 Statistical results of questionnaire surveys

B-203

B.5 BATESL students' philosophies of teaching

B-244

\section{APPENDIX C: Chapter 5}

$\mathrm{C}-1$

C.1 Transcriptions of interviews with BATESL graduates in $1998 \quad \mathrm{C}-1$

C.2 Main points to emerge during interviews with BATESL graduates in 1998

C.3 Lesson observation reports of 1997 and 1994 BATESL C-54 graduates

C.4 Transcriptions of interviews with BATESL course leaders in C-93 1999

C.5 Main points to emerge from interviews with BATESL course leaders in 1999 


\section{Tables}

3.1 Issues and organisations reported on in the English language A-364 newspapers in 1997

3.2a Issues and organisations quoted in Hong Kong English A-365 language newspapers on Mondays in 1997 (January - June)

3.2b Issues and organisations quoted in Hong Kong English A-366 language newspapers on Mondays in 1997 (January - June)

3.3 Comparison of issues/organisations reported before and after A-367 the changeover of sovereignty on July 1 st

3.4 Table 3.4: Language issues in the English newspapers on A-368 Mondays in 1997

3.5 Educational issues in the English newspapers on Mondays in A-369 1997

3.6 Socio-political issues affecting English language education A-369 mentioned in English newspapers on Mondays in 1997

3.7 Composition of interviewees in 1997 and 1999

A -370

3.8 Comparison of major issues in terms of comments made on A-371 them in each interview

3.9 Comparison of major issues in terms of total number of A-372 comments made

3.10 Aspects of the Medium of Instruction issue raised in A-373 interviews in 1997 and 1999

3.11 Aspects of the Benchmarking issue raised in interviews in A-374 1997 and 1999

3.12 Aspects of the Teachers issue raised in interviews in 1997 and 1999

A-375

3.13 Aspects of the Proportion of the Timetable issue raised in A-376 interviews in 1997 and 1999

3.14 Aspects of the Teaching Methodology \& Curriculum issue A-377 raised in interviews in 1997 and 1999

3.15 Aspects of the English language issue raised in interviews in A-378 1997 and 1999

3.16 Aspects of the Putonghua issue raised in interviews in $1997 \quad$ A-379 and 1999

3.17 Aspects of the Changeover issue raised in interviews in $1997 \quad$ A-380 and 1999

3.18 Aspects of the English Teaching in China issue raised in interviews in 1997 and 1999

3.19 Aspects of the Students issue raised in interviews in 1997 and A-381 1999

3.20 Aspects of the Education Policy issue raised in interviews in A-381 1997 and 1999

3.21 Aspects of the Schools issue raised in interviews in 1997 and A-382 1999

3.22 Aspects of the New Immigrants from China issue raised in interviews in 1997 and 1999 
3.23 Aspects of the Characteristics of Hong Kong People issue raised in interviews in 1997 and 1999

3.24 Aspects of the Chinese Language issue raised in interviews in A-383 1997 and 1999

3.25 Comments made on Higher Level issues in 1997 and $1999 \quad$ A-384

3.26 Comments made on Language issues in 1997 and $1999 \quad$ A-385

3.27 Comments made on Professional issues in 1997 and 1999 A-386

3.28 Comments made on Administrative and Institutional issues in A-387 interviews in 1997 and 1999

3.29 Comments made on Socio-political issues in 1997 and $1999 \quad$ A-388

3.30 Comments made on Educational issues in 1997 and $1999 \quad$ A-389

3.31 Comments made on Behavioural issues in 1997 and $1999 \quad$ A-390

3.32 Comments made on Intercultural issues in 1997 and $1999 \quad$ A-391

4.1 Self-perceived ability in written English B-203

4.2 Self-perceived ability in spoken English B-204

4.3 Admitted use of code-mixing with Cantonese-speaking B-204 friends

4.4 Admitted use of code-switching with Cantonese-speaking B-205 friends

4.5 Level of agreement on whether English should be the only B-205 language used in English lessons

4.6 Level of agreement on whether lesson content can be B-206 presented in mixed-code in English lessons

4.7 Level of use of English only in English lessons during B-206 practice teaching

4.8 Extent to which amount of pure English used in lessons $\quad$ B-207 during teaching practice changed during the period

4.9 Situations in which subjects would or might use Cantonese in B-208 English lessons

4.10 Level of agreement on it being impossible to have a B-209 successful lesson that hasn't been well planned in advance

4.11 Level of agreement on whether teachers should write detailed B-209 lesson plans for every lesson they teach

4.12 Assessment of how detailed lesson plans need to be B-210

4.13 Basis on which lessons were planned during practice teaching B-210 
4.14 Factors having most influence on decisions made during practice teaching

4.15 Level of agreement on whether teachers should be more responsive to student needs than the set syllabus

4.16 Level of agreement on whether examination requirements should determine what is taught in English lessons

4.17 Level of agreement on whether students learn English better though interaction with other students rather than through direct instruction from a teacher

4.18 Whether students learned English better through interaction with other students or through instruction from teacher during practice teaching

4.19 Level of agreement on whether a class should be strictly controlled by the teacher and all lesson content directed by the teacher

4.20 Level of agreement on whether a teacher should be prepared to experiment with materials and teaching strategies

4.21 Description of teaching style

4.22 Factors having most effect on teaching of English

4.23 Level of confidence in ability to become successful teacher

4.24 Opinions on status of teachers

4.29 Expectations of which first-year teaching activities would take up the most time

4.30 Views on extent to which they might work closely with other B-228 teachers in the future

4.31 What will be of most help in terms of development as a teacher in first year?

4.32 Opinions as to whether they have a definite philosophy of B-232 teaching

4.33 Factors that subjects felt unsure about

4.34 Most important factors affecting what goes on in the classroom

4.35 Importance of language skills 
$4.3897(3)$ group's opinion as to the extent to which the use of English in secondary schools might be affected by the changeover in sovereignty

4.39 97(3) group's opinion as to the extent to which their lesson planning and decision-making might be affected by the changeover in sovereignty

$4.4097(3)$ group's level of agreement on whether the changeover in sovereignty will affect their approach to teaching

4.41 Extent to which the Yr. 3 (97) group are worried about their future as teachers after the changeover of sovereignty

$4.4297(3)$ group's opinions as to whether their professional relationships or responsibilities will change after the changeover of sovereignty 


\section{APPENDIX A}

Appendix A consists of the following, which should be viewed in conjunction with Chapter 3 of the thesis:

\section{A.1 List of interviewees}

A.2 Outline of research sent to interviewees in 1997

A.3 List of questions sent to interviewees in 1997

A.4 Full list of comments from interviews 1997

A.5 Interview transcripts from interviews in 1997

A.6 List of questions sent to interviewees in 1999

A.7 Full list of comments from interviews in 1999

A.8 Interview transcripts from interviews in 1999

A.9 Tables of results of interview study 


\section{A.1 List of Interviewees (in alphabetical order)}

\begin{tabular}{|c|c|c|c|}
\hline \multirow[t]{2}{*}{ Name } & \multirow{2}{*}{ Position } & \multicolumn{2}{|c|}{ Date(s) of interview(s) } \\
\hline & & 1997 & 1999 \\
\hline Chan Wai Ming & $\begin{array}{l}\text { Education Officer (English) } \\
\text { Curriculum Development Institute } \\
\text { Hong Kong Education Department }\end{array}$ & & 24 Sept. \\
\hline Chan Yuk King & $\begin{array}{l}\text { Teacher (English) } \\
\text { Tsun Mun Government Sec. School } \\
\text { Tsuen Mun }\end{array}$ & 11 June & \\
\hline Daniel Desilet & $\begin{array}{l}\text { Teacher (English) } \\
\text { Japanese International School } \\
\text { Tai Po }\end{array}$ & & 2 October \\
\hline Peter Falvey & $\begin{array}{l}\text { Senior Lecturer } \\
\text { Department of Curriculum Studies } \\
\text { University of Hong Kong }\end{array}$ & 30 May & 22 June \\
\hline Gail Fu & $\begin{array}{l}\text { Professor \& Associate Head } \\
\text { English Language Teaching Unit } \\
\text { Chinese University of Hong Kong }\end{array}$ & 4 June & \\
\hline John Fullilove & $\begin{array}{l}\text { Subject Officer (English) } \\
\text { Hong Kong Examinations Authority }\end{array}$ & 3 June & 15 July \\
\hline Keith Johnson & $\begin{array}{l}\text { Senior Lecturer } \\
\text { Department of Education } \\
\text { University of Hong Kong }\end{array}$ & 30 May & \\
\hline Graham Kennedy & $\begin{array}{l}\text { Subject Officer (English) } \\
\text { Hong Kong Examinations Authority }\end{array}$ & 3 June & 15 July \\
\hline Koo Chau Ming & $\begin{array}{l}\text { Headmaster, } \\
\text { Kwok Tak Seng Catholic Sec. School } \\
\text { Shun Tin Wai }\end{array}$ & 22 August & \\
\hline Lai Mei Ling & $\begin{array}{l}\text { Lecturer } \\
\text { English Department } \\
\text { Hong Kong Institute of Education }\end{array}$ & & 29 June \\
\hline
\end{tabular}




\begin{tabular}{|c|c|c|c|}
\hline Christina Lee & $\begin{array}{l}\text { Subject Officer (English) } \\
\text { Hong Kong Examinations Authority }\end{array}$ & 3 June & 15 July \\
\hline Cynthia Lee & $\begin{array}{l}\text { Native English Teacher } \\
\text { Heep Yunn School } \\
\text { Kowloon }\end{array}$ & & 2 October \\
\hline Nancy Lee & $\begin{array}{l}\text { Head } \\
\text { Support Unit for the Standing } \\
\text { Committee on Language Education } \\
\text { and Research } \\
\text { Hong Kong Education and } \\
\text { Manpower Bureau } \\
\end{array}$ & & $\begin{array}{c}15 \text { July } \\
\text { (in writing) }\end{array}$ \\
\hline Sheridan Lee & $\begin{array}{l}\text { Senior Inspector (English) } \\
\text { Hong Kong Education Department }\end{array}$ & 17 July & \\
\hline Tom Lee & $\begin{array}{l}\text { Lecturer (English) } \\
\text { Hong Kong Institute of Education }\end{array}$ & 6 June & \\
\hline Matthew Leung & $\begin{array}{l}\text { Head of Language Institute } \\
\text { City University of Hong Kong }\end{array}$ & 29 May & \\
\hline Benjamin Li & $\begin{array}{l}\text { Lecturer } \\
\text { English Department } \\
\text { Hong Kong Institute of Education }\end{array}$ & & 29 June \\
\hline Angel Lin & $\begin{array}{l}\text { Associate Professor } \\
\text { Department of English } \\
\text { City University of Hong Kong }\end{array}$ & 27 June & \\
\hline Graham Lock & $\begin{array}{l}\text { Associate Professor } \\
\text { Department of English } \\
\text { City University of Hong Kong }\end{array}$ & & 5 October \\
\hline K.K. Luke & $\begin{array}{l}\text { Senior Lecturer \& Head } \\
\text { Department of Linguistics } \\
\text { University of Hong Kong }\end{array}$ & 4 July & 13 July \\
\hline Ken McMorland & $\begin{array}{l}\text { Panel Chair (English) } \\
\text { St. Stephen's College } \\
\text { Stanley }\end{array}$ & 10 June & \\
\hline
\end{tabular}




\begin{tabular}{|c|c|c|c|}
\hline Irene Mok & $\begin{array}{l}\text { Panel Chair (English) } \\
\text { Kiangsu Chekiang College } \\
\text { North Point }\end{array}$ & 16 June & \\
\hline Shari Nazir & $\begin{array}{l}\text { Teacher (English) } \\
\text { St. Stephen's College } \\
\text { Stanley }\end{array}$ & 10 June & 16 June \\
\hline Frederick Poon & $\begin{array}{l}\text { Teacher (English) } \\
\text { St. Stephen's College } \\
\text { Stanley }\end{array}$ & 10 June & \\
\hline Dominic So & $\begin{array}{l}\text { Headmaster, } \\
\text { Shek Lee Catholic Secondary School } \\
\text { Kwai Hing }\end{array}$ & 19 August & \\
\hline Steve Tauroza & $\begin{array}{l}\text { Associate Professor } \\
\text { Coordinator of BATESL } \\
\text { Department of English } \\
\text { City University of Hong Kong }\end{array}$ & 16 June & 7 Sept. \\
\hline Andrew Taylor & $\begin{array}{l}\text { Associate Professor \& Acting Head } \\
\text { Department of English } \\
\text { City University of Hong Kong }\end{array}$ & & 28 June \\
\hline Simon Tham & $\begin{array}{l}\text { Education Officer } \\
\text { English Inspectorate } \\
\text { Hong Kong Education Department }\end{array}$ & & 24 Sept. \\
\hline Amy Tsui & $\begin{array}{l}\text { Professor } \\
\text { Department of Curriculum Studies } \\
\text { University of Hong Kong }\end{array}$ & 4 July & 21 June \\
\hline Ken Yiu & $\begin{array}{l}\text { Teacher (English) } \\
\text { Kiangsu Chekiang College } \\
\text { North Point }\end{array}$ & 16 June & 30 June \\
\hline
\end{tabular}

In addition, one school principal and one English panel chair answered questions anonymously in writing during the second administration in 1999. 


\section{A.2 Outline Sent to Prospective Interviewees in 1997}

\section{LEARNING TO TEACH ENGLISH IN HONG KONG: EFFECTS OF THE CHANGEOVER IN SOVEREIGNTY}

(Supervised by Prof. Martha C. Pennington, Powdrill Chair of English Language Acquisition, University of Luton, UK)

\section{Dear Colleague,}

At present I am gathering data for a research project which will, I hope, lead to my completing a doctorate. From the above title, you can see that $\mathrm{I}$ am looking into the teaching of English in Hong Kong with special emphasis on how it is affected by the coming changeover in sovereignty.

The purpose of the research is to investigate issues of teacher change and educational change more generally in a changing society. The focus is on the teaching and learning of English as a second language in Hong Kong through the perceptions of individual teachers, teacher educators, administrators and the community at large in the run-up to and the first year after the changeover of sovereignty of Hong Kong from British to Chinese rule on 1st July 1997. The project will be in two stages which together will document the effects of the changeover on English language teaching and English language teacher education in Hong Kong.

The first part of the project will consist of a survey of the views and opinions of people connected with ESL in Hong Kong with regard to the possible effects on the teaching and learning of English in Hong Kong of the changeover in sovereignty. Firstly, the views of the final-year cohort of students on the BATESL course at the City University of Hong Kong will be taken, by means of questionnaire and interview. These views will be compared to those taken from the same group three years earlier when they were in their first year of the BATESL course, as well as to the views of a similar cohort of third-year students on the same course gathered three years previously. Secondly, via interviews, the views of school principals, English panel chairpersons, teacher training supervisors from the tertiary institutes in Hong Kong offering Bachelor and Masters degree programs in TESL, officials of the Education Department/Examinations Authority/Curriculum Development Council, and local academics with particular expertise in the field will be taken.

The findings from these surveys will then be compared with previous studies of English teachers in Hong Kong carried out during the 1980s and early 1990s, with the intention of addressing issues raised in those studies and in the larger field of teacher change and educational innovation in language teaching. In addition, to extend and elaborate these findings, this part of the study will look at how the issues connected with English 
language teaching are reported in the English language press in Hong Kong during the period of the changeover, including language use, language planning and policy, the teaching of language, language teacher issues, and education issues affecting language teaching. By recording the number of times that each issue is mentioned in the South China Morning Post (SCMP) and the Hong Kong Standard (HKS) on Monday of each week, it will be possible to see which issues are most prevalent, whether a pattern emerges concerning which issues are mentioned most frequently, and whether this pattern changes over time. In addition to this quantitative analysis, relevant articles, letters to the editor, editorials and reports will be collected to form a database that will be drawn on in the discussion of the overall results of the project.

The second part of the study will consist of a continuation and extension of the study into the 1997-1998 academic year and beyond attempting to test and refine the findings of the first part of the study. The BATESL graduates, who will be in their first year of teaching in Hong Kong secondary schools, and the other ESL professionals previously surveyed will be surveyed again to see if their views change in any way after the changeover, while also looking at the reporting of educational and language issues in the press in this same period

To this end, I would like to invite you to take part in this project by sparing a little of your time and allowing me to interview you with regard to the issues outlined above. I would take no more than 30 minutes of your time at a time and place of your convenience.

I intend to begin talking to people after the 18 th May. I can come to your place of work or if you would prefer the interview could be carried out via e-mail. I would be most grateful for your participation in what I hope and anticipate will be a fascinating study. Needless to say, I will keep everybody who helps me well informed of my findings. I can be contacted as detailed below.

Many thanks in anticipation,

Alan W. Urmston

$\operatorname{Tel}(\mathrm{H}): 25646279$

Tel (O): 27110862

Pager: 72216095

E-mail: alanu@netvigator.com 


\section{A.3 List of Questions Sent to Interviewees in 1997}

\section{LEARNING TO TEACH ENGLISH IN HONG KONG: EFFECTS OF THE CHANGEOVER IN SOVEREIGNTY}

What follows is a list of questions which I would like you to think about and which we can discuss when we meet in person:

1. What are your feelings as regards the Education Department's decision to force seventy-five percent of secondary schools to adopt Chinese medium education?

2. What are your feelings as regards the Education Department's planned introduction of Benchmark language levels for language teachers?

3. Do you think that there will be any other significant changes in government policy towards the teaching of English in Hong Kong after the changeover? If so, what might these changes be?

4. What changes do you envisage in the proportion of the timetable given over to English language teaching in both English and Chinese medium schools?

5. Do you think that there will be any other significant changes in your school or the education system more generally that might affect the teaching of English?

6. Do you think that the changeover will affect teaching methods or approaches in English lessons in secondary schools? If so, in what ways and why?

7. How do you think that the changeover will affect the status, situation, or morale of teachers of English in Hong Kong?

There may be other questions that come up during conversation or arise due to announcements by the government or Education Department, but the above will make up the bulk of my interview.

Many thanks for your participation

Alan W. Urmston 


\section{A.4 Full List of Comments from Interviews in 1997}

The following are the comments derived from the transcribed interviews with the interviewees in 1997. The numbers refer to the number of interviews in which that comment was made. If a comment was mentioned more than once during an interview, it was only counted once. In those interviews where there was more than one person interviewed, a comment was only counted once, even if it was made by more than one interviewee. For comments mentioned in only one interview, no number is given. The comments are grouped into common themes and the themes are listed in descending order with the most common given first. Within each theme, the comments are divided into Main comments (mentioned in two or more interviews) and Individual comments (mentioned once or in one interview).

\section{The Medium of Instruction Issue - 154}

\section{Government influence - 19}

\section{Main comments:}

The firm guidance is a political decision. (5)

It will be easier for the SAR government to implement the firm guidance than it would be for the colonial government. (4)

The firm guidance is not connected to the changeover. (3)

Individual comments:

As many changes are taking place, the government hopes that the firm guidance will be accepted.

The government may be pre-empting the future SAR government from making all schools CMI.

The Chinese government is more concerned with educational content than with MOI.

The Education Department is giving out conflicting messages on the effectiveness of CMI versus EMI.

Firm guidance means that the government is forcing schools to implement their policy of EMI properly.

The Education Department appears to be promoting CMI but are really promoting EMI. The government may revoke the firm guidance after a few years.

\section{In support of $\mathrm{CMI}-18$}

\section{Main comments:}

Teaching in Chinese can cover the syllabus more quickly. (6)

Students learn more efficiently through their mother tongue. (4)

CMI will remove the language burden from students so that they become more interested in English as a subject. (2) 
Individual comments:

Schools are not against CMI.

The implementation of CMI in most schools will stop many students from giving up.

Most teachers are in favour of CMI.

Most school principals are in favour of CMI.

A mastery of English is not as important as being able to learn better through the mother tongue.

Students who can learn equally effectively through either English or Chinese are exceptional cases.

\section{Schools could be allowed flexibility in MOI - 14}

\section{Main comments:}

Schools should be given flexibility. (4)

Teach some subjects in English and some in Chinese. (4)

Schools will be allowed more flexibility from Form 4 upwards to prepare students for

EMI tertiary. (2)

\section{Individual comments:}

Switching the MOI from Chinese to English in Form 4 will not work.

Schools can introduce CMI gradually.

A gradual approach of teaching some subjects through English and some through

Chinese will not work.

Weaker classes can be taught in Chinese and the better ones in English.

\section{MOI at tertiary level -14}

\section{Main comment:}

English has to be retained for tertiary or further study. (3)

Individual comments:

Some universities could become CMI.

There needs to be a fast track for the best students in CMI schools through to EMI tertiary education.

CMI tertiary education would be seen as second-class.

Support must be provided for students from CMI schools entering EMI tertiary education.

Students studying in CMI schools will be disadvantaged as regards entry into university.

The tertiary institutions are not making any preparation to distinguish between students from CMI schools and those from EMI schools.

EMI in tertiary education will have a washback effect ensuring even CMI schools maintain a strong English stream.

Some students will struggle with EMI in university.

In tertiary, how much is in English? 
The transition from learning through the $\mathrm{L} 1$ to learning through the $\mathrm{L} 2$ will be easiest for tertiary students.

The majority of students in university could be taught through Chinese.

\section{Against CMI - 11}

\section{Main comments:}

The implementation of CMI will result in a fall in the standard of the student intake. (5) The implementation of CMI will result in a fall in the standard of English. (3)

\section{Individual comments:}

The morale of Chinese teachers will be affected by switch to CMI.

Teachers may feel that teaching through Chinese is no better than the old method. If students learn through Chinese, it restricts them to Hong Kong or Southern China.

\section{Views of parents and the public - 11}

Main comments:

All schools should be CMI but are not due to parental and societal pressure. (4) Parents prefer EMI for their children. (3)

\section{Individual comments:}

Parents don't understand the situation as regards MOI.

Parents should be educated as to the reality in terms of MOI in universities.

People don't want the MOI to change so the government has to enforce it.

People have been waiting for the government to make a decision about the MOI.

\section{Background to firm guidance - 11}

\section{Main comment:}

The firm guidance should have been implemented a long time ago. (4)

\section{Individual comments:}

CMI is not a new idea.

In the past the Education Department has allowed schools to do what they like as regards MOI.

The lack of firm guidance on MOI in the past has had a negative effect on English, Chinese and subject learning.

There has always been a seesaw in popularity between English and Chinese in education in Hong Kong.

The government has always backed-off from implementing any firm guidance on language policy in schools. 
The main thrust of the firmguidance is the concern with students' ability in content subjects.

There was a lot of resistance to the implementation of CMI so it was continually put back.

\section{English in CMI schools - 11}

\section{Main comments:}

Students will no longer leam English outside of English lessons in CMI schools. (2) There may be more support and resources for English in CMI schools. (2)

Individual comments:

There will be a need to explore innovative methods to motivate students to learn English in CMI schools.

English teachers in CMI schools will need to provide a rich linguistic and cultural environment in which English has major communicative roles to play.

In CMI schools, only English should be used in English lessons.

For the CMI schools, more English teachers will be employed.

In CMI schools, the students will get increasing amounts of English until Form 6, which will be the transition to EMI tertiary education.

English teachers will have to teach terms that students won't have learned in their other subjects.

English should remain a compulsory subject.

\section{Effect on teaching or curriculum of change to CMI - 8}

\section{Main comments:}

Teachers will have to learn Chinese translations of terms that were previously given in English. (3)

\section{Individual comments:}

Textbooks will be in English but teaching will be in Chinese.

The CMI schools will be highly textbook-dependent.

The enforcement of CMI will mean no more mixed-code.

There will inevitably be mixed-code teaching in CMI schools.

Schools changing to CMI will increase the number of Chinese lessons.

\section{Against firm guidance - 7}

Main comment:

The firm guidance is very unpopular with students, parents and teachers. (2)

Individual comments: 
There is a mistaken belief that the change in the MOI will raise standards.

The firm guidance is inconsistent.

Many schools are dissatisfied with the short notice they have been given as regards the implementation of CMI.

The implementation of CMI will be socially divisive.

The firm guidance is an estimate based on a study in another area.

In support of EMI - 6

\section{Main comment:}

Students shouldn't be deprived of the opportunity to reach a high standard of English through immersion. (3)

\section{Individual comments:}

There are more than $25 \%$ of schools that are capable of teaching through English.

EMI does help improve students' English standard.

English teachers in EMI schools will get a boost as everyone wants to be there.

\section{Against EMI - 6}

\section{Main comment:}

EMI provides students with the opportunity to use English wrongly. (2)

Individual comments:

EMI is not being implemented effectively at present.

Although schools are EMI, they teach mainly in Chinese.

With EMI the whole population of students become second class citizens.

Only the best students will be focused on in the EMI schools.

\section{Time will be needed to judge the effectiveness of CMI - 5}

Main comment:

It will be a long time before the effectiveness of CMI can be determined. (3)

\section{Individual comments:}

It may take some time for the standard of English to pick up after the implementation of CMI.

It will take a period of time for people to get used to the idea of CMI.

\section{Effect of firm guidance on schools - 5}

Individual comments: 
Schools may lose educational goals due to obsession with EMI.

The EMI schools will become élitist.

Some CMI schools will be more famous than others.

The number of EMI schools may be reduced.

Some schools will not apply to be EMI because of the policy of their controlling organisation.

\section{Differences between EMI and CMI - 3}

Individual comments:

There will be different rules for English departments in CMI and EMI schools.

Different strategies need to be taught to teachers who will be teaching in CMI and in EMI schools.

There should be different examinations for students from CMI and from EMI schools.

\section{Effects of change to CMI on society - 3}

Individual comments:

In the future, all high-ranking government officials will come from CMI schools. If the people who are in power came from CMI schools, then they will regard English as less important than Chinese.

The tertiary institutes and local business will defend the use of English.

\section{In support of firm guidance - 2}

Individual comments:

School principals feel that it's not too good being directed by government but it is necessary.

The firm guidance will be easier to administer than the present situation.

\section{Teachers - 83}

\section{Standard of English teachers - 14}

Main comments:

Most English teachers are not qualified to teach English. (4)

The people entering the teaching profession are not the best. (4)

Some language teachers are not of the expected standard. (3)

Teachers are not confident in using pure English or pure Chinese as they were taught in mixed-code. (2) 
Individual comment:

English teachers are good enough to do the job if they have a degree.

\section{Native speaking teachers -13}

\section{Main comments:}

More native-speaking English teachers should be recruited. (3)

Local English teachers need to work hard to compete with native-speaking English teachers. (2)

Localisation will mean fewer opportunities for native-speaking English teachers. (2)

\section{Individual comments:}

Local graduates are being overlooked as teachers in favour of native-speakers.

Some schools don't want native-speaking English teachers.

Local English teachers feel that native-speaking English teachers cannot help with administration.

English teaching should continue to rely on both local and native speaking teachers. Native-speaking English teachers will be employed for extra-curricular activities. Returnees from overseas can become English teachers.

\section{English proficiency of English teachers - 10}

\section{Main comments:}

The English proficiency of local English teachers is inadequate. (3)

Many teachers cannot teach through English. (2)

It is important for the teacher to be better than the students in order to raise the students' language level. (2)

\section{Individual comments:}

Teachers with a low proficiency of English could be asked to teach the lower forms.

Teachers teaching at senior secondary level have a good standard of English.

The teaching method is more important than language proficiency for teachers.

\section{Teachers' retraining - 10}

\section{Main comments:}

Teachers must be trained to teach in Chinese. (3)

Teachers should be made to attend regular refresher courses. (2)

Teachers will take in-service courses if they are related to promotion. (2)

Individual comments: 
There are new ideas in teaching methodology being generated as teachers attend more training courses at local universities.

A lot more courses are available to teachers nowadays.

Non-trained English teachers should be told to get an English teaching qualification.

\section{Situation and status of teachers - 8}

Individual comments:

Many teachers are forced into teaching English by their schools.

Teachers teaching at lower secondary don't want to teach English.

Some people become teachers because the salary is high.

Language graduates only go into teaching when the economy is bad.

The government wants to make the criteria for joining the teaching profession more specific.

There will be less need for so many English teachers.

English teachers will still enjoy a high status.

There will be no change in the status of teachers.

\section{Motivation and morale of English teachers - 7}

Main comments:

The morale of English teachers is already going down. (2)

There will be no effect on the morale of English teachers. (2)

Individual comments:

English teachers lack motivation.

Primary teachers are not as motivated as secondary teachers.

Teachers should be found who are interested in teaching English.

\section{Concerns of teachers - 7}

\section{Individual comments:}

Some teachers are worried about self-censorship.

Teachers in Hong Kong are concerned about equality.

Teachers hope for a reduced workload and an increase in the number of teachers.

Teaching English is like fighting a war against so many obstacles.

Language teachers are being given a hard time.

The teaching environment in Hong Kong is not attractive enough to attract people to become teachers.

Teachers are too overworked to spend time using high-tech equipment to prepare materials. 


\section{Subject teachers -3}

Main comment:

It is the subject teachers who need the most help with language. (3)

\section{Image of teachers - 3}

\section{Main comment:}

There is a culture within teaching that rejects new initiatives. (2)

Individual comment:

The Hong Kong media blame English teachers for students' poor proficiency.

\section{Teachers' practices -3}

\section{Individual comments:}

Teachers are not using new methodologies in any practical way.

Some students or teachers will think to communicate is the only requirement for English proficiency.

Most teachers teach English as a foreign language.

\section{Needs for teachers -2}

Individual comments:

Teachers should be given flexibility to teach within the classroom.

Teachers must be given a lot of support.

\section{Teacher trainers -2}

Individual comments:

Teacher trainers oppose using English to teach trainee teachers.

Many teacher trainers do not have adequate proficiency in English.

\section{Teachers' personality - 1}

Individual comment:

The teacher's personality plays an important role in making students more confident in using language. 


\section{Benchmarking - 64}

\section{Specificity/Details of benchmarking - 13}

Main comments:

If the benchmarks are set too high, there won't be enough teachers. (4)

It is not clear how the benchmarking will be interpreted. (3)

\section{Individual comments:}

The benchmark should not be set too low just to fill posts.

The level of the benchmark should be high to be meaningful.

The vagueness of criteria of the benchmarking is a problem for teachers.

There may be different benchmarks for primary and secondary teachers.

Benchmark must be the same for all bands of school.

The benchmarking will contain a classroom observation component.

\section{Against the benchmarking - 9}

\section{Main comments:}

Some teachers will deliberately fail the benchmark. (2)

The benchmarking is unnecessary because of teachers' pre-existing qualifications. (2)

Teachers do not support the benchmarking. (2)

Individual comments:

Some teachers feel that the benchmarking is an insult.

Benchmarking will have a demoralizing effect on teachers.

The benchmarking will waste a lot of money.

\section{Benchmarking as way to improve teachers' language proficiency -8}

Main comments:

The benchmarking must be done positively, with the emphasis on training. (6)

Individual comments:

Instead of benchmarking, further training should be given to teachers to improve their language levels.

The benchmarking will encourage teachers to improve their language ability.

\section{Benchmarking will put pressure on teachers - 7}

\section{Main comments:}

There will be more pressure on experienced teachers due to benchmarking. (4) 
Benchmarking will put pressure on teachers to perform. (3)

Benchmarking for all teachers - 6

Main comment:

Benchmarking should be for subject teachers also. (4)

Individual comments:

There are plans to benchmark all teachers.

Benchmark levels for subject teachers should not be as high as for language teachers.

Benchmarking for new teachers only - 6

Main comments:

The benchmarking may only be feasible for new teachers. (3)

The benchmark can serve as the graduation standard for new teachers. (2)

Individual comment:

The benchmarking should be introduced gradually and to new teachers first.

The benchmarking related to professionalism of teachers - 6

Main comment:

Through the benchmarking, the government is trying to promote professionalism. (2)

Benchmarking will add to the prestige of the teaching profession. (2)

Individual comments:

If teachers are professional, they will welcome the benchmarking.

The benchmarking will raise the status of English teachers, as not everybody will be able to teach English.

In favour of the benchmarking - 4

Main comment:

Support for benchmarking. (3)

Individual comment:

Reaching the benchmark will result in an improved morale of teachers.

Incentives for those who reach the benchmark - 4 
Main comment:

Incentives should be offered for teachers who pass benchmark. (3)

Individual comment:

Make benchmarking voluntary for existing staff, but they get an increment for passing.

Origins of benchmarking - 1

Individual comment:

The benchmarking initiative is fueled by pressure from the business community.

Focus on English - 34

Status of English in Hong Kong will remain high - 14

Main comments:

The importance of English in Hong Kong will not be de-emphasised. (4)

There is a demand for good English teachers. (2)

More graduates will be needed with good language skills. (2)

Individual comments:

There will still be a need for English in the foreseeable future.

Business people wish to keep the status of English in Hong Kong high.

There will be a greater demand for better English in schools.

An elite group of people having good English will get the best jobs.

English proficiency is still an asset in Hong Kong.

Parents want their children to have a strong command of English.

\section{Status of English in Hong Kong will decrease - 8}

\section{Main comments:}

The status of English teachers will become lower as the emphasis will no longer be on English. (3)

English will slip from first foreign language to second foreign language. (2)

\section{Individual comments:}

There will be a gradual loss of English in favour of Chinese amongst university staff. Hong Kong may not produce enough people with good English.

The prestige of English in Hong Kong is not as high as in the past. 


\section{Question of status of English in Hong Kong - 4}

Main comment:

Hong Kong is not an ESL situation. (2)

Individual comments:

English in Hong Kong should be treated as a second language and not as a foreign language.

English is a means of widening learners' horizons.

\section{English as no longer associated with England - 4}

Main comment:

English is becoming more globally functional. (2)

Individual comments:

The name of English Literature is to be changed so as to de-emphasise its relation to England.

People in Hong Kong tend to pick up American rather than British accents.

\section{English in universities - 4}

Main comment:

There will be an increase in the attention paid to English in the universities. (2)

Individual comments:

Universities will stay international if they can publish in English.

Students in university should be able to read journals and write in English.

\section{Teaching Methodology and Curriculum Issues - 29}

Content -7

\section{Individual comments:}

There will be no cultural content in English lessons.

Oral is not given emphasis in English teaching in Hong Kong.

The Form 5 syllabus is having a washback effect on oral instruction.

Hong Kong students need to be taught patriotism.

Students in secondary schools should be given the choice of different types of English instruction, such as, EAP.

Students should be taught practical English rather than social English. 
Putonghua, Standard Written Chinese and English should be treated as foreign languages.

\section{Possible changes in methodology - 6}

Main comments:

There will be no change in teaching methodology. (3)

There may be a reaction against communicative methodology by teachers. (2)

Individual comment:

The business community will resist any reaction against communicative methodology.

Existing problems with methodology - 4

Individual comments:

New methods have been employed but have not improved learning.

The biggest problem is the narrowness of the education system.

It is recognised in Hong Kong that traditional teaching methods don't work.

To try to train students in a foreign language at university level is too late and too expensive.

Students should learn grammar - 3

Main comment:

Students should learn grammar. (3)

Extra-curricular activities - 3

Main comment:

Schools may incorporate extra-curricular activities to improve English proficiency. (3)

$\underline{\text { TOC }}-3$

Individual comments:

The TOC will bring a greater emphasis on oral instruction.

TOC is the only other change envisaged.

The implementation of TOC would indicate that there might be changes in teaching methods. 
Curriculum -2

Individual comments:

The school curriculum must be revised.

The number of years in primary and secondary schooling could be changed.

\section{Textbooks - 1}

Individual comment:

Textbooks must be designed for Hong Kong students.

\section{Putonghua - 26}

Putonghua as MOI - 13

Main comments:

Teaching in Putonghua will become an issue. (5)

Putonghua should be used to teach Chinese. (4)

Individual comments:

Putonghua will eventually become the MOI in schools in Hong Kong.

Putonghua cannot become the MOI because there are not enough teachers.

Cantonese and not Putonghua should be the MOI in Hong Kong.

Putonghua will not become the MOI in schools for many years.

\section{Putonghua in Hong Kong society - 6}

Main comments:

English will become the common language between speakers of Cantonese and Putonghua. (2)

Putonghua will replace English as the status language in Hong Kong in the near future. (2)

Individual comments:

Most of the work that will be done on raising language standards will focus on Putonghua.

The switch from English to Putonghua will be politically correct after the changeover.

\section{Putonghua teaching in schools - 5}

Main comment: 
The number of lessons of Putonghua will increase. (2)

Individual comments:

Putonghua will only be offered as an extra-curricular activity.

Learning Putonghua can help students when learning English.

English and Putonghua could be elective subjects in CMI schools.

\section{Teachers of Putonghua - 2}

Individual comments:

There are not enough teachers of Putonghua.

There will be more and more people capable of teaching Putonghua.

\section{Proportion of Timetable -23}

Increase in amount of English - 10

Main comment:

The proportion of English on the timetable in CMI schools will be increased. (10)

\section{Decrease in amount of English - 6}

Main comment:

The proportion of English on the timetable will be reduced due to the introduction of Putonghua. (4)

Individual comments:

The number of English lessons could be reduced in EMI schools.

The implementation of the firm guidance will result in a reduction in both English and Chinese on the timetable.

\section{No change in amount of English - 3}

\section{Main comment:}

There will be no change in the proportion of English on the timetable in schools that are designated EMI. (2)

\section{Individual comment:}

Changing the MOI will not affect the proportion of languages on the school timetable as the proportion for content subjects will not change. 
Schools should be allowed to decide - 1

Individual comment:

It is up to the schools to decide on the proportion of the timetable to be devoted to the languages.

Quality is more important than quantity - 1

Individual comment:

The quality of teaching is more important than what proportion of the timetable is given to the teaching of English.

English enjoys privileged position in timetable - 1

Individual comment:

In most schools there is great envy at the amount of English on the timetable.

Any decrease in amount of English will be opposed - 1

Individual comment:

Parents may agitate against any decrease in the time given to English teaching.

\section{References to the Changeover - 15}

The changeover will have no effect - 8

\section{Main comments:}

The changeover will have no effect on government policy towards the teaching of English. (4)

There will be no effect on the situation, status or morale of English teachers due the changeover. (3)

Individual comment:

Teachers are more concerned about personal or contractual issues than the changeover.

There will be changes but not due to the changeover - 6

Main comment:

Advances in technology could be more influential than the changeover. (3)

Individual comments: 
There will be no significant changes in government policy towards the teaching of English but there will be changes on the ground.

The only major change will be the change in textbooks.

Teacher education programmes will affect teaching methods more than the changeover.

There will be changes due to the changeover - 1

Individual comment:

The changeover may improve the status of English teachers due to the international status of Hong Kong.

\section{English Teaching in China - 13}

English learning is very popular in China - 7

Main comment:

Many people are learning English in China. (5)

Individual comments:

People in China have no hang-ups about learning English.

English Literature is very popular in China.

\section{English teachers in China - 3}

Individual comments:

Teachers from China can meet the demand for more English teachers.

There is a shortage of English teachers in China.

Native-speaking English teachers are hired in China to teach oral English.

\section{English teaching methodology in China - 3}

\section{Individual comments:}

The teaching of English in Hong Kong is better than that in China.

The teaching methodology in China is one of vocabulary memorization and grammar rules.

The communicative approach to teaching English is now being implemented in China. 
Students - 12

Criticism of students -7

Main comment:

Students nowadays have a short attention span. (2)

Individual comments:

Hong Kong students have difficulty when performing any task that requires extended language use.

Students entering university are accuracy-focused and afraid to speak.

Students always make the same kinds of mistakes and these are very difficult to change. The standard of English of students in Hong Kong is declining.

Most students can't see the point in learning English.

\section{Culture of Hong Kong students - 3}

Individual comments:

References to the decline in language standards affect the morale of students.

There's a culture within schools which frowns on people being fluent in English.

Students think that if their Chinese is all right and their other subjects are all right then they won't worry if they fail in English.

\section{Affect of changeover - 1}

Individual comment:

The reluctance to show ability in English may disappear.

Comment on language standards - 1

Individual comment:

The fall in language standards is more folklore than statistical.

\section{Education Policy - 11}

Changes due to the changeover -4

Individual comments: 
Time is needed to measure the effects of new initiatives before other changes should be implemented.

Government policy may change in response to changes in demand and the atmosphere in society.

The colonial government would like to be seen to be promoting Chineseness.

China will want to preserve the education system in Hong Kong.

\section{Needs for education system - 4}

Individual comments:

Hong Kong needs to give its children the best education so as to compete with other places.

The conditions in schools must be improved to attract better people to join the teaching profession.

Standards cannot be improved unless resources are committed to the school system. The government needs to assign more resources to extra-curricular activities in both Putonghua and English.

\section{Call for 4-year university programmes - 2}

Main comment:

Universities will revert to 4 -year programmes. (2)

\section{Comment on education in Hong Kong - 1}

Individual comment:

One cannot be revolutionary in education in Hong Kong.

\section{Schools - 9}

Schools' accountability -4

Main comment:

Schools have a responsibility to parents. (3)

Individual comment:

Schools do what is convenient for the school and which suits the parents.

Schools' prestige - 2 
Main comment:

The prestige of schools will become more overt. (2)

Alternatives to government-run schools - 2

Individual comments:

There is a growth in popularity of private schools.

More local students are going to international schools.

Comment on language standards in schools - 1

Individual comment:

The language situation in schools is getting worse.

\section{New Immigrants from China - 8}

Increase in number of immigrants - 6

Main comments:

There will be more immigrants coming to Hong Kong from China. (3)

There will be more lessons to cater for the new immigrants from China. (2)

Individual comment:

More students from China will result in bigger class sizes.

Schools rejecting new immigrants - 1

Individual comment:

Some schools are rejecting new immigrants from China.

Need for retraining for teachers - 1

Individual comment:

The government will hold courses for teachers to help them with teaching the new immigrants from China. 


\section{Reference to Hong Kong People - 7}

Pragmatism of Hong Kong people - 5

Main comment:

Hong Kong people are very pragmatic as regards language use. (5)

Non-negative attitude of Hong Kong people towards English as colonial language - 2

Main comment:

Hong Kong people are not resentful of English as component of colonial rule. (2)

\section{Reference to Chinese - 5}

Simplified characters - 2

Main comment:

Simplified characters will need to be taught. (2)

\section{Standard of Chinese - 2}

Individual comments:

The standard for Chinese will become an issue.

The standard of Chinese amongst Hong Kong students is very low.

Importance of Chinese - 1

Individual comment:

The importance of Chinese will be emphasised. 


\section{A.5 Interview Transcripts from Interviews in 1997}

In the interview transcripts that follow, Roman numerals are used to designate the interviewees that took part in both rounds of interviews (in 1997 and in 1999). The transcripts for these interviewees are presented first. These are followed by the transcripts of the remaining interviews, which are numbered, but are in no particular order. The short form of the year of interview is given in brackets to help distinguish between the two rounds of interviews.

\section{Interviewee I(97): University educator \\ Date: $\quad 30$ May 1997}

$\begin{array}{ll}\text { AU } & \text { Researcher } \\ \text { I(97) } & \text { Interviewee } \\ \ldots & \text { Missing words / Unfinished utterance } \\ \text { - } & \text { Pause }\end{array}$

I(97) The first thing is, many of the points, points 1 and 2 haven't got anything to do with the changeover.

AU True, not directly anyway.

I(97) Not at all, I mean, this is stuff that was started 10 years ago, the MOI business started more, 15 years ago. And the benchmarking is nothing at all to do with the handover. Nothing at all. OK, number one, what are you feelings as regards the Education Department's decision to force $75 \%$ of secondary schools, well number one, force is a pejorative term, you know what I mean? So I would be careful about putting that as research question, but I agree with it entirely, it's been long overdue, and once again it's the culmination of what has been agreed as policy but resisted by the schools, because of parental pressure I think, this is my view. Mainly because of parental pressure and inertia and because of this gut feeling that English is important. But without being able to reconcile the gut feeling, because parents don't know what's going on, nor do many teachers, that what is actually happening in the socalled MOI schools for English is as we all know, code-switching and codemixing, where the kids are, poor children, penalized, really penalized, so I think it's wonderful what they are doing.

AU Yeah, as you say, it's a culmination of what's been going on since ...

I(97) ECR2, yes, since the Education Commission was founded and people started getting into it. And you remember the working party on language proficiency, the chair, the lady, I know her well, the head of the Tung Wah Group, who's 
just retired, and she was the chairperson on the report on the proficiency. Do you remember they floated the idea, just as a discussion, as to whether it should move into Primary 4? People got really upset about it. Now I'm going to be giving a conference paper at the big Lingnan conference on language policy, language issues, all of that, on the 25th, 26th, they've got Michael Halliday coming and Gunther Cress coming from the Institute. I'm talking on the ESL/EFL context, and I'm going to say once and for all can we please stop talking about ESL in the context of HK, and I'm going to propose that we take English out of the primary schools entirely. Just to provoke people, just to get them thinking, why is it there? Think of the billions that could be saved and put into the proper ... because I've got the figures from the benchmarking exercise and from government, I mean you know that in secondary schools only $14.5 \%$ of teachers have a relevant degree and are professionally trained? How the hell can we expect any kind of implementation ... my 1995 paper ... where is it ... here it is, I'll quote you from it ... my 1995 paper in there I state categorically that ... yeah, 'It is not unreasonable to assume that with such a large proportion of the workforce unqualified to teach English either by subject training or by professional training or both, teachers will on the whole have problems implementing any curriculum that requires in addition to all the other general knowledge and sound methodological practices, a requisite amount of subject knowledge and pedagogic content knowledge.' So that's my view on that one. Is that OK?

AU Yes.

I(97) Number two, excellent, and number two of course since the announcement this morning that Tung Chi Hwa and his people are going to be fueled by business considerations rather than by ... this benchmark initiative really came through ECR6 but fueled by pressure by the financial and commercial community. Now, there's background on that in Curriculum Forum, the May issue this year, just coming out, [?] and I have a paper on the English benchmarks, the English language benchmarks. It's Curriculum Forum and it's the May issue so that would make it Issue 6, Volume 6, Issue 2. It's out this week, later this week. And in that I have a paper with [ ] and we talk about the background to the initiative and why it came about and stuff like that. Because [ ] and I are the Principal Investigators for Government on the benchmark project. So, naturally I feel it's a good thing since I got involved in it because of the chances are that four out of five children are likely to be taught in secondary school by someone who is not either professionally or ... and subject qualified. So for those people we need some form of benchmarking, even if we accept that the others are fine. So I don't think it's unreasonable at all.

AU How about benchmarking for subject teachers? 
I(97) Yes, I think that will have to come about because many are not comfortable with Chinese, funnily enough, because of the mixed-code they've been using, and so they'll need to be benchmarked for Chinese as we move over, if number one goes through, and the English ones in the 95 schools, I'm sure they're going to be all right, but they will certainly need to be benchmarked to ensure that they're up to speed, and they we have tuition through two languages and not through one. I am concerned obviously about in question one about the fact that in people's minds the English ones will be the sunshine schools like the old elitist schools, this is the danger obviously and I don't know how we're going to do it, how we're going to get over that. I suspect one way might be to make everybody teach in Chinese, but the parents will then vote with their feet and there'll be surreptitious and there'll be such hell. The principle is that they should all be taught in Chinese but they're allowing this because of parental and societal pressure.

Number three, do you think that there'll be any ... in the teaching of English, the only thing that I'm concerned about is that English might get slightly less on the timetable because we'll probably have to get Putonghua put on. I think it's not unreasonable that Putonghua gets on and therefore English will slip as it was from the first foreign language to second foreign language, because Putonghua, if we call that a foreign language, I mean K.K. Luke says that Cantonese is now a distinct language, although it shares the same written form, there might well be more slippage.

AU How soon do you think that might be?

I(97) Oh quite soon, because how otherwise are the people in Beijing to communicate with the people of HK? They do it in Guangzhou through Putonghua. OK, a lot of people understand Putonghua but they're not really good so they probably need some pulling up and they probably need some form of Putonghua training. So I would imagine, in fact this impinges on four, oh no it doesn't, what change do you envisage in the proportion of the timetable given over to English language teaching? I suspect there might be a slight slippage as I say.

$\mathrm{AU} \quad$ Putonghua as a MOI?

I(97) No, I don't see that, it should be Cantonese. I don't think there'll be a switch for the English schools. There might be some for the children of Mainlanders in order to continue their education in the same way that we are having with the ESF for native speakers of English, the international schools, I see no reason why they shouldn't be ... no, the majority of the population, $98 \%$, are going to be taught through Cantonese, I suspect, and what may happen is they might begin to diminish the number of English-medium schools, although all the top people want to send their kids to the 75 English-medium schools, don't they? 
AU Well yeah, I mean the tertiary presumably will stay predominantly Englishmedium?

I(97) The current vice-chancellor has started talking Cantonese in Senate.

AU Really?

I(97) I don't blame him.

AU True.

3(97) Why should we speak to each other in French?

AU If they want to remain international ...

I(97) What's interesting is that the CU, where I live, my wife works there, in the Faculty of Education, we live there and I commute, are now sending out messages not in English at all, it used to be dual, now it's just Chinese, so people have to get clued-in.

AU How do the expatriate staff deal with that?

I(97) With a great deal of difficulty, they have to say, is there anything here relevant to me? Not only in the Business Faculty but in Education, they've got one, two three, four expatriates in the whole of the Faculty of Education, two of them in the English section, my wife and [ ]. So basically the office staff have to just alert them and say, hey, this is important. So you'll get a kind of gradualism. The thing is that people don't want it to slip, but you'll get a kind of inevitable gradualism, so what we've got is the reality against the desire. The desire of course is to maintain an international university, an international image and international connections and people sincerely believe that, they believe they can do that, but also, if they are together and there are no expatriates present then they talk in Chinese. What happens then to your English language skills? I'm already witnessing that in government. Ten or fifteen years ago, I'd be in a meeting and it was always in English, now, for something like the TOC Coordinating Committee for Assessment Mechanism, which I sit on because I'm chairman of the English subject committee group for assessment, it's in Chinese, I'm the only expatriate there, so it's in Chinese and I have someone translating and I speak in English and everybody's happy then we have a bit of English, then one of the primary teachers will talk and of course, she understands a bit of English, she'll talk in Chinese and somebody translates for me. And this is not simultaneous. Now when we go to AQTEC for the benchmarking, then we all wear the earphones. Then you've got two people in the glass cage giving you simultaneous translations. The government are into that already. So, there again you see it's moved in the last four or five years, likewise the law courts 
now. It's disgusting that the majority of the people in HK have to go to law through a language they don't understand. It's awful, crazy. Let's get on, where are we up to? So, number four, what do I envisage - a little bit of slippage. Is that OK?

AU I just wanted to come back to this idea of Chinese-medium in universities ...

I(97) In the Education Faculty at CU, no expatriates ever join, because the majority of the courses are through the medium of Chinese.

AU I still think to remain on an international footing they need to remain Englishmedium, or at least Putonghua-medium.

I(97) I think what will happen is they'll stay international provided people can understand English and publish in English. The moment they stop publishing in English and the moment they stop getting the visitors in ... so what happens is you get visitors into CU and they give a talk in English and everybody understands, they are more self-conscious over there than they are here, they speak in Cantonese. The problem there is, the Putonghua speakers, whose English isn't very good and whose Cantonese is non-existent, big problems in CU. What we tend to do is hire Putonghua speakers who are pretty good in English. They've been to The States for their Ph.D., they write in English, they don't even write in Chinese. You're right, and certainly the business community want us to stay international. I think what happens is, you will get this gradualism amongst the existing staff, but they, because they are not language-sophisticated, will not realise the knock-on effect down the line in five to ten years unless they hold the line, because for them it's not a problem, they come in here and they speaking Cantonese with us, that doesn't affect their ability to write papers, to get onto the network, phone somebody in America, to bring a visitor over, no problem wit these people because they've come through the system, but it's the kids who are now about fifteen/sixteen who are bright and they go on to do A Level and then they come here, speaking a lot of Cantonese here and then they get a job. Some of our junior staff are not doing terribly good. OK?

Any other significant changes in your school or the education system more generally which might affect the teaching of English? No, no, number one's going to be the big thing. What I would hope is that we actually get greater concentration on English as $75 \%$ of them go over to Chinese-medium and stop pretending to be teaching in English. And it means that there might be more support and more resources for the English lessons. The problem is that the children there, who are already alienated, I'm just reading an excellent Ph.D. report which you must, Elizabeth Walker, 1997, University of HK, unpublished thesis, Ph.D. thesis, she's at the HKIEd., I don't know which campus, very good thesis, which I'll be quoting in my paper at Lingnan, where she just talks about foreign language anxiety, fear of speaking English, 
prevalent throughout the territory, and what is surprising is that it's more prevalent in Form 1 and more prevalent in Form 7 than in Form 6. So these A' Level kids who are coming here are so accuracy-focused, that they are terrified, and you as a secondary school teacher will know damn well. Maybe you've overcome it, or you pretend not to know Cantonese or things like that, you know the heads down and the whispers to each other, refusal to answer, teacher-initiated questions, the traditional Coultard \& Sinclair IRF, Initiated Response Feedback, there's just no talking going on. Now what we might find is that we get the cream rising to the top, like we do in Beijing and Shanghai and Nanjing and elsewhere, you get pretty good speakers, good readers, they work hard on the oral side. At the moment the oral side is not worked on because there's this slippage. Now I think it's getting better and we are seeing washback effect, we are even seeing washback effect in Form 5 , because one of my people, who is just finishing her Ph.D., [] at the OLI, has tracked the washback effect of the new Form 5 syllabus, and the effect of the oral exam. It's definitely changing practices in schools.

AU We actually start it in my school, we start in Form 3 and the teachers have been very impressed wit the way they have adapted to it.

I(97) Excellent, if you focus on it you'll get it. What might happen is, because people were so hung up about the academic side, what we might find is a change for the better by saying that a percentage of year one, year two, year three will be oral, when TOC comes through, God willing it comes through to the secondary schools, task-oriented stuff where they have to use English, integrated skills, a great percentage of oral, then they are getting better prepared for the financial world. So I think those changes might come, but not connected to the handover. Maybe they'll have less hang-ups. I'll tell you one more thing, which I was thinking about since I spoke to you on the phone, people in China have no hang-ups about learning English, except the usual ones, but they're the hang-ups that a German or a Russian might have. People in HK, there's an anomie, there's a culture within the schools which frowns on people being fluent in English and it's not a strong reaction, it's not like in other countries, when you've got this anomie against ... because it's the language of the colonialists, it's the language of the power people. Now once we got rid of that emotional and psychological baggage, it seems to me that then people cannot be afraid. You know that 25 years ago, kids would be speaking English on the bus, because they were from the elitist schools and that was the mark, when there were only 70 secondary schools, you know? It was a class distinction. But that changed and now kids will not speak it on the bus or the train because they'll be frowned upon. Now I suspect that that might disappear and that could be a power for good. There you are, there's an idea for nothing! So the school education system, maybe there'll be changes in attitude and the fear of foreign languages that $\mathrm{Liz}$ Walker documents so well might begin to disappear. 
Methods or approaches in English lessons in secondary schools - I hope not. I do hope that we don't have a reaction against communicative methodology because that's a hangover from imperial times. I do hope we don't go back to practice and manipulation, but I suspect that the business people will, funnily enough as they are represented on committees and things like that, actually say, no that's no good, I think they will listen to reason. Teachers might want to do it, because the easy way out for the teachers is exercises, exercise manipulation, lexical/grammatical workover, no work at the macro level and no work at the oral level. I can identify with everybody because I work in the system; people know that I identify with the education system and the aspirations. So, I hope there's no slippage and there's not a reaction against communicative language methodology. I've just come back from Beijing, from [?] the foreign studies university, they really work hard on the oral side with their people, so many of them go out to be interpreters or translators or work in business, you know? Some of them go out into teaching. I'm impressed. The middle school teachers they had are accurate, fluent, not as good as the majors we get on our PCEd., they are more fluent.

How do you think that the changeover will affect the situation, status or morale of teachers of English in Hong Kong? I think there's been this residual belief that this myth that it's a second language, which it's not, that therefore they were less important these people. I suspect there'll even get a boost in their status, is this something you're finding?

AU It's something I'm thinking about, from what you said before that once the colonial power has gone then this stigma which has developed over the last 20 years perhaps will fade away.

I(97) Well I hope so, it might be a lead that you have in Beijing, you know they're pretty important people, they're the good guys.

AU At the moment in the schools it's very much like, well you have to do this.

I(97) That's why I'd take it out of primary, I mean they won't accept it, Sweden has it in secondary. I've got data from all the consulates. They don't have to teach it in primary. They've got to survive as trading nations. And I would use that money to give people credits so that when they really need it and have the motivation they can use those credits and go to the British Council, or if it's French they want, Alliance Francaise, or the Dante Institute if it's Italian they need. And their employers will say, how much credits do you have left? You get yourself down the British Council and get that certificate or you're not coming back. I find this an attractive notion. I was talking to a French guy, I was just toying with the idea, I said, the billions we waste on unqualified and inefficient and low levels of English type teachers in primary. If you had some of that money to beef up secondary it would give them more resources. Then give the kids credits to go to the British Council or 
somewhere. These people will work damn hard because they need this thing. Primary kids don't know why they are learning English. The only reason we retain it because of the notion that acquisition is better than learning, if we separate the two, and that phonological acquisition occurs before the age of 7 or 8. These kids are not going to acquire native-speaking type phonology; they are going to have, if they are educated, HK variety. They'll get that whatever happens, whether they start at secondary or primary. So my thesis is, let them go where they can see a purpose for it. I quite like the idea. I mean people will hate it, I just want them to think about it, I want them to fight me, then I'll get them to prove why it can't be in primary. There are kids whose parents want to send them to tutorial schools and everything, fine, but don't use state money for it. I'm just sort of pursuing this at the moment, it has to be ready in about 10 days this paper. Let's treat it like a foreign language situation, not like a second language. Let's get it into the secondary schools. There's actually some evidence from Australia, in French, where children who've been taught French in primary school, were then continued into secondary and at the end of two years compared with children who started only in secondary, there was no discernible difference. Now it's a single study and it's French not English, but it's some evidence. See what happens? Primary teachers will hate me because they'll all be out of work.

AU Like you find in primary schools that teachers are not specialists.

I(97) Right, they're generalists. If our population is going to expand to 10 million in the next 10 years, there's work for them. All these kids coming down from China, I mean 50 a day, 54,000 a year, divide them into 30, that's a lot of classes of English. Isn't it? It's about 1300 classes of English. Divide that by 3 , you are talking about 400 extra teachers.

AU Where are they going to come from?

I(97) They'll have to come from the HKIEd. 


\section{Interviewee II(97): University educator \\ Date: \\ 4 July 1997}

$\begin{array}{ll}\text { AU } & \text { Researcher } \\ \text { II(97) } & \text { Interviewee } \\ \ldots & \text { Missing words / Unfinished utterance } \\ \text {-. } & \text { Pause }\end{array}$

AU The first question is what are your feelings as regards the ED's decision to force $75 \%$ of secondary schools to adopt Chinese-medium education?

II(97) OK let's put it that way, the decision to use Chinese-medium education is something that I support $100 \%$. I think this is something that the government should have done a long time ago but didn't have the, I don't know if we should say didn't have the guts, the determination to do it. On the other hand, I can also see why the government is not doing it across the board. I think it's for political and pragmatic reasons. For pragmatic reasons, why I'm saying that is that there are children in HK who are perfectly capable of using English as a medium, and personally, I don't see why you should deprive them of the opportunity of acquiring a very high standard of English through immersion. That's my pragmatic view. There are people who disagree, who think that that can't be true, how can mother-tongue education not be the best thing? And I disagree with them, but I don't think there are $25 \%$ of the schools who are capable of doing that, and in that sense I think it's a political decision in the sense that if the government had made the percentage even smaller, it would have been an even more difficult problem for them. Already there has been such a lot of opposition from schools, so I think it's a bit of a compromise.

AU Do you think the announcement of this has anything to do with the [changeover]?

II(97) I would say that it's certainly because of the handover but that the government is so determined because politically they don't need to impose English-medium, prior to that, you can say since the 1860 s, the reasons for introducing English-medium and for the emphasis on the importance of English was definitely political. And all along the govts. being, despite the fact that educationalists have been saying, look mother tongue is the best medium, government never paid any attention to it, until it got very very close to that, and then they put it's foot down, and I think that is political.

$\mathrm{AU} \quad$ I think you're the first person who has actually said that to me and it's the kind of thing that I have been looking for ... 
II(97) You mean of the 15 people ... that can't be true?

$\mathrm{AU}$ Of course, they've been planning this for a long time, change the medium, but to decide to implement it next year, and to announce it two months before the handover.

II(97) Well I think it was announced in 199... in ECR4, even then the government was not stupid, they could see, well how many years do you have to go, and the very fact that the firm guidance was planned in 1998, and not 1996, is because I think they would see much less opposition after the handover than before the handover. But the very fact that between 1990 and now not much has been done. I mean they have got new CMI courses for teachers, but how much publicity have they sent? You haven't seen a lot of that, so on the one hand you can say to teachers and to principals look, it's already in ECR4, you haven't read it, yes, on the other hand you can also say well, how much publicity have you given. Wouldn't you think that people have good reason to doubt the sincerity of the government, are they really ...

AU Yes. OK, what are your feelings as regards the ED's planned introduction of benchmark language levels for language teachers?

II(97) I agree with that. I guess I'm speaking against the tide, as it were, I know how a lot of teachers feel about it, how a lot of principals feel about it, they feel very strongly about it. The PTU feel very strongly about it, they feel that this is an insult to teachers, but my feeling is that if you consider yourself a professional, then you've got to ask yourself, well what are the benchmarks of the profession? I'm sure that doctors, lawyers and architects, they have the benchmarks so they need all this training. But the very fact that is that HK teachers don't need training to go into the profession means that something's terribly wrong. I think, and I hope, that this is a stopgap measure. OK we say that we've got to have an all-trained profession, that's true, but it takes time, so in the meantime what do you do? You've got all these people, a fairly substantial number of teachers, whose English proficiency is not up to scratch. So by imposing a benchmark on them, it's kind of saying, look, this is the standard, and I hope that this is something that could be turned into something positive, and that is the emphasis lies in retraining and I've been making an appeal in the Chinese press and in various seminars saying, can we please be positive because I don't think teachers have anything to gain by just resisting, because once you've said that, you have no excuse for getting more resources. You know, this is the golden opportunity, grab it. OK, I'm willing to do this test, but what if I don't pass it? I need all these resources to get me up to scratch, and I think there is a lot of room for negotiation and bargaining with the government

AU I believe that even teachers who don't reach the benchmark will be given ten years to do so, within these ten years they are going to have to going for 
retraining, so from that point of view it's a very positive thing, but as you so rightly say, a lot of teachers feel threatened by it.

II(97) I guess its really up to the profession to turn it round, because I think somehow there is this culture which I am not terribly happy about where any measure that is put forward, the first reaction is, no, and there is this terrible inertia. I seem to be very scathing about teachers, when in fact I'm not, but I also feel that there's a lot to be done about professionals, but if you say, well let's take a look at it and let's negotiate, I think this is what the union should do, if you think you've not been given enough opportunities to improve yourself, well this is the time to go for it. To be very honest with you, I said this in 1990 at the Language Fund Campaign seminar, when I pointed out that the percentage of teachers that are both subject training and professionally trained is embarrassingly low, I don't know if you've got those figures, you must have, and I remember at that time it hit the headlines, which said expert, which I'm not but that's how they referred to me, put the blame on teachers, and I knew at that time that some teachers were very upset that I should make a statement like that, it's like putting them down, well I'm not. What I really wanted to do is say, well let's be very objective, let's look at our profession, let's look at what's happening, and at the time, the reason why I did this study, basically what I did was, I took the 1991 teachers' survey from the ED, and I said, look, we've been talking about how teacher's English standard not being up to scratch and all these problems with English language teaching in $\mathrm{HK}$, let's take a look at what our profession is like, so I got the figures from that and I reanalysed it, and I found out that the figure that was released by the ED was very different from my analysis, because they said that nearly half of the teachers were subject training, when in fact what they meant by subject-trained, if in their PGCE or in Cert. Ed., if they said that or if they were trained in English teaching methodology, then they considered that subject-trained, which of course is not true. So you can have a sociology major doing one-year PCEd English teaching methodology, and they considered them graduate subject-trained and fully-trained. And I said this is pulling wool over people's eyes and I'm not going to let you get away with it. And so I reanalysed those figures and released those figures in the Language Campaign seminar and they hit the roof, they absolutely hit the roof, and Cheung Man-kwong put the figures to Legco saying, how is it we've got the figures from HKU saying the figures were so low. And I knew there would be an upset but I kept putting the same message across to them saying, look, you've got to distinguish between the two, and I'm sure there are lots of other people conveying the same message to the government as well, so finally I think they have come to admit that subject-trained means you have actually studied the subject at university.

AU Well as we both know, there are countless teachers who are teaching English who are not trained at all, they have a degree from an English-medium 
institution and they are deemed to be competent to teach English, which hopefully these measures will help to address.

II(97) But this also illustrates my point about teachers not addressing the problem head-on, and I think they should. They shouldn't kid themselves. If you are not subject-trained, it's not your fault, it's a problem with the governments' planning. So what do you do if you've been asked to teach then what you should do then is say, well I'm not subject-trained and I don't feel adequate so if you want me to stay in this profession, then give me all the support you can to get some more re-training.

AU Well hopefully that is what will develop from it. Question three; do you think there will be any other significant changes in government policy towards the teaching of English in HK after the changeover?

II(97) I don't think so, in fact I'm not sure what exactly you have in mind.

AU Well, we've discussed so far the medium of education and the benchmarking, do you see any other significant changes being brought in?

II(97) I think the importance of English will not be de-emphasised, but I think the importance of Chinese will be emphasised, but it won't be done at the expense of Chinese, although people who specialise in Chinese education feel very resentful about the current situation, that they are not considered as important as English. So they would want to make themselves more prominent, and quite rightly so, but I think that there is a danger that they might do it at the expense of English. This is something that $\mathrm{HK}$ as a community has to resist. An example would be the current medium of instruction issue, where some tertiary Chinese educators would say, why not? Why not have Chinese medium in university? Why not make all universities Chinese-medium, why do we want to have English-medium? Which is something that I've argued very strongly against. And they will say, look at Japan, Japan has this huge body for translating things, all you need to do is have a large group to keep translating things into Chinese. I don't think it works, and I think Japan is now coming to the realisation that it doesn't work, so you see a lot more English in Japan, than, say, ten or twenty years ago.

AU I think that one of the major changes will be the introduction of Putonghua into secondary schools, the ones who are not teaching it already. In my school I think they do give them one lesson a week in Form 1 and 2 and then that's it. Presumably it will be throughout the whole curriculum starting next year.

II(97) Well I think learning Putonghua is one thing, using that as a medium of instruction is another thing, and I can also see the danger of pushing that through, probably not from above, it's from people themselves, and I think 
this will not be a good thing for $\mathrm{HK}$. I think it should preserve Cantonese as the medium of instruction.

AU Which kind of leads into the next question, considering the proportion of the timetable given over to English language teaching in English and Chinesemedium schools, can you see any changes in that?

II(97) For schools which are going to go Chinese-medium, I suspect they might have more English language teaching lessons, because they are very keen to keep up the standard or to improve the standard, but it depends how they are going to juggle the timetable. If they are going to out in Putonghua, for instance, then will it be done at the expense of English? I think in the next ten years, I don't think people are going to change their perception about the importance of English. Certainly Anthony Leung thinks that it's very important to keep the status and business people are very adamant that this is what they need. Of course one never knows who is going to be the next Exco member with an education brief.

AU Certainly there is a question if schools are going to change from Englishmedium to Chinese-medium, do they want to be seen at least to say, OK we are going Chinese medium now but we are going to put more effort into English, we are going to increase the amount of English on the timetable, perhaps to placate parents who might be worried that their students are not getting enough English. If they do so where do they take the extra lessons from?

II(97) Or maybe they will build in a lot of extra-curricular activities which will have the aim of improving the English proficiency. I suspect that going to happen, and this is something that I've been suggesting to teachers, which gives them an authentic ... where they have to use the language for more authentic purposes.

AU That's hard to do that, well it's not hard but it's hard because, again going back to if you push something towards teachers which looks like more work, then immediately they say, no.

II(97) Say for example, they could have extra-curricular activities which is ... one thing I said to them is, well couldn't you try and see if you can do it in English? Because with things like that, you've got all kinds of things to help you, it's not entirely cognitive because, well I shouldn't say it's not entirely cognitive but you've got gestures, you've got objects to help you. So even if they could not follow exactly what you're saying, they could guess by the gestures, by the colour, by things like that, you know. And I thought that might be a way to do it, but for subjects which really require reasoning, I said, for God's sake, don't jeopardize their cognitive development for the sake of English, it's the wrong way round. 
AU Do you think there will be any other significant changes in your school or the education system more generally that might affect the teaching of English? It's mainly aimed at secondary schools, although people within the universities have commented on it.

II(97) Actually, the Vice-Chancellor published an article in Sun Bo, Economic Daily, it's one of the Chinese papers about an English-medium university, and I think he's very committed to that, that HKU must stay English-medium, which I think I agree with.

AU I know that City $U$ are implementing a whole new English enhancement programme throughout the university, they are really committed to trying to improve their students' English. It certainly seems a common thing throughout the universities, I'm not sure about Chinese U, they want to maintain English as the MOI, make sure that their graduates as well as being trained in their specialist subjects are able to communicate in both English and Chinese.

II(97) I know some people are suggesting, why can't we have some subjects at the university which are taught in English and some subjects which are taught in Chinese. Actually I don't care what language it is taught in, what I think is important is that students be able to read journals and references in English and they should be able to write in English and if it were an English lecture, they should be able to understand, they should be able to contribute to the discussions and the tutorials in English. Whether you have the odd lecturer who did the whole lecture in Chinese, I don't think it's really that important.

AU From what I can gather I think a great deal of tutorials and so forth are conducted in Cantonese, while lectures perhaps would be in English if the subject is English-medium, but a great deal of interaction goes on in the first language.

II(97) That doesn't bother me at all, but say for example, you've got a native speaker of English as one of the lecturers and you cannot interact with him or her in tutorials and in lectures, I think that's a problem. Because it's very natural, with my students all my lectures are in English, but I can see that if I were to sit down with them and discuss things I think switching to Cantonese is much easier for them. It's just that if I couldn't, if I had to speak English, if I weren't Chinese, then students should be able to communicate with me. Otherwise the university will lose its international flavour.

AU I think that's the desire of the vice-chancellors of all the universities to maintain their international status, it's very important. Do you think the changeover will affect teaching methods or approaches in English lessons in secondary schools? 
II(97) I don't think so.

AU With the change in medium, do you think the change from English-medium to Chinese-medium might ...

II(97) Change the methodology? I don't think so. I don't see that as an issue at all.

AU Okay, and how do you think the changeover will affect the status, situation or morale of teachers of English?

II(97) I don't think teachers of English will feel any less important than before. To be quite honest, they might feel even more important than before, because this whole emphasis of keeping the international status in HK, like if a school turns, changes to Chinese-medium, I mean already l've been asked to go into schools and talk to their staff about how to maintain English standard in schools, I think it probably goes the other way. I don't know if you agree with me, you are in a school you probably know better than I do.

AU I can't really say, my school has done nothing at all for the handover, no celebration or anything, the only way that it has even been recognised is the headmistress in the last assembly said, 'This is the last assembly under British rule.' And that was it! I should've gone back yesterday, presumably on Monday we'll just carry on. I understand it might be different in other schools, ours being one of the elite schools, and the headmistress is very much one of the old school type, an Anglophile, she'd be quite happy to keep the status quo as it is and not want to change it. She will still want to maintain the school as English-medium.

II(97) I guess it really depends on the principal, there are schools where they've got the students to practice the national anthem or whatever for the handover. There are principals who've started to teach the kids how to sing the national anthem and there are principals who have decorated the schools. I guess it really depends on individual principals. 
Interviewee III(97): University educator

Date:

\section{July 1997}

$\begin{array}{ll}\text { AU } & \text { Researcher } \\ \text { III(97) } & \text { Interviewee } \\ \ldots & \text { Missing words / Unfinished utterance } \\ \text { - } & \text { Pause }\end{array}$

AU Good afternoon.

III(97) Good afternoon.

AU Perhaps we can go through the questions as they appear and anything that comes up we can talk about.

III(97) Sure.

AU First question is what are your feelings about the ED's decision to force about $75 \%$ of secondary schools to adopt Chinese-medium education?

III(97) My main feeling about this is ... I myself don't really see a strong reason for it, although I know that in practice this may be one way of going forward, I have to say that if the government is really convinced of the advantage of teaching through Cantonese in schools, then one option would be presumably to ask all schools to go down that direction, although it might take some time before schools can actually change and move into the new system, but by asking some schools to do that while others remain in exactly the same way as they are now, more or less, then it strikes me as being inconsistent, or it could be perceived by the schools or the parents as inconsistent. What I've heard about some schools' and parents' reactions is that they think this is a move to keep some good schools or prestigious schools the way they are, but ask the other schools to do what is supposed to be a better way forward. Now, having said that, I suppose I can also see that if the government was to ask all schools to change into a Cantonese medium, then they would expect to encounter a lot of resistance from some schools and some teachers and from lots of parents as well. That may be the point, but I suspect also that another reason for this move may be the government is thinking of producing a certain percentage of students proficient in English to meet the demand of English-speaking graduates, but meanwhile to move in the direction of mother-tongue education because its psychologically or educationally more desirable perhaps. So the split is perhaps done for that reason. It's a very complicated situation, I suppose, but that's the way it is at the moment. 
AU As you say, the system as it is now is that there is a group of elite schools which genuinely teach through the English medium and the majority which are supposed to teach through the English medium but teach through a kind of mixed-code, with English textbooks and Cantonese being the medium in the classroom, and as you say, perhaps the only way the system will change is that the Chinese-medium schools will then go on to use Chinese textbooks presumably and they won't have any use for English at all, apart from in English lessons.

III(97) That certainly is one possible system, but I think that there is some worry that if all schools were to adopt this system, in which all the textbooks are written in Chinese and the teachers deliver in Cantonese, except for the English language lessons, then the worry is that the standard of English may take another fall. We may not be able to meet the demands of the job market of the employers and so on. I can see some justification in that concern, because to say the least it may take some time for English language teaching and the standard of English to pick up again once you change entirely into Cantonese.

AU Do you think the fact that they've announced this just recently, I know the planning has been going on for quite some time now as regards this, do you think the fact that they announced this two months ago had anything to do with the changeover?

III(97) I myself don't see any direct connection there, I think it's really a conclusion that the education authorities come to after a fairly long process of having meetings within the relevant committees, having consultations with the schools and the public, so it has in fact taken some years for this final decision to come and it is a major change in policy. Some may say that it's not thorough enough, that it's determination to go through with it, still it's a major change in policy. I think any change like this does require a long enough time for implementation, a period for people to get a bit used to the idea that this change may come.

AU Because they've announced that it will come in the next academic year, 19981999.

III(97) When it comes, it does strike one as being rather forceful, but in fact the idea of it was already in the ... some years and the schools were getting prepared for it, or trying to avoid it for some time, but apparently they now have to make some definite move by next year. That was in the previous Educational Report too when schools were given this lead time to get themselves into a comfortable position, when the time comes, I think that was the idea, but as it turned out, I think lots of schools simply left it, not necessarily because they didn't want to do anything, but there maybe real difficulties with a lot of resistance from the parents, some teachers may not want the change, may not prefer the change, some head teachers themselves may have reservations 
perhaps, and so this move was put back for another year then put back for another year and finally the government said let's do something about it next year, we've got to do something about it. So, I don't know actually what might happen come next year.

AU It's just the thought that perhaps that they've announced it just prior to the handover ... it was announced in the press as well, maybe there was some kind of connection. They thought, well there is obviously going to be objections to it, with the majority going Chinese-medium, perhaps the atmosphere in the territory at the moment perhaps they thought it might be more easily accepted, this is the way into the future, to become Chinesemedium, at least for those students who will benefit from it.

III(97) Yes, if that was somehow planned, which took these socio-political changes into account, then it could have been a miscalculation. I don't think myself that any changes we've gone through will have any effect on the schools themselves with regards to the question of what language they should teach in, it's just, the question is a much more long-term one, than any change which may have come about politically or otherwise may have any real effect on ... that's my view anyway. Most parents and a lot of schools and the teachers have more to think about the way education has been understood, in the schools and the system can continue and students can continue to learn and whether any major change in the medium of instruction would have any adverse effect on that education, I think that goes a long way beyond these recent changes. So, now that we have change of government, change of sovereignty, then Chinese is bound to become much more important in the society and also in the schools. We'd expect a lot more schools to offer Mandarin and so on if they have the resources, if they have the means of doing so, but still I think that's somehow that's a slightly different question than whether the schools should become Chinese-medium. That's a different question.

AU I think we'll come to that later. Okay, what are your feelings as regards the ED's planned introduction of benchmark language levels for language teachers?

III(97) Well this is an idea which I would think it would be very hard to say no to, obviously everyone's going to say, 'Very good' if you set some benchmark for language teachers and require them to have a certain level of ability in the language they teach. I suppose it's a reasonable thing to ask of a language teacher. In practice though, there may be difficulties, for example, teachers who have been on the job for many years, there level of English may have gone up, may have gone down, may have stayed the same. Do you test these teachers? And if they don't reach a certain level, what do you do? I can see the benefit of this requirement and I can see that it's a bit more feasible with the new teachers as they come into the profession, we give them some tests or 
some requirements, that probably is okay, even though you need to think how many people will want to join the profession at any point in time, and how many teachers you need every year, and what if you set the level in such a way that a lot of aspiring teachers can't reach that level then you end up not having enough teachers to fill the positions. In practice there's all kinds of problems when you try to implement the policy. But as an idea, I suppose it's a reasonable thing to require teachers of English or Chinese to have a certain minimum standard.

AU I think the idea is that initially it'll be just for new teachers, that's what they'll do. There have been quite a lot of objections to it from the teachers' union, from experienced teachers anyway, they see it as some kind of a threat I suppose. I think also the idea is that if people don't reach the benchmark, then you give them something like ten years to get up to that level, which can be a career, for some people they might stay in teaching for ten years and then quit and do something else. I think that the finer points have yet to be worked out, but that's what I believe. I think most people would accept it.

III(97) I think most people would accept this and it's also the social perception, and I don't know how much truth there is in it, there's this perception that in some schools there are some language teachers who are not up to the kind of standard that is expected of them, and so they pass down features of the use of English and Chinese, so for example, when students come to the university we notice that they are making the same kinds of mistakes all the time and at their age, they've become used to certain patterns of language that it's very hard to change.

AU But you are right in saying that if they are over-zealous in this then they won't have enough teachers to teach in schools if they set the benchmark too high. I think most English teachers would agree that the standard of some English teachers is not as high as might be desired, but what can you do? We need English teachers.

III(97) Well I think it's not a bad idea to continue to rely on native English speaking teachers, and in fact even to extend this system. Because that way you can set you benchmark that little bit higher, because then you are not afraid of not being able to fill the positions because of the number of teachers you need every year, and part of that can be filled perhaps by native-speaker teachers, who have certain things to offer, although who may come up with difficulties in being in a Cantonese-speaking environment and so on, being not always able to communicate fully to their students, but on the other hand they have other strengths to offer. So I think the system can continue to rely on both the local teachers and the expatriate teachers. That way you can perhaps set the benchmark a little higher, rather than to let the market take the lead and simply sort of pay lip service to the benchmarks because then the number one priority is you fill the posts, you know, let the students have a teacher and 
then you require them to fulfill certain requirements or standards. If you don't even have the teachers in the first place, then how's the teaching to be done?

AU I know that people, certainly at the City University actually welcome it on their BA course, BATESL course, that it gives them a benchmark to work to as well, and they can incorporate it within their degree programme. This is a requirement of the Education Department, so you can't graduate from our university until you've reached this benchmark.

6(97) That's very good. It would certainly put some pressure on the students to work harder on their language and to get themselves up to a certain level by the time they graduate. With the English majors I don't suppose that's a problem, because they are interested in improving their English and learning the language and so on, but with the students on other programmes, they don't always take the English too seriously and some people say that by the time an engineering student graduates from the university then it may not be any higher than when he came to the university three or four years ago and that would be a pity, having come to the university and having been given all the opportunities to improve their English and not having done anything about it.

AU I suppose it's whatever is needed. If they don't need a particular level of English to do engineering then they won't do it I suppose.

III(97) Yes, but I also think that university graduates, be they engineers or medical doctors or any kind of professional need some basic communication skills. We should expect them to do not only what they are trained to do in their fields, but also to be able to communicate with other people in the company or other people, up and down in the office in an everyday situation, not necessarily talking about their specialisation. Simply being able to explain to people what they want to be done. I don't think there is any reason that I can see, for example, an engineering student or a science student or a mathematics student should have no ... in fact the whole level of expectation that we should now have of people's, of our graduates' standard of English and that of Chinese too, should simply has to raise, because of the change in our economy and all that because of the greater importance of the service industries, and of the greater need for us to be able to compete with other countries, I think we need more graduates not less graduates who are good in Chinese and English.

AU Do you think there will be any other significant changes in government policy towards the teaching of English in Hong Kong after the changeover?

III(97) I hope not, unless there is any intention to take the present change through in any more thorough fashion, otherwise because already we've seen major changes in the governments policy in this area and not enough time to see the 
effects of these changes, or even time to see if these changes can actually be implemented and carried out in the way that they were planned to be carried out, and so I don't think it's such a good idea to make another, to make any more changes in the short future.

AU Okay, what changes do you envisage in the proportion of the timetable given over to English language teaching in both English and Chinese-medium schools?

III(97) That's a question which I'm afraid I can't really say too much about because I don't have first-hand knowledge of the schools and how they work out the timetables. I suppose every school puts a certain amount of time into the English lessons and a certain amount of time into Chinese, now with Mandarin coming into the picture, this raises the question, for example, of whether a certain number of lessons be taken out of the Chinese subject perhaps and out into the Mandarin, and that means the Chinese lessons will suffer. Or should we take some English lessons out of the timetable and put them into Mandarin? Or should we take some from either direction? Or should we increase the total number of hours given to the languages? In which case the other subjects will suffer in that sense. It's a very difficult problem to tackle because of the limited time that we have on the timetable. There are more and more things that we want the students to learn. I think it's just a very difficult question.

AU At the moment it's about $20 \%$ of the timetable for English, and for Chinese and for maths, the three core subjects, and the other $40 \%$ is for everything else, things like PE and RS and which they have to do and everything else, and so I think there are perhaps two questions, if a school becomes Chinesemedium, which was formally nominally English-medium, will they then increase the number of English lessons they have on the timetable? Perhaps to be seen not to be neglecting their students' English. If they are no longer English-medium, I think that's for the benefit of parents. Or, another idea that people have told me is that if the students no longer study other subjects in English then they have no need, or less need for English lessons. They don't need to learn study skills in English as much as they do in English-medium schools, they might actually reduce the amount of English on the timetable.

III(97) I think they should increase the English lessons, at the same time they should put some lessons into Mandarin, and so chances are that the overall total number of hours devoted to the languages will have to be raised against the other subjects, but maybe that's what we should do. Because after all, a training in ... good basic knowledge in Chinese and English, skills in Mandarin will be have to become a basic requirement at all levels. Originally they have to do their O' Levels, they will have to do these three things and to do their arithmetic and their mathematics, and then whether you pitch in with one thing or another a specialised programme, do your A Levels and 
university programme keeping in mind that they should be able to do certain things in English, Chinese, written Chinese, mathematics, these are the basic skills that they must have. And we can also see that the traditional idea of students beginning to specialise in certain things ... for their A Levels and then to further specialise in their subjects in the university, that may be changing, because first of all, university places have become much more widely available with more students being able to do university degrees and the universities themselves wanting to broaden their curriculum and wanting in fact to have the four-year programme so that the first year will give the students some further language training some broadening of their studies and to give them some knowledge about other fields other than their own specialisations. We haven't had any success so far on the four-year degree, but I think universities across $\mathrm{HK}$ are pretty much agreed on this, that this is seen to be of benefit to students, so that more and more I think will be expecting the students to specialise a little later, actually, in the senior years of the first degree, or even in their graduate or postgraduate studies, that's when they will specialise, but we are expecting more and more students to do some university course to get a first degree, and so at the $O$ Levels, we can expect them not to be terribly specialised in their physics or whatever it is, which we used to do, you know we used to think in fairly high terms of what they should be able to do in this or that subject. But I think rather at that level we should think more in terms of bringing the students up to a certain level in their English and Chinese and certainly their mathematics but these will be the basic things that they all should do when they've reached that level, and then to give them their specialisations further up the scale.

AU Okay, do you think there will be any major changes in your school or the education system more generally that might affect the teaching of English? This generally applies more to school teachers than ...

III(97) Well, in our university anyway, we've already been trying to find ways to reaffirm our desire to keep the level of English high, keep entry requirements high in terms of the English results and to say to students that they are expected to reach a certain level in their English, and I can only see that more effort and more resources are going to be put into the English language teaching. To give the students more attention perhaps, to give them more help, and to provide more resources where they can go and practice and do their own work in their own time to bring the level of English up. I can only see more and more being done. I think we are, or at least we feel we are faced with a bit of a risky situation, because if we don't do something about it because it does seem that the standard of English and Chinese has been slipping a little to say the least, and we if don't arrest this and reverse the direction of change, then we could be in for a very difficult time.

AU So has the university actually implemented any changes? 
III(97) We are very conscious of English. I don't think we have recently made any special change or any arrangement to do anything differently, but I think the determination to give the students more English, to give them more help and to provide them with more resources for them to improve their own English is there. And across the university there is more faculties are now joining the English language programme, which is basically first year, sometimes second year but basically first year English language courses. So more and more faculties are adopting this language policy, and so over the next few years we can expect practically all faculties in the university to be asking their students to do a certain number of English courses in their first year and even requiring them to have reached a certain level. Although you don't get the same level of enthusiasm all across the board, some faculties are less enthusiastic and they say, 'Well, do our students really need so much English?' But I think more and more people are coming out to mean it's not just the professional training that matters, but also the general skills in communication, which language obviously is the most important part of it. As I said just now, I don't think that even professional training offered in some faculties can be doing very well without the language.

AU I think it's the same in ... certainly the same at City University, they're implementing a new English programme for all the students not only first year, but throughout the whole three years. They are paying a lot of attention to it because they ... it's become accepted that it's just become not good enough that there are students graduating from university who are unable to communicate, so they've started to do something with that.

III(97) Yes, I've seen something about City University's changes, I think they are moving in the right direction.

AU Do you think the changeover will affect teaching methods or approaches in English lessons in secondary schools?

III(97) Again I hope not because, of course there is always room perhaps even a lot of room for improvement of English language teaching in the schools, but what I meant to say was that I hope that people wouldn't feel that some change was necessary somehow because now we are part of China, I don't think language teaching has anything to do with whether you are part of China or not part of China. The fact is that we need to maintain our competitiveness, like as a society, and we need to give our children the best education in these days of fierce competition with Singapore and all these other places. With some cities in China coming up to challenge our position we should give our children the best of education, give them the best of our language training. If that means giving them more attention in their English, bringing in teachers who can help us do the job, then we should do it. I don't think there's any benefit to be gained by saying we should teach English by using more Chinese, for example, because we should all speak Chinese. I 
think that would be a wrong move to make. I think we should strengthen the English rather than anything else, and the Chinese, and the Mandarin, as I said before I don't think there is any contradiction in all these things. The only change that perhaps this would imply is that we should get more resources and time, for example, in the timetable for the languages. That may be something that we have to think more seriously about.

AU You don't think that the teaching methods will change in any way, or approaches?

III(97) I don't really think so because at the moment as you said a lot of schools are already using a combination of Cantonese and English ... so it's not as if most schools were using only English. In a sense it would be better for schools to try to use more English in their English lessons. I also see some practical difficulties which teachers will be faced with in the classroom, especially in the so-called weaker schools or the lower band schools, because then the teachers will find themselves faced with a lot of students who have very little English. If they were insist on teaching through English or using a lot more English they may lose their attention altogether, so it's very difficult. I don't see that any change will be necessarily a good thing in this whole situation unless you can be sure about bringing about better results. It may be best to keep using our present methods, although try to improve upon them. Now, I don't know what you might have in mind when you say teaching methods because if the idea is okay, now at the present moment a lot of schools are using a more communicative approach to teaching, although, again how many schools are actually doing that, and to what extent is that put into practice? That's another question. Nevertheless, if that's what we are thinking about, whether we should be strengthening or trying to implement more fully the communicative approach, or whether we should think of some alternative ways of doing the language teaching, then I think that would be a very interesting question that we should talk about and we should keep any interesting discussion alive about, better ways or alternative ways of achieving better results. But I don't think that should have anything to do with 1997 or anything of that kind, but rather the fact that some people may not be happy with or may not feel satisfied with the English language performance or the proficiency of the students. If that's how they feel about it then I think it will be worth talking about possibilities of using different methods or supplementing the present ones with additional methods.

AU Okay, the last question, do you think that the changeover will affect the status, situation or morale of English teachers in HK?

III(97) Again, I don't think so, although the change in policy itself, which I don't think has anything directly to do with the changeover, but the policy itself of streaming the schools and asking a lot of them to become Chinese-medium, and then to keep the rest English-medium, that may have an effect on the 
morale, because some teachers may feel obliged to teach their subjects through Chinese, which first of all they are not used to, and secondly which they may not feel is any better than the old method, and they may also feel insufficiently supported by all the teaching materials and all the reference materials that they used to have when the subjects were taught in English. It's one thing to be able to come up with one or two basic textbooks in Chinese, which the ED and one or two publishers have tried to come up with in order to have this policy, but it's another thing to have a wider, broader base of support when you have to turn to additional materials make your teaching more effective. Then, some teachers may find their job even harder because of this change because they will have to go and look for some more resources, to find if they can find any useful materials in Chinese, and some teachers may have to put time into familiarizing themselves with the new jargon, which they may not have been familiar with, which they now have to teach to the students. So all these things may add up to a feeling of a lot of work, a very heavy work load, this may have an effect on the morale, but I don't think, for me, this would have anything in particular to do with the change, in terms of the change of government, but rather in terms of the educational policy itself maybe putting some teachers in a bad position. 


\title{
Interviewee IV(97): English teacher in secondary school \\ Date: \\ 10 June 1997
}

\author{
AU Researcher \\ IV(97) Interviewee \\ ... Missing words / Unfinished utterance \\ Pause
}

IV(97) Well, first of all my feelings as regards the ED's decision to force $75 \%$ of secondary schools to adopt Chinese-medium, I think most of the students they understand better in Chinese, I agree with the decision basically because even though most of the schools were English medium in name but most of the time they used Chinese to teach the students. I think the decision is quite pragmatic, the ED probably realise that it's a good idea to use Chinese as the MOI.

About benchmark levels, I don't know how I feel about it but there are two reasons for the government's decision. One is that the competition is very keen and they have to make teachers realise that they have to excel. Usually after doing graduation, teachers can just join as GM teachers without any training, so in this way the government is in a way trying to promote more professionalism and also because at this moment they need to, how to say, there are too many graduates at this point, so they want to make the criteria a bit more specific for teachers to join the teaching profession. From another point of view I think it is high time they did it because everywhere in the world, English teachers are becoming more professional. To know English doesn't mean you can teach it. So they should have more professional expertise, especially in a second language context.

AU In schools at the moment there are teachers who teach English and they are not trained at all to teach English, perhaps they are geography teachers or history majors, they have to ...

IV(97) Teach English

AU Yes

IV(97) Actually there are many cases and even in our school there teachers who are not English majors who are teaching English. I think this is one way of making schools know they should have more professional teachers teaching English and I actually agree with the government's decision. I have noticed in $\mathrm{HK}$, people are very practical, very pragmatic, so many teachers are taking a course and you can really notice that even teachers who have been teaching 
for quite a few years, I have a friend who has been teaching for 10 years now and she has just started doing her Masters. It is becoming more competitive and more professional. Although she has done post-graduate certificate, she wants to study. In a way it is a good message for schools that they want to employ more, become more professional.

AU Oh I agree, there are more courses available for teachers, such as Masters courses and Bachelors courses, post-grad courses, and more teachers are willing to do that because if they are going to teach English they are going to be trained to teach it. Perhaps if they weren't trained originally, it's this idea of professionalism and it's a HK thing that people like to do courses, and get more qualifications.

IV(97) I think society demands more experts, in teaching actually, things were rather lax, especially English language teaching. Maybe it's because, my last principal he said that it is easy to find teachers, but it is very difficult to find good English teachers. Actually it's quite difficult, although you may have many English teachers, but very few professionally trained English teachers. In HK I think it's even more important to have training teaching language.

AU So hopefully the benchmarking will increase the level of professionalism, as you say. Maybe those teachers who don't want to teach English, who are forced into it and whose language levels are not up to it, maybe the schools will no longer be able to ...

\section{IV(97) Employ them.}

AU Employ them to teach English, they will then be able to go on to teach their specialist subjects. Perhaps they'll fail the benchmark on purpose.

IV(97) Well, according to what I've heard, the benchmark if you fail, you have ten years to get a pass. I think if you really want a benchmark the criteria has to be stricter if it is to be meaningful. The number of years they give is a bit too long I think, ten batches of students by then will already have been taught by those teachers.

AU Yes, ten years is a long time.

IV(97) If it is to be ten years, now they have a new criteria for PGCE, new graduates must get PGC within four years. I think that is better, four years is a good time, ten years?

AU And how many teachers will stay in teaching for ten years these days? I don't know what the figures are for the length of service of teachers now. Traditionally people would make teaching their career, even within one school, teachers stay for 30 years, but nowadays, I'm sure that it's actually 
less. Teachers have a go at it, they train for it and then maybe do it for 3 or 4 years and then try something else.

IV(97) Yes, because they haven't ... some people join teaching because it is a very good salary, starting salary, but they don't really have the motivation to be teachers, that's why it's a good idea to have benchmark, but the criteria needs to be a little bit, if you set a benchmark you don't need to give 10 years, it dilutes the effect.

AU Do you think there will be any other significant changes in government policy towards the teaching of English in $\mathrm{HK}$ after the changeover?

IV(97) It is a very difficult question. I think it is quite difficult to foresee, there may be changes in the content but the methodology ... it may not change. Even though communicative approach has been introduced for such a long time, and even though HK has a lot of resources and a lot of ELT specialists coming here from all over the world, having so many seminars and courses, I don't think teachers are really using new methodologies in any practical way. Maybe teachers are not trained. I think for the coming 5 years, there will not be any major change in teaching methodology. What I can see is that schools are forced to adopt CMI and they had the English-medium before, I think the number of lessons may increase, so those schools may have changes in syllabuses, in teaching methodology. Like our school, we have only 7 lessons per week and if we change to Chinese-medium I think we have, I don't know, but I think we should have from 9 to 11 lessons. My last school adopted Chinese-medium, it is a Catholic Diocesan school and Catholic Diocesan schools have this policy that they want to have mother tongue and they want to teach English in a better way, so they are focusing more on English as a subject and to teach other subjects through the mother tongue medium. In that school I taught 11 lessons per cycle, a cycle is 6 days, so I think it's better to have more lessons.

AU It certainly is, 11 lessons per cycle, if you think that probably corresponds to 9 lessons per week. The other argument has been that within English-medium schools there's a need to teach English to give the students ...

IV(97) More exposure?

AU More exposure to the language and teach then study skills within English so that they can cope with their other subjects through the medium of English, and that if schools become Chinese medium then there is no need for that, so the proportion of English on the timetable may actually decrease.

IV(97) Do you think so?

AU It's what other people that I've talked to have said. 
IV(97) To me, actually people think that the importance of English, actually to be very honest, the attitudes have changed. People before, a change has taken place. For me it is very sad because the attitude has already switched back. Not our school, we really want to continue with English-medium, and most of the parents and the teachers want it, but they accept the fact that it will be Chinese-medium ... and actually I don't know what is the difference between Japanese society and HK society but it may happen that because they don't need English in their everyday life, so we will have a group of people having very good English, as people say, the elitists, and they will fulfill the jobs where it is needed, and the rest will still continue. It is a general idea that they don't need English in their daily life. On the other hand, personally, I won't have much worry about the commercial world as such, but looking at the F6 syllabus we can have an insight into what they really need in their commercial life. HK is a service-based city you could say, most of the ... it depends on international business. Now it is becoming more of a financial centre rather than based on industry, so they have to depend on other countries and to me a lot of jobs in HK, apart from sales, even commercial field, blue-collar jobs need a lot of English to communicate with other countries. So I don't know if HK will still produce enough number of people to satisfy the needs.

AU This actually goes into Q4, the idea of the proportion of the timetable given over to English teaching, I actually agree with you that in Chinese-medium schools, in some of them anyway, to balance not being English-medium anymore, they will increase the emphasis on English, if only to placate the parents who wish their English level to be good enough to give them a chance to go into tertiary education. If they are not able to get into what will be fewer English-medium schools.

IV(97) I think they should do it in first year, I think this is really necessary because English, although it may become like a foreign language, still it cannot be like learning French, or Spanish, it is still an international language, you cannot just teach it as a foreign language altogether. And it has to be kept a compulsory subject, a subject that has to be taught to every class at every level, so they must give more time in the timetable. I don't know if they will do it or not. Based on my experience in a Chinese-medium school, I think they will, so this is an area where I'm not sure what the ED will do. I think they should. In this school, some of the weaker classes we cannot, I would like to have 2 more classes a week with the weaker classes. I think even in socalled English-medium schools, 7 lessons is not enough.

AU Do you think there will be any other significant changes in your school or the education system more generally that might affect the teaching of English?

IV(97) I think curriculum certainly will undergo some change. I don't know whether it satisfies your question? 
IV(97) Curriculum, subjects like history, even languages content, I think it will undergo some change and to me it is necessary because HK students they need to be made aware of being citizens, not citizens, citizens of a country rather than a city and they also lack a lot of, how to say, people in the USA , how they teach their students a love of their country etc. I don't know, personally, patriotism is not one of my ideas. I don't know what part it will play in the future because the world is becoming smaller, but I think this will be one major part that will undergo change. Teaching methodology may not have any drastic or radical change. To be very honest, most of the things are taught in a very traditional way. Even in $\mathrm{HK}$, although it is a very modern city, still in the field of teaching, in one of our meetings, the principal pointed out that we are still backward in the way that we are not using technology to teach our students, that's why we find teaching very boring. I don't know how much people in our school or the $\mathrm{ED}$, how much change they can bring. At this moment it seems that the budget for teaching is decreasing. I don't know whether this aspect can be changed or not.

AU That I think would make the biggest difference, I mean it's not the only difference of course, it's not the only answer to education's problems to throw money at it, but certainly in a lot of ways it is necessary, just to bring the environment of schools and the facilities up to date.

IV(97) Teachers are so already overworked, how can they spend the time preparing materials with high-tech or audio-visual things, they are given more courses, even then it will increase their workload, they will have to spend more time. But I think at least they should give more facilities to schools if they want to keep up with the rest of the world.

AU Okay, teaching methods and approaches I think we've covered, so teachers' situation, status and morale?

IV(97) Status, to me the status of English teachers even now is not much different and as I told you what I notice in HK is that the changeover is affecting people before ... because HK society to me is a survivalist society ... it is clear that morale of English teachers is already going down, even myself, I think that if we change to Chinese-medium the intake will drastically drop as far as English is concerned, and not only English actually, good students will go to English-medium schools, and then not only English, it is so inter-related teaching of English nowadays. To me the quality of students will deteriorate, not only English but other subjects, so it is already very difficult to teach, to keep their interest and to teach them, they are not going to make it themselves. Certainly we can foresee, English teachers can foresee, this is bound to happen, so there is a drop in morale. That's why I say that government should realise and in order to balance should realise and in order 
to balance they should neutralise that they should increase the number of lessons, more incentive for English teachers from courses. Already I think English teachers have ranging from 40 to $70 \%$ more workload. So I think certainly it will affect, but on the other hand it depends what really happens after the changeover.

AU Of course, yes, I mean not only within schools but in society generally. Anything which affects the situation or status will automatically affect the morale as well.

IV(97) In my last school the first year was difficult, the second year was quite good, but classroom communication is much better here. I have not faced any serious problem and I'm quite satisfied this year. On the other hand, if the intake of students, it deteriorates then I think I will have problems. 
Interviewee V(97): University educator

Date:

16 June 1997

$\begin{array}{ll}\text { AU } & \text { Researcher } \\ \text { V(97) } & \text { Interviewee } \\ \ldots & \text { Missing words / Unfinished utterance } \\ - & \text { Pause }\end{array}$

AU What are your feelings as regards the ED's decision to force $75 \%$ of secondary schools to adopt Chinese-medium education?

V(97) Well I think it's a bad decision on the basis that it's going to be very socially divisive. It's going to emphasize how education plays a role in dividing classes within society and it's going to do it at a very early age, at around 11. At the moment you still have the divisions but you still have the permeability with the present system where the schools can opt for their MOI and even though the present system has a lot of flaws, one of it's great advantages is that it's removed a lot of the social divisions that were exacerbated, or it's lessened the social divisions that were exacerbated by the previous education system we had with the elitist English language schools, and Chinesemedium for the rest, I think you are going to be going back to that style. So I think that in terms of the social purpose of education, that it's a bad move. Regarding the issue of MOI and it's value of getting schools teaching in either English or Cantonese, again I don't think it makes particular sense, this fabled research showing that $30 \%$ can benefit and $70 \%$ can't and it's going to be much better for $30 \%$ to have English-medium and better for $70 \%$ to have Chinese-medium, but it's not particularly substantial, it seems to be an estimate based on a study in another area, based on inferences and not enough certainly to make a major social educational decision. I know that they've got some study coming out that should be published in the next 12 months indicating that of the various types of education or MOI in the education system the one that on the balance seems to be preferable in HK in terms of maintaining levels of educational attainment across the curriculum, while helping HK develop as a bilingual society through English and Chinese is the one that's closest to the Philippine model, where they have certain subjects taught in the mother-tongue and other subjects taught in English. And okay you don't get as many people getting as high scores in English as you do in totally English-medium, but their scores in other subjects are pretty good in comparison, and for the Chinese-medium instruction, obviously they get better scores in Chinese than in the other two ... but their levels of attainment in the other subjects are lower, so it seems like a decision that wasn't based on academic findings or research, so it's appalling that such a major decision should be as you put it forced on the education system on that basis. 
AU Perhaps force is too strong a word. They've actually said, they call it firm guidance.

V(97) To induce, let's say. So I don't think it's particularly sensible at all. I support Joseph Boyle's line, so I think it's a bad decision.

AU Do you think there's any significance in them announcing it now, I know that the background to it has been going on for a long time through the Education Commission, but the fact that it's been announced to be implemented in the next year, do you think that has anything to do with the changeover?

V(97) Perhaps in view of the fact that some of the people involved in the decision won't be around to be held responsible for the consequences, there may be an issue there. It may be an issue that in the colonial government they would like to be seen to be promoting Chineseness in some way, but whether those are sub-conscious motivations rather than conscious motivations I don't know. I would have thought they would have been better with the system they have at the moment where it largely depends on the headmaster and the headmaster's responsive to the population as, if you like, you are looking for a political motivation or something like that, say, look at this highly democratic way that we have it. What I really think is that you have inside the ED new people who saw that they could be identified with this make it either fish or fowl, no more of this red herring mixed-code, and people like John Clark were able to argue very vociferously and convince people that what they were proposing was worthwhile, it was a clear cut and clean solution and I don't think it has any substantial research backing, it was one of those internal people mailing their colours to the mast, saying this is the thing to do and whacking for it. I don't think it's tied in greatly with 1997. There were those concerns that kids were neither achieving particularly well in English or in Chinese, but were they preparing them to monolingual achievement? I know that John Clark was beating that particular drum about six or seven years ago when the handover was still a distant glimmer on the horizon and people were not sharply focused on it as they have been in the last three years.

AU Okay, what are your feelings as regards the ED's planned introduction of benchmark language levels for language teachers?

V(97) I think it's a good thing that there's an attempt to specify benchmarks for language teachers. It's quite a difficult thing to do. From what I've seen there are quite lenient or not particularly demanding levels set, although it's quite subjective as to how to interpret the particular criteria that are being set. I think it's particularly useful for us at the BATESL because we can have guidelines saying to our final year students, this is what you should reach, because language proficiency is always a worry. With the BATESL, some of the students, are they good enough? And it's nice to have the external criteria and someone doing the donkeywork for us. With the notion that currently 
employed teachers who have been given their contracts and accepted as qualified teachers and teachers of English, whether they should be required to do it is a different issue. On the one hand you can see quite objectively at someone just planning a system, it's attractive having it and if people don't meet that benchmark they we ply them with supplementary courses, if you look at it purely as a mechanical systems manner, however, you are dealing with people here and if they've been given a contract and recognised as qualified it's strange when some of them will be sixty minutes through the game, some may be forty minutes through the game, to suddenly say, hang on we'd now like to see if you are actually qualified to be in this match. I think what they should do is basically make it voluntary for existing staff but offer any staff who has it gets an increment in their salary, like they have in the police force, except they have them wearing little red flashes on their shoulders to show they have got their English proficiency level.

\section{AU Or Chinese?}

V(97) Or Chinese, yes.

AU It's interesting what you say about the BATESL, for example, there being that benchmark for them, what happens if at the end of their 3rd year they don't pass? They don't reach the benchmark, then what do you do? They are not qualified to teach, they can go away with an English degree.

$V(97)$ We would build that in as part of the proficiency programme, so if they didn't pass that they haven't completed the requirements of the degree.

AU Do you think they could do that as well at the I of Ed?

V(97) From what I've heard all new entrants to the teaching profession will have to ... that's the plan at the moment. Even though they are still working out the test and the whole thing has been, from what I've seen today, it's been worked out at tremendous high speed. They are fortunate that they've taken on somebody who was involved, Peter Falvey, who was at the University of Cambridge examination for language teachers and piggybacked on his experience to develop it.

AU The interesting thing is that teachers within schools who are not trained English teachers but who are teaching English, people who are perhaps unwillingly told, you are teaching English next year. But I'm a geography major! Too bad, you are teaching English.

V(97) You are the least worst English teacher.

AU But then it could be a get out clause, they might even deliberately fail. It doesn't mean they can't teach, they'll be given a grace period. Personally I 
think it's a good idea, although it doesn't affect me. That is a problem. I've had student teachers from the Institute and from here and I've watched them and they are great, they are full of ideas, but their language proficiency is just not up to it. That's the main problem, I think. A lot of are good teachers, they have good methodology and they like the job, but they are not a good model for their students

$\mathrm{V}(97)$ I think part of the problem may be the vagueness of some of the criteria making it difficult for the teachers to actually have a clear goal to aim at. If they had a clearer statement of the criteria, then it would be very good in the HK situation if people see a goal and objective then people seem to crack on and achieve it. But it's not that kind of set criteria, or it wouldn't be applicable in the way that they plan to apply it.

AU Maybe eventually they'll have benchmark language levels for all teachers.

V(97) I think in a lot of subjects you are going to have mixed-code inevitably, even if you say you are going to teach in Chinese-medium, there must be so many words in English.

AU Do you think there will be any other significant changes in government policy towards the teaching of English in HK after the changeover?

V(97) I can't see any other major changes, nothing like the Singaporean decision where they want more native speakers to follow the extra-normative model. What I can see happening is there being a lot of lip service paid to English language teaching and maintaining English standards, and most of the attention and work being done on spreading and raising standards and knowledge of Putonghua in the Territory. Putonghua is the link language to link the nation together and despite all the things I hear I find it impossible to believe that there won't be greater emphasis on teaching Putonghua and English, while it may not formally have hours lopped off, may be left to the decision, how do you expand the timetable and where do the hours come from, being left to a more school level, that people will find it too politically sensitive, teach 2 hours less English per week. And I think you will get some cases where the schools will have been coming back on their English, surreptitiously going over to Putonghua will be, their voices will be heard more clearly calling out for that option. And it will be seen as a politically correct thing to do, rather than at the moment it's being done surreptitiously.

AU Oh absolutely, Putonghua is coming in, no doubt about that, and schools are going to have to find a place on the timetable to fit it in.

V(97) And then you have other things like Chinese culture and civilisation, maybe that will take some time out of the history or the geography area. We've had a thing where all our students here now on the BAs, they have to do the 
equivalent of 6 credits, that's the equivalent of 2 modules, on Chinese culture and civilisation, and we've all had to look at ourselves, course planning teams, where do we cut in order to make room. Some people have chosen to just increase the workload on the students, although it's been said for years that we should be decreasing it. Other people having been looking for ways in which they can cut or trim.

AU So even on the English courses they have to Chinese ...

V(97) Culture and civilisation, yes it's a university requirement, it being designed in part by Beijing and in consultation with the Faculty of Humanities and Sciences here, social studies, public and social administration. Originally the idea was that it would be entirely planned and constructed by the people in Beijing and taught by the people here, but there was understandable reaction to that because we do have pretty good social studies and public and social administration, and Chinese, Translation and Linguistics already in the Faculty. But you'll get what isn't unusual in a post-colonial situation where the features of the society that identify most strongly with the colonial power are de-emphasised. In some cases there's a reaction against them, say in countries like Malaysia or in India, where they planned to phase out English and have Hindi as the one official language. In Malaysia they didn't really phase out English, the same with Sri Lanka. Then in all those societies you've then got a bounce back after say 15 years, when, oh, we actually need the language. I think here we are going to see things more like Pakistan, where everybody realised the importance of English to the society, but it wasn't really regarded as a priority issue to do that much about, and they just let things drift and then after about 25 years they got alarmed about the state of their English. I think here you are going to see that kind of drift, definitely in the short and medium term, maybe for longer, but definitely in those first 5 years.

AU The next question is concerning the proportion of the timetable, which we've touched on already.

V(97) I think probably in the English-medium schools, if by that you mean the $30 \%$ genuine English schools, then probably as is. In Chinese-medium schools, the ones who are declared Chinese-medium at the moment will maybe have a slight decrease and the ones who are made to go over to Chinese-medium, I'm not sure. I'm unsure about the number of hours they devote to English at the moment.

AU I've heard both views expressed, that schools previously English-medium or nominally English-medium who decide to go Chinese-medium, either they could increase the emphasis on English, they could timetable time to it, hoping to placate parents who are worried about their children's falling English standard if they are no longer at a so-called English-medium school. 
On the other hand people have also said to me that the whole point of having English lessons anyway for students is for them to cope with English-medium education in the rest of the school curriculum. If you don't have that anymore then the point of doing the English is taken away. A lot of the kids within Chinese-medium schools are not going to use English at all.

V(97) That's right and you could have the throwback to the system you had in the $60 \mathrm{~s}$, which is also current in India at the moment, where they have mainly mother-tongue education until the equivalent of sixth-form college, and in the mother-tongue education they get increasing amounts of English and the sixth-form college is a transition to prepare them for English-medium tertiary level education. I think in the tertiary sector they are going to remain Englishmedium in the science and technology faculties, especially if they've got any knowledge at all of the world around them. Malaysia with it's policy of phasing out English throughout the education system and phasing in Bahasa Malaysia, have had to climb down, about 4 or 5 years ago they realised the problems that was leading to their plans to modernise, and they decided about two years ago to switch the science faculties in most of the universities to English-medium, and I think the only complaint I've heard or read about is from the professors in the arts faculties, complaining if the point of the policy favours vision 20-20, it was to enable citizens to share in the global culture of science and technology and be part of that, and surely the arts are also part of it and they also teach in English.

I guess that's getting on to Q5, significant changes, yeah? We already have the thing that in the BA in social work degree that can be done largely in Cantonese and the exams in written Chinese. At the moment there doesn't seem any likelihood of that spreading, there seems to be greater emphasis on English. Certainly under this new president there are more initiatives being launched to boost the teaching and English proficiency university-wide than at any time in the 8 years that I've been here. Again, whether that is tied into the handover in that obviously the courses in Chinese culture and civilisation were tied into that, and whether this is to show that we are not losing our international focus, even though we are acquiring a greater emphasis on our Chinese heritage. We've had this peculiar thing in the last year with the new president, this very strong emphasis on the need to improve levels of English language proficiency, so we've got that entire English re-engineering programme coming.

AU That's right, the programme that the university is proposing, I know they wee advertising for teachers for it on Saturday.

V(97) That's right, the English Enhancement Programme.

AU Will that affect this department? Is it run from the Language Institute or what? 
V(97) Yes, it's mainly run from the LI, with some people giving input from the college, but it's not being run from the department. What you are having is six credits, so the equivalent of two modules of English and the idea being that all students who have got below a $\mathrm{C}$, that it's open to them with an idea of moving them up one grade equivalent in $\mathrm{U}$ of $\mathrm{E}$. So it's pretty much like the English Foundation Programme which they have at the moment, which is available for all D-grade, students getting D and below. But I think there's the feeling that it should be an improvement on the English Foundation Programme. This department will still be having students doing their ESP, say if they are doing technical communication for computer science, most of those modules will still be run from this department. This will be part of the core degree programme for computer science students. It will probably mean that those ESP modules get taught later and the English Enhancement Programme will come in the first year. Are you tempted to ...

AU To apply?

$\mathrm{V}(97) \quad$ Yes

AU I'm tempted to apply, I've been applying for a lot of jobs in tertiary at the moment, because that's what I want to do. The salary is not too attractive to be honest.

Okay, do you think the changeover will affect teaching methods or approaches in English lessons in secondary schools?

V(97) I find that a very difficult one to answer because there's such a range of methodology going on at the moment and people labeling their methods, and a certain label doesn't always fit what they are actually doing. I can't see people actually getting back to traditional methods more than the ones using those traditional methods at the moment, or becoming more so-called progressive in their methods. So I can't see it having a major impact on the teaching methods. Am I an odd man out with that?

AU No, most people have said pretty much the same thing.

V(97) Especially in the medium term, in the short to medium term, in the next 5 years.

AU Unless you look at it from changes in the curriculum generally or the MOI, perhaps. If schools change from English-medium to Chinese-medium then maybe they will need to change their approaches within English lessons. In the Chinese-medium school, if they become the only source of English to the students then will they be teaching it more as a foreign language than as a second language. 
$\mathrm{V}(97) \quad$ Yes, but from what I see at the moment, a lot of what I see is teaching English as a foreign language. Very little use is made in schools that if they teach imperatives that the students will be walking by shops saying, buy now, or big sale now, than if they are doing comparatives or superlatives then very little use is made of the fact that around school they may see things like, best bargain in town, or biggest meal for the least money, things like that. I think already most teachers teach English as a foreign language, and the kid of $30 \%$ overlap between the English on the streets in HK and the English in the syllabus is exploited to a minor degree. You get the use of pamphlets and things being brought in, occasionally newspapers, but the mass of English that is around that the students will see when they are on the MTR, going through the station or going past any place where there is a Wendy's or McDonald's, who like to put their branches near schools, most teachers find it a very alien concept to say to them look, why don't you take a look at what your students walk by on their way to school. For the last three years I've been having students do projects on that, doing 500 metres around schools, paying particular attention to transport routes, what do you find and how does that, how can that be related? The students all look as though it's something from outer space, they've certainly never had it done to them. My impression has always been that in HK English is taught as if it's a foreign language. That's why I was surprised when Tsang Yok Sing had his article in the paper saying we should teach English as a foreign language rather than as a second language. But when you asked about the changeover I though about the situation in the mainland and whether there would be anything coming down from there. I think Mainland China's problem is that it's got ... shortage of English teachers, a tremendous desire, so they've got the common methodology being one focusing on vocabulary memorisation and grammatical rules and the students tens to find they're very very happy with mainland teachers doing that. If they can then get native speakers doing their oral work, and I think the few surveys done in Mainland China indicate that the students feel that's where they are getting the best deal, getting the kind of balanced approach to the teaching of English. Is that what the government is trying to do with the expatriate teachers scheme?

AU I think so.

V(97) I think is likely to continue then you've got the situation the Chinese educationalists in China would be opting for. Unless you've got a political campaign where the leftists acquire power and they go anti-modernisation and you get these anti- Western spiritual pollution campaigns that they have coming down, in which case you have being an English language teacher isn't going to be a wonderful thing, anywhere in China you will be regarded with a bit of suspicion, and I don't think there are going to be any great changes, which brings us onto the next thing about the status, I think English teachers will begin to feel that they are a little less important, especially in the lower band schools and the students who have been poor achievers who have 
been able to salve their egos through saying, English isn't important anymore because we're going back to China and it's just part of this elitist foreign influence and after $1997 \ldots$ and you've already had that happen in some schools already. I think in the schools that are the higher band schools, the students are doing well and they like the things they are good at, so us teachers won't be that affective there, I don't know. I wouldn't like to guess the situation in the middle, Band 3 area. I think already in Band 4 you've had a suffering of morale with English being given a ...

AU Of course there is a possibility, something I've thought of, of once the changeover has come and gone, that English will no longer be the colonial language, it will no longer be something that is pushed down the throats of the native people, like this is our language and you are going to learn it, and the backlash has always been, why the hell should we learn it? Now it's the case that it's no longer the colonial language, the colonialists have packed up and got on their ship and gone, it's now the international language, it's the language of the rest of the world. You either learn it or you don't. If you want to be international, you learn it, if you want to stay here all your life, you don't. I suppose then a lot of the population will say, I don't. Then they'll have their English lessons in the Chinese-medium school, which will be just like anybody else learning a foreign language. It's there if you want it. Regarding the status of English teachers, perhaps they will no longer be seen as forcing ... the way we used to see French teachers at school.

$\mathrm{V}(97) \quad$ But there again, where I went to school French was an obligatory part of the curriculum, everybody had to do it.

AU Oh it was for us to.

V(97) Whereas you could choose whether you wanted to do German or chemistry or another topic and choosing German had a very different feel to the lesson, so there will be that difference whether it's a choice or not. How quickly that will come in, whether there will be this mass movement, if they carry on through their programme with their 30\%, which they seem likely to do, 30\% English-medium and 70\% Chinese-medium then you are going to get a boost among the teachers in that $30 \%$ of schools as everybody there wants to be there, and then for the teachers in $70 \%$ of schools, I find it hard to imagine what their situation will be like, whether you will have the majority of them saying, you know this is just an important subject, let me try and do my best at it and make the best of the opportunity, we are Chinese-medium, we've already flunked on the English level. Why am I getting more pay?

AU Presumably there will be students from the Chinese-medium sector who will go into tertiary education in the English-medium, so there will still be a need for English within the Chinese-medium sector. Perhaps even there will develop an elite within the Chinese-medium sector. 
V(97) It's such an advantage for them having an English-medium tertiary level education in science and technology subjects. It means they keep abreast with the latest developments very easily. It's easier to go on to mater's course, if the students are required to go to major universities overseas they've got that advantage, so I can't see, unless there's a major political upheaval and a break on the Chinese modernisation programme, I can't see there being pressure to make the science and technology ... [tape ends] ... less as a spoken language, but until they've got the vast majority of the staff all teaching in Cantonese, you are still going to have the need for comprehension of the spoken language at tertiary level. And as education in the British model system is geared mainly to those going to university to do well there and that has a major washback affect on the schools maintaining that even those who go over to Chinese-medium maintain a strong English stream. 


\section{Interviewee VI(97): English teacher in secondary school \\ Date: \\ 16 June 1997}

$\begin{array}{ll}\text { AU } & \text { Researcher } \\ \text { VI(97) } & \text { Interviewee } \\ \ldots & \text { Missing words / Unfinished utterance } \\ \text { - } & \text { Pause }\end{array}$

AU What are your feelings as regards the ED's decision to force $75 \%$ of schools to adopt Chinese-medium education?

VI(97) I think it's kind of politicism that comes up once again. In the past years there were famous schools and English was regarded as a very important language to people in the years before and after that a more general education, more students have been allowed to study English and they have better chance to seek for a higher position in the society, but here in the run up to 1997 the ED has changed its policy from encouraging schools to adopt English-medium of teaching to adopt compulsory education in Chinese. It is a kind of action which apart from politicism is inconsiderate of the situation.

AU How do you think it's inconsiderate?

VI(97) I think students have to have a lot of chance to expose to English, not just their English subjects, they have to have more exposure in other subjects because there's a linkage between their further studies and their work, and I worry that we HK people will be like Japanese. In Japan, all the textbooks are in Japanese and so the English standard is not as good as that in HK, I'm afraid that one day we will go to that situation.

AU How about your school, is it now English-medium or Chinese?

VI(97) A mixed-code of teaching using English textbooks and teachers mainly teaching in Cantonese and sometimes using the English terminology to bring the concepts or ideas.

AU Do you know what will happen to the school? Will it change, will it become Chinese-medium in 1998 ?

VI(97) It has not yet decided, but as our school is a self-subsidised school we have more autonomy in our reaction to the language policy of the government $\mathrm{We}$ haven't decided yet. 
AU OK, what are your feelings as regards the ED's planned introduction of benchmark language levels for language teachers?

VI(97) To me, I think it's unfair to teachers who have already entered the field. Just like lawyers, they don't need to have some sort of test to them whether their professionalism is recognised. I think there should be more emphasis on the assessment of teachers in training, maybe they are attending a certificate of education, then according to reach the level of professionalism then more emphasis can be put on the assessment. Instead of providing a language benchmark to English teachers on their job, maybe more encouragement should be given to teachers, say like some refreshment courses, some chances for further studies overseas so that they will encourage their level of English.

AU I think the point of the benchmarking is that it will then if teachers don't reach a certain level then they will have to take some courses or some further training. I've been told that they will be given something like 10 years to reach the benchmark.

VI(97) I don't quite worry about that as there is 10 years to go, but I think the policy is not so fair, I don't mean that it is quite difficult to pass the test but it is ridiculous to me, compare with other professions like lawyers or doctors. I don't think if you are a doctor, you need to have a test to see of you can continue to be a doctor.

AU Well I don't know, certainly I don't think they have tests of their language skills, but you are right, I don't think they have continuous tests of their ability once they have qualified as a doctor ... or as a lawyer.

VI(97) They do have testing, but the result can lead to further advancement in their careers. I know that doctors have to study a lot to keep abreast of the advancements in their field, and some of them maybe take some studies.

AU But don't you think that having these benchmarks is a kind of way of raising the standard of teachers? I think what a lot of people are worried about is that there are a lot of English teachers who are not English majors, who are not trained as English teachers who are teaching English, they are perhaps geography majors or history majors and because they went to university and did their university degree through the medium of English, then principals or panels think that their English must be good enough and they are a teacher so they can teach English. I think the point of the benchmarking is to try to even that out and to get rid of that situation and that only people who are competent, at least in their language level, should be teaching English.

VI(97) So, it won't apply to all English teachers? 
AU Oh I think it will apply to all English teachers, but presumably those who are trained as English teachers either from the I of E or BATESL or another programme like that, their language should be good enough. It's perhaps the other teachers of English or of Chinese whose language levels are not up to scratch, who they are targeting.

Do you think there will be any other significant changes in government policy towards the teaching of English in $\mathrm{HK}$ after the changeover?

VI(97) I think more money will be spent on teachers but I think it's related to Q5. I think we have to talk about the kinds of teaching that will change first because the government will spend the money on them. I think more remedial classes will be given to those schools who follow Chinese medium of teaching, and also there are courses for new immigrants to have a match to the local students at the same age, so that they can catch up with the English intensive courses. For the remedial classes for the local students attending local schools, they will have more chance, they will not just study the knowledge from textbooks, they will be more encouraged to read newspapers, magazines, things like that, it's a part of their curriculum, not just the textbook, to top up the contents. So the government will spend more money on these two main aspects. And for those students taking Englishmedium schools I think not many changes will be taken because the students exposed more or less the same they did in the past.

AU So English-medium schools, the curriculum will stay pretty much the same, the changes will be in Chinese-medium you think?

VI(97) Yes and those schools giving classes for new immigrants, so the government will spend more money on some refreshment courses for teachers so they can hold some intensive classes for the immigrants and some kinds of materials for them to develop to teach students to have more exposure to English apart from the textbooks. Kind of tailor-made lessons.

AU So you think more money will be put into more resources?

VI(97) I don't know what kind of policy is more important.

AU The main policies are those in 1 and 2, the main policy changes that we know so far, but certainly more immigrants coming from China will be a factor in HK in the coming years, and you are right, something will have to be done to cater for them to bring their language level up to a certain ...

VI(97) To their counterparts.

AU I don't know how easy that might be, you might have extensive courses but an intensive course for a few weeks might not change very much. They might 
need remedial classes; they might need classes throughout the year. But I think that in that situation, schools wouldn't want to isolate those students, they would want to integrate them totally.

VI(97) I think they have to attend lower form compared with the local students maybe.

AU Well that's what usually happens, isn't it? What changes do you envisage in the proportion of the timetable given over to English teaching in both English and Chinese-medium schools?

VI(97) For the change of timetable, I think for English-medium schools, more or less the same the timetable. For Chinese-medium schools, yeah, so it is I think more lessons will be held on English because the students lose something and they have to gain something to have a compensation.

AU I think that is what a lot of people think. Of course the other factor perhaps is Putonghua. Although I've not seen any instructions or any plans about it, it will be taught in secondary schools starting from this year I would think.

VI(97) Just the Chinese language subject?

AU Because having talked to the EA people, Putonghua will be a HKCEE subject in the year 2000, so they must start it now in order to bring the students up to standard.

VI(97) Will it be a separate subject or mingled with the Chinese language subject?

AU I think it will be a separate subject.

VI(97) Just like French?

AU As a foreign language, yes, which is interesting because the idea of the timetable comes in, and the proportion of the timetable. If you're going to tech Putonghua as well as English and Chinese, as Chinese is taught now, then where do the extra lessons come from?

VI(97) So you can say about it, in proportion for English-medium schools, they may lose 2 lessons, not just from English lessons, maybe some other lessons, Chinese language lessons, and for Chinese-medium schools in that case ... I think they may also lose 2 lessons. They won't lose more or less than 2 lessons. And for the extra lessons, it's a pressure, how to squeeze out ...

AU The Chinese-medium schools, they don't want to decrease their number of lessons on English, that will be the only English the students get. 
VI(97) Maybe the students will have less choices on other subjects, apart from language ones, yeah, like in my school, for arts stream students, they have to take economics, geography, history, English, Chinese, mathematics, and then Chinese history. Four subjects, Chinese history, history, economics, geography, they have a choice of 3 of them, instead to take 4 of them, so that one more subject's time can be allocated to Putonghua, and for those Chinese-medium schools, I think maybe they can shorten their lesson time for other subjects, because now they are teaching in Cantonese, the students can learn much faster than they could before. They can shorten the lessons, or simply give extra burden for students to stay longer in school.

AU Add 2 extra lessons in a day, perhaps, or an extra 1 lesson per day. Yeah, make the lessons 5 minutes shorter and add one extra lesson.

VI(97) If two extra subjects have to be focused at the same time, there would be sacrifice, either in shortening the time for teaching other subjects, or excluding some subjects.

AU Something will have to give, unless they make Putonghua the MOI.

VI(97) It is a long way to go.

AU I think so, yes, maybe in 10 or 20 years. Do you think there will be any significant changes in your school or in the education system more generally which might affect the teaching of English?

VI(97) I think, more remedial classes will be held in our school. I can't say for sure, probably my school will follow the teaching in English medium, and actually there should be less changes in the kind of teaching and the time spent on it, I mean English of course, but the level of our students is not so good, so just like before, the students will still need to attend remedial classes. I mean more remedial classes will be offered to students. This arrangement does come from the change in medium of most schools; it comes mainly from more teachers' resources. In recent years there has been a drop in students going to my school because my school is a subsidised school and it doesn't follow the arrangement for primary school students, so more teachers' resources can be allocated for remedial teaching.

AU Do you think that the changeover will affect teaching methods or approaches in English lessons in secondary schools?

VI(97) This one, I don't have any idea about that, to the methods or the approaches, as I mentioned for Q5, more remedial classes will be offered to students in Chinese-medium schools, and intensive courses for new immigrants, so there should be some kinds of changes in the methods in teaching. 
AU Because of having to teach more remedial classes. I suppose the change in the MOI might change things. If schools become Chinese-medium, then that may change their methods or approaches to a certain extent. If they have to teach English as a foreign language, as opposed to as a second language then maybe the approaches will change, because the students in the Chinesemedium schools will not be getting any backup from their other classes.

Final question, do you think the changeover will affect the situation, status or morale of teachers of English in HK?

VI(97) For the morale, I think there's not many changes because the morale of teachers of English does not depend on the change of emphasis on the language of teaching, yeah. Morale comes from the teaching environment, the workload and the kind of support from the colleagues, things like that.

AU That will change?

VI(97) I think not many changes because for English-medium schools, as I have said before, there will not be a lot of changes. For Chinese-medium schools, the government has proposed that more English teachers will be employed to help the students so that the workload will be more or less the same. Maybe the beginning years, I don't know if it will be better or worse, I can't say exactly because there's some kind of adjustment and teachers in Chinesemedium schools are a kind of guinea-pigs. But anyway, there are not many changes because more teachers will be employed to help them, to share the workload. The status or situation may be a little bit worse only, because the emphasis is no longer on English. Of course the status will be less important as before. 


\section{Interviewees VII(97), VIII(97) \& IX(97): Education Officers \\ Date: \\ 5 June 1997}

$\begin{array}{ll}\text { AU } & \text { Researcher } \\ \text { VII(97) } & \text { Interviewee } \\ \text { VIII(97) } & \text { Interviewee } \\ \text { IX(97) } & \text { Interviewee } \\ \ldots & \text { Missing words / Unfinished utterance } \\ \text {-. } & \text { Pause }\end{array}$

AU The questions that I've written perhaps apply more to people in schools than perhaps the government sector. The first question is what are your feelings as regards the ED's decision, I've put to force $75 \%$ of schools to adopt Chinese medium of education, it's actually called the Firm Guidance.

VIII(97) It's a bit like voluntary repatriation.

VII(97) What was the percentage before $75 \%$ ?

IX(97) I mean before it wasn't forced at all. There are those schools that have been middle schools for all these years. I'm not sure of the proportion of Chinesemedium to English-medium schools but there are definitely fewer, from our candidature for example in 1997 we have something like 11,000 Syllabus A for Chinese-medium schools and 115,000 Syllabus B for English-medium schools.

VIII(97) The ones that take Syllabus B are not necessarily in English-medium schools, the ones who take Syllabus A are not necessarily in Chinese-medium schools but it's a rough indication.

AU So the English-medium, at least in name, far outnumber the Chinesemedium? In fact I think the schools are actually afraid to come out and say we are Chinese-medium.

IX(97) From what we've heard from teachers, for example, Daisy was talking about it the other day, her school because they change from English-medium to Chinese-medium, not the whole school but three classes of Chinese-medium and two of English-medium and at once the banding of the school dropped. It was a Band $1 / 2$ before and once they' $d$ made the decision parents were reluctant to send their children to their school so at once the banding dropped by one or two bands so now it's a Band 3/4 school and parents who actually register their children for that school year tried to get another school. So in the middle of the school year they lost a lot of students. Parents were really 
anxious about getting their kids into the English-medium classes, so that's the situation now, that parents prefer English-medium. And I suppose that market forces would force the principals to go English-medium.

VIII(97) But now they won't have the choice.

IX(97) Well they have the choice, I think what they do is they apply. If they think that they've got the right intake of students that are qualified to learn through the medium of English, they can apply to have $85 \%$ who are in that category, then they can apply to be English-medium, so it's not automatic, they have to apply to be English-medium and if you do not qualify then definitely you cannot stay English-medium.

VIII(97) From what I understand, the exact number hasn't been set, this figure of 96 because of that article in Apple Daily or whatever. The actual figures haven't been ...

IX(97) And also because they don't know how many schools will apply to be English-medium, or to stay English-medium. I don't think the Chinesemedium schools now will want to change to English-medium, so people who apply to be English-medium will be Anglo-Chinese schools now. Although I've been told on Saturday that some quite good schools, I suppose I shouldn't mention the names of schools here, some quite good schools are going to not apply to be English-medium because it's the decision of some board or some overall body like some Christian organisation for example have decided that all schools should become Chinese-medium, so even if they qualify, they are not going to apply to be English-medium. So some schools which say would be English-medium because it's a well-established school, it doesn't mean that they will remain English-medium next year.

AU That's interesting. You would normally expect them to stay English-medium, wouldn't you? It seems that most parents still believe that English-medium gives the students the best chance of advancing into tertiary education.

VIII(97) But maybe they are basing their decision on pedagogic grounds, that it's better and healthier to be taught in the mother tongue which I think there are a lot of arguments for that.

IX(97) So actually, I think all the catholic Diocesan schools are opting to be Chinese-medium, with the exception of two, that's what Brenda said, and her school is one of them, that's why she knows. I think people do it, as John said, for pedagogic reasons. Lots of people are pro Chinese-medium, for example, the teachers' union, they are definitely for Chinese-medium.

VII(97) Presumably the main thrust behind it is less concerned with their English ability, but their ability in other subjects, where they are studying in English 
and where their performance is affected by the fact that they are not studying in Chinese, so I guess there must be some motivation behind it to give them more of a chance in other subjects as well.

AU But still the question will arise when it comes to tertiary education, which presumably will remain English-medium.

IX(97) I read a report that actually from Form 4 upwards, so from Forms 1 to 3, the lower secondary, it's compulsory, Chinese-medium. So from Form 4 upwards they are saying that the schools will have more flexibility to put more English in to prepare their students for tertiary. That was actually in yesterday's newspaper, I think, what's his name, Mr. Leung, Tung Chee-hwa's advisor for education. What's his name, Andrew? According to him, that is what will happen, schools will be allowed more flexibility from Form 4 upwards to get students ready for English-medium tertiary education.

VIII(97) But even in tertiary education, there's a question of how much of it is actually conducted in English.

IX(97) I think the lectures are probably all in English but the tutorials are very often in Cantonese.

VIII(97) Oh it depends on the subject, in some of the more specialised, the sciences or engineering, things like that ...

AU My focus is the changeover and the planning for this has been going on a long time and is nothing to do with the changeover as such. How do you feel about the MOI in say 10 years time, do you think that will change and maybe Putonghua will come into the equation?

IX(97) I don't know, I think there will be quite a lot of resistance, if Putonghua, you say Chinese-medium, so is it going to be Cantonese or Putonghua? I mean a, they can't get the teachers, there won't be enough teachers who are fluent enough in the language to teach, unless they have lots of people coming from the Mainland teaching in HK. But what are the local teachers to do, I mean do they all lose their jobs? I don't think it's that easy for Cantonese people to become fluent in Putonghua, I mean fluent enough to be able to teach in that medium, because I know that even in Guangzhou, in Canton, the schools are in Putonghua, the language of the students, the language of the playground is all Cantonese. So I think it will be very difficult.

VII(97) It could be, encounter the same problems as with English-medium schools here, there'1l be cases where being taught in Putonghua will inhibit their ability in other subjects. 
IX(97) Except that with Chinese, I suppose because the characters are the same and they are used to the Modern Standard Chinese characters, that it would create less of a problem than if they were studying in English, they can read. I mean, Cantonese students now have that same problem, that they read things that are different from their spoken language and some people have said that maybe the Chinese will benefit if you force them to use Putonghua, that their written Chinese will improve, but as Graham said, it won't be more difficult but it might be just as difficult as learning through the medium of English. Although the reading is $\mathrm{OK}$, but the pronunciation is very different and the grammar is sometimes different so there will be problems there as well.

VII(97) There would also be, I guess, the problem of building up the prestige factor, I mean the thing which sells English is the international language and to actually institute Putonghua-medium teaching would be a lot more difficult because the students would have to be convinced in some way that this is going to be useful in a global context.

AU It would certainly be more useful than Cantonese, I mean Cantonese is of no use outside of southern China. Perhaps the patriotic angle would help, China and Taiwan as well being Putonghua-medium.

LX(97) I suppose if they make it a prerequisite for the civil service for example, then it would add to the prestige of Putonghua and then there is reason to learn it, and I think certainly, things may go in that direction with closer ties with the Mainland. Now people are learning Putonghua for business, but Mainland people are learning Cantonese as well, it's very popular in Shanghai or Beijing for people who come to HK to do business.

AU At least if Putonghua doesn't become a medium, it will become an important subject in secondary schools, presumably starting this year?

IX(97) From Form 1 I think, but it's only like two periods a week. In primary schools they've had it for many years in fact. I think from Primary 4 onwards they do it for two periods a week.

AU They do it in my school, then after Form 1 they stop.

IX(97) That's actually quite odd, I mean what good does it do for one year? Most students will have had it for two years when they come to secondary school, no, four, five six, it's actually three years.

AU So it's not an introduction?

IX(97) It's not an introduction, it's actually a continuation, but it's strange that it will stop at Form 1. I think some schools actually carry on to Form 2 because in 
the year 2000 they have this Putonghua exam at the CE level, so people will go on I think from Form 1 last year then there will be this exam.

AU OK, next point is about the benchmark language levels for language teachers, how do you feel about that? Language teachers taking proficiency tests to see if their language levels are up to scratch?

VII(97) No comment.

VIII(97) Well we're in a difficult position with regard to that because the Exams Authority is actually going to be administering it, particularly the pilot for it, so I don't think it's really appropriate for us to go into detail about it, our sort of personal views. Quite honestly it's so sort of up in the air I don't know that we have any strong feelings about the details of it because the details ...

IX(97) I myself am for the idea of having a benchmark. I think it adds to the prestige of the teaching profession because everybody has to be qualified to teach because very often some university grads who can't get any other job say, oh well I'll teach for a year and see what happens. I think that's not very good that people see teaching not as a profession but as a stepping-stone towards other things better, so having exams, sort of professional qualifying exams like that is actually good for the profession. But at the moment we don't know exactly what the benchmark exams be like.

VII(97) We're being very technical minded about it really, we are only really concerned with setting the tests and the finer points of administration, but the actual interpretation of the results is the area which is still under discussion, like what are you going to accept as a benchmark? What does a certain benchmark mean? And that I think is going to take quite a while to establish.

AU Presumably it'll be quite a complicated thing to do to work out what a benchmark should be, depending on the situation a teacher is in. Is somebody teaching in a Band 1 English-medium school going to have to have a higher benchmark than someone teaching in a lower band Chinese-medium school? I'm not saying that the second one should be any less competent but ...

IX(97) But the argument against that is that if you have worse students you should get better teachers.

VIII(97) There may be a different benchmark for primary teachers than for secondary, but I don't think for the band of school, for one thing that would then prevent you from moving from school to school.

IX(97) I think probably there will also be a distinction between teachers who teach through the medium of English in other subjects and teachers who teach English, there will be a different requirement. 
VII(97) There are students who are all from different band schools and they are all assessed the same level, so there's no reason why teachers should have anything different in terms of banding.

AU I suppose in the future perhaps benchmarking for all teachers? Why only benchmark language teachers?

IX(97) I think language teachers is only the first step, there are in fact plans to benchmark all teachers. If they teach through the medium of Chinese then their language, their Chinese language will be benchmarked.

AU Do you think there will be any other significant changes towards the teaching of English in secondary schools in HK after the changeover?

VII(97) From our perspective, we've not had any indication of any changes in examination syllabuses or the format of our papers.

IX(97) Just because of the changeover, I mean there are changes because of curriculum changes, for example.

VII(97) But if we want to change the syllabus it's a good few years in the ... it takes quite a few years for the whole process to complete itself and there is nothing that we've started in the last year because of 1997.

VIII(97) I think it changes, the only changes on the horizon are the TOC, which has been in the pipeline for a long time, but they won't be examined until 2004/2005, but partly in anticipation, our syllabus was changed in 1996 to make it more task-based and practically oriented, and whether it will require more changes, I don't know. But I don't think we have any plans at this point to change or revise the syllabus. I can't really think of any other changes other than medium of instruction.

IX(97) Because at all these CDC meetings, they are sort of changing the syllabus because of the TOC initiative, not because of the handover.

AU Well maybe not because of the handover but ...

VIII(97) But at the same time. But I think the TOC stuff is not really designed to upgrade the importance of English and it's not designed to downplay it, it's designed to make it more practical. If there were a syllabus to downplay English, I don't think that would be compatible with TOC.

IX(97) And in fact TOC is not just for English, it's for Chinese and Mathematics, these major subjects. 
AU There have been arguments that TOC is basically a European/British idea imposed on HK. Is it suitable for Chinese children?

VIII(97) Or is it suitable for classes of 40 kids?

AU . Maybe there might be more changes in other subject areas rather than English or languages, in History or GPA?

IX(97) I think eventually they want everything to go the TOC direction, but now they have their hands full with just Chinese and English and Mathematics. I think not much has been done in the other subjects about the TOC, how to change the curriculum.

VII(97) The only thing I can think of is, one of my papers is the CE English Literature, and their seems to be a lot of pressure from somewhere to try and change the title to anything other than English Literature, to Literature in English or Literary Studies is another suggested last year. And one theory is that English Literature would sound too, colonial is not the right word, it's foregrounding English and Englishness too much and I found myself having to argue that it doesn't mean English writers, it means writing in English. Changing the title from our administrative point of view is not sensible when you're not going to change the course content, because it just confuses students. It's amazing how much time has been spent on discussing the title of the course over the past two years.

VIII(97) But didn't you say [IX] that in Chinese ..

IX(97) In Chinese, the literature of England, it could be interpreted that way. If anything they should change the Chinese name.

VII(97) I don't know how much truth there is to that theory because I read in the paper last year that English Literature is extremely popular in the Mainland. The number of students has been rising dramatically over the past couple of years. But maybe they want to change the title to try and attract more students.

AU They say that in schools it's becoming less and less popular, very few schools actually do English Literature any more. You would know that, our school does because our principal is very keen on it.

VIII(97) Which school?

AU Heep Yunn, girls' school, but I know a lot of secondary schools don't. We do it throughout the school. 
IX(97) More girls' schools do it than boys' schools because I think the teachers think that they will have to have a certain standard of English before they can handle it, so they don't offer it if they think that the students are not good enough.

VII(97) But you do end up with students who are really really weak because they are not able to take whatever the other option is, so you get incredibly weak students in English Literature as well.

IX(97) Really?

VII(97) Yeah

AU Personally, I see nothing wrong in them doing English Literature, certainly at the lower school level, even if their English ability is low.

IX(97) I mean a lot of schools do have readers and things like that, but it's not literature.

AU I mean if they are good enough they can do the certificate, but if they can't write it's hopeless.

VIII(97) But it must be terribly frustrating for a kid because when I first came to HK I taught in a private school and it was pretty bad, and all the kids in that school did English Literature and they couldn't spell cat and they were supposed to be studying Shakespeare, it was absolutely ridiculous. So it led to great frustration on their part, on the teacher's part.

AU OK, changes in the proportion of the timetable given over to English language in English and Chinese-medium schools, do you see any changes? Perhaps it will change in the Chinese-medium schools? At the moment it's about a quarter of the lessons are English and Chinese.

IX(97) I don't know enough about this to make a comment really because I don't know whether there is a difference between Chinese and English-medium school, you say a quarter?

AU In English-medium schools yes.

IX(97) And in Chinese-medium schools?

AU I'm not too sure.

VIII(97) So you're talking there about actually teaching the language and not teaching in the language. 
IX(97) I would imagine it would be the same, because for these other subjects, let's say Economics, the CDI they have this recommendation about how many lessons you have a week for let's say Economics and it's the same number of lessons, whether it's taught in English, or in Chinese. So changing the medium shouldn't affect the other subjects, so I wouldn't imagine that it will make a difference to English Language as a subject.

VII(97) When Putonghua becomes compulsory ...

IX(97) Then they might add a couple of ...

VIII(97) I remember when we added the Use of English oral, one of the biggest complaints after the first year when they started preparing for it was that teachers complained to us that, we don't have any extra lessons for oral because we haven't been allocated, so what do we do? And we tried to say, well you don't need to have a special lesson for oral, you can use your writing lessons or things like that, which is all true, but I can appreciate their point, was that they were being asked to do more, with no expansion of time. On the other hand, there are only so many hours in the day and the other subjects are going to be jealous as well.

AU We have six lessons of English for F6 and 7.

IX(97) And I think that varies, some schools will have like 8 lessons per week, depending on their emphasis or whether they think their students need more time for that particular subject. And I think the ED is quite flexible, they suggest a number of periods for that particular subject. The principal has the discretion, or the teachers in the school try to fight for more time.

AU It is tough, doing the AS Level in six lessons a week and covering all 5 papers is very tough.

IX(97) Some schools have eight lessons a week I think in F6 for English, so I think it varies.

AU Perhaps schools who were English-medium change to Chinese-medium, maybe to keep parents happy, they are going to say, OK we are going to increase the amount of English on the timetable.

VII(97) If they are Chinese-medium schools and they are being taught Biology or Geography or whatever, I spoke to a teacher some months back who attended a course, a package that the British Council had designed to help teachers of non-language subjects teach their subjects in English and he went on this course and said that the main problem most of the teachers on this course had 
was that the volume of material that they are able to get through in English was very small compared to what they would have been able to get through in their native tongue. The focus of it was doing interactive group work but with geography or biology or whatever and the main feedback that they got from the teachers was that they were all great techniques but we've got to get through this amount of material on the syllabus and if we do it this way, we won't get through a third of it. So if they are going to be taught in the Chinese medium, presumably they will be able to achieve a lot more in terms of syllabus content.

VIII(97) One of the criticisms that I've always found difficult to answer of modern TEFL methods is that you can spend a whole lesson teaching people how to interrupt politely. It's broken down into such small chunks that in the end they may or they may not know how to interrupt politely but it takes an accumulation of a lot of little bits to aquifer a language. If you apply to same methods to content subjects, they may learn the little bits well, but it's difficult. But also the schools may feel that if they go over to Chinese medium to placate the parents they'd have more English lessons, presumably that's one of the reasons it's being handled as it is. It's the heat that going to be handled by $\mathrm{ED}$, not by individual schools. It used to be that schools voluntarily opted to change from English-medium to Chinese, they then ran the problem of losing parents' support and students', but if it's being imposed from on high, then they don't have to worry so much about that, do they?

AU Certainly as regards the subsidised schools, they exist an awful lot on donations from parents. The next one is about changes in your school or in education more generally which I guess doesn't really apply. Do you think the changeover will affect methods or approaches in English lessons in secondary schools?

VII(97) People which we've occasionally met from the mainland seem to like our exam papers and the way they're put together and the washback that that produces. One thing I was thinking we could mention is the modular approach which is something which we tried to launch last year or two years ago, which is an examination where the ultimate aim is that some recognition is provided to lower ability students, so that might affect, not the methods or approaches but maybe they'll be more lessons geared to much simpler levels of English.

IX(97) But that's not specifically because of the changeover.

VII(97) No, it's not. This is something we were going to do a while back but didn't get much support from teachers.

AU It can be said that these changes have been mooted for a long time but whether or not they were dons with one eye on the changeover is impossible 
to say I suppose. OK, how do you think the changeover will affect the situation, status or morale of teachers of English in Hong Kong?

VII(97) It's quite difficult to say.

AU Perhaps the benchmarking will come into this as well?

IX(97) I can only say what teachers have told me and again, she told that her school is going to go Chinese-medium from 1998 onwards, and she said there is more pressure on the English teachers to maintain the English standard because all the other subjects are taught in Chinese then the principal will expect the English teachers to work extra-hard to maintain the standard, so she thinks it will actually have more pressure on them to try and maintain the standard, to work harder to keep the level of English the way it was before the change of medium. So she thinks it's going to add to the work, or the responsibility or the expectation.

VIII(97) How much will, in practical terms, changing from the English-medium to the Chinese-medium actually have? Everything you here and read about the socalled English-medium schools, a lot of the classes are conducted in Cantonese anyhow. It seems likely that the only big change is in the change of textbooks. If they go from the English language textbooks to the Chinese language textbooks, there will be a difference.

IX(97) Test papers, all the exams will be in Chinese.

AU They'll still have to learn English terms presumably?

IX(97) For certain subjects.

VIII(97) [IX(97)]was saying that she taught Biology and she would find it difficult to teach in Cantonese because she doesn't know biological terms and things in Chinese.

IX(97) I think they are now running courses for teachers who will have to teach their own subjects in Chinese medium and in fact it will be difficult for teachers as well at the beginning, to get used to all these Chinese terms, because I think most teachers here will have gone through the Anglo-Chinese system and they themselves will have learned their own subject matter in English rather than in Chinese.

VII(97) Thinking about question 7, one of the teachers who said, well I want to get my pension before 1997, and have since emigrated or whatever, I mean there was a point last year when there was a lot of press about the teachers in the mainland not having been paid for months and months, but they are all very personal reactions and when I joined here I imagined that there might be a 
gradual loss of exam personnel and eventually we might have to settle for people who were not quite as good or as competent as those a few years before, but there always seems to be enough people.

IX(97) People have come back.

VII(97) I would imagine that the Mainland will want to look at what goes on here and preserve it. Certainly as far as exams go, I remember seeing a paper last year at UST about an oral exam they've developed in the Mainland which is very similar to ours. It's obvious that they've looked at what we were doing and said, yes, that's interesting and worthwhile and they seem to have quite a lot of respect for what's going on in HK.

AU It's interesting to see that they are pushing the Expatriate English Teachers Scheme. Will the status of English teachers improve? Perhaps as English is seen no longer as the colonial language once the colonists have gone and it becomes the international language. Will the status be higher? And the morale of English teachers, I guess we can only judge that once we see what the situation and status is.

VIII(97) Well all teachers complain, not just English teachers because it's not an easy job.

IX(97) I think that most people think that they will have a harder job because they are now, students won't learn any English outside the English lesson whereas before in an integrated science lesson they would learn words like 'thermometer' or in geography they would learn geographical terms, whereas now it's up to the English teacher to teach them all of these.

AU It's going to make things more difficult presumably, they don't know what a thermometer is in English, or simple, everyday things perhaps.

IX(97) And the then the English teacher will have to teach them all those words, they cannot assume they have learned them from other subjects.

VII(97) That used to be how we taught in Oman, they would have their technical subjects and then they would come to, it was really like ESP I suppose, they would come to the English class and want to know what the word for thermometer was, in English, so it got very technical specific, but I can't see that happening here.

AU Perhaps to a certain extent it does already, but the English teacher will become the master of all knowledge in English. People will come to the English teacher saying, how do you say this in English? The local teachers will become translators as well as teachers. 
IX(97) One of the questions which was raised by this HKIEd group was will there be a translation paper? There used to be translation papers for High Level and CU Syllabus A a long time ago for the Chinese-medium schools, so they have a translation paper or section in the English language exam, so they are saying will you revive that because if schools go Chinese-medium will you bring that paper back, so I suppose the answer is no. 
Interviewee 10(97): University educator

Date:

\section{May 1997}

$\begin{array}{ll}\text { AU } & \text { Researcher } \\ 10(97) & \text { Interviewee } \\ \ldots & \text { Missing words / Unfinished utterance } \\ \ldots & \text { Pause }\end{array}$

AU Good afternoon.

10(97) Good afternoon.

AU The list I gave you, I think we'll just go through those questions and if anything else comes up we'll ...

10(97) Well, about the first one, about the ED decisions I think it creates a very difficult situation for the schools. I support of course the spirit of the whole thing the idea of Chinese medium instruction [and] all that sort of thing but using some sort of executive decisions is going to cause a lot of problems. I don't think the schools are against the idea of Chinese medium education, but they have a responsibility to the parents as well. And I would prefer something like in the schools for example, they have certain choices for example, certain subjects can be taught in Chinese, other subjects may be taught in English. Maybe in a number of years, the number of subjects being taught in Chinese could be increased, when the students actually see, they study in Chinese they get better results. That can be reflected in the end of year results, in CEE exam results, and the parents will start to buy the idea. But now they say well this school is English this is Chinese and that in English schools all is in English, other than of course Chinese language and Chinese History perhaps. I think that created a very difficult situation in schools. You have schools where starting this year maybe Geography or General Science may be taught in Chinese, where History, Western History for example, may stay in English. That kind of scheme may help rather than saying 30\% English, 70\% all in Chinese. All the parents, in fact I'm a parent myself, if you ask me, mother tongue education or education in English I will say yes, mother tongue education, but the question comes up, well, what about your boy? And I will think about my boy must be better than all the others and he must be competent in 2 languages. No one can say that my boy is not as good as the other, in terms of learning language, not about general intelligence, just the fact that can he study in 2 languages or just study in one language. Most will feel that my boy can do 2. Now his or her may not be able to do that, then well he should go to a Chinese medium school, but my boy can do that. Perhaps you have the time, the money or whatever I'll create 
a situation where in fact my son can in fact function in 2 languages. There are lot of examples in the world where people can function in 2 languages, nothing miraculous about the whole thing, if you can provide that kind of environment. If you can't, well then maybe yes, maybe those children should go to Chinese medium schools. That's very difficult, you have to convince the parents, especially ... people who themselves have gone through the system, they know that's very hard, but ... these are the people in the middle class who are willing to spend the money, and a little bit of their time on their kids. Maybe it puts a lot of pressure on the kids but when we are parents we have a lot of pressure. So I think if the Education Department does not allow a bit of flexibility for schools then the schools have a lot of trouble. I read a couple of days ago that a lot of schools do not want to change to Chinese and then you can expect a lot of schools will request to be English medium schools, and of course, some are not going to get it, and it just creates for a lot of schools frustration, well this year I didn't get it, maybe try again next year or the following year and maybe all these educational goals may be thrown overboard. The main thing is, you create the kind of results, academic exam results, that would qualify you to teach in English and we have seen this in the last 10 or 20 years, where a lot of schools have tried to become what is known as the elite schools, the former Catholic schools or whatever. Now we have some in the housing estates that have the aspirations to be one of the elite's, I have friends who have taught there and they just push the students very very hard to get that kind of results and every class is just preparation for examination. This gears the students towards ... I think that would create, in the end, a lot of problems.

AU But don't you think that effectively most of the secondary schools are Chinese medium now anyway and it's only putting the stigma of a name on them that they are worried about?

10(97) But this is the fact, they don't want to come out and say that.

AU And its creating, as you say, there's already a certain number of elite schools, by having an even smaller number of elite schools then they will become the elite schools of the future.

10(97) If I had the money, I would go into business and I would come up with my own private school. I mean, the international schools in some ways are like that. So now if you say only $30 \%$ then there you have a ready market. OK, I'm not willing to take the government subsidies, I run my own, I charge a lot, and already you may see a growth in these private tuition classes in English, so there is a market and someone will come in and exploit that situation.

AU So you think we will see a growth in English medium schools, private schools, international schools? 
10(97) It's possible yes, at least when they see that a lot of parents are asking for these schools, they couldn't get in for example, the government designated ones, then international school is one possibility and ... international schools now are filled with Chinese students, basically are Chinese students, not so international schools 20 years ago where they served a very clear function, now they have all changed. So in a way, it just forces the situation to go back 30 or 40 years ago where you have these so-called elite schools and then you have a whole bunch of whatever happens will be looked upon as somewhat secondary.

AU How about the actual situation within schools? A lot of teachers have complained that they are only able to teach in English medium, or at least in a mixed-code. They haven't been taught to teach in Chinese medium, I mean the subject teachers such as Science or Geography, History or Mathematics?

10(97) The thing is quite a number of years ago the government set up a fund or whatever to look into having Chinese textbooks, well, if you don't have good Chinese textbooks on Geography, Biology, General Science or whatever, then if the textbook is still in English, then what's the point of using Chinese as the medium of instruction? You have to come up with some good textbooks, and not just translations of English. You have to have textbooks written for students in Hong Kong, with that kind of background and written in Chinese. And the teachers have to be trained, retrained because most of them they learned a lot from English books, a lot of the terminologies, and the way of doing things is somehow very much influenced by the language we learn in. I did my MA in Linguistics. If you ask me now to stand up and give a lecture on linguistics in Chinese, I would have a big problem. It's not a question of what is this term called in English, it's not just one or two technical terms, that you can easily make the conversions, but the whole approach, the whole way of talking about it, the methodology, the concept would need to be, I think, retrained. That is not insurmountable, I think we can obviously do that, but then you still have this additional problem that you teach in Cantonese, the textbook will be in written Chinese, you still probably need to learn some of the vocabulary in English. I mean it can be done. It will take a number of years to get the translations. As I said at the very beginning, I'm very supportive of the idea of using the mother tongue to teach, but why not let the schools decide on using a more gradual approach. This year all the General Science will be in Chinese, next year other subjects will be taught in Chinese. Gradually, that would be easier I think for people to accept. I don't know why they don't reconsider this option. AT one point they considered an option like having 2 streams, that is like even more problematic, they have an English and a Chinese stream within the same school. Then you have real problems. But subject, why not subject by subject. Even I think in an English school, what about sports? Do they do sports in English or in Chinese? When I was in school the teacher used English. 
AU They used English? They don't anymore.

10(97) Well that was 30-odd years ago. That was an English school, so everything was in English.

AU To what extent do you think is the changeover itself, has that affected the language policy?

10(97) I don't think it will affect the schools now. Indeed I think it is an area they will leave the schools alone on this.

AU Do you think, well ... the medium of education policy they've been going since about ' 92 since the Education Commission Report No. 4 they've been mooting this, do you think they were looking long distance and looking at the changeover in sovereignty and we should be moving towards Chinesemedium education or ...

10(97) Maybe, maybe that was at the back of some people's mind but I think this idea of mother tongue education is something which cropped up much earlier, its not something which suddenly come up in the last five years. When I was a student, they already had talk of mother tongue education and that sort of thing so it happened for a long time. So maybe yes, this could be a political decision and say well, now is the right time to change. But I doubt that the Chinese government is particularly interested in this debate, they will be more interested in, for example, what to teach, not the language, English, Cantonese whatever, but the subject matter, the content, that will be much more important. What you have in the history books, is more important. I don't think that will be their first priority, they know they have to tolerate us. In China, a lot of people are learning English so why do you want in this case to come to Hong Kong and mess things up. This is the least of their worries. But I don't know, Hong Kong people sometimes try to guess what China wants. But it's been talked about for a long time, so sooner or later you can see that coming.

AU How do you feel as regards the introduction of benchmark language levels of language teachers?

10(97) I think that it helps people to look at this question seriously. I have friends who teach in secondary school right now, and they are History majors and Biology majors and somehow they are drafted into teaching English because their aren't enough English teachers and there aren't that many English majors going into English teaching. There are a lot of English lessons, from Form 1 to Form 7, everyone has to do English, so the school principal has to look for someone who seems to have a reasonable control of the language and they become English teachers. And some of them may become English panel. Some of them they do not like that, teaching History is much easier than 
teaching English, you have to do all the marking. Language teachers have a lot more work to do. When you have people who have that kind of attitude, they are not going to make English language teaching something interesting. I think that is one of the reasons why English language teaching is in such a bad state at the moment. All through the system, you are taking people in who are not that interested and doing a job which they find is much more difficult, requiring more work. Marking an English paper is much more difficult than marking a History paper. A, History paper just tick, you've got the point, all right good, with English, you've got to rewrite every sentence.

$\mathrm{AU} \quad$ So teachers have no control over it, they are told to do it and they have no choice. They can't say no.

10(97) So some people are very happy now you've got this benchmarking, they're deliberately going to fail it, they will not even qualify to teach English, so they can go back and teach their major subject and the school cannot force them to do it. They are not qualified, so how can they teach that? They will go back to what they like to do.

AU So there'll be even more of a shortage of English teachers.

10(97) So I think good English teachers will be in big demand.

AU But what's the incentive? What's the incentive to draw in English majors?

10(97) They have been talking about some form of incentive for people who let's say pass with a distinction or whatever do very well in their thesis or whatever assessment method they're going to use. But I think they have to think seriously about this now and I was told more schools are now going to get expat teachers, they will have less of a problem with the language and so on.

AU Well they are certainly advertising for them, expat teachers on the scheme that they've set up.

10(97) But if they are bringing expat teachers in, into a school in HK, they have to be very careful. For certain schools there's no problem, but for other schools it's a really big problem. A school doesn't want to have an expat teacher because the principal doesn't want to have the meetings in English. If you have one expat teacher, then you have to start conducting your staff meetings in English, and the school doesn't want that, and probably all the other teachers don't want that.

AU There are ways of getting round it, I had that problem. I was in a Band 1 school and most of the teachers understand English, but when they have staff meetings, which they don't very often to be honest, the headmistress will explain the content of the meeting to me beforehand and tell me what's going 
to go on, and then say well now you know you don't need to come. If you have any comments on this you can tell me later.

10(97) You have to handle this carefully, you may be offended by this as well, not just the others, it can create tension in the staffroom and so has to be carefully done. But I am optimistic. Once you isolate those teachers who are interested in teaching English, then I think you will have maybe a smaller, but a much better group of teachers and that will ultimately help the teaching of English in secondary schools. And also by removing that burden from the students, you may actually get students interested in learning English as a subject, rather than something they have to learn like they have to learn Biology and Chemistry which are the subjects they are interested in.

AU As a foreign language.

10(97) Like clearing the plate, in fact you might have a better change of improving and helping students to learn a language.

AU Do you think there will be any, or can you see any other significant changes in government policy towards English teaching?

ML No I don't think so, the main thing is teaching in Cantonese or teaching in Putonghua, sooner or later someone's going to raise that question.

AU Teaching in Putonghua?

10(97) Well, that is the language they use to teach in China.

AU Do they teach in Putonghua in Guandong?

10(97) I think yes, probably not in primary school but in secondary school, at least in the cities, in the villages no control ... but by the time they reach secondary school ... if not, what's the point of having Putonghua as the national language?

AU And then we'll have a whole new ball game.

10(97) Yes, because who can teach in Putonghua?

AU And teaching in a medium that the students do not use outside of school.

10(97) The situation may not be as serious as English but there'll have to be another transitional period. In English, they'll probably leave it at that, 70\%/30\% if they can force it through, but this question of Putonghua is going to come up sooner or later. Already people are talking about it - teaching in the mother 
tongue, that is in English all right? In Chinese, we say [ ] or [ ], so what do we mean by Chinese?

AU And then of course there's a different system of writing, there's the simplified system on the mainland.

10(97) Well at least China, mainland China has not been pushing that very hard, but you have to get used to that sooner or later if you want to know about what's happening in China. And later on when you are dealing with officials with a different style of writing, they won't understand what you are writing. So I think the students are going to have a lot of things that they will have to learn.

AU What changes do you envisage in the proportion of the timetable given over to English language teaching in both English and Chinese medium schools.

ML I don't really see this as a big issue, I think they will schedule the same amount of time, if not more, to English.

AU At the moment a quarter of the timetable is given to English, a quarter to Chinese, a quarter maths and the rest for the other subjects.

10(97) I can't see how they can change that, unless they can see that teach Biology in Chinese, they can cover the syllabus in less time but I don't think the Biology teachers are going to show that is the case.

AU Unless perhaps they bring in other subjects, perhaps in which they will learn more about the mainland.

10(97) Well, they have Chinese History and they will probably have Civic Studies ...

AU Government and Public Affairs ...

10(97) I don't see that as a real ... I think that most schools will try to keep the same, if not more time for English.

AU I mean, Chinese-medium schools, you would think they would want to have more English lessons.

10(97) Maybe, because remember, at the end of the road you still have all these eight universities saying that English is the medium of instruction. If you want to go overseas, of course English is the medium of instruction, so it will be necessary for them to keep the students' ability in English to a certain level, if not then any chance of further study will be closed to them.

AU And I suppose to appease parents as well. 
10(97) Yes of course.

AU They will say yes we are Chinese medium but we will have more English lessons to compensate. The next question is do you think there will be any other significant changes in your school or the education system more generally, which might affect the teaching of English?

10(97) I take a more long-term view on this. The general education system in Hong Kong, I think sooner or later, the question will be raised as to whether all universities have to be English medium. I think some people will raise that question sometime. Why not some having English as the medium of instruction and some having Chinese as the medium of instruction? All the universities in China teach in Chinese, so why do you have to have all the universities taught in English? You will definitely have students who will have a lot of problems struggling in English, if they have to make that transition only when they get into university, especially now with more and more students having opportunities to get into universities. Then you do have students who in terms of their knowledge of the content subjects, Biology or whatever, may not be as good, and if they have the additional problem of having to learn in English in university, their potential may not have a chance to actually really develop. Once again, I see it more in terms of disciplines, why do you want to force all the mathematicians to work in English? They have their own language, so what's the point?

AU I suppose it's all part of the internationalization of tertiary education.

10(97) Oh yes, I can understand why the universities want to keep that, they will have no problem trying to bring in famous scholars from overseas who can only lecture in English. Maybe you can do it with a handful of postgraduate students who will be able to understand English or whatever. Well, what about Japan for example, how good in English are the university students? If you have let's say an excellent translation system and obviously you are the top of the top and you want to read the latest journal in English, or go overseas to international conferences and give papers in English, but you're really talking about a very small quantity. I'm talking about a much larger group of people who are just getting a tertiary education, why should we force them? If some of the key papers can be translated into Chinese, they can read that in Chinese. They are not the ones who are chasing after the latest research data, they are not in that league, why do want to force them into that league when in fact only a very small percentage of people are ready to do that.

AU Where do they come from?

10(97) Well, there will always be people who are better, whatever you say, there will always be some people who are better. 
AU Why?

10(97) More motivated maybe, maybe nothing to do with intelligence, more motivated. I want to be like this. I want to go overseas and give papers at international conferences, so I'll study English. Here for example, we have quite a number of postgraduate students doing Ph.D. or M.Phil. Initially they're from China. Obviously they have studied English in China, some of them have a lot of grammar rules in their heads as well, but obviously they can't give a lecture in English, they can't give a paper in English. They may be able to write something, write a technical paper in English, but these people are extremely motivated, they put $\mathrm{n}$ a lot of effort while they are here to try to improve, because that is what they want, to be able to go overseas or write a paper on their own, rather than having this advisor to help them correct their English. To be able to go to international conferences and deliver a paper, not just deliver a paper but even answer questions on their field. That is what they want, that is their goal and if that is their goal, then I think they will have a lot of motivation. But we are not talking about the highly motivated, we are talking about the average students, average intelligence, average motivation, they want to have a tertiary education, they will be able to do that, they will have no problem doing that, but in English it becomes a hurdle. Why should we put a hurdle there? Now the presidents of the universities they will have this in mind, the lecturers they bring in from overseas or come back form overseas can probably only lecture in English or a mixture of English and Chinese. Because one university may say, well, we'll be the first Chinese medium university, of course they will still provide ... for students who want to improve their English or for the ... they go for the academic career, that's very well, they can make use of the resources available in the university to help them improve their English. They've got three years to do that, and if they are motivated, they will improve. But I don't know, will anyone dare to come out and say that.

AU Do you think that the changeover will affect teaching methods and approaches in English lessons secondary schools?

10(97) In fact I would like to ask you why you have that type of question, how will the changeover affect teaching methods? Are you saying that teaching methods and approaches will not be acceptable to the Chinese government and so because of the changeover and then some of the approaches will be discouraged?

AU Possibly, although I can't think of too many reasons why they should be changed. By approaches, I suppose the things we were talking about before. Perhaps not due to the changeover but due to the changes which are happening which are maybe coincidental or maybe not because of the changeover. Perhaps the changeover might bring a different mood within schools, a different political climate might bring a different mood within 
schools, different aspirations, perhaps teachers might have different ways of thinking, perhaps not expatriate teachers like myself but maybe local teachers.

10(97) Probably more in universities than in secondary schools. In a way, secondary school students are easier to control; it is the university students who could be the troublemakers.

AU Politically?

1(97) Or socially, whatever. These are the group, this group of people are more mature, who could be affected by certain ideas and I think if you look at it from a political point of view, that will be, if they have a ... it will be at this level. It will be not at the secondary school level.

AU I suppose from what we were saying before with the benchmarking will mean a lot of English teachers, local English teachers who don't particularly like teaching English, they never majored in it, perhaps the changeover might strengthen feelings of anti-English or something like that.

10(97) Obviously, we are talking about people in their twenties, thirties think about this, but people of my generation will always appreciate the fact that whatever we say about English, colonial power, that sort of thing, then it will just represent a way of opening up a horizon for us. It does allow us to know a lot of things if I were only Chinese speaking, I would never know, I would never be in contact with. But Hong Kong has become such a cosmopolitan city that I don't think that they are actually now having anarchistic feelings about English.

AU I suppose so, but there again very often simple incidents can affect the mood, I mean the June 4th incident affected the mood of everybody here, 'till it blew over, at least for a year or so. There's a great anti-Chinese, at least antiChinese government sentiment here. Perhaps something could happen to change things the opposite way, it might make people anti-British or antiEnglish feeling, which the Chinese government could use through the media to stir up something.

10(97) Obviously it's possible but I doubt that, not in Hong Kong, not in the next ten or twenty years. Really if you look at all the people in government or in power, they are all western-trained, obviously some of them may in fact look back at history and recognize the fact that they have been living under a colonialist system and a lot of injustice may have been done, maybe not to them personally but maybe to friends, other people but I don't think they will go to that extreme. In Hong Kong we still have a lot of ... and Hong Kong will stay as a financial centre. If your livelihood depends on trade, then no matter what feelings you may have inside you wouldn't express it. 
AU I suppose what we mentioned before that benchmarking could actually improve English teaching by limiting it to those who are actually trained in it, that could then affect the teaching methods or approaches. Whether it is actually a direct effect of the changeover is unclear.

10(97) I still say that the benchmarking will have a demoralizing effect on some teachers. In fact we do have some teachers who come out as English majors who are still not really competent to teach English as a subject, especially people who have come out in the last five or ten years, they have ... I think a lot of English majors who are potential English teachers have gone overseas, so-called the Arts students and so people going into English departments nowadays may not be the best students. Of course English is not always the most popular of subjects, it's Business which attracts all students nowadays. Science will have their own band of students. Some of the teachers for example, when we interview for positions in the last five years, some in fact already have qualifications in English, may even have an MA in TESL and you still see that they have problems expressing themselves in English in the letter they've written to us, so I think there are concerns for teachers, they may think they may not be able to pass the exam and when you have benchmarking, it means that some are going to fail. If no one fails then it's a total farce.

AU Then it's a case of how high you set the standard.

10(97) Yes, how high you set the standard. But however low you are going to set it, you are still going to have a certain proportion failing, if not then why all the fuss? So some would be, a lot of pressure would be put on them to perform. So even though you say $\mathrm{OK}$ you fail so you are given more training but who would like to be sort of labeled as someone who couldn't pass the benchmark the first time, and had to do it at a second attempt.

AU Perhaps, the trainee teachers, when do they take the benchmark? Perhaps when they finish their training they take the benchmark and then they fail? It like, well they can't teach English but they can teach another subject, it's like their whole course has been a waste of time.

10(97) Yes, probably they should not be tested at the end of their course, but I understand the benchmarking will not just be a language test, they have classroom observation. So the students from the Education Department or whatever should be given a chance to actually function in a school environment. But whenever you set up benchmarking, when they test you after a number of years, that is the scary part.

AU So finally, do you think the changeover will affect the situation, status or morale of teachers of English in Hong Kong? 
10(97) I don't see any major effect in Hong Kong on these, on the status or situation. Morale and the changeover, I don't see that affecting it.

AU I suppose from my point of view, for expatriates, the chances for promotion might be blocked, with localisation. Positions of authority will not be given to expatriates.

10(97) Yes, in those terms. I think it depend whether the expat community will show the same kind of commitment as a local person can show. It will depend very much on the particular school, I suppose. No doubt about that, localisation is going to stay. It does mean less opportunities for expats for senior positions. There is a problem of communication with expats among a lot of local Chinese so very often they just take the easy way out and say well. Better not.

AU I know my headmistress has told me, I mean it's in my contract, promotion prospects nil - we don't expect expatriates to stay. I've been here eight years, how long do you want me to stay?

10(97) I know. I mean it's probably unfair, that's why they have to show the same amount of commitment or even more. You may stay while a local Chinese may emigrate. But I think the general perception might be that you are going to leave because you do have connections overseas, you may still have certain ties and responsibilities overseas and when something turns up you may have to go back. A local, you still have the perception that they have local ties and so on, it may be less easy for them to move. But the last ten years may have changed that. Most of my friends are overseas now; I have more friends overseas than in Hong Kong. Most of my university classmates are overseas, all over the world. So, the situation a little bit different now. If you ask someone in the street do you have a friend or relative living overseas? They will say yes.

AU Well, that's about it, thank you. 


\section{Interviewee 11(97): University educator \\ Date: \\ 30 May 1997}

$\begin{array}{ll}\text { AU } & \text { Researcher } \\ 11(97) & \text { Interviewee } \\ \ldots & \text { Missing words / Unfinished utterance } \\ - & \text { Pause }\end{array}$

AU Good afternoon.

11(97) Right OK, what do we do? Do we work our way through the questions?

AU Yes, I guess so and see if anything else comes up.

11(97) Yes, this decision to force schools relates to the firm guidance that has recently been issued, OK. Now, I mean all they are doing is now starting to require schools to come into line with the policy which dates back as far as '84. In 1984, Education Commission Report No. 1, they said $20 \%$ of schools. They revised that upwards to $30 \%$ can be English-medium in ECR4, and now I don't know that they have put any actual percentage on anything and what they are saying, if I've got it right, is that all schools will be regarded as Chinese-medium unless they can demonstrate that they can be effectively English-medium. So there's no actual percentage on that, they've certainly said what they think might be an estimate, but in theory, at least, if $50 \%$ of the schools could demonstrate effective English-medium, there's no reason within the guidance why $50 \%$ shouldn't be English-medium. But what is clear is that since 1990 when Education Commission Policy on Language was made, nothing has happened in the schools to implement English-medium effectively and all the evidence is that the situation is getting worse, especially in the lower band schools, but arguably in the higher band schools as well. So I really think if they are forcing them to do anything, they are forcing them to do what they say they are doing, they are not forcing them to adopt a new policy, they are simply asking them this is the policy, come into line or move to Chinese-medium. I just don't see any alternative quite honestly, I think the education system has been putting itself in dire jeopardy by letting schools do whatever they felt like doing basically, with the medium of instruction for twenty-odd years or more and I think they are now beginning to realise that this is having very serious effects on English, on Chinese and on subject learning, content learning. We'd always done very well on comparisons, international comparisons in maths and sciences, until the last one. Suddenly we went woof? Down the list and an analysis of the results shows that it is anything that involves extended language use which is really hitting our students. They can't do it. They can do the multiple-choice answers, yeah. So I think enough evidence has now come in from enough 
places for the iron to enter the soul. The interesting thing is, let's see we've got ... erm ... yes it's in your heading there, the effects of the changeover in sovereignty, because I don't think it has anything to do with the changeover in sovereignty. Ironically, it might just be the case that the HKSAR Government is in a better position to enforce language policy than the colonial government was. So one might be able to argue that therefore this has some kind of relationship to the changeover, but when people have said to me and they often have, is this because of the changeover I have to say no, this policy goes back to ' 84 and before that. And its most recent form precisely to 1990 and the date for implementation of September 1998 was laid down back in 1990, it's nothing to do with the changeover.

AU In fact the way the news of the firm guidance was put out in the newspapers made it appear as though it was out of the blue, it was reported on in the past but they wouldn't have known.

11(97) Yes, language is in the newspapers all the time, it's not often ... it's become a slightly more informed discussion over the years, but still it's the tendency to say it's the English teachers' fault; if only we could get some decent English teachers into our schools we could solve all our problems, without sort of getting very much beyond that, and even ECR6 has slipped back, because whereas ECR4, the only report to do so said medium of instruction is a problem, ECR6 said yes yes medium of instruction is a problem, let's look at language teachers. They talk all about English teachers and Chinese teachers and they don't say anything about the medium of instruction. They are setting up benchmarks for English teachers and Chinese teachers to meet in terms of proficiency, but they say oh sometime we'll get around to the content teachers, but it's the content teachers who really have the problem ... so right through to ECR6 I thought this one would slide away just like all the others and slip under the carpet, so it is interesting to speculate that it is more possible for an SAR government to take a firm line than it was for the colonial government. I don't really know why that should be, but maybe there's some sense of Chinese people ruling $\mathrm{HK}$ or $\mathrm{HK}$ people ruling $\mathrm{HK}$ which makes that more possible, because they have got it in the neck from the parents they will get it in the neck from the parents, the pressure will be right on the schools to continue to pretend to be English-medium and it'll be even tougher now because the chances of getting a little bit of extra kudos from pretending to be English-medium will really pay off, because if the other are switching to Chinese and you stick to English, you'll get the better intake, no question about it. So there's going to be a big shakedown period and we are still in the consultation document stage at the moment. We have had consultation documents before, right back to the sixties in fact, saying that we must get Chinese-medium education in $\mathrm{HK}$ and actually back to the thirties, it goes back all the way through, not just HK colonial history, always the seesaw between English and the vernacular language whatever it was with virtues seen on both sides. But it will be interesting to see whether the muscle 
has come into the issue or not, or whether finally the politicians will just back off on this as they always have in the past.

AU At the moment there's an elite system, there is a certain division within schools where some schools are classed as elite schools, my school is one of them, and having a definite division between English-medium and Chinesemedium is just going to exacerbate that.

11(97) Yes, that's one of the arguments, that this is elitism, then elitism is well and truly fixed in the system. Everybody has a rank order, every parent has a rank order in their mind, all the way through the secondary schools and whereas it has been covert, it will become overt and that's the difference. The problem then is to make sense out of the Chinese-medium education so that talented late-developers, because their won't be many Band 1 students going into Chinese-medium schools, but their will be late-developers and there will be bright kids and kids who will benefit far more from Chinese-medium than many of the kids who manage to squeeze into English-medium without being committed etc. So there's got to be a fast track for them through to Englishmedium tertiary education. It won't solve the problem to offer them Chinesemedium tertiary education because it will be regarded as second class, it will even be regarded as second class in China because what use is a Cantonesemedium institution degree? Absolutely nothing anywhere actually except arguably HK. In HK it will be seen as second class to English and in China second class to Putonghua. So, that isn't the solution. The only solution is to get the secondary education sorted out and really provide the support for those students when they go across to English-medium tertiary and prove they can do it. The trouble is that the time-lag is a long one, they've got seven years before they get into tertiary education, another two or three years or more before parents finally start to see hey, maybe there is something in this. So you're talking ten years before you can really say to parents, cross your heart supposing you can, we've got it in place, they really are doing well out of the Chinese-medium schools. But I don't think so, the ED speaks with forked tongue on this, it says one minute, yes yes it's just as good to be going through the Chinese-medium system, the next minute they're saying it's really only for the weaker students, so they haven't really got it right. The tertiary institutions are not making any preparation to distinguish between those who come from CMI or EMI schools, and they won't because they know it's a problem that they won't have to deal with, but they will. HKU hopes it won't because we will continue to have a higher entry admission level for English than the other institutions and we hope we won't be having to deal with this problem, thank you.

AU Do you think it might lead to four-year degree courses?

11(97) No, because that's been explicitly ruled out by government. They said this was just empire building by the tertiary institutions and they were just not 
having it, that was that. Because they decided to extend Chinese-medium secondary education to seven years, at that point it really because impossible. I mean with hindsight, you then have students going into four years of tertiary education after thirteen years in school, there's no system that does that. So you either shorten your secondary and lengthen your tertiary, or you lengthen your secondary and shorten your tertiary, which is the British system, that's been inherited here. But with hindsight what they could have done is six years to A Levels for Chinese-medium students, who will then get a year to pull up their English if they qualify for tertiary education; seven years for English-medium students on the basis that they're working through the second language and they both go into tertiary education at the same time then. But the Chinese-medium students could work faster, the good ones, they could have a two-speed system in the Chinese-medium schools - fast track and slow track - fast track for the ones who finish in six years and then get an extra year. At one time I was arguing that the solution was to impose on the tertiary institutions there'll be a sliding scale for the admissions, next year it'll be $20 \% \mathrm{CMI}$, the next year $30 \%$, the next year 40 , it will go up to 70 , and watch the schools switch over to CMI under those circumstances. It doesn't solve our problem of maintaining English as the MOI at tertiary level, which is quite serious now.

AU It has been suggested that within the Chinese-medium schools then do it stepby-step, have certain subjects which are Chinese-medium rather than change over straight away.

11(97) This gradualist sort-of approach is frequently talked about, there's no evidence from anywhere and certainly no theoretical position which says that this has any foundation whatsoever. The problem comes at the beginning of the switching of the MOI. You've got to get them through that fast and the only way you do that is through really intensive work with them on their language and get them moving. Having one subject in the new language, it'11 certainly be better than having no subject, but as a way of getting them right into using the medium effectively, just no way. Most of the literature and most of the research has said that the best way is to start flat out $100 \%$ in the target language, I don't think it will do that in HK, this is just from the experience elsewhere and the theory, go flat out $100 \%$ in the target language from the beginning of primary school, and you're certainly not going to do that here, and then by the end of secondary you're starting to bring in the first language, just starting to, and then by the end of secondary they'll be doing most of their studies in their first language, but then they go into university in their first language, not their second language. But you break the problem of the second language fast and early, that's the theory, this kind of add a little bit, then a little bit, then a little bit more, it actually isn't the way language learning seems to operate, you get to a certain point and then you have really got to be immersed in it like immersion education. You've got to really take a bath and then it comes, and it can come in one or two years, very fast 
actually. But the notion that a three month bridge programme, very inadequately implemented, is going to do the job, is pathetic. And the idea, well let's use lots of Cantonese in the first year and a bit less in the second year and a bit less in the third year just doesn't happen. Once you're locked into an expectation in the students that they're going to be hearing Cantonese all the time, they are not going to give up on that. What you find is actually more, as you go up though the school, more mixed-code anyway, Cantonese doesn't demoniac, but the amount of English terminology increases, so that is what you get as you go on, a lot more English. So all the arguments, that particular argument, that you start off with them when they are weak, as much as you can, then reduce the amount of Cantonese, it just doesn't happen. There is no evidence for that and massive evidence that once you've started doing that, you cannot back off from it, you have to keep on doing it. Then on particular subjects, I mean here they're actually doing this in schools, they are not supposed to, but there are schools that are switching students from Chinese-medium to English-medium in some subjects, they are taking some subjects in Chinese in Forms 1 or 2 or 3 and then they bang them into English, it cannot work. The curriculum is more advanced by that stage, it's more abstract, the proficiency gap is greater, but they've got to jump, and they're now within shouting range of their examination in English. They are absolutely crippling those kids, it's disastrous. So I'm afraid what schools do is largely what is convenient for the school and what is convenient for the teachers and suits the purposes of the parents without thinking about it too much. I've got more sympathy with the parents, it's a rat race out there, English is certainly a factor in staying ahead in the rat race, and while they may realise their kid is not getting a good deal, if their kid drops out, he drops out of the rat race, and where is the percentage in that? So I don't think many of them are fooled much by what's going on in the schools but they still think they are in there with a bet and might as well bet on your horse as refuse to bet or pull him out of the race, it's no good, because they really see it as only one race and the kids see it one way, and another good thing about the firm guidance is that there will now be massive numbers of kids in CMI and they can't all just give up hope, which is what they are doing at the moment, with a very few exceptions to that I guess. But I mean the ED, and I've been involved in this research too, we've been looking at the comparative performance of kids continuing to study in Chinese and who've switched into English and it's very hard to get legitimate comparisons because the kids who have switched into English include all the higher ability students and the kids who haven't switched into English include all the weakest, least academically motivated students. But what you would still expect to see is anytime you can get a comparative proof, the Chinese-medium ones should be miles ahead, because these have switched MOI, everything should be grinding to a halt as they get their MOI sorted out, and so of course they should be way behind. Is there any evidence of this? No, because the kids who are really continuing in Chinese have given up. They gave up way back in primary school actually, they haven't just given up. They didn't learn much in primary school and it's 
clear they are not going to learn much in secondary school either. Where does that get us to?

AU Well, the benchmarking issue is next.

11(97) Yeah, well OK I've said fine, by all means let's do that, but why for language teachers? Why aren't they doing it for content teachers as well? Why didn't they do it years and years ago? The teacher training institutions are guilty, I mean we should have I guess the best of them, but we know we are certifying people who can't teach through English, we know that and in the days when there wasn't a language policy, maybe we could have cheerfully said, well there you are do the best you can, but once there was a language policy it has been really quite criminal I think for HKU and the Chinese U to simply ignore the issue. HKIE has to some extent taken it on board, but it is actually in the weakest position to do that, and to what extent what they're doing is tokenism and to what extent it's real, but at least they've acknowledged that the situation does exist.

AU There are certainly a great number of English teachers as you were saying before who are not legitimate English teachers.

11(97) They are certainly not trained English teachers and there are several reasons for that the kind of person who becomes an English teacher is often literature oriented, there's no schools really teaching literature any more in $\mathrm{HK}$, so that side of it goes out. So they have to be majors in something other than language, because they can't be language majors at school, so there are no real English language programmes in the tertiary institutions. To support large numbers of graduate majors, then if you have got people who are very interested in language and get their language very good, they are very much sought after out there in the market place anyway. So why would they come into teaching? They come into teaching when the market is tightening up and it's harder to get jobs, but they flow out again pretty fast when the market opens up again and there's demand for them. So, those are some of the reasons anyway why it's hard to get initially and hard to keep people who've got an academic background in language. We teach methods courses in our PGCE, where we admit people who are teaching English even though they have no English language background, whereas Maths Methods would not admit people who haven't got a maths background. Geography wouldn't allow somebody to come in and be a geography teacher who hadn't done a substantial amount of geography at undergraduate level. But in English, they're out there, they're in the schools, we've got to do something.

AU I think a lot of people who are teaching English, perhaps their major was in geography or history who are actually history teachers ... 
11(97) Yes, anything. Sometimes they've been overseas, the principal says, you've been overseas, your English must be good, you went to HKU, your English must be good, surprise surprise, but anyway, and then, you teach English.

AU Isn't there a possibility that giving the benchmarking to these people who perhaps don't like teaching English, they don't want to teach English, they will deliberately fail it?

11(97) I think if they are really feeling that badly about it they could move to another school, but maybe they are in a government school so they get better conditions, but that same argument would then lead to them pushing themselves a bit to make sure they pass it. I think that this benchmarking can be done very positively, where teachers are given a reasonable period of time to and support in coursework and things of this kind, to get them up to the benchmark standard. If it's done that way then it's going to be fine. If it's simply a big stick for whacking people who are inadequate with, and threatening them, then it's not going to work very well at all. But it should be done and there's no reason it can't be done in a positive way, certainly there is a kind of evaluative point to it, but if it is simply we all recognize this as a problem, we all want to get English teachers' English up as high as possible, Chinese as well, it applies to Putonghua, and content teachers to. To a large extent we are saying you must improve your English you must improve your English and doing nothing to help them, which is ridiculous. We get assignments in, I've just been doing my marking, and you think, dear God is this person going to be teaching English? Do I go through and correct it; do I get the student in and take him carefully through? No, I don't. I haven't got the time and if I'm feeling particularly cynical I'll write on the bottom, 'You must work hard on improving your English'. That must amuse them enormously. They know their English is no good, what are they supposed to do?

AU It's very difficult. We've had student English teachers, I had one this term who took my Form 2 class, she was very good, very enthusiastic, lots of good ideas.

11(97) Where was she from?

AU The Institute of Education, but her actual spoken English didn't seem too bad, but when I looked at the compositions she'd marked, she'd missed loads and loads of mistakes, even some of her corrections were wrong. It's worrying.

11(97) It is worrying. Certainly it would be ridiculous to say that they should be equivalent to a native speaker, that isn't required, but where exactly you draw a line and say, hey, come on! This is going too far. 
AU OK, do you think there'll be any other significant changes in government policy towards the teaching of English after the changeover?

11(97) I wouldn't think so. I think as far as education is concerned, apart form a few history texts which seem to have come up as an issue, the Basic Law is absolutely clear that all policy decisions are the business of the SAR Government and nobody else's, and I don't really think there is any reason why the changeover, the One Country Two Systems, why the changeover should have any impact at all. I should imagine it will have an impact on Putonghua in that it already is having, very very late, much later than it should have been, people beginning to have the idea that Putonghua need to be taught in schools a little bit seriously, which was a joke before. So, I would imagine some strengthening there, I would think they are going to have to look at simplified characters somewhere in the system and they may need to get a severe grip on the kind of Chinese that people write in schools. In terms of English, I don't really see it, I don't see where the momentum or the impetuous for any kind of change would come from. My best guess was, this used to be, that absolutely nothing would change and we would be so terrified of any appearance of interference in the system that absolutely nothing would change and that would be a disaster, because things definitely need to change. The evidence of the firm guidance is that they are still prepared to push things along in directions they think it needs to go in. I cannot see at the moment, in relation to language education, there is anything coming from the changeover. Give it another ten years, fifteen years and it may be Cantonese that's being threatened as the medium of instruction and Putonghua will be the move, that will be where the pressure is coming from. I don't see it threatening English. The push for English in China is just so strong that why on earth would they be pushing against it here? Who would benefit from it, there's this sort of who stands to gain? There is always a good way of sussing out what might happen and I can't see who would gain from sacrificing English in the expense of Cantonese. Cantonese is going to be a problem for HK. In HKU, about 1982, I'd been here just a little while, there was a lot of pressure for a change to Chinese-medium, eliminate the requirement for English to get into the university. Well, then it became very clear who stood to gain, local academics benefited from this being a bilingual university in the way that Chinese $U$ was, you could use any language you wanted it was OK, let it all hang out, but it meant that it would become more and more difficult to employ expatriates under those circumstances. And then as soon as the agreement for 1997 was signed, that pressure just disappeared because it was English or Cantonese command, we're going back into China, it got to be English and Putonghua. And if you are pushing for Chinese, you are then competing with people from China and really not justifying the whole difference in salaries, in research facilities and all the rest of it, which you can justify it with English as an international medium of instruction. So, the pressure just disappeared. I cannot see a group that would benefit from pressure on English in HK and I can see the groups who will scream and 
scream to defend it and they're the tertiary institutes and big business and they carry very big sticks. And it isn't just the parents where the pressure will come from against the firm guidance either. Quite mistakenly there will be a lot of pressure from big business as well, saying this is a threat to English standards without realising that that they can't get much worse than they already are at the moment, they would still think, oh dear, maybe we are going to get even worse. It's a comfort to them to know that things could not actually get much worse but they couldn't.

AU The changes in the proportion of the timetable given over to English language teaching in Chinese-medium schools?

11(97) They're both very high, I think it's about a quarter of the periods is it in your school? It's higher than it should be, it's higher than it needs to be in the English-medium schools if they were English-medium they wouldn't need that number of English lessons. They could make do with five or six a week maximum and really diversify what they teaching in those lessons, if the other teachers were really teaching through the medium of English. Chinesemedium schools? Well I think they will probably keep their number of periods up simply because they don't want to appear to be neglecting English. They want to be able to maintain the standard of English that they produce is better than, well it used to be, it can't be, not if English is done properly in the EMI schools, but the Chinese-medium schools will want to say that they are paying a lot of attention to English and the credibility of their students moving into tertiary will depend on a very high level, so I doubt if they'll reduce the number of English periods they have. I don't suppose the EMI schools will be intelligent enough or smart enough to work out that as they implement English effectively, assuming they do, they really can afford to back off on the number of periods they are using for English teaching and give those lessons to supporting the content subjects which have to move more slowly as a result of the English medium. So I think we'll probably stay exactly as we are actually.

AU The next question, maybe it doesn't apply to you, it's any other significant changes in your school or the education system more generally.

11(97) I think in terms of our faculty, we are going to have to do something about the medium of instruction. We've always assumed that we are training English-medium teachers. Now we can go on assuming that and cater for what might well be a very small market, in which case we might have to contemplate having smaller numbers of students, which means having smaller numbers of staff, which means cut-backs, nobody likes that. Or, we're going to have to start thinking about training for Chinese-medium schools, which I think most of our methods teachers would be very happy to do. But either way, we are going to have to face up to the MOI issue that we never have done in the past. If you simply train teachers and assume that they 
can go and do it in Chinese or in English or in any mixture of the two, and they don't need different skills for teaching through different languages when quite clearly they do.

AU Presumably then that would affect all the teacher education departments.

11(97) I think it will affect us more because we don't train primary teachers. In CU it has definitely always been maintained as a sort of bilingual and therefore user-friendly to Chinese-medium, teachers could more easily go from their programmes to Chinese-medium I think than they could from ours. So, we have always assumed that our teachers could teach in English-medium schools, would teach in English-medium schools and now I think we will have to come to some conclusion on that and then having done that, we will have to have a look at our programmes and quite radically change those to cater for two different kinds of teacher.

AU Have different streams?

11(97) Yeah, which then becomes inefficient from the point of view of costeffectiveness, with a lot of questions then about, well, do you say that your Chinese teachers that you are preparing for Chinese-medium should still be able to read the books in English etc., and attend the lectures in English and the discussions and seminars, otherwise they're missing out on all sorts of international aspects of education, in which case, that takes you one way, which is you save a lot of time and effort on that and just their methods courses split. But there are certainly a lot of things that people will have to think through.

AU I mean, if people are trained to teach English in a Chinese-medium school then will that block their opportunities to move into an English-medium school?

11(97) I don't think it would, I mean ideally there should be a different role, from English-medium schools where the English department has a kind of supportive role for English across the curriculum, whereas in a Chinesemedium school they can actually have more fun, they can do anything it doesn't matter, there is no concept associated with English in that school. So they don't really have to play that kind of academic English support role, but in practical terms I wouldn't think English teaching is the problem or Chinese language teaching, rather, it would be the science teachers, maths teachers and so on, and social science teachers, and one group will be looking to exploit the language that the students are strong in, the other group is supposed to be looking at ways in which you help them, support them, develop the language in which they are weak. And this does imply two totally different kinds of strategy I think, and again we have never actually acknowledged this. 
AU It's going to be interesting. Do you think the changeover will affect teaching approaches or methods in English lessons?

11(97) I don't think so, no. I don't think so. I mean again ideally it should. Not the changeover, but if the firm guidance comes in, in that, students in Englishmedium schools ought to be way ahead of the students in Chinese-medium schools, so they ought to have different targets set for them in the two different schools, they should have different examinations. It would be criminal if they go on putting poor Chinese-medium students into the Use of English examination against the English-medium students, so the Englishmedium students can be guaranteed all the As, Bs and Cs and the Chinesemedium will get all the Ds, Es and Fs. There should be very little overlap there, there should be very little, if the English-medium schools are doing their job only half well, there should be no overlap actually.

AU They would have to create two exams?

11(97) I think they would have to, and then they would have to accept that the Chinese-medium students have got to have something that gets them over the gap before they go into the tertiary institutions. But they don't want to do that because that would penalise the CMI students by putting them a year further back, if they did it half reasonably. Or it would be enormously costly if they were supported on a kind of individual basis once they got into tertiary institutions, I don't know. I think there are a lot of problems that simply haven't been addressed. Firm guidance for example, really is saying, we must do it right, but nobody has revised the curriculum. It is still assumed that the English-medium schools and the Chinese-medium schools cover exactly the same curriculum at exactly the same pace from the beginning of Form 1. You can't change MOI and do that. So if schools are to follow the firm guidance they must cut the amount of content to be covered in that first year at least, probably the first two and even three years have to be restructured on that basis. There's no evidence that anybody's actually doing that, or even knows that it's really necessary. Even the bridge programme was interesting because originally that was supposed to be a recoding of the primary curriculum; this is what they've learnt in Chinese, this is what they don't know in English, but it's familiar so they can recode it pretty quickly. Let's have a programme which tries to recode what they already know from primary education before they start the secondary curriculum. Soon as this was put to schools, the principals said, this is old stuff, they've already done that, we want them to do the secondary curriculum. So the publishers in the end caved-in and did a simplified version, a cut-down simplified version of the first term secondary syllabus, which actually achieved damn all.

AU They don't use it at our school, they got some poor soul to write the course themself ... 
11(97) No, it was never actually going to work that well because what was needed was a restructuring of the curriculum. As long as they had the whole curriculum lined up there waiting for the bridge programme to finish and then you had the transition, not just from primary to secondary but from the end of the bridge programme into another textbook and then you had to catch up. So even if the teachers used more English during the bridge programme, supposing they tried, they've then got to spend the rest of the year trying to catch up with the rest of the curriculum so they can use more Cantonese to try and get it through, so there were a lot of problems, a lot more than we first thought when we were think about it. It's very much my baby, the bridge programme so that was a bit of a disaster.

AU At least it set people actually doing it.

11(97) It got people thinking about the issues that perhaps they hadn't before, yes.

AU How do you think the changeover, I keep saying the changeover because that's my focus, or at least the future will affect the situation, status or morale of English teachers in HK?

11(97) I wouldn't think it will affect it negatively at all. Again, if we are looking for people whose morale is likely to be under siege, it will be the Chinese teachers because a lot of written Cantonese now is into the writing of $\mathrm{HK}$ and it is not acceptable outside HK, and much sneered at by the Chinese in Taiwan and China. And at the moment HK has been splendidly isolated and arrogant and what we do is fine by us. It's the teachers of Chinese, across the curriculum too, accepting what is the standard for Chinese in $\mathrm{HK}$ is going to be a lot tougher in many ways than determining what the standard is for English. That is an issue that nobody is touching, but I don't think anybody is going to feel unpatriotic because they are teaching English. They might have done if the changeover had come 30 years ago, or 20 years ago, but not now, I mean the notion that English is sort of the colonial language is not something most HK people regard very seriously, hell, it's the language of America and Canada and Australia, that rather more important than whether its the language of the colonial government.

AU I think you're right here, that certainly once the changeover has gone, then even more so it will be English is just the international language and not necessarily the language of the colonial power.

11(97) I think it already is, because there is no English cultural content, for example, no English Literature taught in the schools, none that I know of anyway.

AU I teach some. 
11(97) Do you? It must be a girls' school. You might have one form throughout the school.

AU We teach it throughout the school, from Form 1.

11(97) Do you? What do you mean by Literature then?

AU We teach them poetry and appreciation.

11(97) But is this Literature with a capital $\mathrm{L}$ or is it modern stuff?

AU It's Literature with a capital $\mathrm{L}$, we teach them traditional stuff.

11(97) You know that the Exams Authority has for a long time said that it's just going to cancel English Literature exams altogether. Schools wanting to put students into English Literature can sit them with the English, British examination boards because the numbers are way down, below 500 now and it's just not economical to run the examinations. So there are just a very few schools that do it. But this is part of the thing. Look at the textbooks, what is the cultural content? It's trips to Ocean Park and things like this, it's not London Bobbies and Big Ben, and you cannot sell British-oriented textbooks in HK, nobody wants to know. The colonial thing has gone long ago I think. You also can't get American materials or any other materials, people are very much into what $\mathrm{HK}$ is all about, that and learning English in order to enter this group in society in $\mathrm{HK}$, that uses English and is big wheelers and dealers. Some of them want to emigrate, but they're gone anyway.

AU Well, that's just-about it, thank you. 
Interviewee 12(97): University educator

Date:

4 Jun 1997

$\begin{array}{ll}\text { AU } & \text { Researcher } \\ 12(97) & \text { Interviewee } \\ \ldots & \text { Missing words / Unfinished utterance } \\ \ldots & \text { Pause }\end{array}$

$\mathrm{AU} \quad \mathrm{OK}$, what are your feelings as regards the ED's decision to force schools to adopt Chinese-medium education?

12(97) I think it's realistic to be teaching in Chinese and I think parents don't understand the situation fully. Parents are voting with their feet. Parents are saying, I want English. ED is saying that's probably not the best way to go and I brought with me an article which I wanted to ask you ... are you familiar with Joe Boyle's article? And if you don't have a copy I'll give you this copy, because Joe has said a lot of what I believe. He's talking specifically about mixed-code and outlining some of the ED's decisions and policies, the Education [Commission] Reports and talking about how they seem quite inflexible in the things they are recommending to schools, but from the teacher's point of view it's just not realistic. I feel that I'm very much of the same opinion that Joe expresses in this article. He seems to have a good sense of what teachers are dealing with. I know what I'm dealing with here at the university level and I know that my situation as a native speaker of English with very inadequate Cantonese, puts me in a different classroom position that say even my HK colleagues here and definitely HK colleagues in secondary schools, where students can 'take advantage' as it were of the teachers sharing the first language with them, right? And some of my students will say, well gee, I know when I'm speaking to you, Mrs. Fu that I have to use English, and I have to work out strategies if I don't know this word I have to figure out another way to say it, to repeat myself or to do it a different way, right? And I think about how would I be in my classroom if I were a fluent speaker of Cantonese? And my students could take advantage of falling into Cantonese when they couldn't think of an English way of saying things, right? So I think Joe's being very realistic. He's talking about code-switching there and he also talks about his view, and I share the view, that maybe the ED is speaking, in terms of policy, a little inflexibly and that a teacher in the classroom just need a little more flexibility than that. Actually, I have a question for you because I believe you're focusing on the English language classroom, is that right? Some of the questions lead a little bit on media of instruction, your primary interest is the teaching of English, is that right?

AU Yes 
12(97) So, those are my thoughts on that one.

AU It's not a new decision; it's something they've been building on for the last 10 or more years.

12(97) It almost feels like an acknowledgment of reality.

AU In most English-medium schools, the teachers use mostly Cantonese anyway.

12(97) Yes, and my students tell me about English language classrooms, where most of it is in Cantonese.

AU I can believe it, my school is supposedly English-medium and one of the socalled elite schools and yet most subjects are taught in Cantonese, perhaps $60 \%$.

12(97) And part of me says, why not, you know?

AU But then are you cheating parents who believe that they are sending their kids for an English-medium education?

12(97) My own feeling is that parents want their children to have a strong command of English. They may not understand what the best way is of getting that strong command and Chinese-medium of education is not necessarily counter to a strong command of English as you and I well know, but parents don't well know. So if that could somehow be demonstrated, that a strong English language program can produce the English language results without having the medium of instruction. I heard things on the radio about taking advantage of tertiary education, their concern about forcing schools to go Chinesemedium, they are concerned that their child would be disadvantaged at university, where they, the parents, thought much of the education went on in English. I wanted to say to that parent, over the radio, that's just not true, here at our university, yes we are Chinese University, but much of the teaching goes on in Cantonese and again, why not? I think it's perfectly realistic so I think if anything, one of the tasks perhaps is good PR approach for parents, educating the parents. I've been in HK for 30 years but as an American citizen wasn't able to go to China until the end of the '70s and in those early visits to a number of places in China, I was often struck rather powerfully by the strength of the English I was meeting in China.

AU Even back then?

12(97) Even back then. We were meeting colleagues in universities, or what they were called then was Foreign Language Institutes. I think most of them are now called International Studies Universities or something like that. So granted that we might have been meeting the best that China had to offer, but 
then hearing their language classroom background and I'm thinking, and it worked! And they were all saying to me, oh everyone in HK must speak such wonderful English, and I would point to them and say, actually, you're better. Of course they would not wish to accept the compliment, but MOI and strong English are not necessarily like that.

AU Next point is benchmarking language levels for language teachers which they've said they are going to introduce this year or next year. Do you think that's a step in the right direction?

12(97) Is it fair to say a student can only be as good as the teacher? So it's important for the teacher to be better than the students in order to raise the students' level of language use. So it's important to have good teachers in order to have better improvement in students. So good teachers and teachers who have a good competence in English, we are talking about teaching English, right? A good command of English as their second language, so the question is, is the benchmark a way to do it? In other words, we need people in the classrooms with strong English. Is benchmark the way to do it? We know that this society is an exam-driven society and people seem to rise to the occasion, if they have to do it, they do do it.

AU So it's a question of how they will do it. Will they respond to market forces? If they set it too high then they are not going to have enough teachers?

12(97) Well, since your title is the effects of the changeover in sovereignty, let me just throw this piece in. Following on from what I've just been saying about having met people in China, as I say, that was my first impression, a long time ago, but you have probably met other such people and I have met other such people since the '70s from China with really really strong English, accurate English, good pronunciation, good fluency, question - will these people be available to our system?

AU I see no reason why not.

12(97) In which case, a concern about not having enough teachers may not be a problem. We have all the workforces in China available.

AU Perhaps if they want to come here because they could earn more ...

12(97) A couple of years ago now, I know it's currently in the Chinese press, I haven't seen anything in the English press, my husband was reading something to me from the Chinese press, and they're on the same subject and that reminded me I saw maybe two years ago or three years ago I saw in the Chinese press a cartoon so it was very clear what the message was, and the cartoon was of a track, a sports track and there's a figure at the forefront of the cartoon sort of loping along, he's got sunglasses on, he's got a walkman 
over his ears, he's listening to music, he's jogging but he's just kind of taking his time and the T-shirt says 'The Hong Kong Graduate'. And then, not too far behind him is a hoard of other runners, running to the extreme of their capacity, overtaking the HK graduate, and of course that was the 'China Graduate'. I know our students are concerned, I have that thought in my mind, I have that picture in my mind, my students didn't even want to look at it. I think there was something this week, we're interviewing employers of jointventure companies, just characterizing Mainland university graduate employees and $\mathrm{HK}$ graduate employees, and the Mainland employees came off very much better. So, is the teaching profession going to be open to ... well of course it is, we accept applications, people can apply, for posts, and if their English is better, they will presumably get the job. So maybe that's one thought to think about.

AU I suppose China's so big, I was going to say if English teachers from China come here, who is going to take their place in China, but there will probably be people to do that, perhaps the elite will come here.

12(97) If we're teaching other subjects well in Chinese and teaching English well in English, I don't personally have a problem with that, but as you know, you're speaking to an English-speaking mother who chose to put her daughters into a Chinese school for reasons of bilingualism, so I can relate to parents wishing their children to be bilingual. But, I do not think that many parents in HK understand the best way to do that.

AU Do you think that they are becoming more aware of the best way to do that? Is there enough information being given to parents to perhaps educate them?

12(97) I really don't know. Joe Boyle's article talks about what seems to be an inflexibility in the EC's reports, trying to keep Chinese separate from English. I don't know if the ED does do enough for parents at all, you'd be in a better position than me to know that.

AU I've been covering the English press and there's not much in there, maybe in the Chinese papers.

12(97) Do you have any facility for covering what's in the Chinese papers?

AU No

12(97) Maybe next time. So I'm all for improving language levels for language teachers, because the better the language the better the model and the faster and the better the students will be, but the mechanism for doing it with these teachers, how would these benchmarks be determined, I don't know completely how that would work. 
I was speaking with the EA people yesterday and they are involved, they are going to be doing it, they wouldn't comment on the technicalities of it, seems nothing's been finalized yet.

12(97) We all know that HK people are very pragmatic, if this is what they need to do, they'll probably do it if it's going to lead to a better job or promotion.

Do you think there'll be any other significant changes in government policy towards the teaching of English in HK after the changeover?

12(97) I'll skip down to maybe number 4. You're talking about matters of timetabling or matters of the education system. I have no way of talking about the proportion of the timetable because as a tertiary teacher I don't really know what the proportion of the timetable is.

AU At the moment it's about a quarter of the timetable for English.

12(97) For English language, for English-medium?

AU In English-medium schools and I think it's the same in Chinese-medium schools as well. The only thought I had was that perhaps with the change in the MOI Chinese-medium schools might increase the proportion of the time given to English. If they are not going to be officially English-medium anymore once they change to Chinese-medium, then the amount of English classes might increase as a way of reassuring parents that they're committed to English language teaching.

12(97) So I guess the real answer is, I don't know, I don't know, I don't know. The other piece of course is where does Putonghua fit into this? We've got that whole thing about the medium, Cantonese and English-medium, and later over that is the growing importance, perceived importance and probably real importance of Putonghua, so how's that going to fit into the curriculum, and how's that going to fit into parents' and students' sense of priority? So whether that will be a change in government policy towards the teaching of English, maybe that's one thing to think about, having to share time with what's really another second language, right? And then when you say significant changes in your school, meaning my school, maybe this is question 6, will the changeover affect teaching practices or approaches, you're asking about secondary, but how it might affect your school, which is university, I just wanted to say that a concrete piece of information that I could give you is that at a faculty meeting within the last 3 or 6 months we were discussing plans for next academic year, and one of the teachers expressed a reluctance to teach a course that she has been teaching for three or four years. We have a number of elective courses in my department for any student in the university. The English Department teaches English majors, and my department, the ELTU, teaches all the other students, we do the SL 
work for the whole university, and we have a number of elective programmes which try to help students in various aspects of their English, and this teacher had developed a course called Current Affairs and Issues, so basically a reading and discussion course which she had organised around basically newspaper, print medium, not radio/TV, mostly print, and this teacher had expressed a reluctance to teach it next year because she didn't want to be put into a position discussing something that might be sensitive. And I heard her say that, and I can understand her concern, and later on she eventually came to a decision that she would teach it, but her initial reaction was, I'm not sure I want to teach this course next year. It was basically a case of selfcensorship, right. I don't want to be in a situation where I might have to discuss political issues, or sensitive issues, current affairs, right? In the end she decided that she would do it, but that was the first time I had heard something in my university, very directly articulated. I've brought another article that I wanted to give to you just in case you might not have seen it, but it was in Newsweek recently, it speaks about self-censorship and how in this person's view, the media and the universities are already doing this, and I thought of my colleague who had started doing that and then came back and if we're self-censoring even before the changeover, I like your word, changeover, we are not handing over, we're just changing.

AU I actually teach a similar course, it's AS Level Liberal Studies, The Modern World, which is basically current affairs, world affairs, and I haven't thought of that.

12(97) Well good for you.

AU Because we deal with international news. I wouldn't expect there to be any change in international news coverage

12(97) How is the university going to change? How is your school going to change? How is the city going to change? I don't know.

AU Presumably it'll continue as it is and will stay English-medium, not much will change. As far as other subjects in English are concerned, that's where there might be changes.

12(97) I just wanted to mention to you that, whether it's relevant or not relevant, I just wanted to let you know that a month ago we had a visitor to our university, Doug Brown, who is at San Francisco State, and a prolific author and a fine teacher amongst many other things, and he was here speaking at our university and we have a video of his speech if you have any interest in seeing it. His topic was dealing with moral, ethical and political issues in the classroom. So I just wanted to let you know that, but it was thought provoking. And the other thing, I just wondered if you know [ ]? She deals a lot with schools. 
12(97) It's just that [ ] might be in a better position to help than I. Anyway, your question is what changes will there be at $\mathrm{CU}$, and one thing that we hope perhaps is that we will go back to 4 years. We never wanted to go from 4 to 3 , which happened for us very recently and I think it's this year, we graduate our first 3 year graduates and I was thinking the first year we graduate our 3 year graduates we become an SAR, and all of the Chinese universities are four years, right? We are out of step with all the tertiary institutions in China so we would certainly like to go back to 4 and perhaps will go back to 4 . One other thing I've heard, which is a fact, is the hope that the funding arrangements remain as they are now, which is not the ED, it's called the UGC, and that the whole arrangement will continue so that we are not, in fact civil servants but a separate tertiary system, so those are the two very large matters that are of concern. I personally don't imagine that my teaching will change, I don't know, ask me again next year. Maybe the concern, partly in that article and the concern that my colleague was manifesting is that kind of watching what you're doing,, watching what you're saying, who am I talking to and what am I saying. Is there a video camera? Call it paranoia, call it selfcensorship. Would I choose this more neutral topic of discussion rather than that perhaps more dangerous subject. That's how I found Doug Brown's presentation, very thought provoking because he was saying that language is not neutral and perhaps we have a responsibility not to be safe.

AU Safe is boring.

12(97) Right, just water it down so that it's so bland it's ... boring and not particularly productive for anybody. But then a colleague, a first language speaker of English who had worked for a number of years in China, spoke during the discussion, spoke very powerfully during the discussion session, what it meant for her in the teaching situation she was familiar with in China, so that it's one thing for us in HK with HK students, Doug Brown with international students, or Spanish speakers in California, and this colleague was saying, but for my Chinese students in what what university in China, what does it mean? It's dangerous, it's dangerous for me as an English teacher to ask them to do this activity, or to discuss this, because she didn't even know who all her students were, for one thing, right? But she used the word dangerous, and Doug took her point, right? It is dangerous, but he was saying it can be neutral also; there is a content to everything we discuss in the English language classroom. I somehow think the answer to number 3 is no significant changes in government policy towards the teaching of English, but will there be changes on the ground. And for the timetable, the whole matter of the Putonghua, number 5, I've already mentioned the two changes, the concerns in our school. I needed to talk to one of our administrators, just some contractual issues regarding a new teacher and I said what do you think the changes will be, I said, do you know something, guidelines that would 
cover this kind of situation and he said, no, he didn't know anything from the ED or the government, he hadn't received any guidelines, and he said, we're just assuming that it's going to continue just the way it is now, and I laughed out of one side of my mouth and said, well that's a big assumption isn't it? He was a high position administrator and so I figured if he had not been told differently then what could the rest of us do but assume that it would continue. And I hope it can and does and will.

AU Everybody does.

12(97) I suppose generally in life we should expect the unexpected. Well one thing from me not as a tertiary teacher but for schools is the growing population of Putonghua-speaking students, right? Children who live are now on the mainland who are children of HK residents, coming into the classroom, and that might be a good thing, because English will then be the common currency between a Cantonese speaker and a Putonghua speaker, right.

AU It will be leaning a little more towards an international classroom. And as regards the timetable, Putonghua, they are obviously going to have to start teaching it, probably from next year, and it will have to be scheduled somewhere, there is only a limited number of hours in the day or periods in the week. If they take it from somewhere else from the other two languages.

12(97) It is distinctly different, am I correct? I mean spoken Putonghua would be distinctly different from the normal Chinese lessons students have now, is that correct? Do I understand that Chinese lesson is reading and writing characters? But spoken Putonghua is another layer.

AU They do some in primary schools, I think they have an introduction, at our school they do it in F1 and they drop it in F2.

12(97) I have a feeling that will probably change, yeah.

AU They'll continue throughout the school I suppose, but in our school they only have one period a week.

12(97) So the question is, will that come from English?

AU Or Chinese?

12(97) Or become incorporated into what they do in Chinese classes now? Add a spoken element.

AU Putting English aside, the common feeling is that standards of Chinese are dropping too, so schools will be reluctant to reduce the amount of their teaching of Chinese. 
12(97) Did you speak with the EA people about that?

AU Yeah, they said that Putonghua will be taught from next year, but they were not sure how much of the timetable would be given to it, I guess there'll be directives.

12(97) Right, and what about the decline, the much discussed decline, did they speak to that?

$\mathrm{AU} \quad$ Of English or of Chinese?

12(97) Both, I guess.

AU Not really.

12(97) OK, I know that's the common currency.

AU I guess from their point of view there's no evidence of it, our results are just the same as they've always been.

12(97) Right, right, I've heard Rex King speak, and that's why I asked you, I've heard Rex King speak to that and say it may be more folklore than statistical.

AU In tertiary, there have been claims that the students just don't have good enough English, the Law Department at City $U$ for example.

12(97) Well, we have to balance that against the very rapid advancement of tertiary places, so when I hear tertiary teachers speak to this, my first reaction is to say we broaden the number of places so we have to go deeper into the pool to fill the places, right? So of course not everybody is getting the cream anymore because there's not enough cream to go round all the bottles.

AU In the past all the university graduates came from HKU.

12(97) Right, right, and my sense is that some people are remembering the perfectly flawless English and making some assumptions about what any graduate should be. It's really quite an incredible amount of pressure on our students I think because I sometimes wonder for a new student coming into our university and everything is new and kind of real scary anyway and kind of like, university students are declining in this and they're declining in that ... I think it must be demoralizing for any of our students, especially a new student starting out.

AU I guess so, but they were saying the same kind of thing when I went to university. 
12(97) Yes, right, this younger generation ...

AU Always seems to be the way.

5(97) Maybe it's just a part of being a different generation, right?

AU All university students are layabouts.

12(97) That's right, that's right.

AU The last question talks about the situation, status and morale of teachers. Do you see any changes in that?

12(97) Well, I guess all along, people have been asking me, what are you going to do in 1997? People have been asking that for ten years, right? The short answer just to cut the discussion because it could go on forever right, well there are English teachers in China too. Assuming there are English teachers in China, there will continue to be English teachers in HK. I'm an English teacher and presumably my experience and efforts will continue to be needed and done. I guess that's the short answer, I think there will still be the need, and I think English is enough recognized as an international language in business and commerce and e-mail that it will be a needed skill for a foreseeable future, maybe the not foreseeable future, so I don't personally think the situation will change very much among teachers of English as a group because we are teachers of English. I don't think there'll be a change. Whether there'll be a change in morale, I don't know. There'll still be a need for teachers of English, when my students say, oh my ... terrible, I make so many grammatical mistakes, I don't know enough vocabulary etc. etc., just to lighten the ... when they feel down about their English, I just say well, I'm glad that you have problems with you English because if you didn't have problems with your English then I wouldn't have a job! Oh well, there's some good in it, kind of thing. Do you see it affecting the morale?

AU I don't think so. I guess it's tied in with all the other things, which are not directly connected with the changeover anyway, the MOI, the benchmarking. My focus is on the changeover but a lot of changes are not connected with the changeover, they are concurrent, so that's probably one of the big conclusions I'll come to.

12(97) Maybe except for the Putonghua, maybe all those other things were happening already, like expansion, and standards and code-switching and MOI.

AU The only thing perhaps is the idea that English is the international language and it perhaps will no longer be seen as the colonial thing, that's being forced on students. I don't think it is so much now, like it might have been ten or 
twenty years ago and once the colonising power has gone then we can get on with teaching the international language.

12(97) I was at a conference recently in Kuala Lumpur and a woman from Singapore spoke very provocatively deliberately about that and I learned later that some of her Singapore colleagues thought she went a bit too far, maybe she didn't go too far but maybe she simplified the Singapore situation in a way that I didn't recognize but they recognized, whatever, but basically she was saying, well English is an international language and if it wasn't English what else would it be? If there's going to be something out there that help's us to communicate, then it's going to be $x, y, z$ instead of $a, b, c$, right? I took that point, we're globalizing and talking to each other and it just happens to be English. People who are speakers of English just happen to be speakers of a language which is becoming more broadly functional, and the reasons why that happens are bigger than us, right?

AU So perhaps in some ways maybe the status will rise? People tend to look on the changeover pessimistically and say, what are you going to do? Perhaps from our point of view the status will rise. HK will have to remain international, as it won't be a dependent territory anymore, so it's knowledge of English that we have and as you say, we'll still be in great demand.

12(97) Maybe I've taken some shelter in the fact that I am American and haven't felt myself particularly colonial, right? Maybe if I were British I would have felt that way, I don't know. Many of my British colleagues never have, do or did, but it seldom comes up in conversation with my students but if ever it does then I say, well America was a colony too.

AU From the point of view of me and other British teachers, perhaps, after the changeover we'll have to get a work visa just like anybody else, we won't have any special privileges; therefore perhaps we won't be resented so much.

12(97) Well actually, you ask this question about how the changeover will affect certain things, my own question marks are not about the actual teaching or my classroom, it's about other things. What ID card will any of us carry? I have an $\mathrm{D}$ card, will this keep working? My visa, will it keep working? What about my retirement benefits, will they keep coming? Kind of the contractual things or aspects of my work, not the actual teaching or how I would conduct myself in the classroom or even very much the content of my classroom because the courses I teach don't happen to lend themselves to discussion of the CE Designate, or the Provisional Legislature, it's just not particularly relevant to the work we're doing, so I think my concerns as an individual employee here have to do with those other things, the status of my stay, right? And in fact that was part of the call that I made to the administrator earlier. I am going off to visit my family in the US this summer, do I need to do 
anything before I go off in order that my coming back will be smooth? We assume things will continue as they are.

AU Yes

12(97) ... and how do you feel dealing with a subject which is just so incredibly current?

AU It means that I have to do it now.

12(97) Well there is that ... and do you have any concern about the rug being pulled out from under you as it were, there's no way that you can have any sense of perspective on the whole topic, you can't sort of work out a structure or work out an outline and say well this is how I'm going to approach this project, because your project is evolving as you're doing it, right? If it goes off in that direction then you don't have a project, or is it that you follow whichever direction it takes, is that it?

AU Yes

12(97) So even if it turns out absolutely nothing changes, that's fine? OK

AU Obviously it will be more interesting if things do change but if nothing does change then so be it.

12(97) And to be able to say, gee, nothing changed. Actually, from the very early meetings of the JLG and the Basic Law and the 'One country two systems' and there'll be no change in HK for 50 years, and I would think, how could anything not change for 50 years? You can't mandate no change for 50 years, you just, my impression is that changes have been happening faster in a number of spheres, just take the computer, it's changing every night. How long have we had computers? How fast are things changing now, they are changing twice as fast in 6 months than they did in the past year, you know? The rate of change is speeding up, that's all I'm saying, right? I think what we have now will not function in 50 years, right? In fact that would be the worst-case scenario because that sounds like stagnation, right? I didn't find that reassuring or encouraging at all.

AU I guess they meant there'd be no essential loss of freedoms or what people hold of value.

12(97) That seems to be happening even before the changeover, right?

AU I suppose within the timeframe I have, within two years, you can't expect that much to change, but who knows? Things may change, technological changes 
12(97) You know, thinking about no. 5, significant changes in your school which might affect the teaching of English, for a teacher in a secondary school, in the classroom, how might things change in the classroom as a direct result of the changeover? My own thought was, probably not much, but what you just said right now, what about other changes, not changes of sovereignty, but other changes that might affect the profession of language teaching in many different ways. I know that the new grads that I was meeting at this conference in KL, they were abuzz with MSC? Multi-media superhighway or ... just an amazing computer link where everybody can be talking to everybody else? What is that called? Apparently this is all the buzz in Malaysia, everybody's got to be on this superhighway, sitting at their own little terminals and squirling away and they asked me, what is the situation with that in HK, and I said what?

AU In a similar way I teach a class of Liberal Studies and there are only 7 in the class and they all have e-mail so I can contact them and download articles for them, fantastic.

12(97) So they all have e-mail at home?

AU Yes, on the Internet, so they can do their own research as well. Or they can send me messages; in fact quite often they prefer to send me messages that talk to me in person.

12(97) They can double read their message, right?

AU Or they can have me correct it.

12(97) Right. All of the convenience and the advantages that the new technology opens up to us, and some of my colleagues who are miles ahead of me, and I say well what price are we paying? Yes there is an advantage and yes it is convenient and yes I can leave an e-mail message which a person can read at a convenient time and yes yes yes yes, but what price are we paying? That's a whole 'nother project and a whole 'nother thesis, perhaps your next Ph.D. Maybe those are the sorts of things which are going to change our language classrooms rather than the changeover. In your school, is the English language classroom going to be much different five years from now?

AU I can't see it, not unless there's a huge influx of cash to update the facilities. 


\section{Interviewee 13(97): Education Officer \\ Date: \\ 18 July 1997}

$\begin{array}{ll}\text { AU } & \text { Researcher } \\ 13(97) & \text { Interviewee } \\ \ldots & \text { Missing words / Unfinished utterance } \\ \text { - } & \text { Pause }\end{array}$

13(97) Well some of these questions will involve a certain amount of crystal ballgazing, I think Mr. Leung has yet to announce his plan, so any comments will be conjecture on my part. First one obviously is kind - of controversial. We do believe in the benefits of mother-tongue education, it's a matter of whether we see language use in teaching is of greater importance than educational considerations. From my point of view, I consider it important to make sure that students do benefit from learning through the mother tongue, even though mastery of English is important, but it is not as important as them being able to learn better and more comfortably through the mother tongue. I should like to suggest that perhaps schools could be allowed to maintain CMI up to S3. S4 upwards could be English-medium to provide a better bridge between senior secondary and post-secondary, sixth-form and tertiary, because after-all, tertiary is English-medium. So if you have sixth-form English-medium education, that may not be enough for the majority of students. And I thing the Use of English examination results testify to that, I mean there are a lot more students who fail to obtain grade $\mathrm{D}$ or higher, in ... some 2000 students would be entitled to enter university anyway despite not being able to scrape an E, or not being able to score a D in the exam. So, I see basically, a good change if we have to S3 CMI. I do believe in maintaining, in some of the more able schools, the EMI tradition, because after-all there are students out there who are perfectly capable of learning in either medium, whether it be English or Chinese, so my very personal view would be for those who are able to benefit obviously they should be given the chance. I will not venture into the administrative details about how best to get it done, because obviously schools will argue from their own point of view in terms of student enrollment because they will expect as soon as you declare yourself CMI you are not getting the more able students into the school and so on, this is their consideration, but from my educational viewpoint, I would support mother-tongue education up to F3 and to maintain EMI for the more able students. I think it has been explained before why ... was made, based on statistical analysis, because it's not statistically significant to do it otherwise, I was told, now I'm not a statistician myself, but I think there is some point in making that decision. What really matters more perhaps has to be linked with the competence of subject teachers in using that particular language as a medium. 
So I could actually move on to $\# 2$, benchmark language levels. Again, I am in support of this. I would like to suggest that the primary spirit of the benchmark exercise is to ensure that teachers do have the language competence to deliver the curriculum, and that's why it is an exercise to be operated on all teachers and not just language teachers alone. And I think our emphasis is on training teachers up so that they meet the requirement. We are in the middle of striking a balance between asking people to tick the benchmark, or requesting them to take up training. We would like to say that it's the training that we want to aim at, because once they receive training, part of that training will embody a sort-of exit test so that once they've undergone the training they are in fact certified to have obtained or to have reached the benchmark proficiency level, and therefore they don't have to take this so-called benchmark assessment. It is important because we believe in speech models, to say the least. If the teacher is competent, then the chances of his or her students being competent will be high. But if a teacher has difficulty in expressing himself or herself, then we could not expect their students to be able to do likewise. So it is a must I would say to ensure that teachers have attained the benchmark levels. In the HK situation, what happens is that a lot of teachers have not receive professional training either in the subject of English or in the teaching of English so it is understandable in a way that their level of proficiency may not be as high as their counterparts who specialised in English language teaching. So in a way we will have to find means to ensure that they have the training required, posses the competence to deliver, whether it be a language curriculum or just subject curriculum.

AU So there are plans to bring in benchmarking for subject teachers as well?

13(97) Yes, it will begin first of all with the language teachers. The subject teachers also need to go through the benchmark exercise, but the level of requirement will not be as high as that required for language teachers. And therefore, as a language teacher, once you have taken the language teacher benchmark, you will be exempted from the subject teachers benchmark, because it is assumed that you can perform at a higher level then their is no need to perform at the subject level. All these are being research upon, for the time-being we will concentrate on language teachers at junior secondary level of English, primary level Chinese and Putonghua, and gradually we will move to cover senior secondary, primary for English, secondary, lower secondary for Chinese and Putonghua.

AU There has been some objection to the benchmarking though hasn't there from some teachers.

13(97) Graduates from the former colleges of education, yes, that is why we have suggested officers to put on training, now, if you think you posses the qualifications, then taking that assessment should not be a problem. After all, 
there are changes introduced in terms of methodology, curriculum strategies and so on. As a teacher, we believe in sort-of life-long education, so we need to periodically, regularly updating yourself. So from that viewpoint, we don't see any counter-arguments or a teacher to be sort-of complacent with his or her whole level of English. I did suggest that a lot of our teachers, possibly up to about $45 \%$ at the secondary level, are not subject trained, so we do need a guarantee that they do possess the competence that we require from an English teacher. So I think in the near future, announcements may be made regarding that you can simply come forward for the benchmark if you like and thus spare yourself all the training, otherwise there will be training for all teachers whether you be subject trained or otherwise. So training we offer, if you want the training, then do so, and having gone through training you will already posses the benchmark qualifications required. If not, if you think you don't want the training, you want the proof that you are already capable, competent and so on, why not take the benchmark? It is a very good sort of exit test. So we hope there will be less resistance. Basically we are all aware of the fact if you need to take an examination, that sort of gets people on their nerves, because the syllabus has yet to be publicised, it hasn't been finalised, we still need a lot more teacher input before we finalise any syllabus for the benchmark exams, but we don't want to over-stress that because basically in HK we all know that it has been sort of exam-driven to a large extent. So we would like to think that after all it's a measure of communicative skills that we are looking after, not just dry pen and paper tests and that's why in the benchmark there will be a classroom English component where teachers will be observed in action and then be assessed in terms of delivery.

Shall I move on to \#3? Significant changes in government policy towards the teaching of English in HK. There won't be great changes. I think Mr.

Anthony Leung may think about school organisation, altering the primary and secondary schooling in terms of how many years you spend in. I mean how many years in secondary. I'm not sure about the details, but I think if we talk about change, that could be one of the changes. There used to be a debate some years ago about the desirability of introducing English at a later stage, say in upper primary, I think again there will be heavy resistance from parents. On the one hand there is the early immersion exposure theory, the earlier you are exposed to second language or foreign language the better, because as a child you are perfectly capable of learning a number of languages at the same time, on the other hand we have to juxtapose that with the supply of teachers. Whether we have that many qualified or capable teachers at the primary level, because if you are exposed to inaccurate English at the very beginning, you may have a very difficult task in undoing or unlearning all the incorrect elements that you picked up in the early days of your language education. Somehow there are suggestions that it is not that difficult to amend the mistakes in the past, I don't know how true that is. Merely from the clients' point of view, I don't think that policy will be supported if we introduce English only say Putonghua P4 upwards, I don't 
think that will be a welcome change. So I think more or less we will stick to our existing practice of putting in English from P1 onwards. The TOC actually does that. The emphasis in P1-3 is on enjoyable learning, pleasant or pleasurable activities associated with English - all the songs, poems and so on. Quite recently we have announced that we will try to extend extensive reading all the way down to $\mathrm{P} 1$. So we believe that with greater exposure to English books, there is a better chance of getting students interested in the language through the kind of books that they read and through the visual materials presented in books and so on, help them actually acquire the vocabulary they need. So we should like to think that the extensive reading programme will contribute much to improving motivation for learning English as well as the actual learning of English in primary schools. Apart from that, I don't see much big change in the teaching of English. The TOC actually built on very sound ELT principles, so even tough there was some resistance at the beginning from teachers who have more problems with the meta-language really, the TOC rather than the spirit of it all, and the assessment related to TOC, they have now been won over, I would say, by very thorough teacher training programmes being run by the ED. With greater understanding there is much less resistance, so again, I don't see much significant change in terms of the system.

\#4 relates to the proportion of timetable. Now I must say at the very outset that the amount of time devoted to any subject, not just English is a sort of school-based decision. The CDC are merely making recommendations as to the number of periods a school should allocate to English, or any other subject for that matter. So for instance, at secondary level we recommend that 7 to 8 lessons a week would be quite sufficient. In actual fact, I think that a lot of schools allocate up to 9 or 10 lessons per week, so that's 2 or 3 lessons more than what was recommended. Again, we have no objections whatsoever, particularly when you assign more lessons than we suggest, that is from a very narrow, selfish, English language point of view, but it's really up to the school themselves to decide how much time they like to spend on English. We tend to feel that having more English lessons will necessarily mean that students will learn more, but on the contrary, we feel that some of them may be having an overdose of English. We believe in quality time. So if you can do something well, it doesn't matter if you spend an extra 30 minutes or an hour on that particular subject. So this depends on the capability of the teacher, if he or she is a good teacher, then 30 minutes will be very useful, but if the teacher has to wrestle with the language herself, then probably 3 hours won't make any difference.

AU Do you think Putonghua will make a difference to the proportion of the timetable given to the other two language?

13(97) At the very outset I would say no, simply because we don't have enough teachers of Putonghua. Now, in a few years' time, when we have more 
qualified Putonghua teachers, then we may have a full swing in terms of Putonghua curriculum. So far we have to make do with offering Putonghua as an extra-curricular activity, or as an intensive programme over the summer, simply because there aren't enough teachers to teach it as a formal curriculum, but obviously the changeover affects people's sense of importance as far as Putonghua is concerned. Everybody's trying to learn Putonghua from informal sources or look for the opportunities, but as a school subject in the curriculum, I think teachers is the key to the whole issue here.

\#5, again, significant changes in schools ... affect the teaching of English, well, my immediate feeling is that English teachers will feel the pressure in that if there are more schools switching to CMI, then obviously English becomes the only opportunity for their students to be exposed to the language, it will be even more difficult to have students with higher abilities in English. I think the ideal situation will be that you have one or two subjects which can be taught in English, so that what you learn in English lessons will be applied, will be used, will be recycled in one or two subjects. These subjects, one of them could be social studies, because the vocabulary can be readily applied and so on. Possibly not the sciences, but the sciences you require specialist vocabulary to understand the ideas and concepts. English teachers are fighting a sort of uphill battle already. Now with CMI introduced, we really need to explore innovative methods, more effective methods, to motivate interest. One of the key issues at the moment is a lack of motivation among students. They are generally not motivated enough to learn anything, let alone language. We have done quite a lot to make sure that learning materials are interesting, but I have a feeling somehow that we have done far too much, and so there is less demand on the learners to do their own part. We have made things interesting, we have made things simple, but they are not able to retain knowledge, language, ideas that they've picked up in the lesson, and that is a shame, that is a pity. They may have enjoyed the lesson, but at the end of it they have forgotten what they were supposed to have picked up. I am referring to this as a general case, not just in the case of English. That is true for any subject, even for science, even for mathematics. They were having a very enjoyable time, yes, but very little learning takes place. I can also think of one significant change which may have very dramatic results, and that is the introduction of information technology (IT), i.e., they use computers. Because we are shopping for ideas, software packages, which will make language learning easier, more motivating, with the availability of more computers in schools, I think we are trying to provide one computer among every five students at the primary level, or one among three at the secondary level. If that becomes a reality, then there becomes a bigger possibility that students will have access to all these software for language learning, self-access learning and so on, and that may have a very dramatic impact, we are not sure, we are working towards that end. So hopefully in 3 to 5 years time, when computers are popularised, when 
students are so hooked onto computers that they actually have their own PCs at home, then they will be able to learn at home. That could be something very positive for English language learning. And strangely enough if you use e-mail, you are more likely to use English than to use Chinese. When you start your e-mail you will be communicating through English and that may help.

AU It has its own genre though, e-mail.

13(97) True, but at least you get them into a habit of writing in English. Basically you have the motivation there. Of course what is more effective is the sort of programme learning built into it or other software.

The sixth question - changeover, will it affect teaching methods? I don't really think so. I think teacher education programmes will affect the way teachers teach, rather than the changeover. As I said, there will be greater demand for better English in schools, and too many people have already said that they are dissatisfied with the level of English that our students have. I don't want to get into the argument of whether standards have been falling or have been maintained because it's really a numbers game. If you like to look at the absolute numbers, then there are more people using English than before. Percentage wise, of course, we do not have any reasons for complacency.

Last question, changeover ... status, situation, morale of teachers of English in HK. I think I've more or less answered that. Tie that in with the benchmark exam. If you can pass your benchmark exam, I think that in a way is a morale booster. I believe that when they develop the benchmarks, they are sort of competency indicators, so you will end up emerging as a sort of grade $\mathrm{A}$ competence. It's nice to know that. If you can make it, then you get your recognition and people will respect you more. If you don't have the benchmarks, then you are just a teacher doing English. I think that should help. On the other hand, if a teacher really fails, then it simply means that a teacher need to do something about his or her command of language, to put it very simply. Government is willing to provide a training opportunity, free of charge, I think, so there is no reason why they should be unhappy. After all, so long as you remain a teacher you'll be benchmarked whether you like it or not. So I think that should be something positive, even though we are only at the very beginning of the benchmarking exercise, nothing has been finalised yet.

AU Will the results of the benchmarking be publicised? Will other people know that this teacher has passed the benchmark while this teacher hasn't?

13(97) No, because of this privacy ordinance. You really should not expose this personal data to anybody who does not have a purpose for acquiring that piece of data. Now if you apply to join a school, obviously you need to 
present your qualification to the school, and the school can only make use of that bit of information for recruitment purposes, so I guess it will be up to the individual person how that data will be used. So it will not be announced in the paper, but I think the school has a right to know so that they are able to make some administrative decisions, for instance, if you should fail the benchmark, at least the school should know, but to begin with they shouldn't really ask you to become a language teacher in the first place. So that sort of information should be of use to schools.

AU My focus is the changeover, regarding the changes in MOI and benchmarking, the fact they were announced pre-handover, any significance in that do you think?

13(97) No, you see the benchmarking stems out of ECR6, so that was 2 to 3 years back. Of course it took them one year and a half to work on Report \#6. There was a preliminary working group report, went through the consultation process and then it was redrafted, another consultation period and then it was finalised. But actually, for instance, I think the MOI issue dates back to 1984, so there has been a very long-term planning. It's a matter of government tightening its monitoring, where as before trying to encourage schools to adopt the use of Chinese in one or two subjects and so on. Having given 10 years or more and result in not that satisfactory, the government has to step in and do something. I would say it's sort of coincidence, if you like, that we have step up everything, firm guidance in ' 98 and so on, it's a sort of very long-term thing. Benchmark is relatively recent, and again it's not anything to do with the changeover. TOC also.

AU And the question of Putonghua, as you said, is shortage of teachers.

13(97) That's right.

AU It's planned to be implemented in schools with the cert. in the year 2000, but that won't be compulsory in schools?

13(97) Not as yet. It will be offered as an examinable subject in the school cert. exam but again we can't push it too far because if you don't have the teachers you simply can't teach it. We can't expect everybody to use self-access tapes and become proficient in Putonghua.

$\mathrm{AU} \quad$ Presumably, is students are going to study it to cert. level, they are going to have to have had the background in it, they can't suddenly begin it in F4 and expect to get the ...

13(97) Take a comparable case, literature is an examinable subject, how many schools are offering literature? Why aren't they offering literature? Because there are not enough literature teachers around. so we have a handful of 
schools entering students for the literature exam, and I think this is quite similar to the Putonghua situation. If you have the teachers there, then there isn't much problem entering students for the Putonghua exam, on the other hand, if you don't have the teachers you will not even contemplate entering students for the exam. If students are so good at it, they will always be able to enter as a private candidate, this also happens, so there are candidates entering for the literature exam although they are not offered in their own schools.

AU According to press reports, more and more Chinese teachers are doing courses in Putonghua.

13(97) I think the Department is offering more courses in Putonghua fir teachers, and somehow, we think that there could be a lot of Putonghua teachers around who may be recent immigrants, although they are no longer called immigrants because we are part of China now. There is reason to believe that will be more and more people who are capable of teaching Putonghua, but we will have to look at their basic qualification to see if they are actually qualified to become teachers in the first place. 


\title{
Interviewee 14(97): Panel chair of English in secondary school
}

Date:

\section{June 1997}

\author{
AU Researcher \\ 14(97) Interviewee \\ ... Missing words / Unfinished utterance \\ - Pause
}

AU Perhaps we can go through the questions and then anything else that comes up ...

14(97) OK

AU What are your feelings as regards the ED's decision, I've put to force $75 \%$ of secondary schools to adopt Chinese-medium education?

14(97) Well, I think this is a stupid decision, even in China now, many schools are recruiting English and American teachers to help the students to improve their English. I think it is totally unnecessary; they are making this decision simply because of July 1 st 1997 . I think they are very stupid. It's very unpopular.

AU Unpopular with whom?

14(97) With parents, students, teachers too, school authorities too. Very stupid.

$\mathrm{AU} \quad$ But it is something that they have been planning for quite a long time.

14(97) I wonder why.

AU Through the Education Commission.

14(97) Even China realises that if they are to keep abreast of the developments in the rest of the world, English should play a very important part, and now HK seems to be going backward. I disagree.

AU Don't you think that there are a lot of students who will benefit more from Chinese-medium?

14(97) Well I think it is up to the school authorities. Why do you have to make them change into Chinese-medium schools? If they feel that a lot of their students want the Chinese-medium, want lessons to be conducted through Chinese 
then OK. Why do you want to make them do that? Mind you, there will be trouble coming.

AU Well I think that's the point. I think they feel that if they leave it up to the schools then the situation will stay as it is.

14(97) Well they talk as if the school authorities are fools. They should know. If they feel that the students are not good enough to be taught through the medium of English they will change into Chinese. They don't have to make them. Now $25 \%$ of the students are at the advantage of being able to go into the university more easily, then the $75 \%$, so unfair. I think the schools should be allowed to make their own decision.

AU OK, what are your feelings as regards the ED's planned introduction of benchmark language levels for language teachers?

14(97) Well, on the one hand, I don't think it's such a bad idea actually, if you expect students to be able to speak and write good English, teachers should be able to teach good English. On the other hand, some teachers complain that this is not respecting them; after all they did pass their exams, especially those who have been teaching for many years, they feel that is an insult. I've been talking to my own colleagues about this. The truth is there are some teachers, even in our school, who are teaching English, whose English is really not as good as we expect, and well if you are really a good teacher of English, I don't think you should mind this sort of thing. What I am trying to say is that it's no good complaining that the students' standard is low, when you yourself can't speak reasonably good English.

AU So you think it's a good idea?

14(97) Not a bad one. Why should you fear if you are good?

$\mathrm{AU} \quad \mathrm{I}$ think the feeling is that there are a lot of people teaching English who are not trained to teach English.

14(97) It's not their fault mind you.

AU No, not at all, when school principals or whoever is in charge of staffing says we need someone to teach English, you did your degree in English medium, you can teach English.

14(97) Mind you in past years we had mathematics teachers teaching composition, and then of course there were complaints, and after all you can't blame them, I didn't want to teach English, the school wants me to. 
AU That's it, and the thought is that these people undergoing the benchmarking will be happy to fail it if it means that they don't have to teach English any more.

14(97) That's right, and then the school authority couldn't make them teach English, yeah. That's one way to look at it, yes.

AU Although I don't think it's quite that simple. I think if people don't reach the benchmark they will be given a certain amount of time to improve their English.

14(97) And if they don't? If only $10 \%$ of the teachers pass, then there won't be enough English teachers.

AU Exactly, and then what do they do? I'm sure they will set the benchmark at whatever level they see as being feasible.

14(97) Then what's the point?

AU Exactly, they know if they set it too high they will eliminate half their teachers.

14(97) Then what's the point of going to all this trouble and wasting the money and time?

AU I think perhaps just to encourage teachers to improve their language levels if they find ...

14(97) It has it's merits, but think of the amount of work the ED has to prepare. Do you think they will go through with it?

AU They will yeah, I've spoken to the EA people, they are the ones who ...

14(97) I'll be most interested to see how it goes.

AU And for Chinese too.

14(97) Of course, both languages.

AU Do you think there will be any other significant changes in government policy towards the teaching of English in secondary schools in HK after the changeover, and if so what might they be?

14(97) I hope that after a year or two, when they realise that this so-called new policy is not working, they'll change back and allow schools to make their 
own decisions. That's what I hope. I hope the sooner the better that they'll change back. Chaotic, chaotic, so this is a change I expect to see.

AU What changes do you envisage in the proportion of the timetable given over to English language teaching in both English and Chinese-medium schools?

14(97) English-medium schools I don't think there will be any changes, it won't be necessary, they can go on the way they have been going. In Chinese-medium schools, I don't know. If the school principals are sensible, instead of just one and a half hours English lesson per day, there should be a bit more, at least in the form of after-school activities. Perhaps some native-speakers can be recruited to help the students after school or on Saturday morning. If they want to maintain the standard of English, after all even Chinese-medium schools will want the students to go to university, won't they? So I hope if they are really made to change to Chinese-medium schools this is what will happen, that there will be ... well in one of my 4 I class 3 English lessons a day, two in the morning, one in the afternoon, but they can't do that everyday I suppose, then they can have more English activities conducted by native speakers to help them with their spoken English. After all, if they can speak reasonably well, they can write reasonably well. So that is what I hope will happen, if the worst comes to the worst.

AU I think the other factor here is Putonghua, whether that will fit into the equation somewhere. It will have to be taught in schools.

14(97) Well I don't think it's a very big problem because even the Cantonesespeaking students can understand some Putonghua. Maybe they can use Putonghua-speaking teachers to teach Chinese, that should solve the problem. Because after all they can understand the written words, you see, and we socalled Cantonese, we think we can speak Putonghua by slightly changing the sound of the words, but I don't think it's a problem, I'm mainly concerned about the English. I feel that if there are more activities, that's the way to maintain the standard.

AU Do you think there will be any other significant changes in your school or in the education system more generally which might affect the teaching of English?

14(97) Our school is different, our school is a direct subsidy school, and as far as I know, there was a meeting two weeks ago during which the principal said he thinks the ED is going to allow us to do whatever we like, so most probably we will be able to go on being an Anglo-Chinese school. Not really actually in our school, in our case, most of our students are Band 5 students and quite a big number of them are young immigrants from China, so apart from Chinese and Chinese History, all the other textbooks are English textbooks, but the teachers very often use Chinese when they are teaching those things. I 
insist that our teachers of English use English when they teach their lessons, but still there are some who come complaining to me saying that there are students who misunderstand and so on, but that's beside the point, but as I was saying, our school doesn't have to change, if we don't want to. So, I don't think there will be a lot of changes here. Probably more Putonghua lessons perhaps, but not other things. In that way we are quite lucky.

AU So you are not directly under the control of the ED?

14(97) No, not directly. Mind you, they say 50 years without change, that's rubbish, we are changing already, but I hope the changes will not be drastic.

AU Do you think the changeover will affect teaching methods or approaches in secondary schools?

IM As I was saying, if after the changeover some schools are forced to become Chinese-medium schools, then the school authorities should have the good sense to first of all insist that only English be used in English lessons. Secondly, if they cannot have more English lessons in a day, then there should be some more English activities after school and on Saturdays and that the best method is to recruit native speakers to teach English. I think that would be a very good solution. If not all, then at least more native speakers to teach English lessons, then students will have more exposure.

AU I think that's what they are trying to do now, isn't it, with the expatriate English teachers scheme.

14(97) It does help you know because now I get students coming to me after school speaking to me in Chinese and I'm not a very harsh teacher, I'll simply answer in English and eventually they smile and ask me in English, but if they are speaking to a native speaker, at once they'll change. And although they are not speaking in perfect English, at least they have the confidence to speak in English and that makes a lot of difference.

AU OK, and finally, how do you think the changeover will affect the status, situation or morale of teachers of English?

14(97) I am optimistic. I think society will continue to look up to people who can master the English language. Look at China, my husband goes to China very often, and he says that civil engineers, they are both Chinese, but because he speaks Cantonese and they speak Putonghua, and he doesn't understand Putonghua, they are conversing in English. I feel that if HK is to maintain its international position, we should remain a bilingual society in spite of all the rubbish they say, society will still look up to people who have mastered the English language and the people sitting at the top of the firms can always speak English very well. If you want someone to represent the firm to go 
abroad, you want someone who is fluent in English, don't you? That's what I feel. We should be optimistic.

AU So the status of English teachers will still be high?

14(97) Should be.

AU One thing which has occurred to me through talking to people is once HK is no longer a dependent territory and English is not the colonial language any more, there's not the feeling that it's being forced on students, it will become truly the international language without the colonial baggage attached to it.

IM But I feel that English should not be a foreign language but a second language, people should feel at home with the language, like Japan, they can understand you and yes, yes, yes ... no, people should be able to use it comfortably.

AU Like Singapore?

14(97) Exactly, but whether it can happen or not, we'll have to wait and see. 


\title{
Interviewee 15(97): English teacher in secondary school \\ Date: 10 June 1997
}

\author{
AU Researcher \\ 15(97) Interviewee \\ ... Missing words / Unfinished utterance \\ - Pause
}

AU What are your feelings as regards the ED's decision to force $75 \%$ of schools to adopt Chinese-medium education?

15(97) I think it is a good idea because nowadays even some schools claim to be using English as the MOI, but actually, they do not so it like this. There will be a mixed-code in the classroom, so if the ED really force the schools to adopt $\mathrm{CMI}$ in the classroom, then the schools will really follow what they suggest. So it is a good idea and it is good for students as well. And also, the standard of English of the English of the students in $\mathrm{HK}$ is declining in the past years and I think learning through the mother tongue is much better than English if their English level is not so good, but those who can learn through EMI, they can use EMI in their own schools. So it is a good idea to follow this policy.

AU How would it affect your school?

15(97) My school is now using English as the MOI, and we haven't been told which language we will use in the 1998/1999 academic year, but recently in a staff meeting, most of us proposed to use English as the medium, but it depends on the ED's decision to see whether we can use English or Chinese. But we want to use English for all subjects, except practical subjects.

$\mathrm{AU} \quad$ And Chinese subjects as well?

15(97) Yes, only Chinese and Chinese history and home economics and D \& T, all other subjects will use English.

AU Do you think your students benefit from English-medium? Or do you think some of them might benefit more from Chinese-medium?

15(97) Some of them might benefit, for the best, they benefit with the using of English, but the worst class, especially the remedial class, it will hinder their learning process, so it is allowed, some classes should use EMI and some classes should use CMI. It will be the best method, to teach in this way, because some can really do well in English and some don't. 
AU What are your feelings as regards the ED's planned introduction of benchmark language levels for language teachers?

15(97) Personally, I don't think it is necessary because we have got the certificate, or we have got the diploma or the degree already, from the I of $E$ or the universities, so our standard should be good enough for teaching the students, and there's no need to have such a standard for language teachers. So, I do not support this proposal or policy. But I think it is the best way to test the new teachers before they enter their career, but present teachers, they have already been given their qualifications, they don't need to have any test or examination in order to get the qualification again. But for the new teachers I think it is a good idea, because those new teachers, their English may not be as good as those who are now teaching.

AU How about teachers who were never trained to be English teachers, who for various reasons find themselves teaching English.

15(97) For those teachers, I think the school should tell them to attend the post-grad cert. or diploma in education, so that they can learn some of the methods in teaching, but for their standard of English, I think they are good enough if they can get a university degree. But as I mentioned before, the standard of English of the university students is not so good so they need to have tests first before they enter the career. So the standard of English of teachers and the training of being a teacher is two different things, I think, that's why these two things should be done separately.

AU But I think the idea behind it really is a way of raising the level of language teachers. If they don't reach a certain mark, a certain benchmark, it doesn't necessarily mean they won't be able to teach, but they'll then have to go through some extra training, perhaps. How effective that might be, I don't know. I think that's the intention. Of course it may also mean that there are teachers within the English-medium schools who have to teach their subject in English, but their English is not good enough.

15(97) But in this case the schools may ask these teachers to teach lower forms, they can still teach in this school. So I know that some teachers who are graduate master, they just teach lower forms, but I'm not too sure the reason might be the standard of English or not. The CM are asked to teach the lower forms, if the GM are asked to teach the lower forms, it may be because of their language standard.

AU I think on benefit of the benchmarking is that schools will be able to define which teachers can teach which levels, rather than the panel chair or the principal using their judgement to guess, what their level of English, or Chinese even, is. I think the general idea is to raise the standards, because 
people feel that the standard of new teachers, their English level is not high enough, and their Chinese too.

Do you think there'll be any other significant changes in government policy towards the teaching of English after the changeover?

15(97) I think there will be not so many changes in the coming 5 years, as HK is still an international city, they must produce graduates whose, their English must be at a certain level so that they can communicate with the other people. But as the trading between HK and China will increase in the coming years, the importance of Putonghua is also important, so that's why the ... may be put on Chinese as well. So there will be a balance between the teaching of English and Chinese in HK secondary schools and primary schools as well. Slight changes, there will only be slight changes in the coming 5 years.

AU You said that there will be more emphasis put on Putonghua, do you think that will affect English? Presumably schools will teach Putonghua.

15(97) No, I don't think this will affect English very much, but I think this will affect Chinese. If they learn Putonghua, then their written Chinese will be better. There will not be so many slang or informal words they use in Chinese. So as their Chinese standard is becoming better, this may also influence their English standard as well. Actually I think it is a good idea to learn Putonghua. Sometimes their pronunciation in Putonghua may help them with their pronunciation in English to a certain extent.

AU Why do you say that?

15(97) Because some of my colleagues they are learning Putonghua now, and they say, why the foreigners can learn Putonghua very well, much better than Chinese, because the pronunciation between English and Putonghua is quite similar, according to what they say. That's why if they know Putonghua well, that can help them with their pronunciation of English. This is what I heard from them, after we attend the Mandarin class at HKU, so that's why you foreigners can speak better Putonghua than us if we learn at the same time.

AU Perhaps the tendency of Cantonese speakers is to rely on Cantonese pronunciation, for it to influence what you say in Putonghua, since the writing is basically the same. This is just my idea, maybe non-Chinese speakers, when they come to learn Putonghua, it's like an empty slate, they go into it fresh, if you like, without any influence of any other language as it's so different from European languages. What I was thinking was that, and this actually goes into Q4, that if schools are going to teach Putonghua, they are going to have to get the time on the timetable from somewhere to fit it in, already timetables are full. If they are going to introduce two lessons of 
Putonghua a week, where are they going to take those lessons from? Are they going to take them from Chinese, or English, or from somewhere else?

15(97) In our school we don't have Putonghua lessons now, and next year, I don't think we have. We don't have any final decision yet.

AU It's going to be a certain exam in the year 2000, and so schools are going to have to start teaching it.

15(97) I don't know because I'm not a member of that department. We have nine periods now, so if they want to add one, maybe we'll have ten periods, but I think ten lessons would be too much, they would be too tired. Maybe, they will take one of the Chinese lessons.

AU That's a possibility, but I think people generally feel also that the standard of Chinese is becoming worse, so many Chinese teachers may say, we don't want to lose any lessons either.

15(97) Yeah, just like the case with English, we don't want to lose any lessons of English.

AU Unless of course, they teach Chinese through the medium of Putonghua.

15(97) This is what our principal suggests, I think. If you really enforce Mandarin in schools, then use Mandarin in Chinese lessons as well.

AU It's interesting, I think it all depends on what people at the top regard as important for HK society.

15(97) I also think that if the Chinese lessons used Putonghua it would be much better, because if there is just one or two periods of Mandarin a week, it's not the best way. If we use Mandarin in Chinese lessons, so that the students can use Mandarin in class in conversation and other things, it will be much better than one or two lessons a week.

AU So no other significant changes in government policy? I think the main things so far are the MOI and the benchmarking. If they are going to make any changes in schools, they tend to make changes in the examinations first and they washback into the curriculum.

The proportion of the timetable given over to English language teaching, as we've just mentioned, Putonghua adds into that equation somewhere, but also perhaps, if schools become Chinese-medium, schools which were previously English-medium, would they then feel that they wanted to perhaps increase the number of English lessons, increase the proportion of the timetable given 
over to English seeing as they will be teaching all the other subjects in Chinese?

15(97) I think there's a need to increase the number of English lessons if the EMI school will be changed to a CMI school, but it will also be a problem for the timetable, where can we get more English lessons in such a tight schedule, it's difficult, unless we have one more period each day.

AU Add one more period, maybe shorten the other periods by 5 minutes to squeeze another one in, which is possible. Then they would need to have more double lessons.

Do you think that there'll be any other significant changes in your school or in the education system more generally which might affect the teaching of English?

15(97) I don't expect too many changes in our school. I think we'll just wait and see what happens first. So far I haven't heard that there will be great changes, it will still be the same thing as in previous years.

AU Do you think you'll be getting any students from China?

15(97) More students from China, maybe. I am teaching some in F1, they only stay for two or three years. They are holding a Certificate of Identity. There'll be more students of this kind. But this doesn't mean that their English will be bad, they learn very fast, and their attitudes of learning are sometimes much better than the local students. But at first it may be difficult for them to fit into the ... system, but as time goes by, they can survive. The first term their results are not so good, but in the second term, this will change.

AU Any other changes in the school?

15(97) I don't think there will be any changes, everything will stay the same.

AU Do you think that the changeover will affect teaching methods or approaches in English lessons in secondary schools?

15(97) I think for the CMI or EMI school, the ED enforce such policy, that every school should follow what they suggest to do, then both EMI and CMI English lessons will be more or less the same, except the CMI English teachers, will spend more time with the students for English language because they can only contact with English within that period. So, I don't think there will be a great difference between these two kinds of school as long as the teachers teach the students well in English. But for those EMI schools, they are in a better position because their English is quite good, and they will learn easier than in those CMI schools may not be totally different. 
The teaching methods, I think it depends on the teachers. Now, I think with the more teachers attending the cert., the dip. ed., and also the teacher education courses at City $U$ and Poly $U$ as well, those teachers will have some fresher ideas in teaching methods. So the changeover may not affect at all, I think, just in teachers' teaching methods.

AU Although my focus is on the changeover and how English teaching is affected, I'm not expecting the changeover to have any dramatic effect as such, but it might. We'll have to wait and see for that. Most of the changes we are discussing now have been planned and discussed for quite along time anyway, and as you say, the fact that there are more teacher education courses now than there were 10 years ago, the courses at City U, Poly U, I think they are having an effect. There are now more highly trained English teachers, more specialists than there used to be, and obviously this affects their methods and approaches.

OK, how do you think the changeover will affect the situation, status or morale of English teachers in HK?

15(97) The status, as I mentioned before, English is still important in the society, and the society still need us to produced graduates of good English, but as the importance of Mandarin is now becoming much more important, our status will be more or less a little bit lower down because maybe more money will be spent on Chinese subjects, reducing the amount of budget for English. And people will continue to learn Chinese or Putonghua because they think it is practical to be a good user of Mandarin because they need it to trade with people in China, so they think that English is not so important now, if they just think about trade with China instead of the western countries. And for the situation, what do you mean by the situation?

AU Within schools, the job that the English teachers are expected to do. At the moment, as an English teacher in a secondary school, teachers are not only expected to go in and teach English lessons, they are expected to train students for the Speech Festival, for example, and perhaps take part in debating, or anything else connected with the English language, school magazines and newspapers, that kind of stuff. I just wondered whether that is going to change in any way.

15(97) We still have the English Speech Festival, we still have debates, so it will be the same.

AU So English teachers are not only teachers of English, they're seen as being specialists in the English language.

15(97) They also need to train the students during the Speech Festival and sometimes there are some poems, English writing competition. We have got 
some activity like treasure hunt for the students. I don't think there will be changes in this aspect; we still do the same thing. And for the morale of the teachers, for English teachers, I think there will be no change as well because we still need to teach English, we still of equal importance on both Chinese and English, but the morale overall, of all the teachers, this may be affected. As we heard that, although we may not be so sure, our salary may not be so high after the changeover, or the present promotion system may change, this is what we've heard, we don't know the exact formation, so this may affect the morale of all the teachers. For the English teachers, I don't think there will be a change.

AU Unless of course, as you said before, due to trade with the mainland, Chinese and Putonghua become more important, so English will become less important, and the status and therefore the morale of English teachers ...

15(97) The parents also want us, the parents prefer an EMI school, so with the parents' support and the society's needs, English still has a very key role in the society. So, as English has a key role, we have the responsibility to maintain the teaching of English. I am very positive as regards the status.

AU I think everybody else I've spoken to has been positive, which you have to be.

15(97) But I don't know about one year later, we'll have to wait and see. Maybe we can't see the changes after one year, maybe not change so quickly.

AU Perhaps not, but perhaps after one year we can see more clearly how things might change, a year down the road after the changeover, whether the change in the MOI will have any effect. 


\section{Interviewee 16(97): English panel chair in secondary school \\ Date: 10 June 1997}

$\begin{array}{ll}\text { AU } & \text { Researcher } \\ 16(97) & \text { Interviewee } \\ \ldots & \text { Missing words / Unfinished utterance } \\ \text { - } & \text { Pause }\end{array}$

$\mathrm{AU}$ What are your feelings as regards the ED's decision to force $75 \%$ of schools to adopt Chinese-medium education?

16(97) Well first of all I can understand why they want to do this, there has been no move towards native-tongue education over the last 10 years, even though the ED has been cajoling and encouraging people to move in that direction, so they have obviously decided that now is the right time to enforce such measures. In the past they would probably not have been able to get away with enforcing something like this, but they now obviously feel that they can, that it's the best interests of HK education if they do so. As regards my feelings, it's a mistake in the sense that it's going to prove a disappointment I think when there is a major switch the native-tongue education because I think there's going to be a mistaken belief that this is going to automatically raise the standards of the students in HK secondary schools, which is a big mistake, because in any system there will always be a certain number of, it may be unfair to call them this, but failures, to a certain extent there will always be a certain number of failures and that doesn't seem to be expected in HK. This seems to be seen as being the savior of HK education and everybody will receive a higher level, a higher standard of education, which I think common sense and previous experience has told us will not be the case. So I think it's a mistake to go about it in this way. I don't think it's a mistake to encourage the use of mother-tongue education, but I think it's a mistake to go about it in this way and it raises false expectations. I think a further campaign of persuasion would have been far better than any plan of forcing people to carry out native-tongue education.

AU Do you think they've got away with this enforcement because of the changeover?

16(97) Oh definitely, I think there would have been a far greater outcry in previous years if there had been even a minimal tinkering with the system. There's usually a great outcry both in the media and through the media from public personalities and also from the public themselves, parents, who were concerned about what this meant for their children's future. They were also up in arms about it and were very public about their complaints, whereas now 
because it's just one of many changes taking place this has obviously proved to be the best time for the ED to try and do this. It's very cunningly planned, and obviously from their point of view it's the best time to try and carry it out.

AU I think the feeling is that, well as we know most schools teach through the medium of Chinese anyway, or at least mixed-code, I think the feeling in some quarters is that it is the better students who go to the English-medium schools anyway, and students who learn though the medium of Chinese will not necessarily succeed because they have been learning through the medium of Chinese for the past ten years or more anyway, it hasn't helped them. particularly. If they are going to fail, they are going to fail; the medium does not make that much difference.

16(97) I think the biggest problem with education in HK is that isn't often addressed, because there are so few professionals involved at the decision-making level. The biggest problem is in fact the narrowness of the focus of the education system here, it focuses on a few particular subjects and tries to establish some kind of excellence in those areas, much to the disadvantage of other areas and much to the disadvantage if not the majority then to a very large number of students in HK, and this constant focus on the academic subjects, again because of the figures of those who can succeed, is only of advantage to the top sort of 20 or $30 \%$ of students, so it's obviously to the disadvantage of 50 to $60 \%$ of the students in HK. And I think this needs to be resolved, this is a problem that needs to be resolved, to try and open out the system of education, you see it mentioned more and more often in the newspapers these days because there is a focus on the main policy areas and how they are going to change after the handover. There's a lot more discussion of these issues in the press and the media than there used to be, and some people are being very positive in the comments that they are making, for example in the newspaper this morning I saw that the PTU was proposing that more waiting be given to other subject areas at primary level, when deciding which schools the students should go to when they move into S1, which I think is a very positive move, but then at the end of the article which I read, there was a civil servant quoted as saying, well this is a very fine aim but I'm sure that in reality it cannot be achieved, which I totally disagree with, I mean how do you know it can't be achieved if you don't try to achieve it. So I think that is the major problem that needs to be resolved in the education system in HK, is the narrowness in the syllabus and the curriculum in the school system.

AU OK, how do you feel about the ED's planned introduction of benchmark language levels for language teachers?

16(97) Again, being in a very invidious position in that I'm one of the few nativespeaking panel chairpersons of an English department in a HK secondary school, I try not to be too critical of the standards of the language teachers, 
but from what I've observed myself in the two schools that I've taught in HK and the schools that I've visited and I know of from friends and colleagues, at the upper secondary level I don't think there's any problem at all with the language standards. The teachers who are teaching at certificate or AS Level, their standard of English is actually very good, and in the majority of cases they have been educated abroad and have developed fluency in the English language. I think it's at the junior secondary level that any problem lies, from $\mathrm{F} 1$ to $\mathrm{F} 3$, where the problem is that the teachers teaching language at that level may not really be wanting to teach language, it's just one of the subjects that they have to teach in order to have a full timetable in their school. You can have teachers teaching such subjects as far apart as English and mathematics and science and Chinese, we have this in our school for example and it's completely absurd that the teachers should be placed in this position. And so you have teachers who don't want to teach English, who don't have confidence in their own abilities who are actually teaching the subject, so I think that the problem lies at this junior secondary level. Introducing language levels may help in that it may encourage these teachers to take further courses themselves to try and improve their language abilities, and that may encourage schools to release those teachers to take those courses, which is the most important factor as far as I'm concerned. When it comes to in-service training is how little time is actually given to teachers to take part in in-service training. It's espoused at all levels and every school will say that they do encourage their staff to take part in in-service training. Then very little is done to allow them to take time of to take part in it, so in that sense perhaps the planned introduction of the benchmark levels will be a positive thing, if it does encourage teachers to voluntarily take new courses, if it does encourage schools to allow teachers the time to take part in new courses then I think it can be very positive. But it could also be very negative in the sense that it will affect the confidence of the teachers at those levels who will feel that they are being threatened, that they are being put under pressure or criticized without being given the opportunity to do anything about it.

AU I think the idea is, as you say, to improve language levels and should teachers not reach a certain benchmark then perhaps they'll then have to undertake certain courses to help raise their levels.

16(97) It creates another problem, doesn't it, in that where are the replacements for those teachers going to come from? Because there is a shortage not only of English teachers, but of fluent Chinese teachers to teach the Chinese subjects in many secondary schools. It is a major problem. So if these teachers are forced out of teaching languages because it is felt that their language abilities aren't up to the appropriate level, where are the replacements for those sorts of teachers going to come from?

AU From China? 
16(97) Well yes, but as far as I'm concerned the best thing that the ED can do is provide courses for those teachers, to provide further incentives for them, to take part in further training.

AU Oh I quite agree, I spoke to the people from HKU and the EA, whose job it is to implement this, basically that's what they are looking to do. Those people who are not specifically trained to be English teachers are having to go in and teach English, or Chinese to a certain extent. Perhaps for some of them it will be a way out, hopefully if I fail this I won't have to teach English any more.

16(97) Yes it could function that way it certainly could, yes.

AU Do you think there will be any other significant changes towards the teaching of English in $\mathrm{HK}$ after the changeover?

16(97) There only does seem to be one policy as regards the teaching of English, that's whether it's done or not done, anything else that affect the teaching of English in HK seems to depend upon the goodwill of the schools and the amount of funding that does come from the government. One area in which I can foresee a change is in the native English teachers scheme, which I think was previously the EELTS scheme or something similar, it's now become the NETS scheme, but the benefits of those teachers have been cut and I think a result of that is that they will not attract as many experienced teachers to $\mathrm{HK}$ as they perhaps did previously, and as a result less schools will be interested in participating in this scheme, so perhaps you will see less native speakers teaching in secondary schools in HK. The majority of them will remain in the tertiary level from vocational college level up, there will be very few who will remain in the secondary system itself. I don't know if that will be a policy change on the part of the government, or if it will just come about because of the lack of funding available and the lack of goodwill on the part of the schools to appoint native language teachers, because after all, the majority of the native speakers can only teach English, the one subject and many schools would like them to be able to do more than that. Because I'm not part of any scheme, my job in this school involves being a form teacher and taking care of the pastoral side of the education for these students, whereas in the majority of schools who have native-speaking teachers that is not done, because the ED they feel that it wouldn't benefit the teachers and it wouldn't benefit the students.

AU Not in my school, I am on the scheme but I'm also a form teacher. I've been in the school for six years and I've been a form teacher every year. I have to take extra-curricular stuff as well.

16(97) But in other schools that isn't the case, the native speaking teachers are exempted from those duties, which then, I think, maybe not consciously, but it does put up a barrier, it causes some resentment as it's seen as being a 
different case for those teachers. As I said, the government policy towards the teaching of English, to me it only focuses on whether to do it or not and I can't really see any major areas of policy that would be affected by the changeover regarding the use of English.

AU Do you envisage any change in the proportion of the timetable given over to English language teaching?

16(97) Yes, I think that will definitely take place, rather than on a wholesale basis across every school, I think individual schools will perhaps put pressure on the ED to change the stipulations in the curriculum guides so that not so much time has to be devoted to the language subjects. After all, English language as a subject only has the number of periods in the timetable that it does have because it is seen as being the MOI, therefore the extra time is required to bring the students up ... [tape ends] ... the other subjects, the heads of those departments and the teachers of those subjects will put pressure on the principals in those schools to change the weighting of the timetable so that they have more time for their subjects. As a result, the subjects which will lose out will be English first of all then Chinese language secondly. But yeah, I can see a major cut, maybe $20-25 \%$, maybe two lessons on average per week would be cut from the timetable for the language subjects.

AU One thing I thought of is perhaps schools do change from officially English to officially Chinese medium, which a lot of parents are against as they still believe that English medium gives the best chance for their children to progress into tertiary education, that maybe they would want to emphasize that English was still very firmly on the curriculum, even though the rest of it might move over to Chinese.

16(97) I think initially that could happen, and I think a lot of that if it does happen will purely be lip service because I know that in the majority of schools there is great envy of the amount of English on the timetable, and I know that in many subject areas the teachers involved don't feel it is necessary to do so. And if English is dropped as the MOI then there is even less need to do so. So I think within the schools, from without the schools as you say the parents may be worried and may agitate against any decrease in the time for English, but within the schools I think there will be great agitation for decreasing the number of periods allocated. I know that's the case here, it gets very repetitive trying to come up with the same arguments all the time to heads of other departments to try to explain why it is necessary to have so many periods of English on the timetable.

AU Of course the other possibility is Putonghua, not possibility, definitely, it will have to be included on the timetable. 
12(97) And just how much emphasis is put on that is another problem area, yeah, I agree.

AU And where lessons will come from.

16(97) Uh huh

AU Do you envisage any significant changes in your school or the education system more generally, which might affect the teaching of English?

16(97) This school's in a very invidious situation in that it's partly boarding school. We've got about a third to two fifths of the students who are boarders, and their parents send them here because of the reputation of the school for allround education, for the boarding facilities as regards looking after the children when they're at home, and thirdly for the English language education. It is English language education at St. Stephen's, and if that changes, I think it will have a major impact on this school because it will mean that there will be less parents interested in sending their children here, of the standard that we receive at the moment, and once those standards begin to drop, their will be a knock-on effect and more parents will be less interested in sending their children here. The boarding section will begin to suffer and may eventually not be cost-effective to keep it running, so I can see a major change in this school as a result. To a lesser extent I can see changes in other schools as a result particularly schools which are not Band 1/ Band 2 schools, they may be Band 3/ Band 2, something similar to that, or a mixture of levels, and if those levels begin to drop they may see themselves slipping down the ladder if you like, slipping down the league table, so that they go into the bottom half of the table, as it were. So I can see those changes happening. What the schools will do to respond to that situation, I just don't know. Once the ED sets these changes in motion, I don't see what the schools can do to turn it around. I think it will take a long time before there's actually a settling down effect in the system so that parents can actually see using native-tongue education, they can actually see some schools achieving more than others, and then there will be a focus on others, and those will be the schools that they will want to send their children to. But that will take some time to sort itself out.

AU So there will be like an elite within the Chinese-medium?

16(97) Yes, there will be an elite group of English language schools. There will develop an elite group within the Chinese system. Then there will be the, inverted commas, also-rans at the other end of the system. So you'll have a very strange system, you'll have two groups of elite schools emerging as a result. It will be very strange. 
AU Can you see the changeover affecting teaching methods or approaches in English lessons in secondary schools?

16(97) I don't think so. It is well documented now that the traditional methods do not succeed in producing students with the capability of moving from area to area, as is seen as being necessary the way that society has moved now. If that mobility is seen as being necessary, that flexibility is also necessary, and these are the two things that this system least develops in the students is the mobility from one area to another and the flexibility to deal with problems from different areas because of the focus on the few academic subjects. So I think there is recognition that if the traditional methods continue to be used in $\mathrm{HK}$, it will actually hinder HK. I was also interested to see last week in Singapore that exactly the same thing is being said, that Singapore often holds itself up to be the perfect example of where Eastern and Western methods come together, but even in Singapore they are realising that the traditional East Asian, Chinese, all them what you will methods are not suitable for this society any more, and they themselves are trying to promote change in their own education system. So I think that in HK it is generally recognised that traditional methods do not pay dividends in the modern world, I don't think there would be any move back to traditional, Confucianstyle of teaching methods, even with the influence of mainland China, I still don't think it will move back in that direction. I think it's generally agreed that different methods and techniques are required nowadays, and there will be a move still in that direction. A move in that direction many people would see that as HK cutting it's own throat, and there are very few people who are willing to allow for that.

AU All I was going to say was there are more courses for people to take, courses at $\mathrm{HKU}$, City $\mathrm{U}$, that are teaching English teachers to use innovative methods.

16(97) The students themselves wouldn't sit still for it either, in the UK, in Australia, in the US, in any country, it's very difficult to hold students attention and concentration in classes nowadays because they are used to short, sharp feeding, if you like, they get their entertainment in 10 minute snatches, so that tends to be all that they are capable of digesting nowadays. So I don't think the students would be willing to sit for an hour of traditional methods of teaching.

AU Yes, you walk into a classroom and it's kind of, okay, entertain me, and they want to do group work and they want to do interactive stuff, they don't want traditional stuff.

16(97) In one way I can see a slight change in emphasis in that I think that personality is more of a factor in teaching nowadays, than it has been in the last 10 or 20 years, and I can see that the teacher's personality will be a very important factor in whether the students are interested in learning or not. So I 
would then hope that this will be recognised in teacher training colleges so that the teachers themselves won't be afraid of using their personality in that sort of positive way. I think particularly for language teachers that is a very important factor, actually making the students feel comfortable enough so that they will use the language, is very important. The teachers' personality plays an enormous role in that respect, in making the students feel comfortable and confident in using the language.

AU Do you think the changeover will affect the situation, status or morale of English teachers?

16(97) The other ones were probably easier to answer than this one. Initially, I don't think so, particularly status because the English language schools, schools who are teaching through the medium of English, will be the elite schools, as I've said already, therefore the status of English as a result of that will remain quite high, and as you have mentioned, the majority of the parents are still of the view that English language education is what will be best for their children. Therefore, the status of English teachers would not fall as a result of that. It's very difficult to say what would happen to that over a longer period of time, if it became generally accepted that native tongue education could succeed, them maybe the status of English teachers in those schools would fall as a result. As I said before, it definitely wouldn't in the elite English schools, but maybe in the elite of the native tongue schools, maybe the status would fall there. The situation may change in the sense that there may be less need for so many English teachers, if there is a change in the timetable, every school will be dropping, every school which is forced to adopt native tongue education, maybe dropping one or more English teachers, so maybe the situation would change in that maybe those teachers that we've mentioned who won't be too comfortable using English, maybe they are the ones who could drop out of teaching English and get into teaching the subjects that they are more comfortable with, and more able to teach. I'm trying to think of anything else that would come under the situation of English teachers ...

AU Things such as the English teacher is often seen as being the expert in all things to so with the English language in schools, training students for speech festivals and essay competitions and all kinds of things.

16(97) Then in that sense yes, I could see a change there, but then again that's a change I've tried to put into effect here anyway because I'm not a great fan of the speech festivals, I don't think that they are good for the average student, that they pay any benefit at all, and being forced to participate doesn't have any benefit, sometimes it has the opposite. I see that it only has benefits for the students who are already comfortable using English, whereas our focus should be on the majority who aren't comfortable using English. So we actually only enter a minimum number of students for the speech festivals every year, and those students are entered only for the individual categories, 
we don't go in for the choral speaking categories at all. What we do try to encourage is more participation in public events like debates, if possible, and in the radio programmes partly organised by RTHK and partly by the newspapers. SO we encourage our students to take part in those, rather than worry about speech festivals. After all, what they do in the speech festivals is directly related to literature, and very few of them have English literature experience nowadays. I think the numbers entered for the certificate literature exam is down to something like 3 or $4 \%$ of the students in senior secondary in HK, so I don't see any advantages in that. Therefore if that was one of the effects because of this lack of pressure to participate in speech festivals etc., I don't think that would be a bad thing, and I think English departments could find other ways to shore up their situations within schools.

AU The time can be better spent.

16(97) Oh definitely, most definitely, and as for morale, very little will change for those teaching at certificate and A' Level, I don't see any impact on their morale at all, and those who are teaching at junior secondary level, some of those don't want to be teaching at that level anyway. Therefore, any change for them may be a change for the better. For others, there may be a slight impact on their morale, but teaching at junior secondary level, I don't think they see themselves as being the elite anyway, they don't see themselves as being the experts. I think they take pride in the job they do, and they see themselves as being important, but I don't think they see themselves as being experts in any way. And they perhaps don't aspire to being seen as experts. Therefore, I don't think it will have any major impact on the morale of the teachers. 
Interviewee 17(97): Principal of secondary school

Date:

22 August 1997

$\begin{array}{ll}\text { AU } & \text { Researcher } \\ 17(97) & \text { Interviewee } \\ \ldots & \text { Missing words / Unfinished utterance } \\ - & \text { Pause }\end{array}$

AU The first question is, what are your feelings as regards the ED's decision to force $75 \%$ of schools to adopt Chinese-medium education?

17(97) I think they should have done this long ago. It's been delayed, this decision. I support this decision. Actually, I should say that all schools in HK should use Chinese as the MOI, yes. But I don't think the ED, Manpower Branch, they will allow all schools to use Chinese, but $75 \%$ is better than 10 to $20 \%$.

AU Right, which is what it is at the moment. I suppose a question would be, how about the tertiary education on that point, do you think the students ...

17(97) Can they catch up?

AU If they were in Chinese-medium schools, do you think their English would be

$14(97) \mathrm{OK}$, as at the moment only $18 \%$ of students can obtain a degree place, they should be theoretically, the cream of the age group. It won't be too difficult for them to use English in the tertiary schools, tertiary institutions, even if they're using Chinese in their secondary. And some of the schools, even ED, I learned from the newspaper I think the day before yesterday that the ED will allow schools to choose their own MOI in the senior secondary, which in that case is even not a problem.

AU So you think it will be possible for them to have Chinese-medium in the lower secondary and then go into English-medium in the upper secondary.

17(97) Yes, the transition is very difficult especially for the Form 1, it will be easier for the Form 4 students. It will be most easiest for the tertiary students, if you need the transition.

AU I've put force there.

17(97) They need to add a few more. The best theory is to have no transition at all, so either you have using your own mother tongue for the whole period, 
primary or kindergarten to undergrad and grad school, which is the case for most countries, or you start to learn English in your nursery, no transition. But if you need a transition, the later the better, it's my experience, yes. I came from CMI schools and then when I joined the university I had to learn it all in English. One or two month's hard work and then it's OK.

AU In tertiary the people I've spoken to have agreed that they have to remain English-medium.

17(97) Good, it's OK, I mean most people in HK, I mean educators, got the illusion that if you learn in Chinese you are, you know, in theory, I couldn't agree less.

AU I think the point is just the internationalisation idea, I think, if they learn through Cantonese then it restricts them to a certain extent to HK or to southern China.

17(97) But using Chinese as the MOI doesn't mean that your English must be poor.

AU No, of course not.

17(97) You use English as the international language.

AU Parents worry don't they?

17(97) They worry because all they learn about MOI and CMI are from so-called educators, the majority thinks that English is much better, a better life, you earn more. I had two applications today, two interviews with two students, one a local S1 girl from a CMI school asking for a place here, another one a new immigrant, came here only four days ago, she finished senior middle one in China. I talked to her for a long time and I told her about the problems she's going to face in this school. I gave her a written test to tell me, to show her what the actual situation is. And I point out that if she applied to a CMI school, she can continue in S5, she's got quite a good academic result in China, and she said no. She still wants a S3 place.

AU In English-medium?

17(97) Because she will study in English. You see? Even a newly arrived immigrant got this idea.

$\mathrm{AU} \quad$ In China, English is really being pushed now isn't it?

17(97) Yes, they are pushing so hard. I don't think she'll do really poorly. 
AU It's very interesting. On another issue, do you think ... this issue of new immigrant children; will it affect your school?

17(97) Actually it shouldn't because we are fully enrolled, no vacancy, not even one, but I don't want to say no to the applicants. I even promised her this morning that if she did the test well, I might get another extra place in one of the classes. I have 5 S3 classes, altogether 20 students, 200 students, fully occupied. She told me that she heard all the statements from the schools she went, they all speak to her, well no place. I have no place here but if you are doing well and you are eager to learn, I might give you a place, it all depends on how you did the test. They have to have a place to learn, right? And the time is short, you know, it is late August. I don't know, but people are rejecting new immigrants all over the place.

AU What will the ED do in that situation, if they find they have a group of students without anywhere to study?

17(97) Most probably they will go to schools with, still with places, the least sortafter schools. Or in the primary schools, they will go to those endangered schools, schools with very few classes, going to be phased out, they will welcome those newly immigrant students.

AU OK, second question then is about the benchmark language levels for language teachers.

17(97) It's good, although it's not well received by the language teachers. I think if the language teacher received his or her education in language, I think he or she is qualified, no benchmark examination, nothing is needed. But if a teacher is going to teach language in primary or secondary school, well, if she has no proper training in language, then a benchmark is needed. But the point is that when the Ed first bring out the benchmark idea to the public, they didn't mention about two different kinds of language teachers, they just mention language teachers should be tested. It's humiliating for those properly trained university graduates in language. Why should I need a test?

AU Although having spoken to one or two people, HKU people included, I have told that only $14.5 \%$ of English teachers in secondary schools are actually qualified, trained as English teachers.

17(97) I don't know the percentage is that low.

AU Of course what you say is right, if they are trained as language teachers then they should be exempt from that, at least those who are practicing now. The authorities perhaps bring it in now for the new trainees. 
17(97) Certificate course, diploma courses - one of my English teachers is not qualified, but when she joined this school she took all the courses available. I think she's over-qualified now. Still no degree, but certificate in two-year course, workshop, I think that's OK.

AU I think the point of the benchmarking is not to hunt out those teachers who language is not good enough, I think the point is just to raise standards basically. People who don't come up to the mark will be given the help they need.

17(97) Positive thinking eh? It should be for education.

AU Whereas I think as you say, teachers look on it another way.

17(97) Even the PTU got this idea.

AU As a challenge to their competence.

17(97) The point is, we allow ourselves to be challenged, so we need quite a large space for the others to push us.

AU Certainly English teachers, probably Chinese teachers too, would say well we'd love to take in-service course to advance ourselves, but we don' have the time, you know?

17(97) If the courses are not related to promotion then very few will take these courses, yes, but if it is related to promotion then everyone got time.

AU That's it, if they give teachers time to do these courses, give them an extra scale point if they pass it.

17(97) If you have to, yes, as an incentive. Actually, that point has been raised in discussions, and then someone will say, what about those non language teachers, should they get the chance to improve themselves so that they can get an extra point? It's the case here, you know, we are always thinking about equality.

AU Well, I suppose language teachers may argue, if you worked as hard as the language teachers do then you can get equal pay.

17(97) The PE teachers, they work hard, they have to stay after school a long time for trainings, and science teachers, they are in danger of toxic substances. So, in this school, they have long ago told me that they are not going to count all the different tasks, you can't do that. Who is working more, no you can't do that. 
AU It is impossible to count, it's just part of the profession.

Do you think there will be any other significant changes in government policy towards the teaching of English in HK after the changeover?

17(97) I don't know. As it is planned that only 25 or so $\%$ of the students will be taught in English, that full scale English teaching will be for those green students, you know elite students, top students only. Then it might be a good point for the majority students to spend most of their time in other subjects, they don't have to waste their time in, you know, Chinglish learning, and English learning will become a subject not a language, not a daily life learn language. I would say that the overall standard will become higher in this way. They can learn a subject well, but they cannot learn a language well because they are not using the language every day, and they are not putting an equal amount of effort in this as a subject, in English as a subject, and so English in HK, even for me is not a good subject. So we may tell those people, try to think of English as a subject. Let us ask ourselves how to teach this subject better. Learning method will be changed, teaching strategies will be changed. Maybe we should not use so much communicative approach. There are always two different kinds of teaching methods argue with each other, should we learn English by grammar, by rules, by drills, or we should use it in daily life. We are using communicative approach now, thematic approach, all these modern things. I support modern things, but the result is no one is using English as they should be. A F2 boy of this school asked for a pre-examination because he was asked to be detended [?] [Cantonese], okay, $I$ give the pre-examination the day before and the grammar is very very poor. I look at the sentence and I translate every word into Chinese, what does it mean? A F $2 / 3$ student, it's average, even for school cert. scripts, full of these Chinglish. We are talking about English learning, change of sovereignty should be a chance to change better, if not change of sovereignty is not to do with daily life in English because it provide a change for us to change differently what is English.

AU That is probably one of the things I was aiming at, this idea that now that we have changed sovereignty that English is no longer the language of the colonial ruler, it's now the international language, so there's no difference between people learning English here, and people learning English in Mexico or in Vietnam or anywhere else.

17(97) Or even in mainland China?

AU Or even in China, precisely. As it is now, you must take English, you must take Chinese, you must take maths, it's a core subject, I think what you are saying is that it shouldn't be a core subject that they are forced to learn, okay they'll still have to do it in secondary schools, but it should be a subject, like history or science. 
17(97) What I'm thinking is, with EMI the whole population of students become second class people, because they have to, just like I'm doing now, you have to do a translation in your mind for everything you learn, so how can you learn it efficiently? So from one year to two years, from S1 to S5, the student is stuck, is stumped for so long that he couldn't even recognise his own capacity of logical thinking or ... so if you put aside EMI, you learn in your mother tongue, you think straightly. You are now your ability to throw into all directions. I would say that the student become a better one, a more able one, when you learn English as a separate subject. The result will be much better, that is always my feeling. But I'm not going to change this as a CMI school, because the students are qualified to learn in EMI. So the management committee, the majority teachers, the majority parents will say to me please apply for exemption from CMI, so I don't like the job! I love doing what I think is the best. Today I told the managers in our management committee, one of the managers asked me, what do you think ... CMI is definitely better, but I will ask you to consider EMI because my colleagues, parents, on their questionnaire I have to present you with these requests, which is not my ... but I have to do it, which is as a matter of fact in line with the manager's ideas so I think we can apply for exemption. And the government will definitely push at least $25 \%$ to learn in English, they will not push $100 \%$ CMI, no.

AU No, they can't, can they? I think ...

17(97) They don't believe themselves that CMI is best, they don't believe that. Yes, I've been talking to Mr. Leung, to Helen Yu, I told them ... you are not promoting CMI, you are promoting EMI instead.

AU The wording of whatever they put out is that they believe that the majority of students are best learning in CMI, they never say all students are best learning in CMI.

17(97) The majority students are not the best students, right? We are covering a few questions.

AU Yes, I think so. So, perhaps no other significant changes in government policy at the moment.

Proportion of the timetable ...

17(97) For the CMI schools, more periods in English, because want to keep ...

AU If they're learning their content subjects through Chinese then they ought to be able to learn them quicker, allowing perhaps more time to spend on English. 
17(97) That's the logic. So that English will become the subject with most periods allocated, as much as two or three other subjects put together.

AU Something that I've not mentioned there is Putonghua, something else which is going to come in.

17(97) It's coming, I don't know, some say it's going to be a core subject, which means that you have to teach, no matter what.

AU So again, space will have to be found on the timetable for that.

17(97) I don't know. We are going to start a two year project in this school but not starting from September, we've got too much to do, maybe January I'll start a two year project for curriculum review. So we are going to spend more time in this subject, in that subject, we going to teach ... civic education, patriotic education, more about China. Okay, what can we do now? We are fuller than full house. So we have to rate those different subjects, maybe some traditional subjects have to give way. We have to re-arrange our priorities. We are going to do it with a committee, a team. It's not an easy job. You know, I am reading some books about curriculum reform in HK, unfortunately it's in Chinese, but I don't think I can use the ideas in the book because they are very revolutionary and you can't be revolutionary in HK. In the end, we have to take part in the only kind of evaluation is available, is CE. So far I've read this much, already I think I can't finish the book, I don't think I can use all those ideas.

AU What kind of guidelines do the ED give you as regards how you design your curriculum?

17(97) I've never seen one. Even if they do have one it's not practical.

AU You mean they tell you now you have to teach Putonghua but they don't tell you how you are going to fit it in?

17(97) No, if you put all those suggested time allocation together, you see that you need nine days week. I'm not saying nine days cycle, no, nine days week, to meet all the demands. Home economics should have two to three periods a week, probably preferably three periods, we can offer only two. You know the story?

AU Yeah

17(97) Nobody cares about how you teach the subjects, they just care about how they put forward, what they suggest to the public, to the ED. So we have to do it ourselves, we have to tailor our own curriculum. 
AU. Which I suppose could be how you would like it. I mean you would like that flexibility to tailor your own curriculum to suit your own students.

17(97) So what I demand is that we try our best to tailor the junior forms, senior forms our hands are tied. So if you can do it better in the junior forms, okay we'll have our students in better shape to enter the senior secondary. That's why we are still thinking about curriculum reform.

AU The next one is do you think there will be any significant changes in our school, which we've talked about quite a lot already, or the education system more generally which might affect the teaching of English? Again, we've talked about Putonghua and how that might fit in, and you've mentioned civic education as well, which is something new.

17(97) Do you think there will be any significant changes in my school, yes, if we apply for EMI then we should use more English in school. I have to speak more in English, you know? I seldom speak in English.

AU Are you EMI now, or CMI?

17(97) We are using English as MOI in junior forms, they are very ... in junior forms, not in senior forms, because in senior forms, when you teach physics, you know, you've got a lot of very complicated ideas. Teachers are saying, please tell us to use Chinese to teach complicated ideas, so in senior forms, Chinese as MOI using English textbooks, but in junior forms, we use English textbooks, we use English in the classroom. But if we apply for exemption, then I would say that from S1 to S7, English. Please improve your English; clearly, they have to speak more. Assembly in English, school notices in English, otherwise, well, we are cheating ourselves. We are EMI but English is still only a subject, not a language in school.

AU It's a question of whether English is a true medium in English-medium schools.

17(97) An English environment.

AU An English environment sure. My school, I've just resigned this year, I've been teaching for six years at Heep Yunn, which is called an English-medium school and generally speaking it is, but of course a lot of teaching is done in Chinese.

17(97) I didn't know that. I have an idea that it is an English-speaking school.

AU It is but a lot of the teaching is done in Chinese.

17(97) Complicated ideas? 
AU Well yes, from what you were saying, upper forms in physics, chemistry, mathematics, economics, principles of accounts, the teachers all do it in Chinese now. They use English textbooks. A lot of students, and they are good students, Band 1 students, a lot of them would struggle if you did it in English, purely in English.

17(97) Heep Yunn, very good school.

AU Standards of English are still pretty high, although they are probably dropping a little bit. I would think amongst that band of elite schools, we've probably dropped a bit

17(97) The situation will become better once the CMI is enforced, because in that case the best students will not be distributed into many schools, they'll be focused into the $25 \%$ good schools. So in this $25 \%$ schools, the ability of the students will become better.

AU That's the theory I suppose. I know that at Heep Yunn, most of their intake comes from their primary school. Those from outside are given entrance exams. And the lower ability students, some students are probably Band 3, not very many ...

17(97) They are not allocated?

AU They will come from the primary school. You say that the best students will go to the $25 \%$ EMI schools, perhaps over time there will develop like an elite within the CMI schools.

17(97) Yes, going to, should be. I don't see any problem there. Even for CMI schools, there must be some who are more famous than the others.

AU Okay, teaching methods or approaches, you already mentioned that the teaching methods or approaches could well change.

17(97) To teach English as a subject, maybe more drilling, less communicative. There are certain rules you have to learn.

AU But in the end it's still a language, isn't it? If they are going to use it, it depends on what the students are going to use English for. Probably they won't use English to chat to people. If they use it once they leave school, if they use it in their work, then it might be predominantly written.

17(97) Okay you use language in many ways, you write it, you speak it, and if you learn grammar, you learn tenses, you can write better sentences. Nowadays students do not write correct English. If you do not write correct sentences you cannot speak correct sentences. So, the so-called EMI schools provide 
chances for the students to use English wrongly. That is why the English standard of HK students is so poor; even they spend so much time in the socalled EMI, because the EMI provide chances for them to use English wrongly.

AU So they can practice, but they practice wrongly. They make mistakes but their mistakes are never actually corrected?

17(97) We don't correct them, we can't correct that much, so they think that they are doing right.

AU I suppose that's the idea of having native speakers as English teachers.

17(97) But then when you have a native speaker in the school, you know that in our school we conduct all our meetings in Chinese, we emphasis the different aspects of a teacher's duty, teaching in the classroom, counseling after school, talking with the parents over the telephone. A native speaker cannot do all these. ECA okay, he can participate in extra-curricular activities, to take care of the English Society, but it's not easy for you to ... in other aspects, parents cannot come to see you comfortably. That's why I'm always hesitating to employ native speakers.

AU I have the same experience in school.

17(97) But in Heep Yunn I don't see these kind of difficulties.

$\mathrm{AU} \quad$ Not to the same extent perhaps as it might be in other schools, but staff meetings, although we rarely have staff meetings, but they would be in Chinese, the headmistress would simply ...

17(97) Do you speak Chinese?

AU Not well enough to take part in a meeting.

17(97) Chinese cannot be learned in 8 years, unless you spend a lot of time and effort.

AU So I wouldn't have to go to the meeting, after the meeting she would just tell me herself what happened.

17(97) So you can never be a full participant?

AU Not $100 \%$ no. I mean I took extra-curricular activities, I was in charge of the hockey team ...

17(97) You are included in a curricular reform committee? 
AU No, even in meetings, perhaps promotion meetings, they would take place in Cantonese, which is fine, I understand that, but I know that all the teachers there speak English, maybe 70\% of them are English teachers, yet, it's very strange. Some political things going on as well. So the idea of having an expat teacher, they are a language resource but they can't, they are always going to be different; they are always going to be separate.

17(97) Now I am involved in promoting CMI in diocesan schools, I'm a committee member, and we spend a lot of time and resources in promoting standard of English in diocesan schools. We employ quite a number of expatriate teachers, native speakers, but in the extra-curricular activities, in the immersion, in conducting English-speaking classes after school, during weekends, immersion camps.

AU So, out of six years I spent at Heep Yunn, I enjoyed it very much, but I always felt not quite $100 \%$ part of the school because of the language. Sometimes it was advantageous, sometimes if there was gossip I kept well out of it.

\section{7(97) Maybe that was good!}

AU Yes. And the final thing is, how will the changeover affect the situation, status or morale of English teachers? Again, we touched on this when we talked about the benchmarking.

17(97) Question 7, it's the only one I've no idea. I've put down nothing.

AU Well, the status of English teachers will I suppose depend on the status of English.

17(97) It should not be changed, the change of sovereignty should not change our language, should not change our values. Maybe most of the students will learn in CMI, then English teachers got a higher status. Maybe, or maybe worse, I don't know. My English Panel keeps asking me to use more English in school to help them in their work If the school is CMI, what become of that, I don't know. What will they think of themselves? Elite group among colleagues? Or a downgraded grouping? I've no idea. It's for you to find out, right?

AU Yes, but it won't be until next year that the changes come in. Whether they are actually affected by the changeover, whether it's to do with the changeover, again is open to question anyway. The change in the MOI is nothing to do with the changeover; it's been planned for years.

17(97) Anyway, you've got a lot of data on this topic. 
AU Yes

A -168 


\title{
Interviewee 18(97): English panel chair in secondary school Date: 11 June 1997
}

\author{
AU Researcher \\ 18(97) Interviewee \\ ... Missing words / Unfinished utterance \\ - Pause
}

AU What are your feelings as regards the EDs decision to force $75 \%$ of secondary schools to adopt Chinese-medium education?

18(97) I think for the majority of students here in HK it's the right step, the right policy. Well I think actually most students may have difficulty using English as the medium because actually most of the students are struggling hard to learn English and if they have to use English for other subjects it may really ... affect their performance in their studies. But actually I think most students or parents they agree that we should use mother tongue to be the medium but just people may worry that students may get into a school of less ability or a lower standard so nobody would like to change, so the government has to do something to enforce it, to take the lead. That's what I think. But actually teachers are facing difficulties too because some teachers, frankly they are not confident in using English totally, or in Chinese because they were brought up, they had the education in English, so actually everybody's suffering.

AU Is your school English medium?

18(97) Yes, supposed to be and we plan to apply for EMI in the coming years. But already some teachers are expressing fear, they worry about the preparation, but I think they will manage. But of course they have to do some hard work preparing. We hope that the students will be able to cope with it so we are trying hard to promote the atmosphere for the preparation of coming years.

AU So that means that if the school becomes EMI then the new EMI will be strictly enforced?

18(97) Yes

$\mathrm{AU} \quad$ Even though it's officially EMI now, like many schools.

18(97) So we have to put double effort, to train the students and even help our teachers prepare for it. Not only within lessons, but even extra-curricular 
activities or the whole atmosphere of the school should be enforced towards that direction. So it's not an easy task but, well, we have to do it, don't we?

AU As you've said, some people do think English medium gives the students a better opportunity if they should go on to tertiary education.

18(97) Yes, yes, actually there is really a gap between secondary school and the university education, so if they are really bright students then they can cope with it and they are happy learning English, then it's a good choice for them. Because our school is a Band 1 school, supposed to be, we suppose that we should have the very bright students, so if they can't do EMI, then I think many other schools cannot cope with it. So that's why our principal decided to try with EMI, really enforce it, but I think our students will be really weak in speaking and really communication with teachers, but for written work and exams, they can cope with it. For real communication, there's a lot more to do.

AU I've said force $75 \%$; it's perhaps not quite that number.

18(97) Well I really haven't thought about the figures.

AU It's something like, I've heard a hundred schools with EMI, I've heard 96 with EMI.

18(97) Yes, but I think it should really be up to the school to decide. I don't care about the percentage but I don't think the government will really restrict the number to that percentage, I don't know. Maybe fewer schools than this percentage will apply.

AU Do you think there is any significance in the timing of the decision, to do it ...

18(97) Well maybe it's because of the handover of HK to China, maybe it has some effect, yes if you really have to do something you have to choose this time. Otherwise it will just drag along and everybody is waiting for someone's decision.

AU Okay, what are your feelings as regards the planned introduction of benchmark language levels for language teachers?

18(97) I definitely oppose this, I don't think it is the right direction to raise the standard of the language teachers. You do not just give them a test and they can improve themselves, you really have to give them a lot of support, training courses, better materials, more sharing workshops, support from schools, among schools. I don't think giving a test will work. A lot of people may not feel good about this. Do you have a test in England like this? 
$\mathrm{AU} \quad$ For language teachers?

18(97) Yes.

AU No, I don't think so, I was never a language teacher.

18(97) I mean foreign language, you may teach French or Spanish in England.

AU I don't know actually whether there is a test for foreign language teachers to test their competence. It's an interesting question.

18(97) So maybe if you really want to raise the standard of English teachers or language teachers, you have to be stricter when you recruit them, in the training, the teacher training. So f you think that they are not good enough, then why do you accept them as language teachers? So you just enforce that the teachers should be up to standard and then the schools should give the teachers the teaching duties, otherwise ask the teachers to teach other subjects. At this moment, to give them a test or several tests, I don't think so.

AU I think perhaps the idea is they be given a test and then they ...

18(97) Rank them?

AU They'll be given, I have heard a figure of 10 years to improve. So it might just be an incentive, or a disincentive.

18(97) I think it is better to say, after 5 years or 10 years, you must attend the refresher course, that kind of thing, or you provide more courses for teachers to upgrade their standard. Well actually it's really a problem. Is it actually about language teachers? You know some other subjects' teachers; I don't know if they are really up to that standard. It seems just discrimination, just penalise language teachers and give them a hard time. Actually language teachers are already working very hard at school, but if you think that they are not good, okay give them training, give them support, free them from school and then. I think it's a better idea. And actually you have to administer the test for so many teachers, you also waste a lot of money. And you need a lot of staff to set the paper, mark the paper, the running cost is not little, I think.

AU It will be run by the EA.

18(97) Yes, but it still needs manpower, money.

$\mathrm{AU} \quad$ Certainly the idea of there being language tests for all teachers ...

18(97) All teachers, yes, all English teachers and Chinese teachers. 
AU Whatever medium they have to teach in, particularly if it's not their first language. Teachers of other subjects in English-medium schools ...

18(97) They also have to take the test?

AU Not at the moment, no.

18(97) But in the future?

AU I think that's what they are looking at.

18(97) Will it include speaking and also writing? That will be a terrible thing. When actually is it formally announced that it will take place? In the coming year, 1998 perhaps?

AU I talked to the people at the EA and they wouldn't comment.

18(97) They wouldn't comment. Maybe from the administrative point of view it's right, but for the teachers on the whole, I don't think they like it. But even the language teacher has very good skills, very good language, it doesn't mean that he or she is a good teacher. What about the teaching method and how are you going to raise standards? From my experience the main problem is the teaching method, yes. We have teachers with very good standard, but I don't guarantee they are teaching very well. The main problems I think, especially when they are teaching primary school students or even lower form students, secondary school students, your language skill is not really so demanding, but teaching method, that matters I think.

AU Perhaps the thought is that certainly in the I of Ed, and with there being a lot more courses for teachers, BA courses, MA courses, to improve the teaching methodology, they still need language levels to be improved.

18(97) If they run courses really that can meet the need of the teachers either to improve their skills or upgrade their standard or status after getting the degree, naturally teachers will go for it, will join the courses, but the test ... it's not a positive way. That's what I think, and we all look to the Institute and we hope that they train good teachers and believe that teachers should be very important in education.

AU Do you think there will be any other significant changes in government policy towards the teaching of English in HK after the changeover?

18(97) I really can't think of any but we just hope that they can really cut down the workload and have more English, I mean language teachers, but I really don't know if it is related to the changeover but we hope that the direction should be like that. But nobody can predict policy. But we also worry that there may 
be more students, I mean more children coming from China, and it's difficult to predict the whole number and it may affect the whole education system. We don't know what's going to happen.

AU If there are more students and they are demanding higher and higher levels of competency from the teachers, then more and more teachers will be needed.

18(97) Actually we are striving for fewer students in each class and possibly the picture won't be true if more children are coming from China. But definitely teaching is not easy. This is going to be more and more difficult, and if the funding is not increased then it will be more and more difficult. It seems the picture is not so good.

AU What changes do you envisage in the proportion of the timetable given over to English language teaching in English and Chinese-medium schools?

18(97) I really don't know because actually for our school the school is taking our English lesson to make way for Putonghua, so even though you may be using EMI you have so many subjects struggling for time. I don't see any positive change in the timetable for teaching English. But for the Chinese-medium school, if I planned the curriculum for the school, if they had other subjects in Chinese I think they can spend less time for the individual subjects and they should give more support and emphasis on English teaching lessons, because if they have Social studies or history or science taught in Chinese, the children need less time to understand. If so they need not struggle with the English, the vocabulary, then they have better comprehension and if they still teach the same amount of content, they need less time, and then the school should have more emphasis on English, more lessons or whatever.

AU I've heard both views actually, I've heard that, people tell me that, for example, in English-medium schools they need a level of English so they can cope with their other subjects, whereas in the Chinese-medium schools they don't need the English because their other lessons would be taught in Chinese, so the amount of time spent on English may decrease.

18(97) That is another view, right? It depends, if they no longer have contact with English in other subjects, if you really think that English is an important language, is an international language, if the principal thinks their students should learn English well, then you have to give them extra support. If they think, okay, English is just one language, either you learn it or not, the emphasis is not that heavy then you may just cut the timetable for English. But it's really true that for a school just like this, if they have other subjects taught in English, actually students need more training or time to practice English. But I don't think the school will give you extra time in the timetable. Possibly then you have to think of other activities, of other whatever outside the timetable, so possibly the English Society or the whole English 
Department should think of other ways or English-speaking weeks or whatever.

AU Of course the other question is that of Putonghua. As you've said, they are actually going to take lessons away from English to fit Putonghua onto the timetable.

18(97) Yes, so from F1 gradually to F2 and 3 and even up to F5. But I'm thinking if it's really possible if some students, they really don't enjoy learning English, they don't think it's useful to them, why don't they just choose English and Putonghua as electives, either one? Then nobody's struggling for time. Don't force them to learn it if they don't feel like it. If they have the CMI at that school, and if some students don't really see the point of learning English, then they just don't take the class. I don't think whether it's possible.

AU As the system stands now, all students must take English, otherwise they'll certainly have no chance of further study.

18(97) But maybe after adopting the CMI, they don't have to worry about other subjects and they don't hate English so much because it affects their academic results in other subjects, possibly some students will enjoy learning English and if the teaching method can improve and all the teachers are happy with the workload and they have more creative ideas in teaching then possibly the picture will change. But it's not going to change overnight, you have to wait and see and adjust and improve and every time you change to fit the situation.

AU That's certainly going to be interesting, where Putonghua is fit into the timetable because presumably it will be taught from next year because they plan to have the certificate exam in the year 2000 .

18(97) Certificate exam in Putonghua? Well actually most students will see the point in learning Putonghua rather than English because we have contact with China and you can see the trend, not only in the business field more people are learning Putonghua. Most students they can't see the point in learning English, when they go out they never use English, they don't see their future going to England to work, or America. So most students, even F1 students, when you ask them do they want to learn Putonghua they say yes. What about English? They should say.

AU Although it probably would be their parents who make the decision not they themselves. Okay, do you think there will be any other significant changes in your school or the education system more generally, which might affect the teaching of English? 
18(97) Changes in our school? Possibly there may be some students from China. So far we do not have many but possibly in the future we'll have more students of various standards, I mean English standard coming.

AU Do you think being in the NT you are likely to get more students from China?

18(97) Possibly, yes, and if we have EMI there may be changes in our school also. We don't know about the choices of the parents of the students in our neighbourhood. But people predict that the schools adopting EMI will be more prestigious, so possibly we hope that we can have more, I mean brighter students, we hope so.

AU I think that's what people think. Certainly there is this group of elite schools at the moment caused by the banding system, so a Band 1 school is generally English medium, and they form an elite group. I suppose if you make the English-medium schools a smaller group ...

18(97) Yeah, people will be competing to get into these schools.

AU But I suppose among the Chinese-medium schools, over a period of time there will become an elite group of Chinese-medium schools and they will accept brighter students.

18(97) But I think if you can group the students of the same kind, it's easier than pooling some students with really difficulties. Though if they are really good in English and they all come together and we can create an atmosphere and help them and educate them. It's a good picture. But the results may not appear so soon, so we have to wait a few years until especially the exam results will show something. What other changes, I don't know, of course we hope there may be more emphasis and help on the teaching of English, but it's also a long way to go. But the trend is we hope that we will have more computer-assisted learning in school. Actually these few years we have more equipment, well actually everything is improving. We hope it will go on after 1997. So if we have more self-access learning and so we can help students with English outside the classroom and when they go back home they can still help themselves. But maybe it's a bit too ideal but the direction should be correct I hope.

AU I suppose the only thing on the horizon is the TOC.

18(97) Everybody is worrying. Well actually I haven't looked at the materials, I just have a brief idea of the rationale. Well actually I think the trend should be correct. We just hope that it will not give us extra workload to prepare the materials or to do the marking. The trend should be correct, because when you look at the textbook now, I don't think they are really training the students some specific task. Possibly just understanding some comprehension 
and you learn some vocabulary. If you ask them to perform something, it's not good. I don't know about your school and your students, but possibly, we really need to improve that aspect.

AU Yes, but the fact is I suppose that it is being brought in at primary school level so the students will have been brought up with it.

18(97) But actually what's the feedback of the primary school teachers, have you got any?

AU Only a little bit, in fact a lot of primary school teachers are unhappy, they've not been given sufficient resources to do it and a lot of teachers are too traditional, they don't want to change.

18(97) When actually the main problem is the teachers, they are not ready to accept changes themselves, and while the education policy the teaching methods, everything is changing, they are just not ready to change and that's the main problem. But it's true that they need extra support, resources, training sessions, that kind of thing.

AU Do you think that the changeover will affect teaching methods or approaches in secondary schools?

18(97) I think the teaching method should change, the approach should change, but whether it's because of the changeover, I doubt it, unless there is a policy from the authority, they really want to change it, otherwise I just hope that we follow the trend of the international changes. The trend, I hope that we will not go back to the traditional training because especially the teaching of English in HK is much better than China, and we should accept changes, adopt new methods. We should look out for any brilliant research or whatever, findings, internationally to integrate them into ours, soon I hope. I hope it's not the changeover that is affecting our changes.

AU Although my focus is on the changeover, a lot of the changes that are being brought in are not directly related to the changeover.

18(97) Well actually from the ED and the government and the public, people seems to focus on the language, both English and Chinese, so we hope they will see the importance and they are trying to improve and help us instead of giving us difficult tasks.

AU I think, as I mentioned before, there are a lot of in-service courses for teachers now and was in which teachers can improve their own qualifications, and these things feed back into the classroom. It's amazing to me these days just how many English teachers have masters degrees. The days when 
teachers were simply trained at the I of Ed and the education colleges seem to have gone to a certain extent.

18(97) Yes, yes, yes

AU Even those teachers who are coming out of the teacher training colleges, they later plan to do a degree, simply for financial reasons to upgrade their status.

18(97) One reason is that there are a lot more courses provided nowadays. If you say 10 years ago, if you wanted to study, there were few choices, nowadays there are many courses, local, overseas, combined, Open University, it's a lot more easier nowadays. Of course you can't deny that there are many conscientious teachers that are really trying to improve themselves and that is a very good sign in $\mathrm{HK}$.

AU I think as regards approaches in English lessons, I think the students are very much aware these days of the possibilities of teaching English in a more interesting way. I think you now go into a class in some schools anyway, they expect you to do something. They want to do activities, if you say, okay we're going to do this reading comprehension ...

18(97) Oh they don't like it.

AU They want to do group work and so on.

18(97) Maybe in your school the students are ready for your approaches, maybe they have some teachers to compare with, before that they have everybody like that, then they have no comparison, especially now, students have very little attention ability.

AU A short attention span.

18(97) Yes, a short attention span, and they have so many things so exciting outside. If you are so boring then they just don't like it. It's good that they are ready to accept it.

AU The last question, how do you think the changeover will affect the situation, status or morale of teachers of English?

18(97) Well I don't see any change in the status, but I think the workload will be heavier, and we have just discussed the problem English teachers will be facing, so possibly it is a difficult task. But for the morale of English teachers, I think actually there should not be any effect, as compared with other teachers, sorry, other subjects' teachers may have worries over their morale because they have less freedom. Possibly it is quite optimistic, and we hope 
that people may find English important and they will have more respect to English teachers, I hope.

AU We all hope that. Again, how the changeover itself will affect English teachers is hard to say. We won't know for a year or two at least.

18(97) I have no definite answers to that. I just hope that the status will be higher. I think it will be higher, because as people are focusing on language learning and more people are aware of the techniques of English teachers having, that they should have, then I hope that we will have better status. But one worry that local teachers should really work hard because it seems that the ED and even principals they have more confidence to have English native speakers as teachers, and we of course see the advantages, we really see that native speakers should help, but that's why local English teachers should really work hard so that they should come up to the same level. If their language skill is not that high, they are not native speakers, but at least their teaching methods and their understanding of HK students should make up for difference. But actually we like to see the trend of having more native speakers in our schools, because they may bring in different views, different methods and also they give us some language support really.

AU Certainly the ED is trying to push the native speaker scheme.

18(97) Yes, we have two here this year.

AU They had the expatriate scheme before, now they have the expatriate scheme with less benefits.

18(97) Because they are going to have more teachers. But I don't know if the benchmark language levels will affect the teachers. I hope that the teachers on the whole will see it as a positive stimulus to them and so they will just accept it. I just think that teaching English in HK is a very challenging job. Although it's very difficult but it's challenging. It seems like we are fighting a war against so many obstacles, but we really have to work together, and you have to convince all the English teachers and ask them to try and that's the trend. But I don't know if the changeover will affect anyone. Anyway, people now are affecting the change actually, all of them can foresee not very negative changes on the whole, I mean to the society of $\mathrm{HK}$ and people are staying here quire ready to accept the new government. And so I don't think the change will be too much on teachers themselves. 
Interviewee 19(97): University educator

Date:

6 June 1997

\begin{tabular}{ll} 
AU & Researcher \\
$19(97)$ & Interviewee \\
$\ldots$ & Missing words / Unfinished utterance \\
\hline & Pause
\end{tabular}

19(97) The first one, my feelings as regards the ED's decision to force $75 \% \ldots$ I think it's the right thing to do. The government should have done something about it a long long time ago. I think the reason why most of the people within the ED now are native Chinese of HK, I think probably the people inside the ED thought of this a long long time ago but nobody had the courage to speak up because the British were still in control and as the British are going the people inside the ED think that now it's time for them to do something. Although it's a bit too late, I think this is the right thing to do. Seventy-five percent ...

AU It's an estimate, I think, it's probably more like 70. I don't think they've given clear figures yet, but I've heard that about 100 schools will remain English medium, 96 has been quoted to me, so it works out at about $70 \% / 75 \%$.

19(97) To my knowledge the figure is based on a study done by Keith Johnson, at HKU. If indeed it is true that only about one third of the students in $\mathrm{HK}$ are capable of learning through English, I think that's the right thing to do.

Second one, what are your feelings as regards the ED's planned introduction of benchmark language levels? I don't think this is quite realistic since most, or I would say a large proportion of English teachers in HK are not up to the ... as a teacher trainer maybe I shouldn't say it but I have to admit that many of our teachers, many of the trainee teachers, I'm afraid are not quite capable of teaching through English, even though they are English electives. To ask them to take this test is okay, but I'm afraid most of them would fail, and what would happen if most of them failed, as we still need teachers in HK? It's a very realistic problem facing us in HK, and not only HK but many countries around the world, in New Zealand for example, they are very short of teachers and they have to allow people who have not been trained to go into their schools to teach. So that happens everywhere. So, I'm sure that it is a very well designed test and is capable of assessing whether teachers are capable of teaching up to that level. Many of them would fail the exam, and what would happen if the government says, hey you can't teach, I give you 10 years time, I give you 5 years time to try to bring yourself up to the right level. That may not happen, let me say we still have to allow teachers who 
are, who have been proved to be capable, to continue teaching through the medium of English. That may put a little pressure on teachers, but I don't think that would change things very much, unless the government is prepared to spend a lot more resources on the training of English teachers in HK. Unless they are prepared to make the working environment in schools in HK more favourable for English language teachers, for teachers teaching through the medium of English. I don't think much will change.

$\mathrm{AU} \quad$ Or to attract better graduates?

19(97) Yes, right now I don't think the working environment is too attractive.

AU Although having said that I think for many young graduates, certainly the starting salary for new teachers is quite attractive, compared to if they went into business. Potentially they could rise a lot quicker in business, but if someone is looking is looking for an attractive starting salary then teaching is a good option.

19(97) Maybe that's only one thing but maybe the only one thing that makes the job a little bit attractive to some young people, but in the long run, once you get into a school, I mean one of our fresh graduates here, when a fresh graduate from a teachers' training college gets into a school, although the money is good, but you can't face the students, you can't control them in class, you find yourself so inadequate the moment you get into the classroom you panic, you don't know what to teach. No matter how good the money is, you can't survive. If you can't survive you have to think of leaving. You can't go in there being ridiculed by the students, finding out that your confidence is shattered. Although the money is good, as I say, they can't survive long.

AU Do you think the benchmarking will have a washback effect on you, on teacher trainers that it would force you into a situation where you would have to try and improve their English standard, and therefore the government might then give you more resources to do that, so it might work in your favour?

19(97) I think we are trying, at least the people in the English Department are trying, but we need the cooperation of our colleagues in the other departments, who I'm afraid may not be too much in favour of this. I don't know how much support the English Department here is able to get from the other departments of the Institute, but as far as I know the people in the English Department are working very hard to help other people in other departments realise how important it is. If we say that we have, you see that our Institute here is divided into two streams, one preparing themselves through the medium of Chinese, the other streams having students prepare themselves through the medium of English. So things are a little bit funny here. 
AU That is for English teachers, or subject teachers?

19(97) Subject teachers teaching through the medium of English. But things are a little bit funny here because we have, I mean our colleagues in other departments, in the morning they may be teaching a class of students who are preparing themselves to teach through the medium of Chinese, now in the afternoon, they may be teaching a class of English stream students. You can't possibly expect them to switch the language. In the morning they lecture through Chinese, in the afternoon they lecture through English. So, when we ask our colleagues, if you are training students who have to teach through the medium of English, when you get out you will have to use English, no matter whether you have the confidence to use English or not. The opposition is just enormous.

AU How long has this programme been going on, is it something that's always happened?

19(97) Yes, always, since the establishment of these training colleges.

AU I mean so few schools are actually officially Chinese medium anyway.

19(97) That's what many of our colleagues are saying.

AU So how can, it seems strange to me. If it had been brought in recently with the new guidelines that the majority of schools are going to be told to teach in Chinese, then okay, but previously actually very few were officially meant to be teaching in Chinese and yet the Institute has been teaching teachers to teach in Chinese, although we all know that most schools teach through the medium of Chinese anyway, although they are not supposed to. One more question on this before we move on, it has been suggested as you pointed out, that with the benchmarking, quite a few teachers would not get through it, and we still will need English teachers, the other possibility is that more teachers will come from outside of HK, the expatriate scheme and teachers from China, it's been suggested, that there are very competent teachers with very high levels of English, who might be attracted here to fill the gap.

19(97) That might happen. That might take place, but I don't know, I'm not the government, but from the government's point of view you will find that a lot of the teachers that we are training here locally are on the job. You spend a lot of money training local people to teach but you are employing people from outside of HK, whereas our local graduates are being unemployed, so ...

AU An interesting situation. 
19(97) That might happen, I don't know, unless the government can convince people that it is the right thing to get people from outside while our local graduates are being unemployed.

AU As a teacher I've had experience of seeing student teachers, novice teachers, both from the Institute and from the City U who do a BATESL programme, and I found that in general, the ones I've seen, have been very good teachers, they are enthusiastic, full of ideas, which they might well be at the beginning, but their language level is a problem.

19(97) Yes, that's a problem with many of our teachers in HK. That's why I think most of the teachers in HK have to resort to some kind of mixed-code. When they themselves went through school, their teachers taught them in the mixed-code, that's why many of our teachers still believe that mixed-code can work., there's no problem ... because they learn it in that way, so why not their own students? If they went through matriculation, if they went through university, like teaching in secondary schools nowadays, many teachers have higher degrees, so why not? I've been through all these institutes and schools, why can't my students? That's why it's so difficult to convince teachers to change, to tell them it's no good using the mixed-code because you are smart, of course, you are different, but your students may not be as smart as you. So they can't talk about their knowledge, they can't generate their own knowledge, they can only reproduce, they can only become pigeon-ducks, yes, if you are satisfied with being pigeon-ducks, it's okay.

AU Within the different subjects perhaps, but I suppose English, you're expected to tech in English, rather than in mixed-code. Even in English lessons, we know that certain amounts of mixed-code go on, even though it's not supposed to. I think it's accepted in other subjects, but in languages, you wouldn't expect that. To actually teach in a mixed-code, sure they might interact with the students in Cantonese, but when teaching English, using Cantonese goes against what everybody's been taught.

19(97) Not in English classes, I mean not in English language classes.

AU Perhaps the change in the MOI might change that as well. Teachers going now into officially Chinese-medium schools, for an English teacher perhaps, the pressure to no longer be seen as a model, so to speak, is good. As a teacher of English, they go into the English class to teach English, then outside of the class they can use Cantonese all the time, without any worry. I think perhaps English teachers in English-medium schools are expected to use English all the time, even with the students, although they tend not to.

The next question is any other significant changes in government policy towards the teaching of English after the changeover? 
19(97) I really haven't though about this question. Offhand I would tend to say right now, not in the coming five years. There won't be much change, I think. Even though there may be changes, changes will be very gradual. They will be very piecemeal and gradual, I think. What changes might there be? I you would ask me my own opinion, I definitely would in secondary school and primary school, when we teach English we would teach English for much more specific purposes, to prepare our secondary students ... for employment. Say, teach them office English, teach them academic English, and I would allow students to make a choice, whether they want to learn office English, maybe they are not prepared to pursue further studies after secondary school. Those who are prepared to go into university etc., offer them academic English. But I would try not to teach students English for social intercourse, that may not be too useful for students in HK. Let me say English for practical purposes, rather than for social purposes. If you ask my opinion, that's the kind of changes that I would introduce into schools in HK.

AU I agree, that would seem to be a logical thing to do, but it's a case of how it fits in with the examination syllabus, isn't it? The certificate and the AS Level. I think the AS Level is trying to teach a mixture of EAP for those going into further education, lower down the school; the certificates are a necessary qualification for high school graduation. It will have to be fitted in with the examinations. They will have to teach more ESP. What we do now is teach the lower forms politeness strategies and things like that, and they are not going to use that.

19(97) They are not going to use that, you're right.

AU We hope that it will all add to producing a competent speaker, who can communicate on any level, but we find that the opportunities for students to find themselves in a situation where they are going to be able to do that are very small. One thing perhaps which is connected with this which is significant changes in government policy, which is not directly towards the teaching of English is the question of Putonghua and where that will come in.

19(97) Of course, but as far as I know, the syllabus for secondary schools is already rather full. I don't know whether we spare any time for any other subject, unless time is taken out from English. I don't see where we can find time to squeeze in Putonghua.

AU I think presumably they must. It's going to be a cert. exam in the year 2000. Where they get the time form is not clear, the timetable is already full, at lower secondary level, unless they take it from English or from Chinese.

19(97) Or from Chinese.

AU A lesson from each will be only two lessons per week. 
19(97) But the general standard of Chinese of our students is already very low. You can't take any more time from the Chinese lessons. But Putonghua is definitely being brought into the curriculum.

AU I can see other subjects being, if not dropped, then edged aside, subjects such as geography, the traditional subjects, history even, things that we would regard as essential for a student's all round education.

19(97) One solution could be, and a practical one I think, is to use Putonghua to teach Chinese, rather than to use Cantonese. So maybe in about 5 or 6 years time all our Chinese teachers must be trained to teach Chinese in Putonghua. So, another benchmark exam for Chinese language teachers, they must pass the benchmark to be able to teach Chinese. I don't think students need to learn three, well Putonghua is not really a separate language, we can still regard it as one. I don't think there are many places in the world where students are required to learn 3 languages all at the same time, compulsory languages.

AU Oh I think there are, countries in northern Europe, for example, such as Germany, Scandinavian countries too.

19(97) All three are required subjects or the students are offered a choice?

AU They are required, certainly at the lower level, later on ... We have exchange students, this year we have one from Italy and she studies Italian at school, which is her native language, and she speaks English, French and she knows bits of Spanish as well. So certainly they study three. So the teaching of Chinese through Putonghua, because it's something I know nothing about, the teaching of Chinese, I have no idea what goes on in a Chinese lesson. I can only think that it's similar to what went on when I learned English. I would say rules of grammar only to a small degree. We were never really taught grammar, we were given practice in writing, how to write letters, basic things like punctuation. From what I can gather, the problem with Chinese now is that standards are falling and students' written Chinese is basically Cantonese, which is none, pure, if you like, it contains a lot of slang and the stuff they read in magazines and newspapers. It's very idiomatic. So no question Putonghua will come in, and as a MOI in 10 years, and will English be shoved aside, or will English maintain it's status as the international language which all students must learn.

19(97) It depends ... Why did I say it depends? I think it depends on who will be running the ED, who will be running, who will be the Chief Secretary or the Governor. Right now these people, all those people, all those high-ranking officials in the government came through English-medium schools. As you say, maybe it will take 10 years. I think in about 10 years time all the highranking officials will come from Chinese-medium schools. If people who are in-charge, who are in-power, came from Chinese-medium schools then 
probably they will regard English as not as important as Chinese, or much less important than Chinese.

AU If they do go to school through the medium of Chinese then they'll go to university through the medium of English, though not necessarily, that may change too.

19(97) I don't know but I strongly suspect that in our universities in HK, what goes on in the classroom is what is being done through Chinese rather than through English. Even many of our fully-fledged lecturers from European universities are not that proficient in delivering their lectures in English.

AU Or they can deliver a lecture in English, but away from their specialist subject, they struggle in English. They can deliver a lecture in English, but tutorials are in Chinese.

19(97) Or in mixed-code. The reason I say this is that I spent some time at CU and my experience there was that the lecturers taught through Chinese. Of course, the course didn't specify what the MOI was, that was very much up to the individual lecturer. The paper, the exam paper the students had to take was in English, but the lecturer had chosen to lead the discussion in Chinese. So maybe that would not happen in a few years time.

AU Next question, what changes do you envisage in the proportion of the timetable? I think we've probably covered that already, with the idea of Putonghua coming in perhaps.

19(97) Do you see the teaching of English ...

AU This question applies more to people in schools, but also we can regard your school as being your department, your Institute. Again, perhaps we've covered a few things already, the idea of the benchmarking perhaps aiming to push the levels of competency.

19(97) Maybe that's the only thing I can mention, what' being done by us, in this Institute, I mean by the people in the English Department in this Institute is to implement an Institute-wide policy that lecturers who are teaching in the English stream, must use English when they deliver the lectures, when they lead the discussions. As I said, we are running into a lot of opposition, and the Institute has accepted our recommendation that it should become an Institute-wide policy, that if people are teaching in the English stream must use English. But we don't know how well that could work out, we don't know, but we are running into rather severe opposition from many sides.

AU Presumably if you have an English stream and a Chinese stream then the English stream should be in English, or what's the point of having it? Do you 
separate the two, or do you have an English stream, a Chinese stream and a mixed-code stream?

19(97) The so-called argument trend followed by so many of our colleagues in other departments is, although many of the schools are in name English schools, actually they are Chinese-medium schools. So what's the point of training our students to teach through English when they don't have the chance to teach through English? That's their argument, but of course there is another reason why they are fighting back, that is they don't have the confidence, they don't have the proficiency for it. It's quite natural if you don't feel up to the job, you will say no.

AU Although some do of course. Perhaps other teachers might have a bit more confidence or they might have their own misplaced idea about their own standard and might actually think that they are better than they really are. I know some teachers in my school who do teach through the medium of English and whose English, from my point of view, is quite poor. They battle away, in fact we have students who have better standard of English than some of the teachers, some of the F6, even lower school. I know that as an aside, one of my colleagues and friends, he teaches economics, he and another teacher they both teach economics and share the teaching of the cert. and A Level, and he teaches in Chinese, she teaches in English. They don't get on very well and so he teaches F5 and 6, and she teaches F4 and 7, so they swap over. The students get half their course in English and half in Chinese. It seems very bizarre. But the one who teaches in Chinese gets excellent results, every year A' Level, a lot of As in the class.

19(97) You can't deny the fact that students learn much more efficiently through their mother-tongue. I think its' really a very exceptional case when someone can say that he or she learns through a foreign language just as efficiently than through the mother-tongue. It is an exceptional case, and there are not too many exceptional cases in HK.

AU Okay, do you think that the changeover will affect teaching methods and approaches?

19(97) No I don't think so, if you are thinking of the English language alone, I don't think there will be much change, unless the teachers here in the Institute change. I think we need to change. So if you are thinking of the English teachers, then I don't think there will be any changes after 1997. Other subjects, if the government's plan to enforce their so-called recommendation that schools, that two-thirds of our local schools must switch to Chinesemedium, if that really can work, then yes, many schools will have to switch to Chinese-medium. If there would be any change in the English language subject, the only thing that I could envisage is say the revival of the teaching 
of grammar. After all, we do admit that this is not our second language, this is a foreign language. We need to learn more grammar.

AU It would be if it's in Chinese-medium schools, and English then will become a foreign language within the school system.

19(97) I think what happened in the past was we tried to teach English more or less as a second language, which is not quite realistic. That's why we teach people to show people direction, to make an invitation etc. etc. We've been trying to teach our students English as though it were our second language, whereas it is not, it's quite a foreign language. So the only change I could envisage right now is the revival of the teaching of grammar.

AU I think it also depends on the examinations. The washback from the examinations is so strong. If the examinations don't change then presumably the teaching methods won't change either. If schools change to Chinesemedium, then perhaps within schools English teachers may become more valuable, because even within Chinese-medium schools, in subjects like geography, history and science, there'll be English terms that they have to use, and if the teachers teach through the medium of Chinese, maybe then the English teacher will need to help out with certain vocabulary, or the subject teachers might come to the English teachers more than they do now.

19(97) By the way, have you heard of the TOC? Again, I don't know how well that can work out, hopefully that can work out well. Although the government has made it it's policy to implement TOC and there has been very strong opposition from schools and from teachers ... [tape ends] ... teachers in schools are prepared to co-operate with the government to implement the TOC. That would imply there might be changes in the teaching methods.

AU I think that teachers and schools feel they are not being given enough resources to carry through TOC. I think everybody agrees that it's a good thing to do and it's a step in the right direction, but you've got to have the backup to do it. Whether it works in a class with 45 students with very limited facilities, you can't suddenly change your whole teaching method if everything else doesn't change.

19(97) But it must work first in primary schools. Right now it's very difficult, especially with primary teachers who are not as ambitious as secondary school teachers, they are not as motivated as secondary school teachers. They have quite low self-esteem compared to secondary school teachers. They are quite ready to accept new ideas. That's why the opposition especially from primary school teachers has been very severe. I think more severe than the government anticipated. So again I don't know how that can work out, I will only pray that it will work out. As you said, many realise that it's the right 
thing to do, but if it can't work out in primary school, it can't go on to secondary school.

AU Okay, the last question is concerning the status, situation or morale of English teachers.

19(97) That will I think have some effect on the status, situation and morale of teachers in HK. How long have you been in HK?

AU Eight years.

19(97) Eight years! Such a long long time? Probably you realise that not too long ago, probably a year ago, that our Legislative Councilors when they spoke up inside their chambers, they spoke up always in English. Right now whenever they speak up, they always do it in Chinese. So people like Lee Chu Ming, many people, the moment they find the camera facing them, they start speaking up in Cantonese. So right now I think that whenever any debate goes on inside the Legislative Council, it's in Chinese, not in English. So you see the change, the change is quite obvious. In my generation, we sort of, we felt very proud when we found ourselves speaking to other people in English, but right now we don't feel that way.

AU Tve heard this from others as well, twenty or thirty years ago HKU students would chat away in English and feel very proud of themselves, whereas nowadays to be seen talking in English will be frowned upon.

19(97) So that's why to be an English teacher is becoming increasingly difficult, to try and motivate students to learn.

AU Will that affect the status or morale of English teachers? English has become a means to an end really, instead of a status symbol.

19(97) Yes, yes. I think Putonghua is replacing English as a status language for the Chinese community in HK in the near future. But English will still, I would say, English will still be regarded as an important asset. After all it's an international language, when you do business with people from outside of HK it has to be done through English. If you want to pursue further studies, then you need to become proficient in English. People in HK have lots more chances to travel abroad, even people in China are able to travel abroad, to HK to Thailand and to other places, so I think people feel the need to learn English. I think a lot more people in Asia, in countries like Japan, Korea, Taiwan, HK, Thailand, many people are learning English, more people are learning English because people feel the need.

AU I think that is what we call globalisation, we are not talking Asia now, we are talking Asia-Pacific. 
19(97) Many people would tend to pick up an American accent more readily than a British accent.

AU That's true. 
Interviewee 20(97): Principal of secondary school

Date:

19 August 1997

$\begin{array}{ll}\text { AU } & \text { Researcher } \\ \text { 20(97) } & \text { Interviewee } \\ \ldots & \text { Missing words / Unfinished utterance } \\ \text { - } & \text { Pause }\end{array}$

$\mathrm{AU} \quad$ What are your feelings as regards the EDs decision to force $75 \%$ of secondary schools to adopt Chinese-medium education?

20(97) I should say, as a professional education worker, of course most teachers and principals would have a negative reaction towards any sort of firm directive by the government, however as an education worker who has worked for three or four years for Chinese as the MOI, and yet not really satisfied with the progress, I should say that this measure from the ED, however unpleasant it might be, is still needed, and in your question you mentioned the word 'force', I don't know how much the portion is, many schools actually welcome this decision. Many principals, I can quote a meeting of the $\mathrm{HK}$ Subsidised School Council, and we had a voting at that time and amongst the 300 principals present, more that 280 , more than $90 \%$, voted that if we could do it together, we want to adopt Chinese as the MOI. So for some schools, they are being forced by the government, especially many schools are not satisfied with the ... very short notice, very short period for them to prepare for the transition.

AU I mean I've put the word 'force' because the feeling that as you said, some schools don't want to have anything forced on them at all, they want to make their own choices.

20(97) Maybe since you just mentioned that you are collection of press coverage mainly from the English-medium paper, maybe some schools voice their dissatisfaction being forced by the government through the English paper. Amongst some sort of informal sharing and discussion with other principals, I can notice that most of the principals just sort of said that it's not too good being directed by the government but it is necessary.

AU Perhaps you're right that in the English press they favour coverage from schools that perhaps think that by losing their status as an English-medium school, might feel that they are not going to attract the best students. The parents may worry that their children are not going to get a standard of education that is going to allow them to enter further education, tertiary education. 
20(97) Yes that's the main worry, even among our teachers, some of them also worry that if we adopt Chinese, while other schools of similar banding are still claiming to be English-medium schools, that will certainly affect our intake of students.

AU And also I think the fact that the tertiary education issue has been raised a great deal, I think a lot of parents are worried about that. Perhaps a lot of teachers as well, talking to other people, they feel that teachers have been trained through English and although they may not teach purely through English nowadays, the fact is that they are trained to teach through English and use English materials, having to change to use Chinese materials, which they are not trained to do, I think some teachers worry about that.

20(97) I think they teach the English vocabulary more than the sort of presentation, the sort of reasoning really in English. I went to do classroom observation in my school, and I see that most of them are using Chinese, and on some occasion when teachers tried to use English when I was in the classroom, so that it is very unnatural, and the students had no response to their teaching. After the lesson I asked the teacher whether she used English or she used Chinese, she told me that normally she just used mostly Chinese.

AU So possibly the only difference would be that the materials would be in Chinese, as you say, in many schools most teachers use predominantly Chinese anyway. Your school is English-medium?

20(97) Claim to be, but starting from three years ago we try to use Chinese in some subjects in lower forms, and up to now more that $50 \%$ of lessons are done in Chinese.

AU Have you noticed ant improvement in the performance?

20(97) In the subjects? There's improvement in the subjects integrated science and geography, it was marvelous. When we used English as the medium, about 20 to $30 \%$ passed, and then after the change, this rose to $80 \%$. And for EPA, the rise was not significant, but the setting of the paper was completely different. We asked questions, some sort of opinions or newspaper cuttings, asking them to apply the knowledge to answer the questions. So the setting of the paper is different. And concerning English standard, I do not see any significant decline in the English passing percentage, so I just wonder how had the English teaching in subjects really helps students in improving their English standard. So it seems we didn't help the students' English standard by teaching them in English.

AU Maybe just confused them?

20(97) Yes 
AU Okay, next question is, what are your feelings as regards the ED's planned introduction of benchmark language levels for language teachers?

20(97) On a micro level, concerning the case in my school, I would rather encourage them to improve, rather then having this sort of benchmark, and I feel that it works, but of course it's only a micro level. So I'm not quite fully aware of the unsatisfactory language level of the language teachers. So I think if possible, to encourage them to improve is better, and if you say that teachers are not, I mean incompetent in teaching, not really in language level, this will be another problem. Can the school not do anything to deal with those teachers who are not so capable, and not only in language teaching, but also in other aspects? Actually I would think that if the school can do more, then we really don't need the benchmark language levels, and the school can see whether this teacher can really help our students, can really perform their duties in a satisfactory manner.

AU Oh of course, a lot of teachers have said that there is more to teaching then having a certain language level, but a lot of teachers are worried about it, they think that it is something that they shouldn't have to prove. Other teachers don't need to prove their competence, why should it just be language teachers? But also others think that it is a good idea. If they don't actually reach the benchmark, I think the plan is they'll be given support then to reach the benchmark and they'll be given plenty of time to do it. So it won't be that difficult. It's not a case of if you fail you are out.

20(97) But maybe the teachers will feel, just as you mentioned, why do teachers of other subjects not need this benchmark? For computer, physics or economics, which I taught 20 years ago, and I notice that the syllabus has changed so much so that you couldn't stop learning or you just couldn't teach.

AU I suppose one feeling is that subject teachers have to be graduates in their discipline, whereas a lot of English teachers are not English graduates, they have not studied English, they may have studied something else. They may have a teaching qualification in English, but they are not actually English majors. Maybe at a later stage they will have the benchmarks for subject teachers. I think the main point is that it's not a witch-hunt, it's not a way of getting rid of teachers whose language levels could be better, it's a way of raising standards.

Do you think there'll be any other significant changes in government policy towards the teaching of English in $\mathrm{HK}$ after the changeover?

20(97) I think that government policy may change, not really initiated by the government but in response to the changing in demand and the atmosphere in society. Now when we ask the students to improve their English, some of them will say, oh Chinese is okay. Even if I fail in English, I can still get a 
good job in Mainland China, but maybe years ago we could say that if we fail in English, that will be finish, you cannot get any kind of management job or administrative job. But nowadays they will think that if their Chinese is all right, if their other subjects or their ability to work is all right, they don't worry if they really fail in English. So there may be some sort of change of the attitude from the students, and so I don't know how the government will respond to this or the government will think that Chinese is more important than English. Now we can see that the government circulars, or the government officials making speeches, they have to communicate well in Chinese.

AU So whether they'll be any other changes, as you say is unclear.

20(97) In the teaching, towards the teaching? Well there should be others.

AU I think the government, or the provisional government and the person they put in charge of education, which is Andrew Leung, has very much stressed the importance of English, he's very keen to do that, so I think English will still remain ...

20(97) English proficiency and English ability is still an asset in HK, otherwise people from Shanghai and people from Beijing, they are smarter than the people from HK because there are so many people, they can just get the cream of the people and compared with $\mathrm{HK}$, they are much smarter. But the English proficiency, this sort of bilingualism in HK, it will be important.

AU And perhaps the other factor is how important Putonghua will be, whether that will take on a higher profile to the expense of English. People may say, why should I learn English, Putonghua is more important. HK will remain an international city and English will still be of great benefit, but if their English is no good then they can say, well I can go and work in China where I don't need English.

20(97) And maybe, how shall I put it, the sufficiency of communicating in English, when HK remained a colony, speaking good English, perfect English, Oxford English, was important, but nowadays, so long as we can communicate, so if you get in a taxi and the taxi driver understood where you wanted to go, you can just talk with the business people from other countries, they are not so good at English really, so there maybe some students or teachers will think that's okay, even I make many mistakes in the grammar and the tenses, so long as I can communicate it's all right. And for students getting an F in their school cert. or Use of English AS Level, I think most of them can still communicate, or after some years of experience in their job, they can communicate no problem. But years ago it wasn't the case. If you can speak good English, then you can get a high position. 
AU So the focus has changed, whether the government will actually seek to change that, again only time will tell.

What changes do you envision in the proportion of the timetable given over to English language teaching in English and Chinese-medium schools?

20(97) I'm a member of a working group promoting in Catholic schools Chinese as the MOI, and we propose that when we change the MOI to Chinese, then actually we can save some teaching time. We do not need to teach them the very difficult vocabulary and presentation, but we can devote more time in teaching English, increasing the exposure, giving the students more practice and doing some activities in the language lab or using the multimedia, and I believe it should be the way, the direction, and together with some proposal that we should try to integrate subjects. Now, for example, in our junior forms we have so many subjects and we are always thinking of trying to integrate them, and again we can save the teaching time and devote them to English. I always believe that we should do this. So after the changeover we still take English as an important subject ...

AU Going back to the first question and the worry perhaps for some parents with their children's schools changing to Chinese-medium is that their English will be ignored, but also the feeling is that if they study their other subjects in Chinese, they should get on much quicker without the hindrance of the language they struggled in before, allowing more time then perhaps for English to be in the timetable. But as you say also, there are so many subjects to do that finding space on the timetable is very difficult.

20(97) From my experience, in the past we had five periods of integrated science in the junior forms, and after the change in the MOI we cut the number of periods to four, and still the teachers found that they could manage, no problem, and then we can use that extra lesson for English.

AU In English-medium schools, maybe nothing will change.

20(97) Yes, unless they integrate the subjects, but even after the integration, there are still other demands, for example, Putonghua, computer literacy and that depends on how the school rates the importance of the subjects.

AU Yes, well Putonghua will presumably become a fixture on the timetable, it will be a cert. exam in the year 2000 , so that will have to fitted into timetables if it is not already, and something will have to be lost. Will it be taken away from Chinese or will it be incorporated into Chinese?

20(97) I think the best way is to teach Chinese in Putonghua, but again teachers are not well-trained in this aspect, so still Putonghua is a separate subject and we 
have to give lessons to this subject ... so different schools have different ways to handle that.

AU Do you think there'll be any other significant changes in your school or the education system more generally that might affect the teaching of English?

20(97) I can think of one of the issue is that the newly arrived immigrants. Even before the changeover, we did admit some. They are really newly arrived, first attending this school, our school. They may have arrived two or three years, they started in P5, and yet their English standard is still lagging behind. They are admitted to our school, and even say the standard of our school is medium, we're not really the top school, but students if they are quite good at mathematics, then they can get a not too bad banding. They are admitted in our school but they just learn English for one or two years. I think that really affects the normal schedule of our teaching, since the teachers prepare the schedule based on the students having completed P6, and we presume that they have learned English for at least 5 or 6 years. So that may affect, and in the past we only admit two or three students like that, really weak in English, but now we find that the number is increasing. So we have to design some small groups for them, sort of extra remedial groups, not really the remedial class, which we have had for more than 10 years.

AU What kind of numbers are we talking about?

20(97) This year we have much more, because after the summer vacation begins, we still admit some students, and this year I find that the number of students applying for F2 or F3 places, who are newly arrived, this number has increased. When I gave them a test using our own test paper, they performed very well in mathematics. But English, that depends, some of them did it very well and we grant them straight admission. For others, I will still admit them but I advise them to do something in the summer vacation to catch up to the normal standard, and we plan to help these people as well. At least we have around 10 who are newly arrived immigrants. But those who have arrived for two or three years, at present I do not have the figures. But the form master or form mistress, sometimes they will report to me that there are some students who could not manage the $\mathrm{ABC}$. We still have students like that.

AU The fact that you have more new immigrants in your intake mean that the overall intake has expanded?

17(97 I should say the number is expanding. I might say that this area, Kwai Ching, is an area taking more immigrant children.

AU So do you think your overall intake will increase?

20(97) No, no, no. 
AU So the new immigrant children will be integrated into the intake?

20(97) Yes

AU Because they have said that they are going to have to build a lot more new schools to cope with the intake.

20(97) But I think the problem is more serious with the primary schools, and of course this will apply to secondary schools some years later.

$\mathrm{AU} \quad$ Yes, there is something like they will have to build 600 new schools.

Question 6, do you think the changeover will affect teaching methods or approaches in English lessons in secondary schools?

20(97) Maybe in the past, the ED asked schools to use the communicative approach and not encourage schools to teach grammar, or structure, and they had the presumption that we can provide exposure of the language for students, but that of course was not the case. So I think that for students to really learn this language, of course this sort of exposure, communication is important, but to know the structure, the grammar part of the language, is also important. I think that for the methods in teaching English, the stress will be turning to teach English as a subject, and they you have to know how to make the structure of the language, and on this basis you can try to apply that and try to associate what you have learned with daily language.

AU So English will be taught as a foreign language, as opposed to being taught ideally as a second language.

20(97) I think in the past the ED tried to ask the teachers to teach English as a first language.

AU In English-medium schools, the hope was that during the rest of their schooling they would get the support, the language support and they would have the chance to use it and to be exposed to it. And even outside of school as well. So whether the changeover will affect it, the fact is that schools will be changing, some will be changing to Chinese-medium.

20(97) But I think the changeover actually ... the situation of schools changing to Chinese-medium, plus at least indirectly, since we are using more Chinese in our daily life in the official part, and also another reason is that students using Chinese to learn is much more effective. Both of these affect the teaching of English in secondary schools.

AU So for English, the approach to it will change, won't it? Chinese will become the official medium of education and of many things for most of the people 
here. In the past perhaps English has been seen to be a secondary medium, perhaps even a primary medium in many cases. Students go to the elite schools and perhaps want to go on to tertiary education, the reality being that that doesn't affect most of the students in HK.

Okay, finally, how do you think the changeover will affect the situation, status or morale of teachers of English in HK?

20(97) I think teachers of English will still enjoy a high status, but overall society will still take English as very important, and also I think the status will also be affected by the sort of supply and demand situation. If the cream of the English-trained professionals, if they take up English teaching as their career, the other people will think that they enjoy a high status. But some people will say, oh the English teachers, their language standard is not satisfactory, and also anybody can become an English teacher, this will affect their status. And of course the demand side may deteriorate after the changeover because people will think that Putonghua is also important. For our students, some of them just got a D in UE, and we think their standard is not so good, and still they can be admitted to an English department. Of course we believe that the training after 3 years can help them, but sometimes we may wonder, how about the As and Bs, do they go to the law or business administration, instead of going to the training of English?

AU My students have said to me, I'd like to study English, or English literature, but my parents are not happy, they want me to do business, they want me to do medicine. They say English, what kind of a job can you get with English, or English literature? And you can understand their feelings, but at the same time as an English teacher I've got to try and persuade them that there's plenty of openings for English graduates.

20(97) I believe that you can transform them.

AU Well certainly you can do a lot, depending on how motivated they are. So perhaps we need to improve the status of English teachers. Being an English teacher myself, of course I always say that we should be paid more because we work harder than anybody else.

20(97) And they are also faced with the competition from the expat teachers.

AU Which in the past has been great for the expat teachers, but I'm sure it's been a cause of resentment. But I don't think this is the case now, expat teachers do not get so many benefits.

20(97) Do you find that they can attract the best teachers? Because we do not have the experience to employ expat teachers. The reduction of the benefits, will it affect the quality of the teachers? 
AU I think for the expat teachers who are here, the attraction is no longer there. The expat package was a big attraction. Now that they can no longer get such good terms, perhaps you will no longer get the best teachers.

20(97) Sometimes the local teachers will think that the expat teachers cannot help much in the administrative work, for example in my school I have a teacher from South Africa employed on local terms and he's a native English speaker but a born Chinese, but he picked up Chinese very quickly. When he first came some students turned to him as a teacher helping them in speaking, in oral English, but nowadays the students speak to him in Chinese. So his image has changed.

AU I suppose it's good for him, it helps him with his Chinese. 
Interviewee 21(97): University educator

Date:

28 June 1997

$\begin{array}{ll}\text { AU } & \text { Researcher } \\ 21(97) & \text { Interviewee } \\ \ldots & \text { Missing words / Unfinished utterance } \\ \text { — } & \text { Pause }\end{array}$

21(97) ... the ED's decision to force $75 \%$ of secondary schools to adopt Chinesemedium education? It's actually about 75 , I read in the papers, but of course the actual figures are not out yet. I think it's wrong. I think the ED should adopt a more flexible approach. Yes, they are right to say that we shouldn't have too much code-switching or code-mixing going on, but we should allow some subjects to be taught in English, some subjects to be taught in Chinese, some levels like junior form levels to be taught in Chinese and some levels like senior form levels to be taught in English and there should be more flexibility in ... so I think a more flexible approach should be taken to take into account we don't have clear cut proficiency batches, saying that this batch of students is totally not suitable, while some are more and more and they can progress in F1 and F2. Maybe more subjects in Chinese, F3, F4, more subjects in English certainly should allow for flexibility.

AU Why do you think they are so strict in this division of totally English-medium and totally Chinese-medium?

21(97) I can only speculate about their reasons, so one reason may be administrative, administratively it's easier. Administratively it's easier because clear cut black and white. If you allow me to speculate I would say that they want to pre-empt the Chinese government from doing anything to the MOI by saying, look, we have already forced the majority of the schools to adopt Chinesemedium, so don't do anything more. This is just my speculation. There might be this worry that the Chinese government, or the SAR government, which will be under Chinese influence anyway, will say change the MOI in all schools into Chinese, so they can pre-empt that by doing this. By having a clear cut policy that saying the majority is in a Chinese-medium, a pure Chinese-medium, so don't ask us to do anything more, it's already in the Chinese-medium. Then they can retain the $25 \%$ as an elite English-medium schools. If they don't do that, they may fear that the Chinese government may say, oh, change across the board, into Chinese. But if this is the real case then this wouldn't be the case because Chinese, the Chinese government is unlikely to do that. 
AU I can see that if your speculation is true then it is affected by the changeover, to a certain extent.

21(97) If my speculation is true it is because they have been saying code-switching isn't good for two decades, at least one and a half decades, and they don't enforce it until now. Why? They could have done it in the 80s. In the mid$80 \mathrm{~s}$, in the late $80 \mathrm{~s}$, in the early $90 \mathrm{~s}$, but they weren't doing it until now, so ...

AU They have decided to do it next year and they have announced it in the last two months so it is curious as to why they have ...

21(97) It is due to the changeover I speculate, but nobody knows, these are highly political decision-making processes. So my feeling is that they want to leave behind a small percentage of elite who is educated in pure English medium, not mixed-code. So there might be some political reasons behind this sharp division, right?

What are your feelings as regards the ED's planned introduction of benchmark, yes I think it's good because in every profession you need to have professional ... to pass. In teaching, especially teaching, we should make it more professional. And it certainly will have an effect on new teachers, and in the long run will have an effect on the old teachers as well, when they compare themselves with the new teachers, when they feel they are not as adequate as the new teachers. It will also raise the status of English teachers so that not everybody can teach English. Right now it gives the impression that everybody so long as you have a bachelor's degree can teach English, which is absolutely wrong, I mean you can't teach physics with a degree in math or chemistry, but now you can teach English with degrees in history, physics in any disciplines, that's absolutely wrong.

AU A lot of teachers are unhappy about the benchmarking.

21(97) They are not happy. I don't blame them because they are already doing the job and they fear that they will lose their job or something like that. But I think those really professional teachers they would welcome that. They would welcome the chance to raise their status to prove that they are professional. If they are good, they have nothing to fear. If they are not so good, then I think the government should provide then with chances, like time off to take refreshment courses, time to brush up their proficiency and their teaching techniques. So if they don't face it then they are being unprofessional, and the teaching profession will not have a high status. If anybody can teach, then it's not a profession at all.

AU Presumably if the benchmarking results in some teachers not being able to teach, it may create a shortage of teachers? 
21(97) That's right, so I'm not for a drastic implementation of this, it should be gradual and it should apply to new teachers first, so gradually we will upgrade the teaching profession. or the teachers' status.

Next question, do you think there will be any other significant changes in government policy towards the teaching of English in HK after the changeover? If so, what might these changes be? It's difficult to speculate about what the new government will do. It's easier for me to predict what the old government would do. The new government is totally unpredictable. So, my guess is that in the first five years they will try to leave things unchanged because they don't want to change things in the first few years, at least the first three or four years they will make minimum changes, I think the government And I think as far as I know from Anthony Leung, the speeches of Anthony Leung, who will draw up the new education policy of the future SAR government, English is going to be very important in the new HK, and all the universities, I mean most except Chinese U, will retain English as the main MOI. So, that policy has already been announced by Anthony Leung, so we know that English will remain very important in the school system because the universities will use English as the MOI. So, I think the new government might put more resources into the teaching of English, might, because there are other priority areas for the government to assign resources to.

Next question? What changes do you envisage in the proportion of the timetable given over to the teaching of English in both English and Chinesemedium schools? I don't think there will be any changes, as I said, in the first 5 years, the government will try to minimize the changes. Maybe more in both English and Chinese-medium schools, maybe more in the Chinese subjects, maybe more lessons might be assigned to the Chinese subjects, difficult to say.

AU It might change if the school changes from being English-medium to Chinese-medium ...

21(97) Then they might increase the number of Chinese lessons, yes, it sounds logical, sensible to do.

AU The other factor is Putonghua.

21(97) That will take up some time, yes, some timetable, yes Putonghua will certainly take up some time. Whether it will be at the expense of English or of other Chinese subjects, we just don't know.

Next question, do you think there will be any other significant changes in your school or the education system more generally that might affect the teaching of English? What do you have in mind when you wrote that? 
AU Any other changes, not specifically geared towards changes in language education, changes in other aspects of education, or in what is taught in other subjects, for instance.

21(97) I think in those Chinese-medium schools the students might have less exposure to English, so they will be functioning mostly in the Chinese environment, in the written and spoken environment. Right now they function in an English written environment and a mixed-code spoken environment, but after the change to the Chinese-medium they will function mostly in written Chinese and spoken Cantonese ... so, it might not be bad because other subject teachers might not be able to do a good job teaching their subjects in English, so they may be getting the wrong kind of input, so it might not be a bad thing in the teaching of English. Then the teachers, the English teachers, need to provide a rich linguistic and cultural environment in which English has major communicative roles to play for the students, like in extracurricular activities instead of in physics, math and other subjects. So we might say that the government needs to assign more resources to extracurricular activities related to both Putonghua and English because you won't have Putonghua or English in other subjects except for that small subject. Immigrant children, yes that could be a problem for primary schools because they do not start English as early as HK students, so we need to have enrichment programmes for immigrant children so that they can catch up with the syllabus. I think good things might be we have more returnees from English-speaking countries and if they cannot find jobs in the business sector then they might find jobs in the schools, and then provided that we teach them the skills of teaching English, they might be very good in English, they might speak English well, but they might not know the techniques. Classroom teaching is different from teaching a language well. So provided that we can give them teacher training then they can improve the general quality of teaching in schools. So we may have more of that happening.

The next question, do you think the changeover will affect teaching methods and approaches in English lessons in secondary schools? If so, in what ways and why? I cannot see any direct, we won't be taking any mainland Chinese teachers in our schools, unless we do, and they may bring along their methods, which might be audio-lingual or grammar translation or maybe communicative because they are implementing a communicative approach in mainland China as well. So, if that isn't happening, if our teachers are still HK teachers, then I don't see any major effect on teaching methods.

AU Do you think the change in medium might affect the teaching methods in any way within any particular school? If they ...

21(97) I don't think so. Why would you think there is ... 
AU I think the idea behind teaching English within English-medium schools has very often been so that the students could cope with learning their other subjects through the medium of English, and if they then become Chinesemedium schools then that need will be taken away. So perhaps their needs will change, the functions they need English for will change. A lot will depend on whether the curriculum changes.

21(97) Right, and if the exam curriculum, the exam syllabus doesn't change because HK teachers and learning is very much exam-driven, like it or not, so especially when all students want to enter the university and they want to learn an study in English in the future, so EAP, English for academic purposes will still be important for all students but not all of them can enter the university, but all of them want to so they will want teachers to help them to.

AU It's fairly much true now that the students who enter the universities come from the elite schools.

\section{1(97) That's right.}

\section{AU Perhaps even more so.}

21(97) Yeah, so it depends on the schools, so for the English-medium schools you won't expect any changes. For the Chinese-medium schools, they will follow the exam syllabus anyway, and the textbooks anyway, they are highly textbook-dependent, especially in the non-aided schools, so a lot needs to be done there but the government is not willing to commit the resources. The elite schools can function well even with the current level of resources because their starting point is higher and teachers are generally more well trained. This is a generalisation of course, there can be highly qualified teachers in non-aided schools to, but generally ...

So how do you think the changeover will affect the situation, status and morale of teachers of English in HK? So back to the same question, situation, status, morale, all these have to do with whether resources are channeled to the school system. Given the current status, anybody can become an English teacher, how can you feel that you are a professional, you have a high status? Anyone who can speak English can teach English, that's totally nonprofessional. So if the government is not willing to commit resources into the school system to reduce teachers' workload, like teaching 40 students is outrageous. All over the world, to have language classes, foreign language classes, in Canada, teachers will all go to the streets to protest for large class sizes. For them, large class sizes means 30 students. So if the changeover doesn't bring along a new kind of government commitment to language education, foreign language education and English, Putonghua, Standard Written Chinese, all these are close to foreign language education in HK 
given the dominant Cantonese-speaking environment in HK. But this cannot be changed, you can't blame people's mother tongue, you can't say you are too Cantonese, what do you expect? I'm Cantonese, right? You speak too much Cantonese, what do you expect! The family speak Cantonese, the media speak Cantonese. Okay, you can change like Singapore, change the media to Putonghua, then you will have private channels speaking Cantonese, unless you have a totalitarian society, you control the language of the media, the language of the public places, don't speak Cantonese in public places. We don't want to have such a highly policed society. So, in any democratic free society, relatively free society, we expect people to speak the language they are most familiar with, which is their mother tongue, which is Cantonese. So Chinese, meaning Written Standard Chinese, Putonghua and English, should be treated like foreign languages and foreign language education is expensive. It's not something anyone can teach. It's not something anyone can succeed in, if not given a rich environment, a rich cultural environment. So, if the changeover doesn't bring along this realisation of the importance of committing resources into the school system, especially in the primary and secondary, not just in the tertiary where you can build beautiful language centres, self-access centres in universities, it's too late and too expensive to do it at such an old age. To train a person in a foreign language at 19/20, when the student has to deal with all the highly academic subjects in the university. You cannot expect an engineering student spending hours and hours learning English. It's too late and too expensive. So if the new government and the changeover doesn't bring along such a change in the policy and in the allocation of educational resources into the primary and secondary school system, and in terms of reducing class size and reducing teachers' workload, in terms of raising the professional requirements for the teachers so that not everybody can become a teacher, and those who become a teacher have those professional requirements and receive the rewards for having acquired them by having raised salary, by having passed the benchmark, for example, and the status and the morale and the situation of teachers and language teaching in HK will improve. But without that, all this empty talk, without the resources, you can impose a benchmark but if you don't create opportunities, give them the resources, time off to take courses, you will have a shortage of teachers. And then you will complain, the benchmark doesn't work. It's not just the benchmark, it's the benchmark coupled with professional development resources, so you provide resources for those teachers to upgrade their proficiency and their teaching techniques. And to attract those qualified people to join the profession by improving the professional status, and improving the income and improving the professional situation, smaller class, less workload, then certainly conditions is more attractive, you can attract better people to join the teaching profession. But without all these things, you can talk and talk and talk, raise the standards and it's all empty talk without the money. You can complain at the declining standards. You can complain at the teachers' quality, the teachers speak poor English, the students speak poor English, you can do all that kind of stuff, but 
you will change nothing because you haven't committed any resources to the school system. 


\section{A.6 List of Questions Sent to Interviewees in 1999}

Below is a list of questions which I would like you to think about and which we can discuss when we meet in person:

1. How do you think the change in the government's policy on medium of instruction in schools has affected education in Hong Kong?

2. Have you noticed any changes in the status, situation, or morale of English teachers since the changeover?

3. Have you noticed any significant differences in students' attitudes towards the learning of English since the changeover? If so, what are they?

4. How do you think the changeover has affected the attitudes of the general public towards English and the learning and teaching of it?

5. Have there been any changes in the curriculum or examination syllabuses for English since the changeover? If so, what are they and why do you think they have been made? What effects do you think these have had or will have?

6. Can you envisage any changes in English language teacher education as a result of changes which have occurred since the changeover?

7. What do you foresee as the future of education in Hong Kong?

There may be other questions that come up during conversation or arise due to announcements by the government or Education Department, but the above will make up the bulk of my interview.

Alan Urmston

June 1999 


\section{A.7 Full List of Comments from Interviews in 1999}

The following are the comments derived from the transcribed interviews with the interviewees in 1999. The numbers refer to the number of interviews in which that comment was made. If a comment was mentioned more than once during an interview, it was only counted once. In those interviews where there was more than one person interviewed, a comment was only counted once, even if it was made by more than one interviewee. For comments mentioned in only one interview, no number is given. The comments are grouped into common themes and the themes are listed in descending order with the most common given first. Within each theme, the comments are divided into Main comments (mentioned in two or more interviews) and Individual comments (mentioned once or in one interview).

\section{The Medium of Instruction Issue - 126}

\section{Views of parents and the public -22}

\section{Main comments:}

Parents still see EMI as the way to economic success. (5)

More parents are accepting CMI due to publicity in the media. (3)

Parents may want their children to go to EMI schools but students prefer CMI. (2)

Change in MOI has caused a lot of reaction. (2)

Some parents and students welcome the change in MOI. (2)

Parents are now more concerned that English is being taught effectively than whether the school is EMI or CMI. (2)

There is a certain number of parents who really want EMI for their children. (2)

\section{Individual comments:}

People in business are afraid that change in MOI will result in decline in English standards.

There is a certain proportion of the population who will always want their education in English.

The people who want EMI for their children are getting it and those that don't aren't. Some people have the wrong idea that CMI schools are second class.

\section{Effects of change to CMI on teachers - 17}

\section{Main comments:}

Teachers in CMI schools are finding it harder to teach as students have many questions they can't answer. (2)

It is not practical to expect teachers to switch from teaching in English to teaching in Chinese. (2) Change in MOI has had a demoralising effect on teachers in schools who failed in their application to be classed as EMI. (2) 
English teachers in CMI schools will be seen as more specialist. (2)

Individual comments:

English teachers in CMI schools do not have the support of other teachers.

There is pressure on English teachers in CMI schools as they have to compensate for students' reduction in exposure to English.

There is a need to provide training for teachers working in CMI schools in English enhancement and ECA.

English teachers in CMI schools will not have to unteach incorrect English taught by content teachers.

Teachers in EMI schools are being checked much more by superiors than before the change in MOI.

Some content teachers in EMI schools have been working to improve their English language skills.

There has always been pressure on English teachers in EMI schools to maintain students' English standards.

The morale of English teachers in EMI schools has fallen as a result of the change in MOI.

There will be increasing pressure on English teachers in CMI schools to maintain students' English standards.

\section{Effects of change to CMI on students - 13}

\section{Main comments:}

Students in EMI schools feel lucky to be there. (2)

\section{Individual comments:}

Change in MOI has had more effect on primary than on secondary school students.

Students are more aware of divide between CMI and EMI schools.

Only students who can benefit from EMI should stay in EMI schools.

Students in CMI schools will regard English as less useful in the short term.

Doubts over how students will cope with Chinese terminology in CMI schools.

Some students in CMI schools will welcome not having to study through English.

Some students in CMI schools will feel that they have been labeled as being less able. There is a common feeling that because of the change in MOI a drop in the standard o English of students is acceptable for the first five to ten years.

The change in MOI is intended to help the majority who were previously overlooked. Parents and students in primary schools are under stress to get into EMI secondary schools.

Students in EMI schools do not feel prestigious. 


\section{Effect on teaching or curriculum of change to CMI - 11}

Main comments:

A lot of resources have been put into English in CMI schools. (6)

Change in MOI has resulted in content classes in CMI schools becoming more interactive. (3)

Change in MOI has had no effect on ELT. (2)

\section{Effects on education system of change to CMI - 10}

\section{Main comments:}

The change in MOI will result in a strengthening of the elite nature of education in Hong Kong. (3)

Change in MOI has had a significant effect on education in Hong Kong. (2)

Individual comments:

Change in MOI has had a much bigger effect on education than the changeover.

Change in MOI has focused questions on language.

Change in MOI has had a positive effect on education.

Change in MOI has caused a lot of division in the education system.

Change in MOI has not had as big an impact as anticipated.

\section{English in EMI schools - 10}

\section{Main Comments:}

Students and teachers in EMI schools are having to work hard to maintain their EMI status. (4) Standards of English in EMI schools are being monitored closely. (2)

\section{Individual comments:}

Students will do well in English in EMI schools.

The change in MOI has resulted in a raised commitment to EMI in EMI schools. Students in F1 in EMI schools are now getting a purer form of English than before. In the past, English teachers in EMI schools taught mechanically as they felt they were not solely responsible for improving students' English.

\section{Effects of change to CMI on schools - 8}

\section{Individual comments:}

Change in MOI has affected policy in schools.

The status of EMI schools is rising. EMI schools are still more prestigious.

Some schools feel that they have been labeled as second class.

There are some well-established CMI schools that are equal to EMI schools. 
It is now more difficult to get permission to observe lessons in schools as they feel that you might be checking up on their language use.

The Government's policy on MOI has created elite schools.

Resources need to be distributed evenly to both CMI and EMI schools.

\section{MOI at tertiary level - 7}

\section{Main comment:}

There will be problems for students going from CMI schools to EMI tertiary education.

(2)

Individual comments:

Money needs to be invested in ELT at secondary level or universities will have to go CMI.

University students are finding it harder to cope with the curriculum in English.

University students are less prepared to learn things through English.

More teachers at tertiary level feel that they need to teach some parts of their lessons in English. The majority view is that English should be the MOI at tertiary level.

Time will be needed to judge the effectiveness of CMI - 6

Main comment:

It is too early to tell if the change in MOI is affecting education. (6)

\section{English in CMI schools - 5}

Main Comments:

Whatever English that students were getting from mixed code in content lessons they are not getting at all now. (2)

English will become worse unless enhancement measures are taken in CMI schools. (2)

Individual comment:

Mixed code will continue to be used in CMI schools.

\section{Effects of change to CMI on society - 5}

\section{Main comments:}

Over the long term, if most schools are CMI, then standards of English will inevitably go down. (2)

Individual comments:

Change in MOI has created a lot of anxiety. 
The change in the MOI has raised awareness of importance of learning English.

People do not know how to react to the change in MOI.

\section{Government influence - 2}

Individual comments:

Change in MOI was a political decision in that the Government thought that it would be easier to impliment after the changeover.

EMI schools are very conscious that the Government wants to move towards CMI.

Schools could be allowed flexibility in MOI -2

Individual comments:

If CMI schools prove themselves to be competent in English, they might be allowed to run parallel streams in English for certain subjects.

There needs to be a bilingual or trilingual education system rather than a division between EMI and CMI.

\section{Against EMI - 2}

Individual comments:

There has been less defence of EMI than there would have been ten years ago.

EMI as a system in most schools had to go as it wasn't working.

\section{Effect of firm guidance on schools - 2}

Individual comments:

There is still confusion over what was expected by inspectors of schools who applied to be EMI.

Relatively few schools objected to the change in their MOI status.

\section{Differences between EMI and CMI - 2}

Individual comments:

Research is showing that comunication is getting in the way in EMI classes compared to CMI.

The education system in EMI schools became one of rote learning from textbooks. 
In support of EMI - 1

Individual comment:

EMI schools are necessary to maintain the English standard of students.

In support of CMI - 1

Individual comment:

Students will be able to make greater progress at a faster pace when learning through CMI.

\section{Against CMI}

Background to firm guidance

Against firm guidance

In support of firm guidance

Teachers -72

Native English teachers - 15

Main comments:

NETs need to be used more effectively in schools. (4)

Individual comments:

The NET scheme has not been well thought out.

The NET scheme is bad for teachers' morale.

Local teachers see the NETs as a resource.

The NET scheme has had difficulty recruiting teachers.

The NETs are useful as they bring in a foreign culture to a school.

Students find the NETs have very interesting ideas and approaches.

Students find it more comfortable to talk in English to NET teachers.

There needs to be more NET teachers put into CMI schools.

It will take a long time before any significant impact is seen due to the NET scheme. The Government did a lot of bad PR work in regard to the NET scheme.

The Government should have done more for local teachers to lessen the aggravation caused by the NET scheme. 


\section{Teacher training -15}

\section{Main comments:}

There is going to be more demand for English language teacher education. (3) There needs to be a stronger component in IT in teacher education courses. (2)

\section{Individual comments:}

An English enhancement course will be brought in for BATESL Year 3 students. The BATESL course is taking in more non-JUPAS students to try to raise overall English standards.

The number of places on PgD or Cert. Ed. courses does not meet the demand.

There is a big demand from student teachers for instruction in how to handle students on low-band schools.

The weighting given to methods in teacher education programmes is declining.

The trend in teacher education is reflective learning.

There has been no change in teacher education.

Courses in multilingual education will need to be included in teacher education programmes.

Teacher education courses are going to have to offer streams which are Chinese medium.

In teacher education, more emphasis is being put on developing teachers who are multiskilled.

\section{Motivation and morale of English teachers - 12}

\section{Main comment:}

There has been no noticeable change in the morale of teachers. (5)

\section{Individual comments:}

Some teachers have doubts about the value of their work.

Teachers need to decide whether or not they are committed to a career in teaching.

New incentives affect the morale of teachers.

Teachers involved in the QEF are really excited about it.

Teachers prefer an unchanged syllabus because they don't like changes.

The morale of English teachers is greater than before.

People in schools are very demoralised.

\section{$\underline{\text { Standard of English teachers - } 9}$}

Main Comments:

There are people teaching English who should not be. (2)

Individual comments: 
English is being taught rather poorly in primary schools by people of less than minimal competence.

Teachers should be just as accountable to the public or parents as other professions to get themselves certified.

Teachers do not want stricter rules over who can teach and who can't.

There will be more emphasis on the competency of English teachers leading to them being subject trained.

The standard of English teachers in Hong Kong is not high.

There are still large numbers of untrained English teachers.

Parents feel that teachers of English are not good enough.

Situation and status of teachers - 9

Main comments:

There has been no change in the status of English teachers in EMI schools. (3)

Hong Kong English teachers have become more prized since the changeover. (2)

The economic downturn has resulted in more people wanting to become teachers. (2)

Individual comments:

Teachers are worried about their status.

There has been a rise in the status of primary school teachers over the past few years.

\section{English proficiency of English teachers - 3}

Main comment:

There needs to be more emphasis placed on the language proficiency of prospective

English teachers. (2)

Individual comment:

New English teachers should be proficient in American English.

Image of teachers - 3

Individual comments:

English teaching is seen as unattractive by school leavers.

English teachers in Hong Kong have a limited view of what students can learn.

The mobility of teachers is much lower than it was before.

Teachers' retraining - 3

Main comment:

If teachers are to survive, they must develop professionally. (2) 
Individual comment:

Teachers don't often get the opportunity to go out and have professional development.

Teachers' practices -2

Individual comments:

New teachers do not want to use English in their lessons.

There is a big gap between teachers' beliefs and their actions.

Needs for teachers - 1

Individual comment:

Trainee teachers must have pedagogical content knowledge as well as knowledge of language.

\section{Concerns of teachers}

Subject teachers

Teacher trainers

Teachers' roles

\section{Teaching Methodology and Curriculum Issues - 39}

Curriculum -11

Main comment:

Changes in the curriculum are the normal process of curriculum development and not because of the changeover. (3)

Individual comments:

Changes in society have required a change in the curriculum to more skills-based.

Changes in the curriculum must be matched by changes in the examination system.

The English syllabus for schools has been changed because they wanted to bring it up to date with modern thinking and pedagogy.

It is desirable to have a continuum from primary into secondary.

Hong Kong English syllabus is too localised and not given an international context. There has been a good response to the new English syllabus for schools from teachers. Elements of culture will come into the new English syllabus for schools. 
Students in Hong Kong specialise too early.

\section{Examination syllabuses -9}

Main comment:

Examination syllabuses haven't changed. (3)

Individual comments:

The examination syllabus is only changed if there is a specific reason.

Changing the exam syllabus is a very long process.

There is a reluctance to change the exam syllabus because it is comprehensive.

School-based assessment is becoming a more important issue.

The examination system is moving away from a pass/fail system towards a set of descriptors of what students can do.

The examination syllabuses will change but we cannot predict what the changes will be

$\underline{\text { TOC }}-6$

Main comment:

The new syllabus for English is a continuation of TOC. (3)

\section{Individual comment:}

Changes in HKCEE English syllabus in 1996 were in anticipation of TOC.

There haven't been any fundamental changes in the classroom as a result of TOC. The TOC has sreated a lot of unhappiness in primary schools.

$\underline{I T}-6$

Main comment:

IT will be imposed more widely in schools in the future. (5)

Individual comment:

Education has to become more IT-orientated.

Content -3

Main comment:

Education in Hong Kong is becoming more practical and skills based. (2)

Individual comment:

There is too much percieved importance placed on examinations. 
Possible changes in methodology - 3

Main comment:

Self-access learning will be an important approach in the future. (2)

Individual comment:

In English, there will be more developmental writing and portfolio work.

Existing problems with methodology -1

Individual comment:

There has been a reduction in the regard given to the teaching of listening.

Students should learn grammar

Extra-curricular activities

Textbooks

Focus on English - 35

Status of English in Hong Kong will remain high - 24

Main comments:

Parents still think English is important. (5)

Students feel that English is as important as before. (4)

Students' attitudes towards the learning of English haven't changed much since the changeover. (4)

Employers/business people think that English is important. (3)

There has been no noticeable change in the status of English. (2)

Individual comments:

Teachers still tell their students that English is important.

There has been no noticeable change in the attitudes of the public towards English.

If attitudes of the public have changed, they are in favour of English.

People in Hong Kong seem more willing to speak in English.

English has become the necessary accouterment for an educated person.

English has an even higher profile than before. 


\section{Question of role of English in Hong Kong - 4}

Individual comments:

English is a foreign language in Hong Kong.

There is no longer the stigma of English being a colonial thing.

University graduates only need to be able to read and understand English so that they can use the Internet.

Employers' requirements of employees' language abilities are broader than they used to be.

Status of English in Hong Kong will decrease - 3

Main comments:

Students are less sure about the importance of English than previously. (2)

Individual comment:

Some students feel that English will become less important in the future so they feel they should do something themselves about it.

Concern about falling English standards in society - 2

Individual comments:

The Tourist Association are concerned about English standards.

There is concern about the need for improvement in English in the workplace.

English in universities - 2

Individual comments:

Students feel that they only need to be able to read and listen in English unless they want to go to university.

As standards of English fall, the universities will have to admit students with lower English standards than they do now.

English as an international language

\section{Benchmarking - 20}

Questions on implementation of benchmarking - 7

Main comment: 
There is uncertainty about the procedures involved with the benchmarking. (2)

Individual comments:

EMI schools fear the Government will use the benchmarking as a way of removing their EMI status.

It is more sensible to spend money on training teachers than on testing them.

The Government's propaganda on the benchmarking has not been very good.

The benchmarking is unlikely to be done sensitively.

The benchmarking should involve teachers getting a profile of their competences in the different areas.

Benchmarking will put pressure on teachers -5

Main comment:

The benchmarking is putting pressure on teachers. (3)

Individual comments:

The benchmarking is going to have a very important impact on teachers.

Some teachers are feeling anxious due to the benchmarking because they have gone past the time when they could change careers.

Against the benchmarking - 2

Individual comments:

Some teachers oppose the benchmarking because they think it is an interference in their professional independence.

Some teachers might fail the benchmarking in order to have a week off at the Institute of Education.

Benchmarking as a way to improve teachers' language proficiency - 2

Main comment:

The benchmarking is an attempt to raise the standard of existing teachers. (2)

The benchmarking and teacher education - 2

Individual comments:

The benchmarking will lead to changes in language teacher education.

The benchmarking needs to be built into teacher education courses. 
The benchmarking related to professionalism of teachers - 1

Individual comment:

Some teachers oppose the benchmarking because they think it is an interference in their professional independence.

In support of benchmarking - 1

Individual comment:

There is big support for the benchmarking from the public.

Benchmarking for all teachers

Benchmarking for new teachers only

Incentives for those who reach the benchmark

Origins of benchmarking

Education Policy - 16

Educational reforms -13

Main comments:

Education in Hong Kong is in the middle of a huge transition period. (2)

Individual comments:

The Aims of Education document is too general.

The Chairman of the Education Commission is very ambitious.

Changes in education are being made on all fronts resulting in a loss of the main focus The Government has not made up its mind as to the way ahead.

Educational decisions are now being made based on research rather than popular trend The Chairman of the Education Commission makes decisions like a businessman rathe than based on research.

The Government is showing great determination to enforce changes in education. The extension of schooling from five to six years could free up teacher time provided there are no cuts in manpower or resources.

The emphasis and spending on IT in schools could be a smokescreen to cover up problems involved in implementing other changes.

The Government's method of consultation with the public on educational reforms is seriously flawed. 
The Government do not involve the grass roots in any educational changes.

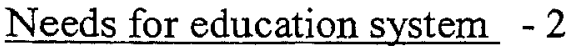

Individual comments:

The number of post-secondary places needs to be increased.

Space needs to be found in the timetable for teachers to meet to reflect.

\section{Comment on education in Hong Kong - 1}

Individual comment:

The education system will become less elitist in emphasis.

Changes due to the changeover

Call for 4-year university programmes

\section{Students - 15}

\section{Culture of Hong Kong students - 7}

Individual comments:

Students have realised the importance of 'soft skills' in communication.

There is an ambivalence amongst Hong Kong students as to whether to fully embrace Chinese culture.

Students now are required to do more than they were in the past.

The future world will expect different things from students.

Students are more aware of their rights and that they don't have to accept what is given to them.

Students are using English for communication with each other via ICQ.

It is very hard for most Hong Kong students to understand humour through English.

\section{Comment on language standards - 7}

\section{Individual comments:}

The gap between students' enthusiasm for English and the effort they put into learning it is getting wider.

The English standard of students in Hong Kong is declining.

Students are able to solve problems but their communication skills are weak. 
There has been no noticeable drop in standards of English proficiency of students over the past two years.

The majority of students will still try very hard to keep up their English.

Students expect their teachers to explain things in Cantonese.

Standards of English in secondary schools in Singapore are higher than in Hong Kong.

\section{Criticism of students - 1}

Individual comment:

Students don't do well on UE oral exam when faced with non-local topics.

\section{Affect of changeover}

\section{References to the Changeover - 12}

The changeover has had an effect - 9

Main comments:

As a result of the changeover, people feel that they need to question the role of Englis and feel duty bound to emphasise Chinese and Chinese culture. (3)

Students are using the changeover as an excuse for not trying as hard as they used to $d$ in English. (2)

Individual comments:

To say that the changeover has had no effect is an interesting statement.

Mass changes are the result of post-colonial syndrome.

Education will become more diversified as a result of the changeover.

There was an initial increase in Chinese ethnocentrism after the changeover but that soon changed as people realised that English is becoming more important.

\section{Reference to the changeover -2}

Individual comments:

Educators were worried about the status of English changing before the changeover. Since the changeover, education has become less functionally oriented.

The changeover has had no effect - 1

Individual comment: 
Since the changeover there has been a combination of changes in education that were not related to the changeover but were part of the general trend during the last years of colonial rule.

The changeover will have no effect

There will be changes but not due to the changeover

There will be changes due to the changeover

\section{Putonghua - 11}

\section{Putonghua in Hong Kong society - 5}

Main comment:

Putonghua is becoming more important but not at the expense of English. (4)

Individual comment:

Putonghua has taken over some of the importance of English.

Views of students on Putonghua - 3

Individual comments:

Students feel that there is less requirement for English than for Putonghua.

University students are more aware of their deficiencies in Putonghua than in English. Students who resent learning English might think they won't need it in the future as Putonghua will be more useful.

Putonghua teaching in schools -2

Main comment:

The introduction of Putonghua as a HKCEE subject will have an impact. (2)

Teachers of Putonghua - 1

Individual comment:

Putonghua will probably not be a compulsory subject because there are not enough teachers. 
Putonghua as MOI

\section{Reference to Hong Kong People - 8}

Views of the public on education -8

Main comments:

The public are becoming more involved in education. (3)

Many parents are confused about the changes in education. (2)

Individual comments:

Parents are more concerned about their children than the general decline in English standards.

The views of the public on education are very negative.

Many people in Hong Kong think that one must go overseas to achieve a good English level.

\section{Pragmatism of Hong Kong people}

Non-negative attitude of Hong Kong people towards English as colonial language

\section{English teaching in China - 6}

English learning is very popular in China -4

\section{Main comments:}

In China, English is becoming more and more important. (2)

Hong Kong will need graduates with good English proficiency just to keep up with the Mainland. (2)

\section{Universities in China - 2}

Individual comments:

Students may think that even if they cannot get into a local university they may be able to get into a university in China.

Universities may become more like their counterparts on the Mainland.

English teachers in China 


\section{English teaching methodology in China}

\section{Reference to Chinese - 3}

\section{Importance of Chinese - 3}

Individual comments:

People now feel that using Chinese (in Government Departments) is more acceptable than before.

Chinese only is being phased in for documents that used to be bilingual (in Government Depts).

Research seems to show an increase in Chinese ethnolinguistic vitality.

Simplified characters

Standard of Chinese

\section{Schools - 2}

Schools' accountability - 1

Individual comment:

Some Band 1 schools may go private as a result of changes in the banding system.

Alternatives to government-run schools - 1

Individual comment:

It is necessary to have a variety of schools for parents to choose from.

\section{$\underline{\text { Schools' prestige }}$}

Comment on language standards in schools 


\section{A.8 Interview Transcripts from Interviews in 1999}

In the interview transcripts that follow, Roman numerals are used to designate the interviewees that took part in both rounds of interviews (in 1997 and in 1999). The transcripts for these interviewees are presented first. These are followed by the transcripts of the remaining interviews, which are numbered, but are in no particular order. The short form of the year of interview is given in brackets to help distinguish between the two rounds of interviews. Three of the 'interviewees' submitted responses to the questions in A.6 in writing and copies of these responses are provided after the interview transcriptions.

\section{Interviewee I(99): University educator \\ Date: \\ 22 June 1999}

$\begin{array}{ll}\text { AU } & \text { Researcher } \\ \text { I(99) } & \text { Interviewee } \\ \ldots & \text { Missing words / Unfinished utterance } \\ \text { - } & \text { Pause }\end{array}$

AU First question then, how do you think the change in the government policy on medium of instruction in schools has affected education in Hong Kong?

I(99) I think it's had a significant effect, it's focused questions on language, it's certainly affected policy in schools. The EMI schools are very aware of the fact that their teachers are going to be benchmarked and in fact by March 2000, any new teacher going into an EMI school, let's say somebody in Canada with a degree in physics or chemistry who's being hired by an EMI school will have to be benchmarked before they go in. So what they're trying to do there is that certainly the standards of English in EMI schools are being monitored very closely and there's been a report from the Inspectorate on this. They've said that on the whole people were coping but that there were some problems. It's affected education in the EMI schools in that they are very conscious that government wants to move towards Chinese-medium education, which I believe in. And there is a fear that they will use the benchmark as a way of telling schools they are no longer able to offer English-medium. That's one thing, plus government are committed to the CMI. On the other hand, government are aware of parents' views, that parents are not educated about the facts of education that they do not know, they think that a school calls itself English language then that's what's happening to the kids and we all know ... it's not. So, on the one hand they might be a little bit loosening and if they can find CMI schools who are competent in English, they might have parallel streams for certain subjects. So I think certainly it's affected education considerably. It's too early to tell whether the 
focus on CMI in all other schools now is affecting education, and that'll take some time to work through as we see the results of the CE exams in about three or four years time, kids who are coming into Form 1, taking the exam in four or five years. Also the fact that now there is CMI has led to the setting up of a number of centres and we are now starting to get really serious research on what happens in a CMI classroom and an EMI classroom. So in our CMI support centre for teachers there's some fascinating evidence where two classes in the same school, one taught in Chinese, one taught in English, by the same teacher, clearly reveals that minimal communication's going on in the EMI school, in the EMI class, and that concepts are explained at the basic level, it's just what they need to know, there's no extra in-depth, there's no extra explanation, there's no elaboration, there are no exemplars because communication is getting in the way. So there's some stuff coming out now which I think is quite interesting.

\section{Do you think that it's affected English teaching?}

I(99) Well yeah, because whatever little they might have got from mixed code they are not getting that now at all, and I think it simply clarifies the paper that I did, remember for the Lingnan conference just on the eve of the 1997 transition, and I noticed a NET teacher talking about it the other day, people still in Hong Kong consider it a second language, and it's not, in spite of the fact that government uses English so much. As I said two years ago now, exactly two years ago this week, and as I've been saying for some time, to all intents and purposes English is a foreign language in Hong Kong and we know that school tuition in a foreign language, whatever country in the world, is not enough by itself, there must be extra. And this is why there must be enhancement measures and all kinds of measures. So I'm convinced that English will become even worse than it is now if it's left by itself in CMI schools. I think what's happening in the EMI schools, the ones that are left, the 114 , is focused on properly and those kids will do well, and that's what parents are worried about, of course. It's an anomalous situation, they want to be educated in Chinese, but because they are focusing on it in the EMI schools, they'd better get their ... Another bit of fascinating news which came out this morning and this is something that you guys will be involved in, English Centre here, other places, government want to ... a significant gain. People who come to your English Centre and ours it seems to be ... this is off the record I'm afraid but you might want to know, the good ones make progress, those with As and Bs in Use of English, the poor ones get worse. That's going to exacerbate the situation, so they either invest more money or we go CMI in the universities.

Do you think that's likely?

I(99) Not for the next 5/10 years ... until we get a democratically elected government ... because the powers that be are too strong. As I've always said, 
it doesn't stop Japan from trading the fact that everybody gets educated through Japanese, or the Russians from trading, as long as you've got a cohort of good speakers.

AU I know that in the CMI schools they have been offered resources to improve their English teaching.

I(99) I think they're focusing on it and I think one of the good things is we ticktacked along with mixed code with this lip service to English and now they realise how bad it is and the research is coming out, so they will probably put the resources in there and get the teachers better through benchmarking. So I'm quite hopeful because we were in an FL situation which nobody recognised. Parents thought that everything was all right because schools called themselves English schools, they weren't. But now they realise the problem in CMI if they've only got these lessons a week. It's like expecting kids in England to operate at age 16 successfully in French. We know that's not possible. This is an anecdote, I don't mind you quoting it. We have this wonderful helper ... Chinese ... who has a 16-year-old girl who's just done the Hong Kong Certificate, and it's only one anecdote, but I suspect when we investigate it further because there is some research to back it up, $\mathrm{Liz}$ Walker's research on anxiety and sixth-formers about English, and the fact that they have developed avoidance strategies to talk in class in sixth form. And this 16-year-old child who came along, two girls came along with our helper's daughter, she's been coming along and my wife talks to her, she's now grown in confidence. Margaret was having a problem with one of them in that she just wouldn't respond. After about 2 hours, because my wife's a professional, the girl started saying the odd word and another word ... it emerged that this child has never in her 16 years of existence ever spoken a word of English in the classroom. And when asked what happens, they do what they do, they stand up and put their head down. Teacher knows they're shy and teacher turns to somebody else. You know the thing, stand up, ask the question, doesn't answer, ask somebody else. Avoidance strategy, the point is that the child has never used a word of English in her life!

AU I can believe it.

I(99) But it's an indictment of the system, isn't it?

AU Yes it is.

I(99) It's only an anecdote, but you can quote it.

AU I guess it's always the case, oh she's a bit shy today, she'll get a chance later 
I(99) That's right and when anybody ever comes in, oh she's a bit shy. And the teacher then starts avoiding her and you turn to the ones who are willing. That child had a hell of a lot in there [points to head]. It needs to be activated and with large classes I'm afraid that's simply not possible ... forty in a class.

AU Okay, have you noticed any changes in the status, situation or morale of English teachers?

I(99) No, certainly we're getting massive applications for our B.Ed. in language, like massive, and for our PCEd., from teachers who want to be teachers because of course the economic downturn. No I haven't.

AU The only thing that I have sensed a little bit when I have been visiting schools amongst local teachers is this slight animosity, maybe not animosity but jealousy towards the NET teachers.

I(99) You should seek information from David Bunton on that because he runs the TELEC and they have an open net and that's a wonderful source, where teachers actually come in, NET teachers and local teachers and all of that. Sure they don't like it but I don't think it's affected their morale or whatever because I think the NETs have been pretty well briefed this time, they didn't come in like the last time, do you remember in $89 / 90$ ? When they thought they were coming in to save the situation. They've been told what to do. The teachers that I've talked to see them as a resource, unless they're stroppy. And I think the heat went out of the situation when it went down to, what was it, $\$ 13,000$ for the housing allowance. I think those who are threatened, those who are on the defensive will feel threatened or defensive about anything. Those who feel reasonably confident about themselves as language speakers just take it on board.

AU When I ask to teachers to what extent do you use interactive strategies in you teaching, they say, well, I teach grammar and the NET teacher does all the speaking activities. They have all the fun while I do all the drilling, so to speak.

I(99) That I think is lack of knowledge about the principles. I don't know if you saw the letter to the paper that my group ... we said it's a waste of a resource to give them 30 lessons a week where they see a class of forty once, for English, for communication. These people are well qualified, some of them are superb, they really are a good bunch and some of them are doing superb work and the schools, once they get over their first contract ... people have been assimilated and moved in and on to higher education. Some are still there, once they get beyond the first contract then they get trusted. I wouldn't give someone who doesn't know the situation an exam class, when exams are so important the parents would kill. You know this ... but I haven't noticed a dreadful reaction, I must say. 
AU No, I wouldn't say it was dreadful, no. Any changes or differences in students' attitudes towards learning English?

I(99) I think in the EMI schools they are now certainly getting English all the way through, there is still a little bit of mixed code still but they are getting reinforced and they are going to get on fine. In the CMI there's a little bit of feeling, now I haven't got this personally because I'm not working with the PCEds these days, I'm mainly doing Masters and PhDs but from my teachers I'm getting the idea that students feel, students not their parents, students feel there's less requirement for English and that Putonghua is really a very useful skill to have. They think it's necessary but it's jam tomorrow and ... they always want jam tomorrow whereas English is jam five or six years down the road and oh, it'll wait.

AU Do students feel like that, that oh, we're part of China now and so English is not so important?

I(99) I don't think it's so much that we're part of China but it's our biggest trading partner and that's where the work will be, most of my work, so long as I've got a reading knowledge. I think there's a feeling that if you can read, the speaking bit can be left to others. As long as you can read and understand and watch the movies, listen to the songs, the passive skills, you don't really need to do the writing and the speaking unless you want to go to university. Certainly at the universities there's much more Cantonese being used for lecturing, much more, Chinese $\mathrm{U}$ is now ... hundred percent at Chinese $\mathrm{U}$ almost, except for foreign lecturers.

AU I'd say it's probably sixty, seventy percent at our place, maybe even more.

I(99) And again, the kids are not going to get the reinforcement there. So there is a massive problem with English, we are never going to be out of a job, because they know they're going to have to have a cohort who can operate ... but I wonder how much of it has to be, being slightly provocative, but provided they can read and understand and listen and understand, they don't have to be totally fluent in the writing and the speaking. They need to be able to absorb, to read, to do stuff on the net. How much do they need to be able to operate at civil service standards for writing? The civil service are worried about the standard of graduates coming in and are even talking now about developing a new test to really suss them out. So they're worried. And I think also that students feel more that they're in a Chinese-medium environment, so I've noticed a lot more notices going up in Chinese than in English. Oh yes ... our external has noted that students feel less aware of their weaknesses and deficiencies in English but they are very conscious, this is our recent external examiner's report, very conscious of their proficiency and deficiencies in Putonghua. So there's great awareness there and less awareness of English. 
It's something that fairly new, I suppose.

I(99) Oh yeah, and they're willing to pay a lot for their Putonghua, considering going to China for two or three weeks. They know the economic advantages.

This leads on to the next question, we may have even talked about it already, the attitudes of the general public towards English.

I(99) I think the general public, the parents, that's not the general public, the parents still see EMI as the way to economic and academic success. For them it's quite clear. They're very suspicious of CMI schools, they don't want their kids to go to them, this is the general public, and they wish for a relaxation and a back to where they were before, because they knew where they were, status-wise, and how the kids could get through the exams. I don't think it has changed. It's changed towards maybe English people, but not towards the learning and teaching of English, no. They feel that the teachers are not good enough, teachers of English. They're right. There's a percentage of people teaching English who should not be teaching English. I can tell you that from benchmarking. It's not massive but there are some who should not ... there are some who are not doing any harm, but they're not doing any good at all. And there are people who I think are actually doing harm to the kids, people who should not be drawing a salary for teaching English.

Are the public aware of this do you think?

I(99) Yes, the public think there're low standards. There's big support for benchmarking from the public.

You said towards English people there's been a change.

I(99) Well this is the NATO stuff, the bombing, since the bombing there's been a certain amount of attitudinal change, and Americans, but that doesn't stop them doing business with them or whatever, and less in Hong Kong than in the Mainland.

AU Changes in the curriculum or examination syllabuses since the changeover?

I(99) Yes and no, nothing has happened yet, but the ROPES report, Review of Public Examination Systems, 1998, the Baptist University review? Clearly we'll be seeing big changes, we'll be seeing a lot more project work, a lot more formative evaluation a lot more continuous assessment. They want to build on that in the lower schools and then start to build it into the syllabus and I think the Government is responding favourably to that. As far as the curriculum itself is concerned, the preliminary syllabus for secondary has come out and is now being discussed. It's too early to say, it's been published, you've seen it. There's also one on sixth form Use of English, 
which has just come out again, some guidelines. Why have they been made? Normal process of curriculum development. It's not affected by politics ... do you think these have had or will have? I think they are absolutely for the better and I think it's a slow movement which has taken a long time. There's the TOC and that's been slowed a bit, but that's been built into the ROPES review and I believe that the review of education will also move towards other modes of assessment, not merely the old exam, norm-referenced itembased stuff. There'll be much more in the sciences, there'll be more project work and I think specifically in English we'll see a lot more of developmental writing, of portfolios of work. We've already introduced it for English Literature but it's such a small candidature, that all of this stuff will come and I think it's absolutely for the better. And I don't think it's got anything to do with the transition.

AU No, no, that's why I put since the changeover, as that's my time frame.

I(99) Exactly, you've got a nice point, and I think this is purely curriculum reform, changing paradigms in assessment, which people are catching up on. They've been on the move since the eighties and we're finally getting round to them.

AU Can you envisage any changes in English language teacher education?

I(99) Yes, a couple of things, which I think, are quite good. One is the notion of the whole teacher has entered language teacher education as opposed to a more narrow focus on competency and methodology and what have you and a language knowledge and a language awareness. Those are still stressed but more and more we're being encouraged and persuaded and beginning to put up electives and adoptions for things like counselling and guidance, making them more aware of that, environmental issues, extra-curricular activities, that slight move which I think is good so that you're not just a teacher of English who goes to those classes and does that, you're a teacher in a school within an educational framework and you'll be a specialist in English but you'll also have an awareness and knowledge of other things as well. And one of the things that's changing with ours is being forced upon us, it's quite interesting, principals'are saying, we don't want somebody who's shit-hot, a B.Ed. in language education only, they're looking for the extra strings to their bow because often they are looking for someone who can do maybe three or four lessons in English but one of economics or one of history. Now there's no way we can build that into our curriculum because we want to get our people from a high standard and from my perspective I want people who are really very very good in English, aware of, have the use of, might never have to use all that in the classroom but they need to have it as a repertoire of skills and knowledge and Michael Halliday, our external, has just said that in a report I've got today. And I believe this very strongly and that's why in the benchmarks, we're not just testing the language that you need for the classroom, but also testing the language that you need to be a language 
teacher. So I think that we are finding changes in the sense of ... in two ways, maybe broadening those teachers and the second one is more and more increased emphasis on language awareness, not just language knowledge but that awareness, that further step and then moving that into pedagogical content knowledge, not just content knowledge but that awareness that is slightly different from just knowing the rules, being aware of the register and the sociolinguistic features and the varieties, all of those things as well as the syntax and the morphology, which they have to be rock solid on and which we try to work towards. I think that the changeover was an effect because I think the educationalists have felt slightly less functionally-oriented now. The functions still strong but I think they're moving towards the whole person in the schools and that means more of a whole person amongst the teachers.

AU And the last one is a very open question, what do you see as the future of education in Hong Kong?

I(99) The rag-bag question! I think Putonghua will assume greater significance. I don't think English, the need for will diminish. I think we'll continue to get the businessmen crying out that we haven't got enough people of sufficient standard. I think there's a chance that things will improve because of the focus now being given and I think two big things: one is, the Government for about the last four or five years been funding research specifically to find out so that decisions are being made on at least a research basis, not just the latest prejudice that somebody has. I'm still concerned about the Chairman of the Education Commission, Anthony Leung, who gets all the data and stuff presented but still goes with their gut instinct, as businessmen do. That's a worry. I think the focus on CMI and EMI and all the data will at least give us a way forward and I think that's good. I think there's a danger that unless they put massive resources in, which they won't, especially if economic times are tight, into CMI schools to give them that extra bit. They might get some sense, I don't think this will ever happen, do the sensible thing and take English out of the primary schools or at least out of the first three years and give the kids a solid grounding because to be honest it's being taught so dreadfully in the primary schools by people of less than minimal competence on the whole, our evidence proves this. It's a waste of money. They might as well spend the money on improving the teachers they have got of English or ensuring high standards. I very much hope that it will change for the better when they move towards a qualified graduate certificated profession because our benchmarking has revealed very clearly ... when're you going to publish this by the way?

AU Probably not for another year or so.

I(99) Right, our evidence shows clearly that head and shoulders above everybody else, guess who? People with a relevant degree who've majored in English in their PCEd, and everybody else lags behind. That's what they should be 
aiming for. Once they do that you can do something about English. But at the moment, guess who's at the bottom? Primary teachers, who are trained but haven't done English as a major but have to teach it. These people have less than minimal competence.

AU Oh I know, I know that from personal experience.

I(99) But we've now proven it. Tell me who you think is second. People with a relevant degree but no professional training or people with a non-relevant degree and professional training, with the professional training in their relevant major?

AU People with the professional training?

I(99) Correct, now that's interesting, across the board. We thought experience wouldn't count, we thought it might be those between five and ten years experience but experience is a factor. So maybe the more they do it the more they get used to it. This is with a cohort of three hundred, it's good enough. The report will be coming out ... I hope they publish it and don't mess around. They kept our 1996 report hidden because we were proposing prototype benchmarks then and they didn't want people to think those were they, because we've changed them considerably. We're at the recommendation stage now ... and I hope they publish it, I mean that's chapter two, you can see the title of that, and this is the report, you can see we're more or less there ... there's fifteen chapters and all research-based. So I think providing the economy doesn't go bust, I think they're targeting, they're focusing, it's not just the functional approach that the British had it's get the job done and make the money, they are now thinking more widely. I think this review of education is a very good thing; it's getting people to think and question. 
Interviewee II(99): University educator

Date:

21 June 1999

$\begin{array}{ll}\text { AU } & \text { Researcher } \\ \text { II(99) } & \text { Interviewee } \\ \ldots & \text { Missing words / Unfinished utterance } \\ \text {-. } & \text { Pause }\end{array}$

AU My first question is how you think the change in the medium of instruction has affected education, although it's the first year.

II(99) Has affected education in what sense? What do you mean by that?

AU Affected English teaching primarily plus maybe the way the schools are run in the language that is used within the school, whether it has freed a lot of teachers so that they don't have to use English anymore and they can use Chinese for all purposes.

II(99) But that's very different from English language teaching so I think what are you talking about here?

AU Well, English language teaching primarily.

II(99) I don't think it has any effect on English language teaching at all. I think English language teaching just goes on as they have always done. English teachers in Chinese-medium schools just carry on with their work. I don't think that the major impact of the medium of instruction is on English language teaching at all, it has a lot of impact on content teaching but not on English teaching.

AU What I've found, I've been into a few schools and observed some teachers and compared to observations I did a couple of years ago, it seems more Chinese is being used in English lessons. My feeling is that it's more acceptable to do that now, since the change in the policy.

II(99) Have you compared before and after, I mean have you looked at the same school before and after?

AU No, I haven't, it's more of an impression. I have looked at some schools before and after but they have haven't really changed. One was an Englishmedium and is still English-medium, so there's not been any change. 
II(99) I think you might want to be very careful about making a statement like that because it really depends on the kind of school you're looking at, I mean if you are looking at pre-vocational schools and that type of schools, there has always been .. I mean they have always used Chinese and in fact in the recent English language teacher benchmarking we find that in some cases you just cannot ... it's a case of no evidence because for the classroom language component you just can't assess because they've been using $80 \%$ Chinese in English lessons. So that kind of thing does happen. But we could also say that in a lot of CMI schools, they have put in a lot of resources for extra-curricular activities. I think that's definitely the change, basically because of the availability of resources from the government to support CMI schools.

AU I have seen that, plus the NET teachers as well.

II(99) Yes, the NET teachers, if anything that is definitely something that affects English teaching, but in terms of the curriculum, I don't think it has changed.

AU No, you said that you think that content teaching has changed a lot.

II(99) If you are talking about content teaching, about what goes on in the classroom and also the kind of work that students are engaged in, I think in some schools there are some very fundamental changes going on there. It's still in a small number of schools, but they are very indicative of the future trend, there is more project work, students go and do field work, investigate and do reports. In terms of teaching in the classroom it's much more interactive. Students are beginning to find that they are bored because the curriculum is not challenging enough and teachers are finding it much harder to teach because the students have got so many questions that they can't answer. So those are the very important changes.

AU I see.

II(99) You've probably heard about these changes.

AU Yes, to a certain extent. So English teaching you think has not really changed?

II(99) I think English teaching in the classroom has not really changed but outside the classroom is something that you might want to explore? So you might want to ask CMI schools how they spend their resources on that. Some schools have set up English corners or rooms, debates, whatever ... extensive reading ... I think that is the place where interesting things are going on. But in the classroom it's still very much the prescribed curriculum.

AU I notice that with the NET teachers, and this is just the three or four schools that I've been to, they are given the task of teaching oral English and one or 
two of the teachers, of the local teachers have remarked to me that they then focus on teaching grammar and writing, which they then say ... 'cos I've been saying, 'Well, how interactive are your teaching activities?' And they say, 'Well not really because I just teach grammar from the textbook, maybe the NET teachers does activities and games and so on.'

II(99) Yes

AU There's a little bit of dissatisfaction; I sort of felt it there, not actually expressed as such.

II(99) You mean by local teachers?

AU Yes

II(99) Oh yes, I mean the whole NET scheme is a very political issue but you might want to find out from the NET teachers what they are actually doing with the students and whether what they have done has had any impact on the rest of the English teachers.

AU Have you noticed any changes in the situation, status or morale of English teachers? That's one aspect.

II(99) No, apart from the NET scheme, but of course it's got nothing to do with the changeover, it took place before the changeover, the fact that the NETs are getting better treatment than the other English teachers ... in fact some of them are really local and they are Hong Kong residents, it's just that they were brought up overseas and some of them have their family in Hong Kong. Why are they getting different pay? It's a very tricky issue.

AU There definitely now seems to be a divide between local teachers and NET teachers.

II(99) Yes, absolutely because, I mean if the pay is different, the terms of service are different, you can't really blame the local teachers.

AU I taught in secondary school for six years but I was employed on local terms for the first four years and then I qualified for NET terms.

II(99) And then you also have issues, for example, you have overseas Chinese, who they say, well, I'm sorry you don't qualify, because I'm not Caucasian or what, you know? And then you also have the problem of some local teachers whose English is super, excellent English and I would say ask them about their subject matter of English, and I would say they are I don't know how many cuts above some of the NETS. I just feel that the whole scheme is not well thought out. It's not very good for morale. 
AU No, no

II(99) So, students' attitude, you want to talk about students' attitude towards learning English?

AU $\quad$ Yes

II(99) I think the medium of instruction, the fact that everybody wants to get into EMI schools, has a much stronger effect than the changeover. So the changeover doesn't really, as far as the school sector is concerned, it doesn't actually have that much impact as [the] changeover per se but it's because of the streaming that people say it's so important, you know. And the effect is probably more pronounced in primary than in secondary because once you are in secondary school there's very little you can do about it, you're either CMI or EMI, but in primary it's what the results you can get in your English test...

AU The push to get into those schools in the first place.

II(99) Yes, and certainly if you are thinking about students' attitudes as being influenced by their parents, then this is certainly a very very strong concern for parents.

AU I was just think whether students in secondary schools, whether ... I mean I put 'since the changeover' it's merely putting it in a time frame, it's meant to be over the past two years not because of the changeover. Whether the actual changes in the medium of instruction policy and so on, whether students' attitudes have changed significantly or whether there's been a trend over the last number of years that has continued, or maybe ...

II(99) I think students are certainly made more aware of this divide between CMI and EMI schools and that they feel that their parents would want them to get into EMI schools but they themselves do not necessarily think that this is the case. I think there are some students who say, 'If you want me to choose, I think my parents want me to go to an EMI school, but I enjoy CMI classes.' I think there's a lot of that actually.

AU So they try for their parents' sake, I suppose.

II(99) Yes, but for themselves I'm sure they'd find CMI lessons much more enjoyable.

AU But as regards actually learning English, has there been any change there do you think? 
II(99) Not that I'm aware of. I don't think that there's a feeling that since the changeover English is not important anymore. I don't think there is this feeling at all. I think they still feel that English is very important.

AU And how about the attitudes of the general public towards English?

II(99) It depends on who you mean. I think for business, people in business, definitely still insist that English is very important; they are very worried about the medium of instruction policy, that it might cause a decline in English standards. And I think SCHOLAR was recently talking to various big business firms about English in the workplace and we're trying to get together a consortium to look at how we could look at English in the workplace and make sure that there are channels for training people of different levels, so if you interview Nancy Lee, she'll be able to tell you the things that we're trying to grapple with. So, I think business definitely, people like the Tourist Association is very concerned, definitely parents are very concerned. Parents are concerned, not about the general decline in standards, they're concerned about their children. Business is concerned about the general decline because Hong Kong is going to lose its competitive edge. Tourist Association is concerned because tourists complain they come to Hong Kong they just can't talk to them in English, they can't even understand emergency calls and things like that, so you can't make a blanket statement about the general public, you have to look at the different sectors.

AU I just wonder whether there's been a sort of shift in approach, this feeling that English is always important for getting on, for career and so on and that now with Hong Kong being part of China that it's perhaps not seen as quite as important as most business seems to be done with the Mainland and that Putonghua will become more important.

II(99) I think there's certainly a feeling that Putonghua is becoming more important, but it is not at the expense of English. I don't think people will feel that it's less important, less ... I don't think it's happened, and it's also because even in China, English is becoming more and more important so ...

AU Okay, any changes in the curriculum or examination syllabuses for English, what are they, why do you think they have been made?

II(99) You know they've come up with a new syllabus, a new English syllabus, are you aware of that?

AU The new curriculum.

II(99) The CDI?

AU Yes 
II(99) They just sent me a copy last week, but actually the new syllabus has got nothing to do with the changeover, it's a continuation of the TOC trend. It started with primary, now they're thinking of secondary and task-based learning and that kind of trend, more than the changeover. I'm sorry to keep ... I don't want to be a wet blanket but I really don't think that it is an issue here in terms of English language.

AU The changeover you mean?

II(99) Yes

AU You're right, I should probably have taken ... it's in my title, I should probably have taken it out, that's why I put 'since the changeover' rather than 'as a result of the changeover', just within the last two years, I guess, that's the time-frame I'm using, and whether things are following trends that have developed over the last ten years, say, or whether there has been any sudden changes.

II(99) But on the other hand if your conclusion is that the changeover has had very little impact, that itself is a worthwhile statement because there was such a lot of speculation before the changeover, and if your research says, look all these speculations were unfounded and ungrounded, I think that's an interesting statement to make. I remember the Lingnan conference, which I don't know if you were there, which took place right before, and there were a lot of discussions and people were ascertaining each other and were reassuring each other that English is not going to change, I don't think the status is going to change, let's not worry about it. I just thought it was ... to me I just don't know whether it's because people were worried and that's why they keep reassuring each other that nothing is going to happen.

AU Maybe even people if they've been here are unable to see the changes...

II(99) I think the only ... if you were saying in terms of the way in which language is taught and is learned then I think if anything it will only be obliquely influenced by MOI. Of course then you could ask, why did the medium of instruction policy change after the changeover, because it was something drafted in 1990 and they laid down the timeline for it to be implemented in 1998. Now that obviously is a political decision, that they thought that that's going to be a lot easier to implement when there's a changeover, but I think that they completely miscalculated. So they just did it in a top-down fashion and in reaction to that, a lot is happening ... the NETs, the extra resources and things like that, so it might be useful for you to just take a few cases and really look at what is actually going on in schools, which may not come through at a macro level but maybe something very interesting is coming through. 
AU My main focus is looking at a selection of teachers who graduated from the BA course at City University and I've followed them, two groups in fact, since 1994.

II(99) Right

AU And so what I'm doing now is getting the macro view so that I can put my other findings into context.

II(99) It could also be I'm so close to ... that maybe if you asked somebody who's not so involved in the field, I mean this whole business of language benchmarking ... again it's something which happened before the changeover and not because of the changeover.

AU Yes

II(99) I'm conscious of the time ... English language teacher education as a result of changes which have occurred ... not really ... if you think of language benchmarking and the subsequent refresher courses that's going to be offered to teachers as a way of upgrading their language competence, then that is going to have a very important impact on teachers but as yet nobody knows what the content is going to be, but when it does happen then it will be quite interesting to see how many teachers get the training, what happens to them after they've been trained and whether the benchmark exams have any washback effect on their teaching. So that's something and Peter is the person

AU I'll be speaking to him tomorrow.

II(99) Okay, you'll get a lot of information from him.

AU I was actually involved ... I was involved in the pilot for the oral benchmarking; I was one of the examiners on that.

II(99) Wonderful, that's great. That gives you sort of first-hand experience.

AU $\quad$ Yes

II(99) So what do I foresee as the future of education in Hong Kong?

AU It's a very big question and I wouldn't like you to try and answer it in a minute or two but anything off the top of your head which you thought ... a direction in which you think things are going?

II(99) It's very very difficult to say at this stage because if you look at the macro picture about the new chairman of the Education Commission, who is 
obviously very ambitious and he wants to perform and so he's started this discussion about aims of education, which to me the document is full of very general statements and it's basically motherhood and apple pie. You can't really respond to them. If he says you should study happily, and things like that ... but what I think the effect that it has on teachers and the community as a whole is that I think for the first time they're involving parents, they're involving business people and teachers and the response from the people outside education is very enthusiastic because then they get to air their views. But their views are very negative ... about the education system, and of course people in schools are very demoralised because you constantly find people you're hammering on the head you're saying, 'you're not doing a good job.' But on the other hand in response to that people start saying, 'okay let's do this, let's abolish the public examination system, let's revamp the whole secondary school placement, let's look at the curriculum, let's revamp the curriculum.' So what is going to happen is you start making changes on all fronts and then you begin to lose what is the main focus, what are the major issues that you must tackle? And I think that this is some kind of handover, post-colonial syndrome, which is very much in line with what the Chief Executive is doing, because the Chief Executive is saying let's take a look at everything we're doing, so civil service you have to reform, the medical welfare you have to reform and you're beginning to have reforms everywhere. And of course civil service you'll find that everywhere there are reforms. So it really depends on how things are steered, and of course in terms of the medium of instruction policy we need recommendations. The government is asking for recommendations. I sit on the working group and we've been meeting principal representatives from subsidised schools ... Chinese-medium schools, English-medium schools, government schools and what have you, which I strongly advise the Government, the working group to do is go and talk to these principals and get the feel of their reactions. At first the general reaction was, okay, let's just steamroll, why not all compulsory mother-tongue from Form 1 to Form 3, and then they think of an alternative, why not ask all government schools to go mother-tongue, which you probably picked up from the news as well, but as soon as you go and confront these people and talk to these people you find that it's not possible. It's electric, you know, like the minute you go into the meeting room it's electric, they start hitting back at you, so I think it was a very useful exercise and the government is really beginning to say, well what is the way ahead? They haven't made up their minds, we haven't made a final recommendation, so it would be a very interesting period to track all the different forces. So that's all I can say about the future.

AU What's making it so interesting for me is that there are so many changes happening and potentially going to happen as well. 


\section{Interviewee III(99): University educator \\ Date: \\ 13 July 1999}

$\begin{array}{ll}\text { AU } & \text { Researcher } \\ \text { III(99) } & \text { Interviewee } \\ \ldots & \text { Missing words / Unfinished utterance } \\ \text { - } & \text { Pause }\end{array}$

AU The first question is how do you feel the Change in the Government's policy on the medium of instruction in schools has affected education in Hong Kong?

III(99) The Government's policy as regards the medium of instruction as we all know has been in the last two or three years especially been to change the system from basically an English-medium one, or one which is dominated by English-medium schools, into a Chinese-medium one. And this change being made in a rather short span of time, the Government obviously wanted to move ahead in this direction rather quickly. One effect that this seems to have on the education system is that it's created a lot of anxiety, as far as one can see in the sense that some schools may not have really made up their minds as to whether they want to stay with the old system or how fast they want to move into the new one, but at the same time they feel the pressure from the Government to make a decision and to make a certain kind of decision. So, at the time when lots of other things are changing, I think this would have put quite a bit of pressure on the schools to come to terms with all these changes and demands. Also for the students and the parents I think the change has been a rather strong one, for example some parents have been trying to make up their minds whether to change schools for their children because the school may be changing their medium of instruction. Meanwhile of course some parents and some students welcome the change, they want to study in Chinese and they feel more at home with the language and so on. I think the overall effect of this seems to me is that the education system in the very broad sense including the parents and the students and the teachers has become a bit split up in a way by this change of policy, and there's been a lot of debate and a lot of discussion about where things are moving. In a way I think it's good that people should talk about it and discuss and debate these questions but on the other hand some schools may be under some hardship trying to cope with all these things at the same time. So for me I think that's the main kind of effect that the policy change has had on the education system.

AU So it's created confusion, would you say? 
III(99) It may not be as bad as that but it certainly has created some anxiety and some stress, I suppose, on everyone that's ... and also I suppose, at least at the earlier stages, before now, I mean more recently the Government has shown even greater determination to change and to make it very clear to everybody what it really wants to do, but before now there was a time when schools thought maybe they could have a little breathing space and maybe the change wouldn't come quite so drastically. So at that point if some particular schools weren't actually feeling any pressure or anxiety at least they were feeling some uncertainty, not knowing exactly where to go, but now they know that this is the direction that they're expected to go and it becomes a different situation again.

AU It's very much a transitional period, isn't it? I think a lot of schools, there isn't that uncertainty that there was before and now they know, this is the direction in which you have to go, you have to be a Chinese-medium school for example, and then ...

III(99) And only a very small number can remain English medium. So I think that message has come across very clearly now.

AU I think so, and recently there's been something in the paper about the fact that some of the schools who were designated English-medium, have not been teaching through English-medium as they were expected to, and so there's a possibility of them losing their status as a result of that.

III(99) The message now is that the requirements are being set at a higher level for schools to qualify to stay English medium. And any school below a certain level of expectation may be forced into Chinese even if they don't want to. Their argument being of course for example the students are not prepared to learn in English, even some teachers may not be prepared to teach in English, so in spite of some parents or some teachers' subjective wish to have the teaching done in English, it should not be done, that seems to be the idea.

AU And I guess it's too early to tell how this will affect other aspects of education.

III(99) It is rather early at this point to know what will be the actual outcome and again trying to assess the outcome may be a complicated thing, isn't it?

AU Yes, it's too early to tell how that might affect examination results further down the line. I think the trend though is for, well they keep telling us the trend is for more students to keep taking their examinations through Chinese, but it's still a small proportion compared to those who take them in English. Talking to teacher trainers who've been into schools and they've said that watching lessons conducted through English, content subjects, mathematics for example, sorry watching them conducted through Chinese and comparing 
them to lessons conducted through English, there's a very big difference. Through Chinese the students are attentive, they're interested and motivated and so on, whereas through English they struggle.

III(99) Yeah, I've heard anecdotal reaction to this, students seem to be more involved in what's going on and teachers are more confident about what they do and in some cases reports have suggested that some teachers feel that they were in a way hindered by trying to use English. Teachers say that now that they are using Chinese they can move along much more efficiently to such an extent that some of them can now finish what they need to teach for the year before time and have time to talk about more advanced things and other things whereas before they had to struggle to try and finish the teaching. But it's all anecdotal, there's no systematic study that I know of that actually shows any of these conclusions.

AU Yes, it will be interesting if somebody does that kind of study, which I'm sure they will.

III(99) I'm sure they will, yes. I think it's absolutely essential that we assess the situation regularly and of course at the same time we need to give it time, give it a few years to see how people cope with the changes and how the educational process might be affected one way or the other. This will take time but it's essential that we keep a check on the situation and try to have a long-term feel of what might have become and how.

AU Have you notice any changes in the status, situation or morale of English teachers since the changeover? This might be hard for you to answer.

III(99) This is hard for me to answer because I don't have first-hand experience but I've spoken to some friends and to students and some of them are teaching in schools. I feel that there's now a distinct feeling amongst the English teachers that they need to keep up with the latest of the English language teaching methodologies or the resources and also to make sure that their own English keeps up with the times. This benchmarking thing is making teachers feel that they're being assessed; they're being evaluated with reference to English and Chinese medium for example, whether they are good enough to teach in an English-medium school, for example. That's put some pressure on them. Again, it's good and bad, I suppose, maybe it's good in the sense that this will give an incentive to teachers to improve their teaching, to improve their own skills and to make them more able to teach their subject. On the other hand, the anxiety may make people feel less generally motivated, although they may be motivated in some other ways, for example, they need to keep their job or they need to find a better job, but otherwise ... it's a type of changes for them, it's a type of changes, isn't it? So, with the changes come all the uncertainties and apprehension, even that some people seem to be feeling. 
AU I think most teachers, we're both teachers as well, whether we teach secondary or whatever, feel that they welcome anything so long as it doesn't involve any extra work, any extra thing on top of their already heavy workload. I think that I agree with you that teachers ... there is a lot of uncertainty with the benchmarking ... I think they can see that it's leading towards more professionalism and most teachers welcome that. I guess it might end up sorting out those teachers who are genuinely looking at a career in teaching English from those who are perhaps undecided, who are doing it at the moment but they are not that happy doing it. Perhaps when the economy gets better they'll do something else.

III(99) Yes, I think there's a question of commitment here, so it may not be a bad thing for teachers to make up their minds, especially the ones who have only joined the teaching career for a shorter period of time. Mind you there of course many teachers who have been in the profession for a long time and they may for example, they may not have a lot of opportunities in the past to keep up with things or to make any major improvement of their skills of teaching, those people may be feeling a bit more anxious because they've gone past the time when they could try and do something else, for example, so they've decided to stay in the teaching profession anyway. But the rules seem to have changed, so those people may be in a difficult position.

AU People who've been teaching a long time maybe never actually qualified to teach English as such it her than a PGCE ... so hopefully it will have the effect of harvesting the profession in a way and those who are perhaps not fully committed will make way for people who are.

III(99) Possibly, yes.

AU Third question, have you noticed any significant difference in students' attitudes towards the learning of English since the changeover?

III(99) Now this one I know even less about because my own students go out and become teachers in the schools. I hardly have the chance to talk directly to students in the schools about their feelings, but as I said, anecdotally I've heard the students saying indirectly that they welcome the change and I'm not surprised because for good or for bad students being younger children or younger people would perhaps tend to welcome an easier approach to learning. Now whether they become better learners or whether they actually learn more things as a result is not the main concern, they just want an easier time, basically, so that they don't have to try and speak in another language and they don't have to put more time and effort into their study. So I'm not surprised that many students do welcome the change and feel that this way they can cope with things more easily. On the other hand there's nothing wrong about making students feel more relaxed and motivating them more to get them more involved. For example, one difference that this changed policy 
seems to have made is that as we all know being teachers in the Hong Kong education system, students here one major drawback or characteristic is that they don't ask questions, or they ask very few questions and they tend not to say very much, try not to say very much in a public or classroom kind of situation. Apparently in the schools more students are now asking questions and making comments and even making some contributions to the classroom discussion. This seems to have come about with the use of Chinese. So again, it's very hard to know at this stage whether in the end it's ... get more good out of this situation or not.

AU I think so, intuition tells you it should do. The majority of students are better learning through their mother tongue. It should benefit them, and those who will benefit from learning through English will still benefit from it. I guess the only ... hiccup on that is the tertiary sector, which is still mainly English medium. The students will learn through Chinese and then if they want to go to university making the transition. In my experience, I think if they go on to do science, engineering then they'll find that they're taught mostly through Cantonese, certainly at the Poly $U$ where I work, although their notes and books and exams will be in English. So it might not be too much of a jump for them.

III(99) But in some other subjects in the arts and humanities, in social sciences, certainly in law, for example the law schools are getting a lot of pressure in that way because students are finding it harder and harder to cope with the curriculum, and harder and harder to express themselves in a clear and correct way in matters of the law when everything's written in English. So it's a problem, it seems to me, for the system because you may get a lot of students feeling perfectly at home and very happy learning lots of things but when they come to the university that's when we feel, in the university, that we are being given a harder and harder job as time goes by because the students are less and less prepared to learn the things through English. Does this mean that we're being drawn in that direction even at the tertiary level? In a way we are feeling that pressure too that more and more people feel that they need to do some of the teaching, at least the informal parts of the teaching, in Cantonese. Is this what we want? Apparently the majority view, I mean the consensus is that we want to keep the English-medium system at tertiary level, for all kinds of very strong reasons.

AU We have students come to us and we teach them English for academic purposes and so on, which they need because they have to write reports and they have to give presentations and so forth in English, and yet the majority of the time they're taught through Chinese, their teachers discuss things with them in Chinese, so often they say we know we have to do this but we're not really sure why. It just seems like some kind of punishment, making us do this when they will use Chinese in their labs and workshops and they'll use Chinese in the workplace. 
How about the next question ... is about the general public, I suppose that leads into that ... the possibility that those studying through the Englishmedium schools, they always have had the advantage as regards going on to tertiary and they'll be seen to have even more now. Do you think the attitude of the public has changed in any way over the last two years towards English?

III(99) I suppose, maybe not towards English but their resistance towards Chinesemedium teaching seems to be easing up because there's been a lot of publicity in the media, so some parents seem to have become more receptive to this idea than before. Though again a very important consideration is university education. If the parents are looking to send their children to university, then somehow in one way or another they've got to face up to this question of can my child do whatever it is that he wants to do at the university level, especially for example if the parents want to send their children overseas to universities elsewhere, can they cope with the teaching? So I think parents are just a bit confused, if you ask me, being a parent myself what am I supposed to do? There's all these changes going on I don't know if my son's school is going to change. At the moment it's English but there's been a lot of talk that they're being asked to change to Chinese but they really don't want to do it. So I have to be sure that the child can learn things at the tertiary level in English somehow, I don't know how. If the school becomes Chinese, are they going to be able to learn as much English? All these questions.

AU I think the idea in Chinese-medium schools is that their Form 6 and 7 will still remain predominantly English to prepare them for university and hopefully with the CMI schools being given more resources, that is the theory, that they are given more resources to put into English teaching, so that their English level won't actually drop any, won't actually fall and they'll maintain at a certain level so that hopefully they'll be able to cope when it comes to doing A Levels and going to university.

III(99) Well, I hope things will work out in that way because ... there were times when ... even long before now when the Government was in a way subsidising or compensating for the use of Chinese with resources and funding for hiring more English teachers, but from what I've heard, these measures haven't always produced the desired results. They may get another teacher but it's not enough to be shared out by all the classes. I think somehow in order for this idea to really work, the Government I feel should put a lot more resources into language. I don't know how much is being out into it now, presumably more than before but still you are talking about a situation where most schools will now be doing almost all their teaching in Chinese. In this context, you want to make sure that the English level stays up. It's a very challenging task and will at least as a basic requirement need a lot of resources, lots of teachers with good English, for example, who can 
actually stay for a long time and who can actually become part of the school, rather than a stranger or outsider coming in just to be shared out, just going along and speaking some English to the kids and not feeling part of the school. I feel they need to integrate and put more resources into the system so that we'll be actually hiring more teachers into the system proper and taking the long-term view so that these are teachers who we should rely on to keep up the English level. In fact to improve it because the level is not that great at the moment anyway. I think there's a major resource implication there, which they may not be phasing out.

AU I think no matter how much in terms of resources the Government puts in, somebody somewhere will say that it's not enough. It is a massive undertaking.

III(99) For sure, but if you're talking about two Native English Teachers per school, you're talking about too few, I think.

AU That's the target, I think, two Native English Teachers in each school, in each CMI school. Still, it only gives one lesson a week with each class.

III(99) There are so many classes using this teacher for one particular purpose, therefore making him or her different from other teachers, which is not, maybe not the best thing in the long run.

AU I know a lot of schools who are doing that. There are some where the teacher takes on the full role and takes on the same role as any other teacher, but certainly a lot are teaching only oral classes once a week and a lot of the local teachers are a bit ... not too happy about that in a way, they see the Native Teacher, they play games while they have to teach grammar and so on.

$7(99$ So either way it may not be producing the kind of effect it ought to be producing, and I think maybe one basic difficulty there may be insufficient numbers.

AU Well they've said it's hard to recruit Native English Teachers. I'm sure that there are great numbers of teachers in Hong Kong who are native or near native speaker standard and could be employed.

Any changes in the curriculum or examination syllabuses for English since the changeover, and if they have whether they have had any effect?

III(99) I really don't know, has the exam syllabus changed?

AU No, the Certificate and the A Level exams have not changed. The only change on the horizon is the Target Oriented Curriculum, which I think is in Primary 5 now. 
III(99) I'm not aware of any changes.

AU Okay, let's go on to the next question. Some of these apply more to some than to others. Can you envisage any changes in English language teacher education as a result of any of the other changes?

III(99) I think so, it wouldn't be too far off the mark to imagine that more and more English language teacher education is firstly going to be in greater demand, schools are going to be sending their teachers for in-service training or teachers will themselves want to do this, and the curriculum, the things that we teach, will also be updated in a fast changing scene. And so I can imagine seeing this whole area becoming more and more active and more and more professional, and because of the demand for it, the self improvement that's going on, I think there's a kind of good circle going on, which I think in the long run will benefit the schools and the students.

AU Which is good for us, or good for teacher educators anyway

III(99) I think in the past university graduates can just go off and teach, but things are changing now, people are saying, well it's all very good having your degree in physics or chemistry, or even English, but do some further teacher training before you go out, or at some time during your career you have to come back and do some refresher courses and pick up some new skills and resources. I think that's good. As with any other profession, even medical doctors are faced with this demand now, some of these doctors have been family doctors for years and years and they never did any further study or gain any new information. I think that's certainly very reasonable for a modern society like ours to expect the professionals, that includes the teachers, to keep up with things.

AU Absolutely, and again it will all increase the professionalism of the profession. Okay, as a final question which is meant to cover any thing that we haven't already covered, what do you foresee as the future of education in Hong Kong?

III(99) I can see some reason for seeing a bright future, for example, the Government is putting a lot of emphasis on computer technology. We have already mentioned information technology and schools are being equipped with the latest of this technology and teachers are being given all kinds of help to learn these new things and to run their lessons and their courses with the help of computers and the Internet. And so I think the infrastructure is going to improve over the next five to ten years and that the Government is very conscious of the need to move ahead in these areas. For example, we read about how schools in Singapore or even Taiwan and China are making improvements in these ways and we must not lose out to them. So that's all very good, but on the other hand, students' motivation to learn and their life 
pattern, really, it's not their fault but we're going through a stage in the world's development where less and less time is being spent on reading and writing, for example, there's a lot of visual things, films and movies, computer graphics and action games, all kinds of distractions, so that students hardly sit down and read a book, let alone write one, people don't seem to write books any more, in Hong Kong anyway. Of course maybe some of these things are getting a bit out of date so in future education needs are going to be different [Tape ends] Somehow I think there's something about education which says that students should be able to read and to write, basic literacy. I can't see this basic ability being taken away from the meaning of education. Whatever you say, these kinds of things are supplementary or additional, to me, supplementary to the basic requirement or core of what it means to be an educated person. But with all these other things, students today have a much more demanding job to do because we ask them to do this and this as well and we're asking them to do three or four things, whereas before we were asking them to maybe one thing. So I suppose on the whole I am optimistic, the children that I see, many of them are very bright, they are very quick, they can learn new things, they can solve problems. Now their language standard isn't very good and you frown on what they say and how they write letters and reports and you worry about that. I don't know, maybe we worry too much.

AU I agree, there's more media for them now than there ever was before and of course when they have access to these media it takes up a portion of their time. At the moment we're teaching a group of students from the Mainland, from Shanghai and Nanjing, twenty-four of them who are going to be studying at the University full time, so they are doing an English course. They spend the morning doing English and the afternoon studying Cantonese. We find that their reading skills are excellent and their writing too. Speaking they're not so strong, but they have a big vocabulary because they have obviously been taught to read all the time and so they read vociferously, and they write fairly well because of it. Perhaps they haven't had the access to computers and so forth or the other things that Hong Kong students have had.

III(99) That would be my guess. In some parts of China, where life is still fairly quiet, of course they do have television sets, but still life is relatively traditional, unlike Hong Kong where children are just let loose on all kinds of visual and non-linguistic things. So typically for example, many of the students that we come across in the University, they are very good at computers, they can find things very quickly, they can solve all kinds of problems, but they can't tell you what they're doing. You ask them to explain to you what's going on and they can't do it. Or write me a report to say step by step in a very clear or methodical way what they set out to do, they generally can't do it very well. Maybe that's the weakness in a way, they've got their strengths too. Perhaps the future world expects different things from them maybe. 
AU Perhaps we somehow have to balance the two, obviously let them explore these new media, while at the same time as teachers we are always trying to pull them back and get their basic literacy up to scratch and marry the two. Try to keep hold of them before they skate away and ... well they do now, they spend all their time in front of a computer screen, which is using language to a certain extent, they are sending ICQs and e-mails to each other, but it's not as we know it.

III(99) Other times they'll just be clicking on the icons and pictures without even thinking about the language. So I guess I really don't know about that, but we'll try and do our best, as educators ourselves we must try and be optimistic.

AU Okay, thank you very much. 
Interviewee IV(99): English teacher in university

Date:

16 July 1999

$\begin{array}{ll}\text { AU } & \text { Researcher } \\ \text { IV(99) } & \text { Interviewee } \\ \ldots & \text { Missing words / Unfinished utterance } \\ \text { - } & \text { Pause }\end{array}$

AU How do you think the change in the Government's policy on the medium of instruction in schools has affected education in Hong Kong?

IV(99) I think it's too early to notice any significant change at this stage but my personal opinion is that it has made school authorities more aware of the fact that they need to put more resources into teaching English language and it has also coincided with some IT resources being given to schools and as far as I know, because I am no longer in too much contact with secondary schools, as far as I know they are hiring native speakers and they are buying a lot of IT resources for English language teaching as well. I don't know if there's any significant change by changing medium of instruction because as far as I know even before many so-called English medium schools did not really stick to the policy of using just English in English lessons. So, it's too early to notice any significant change.

AU It is, there's been only one year, it's the end of the first year since the change. I think my feeling is that not too much has changed. Some schools are now Chinese-medium and so they're likely to have noticed the most changes in ... schools that are EMI, that have always been EMI have probably not changed at all. I spoke with [ ] at [ ] and he said that nothing's changed.

IV(99) Quite unlikely.

AU Okay, have you noticed any changes in the status, situation or morale of English teachers since the changeover?

IV(99) Status, quite significant changes sometimes. I don't know what you mean by status actually.

AU Well, the perceived status really, whether teachers feel that they are more important or less important than they were before.

IV(99) Well, before the handover there was some kind of feeling amongst teachers that English is going to go down in importance and they are going to ... some of them are even going to lose their jobs, there'll give less hours to English, 
and this happened in BATC as well and I have some information, but I don't think it has happened, it has been rather the other way round because they have given more importance to English, even in Chinese-medium schools, they have hired one or two native English speakers to try to at least help. And also I think if you look at benchmarking, is it called an examination, the benchmarking practice of the Education Department?

AU The benchmarking assessment.

IV(99) Assessment ... in a way they are trying to raise the standard of existing teachers, which sometimes threatens some of the teachers and they feel that ... many of them don't agree with it. But as far as significance is concerned or importance is concerned, I don't think it has gone down. And especially among other sectors of society like parents, they are not very happy with this change and they feel it is even more important to provide extras and ... as I've said, they are not very happy about the benchmarking assessment and to be really honest there is something about English teachers in Hong Kong that is very disturbing for me ... I think that they have a very limited view of what students can learn and they tend to provide students with what they can do and they screen out a lot of materials. I think their attitude towards English, it has something to do with it, they think for example, these vocabulary items are not needed, this kind of literary text, who will use them in Hong Kong? So they screen out ... I don't agree with them at all, they make, they leave very boring or very limited materials or approaches to teaching. This is something I've noticed with a lot of teachers I've talked to. Whenever I discuss, we can do this, 'It's impossible, how can you do that in Hong Kong?' So, I don't know, there is not much change as far as that aspect is concerned. I don't know if it's relevant what I'm saying?

AU Oh yeah, I know it's difficult to judge it as neither of us are in secondary schools anymore. It's more of a feeling of how things are going with talking to former colleagues and so on.

IV(99) Actually what I said is because I talked with a former colleague, she decided to attend some of my movie courses, she attended three because she is doing extra-curricular activities.

AU Yes, it's a perennial thing with school teaching. The knee-jerk reaction is to say, 'We can't do that, it's impossible,' without even thinking about how to make it possible.

IV(99) Exactly

AU Or if it's not exactly possible the way we think ... at least do it in some form of direction. But I think the benchmarking is one of the main things that is going to affect English teachers. 
IV(99) Do you think so?

AU Yeah, but as yet it hasn't because it hasn't started yet. There's the potential threat of it. For most teachers it shouldn't be a problem.

IV(99) It shouldn't be ... that is another question, how are they really going to benchmark, what kind of criteria are they going to use and as I've heard over the years they will implement this policy and if a teacher is inadequate according to the benchmarking, they have like ten years to upgrade?

AU That's right. They will give them plenty of time and the opportunities are there, they will offer in-service courses and so on, which all should help raise the standard. I think most teachers agree with it, although some think that it's questioning their professionalism.

IV(99) And I think there are some doubts about the procedure of benchmarking, they are not really sure what is going to be tested and things like that.

AU Have you noticed any differences in students' attitudes towards the learning of English since the changeover?

IV(99) Again as you know I've moved from secondary school to tertiary but before that, like in 1997 I joined Poly U, I noticed that some students do voice out that Putonghua is going to become a major language and that English is not going to be very important and you could also see a lot of Putonghua courses mushrooming in society. However, I think students, especially higher form students, their attitudes have not changed much, I don't think this changeover has caused any significant change, as they feel English and a lot of it comes from their home and family, I think. I think it hasn't affected students' attitudes. The only thing is that some of the students who resent having to learn English all the time, they might think that they don't really need English in future; they can get along well by using Putonghua. Maybe it is easier for them to learn Putonghua because they have a shared written system. But I think students who are good at languages are good at both languages.

AU I think that's true. The feeling was, I think that perhaps students would think that English was not as important as it might have been at one time. I've had other people tell me that there's no difference in students, parents still impress on them that English is important.

IV(99) To me it has had a kind of ... there are becoming aware of the fact that it's going to be reduced in importance and sometimes they get panicked and they feel that they should do something themselves, which is a rather opposite effect sometimes, raising the awareness. 
AU And all the talk about English and learning languages came up because of the change in the medium of instruction, which raised awareness of the issues. Previously people probably didn't even think about it too much, so on the surface English is important, you have to study, now it's become even more crucial to the point where ...

IV(99) And many of them don't agree with government, it's so evident that ... many parents don't agree at all.

AU Which brings us into the fourth question, whether the general public has changed its attitude towards English and the learning and teaching of it.

IV(99) Again I think if attitudes have changed, they are in favour of English rather than against it, as you just said and I agree with it that they have actually become more aware of the importance of English and this issue of the changeover and medium of instruction change actually raised their awareness and they started think about it and the more they think the more they realised how important it is to learn English. And even when they look at Mainland China, China is becoming aware of English and they are doing so many things in Mainland China. So wherever they see it they see that people are learning English like anything. So I think the general public, if there has been any change it has been in favour and this is what I know. They all will agree because being Chinese they tens to agree that mother tongue instruction is good, it's important, but I don't think they confuse having mother-tongue education with having a good standard of English. I think even though many schools were English medium for a long time but many of them were in name only. I don't know how much difference it is going to make. Textbooks were in English. I learned through English medium and I was also in an English as a second language context and I was one of very few privileged kids who went to English-medium schools. Once I had to change over to Urdu medium because my father moved to another city and I couldn't get admission in an English-medium school very quickly, so I went to an Urdu-medium school for a few days and it was a disaster. I could not understand maths and science subjects because they used Urdu terms and I just remember that if that is going to happen in Hong Kong, I don't know how students are going to cope with Chinese terminology in Chinese-medium schools, but my personal experience is not a good one. It might be an area worth exploring, how students feel about science subjects being taught in Chinese.

AU I think the case in Hong Kong as you know with your previous experience, many schools were supposedly English-medium and the teaching was not English medium, it was greatly Cantonese and mixed-code, so if there's going to be a change in the medium of instruction, it's not going to be from purely English medium to purely Chinese medium. It's probably going to be from mostly Chinese medium to all Chinese medium. So I think most students will actually benefit from it. 
IV(99) How about writing, in exams, do they actually have to write subjects like science and maths in Chinese? I don't know.

AU Presumably, yes, although technically it's only been from Form 1 this year anyway, so I think most of the schools not much will have changed. But presumably in CMI schools all their examinations will be in Chinese. But I've certainly noticed this raising of awareness thing has led to parents queuing overnight to get their children into certain kindergartens because they know it will lead on to the good primary and secondary schools. Their future is all mapped out.

IV(99) I have a friend and she is an English teacher and she is hired to give tutorials to a boy whose school has changed from English to Chinese medium and the parents of the boy thought that he needed extra help for English, so they actually hired tutors. It might be happening elsewhere as well.

AU So if anything the attitude of the general public has become stronger towards English because of this fear that it might decline. Five, have there been any changes in the curriculum or syllabuses since the changeover.

IV(99) Well, I've had a cursory look at the new syllabus and if they're going to do what is written here it seems very impressive. It seems that this new syllabus is not only communicative as such; they have a few terms which describe this syllabus quite well. They have learning targets and those targets incorporate like interpersonal dimension, knowledge dimension and experience dimension and they have certain goals which are really quite, I don't know if they are really going to incorporate it or not. I'm quite impressed by things like developing their ability to express, respond and express their experience and to involve them in imaginary experiences. It also mentions how in order to develop their interpersonal dimensions they will introduce them to literary texts and ... throughout the stages and different stages, they are called Key Stages, I think, and it seems that they are going to incorporate imaginative literature including dramatic texts and poetry and things like that. I am quite happy about this change, it seems that this is something new, this is Form 1 to 5. I hope they have similar things happening in primary schools as well. In Pakistan we are using an ancient syllabus, even now, but our syllabus ... like every year we have to have like six or seven poems, at least four or five literary texts in our reading. I don't know why they have changed this syllabus to include more ...

AU Well it's based on the Target Oriented Curriculum, the TOC, which will have reached Primary 6 next year, so the following year it will go into Form 1, so in Form 1 will have the Target Oriented Curriculum. 
IV(99) We want to have a continuum of education from primary into secondary and that's why they are bringing it all together. This is quite a good plan but I really don't know how they will develop materials.

AU Well, yes, it really involves a whole range of new input, doesn't it? Teaching methodologies and textbooks, all kinds of other things, a lot of administrative things as well. Each student has their own profile because they are each working towards individual targets and so the teacher has to keep a record of all of this.

IV(99) This is being done in primary schools?

AU This is being done in primary schools.

8(99 If this is what is going to happen in secondary schools it seems quite promising. We went to Singapore once and I visited some of the classrooms, I know they have a very long tradition of teaching literature and poetry and imaginative fiction and integrating it into their syllabus. It is a completely different syllabus to the one we have here in Hong Kong. I think I attended the Secondary 2, Grade 2 classroom and it was a lot better than our Form 6 and 7 , the level of discussion taking place, it was so impressive. I actually ... to good samples of language, good literary language and I don't know how Hong Kong could have overlooked such an important resource of language.

AU Well it's gone in different ways, English has always been seen here from a functional point of view, I think, English is used as a tool to progress, to get somewhere, to get a better job, to get a university place, so ...

IV(99) Basically it's a tool for communication and expression but there are like a hundred ways to express which varies from the very sophisticated to the very simple. If you just teach them a very basic way they are not going to understand idiomatic language and communicate beyond a very surface, practical level. Language is a lot more than that, I think. It's very hard for a lot of Hong Kong students to understand humour, for example.

AU Yes, we laugh at the pun, the play on words, but they can't understand it, they can't see why it is funny. How do you explain why it is funny?

IV(99) That's what I mean when I say that language can be interpreted at various levels of communication, that is what is lacking here.

AU Yet within Chinese they have a similar kind of thing. They have things that are funny because the written characters can't be confused with anything else because they are so intricate, but the sounds can. They'll say something which has a very similar pronunciation to something else and that can be hilariously funny. 
IV(99) Exactly

AU And you get the English subtitles on a Chinese movie and it doesn't make any sense at all and the Chinese are laughing their socks off. I guess it's just culturally and linguistically very different.

IV(99) It is but again I think that language should be seen as a very cultural thing, you can't translate it in another language, that's what makes it even more important to expose them to specimens of language that are culturally significant and by cutting out literary texts they are cutting out a lot of material.

AU But in the limited time it's hard for us to teach them British culture, American culture and cultural things through the language whereas I guess the trend now is that English is the international language and that's what we have to teach, English as an international language and not any cultural baggage which might put students off learning.

IV(99) Well in a way it's fair enough but there's so much of language that comes from culture, but ... I find the syllabus too localised. Even if it's an international language it should have international context, for example, in Form 1 and 2 textbooks, Oxford English, you will see even the biographies given of pop stars they are Hong Kong people, they could choose some international figures to look at. I don't know if you agree with me or not?

AU Absolutely, it all depends on the needs of the students, I think, and their ability as well for those who want it and have an interest in it. I was talking to the examiners from the Exams Authority yesterday and a question came up which we talked about two years ago and that was the name of the English literature exam. They were considering having to change English literature because ... to change the name so that it gets away from it being basically English or British culturally-based. They did a bit of research and found that most countries in the world don't call English literature English literature any more.

\section{IV(99) What do they call it?}

AU They call it literature in English or something of that kind. English literature sounds like it's all Shakespeare and all that but of course we know that it's not, it's whatever can be described as English literature through the English language.

IV(99) Which is very interesting. When I studied literature there were only ... there were two things that were not British in origin but one was translated into English by British poets ... Omar Hyaam is a Persian poet, and I think Spencer, Matthew Arnold has translated Omar Hyaam's portraits into English 
and we studied those beautiful ones. He has made them even more beautiful. Again, I don't understand Persian much, my mother doesn't agree with me, she understands Persian ...

AU Okay, next question, can you envisage any changes in English language teacher education as a result of any other changes?

IV(99) I think the intensity or the frequency will increase. I don't know whether the way they are trained will change or not but definitely a lot of refresher courses are being introduced and I know a lot of teachers who've gone and joined Institute of Education refresher courses already. So definitely such kinds of training courses at I.Ed. or Hong Kong $U$ have responded to these need and I think they will introduce more and more courses to respond to the growing need for training.

AU There's Hong Kong U, and there's City U as well, they've been asked to open a part-time BA course in teaching English.

IV(99) Oh yeah, I know, one of my friends ...

AU And cert courses, I'm not sure if they can but there is such a big demand now. What are now CSM teachers, teachers who don't have a degree, are now being phased out and so it's worth it for those teachers who perhaps graduated from the Institute of Education maybe five years ago with a cert. but no degree they don't have to get at the moment but they're probably thinking it's something they'd better do.

IV(99) This is something I heard as well, secondary schools will have all masters' teachers, the GM there, and even primary schools are aiming towards degree holders.

AU It's the Government's plan, in Tung Chee-hwa's address last year he set a time frame by which all teachers would be degree holders. They should be degree holders and subject trained as teachers as well.

Lastly, what do you see as the future of education in Hong Kong?

IV(99) Well, I think like all cosmopolitan cities, people in Hong Kong are aware of the importance of education. You can see that the institutions like the Open University are getting more and more people who perhaps join their degrees and so it seems that the profile of an average Hong Kong citizen continues to rise. I don't know with the merge with Mainland China, I don't know how the statistics will do because many people will come from China but I think people who are really going to work in Hong Kong because it changing from an industry-based economy to more of a services and financial institutions based economy maybe more people will have a higher educational profile 
than before in future, and most of the workforce of manufacturing industry is moving to Mainland China. If people come from Mainland China to Hong Kong they will have maybe, they will only be able to survive here if they have a higher education level in Hong Kong. From educational institutions' point of view ... do you mean from language or just from general education?

AU Well generally.

IV(99) Maybe becoming more and more, as Poly U., more and more universities will have more practical courses and things like English literature are phasing out maybe, so that the nature of education here will be more practical and skillsbased and vocational. That is another sad fact as far as I see it. I think it reflects in how people behave and I think Hong Kong is in a dire need of more arts and more literature. From my point of view they just need to be given real education as far as arts, but again a lot of people may not agree with me. I think that is kind of a consequence of too much practical education. People don't even understand abstract concepts, they don't understand them.

AU Yes, no appreciation of beauty.

IV(99) Yeah, or human relationships, for example. It seems that when teachers talk about these things with them, they just sit spellbound. They just want to do it; they just didn't know that there is such an area that they were never exposed to it seems sometimes, especially at tertiary level. They feel the need for that. Some of the things in the syllabus are promising if teachers know how to do them.

AU I would agree. I think education is becoming more available for more people. Some people are naturally enough taking advantage of it for what they need and the old idea of basically having a good, sound education and being able to go into anything, which may have been true 20 or 30 years ago, if you went to a good university and got a good degree you could go into anything really, it didn't matter what. If you've got a history degree you were taken on in business, it didn't matter ...

IV(99) I agree with you here, I think a well-educated person is a person who ... it should be a sound foundation before they get into vocational training.

AU They should have a good all-round education ... they should have a knowledge of maths, a knowledge of science, of literature ...

IV(99) Contemporary maybe...

AU All these things when I was at school you learnt up to $O$ Level. It seems that here in secondary schools they specialise so early. 
IV(99) They do, look at the Form 6 and 7 syllabus ...

AU They are doing very vocational things in a way, computer science and IT, which is great and the skills are useful. Maybe it's just the world is changing. I noticed that in the newspaper yesterday, there's many of them every day, they're advertising distance courses, business courses, MBAs, and now as well as MBAs there's DBA, Doctor of Business Administration, in two and a half years, and I'm thinking how can you do a Ph.D. in two and a half years?

IV(99) It takes me two years to write a proposal!

AU Exactly and I don't think these things will be worth the paper they're written on really.

IV(99) That's another thing that's coming into education, if they're producing these ... products, selling products these different degree courses.

$\mathrm{AU} \quad$ And then people get a DBA to go one better than the other 60 people who've applied for a job ... and where will it all end?

IV(99) Exactly

AU And fair enough to the people who are doing it, they're working hard at it and investing their time...

IV(99) And money

AU And money, to get ahead

IV(99) Especially in Hong Kong, so many degrees ... everyone wants to go to UK and do something, no matter what. English teachers will just go and do a refresher course if nothing else. Once I interviewed a ver smart Hong Kong student who seemed to have very good English and I said why don't you go abroad and she said, 'I don't think there's a need.' Many people in Hong Kong think that can achieve a good level of English only if they can go somewhere and get something done. In the movie course we chatted and some students asked me, 'How can your English be so good, did you study in the UK?' I went to UK to study because my English is good and not to make English better. If you can't go to UK it doesn't mean that you can't improve your language. It is an international language and we have a lot of exposure and media these days to work on it.

$\mathrm{AU} \quad$ It's more a case of exposing yourself to different things, not only language. I have students who also went to study overseas in the UK or the States or Canada and they were good already, they were excellent [Tape ends] 
IV(99) They already had a certain level of the language.

AU And the opportunities they've had they've obviously gained from, wherever it may be has really helped them and now they have the option ...

IV(99) Good education is such, my father was a person who would not save a single penny for tomorrow. He was a lawyer, earned a lot and spend immediately, and my mother focused al her attention on our education ... good education is the greatest thing to have nowadays. 
Interviewee V(99): University educator

Date:

7 September 1999

$\begin{array}{ll}\text { AU } & \text { Researcher } \\ \text { V(99) } & \text { Interviewee } \\ \ldots & \text { Missing words / Unfinished utterance } \\ \text { - } & \text { Pause }\end{array}$

V(99) Okay, change in the medium of instruction has affected education in Hong Kong, well, my initial opinion was that it was going to stratify the system very clearly into these are the elite best schools, with English language medium of instruction, as opposed to second class schools. But then I realised that effect wasn't of much consequence anyway because they were nearly all Band 1 schools that remained English medium and that it was just instead of having the academic stratification, you now had both academic and linguistic stratification. For a society it wasn't the sort of sense of divide which you had from say having the grammar schools and secondary schools in Britain, it seems to be just saying that the reality is in these lower band schools where not much English language teaching was occurring anyway, let's just scrap the pretence and make sure they're clearly labeled Chinese medium and then in these Band 1 schools that were always the ones that the parents desired their kids to go to, they just remain in that position. I know that a few of the schools that were on the cusp, it would have made a big difference to them to be included in the English medium but were excluded, in those particular instances it had quite a big impact and was quite demoralising for the teachers. Otherwise I think the amount of change wasn't that great. You've had a change of labeling. Some of the schools that have remained English medium but previously may not have focused so much on being thoroughly English medium, it made them aware of, OK, those cases where we're not, let's tighten it up to make sure we get classified. Otherwise, I think in a lot of the Band 5 schools, it was just a labeling of what had occurred anyway. So I don't think there was as big a change as I thought there would be. It's just that whereas previously everybody would wish to get into the Band One school, now they're forgetting that these are the old Band One schools and they're saying, oh we wish to get into the English-medium schools. The pressure on kids in primary schools, I think it's much the same as it was.

AU I agree, I think it's clarified things in a lot of schools. As you said, this pretence that they were English medium and trying to attract the better students has been taken away. If they're Chinese medium now, they're able to go for that and say, right we're Chinese medium, but we're putting these resources into English teaching as such, they're not going to neglect English, but it'll free the rest of the system to teach through the mother tongue. 
V(99) As I say, I think there's been this clarification, this stripping through of pretence. We all knew for years that there were a lot of so-called English medium schools that were using English medium textbooks and glossing a lot and there were a lot of students who were having double problems because of that, that they were not particularly academically able or academically inclined kids and having English medium textbooks gave them another reason for rejecting or excusing their failure in the system.

AU It is early days yet, they've still only had one year of the new system, it's only really been introduced into Form 1, but I've been into one or two schools and talked to teachers and that's seemingly the ways that things are going. As regards English teaching, and I guess this might come up in a later question, in fact it is the second question, any changes in the situation, status or morale of English teachers?

V(99) Since the changeover? Well, one very clear objective effect has been that with the end of the easy visa access for British passport holders, suddenly Hong Kong English teachers have become far more prized, especially when it comes to doing summer vacation courses that previously could only get their teachers by recruiting internationally and suddenly that pool of recruitment's been blocked off and there's been a greater awareness of other private organisations or university organisations when they're running summer courses that they have to use staff that are locally available, they can't just depend on filling in positions, getting teachers from overseas. And this means that more of a range of teachers are getting more possibilities to expand their careers than they had in the past, experience at other levels even if it's just pre-university intensive summer courses. As for the morale of English language teachers, I know that some had complained about the fact that kids were saying round about the changeover that English isn't important now but that didn't seem to have a depressing effect on them because virtually everybody who I knew who said that would say that the students didn't realise that English remains just as important. So I get the feeling from most of the school teachers I've talked to and all my colleagues that English is as important as ever. There's no sense of being downgraded or underappreciated. You sense that there's the additional necessity for more Putonghua but there's not this sense of English being downgraded or swept out or any less of a necessity.

AU Of course there was that possibility around the changeover time that English teachers would feel that they were being downgraded, that they were going to be usurped by teachers of Putonghua, perhaps or other Chinese subjects. But perhaps there's the sense that with most schools becoming Chinese-medium that there even more important. There are seen as being, in a way, a bit unique now, the English specialist. Whereas before when schools were English medium, all the teachers were teaching in English and it was a case 
of anybody can teach English, especially lower form English ... now I guess the English teacher is more isolated.

$\mathrm{V}(99) \quad$ Isolated in the sense that previously students were regarded as getting immersion, at least in terms of vocabulary and exposure in the other subjects and now the English teachers in the schools tend to be seen as one, somewhat more specialist and also more special, in that now they are not just one of a group of teachers doing English and that they've got the necessity to be English teachers, they don't get it if that person's not on the ball, then the students really have problems with their English.

AU I suppose the only thing affecting ... I suppose the Native English Teachers scheme has been running for a few years but it's been more high profile in the last couple of years. How do you see that ... do you think that it's been handled well or ...

V(99) I haven't kept up with the scheme that well at a detailed level ... I think the Government did a lot of bad PR work, they could have done a lot better PR work for it. It's very clear that they are just copying the Singapore model and I think they could have made it explicit. They could have said, look in Singapore there's a general level of English in society on the whole, English is more widely used than in Hong Kong. They have exactly the same type of scheme and they've had it for a few decades and it seems to have been a success there; we would like it to be a success here; we regard it as a supplementary feature and also they should have realised that the recipient staff, the success of the scheme depends a lot on the recipient staff, how they treat their colleagues and therefore with this business over the extra allowance, the housing allowance ... there's need for them to have made some gesture, especially when they cut the housing allowance and reduced it they should have said something like, we realise that it's going to take work to make this a success, it depends on the induction of the member of staff into the school and that this is an ongoing process throughout the first year and therefore they should have offered some kind of monetary recognition of the fact that getting in an expat into the school does place an extra work burden and a new type of work burden on the colleagues. Given the fact that it was clear that there was resentment at the wage differentials, then they should have made some gesture towards sweetening or lessening that difference by saying, for each expatriate scheme teacher in there there'll be ... five thousand dollars a month to be shared amongst whoever does the inductions, so if they're going into a school and they decide that there's going to be three colleagues working closely to help with the induction of the expatriate teacher then they split it up between them. It's recognition that it's extra work. The English language teachers in Hong Kong during the term time have got a hell of a lot on their plate with the marking and homework correction so even having just a little extra burden of something that maybe an hour each week or three or four hours at the beginning can be quite painful 
if it comes on top of other resentments. I think the Government should have been more willing to lessen aggravation there, especially as the success of the scheme does depend tremendously on how the teachers are received. The other thing they should have done was made sure the person wasn't just stuffed up with oral language lessons. I don't know how many teachers, this is what I mean I don't know the details, but there were cases where the NET was just given an entire schedule entirely of oral classes, which was something that never really used their skills and abilities and led to people getting rather bored. It didn't seem appropriate usage there because there's probably also the sense at the end of that maybe from the local staff that here's this person getting paid more money and getting out of marking.

AU Well that's true and I've seen it in schools that I've visited that it happens, the NET teachers take the oral lessons and the local teachers take the grammar and all the other lessons, the native teacher does the speaking and listening and they do the writing and grammar and so on, which again seems an unfair burden as the local teacher does what might be looked at as the more down to earth grinding sort of English teaching, whereas the NET teacher does activities and games and as you say less marking, and get more pay for it.

V(99) It's not clear why that situation arose either. Some NETs were very happily integrated.

AU Oh yes, it depends on the school, how they're used.

V(99) Any significant difference in students' attitudes towards the learning of English since the changeover, do you mean my students in the university or school students?

AU I'm basically thinking about school students but your students, at least when they first come and are fresh out of school, they probably change over time. Well, thinking about students in general, mainly in secondary schools, whether since the changeover, maybe not directly because of it but since then anyway, whether there's been this feeling that English is not as important as it used to be, as we said before.

V(99) Some teachers reported that but I haven't seen it, especially not at university but we're now running a load of completely optional courses university-wide, they have got this credit system, where, say I do a course English in the Welding Field, and it's optional, students could choose any one of hundreds of these out of discipline courses. I operate in places and it's chock-a-block. There doesn't seem to be a lessening for English.

AU I guess at the tertiary level we are talking about the top $18 \%$, whether overall, in the whole population of students in Hong Kong, whether there's been a 
change in the attitudes towards English. Those at the bottom were probably never interested in it in the first place and still aren't.

V(99) My students haven't reported any drop in demand for private lessons, they teach English private lessons.

AU I think parents still consider it important, that comes up in the next one about the general public, so they're still prepared to push their children to do well in English and to get into the English-medium schools.

V(99) I think a lot of these changes are fairly slow moving things ... ten years ago I was hearing reports from teachers in schools out in the New Territories that had been using their English periods for teaching Putonghua. These schools obviously realised their students never had that much exposure to English, were unlikely to succeed in it and given the kind of area they were in and with parental sympathy they just decided unofficially to do that. I don't think there was an increasing number of schools doing that ... great surge around the changeover period, I think you've got ... we have little waves occurring but ... the students' attitudes I've certainly not seen any big change over the last two years.

AU I think maybe at the time of the changeover it was high profile and big news, if you had taken a poll then about students or public opinion about the value of English, Cantonese and Putonghua, there may have been an increase in Putonghua and an increase in English, but I think that's leveled off again now, I think business with China is growing and Putonghua is growing but the need for English is as much as ever. I think those students still realise that.

V(99) The university over the Handover period, it planned it's move to credit unit system. The credit unit system, it's just put out 6 units language requirements which every student in the university has to get. If the student has below a grade $\mathrm{C}$ at Use of English then they have to take a university course that's aimed at moving them up a grade ... if they've got a grade $\mathrm{C}$ or above in Use of English then they're free to pick their courses that go for the university language requirement.

AU We have a supplementary programme in which students who get ... generally we're looking at Es and below, students who've failed particular papers, particularly in speaking or writing, again aiming to bring them up perhaps one grade. At the same time they have to do the EAP course, all university students have to do the EAP course.

V(99) With regard to your Question 4, the general public, my personal impression is that people have been more relaxed and more willing to speak English, I don't know if that was a change that was growing or else I've just been here in Hong Kong so long that there's a certain style of interaction that I've got 
into that makes people more prone $t$ speak English to me but all these little service encounters, like here, people that years ago would have been frightened even about just the ordering and not wishing to go beyond that, they try to initiate conversations and quite a few people when I'm off walking in the hills and quite a few people ... if anything I've found that there's more of a willingness to use English. Whether that's ... maybe I've more time or more time for teaching...

AU I certainly think that the development of this kind of place, this kind of fairly up-market shopping area, whilst still the majority of customers are local Chinese, you're getting more of this kind of Western-style ... that they probably make an effort to train their staff in the basics of service English.

$\mathrm{V}(99) \quad B u t$ these people are going beyond that, you're talking about a service industry, but you get staff wanting to talk to me and ask me about my family and so on, willingness to talk about other areas beyond work that I've not noticed in the past so much. Unless you've got the people who may have a certain level of English, perhaps they've been abroad or overseas or worked in contact with English speakers in Hong Kong for a long time. Here what I'm talking about is people who have maybe been out of school for a few years, your typical Hong Kong restaurant workers, service workers, without a great level of English but a willingness to come forward with what they've got, which is something in the last few years that I've found has happened more and more.

AU I still find most local people reluctant to use English. I found this when I was on my holiday. Of course most of the people on the tour group, well they were all Chinese apart from myself, and I would say that about $75 \%$ were well educated, a lot of teachers I think, civil servants perhaps, and when you asked them questions they will answer you quite fluently but then that's it.

V(99) Maybe the connotations of English for work, Cantonese for leisure.

AU Yes, so they don't volunteer. I kind of think that other nationalities would. If they were European perhaps with a similar standard of English I think they'd be more open.

$V(99) \quad T h o s e$ people would be thirty and above, wouldn't they? ... age group around twenty and below, the seventeen, eighteen, nineteen, twenty year-olds who are service workers. Maybe a generational thing. It may be nothing, it may just be my own individual experiences.

AU I think in general the push for people to learn English is still as strong as it ever was. This feeling among the average person that, yeah English is important. If they have children, they want them to do as well as they can and 
get a good grasp of it because it still furthers your career, gives you more options.

V(99) Well, as the years have gone by English has become more and more the necessary accouterment for an educated person, that it's difficult to be seen as a totally educated if you don't have a moderate knowledge of English, in the way that in the 1950s in England, you could say the same about Latin. An educated person would be expected to have a smattering of Latin and that was something that endured for three to four hundred years, in Europe, and the place of Latin has gone, not to be replaced by another language in the Anglophone societies but to be replaced by English in the non-Anglophone European societies and has spread and had the same effect in the other societies in the world. I started secondary school in 1965 ... I had to study Latin in my first year because it was only at the end of that year that the major top class universities in England decided that an O Level in Latin was not a necessity as an entrance requirement. Once they dropped that, then the schools started phasing Latin out.

AU I did Latin as well. Okay ...

V(99) Changes in the curriculum and examination syllabuses since the changeover, well, in the curriculum they're working through it now, they've got the draft syllabus which has been discussed. I've got to admit I still haven't got around to reading it, I can't comment on those changes that are coming through there. In the exams, almost coincidental the coinciding of reading writing and listening of Syllabus B came in. I think that was done for examination rather than changeover reasons. I think one of the effects has been a slight diminishment in the regard ... even for the teaching of listening, which was never that great anyway. Now it's become a fraction of a fraction of a paper, it's decreased even more.

AU I think the only change, I've had a look at the syllabus, they're gearing it towards the TOC, which I think will come in next year. I think they tend to revise the syllabus every five years or so.

V(99) Maybe even longer, I think the previous, I don't know about revisions, but the last major syllabus change was I think in 1984, and they've just tinkered with that so this is the next major change.

AU And the examinations haven't changed for the last two years. There will be a certificate examination in Putonghua this year, the year 2000. I think that's the only major change. Can you envisage any changes in English language teacher education; this is more up your street, as a result of these other changes? 
V(99) I can see changes due to issues that we haven't discussed that have occurred recently ... with the emphasis on mastering information technology skills at all levels. There has to be a much stronger component in information technology or using educational technology in teacher education courses. Our students always had a basic course of information technology skills, using word processor, database, spreadsheet, as well as getting access to the Internet and using CD ROM databases, but aside from this compulsory basic course, we've now put in new options in educational technology and new technology for teachers. But when I think about that first policy statement of Tung Chee-hwa, where there was this tremendous emphasis and highlight placed upon technology and information technology in schools. I can see this emphasis in education courses just grow stronger because there's just more that you can do with technology, and I can see that traditional technologies like video, now that they are seen that are not just things that are intermediate technologies that are going to disappear, there's also the need for far greater time to be spent on how do you maximise their use, even when you know that the schools won't be that well-equipped, you know that there is going to be a decent, minimal amount of equipment that will be available.

AU In the schools that I've visited, two out of five, have multimedia language labs, computers, big screen, and they're using them. One was a private school and they paid for it themselves and the other one they raised the money though the Quality Education Fund, so it put a lot of work from the Panel Chair to put forward their case. Of course there is s lot of classes in the school and each class can only get a chance to use it perhaps once a week or once a fortnight. The kids love it.

V(99) The other thing that I can see that has actually occurred, and again I find myself stumbling over this notion of since the changeover or ... because I think since the changeover there haven't been any, besides the medium of instruction, which was a clarification, there haven't been these huge, drastic ... we've had these things progressing for years. The information technology, the emphasis on that in Tung Chee-hwa's policy address, that was something that was a rapid change of a very decisive ... we're going to put more this and bang, it's begun to impact the schools. I think self-access, you're going to see that becoming a standard part, a giving some knowledge of self-access in its role in language learning as just a standard rather than a specialist part of teaching, and I think it ties in with the fact that self-access has been around long enough now that it's shown it's value as an aid for language learning, that it's been introduced in all the universities so quite a few graduates themselves, who are going through the system, are having contact with selfaccess and I've found that there are more teachers who are doing things off their own initiative and ... some elements of self-access learning within the schools. In fact, there's one colleague who's putting forward for a Quality Enhancement Fund grant to set up a self-access centre there. But again I feel a bit uneasy in talking in terms of changes since the handover, unless I was 
going to tie it in with ... you're getting more and more multimedia equipment within the schools and one of the things they can lend themselves to if teachers know ... but otherwise I think there's more work with Putonghua and English remaining the much-desired fruit that is rather elusive, for the majority of the students, or if not for the majority for a large minority.

AU The fact that most English teachers that have either graduated from here from the BATESL or from the Institute of Education will be teaching in Chinese medium schools, has that had any effect? In the past you might have assumed that most of them would have gone into English medium schools, now most of them will end up teaching in Chinese medium schools.

$\mathrm{V(99)}$ Well in the past most students ended up going into Band 3, Band 4, Band 5 schools, which were ... virtually Chinese medium anyway. We have had a course on bilingualism and bilingual education that's transformed since the changeover into multilingualism and multilingual education and that covers some of the differences between teaching in a ... as part of a bilingual education system versus teaching English in more or less a foreign language situation. I should imagine on any degree programme where they don't have a component like that, they'll have to introduce that into it. But given the reality of the changing of names, for our students, there's not going to be a big difference gearing towards going into Chinese medium because as I've said, the changing of labels for the Band Four and Band Five schools is just a changing of labels and a changing of textbooks. Whereas the actual techniques of the teachers [tape ends] whereas for years we have trained students to make better uses of the language resources that there are in Hong Kong to get their students to have more exposure or to have them focus their attention on the English around them, we've always worked on the basis that the students have not made a great deal of use of the English around them and the problem is that they've had a very limited exposure to English anyway in schools, getting even their English coursebooks glossed in Cantonese. So it's not going to make a big difference there. What we have to think about is the fact that now these hints that we've had of how the students can make use of this additional English around them, then obviously it's not going to be a major factor. Maybe you get one of our electives, teaching English through content areas, has to be modified.

AU Well, unless they're going into ...

V(99) Unless they're going into Band One schools, yeah?

AU Or if they're teaching at Form 4 level or above, then they will still teach in English. Certainly in Form 6 they will.

And so the last question is what do you foresee as the future of English in Hong Kong? 
$\mathrm{V}(99)$ Well what I can see is that they are going to go far more onto using their Task or their Target Oriented Curriculum or their grades in order to set up a system of assessment whereby they're emphasising what the students have done and that they are going to move away from this very heavily norm-referenced examination system and to having the label of $x$ percentage just failed and failed totally towards it being, and it'll still be norm-referenced with respect that they will expect that within any set of tasks that are assessed, they will expect $x, y, z$ percentage to perform in a certain manner. But what you are going to get is far more descriptors so that you are moving away from pass/fail towards a set of descriptors, the person being able to do this, this and this. And maybe their list will be that long and you'll know that at the moment they're not fit to go onto sixth form study and the university because in order to do that they need to be able to do so many other things. I can just see that being one of the major changes, that there are going to be these four descriptive targets, benchmarks. I think educationally there are good reasons for it, you want to know what people can do, you want to have clear targets for them to achieve. Socially there are good reasons for it ... employers want to know again what the person can do, they don't want to know they've got seven GCSE's or five GCSE's, it's so much more useful if they've got a descriptor. And I think the loss of face thing that exists here, not to have failure ... we're a kind, enabling society ... so I can see that as being the biggest educational shift that we're going to get ... within the next decade, I'd say, the next decade or fifteen years

AU Moving away from this very black and white exam system that we have... they're on a path to success, the rest of you find something else to do.

V(99) That's right and because the education system is based on the British model with its emphasis on the elite, you're the ones who are going to have success, you can do this and this, you can go through the university system, yeah, or the school system and all the emphasis is on them and not that dark shadow, that large number that then are failures. There was in Britain for a very long time just the thing above, you look after yourself as best you can and the resources were poured into the elite, and I think we are going to move away from that. People are still going to have their elite that they want to do well, but we are going to have a system which gives more emphasis to the other people and one of the ways to give it that greater emphasis is to show what they can do. 


\title{
Interviewee VI(97): English teacher in secondary school
}

Date: 16 June 1997

\author{
AU Researcher \\ VI(97) Interviewee \\ ... Missing words / Unfinished utterance \\ - Pause
}

AU What are your feelings as regards the ED's decision to force $75 \%$ of schools to adopt Chinese-medium education?

VI(97) I think it's kind of politicism that comes up once again. In the past years there were famous schools and English was regarded as a very important language to people in the years before and after that a more general education, more students have been allowed to study English and they have better chance to seek for a higher position in the society, but here in the run up to 1997 the ED has changed its policy from encouraging schools to adopt English-medium of teaching to adopt compulsory education in Chinese. It is a kind of action which apart from politicism is inconsiderate of the situation.

AU How do you think it's inconsiderate?

VI(97) I think students have to have a lot of chance to expose to English, not just their English subjects, they have to have more exposure in other subjects because there's a linkage between their further studies and their work, and I worry that we HK people will be like Japanese. In Japan, all the textbooks are in Japanese and so the English standard is not as good as that in HK, I'm afraid that one day we will go to that situation.

AU How about your school, is it now English-medium or Chinese?

VI(97) A mixed-code of teaching using English textbooks and teachers mainly teaching in Cantonese and sometimes using the English terminology to bring the concepts or ideas.

AU Do you know what will happen to the school? Will it change, will it become Chinese-medium in 1998 ?

VI(97) It has not yet decided, but as our school is a self-subsidised school we have more autonomy in our reaction to the language policy of the government We haven't decided yet. 
AU OK, what are your feelings as regards the ED's planned introduction of benchmark language levels for language teachers?

VI(97) To me, I think it's unfair to teachers who have already entered the field. Just like lawyers, they don't need to have some sort of test to them whether their professionalism is recognised. I think there should be more emphasis on the assessment of teachers in training, maybe they are attending a certificate of education, then according to reach the level of professionalism then more emphasis can be put on the assessment. Instead of providing a language benchmark to English teachers on their job, maybe more encouragement should be given to teachers, say like some refreshment courses, some chances for further studies overseas so that they will encourage their level of English.

AU I think the point of the benchmarking is that it will then if teachers don't reach a certain level then they will have to take some courses or some further training. I've been told that they will be given something like 10 years to reach the benchmark.

VI(97) I don't quite worry about that as there is 10 years to go, but I think the policy is not so fair, I don't mean that it is quite difficult to pass the test but it is ridiculous to me, compare with other professions like lawyers or doctors. I don't think if you are a doctor, you need to have a test to see of you can continue to be a doctor.

AU Well I don't know, certainly I don't think they have tests of their language skills, but you are right, I don't think they have continuous tests of their ability once they have qualified as a doctor ... or as a lawyer.

VI(97) They do have testing, but the result can lead to further advancement in their careers. I know that doctors have to study a lot to keep abreast of the advancements in their field, and some of them maybe take some studies.

AU But don't you think that having these benchmarks is a kind of way of raising the standard of teachers? I think what a lot of people are worried about is that there are a lot of English teachers who are not English majors, who are not trained as English teachers who are teaching English, they are perhaps geography majors or history majors and because they went to university and did their university degree through the medium of English, then principals or panels think that their English must be good enough and they are a teacher so they can teach English. I think the point of the benchmarking is to try to even that out and to get rid of that situation and that only people who are competent, at least in their language level, should be teaching English.

VI(97) So, it won't apply to all English teachers? 
AU Oh I think it will apply to all English teachers, but presumably those who are trained as English teachers either from the I. of Ed. or BATESL or another programme like that, their language should be good enough. It's perhaps the other teachers of English or of Chinese whose language levels are not up to scratch, who they are targeting.

Do you think there will be any other significant changes in government policy towards the teaching of English in HK after the changeover?

VI(97) I think more money will be spent on teachers but I think it's related to Q5. I think we have to talk about the kinds of teaching that will change first because the government will spend the money on them. I think more remedial classes will be given to those schools who follow Chinese medium of teaching, and also there are courses for new immigrants to have a match to the local students at the same age, so that they can catch up with the English intensive courses. For the remedial classes for the local students attending local schools, they will have more chance, they will not just study the knowledge from textbooks, they will be more encouraged to read newspapers, magazines, things like that, it's a part of their curriculum, not just the textbook, to top up the contents. So the government will spend more money on these two main aspects. And for those students taking Englishmedium schools I think not many changes will be taken because the students exposed more or less the same they did in the past.

AU So English-medium schools, the curriculum will stay pretty much the same, the changes will be in Chinese-medium you think?

VI(97) Yes and those schools giving classes for new immigrants, so the government will spend more money on some refreshment courses for teachers so they can hold some intensive classes for the immigrants and some kinds of materials for them to develop to teach students to have more exposure to English apart from the textbooks. kind of tailor-made lessons.

AU So you think more money will be put into more resources?

VI(97) I don't know what kind of policy is more important.

AU The main policies are those in 1 and 2 , the main policy changes that we know so far, but certainly more immigrants coming from China will be a factor in $\mathrm{HK}$ in the coming years, and you are right, something will have to be done to cater for them to bring their language level up to a certain ...

VI(97) To their counterparts.

AU I don't know how easy that might be, you might have extensive courses but an intensive course for a few weeks might not change very much. They might 
need remedial classes; they might need classes throughout the year. But I think that in that situation, schools wouldn't want to isolate those students, they would want to integrate them totally.

VI(97) I think they have to attend lower form compared with the local students maybe.

AU Well that's what usually happens, isn't it? What changes do you envisage in the proportion of the timetable given over to English teaching in both English and Chinese-medium schools?

VI(97) For the change of timetable, I think for English-medium schools, more or less the same the timetable. For Chinese-medium schools, yeah, so it is I think more lessons will be held on English because the students lose something and they have to gain something to have a compensation.

AU I think that is what a lot of people think. Of course the other factor perhaps is Putonghua. Although I've not seen any instructions or any plans about it, it will be taught in secondary schools starting from this year I would think.

VI(97) Just the Chinese language subject?

AU Because having talked to the EA people, Putonghua will be a HKCEE subject in the year 2000 , so they must start it now in order to bring the students up to standard.

VI(97) Will it be a separate subject or mingled with the Chinese language subject?

AU I think it will be a separate subject.

VI(97) Just like French?

AU As a foreign language, yes, which is interesting because the idea of the timetable comes in, and the proportion of the timetable. If you're going to tech Putonghua as well as English and Chinese, as Chinese is taught now, then where do the extra lessons come from?

VI(97) So you can say about it, in proportion for English-medium schools, they may lose 2 lessons, not just from English lessons, maybe some other lessons, Chinese language lessons, and for Chinese-medium schools in that case ... I think they may also lose 2 lessons. They won't lose more or less than 2 lessons. And for the extra lessons, it's a pressure, how to squeeze out ...

AU The Chinese-medium schools, they don't want to decrease their number of lessons on English, that will be the only English the students get. 
VI(97) Maybe the students will have less choices on other subjects, apart from language ones, yeah, like in my school, for arts stream students, they have to take economics, geography, history, English, Chinese, mathematics, and then Chinese history. Four subjects, Chinese history, history, economics, geography, they have a choice of 3 of them, instead to take 4 of them, so that one more subject's time can be allocated to Putonghua, and for those Chinese-medium schools, I think maybe they can shorten their lesson time for other subjects, because now they are teaching in Cantonese, the students can learn much faster than they could before. They can shorten the lessons, or simply give extra burden for students to stay longer in school.

AU Add 2 extra lessons in a day, perhaps, or an extra 1 lesson per day. Yeah, make the lessons 5 minutes shorter and add one extra lesson.

VI(97) If two extra subjects have to be focused at the same time, there would be sacrifice, either in shortening the time for teaching other subjects, or excluding some subjects.

AU Something will have to give, unless they make Putonghua the MOI.

VI(97) It is a long way to go.

AU I think so, yes, maybe in 10 or 20 years. Do you think there will be any significant changes in your school or in the education system more generally, which might affect the teaching of English?

VI(97) I think, more remedial classes will be held in our school. I can't say for sure, probably my school will follow the teaching in English medium, and actually there should be less changes in the kind of teaching and the time spent on it, I mean English of course, but the level of our students is not so good, so just like before, the students will still need to attend remedial classes. I mean more remedial classes will be offered to students. This arrangement does come from the change in medium of most schools; it comes mainly from more teachers' resources. In recent years there has been a drop in students going to my school because my school is a subsidised school and it doesn't follow the arrangement for primary school students, so more teachers' resources can be allocated for remedial teaching.

AU Do you think that the changeover will affect teaching methods or approaches in English lessons in secondary schools?

VI(97) This one, I don't have any idea about that, to the methods or the approaches, as I mentioned for Q5, more remedial classes will be offered to students in Chinese-medium schools, and intensive courses for new immigrants, so there should be some kinds of changes in the methods in teaching. 
AU Because of having to teach more remedial classes. I suppose the change in the MOI might change things. If schools become Chinese-medium, then that may change their methods or approaches to a certain extent. If they have to teach English as a foreign language, as opposed to as a second language then maybe the approaches will change, because the students in the Chinesemedium schools will not be getting any backup from their other classes.

Final question, do you think the changeover will affect the situation, status or morale of teachers of English in HK?

VI(97) For the morale, I think there's not many changes because the morale of teachers of English does not depend on the change of emphasis on the language of teaching, yeah. Morale comes from the teaching environment, the workload and the kind of support from the colleagues, things like that.

AU That will change?

VI(97) I think not many changes because for English-medium schools, as I have said before, there will not be a lot of changes. For Chinese-medium schools, the government has proposed that more English teachers will be employed to help the students so that the workload will be more or less the same. Maybe the beginning years, I don't know if it will be better or worse, I can't say exactly because there's some kind of adjustment and teachers in Chinesemedium schools are a kind of guinea-pigs. But anyway, there are not many changes because more teachers will be employed to help them, to share the workload. The status or situation may be a little bit worse only, because the emphasis is no longer on English. Of course the status will be less important as before. 
Interviewees VII(99), VIII(99) \& IX(99):

Date:
Education Officers in Hong Kong

15 July 1999 $\begin{array}{ll}\text { AU } & \text { Researcher } \\ \text { VII(99) } & \text { Interviewee } \\ \text { VIII(99) } & \text { Interviewee } \\ \text { LX(99) } & \text { Interviewee } \\ \ldots & \text { Missing words / Unfinished utterance } \\ \text { - } & \text { Pause }\end{array}$

AU Thanks for agreeing to be interviewed today. The first question is how do you think the change in the medium of instruction in secondary schools has affected education in Hong Kong?

VIII(99) It seems to me that it's a bit early to tell if there's been any definite change, it's only been since September and even there it's introduced sort of gradually, as you were saying yesterday, it starts with Form 1, so I think it's still the potential effect rather than any actual effect. And I don't think at the Exams Authority we've noticed any effect. I don't think it's affected the number of candidates taking Syllabus A, for example, it was up slightly this year but not much.

IX(99) I think it's too soon because if those kids are in Form 1 only, it'11 take another four years for them to get up to Form 5 when they will take our exams. So I don't think on our part that there has been any changes. The sort of usual rise in numbers for Syllabus A candidates ...

VIII(99) It was only marginal this year, it was something like, it was up from fifteen thousand to sixteen thousand, so maybe it was up by four or five hundred but it wasn't a huge jump at all.

VII(99) There could also be some repeaters.

IX(99) And that's usual.

AU Just as a general feeling about things, do you think it's had any marked effect on any aspects of education?

VIII(99) I mean actually the only thing that I've really noticed are the individuals that we know who were in schools that were initially not granted EMI status and who were very vocal and vehement about their wish to retain EMI status and they lobbied and petitioned and all of that, so in a sense it's raised their commitment to English as a medium of instruction. 
IX(99) And I suppose at Form 1 level now EMI schools are more conscious that English must be used as the medium of instruction and not mixed code because there are inspectors going round checking on whose schools are ... I think for those students at Form 1 level they do get maybe a purer form of English instruction rather than mixed code.

VII(99) There's still quite a lot of confusion over what was expected of schools when the inspectors went round, as in what sort of things do you have to present in order to support your case being an English-medium school. I think that was the source of some resentment as well. There didn't seem to be any kind of fixed pattern or expectations of the schools to state their case. Some schools would get their status renewed on something that seemed to be much slimmer than say another school that had more of a struggle to get accepted for English-medium.

VIII(99) Wasn't the fact that they didn't consider value-added in the sense that they looked at the intake of the students and the level of the students that actually went into the schools. The schools were arguing for whatever, for historical reasons or geographical reasons or whatever, they may have had students that were weak in English but their claim was that they actually improved their English; they were saying that they should look at the result rather than the intake. Maybe in that appeals process they changed and looked at results, or took that into consideration.

AU I know that there's been some talk recently of reassessing those schools that have been granted English-medium status and if they are not actually doing it the way they are supposed to then they could lose it.

IX(99) I'm sure they will do that just to keep people on their toes and make sure that they are really English-medium throughout and not sort of mixed code again.

AU Okay secondly, have you noticed any changes in the situation, status or morale of English teachers since the changeover, or in the last couple of years?

VIII(99) You don't mean the changeover in the medium of instruction, you mean $1997 ?$

AU Yes

VII(99) I don't think so, I mean the teachers that I have contact with through the committees seem to have the same degree of morale as they always had.

VIII(99) Morality 
IX(99) Well, if there's anything that affects the morale of English teachers it's new initiatives, like the quality education thing ... [ ] was telling me that the schools have gone through the same process ... it's other initiatives that have to do with overall quality of the school, rather than just English, it's sort of like the driving force behind improvements or plans to improve schools, not just the English department but the school as a whole.

VIII(99) And also in a sense we see the people who are the most motivated and the most committed and have the highest morale ... they are the ones that are interested in working for us and we see two levels, we see the ones who are just markers and they are more representative of the average teacher, but the ones that we have most contact with are the ones who sit on our committees and do special work for us because they've shown a commitment and they've shown an interest and they've shown an ability so ... and I don't think they've been affected, they seem to be the same as before.

AU Yes there are a lot of other changes like the medium of instruction, the Quality Education Fund, the TOC, which is coming along, and the benchmarking of language teachers as well, which hasn't actually started yet but there's the potential threat, if threat is the right word, of it. Do you think teachers are worried about these kind of changes?

IX(99) I think the teachers we know mostly are good enough so that they don't think the benchmark will be a hurdle for them but of course there are other teachers who may think of that as a sort of obstacle to their career because we do know of some teachers who may not meet the benchmark.

VIII(99) But I don't think the objection to the benchmark is based only on your personal assessment of whether you can pass it or not, I think there is an objection that it's not a fair, whether it's true or not and whether it's a fair assessment, some teachers oppose it because they think it's an interference in their professional independence. Once they've gone through their education system and they've been certified as good enough to teach, then they shouldn't have to continue to prove themselves. But if you have a personal stake in it and you actually don't think you're going to pass it then I think your feelings will be stronger.

IX(99) So I actually don't see why people should object at a professional level because doctors don't object to having to pass certain criteria.

VIII(99) In the paper there've been recent things about doctors having to do not the same thing but are going to have to be recertified. I think initially it's voluntary but there is the same notion in other professions.

IX(99) And I don't see why, I mean teachers as a profession should be just as accountable to the public or parents as other professions to get themselves 
certified. I mean it was before that you didn't have to do it, I mean you need just a degree in fact and then you can be a permitted teacher without any training and I think a lot of the teachers, at least the principals will know that's not satisfactory, but I think people are only too happy with the status quo which is quite relaxed in that respect and they don't want any more stricter rules about who can teach and who can't.

VIII(99) But actually, when you mentioned that there'd been lots of other changes and you mentioned the quality education thing, again it is only the small number of teachers that we have contact with. The ones who are involved in that seem to be really excited and they've done really interesting projects ... I don't really know if it's earth-shaking but it sounds really like full of interest, for the teachers.

LX(99) And for the kids, mostly, I think for the teachers it might mean more work but for the teachers if they are interested in those projects and things they are really motivated.

VIUI(99 Well at least it takes them out of doing just classroom, only classroom activities.

IX(99) And I think that's actually kind of boosting the morale of teachers, they're now given this money to do other things, which they might have wanted to do but didn't have the resources for.

VII(99) I guess things like the conference we went to at UST where one of the days there was supposed to be like three hundred teachers and I'm not sure whether that kind of thing happened in the past or whether we were allowed to ...

IX(99) I think the ILEC conference teachers are allowed to go, but I think at the conference at the UST they've got this special day for teachers.

VIII(99) Special rate

IX(99) I think that's good because teachers don't often get the opportunity to actually leave the classroom and the school and sort of go out and have some professional development. The teachers we met there were certainly happy to have that day for other things than teach.

AU Any significant differences in students' attitudes towards the learning of English?

IX(99) We don't see students. 
VII(99) The only way that we would be able to gauge that would be through an increase in higher grades or a drop in standards, something like that. That's really the only way we could tell ...

IX(99) ... and we haven't seen that in the last couple of years, it's been quite stable judging from the percentages of people passing and getting As and Bs. We actually really don't see students.

VII(99) There's still a huge market for extra English tuition, they're still battering the tutorial schools.

IX(99) I think if anything the students are just as keen judging from the attendance at the tutorial schools, they are just as keen to try and improve their English, in the right way or not, I wouldn't comment on that, but they are certainly keen to get a good grade in their English exams and I'm sure that they realise that that's an important thing for higher education and all that.

VIII(99) I think in a way, and it's not just English, I think students are more assertive now. I remember [former head of EA] used to say that Hong Kong students had changed since he started here thirty years ago, they no longer meekly accept the grades they get on exams, they appeal or they complain, more of them do it, or they call before the exam and want to find out more details about exactly what's expected of them, things like that.

VII(99) They know the regulations.

VIII(99) They know the regulations, which is fair enough, they're more aware that they do have rights, that they can challenge and they don't just have to accept what's given to them.

IX(99) We don't see students but we do get calls from students sometimes and they say, what's the right thing to do, my tutorial school says this and my teacher says that, so sometimes they do know to call and check.

AU I think the feeling that I have perhaps is that in CMI schools that they may feel that there's a moving away from English, and it's still a limited number who are now perhaps freed of this having to learn through the medium of English, that it's now all Cantonese that they may feel that English is not as important now as it used to be. I think those taking the exams are still at the top end, probably haven't changed that much in their attitude.

IX(99) But as we said before, it's only the Form 1 students so it's more difficult to see the effect because really we don't meet Form 1 students very often. I think we have to wait a few years and when they come up to the school Cert. level, Form 5, before we see an effect, because I know for a fact when I was a teacher students do have priorities so like science students will say, well I 
spend a lot of time doing maths and lots of practice but English is less important, for the science students they feel that way, so I'm sure that students have their own priorities, when they have only so many hours to study they'll say, I'll do this first and then I know that some science students will fit English last. But I don't know if Chinese medium is going to have that effect that they are going to put all these subjects in front of English, I don't know.

VIII(99) Also, if the medium of instruction is not in English then it stands to reason in terms of their short-term view of English as a useful thing, it must be less. In the long term if they see that it's useful in society to know a second language well, eventually when they get out into the workforce it will be useful, then that's something that is not immediate at all.

IX(99) For a twelve year old.

VIII(99) For a twelve-year-old it's very hard, so in terms of the immediate usefulness then surely it must be less useful. I assume that the textbooks will eventually become Chinese.

IX(99) But they do have Chinese-medium textbooks.

VIII(99) But that was always one of the alleged problems of changing to Chinese as a medium, was that there wasn't sufficient good textbooks in Chinese. I don't actually know now in the Chinese-medium schools, the ones that have been mandated to go, is it mandatory that they use Chinese texts? I assume that they would.

IX(99) They will check the EMI schools to make sure that they use English medium.

VIII(99) If the texts are in Chinese then what's a twelve-year-old ... why should he think that it was particularly useful to learn English?

IX(99) Except for the subject of English.

VIII(99) Except for the subject of English.

AU I think there may be the possibility that schools that had become Chinesemedium, although the change is only supposed to take place in Form 1, that it's been very much a mixture as we know, some teachers will actually use a lot of English and other teachers will use all Cantonese and I think now that even higher up in the schools that teachers are now switching from ... those who would use English are now switching to Chinese. And from what people at the Institute of Education have told me, they've seen differences, they've seen maths lessons taught through Chinese compared to English and said there was a total difference, students were much more ... 
VIII(99) You're going to be called over the carpet for using Chinese to teach math even in the higher forms in a school that's been told ultimately you've got to teach in Chinese. If I'm one of the last ones that's teaching in English for Form 1, 2, 3, 4, I might slip into Chinese ...

IX(99) I think for science subjects and maths very often in the old days with the Anglo-Chinese schools very often they would have those lessons in Chinese because the schoolchildren will find it easier to understand and the teachers like to supplement explanation in Cantonese. They may do it once in English and do some more explanation in Chinese and that's quite common in the science subjects. And I think for the science subjects even in Chinesemedium schools, they will use some English, like in chemistry there are lots of terms that's easier in English than in Chinese, so I think the mixed-code will go on whatever you say, except maybe in the English-medium schools if they really do a good job checking and people may, okay they have to use English all the time. But I'm sure in the Chinese-medium schools some English is used for teaching of certain subjects.

VIII(99) But I remember you talking about, Christina taught biology, you were saying that you actually find it difficult to teach in Chinese.

IX(99) So I'll lose my job, if the school says we'll change to Chinese-medium next year then you have to start teaching in Chinese then I'll have to learn all my terms or maybe study the textbook ahead of the students to see what those terms are. I mean there are glossaries and you can learn things again but it's not going to be easy, especially for teachers who've taught quite a number of years in one medium.

AU That's the point, now we are Chinese-medium so you've got to teach in Chinese when you've been teaching for so many years in English.

IX(99) And I think people still assume that it's automatic for the teachers and they can switch and say now I use Chinese and only Chinese. It's just not practical to expect that teachers can do that, switch.

VII(99) I suppose if you have a content-based subject as well and you've taught it in English, say, and you know that you can get through everything on the syllabus, if you have to teach in Chinese there might be unforeseen problems in that, whether or not you could get through everything or something needed more explanation.

IX(99) And all the materials have to be redone in Chinese, you know, the handouts and worksheets, whatever. It's not easy for the teachers as well. Most primary schools in Hong Kong are Chinese medium, and I'm talking as a parent now because my boys are both in primary school, so they are Chinese medium so the kids learn everything in Chinese, maths, general studies, all subjects but 
English, but they still pay quite a lot of attention to English because the school say English is very important, if you don't pass English you'll be detained, you can't be promoted to the next class. So the kids know. I think it's what the teachers tell them or what the focus of the school is. You can say, every subject is in Chinese but if you don't pass English, you can't go on to a higher level. Then the kids will know that English is important. It's not that they do everything else in Chinese so English is not important, they realise the importance. I think teachers in Hong Kong tell kids that English is important if you want to get a job, even from primary school onwards they get told that. Or if you don't prepare well you won't get into an Englishmedium school and English-medium schools are still more prestigious. So they get told things like that from very early on, and I think any student who considers himself above average would try and cope with English and make an effort to try and learn English well. Of course they are always some who find it too difficult or can't learn from the start and then they may give up, but I think the majority will still try very hard to keep up their English, even if it's just a subject.

AU Okay, any change have you noticed or you believe in the attitudes of the general public towards English and the learning and teaching of it?

VII(99) There were quite a lot of letters in the press when the medium of instruction thing came in, people voicing their support for mother tongue, and then the next day there would be a response saying English is more important. But I think those attitudes have always been there, the two opposite points of view. I'm not sure that it's been any different since the changeover.

VIII(99) But I think there's less adamant defence of English as the medium of instruction than there would have been ten years ago, it's a fairly shaky argument that all kids should be taught in English as a medium of instruction. It's not logical that you could do as well in a foreign or a second language than you could in your native language, so I think there's less of this adamant $\cdots$

VII(99) Certainly at the education level but I think with the recession, I seem to remember there being quite a lot about Hong Kong's going to lose out unless it improves it's English proficiency as it's not competitive in the international market and English is the ...

LX(99) I guess certainly from the parents' point of view they would like their kids to be in the top fifth because the top fifth it is who get to English-medium schools. Just last week the results of the Primary 6 assessment were released and there were several reports in the newspapers, these new immigrant kids they get into English-medium schools, and that's a big thing you see, getting into an English-medium school, whereas nobody really publicise kids who get into a Chinese-medium. So I think it's quite clear the attitude of the 
general public I don't think has changed very much in the last couple of years in that parents are still aiming for the prestigious schools that they want their kids to go in and they think that it's an honour to be in an English-medium school. They would rather be in an English-medium Band 2 school than in a Chinese-medium Band 1 school. But of course there are not very many Chinese-medium Band $1 \mathrm{~s}$.

VIII(99) But I think the attitude towards English, not learning it or teaching but towards English has actually changed, not really drastically but I find being in more and more situations where handouts or at meetings where ... I mean our meetings here used to conducted virtually $100 \%$ in English, whereas now they are conducted in $70 \%, 80 \%$ Cantonese, which is fine but I think it has changed.

IX(99) I suppose that has to do with [ ]. Before, our section head was actually nonChinese, like when [ ] was here all the meetings had to be in English.

VII(99) So the policy hasn't changed.

IX(99) The policy hasn't changed it's the people who have changed, or people now feel that using Chinese is probably more acceptable than before, whereas before they would think that as English is the official language we should try and use English all the time. Even with government, there are more bilingual things and so ... we used to have everything bilingual but sometimes I think they are phasing in Chinese only, like with the welfare committee, newsletter

VIII(99) But I don't think that is all to do with [ ] not being here because [ ] wasn't SSO for quite a few years and he didn't always come to the meetings, he was always a lurking presence in the background and yet they were conducted in English. I think it's more of a fair notion that it's perfectly acceptable. The Government is no longer English dominated ... but I think other companies, I think where my wife works, which is Hong Kong Telecom, they still conduct everything in English because it's an international company and they've got so many expats in the workforce that I think certainly all their written stuff is in English and most, a lot of the meetings ...

IX(99) Whereas I suppose at the Exams Authority there's not so many expats.

VIII(99) There are only three of them ... I suppose things like all of the written documents will go out ... the meetings are in Cantonese but the minutes are in English and except for [IX] taking notes is difficult.

AU What we've found in our institution, the Poly U, is that everything is done electronically now which necessitates it being in English. 
IX(99) Well you could do that in Chinese as well, it's more difficult but ...

AU So all notices are sent round via the internal e-mail system. You could send them as attachments in Chinese and some are but generally everything is in English.

VIII(99) I do think actually ... I've talked to friends who work for Government agencies and they say the same thing, the written things have continued in English but most of the actual face to face communication ... but not just the one to one ...

IX(99) I thinks it's very interesting, we were talking about it at lunch once, now the kids they have these ICQs and chatrooms and they do that in English where normally they would never speak to their friends in English, but they would be using English because that's the medium ... I mean they can type in Chinese but that's so difficult to learn and so they use whatever English they can and the English is usually quite appalling and so the fact they are using English all the time in those situations may not actually help their composition writing but they develop their own form, short forms and abbreviations but I'm sure the grammar is terrible.

VII(99) That was one of the objections to the original draft of the composition paper last year where the context was you sent an e-mail to a friend and we decided not to use that, a, because they had to hand write it and $b$, because we were worried that they would come up with all these short forms.

IX(99) It's a different language. So in a way some kids are using more English than before as they're using computer.

AU Those with the means.

IX(99) Yes, it's quite common in some schools, actually, I'm sure not in all schools yet.

AU University students all the time, it's all they do every night, ICQ, into the early hours.

IX(99) Really? Some secondary schools ...

AU Okay, this is one which is more up your street, any changes in the curriculum or examination syllabuses for English since the changeover, probably no, and if so what have they been and why have they been made and what effects might they have had? 
IX(99) Not our exam syllabuses, our most recent change was 1996, so that's before the handover, and we've been using that syllabus up till now and we don't have any plans for changing the syllabus.

VIII(99) But the curriculum the CDI has changed both for sixth form, or they're in the process of changing for sixth form, and for $\mathrm{CE}$.

IX(99) Well actually for Forms 1 to 5 . Will you be talking to the CDI people as well?

AU It's very difficult; they don't want to speak to me. I've tried before and they've said, 'I'll have to ask my boss,' and they come back and say they can't talk to me. I will try again.

IX(99) Have you talked to [ ]?

VIII(99) She's really a nice, open person, I think we can find her telephone number for you.

IX(99) And I think because they are now in the process of promoting the new syllabus for Forms 1 to 5 , I think she'll be quite happy to talk to you about the syllabus, why they are changing it and so on. Or we can talk about those curriculum changes but I think [ ] will still be the best person and she's involved in both $\mathrm{CE}$ and $\mathrm{AS}$ Level, right?

VIII(99) For the sixth-form change, that Education Department syllabus, because I was on the committee that looked at that, it didn't seem ... basically it seemed that they decided to do it because it had been a long time since ... and they thought the old one was out of date ... they thought the old one wasn't detailed enough, that it didn't give enough support for teachers or actually explain what they wanted or what should be included and they wanted to bring it up to date with more modern thinking and pedagogy. I don't think it had anything to do with the handover.

IX(99) The same with the CE syllabus, it's really to try and maintain this link with TOC because now the primary kids who are using this TOC syllabus they will be in Form 1 soon, I think, if not next year, so that's why they think that there should be some continuity there and that's why they are updating the syllabus so that it would link better with the primary syllabus. I think that's the reason for the change and it has nothing to do with the changeover.

AU Oh no.

VIII(99) And in fact the exam syllabus, the last change in 1996 was in CE and I suppose to a lesser extent to changes that were made to the oral in UE, well they were, I mean the changes in the $\mathrm{CE}$ were a pre-emptive strike designed 
to anticipate the introduction of TOC and therefore make it unnecessary to have to change our syllabus, so it was made more task-based, more ...

IX(99) So we had to change ahead of that.

AU Rather than make a sudden drastic change ...

VIII(99) Yeah, and rather than having to react to whatever TOC eventually brought about.

VII(99) Is there like a regular period when people think that it's time to change the syllabus? There's always some specific reason, isn't there?

IX(99) Yes, there's always some sort of specific reason. I don't think that people say that it's been four years so we should change the syllabus. Normally we don't change syllabuses for the sake of it, because actually teachers prefer an unchanged syllabus because they are used to it and they don't need to change and have taught the same syllabus for years and they don't like changes, but we only change for specific reasons.

VII(99) It's a very long process actually, to change the syllabus.

LX(99) So it will be what, three or four years?

VIII(99) Actually the one in '96 was actually a fast track and that took us at least three years because it was one year with a working party with proposals and things and they had to send it to schools.

IX(99) They put it through the subject committee first and then the schools.

VIII(99) But the schools have to have a two-year gap, don't they?

IX(99) Because they start teaching in Form 4 and the exam is at the end of the fifth form, it needs two years, actually, and then the publishers need to be given enough notice to get textbooks printed and new editions and things like that.

AU You said that when you are setting the questions you have to take a lot of things into consideration, how does that reflect the context of society at the time?

IX(99) If anything we try to avoid tutorial school tips.

VII(99) Well the thing is with some papers, with all the papers I suppose, is that you want to be reasonably topical but you can't be too topical because then they will predict what the subject will be. 
IX(99) It's actually quite difficult because we would like the students to be reading about current events but [VII]'s right, if you use topics that are too topical or too hot, then all tutorial schools will predict those as composition topics or topics in passages.

VII(99) It's like the handover was a classic theme ...

IX(99) Or the medium of instruction and things like that ...

VIII(99) And we've had in UE C, we've had reading passages about corruption in the distribution of coal or about a fire in Shenzen ...

IX(99) So it's not all about ... we always try to keep these different perspectives so that we have some local and some topics which are more international or general.

VII(99) We still get quite a lot of resistance, in the UE oral, for example, there's eighteen sets with four passages per set, so it's impossible to make and not even desirable to make everything locally based and yet there" always a reaction from examiners on the passages that are about other countries or global themes, our candidates don't do so well on this because it's something that they are not familiar with.

VIII(99) I had something recently in a question which mentioned something about South America and for the kids to get the right answer they had to realise that Brazil was in South America and I was told in no uncertain terms that we can't have that because a lot of them won't know that.

IX(99) It's the same thing with the listening, isn't it, I mean the rainforest or whatever, we had to change it to an Asian country.

VII(99) Originally it was the wrong country and then it was changed to an Asian one because it's more relevant to the local scene, but again it's ...

IX(99) Still too difficult for the local kids?

VII(99) Well not too difficult, it's just that they don't know anything about this, which is no good reason not to have an exam passage about it.

IX(99) But we do keep an eye on ... we have teacher members on our committees and then the teacher members will let us know, the kids won't know anything about this or that or they'll definitely know something about this.

VII(99) I think there are always changes taking place but within the context of the syllabus. Every year we have subject committee meetings where there are teachers, there are people from tertiary, ED people, and everybody has 
suggestions about ways in which things can be improved, but they are improved from the perspective of we don't really want to change the syllabus because it seems to me to be quite comprehensive.

IX(99) And I don't think that people have ever suggested that we should be politically correct.

VIII(99) We went through a period when we were unsure what to call was it the Mainland of China or the Chinese Mainland and actually the Poly U seems to have something about that as well.

AU We've had messages sent round informing us.

VIII(99) The China Mainland was what we seemed to have at one point but it didn't seem to be grammatically correct.

IX(99) But also we say that we do whatever the curriculum institute says because that's what the students get in the textbooks, certainly they should get the same terms in the exam and not be confused by different versions. But apparently there is no sort of fixed term for different subjects that might come up for different technologies.

AU Okay, six, can you envisage any changes in English language teacher education as a result of any other changes which have occurred?

VII(99) I suppose the benchmarking would lead to changes in language teacher education.

IX(99) Because they've got this money to provide training. I don't know if it's now in preparation for the benchmark or instead of the benchmark.

VII(99) Well I think originally if you don't reach the benchmark then you automatically qualify for a training course where the whole purpose is to get you up to the benchmark.

IX(99) Whether you take an exit test or you get an exam afterwards, it's not very clear.

VII(99) But I think people are already bidding for these courses.

IX(99) Because they've already got the money and I suppose that's the right place to spend the money, rather than spending lots of money on tests, maybe it's more sensible to spend money on the training.

AU Last question then, which is meant to cover anything that hasn't already been mentioned, what do you foresee as the future of education in Hong Kong? 
Any trends you can see or directions in which you think things might be going?

IX(99) From my point of view, one initiative which is in the process of starting consulting schools is whether school-based assessment will be a more important part of public exams. It's been talked that we should include teacher-assessed components or school-assessed components, but that's only being discussed and schools will be consulted I believe next year? The reports been out but they are only now ... I think it has to go through the Authority first and then they will consult schools, so the Authority has to decide what form these consultation document takes and I suppose that will be in September and not earlier than that. For us that's going to be quite an important change and for teachers in school as well of course.

AU Yes, I can see quite a lot of pressure being put on teachers in the future, with school-based assessment and then benchmarking, with the Authority demanding certain things already it's a difficult job being a teacher with all the marking.

IX(99) And all this talk of four years university and six years secondary again. There was quite a lot in the newspapers at one time and then suddenly it's all gone. At that point people though it's going to be real and that's going to happen next year and then suddenly everything is dropped and nobody talks about that anymore so ... they proposed it and it sounded so real and the people [tape ends]

VIII(99) I think also there seems to be a sort of general agreement that exams as they now exist, there seems to be too much perceived importance placed on exams and there's too much attention paid to rote learning.

IX(99) Which is probably not true really.

VIII(99 Probably not the truth and too much rides on what is essentially a snapshot, so it's a one-off exam rather than an assessment over time and also there's the notion that Hong Kong because of this push for a cyberport and all that that it has to become, education has to become much more IT-orientated. So I think those things are going to have effects. Whether they will change or improve anything I don't know.

IX(99) But I suppose the introduction of Putonghua as a CE subject might have an impact as well because it was never a Cert subject and now it will start in, what, 2000? In fact we have a new subject officer who's going to be in charge of that subject. But I've heard that there are not that many schools now who are actually offering that course do they don't really expect a very high candidature. I think schools would like to wait and see. 
AU See which other schools are doing it.

IX(99) Or see what is the passing rates are, because you see there are only so many subjects that students can take and if they take Putonghua and Chinese Language, which is compulsory, then they have two Chinese subjects, so it depends. And I think that soon they might want Putonghua at AS Level or A Level. But I think initially it would not be a very big candidature and it will probably not be a compulsory subject for a long while to come, because they don't have the teachers. 
Interviewee 10(99): University educator

Date:

28 June 1999

$\begin{array}{ll}\text { AU } & \text { Researcher } \\ 10(99) & \text { Interviewees } \\ \ldots & \text { Missing words / Unfinished utterance } \\ \ldots & \text { Pause }\end{array}$

10(99) Unfortunately, the first question I wasn't sure that I have anything specifically to say so maybe we can move onto the others and in the course of that the first one may get answered. The second one, let me say that my knowledge of English teachers is mostly about the ones here and not about the ones at school. What information I get about school teachers is kind of filtered down through the staff here that have been out supervising practice teaching and so on, but as just overall comments, number two, I wasn't sure about changes in status or situation, but I do think that morale seems to have been affected in that there seem to be doubts some teachers have about the value of the work they've been doing and support for it. That might seem strange when the government has been throwing a lot of money, but I think there's still an uncertainty about things, possibly because Chinese is getting also more emphasis than it did get and the move to have sort of what seventy to eighty percent of schools Chinese-medium, I think has made some teachers, well, reassess what they are doing and they may not be too sure just what the value is as seen by those above them, in spite of what's said.

AU So they feel that English is on the downward slide, as it were?

10(99) I wouldn't say a slide, but at least there's been a step down, perhaps, I mean I'm not sure that they see it continuing on sliding but a step down and it may be a bit more time until the smoke clears and they might see that okay it is going to work out after all. But at the moment I think there are some that aren't sure about that because there are all sorts of political and judicial uncertainties in general which I think maybe unsettle people anyway and that might affect what else they're doing.

Students' attitudes towards the learning of English, in what I do, which is with people in the BAEPC, I haven't seem any significant change, there seems to be much as before, but the BAEPC students, as I've started to find out, are by no means typical and I meet a number of lecturers in the department who say, the students are sort of ... don't have much contact with the outside world, very narrow and so on but I quiz my students at the intake each year about what they've done in the summer and in a class of 20, I've been finding over half consistently travel overseas during the preceding 
summer and I'm not counting Mainland China. So the States, Australia, New Zealand, Europe, some of them in group tours, you get some of them just going themselves doing all sorts of strange things, it's very interesting, and during the recent break between the two semesters two of them each other by accident on ski tours in South Korea! This is the economic downturn. So these students are not ... they are pro-English, I think and they are determined to get out in the world and they're doing that. Some of them are doing it now instead of looking for jobs, they've said they are off to travel first, so they're not too typical I suspect, I'm pretty sure about that. And they saw opportunities before to get into the course if they could English would help. Another thing occurs to me while I'm talking is that our intake into the BAEPC and the number of applications is strengthening a bit, it's not weakening, there does seem to be more interest. Not so much in the BATESL, while the teaching brings in good money in the first couple of years, better than other jobs, I think the English teaching is still seen as a bit unattractive, but people going for an EPC degree have in mind that English plus communication skills in general ... communication production, bit of journalism, bit of PR, bit of tech writing, whatever, and multi-media stuff now, this is worth a go. So perhaps even a bit of strengthening, they do see opportunities.

The fourth question, the general public, there did seem to me a distinction between parents and their children, and their youth, in that parents still seem fairly well-persuaded and that their kids should learn English preferably in an English-medium school, that seems still to be pretty strong because they see the value of English, at least ... or they've seen it and they still think it exists ... whereas the impression I get among young people is that they're a bit more ambivalent or at least less persuaded about the value of English than they were. That is in general not the EPC intake I was just talking about, but in general that's the impression I get from talking to staff about their visits out to schools and so on, because at that level in schools they are facing the English and Chinese situation and trying to sort out which language they should ... pay most attention to. I don't know what they're told in schools about their knowledge of Chinese but I hear people telling them that their Chinese in weak ... I don't know if they're told that to their face in schools, if they are then that's going to unsettle them ... I don't know what goes on.

Changes in exam syllabus or curriculum since the changeover, here, it preceded the changeover but sort of revved up since it we have this English enhancement course and the setting up of an English Centre, which is just two years, sort of close to the handover but the planning preceded the handover, so we have a whole big system where $75 / 80 \%$ of the students get a lot of English ... 150 to 200 hours ... at least all the full-time students. So the university felt this was worth ... they wanted to get the message across that English continued to be important ... apart from wanting to raise standards I think the idea that English was important was in there as well. Because it's so 
much extra English, you're asking what effects the changeover might have had, it's a bit early to assess the effects. I think that they probably feel now they have had too much English. When they look back a year after graduation, in a year or two, they might feel that it was pretty helpful, but I'm not sure they think that now, I mean we have staff teaching students in the part-time evening BAs and they're teaching two nights a week for two hours because it's only a ten-week term, then I find out that the other two nights a week, they come in four nights a week, they're getting English enhancement courses ... they're getting four nights of English ... well that's guaranteed to put students off. If the other two nights were psychology or government or politics or something, which it could well of been in that programme, that would have been better. So there are students in situations like that who ... the institution thinks they're doing the right thing but it's pretty painful for the students at the delivery point. In the BATESL, we wanted to introduce for this coming year an English improvement workshop for BATESL students who are identified by the third year as still in need of help, because they're the weaker half probably. We're not running it this coming year because planning was a bit late and we have resource problems but it will be run in the following year, I'm pretty sure of that ... because our BATESL intakes are not as strong as our BAEPC, on average, 1997/98 was a bit weak, this year was a bit better. One way we have done that is to take in more non-JUPAS students. The JUPAS system takes students in from ordinary Hong Kong schools. Non-JUPAS originally was to get students in who had been working, but we're using it to get people in from international schools in Hong Kong, ESF, Chinese International School and so on ... they are still in Hong Kong, they haven't got kids, they are mostly Hong Kong Chinese we are talking about, they don't have to be but the majority still are, and we target the ones with the good English results. We were doing this before the handover and we continue to do it for both of our degrees, not just to get the average higher, which looks good on statistics, but also if we get these good students in it helps the others because there's a better overall standard in the group and the weaker ones get, hopefully, a bit more exposure to other students bringing better English, they can be a reasonably good role model, and that does seem to be working. So we've been doing that for a while but we're having to work very hard to get the BATESL intake' English standard greater, after all they do go out and teach English.

AU Have you noticed an increase then in these non-JUPAS students?

10(99) We're taking more in. We weren't sure what percentage the university would stop us at. Initially I thought they didn't want any taken in as we were told we were set up basically to cater for Hong Kong school-leavers, without realising that Hong Kong school-leavers these days can be defined more broadly, they don't have to come from regular Hong Kong schools, because they are still Hong Kong kids, in this other system, and very often because they've been over to Canada or New Zealand or somewhere with the family and the 
family's come back to Hong Kong and put them in one of these schools because they've become out of touch perhaps with the Chinese language requirements or fallen back, or they've got used to a different education system ... slightly more relaxed perhaps ... slightly broader ... and feel more comfortable with that. Or they want them to have ... say a Canadian high school diploma rather than A Level because the family still favours going back to Canada. So we have increased our percentage a bit, it's $15 / 20 \%$ of the intake, not 3 or 4 , and it's brought in good students.

AU In my experience there always has been ... I don't know what the percentage is ... people who are teachers who've been teaching before they ...

10(99) Right, we're still getting a small number and we still do take people in who've been out working but that's more likely to be one or two a year in each programme, not half a dozen. So in the BATESL if we took in six nonJPAS people, maybe one or two of them working and four coming from the non-standard school system. It's a breakdown something like that. I think with the economic downturn rather than the handover, we're getting a few more people who have perhaps lost their jobs who think, well I'd better go for something else, who've been working and at least are applying, l'm not sure we're taking them in ... we did get more applications from non-JUPAS entry this year. So the university has made changes in terms of the introduction of the English enhancement course, which isn't a cause and effect of the handover but certainly has gone alongside it, and the English improvement workshop is since the handover and we want to ensure the graduates go out from the BATESL with as high a standard of English as possible. We don't need to be persuaded that English teachers need to be as good as ever irrespective of the handover because we know what government policy is.

Can I envisage any changes in English language education? Well, we know that the government has brought in this benchmarking idea and you've no doubt talked to people about that. We are concerned about it as people who are graduating from the BATESL need to have something ... they need to be prepared for the benchmarking exercise and hopefully be successful. Also one of our staff, [ ] is involved in one of the benchmarking committees or sub-committees and [ ] who was the BATESL external examiner up to last year is on the benchmarking committee and is involved in it. He's given us some feedback and last year he spoke to our graduates about how it's going to work and so on, so that's important. And because the government is saying that teachers need to be fully qualified otherwise they can't go ahead and because the number of places in post-graduate diploma in education or cert. ed. at HKU is limited and nowhere near meeting the demand, we've thought about offering one ourselves. It would probably have to be self-financed but we haven't gone ahead with that yet but we are certainly giving it very careful thought, maybe not just for our BATESL graduates here but certainly they do need an opportunity to get fully qualified. At the moment just a few 
get admitted to the other programmes. Because of the competition, I understand that they do tend to take in teachers who've been teaching longer, the graduates have all been teaching for one to five years, so they're less likely to be selected, unless they do it full time, then there's less competition. So those government decisions do affect us on the BATESL.

I've written something here which I can't read ... what do I foresee as the future of education? I keep getting things like this ... this is from the SCMP Careers Post a week ago ... so here's the Labour Commissioner and he's bashing away at IT and com skills, communication skills and I'm not sure ... does he say ... yes, 'IT literate in Windows and word-processing in both English and Chinese.' Another area is soft skills in communication, I mean communication skills as such without necessarily the language, I mean independent of the language being used. Apparently this was continued on the back page and I missed that. So we're being, sort of, I mean the community's getting a barrage of this stuff, I see the English press and I'm sure there's plenty in the Chinese press. I do get copied to me the press cuttings every day, a short summary of things, not of everything, so I get a reasonable look at what is going on ... but there's a lot of it. I think that people will get the message about this and that the job situation will force them on people, whether they like it or not and my impression is students have already worked this out, that's one reason why the EPC degree is more attractive these days. So, on the one hand I think they're going to look for things like that. On the other hand they've got to ... simple things ... first of all ... the English teaching profession is still in trouble, I guess there are still too many people teaching English who are not qualified to do so and the numbers are just huge. Until that's changed or overcome, and that's going to take a long time, it's not going to be done overnight, then there can't be the kind of improvement they want in English teaching. So in English teaching you understand that anyway there's clearly that problem. So for a long time people'll still be moaning about English, I think, the situation if it improves will improve gradually. I think that costly though it is the percentage of kids getting post-secondary education needs to be increased. The unemployment rate for school-age youth, under 19, who are not in tertiary education is $25 \%$ and the government admits to $25 \%$. Based on similar figures in the UK and Australia, it could be more than $25 \%$. That's a lot of young people hanging around New Territories shopping malls and so on or worse, the pool halls and so on. To me that just spells trouble in the long run, especially the young men, they're not going to get jobs in manufacturing or labouring in the same way as they used to in the past and so they ... I know they're trying to do something but with it moving more into IT, there's no point doing that just with the top $20 \%$. I guess if they bring in TIs and so on it increases the number, but I think that's going to be very important, which I don't see them talking about too much ... they are talking about a fourth year at university but you are still talking about small groups. They need to look at a wider group to provide something, even if it's more TIs or something at that level, 
but it's going to require an effort. The other thing of course which has occurred to people and this is definitely since the handover and that is there's more competition from the Mainland and how do they ... I don't think they can talk about being in front but I think how do we keep up? I think that's the problem. And clearly I don't think good English is what separates Hong Kong from the Mainland, there are plenty of people over there with good English ... but they will need people with a good level of English proficiency amongst graduates here just to keep up plus the cultural breadth that somehow needs to go with it. My daughter works for Cathay Pacific ... she was telling me yesterday that I think all of Dragonair's flight attendants; nearly all are from the Mainland. I just thought they were all from Hong Kong. If she's accurate, there's a place where Hong Kong people have missed out. They fly a lot to the Mainland, they need a lot of Mandarin speakers, I don't know how good their Cantonese is, but there are places around where that's sort of happened, I think, where Hong Kong has missed out. So I think this is ... I'm sure people are very well aware of that, one of the things they're most aware of. Except for the handover and recent court cases, that sort of reminder of this general issue of competition with the Mainland, and numbers of people and soon and what side of the border they happened to be on at the time, and English proficiency will be one element in that, better IT skills etc. will all be part of that. That somehow international contact and being able to cope with that I think's important and is complementary to the soft communication skills because that's something Hong Kong students could have if they were willing to go for it. With some of them ... I think there is an ambivalence generally about whether to throw in their lot entirely with Chinese culture. But I think there are people on the Mainland who realise that, we feel confident with that, we've grown up with it, but the opportunity is ... for the international side of things, for the better jobs and so on and that's more blurred here. But I would hope that it becomes clear quickly because the longer it's blurred the further behind they'll be when they start to want to get through it, that's my feeling.

AU Originally my ... effects of the changeover and when I've asked people they've said that the changeover has had no effect as such but a lot of things are happening anyway so I've kind of reworded things 'since the changeover' rather than 'because of the changeover'.

10(99) Yes, it's difficult to say there's an actual cause and effect relationship. The changeover means that there is a change in sovereignty and people feel that they need to question the role of English and feel perhaps more duty bound to emphasise Chinese and Chinese culture, certainly of Chinese civilisation taught here, two courses for everybody ... it hasn't reached everybody yet but the new intakes, and this is felt very strongly by the leadership and I see a lot of that. They really do want to strengthen the appreciation of Chinese culture in a way that I couldn't believe anyone trying in Australia, except in a deliberate way if it was done more subtly perhaps. Perhaps it doesn't take as 
long. So I think you can say that's definitely since the change of sovereignty, right these things have happened, but the other things I mentioned, the benchmarking and so on and the big push for upgrading the English teaching profession, that's kind of around changeover time but was started before it and the need was realised. Perhaps they were looking ahead to the changeover but I think you have to say, well I knew the problem was there anyway and if we hadn't had the changeover they' $d$ still be doing this, whereas some of the other I think is more directly related and of course there were people coming over from the Mainland each day before the handover but their numbers have increased ... the official allowance or quota, and there is more freedom of movement, a large number of Hong Kong women are going over the border to shop. I mean there were before but now it seems a much more normal thing to do. So you might be wise to limit it to direct cause and effect. There might be some things but not so many perhaps.

AU This year at the Poly U we have an intake of 24 students from Mainland China who are to start degree courses in September and they are undergoing English classes at the moment and Cantonese as well. I'm sure they have been hand picked because they are excellent, their attitude and motivation are superb compared to many local students.

10(99) Yes, we get some as well, although we haven't got any in this department. I think if you know you are one person in 1.2 billion you are more motivated to get ahead, or take the opportunity than if you're one in 6 million. There's a big difference and there's a lot of you anyway in a similar situation, but I can just imagine if you get an opportunity and somehow get picked out of the system and it's such a huge system that you are likely to be more appreciative of that chance and I think it toughens you up a bit. Not that we don't have students like that, but it's not universal in the classes that I teach.

AU Has the department changed in any way? I know the English Language Centre has developed and has taken on a bigger role in the university?

10(99) We lost about 30/35\% of our ESP teaching, basically first year stuff, so what has happened is that we continue to teach the $20 / 25 \%$ of first year students who are not taken into the programme because their results were already good enough, mostly it means they got C in Use of English, in some cases D, then we're also in second year and third year. In some programmes we were hardly in at all and they got rid of us. Others, like building, computing, we're in all three years, so they kept us in second and third year. And in the BBA, which has an intake of about 800 and something, we get about 120 something who didn't have to do the English enhancement course and the other 700 odd come to us in second year. After they've done the English enhancement course they do a business English course with us in the second year. So we've not lost the lot and there's no move to throw us out. But on the other hand, we have added two MAs, an MA in Communication and New Media and an MA 
in ESP, which is self-financing, so it is from my point of view we have to ensure that we don't neglect the service teaching ... we do but we have to make sure that we don't because our own programmes tend to be more attractive to staff. The MACNM, which has a really heavy IT and English isn't in the title, we've had excellent numbers of applicants and when we've made offers of places virtually nobody says no. We had an intake of 25 this first year. It's part-time evening and during the year only one person dropped out, which is very ... and that person got transferred back to America. Maybe there's one other, there might be two. Numbers are kept up, people are very keen, we are able to get good people, and that ties in with this guy's comments on IT, but these are people with first degrees, but they see the opportunities. So the department's now got three Masters degrees, two BAs plus we provide the leadership for a BA in primary education ... we provide the programme leader plus we provide one of the majors in teaching English, though it's technically a faculty level degree. So we're close to having three BAs as well. And then there's the servicing which is still a large amount of our teaching but it's not quite so much of our interest. But if we're going to run a BATESL and we're going to run an MAESP, it's important that we show the practical outworking of that in our service teaching. Some people do teach on both, they teach ESL methodology plus they teach English for engineers and the two kind of go together to some people. So there has been a change but the change has slowed down a bit. There was just one year with a bit of a dip but it back up again, it's coming in and without the resources quite matching the increase, in fact it's not matching at all, so it's a reasonably even keel. But the department's interests are now fairly wide, I mean they did go from ESP and English literature, which there's a small but increasing amount because we are now running out of discipline courses for everybody who wants to take them to mass media, new media, the far end, the other end I should say. There are other departments that are pretty much that wide, certainly in Australia there's a department of English that's nearly as wide as this and another one which has introduced a course in English and communication studies. There seems to be a tendency in the hope that it'll save money at least in Australia group disciplines together rather than split them up. 


\section{Interviewees 11(99) \& 12(99): University educators}

Date:

29 June 1999

$\begin{array}{ll}\text { AU } & \text { Researcher } \\ 11(99) & \text { Interviewee } \\ 12(99) & \text { Interviewee } \\ \ldots & \text { Missing words / Unfinished utterance } \\ \text { - } & \text { Pause }\end{array}$

AU How do you think the government's policy on the medium of instruction has affected education in Hong Kong?

11(99) Actually, personally I think the education in Hong Kong will be more diversified. What I mean is that the prestigious schools will be more prestigious, whereas the CMI schools will have to suffer, because parents will be fighting for the places in the prestigious schools on the one hand, more schools are taught in Chinese, I mean the medium of instruction. But the point is parents still know that English is very important, so before anything can be done to the exam, the format of the exam, and the people's attitude towards English say in finding jobs or in offering jobs, otherwise ... it will be more diversified I would say.

12(99) I think it's something good, too, in a way, because parents and students and schools they are led to look at education, look at the merits of education itself, instead of looking at the medium of instruction, whether somebody can speak English or not, they'll really look at what one can get from education, look at the educational development, cognitive development and so on instead of just the medium of instruction. But then this is a transitional period and this is a period of pain for everybody, for the policy-makers and also the followers, so I think in the long run things will be good, yes.

AU Very much it's a transitional period, that's why I'm studying the transition and everything and that's why there's so much depth to it. So you think that before there was still elite schools, English-medium schools, but there was still a sort of morass of schools where ... who were supposedly Englishmedium but we all know they were effectively that, but now it's very clear cut. That's what the government wanted it to be.

11(99) In a way I think it's good. What I mean is the government, I mean what I should say is the view is that only those who can benefit from EMI should stay in EMI schools. I think the idea is good, the whole idea is good, I mean in the past, you know those who were still not proficient enough were still put in EMI schools; they don't benefit at all. I think it's good. And to me 
personally, I think CMI is equally good. We supervise 60 lessons, TP lessons, we pay TP visits every year, I know the situation. Take for example, I went last month to an EMI school, no no last year, it was a mess, no student was interested in the lesson, a mathematics lesson taught in English, the students were just not interested. Whereas, right following that lesson I went to a CMI school ... wonderful ... all students were totally absorbed and they were even challenging the teacher, of course in a polite way. They were attentive, they were interested in the lesson, they were willing to learn, they understood every word that the teacher said, they were able to throw answers to the teacher, you know, wonderful. And why not? Both students and teachers benefit.

12(99) In terms of effectiveness, it's good, and efficiency as well. If you give the best and you make the students study in the medium of instruction which is not suitable for them, don't twist them in a way that you force anybody to do it in a difficult language.

11(99) I taught in a so-called EMI school years before ... I taught them EPA, Form 1 ... all those vocabulary ... commander-in-chief, attorney general ... to the students, all these are Greek to them, just not interested.

ML A few days ago there was a feature on TV, it's on the Chinese channel ... it's a teacher, a secondary school teacher commented on the lessons because their school has changed from CMI to EMI, and he said they'd now changed to CMI so the textbook was, you know, relatively easy now, it's too easy for the students now because in the old days when it was all in English, they learned something very factual, they learned terms, the spelling of the equipment etc. ... it's science, integrated science. But now that book was too easy for them. The teacher just abandoned the book and designed his own materials and tried to stimulate the students' discovery kind of interest.

11(99) Something more challenging.

12(99) Yes, something more challenging, and then he's faster forward actually.

11(99) So you can tell language matters.

12(99) Does that answer your question? It's a positive effect on education.

AU Have you noticed any change in the status, situation or morale of English teachers since ... over the last two years?

12(99) A lot of our students, well some of our students ask me whether I think the status of English is going to change, whether they will still have jobs after they graduate because the language, you know, is fading out of the scene. But then I told them that I thought it was still an important international language. 
But then they have this kind of worry and they think that, well perhaps the status is getting lower and is being, well will be taken place by Putonghua or Cantonese. Well they have this kind of initial worry, but it's not ... the feeling is not strong. I think it's fair enough for people to think that way.

11(99) I agree.

12(99) But I don't notice any special drop in the status.

11(99) It depends, I mean it's inevitable the general feeling that English is not as important as before, whereas you can see even in the Legislative Council, the meetings, right, all these official government meetings and documents, you can tell the importance of English is a bit declining, right? I mean inevitably, people have a feeling that it's, that the Putonghua, you know, will become high flyers. But for English teachers it depends, I mean just last month we went to supervise a number of English lessons and I had a feeling that the CMI teachers have really suffered a bit.

12(99) CMI ...

11(99) CMI, English teachers in CMI schools, they suffer more this time because they do not have the support of the other teachers, whereas in the past some of these EMI schools, you know, there were more EMI schools at least those other subjects were taught in English, but now it seems that they are fighting a lonely battle, you know, without the support of other teachers. I feel that now they have some hard time. The lessons I supervised were taught by experienced teachers, still teachers suffer, you know they were experienced teachers, some of them were actually very experienced teachers, I can tell. It's difficult to motivate the students at CMI schools.

12(99) But we have change in our institute as well. In our institute, Putonghua is compulsory for every student, but not English. In the old days doing English was compulsory, doing English language skills is compulsory for every student. But now is not compulsory, doing Putonghua is compulsory, everyone takes Putonghua. So in a way changes have taken place, but then when you are talking about worries of teachers, then we don't think ... are we worried?

11(99) It depends on which type of teachers you are talking about, lecturers or secondary school teachers.

12(99) Talking about us, we are worried that some of our course will shrink, some of our courses, is that right? Some of courses like EMI.

11(99) No, if you are talking about, I also teach what we call General Practicum, that is teaching those who are going to use English to teach History and PE and all 
these. Again, I feel that the morale and attitude of these so-called future teachers in using English is poor really, the attitude. They don't want to even use English in the lesson. These are teachers, potential teachers ...

12(99) They are not proficient themselves ...

11(99) It's not just proficiency, it's the attitude. To some, the proficiency may not be good but the attitude is good, they try hard to use English, but the general picture is year by year I feel the students' behaviour towards English is disappointing. They are just not willing to use English just to interact in class. They know that they are going to use English, they may have to use English to teach, if they can find a job in an EMI school. I don't think their attitude is good, they keep on saying, 'Ben, can I use Cantonese for group discussion?' In a way I think we as English tutors suffer, the feeling is not that good.

12(99) I think the problem ... some people at City University, [ ]? They study motivation and said there is a gap between the desire to learn the language and then the real effort, the actual effort that they put into the subject. I think there is a wider gap in between, so if you ask the students whether they like to learn English, whether they still think English has got high status, high prestige, they will say, 'Yes, definitely.' But then, well maybe in the old days more students would transfer this kind of desire into actual kind of effort and they join in extra courses and read newspaper on their own, keep vocabulary books and really work hard for it but now they are more passive, the gap between is wider.

11(99) Why? Have you thought about why the gap is wider?

12(99) Because they don't need it in real life, because in the old days, well your work requires a lot of English, but now a lot of schools have turned CMI and Cantonese has become, well according to Pennington, the ethno-linguistic vitality, is it, of Cantonese has taken the place of some functions of English. So there is a gap between, yeah, so idealistically they know that.

11(99) So it is unlikely they can get a job in an EMI school, right? So there's no desire for them to ...

12(99) Because there's getting fewer and fewer EMI schools.

11(99) That's right.

AU They're not looking at that, are they? They're looking at the majority.

12(99) In real life they don't need English at all, actually. 
11(99) And also the proficiency level is a reason ... they're so weak in spoken English. They don't have the confidence to speak up.

12(99) My feeling is that English is still a goal for them, a very high goal ... that is still the ideal ... the distance is getting wider and wider. For my research I did an interview of students in Band 5 schools, whether their attitudes to English, Putonghua and Cantonese had changed after 1997 and very surprisingly all the Band 5 students they told me, 'Yes, English is good,' they'd like to learn it, just like the Band 1 students, no difference at all. But their standard is still very low. So, the distance is higher but the goal is still up there, they still worship that thing.

AU And perhaps it's becoming harder and harder for them. Okay, perhaps the opportunities to learn English in school are still there but outside of school and the environment it seems is getting worse and worse. Their entire life is in Cantonese now, they don't need English at all.

11(99) They don't need to use English at all once outside school in Hong Kong without having to use English.

[12(99) leaves]

AU Have you noticed any changes, significant differences in students' attitudes towards the learning of English? I suppose we've been talking pretty much about that already. Do you think the changeover has affected the attitudes of the general public towards English ... and the learning and teaching of it? The general public and parents might be included in that.

11(99) As has been said earlier I think most parents still think English is important, right, despite repeat announcements by the Education Department that, well, why don't we use our mother tongue, right? The problem still is, when you're talking about government jobs, when you're talking about furthering study, when you're talking about even jobs in the commercial sector, plays a vital role, right? That's the reality, so I think that the feeling of the general public is that still English is very important. As far as people in the education field, we all know it's true that the mother tongue is a good tool for teaching and learning. As ML said, it stimulates students' thinking, their cognitive development, all these, but the general public, which comprises the majority, well, have different views. That's what I think.

AU Do you not feel that like ... that like student, that the status of English has fallen since handover?

11(99) I think all along, to be honest, students have found it not easy to learn English, but in the past they did not have any say because English was the deciding factor and during their education, the students were repeatedly 
bombarded by parents English, English and English, you know they had no excuse. Now it seems since the changeover, students got sort of an excuse, that ah, English is not that important now, I don't have to put everything into it. It seems that there is an excuse for that. But as I said, it's just an excuse, right? So as far as the students are concerned, that gives them something that they can relax a bit in English. To be honest, as someone who always has to go to the English classroom, I think I've witnessed a very fast going down of the standard of English in Hong Kong. Even student teachers here, it's really terrible, you know. If you had a chance to go and witness their lesson, you would be horrified, you know, by their standard of English. I mean students in Poly $U$ or City $U$ are even better compared with ours ... ours are subdegree level ... lots of mistakes in their written and spoken English.

AU Some of our students as well, we have diploma and higher diploma students.

11(99) It seems that we are fighting a losing battle.

AU I think perhaps in the past with their being more English-medium schools, the majority or at least a great many more than there is now, parents then felt with their kids that English is important and generally their children were able to go to nominally an English-medium school, whereas now with a smaller percentage being English-medium, maybe those parents with kids in the middle thinking the kid's realistically got no chance of going to an English-medium school so fair enough. So, the point being that just because they go to a Chinese-medium school, they are not going to learn English, but obviously they are, the government is putting more resources into CMI schools, in theory, so that could possibly have affected parents' views. There is a kind of cut off now, if your kid isn't bright enough then they are not going to go to an English-medium school, and in a CMI school they can still have a good living and not need English. So the feeling is that it is not as important for everybody as it used to be.

Have there been any changes in the curriculum or exam syllabuses for English and if there has why do you think they've been made and what effects have they had or will have?

11(99) Well, we all know that for the primary schools a new curriculum has been implemented ... the TOC, but they don't call it the TOC anymore, just the primary curriculum. The idea is I think, or let me say the people in the Education Department are also fully aware that the standard is declining, that there must be some way out, that we have to do something so that the demand of the market has changed from in the past ... really kind of ... industry demand for skilled workers. Now the focus is for service industry which requires a good command of English. I think having witnessed all these changes I think it's really time for a change in our curriculum. I won't say it's much to do with the changeover, it's just the change in the society which 
requires a change in the curriculum and then this sort of task-based learning or task-based approach will be implemented in the secondary schools in two years time. I think there is a need, right, rather than focusing on the exam also take into account students' performance as a form of continuous assessment. All these, I think will be useful; will be helpful to the teaching and learning in our schools. So as I've said, it may not be much related to the changeover.

AU No, I don't think it is, no.

11(99) And I think the effect, it's too early to say, right? Say in the primary schools the TOC has been implemented for four years now. I know there is an interim report on its effectiveness ... so I think I am looking forward to something positive. I think it's a good change. What I mean is if we were to sacrifice a lot, I mean if the existing one is good, is very good, then we might have to sacrifice something, but if the existing one is proved to have been a failure now that the standard of English of our students is going down, and I mean there's no harm done to try out something new. But whether it is effective or not depends on the exam system, I mean the public exam will be changed according to the curriculum. We know there is a backwash effect. If the exam system is going to be changed, then it's a joke. If the curriculum doesn't fit, the exam doesn't fit the curriculum then it will not be effective.

AU Of course there will be pressure on the Exams Authority or the education system from businesses and tertiary institutions to keep the exams system the same, presumably, because it's a system they know and they know if a person's got a grade C or grade B in Use of English, they know what they're getting. That's why I guess you always get opposition to changes in the examination system.

11(99) But I think the curriculum is good, it encourages students to ... rather than just focusing on this pencil and paper thing, students are involved in tasks and that's more related to their daily life. That would prepare students for their jobs more, right? But last month a team from the Education Department came to our campus to introduce the new curriculum and at the end of which someone from the audience asked whether the exam format would change according to the curriculum and the answer was negative, at least at the moment. They don't have any plan to change the exam format because they think that it's already taken into account all these task-based things. It was a bit disappointing I would say.

AU I think that even in a task-based curriculum, at the end of it you still need some measure of the students' ability, or at least whoever is going to take them on after they leave school is going to need that, whether it be business, industry or higher education. 
11(99) But still, is this kind of one-off exam totally reflecting the ability of the students?

AU Oh no, I think anybody in education would ...

11(99) ... add a component, make it a bit at least continuous assessment and taking into account the students' performance in school ...

AU There has been opposition from teachers though.

11(99) Yeah, I know.

AU It just creates a lot of work, a lot of administration to do, and at the same time they still have to do the same things, if they teach, they still have to do the examination system, the SSPA ...

11(99) Well actually we all know we are following the National Curriculum implemented in the UK some years ago, right? Again, there was lots of opposition from teachers.

AU Why do we have to follow a British system, it's not applicable to Hong Kong, that kind of thing.

11(99) Yeah, yeah, it's quite a controversial issue actually.

AU I guess we'll see once it's gone through the entire school system, once we get through the six years then the whole school will be TOC, then beyond that things will have to change, I think.

\section{1(99) Hopefully}

AU The transition period is always the hard part.

11(99) Is it true or rumours, just rumours, that the National Curriculum is going to abandon this task-based thing?

$\mathrm{AU} \quad$ In the UK?

11(99) Yes, I took part in a summer school 2 years ago in Durham and there was a guy from OFSTED, I don't know if you know OFSTED? He said, 'Oh, your Education Department is sending 200 inspectors to the UK to learn this type of task-based learning thing, but we are going to scrap it. I don't know whether it is true or not. 
It's hard, isn't it? Because it was obviously brought in by the last government. A new government comes in and obviously feels different about it. The politicians are new, the civil servants are usually the same and policies change. The government decided to look at education and they decided, well obviously it wasn't working. That's probably the worst aspect of having a parliamentary democracy, change from one government to another and they have ... no stability.

Okay, this affects you more than many other people I've talked to, any changes in English language teacher education as a result of changes which have occurred since the changeover.

11(99) I would say change is related to the change in language policy rather than the changeover.

AU That's what I mean so that's why I didn't say directly as a result of the changeover, the result of other changes.

11(99) Yeah, I'm talking about again the CMI and EMI thing. I would say, it's not obvious yet, but I would say there is a demand and the demand is huge. And courses or modules which can help students to handle the low-banding schools, the demand is so great. In in-service courses, in the pre-service courses, because I am talking specifically this institute, you know we are not taking students who are going to teach in Band 1 schools, you can tell we are producing teachers who nine out of ten can only find a job in CMI schools. So what they need is not something we would offer the ... how to use the communicative approach in an ideal situation, but rather they are acquiring something which can help them survive in low banding schools. To be honest at the moment we are not able to help them to solve the problem. We know the demand is huge, we know that it's important for them to learn how to teach in Band Five schools. Interestingly, in the evaluation, post-TP evaluation again we've received lots of comment on these, 'Goodness me, the things we've learned in there, on the campus, it's useless! It doesn't help me handle these Band Five students.' Still, at the present moment what we can do is to just invite some experienced teachers teaching Band 5 schools and Band Five schools come over and give a talk, give a seminar, give a workshop and that we can do. To be honest, nine out of ten lecturers did not have experience teaching Band Five schools. It's a problem. So we have to do is we have to, not just for this institute, I mean even in universities in the teacher-education programmes, I think there is a demand for ... training courses which can help teachers survive in these middle or low banding schools. In Band 1 schools, you don't need any strategy actually, because those students are motivated without you, a lousy teacher even can teach well. That's what I think.

AU Do you think though if you show student teachers what it's like in Band 5 schools, then they'll decide, well teaching's not for me in that case? 
11(99) So that's one aspect. Another aspect I envisage, that is I don't know if it's the case Polytechnic or what, we're beginning to offer B.Ed. courses and there is a trend that the methods component, I mean the weighting given to the methods component is, comparatively speaking, very little. There's this trend that we are not telling students what to do, we are not spoon-feeding them with the model of a lesson, model lessons, the format of lessons, well you must begin your lesson with presentation followed by practice and followed by application. But there seems this trend that we are encouraging students to explore, to reflect, to think, to behave as professionals. We are enabling them to develop as reflecting practitioners, rather than the past. I'm not sure if that's the right trend, If the students are weak, for example, whether we should at least provide them with some survival kits, right? Personally I believe, take the case of learning how to swim, you just can't throw them into the deep end, at least you have to, you know, enable them to practice ... step by step, things like that, but it seems to me the trend is reflect, you reflect on your own teaching. But if they don't even achieve the threshold level, what can they do? Nothing. Take for example, we've just worked out some of the modules of our B.Ed. English course, and the hour given to methods is just nine hours out of a couple of hundred hours. Over the whole course you can tell how insignificant it is. Nine hours for teaching English, for the methods course, for the methods module, out of three hundred and fifty something. You can tell how people out there think. So methods is not important at all to them. Most of the time is spent on life skills, education psychology, things like that. So the trend is like that. It may not have anything to do with the changeover.

AU Where are these changes coming from, popular or current theories of education or teacher education?

11(99) Popular theories of education

AU There's a lot of this reflective teaching and reconceptualizing the roles of teachers at the moment.

11(99) There has been a lot of research on this, right, and I'm not sure if we can just directly copy their research findings in overseas countries, right? In a way it's grand, but not at this stage, again, these are beginners, they have no knowledge in education or teaching at all. At least I think we should feed them with something for their survival, just like [ ] who devised sort of model of development of teacher development with a couple of stages. I don't think students are able to reflect much in the first stages of ... you know very novice level.

AU Okay, the last question is a very wide one, what do you foresee as the future of education in Hong Kong? 
11(99) Not necessarily to do with language education and all that?

AU Yes, not necessarily

11(99) My feeling is that is the element of marketing is taking centre stage ... the education is as the Education Commission Report No. 7 states that we are moving towards quality education, I would say unlike the past when teachers were seen as holding an iron ball, right? You don't need to change, you just don't make mistakes and you can survive for your whole life and you can keep your job, and I think the future there is very different schools they have to develop. They have to adopt on-going development. To survive you have to develop. If you don't develop, you don't get good students, you don't have to ... you can get only low-banding students, you don't even get enough students, you don't get enough resources and sooner or later you have to close down. I'm not sure if a league table will be established.

AU It has in the UK.

11(99) I'm not sure. 


\title{
Interviewee 13(99): University educator \\ Date: \\ 30 July 1999
}

\author{
AU Researcher \\ 13(99) Interviewee \\ Missing words / Unfinished utterance \\ Pause
}

AU Our first question is regards the medium of instruction in schools, do you think the change in the policy has had any effect on education in Hong Kong in terms of English teaching?

13(99) There doesn't seem to be really any dramatic change regarding the medium of instruction in schools. The dramatical change which has occurred in schools was planned a few years ago. The major change that I just mentioned is the change in policy that most of the schools use Chinese as the teaching medium and about one third of the schools can use English as the teaching medium. For primary schools there hasn't really been a lot of changes. Having said that, there has been small changes and gradual changes. We can see that Putonghua is becoming more important and it has become a formal part of the curriculum in primary schools and secondary schools. Now it's normal that a school has Putonghua in the curriculum and some schools are attempting to have it even at Form 5 level. As for English as the medium of instruction, a lot of schools because of the new policy and political change in secondary schools in terms of teaching medium, two thirds of the secondary schools must use Chinese, mother tongue as the teaching medium, so people are getting more and more concerned with these students ability or confidence in using the English language. People on the one hand feel that it is natural that Chinese be given an important status, at the same time people still keep very strong confidence in the English language and feel very strongly that to succeed in future, whether in career or studies, one must posses a reasonable level of the English language.

AU So you think that, and this comes later, that it hasn't changed people's attitudes towards English? People still regard English as being as important as it was?

13(99) Yes, you're right, but it has got a stronger competitor, Chinese.

AU So Putonghua has now become, has taken over some of the importance of English? 
13(99) Yeah, it has taken over some of the importance and shares a lot of the attention.

AU I think the case is that the change in the medium has only come into effect this past year and so I'm not expecting a great deal of change, as it's only come into effect in the last year and in most schools only in Form 1.

Have you noticed any changes in the situation, status or morale of English teachers since the changeover?

13(99) I guess when we talk about status it's more related with the image of the teacher in front of his or among his colleagues and especially his image in front of the principal and this relates to the extent to which this school has to rely on that particular teacher. To put it in another way, it is related to whether the teacher can do something that most other teachers can't do. In this respect, there doesn't seem to have been a lot of changes and I don't see any significant change in this respect. For both situation and morale, there doesn't seem to be any significant change. In the past two or three years, English teachers are still English teachers; they're still doing the same job. There's been changes in the curriculum but basically the nature of their job ... their role in the school is the same.

AU I'm thinking that a couple of things may have changed the situation and the morale of teachers, even the status, and that is the NET teachers, the introduction of the NET teachers, and the planned benchmarking of language teachers

13(99) It seems tome that both could have some effect but I wonder if the effect could be really significant in the actual operation of the school. For the NET teacher plan, the only difference is that one of the teachers within the school is a native speaker. In a school, normally there are more than 10 English teachers, so there might not really be a lot of dramatical change under such a plan. As for the new benchmarking policy, it seems to me that the system is just going to control the minimum language proficiency of language teachers and that applies to new teachers only. So, in future, if the system is successful, we could be quite confident that the new language teachers will have a minimum level of proficiency. But still the point is for a school to have new English teachers, every year there might be only one English teacher, even less than one. In the past year quite a lot of schools have had zero mobility. The mobility of secondary school teachers and primary school teachers is far lower than the case we had five or six years ago, so the situation is quite different now. The impact still needs to be seen and it seems to me that it will take quite a long time for us to see any impact, especially significant impact. 
AU That's a good point. There was a big turnover about five years ago ... but now the economy is not so good so people are hanging onto their jobs.

13(99) I am on the management committee of six secondary schools, so I am involved in managing six secondary schools. From the reports of the principals, every year it seems to me that quite a lot of the secondary schools, I have a small sample size and within the context of my friends it seems to me that the mobility is very very low in the last few years, especially the last couple of years, it's very difficult to find a teaching job now.

AU For the new graduates yes it is, especially when teachers are trying to hang on even when they're past retirement age. Have you noticed any differences in students' attitudes towards the learning of English? Has the change in the medium meant more emphasis on Chinese, do you think?

13(99) Not really any significant changes, because the education system is still operating in basically the same way and students just react to the system in a certain way. I haven't really observed any significant difference but if I need to mention anything, I guess one possibility, and I say possibility because I would really need some evidence to really say for certain about this point, the possibility is that the low ability group would feel more strongly that even if they give up the English language, it won't cause them too great a harm. Of course almost all students think that English is important, especially when they want to succeed, but the low ability group, they know that they can't succeed very far and there is probably a small tendency for them to find an easy way out. As they know that Hong Kong is no longer a British colony, everything could be in Chinese and fact is that they can survive fairly well without English. If they don't want to go to university, they don't want to be a top student, they can still survive without English. So they might be less motivated in making an effort to learn the English language.

AU I tend to think so too. Despite a certain proportion of the population, parents, teachers and so on telling them that English is important, they are quite realistic and they think that they're never going to get into a situation where they're going to need English, why spend hours and hours struggling over it, when I can spend my time improving my Chinese or my maths? And the change in the medium of instruction is only going to give them more justification. They can say well, they want us to learn in Chinese, perhaps introduce Putonghua as well as a second Chinese language.

13(99) So students may think that even if they cannot get into a local university, they may be able to get into a university in China. In that case they probably don't need so much English. A lot of universities in China are now recognised by the government and the private sector in Hong Kong. 
AU And the general public, how do you think the changeover has affected the attitude of the general public towards English and the learning and teaching of it?

13(99) I think the major impact is the same as I just mentioned and that is there is a very strong competitor. In the past people focused mainly on the English language, now, part of the attention is shared by the Chinese language.

AU Parents, I think, still think that it's important to get their children into English-medium schools. You can tell that by the queues outside the kindergartens.

13(99) The parents is probably an important group. For employers, they are probably the same. They think that English is important in business. At the same time attention is also shared by Chinese, they now think that Chinese is also important, especially for organisations or companies who need to trade with China or do business with China. Or they trade with organisations in other parts of the world, with Chinese. They need to use Chinese. It's quite natural in the past that when a company looks for applicants look at their English language proficiency, some of them even look at the English language proficiency exclusively without looking at their candidates' ability in other languages. Now, most companies will look at English language proficiency and Chinese language proficiency. Take our clerical staff for example. In the past, of course we expect even our clerical staff to have passes in both English and Chinese. Now, we not only require our clericals to be proficient in both English and Chinese, we require them to be able to converse in Putonghua, and to be able to input characters into the computer, Chinese characters into computers. So, the requirements related to language is broader.

AU That's a danger, teachers of other disciplines might see it as a danger that language proficiency just overrides everything else. Okay, the next one is to do with changes in the curriculum or syllabuses for English, whether there have been any, why they've been made and what effect they might have had. I don't think there's been any changes in the exam syllabuses, but there have been some changes in the curriculum, or at least some potential changes.

13(99) I don't really have any close contact with the primary and secondary curriculum.

AU I think the main thing is the coming of TOC, which I think is going to be up to Primary 6 next year, so the new secondary school syllabus reflects the TOC.

13(99) But the change doesn't come from the changeover in sovereignty. 
AU No, it doesn't.

13(99) It's part of the ongoing process of curriculum innovation.

AU But the TOC is based on the system that they use in the schools in Britain, so it came from a British education background and now it's being continued on, so despite the fact that the British have left, we are still getting this Britishbased education system coming through. It will be interesting to see whether it actually succeeds and whether it continues or whether it develops more local or Chinese characteristics.

Number 6, can you envisage any changes in English language teacher education as a result of any of the other changes which have occurred?

13(99) Not really any significant changes. If there have been any significant changes, they don't seem to me to have been related to the changeover.

AU Let's say as a result of the change in the medium of instruction or the benchmarking.

13(99) Not really, I would be interested to find out what are the interviewees of yours have mentioned. There doesn't seem to be any significant changes. I also participate in the teaching of the post-graduate cert. in education at Baptist U. a few years ago. I had groups of English language teachers, teacher students, so I'm quite interested in English language teacher education. It doesn't seem to me that there's been any change in ... perhaps I'm ignorant about this.

AU Well, I think the main thing that's come to my notice is that the change in the medium of instruction will affect it because teachers will now have to be taught through the medium of Cantonese, whereas before most of their teachers were going out into English-medium schools. Whether or not they actually taught through English is debatable but now they have to go out and teach through Chinese medium. Even English teachers are going out to teach in a Chinese-medium school.

13(99) But still we are not talking about the training of teachers for other subjects, we are talking about training teachers of the English language, so those teach they teach the language as a subject, rather than other subjects such as biology or chemistry or other subjects.

AU But I've gone in and I've observed lessons where they're teaching English and they're using Cantonese in English lessons. And when I've said to them, is this okay, how does your principal feel about you using Chinese? Is there any policy? And they say, well they try and encourage us to use English as much as possible but it gives them that flexibility because they know that ... 
13(99) It seems to me that the situation has always been the same. I guess in most of the English language teaching programmes, the instructors will encourage the student teachers to teach through the medium of English, teach the language in the medium of the language to the greatest possible extent but of course there are limitations and there are constraints.

AU But in a real-life situation, they might just have to face their students and ... but it's okay for us to say that, to teach English through the medium of English, but sending you out into schools knowing full well that you won't be able to and so then do we say that we've got to teach you some methods whereby you can use the mother tongue in an English lesson at a minimal level so you keep the students interested in the English language, rather than burying our head in the sand and saying you must use English in an English lesson.

Okay, the last question is meant to cover anything else that hasn't been covered already, what do you see as the future of education in Hong Kong? Any trends or directions in which you can see things going?

13(99) A very big question ... I believe the most observable change in the next decade will be the use of technology in education, so computer-assisted learning, the design of software for teaching and learning English, the use of software for assessment and recording students' progress will become more and more important. Other changes would be gradual, maybe far less dramatic. The TOC is one example, the Government has invested a lot in it to implement TOC. It seems to me if we want to change things in the classroom, we need to have the right people in the classroom. The right people includes the people having the right knowledge, the right mind, the right attitude so that they can do the right things in the classroom. The teachers need to have the confidence to implement the policy. TOC seems to be something that upsets people, at least it upsets me. It seems to me that hasn't really been any fundamental change in the classroom because of TOC. The textbooks have TOC on the cover and claim to be using TOC methods, the teachers and the schools claim to have been using it, but the substance of the lesson, what is happening in the lesson, what the students go through doesn't seem to have gone through the expected changes. This worries me, but I guess it will take time for it to be implemented properly.

AU It's an indictment of teachers to say that they will resist anything that they are not familiar with. Where it involves more work or more administration, teachers don't like that. 
Interviewees 14(99) \& 15(99): Education Officers

Date:

21 June 1999

\author{
AU Researcher \\ 14(99) Interviewee \\ 15(99) Interviewee \\ Missing words / Unfinished utterance \\ Pause
}

AU First of all, how do you think the change in the policy on medium of instruction in schools has affected education in Hong Kong? Or has it, so you think, had any effects, specifically on English teaching?

14(99) Well I think colleagues of our planning and research section, or division, would be able to give you figures on research findings about students' improvements in academic studies and so on and so forth. But obviously, one can generalise to say that if a language is used as the medium of instruction, then the exposure to that particular language will be much greater, when compared to the time that your students can get if it's only taught as a subject. I think that fact we've got to acknowledge. But on the other had we have introduced a lot of measures to try to make up the exposure, to enrich the language environment in those schools which are now using Chinese as the medium of instruction. So I'm thinking of various aspects involved. First of all, the actual data you can collect from the research probably will tell you that academically speaking a lot of students will be able to make much greater progress and at a faster pace in other subjects. On the other hand, there might be the psychological effect on the students which to some may be a welcoming fact that now they don't have to study through the medium of English. To some, they will feel very worried because they think that they might have been labeled as the less able group. Simon can probably top up my response by pointing out the kind of resources we're now putting into the schools.

15(99) Yes, last year the government initiated what we call ... further support measures for CMI schools ... where we gave a grant to all CMI schools who had applied, it was on a voluntary basis, they apply and they can set up their own English enhanced programmes for their intake of S1 students, preferably S1 students. The response was very good, we had over two hundred and forty-odd schools participating, no more than that, four hundred and something schools taking part in that programme. Apart from that, each school had a maximum of, I don't have the exact figures but they can have, they can cater for eight individual groups, each group about twenty-five students each. That of course is extra apart from the timetabled courses, these 
courses can be done either after school, during lunch hour, during long holidays or during the summer vacation, so it was for a whole year and the responses were very good. That was one measure. The other measure was that each school, each CMI school, they were given a sum of thirty thousand dollars to set up an English corner. They could identify a room, it could be something that's mobile, some space in the library or anywhere and it would be stocked with reading materials, software, hardware, to help enhance, to help provide an environment for an English corner. They could also use what we call a language grant. A language grant is also given to schools. For every class, S1 to S3, they're given six hundred dollars per class, and S4 to S7; they're given nine hundred dollars per class. They use that language grant to top up the thirty thousand dollars that they have been given to set up the English corner. So that's one thing. The other measure is that the government, as you can read from the papers, we have recruited what we call NETs, native English speaking teachers to again provide additional help and act as a resource person for schools. All CMI schools are eligible to two NETs. Of course we have what we call reading grants, an extensive reading grant as well, so that schools can use the grant to buy library books for the students.

AU But do you think there've been any changes in the schools themselves, let's say the English medium schools? I would anticipate that very little has changed within these schools, they were English medium before and they're still English medium, but perhaps those schools that have become Chinese medium from English medium, do you think there've been any internal changes in the schools, any changes in attitude or the way that they organise their teaching, other than the extra facilities and help?

14(99) I wouldn't be able to say anything concrete, I must admit, because we have not done any research or studies on actually what has taken place after the switch and one can only use one's common sense to think that there might be those who still want to go back to the English stream and therefore try very hard to push for an improvement in the quality of their students' learning. Those who wish to maintain or switch back to the English medium might take a stronger line in terms of strengthening their own English programmes, adding extra lessons to make sure that the students are going to be able to cope in the future. Then there will be those which are very ready to accept the change and feeling that the students might really gain from the reduction of pressure in learning through a foreign language.

AU There hasn't yet been any actual study of that from observation, from people who have been talking about things and so on, they do say that there is more interaction in the class because of the fact that the language barrier has been removed.

14(99) That would be a very interesting research topic, of what actually happened after the switch. 
15(99) That's what he's doing, isn't it?

AU In a way, yes. I have spoken to people at the Institute of Education who've said that in the past they had witnessed content lessons taught through English and now they've seen them taught through Chinese and the difference is huge. The children are much more motivated and the whole thing is working much better. So I can see that there are schools who are still hoping that they might gain EMI status if there's a review of the situation so they're maintaining a high profile.

15(99) As far as the morale of English teachers is concerned, I think it is even greater now because they are more motivated to teach the language itself because it seems that it's on their shoulders now to ensure that the standard will continue to be as it is, that is there won't be a drop in standards and probably they don't have to unteach a lot of stuff that has been taught wrongly or mispronounced in the other subjects.

$\mathrm{AU} \quad$ In a lot of situations, as you say, they are the sole provider of the English language, or the English department is.

15(99) I think we've answered question three.

AU Pretty much ... the status, situation and morale of English teachers, the status

15(99) Probably as I was saying before you switched on the tape, the emphasis now is even greater as we don't have the stigma of English being a colonial thing now, we recognise the fact that English now is a world language, an international language and in order for Hong Kong to continue it's present status, English plays a very important part as well. I suppose you have read from the papers the interest that the business community has and other areas of the community have in English as well.

14(99) The Secretary of Education and Manpower has come out very strongly now to say that for the whole community there is a need for English and hence they are talking about competency of trade practitioners in terms of English standards, so there is a movement in this community about English learning and the importance to be attached to English. So status wise it hasn't dropped at all. It has an even higher profile!

AU An even higher profile, yes. And looking at the students' attitudes towards learning, how ... also, from question four the general public, by the general public we are mainly talking about parents. Have their views sort of kept in step with what we were saying, that English is no longer seen as the colonial language, they are coming to realise that it's the world language. 
15(99) I think with parents, the importance that they attach to English has always been there, it hasn't diminished at all. This is only an observation but if you have followed the media closely you will probably have realised that there are a few who are now saying that Hong Kong cannot keep up with its English standard or in terms of producing people who can speak fluent English and very soon we are going to lose out because major cities in Mainland China are producing people of high quality, including their command of English and obviously this sense of crisis is coming along and coming along very strongly among the business sector. Parents will get that message as well.

$\mathrm{AU} \quad$ And looking at it from that point of view that parents still, I think, want their children if possible to go to an EMI school. Can you see that, there's still this big demand for an English-medium education?

14(99) I think gradually the parents are now seeing that not really the name EMI or CMI but whether English is being taught effectively, the children can actually learn English effectively. We don't know when the policy will change again. Pardon me for saying that, I'm not gazing at a crystal ball but things will change and maybe the distinction is not that important any more. But okay in the past, parents might prefer sending kids to EMI schools only because they were under the impression that EMI schools would be able to give lots of exposure to the children and therefore they can learn better.

AU Do you think parents are becoming better educated now as regards the best learning situation for their children? Do they now see that if their child doesn't have the capability to learn through the English medium that they are better in a Chinese-medium school?

14(99) We saw that opinion being expressed in our medium, in the Chinese press, so gradually they are.

AU So whether it's English medium or Chinese medium, for the so-called famous schools, you still see the long lines of parents queuing up to register their children into the good kindergartens.

14(99) It's very interesting. The reason there is coverage in the Chinese media that there is one parent that made a complaint to the media about the English books being used in the prestigious school that her daughter registered. It is interesting because obviously she has chosen the school for the child knowing what sort of programme she is going to get and now she is finding that the English programme is too demanding for her daughter. So, you always got that sort of people, who are queuing up not knowing what they are going to get or knowing what they are going to get but still won't be satisfied 
AU This next question is probably up your street a little bit, any changes in the curriculum or syllabuses for English since the changeover. Why do you think they have been made if they have been and what effects you think they might have?

14(99) As I was talking to you at the very early moment when we met, I think it's just coincidental because as I was explaining to you, any changes to be made to the curriculum or the syllabuses takes a long period of time and the thought probably began when the committee members saw the need for revision rather than really because of the changeover. Yes there are changes but they are not because of the changeover.

AU I'm looking since the changeover rather than because of the changeover, within the last couple of years. I know that there is a new syllabus for secondary schools.

14(99) The one for the primary was issued in 1997, which was an amalgamated one because in 95 we had the programme of studies for TOC. We incorporated the principles of TOC into our 97 version, including other teaching suggestions. Then the secondary syllabus, the current one was issued in 1983 and again was long due to be revised. So we have now a version being printed by the Government Printer, due to be sent to school in October this year. So this is a 1999 version, which actually uses or has kept the framework for the primary syllabus and then stretching it up to the secondary level. So that's the new development and we are seeing very good response from the teachers about these changes and we are also bringing in new elements like, highlighting the experience dimension. One of the purposes of learning English or learning a language is being able to respond and express one's view, one's experience, or towards other people's experience, you try to respond to other people's experience. And the cultural element will come in obviously because if you choose texts, no matter whether spoken or written, about other cultures, other people's ways of life, then elements of culture will probably come in.

AU And the secondary syllabus, which seems to me, not as a layman exactly but it seems that the influence of the TOC is being anticipated there and that will come in in a couple of years. And the examination syllabuses so far haven't changed.

14(99) No, they haven't announced any changes because every time we ... when teachers ask whether there will be any changes to go along with the new secondary school syllabus and they were saying that if there is any need for changes, it will be reviewed in due course, which probably will mean 2004. But, they are now under such a big pressure of reform; the public examination system has been looked at very closely by consultants and other 
people that one cannot predict what is going to happen except that we know that they are going to change.

AU And, any changes in English language teacher education? I don't know if you'll be able to express any opinion on this.

14(99) Not really, the I.Ed. people and the Hong Kong U people.

15(99) Well they do look closely at our syllabus as teachers will be going into schools and making use of our syllabus because they will be training our students towards that end. I think where English teachers are concerned there will be more emphasis on the competency of the English language teachers as I think there will be more emphasis on the fact that teachers will be subjecttrained. We can look forward to that.

14(99) And the benchmarking.

15(99) And the benchmarking, yes. Teachers will be benchmarked.

14(99) Have you been in touch with those people who designed the benchmark?

AU I took part in the pilot scheme. I was an oral examiner. So that is obviously going to come in and the wish expressed by the Chief Executive for all English teachers, for all language teachers to be subject-trained and degree holders eventually. So a general increase in professionalism of the profession is the trend.

And the last question is what do you foresee as the future of English in Hong Kong. I know it's a very wide question but the intention is to ...

15(99) I think we can narrow it down to languages.

AU $\quad \ldots$ is anything that hasn't been mentioned already.

15(99) Well I would hope and I would like to see that be more student-centered. The profession will be more professional as you said. Teachers will be more proficient and they will be trained, degree holders and that they will be able to train students to be able to have life-long learning. It's not just information in isolation but be able to find ways of looking for information. They will be, what's the term, they will be IT ... what's that?

14(99) Supported in language learning?

15(99) No, literate, IT literate, I'm getting lost for words. That sort of thing. 
14(99) I would like to see language teachers become really student-oriented, rather than just thinking that a certain way of teaching might help. They need to have action research in their own classroom to verify their belief and align their belief, where's their action. There's such a big gap between their action and their belief and sometimes they might think that they are doing the good things for their students but actually the effect is not there.

AU It's an interesting thing you say about action research, I think a lot of school teachers feel that research has nothing to do with them. Research id for researchers, I'm a school teacher, I go in and teach and that's it, rather than the two marrying together and feeding into each other. I think a positive thing is that there are more and more in-service and more and more courses, BA courses in TESL and MA courses that can make serving teachers more aware of different aspects, and as you say, let them explore their beliefs and bring things into the classroom that otherwise they might not do. A lot of the time they are under so much pressure...

15(99) Yes having said that it's ideal that they could do action research but we also have to take into consideration the workload of teachers.

14(99) But I think action research, the term, sounds very demanding for local teachers, especially when it's translated into Chinese. In fact you don't really need a huge, or large scale one. A small one like analysing the learning style of your students within a class and then trying to match your teaching style with at least one or two, if not all of their learning styles and then you're helping more than the imagined average ones in your class.

AU Perhaps if you have one teacher within each department whose brief it is to do something about that, to encourage action research or to encourage teachers to do extra training ...

14(99) Reflection? If not large-scale research.

AU Because I find that in Hong Kong, compared to when I taught in the UK, that teachers very rarely meet together. In the UK I used to find that teachers would have regular meetings just to say, where are you up to in the syllabus, what have you been doing, what sorts of methods have you used that have been successful? It doesn't seem to happen here, you have a meeting at the beginning of the semester to work out the syllabus and who's responsible for this and this and this and you have a meeting at the end to sort out the examination results. There doesn't seem to be regular meetings to share ideas.

14(99) The workload is a problem. The timetabling can help, if a school really wants to encourage that trend. We have witnessed at least one school trying to do that by making sure that there is a common free period among the teachers teaching the same level, so that they can sit together and talk. You can't 
expect teachers having different free periods and be able to squeeze their lunchtime, which is already used for meeting students and be able still to talk shop.

AU And there's more and more subjects being introduced and putting pressure on the timetable, there's IT, there's Putonghua, there's lots of things coming in. And as a last point perhaps, the idea just mentioned this week of abolishing the certificate examination and having four-year degree courses and having students matriculating in Form Six.

14(99) I went through that system.

AU And then went to CU?

14(99) Yeah.

AU And you found that to be ... good?

14(99) Well I haven't been able to experience the other way, let me put it like that, I haven't been through the two-year matriculation course so I don't know what I have missed, but certainly I survived. It's still in the consultation stage, we have to wait and see what the public can accept. Today's paper carries coverage, like, no yesterday's ... there are school heads and sponsoring bodies who are worried about that sort of suggestion.

AU Worried about it? But it would take students away from the school perhaps and free some teaching hours for the teachers then to spend more time on other things.

14(99) They are thinking perhaps of what if students want to exit at a certain point and now they have to wait until the end of the sixth year, rather than the fifth year. When you say that there might be more time for certain things or more manpower for smaller classes, that sort of prediction is premised on the assumption that there won't be any cut in manpower, in resources and so on. A lot of other things will come in. 


\section{Interviewees 16(99) \& 17(99): Native English Teacher and English teacher in international school \\ Date: 2 October 1999}

\begin{tabular}{ll} 
AU & Researcher \\
$16(99)$ & Interviewee \\
$17(99)$ & Interviewee \\
$\ldots$ & Missing words / Unfinished utterance \\
\hline & Pause
\end{tabular}

AU Let' start with the first question, how do you think the change in the Government's policy on medium of instruction in schools has affected education in Hong Kong.

16(99) Okay, first of all I think there's a lot of money being spent and in particular there's a lot of money being poured into CMI schools because the Government is out to prove that CMI can work. Just to give you one simple example, in the case of NETs, I don't know if you know this yet, but starting next year there will only be one NET in each EMI school and there's going to be two in the CMI schools. So like in our school, one person has to leave. That's just one example. According to the principal, I had a very long chat with her, that's just one example of how the Government is pouring money into CMI schools. I think that the EMI schools are in a huge panic because they have to prove that they are good enough to be EMI, as is the case in our school, everybody is on pins and needles constantly this year especially and everybody has been told outright, speak English or leave. That's what's happened, more than ever, and of course we're having ... the teachers are being checked more and more by the superiors. Up until last year, nobody ever walked into my classroom, or anybody else's and now they're having regular checks on classrooms, going in and watching teachers teach and telling teachers that this year they will walk in on their classes any time that they wish, without notice, and other teachers will be allowed to walk in on their classes as well. So just sort of more, coming down from the top. Also, everything is being checked, textbooks are being checked, notebooks are being checked, every book that the students own is being collected twice during the year, just so that they can be checked to make sure that everything is up to par. It's a huge logistic nightmare. At the end of last year, every textbook was collected, absolutely everything.

$\mathrm{AU} \quad$ Is that in all subjects?

16(99) All subjects, not just in English and Chinese anymore, everything, and they go through it with a fine toothcomb, and they give you the feedback and tell 
you what's not happening. As a result of that this year all the teachers are working harder. Everybody is working harder, everybody is stressed, because every individual is now responsible for proving that the school is up to par.

17(99) They're probably concerned about their own job, too.

16(99) Yes, and everybody's been given ... in the English Department we've been given more work again. Now we have to do more compositions and we have to do more of everything and more things are going to be checked, so ... but not just among the English teachers, all the teachers in the school. So, they're definitely feeling the pressure. Here's another example, there are some teachers in our school that over the past year have clearly been studying English and improving their English outside of the school, clearly. There are some teachers whose English was so bad before and now suddenly when you hear them speaking in assembly, their English is so much better. You know that they've obviously been studying. What else? Status-wise, I think it's a bit of a mixed bag, at first everybody was sort of into the Chinese, but now ironically I suppose the Government doesn't mean for it to be this way but it is in fact, the status if the EMI schools is actually going up.

17(99) Is that question two?

16(99) Oh, I'll shut up.

17(99) It's all right. I just want to say that, for question one, it's clear that there is a certain demand for English schools, since the schools are full, right, so as the schools are changed to Chinese medium, there will still be a certain population that will want to have their education in English and their only other option is to go to an international school. I'm assuming, I don't know if this is true in all cases, I'm assuming that the international schools are more expensive than the regular public schools.

AU Well the public schools they just pay a nominal fee.

17(99) If that's the case, having an education in English will become an elitist thing, because only the people that can afford it will go, seeing that there are fewer schools. They'll be forced to go to the international schools and the international schools can only accommodate $\mathrm{x}$ number of students at a time. So a lot of people that want to have an education in English will not be able to go because the schools won't be there and the ones that can go will probably be the ones that can afford to go to the international school. So there'll be a lot of people cut right out just there.

16(99) I think also that it's causing lots of confusion among parents. Before most of the schools were English and English was important and parents didn't have to think about it so much. Now the Government's saying that CMI schools 
can produce competent English speakers but the parents are sure about that so they're flocking to these one hundred and fourteen EMI schools, but of course they can't all get into those. I think they feel that they should have confidence in the Government and they feel that it should be okay to go to CMI schools but I think they're really not sure yet. And they won't be sure until it continues and it's twenty years down the road.

Well of course the change has only been in effect for one year.

16(99) Exactly

AU And it's only affected Form 1, so it is early days. But as you say, it does affect parents who are putting their kids into school now. If they want that option, English medium, then there is less opportunity.

17(99) Are parents allowed to apply to more than one school? Can you apply say to your school and an international school and also a third school?

16(99) International school maybe but I think with government schools, isn't the entrance exam around the same time? I think logistically it's not possible to apply to a number of different schools.

AU I think it is but there's obviously a limited number of places because the majority of what are EMI schools now, which are the elite schools, have their own primary schools and the number of places that are available is small, like it is at your school, but it is possible. They have to live within the area though, whether it's Kowloon City or Yau Tsim.

17(99) Right. Question number two?

AU Yeah. Thinking of the teachers, any changes in their status, situation or morale?

17(99) At the time of the handover I was a tutor, a part-time tutor and at the time a lot of people left Hong Kong, a lot of people from the UK because their visas were running out. They left Hong Kong and they left a humongous vacuum for home tutors so for people who are not school teachers, there's a lot of work out there. So it's a positive thing for tutors at least.

16(99) In the schools, I'd say from a status point of view, in an EMI school, the status hasn't been raised per say, as much as more pressure is put on them to perform. I think all along a lot of pressure has been put on the English teachers for keeping the standard of English up, something that I've always been opposed to. It's something in the EMI schools that I don't understand why so much pressure is put on the English teachers when we're just a fraction of what's going on in the school. So now like I said before, the other 
teachers are being held responsible for teaching in English, but they're still not being held responsible for teaching those girls English. It's still the responsibility of the English teachers, it seems, to do that, which as far as I'm concerned is completely the wrong attitude, But yes, even more pressure put on the teachers, so now, the English teachers and the Chinese teachers have always had more work, but now after school, instead of there being two or three English teachers there until five or six o'clock, there's about eight or ten English teachers staying after school on many days of the week. People feel like they just can't go home because there's just too much to do. So situation wise, yes, more work, more pressure, and the morale is definitely down, definitely down, really big time down.

17(99) Is it because they're overworked or ...

16(99) Because they're overworked and as one teacher put it the other day, I feel like a child, I feel like I'm going to be punished, I feel like somebody's watching me all the time, like big brother's watching me and I'm going to be punished for every little indiscretion. I feel like I have to prove myself, and in her case she's been a teacher for like eight or nine years and it feels like nobody is giving her any credit for her qualifications or her experience or the job that she's doing on a day-to-day basis. Of course I feel a little bit different to what they do because at the end of the day I don't care. What are they going to do, fire me? I don't care, go ahead and fire me, because I know that compared to the other teachers I'm not doing that bad a job. I keep telling them, look, compare yourself to all the other teachers around you, do you really think you're that bad? You're not that bad, they're not going to get rid of you. At the end of the day you are better than ninety percent of the people out there. But they don't see it that way, they have this feeling of inferiority, they feel like someone's just going to come along and fire them because they're not good enough for an EMI school.

17(99) Are they native teachers or ...

16(99) Chinese teachers of English, most people are.

17(99) So they are competing with each other, because they think that eventually somebody's going to get the axe and it's going to be one of them.

16(99) Nobody's going to get the axe, later on the question but the standard of English teachers in Hong Kong is not that high, as far as I'm concerned, there is not that competition out there, they are desperate for teachers. And there are headmistresses who say so. When heads of departments complain about the quality of teachers, the headmistresses will say to them, she's the best I could get. What do you want me to do, there's nothing else out there. So people, they don't have to worry about losing their jobs, they only have to 
worry about somebody breathing down on them and saying, look, you better shape up.

17(99) In that case what are they going to replace them with?

16(99) They can't, that's the point.

17(99) So then there's no need to worry.

16(99) There is nothing to replace them with, especially when the Government won't allow them to have any more foreign teachers. From now on EMI schools can only have one foreign teacher, that's all the Government's going to pay for. The school can get more but of course no teacher is going to work alongside a teacher who's making twice as much as she is on a local contract. It's not going to happen, not these days.

17(99) So the pressure they're feeling is only the pressure to maintain the school as an English-medium school. They're not feeling the pressure for their job.

16(99) They are but they shouldn't be, that's the point. They feel it in so many different ways and it's because so much pressure is put on English teachers in particular. It's up to you to keep this school EMI, if we fail it's your fault. Forget about all the other teachers who are supposed to be teaching their subjects in English and therefore should be teaching kids how to write essays and whatever it is they're supposed to do in English, why is it my responsibility to teach them the English skills when all the other subjects are being taught in English? It's ridiculous, but that's the attitude. Therefore, morale, I would say, is at an all time low, this year especially. It started last year but this year, it started right from day one, everyone's been down down down. We're one month into the term and everyone is tired, really tired and complaining constantly.

AU That's interesting, I've had teachers in CMI schools say that they think that their status has risen in that they are now seen as the source of English in the school and so they are held in some kind of esteem there. And yet you would think that they would suffer that same kind of pressure there, that it's up to you to keep the standard of English high, although we're Chinese medium, we still want these kids to have a decent standard of English.

17(99) They've got nothing to lose.

AU That's it.

16(99) That's it.

AU The feeling is, who cares about English now? 
16(99) I'm sure psychologically, no matter what people say, I'm sure that everyone is thinking, okay, we've switched overnight from EMI to CMI, of course a drop in the standard of English is acceptable for the first five or ten years and after that we'll work our way up again. So they have got no pressure, absolutely none as far as I'm concerned. Whereas the Government has as much as said, we're out to get the EMI schools, we don't want you, so if you want to stay where you are, you bloody well better prove that you're capable of being there. All the pressure is on the EMI schools, believe me, according to the principal.

AU Okay, have you noticed any changes in the students' attitudes towards the learning of English in the last two years.

17(99) If I may, this question does not apply to me as I teach Japanese kids so I'm going to pass the buck to [16].

16(99) I think I haven't ... the first year, right after the handover, I think it was part and parcel of the handover fever and everybody was of course pro Chinese in some ways, I mean pro Chinese language in particular. So immediately after the handover I think I noticed sort of more ... less status for English and they were more proud of their Chinese, so to speak. But it quickly changed over, it really quickly changed over because the Government of course said you are changing to CMI but of course you must not forget that English is still the international language and you will still need English to survive in Hong Kong in the business world and so on and so forth. Although there was that initial euphoria of, yes we are Chinese and we want Chinese, it quickly swung the other way again, to where people realised that English is becoming more important, so I am really special because I am in an EMI school and every day they are told now, you are in one of the one hundred and fourteen EMI schools, you are lucky, you are privileged, you are special. They are told that. And they feel that as well. So when they write in their journals, they constantly write how lucky they feel they are to be in an EMI school and how proud they are and they must work really hard in order to maintain that right to stay there because they don't want to get kicked out, they don't want to go to a CMI school, because despite what everybody says, it's still a better place to be. So I think that they still consider it a much better place to be, especially now. Before it was, well we're one of the better EMI schools. Now it's, we're one of the EMI schools and we're one of the better EMI schools.

AU It's created even more of an elitism, even more of a gap?

16(99) Even more, yes.

AU There always was, as we know, a gap between the Band 1 schools, the famous, well-established Band 1 schools and other schools, but now ... and that was a sort of unseen thing, wasn't it? It was something people knew but 
didn't really mention, but now it's actually recorded, against everything that was supposed to happen, it was not supposed to happen like that.

16(99) But do you feel that they are now trying to counter that? There's talk of abolishing the band system?

AU Yes, absolutely.

16(99) Logistically I don't know how they'll do it, but it seems to me that if they get it into their heads that they're going to do it, it's going to be just like this and they're going to do it very quickly.

AU Yes, once they agree to do it they'll do it.

16(99) And it'll be what, zoning like in other parts of the world or what?

AU Yes, I would imagine so.

17(99) I don't ...

16(99) They're thinking about abolishing the band system altogether so it'll be like Canada, wherever you live, that's where you go to school. Well that's one option, I don't know how they'll do it exactly.

17(99) With possible exceptions.

16(99) Yeah, but then again I think there'll be a lot of schools that might opt out of it and go private again. That's what I foresee happening. All the higher level schools, like DBS, DGS, there's no way they're going to succumb to such ... I don't think the church would allow it, the church would just fork out the money and set up some kind of private system.

17(99) Your school might do the same.

16(99) Might, my school's ...

AU They don't have any money. They never have any money.

17(99) Well they're coming up with some money to buy computers so they might be able to go private.

16(99) Oh, later on we'1l talk IT, what's happening.

AU Anyhow, more generally, how do you think the changeover has affected the attitudes of the public towards English and the learning and teaching of it? 
17(99) As for the general public, I think the general public is pleased to be told that the English schools will be switched over to Chinese schools must make them proud, must make them happy because basically they're all Chinese and they all like to see their culture and heritage promoted through the school system. So I think on a very base level everybody's kind of happy about it. But, as you said, after the euphoria, I think a lot of people realised that, yeah, English is quite important, especially if you want to be in business and Hong Kong is quite the business city. So I think it's a mixed reaction, I think people are first and foremost happy. Happy that there will be more Chinese-medium schools and they also feel that English is important and there should be a few English-medium schools out there. You can't wipe them out altogether.

16(99) I think I feel the same way you do. I think right after the handover there were a lot of people who ... at that time we were still living in To Kwa Wan, and we would walk around the streets and right after the handover people would kind of make these gestures, oh we're Chinese now, we don't need these British anymore, because they figure every white person walking along the street must be British. And they would make a point of telling you ... ah ha, remember that old man who said to us, ha ha ha ha, 1997! As if to say ... and that sort of feeling was there for a while. And so it should be, as far as I'm concerned. And in the education system, because there are too many bad schools out there and too many kids getting a sub-par education because they were not ... teachers were not fit to be teaching in English and therefore these kids could not learn in English, everybody was getting a really bad deal. It's a good move to switch it over. But of course, like you say we're in that transition period, it's only been one year and so nobody really knows what's going to happen.

17(99) You can sort of predict that over five or ten years, if everybody's in a Chinese-medium school, it's hard to imagine that the quality of English will stay the same, or even improve. It's next to impossible. If everything's taught in Chinese and the only English that you're exposed to is what, three hours a week? How is it possible? How is it possible that the level of English that exists now, how is it possible that actually stays the same or actually gets better? Impossible. So over the long term, if most of the schools are Chinesemedium, it's inevitable that the quality of English will go down. And consequently the new young students that want to go into education, is their English going to be the same or ...

16(99) I don't know that's true because I've got girls in Form 1, and it is a Band 1, 2, 3 school, but I'm teaching girls in 1B and 1D and I'm really amazed at how good their English is.

17(99) But they probably went to an English primary. 
16(99) No, a lot of them didn't. Most of them went to Chinese-medium primary schools. Heep Yunn Primary is a Chinese-medium school, and a lot of the girls that come into ... of course you are getting the best from the other primary schools, I suppose, but still ... of course that's the key, the key to learning English is to learn young. If they can get them in the primary schools and teach them well. That's where you have to start teaching the teachers, in the primary schools. If they can get them there, then it's quite possible for them to go to a Chinese-medium school and come out being quite confident in English.

17(99) You're right, I think you hit the nail on the head there, you have to start them young. If we remember back to the time when we were in Japan, where it would be the equivalent, everything's taught in Japanese, except for English. English is of course taught in English, by and large. If you look at Japan, and the people that go through six or seven years of English education, and yet they can't string two sentences together at all, not even one sentence.

16(99) They don't start until they're in Form 1.

17(99) But that's right. They start in junior high, they're about eleven or twelve years old and then they go through all of high school studying English and some of them actually go to university and study English and yet they still cannot speak. So why would Hong Kong be any different? In ten, twenty years from now, it'll be just like Japan. People will go through school taking English courses for six or seven years and the end result will be, probably the same as Japan.

AU I think it's not quite as simple as you would make out. The idea being that sure now most of the schools, seventy, seventy-five percent of the schools are now nominally Chinese-medium, but I think previous to that schools, even though they were called English-medium, were pretty much Chinese-medium anyway. Before they changed name very little was taught through English.

16(99) Yes, we are an English-medium school but up until two years ago, no one could claim that all subjects in our school were being taught in English.

17(99) It might have something to do with ... it probably has something to do with the fact that Chinese is grammatically similar to English, whereas Japanese is not at all. It might be easier for Chinese people to learn English than it is for Japanese people to learn English, because the grammars are quite similar, or so I've been told.

16(99) But basically I think that the same people who were sending their kids to those one hundred and fourteen EMI schools before ... those group of parents are the same group of parents that still feel that EMI is the best place for their kids. I think there are a lot of parents out there who are happy that it's no 
longer ninety percent EMI, but you've still got that twenty-five percent of the educated population that wants their kids to have that EMI education, and they're getting it! As far as I'm concerned the people who want it are getting it and the people who don't want it aren't getting it.

17(99) You really have to survey them to find out what percentage of the parents actually want that.

16(99) How many people complained right after? When the Government overnight said, we're switching from ninety percent EMI to twenty-five percent EMI, how may people complained? Not that many. There were a couple of schools that lodged a complaint, they wanted to have the Government change their mind. Not that many schools complained really.

AU Not that many, no. Most schools were happy to change over because basically it just clarified things. They knew where they stood, or they know where they are now.

16(99) It was just a couple of borderline cases that showed up in the news, and they got what they wanted in the end.

17(99) You know the reality in her school, it's supposed to be an English-medium school but you know that deep down a lot of the teachers are uncomfortable with teaching their subject in English. They are probably more comfortable teaching it in Chinese. So if that's the case in your school, it must also be the case in a lot of other schools, so the teachers are probably quite pleased with the fact that ...

16(99) Which is one of the reasons why it happened relatively smoothly.

AU What the reality is I think and what I've found is that the majority of teachers are happy teaching their subject through a mixture of English and Chinese because that's the way they've been taught to teach. They were taught to teach in English but when it comes to clarifying or explaining things they switch into Cantonese, and the whole point of the change in the medium is to eradicate this mixed-code teaching ... this situation where you have an English textbook and the teacher's there writing things on the board in English and then reading from the textbook and explaining it all in Chinese, thereby meaning that all the stuff that they're reading and looking at in English is a waste of time.

16(99) Rote

AU Just hit it straight, go for the Cantonese straight off and then you can get . through the syllabus much much quicker and then you can devote that extra time you have on more and more English. So that's the thinking behind it. 
16(99) I think that's definitely what the education system became, it became rote, memory learning, in just about every subject and that's why this whole idea of English is taught by the English teachers developed because in the English and English literature class to a degree that's true, it's the only place where they are forced to actually produce something outside of a textbook. I've quizzed the girls, when they have a test I'll ask them some questions from their other subjects and they'll stand up and they'll reel off gibberish, because they've tried to memorise something from a geography textbook and they can only remember a certain number of words and the rest they fill in and they don't really know how to pronounce them and they stand up and they sound ridiculous! I can only imagine what it looks like on paper, it's just phenomenal, but they get away with it on those test papers. As long as it vaguely looks like what is on the textbook then they get the points for it. What kind of education's that? It's ridiculous.

AU So would you agree that the attitudes of the general public are pretty much as they were ... we were saying that the middle classes will still prefer an English-medium education, if they can afford it or they can get it, is better for their kids?

16(99) Yes, I think the people who are getting it are the people who wanted it all along and the people who are no longer getting it are quite happy not to get it. Of course there are some people in between, but the majority I think are content right now as far as that goes.

AU It depends on their expectations for their kids, if they want ... if they're thinking they might get to university then obviously university is through English medium.

17(99) I would imagine there's a certain number of parents out there who really want an English medium education for their kids, but as there are fewer and fewer schools either A, they can't get in at all or B, they can't afford it. I don't know if these kids would be very vocal or not, maybe they are of the lower classes, maybe they don't feel that they can express that they want their children to go to school in English. Maybe they're not saying anything at all but deep down they probably do.

$\mathrm{AU} \quad \mathrm{I}$ think there are probably a lot of parents like that. Okay, have you noticed any changes in the curriculum or examination syllabuses for English since the changeover?

16(99) No, not in the lower forms. In the upper forms I'm not sure.

AU Well the syllabuses have not changed, the exam syllabuses have not changed. Changes in the curriculum, there is a new English curriculum for Form 1 to 3, which has been through the consultation stage, they released it for ... 
16(99) Do they have a pilot school?

AU Not that I know of yet. I've had a look at it, I have a copy. Basically it looks towards the time when the TOC, Target Oriented Curriculum comes into secondary schools. It's now in Primary 6.

16(99) Right, so we had a talk about that last year, the Target Oriented Curriculum.

AU They change the curriculum about once every fifteen years. so they are looking to when the TOC comes into secondary schools, the target oriented idea.

17(99) So it hasn't affected you yet but it will next year.

AU Can you envisage any changes in English language teacher education as a result of any other changes?

17(99) Like I said earlier on, over time if all the schools are Chinese medium, I find it very hard to believe that the level of English will be the same or better as it is now. So if I could predict, I would say that the level of English for most people will be quite low, or lower than now, so the universities will have no other option than to admit into the programmes students who have lower abilities than they do now.

16(99) That's one possibility, but God forbid that there should be any lower quality than there already is. I would hope that the government will spend more money, not even more money, just concentrate on better quality teaching of teachers, better quality preparation of teachers. I'm not sure that this whole rumour that ... this educational institute, where does this fit into the scheme of things? It's not a degree programme per se, is it?

AU Yes, it will be.

16(99) Oh, will be?

AU Yes, I think from this year. Traditionally it's always been just education certificate, two-year education certificate, and then teachers could go into schools as what they call, C.M.s, Certificated Mistress or Master, but the plan is, or at least what Tung Chee-hwa put in his policy address two years ago, that all teachers would be degree holders.

16(99) So would the Institute of Education produce teachers of all levels, primary and secondary?

AU $\quad$ Yes 
16(99) In that case I wasn't sure whether, I didn't know whether that was a degree programme and how that would fit into this whole scheme. So now that I know that I would imagine that it is all a part of Tung Chee-hwa's plan that he endorse the whole Institute in the first place, obviously because he thought that it would help matters. I'm not sure. I don't know that many people who have come out of these programmes, but ...

AU Well, the Institute was born out of the amalgamation of all the teacher training colleges, which existed already and that was basically where all the teachers came from, if they weren't university graduates. So people who were teaching in schools either came from the education colleges, perhaps doing two years after they finished their A Levels, or they were graduates from Hong Kong $\mathrm{U}$ or the Chinese $\mathrm{U}$, before the other universities were formed.

16(99) But I imagine these English teachers, also in the EMI schools but particularly in the CMI schools, I know that we were saying earlier that their status has been raised, but I'm sure that soon their pressures will increase because they will be in the same boat, they will be responsible for teaching these kids English and the Government expects results. The government's going to pour a lot of money into the system. Hopefully, the Government will also do something to improve the quality of the teachers that are coming out and I don't know how they're going to do that, as you say because they are going to have poorer quality coming in, maybe, I don't know.

17(99) But that might be counter-balanced by the fact that all teachers will have to be degree holders.

$\mathrm{AU} \quad$ And as far as language teachers are concerned, the government will be introducing the benchmarking of language teachers and eventually the benchmarking of all teachers in which all teachers will have to reach a certain level in terms of their language ability to be able to teach.

17(99) That would be nice to see.

AU The raising of standards is something that the government has been paying attention to.

16(99) I think a lot of teachers, because most schools were EMI schools in the past, I think a lot of English teachers were really mechanical about how they taught English in the schools because although pressure was put on them, in their mind it was always, well, I'm just a little part of this whole picture so not too much responsibility either, even though everybody else is expecting it to be from me, I'm not expecting it to be from myself because I'm just one of many teachers responsible for teaching this kid English. So I think it was sort of mechanically pushing things out and I don't know if that's what will work. I don't think it worked in the past and I certainly don't think it will work in 
the future if they are the only ones teaching in the schools. So probably the Government will seriously take a look at how they're educating those teachers and make sure they do their best to produce higher quality and hopefully significantly higher quality teachers.

AU But I also think that a lot depends on the economy as well. We often find that graduates, university or school graduates will think about teaching if the economy is low, if there's no options, if business is bad and they're not going to get a decent career or it looks as it's hard to get jobs in business so they'll give teaching a go. Teaching is secure ...

17(99) A pension ...

$\mathrm{AU} \quad$ Yeah, long holidays and all the rest of it.

16(99) But all you have to do is look at the old girls who come back and teach in our school. They're teachers, they went through our system. They graduated from university and came back teaching in our school and they cannot speak English. They can function in English, yes. They can survive in English, yes. But how did they get a degree in English? How did they graduate from high school in English? You're really not sure about that. Now maybe their written English is a heck of a lot better than their spoken English, and that's all that's required, isn't it?

17(99) That's where the benchmark comes in, to be able to function orally.

16(99) Does that mean you can teach in English? No, it doesn't, does it? So they have to have that as an integral part of the whole system, definitely.

AU Well, the last question is meant to cover anything we haven't said already, what do you foresee as the future of education in Hong Kong?

17(99) Again, I jotted down elitist as the first thing. I think for those out there who really do want education in English, only those who are able to afford it will get it and I think a lot of people will be cut out, basically because they can't afford it.

16(99) You still have twenty-five percent of the schools EMI.

17(99) But it used to be ninety percent, did it?

16(99) Ninety percent

17(99) Now it's twenty-five. 
AU It's been nominally ninety, in name but in reality most of the schools taught through Chinese.

16(99) But their textbooks were still in English, their exams were still in English, so that's the difference. I see a huge transition period coming up. I think all of these questions that you've asked are going to be up in the air for at least the next ten years, at least. You're going to have to finish this in ten years, good luck, because there are so many things going on. The government is making more and more changes, every day it seems there is something new coming up and the latest thing in our school is IT. Last year we went to a ... remember the former vice-principal who was an economics teacher and he has his own cram school over on Hong Kong side that all the girls go to?

AU Yes

16(99) The missus still worships him, like whatever, so one of our in-service days was over at his school and he was showing off all his toys to us. And we all sat there like, yeah? So you want us to drool over something we're never going to have? Why are we here? And we all just brushed it off and forgot about it. This year we come back and we have a staff meeting and in the staff meeting we're told that by next year we are going to have so many computers in the school you just can't imagine. They're going to be computers everywhere, up to the point where by the end of next year every single teacher is going to have his or her own laptop computer and the government is spending twelve thousand dollars per teacher and the whole sort of projector system is going to be in every classroom ... [tape ends]

17(99) ... beautiful computers, which I'm sure cost millions of Hong Kong dollars and they're supposed to help the quality of teaching and learning I suppose, but they can't seem to find enough money to support a second native English teacher in the school, a live, walking, talking teacher.

16(99) Which is a fraction of the cost of the new computers.

AU It's politics, isn't it? It's how money is allocated.

16(99) I don't know if it's just a coincidence that the whole EMU/CMI transition and this IT transition is taking place at approximately the same time, if it's the Government's big smoke screen to cover it all up and not only cover it up but give a different focus to things that are happening, or is it to add to it, to say, look what we're doing, we're catapulting into the twenty-first century. We're spending billions of dollars and we're going to provide your children with the best education possible. That's our policy and how we choose to do it may be controversial but we are doing this for you and we are spending money for you, we are giving you all of these things. So why would people question what they are doing? 
17(99) People are quickly impressed with gadgetry but the point of the matter is that people have been educated and taught for thousands of years without any of this.

AU Well people are taught and educated to deal with society as it exists at that time. There was no need for IT in Confucius' time because no one was using it. But you're going to get computers in every room and projection systems in every room and there'll still be spiders on the wall in the chapel.

16(99) Are you kidding, we have air-cons in the chapel now.

$\mathrm{AU} \quad$ Is that Christian?

16(99) I'm not sure. Things are changing now; you can't imagine how things are changing. Basically I see lots of change in a little bit if time and confusion for at least ten years and things will maybe settle after that. As I see it it's probably a good thing, the EMI thing had to go, it wasn't working. Now the Government is struggling to find something to replace it and of course it will take time because they haven't had control of the education system for a very long time. The Chinese have been educating people for a very long time and I'm sure they're going to come up with something that works and works well.

17(99) Maybe the intention is to keep the better English medium schools English medium.

16(99) Of course that's the intention, yes, and it's happening. That's the way it should be and our school is still on the borderline.

AU It's recognised that there is a need for an English speaking population.

16(99) But not the whole population because they have to deal with the outside world to maintain Hong Kong as an international city.

17(99) But Hong Kong is what it is and some people would argue that it's quite successful. It's an international city already and it's doing quite well on a business level, although there was of course the economic slowdown, but for the Government to change the education system so drastically, the Government must be assuming that there's something wrong.

16(99) They're not assuming, there is evidence, it's a fact, the quality of education for the majority up until two years ago was painfully obviously lacking.

17(99) But the end result, isn't that reflected in society? Isn't that reflected in what Hong Kong is? 
16(99) I think that's what I'm trying to say. The people who are the English speaking people for Hong Kong up until two years ago are still going to be those people twenty years from now because they're still going to EMI schools and they're still getting that education.

17(99) But by doing this aren't you restricting who can enter the business world, by reducing the numbers?

16(99) There will always be that crossover of people who no matter which system they go to, if they really want to perform in the English speaking system they will, they will do whatever it takes to get there.

17(99) The sink or swim school system.

16(99) But this whole thing is not there to help them, I don't think, it's there to save those people who were left to drown up until two years ago, which was the majority of people, at least eighty percent of the population, which was getting a really bad education.

17(99) Because they had A, no interest in English or B, no intention of entering the business world.

16(99) Not even that; think of the majority of people out there who are language idiots. I'm one of them. If the Government had to depend on me to learn Chinese to save its soul, forget it, we'd all go to hell. But that doesn't mean I'm an idiot, I'm an intelligent person ... there are people out there for whom learning another language is quite difficult.

AU I think they've come to realise that there's no point in teaching the majority of people through the medium of English when they're never going to need it. Society has changed because we are now doing more business with the Mainland and more business within Asia and so people can function quite reasonably within just the Guangdong region and all they have need is Cantonese and written Chinese.

16(99) Cantonese and Mandarin, they are spending more and more money on Mandarin, We've got more Mandarin teachers now and more Mandarin classes now. We've got already, overnight Putonghua is going to be a Form 5 exam, is it this year or next year?

AU Next year

17(99) It's about time, Mandarin is the ...

16(99) Mother tongue 
17(99) Mother tongue and it's spoken by more people than any other language in the world and China economically is on the rise. It's a good time to learn Mandarin, definitely.

16(99) My goodness, if they could speak Mandarin and Cantonese and Japanese, they'd be doing really well, wouldn't they?

AU Well, yes, and English.

16(99) And English

17(99) I think that's why the private tutoring schools do quite well, I mean they have a lot of students, and there is a lot of demand for lessons on the side in languages. Japanese seems to be quite popular.

AU There always has been and there always will be. I think parents see them as a place where they can make up for what they might not be getting at school. You look at the kids who go to the tuition schools and they're not the kids from the worst schools, they're the kids from the best schools, they'll do anything to get ahead, anything to get an advantage over the next kid.

17(99) The competition factor is prevalent, isn't it? When I went to school I never really felt competition on that level.

AU And, it takes the responsibility away from the parent to educate the kid. The parent thinks, I don't have time to spend with my child, helping them with their homework, tuition school, I'll pay for that.

17(99) Under the guise of helping them.

AU They can go to the school in the evening. When I come home at eight o'clock after a busy day I want to put my feet up and pay somebody else to ...

16(99) But do you know what, this is just an aside but I was correcting newspaper cuttings, Form 1 and it was just about this topic, tuition schools, and this eleven year-old was giving this very lengthy essay on the pitfalls and the advantages of the tuition school and the pitfalls, she was quite aware that the tuition schools prepare you for an exam and they teach you how to answer a question for an exam, they give you all the common questions on the exam, whereas the schools of course give you an education. And she said you can sit down and memorise all the answers but are you getting an education? No. Thereby the tuition schools have a disadvantage. She also quoted a case and I have never even thought about this and you probably know about it, a tuition school teacher who had somehow got a hold of the English composition topic for Form Five and had given it to his students and told them to prepare and he 
even collected it and corrected it so that once they got into the exam it was ready, and somehow it got discovered.

AU I guess that's Hong Kong. Tuition is a business and you build a business around education and people make money from it and there's a demand so supply will come about.

16(99) Doesn't seem to be a very difficult formula though. If you get all of the exams from the past ten years and you start going through all of the answers to all of those questions. How difficult is that?

AU Well that's what they do in school anyway, Form Five and Form Seven, that's all they do, exam practice. Okay, I think we'll wrap it up there, thank you for your help. 


\title{
Interviewee 18(99): University educator \\ Date: $\quad 5$ October 1999
}

\author{
AU Researcher \\ 18(99) Interviewee \\ ... Missing words / Unfinished utterance \\ - Pause
}

AU Firstly, how do you think the change in the government's policy on medium of instruction in schools has affected education in Hong Kong?

18(99) Well obviously it's affected it very greatly because schools which previously were teaching in Cantonese in a de-facto sense are being forced to bite the bullet and really come down and consider what it means to use the mother tongue as the medium of instruction, what it means in terms of classroom language and also what it means in terms of textbooks and students and so on. And, like so many of the changes which have taken place, you get the feeling that people are not ready, they don't really know what to do. A lot of teachers from my understanding, which is limited because I'm here in the ivory tower, but a lot of teachers do not seem comfortable in using Cantonese as entirely the classroom language, they haven't had training in Cantonese as the classroom language, for classroom interaction. But I would see these as shortterm problems. It's too early to say what the effect will be, in the long term, if it goes on like this, I suppose a major effect will be the strengthening of the elite nature of Hong Kong education. It always has been like that but it will be even more so to come from the prestigious English language medium schools will have all the advantages. That's the danger and I think it's been pointed out. But I think it's too early to actually tell at the moment although there's that feeling around certain schools that they've been labeled second class. It's really your second question, the main change as I see it is in the morale rather than ... it's too early to tell.

AU It is too early, it's only been going one year and from the teaching point of view, only in Form 1, but I was kind of looking to see if there were any sort of trends that have started and I think you have hit one of them with the fact that the EMI schools now are even more elitist than they were before.

18(99) There's certainly that feeling. I think there's some exceptions, there're some very good Chinese-medium schools that have always been Chinese-medium schools, equal to a lot around. 
AU And within the schools what I've been told is that in the EMI schools there's pressure on the teachers, and this probably comes up in the second question, to remain EMI so they don't lose their status.

18(99) On the students and on the teachers, language police to make sure they ... I suppose one way it shows is that it always was difficult to get into classrooms and observe but it's even more difficult now because they feel that you're checking up that they're using English

AU I must admit that as part of this survey I've tried to get hold of school principals to talk to but they won't.

18(99) It's affecting your study as well.

AU The ones that I talked to two years ago don't reply to my messages. A few that I tried on spec are very suspicious, I think, even the CMI schools as well, maybe they feel that they've had enough interference and they see me as perhaps as something else.

18(99) This comes later but there's a lot of worry on teachers, benchmarking is another thing that's frightening them. I don't see a representative sample of teachers but those I do see are unhappy and some in the EMI schools are under pressure ... [ ] one of the BATESL graduates, his school just scraped in to teach in EMI and he's under terrible pressure to make sure they stay English medium. And schools which want to be English medium and are not are unhappy. So there's certainly a problem of morale and as I said there's the problem of the benchmarking, they're angry about it. I must say that I think a lot of it is based on rumour and misunderstanding of what's going to happen. But again it's become faulty because even when they do the right thing they make a mess of it because their propaganda's not very good because they don't explain what they're doing and what they think is all they have to do is issue the ordinance. And these consultations that Anthony Leung has been doing, you know [ ] from Poly U, she's been doing ... we're all here to consult with you and this big platform at the front with all the important people sitting on it and if you want to consult and give your opinion you have to give your name, your institution and come to the front under the lights and the cameras to give your opinion. They've no idea of consultation. So it's not surprising that teachers are worried about their status.

AU Do you think that within Chinese medium schools, schools that are now Chinese medium, that their status may have risen, as they're now seen as being totally in possession of the means to teach English?

18(99) Possibly, I really couldn't comment, all I notice is over the last few years is a rise in the status of primary school teachers, more and more coming though with degrees and going on to do MAs and having more confidence. I don't 
know how widespread it is because we don't get anything like ... do you know, our MATESL used to be one hundred percent secondary school teachers and it's creeping up to thirty or forty percent primary, that's quite a change.

AU Are they people who want to stay in primary or ...

18(99) Possibly they want to get out, but now with these B.Ed .programmes, parttime programmes, B.Ed. or BA, BA, there are more opportunities for them.

AU Within the EMI schools, as we touched on, the English teachers are under a lot of pressure now to keep the standard of English, whatever the ED requires, to keep it up there. I've heard teachers from my old school, which is one of the most prestigious schools, the teachers are really scared because they're worried they are going to lose their job because they are not up to scratch. And even subject teachers within the school, I've been told that subject teachers have been brushing up their English, they've been told, if you can't teach your subject through English then you're out, so they've been going to classes or going home and listening to tapes or something. It has had a side-effect, I think, perhaps not in all schools because as you say there were a great number of schools who were de-facto Chinese medium anyway and it just served to clarify things and get rid of a lot of headaches for them.

18(99) But there's still this pressure to do away with code-mixing and use technical terms in Chinese and things like that.

AU And students' attitudes towards learning English?

18(99) I haven't ... since the handover, you're talking about two years, talking about twenty years, I would say very strong motivation in the seventies, decline and now going up again. That's my kind of feeling. But that's over the long period, it's not ...

AU No sort of reaction towards the changeover, sort of ethno-centristic ...

18(99) Ethno-linguistic vitality as well, to use the technical term, I don't know, I would expect it but I think that was happening anyway. One of the things about the changeover, it's a marker but since the changeover there's been a combination of a lot of things like the benchmarking, like the change in the medium of instruction, none of them are directly related to the changeover but they are part of the general trend during the last years of the colonial ... education policies put in by the colonial government. After all, the change in the medium of instruction, the debate's been going on for years. It's surprising they still haven't got it right, perhaps not surprising, I don't know.

AU You're saying that attitudes towards English did decline? 
18(99) When I first came, it did seem that there was a reaction against English, which I think has maybe gone a bit. You really should go by the research, I mean other research., but it does seem to show increasing ethno-linguistic vitality. I haven't noticed it particularly in the students here.

AU And the general public?

18(99) I haven't noticed a change, but you can tell from the reaction to the change in the medium of instruction that there's still strong feeling that English has value in economic terms, it has capital. I don't think that has significantly changed. What has changed is Putonghua, there's much more recognition of the importance of Putonghua compared with even five years ago, but I don't see that as particularly threatening English.

AU No, you still get parents queuing up over night to get places in the kindergartens.

18(99) The dreadful tutorial schools are still going, well, they're not all dreadful.

AU Have there been any changes in the curriculum or syllabuses for English since the changeover?

18(99) Since the changeover? Again, I don't think the changeover has marked anything. What's happening now is that TOC came in ... before the changeover, wasn't it? And it's being extended to secondary schools I suppose in the teeth of a lot of opposition, next year? So they're the major changes from the teachers' point of view, I think. Of course there've been changes in the UE ...

AU Not since the changeover, and the cert was changed in 95, I think.

18(99) Again, these were changes that have been in the pipeline for a long time.

AU I know that the syllabus for schools, the draft that they released a few months ago, reflects the TOC.

18(99) It's created a lot of unhappiness in primary schools. Again, it's a good idea that doesn't seem to have been very well implemented.

AU My wife is in a primary school and she's said that the ideas are great but it's always a case of do the best you can.

18(99) It's the same with UE, I think all the teachers are having problems with the new format, discussion group and so on. It's probably having a really good washback effect but some teachers really don't know how to handle it. I hope in the long term it will have a good effect and make the curriculum more 
interesting and less controlled by the textbook. But there's got to be a lot of in-service to make it work.

AU Which brings us on to language teacher education.

18(99) Well, I can certainly see changes. One change that is going on right now is that the new I.Ed. diploma, which at a guess is going to be allocated, within a year or so is going to be Chinese medium. As far as I know the Chinese University Dip. Ed. and Hong Kong University Dip. Ed. are English medium and Baptist University do one now, don't they? But my impression is that I.Ed. is going to be the first Chinese medium, which makes sense, I suppose.

AU Yes, most teachers will be teaching in Chinese-medium schools.

18(99) And are they going to build in modules of using Chinese in the classroom ... and they're going to have one on using English in the classroom as well. So I think that's an interesting change, I suspect. Other teacher training courses are going to have to offer at least streams which are Chinese medium.

AU To teach the teachers how to teach through Chinese.

18(99) I really don't know how much of this has been done. It should already have been done somewhere ... but as far as I know all the major teacher-training programmes are in English. So that's quite surprised me. No reason why 1 should be surprised but I am surprised. The other major thing is Tung Chihwa's promise to fully professionalise the profession, so there should be more teacher education and plenty of it.

AU I was under the impression that eventually the target was a fully graduate teaching force.

18(99) Graduate with the postgraduate diploma.

AU So the diplomas that they're offering would be postgraduate diplomas, and the cert. will be phased out and they then just offer B.Ed. courses.

18(99) Yes, so it's a problem for us, we've really got to push to get either a fourth year or a diploma and it's difficult without an education faculty and the Government's clearly decided it's the I.Ed. where they're going to put their money, but there're enough places around.

AU So your graduates will go to the I.Ed. presumably to get a diploma.

18(99) Yes, I'm sure Hong Kong U would be willing to do it but whether we'll get our own ... we might, because there are still large numbers of untrained English teachers out there. 
AU And then add another year in-service to the BATESL?

18(99) Something like that ... it would have to be the same model, I think, it would have to be one year full time or two years part time. It does seem ridiculous to give them two years on top when they've already done so much, but there'll be a big argument that we can't offer sufficient education. At the moment the majors only take between a quarter and a third of the time and two thirds on the minors plus education papers.

AU And the benchmarking, will that have an effect on the ...

18(99) I hope so, I hope a positive one but in the short term they're scared of it. It depends on whether the Government does as it promised, it promised that money would be available to provide in-service courses for teachers who failed to meet the benchmark in any of the areas. In many senses teachers might even fail the benchmark in order to have a week off at the I.Ed. or something. I doubt that. It's also important that the principals don't demand to see all the results. They might, you know what principals are like. But if you're saying you want to have a fully professionalised teaching force, I don't see the objection to benchmarking, so long as it's done sensitively, which it won't be.

AU I took part in the pilot scheme for the oral section and you could see that the teachers in it were worried, even though they knew it was only a pilot.

18(99) Was the one where they have to listen ... the listening test did you do?

AU No, the speaking, they had to read a poem and a piece of prose and have a discussion.

18(99) I marked the reading one.

AU There was a big variation.

18(99) Certainly was, I don't know, I got my package and it seemed to be arranged school by school. I could feel it when the school had changed and the whole ... I mean some people are going to fail, but the whole ... it's not like failing a degree exam or something, it's just saying you haven't reached the benchmark in this area. I think it's very important to get a profile, I mean your oral needs work so you can take some time off to ... that's the ideal situation and if handled well it should be good. And I hope our BATESL students will pass. Because I think the first cohorts are going to be teachers entering the profession, they're going to be the first cohort to be tested. I think it needs to be built in; I think we need to make sure that benchmarking is built into the course. I'm surprised the I.Ed. are not planning to do that. 
AU Prepare them for it.

18(99) Mmm ... and in the long run get the committee to write it themselves rather than the Exams Authority as it is now. I think that's on the cards. But it will be embarrassing if our graduates fail. I don't think they will though, I think they'll reach the minimum pitch. So there are a lot of changes.

AU Potentially, yes.

18(99) But again I would say that they're all things that have been in the pipeline for a long time.

AU The future of education in Hong Kong.

18(99) Long term I think like Singapore we are going to see that the division between English medium and Chinese medium is not a good idea and that they're going to have to come up with some new bilingual education system, or trilingual education system. Maybe like Singapore they'll preserve some elite schools. Do you know the Singapore system, where basically everyone takes English as their first language and their mother tongue as a second language, apart from the so-called elite schools, ten percent, twelve percent, where they take both languages at first language level. That's the theory. I'm not advocating that but I do ... you asked me to foresee what I think might happen. I'm not terribly optimistic, are you? I'm not blaming the government but the colonial government had a kind of engineering approach to education, if there's something wrong you can fix it with some kind of rational plan, which you then kind of impose on everybody. They don't seem to be involving the grass roots in any change or piloting things properly.

AU It's very much top-down, isn't it?

18(99) I guess for a while things will go on as they are ... the elite will still come out of the elite schools, Hong Kong University will still be regarded as the best, whatever the quality of its courses are like. Maybe in the long term what will happen is that finally the universities will get their four years and we'll move over to more like the Mainland pattern, which might be a good idea.

AU Credit-based and credit transfer.

18(99) Yes, and a first year of a more general education to take the pressure off the tertiary. 
In the following pages are copies of written responses to the interview questions sent in by three respondents:

Interviewee 19(99) - English Panel Chair in secondary school

Interviewee 20(99) - Principal in Secondary school

Interviewee 21(99) - Government education officer 


\section{LEARNING TO TEACH ENGLISH IN HONG KONG:}

EFFECTS OF THE CHANGEOVER IN SOVEREIGNTY

Below are seven questions which I would like you to think about and respond to in any way you wish. Should you require more space, please feel free to continue on the back of the form.

1. How do you think the change in the Government's policy on medium of instruction in schools has affected education in Hong Kong?

The number of EMI schools are relatively small, there is keen competition in the entry into EMI schools. Consequently, both parents and students in primary schools are faced with great stress. Some people have the wrong idea that CMI schools are second class. However, students in EMT schools do not feel prestigious.

2. Have you noticed any changes in the status, situation, or morale of English teachers since the changeover?

The status, situation or morale of English teachers have not changed much. However, they would have to put up with the demands in their job: to sit for the Benchmark Examination, to drain for new ideas to apply for the Quality Education Fund, to attend seminars, to go for IT training courses. etc.

3. Have you noticed any significant differences in students' attitudes towards the learning of English since the changeover? If so, what are they?

No. As we are an EMI school, students feel honoured to be placed here, and they, generally, are more motivated to do better. 
4. How do you think the changeover has affected the attitudes of the general public towards English and the learning and teaching of it?

The public still regards English as an important international language. There is not much difference in the learning and teaching of it after the changeover.

5. Have there been any changes in the curriculum or examination syllabuses for English since the changeover? If so, what are they and why do you think they have been made? What effects do you think these have had or will have?

There is no fundamental change in the syllabus but the approach has become task-based and the stress is placed on the practicability of the language, interactive and communicative skills development.

6. Can you envisage any changes in English language teacher education as a result of changes which have occurred since the changeover?

Student Teachers should know American English well because the English used in computer programmes is Americanized and most of the well-established business corporations are US-based. In order to help our future generations to maintain Hong Kong as a major financial and commercial centre, American English has to be taught. English standard has to be raised. Besides, they need to be well-equipped in IT teaching. 
7. What do you foresee as the future of education in Hong Kong?

If the Education Department continues to enforce mother-tongue teaching, English standard will surely drop, and it is very difficult to maintain Hong Kong as a metropolitan city. Students with poor English standard will find tertiary and postgraduate education very difficult, as there is language barrier Unless the Education Department changes its policy of giving financial support and IT resources evenly to all schools, otherwise EMI schools will be greatly in short of provisions that CMI schools. On the whole English standard has to be raised by. effective methods.

Name: (Optional)

School: (Optional) Position: English Panel (Optional)

Once again I wish to thank you for your time and help. Should you have any questions or further comments please do not hesitate to contact me. 
EFFECTS OF THE CHANGEOVER IN SOVEREIGNTY

Below are seven questions which I would like you to think about and respond to in any way you wish. Should you require more space, please feel free to continue on the back of the form.

1. How do you think the change in the Government's policy on medium of instruction in schools has affected education in Hong Kong?

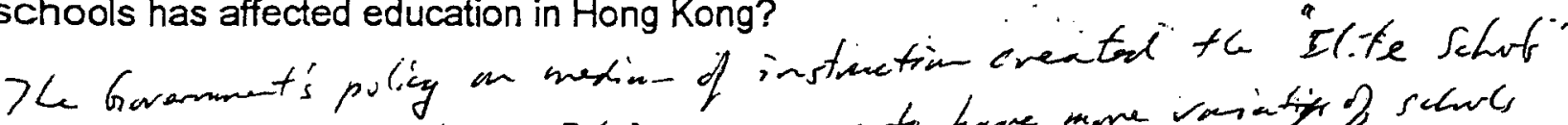
in the education system. It is ne essay to have mo me vaintify of clutch

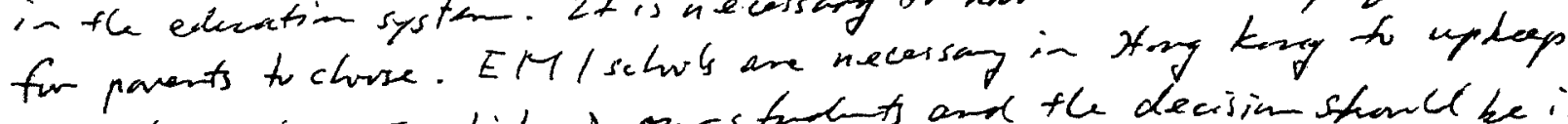

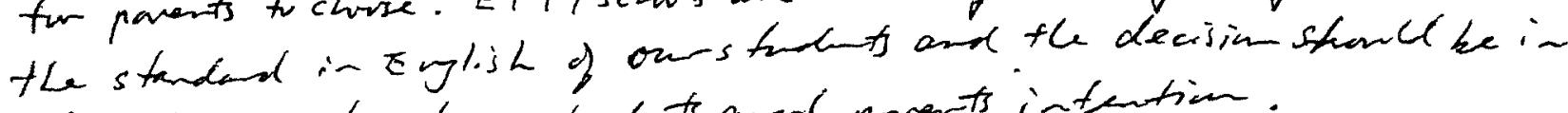
school base on teachers, students and parents intention.

2. Have you noticed any changes in the status, situation, or morale of English teachers since the changeover?

Wo, they keep the same statues andimovale as we are stick using English as our median of instruction.

3. Have you noticed any significant differences in students' attitudes towards the learning of English since the changeover? If so, what are they?

There sears no sijerficiont changes in the i attitudes trans the learning of English. As usual for the hyson previous years, they use Cantmese most of time in tree time out rice classeorsoss

A-359 
4. How do you think the changeover has affected the attitudes of the general public towards English and the learning and teaching of it?

Overly speaking, move parents accept the ideas of using Chinese as medic h of instruction in schorl. But they stich urge th have move practice to

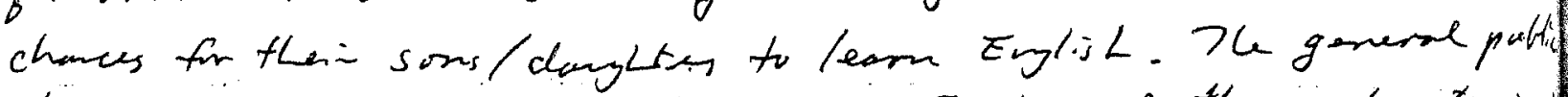

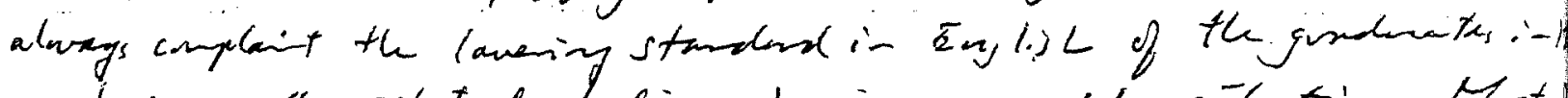
and urge th welted bodies to improve the situation . Mast them, especially those form the middle lager classes of public still thill the English $L$ is an inguntat mediven of communication: future.

5. Have there been any changes in the curriculum or examination syllabuses for English since the changeover? If so, what are they and why do you think they have been made? What effects do you think these have had or will have?

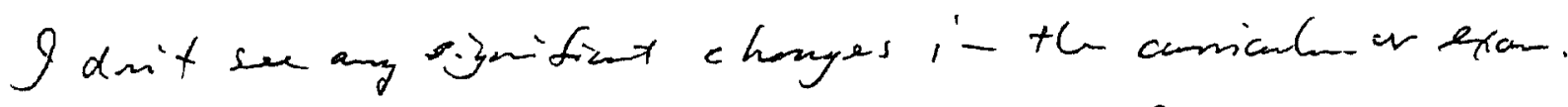

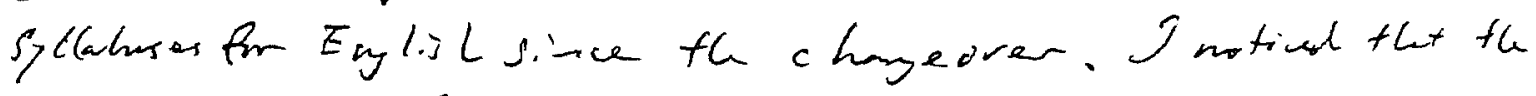
oral examination for both Eolic a nc chinese charged $19 \mathrm{~m}$ not sure what h if is stoptio from $1997 \mathrm{or}$ wot) and the new wry seems better then tu o ll style.

6. Can you envisage any changes in English language teacher education as a result of. changes which have occurred since the changeover?

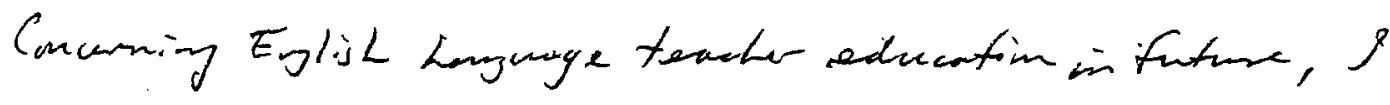
suggest to build up the grammar foundation is primary shul and concentrate move in communication stick, bath

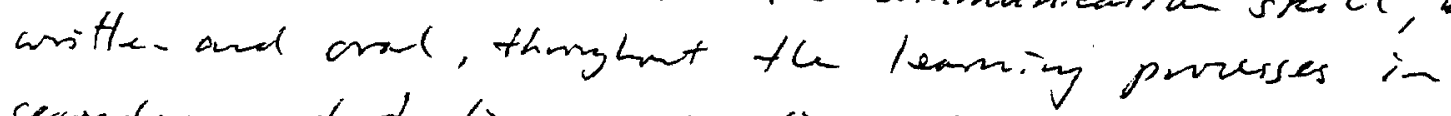

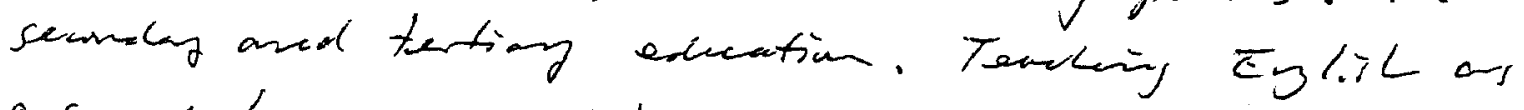
a second language wick became the mari divation after the changeover.

$A-360$ 
7. What do you foresee as the future of education in Hong Kong?

There were inorg eager in the eleatic syst. in HK. since ECRN.l

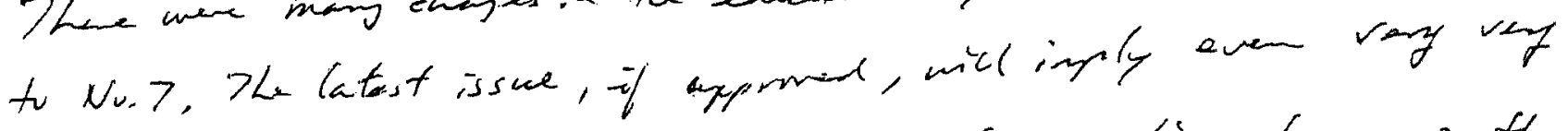
by changes. HK. should prepare a long term poling to move the education system tours national edcectin. At the same tire,

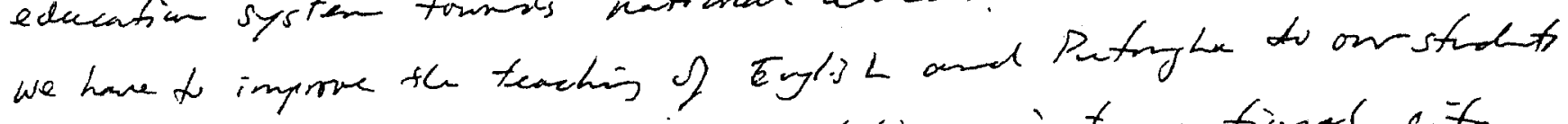
as a any to keep $H^{\prime} k$. a competoxte international c. Fy. I can foresee there with be mary charges i- the elevation system within the fate 10 year but $I$ canst comment until the final version of the latest issue firebird.

Name: (Optional)

School: (Optional)

Position: Primine (Optional)

Once again I wish to thank you for your time and help. Should you have any questions or further comments please do not hesitate to contact me.

Alan Urmston

January 2000

A-361 


\title{
Support Unit for the Standing Committee on Language Education and Research
}

\author{
17/F, Murray Building, Garden Road, Central, Hong Kong
}

\section{FACSIMILE TRANSMISSION ${ }^{1}$}

\section{Dear Mr Urmston}

Thank you for your fax dated 25.6.99. I apologise for not replying earlier but I have prepared some answers to your questions which are indicated below :

(1) The change in the Government policy on medium of instruction in schools has unfortunately split the status of EMI and CMI schools into élite and non-élite schools. The Board of Education and SCOLAR have formed a Joint Working Group to look into the situation with a view to the further implementation of the medium of instruction policy in the next stage. However, recent newspaper reports indicate an acceptance by parents and the public of mother tongue medium of instruction.

(2) I am afraid I am not in a position to answer question (2) as I am not informed about the schools' situation or English teachers.

(3) Again I am not in a position to answer question (3) for the same reason as stated in question (2).

(4) I should like to state my response as a personal opinion and not an official response from the Government. There appears to be a move towards more Chinese within the general public. However, the Government is trying to redress the issue and have adopted measures to improve English Language teaching in schools particularly in Cantonese Medium of Instruction schools within the mother tongue Medium of Instruction policy.

(5) I think this question is best put to [Education Officer - Interviewee 14(99)] or [Education Officers - Interviewees VIII and IX).

(6) There will be forthcoming improvements in the measures to be adopted by the Government such as benchmarking of English language teachers which will impose a standard of English Language proficiency in the teaching workforce and some forthcoming SCOLAR schemes which have not yet been published.

\footnotetext{
'This response was originally sent in by fax and has been retyped as the original has faded and is difficult to read.
} 
(7) I think this is a very big and wide sweeping view of education which may not have much to do with language education.

Please note that my response to the above is given as a personal opinion and not an official view. I apologise again for the delay and shall be happy to answer any questions on the telephone rather than a personal interview. As you can see I am not really in a position to answer all your questions and hope that you will find better luck with my colleagues in the Education Department/HKEA.

\author{
Yours sincerely \\ [Interviewee 21(99)]
}

SCOLAR Support Unit 


\section{A.9 Tables of Results of Interview Study}

Table 3.1: Issues and organisations reported on in the English language newspapers in 1997

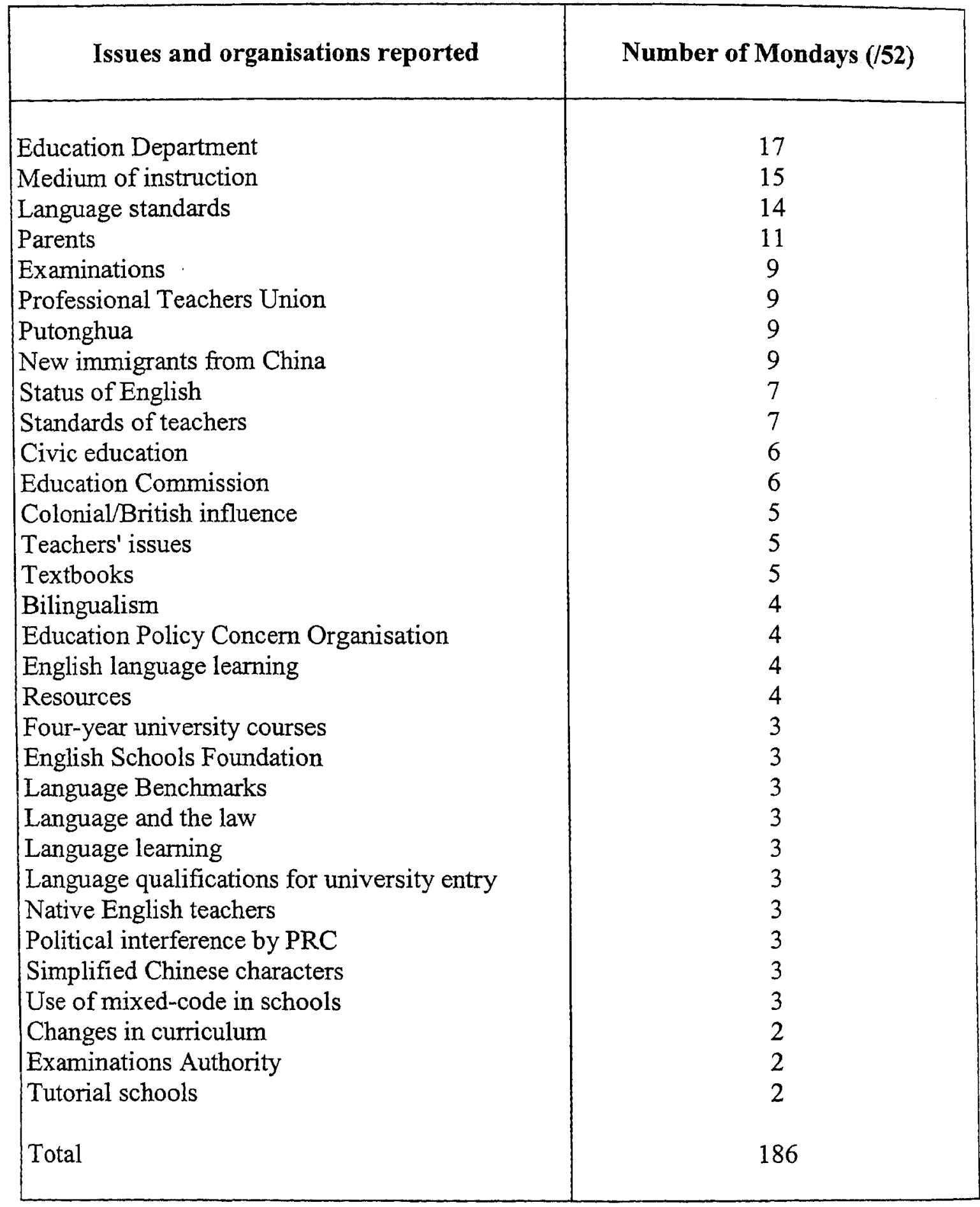


Table 3.2a: Issues and organisations quoted in Hong Kong English language newspapers on Mondays in 1997 (January* June)

Language standards (8)

Education Department (7)

Medium of instruction (6)

Parents (5)

Putonghua (5)

Teachers' issues (5)

Civic education (4)

New immigrants from China (4)

Status of English (4)

Bilingualism (3)

Education Commission (3)

Colonial/British influence (2)

Education Policy Concern Organisation (2)

English language learning (2)

English Schools Foundation (2)

Four-year university courses (2)

Language Benchmarks (2)

Language learning (2)

Language qualifications for uni. entry (2)

Professional Teachers Union (2)

Simplified Chinese characters (2)

Standards of teachers (2)

Textbooks (2)

Use of mixed-code in schools (2)

Changes in curriculum (1)

Examinations (1)

Examinations Authority (1)

Language and the law (1)

Political interference by PRC (1)

Resources (1)

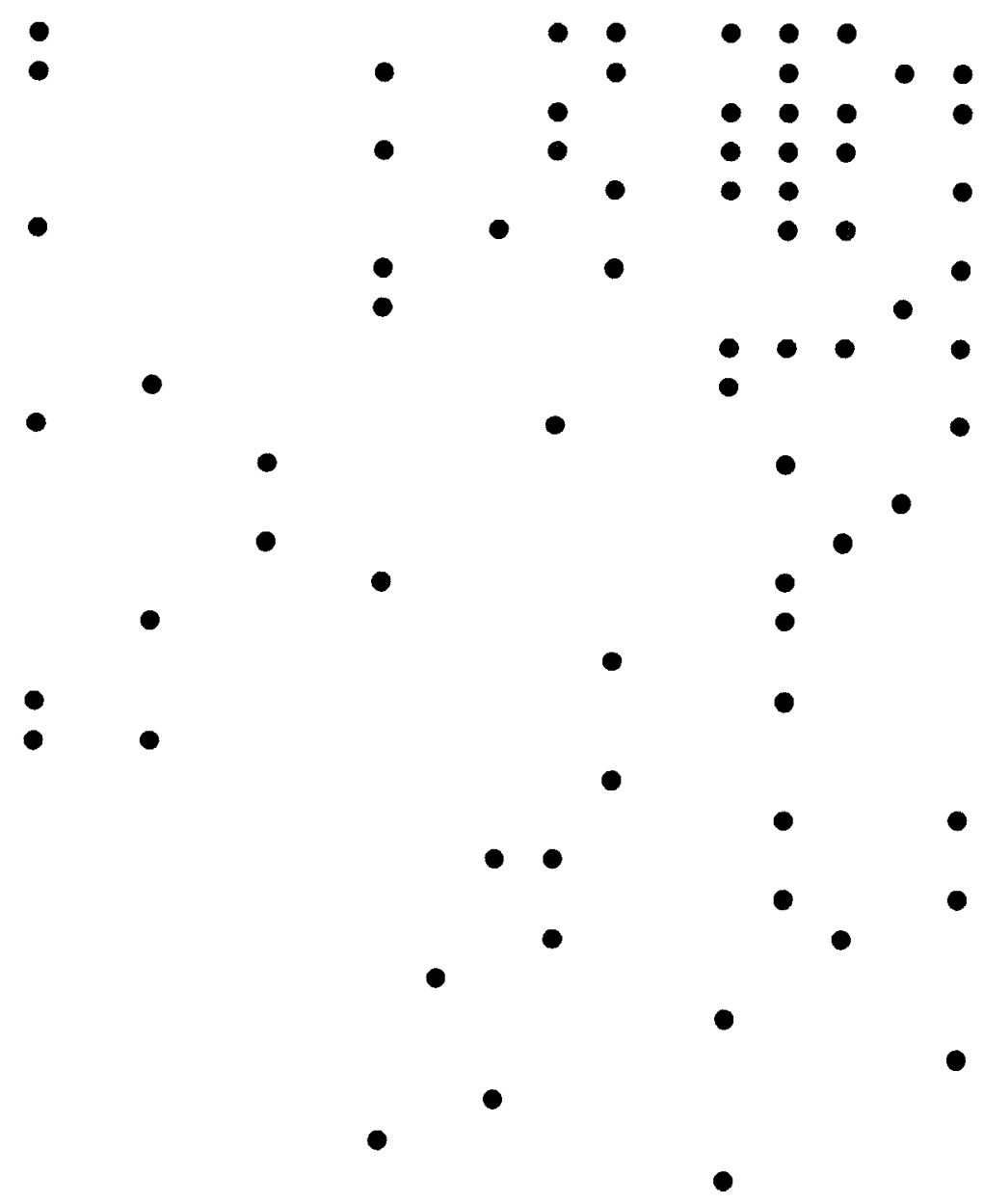

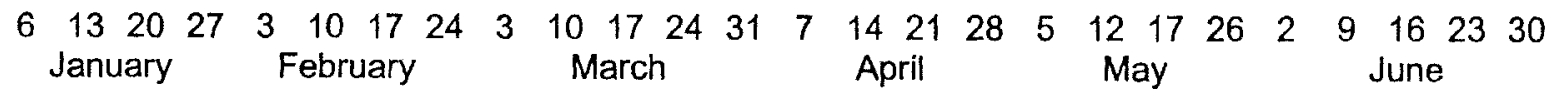


Table 3.2b: Issues and organisations quoted in Hong Kong English language newspapers on Mondays in 1997 (July December)

Education Department (10)

Medium of instruction (9)

Examinations (8)

Professional Teachers Union (7)

Language standards (6)

Parents (6)

New immigrants from China (5)

Standards of teachers (5)

Putonghua (4)

Colonial/British influence (3)

Education Commission (3)

Native English Teachers (3)

Resources (3)

Status of English (3)

Textbooks (3)

Civic education (2)

Education Policy Concern Organisation (2)

English language learning (2)

Language and the law (2)

Political interference by PRC (2)

Tutorial schools (2)

Bilingualism (1)

Changes in curriculum (1)

English Schools Foundation (1)

Examinations Authority (1)

Four-year university courses (1)

Language Benchmarks (1)

Language learning (1)

Language qualifications for uni. entry (1)

Simplified Chinese characters (1)

Use of mixed-code in schools (1)

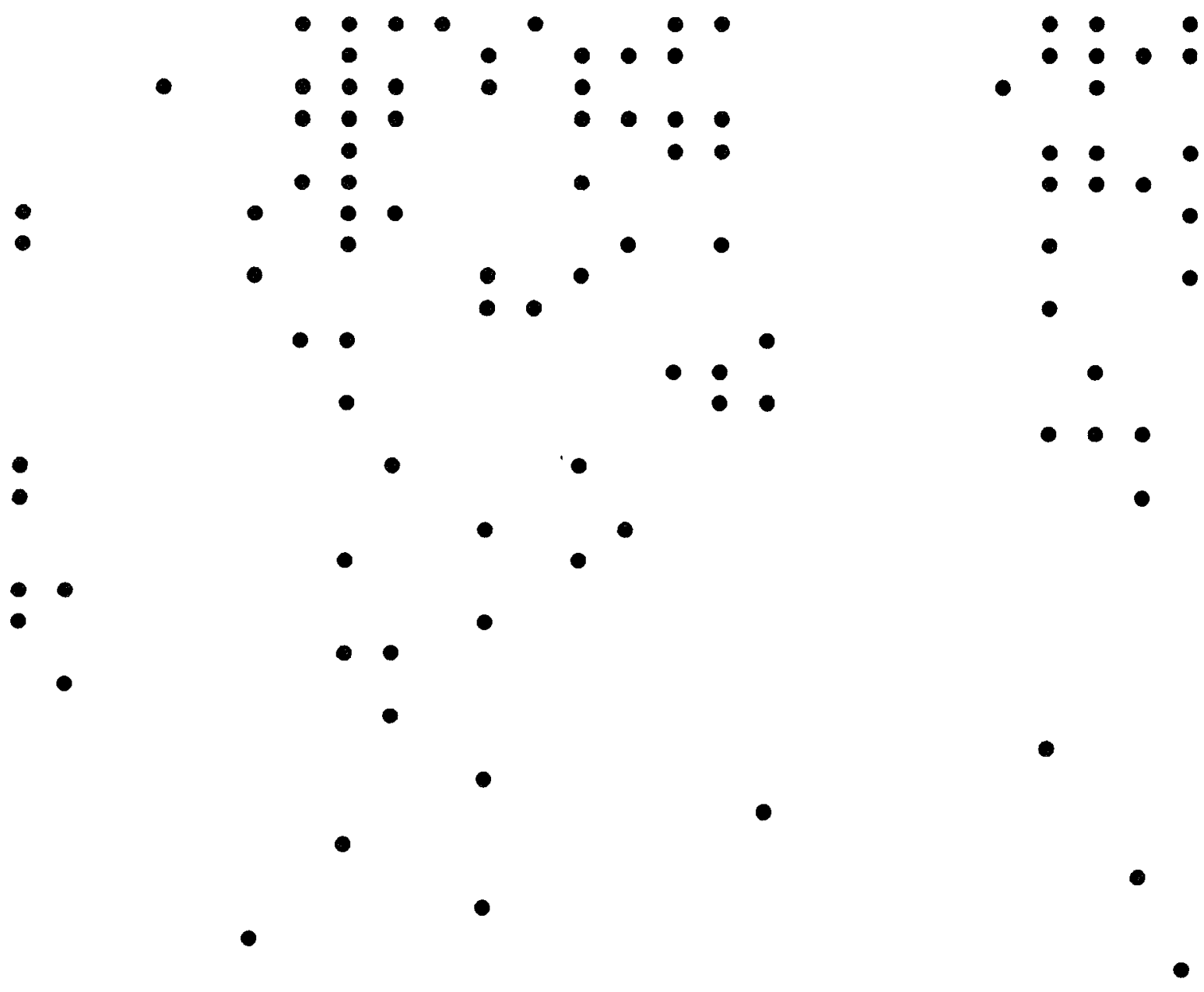

$\begin{array}{llllllllllllllllllllllllll}7 & 14 & 21 & 28 & 4 & 11 & 18 & 25 & 1 & 8 & 15 & 22 & 29 & 6 & 13 & 20 & 27 & 3 & 10 & 17 & 24 & 1 & 8 & 15 & 22 & 29\end{array}$ July August September October November December 
Table 3.3: Comparison of issues/organisations reported before and after changeover of sovereignty on July 1 st

\begin{tabular}{|l|l|}
\hline \multicolumn{1}{|c|}{ January - June } & \multicolumn{1}{|c|}{ July - December } \\
\hline Language standards (8) & \\
Education Department (7) & Education Department (10) \\
Medium of instruction (6) & Medium of instruction (9) \\
Putonghua (5) & Examinations (8) \\
Teachers' issues (5) & Professional Teachers Union (7) \\
Civic education (4) & Language standards (6) \\
New immigrants from China (4) & Parents (6) \\
Status of English (4) & New immigrants from China (5) \\
Bilingualism (3) & Standards of teachers (5) \\
Education Commission (3) & Putonghua (4) \\
Colonial/British influence (2) & Colonial/British influence (3) \\
Education Policy Concern Org. (2) & Education Commission (3) \\
English language learning (2) & Native English Teachers (3) \\
English Schools Foundation (2) & Resources (3) \\
Four-year university courses (2) & Status of English (3) \\
Language Benchmarks (2) & Textbooks (3) \\
Language learning (2) & Civic education (2) \\
Lang. qualifications for uni. entry (2) & Education Policy Concern Org. (2) \\
Professional Teachers Union (2) & English language learning (2) \\
Simplified Chinese characters (2) & Language and the law (2) \\
Standards of teachers (2) & Political interference by PRC (2) \\
Textbooks (2) & Tutorial schools (2) \\
Use of mixed-code in schools (2) & Bilingualism (1) \\
Changes in curriculum (1) & Changes in curriculum (1) \\
Examinations (1) & English Schools Foundation (1) \\
Examinations Authority (1) & Examinations Authority (1) \\
Language and the law (1) & Four-year university courses (1) \\
Political interference by PRC (1) & Language Benchmarks (1) \\
Resources (1) & Language learning (1) \\
& Lang. qualifications for uni. entry (1) \\
\hline Simplified Chinese characters (1) \\
All issues/organisations (86) & Use of mixed-code in schools (1) \\
\hline
\end{tabular}


Table 3.4: Language issues in the English newspapers on Mondays in 1997

\begin{tabular}{|l|c|c|c|}
\hline \multirow{2}{*}{ Language Issues } & \multicolumn{2}{c|}{ Number of Mondays mentioned } \\
\cline { 2 - 4 } & Jan-Jun & Jul-Dec & Jan-Dec \\
\cline { 2 - 4 } & & & \\
Medium of instruction & 6 & 9 & 15 \\
Language standards & 8 & 6 & 14 \\
Putonghua & 5 & 4 & 9 \\
Status of English & 4 & 3 & 7 \\
Bilingualism & 3 & 1 & 4 \\
English language learning & 2 & 2 & 3 \\
Language Benchmarks & 2 & 1 & 3 \\
Language and the law & 1 & 2 & 3 \\
Language learning & 2 & 1 & 3 \\
Lang. qual. for uni. entry & 2 & 1 & 3 \\
Simplified Chinese characters & 2 & 1 & 71 \\
Use of mixed-code in schools & 2 & 1 & \\
& 39 & 32 & \\
Total & 39 & \\
\hline
\end{tabular}


Table 3.5: Educational issues in the English newspapers on Mondays in 1997

\begin{tabular}{|l|c|c|c|}
\hline \multirow{2}{*}{ Educational Issues } & \multicolumn{2}{|c|}{ Number of Mondays mentioned } \\
\cline { 2 - 4 } & Jan-Jun & Jul-Dec & Jan-Dec \\
\cline { 2 - 4 } & & & \\
Examinations & 1 & 8 & 9 \\
Standards of teachers & 2 & 5 & 7 \\
Civic education & 4 & 2 & 6 \\
Teachers' issues & 5 & 0 & 5 \\
Textbooks & 2 & 3 & 5 \\
Resources & 1 & 3 & 3 \\
Four-year university courses & 2 & 1 & 2 \\
Changes in curriculum & 1 & 2 & 2 \\
Tutorial schools & 0 & 25 & 43 \\
Total & 18 & & \\
\hline
\end{tabular}

Table 3.6: Socio-political issues affecting English language education mentioned in English newspapers on Mondays in 1997

\begin{tabular}{|l|c|c|c|}
\hline \multirow{2}{*}{ Socio-Political Issues } & \multicolumn{3}{|c|}{$\begin{array}{c}\text { Number of Mondays mentioned } \\
\text { (n=8) }\end{array}$} \\
\cline { 2 - 4 } & Jan-Jun & Jul-Dec & Jan-Dec \\
\hline Colonial/British influence & 2 & 3 & 5 \\
Political interference by PRC & 1 & 2 & 3 \\
Total & 3 & 5 & 8 \\
\hline
\end{tabular}


Table 3.7: Composition of interviewees in 1997 and 1999

\begin{tabular}{|l|c|c|}
\hline \multirow{2}{*}{ Position of Interviewee } & \multicolumn{2}{|c|}{ Number of Interviewees } \\
\cline { 2 - 3 } & 1997 & 1999 \\
\hline Senior position within teacher education department & 5 & 2 \\
in tertiary institution & 4 & 4 \\
English teacher & 3 & 5 \\
Senior position within language department in & 3 & 3 \\
tertiary institution & 2 & 1 \\
Subject Officer (English) in Exams Authority & 2 & 1 \\
Panel chair (English) & 1 & 2 \\
School principal & 1 & 2 \\
Education Officer (English) in Education Dept. & - & 1 \\
Teacher trainer & 21 & 21 \\
Head of government language support unit & & \\
Total & & \\
\hline
\end{tabular}


Table 3.8: Comparison of major issues in terms of comments made on them in each interview with percentage of total in parentheses (" topics on which questions were specifically asked)

\begin{tabular}{|c|c|c|c|c|c|c|}
\hline \multirow{2}{*}{$\begin{array}{l}\text { Major Issue Identified } \\
\text { Medium of Instruction }\end{array}$} & \multicolumn{3}{|c|}{$\begin{array}{c}\text { No. of } \\
\text { interviews in } \\
1997 \\
(\%) \\
\end{array}$} & \multicolumn{3}{|c|}{$\begin{array}{c}\text { No. of } \\
\text { interviews in } \\
1999 \\
(\%) \\
\end{array}$} \\
\hline & 19 & $(100)$ & * & 16 & $(100)$ & * \\
\hline Benchmarking & 19 & $(100)$ & * & 8 & $(50)$ & \\
\hline Teachers & 17 & $(89)$ & * & 15 & (94) & * \\
\hline Proportion of timetable & 16 & (84) & * & 0 & $(0)$ & \\
\hline Teaching Meth. and Curriculum & 15 & (79) & * & 14 & (88) & * \\
\hline English language & 15 & (79) & & 13 & (81) & \\
\hline Putonghua & 15 & (79) & & 8 & (50) & \\
\hline The Changeover & 10 & (53) & & 8 & $(50)$ & \\
\hline English teaching in China & 9 & (47) & & 6 & (38) & \\
\hline Students & 8 & (42) & & 6 & (38) & $*$ \\
\hline Education Policy & 7 & (37) & * & 9 & (56) & \\
\hline Schools & 4 & (21) & & 2 & (12) & \\
\hline New Immigrants from China & 6 & (32) & & 0 & $(0)$ & \\
\hline Characteristics of HK People & 5 & (26) & & 5 & (31) & \\
\hline Chinese language & 4 & (21) & & 2 & (12) & \\
\hline Total number of interviews & 19 & & & 16 & & \\
\hline
\end{tabular}


Table 3.9: Comparison of major issues in terms of total number of comments made with percentages of total in parentheses ("topics on which questions were specifically asked)

\begin{tabular}{|c|c|c|c|c|c|c|}
\hline \multirow{2}{*}{$\begin{array}{l}\text { Major Issue Identified } \\
\text { Medium of Instruction }\end{array}$} & \multicolumn{3}{|c|}{$\begin{array}{c}\text { No. of } \\
\text { comments in } \\
1997 \\
(\%) \\
\end{array}$} & \multicolumn{3}{|c|}{$\begin{array}{c}\text { No. of } \\
\text { comments in } \\
1999 \\
(\%) \\
\end{array}$} \\
\hline & 154 & (31) & * & 126 & (35) & * \\
\hline Teachers & 83 & (17) & * & 72 & (20) & * \\
\hline Benchmarking & 64 & (13) & * & 20 & (5) & \\
\hline English language & 34 & (7) & & 35 & (10) & \\
\hline Teaching Meth. and Curriculum & 29 & (6) & * & 39 & (11) & * \\
\hline Putonghua & 26 & (5) & & 11 & (3) & \\
\hline Proportion of timetable & 23 & (5) & * & 0 & $(0)$ & \\
\hline The Changeover & 15 & (3) & & 12 & (3) & \\
\hline English teaching in China & 13 & (2) & & 6 & (2) & \\
\hline Students & 12 & (2) & & 15 & (4) & * \\
\hline Education Policy & 11 & (2) & * & 16 & (4) & \\
\hline Schools & 9 & (2) & & 2 & (1) & \\
\hline New Immigrants from China & 8 & (2) & & 0 & $(0)$ & \\
\hline Characteristics of HK People & 7 & (2) & & 8 & (2) & \\
\hline Chinese language & 5 & (1) & & 3 & (1) & \\
\hline Total number of comments & 493 & & & 365 & & \\
\hline
\end{tabular}


Table 3.10: Aspects of the Medium of Instruction issue raised in interviews in 1997 and 1999

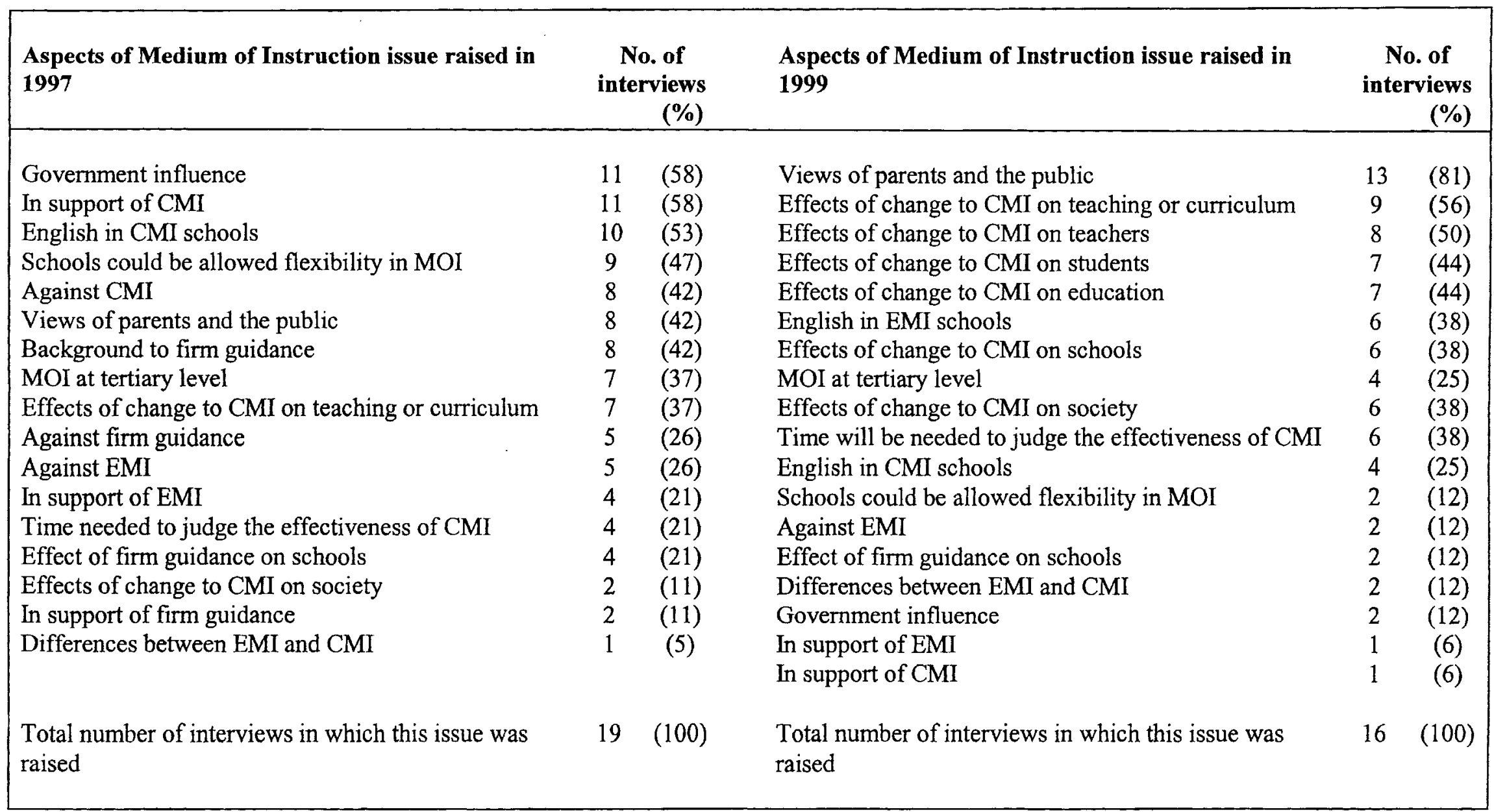


Table 3.11: Aspects of the Benchmarking issue raised in interviews in 1997 and 1999

\begin{tabular}{|c|c|c|c|c|c|}
\hline \multirow{2}{*}{$\begin{array}{l}\text { Aspects of Benchmarking issue raised in } 1997 \\
\text { Questions on implementation of Benchmarking }\end{array}$} & \multicolumn{2}{|c|}{$\begin{array}{r}\begin{array}{c}\text { No. of } \\
\text { interviews } \\
(\%)\end{array} \\
\end{array}$} & \multirow{2}{*}{$\begin{array}{l}\text { Aspects of Benchmarking issue raised in } 1999 \\
\text { Questions on implementation of Benchmarking }\end{array}$} & \multicolumn{2}{|c|}{$\begin{array}{r}\text { No. of } \\
\text { interviews } \\
(\%)\end{array}$} \\
\hline & 10 & $(53)$ & & 4 & $(25)$ \\
\hline Improvement in teachers' language proficiency & 7 & $(37)$ & Benchmarking will put pressure on teachers & 4 & $(25)$ \\
\hline Benchmarking will put pressure on teachers & 6 & $(32)$ & Against the Benchmarking & 2 & (12) \\
\hline Benchmarking for new teachers only & 4 & $(21)$ & Benchmarking and professionalism of teachers & 1 & $(6)$ \\
\hline Benchmarking and professionalism of teachers & 4 & $(21)$ & In support of Benchmarking & 1 & (6) \\
\hline Incentives for those who reach the Benchmark & 4 & $(21)$ & & & \\
\hline In support of Benchmarking & 3 & $(16)$ & & & \\
\hline Origins of Benchmarking & 1 & (5) & & & \\
\hline $\begin{array}{l}\text { Total number of interviews in which this issue was } \\
\text { raised }\end{array}$ & 19 & $(100)$ & $\begin{array}{l}\text { Total number of interviews in which this issue was } \\
\text { raised }\end{array}$ & 8 & $(50)$ \\
\hline
\end{tabular}


Table 3.12: Aspects of the Teachers issue raised in interviews in 1997 and 1999

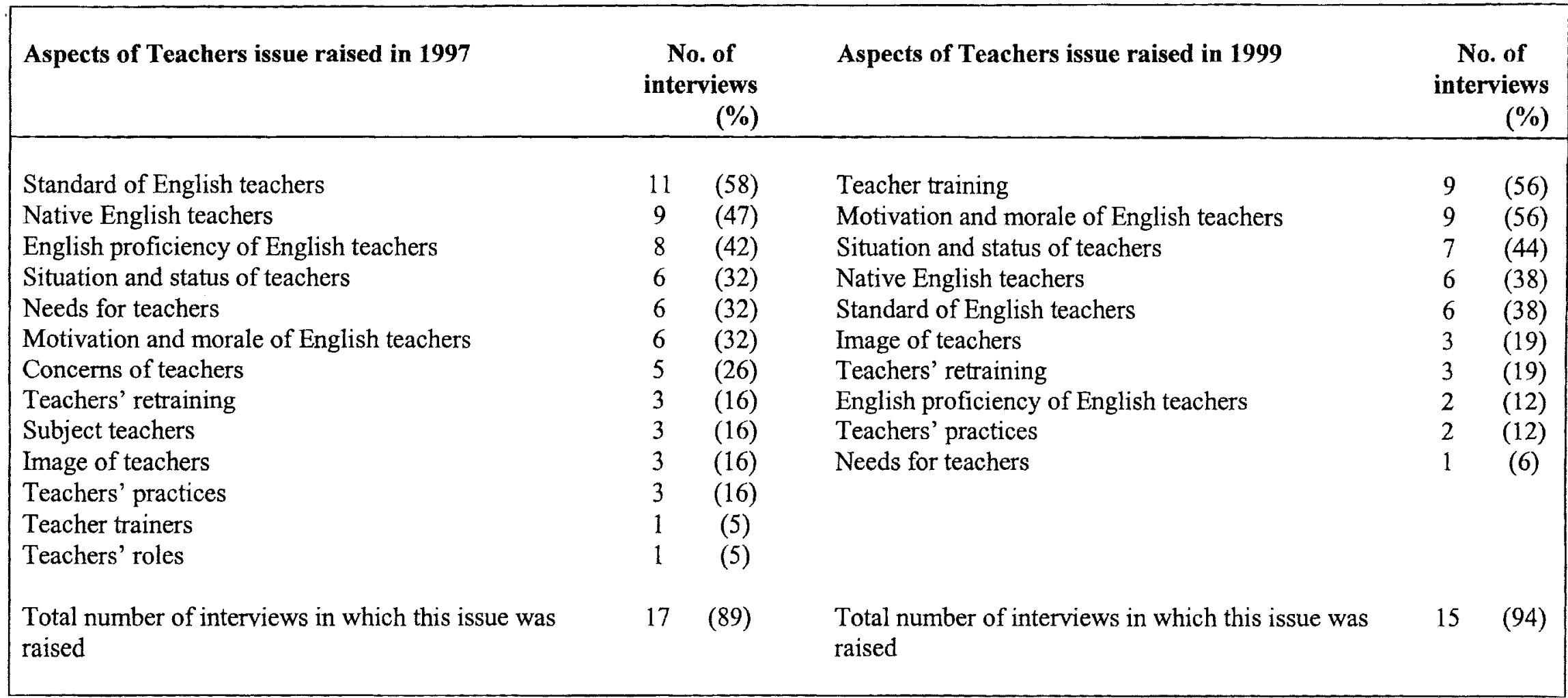


Table 3.13: Aspects of the Proportion of the Timetable issue raised in interviews in 1997 and 1999

\begin{tabular}{|c|c|c|c|}
\hline 10 & 53 & & \\
\hline 6 & 32 & & \\
\hline 3 & 16 & & \\
\hline 1 & 5 & & \\
\hline 1 & 5 & & \\
\hline 16 & $(84)$ & Total number of interviews & $(0)$ \\
\hline
\end{tabular}


Table 3.14: Aspects of the Teaching Methodology \& Curriculum issue raised in interviews in 1997 and 1999

\begin{tabular}{|c|c|c|c|c|}
\hline \multirow{2}{*}{$\begin{array}{l}\text { Aspects of the Teaching Methodology \& } \\
\text { Curriculum issue raised in } 1997 \\
\text { Content }\end{array}$} & $\begin{array}{r}\text { No. of } \\
\text { interviews } \\
(\%)\end{array}$ & \multirow{2}{*}{$\begin{array}{l}\text { Aspects of the Teaching Methodology \& } \\
\text { Curriculum issue raised in } 1999 \\
\text { Curriculum }\end{array}$} & \multicolumn{2}{|c|}{$\begin{array}{r}\begin{array}{r}\text { No. of } \\
\text { interviews } \\
(\%)\end{array} \\
\end{array}$} \\
\hline & $(37)$ & & 11 & $(69)$ \\
\hline Possible changes in methodology & (32) & Examination syllabuses & 9 & $(56)$ \\
\hline Existing problems with methodology & $(21)$ & TOC & 6 & (38) \\
\hline Students should learn grammar & (16) & IT & 6 & (38) \\
\hline Extra-curricular activities & (16) & Content & 3 & (19) \\
\hline TOC & (16) & Possible changes in methodology & 3 & (19) \\
\hline Curriculum & (11) & Existing problems with methodology & 1 & (6) \\
\hline Textbooks & $(5)$ & & & \\
\hline $\begin{array}{l}\text { Total number of interviews in which this issue was } \\
\text { raised }\end{array}$ & $15 \quad(79)$ & $\begin{array}{l}\text { Total number of interviews in which this issue was } \\
\text { raised }\end{array}$ & 14 & $(88)$ \\
\hline
\end{tabular}


Table 3.15: Aspects of the English language issue raised in interviews in 1997 and 1999

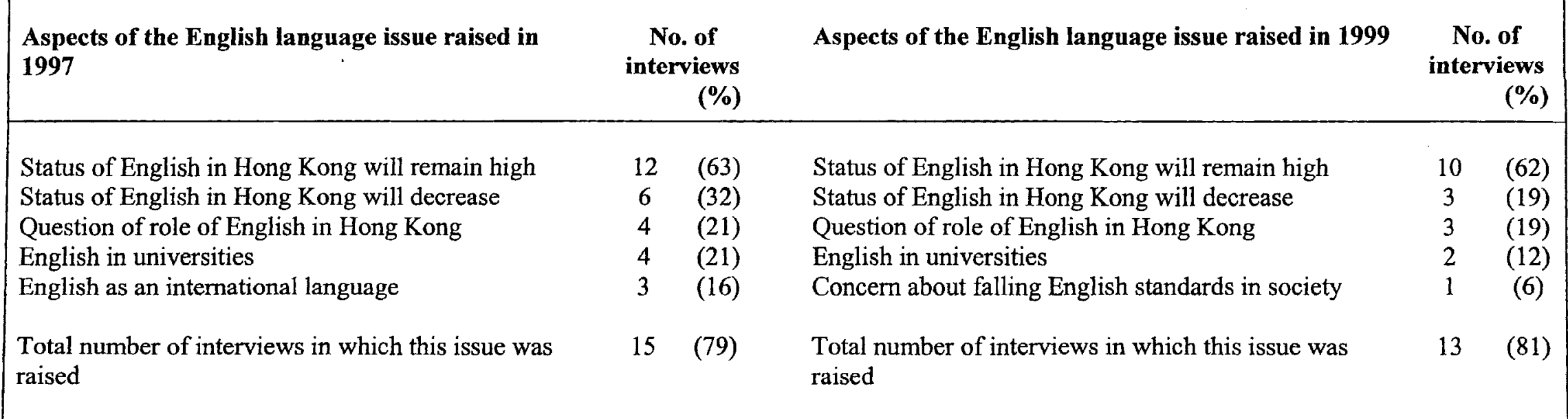


Table 3.16: Aspects of the Putonghua issue raised in interviews in 1997 and 1999

\begin{tabular}{|c|c|c|c|c|c|}
\hline \multirow{2}{*}{$\begin{array}{l}\text { Aspects of the Putonghua issue raised in } 1997 \\
\text { Putonghua as MOI }\end{array}$} & \multicolumn{2}{|c|}{$\begin{array}{r}\text { No. of } \\
\text { interviews } \\
(\%)\end{array}$} & \multirow{2}{*}{$\begin{array}{l}\text { Aspects of the Putonghua issue raised in } 1999 \\
\text { Putonghua in Hong Kong society }\end{array}$} & \multicolumn{2}{|c|}{$\begin{array}{r}\text { No. of } \\
\text { interviews } \\
(\%)\end{array}$} \\
\hline & 11 & $(58)$ & & 5 & $(31)$ \\
\hline Putonghua in Hong Kong society & 5 & (26) & Views of students on Putonghua & 2 & (12) \\
\hline Putonghua teaching in schools & 5 & (26) & Putonghua teaching in schools & 2 & (12) \\
\hline Total number of interviews & 15 & (79) & Total number of interviews & 8 & $(50)$ \\
\hline
\end{tabular}


Table 3.17: Aspects of the Changeover issue raised in interviews in 1997 and 1999

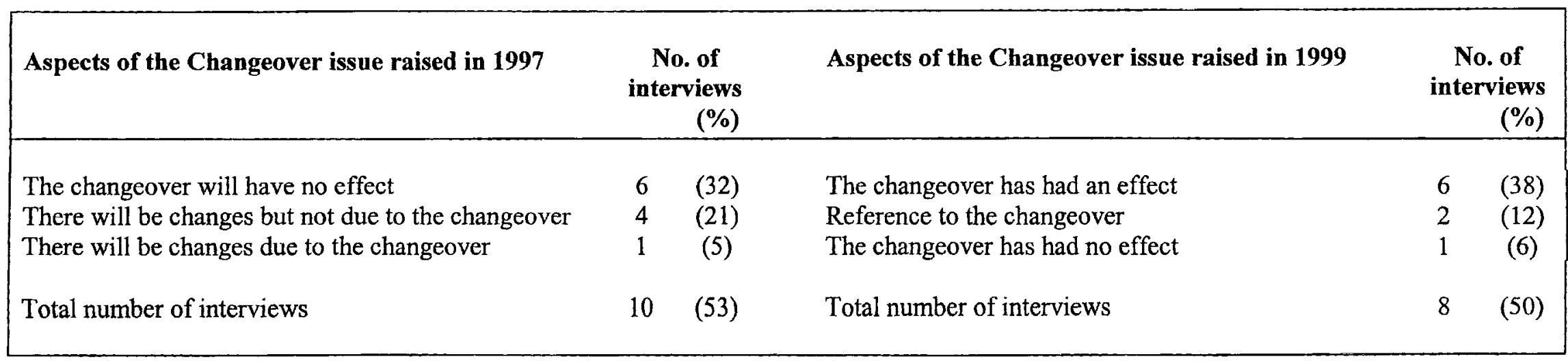

Table 3.18: Aspects of the English Teaching in China issue raised in interviews in 1997 and 1999

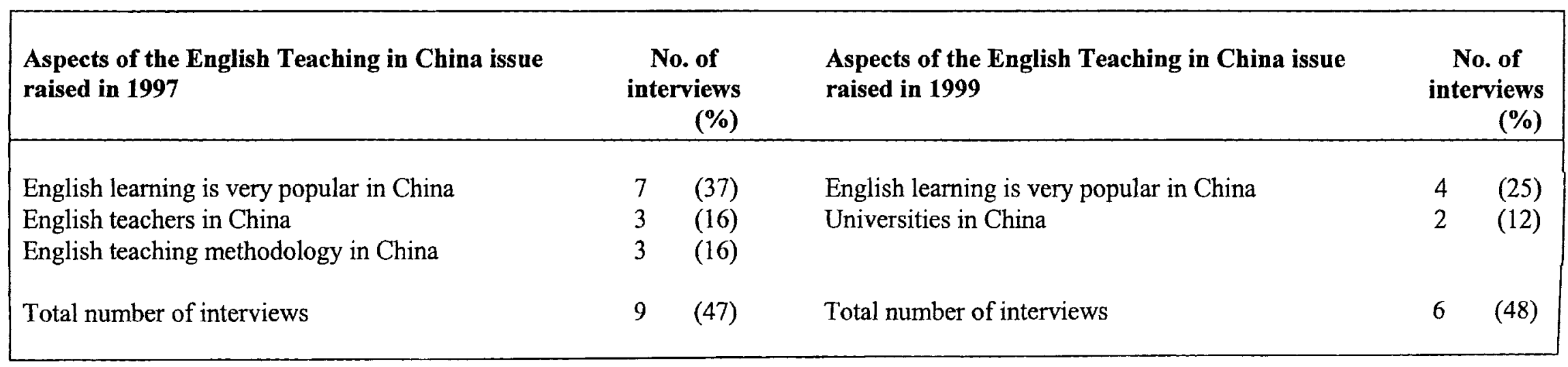


Table 3.19: Aspects of the Students issue raised in interviews in 1997 and 1999

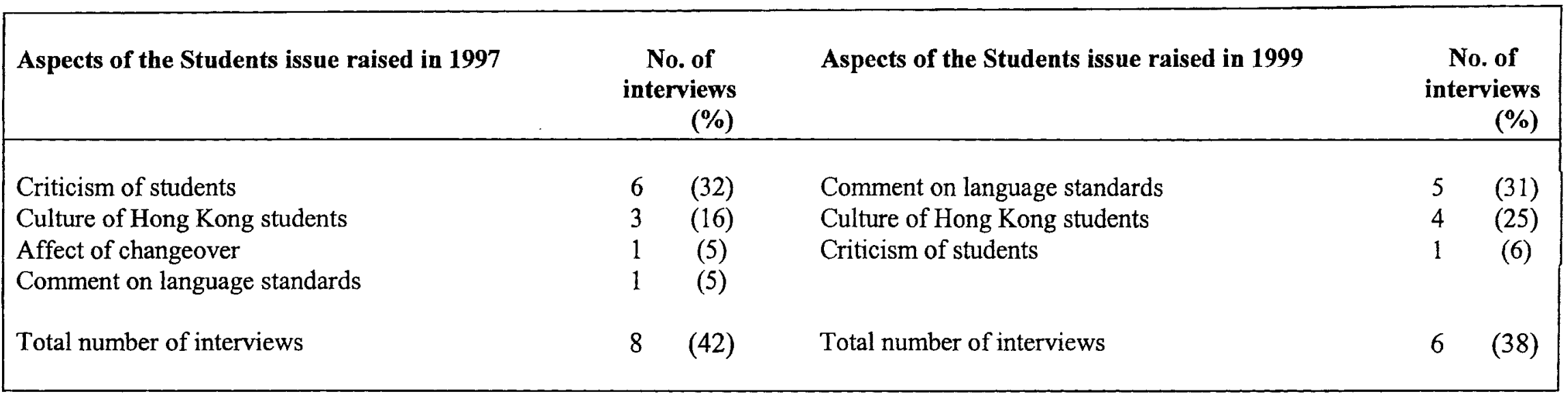

Table 3.20: Aspects of the Education Policy issue raised in interviews in 1997 and 1999

\begin{tabular}{|c|c|c|c|c|c|}
\hline $\begin{array}{l}\text { Aspects of the Education Policy issue raised in } \\
1997\end{array}$ & \multicolumn{2}{|c|}{$\begin{array}{r}\begin{array}{r}\text { No. of } \\
\text { interviews } \\
(\%)\end{array}\end{array}$} & $\begin{array}{l}\text { Aspects of the Education Policy issue raised in } \\
1999\end{array}$ & \multicolumn{2}{|c|}{$\begin{array}{r}\text { No. of } \\
\text { interviews } \\
(\%)\end{array}$} \\
\hline Changes due to the changeover & 4 & $(21)$ & Educational reforms & 7 & (44) \\
\hline Needs for education system & 2 & (11) & Needs for education system & 2 & $(12)$ \\
\hline Call for 4-year university programmes & 2 & (11) & Comment on education in Hong Kong & 1 & $(6)$ \\
\hline Total number of interviews & 7 & $(37)$ & Total number of interviews & 9 & $(56)$ \\
\hline
\end{tabular}


Table 3.21 Aspects of the Schools issue raised in interviews in 1997 and 1999

\begin{tabular}{|c|c|c|c|c|c|}
\hline Schools' accountability & 4 & $(21)$ & Schools' accountability & 1 & (6) \\
\hline Alternatives to government-run schools & 2 & (11) & Alternatives to government-run schools & 1 & (6) \\
\hline Schools' prestige & 2 & (11) & & & \\
\hline Total number of interviews & 4 & $(21)$ & Total number of interviews & 2 & $(12)$ \\
\hline
\end{tabular}

Table 3.22: Aspects of the New Immigrants from China issue raised in interviews in 1997 and 1999

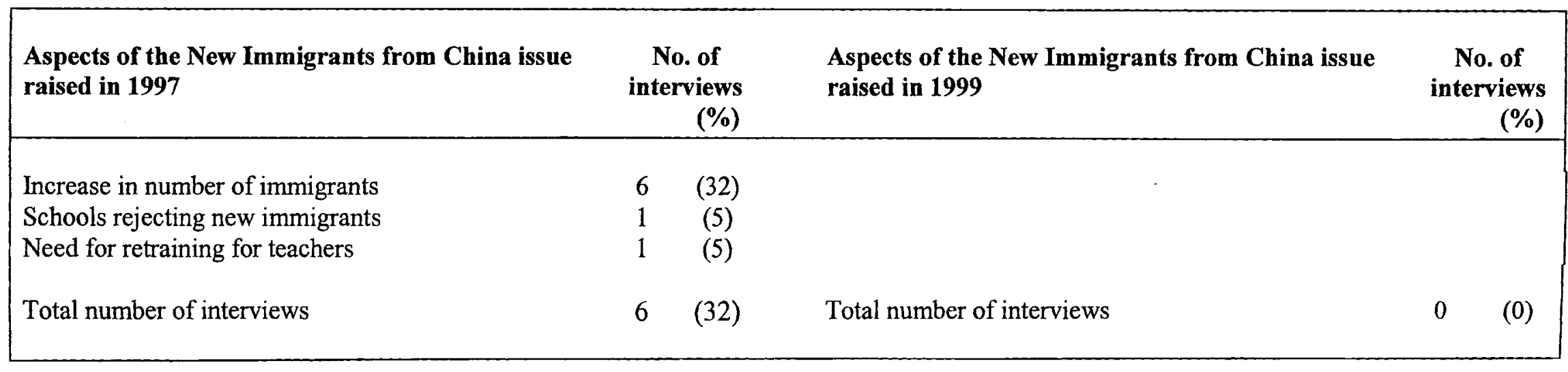


Table 3.23: Aspects of the Characteristics of Hong Kong People issue raised in interviews in 1997 and 1999

\begin{tabular}{|c|c|c|c|c|}
\hline $\begin{array}{l}\text { Pragmatism of Hong Kong people } \\
\text { Non-negative attitude of Hong Kong people towards } \\
\text { English as colonial language }\end{array}$ & $\begin{array}{l}(26) \\
(11)\end{array}$ & Views of the public on education & 5 & $(31)$ \\
\hline
\end{tabular}

Table 3.24: Aspects of the Chinese Language issue raised in interviews in 1997 and 1999

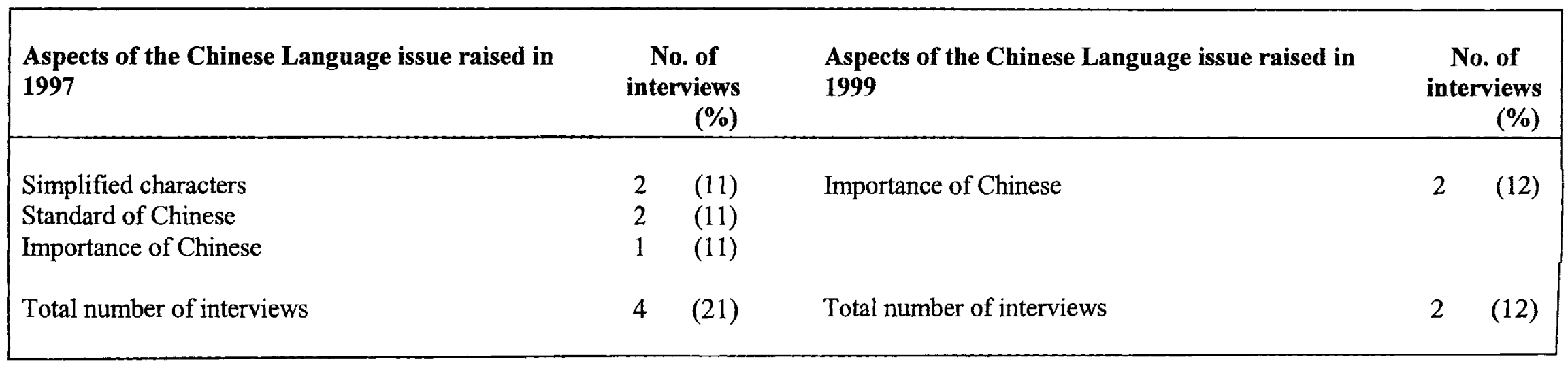


Table 3.25: Comments made on Higher Level issues in 1997 and 1999

\begin{tabular}{|lcccc|}
\hline Issue Categories & $\begin{array}{c}\text { No. of } \\
\text { comments in } \\
\mathbf{1 9 9 7} \\
\mathbf{( \% )}\end{array}$ & $\begin{array}{c}\text { No. of } \\
\text { comments in } \\
\mathbf{1 9 9 9} \\
(\%)\end{array}$ \\
\hline Language & 201 & $(41)$ & 149 & $(41)$ \\
Professional & 89 & $(18)$ & 66 & $(18)$ \\
Administrative and Institutional & 80 & $(16)$ & 45 & $(12)$ \\
Socio-political & 66 & $(13)$ & 47 & $(13)$ \\
Educational & 36 & $(7)$ & 47 & $(13)$ \\
Behavioural & 18 & $(4)$ & 10 & $(3)$ \\
Inter-cultural & 3 & $(1)$ & 1 & $(0)$ \\
Total number of comments & 493 & & 365 & \\
\hline
\end{tabular}


Table 3.26: Comments made on Language issues in 1997 and 1999

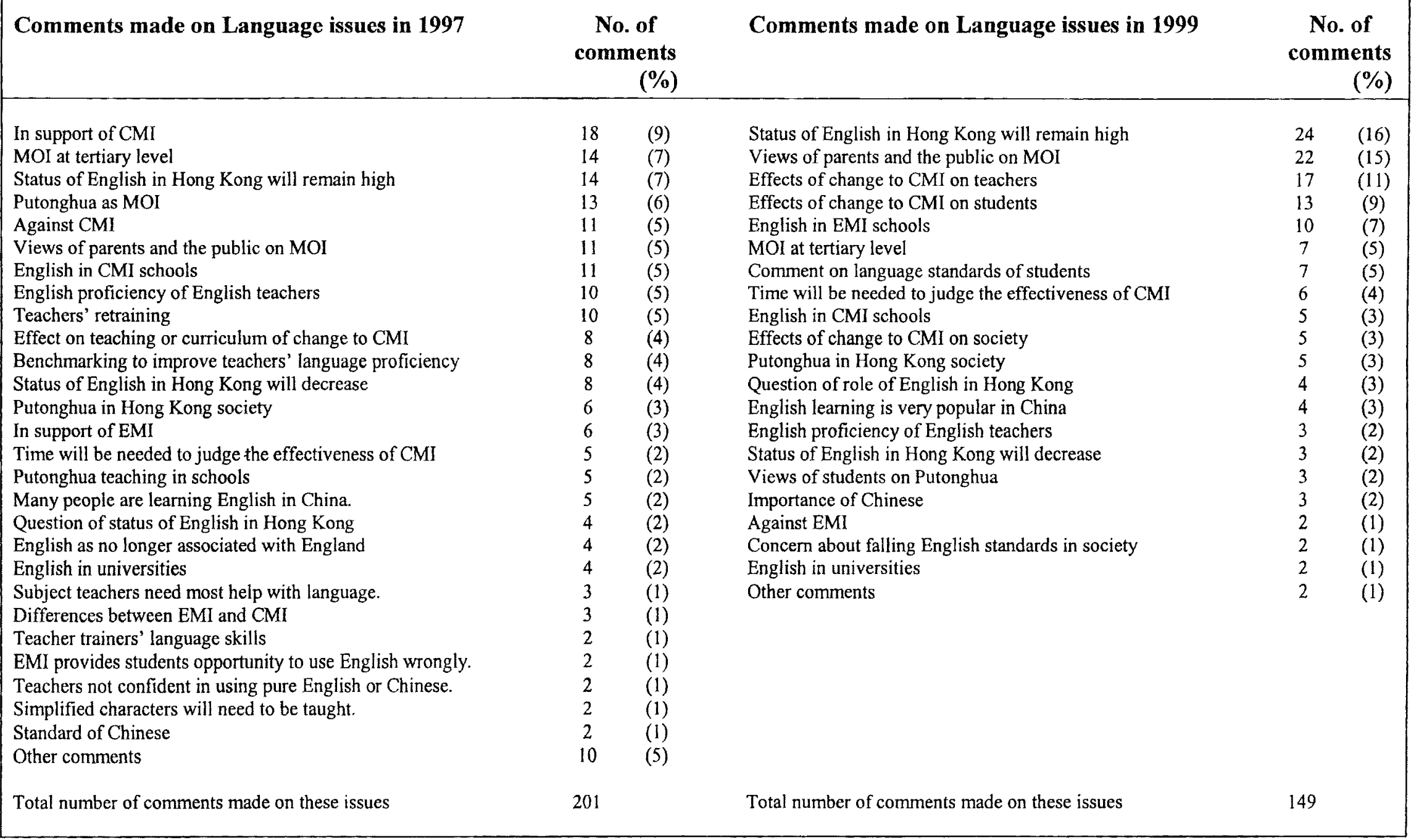


Table 3.27: Comments made on Professional issues in 1997 and 1999

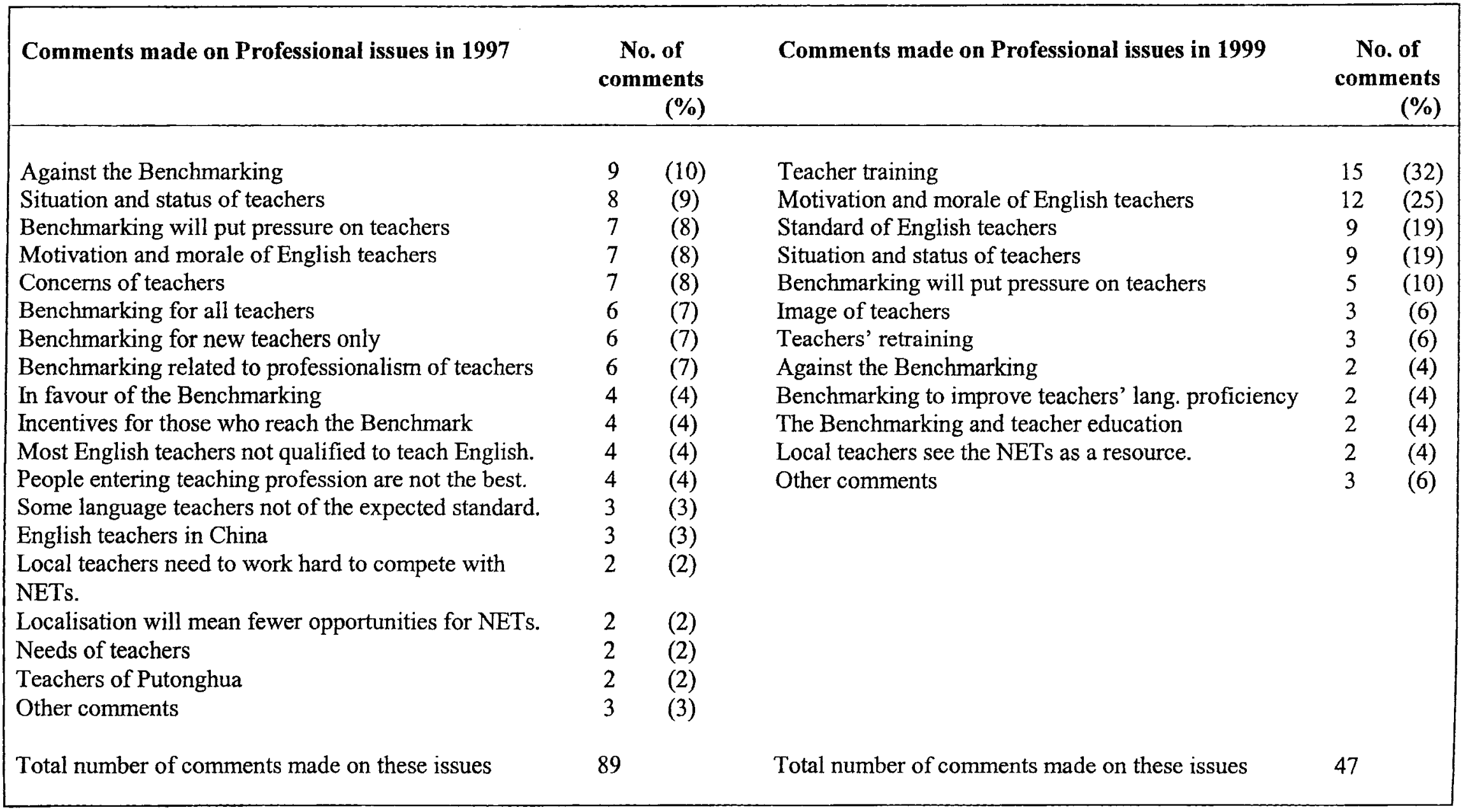


Table 3.28: Comments made on Administrative and Institutional issues in interviews in 1997 and 1999

\begin{tabular}{|c|c|c|c|c|c|}
\hline $\begin{array}{l}\text { Comments made on Administrative and } \\
\text { Institutional issues in } 1997\end{array}$ & \multicolumn{2}{|c|}{$\begin{array}{l}\text { No. of } \\
\text { comments }\end{array}$} & $\begin{array}{l}\text { Comments made on Administrative and } \\
\text { Institutional issues in } 1999\end{array}$ & \multicolumn{2}{|c|}{$\begin{array}{l}\text { No. of } \\
\text { comments }\end{array}$} \\
\hline Schools could be allowed flexibility in MOI & 14 & $(18)$ & Examination syllabuses & 9 & $(20)$ \\
\hline Questions on implementation of Benchmarking & 13 & $(16)$ & Effects of change to CMI on schools & 8 & (18) \\
\hline Increase in immigrants from China [needing schooling] & 6 & $(8)$ & Schools could be allowed flexibility in MOI & 2 & (4) \\
\hline Effect of firm guidance on schools & 5 & (6) & Effect of firm guidance on schools & 2 & (4) \\
\hline Needs for education system & 4 & (5) & Needs for education system & 2 & (4) \\
\hline Schools' accountability & 4 & (5) & Universities in China & 2 & $(4)$ \\
\hline More NETs should be recruited & 3 & (4) & Other comments & 9 & $(20)$ \\
\hline No change in amount of English on the timetable & 3 & (4) & & & \\
\hline Total number of comments made on these issues & 80 & & Total number of comments made on these issues & 45 & \\
\hline
\end{tabular}


Table 3.29: Comments made on Socio-political issues in 1997 and 1999

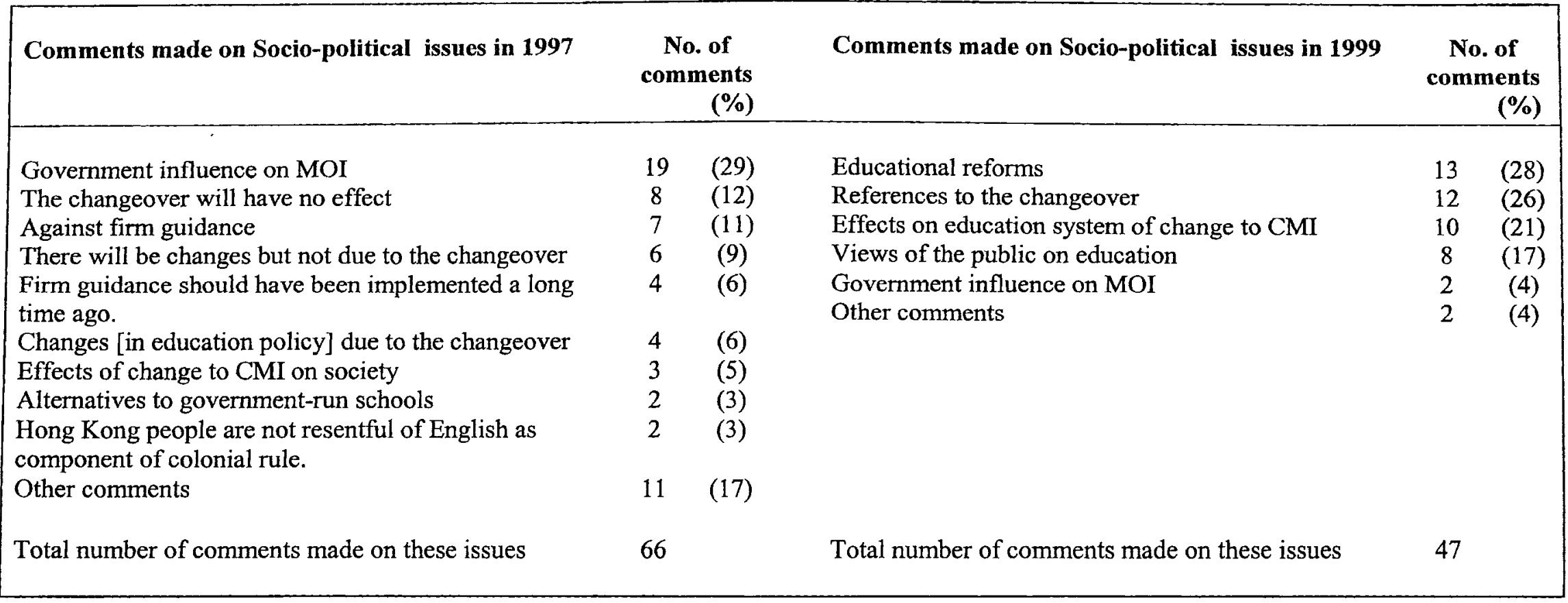


Table 3.30: Comments made on Educational issues in 1997 and 1999

\begin{tabular}{|c|c|c|c|c|c|}
\hline \multirow{2}{*}{$\begin{array}{l}\text { Comments made on Educational issues in } 1997 \\
\text { Possible changes in methodology }\end{array}$} & \multicolumn{2}{|c|}{$\begin{array}{r}\text { No. of } \\
\text { comments } \\
(\%)\end{array}$} & \multirow{2}{*}{$\begin{array}{l}\text { Comments made on Educational issues in } 1999 \\
\text { Effect on teaching or curriculum of change to CMI }\end{array}$} & \multicolumn{2}{|c|}{$\begin{array}{r}\text { No. of } \\
\text { comments } \\
(\%)\end{array}$} \\
\hline & 6 & $(17)$ & & 11 & (29) \\
\hline Content of lessons & 4 & (11) & Curriculum & 11 & (29) \\
\hline Existing problems with methodology & 4 & (11) & TOC & 6 & (13) \\
\hline Students should learn grammar & 3 & $(8)$ & IT & 6 & (13) \\
\hline Schools may incorporate extra-curricular activities to & 3 & $(8)$ & Content & 3 & $(6)$ \\
\hline improve English proficiency. & & & Possible changes in methodology & 3 & (6) \\
\hline TOC & 3 & $(8)$ & Differences between EMI and CMI & 2 & (4) \\
\hline Teachers' practices & 3 & (8) & Putonghua teaching in schools & 2 & (4) \\
\hline English teaching methodology in China & 3 & $(8)$ & Other comments & 3 & (6) \\
\hline Other comments & 7 & (19) & & & \\
\hline Total number of comments made on these issues & 36 & & Total number of comments made on these issues & 47 & \\
\hline
\end{tabular}


Table 3.31: Comments made on Behavioural issues in 1997 and 1999

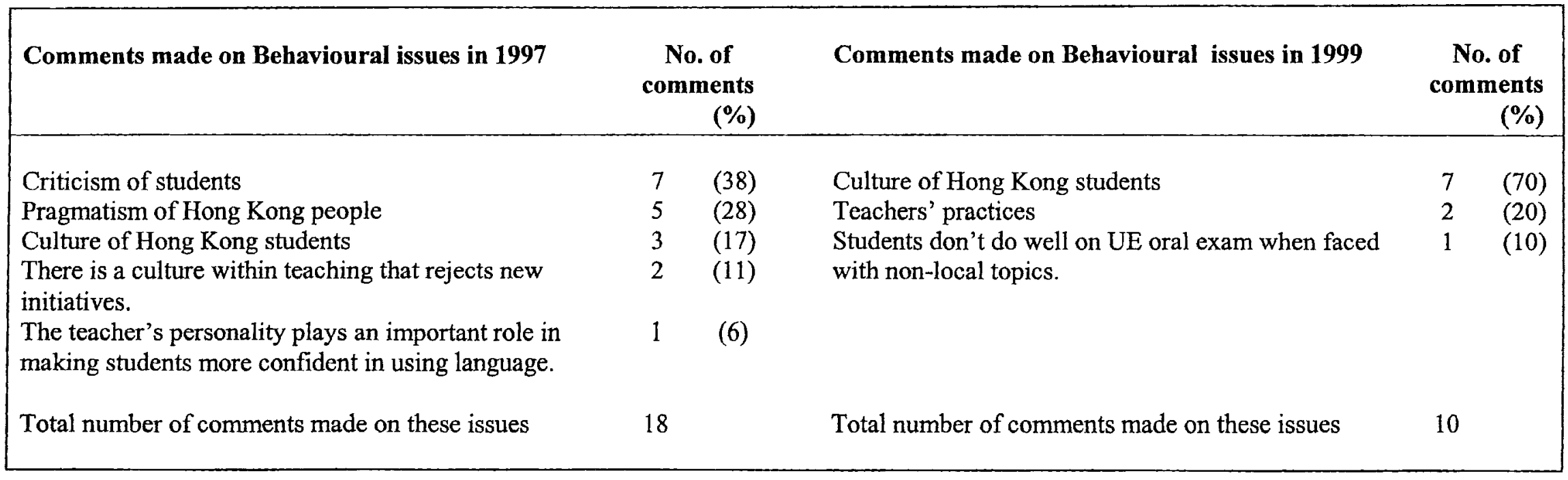


Table 3.32: Comments made on Intercultural issues in 1997 and 1999

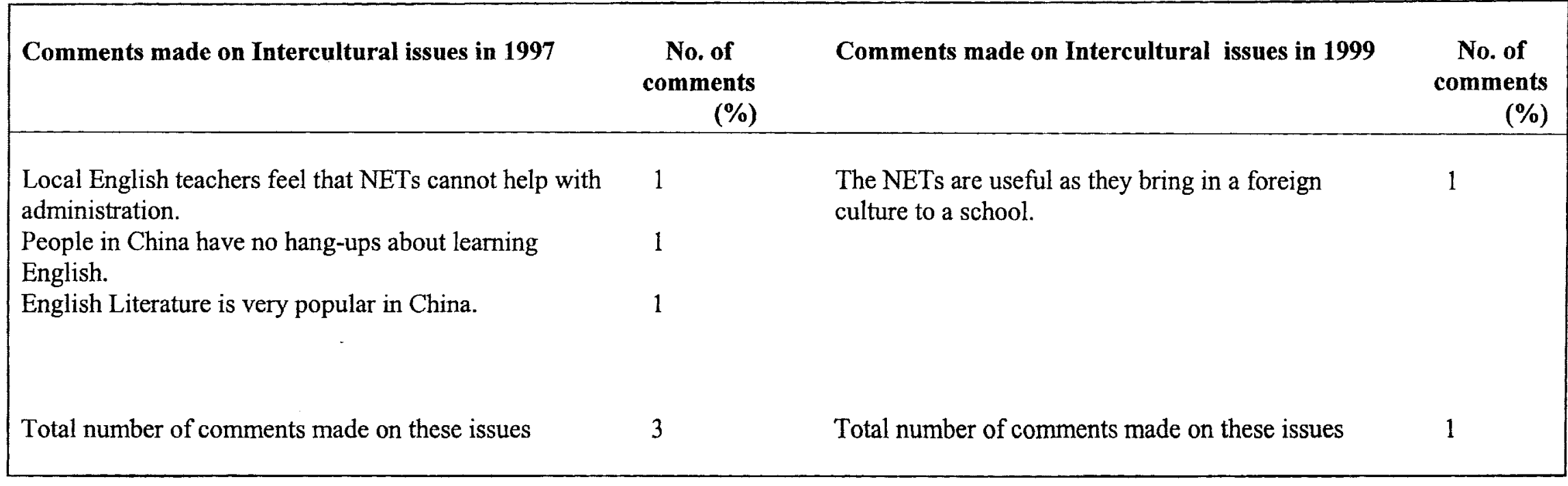




\section{APPENDIX B}

Appendix B consists of the following, which should be viewed in conjunction with Chapter 4 of the thesis:

B.1 Transcriptions of interviews with BATESL graduates in 1994

B.2 Questionnaire administered to BATESL graduates in 1997

B.3 Transcriptions of interviews with BATESL graduates in 1997

B.4 Statistical results of questionnaire surveys

B.5 BATESL students' philosophies of teaching 


\section{B.1 Transcriptions of Interviews with BATESL Graduates in 1994}

\section{Interviewee: $\quad$ Subject $\mathbf{A}(94)$}

Date: 9 June 1994

Female, 22 years old.

Was planning to teach after graduation and would prefer to work in a co-educational school. If she did not go into teaching she would like to work in banking.
AU Researcher
Missing words / Unfinished utterance
Pause

\section{AU Good afternoon}

A(94) Good afternoon

AU Let's begin by looking at Language Use. Do you think that your English, spoken and written, has improved since you started the course?

A(94) Yes, I think so, but writing has improved a lot [more] than speaking because I have to do a lot of coursework in written and I learn more about academic writing and because I have a module called Creative Writing. I learned very much in this module also. Apart from that because I am interested in writing. I think the improvement is better, is more than in speaking.

AU Did you get much chance to speak very much in the course?

A(94) In the course yes, a lot of opportunity because in the class we are encouraged to speak up and a lot of activities required us to speak in English.

AU Did many people speak?

A(94) Only some of them, some of our classmates speak more than the others because some are a bit silent or passive. And the active students, they speak a lot and raise a lot of questions.

AU So let's say in your group of how many?

A(94) Fifteen

AU In your group of fifteen, if the person who speaks the most is number one and 
the one who speaks the least is number fifteen, where would you be?

$\mathrm{A}(94) \quad$ I think in the middle, five to six.

AU When you are speaking Cantonese to friends do you use English expressions, that is code-mixing? You said 'Always'.

A(94) Yes, especially with my classmates because we know some of the terms in the teaching field and we will use a lot of the terms and for our friends, for example, my friends in business field they also use some terms and I use it also, in order to create some, maybe friendship.

AU Do you find that it's just special terms that you would use or do you say other things in English?

A(94) All things in English, only terms, we conduct our main conversation in Cantonese. I think it's only the terms, other than the terms we based on Cantonese for our conversation.

AU Some of the others have said that they might use English for fun, like 'Oh you're stupid!' or for embarrassing things, things that might be embarrassing to talk about, they might use English for them instead of Cantonese.

A(94) Yes, for example, 'Are you crazy [subject's name]!' They will say this, but only for, yes as you say, it's only for fun. But the main content we conduct in Cantonese because we can understand more and we can feel the other's - er we can experience the other's feelings.

AU So you would code-mix a lot but rarely would you code-switch. Would you switch into English for more than just a word or two words.

A(94) Yes, as I said before, all my friends are Chinese and so if I use English to speak to them they will think that I am more superior than them and so I'll use Cantonese always.

AU Can you think of any situations where people might, Chinese people might switch into English?

A(94) Maybe if the two speakers have a certain competence in English and then they switch to English for some situations that they both use English, but I think for their personal opinion they will express it in Cantonese. Maybe for the job purpose they will use English.

AU Okay, now we'll move on to English in the classroom. 'In English lessons, English should be the only language used for all purposes.' You said that you quite agree with that. You commented that 'for some reasons switch to Chinese 
or Cantonese is necessary, for example explain terms'.

A(94) Yes, not just explain the terms to the students but also for classroom management because if I use English the students may not understand what I mean and if I use Chinese they may listen to it more quickly and they will maybe stop talking. They will quickly stop talking.

AU So you think it's okay to present content [in English]?

A(94) Yes, only present content in English and explain the terms in English first. If the students don't understand then I will use Chinese.

AU So, 'How much did you use only English in lessons during your Practice Teaching?' You said 'Usually'. Does that mean that sometimes you would have lessons in which you used only English or would you use some Cantonese in nearly every lesson?

A(94) Er - I think it's 50/50. Yes, because during my practice teaching the students are very poor because they are Band Five students and so I must use Chinese to translate their English content for them because they do not understand and the headmaster said that I have to use both Cantonese and English to teach them.

AU Oh, he said that to you?

A(94) Yes, he required me to do so and I did what he required me to do. Because the secondary school is a Chinese middle school that means Chinese is the medium of instruction.

AU And 'the actual amount of pure English that you used', you said that it increased over the time that you were doing your practice teaching. So you were using more English as you went along?

A(94) More English, maybe $30 \%$ of Chinese and $70 \%$ of English and they can manage to catch up with what I said in class.

AU 'In what situations in an English lesson would or might you use Cantonese?' You said 'To establish discipline.' Is that it?

A(94) Yes, only establish discipline, for classroom management Cantonese is more effective.

AU Right, not to be friendly with students? Or to explain lesson content?

A(94) Yes, also want to create some friendship with my students but I think that the most important is for discipline, it's more effective but I also think use English can also create friendship with the students. If you use English expression it is 
more informal they can understand.

AU There must be some situations where outside of lessons you would use Cantonese when you are talking to students, you would use Cantonese.

A(94) Yes

AU I know there are some teachers who are Chinese but insist on speaking to students in English.

A(94) Yes, there is one teacher in CPHK, he always use English to talk to us. We go to him to chat to him, he use English but we insist to use Cantonese but I think it's a bit odd because we have a friendly discussion on our personal things but he still use English.

AU So you think sometimes it is necessary to draw the line between teacher as friend and teacher as counsellor or whatever.

A(94) Yes

AU Okay, the next section is Instructional Planning and Decision Making. 'It's impossible to have a successful lesson that hasn't been well-planned in advance.' You said that you 'Absolutely agree', 'Planning is absolutely essential for the lesson to run smoothly but the students can have a better environment to learn with well-planned materials and teaching methods.' Do you believe that? That you must plan a lesson well in order for it to be successful?

A(94) Yes, because during my practice teaching, I planned my lessons, every lesson seriously because I think if I prepare the materials well and the lesson well then they will pay more attention to it and they will enjoy it more and also maybe they will [be] more active in the activities if the activity is best suited for them.

AU 'Should teachers write detailed lesson plans for every lesson they teach?' For this you 'Quite disagree' and you said 'I think a brief lesson plan will do as it wouldn't have enough time to write out a detailed lesson plan for every lesson.'

A(94) Yes, as a regular teacher, he or she has a lot of lessons to go and I think he will not write out a detailed lesson plan for every lesson.

AU But obviously planning is important even though it doesn't involve a detailed lesson plan.

A(94) Only for us, I have to go to practice teaching so I have to write detailed lesson plans for our assignment, but if I go to teaching in the future, I wouldn't write a lot of lesson plans. 
AU But you would write something?

A(94) Yes, I think experienced teachers, but I'm not an experienced teacher.

AU I'm quite an experienced teacher, I've been teaching for about six years, but I still write something, maybe a sentence to tell what I'm going to do and a sentence to tell me what I've done.

A(94) But many experienced teachers in Hong Kong, I think they didn't write lesson plans at all.

AU I would forget what I'd done if I didn't write it down.

A(94) Yes, because they just open the text book and teach the lesson, maybe reading comprehension, then check the answers with the students and then, okay finished.

AU 'Teachers should be more responsive to students' needs than the set syllabus.' You 'Quite disagree' with that. 'In Hong Kong teaching is most usually based on the syllabus and school curriculum so not only students' needs should be taken into consideration.' So you say that you have to take both into consideration, the students' needs and the syllabus. Do you think it's possible in Hong Kong to base what you teach on the students' needs? Or do you think the syllabus is too influential?

A(94) I think the syllabus is more influential because if you insisted on teaching based on the students' needs, I think the principal will require you to follow the set syllabus for the students and then you have to do what he said based on the syllabus that you teach. Yes, especially for Form Four, Form Five or Form Six, Form Seven, because we have not enough time to go through the syllabus so we don't have more extra time to deal with the students' needs.

AU 'How detailed do you think your plans should be?' 'Brief and very flexible.' So you wouldn't plan in great detail, you would keep it very brief?

A(94) Yes, I think flexibility is very important because many unexpected outcomes will occur in the lesson. And so we don't have to write the detailed lesson plan because you have to be active in teaching the class and you have to change the plans, maybe give them more time or if they finish quickly, I should give them more activities and so the lesson should be planned in very flexible.

AU Flexible yes, but when you're planning, would you take into consideration these other factors, like having one or two extra activities in case they finish too quickly?

A(94) Yes, one or two, and the timing also. It's not just at 15 minutes so at 15 minutes 
you have to stop and I have to see that all the pupils, they have finished then I will give them more time and so the timing is flexible also.

AU 'How often did you find you had to change your lesson plan?' 'Sometimes', was time the major factor?

A(94) Yes, because when I plan the lesson, I don't know the students will they be active in the discussion but in the class maybe the students will be too active in the discussion and they raise a lot of questions then I have to deal with the questions and so time spent more on the discussion and then I have to cut off maybe one or two activities, in some cases.

AU 'On what basis did you plan your lessons?' You said that the BATESL course guidelines were the most influential.

A(94) This is because the practice teaching only, yes.

AU If it was not practice teaching, what do you think would be the most influential?

A(94) Because in the practice teaching, I have to do what the regular teacher tells me to do and also for the BATESL course, yes because if I do writing then I have to spend more time on doing the writing activity.

AU And 'what factors do you think had the most influence on the decisions you made?' You said 'the supervisor's advice' then 'the regular teacher's input'. Is this because it was more of an assignment than a real teaching situation?

A(94) Yes

AU Teaching Approach, 'Examination requirements should determine what is taught in English lessons.' You 'Quite Agree'. 'In reality, teachers should teach students all that they are require to learn for examinations.' Do you think that's a good thing?

A(94) Is that a good thing? I don't think so but we have no choice because the students have to learn all the things including the syllabus then they can get a pass mark in the examination or get a good mark.

AU 'Students learn better through interaction with other students rather than through direct instruction from the teacher.' Again, 'Quite agree', 'However, students more often need a push to use English in class especially for discussion or communication.' What do you mean by a 'push'?

A(94) Because I think that students are passive and they won't answer questions voluntarily and so only for the discussion or the activity they will think that using English is only for the activity. It is more natural. 
AU 'A class should be strictly controlled by the teacher and lesson content directed by the teacher. You 'Disagree' with that. 'Learner-centered is stressed in my opinion, teacher-student interaction creates a better environment for the students to learn.' So you prefer a learner-centered situation? Do you think that's possible or feasible in Hong Kong schools?

A(94) I think it's possible but if the students are quite active and their language proficiency is better then I think a communicative approach can benefit them. But for the poor students I think we can try the communicative approach gradually.

AU What do you mean by gradually?

A(94) That means, let them experience the communicative approach at the beginning of the lesson and if they have problems then we have to change a little bit to suit them and maybe later we can conduct more activities to help them learn more.

AU 'A teacher should be prepared to experiment with materials and teaching strategies from a variety of sources.' You 'Absolutely agree', 'This can improve our teaching and help us to choose the most suitable methodology to suit our target students.' So you think it's important to experiment with materials and teaching strategies?

A(94) Yes, because what the students learn is mainly based on the materials and the teaching and so the materials should be carefully chosen to suit the students' level and also their interest preference and also their language proficiency. And so because during my practice teaching, I read some books on teaching materials, then I read over them and I choose some to teach the students. And I think the best materials can create the students' interest to learn the language because I can see the students they are very interested in the activity.

AU 'In your practice teaching, did you find that students learn English better through interaction with other students or through direct instruction from the teacher?' You said 'Through direct instruction.' Although you favour a studentcentered approach, interaction with other students, you found that direct instruction was more effective.

A(94) Because for the interaction among the students is only part of the lesson but the overall lesson maybe $70 \%$ is conducted by the teacher and I think although the discussion and the activities can help the students learn the language, but the direct instruction by the teacher is also important.

AU Is it that the students are accustomed to direct instruction?

A(94) Yes, but if I require them to discuss with their partners then they will discuss 
they will use English, but if I don't require them to discuss they will only listen to me.

AU 'Did you find that you controlled your classes strictly and directed all lesson content?' 'Sometimes', 'If the students are interested in the topic, I will allow them to express themselves.'

A(94) If the students finish the discussion then I will go on for the rest of the lesson.

AU So you will allow the students to a certain extent to control what goes on in a lesson?

A(94) Yes

AU 'Did you experiment with materials and teaching strategies from a variety of sources?' 'Very much' 'I tried to find out the best way for my students to learn.' Did you find out the best way for your students to learn?

A(94) Er - yes, in the first lesson, I tried some of the materials and I know that some of them they didn't interested in it and some of them they feel that maybe they enjoy doing the activity. Then after that I will choose those activities or the exercises that they think they can learn the language and they interested in it.

AU So you describe your teaching style as mostly learner-centered. 'Which of the following do you think has the most influence on your approach to the teaching of English?' 'School principal' or 'Education Department policy' being the most influential. Why do you say that?

A(94) Because in the BATESL course, I have to do Practice Teaching and during the two practice teaching the principal and the English panel they both required me to do what they said and so I think the school policy or the principal's policy is more influential to my teaching.

AU Would you say that is true in English teaching in general?

A(94) I think so.

AU And 'Examination requirements' second after that?

A(94) That means the syllabus.

AU And your 'Teacher training', do you think your teacher training will shape how you teach?

A(94) Yes, I think so, but I think the teachers that are graduated from the teaching institutes they have more practical experience than us because I think our 
course is more based on the theory and only a little of the practice teaching. I only have not more than one month teaching experience in our course and I think more practice will be better.

AU That's right, I did a teaching certificate and I did twelve weeks teaching practice.

A(94) But I have only very little teaching experience and I think it's not enough.

AU Even though the course lasts for three years you only get less than one month practice teaching.

A(94) Yes, in the teaching institutes they have two years but they have more practice. They have one month full time in the secondary school but we have one or two lessons only and so I think teacher -training is essential.

AU After this course then if people want to be fully qualified as teachers they have to do a teacher-training course as well don't they?

A(94) Yes, in the university, a cert...

AU Will you do that?

A(94) Yes, I applied this year.

AU Then how much extra teaching practice do you get?

A(94) In the certificate?

AU $\quad$ Yes

A(94) I don't know yet because they didn't send me the course outline.

AU Presumably, you've already learned a lot about theories of teaching and so on so they will give you more teaching practice. Professional Relationships and Responsibilities. You are 'Quite confident' in your own ability to become a successful teacher. Why are you quite confident do you think?

A(94) Because I only have experience in teaching in two secondary schools so I am not very confident in teaching the students.

AU So why did you put 'Quite confident'?

A(94) 'Quite confident' because I'm not 'Very confident' and I'm not 'Not quite sure' so I choose this one, it's not very confident. 
AU Okay, so it's somewhere in between. 'How do you feel about the status of teachers in today's society?' You said 'Teachers enjoy a high status'. Very few people said that teachers enjoy a high status, why do you think that?

A(94) I don't know why but I think the teachers should have a higher status equal to doctors or lawyers because teachers are professional people I think.

AU Teachers should, but do they?

A(94) I think that most people in Hong Kong think that teachers are not so respectful than the doctors and the lawyers because they think that anyone can be a teacher.

AU Okay, your 'best attribute in terms of being a teacher' you would say is that you're 'able to form good professional relationships with colleagues', followed by 'Good command of the language' and 'Good communication skills'. You are not sure whether teaching is a long-term career or not. You don't know, but at the moment you're going to give it a try?

A(94) Yes, after that if I am not interested in teaching maybe I will go into the business field or banking field, but if I'm interested in it then I will continue teaching.

AU Do you think teaching is well-paid?

A(94) I don't think so because the beginning salary is better than the other jobs but after a few years maybe one person in the teaching field and one person in the business field, I think the person in the business field will earn more money. And also for the language teacher, we have a lot of jobs to do, yes, and I think the salary is not high enough.

AU Yes' it's one thing to say that I'll try teaching and after a few years if I don't like it I'll try another job but very often people get stuck in teaching, they get used to the salary and they have a lifestyle and they find that they can't leave teaching because if they went into another field they would have to take a reduction in salary and then they couldn't afford to live on that salary. Okay, "Which of the following activities do you think you will have to do in your first year?' You've circled most. Not 'Meet parents'?

A(94) Maybe, but I think that the parent's day is only one day.

AU It's only one day but you still have to do it.

A(94) Yes

AU 'Attend courses'? 
A(94) Yes, if I do the cert. then I have to attend courses after school.

AU Very often there are courses to do and although teachers are not forced into joining the courses, perhaps short courses on Saturday mornings, that kind of thing, but sometimes there are if there are changes to the examinations then teachers have to attend short courses.

A(94) Yes

AU 'Administer exams', you don't think you'll have to do that?

A(94) I don't think I will do this because only for these experienced teachers.

AU Yes, but you still have to give out exams.

A(94) Yes, ah ha.

AU Perhaps not external exams though.

A(94) I think that is external exams.

AU 'Train students for Speech Festivals etc.', now that kind of thing depends on your school. In my school, they do that. My school's a Band One school so we have to do that.

A(94) And for drama.

AU Yes, drama, debating, that kind of thing.

A(94) Yes, I also agree that Band One schools have a lot of jobs to do. And the history teachers and geography teachers they finish the work in class and then they only mark some workbook, but we have to mark the newspaper cuttings and also composition, reading comprehension, a lot of work.

AU Plus all the extra activities. Okay, 'most helpful to help you continue to develop during your first year', 'Attending conferences or seminars', 'Reading articles', 'Finding new materials'. You put 'Learning from other teachers' the least. You don't think teachers learn from other teachers in Hong Kong?

A(94) Yes, I think they will learn from other teachers and also for me but it's not so important because I think that I have my own ideas to teach the students. Only if I meet some problems then I'll ask some advice from the teachers.

AU Now, Perceptions and Values, 'Do you think you have a definite philosophy of teaching?' You answered 'Yes, quite definite', but nothing there. You have a quite definite philosophy of teaching but you haven't described what it is. 
A(94) Yes, because I just said 'Not quite definite'.

AU You said 'Quite definite', do you have any idea what your philosophy of teaching might be?

A(94) I think learner-centered approach can help the students to, erm, learn the language and also I think the students will be interested in activities, they will learn more language from activities unlike the teachers in the past, they just teach the students using the textbook and I think this is boring.

AU For 'areas which you are unsure about' you said 'Professional responsibilities'. Why do you think that?

A(94) Yes, apart from teaching, I'm not sure I have to do any more jobs required by the school and the principal.

AU 'Which of the skills do you think is most important', you said 'Grammar' is very important and then 'Speaking'. Why do you think grammar is the most important?

A(94) Because it's the most basic thing of learning a language, besides the four skills they should have a good background of grammar then they can do the four skills better.

AU 'Strengths and weaknesses', you said your main strength 'Teaching writing'. Do you think you are skilled at teaching writing or you just enjoy teaching writing?

A(94) Because I enjoy writing, I enjoy teaching writing.

AU Do you have any special way in which you teach writing?

A(94) Only by stimulating materials, with pictures or cartoons and also activity and discussion. The students learn more in writing. And also with detailed guidelines for the students, they know what to do.

AU Do you enjoy marking writing?

A(94) Not much

AU That's it isn't it, with teaching reading, speaking or listening there's not too much marking, but teaching writing you have a lot of marking to do.

A(94) Yes, marking spend more time than teaching. 
AU Okay, 'Are you planning to teach English next year?' You've said 'Yes'. 'Where and in what type of school would you like to teach?' 'Anglo-Chinese secondary co-educational.' You prefer a co-ed. school?

A(94) Yes, not much problems. I studied in girls school and I experienced a lot of problems. Girls are quite troublesome.

AU Really? I teach in a girls school but I don't really have any problems.

A(94) They won't let the teachers know.

AU They won't let the teachers know what?

A(94) They complex and their problems.

AU True, boys are more open.

A(94) Yes, but in co-ed., boys and girls in the same class, this can help reduce problems.

AU You say you might go into banking if you don't go into teaching. Why banking?

A(94) Because I enjoy working in a bank.

$\mathrm{AU} \quad$ You have done that before?

A(94) Yes, especially for the training and the personnel.

AU Okay, that's it, thank you very much for your help.

A(94) Not at all. 


\section{Interviewee: $\quad$ Subject B(94) \\ Date: $\quad 9$ June 1994}

Female, 29 years old.

Had gained a certificate in education and had taught English in secondary school for two years before joining the BATESL course. Had decided to study the course because she wanted a degree in English teaching so that she could teach higher forms. Was planning not to teach after graduation but might come back to it after a few years.

$\begin{array}{ll}\text { AU } & \text { Researcher } \\ \ldots & \text { Missing words / Unfinished utterance } \\ - & \text { Pause }\end{array}$

AU Good afternoon.

$\mathrm{B}(94) \quad$ Good afternoon.

AU I'd like to ask you some questions first of all about Language Use. Just looking at the first few questions on the questionnaire, it seems that you think that your standard of written English has improved from being 'Quite fair', from being fair when you started the course, and 'Quite good' now, and your spoken English from being 'Quite good' to 'Very good'. Would you say that is true?

B(94) Yes, for the written English, maybe I think in my secondary school, my teachers are Chinese, maybe they have a different - er - different perception on the written style of my writing, they may prefer some complex sentence. That means they like you to show that you know how to manipulate the sentence pattern. But in this course, maybe most of the teachers are native speakers of English. I think they may prefer simple sentences, and I like, I prefer writing simple sentences so maybe they accept my style so from the grades, from the results of the assignments I feel that my written English is quite good and it give me some confidence to say that my writing is quite good now. In the past the marks are not so high so I dare not to say that my written English is good. So now I have more confidence is writing English.

AU Do you think because the teachers are expatriates that has made a difference?

B(94) Maybe, but they also teach us some skills in writing academically so I think it helps a lot.

AU And your spoken English?

B(94) I think it's because I have more practice in the BATESL than in the secondary school because I must use English to communicate with my teachers, now. In 
the past I seldom ask my teacher anything and there are too many students in the classroom so teacher may not have the chance to ask me every lesson, so I have less chance to practice. But now I have more chance to practice.

AU You wouldn't have used English very much in secondary school?

$B(94) \quad$ No, because of personality, I don't like talk very much, but after I've taught for two years maybe because the job force me to talk more, so I become more talkative now. So in these years I talk more, and in BATESL course if I want to communicate with my lecturers I must speak English. So I've more chance to practice, and more practice it help me to improve.

AU Good, 'When speaking English to friends who have Cantonese as their first language, do you use some English words or expressions?' You said 'Always'.

B(94) Yeah, because I receive English media of instruction in my secondary school, so usually the terms are written in English, and I can memorise easily, more easily and it comes to me more readily and I try to use them. And also when I'm talking to friends its more leisure so I don't need to try to translate it back to Chinese. Because when I teach I can't use sometimes English and sometimes Chinese and I have to translate all the English into Chinese if I use Cantonese as my .... and so when I talk to my friends I don't need to be so serious, I don't need to translate it so I always will have some English words.

AU What kinds of English words?

B(94) Maybe some terms, - erm - usually maybe some technical terms, it depends on the topic.

AU Would it depend on who you were talking to?

$B(94) \quad$ I think if it's my friends we share similar education so he or she will understand what I'm talking about.

AU And code-mixing, sorry code-switching, you said 'Quite often' you would switch from Cantonese into English.

B(94) Erm - in some case maybe I will find that some phrases or some expressions in English can help me to express myself more easily and I will use that English phrase or idioms or proverbs, so it depends on the situation, and the topic.

AU Would you switch into English for how long?

$\mathrm{B}(94) \quad$ Maybe for one to two sentences.

$\mathrm{AU} \quad$ In what kind of situation or what kind of person would you be talking to? 
B(94) Maybe some close friends or best friends or maybe sometimes we are playing jokes on each other. So, maybe teasing that, I will say 'You're so stupid', then I will use English.

AU Which leads us on to English in the classroom. 'Do you think that English should be the only language used for all purposes?' You've said 'Absolutely disagree', sorry 'Absolutely agree', 'Increase exposure and code-mixing will confuse a student'.

B(94) I think - erm - because it's an English lesson and if you speak Cantonese or you use Cantonese to explain some terms, the student will wait for your Chinese explanation, they will not listen for the English, your English explanation. And usually when you speak a sentence in English and you add some Chinese, that is code-mixing, you will confuse them, because sometimes when I listen to others they use the code-mixing, sometimes I can't get which word is in English, which word is in Chinese if I'm not really listening. So I think that code-mixing is quite confusing. Because it's the English lesson, it's not the content area so I think it must use English.

AU Would you vary the amount of English, if the students are weak?

$\mathrm{B}(94) \quad$ I will speak slower and maybe explain more or use other gesture or examples something like that to help me explain.

AU 'Lesson content can be presented in mixed-code', you 'Absolutely disagree' with that, which follows from this one. Then you said 'English is English not Cantonese-English'.

B(94) I mean the code-mixing because it's an English lesson, so English is the English, you can't use Cantonese and then English because I feel that translation, the method of teaching is not very effective if you just translate a whole sentence because sometimes the sentence meaning cannot be fully translated. You can't translate 'he' that means Cantonese, you can't do that, so English is English.

AU Right, 'During your Practice Teaching module how much did you use only English?' And you've said 'Always', and the amount of pure English you used didn't change over the practice period, so you used English all the time from the beginning right through to the end. And 'In what situations might you use Cantonese in an English lesson?' You circled just one, 'To perform other lesson functions' being 'To give individual guidance to very low English proficiency students.'

B(94) Because, erm, sometimes when I give them the instructions, some classwork, than I allow the students to do that, and then when I check that I will walk round the classroom and check their performance. Some students they don't 
know what I'm talking about, they can't follow the instruction and then, at first I will speak English to them but if they just look at me and show no response then I need to use Cantonese to tell them what to do.

AU To give instructions?

$\mathrm{B}(94) \quad$ Yes

AU Would that involve things like explaining how you want them to carry out an activity?

B(94) For individual work yes, I'll tell them what they need to do, what she or he is going to do, for example, tell the to look at the example and follow the example to do the others and tell them on which page.

AU I mean if you wanted to say 'Ok, form into groups of four and ...' this kind of thing?

B(94) If the whole group do not understand then I will tell them, but before I speak Cantonese I will use English first, but if they really don't understand, if this is group or pair work maybe I will ask the others, his neighbour or the other groups to tell them what to do. But all of them do not know then I will use Cantonese with that group and tell them. If the whole class do not understand then I will go back to the front of the class and explain it again, but also in English but give more examples and slower and break it to step by steps.

AU Would you use [Cantonese for] things like to establish discipline, maybe it didn't occur when you were teaching, but if it did would you use Cantonese?

$B(94) \quad$ It depends on maybe it depends on the standard of the students, if they don't understand then I must use Cantonese but during my practice teaching I still use English to control the discipline.

AU And how would you feel about the students during the English lesson, would you insist that they always use English?

B(94) I tell them to use English but behind my back they will use Cantonese, it's the usual case, but if I know that they use Cantonese then I will tell them to use English. And some of the students they use English during the group activities but some they sit and say nothing.

AU What kind of students were you teaching during your teaching practice?

$\mathrm{B}(94) \quad$ You mean the Band? Two to three.

AU Mixed, boys and girls? 
B(94) Yes, Form Four students.

AU Okay, Instructional Planning and Decision Making, 'It's impossible to have a successful lesson that hasn't been well-planned in advance.' You said 'Quite Agree', 'Materials and teaching aids need to be prepared before the lesson.'

B(94) Erm, if you teach grammar, usually you can teach a lesson without planning, you can just go into the classroom and open your book and then if you know what you are going to do then you can teach it. Sometimes the lesson will be more effective if you have some teaching aids or some handouts or some worksheet prepared, so if you want to improve the lesson, make it more effective then preparation is necessary.

AU From that point of view it is possible to have a successful lesson.

$\mathrm{B}(94) \quad$ Successful, the students they can still get the knowledge, but it will be maybe boring, or you need more time to explain some points but students can still get the information.

AU 'Should teachers write detailed lesson plans for every lesson they teach?' 'Quite disagree', 'Lesson plans can be mental ones.'

$\mathrm{B}(94)$ I mean you don't need to write all the details, for example the blackboard arrangement, or materials you're going to use, you can just mentally and you can jot down some key words in your text book, for example, 'after finish this part you distribute the work sheet', just write down the key word 'worksheets'. So I don't know, It's quite time-consuming to write down a detailed lesson plan.

AU 'Some items of the plan, for example details of the activity are not necessary because the activities were designed by the teacher and he or she should know them.' Okay, so you mean that once you're designing the materials you're going to use, in your mind you're planning how you're going to use them anyway so actually to write down in detail is not necessary.

B(94) It's not necessary to write the details. I'm the college of education [in] which I studied for three years, they said that in your lesson plan you should write down your questions, and then the response of the students you expect. I feel that it's not necessary, which questions you are not reading out from the plan you can teach a lesson more flexible if you have a mental one. And also the time limit, or the time that you spend on that part, for example 'two minutes for asking the question, for finishing this activity' is quite, not practical, because sometimes you will overrun, usually you will overrun. So it's meaningless.

AU So do you think it might be useful if you have a lesson plan, a detailed lesson plan, once you've taught the lesson you can add a post-lesson evaluation of 
how the lesson went then that could perhaps help you teach the same or similar lessons again.

$\mathrm{B}(94)$ Maybe also mental, because you have that experience you will know what to do next time. Maybe some important points you can mark down on the text book, for example some misunderstanding that caused the students, you can mark that down, then when you teach that lesson next time, you know the problems.

AU Personally I don't write detailed lesson plans, you can't do it, not if you have to teach 28 lessons a week, but I have a diary and I do write down brief details of every lesson otherwise I'd forget what I'd taught in the last lesson. I have to write down what I've done so that I know where I am. But as far as detailed lesson plans go, sometimes I might write a lot depending on how much time I have or what mood I'm in. 'Teachers should be more responsive to student needs than the set syllabus.' 'Quite agree', 'However to finish the syllabus is quite important in Hong Kong education system.'

B(94) Yes, need to consider the students, because if they don't understand, the need for more explanations, so you need to explain them you can't just say 'I've planned this lesson, I need to finish that part,' you can't do that, you must make sure all the students understand the part before. But also to finish the syllabus is very important, because the students need to have the examination at the end of the term. And also, there's not only one class in that form, you have other teachers, if they finish faster then you must chase them.

AU Yes, that's why I think some teachers prefer to teach lower forms because you are not tied so much to a syllabus, you have a scheme of work which you are supposed to cover but basically your scheme of work at Form One, Two, Three level covers the same things, you cover the basic things, so you have more scope to experiment, but if you teach Form Four, Five, Six, Seven you're tied to the syllabus, it's up to you how you teach it but it's very controlled. 'How detailed do you think the plan should be when planning lessons?' 'Brief and very flexible'.

$\mathrm{B}(94) \quad J u s t$ include some of the key points or just mark on the textbook.

AU Do you think that's possible for every teacher? That's the way you like to do it but do you think every teacher can do it like that?

B(94) Some teacher may prefer writing detailed lesson plans, if they don't have confidence, because I know that when I first taught in the secondary school, that's my real job, I used to write lesson plans, so at the first week or first two week I need to write the lesson plan but not very detailed one, it include all the necessary parts, for example the activities, the questions. But then afterwards when I used to teaching then I don't write the lesson plans any more 'cause it's 
quite time-consuming. Maybe some teachers they prefer writing the lesson plans to remind them.

AU So you went to education college, is that right? Then you taught for two years, then gave up teaching and came and did this BA course full-time. Why did you do that?

B(94) I want to get a degree, and I like English.

AU 'During your Practice Teaching module, how often did you find you had to change your lesson plans?' 'Sometimes'.

B(94) Because sometimes I can't catch up with my plan, so I have to change it, cut it short, but sometimes, maybe they need more examples or sometimes I feel that the students need another activity other than the activity that I've planned then I will change it.

AU 'On what basis did you plan your lessons, rank the following, 1 being the most influential and 7 the least.' You didn't put 1 but you put two 2s, 'Own ideas', 'Perceived needs of students'. The others as $3 \mathrm{~s}$ and perhaps the least influential.

B(94) I won't consider the syllabus that the Education Department has set, but I will follow the scheme of work of my panel teacher, during my practice teaching, I followed the plan of my cooperating teacher. I never look up the syllabus, so I don't know if he follow the syllabus or he set the syllabus himself. I follow his instructions but sometimes I will use my own way of teaching. For example he had prepared some worksheet, but if I don't want to use that worksheet then I won't use that. He said I can design my own worksheet, my own materials so sometimes I will use based on my own ideas and I will plan my lessons because after one lesson I know their standard of English, then I will plan the kinds of work that should be done.

AU Yes it's easy to do that, once you've taught them. What do you think about the teaching practice, do you think it was long enough?

B(94) No it's too short, because there is only two weeks and the teacher's schedule is quite tight, and you know, in the English lesson there is composition, comprehension, précis, textbook readers, oral, listening, there are lots of things, so for example composition I've got one chance only to do composition with the students so there is not enough practice for me. So the practice is too short. In the college of education, we have six weeks of teaching practice then it's easier for the teacher because then you have a better relationship with the students and you know them more, you understand more and then you can teach them more effectively. I remember in the first week, my students dare not say a word and they give me no response, whenever I ask questions they say nothing, no one raise their hand, but in the second week, the situation has 
improved but it's quite too late.

AU 'The factors which had the most influence on the decisions you made' You said that the most influential was time, you added 'Time' as an extra factor.

B(94) Yes, it's too short so I can't put all my ideas and for example my first lesson of précis writing is not that good, maybe I don't have a chance to correct it because too short the practice.

AU Followed by 'Fellow teachers' advice', 'Student teachers' advice'. Teaching Approach, 'Examination requirements should determine what is taught in English lessons.' 'Quite agree', 'Should be stressed on what students should have learned to evaluate the teaching of the teacher.

B(94) That means - it's not to test what you have taught during the lessons because maybe you have taught a lot but the student really learned so the requirement should be what the student have learned.

AU Rather than examination requirements, although examination requirements, as we said earlier, are important too. 'Students learn English better through interaction with other students rather than through direct instruction from the teacher.' 'Quite agree', 'Students use English in their interaction then maybe a good way but if students' level are low then they can't benefit.'

B(94) If the students have good spoken English and they have more confidence and their English standards are high then they are willing to speak in English, they have interaction with their fellow students, because they know how to use English to communicate. But if the levels are low then how can you ask them to use English? They will just sit there. So if the students' standards are high then to teach English through the interaction with other students is a good way. If their standards are low it will be, it will make them more scared of the English.

AU Is it possible do you think to combine good students interacting with ones whose levels are perhaps not as high?

$\mathrm{B}(94)$ It depends on the activities, because in a group discussion you mix the good students and the poor students then maybe the good students will dominate the discussions and the poor students they will say nothing, or even when you ask them to speak they will say 'You ask him, he's better', so it depends on the activities.

AU 'A class should be strictly controlled by the teacher and all lesson content directed by the teacher.' You 'Quite disagree' with that, 'Teachers should encourage students to produce their own language, increase the chance of practice. Teacher can know if the students have learnt the knowledge or skill.' 
$B(94)$ So if you just the teacher talk through the lesson then maybe the students fall asleep and the teacher will not know did they really get that information. So the teacher should provide time for the students to practice and to use it so that the teacher will know if the students have learned that skills.

AU And experimentation, 'Teachers should be prepared to experiment with materials and teaching strategies.' You 'Quite agree' but you've added, 'But with careful detailed planning before implementing.' Why do you say that?

B(94) If you have designed some new materials or teaching strategies, I think you must plan it very carefully, you can't just [say] 'Oh it's quite good idea, it's quite new, maybe I'll try that, because the students may not accept that strategies and if you teach something wrong or you use the wrong approach then maybe the student they will feel the alien and they will shocked, it will shock them and it may not be effective. It may lead to the students have some bad feelings afterwards.

$\mathrm{AU}$ If they find the activities to be unusual or strange, do you think students like to do unusual activities or do they feel more comfortable with a traditional style of teaching?

B(94) They may prefer a variety but not that extraordinary, so maybe they dare not to use it. For example if you ask them to listen to rock ' $n$ roll songs and they write down the lyrics some of them may like the music but some of them may not, so you must plan what songs to use. So it needs careful planning.

AU 'In your Practice Teaching module did you find students learn English better through interaction with other students or through direct instruction?

B(94) Erm, it's because my group of students were quite passive and I think they are used to the teaching method of the cooperating teachers. The teachers always prepare some materials for them and do a lot of talking and give direct instructions. And all the students will just copy the notes or just do some writing and they seldom speak in the classroom. So I think they will benefit more from direct instructions.

AU Because that's what they're used to?

B(94) Yes that's it, they're quite passive.

AU 'Did you control your lessons strictly and direct all lesson content?' 'Usually'. Because of tight schedule?

$B(94)$ They ask other nonsense questions or if they fool around then I can't finish because I spend time on the classroom management. So I will control them before they fool around. 
AU Right, 'Did you experiment with materials and teaching strategies?' 'A little', 'I had to follow the schedule of the regular teacher.' Was that impressed upon you? 'Okay this is what we are doing with this class and you must follow that. 'Did they tell you that?

B(94) No but he gave me a scheme of work and I have to follow the scheme and he had prepared some materials, so sometimes I will use them and because I feel that sometimes they need to practice the skills that they will use in the examination, the HK Cert. examination, for example précis writing, they have the textbook so I will use the textbook but I will give them some extra materials. So I follow some traditional teaching approach. Because they are Form Four students if I use them as my guinea pigs then maybe they will say that I use them to experiment.

AU Do think that then in retrospect it was not a good idea for you to given Form Four for your practice teaching, would it have been better to have been given a Form Three or Form Two?

B(94) No, because they were not so nervous about the examinations because it's internal examinations, so and usually little children they will be more excited when they have new teachers or some new teaching methods, they will accept them more and the teacher will feel more happy when teaching some little children because they have more response. So I think teaching lower forms is better than teaching Form Four or Form Seven students.

AU You say your teaching style is mostly teacher-centred?

B(94) Because I control the students all the time.

AU And you direct the learning, you don't necessarily allow them to interact with each other too much?

$B(94) \quad B e c a u s e$ I have some activities for them to practice in pairs or in groups but not very often because of the scheme, because of the lessons. For example composition, you can't ask them to do group work, they have write individually. And also the comprehension is explaining the textbook, maybe the questions I will ask them to do in pairs or to do it at home, then I check the answers. And the listening, they will listen to the tape, there is no interaction. Only the oral lesson they will have the interaction.

AU 'Which of the following factors do you think has the most influence on your approach to the teaching of English?' You put a few for 1, 'Class size, educational requirements, personality of teacher, motivation of students and English proficiency of students.'

$B(94)$ Because if there is only ten students for a class then I can give them more 
B(94) So if you just the teacher talk through the lesson then maybe the students fall asleep and the teacher will not know did they really get that information. So the teacher should provide time for the students to practice and to use it so that the teacher will know if the students have learned that skills.

AU And experimentation, 'Teachers should be prepared to experiment with materials and teaching strategies.' You 'Quite agree' but you've added, 'But with careful detailed planning before implementing.' Why do you say that?

$\mathrm{B}(94)$ If you have designed some new materials or teaching strategies, I think you must plan it very carefully, you can't just [say] 'Oh it's quite good idea, it's quite new, maybe l'll try that, because the students may not accept that strategies and if you teach something wrong or you use the wrong approach then maybe the student they will feel the alien and they will shocked, it will shock them and it may not be effective. It may lead to the students have some bad feelings afterwards.

AU If they find the activities to be unusual or strange, do you think students like to do unusual activities or do they feel more comfortable with a traditional style of teaching?

B(94) They may prefer a variety but not that extraordinary, so maybe they dare not to use it. For example if you ask them to listen to rock ' $n$ roll songs and they write down the lyrics some of them may like the music but some of them may not, so you must plan what songs to use. So it needs careful planning.

AU 'In your Practice Teaching module did you find students learn English better through interaction with other students or through direct instruction?

B(94) Erm, it's because my group of students were quite passive and I think they are used to the teaching method of the cooperating teachers. The teachers always prepare some materials for them and do a lot of talking and give direct instructions. And all the students will just copy the notes or just do some writing and they seldom speak in the classroom. So I think they will benefit more from direct instructions.

AU Because that's what they're used to?

B(94) Yes that's it, they're quite passive.

AU 'Did you control your lessons strictly and direct all lesson content?' 'Usually'. Because of tight schedule?

B(94) They ask other nonsense questions or if they fool around then I can't finish because I spend time on the classroom management. So I will control them before they fool around. 
AU Right, 'Did you experiment with materials and teaching strategies?' 'A little', 'I had to follow the schedule of the regular teacher.' Was that impressed upon you? 'Okay this is what we are doing with this class and you must follow that. 'Did they tell you that?

$B(94) \quad$ No but he gave me a scheme of work and I have to follow the scheme and he had prepared some materials, so sometimes I will use them and because I feel that sometimes they need to practice the skills that they will use in the examination, the HK Cert. examination, for example précis writing, they have the textbook so I will use the textbook but I will give them some extra materials. So I follow some traditional teaching approach. Because they are Form Four students if I use them as my guinea pigs then maybe they will say that I use them to experiment.

AU Do think that then in retrospect it was not a good idea for you to given Form Four for your practice teaching, would it have been better to have been given a Form Three or Form Two?

B(94) No, because they were not so nervous about the examinations because it's internal examinations, so and usually little children they will be more excited when they have new teachers or some new teaching methods, they will accept them more and the teacher will feel more happy when teaching some little children because they have more response. So I think teaching lower forms is better than teaching Form Four or Form Seven students.

AU You say your teaching style is mostly teacher-centred?

B(94) . Because I control the students all the time.

AU And you direct the learning, you don't necessarily allow them to interact with each other too much?

B(94) Because I have some activities for them to practice in pairs or in groups but not very often because of the scheme, because of the lessons. For example composition, you can't ask them to do group work, they have write individually. And also the comprehension is explaining the textbook, maybe the questions I will ask them to do in pairs or to do it at home, then I check the answers. And the listening, they will listen to the tape, there is no interaction. Only the oral lesson they will have the interaction.

AU 'Which of the following factors do you think has the most influence on your approach to the teaching of English?' You put a few for 1, 'Class size, educational requirements, personality of teacher, motivation of students and English proficiency of students.'

B(94) Because if there is only ten students for a class then I can give them more 
interaction because it's more easy to control, but if it's a class of 45 students, and if the classroom is too small, you can't ask them to group in small groups, they will overhear the other groups' discussions so it depends on the class size and the classroom size.

AU It was interesting that a great deal of your colleagues who filled in the questionnaire put class size as very low, they didn't think it was that important.

$\mathrm{B}(94) \quad$ It is important I feel.

AU I would feel that as well.

$B(94)$ So teaching the remedial class may be more easy than teach the normal class because there is only a few students in the remedial class, you can give more individual guidance and check them more frequently. But in a normal class of 45 students, how can you give individual guidance to all of them? You can't check all their work.

AU Right, your teacher training was quite influential as well?

$\mathrm{B}(94) \quad Y e s$, if we compare a person who have received teacher-training and one without then it's quite, the result will be obvious. The person with teachertraining, I'm sure that he or she will teach better, more effective than the other one. He or she will know the skills in questioning or how to arouse their interest, or to consider the students' needs, some more detailed points then he or she will be better in that point.

AU Okay, Professional Relationships and Responsibilities, You are 'Quite confident' in your ability to become a successful teacher, but you've been a teacher before, so it's easier I guess to answer that question. 'How do you feel about the status of teachers in today's society?' 'Teachers have no special status.'

$\mathrm{B}(94)$ Teaching is not considered to be a profession, it's not a profession, so people always think that everyone can be the teacher, so a Form Five student can be the teacher of Form 1, they can give private tuition, so everyone can be a teacher unless you know nothing, you are illiterate so you can't be a teacher. So teachers have no special status. And that's why many teachers leave their jobs, change their jobs.

AU Do you think it will be improved, do you think there's any chance of the status of teachers being improved?

$\mathrm{B}(94) \quad$ I know that some of the associations of the, are fighting for the profession, for teaching as a profession, they start fighting in 1988. They try to write out the oath of teaching or the rules, similar to those professions that they have some 
rules to guide the members but it's quite hard for teachers to have such rules or such guides because you can't say if you break certain rules then you can't become a member, you can't teach. It's different from other professions. So it's quite difficult for teachers to have a special status, unless the government can do something to raise the status.

AU What do you think they could do?

B(94) For example the primary teachers, for example the college of education to become a university that offer degrees to the students then maybe it can help to raise the standards, the status, because people with, the teacher are university graduates, they are not just matriculated students or Form Five graduates because a Form Five graduate can enter the college of education and he or she can teach up to Form Three students. So maybe the problem can be solved.

AU 'Your best attribute in terms of being a teacher', you say 'Good command of the language', 'Good communication skills', 'Innovative in terms of teaching ideas'.

B(94) If you are a teacher of course you must show good command of English otherwise the students have no confidence in you, they will tease at you at the back. So I think the teacher himself or herself [needs] to be a very good communicator or a very good English so that they can give confidence to the students, so it's very important. And also, he or she must have some new ideas or teachings because traditional teaching is too boring. So he or she can use some new ideas to stimulate the students. I think the trends in ESL teaching is not very important.

AU 'Do you see teaching as a long-term career?' You put 'Definitely not'.

$\mathrm{B}(94) \quad$ I don't want to be a teacher.

AU So how long have you spent being a teacher? How long have you spent in education?

B(94) Three years in the college of education plus two years as a teacher and three years here.

AU You spent eight years training to be a teacher and you say you don't want to be one!

B(94) I consider this course as an English course only and I want to get a degree, and I apply this course because it's a teaching course then I think if I can't change my job then I must be a teacher again. But after I finished this course I can teach up to higher forms, I'll get a better prospect. So I take the course. But I don't want to be a teacher because I found that teaching is quite boring for 
young people and also because the students, they are not only academically poor but sometimes their behaviour are very bad, and they really scare me sometimes. And I think I don't teach in Band Four or Five schools. And because I taught in Band One schools in the past, so I found the life is easier but I'm sure that there's not so much Band One schools in Hong Kong so it will be quite hard for me to find another Band One school in the future so I hope I can change my job and I don't want to be a teacher. Maybe in the late thirties or forties I will consider teaching again. It's too stable I think, teaching.

AU You don't want to be stable?

$\mathrm{B}(94) \quad$ No, I want more challenge, because you teach the same thing the next year, you speak the same words to other students but you use the same motivation you use the same activities, quite boring. Maybe I'm lazy.

AU Okay, we'll skip through a few questions now. 'Which the following activities do you think you will have to do as a first year teacher?' You having been a first year teacher already are better able to answer this. You've circled nearly all of them except 'Be involved in religious activities' and 'Attend courses'. Personally I found that I had to do all of them, I wondered just how aware the BATESL students were of exactly how many things they would have to do, things that they wouldn't consider, wouldn't think about.

B(94) Some headmaster or the principal will not encourage their teacher to attend courses.

AU At my school we don't have to attend courses but generally people do, you have to attend courses if you're teaching Form Four or Form Five or Form Six or Form Seven. You are expected to attend courses if there's some change in the syllabus that kind of thing. And religious activities ...

$B(94) \quad I$ don't have a religion.

AU It doesn't matter. In my school, it is a religious school, a Christian school so teachers are expected to attend prayer meetings and that kind of thing.

B(94) Maybe during the assembly.

AU Well the assembly is a religious activity, you have to say a prayer and sing a hymn. 'Which will take up the most of your time?' 'Marking examinations'. 'Learn from other teachers', 'Find materials', 'Get feedback' will help you develop as a teacher in the first year. 'Do you think you have a definite philosophy of teaching?' 'Not very definite', what is it, 'To teach students the content of the syllabus in an efficient and joyful way.' 
$B(94) \quad B e c a u s e$ to finish the syllabus is very important for the students, they will [be] anxious about the syllabus, especially the higher forms and they need to face the public examinations, so you better finish the syllabus otherwise they will blame you. But to teach them in an efficient and joyful way, is also good for the students. Actually I take these words from the aim of the Scout Association, they said to teach the children to be a good citizen in a joyful way, from games.

AU 'Which areas are you unsure about?' 'Professional status', 'Working conditions', 'Students' behaviour and standards'. You say working conditions you are unsure about?

B(94) That means the Band of the school and the colleagues, because some of the colleagues they are very old. The old people they do not want to speak to you sometimes.

AU 'Which of the following skills do you consider most important to teach?' You put them all as 'Very important'. Do you not discriminate between them?

B(94) If the students, if I want the students to have good English then I think they should learn all of them.

AU Strengths and weaknesses?

B(94) Discipline, I can't control the class very effectively. I think organisation goes with planning, to plan the lesson and the activities. Relationship with students, yeah.

AU Okay, let's skip through the General Reflections on the Course. Thank you for your help.

B(94) You're welcome. 
Interviewee:

Date:

Female, 31 years old.

Had been working as a part-time teacher of English in a secondary school for two years and decided to become qualified by taking the BATESL course. Would resume teaching at the school full time after graduation.

$\begin{array}{ll}\text { AU } & \text { Researcher } \\ \ldots & \text { Missing words / Unfinished utterance } \\ \text { - } & \text { Pause }\end{array}$

AU Good afternoon.

C(94) Good afternoon.

AU I'm going to ask you about some of the questions that perhaps came out of the questionnaire that you filled in. Starting off with Language Use. We wanted to get some idea of perhaps how your use of English has changed or not since you began the BA course. Do you think your standard of English has improved?

C(94) It's difficult to say, but to me I just think that it's just more or less the same cause I have been teaching before. During the course I also teach in the school and maybe I don't feel too much progress myself, I don't know, but sometimes I think I learned some more vocab. I should say because as a Hong Kong student I really find it difficult to learn vocab, increase my vocabulary power, but here it's different if you are a student again at school, to a certain extent you must learn some more vocab. So in this aspect I will say yes.

AU Right, do you think it's because you have been a teacher previous to doing the BA course, that your standard hasn't improved that much? Do you think that some of the other students might have improved more than you?

C(94) Not because of my being a teacher before I think, it's because the course is not designed to improve the English skills or standards of students, it's a course on TESL, not too much on improving the proficiency of the students.

AU Isn't one strand of the course on improving the English proficiency of the students?

C(94) They have two strands you mean? They, one is equipping the students to teach English and then the other purpose is to help students get better equipped in their own proficiency. Yes, but I don't think it's successful to help the students 
to improve their own proficiency of English

AU I thought on the BA course there were three strands, English proficiency, linguistic theory and education, something like that. One is meant to improve your English standard, the other one is to teach you about linguistics, about the learning of English and the other one is to give you practice in actual teaching. Is that right?

C(94) I find that there are only two strands, without the linguistic, the second one you mentioned, linguistic theory. There are theories on teaching but I don't think they are linguistic.

AU Well, some ideas, some theories in language learning and perhaps bilingualism, that kind of thing.

C(94) Yeah but these cannot improve the proficiency of students either.

AU Oh yes I agree but, it seemed to me that that was the way that the course was structured. So you put 'Fair', 'Fair', 'Fair', 'Fair' for both before and after.

C(94) Yes

AU 'When speaking to friends in Cantonese who have Cantonese as their first language do you use English words or expressions?' You put 'Always', that's very common?

C(94) Yes

AU Let's say, your friends, would they be other teachers or people in similar kinds of professions? Would they be people who would use English in their work?

C(94) Yes, most of them.

AU So they are likely to have English in their minds let's say?

C(94) Yes and most of them are professionals like engineers, judges, and many of them teachers.

AU What kind of English words or expressions would you use?

C(94) Very common English words like 'buffet'.

AU Ones that are commonly used by people in Hong Kong?

C(94) English words? 
AU $\quad \mathrm{Mm}$, I mean there are some words that are, English words that are used quite commonly by Chinese people in Hong Kong like 'buffet' or ...

C(94) 'Canteen'

AU Yes 'canteen' and they are the kinds of things you would use. And codeswitching as well you said 'Always'. So would actually switch into English and have extended discourse in English?

C(94) Yes

AU Any reason why that is or does it just seem to happen naturally?

$\mathrm{C}(94) \quad$ It just happens naturally, just like when I tell them 'Oh I finished my course' then I will use English.

AU Then that would influence whoever was listening to you to respond in English?

C(94) Yes, you know two people of the same nationality won't speak the second language, is it?

AU Yeah, yeah, although to a certain extent they do if they are code-switching.

C(94) Yes, but not every word in the dialogue.

AU I would say so, unless they're doing it on purpose, to practice. Okay, let's talk about the teaching now. 'In English lessons, English should be the only language used for all purposes.' And you said 'Absolutely agree', but then you changed to 'Absolutely disagree'.

C(94) Yes I made a mistake. 'I've been using English since I started the lessons and the feedback from students has been very good.'

AU So you'd use English for all purposes, for presenting material, for practising or even for communicating with the students?

C(94) Yes

AU Okay, which kind of school have you been teaching in?

C(94) I really don't know the Band of the school.

AU Is the English standard of the students good?

C(94) No, they are hard-working students, but their English is not good. They come after Form Five. They come to study with a purpose. 
So they do have motivation.

C(94) Yes

So you said that the feedback from the students is good so they respect the fact that you purposely don't use Cantonese with them.

C(94) Ah ha, and in fact in the first lesson because I started speaking English since the first lesson and the students don't know that I can speak Chinese. Even when I speak with teachers in the staff room or the general office, when I notice that there are some students around I won't speak Cantonese. I do this deliberately. I think they are very curious, they wonder where I come from and they want me to speak Cantonese, but I just keep their curiosity. So even though they are poor in English, even the first few months, they need to get used to my English. But gradually in fact they can get used to it and they find it's useful for them.

So they haven't actually asked you directly yet where you're from or anything like that? That's very good, it's interesting. 'It's the teacher who makes use of methods to present in English clearly', The question was 'In English lessons, lesson content can be presented in a mixed-code.' And you said 'Absolutely disagree'. So you believe that lesson content should be presented in English?

C(94) Yes I mean, sometimes I find that the teachers just like to get some convenient ways, they don't bother to use some methods so that they can present clearly to the students. Then they get a convenient way of using code-mixing. I think it's very bad to the students. They can't learn anything. They may get used to it, 'Oh, sooner or later the teacher will use Cantonese so I just wait, or I can ask my friends'.

AU Right, so they don't actually listen? They switch off and they wait for the explanation. And in your practice teaching you used English 'Always'. 'How did the amount of English you used in lessons ... it didn't change', so you started off using all English and ended up using all English. 'In what situations would or might you use Cantonese?' 'None', okay, if you were in a different teaching situation perhaps with younger students and poor English ability, do you think you would use Cantonese then?

$\mathrm{C}(94)$ Sometimes I think I will because if the students really can't understand you, because they are really weak in English, then how can you continue the class? And sometimes when you explain something, maybe something abstract, the concept etc., it's difficult for you to explain in English. And if you don't have much time to prepare well, it's very difficult, so you need to save time, you need to make the students understand then the best way is to use Cantonese.

AU But again you would, you may have the problem as we said before, the students might not listen to the English. 
AU Mm, I mean there are some words that are, English words that are used quite commonly by Chinese people in Hong Kong like 'buffet' or ...

\section{C(94) 'Canteen'}

AU Yes 'canteen' and they are the kinds of things you would use. And codeswitching as well you said 'Always'. So would actually switch into English and have extended discourse in English?

C(94) Yes

AU Any reason why that is or does it just seem to happen naturally?

$\mathrm{C}(94)$ It just happens naturally, just like when I tell them 'Oh I finished my course' then I will use English.

AU Then that would influence whoever was listening to you to respond in English?

C(94) Yes, you know two people of the same nationality won't speak the second language, is it?

AU Yeah, yeah, although to a certain extent they do if they are code-switching.

C(94) Yes, but not every word in the dialogue.

AU I would say so, unless they're doing it on purpose, to practice. Okay, let's talk about the teaching now. 'In English lessons, English should be the only language used for all purposes.' And you said 'Absolutely agree', but then you changed to 'Absolutely disagree'.

C(94) Yes I made a mistake. 'I've been using English since I started the lessons and the feedback from students has been very good.'

AU So you'd use English for all purposes, for presenting material, for practising or even for communicating with the students?

$\mathrm{C}(94) \quad$ Yes

AU Okay, which kind of school have you been teaching in?

C(94) I really don't know the Band of the school.

AU Is the English standard of the students good?

C(94) No, they are hard-working students, but their English is not good. They come after Form Five. They come to study with a purpose. 
AU So they do have motivation.

C(94) Yes

AU So you said that the feedback from the students is good so they respect the fact that you purposely don't use Cantonese with them.

C(94) Ah ha, and in fact in the first lesson because I started speaking English since the first lesson and the students don't know that I can speak Chinese. Even when I speak with teachers in the staff room or the general office, when I notice that there are some students around I won't speak Cantonese. I do this deliberately. I think they are very curious, they wonder where I come from and they want me to speak Cantonese, but I just keep their curiosity. So even though they are poor in English, even the first few months, they need to get used to my English. But gradually in fact they can get used to it and they find it's useful for them.

AU So they haven't actually asked you directly yet where you're from or anything like that? That's very good, it's interesting. 'It's the teacher who makes use of methods to present in English clearly', The question was 'In English lessons, lesson content can be presented in a mixed-code.' And you said 'Absolutely disagree'. So you believe that lesson content should be presented in English?

C(94) Yes I mean, sometimes I find that the teachers just like to get some convenient ways, they don't bother to use some methods so that they can present clearly to the students. Then they get a convenient way of using code-mixing. I think it's very bad to the students. They can't learn anything. They may get used to it, 'Oh, sooner or later the teacher will use Cantonese so I just wait, or I can ask my friends'.

AU Right, so they don't actually listen? They switch off and they wait for the explanation. And in your practice teaching you used English 'Always'. 'How did the amount of English you used in lessons ... it didn't change', so you started off using all English and ended up using all English. 'In what situations would or might you use Cantonese?' 'None', okay, if you were in a different teaching situation perhaps with younger students and poor English ability, do you think you would use Cantonese then?

C(94) Sometimes I think I will because if the students really can't understand you, because they are really weak in English, then how can you continue the class? And sometimes when you explain something, maybe something abstract, the concept etc., it's difficult for you to explain in English. And if you don't have much time to prepare well, it's very difficult, so you need to save time, you need to make the students understand then the best way is to use Cantonese.

AU But again you would, you may have the problem as we said before, the students might not listen to the English. 
$\mathrm{C}$ (94) Yes but I think in some occasions I will accept that, even though I don't use Cantonese myself, I will accept teachers using Cantonese in some occasions, but not to give the students an impression that, oh you will use English in every lesson.

AU Do you think, presumably if you only use English yourself in lessons, does that mean that you insist that the students only use English?

C(94) No, only myself, I never insist that the students have to use English. In the beginning yes, but I won't make it as a rule that they have to speak in English because sometimes they really can't express themselves. But I will create an atmosphere that they need to speak in English. I encourage them to try first unless they really can't then they will murmur or they will talk with their friends in Cantonese. I understand it right and so I try to help them and then they will make it up.

AU So if you insist that they speak only English you put too much pressure on them?

C(94) Yeah and they will be scared, they dare not speak up afterwards I think. Because I have tried before I come here, in my teaching I did that, the atmosphere is very tense!

AU Yeah, I agree and I don't make it a strict rule that my students should only use English during a lesson even though I understand very little Cantonese. With my Form Six students then I might, if they are using too much Cantonese and I find particular things where they are supposed to be discussing things in English and the whole point of the exercise is oral practice and they start using Cantonese then I will pull them up and say 'Don't use Cantonese', because they are Band One Form Six students and they shouldn't have to use Cantonese anyway. If they do they are just being lazy, but normally with the lower forms I don't make it a hard and fast rule. I think some teachers will do perhaps, if only to create a quiet classroom. If the students can only speak in English then they might not speak at all, so they are not going to be too noisy, but sometimes they need to speak Cantonese to each other if only to ask each other about lesson content or what to do next and that kind of thing. It does make the atmosphere less tense.

Right, let's go on to Instructional Planning and Decision Making. 'It's impossible to have a successful lesson that hasn't been well-planned in advance.' You 'Absolutely agree', 'Planning is very important for every lesson'. So you've said that planning is very important, but how detailed should the plans be? 'Teachers should write detailed lesson plans for every lesson they teach.' You 'Disagree' with that. So one the one hand we've got that a successful lesson has to be well-planned ... but the key word is this word isn't it? 
C(94) Yes

AU 'Depends on the experience of the teachers and I find that I gradually only take a small piece of paper to remind myself.' It's a bit of a leading question I think, no teachers are expected to write detailed lesson plans for every lesson they teach. I think you had to in your teaching practice, is that right?

C(94) Yes, that's why I didn't get used to writing detailed lesson plans, such detailed lesson plans but I think every lesson I have to plan well before I go to the classroom otherwise I really don't know what to do and it's difficult to continue the lesson.

AU Do you think the fact that you have some experience in writing detailed lesson plans has helped in the way that you approach planning lessons generally.

C(94) No, you mean a detailed one?

AU I mean if you, after you've written a detailed lesson plan let's say you're thinking about your lessons do you kind of have a detailed lesson plan in your head?

C(94) I think [in] my lesson plan I will jot down all the things that I will do in class, but not so detailed as in 'Oh this part I should say this and this should be the response by the students'. Not so detailed as the one we have to hand in to our lecturers, but I think with a skeleton of what you are going to do, the things that you need to do you list them out, that's my lesson plan. And for the time allocation, I will also list them out telling ' $\mathrm{Oh}$ this part I will roughly use say ten minutes', something like that.

AU As you go on in teaching then you write less and less lesson plans.

C(94) Sometimes I will omit time allocation because if I list out the items that I have to do, I know I should not put more than five items this time, 'Oh I should not do this, maybe leave number six to the next lesson because I know I don't have enough time for that'. You will know how you will use the time and roughly how much time you need.

AU My lesson plan as such might consist of sometimes just one sentence, sometimes maybe half a page, it depends on what I want to do. If you've taught the same lesson again and again then that's all you need to do.

$\mathrm{C}(94)$ And in reality in fact, teachers don't have time to have detailed lesson plan.

AU 'Teachers should be more responsive to student needs than to the set syllabus', 'Absolutely disagree', 'Teachers should be flexible enough to suit the needs of students, for example slowing the speed instead of teaching the whole 
syllabus.'

C(94) It seems to be contradicting isn't it? Maybe I haven't corrected this one should be ...

AU Should be number one?

C(94) Yes

AU Right, students should be more responsive to student needs than the set syllabus. There are obviously situations I think when the set syllabus if it's public examination syllabus then you are guided by it. 'How detailed do you think your lesson plans should be?' 'Loosely detailed and flexible'. Okay, as we've said, to be very detailed and precise - we'd all love to be, I'm sure we'd all love to be able to sit down and write a detailed lesson plan for each lesson we teach, but probably if we did that we could only teach one lesson a day. 'How often did you find you had to change your lesson plan?' 'Sometimes' In what situations did you find you had to change your lesson plan?

C(94) If I find that I am running out of time then I will leave one part and sometimes I suddenly find that the students prefer another method of doing the exercise then I will change my plan. Just like today I put on the transparency and instead of asking the students to rewrite the whole part, I showed them the lyrics of the song. Instead of asking the students to rewrite into a prose writing then I asked the students to pick out the mistakes in the lyrics.

AU So you changed your plan while you were actually in the lesson, a kind of inprocess decision if you like, was that based on the response of the students?

C(94) Yes

AU Had you thought about doing that before?

C(94) Yeah, I thought of that before, and sometimes it depends on the mood of the students.

AU So in a way you didn't change your lesson plan, you had alternatives in your plan?

C(94) Yeah you can say so.

AU 'On what basis did you plan your lessons?' 'Syllabus', was that the syllabus given to you by the cooperating teacher?

C(94) Yes, but if I'm not having my practice teaching I won't choose syllabus as the first priority. 
AU You would choose 'Perceived needs of the students'? If you have to follow a syllabus then it doesn't necessarily mean that everyone has to teach in the same way.

C(94) Yes, even we are having the same syllabus, different teachers may teach differently. Even I have to base on the syllabus I can use many ways to make the students get interest in it, so I think I can do that, that is make the students enjoy the lesson.

AU 'What factors do you think had the most influence on the decisions you made?' You say 'Supervisor's advice', followed by 'Regular teacher's input' and then 'Students' needs' and then 'Fellow student teachers' advice'.

C(94) I need to be practical that's why I choose 'Supervisor's advice' and then secondly must be the 'Regular teacher's input'. These are the two main criteria that I have to look upon I think, otherwise I will say 'Students' needs'.

AU Right, so during your practice teaching, you'd say it was not an authentic situation as regards what teaching is really like. It was an assessed situation rather than getting experience. Do you think two weeks is long enough?

C(94) Sometimes I will think it's enough if the school is not very good.

AU If the school is not very good then you don't want to spend more than two weeks there right?

$\mathrm{C}(94) \quad$ Yes, and if the supervisor or the regular teacher cannot help me too much then I will say two weeks will be okay. But in my practice teaching we had two times going out. I remember the second time I find that in fact two weeks is not enough, because two weeks I've just got used to the students in the school and then I've finished! I can do more with the students after I know what they want, what I can help them more, but then I can't.

AU Then it's too late isn't it?

C(94) But sometimes like, you need to have a sort of warm-up with the students and students and also you need to get to know more about each other. All these can take two weeks.

AU I agree, I think six weeks would be better.

C(94) I think even if I go to a good school that I want, two weeks in fact is not enough if you really want the students to have practice in teaching. It's not realistic for us to go in the school and do something just for two weeks.

AU Teaching Approach, 'Examination requirements should decide what is taught 
in English lessons'. You said 'Quite agree', 'The exam requirements serve as the basis of the lesson.' 'Students learn English better through interaction with other students rather than through direct instruction from the teacher.' You said 'Quite disagree', 'It depends on the standard of the students, for adults the statement may be more applicable.'

$\mathrm{C}(94)$ I know many people will say 'Oh teaching should be more student-centred', and then a lot of theories have some support on this, but I don't know in other countries but in Hong Kong in fact it's very difficult to achieve. Just now you said if you assign something for the students to do they will shift to Cantonese instead of English. You know, I mean, what's the reason behind, do they really learn much from that? I doubt, but if you say 'Oh then should we give everything to the students or let them learn through direct instruction?' Then I will say it's not healthy for the students either. So it's better to have something balanced, but not to have the students do everything by themselves.

AU So it's some kind of, somewhere in between really.

C(94) And it also depends on the standard of the students, and even how old they are. If you have a lower form, it's difficult for you to control the students and in fact they don't know the purpose of your activity, they won't try to learn what you assign to them.

AU They won't be able to learn from each other?

$\mathrm{C}(94)$ Ah ha, because they can't appreciate what you want them to know. But if you have an upper form the students may be able to appreciate your teaching or using different methods, having student-centred.

AU 'The class should be strictly controlled by the teacher and all lesson content directed by the teacher.'

C(94) Again 'Depends on the level of the class in Hong Kong secondary schools usually yes'. You wouldn't say that students can direct their own learning very well?

AU No, Hong Kong students.

C(94) I'd agree with that.

$\mathrm{AU} \quad$ In fact sometimes when the teacher give activities to the students, the class still is under the control of the teacher. Only that it's not so strictly controlled or in fact I should say it's not done tbrough talking by the teacher all the time but give some time for the students to interact, to talk. But still the class is under the control of the teacher. 
C(94) Yeah, but perhaps not strictly controlled by the teacher. I mean it may be clear from the response of the students that another approach may be needed, so in effect the students do control the lesson.

AU Yes. 'Teachers should be prepared to experiment with materials and teaching strategies.' Yes, 'Very much agree', 'I've been trying new things in my teaching since I started to do this course, it did give me some inspirations.' Where would you kind of look for materials and teaching strategies, you say you get them from the course itself?

$\mathrm{C}$ (94) Yeah some from the course, you know I have a lot of stuff, as a teacher even when I use the same stuff, I can turn it into something new because I have some books, some reference books and I use them very differently this time because I learn that I can use some methods like that. Some materials I get from the library and even from the coursework, the lecturers have given us some interesting pictures, I still keep them and then I think 'Oh this time I can use it'.

AU So you actually use materials that the lecturers have used with you and use them with the students?

C(94) Some of them.

AU In the same way or will you adapt them?

C(94) I'll adapt.

AU But you gain inspiration from the way in which they are used?

C(94) Yes, a lot.

AU I think that rarely will you find materials from a textbook that will work with your own classes, you have to adapt them in some way.

$\mathrm{C}(94) \quad$ And sometimes for example I get a passage and there's no particular strategies or interesting activities from the passage and then I make it up.

AU Do you find that easy to do?

C(94) No it's very difficult but I find it easier after I finish the course, I've become more flexible and more clever in using the materials.

AU Right, do you get more satisfaction from making up your own materials than you do from using those from the textbook?

C(94) Yes, very satisfied after I've finished the class. 
AU 'Did you find that students learn English better through interaction with other students than through direct instruction from the teacher?' 'Through interaction with other students'. You didn't make any comment about that.

C(94) Maybe this is for practice teaching module and usually my cooperating teacher will be in the classroom and do two teachers looking at them they have to speak in English, they have to do the activities. So they learn something. May not be in real situations.

AU 'Did you find that you controlled your lessons strictly and directed all lesson content?' 'Usually', 'I'm not strict but I made the students respect me naturally.' How do you do that do you think?

C(94) It's a very difficult question, it's really difficult to say. I never scold a student. I think I am humorous in the classroom and I am clever enough to know what they want, to know what they want to talk with their friends and before they can do it I can stop them. In fact sometimes I don't intend to stop them but I will make the class laugh.

AU I understand what you mean, I think they call it 'withitness' or something like that, you can spot problems in the classroom before they arise and deal with them in a light-hearted way.

$\mathrm{C}(94)$ The learning atmosphere is very different, the students love it and the students will also like the teacher because the teacher not only know their needs, the teacher can also help them but not force them to learn, especially English which they have no interest at all in fact especially in Hong Kong. And if they don't have much motivation it's really bad for them to sit in the classroom. So the learning atmosphere is very important, the teacher has the responsibility for that I think, to create an atmosphere which the students like and able to learn.

AU Is it easy to do that? Sometimes the students don't like learning English and if the syllabus is not interesting it might be difficult to generate that kind of atmosphere in the classroom.

$\mathrm{C}$ (94) Yes, and just like reading and writing. In the exam they have to read several passages and they need to write two essays for example, they hate that. But in the class they have to do it, they need practice, but what are you going to do? Don't give them any passages or never ask them to write? Throughout the year I need to think every lesson how to make them enjoy reading the passages and then I select the passages carefully, something that interests the students and before I give the passage to them I will ask some questions to arouse their interest or ask the students to do something about it. I remember one passage about neckties, and I planned to get some ties in the staffroom but I failed and when I go to the classroom, some students wear ties that day because they need to take pictures afterwards so that's great and then I make use of them. I think 
teachers need to think of some methods, something that can interest the students and writing is a difficult part because Hong Kong students are very afraid of writing, making a lot of mistakes and every time they get back their work 'Oh full of red marks!' They feel that 'Oh, after I've written so many pieces I still can't have improvement'. It's difficult for them to really get something concrete, so whenever I ask them to write, I won't ask them to write in fact I will make them write naturally.

AU Rather than 'This is the task that you have to write'.

$C(94) \quad Y e a h$, and usually they write with a purpose and then they are very enthusiastic writing it.

AU That's great if you can do that, it's not always possible though but the more you can do that the better.

C(94) Yeah

AU 'Did you experiment with materials and strategies from a variety of sources.' 'I made use of the strategies, invented materials for my students.' Okay, mostly teacher-centred your teaching style?

C(94) Ah ha

AU Okay, 'Which of the following factors has the most influence on your approach to the teaching of English?' Your 'Teacher training'.

C(94). Yes

AU That's interesting, [Subject F], the girl who talked to me before, said the same thing, 'Teacher training'.

C(94) In fact as a student, she finished Form Seven and then she came here and then she wants to become a teacher, so teacher training should be the main factor I guess. But before I came here I've been teaching for some time, but I still find that teacher training is important because in fact now I'm teaching in the same school part-time and then in July I will teach there full-time continue. The year that I teach part-time there is very different from the previous years that I taught there and I find that training here is the main factor that affect my approach to teaching and the methods I use in the classroom and my attitude, everything related to my career.

AU So you'd say you are doing just about everything differently now? 
C(94) Yes, very different and I like it. If a person who wants to be a teacher and she just go directly to teaching, I will say number two, 'Personality of the teacher', is the main factor that affect his or her teaching, because in my previous teaching, I think this influence my teaching very much, personality, and that's why I was very strict and that's why the atmosphere of the classroom was very tense, something like that. And I was not willing to use new materials, I did not know other methods that I could use, but if I know more and learn more I can expose to different things. That's why my teaching is very different now.

AU From what you've learned, from what you've experienced or maybe just from the way it's expanded your awareness of what possibilities there are.

C(94) Yes, that's right.

AU I think that's the main purpose of a teacher training course. They say they can't make people into a good teacher immediately but you learn teaching by experience mainly which makes you aware of different things you can do.

C(94) A successful training course should be like that.

AU Do you think that this is a successful training course, the BA course?

C(94) Er yes, but not absolutely.

AU 'How confident are you in your ability to become a successful teacher?' 'Very confident', okay, 'How do you feel about the status of teachers in today's society?' 'Teachers have a lower status than they should', why do you think that is?

C(94) Sometimes I feel that the teachers are not very much respected in society in a way that they should be respected. People just know that teachers go to school every day and teach, they don't know much about the burden, the responsibility, the work-load of the teachers so much. Maybe they only know 'Oh, being a teacher you have a lot of holidays'. They don't see being a teacher as an important and great job influencing their children.

AU Do you think it's because of the way that people perhaps remember their own teachers? In traditional Chinese society, teachers were well respected, but now they are not as respected as doctors or lawyers, why not?

C(94) Number one, the teachers do not have such a high salary as the doctors and the working environment is not so nice as the clinics or the hospital. The image of the teacher is that they have piles of books to mark.

AU Do you think there's some anomaly in that parents expect the teachers to be professional when they are looking after their children but at the same time 
they're not held, they're not considered as such high status.

C(94) Yes

AU Okay, 'Your best attribute of being a teacher', you've put 'Personality as a gifted teacher', combined with this is that you are very confident in your own ability. Why do you think that is?

C(94) Because I have experience, because before I started teaching, I have tertiary education, not here, I start teaching part-time already, and I find that the students like my teaching, and I find that I could explain quite well, quite clearly, and I could help them. From that feedback, I know that I can do it well. And after I finished my studies and I started teaching and for three years I found that I was successful again and the school and the students gave me good feedback, and after I've got teacher training here, I've become more confident because I have some more as my strategies.

AU So you were tertiary educated already?

C(94) Yes

AU So why didn't you just do a Cert. Ed.? What made you do the BATESL course?

C(94) I did not have a degree, just a diploma.

AU But you could have carried on as you were?

C(94) . Oh yes, that's right. After I've been teaching for two years, I start thinking of continuing my studies. I began to feel stuck in my teaching, 'Am I going to teach every day, in the classroom, in the same boring method?' I can't. That makes me think of getting more knowledge in teaching and then I start looking for courses and in that year in the third year in my teaching and this is the first time that CPHK have the course here in Hong Kong, so I applied for it. I think it's the right time for me to learn more.

AU '... teaching as a long-term career?' 'Definitely', 'Most helpful for you', will be 'More experienced teachers', 'Teacher training supervisor', that's the crucial thing isn't it?

C(94) Ah ha

AU 'Which do you think you will have to do as a first-year teacher?' None of these? 'Meet parents', 'Counsel students', 'Extra-curricular activities'.

C(94) All these should be done in the secondary schools, but because my school has been running post-secondary programs, and so the teachers do not need to do 
these things. And that's why I can concentrate more on my teaching.

AU Very nice! When I wrote this from the point of view of a secondary school teacher, certainly in my school you would have to do all of those, you would have to circle every single one, including such things as 'Religious activities', which you might not expect if you are not a religious person. It's just as a kind of test to see if students are really aware of the kinds of things they will have to do. '... taking up the most time', 'Marking papers', 'Marking exams', marking basically.

C(94) Language teachers need to mark.

AU 'Working closely with other teachers', 'Some', do you think it's particular about Hong Kong that teachers don't cooperate enough with each other, they don't share ideas, they don't meet very often?

C(94) It depends on different schools because I've gone to my secondary school where the English teachers meet once or twice a month and then they discuss about their students and also the methods they use. And they also take turns to look at each other's teaching. But in some other school they really seldom talk about their teaching, they just gossip about their students but not constructive.

AU I've found that too, it's very rarely that teachers actually cooperate with each other, that they have meetings and discuss what progress each is making or have some kind of pool where they share materials. There is some but not as much as there is in England. Teachers here seem to be very insular, they don't like to tell other teachers what they are doing in their own classes. In a way perhaps they are shy or embarrassed.

C(94) You know if you tell what you have been doing in class with the students or what methods you use, maybe they are having different training from you, they have a different background from you and you will make them uneasy. In fact they may not have the interest to listen to you and I don't know if it's the culture of Hong Kong people, they think that they have their own individuality. They don't have to share with the other people even though you are teacher, I am the teacher.

AU They don't think they can gain anything?

$\mathrm{C}(94)$ They just are not open-minded enough to listen. If you tell them the things that you have done, if they haven't done it, they will feel embarrassed, they feel uneasy. If you have done something good, you share with them something sad or something not quite successful, they find uneasy either because they don't know what to help you, they don't know what to do. And some are not willing to give suggestions. 
AU Yes, I've found that too, a lot of teachers [are] very secretive about what they do.

$\mathrm{C}(94)$ And they treat their own class, 'Oh this is my students, this is your students, this class is mine.' I have these materials for them not the other classes.

AU That's funny. Rather than think that all the students are there to be educated by 'us', we're all teachers, we should all educate them, I'll educate my group and you educate your group and never mix the two.

C(94) Ah ha

AU Okay we'll just finish it off very quickly now then. 'Definite philosophy of teaching', what is it? 'A teacher should not only teach what he has learnt but also care about the needs of students.' I think that goes back to the idea of teachers being able to mix what the students have to know in terms of syllabus or whatever they need to be taught as well as whatever you think the students need.

$\mathrm{C}(94)$ Everything include in teaching, is what I think in teaching?

AU Yes, well we've only allowed a very little space, so you can't say very much. We thought about making it bigger but if it's possible to describe in one sentence in one sentence what your approach or philosophy of teaching is then that's it. Okay, 'Most important factors', 'Motivation of students', other things important, 'Class size', 'Language ability'. Least important 'Government', 'Examinations Authority'. Do you think those are not important?

C(94) I find what goes on in the classroom, these are some side factors I should say, not so directly affect my teaching.

AU They might affect what the subject matter is though, mightn't they? They might affect what you teach, not how you teach it?

C(94) Yes

AU 'Which of the following skills do you consider most important to teach?' 'Very important', 'Speaking' and 'Grammar', 'Quite important', 'Reading' and 'Writing', and just 'Important', 'Listening' and 'Pronunciation'. Why do you think speaking is very important?

C(94) Hong Kong students are very weak in speaking.

AU So you would say the same for the grammar too? These are the most important because these are the most needed? 
C(94) Students need grammar, but some people say that it's not important so long as they know how to speak, how to write or understand something. And that's why the English become poorer and poorer, because they don't have enough foundation in grammar. So I think this is very important. Speaking, before they are asked to write, usually they are tested in speaking, so if they can't do this well, they may give a very bad impression to employers or to some other people that they interview.

AU Oh, I see, this would mean in terms of your students?

C(94) Yes

AU 'Strengths and weaknesses', 'Teaching grammar'?

C(94) I like teaching grammar, I like it, the students also like it.

AU Why do you think they like it?

C(94) They need it, they know that their grammar is all in a mess. In their secondary school they know that they don't have a solid foundation. The teachers cannot teach them systematically, but I think I can and I can make my own notes that suit the students.

AU So they can see it's what they know they need, so they are motivated to learn it.

C(94) Yes, and I can use different methods to help them practice and to let them know they can use grammar in their writing, reading or even speaking so that they will get interest in it. They won't think that grammar is a separate thing. so I think I am strong.

AU You find it easier?

C(94) Easy to teach?

AU Do you find it easier to focus activities if you're focussing on grammar?

C(94) Yes

AU I think so too, the other activities, reading, writing, listening, speaking, you can integrate them can't you?

C(94) Yes, but if you focus on a particular point of grammar or a particular area of grammar, I think that gives you direction.

AU So, you're planning to teach next year in the same place, you said you are very happy with your job? 
C(94) Yes

AU So you are going to go back teaching full-time?

C(94) Yes

AU Well, thank you very much.

C(94) You're welcome. 
Interviewee:

Date:

Subject D(94)

8 June 1994

Male, 27 years old.

Had worked in the business field before deciding to enrol on the BATESL course and become a teacher. Did his practice teaching in the school in which he had been a student and was planning to teach there full-time after graduation.

AU Researcher

.. Missing words / Unfinished utterance

- Pause

AU Let's start off by looking at questions about Language Use. Do you think the standard of your English in general has improved since you began the BA course?

$\mathrm{D}(94) \quad$ That means during the process of my studying?

AU $\quad$ Yes

D(94) I think I've improved quite a lot, especially on the building up of my confidence on how to use my language in front of the foreigners, because in Hong Kong I think we seldom have chance as secondary school students to practice using English. But I think we get enough exposure during three years of studies here.

AU On the questionnaire you've said that your written English was 'Fair' and now it's 'Quite good', and your spoken English was 'Quite good' and still is 'Quite good'. So do you think there has been more of an improvement in your writing than your speaking?

$\mathrm{D}(94) \quad$ Actually it's not because of the course, it's because we have lots of assignments and the assignments have to be done in English. So that means I have to get more exposure to writing in English. Because in those days I only do all my composition and such kind of things because of the assignment or for the examination. At that time even though my daily conversation with my partners or with my friends or with my classmates also sometimes use English.

AU Yes, but you don't actually speak much in English, do you get much chance to practice spoken English during the course?

$\mathrm{D}(94)$ I don't think I have much chance especially with my classmates, I only have this chance during the lectures, during the tutorials, with the lecturers, but for the peers that's with my classmates, I don't have much chance to practice it. 
AU Is there much chance within the lectures or the tutorials?

$D$ (94) During the lectures they force you to use English because they say that 'This is an English course so you have to speak in English, okay. If I hear any Cantonese then I will spot you out and say something, okay.'

AU 'When speaking Cantonese to friends who have Cantonese as their first language, do you use some English words or expressions?' That means codemixing.

D(94) Yeah, I think the case is more frequent and more useful for us as English students, because sometimes I find it Cantonese because I have not studied Cantonese for a long time, even after Form Five, then I've no chance to keep in touch with Cantonese again. Even though I speak fluent for a long time but for writing or for reading we seldom read Cantonese or Chinese, so I think my Chinese standard is deteriorating now and but my English standards are still not too good at all, so I don't know what to do.

AU So there are some situations where you would use English, is that because there is no equivalent in Cantonese?

D(94) Maybe, yes because when one start a conversation in English, it become so natural that you keep it on with the English rather than code-mixing or switching from time to time. We don't do this, seldom.

AU Rarely would you code-mix, - er - code-switch. Are there any situations where you think you might?

$\mathrm{D}(94)$ Code-switch? When I get angry, that means when my emotional feelings can't express when I'm using that kind of language I will supplement for example, usually I switch from English to Cantonese especially when I talk with somebody I feel very angry with then I will use some Cantonese to supplement what my feeling is, to say that very strongly because I think if I use the second language I can't express so fully what I feel, how strongly I feel about such kind of things.

AU So if you were normally speaking to somebody in English then you might switch into Cantonese.

$\mathrm{D}(94)$ If I want to stress something, to emphasise.

AU But if you were speaking Cantonese to someone, would you switch into English for any extended period?

$D(94)$ I won't be the initiator, I won't take the initiative to do so but if somebody keep on speaking to me in English I will be more polite, I will talk with him in 
English.

$\mathrm{AU} \quad$ So it depends on the person you are talking to?

$\mathrm{D}(94) \quad$ Yeah

AU 'In English lessons, English should be the only language used for all purposes.' And you've said 'Quite disagree', 'Most of the environment especially in Hong Kong classroom content do not allow English as sole medium of instruction.' So you think it's just not possible to use only English for all purposes? Or not feasible?

$\mathrm{D}(94) \quad$ I think to a certain extent it's not feasible, I don't know what you mean, it's about the daily conversation or it's in the language classroom?

AU Yes, within the English lesson you should not use any Cantonese at all.

$\mathrm{D}(94) \quad$ In the secondary school?

AU In the secondary school.

$D(94) \quad I$ think so far as I keep on teaching with the students I find it is quite difficult to use solely in English the medium of instruction. I've discussed it with the lecturer during this time but before after I finished the questionnaire I also study a module called English in Hong Kong here and this project was about how English is used in Hong Kong and how it is used in the education context. And I think it won't be possible because sometimes students may lose interest, especially for those who are quite weak in English. If you insist on using English as the sole medium of instruction without switching to Cantonese even though they want to do so, for example the students always say 'Teacher, please please speak Cantonese,' or 'Please don't speak too many English, okay'. But I think if you insist on this they will lose interest in learning a language and I don't see any point in our insist on using only English just because you are an English teacher. I think the maximum exposure theory, I learn these kind of terms or hypothesis, I think the maximum exposure theory seems not to be valid here.

AU Right, so there are certain times when you would just use Cantonese, for what kind of purposes do you think?

D(94) I think especially for explaining some vocabulary that is difficult to or the student can't get reach of such meanings concrete so quickly in English, he will make it become more confused about what you are talking about if you keep on speaking English. So explaining vocabulary, or something gives instruction which is difficult to follow and also for some of the classroom management. 
AU Do you think 'Lesson content can be presented in a mixed code.' You said 'Quite agree', 'It's easy for students to understand what the teacher is talking about.' So you see nothing wrong in using some Cantonese to present lesson content in English lessons?

$\mathrm{D}(94) \quad Y e s$, especially when you want to maintain the motivation in language learning. I think mixed-code sometimes is allowed, I don't think it's compulsory for some of the students of very high proficiency, when they get to Form Four or Form Five and reach a certain proficiency then I think one hundred percent English is okay. But I think in Form One or Form Two it's just a kind of transition from primary which is mainly not English medium of instruction. But in English school some Form One or Form Two they have to get used to using more English especially in English lesson.

AU 'During your teaching practice, did you use only English in lessons?' 'Usually', so you found you did use some Cantonese?

D(94) Yes, because you can say I'm quite unlucky or I'm quite lucky because I'm teaching in some quite naughty classes. They have streamed the students into four classes and I take the worst forty students among the form, and then when they group together and I ask them to speak English, how can they get used to it? I think very discouraging when I come to the first lesson. But finally I change my attitude, I understand what is the meaning behind it. I think if I insist on using English they will lose the motivation and I can't change much because the teaching practice only last for two weeks, so I can't do much things to help them.

AU 'How did the amount of pure English you used change over the practice period?' 'It increased'.

$\mathrm{D}$ (94) It increased in the sense that because during the end of the teaching practice because my supervisor will come to supervise me and the supervisor asked me to use English more than Cantonese so I explained to her about the situation and she said that, 'Use as much English as you can even though they don't understand,' so I keep on increasing the amount. But it depends on which subject I'm teaching, for example for teaching it's different, for teaching grammar it's different, for teaching G.E., that means the textbook, they treat it differently.

AU So you might use Cantonese for explaining lesson content and not for any other purposes, not to establish discipline or be friendly with the students?

$\mathrm{D}$ (94) Be friendly, I won't put it in the first factors for me to use English, that means in order to keep friendly we can see outside class, outside class we still can use Cantonese or English, depends on the students, but I think in English lesson if I keep on using Cantonese I will be translator rather than actually teaching them 
English, because for meaning, for vocabulary I explain it bit by bit. For grammar I also explain it in Cantonese, for vocabulary ... there's no point for me to use English any more. So I think so far as I'm concerned I have to use more English and less Cantonese.

AU Right, Instructional Planning and Decision Making, 'It's impossible to have a successful lesson that hasn't been well-planned in advance.' You put 'Absolutely disagree', 'It depends on one's experience in teaching.'

$\mathrm{D}(94) \quad$ Yes I think I have read some article about the novice and experienced teacher, about how they teach and what is the lesson planning, what kind of instructional planning or decision-making, because it coheres strongly with the question you asked so I put it down like this. And I think it's quite possible, it's impossible for us to make a lesson planning in advance because it's quite timeconsuming and the work-load is quite heavy now especially for the regular teacher who have to teach for about thirty lessons a week or a cycle, it's impossible to have every lesson planned beforehand. But for experienced teachers they have already got a kind of schemata in their mind and I think that a well-established schemata may help them to seek some more information to link it up and then to keep track on achieving the goals or objectives of the lesson and then in this sense I think they have already got the planning and then they store in their mind and then that doesn't mean that they have written it out they have a mental lesson plan.

AU Yes I think you're right, there's not enough time is there to write a detailed lesson plan for every lesson. If you teach more than twenty-eight lessons a week, the workload is quite heavy. 'Teachers should be more responsive to student needs than the set syllabus.' 'Absolutely agree', 'It's the need of students that we should aim at in our teaching'.

$\mathrm{D}(94) \quad$ Sometimes I think it's quite ideal, but in my present curriculum I think even though they say a lot about student needs but what actually concern them for student, their needs which have been largely neglected now, because you have imposed [on] them something, the syllabus, the curriculum, lots of things. Even though you may think they are interested some of the students may find it quite boring because it's from the textbook. It's not they want to learn, it's you force them to sit there for thirty-five or forty minutes and ask them to impart them with lots of knowledge, which they are quite unwilling to do so.

AU But very often we have to teach to some set syllabus or scheme of work.

$D(94) \quad$ That's why I very much ... if the set syllabus is as close as we can to link up with the students' needs, then we will have to do a lot of needs analysis before we set the syllabus, but so far as the syllabus is set out now, I see no point here that the set syllabus has so much linkage with the students' needs. At least they don't point it out quite clearly now, so I don't know how do they relate what 
they have learned to their needs and to their daily life.

AU Yes we should be more responsive but in reality often we are not able to. 'How detailed do you think planning should be?' 'Loosely detailed and flexible'. Not too detailed, not too detailed because of the fact that we are not able to write too detailed or because it's better not to be too detailed?

$\mathrm{D}$ (94) One is the time constraint. I think the second factor is even though you have a very rigid lesson plan and you want to follow all the steps in the lesson plan, I think it's impossible. It depends on the situation and the classes and you can't predict what the student response, what you are going to follow, especially for such a novice teacher like us, that means inexperienced teacher. I think if I don't have a lesson plan I will feel very afraid to come to the class and in front of the ... but if I follow all the steps written down on the lesson plan I think you lose the room for students to ask questions to make certain response which is unexpected and then this kind of response I think is very important for us teachers to learn from what the student wants.

AU Yes, 'How often did you find you had to change your lesson plan?' 'Usually', you usually had to change your lesson plan?

$D$ (94) Yeah because many unexpected things happened and you have to tackle it and then you always lacks behind the lesson plan and it's always fall far behind our expectation. You want to finish for example this section within the lesson but it turns out you have only finished one third or just a half of it. It seems that you have to catch up and catch up all the time.

AU 'On what basis did you plan your lessons?' 'Perceived needs of students' you said was most influential on your planning.

$\mathrm{D}(94) \quad$ Yes, and the own ideas.

AU And your own ideas.

$D(94) \quad$ Yes

AU Why do you think that was?

$\mathrm{D}(94) \quad$ You mean the perceived needs of the students?

AU Yes, during your teaching practice were you free to basically do what you liked?

$D(94) \quad Y e s$, because the teacher don't set for me such a rigid scheme of work for me to finish then I can allow to use some extra things to teach them, for example I have to teach them about process writing for one or two lessons and I have to 
correct their grammatical mistakes and written out down on the sheet and ask them to point out what mistakes they have, that is not error analysis but error treatment and ask them to speak more frequently in English and encourage them. I think this is seldomly used by the regular teacher because the regular teacher not has received any English training before, and he keep on using Cantonese to speak during the whole lesson, one hundred percent Cantonese. He told me that 'don't use any English'.

AU Don't use any English in an English lesson?

D(94) Yeah, always use Cantonese because if you use English then they will keep on talking other things in Cantonese and they won't listen to you. If you use Cantonese you can well manage the class so do you can tell what do you want to tell. I think it's his perception about English, about teaching English but I think I have a different ideas of it, so even though I know the needs of the students it's so accustomed to Cantonese but I think sometimes I have to give them more exposure.

AU Right, 'The regular teacher's instructions' would come next?

D(94) Well I put it here because I think sometimes the regular teachers have such an instruction, they ask you to do such kind of things. If I don't follow them and just do it on my own way it will end up in a mess and then they have to remediate or such kind of things like that.

AU 'What were the factors you think had the most influence on the decisions you made?' But you didn't answer this one.

$\mathrm{D}(94) \quad$ I think the first is still keep on 'Students' needs' I think. The second thing I think is 'Fellow student teachers' advice', maybe we exchange ideas from time to time.

\section{AU You did that a lot, shared ideas?}

D(94) Yeah, especially during the two weeks time, during the teaching practice. We have to ask 'How can we teach, especially when we are using the same book, teaching the same chapter and then we explain, exchange lots of ideas about how to teach, what is his experience and how do I compare with the two and see what's wrong with it. And sometimes I think the 'Supervisor's advice', but it won't help much at the time. The first one is 'Regular teacher's input' because sometimes regular teachers are so biased to a certain extent.

AU Okay, Teaching Approach, 'Examination requirements should determine what is taught in English lessons.' You 'Absolutely agree' with that. 'I hope it's not the case but it is actually. Exam presents a strong motive for students to learn language.' So that's what happens in Hong Kong, is that everything is geared 
towards the exams.

$\mathrm{D}$ (94) Yeah, so it means to mentally motivated, yeah, that means if you count marks on a certain assignment or project even dictation or this kind of things they will put more effort on it but if you tell them in advance it's just a kind of exercise they won't do it, they will find 'Oh I'm not so motivated highly motivated enough to do such kind of things, yeah.'

AU 'Students learn English better through interaction with other students.' Again you 'Absolutely agree', 'Peer learning is usually better than mere instructions.'

$D(94)$ Because more than one source of input, that is not just teachers, maybe from the peers. I think it also depends on students, the level of students. If, otherwise it will be the blind lead the blind, if their English poor you ask them learn the poor English with the other classmates then they will get more confused rather than they can learn from their classmates.

AU Do you think there's a way to organise that so that they do learn from their peers?

D(94) Yes, I think there's the most ideal case when we teach Form 6 Form 7 or Form 5 , with the best class, I think it's the case, but I think for the poor class even though they don't trust the peers, they think that the peers will just kidding or do something bad for them, they won't trust them and if they don't build up a relationship that depend on the peers they won't do so.

AU 'A class should be strictly controlled by the teacher and all lesson content directed by the teacher.' 'Quite disagree' with that, 'Teachers can't be in control and monitor all the time.'

D(94) They don't have enough strength or energy to do so, if you keep on to be a monitor or controller you will be exhausted one day, and then you feel very frustrated. Otherwise if I learn from the students sometimes I think it's better. You can't think of something, especially some teaching points or something, you will think that they will took it for granted they will learn it, but they learn it before but it turn out they haven't learn this and I think they give you a more valid reason for this and I think I have to change the lesson plan and change such kind of things.

AU So you have to change things as you go along?

$\mathrm{D}(94) \quad$ Not to be the controller and monitor all the time.

AU 'Teachers should be prepared to experiment with materials and teaching strategies.' 'Through trial and error we can learn to internalise the ideas or strategies or materials.' What do you mean by this? 
$\mathrm{D}$ (94) That means through a well resource of teachings and materials we can try and to see and to test whether the students can be more accustomed or more get used to this kind of sources or this kind of materials or even teaching strategies rather than the others. I think for a group of students they may have different teaching strategy or teaching styles that suit them most. For the other groups it may be different, or even for one class if some of the student prefer this kind of teaching strategies then you keep on insisting lecturing then they will become very bored all the time and sometimes they will play during the lesson and they won't keep on going.

AU So it's necessary to experiment?

$\mathrm{D}(94) \quad$ Yeah

AU 'In you practice teaching module, did you find that students learn English better through interaction with other students or through direct instruction?' 'Depends on the level of the students but poor classes like the one I taught it would only lead to a situation like ... can learn, or weaker students perhaps can learn from better students.'

$D(94) \quad$ It's the class of mixed ability, but I don't know what is the standpoint for streaming the class, that means do you think if it will be better manage or according to their language level I think. I mean it's because they can learn better or it's better well-manage or you just up those who don't want to learn instead of disturbing the class who want to learn. They both have their own standpoints, their own idea, I can't make the judgement.

AU 'Did you find you find you controlled your lessons strictly and directed all lesson content?'

$\mathrm{D}(94) \quad$ No

AU No, 'Rarely', 'Students are poorly motivated and they are categorised to be the worst in the whole form.'

D(94) You really can't control it, if you spend time on control, some of the teachers say, if you can well-manage a class then you can teach, otherwise if it's not well-managed you can't teach. But for my case, then I don't have to teach any more. If I ... well-managed, what certain extent do you think that two people keep on listening to you and the other thirty something keep on talking, do you think that is a kind of well-managed or you think that all of them, one hundred percent keep an eye on you and then listen to what you say is that wellmanaged? I think it depends on the teacher's attitude and expectation.

AU And how did you find it in your school? 
D(94) Teaching practice school? I think at first I wanted all of them to learn but I finally give up. I think that at least they don't disturb the others, that's okay. Even though they keep on doing other homework, I can see that they have done other kind of things, I can't stop them. If I stop them they will continue disturbing the class and then shouting across the class. Even though you lost your authority or even lost your temper or how to do it, how come the students so nasty and so naughty.

AU 'Did you experiment with materials and teaching strategies?' 'Somewhat', 'Sometimes it works, for example the process writing, but I'm sorry to say that most cases fail, perhaps due to time-constraint.'

$D(94)$ Because in two weeks time you can't put lots of things into practice. Maybe it's because of the course, if you spend more than a month it's okay, so on teaching practice, I think it will be better.

AU If you were teaching full-time would you then experiment with materials and teaching strategies?

$D$ (94) I will try but if I find it fail I won't take a small step forward, that means if one strategy fail and I will wonder if the second strategy still fit them most if I try keep on experimenting different strategies. Maybe to a certain extent I'm quite conservative, I'm not too risk-taking person. That's why I sometimes think that they have their own reasons for keep on using Cantonese during the lesson, maybe they can teach the teaching content as fast as they can and in the most effective way.

AU Mostly 'Teacher-centered', your teaching style? Is that purely the way you prefer it or is that because of the constraints put on you?

$\mathrm{D}$ (94) Maybe the constraints that affect me most to have a more teacher-centered class.

$\mathrm{AU} \quad$ Is it easier to do that, is it easier to be teacher-centered than learner-centered?

$\mathrm{D}$ (94) It is easier to do so but it is more easier to do so because you can get hold of all the things in your hand in a teacher-centered class. Otherwise, if a learnercentered class, if you are not experienced enough you will lose control, the students may lead you to a wrong direction, you can't keep the same direction to achieve the same goal and objective as I've said before.

AU 'Which of the following factors do you think has the most influence on your approach to the teaching of English?' And you've put as the first one 'Teacher's attitude towards the students' as being the most influential.

$\mathrm{D}(94) \quad$ Yeah, I think that means the expectation, how do you view your students? 
Because during the teaching practice, I have not experienced such kind of students before, so I think if I know the attitudes and try to put yourself in their own ways and you find that they lose their concentration maybe because for some other reasons and then you understand why they keep on talking or don't pay much attention to you. Then, into a certain sense we don't have to keep so stern or so straight on one hundred percent of students keep an eye on you and listen to what you said.

AU So, 'Teacher's attitude towards students' and then 'English proficiency of students', and 'Motivation'.

$D$ (94) Yeah, English proficiency I think is depend on what strategies do I ... I think teaching the high proficiency classes I think you have more satisfaction than low proficiency classes.

AU More satisfaction with a high proficiency?

D(94) Yeah because you can see how well they progress and when you compare for persons you always want to compare for different classes and different students because for the students' performance it will reflect how well do you teach or how effective is the teaching methods you are adopt. To a certain extent I think the proficiency of students may affect more the teacher's attitude and so will the teaching style.

AU Something like 'Class size' you put quite low.

$\mathrm{D}(94)$ For class size, classroom and noise in the classroom, I think it is the setting, you can't do much on it, that means even though for a smaller class maybe you can adopt different for a smaller group or a tutorial maybe you can to do some more activities-based teaching, but I think you can't do much on this because it will be set already by the school authority, by the principal. They assign you to teach this form and you have already limited the size, the classroom noise level.

AU You mean you have no choice really?

$\mathrm{D}(94) \quad \mathrm{Mmm}$

AU Professional Relationships and Responsibilities, you are 'Quite confident' in 'Your ability to become a successful teacher'.

$D(94) \quad Y e a h$, I think I'm quite confident because what made me so confident is because I'm so eager to teach and to know more about what language teaching is, especially in Hong Kong, I think it's quite unique here in Hong Kong to teach English, especially so-called English as a second language but ... different things. I'm confident in the sense that I want, I don't really want to 
force anybody to do certain kind of things, especially language learning. I know what difficulty they have because I am the person, I'm also the victims, yeah. I understand what they want and I think if I keep on doing so I will be a successful teacher.

AU You think that 'Teachers enjoy a moderate status in today's society.'

$\mathrm{D}(94)$ Because turns out teaching is still not developing into a kind of profession. Like an accountant or the law or a doctor, compared with the social status that the teacher has, it's quite low, but I think the teacher is striving for a more higher professional status to develop in account profession with lots of credit systems or examinations so that ... I think now what the most serious problem is because even though they have refresher training course, ICTD like this kind of things, I think because they are not well-coordinated or well-managed they can't put all the course[s] in a regular or systematic way so that means they are not make use of the teaching resources they have.

AU Do you think teachers should have a higher status than they do now?

D(94) Yeah, yeah, I think.

AU How could the status of teachers be raised?

$D$ (94) By asking him to feel that teaching is not just a kind of job, a career or the first thing is the teacher development ... a profession through a kind of development, that means if they can develop throughout their career, that means they can have lots of chances to be promoted to a higher status. I think it will be better.

AU Okay, your 'Best attribute in terms of being a teacher' you say is 'Good command of the language', followed by 'Good communication skills' and 'Careful planner'.

$D(94) \quad Y e a h$ I think especially for being a language teacher it is of prime importance. If you don't have a well command of the language and good communication skills, students may lose confidence in what you taught, in what you teach, that means sometimes they may feel that the teacher no good or not competent enough to do so.

AU Down at the, ones being the worst attributes, 'Able to form good professional relationships with colleagues'.

$\mathrm{D}$ (94) Yeah, I don't care much about the relationship with colleagues. It is the most important that I deal with the students. Of course if I have a good relationship with the colleagues it may be enhance my teaching because they will help you in lots of ways, but even though I have a bad relationship with colleagues what 
I mainly deal with is the students. If I have a good teaching, that's the most important aspect.

AU 'Do you see teaching as a long-term career?' 'Definitely not', why do you say that?

$\mathrm{D}(94)$ I think because now teaching do not have so-called development, it is a quite stable job. If you don't do anything, nobody can ask to do or force you to do this, unless you are highly motivated enough to keep on searching for brighter prospect, to keep on going for Master degree or such kind of things, nobody will force you to do. That means in such a circumstances I don't think I will carry on too long. Perhaps the situation will change in the future.

AU Do you think there's any chance of it changing?

$\mathrm{D}(94) \quad$ I hope so, but I don't know.

AU 'Who was most helpful to you?' 'Teacher training supervisor' was most helpful, so there's obviously going to be a difference from your Practice Teaching and when you're actually teaching full-time?

$\mathrm{D}(94) \quad$ Yeah

AU 'Which out of class activities do you think you will have to do?' 'You've circled these but you haven't circled things like 'Being involved in clubs and societies', 'Speech festivals', 'Counsel students', 'Meet parents', 'Meetings', 'Mark papers'.

$\mathrm{D}(94) \quad Y$ eah, I don't think that these the kind of activities I enjoy to be if I want to be a teacher, even these are compulsory, you have to do these, but I think we may not be forced to join a club or to be the club supervisor. If you are not willing to do so then you will take it just as a kind if they push you to do something you just do it, they don't want you to do it then I don't keep an eye on this. I don't want to be like this, so I want to do the things what I want to do, I wouldn't want to do, if I didn't want to join the English Club I hope that in the future I find a school that don't ask me to do the things I don't want to do.

AU It's possible, I think you'll find that very often you don't have a choice, when you go into a school, if you're employed by the school or by the education authority. When I made out this list, I made it out because I found that I have to do all of these.

$\mathrm{D}(94) \quad$ Yeah, all of these?

AU All of these yes, and so I was just curious to see whether the BATESL students would be aware of the fact that they might have to do all of these. 
$\mathrm{D}(94) \quad$ 'Be involved in extra-curricular activities', maybe so but I think be involved in the English Club it may not be so.

AU I think these things tend to be, it depends on your school, on the school you go to. I'm teaching in a Band One school so these things are pushed quite a lot, debating, drama, speech festivals.

$D(94) \quad Y e a h$, you have to spend a lot of effort.

AU Especially being an expatriate teacher as well they somehow expect that you're going to be able to do that. 
Interviewee:

Subject E(94)

Date:

Male, 24 years old.

Was unsure as to whether he would teach immediately after graduation as he wanted to take some time away from education. He would have preferred to teach in a Band One school, preferably the school in which he had been a student.

AU Researcher

... Missing words / Unfinished utterance

- Pause

AU Good afternoon

E(94) Good afternoon

AU Let's talk a little bit about Language Use first of all. The first section was about your language use and about how you think your standard of English has either improved or not since you began the BA course.

E(94) Quite good

AU You've written or you've put ...

E(94) I believe that this kind of thing, for writing I've improved a lot because I've a lot of opportunities to do ... as long as writing is concerned I've improved a lot because this is a kind of assessment course, everything depends on projects, assignments. You've got to write a lot, especially in Year Two I believe, I have taken a selective module called Modern Fiction, special topic in children[' $\mathrm{s}]$ literature, stuff like that. For that two modules I have to write diaries for everything that I read. So I've improved a lot in writing. But not in speaking I have to say, it's nothing about the way they taught, it's about the way I absorb this stuff. I don't know what went wrong but l've improved a lot in writing.

AU Right, any ideas why you didn't improve in speaking? Do you think that's true for the other students?

E(94) No I don't think so, I've seen a lot of my group mates improve a lot in speaking especially this year they have this public speaking module, which they have to deliver three speeches in one semester. Yes, what I'm saying is that I've improved in speaking but not as much as in writing, that's the point.

AU That's understandable I think, isn't it? Because, as you say, you've got to write a lot when you do this course, probably write more than you're going to speak 
anyway. Using Cantonese, using English, you've put that when speaking with friends you would always code-mix.

E(94) Ah yes, I guess it's a phenomenon for university or polytechnic students. A lot of them, like the names of those modules, we cannot say them in Cantonese. If we say it in Cantonese it will sound too odd to do so, that's the only way, to code-mix, or use a mixed code of language.

AU So would it just be with certain terms that you would use English?

E(94) Certain terms, names, what we call jargons. We don't use those English items to show that we are university students, we use it because we have to, that's the easiest way.

AU But would you use other words? Would you use perhaps things like ...

E(94) You mean other than those nouns, do I use verbs or adjectives? Sometimes, if I couldn't find the equivalent in Chinese, say like, I can't find an example now but sometimes we do, we do.

AU Why do you think that is? Is it just what people do in Hong Kong? Is it just the linguistic situation in Hong Kong?

E(94) For me I guess I haven't used Chinese for a long long time, even in writing, I mean I haven't used Standard Chinese for a long long time so I have lost track of all the expressions in Chinese sometimes. In that case I have to use English if I have to express that exact meaning, doesn't matter if the other party understand it or not.

AU Sometimes you don't even take into consideration whether the other person would know ...

E(94) Yeah

AU And code-switching, you said 'Rarely', although 'Always' you would codemix but you would code-switch rarely.

E(94) Yeah, that's very rare because it's kind of uneasy to code-switch if you are speaking to a Cantonese, even if we speak to the Chinese lecturers in the department, we bump into each other in the hallway, they have to use English and I feel it's uneasy, let's get out of this conversation in English, we're both Cantonese, we have a common language, we have a mother tongue of Cantonese, why the hell do we have to use English? But then I understand that they're working in the Department, they have to follow the rules that they have to interact with the students in English, so I understand that, but if I'm talking with my friends, we rarely rarely code-switch. I don't think I have any 
experience of code-switching when I am speaking. But that's not the case when we were in classroom contact, we're under the same regulation that we have to use English all the time. So one thing is that code-switching and social contact is very very uncomfortable.

AU Yes, very unnatural, isn't it?

E(94) There's no need to use English, there's just no need, code-switch no need.

AU Okay, 'In English lessons, English should be the only language used for all purposes.' You said you 'Disagree' with this, and you've commented that 'For interaction, Cantonese is more appropriate, it arouses a sense of belonging or safety.'

E(94) But I have to explain. For certain teachers, they can use English for interaction, but not normal English teachers, say like for those girls who have been studying in those educational colleges, they teach very good, they know their subject areas very good, they have teaching points, they have their lesson plan very good but sometimes they don't know how to interact with students. I mean person to person, face to face interaction with the students in what I say colloquial or natural English, the way that I'm talking with you. So for those kind of teachers, it's better for them to use Cantonese to interact with the students, if they force themselves to use English, I would guess they would project a status that 'I'm an authority', 'I'm still higher than you in the hierarchy, although I'm interacting with you, although I'm pretending to be a friend, though I'm not. I'm not using the same language as you students.'

AU I see yeah, that's interesting, I know what you mean. With me, I have to interact with my students in English because I don't speak Cantonese.

$\mathrm{E}(94) \quad$ How does it feel?

AU It's not so bad but you can never get as close to them as the Chinese teachers can.

E(94) There's still a wall, a linguistic wall that you just can't get through. Unless you get to higher level students who feel comfortable using English for social interaction, that will be another case.

AU 'Lesson content can be presented in a mixed-code.'

E(94) Sometimes I think, I think for some students they don't understand everything in English, in that case you have to use Cantonese in order to help student to understand what you're trying to get through. In that special case it's better to use Cantonese. 
AU Do you use Cantonese to present content or to express content.

E(94) Just to make sure, elaborate sometimes. You deliver your teaching points, like say grammar structures blah blah blah, this is an example, they still are confused, that is when, yeah, you can use Cantonese, to fill it in.

AU 'How much did you use only English?' You said 'Always' in your practice teaching.

E(94) Yes because for me, I have two practice I guess, teaching practice I was practising in [name of school]. I was an old boy, I was in [name of school], so I kind of feel, if I don't use English I will be underestimating my fellow, little boys so I have to use English. By the way, when I was in [name of school], when I was a ... all the teachers used English, so I guess that's the way it goes in that school. And if I am practising in that school as a student teacher or whatever they call it, I have to follow the rules. Because I have heard that teachers that use Cantonese in classroom got reported to the principal, they got kind of, I don't know, they got kind of scorned or anything.

AU So that does happen now in [name of school], that some teachers do use Cantonese in the classroom?

E(94) Yes I believe.

AU More so than they used to?

E(94) I don't think so because, okay those teachers, no matter what subject they are teaching, they accustomed to use English to teach, or they may be accustomed to use Chinese to explain some terms, jargons, technical terms. So for most of their talking time, I believe they will use English. That's the problem there.

AU And when they're interacting with the students as well?

E(94) Interacting with the students, it depends. Let me recall. My teacher was a Malaysian, she didn't speak in Cantonese, so there was no interaction in Cantonese, but yes for some teachers, like my Geography teacher, my History teacher, when we were away from those teaching points, they use Cantonese to speak with us. And that kind of produce a more friendly atmosphere.

AU 'How did the amount of pure English you used in lessons change over the practice period?' You said 'It decreased a little bit.' The amount of pure English you used over the practice period actually decreased. So you mean you started off using more pure English shall we say?

E(94) Because I was not making any interaction with the class. Firstly because I'm kind of nervous, secondly because I'm not sure about the discipline of the class, 
so I have to assert a certain kind of authority among the students although they are only Form Two students. But sometimes Form Two kids can get out of hand. So by using English and posing as authoritative or stern teacher, I have to use more and more English. But then when I get to know the kids, they were really good. They were really good, nice kids, hard-working kids, diligent, there's no point I have to use the same persona again. I can switch to Cantonese, be friendly. So it decreased yes indeed, but not when I'm teaching, when I was teaching.

AU And in situations which you might need to establish discipline, to be friendly with students, so although you said that at first you used more English, you used English in order to present this kind of stern, serious image, but in this one you said that you might use Cantonese to establish discipline.

E(94) Ah yes, when they really did get out of time, I remember one time this boy was playing a trick on a kind of weak classmate, and I have to stop that. I have to say it in Cantonese kind of, I didn't plan to use Cantonese but I guess the most direct way to stop what he was doing was to use Cantonese. So that's what I meant by to establish discipline. And also relationship yes, I've explained that.

AU Okay, anything else you'd like to add about language use?

$\mathrm{E}(94) \quad$ No

AU Okay, Instructional Planning and Decision Making, 'It's impossible to have a successful lesson that hasn't been well-planned in advance.' And you said 'Absolutely agree', and then 'That's the way it is.'

E(94) Mmm, because if sometimes I do I understand that I didn't really plan anything, not in Practice Teaching, when I was teaching in an evening school. I didn't plan anything because I was stuck with my homework. I didn't plan anything in the school for the evening class and I just go in, I just went in and the students can sense that I didn't do any preparation. And as time goes by I believe that there's not very good for the students, as well as for me, because at least I have to plan what exercise I'm going to use, after this exercise what I'm going to do. That's the least thing what I meant by plan the lesson.

AU So it's this idea of being well-planned or not which comes out in this question, 'Teachers should write detailed lesson plans for every lesson they teach.' 'Absolutely disagree', 'Spontaneous reaction is what it really counts for in real classrooms.'

E(94) In real classrooms because sometimes you know, you run out of time, you have to change things on the spot. You cannot just stick with that piece of lesson plan you have got, this is not a piece of document or contract you have to follow. The classroom is where you interact with students and you make 
changes all the time, it depends on the situation. So if you stick with the lesson plan, say one time I was in [name of school], practice teaching, I have planned the students listen to a song, they have to fill in the blanks in a lyric sheet. Okay I didn't plan an encore but the students like, do you know Bermuda Triangle by Barry Manilow? The students liked the song very very much and some of them asked if they could have an encore but I didn't plan it. And I was being assessed by the cooperating teacher. It was an Indian lady, so I made the decision if the students like it, if it is fun for them, what the hell let's throw away the lesson plan and do what they want. So that's what I meant by we have to change all the time.

AU 'Do you think it's possible to write detailed lesson plans for every lesson?'

$\mathrm{E}$ (94) It's possible so long as you're willing to devote the time and the effort in writing one, but you have to consider the possibility of changing it when you are executing the plan.

AU Yes, you have to be flexible. 'Teachers should be responsive to student needs.' 'Absolutely agree', 'Be humanistic.' The example you've just given kind of illustrates that. 'How detailed do you think lesson plans should be?' Is that three or four or in the middle?

E(94) 'Quite detailed' and 'Quite flexible'

AU Quite flexible, yeah somewhere in the middle, so as you say, you have a plan in your mind with the option of okay, maybe it will change, you will be able to do something you'd not planned to do. 'How often did you find you had to change your lesson plan?' 'Rarely'.

E(94) Just one, that case, for that Bermuda Triangle case that was the only instance, the only incidence that I have changed the lesson plan, because that plan was kind of unresponsive kids, though they are very hard-working and smart kids. So that whatever I planned for them they will follow it. That Bermuda Triangle was the only case I have to change my plan. So it depends on the students you are dealing with.

AU Is it because students are not responsive? Is that perhaps a reason why you seldom have to change your plans? You go in there and the students are so passive that they just do exactly what you plan them to do.

E(94) That's one possibility, another is that you have to know the students, you have to [be] familiar with the class, what I say is you have to be familiar the teaching of that class. If you have that vague concept in mind and out in that vague concept when you're planning your lesson, you'll know how much time should we spend in this activity, and what should be followed. So it's not just passive, familiar with the class is another point. 
AU Do you think you had long enough to get familiar with the class in you practice?

E(94) Two weeks, yes, after a couple of lessons I realised there's no problem with that class, so I forget about discipline and go ahead with my planning. There's no need to worry about discipline because there's another case when you have a class of poor students, of difficult students, you have to bear in mind what shall I do if this goes wrong and what should I do if this one run out of time? But with that class I can make a very well-planned lesson plan.

AU With more difficult students I guess you have to think about whether certain activities are going to work or not, or if I try doing this game with them there's going to be a riot or something like that. But these kids, they will do that well. 'On what basis did you plan your lessons?' Firstly your 'Own ideas' was most influential in your planning, then 'BATESL course guidelines'. By 'Regular teacher's instructions', that means cooperating teacher. It's quite low on the list, fourth.

E(94) Because I've given a lot of room. For the first time I met that Indian teacher she told me that 'You can use any of your own ideas in the class'. She said that so there's just no point blindly follow her instruction, the way she taught the class because she liked me to give some new ideas to the class.

AU But in terms of materials for example, were you given the materials to use, were you given textbooks?

$\mathrm{E}$ (94) Yes, I was given textbooks yes, exercise books with all those keys and answers, but I didn't really use all the stuff. I have to, because we have to make up activities to teach writing and listening. They didn't really have good writing activities in the textbooks so I have to invent a communicative writing activities, in which they write, in which they have to write a very short letter stating one of their social problems, and they hand it in and I swap them and give them to do answer letters, so that's my own ideas, they don't provide it in the school.

AU Your own ideas, you just made them up or did you use other resources, did you get them from here?

E(94) For that writing, I made them up. For the listening I couldn't make them up because they have to have the tape. But I did produce a short dialogue for the listening exercise for which I asked my sister to record some things so the students can fill in a table. I would say that I made up my own ideas about teaching. I guess because I think it's more time-consuming to go over books to find ideas, it's better to use my own ideas. 
AU 'What were the factors that you think had the most influence on the decisions you made?' 'Supervisor's advice' you've put as the most influential. Second 'Student needs'.

E(94) Because that's kind of silly because I was being assessed in Practice Teaching. If I want to score high I have to follow what he says, so that's what I meant by 'Supervisor's advice'. But I don't take that only as assessment guidelines, I take it as advice because he was a nice guy, my supervisor and he was really good in teaching us. He give us some good advice, so what the hell, let's take it.

AU Oh yes, I realism that practice teaching is very different from real teaching. Teaching Approach, 'Examination requirements should determine what is taught in English lessons.' You put 'Absolutely agree'.

$\mathrm{E}$ (94) That's because that's the way it works in HK and this is I believe a very unhealthy situation, but I couldn't change it from the top because that's the curriculum, and it seems like the only purpose students go to school, or the only reason parents send their children to school is to get high mark in public examination, so that they can have a higher position in job or stuff like that. Yes, for Hong Kong I believe it's true, but for education for the children, I don't think that's very healthy because examination doesn't count for everything that's essential for the children to grow up.

AU Yes, unfortunately it's true, isn't it? We have to teach them what is on their exams, whether it's external exams or internal exams. With the lower forms it's not as bad, you can teach them pretty much what you like. The stuff that's on the exams is the usual stuff anyway, tenses, prepositions, those kind of standard things.

'Students learn better English through interaction with other students rather than through direct instruction from the teacher. 'Quite agree' you said.

E(94) Mmm, interaction with other students because they have to use it. The bottom line is for them to engage students using language and for a teacher handling or dealing with forty students just one at a time and there's not much time to devote to one student, to each student. So it's better for them to interact among themselves in language so that they can get more opportunities to use it.

$\mathrm{AU} \quad$ Is it easy to do that? To get students to interact with each other in English? How did you find that in your teaching?

E(94) Not very difficult I would say. Invent some kind of communicative speaking activities, provide an information-gap in pair work, they speak to each other in order to get the information, fill-in blanks, then ask questions, answer questions. Or for writing, write letter, answer letter. Yes, I think that that [is] workable. And I think teachers should do it more. 
AU Even with big classes?

E(94) Even with big classes.

AU 'The class should be strictly controlled by the teacher and all lesson content directed by the teacher.' 'Absolutely disagree'.

E(94) Yes, we should be sensitive to students' wants and needs. Sometimes .... a very very demanding grammar exercise, teachers should change their scheme of work by doing some kind of language game with the students. I don't know, I'm just looking from the point of view of in practical student teacher but not a real teacher in a real classroom.

AU Oh yes, even if teachers say that they strictly control the classes but generally you can only do what the students allow you to do, in a way, whatever is going to work in the class you do it. If you know that something is not going to work, it's pointless continuing keeping doing the same kind of activities if it's obvious that it's not working, that it's not successful. Students are not learning from it or they find it boring or whatever. 'Teachers should be prepared to experiment with materials and teaching strategies.' 'Absolutely agree', 'That's what makes teaching interesting.'

E(94) Experiment? Take some risks. Take some chances once in a while.

AU Once in a while?

E(94) Once in a while yes, you still have to stick with the curriculum, but it's meaningless to just blindly follow the syllabus without experimenting on students' needs. Because who knows, those areas that you explored may be useful in the next year.

AU 'Did you find that students learn English better through interaction with other students?' 'No difference between the two.' 'Cant tell students' progress within two weeks.' Very difficult to actually tell their progress. Do you think though that they enjoy it better, that they enjoy that kind of interaction?

E(94) Among themselves?

$\mathrm{AU} \quad$ Yes, rather than let's say direct instruction?

E(94) Enjoyed it, I believe I saw that sometimes because I didn't give enough instruction, because the class was not very used to it, interaction among themselves. The Indian lady only used direct instruction, and I believe that she has been doing it for a long long time in the class. But I tried to change it but I didn't give enough instruction at the beginning, but later on in the speaking activities they get the idea. They get the idea and they could follow it. I'm not 
sure if they enjoyed it, they could follow it.

AU I think some students, as teachers I think we imagine that students would love playing games and having these kinds of interaction activities, and a lot of them do, but there are some students who don't like that kind of thing, 'That's not proper learning, we want to be doing exercises, we should be writing and the teacher should teach us'. A lot of students still have a traditional view of teaching.

$\mathrm{E}(94) \quad$ We have to change that.

AU 'Did you find you controlled all your lessons strictly and directed all lesson content?' 'Always', 'It's a Form 2 class and things are all in my hands.'

E(94) I've explained that they're good kids, they are obedient, I have absolute power of the control.

AU 'Did you experiment with materials?' 'Very much', okay, 'Would you describe your teaching style as learner-centered or teacher-centered?' 'Mostly teacher-centered'.

E(94) Yeah, that's ... we should consider students' needs more.

AU I guess it's easy to say learner-centered, but you can only teach what you're allowed to teach. In an ideal world we'd all be learner-centered. 'Which of the following has the most influence on your approach to teaching?' 'Motivation of students' you've put as the most influential. Do you think that's the most important factor? 'Motivation' and 'English proficiency'.

E(94) Yes, motivation, what do they want to learn? What can motivate them? What can make them interested in learning English? That's the most important thing. No matter how good you are as a teacher, no matter how good your teaching material is, if the students are not interested in you, your teaching or your materials, you're bound to fail I believe. And also, you have to also consider those students' English proficiency. If they have a very low English proficiency, you have to adjust your activities and teaching points.

AU 'Teacher training' you've put quite high as well.

E(94) Yes because I believe I went through this kind of training for three years, I believe it helped. I believe that teachers, all English teachers should go through some kind of training, because if you're only good in English, it doesn't mean you're good in teaching English. And some teachers doesn't very fair, I have to change that too.

AU Okay, other things are considered not important, 'Current trends in ESL'. 
$\mathrm{E}$ (94) That's too remote for school teachers to get in touch or to implement the trends, those new ideas that are still in the research stage. It's too risky to carry these ideas in the classroom, I believe.

AU And 'Class size' you put quite low down too.

E(94) Class size yeah, I believe average class size is for students 35 to 40 , and we cannot change that, we cannot, only in very rare occasion can we change, can we split the class into two and take one class out to teach. So that's not very important for Hong Kong teachers.

AU It's not important because it's not a choice that we have.

$\mathrm{E}(94) \quad$ Yes, beyond our control.

AU Professional Relationships, 'How confident are you in your ability to become a successful teacher?' 'Quite confident', what makes you quite confident?

E(94) Partly because of the response I got from all those cooperating teachers. After I left [name of school], that Indian lady kept saying, kept saying to people that know me that I was good teacher because in the last lesson I really did a very very good lesson by playing the songs, and interacting with the students and having the students having a good time. And also as well as for students reaction, in spite of the stern image that I project in classroom, most students tended to come to me after class, not just to ask me questions about English, but also questions about the social life. So I believe that if I continue to be a teacher, I will be very good, I believe.

AU 'Status of teachers', 'Teachers have no special status'.

E(94) Yeah, in HK yes, unless you are a lecturer or professor or you teach in a polytechnic or university. If you are a secondary teacher you are nothing, just a teacher, except that you get paid very well. That's the only point that's good for being a teacher.

AU You think teachers get paid very well?

E(94) Yes I believe yes, for business first of all it's a very keen competition, you get fired all the time, you get a lot of pressure and you don't get promotion if you're not smart enough. But for a teacher, if you get a degree, you are guaranteed that you will get at least thirteen thousand for a month. And there's not very a keen competition as long as you get a degree. You can teach wherever you want to, I believe that, and you get promoted if you don't screw up your students, after a few years, so I guess if I am kind of a steady stable person, I will stick with the teaching profession for the rest of my life and bring up a family and stuff. 
AU Do you think you will? Do you think you are a steady, stable person?

E(94) No, at this point of life I'm not, not yet.

AU 'Your best attribute in terms of being a teacher', 'Careful planner' you've put as ... and then 'Good command of the language'.

E(94) Careful planner, that is you plan what you are going to do, on which ground you are going to make compromise with the students as well as with the syllabus. You have to have at least a vague idea of that, and good command of the language, that's I think the most most important thing for being a teacher. Sometimes, at least for us, those BATESL students, they are good students themselves, okay there's no doubt, but I don't think they can use the language so good that they will make a good impression on the students. What I'm trying to say is that they have to try and improve their English a little bit more before they go out to teach. The most important thing is the speaking skills. Some of the teachers still speak very Chinese accent English, even for me when I was listening to them in class, I will feel kind of uneasy, as a classmate. And I believe if they don't have a good speaking skill they will have a bad influence on the students too, I would say. The students pick up whatever accent they are exposed to. That's the second important thing.

AU Definitely you don't see English [teaching] as a long-term career?

E(94) Because I'm not that kind of stable person, I not the kind of person who will stick with one profession, unless you really like it. Of course there are girls, there are even guys that like teaching, they like it a lot, take it as a lifetime career, but not for me. If I teach probably first because there is no way out, second because I am good in it.

AU 'Which of the following out-of-class activities do you think you will have to do as a first-year teacher?' You circled all of these, but not these. I'm more interested in the ones that people don't circle.

E(94) 'Train students for speech festivals', 'Be involved in religious activities', I'm not Catholic, I'm not Christian so I don't think I'll be involved in them. 'Counsel students', I don't know, counsel students you got to have some kind of training.

AU Supposed to be.

E(94) Supposed to be but it's kind of risky if you give inconsiderate advice, say like if students have problem with their love life or with the love affair, you give one wrong advice, boom, they jump out of the building, that's it. So it's kind of risky. 'Meet parents', oh I understand, we have to meet parents once in a while those meetings. 'Attend meetings at school with ... ', once a year I guess, you 
have meetings once a year, normally. 'Attend courses', it depends on whether you like to attend courses.

AU Yeah, oh certainly they're voluntary, to attend courses, sometimes you find that you're kind of expected to at least attend something. I they keep pushing courses and you keep saying no no no ... and then some times it happens panel chairs or heads or principals they start to wonder why don't you attend any courses. It depends a lot on the policy of the school. I put these and when I put these I found that personally I had to do all of them, and so I was wondering if the BATESL students were aware of the fact that they might have to do all of those things. Even things like be involved in religious activities, although I'm not religious, if you're at a Christian school then you have no choice, you have to go to prayer meetings. I'm lucky, I don't have to because being an expatriate and I don't understand Cantonese and all these things are in Cantonese, we very often get excused from them. But there are one or two that we have to go to, simply because the other teachers would complain. 'Train students for speech festivals', again that depends on your school. If your school is keen on that kind of thing, which my school is then you have to train the students.

'Which out-of-class activities do you think will take up the most time?'

'Marking', 'Preparing', 'Depends on teaching environment'. 'Do you expect to be working closely with other teachers?' 'Some'

E(94) Yeah because sometimes you get into a traditional school, with all these very very very experienced, almost obsolete teachers, you cannot get into their circle, and they don't see you. They only see you as the new kid on the block, that's it, it ends there. But if it's a new school, everyone is new, everyone is kind of exploring, you get a lot of opportunities to share with other teachers, so it depends.

AU What I find usually happens, my school is an old school and a lot of the teachers have been there twenty years, but you get the new teachers coming in and they tend to stick together and then if they stay for a couple of years they stay friends and then a new generation come in and they stay as a group so you get little groups around like this.

Okay, Perceptions and Values, 'Not very definite philosophy', 'Unsure about personal career goals', your teaching philosophy 'Be a sensitive and sensible teacher.'

E(94) Mmm, sensitive to the students' needs, sensitive to the environment in the school and the environment in the staffroom and the environment among the students. Sensible, you have to be smart handling relationships with the students as well as the staff. At least you have to survive.

AU Survival, is that the main thing?

'Location of school', do you think location of school is important? 
E(94) Yeah because if you teach in a New Town, you're bound to have Band Five students, so your only mission is to maintain discipline, but not to teach.

AU Right, 'Which of the following skills do you consider most important to teach?' 'Reading', 'Writing' and 'Grammar', the 'Pronunciation' and 'Speaking' and 'Listening' lower down. Why would you say speaking is less important than the other three?

E(94) Maybe I'm speaking from my own point of view because I'm good in writing, reading and grammar. All these things are important indeed, all these things are important, but I'm most skilled in these three areas. I'm not very skilled in speaking and in listening and in pronunciation.

AU So it's really from your own point of view. 'Which of the following best sums up your feeling as regards the relationships a teacher should have with his students?' 'Teacher should be a transmitter of knowledge', '... should be a facilitator', '. ... should try to become friends', '... should refrain from punishing, ' '... should be prepared to devote as much time as necessary'. Okay, should refrain from punishing, you believe that?

E(94) Er yes, it depends on why do you have to punish them. If they don't do any homeworks or if they don't do well in school, it may reflect some kind of family problem or attitude problem, or it may reflect simply that they don't like to study. So if you punish them to study more, to pay more attention to study, I believe that they will end up hating study more and more. The solution is to get to the source of their problem, if it's a family problem, talk with them, talk with the students, try to find a way out, let them talk about it, let them let it out, don't force them to do something they already hate.

AU Okay, and 'Strengths and weaknesses as a teacher'. 'Organisation', 'Planning', 'Voice', 'Strength of personality', 'Teaching reading', 'Teaching writing', 'Teaching grammar'. And lower down, 'Communication', 'Discipline', 'Relationships with students and colleagues', 'Motivating the students'. All of them really, they just give you points. Even four is strong, it's not saying 'weak' so these are your stronger ones, planning, voice and strength of personality.

E(94) Ah ha

AU Okay, I won't ask you about your feelings for the BATESL course as that's not my department really. 'Are you planning to teach next year?' You said 'Not yet sure', so you haven't applied for any jobs or anything?

E(94) A job, teaching job? I have kind of left a letter when I was in [name of school], they said that when they have an English teacher vacancy they will call me. But what I have heard so far they don't have an English teacher vacancy in [name 
of school], and as I said, I would like to see things other than teaching for one year or two before I decide if I really have to teach. So it's kind of a crucial years, these couple of years. It depend whether I'm going to be a teacher.

AU It wouldn't bother you taking time away, doing something else for one or two years? Do you think you would forget what you have learned here or you would lose the motivation to teach?

E(94) Maybe yes, maybe, or maybe not. Maybe I would end up being a total failure in other areas so I end up that teaching is the last resort so I will be more motivated.

AU And you've said if you do decide to teach you said 'Band One for sure.'

E(94) Yes, because I don't want to deal with any discipline problems and Band One school is perfect, why they have high-proficiency students, well-established, I don't think any new school will be Band One. So Band One school.

AU Probably [name of school]?

E(94) Yes, if they have any vacancy they'll call me. Because I believe that they have a vacancy and I'm an old boy and I have practised there for two weeks. I've got a pretty high possibility, a likelihood of being asked to teach there. Band One school yes.

AU Thank you 
Interviewee: $\quad$ Subject F(94)

Date:

8 June 1994

Female, 23 years old.

Was planning to study for a postgraduate diploma after graduation and then find a position as a teacher, possibly in a lower band school near her home.

AU Researcher

Missing words / Unfinished utterance

Pause

AU Good afternoon

$F(94) \quad$ Good afternoon

AU I'd like to ask you some questions about the BATESL Course Questionnaire.

F(94) Certainly

AU First of all about Language Use, I think one third of the BA course is on improving your English standard, how do you feel about your standard of English after doing the BATESL course?

F(94) In all four skills? I think for speaking, I can have more confidence in speaking out the language but still I think I can't have great improvement in pronunciation or using the vocabulary in speaking aspects. And for writing I think I can improve much because I have the module Academic Writing, I can know how to write a good academic writing. And for listening, I think I can improve much because the lecturer carries out in English, I can listen more and more. And for reading, I think a little bit because in my secondary school I also read many books so there's no great improvement in my reading, because similar workload in reading I think.

AU So you would say it's your written English that's probably been the one that's improved the most?

F(94) Er huh

AU Okay, you put 'How would you describe the standard of your spoken English now?' as 'Fair'. Before the BATESL course, 'Rather poor'.

F(94) Not 'Rather poor' in fact 'Poor', not 'Rather' but there is no choice. 
AU Right, do you think there's any way in which that could be improved within the course, do you think?

$F(94) \quad$ Improve what?

AU Let's say your spoken English.

$F(94)$ I think the lecturers should give the students more chance to speak in the class, and also give them more confidence, instead of only doing some assignment, for example Public Speaking, I need to be assessed so I need to speak only for that assignment. So I think this motivation is not good in that way. Do you know what I mean?

AU Yes, usually how many would be in the group?

F(94) You mean the number of students? In the tutorial I think twenty, but only one hour. And also half of the lesson is the lecturer speaking, so I don't think we have more chance to speak.

AU Right, it doesn't give you much chance.

F(94) And also one week only one lesson for that module.

AU But I mean in other tutorials you would be expected to speak?

F(94) No, just for most of the time just listen to what the lecturer say, talk about the content of that module.

AU Is that because of the way the modules were structured, the way they were taught?

F(94) I think only the modules Speaking and Public Speaking I can speak, for the other modules we only just listen. Or sometimes maybe the lecturer asks us some question and maybe I respond.

AU Were many of the BATESL students keen to speak out in class?

$\mathrm{F}(94) \quad$ No because also I think there are some peer pressure, and also for me I'm quite afraid that because I think that my language proficiency in speaking is not good, so I'm afraid I'll make other students laugh at me. Because I think the lecturer not quite care about the self-esteem of the students in speaking, do you know what I mean?

AU Yes, you mean that they don't take that into consideration?

F(94) I think at the beginning they think that we already have the motivation to 
speak, have the confidence to speak, but I don't think so.

AU I see, it's a common problem. It's a problem I have with my students, secondary students, to get them to speak out in class is very difficult. Do you think most of the other students feel the same way as you, they feel too self-conscious?

\section{F(94) Yeah}

AU Do you think it would help if there were smaller tutorial groups?

F(94) Ah ha, and also it's better to build up the relationship between the lecturer and the students, also the cooperation among the students.

AU How could they do that?

$\mathrm{F}(94) \quad$ I think that depends on which type of person that the lecturer belongs to.

AU Sometimes I think that some of the lecturers set up the authority, so it's very difficult.

F(94) Right, some lecturers have different ways don't they. Some lecturers just like to talk and they don't seem too bothered about the students, whether the students contribute anything to the lesson. Whereas others try more to get the students to join in.

AU Yeah, I think more learner-centered.

F(94) More learner-centered exactly.

AU Okay, 'When speaking English to friends who have Cantonese as their first language do you use some English words or expressions?' You've put 'Quite often'.

$\mathrm{F}(94) \quad$ Yeah I think it's very common.

AU What kind of English words would you use?

F(94) Code-mix do you mean? 'Happy', 'lunch', 'bye - bye', 'good morning' and ... oh it's difficult.

AU The ones you've quoted seem to be very general words you would use every day.

F(94) Commonly for express the feelings and also maybe for talking about some embarrassed term, for example 'underwear'. 
AU I see, so that's easier to do that in English than in Cantonese.

F(94) And also some terms it's difficult to be translated into Chinese.

AU Things like technical terms and things like that, things to do with language or linguistics or things like that?

F(94) Yeah

AU The next question is, you said that quite often you code-mix, but codeswitching, when you switch into Cantonese for a few sentences and then back into Cantonese, you said you 'Rarely' do that.

F(94) Yeah, very odd I think. One sentence English, one sentence Cantonese, I think it's very odd. And also maybe my friends don't understand don't understand the English sentence.

AU What kind of people do you think would be likely to do that?

F(94) Er ... maybe because I teach ... [name] Cantonese, so sometimes when he can't express in Cantonese he switch to English. I think under that situation.

$\mathrm{AU} \quad$ In a lesson situation ...

$\mathrm{F}(94) \quad$ Not very common I think..

AU I suppose the only thing I was thinking of was for people who are bilingual, if two bilinguals are talking to each other.

$\mathrm{F}(94)$ Is it more common to use code-mixing rather than code-switching, for bilingual persons?

AU I think code-mixing is more common for all kinds of people really, for people whose English is not bad.

F(94) Maybe in the lesson, because I studied the module Bilingualism, and they said that in the lesson when doing some classroom management they switch to Cantonese, and then when teach the lesson they switch to English.

AU Yes, that's a possibility. Did you find you did that in your teaching practice?

F(94) I think that depends on what class I teach. If the students' English standard is low I will use more Cantonese. Even teach the contents of the textbook.

AU We'll come back to that. Okay, 'Do you agree or disagree with the 
following statements?' 'English should be the only language used for all purposes.' You said 'Depends on the levels of the students, also for classroom management, Cantonese may be better.

F(94) Yeah

AU So what is it, English should be used for all purposes or not?

F(94) Only quite agree because you have no middle for me to choose.

AU So it could have been somewhere in-between 'Quite agree' and 'Quite disagree'?

F(94) Because when I use English I increase the students' exposure to English, so I think better to use English because for classroom management you want to settle down something as soon as possible, use their native language is better.

AU Okay, but how about lesson content, the next question is about lesson content. 'Do you think lesson content can be presented in mixed-code?' And you've put 'Quite disagree', 'Depends on the level of the students.' Do you think that's reasonable? Some teachers perhaps might present some parts of the content in English and then explain things in Cantonese.

$\mathrm{F}(94) \quad$ Is it code-switching rather than code-mixing in that case?

AU It could be both I suppose, probably it's going to be more like codeswitching ... Do you think it's okay to do that?

F(94) Code-switching or code-mixing?

AU Either

$\mathrm{F}$ (94) I think if the students' level is low then to explain the contents in Cantonese is good, but I think code-mixing is not good because I think they cannot receive, how to say, maybe two language make them interfere something and maybe make affects their written in code-mix. And also I think the organisation of the language cannot be learned well.

AU I probably agree with that.

$\mathrm{F}(94) \quad$ In my practice teaching I teach Band Five so I need to use Cantonese.

AU 'How much did you use only English in lessons?' 'Rarely' did you use only English. 
F(94) Yeah, I tell you, Band Five so ...

AU 'How did the amount of pure English you used change over the practice period?' 'It increased', at the beginning you were using more Cantonese and as you went on you started to use less Cantonese and more English.

F(94) I think there are two reasons. The first reason is that I think after learning a period the standard of the student maybe increased so I can use more English. The second reason is that because at the beginning when I come to the class, I don't know the students quite clear about what standard, so after a certain period I know they belong to what standard so I think they can cope with increasing English.

AU Do you think their standard actually improved over the time you were with them?

F(94) Not greatly because I only teach them two weeks in Practice Teaching, but they have some improvement in speaking and listening.

AU Do you think it's long enough, two weeks?

F(94) No, I think better to have two months.

AU Better for you or better for the students?

$F(94) \quad$ Both I think.

AU I think I would agree with that, two weeks is not really long enough for teaching practice.

$F(94) \quad Y e a h$, you need some period to know more about your students, and also I need to be familiar with the classroom environment and the school.

AU Of course yes.

F(94) And also at the beginning I'm a stranger to the students, maybe the cooperation is not good at the beginning.

AU Yeah, or it takes them time to get used to you, they have to see what you are like. So the last question in this section, 'What situations in an English lesson would or might you use Cantonese?' 'You've circled 'To explain lesson content', 'To establish discipline', and 'To be friendly with students'. We've virtually explained all that already. But not 'To explain lesson content'? Is that what you would hope?

F(94) Hope? No, actual experience. 
AU So you didn't actually present lesson content in English, but you did explain it in English.

F(94) I tried to minimise code-mixing.

AU No other lesson functions?

$\mathrm{F}(94) \quad$ Like what?

AU Well, if you want them to form into groups for example.

$F(94) \quad$ You mean instructions?

AU Yes

F(94) Well for simple ones I will use English, but for complicated ones I think I'll use Cantonese, in order to save time.

AU Would you use, would you give the instructions in Cantonese immediately or would you perhaps give them in English and if they didn't ...

F(94) Rephrase I think, rephrase in another wordings, in English, but if [they] still cannot understand, I use Cantonese.

AU Right, so Cantonese is a last resort?

F(94) Yeah, because I think it's an English lesson, I should use English.

AU When you were doing your English lessons, did you insist that the students use English all the time?

F(94) I think very difficult to control they use English, because the class have forty students, you cannot make sure each one speak in English. And also, I think peer pressure still exists, also they rather not speak, that means they prefer not speaking rather than speaking in English, so they shut up, not speaking anything.

$\mathrm{AU} \quad$ Some teachers like that, they think if they make them speak only in English then they'll get a quiet lesson.

$\mathrm{F}(94) \quad$ Maybe the teacher can give them some interesting activity in which they can speak in English. I think it works.

AU Okay, let's go on to Instructional Planning and Decision Making. Talking about planning, 'It's impossible to have a successful lesson that hasn't been well-planned in advance.' Which you 'Quite agree' with and you've added, 
'If the teacher is full of teaching experience the lessons may not be needed to be planned in advance.' Can you explain a little bit what you mean by that?

F(94) Because they can follow the same planning, in the past they use, so I think not need to plan in advance, only modify something. It depends on which class you teach.

AU So you're saying an experienced teacher wouldn't need to write down everything?

$F(94) \quad Y e a h$ possible I think because they already have some teaching experience, they need to know how to cope with some sudden events.

AU So they're experienced not only in planning but in decision-making ...

F(94) Yeah but I think for the new teacher they need to write out the alternative when something suddenly happen.

AU Yes, if they've not experienced it before, they don't know what might happen.

'Teachers should write detailed lesson plans for every lesson they teach.' Which you 'Quite disagree' with, 'A mini lesson plan is okay in actual situations in order to be workable.' What's a mini lesson plan?

$F(94) \quad$ The outline I think, if I need to write a detailed lesson plan for each lesson plan I think the workload is too heavy.

AU Of course, during your BA course were you given any instructions on how to write lesson plans?

F(94) Yeah, detailed and mini lesson plan, but we only need to write one lesson plan for one lesson, that means when my lecturer come to see what I teach in the class, then I need to give the lesson plan for that lesson only. For the other lesson they don't come to see me so I only use mini lesson plan.

AU So that was part of your assessment, you didn't have to write a lesson plan as an assessment without actually teaching that lesson?

F(94) No we need to follow the lesson plan, the detailed lesson plan to teach when the lecturer come to see me.

AU My lesson plan might consist of two lines.

$\mathrm{F}(94) \quad$ Two lines? No, for the detailed lesson plan at least four pages. For this one I think one pages. 
AU A mini lesson plan?

F(94) Yes

AU And what would it include?

F(94) Date, time, rationale for each step, warm up, the actual content of the lesson and follow up, and materials, the focus, the language form and language usage and something like that.

AU Would it be step by step?

F(94) Yeah, and also I need to state also the interaction between teacher and students, that means, how many students for that activity, if that is group work or individual work, or the interaction between the student or the interaction between the teacher and the student.

AU Yes, I think after you've been teaching for maybe a term, you would need to write very little. 'Teachers should be more responsive to student needs than the set syllabus.' 'Quite agree', 'But the syllabus be taken into account, as the students need to cope with public examinations.' So it's somewhere between the two. You know that most schools would have a set syllabus for the lower forms as well?

F(94) Yeah I think the syllabus is also for lower forms and also in school they have the scheme of work..

AU Would you follow that strictly?

F(94) I think it is a must, but I think I can modify in which way I teach the students. That means I can introduce the new activities, maybe change the sequence of teaching the items.

AU Okay, planning lessons, 'During your Practice teaching module, how often did you find you had to change your lesson plan?' 'Sometimes'.

Okay, 'On what basis did you plan your lessons?' 'Perceived needs of students' you ranked as the first and then 'BATESL course guidelines'. Last of all was 'Regular teacher's instructions', why was that?

F(94) As I say before, I think it depends on the students' needs, not fixed to the scheme of work, I think it is better to be flexible.

AU So you were able to perceive the needs of the students?

F(94) Okay for me yeah. 
AU In such a short time you were able to do that?

F(94) Because I'm good at building up the good relationships with the students, so I can actually ask them directly what they want, or what problem they have. So more easy to perceive the needs of the students.

AU Okay, 'What were the factors that had the most influence on the decisions you made?' Again 'Students' needs'.

F(94) Yeah

AU Teaching Approach, 'Examination requirements should determine what is taught in English lessons.' You've circled both, 'Quite agree' and 'Quite disagree'.

F(94) Yeah, two point five.

AU We purposely don't put a three in.

$\mathrm{F}(94) \quad$ Yeah I know that, if you put that then people will choose that.

AU Yes, 'It also depends on the needs of the students.'

F(94) Maybe the examination requirements set too high, the students cannot meet so it's meaningless to teach the content of examination requirement.

$\mathrm{AU} \quad$ But at the same time if they are going to take the examination then they need ...

F(94) Yeah they need ...

AU 'Students learn English better through interaction with other students than through direct instruction from the teacher.' You 'Absolutely agree' with that, 'Students may be more responsible for their learning and they can learn from each other.' Do you think there are any situations where students would learn better through direct instruction?

F(94) Maybe when teach some new things for them, that means the presentation stage, then the students can learn through direct instruction from the teacher. But for the practice stage and production stage better increase the interaction among the students. And also I think if everything done through direct instruction from the teacher, they try to keep quiet because they think that teacher's standard is much higher than theirs. So they daren't speak something wrong, their confidence is low in that case, because similar level. 
AU Will they learn from each other?

F(94) Depends, depends which partner you cooperate with, but I think this belong to the group organisation. I think for learning from each other, I can pair a strong one with the weak student, that mix the ability of the students.

AU So the weaker one will learn, how about the stronger one?

$\mathrm{F}$ (94) Mmmm, that means nothing is perfect I think. What's your solution to that?

AU My solution to that?

$\mathrm{F}(94) \quad$ Maybe the teacher can give some help to them.

AU I think you have to mix it ..

$F(94) \quad$ Also maybe increase the number of the students, several strong one with several weak one then strong can learn from strong. But something bad is that the strong student may be dominant in that case, especially in speaking. But that will always happen, hopefully the students who are weaker will be influenced by those who are stronger and it will give them more encouragement.

AU 'A class should be strictly controlled by the teacher and all lesson content directed ...', 'Absolutely disagree', 'Lessons will be boring if students' participation are too few. Relationship between the students and the teacher may not be good.' In what ways would lesson content not be directed by the teacher?

F(94) For example, using inductive approach to hear this term, especially for grammar, you can give some problem-solving task for them to find out the rules of some grammar items, yeah. For example, give them some grammar item and ask them to generalise the rule out by themselves.

AU Do you think that's possible in a class with forty students?

$\mathrm{F}(94) \quad$ Yeah, why not?

AU Don't you think it's more difficult with a bigger class.

$\mathrm{F}$ (94) Through that way the rules is more impressive, yeah.

AU 'Teachers should be prepared to experiment with materials and teaching strategies from a variety of sources.' 'Quite agree', 'In order to see the good and bad points of the materials then some improvements can be made in order to make the set of materials more perfect.' 
$\mathrm{F}(94) \quad$ And also I think the same set of materials may not be suitable for some class, may suitable for the other class, yeah.

AU I think that's a bit of a leading question, isn't it? It's the kind of question we would expect most people to agree with.

$F(94)$ If I don't experiment the materials I can know the use of the materials, how useful it is, something like that.

AU 'Did you find the students learn English better through interaction with other students or through direct instruction from the teacher?' 'Through interaction with other students.' No comment, is that because you didn't think there was anything else to say about it or ...

F(94) Yeah, and also I think the time is not enough for me to fill so many things.

AU I see, but you would have said something you think if you'd had more time?

F(94) Mmmm, I think the teacher can also be one of the participants in some activities, so the students can interact with the teacher.

AU Is that good do you think?

F(94) Depends on what relationship between the teacher and the students.

AU Yeah, I'd agree. 'During your Practice teaching module, did you find you controlled your classes strictly and directed all lesson content?'

'Sometimes'.

$\mathrm{F}(94) \quad$ In fact in my case I tried to reduce to punish the students because I think this will break the relationship between the teacher and the students, and the classroom atmosphere is not good in that way. But if there is a great discipline problem, then this will affect the other good student to learn so in that case I need to control my class strictly. And also because my belief is learner-centered so I don't quite agree to direct all lesson content, I try to make them responsible for learning by themself.

AU 'Did you experiment with materials and teaching strategies from a variety of sources?' You did.

F(94) Yeah

AU Where did you get your materials from?

F(94) Modify some activities from a good reference book. 
AU Where did you get the reference book?

$\mathrm{F}(94)$ In the library, and also the reference books suggest by the lecturers and also find by myself in the library. But I think most of the activity are suitable only for foreigners because most of the books are written by foreigners.

AU Most of them are written for teaching English as a foreign or second language in England, aren't they?

Okay,'Which of the following factors do you think has the most influence on your approach?' And you've put 'Teacher training'.

F(94) Yes, I think that through this teacher training I can learn how to teach correctly, so I think it affects me much, the most.

AU So let's say if you didn't have any teacher training, put into a class and told to teach ...

F(94) Then follow my previous teachers' way of teaching, too traditional. Because I need some example to follow.

AU Professional Relationships, 'How confident are you in your ability to become a successful teacher?' 'Quite confident', okay. 'How do you feel about the status of teachers in today's society?' 'Teachers have a lower status than they should.'

F(94) Yeah, because ones with good English they will go to business sector and so fewer teachers to teach, and in that case the Government try to reduce the requirements of the teachers in order to study in for example education college and something like that. That means the society doesn't expect the teachers will be as good as in the past.

AU How do you think they could raise the status of teachers?

F(94) Maybe the Government can give more good promotion and salary so the status will be increased.

AU Do you think they will?

F(94) I don't think so.

AU I don't either.

'Your best attribute of being a teacher', you said is 'Empathy with students'.

$F(94)$ Yeah, because I think we are deal with the human beings, we need to care about their feelings. 
And your worst you'd say is 'Strict disciplinarian'.

F(94) Yeah, I have already said that this will break the relationship.

$\mathrm{AU} \quad$ But don't you think that sometimes you have to be?

$F(94) \quad$ Sometimes yeah.

AU 'Able to form good relationships with colleagues' is fairly low as well.

F(94) Yeah, because I think in my Practice teaching I think the colleagues is not good. They don't care the other colleagues, they only do their own things and also I think many of them do not really want to teach.

$\mathrm{AU} \quad$ Bad atmosphere was that? Low morale?

F(94) Yeah

AU I can see how in a Band 5 school the morale will be quite low. The teachers don't want to teach but they have no choice.

F(94) Yeah, they think that the student is too bad, so they have ...

AU Okay, 'Do you see teaching as a long-term career?'

$\mathrm{F}(94) \quad$ Yes

AU Okay, 'Who do you think will be most helpful during your first few years?' 'The teacher training supervisor'.

F(94) Yeah, they are experienced teacher, I think.

AU Not other teachers within the school you're teaching?

F(94) They may be, I think they may protect themself. And also they receive different training and so difficult to communicate.

AU Some teachers, maybe panel chairs etc. can be very helpful.

F(94) Yeah, depends on which colleagues I meet, but this one will be surely willing to help you.

AU 'Who was most helpful to you?' 'More experienced teachers'.

$F(94) \quad Y e s$, I can learn from them. 
AU So during your practice teaching module it was the more experienced teachers who were most helpful but you think during your first year of teaching it will be your teacher training supervisor?

$F(94) \quad$ Because during my practice teaching we only strictly to go to the school, not come to CPHK so it's very difficult to contact my supervisor.

AU I see, then 'Which of the following tasks do you think you will have to do as a first-year teacher? Again this was a trick question, in my experience you would find that you would have to do all of them.

F(94) No, depends on which school you teach, maybe some school doesn't have any religion.

AU That's a good point.

$F(94)$ I think better to get some person who really have a religion to be in charge of that activity.

AU Yeah, 'Which will take up the most time?' 'Preparing lessons'.

F(94) Most important

AU Not marking

F(94) Not marking, maybe only once a week.

AU Marking assignments, marking books?

F(94) Commonly I think in HK schools, the students have one week to do the homework maybe, or two days. Depends on which form I think, how difficult the assignment.

AU Okay, Perceptions and Values, 'Do you have any philosophy?' 'Yes, very definite.' And what is it? 'I strongly agree that the lessons should be student-centered. The good relationship between students and teachers is very important. In fact teachers can learn from students. Also, I think teaching English should be creative and funny.' That's very clear.

'Most important factors which affect what goes on in the classroom.' None of them you've put as most important, you've labelled them either as 'Very or Quite important'. 'Location of school' 'Not important'. Any reason why you didn't choose any to be 'Most important'?

$F(94) \quad$ Because I think similar priority for these.

AU You've only put one as 2, 'Motivation of students' and all the others as 3. 
$F(94) \quad Y e a h$, most of them relate to the school policy is it?

AU 'Which of the following do you consider most important to teach?' You've put 1 for all of them.

F(94) Yeah, but I think in Hong Kong society I think speaking may be less important, because in public examination they only, that means only a few marks given to speaking skills.

AU How about daily life?

F(94) Daily life I think all are important, but I think speaking again, less important in HK society. Why did you separate speaking and pronunciation?

AU Well, people can speak and be understood but maybe their pronunciation is not good.

$\mathrm{F}(94) \quad \mathrm{Oh}$, then I think these two because the important thing about speaking is to communicate.

AU ' $\ldots$ best sums up your feelings as regards the relationships a teacher should have with his or her students?' 'The teacher should be prepared to devote as much time as is necessary to helping his or her students.'

Okay, 'Your strengths and weaknesses as a teacher and number them according to the following key.' 'Innovation' as being the strongest, followed by 'Organisation', 'Planning', 'Relationships with students', 'Motivating students', 'Teaching reading', 'Teaching writing', 'Teaching grammar'. 'Teaching listening' you've put 'Weak', that's interesting.

F(94) I think in this aspect ... material can help me, and also I think my listening skill is not good.

AU You think your own listening skill is not good so you have more difficulty teaching it?

F(94) Even my speaking is not good but for this aspect I think more material can help me.

AU Better materials?

$\mathrm{F}(94) \quad$ Yeah, I think listening, most of them are traditional and boring.

AU I think nowadays we are getting better materials on the market.

$\mathrm{F}(94)$ And also I think the equipment is not good in school for listening. 
AU I Okay, finally, 'Are you planning to teach English next year?' 'No, but I decide to teach English in future.' What does that mean?

F(94) Because next year I need to study Postgraduate Diploma in Education.

AU Oh, 'If you decide to teach, where and in what type of school would you like to teach?' 'The school is better near my home.' That's why we included 'Location of school'.

F(94) Better, but it's not my priority. I will teach Band Four or Five students, they are the ones really need the teachers' help.

AU So you're looking on teaching as the students need you so you want to be a teacher.

F(94) Ah ha.

AU Okay, thank you very much.

F(94) You're welcome. 


\section{B.2 Questionnaire Administered to BATESL Graduates in 1997}

\section{BATESL Course Questionnaire}

The following questionnaire is to be used as a part of a research project which aims to look at the teaching of English in Hong Kong. This is the same questionnaire you filled out when you first started here as a student on the BATESL course. I am now asking you to fill out this questionnaire, with a few added questions, to find out how your outlook might have changed over the last three years. Your responses will be kept completely confidential. Please take a little time to consider each item carefully and respond as fully as you can.

\section{Language Use}

1. How would you describe the standard of your written English before you began the BATESL course?
1. Excellent
2. Very good
3. Quite good
4. Fair
5. Rather poor

2. How would you describe the standard of your written English now?

1. Excellent

2. Very good

3. Quite good

4. Fair

5. Rather poor

3. How would you describe the standard of your spoken English before you began the BATESL course

1. Excellent

2. Very good

3. Quite good

4. Fair

5. Rather poor 
4. How would you describe the standard of your spoken English now?
1. Excellent
2. Very good
3. Quite good
4. Fair
5. Rather poor

5. When speaking in Cantonese to friends who have Cantonese as their first language, do you use some English words or expressions (code-mixing)?
1. Always
2. Frequently
3. Quite often
4. Rarely
5. Never

6. When speaking in Cantonese to friends who have Cantonese as their first language, do you switch to English and then back into Cantonese (code-switching)?
1. Always
2. Frequently
3. Quite often
4. Rarely
5. Never

Questions 7 and 8

Do you agree or disagree with the following statements? Please indicate by circling the numeral that corresponds to ONE of the four statements:

1. Strongly agree

2. Agree

3. Disagree

4. Strongly disagree 
7. In English lessons, English should be the only language used for ALL purposes.

Comments:

8. In English lessons, lesson content can be presented in a mixed-code CantoneseEnglish.

$\begin{array}{llll}1 & 2 & 3 & 4\end{array}$

Comments:

9. During your Practice Teaching module, how much did you use only English in lessons?
1. Always
2. Usually
3. Rarely
4. Never

10. During your Practice Teaching module, how did the amount of pure English you used in lessons change over the practice period?
1. It decreased
2. It didn't change
3. It increased 
11. In what situations in an English lesson would or might you use Cantonese?

Circle all those that apply.

1. None

2. To present lesson content

3. To explain lesson content

4. To establish discipline

5. To be friendly with students

6. To perform other lesson functions (Please specify)

12. To what extent do you see the use of English in secondary schools being affected by the changeover in sovereignty of Hong Kong from British to Chinese rule?

1. To a large extent

2. To some extent

3. To little extent

4. To no extent

Comments:

13. What (if any) changes do you expect there to be in the use of English in secondary schools as a result of the changeover in sovereignty of Hong Kong from British to Chinese rule? (Please comment) 


\section{Instructional Planning and Decision Making}

Questions 14 to 16

Do you agree or disagree with the following statements? Please indicate by circling the numeral that corresponds to ONE of the four statements:

1. Strongly agree

2. Agree

3. Disagree

4. Strongly disagree

14. It is impossible to have a successful lesson that hasn't been well-planned in advance.

$\begin{array}{llll}1 & 2 & 3 & 4\end{array}$

Comments:

15. Teachers should write detailed lesson plans for every lesson they teach.

$\begin{array}{llll}1 & 2 & 3 & 4\end{array}$

Comments:

16. Teachers should be more responsive to student needs than the set syllabus.

$\begin{array}{llll}1 & 2 & 3 & 4\end{array}$

Comments: 
17. When planning lessons, how detailed do you think the plans should be?

1. Very detailed and precise

2. Quite detailed and precise

3. Quite detailed and quite flexible

4. Loosely detailed and quite flexible

5. Brief and very flexible

18. During your Practice Teaching module, how often did you find you had to change your lesson plan?
1. Always
2. Usually
3. Sometimes
4. Rarely
5. Never

19. During your Practice Teaching module, on what basis did you plan your lessons? Rank the following with 1 being the most influential on your planning and 6 (or 7) the least.

Syllabus

Regular teacher's instructions

BATESL course guidelines

Textbook 'Teacher's Instructions'

Own ideas

Perceived needs of students

Others (Please specify) 
20. During your Practice Teaching module, what were the factors that you think had the most influence on the decisions you made. Rank the following with 1 being the most influential and 4 (or 5) the least.

Students' needs

Regular teacher's input

Supervisor's advice

Fellow student teacher's advice

Others (Please specify)

21. To what extent do you think the changeover of sovereignty of Hong Kong from British to Chinese rule will have an effect on your lesson planning and decisionmaking?

1. To a large extent

2. To some extent

3. To little extent

4. To no extent

Comments:

\section{Teaching Approach}

Questions 22 to 25

Do you agree or disagree with the following statements? Please indicate by circling the numeral that corresponds to ONE of the four statements:

1. Strongly agree

2. Agree

3. Disagree

4. Strongly disagree 
22. Examination requirements should determine what is taught in English lessons.

$\begin{array}{llll}1 & 2 & 3 & 4\end{array}$

Comments:

23. Students leam English better through interaction with other students rather than through direct instruction from a teacher.

$\begin{array}{llll}1 & 2 & 3 & 4\end{array}$

Comments:

24. A class should be strictly controlled by the teacher and all lesson content directed by the teacher.

$\begin{array}{llll}1 & 2 & 3 & 4\end{array}$

Comments:

25. A teacher should be prepared to experiment with materials and teaching strategies from a variety of sources in his/her teaching.

$\begin{array}{llll}1 & 2 & 3 & 4\end{array}$

Comments: 
26. In your Practice Teaching module, did you find that students learn English better through interaction with other students or through direct instruction from the teacher?

1. Through interaction with other students

2. Through direct instruction

3. No difference between the two

Comments:

27. During your Practice Teaching module, did you find that you controlled your classes strictly and directed all lesson content?

1. Always

2. Usually

3. Sometimes

4. Rarely

5. Never

Comments:

28. During your Practice Teaching module, did you experiment with materials and teaching strategies from a variety of sources?

1. Very much

2. Somewhat

3. A little

4. Very little or none

Comments: 
29. Would you describe your teaching style as learner-centered or teacher-centered?

1. Very learner-centered

2. Mostly learner-centered

3. Mostly teacher-centered

4. Very teacher-centered

30. Which of the following factors do you think has the most influence on your approach to the teaching of English? Rank the following with 1 being the most influential and 12 (or 13) the least.

Class size

Classroom size

Noise in classroom

School/Principal's/Education Department policy

Examination requirements

Current trends in ESL teaching

Personality of teacher

Motivation of students

English proficiency of students

Availability/quality of teaching materials

Availability/quality of teaching aids (audio/visual, lang. lab. etc.)

Teacher training

Other factors (please specify)

\section{Question 31}

Do you agree or disagree with the following statement? Please indicate by circling the numeral that corresponds to ONE of the four statements:

1. Strongly agree

2. Agree

3. Disagree

4. Strongly disagree 
31. The changeover in sovereignty will affect my approach to teaching.

$\begin{array}{llll}1 & 2 & 3 & 4\end{array}$

Comments:

\section{Professional Relationships and Responsibilities}

32. How confident are you in your ability to become a successful teacher?

1. Very confident

2. Quite Confident

3. Unsure

4. Not very confident

5. Not at all confident

33. How do you feel about the status of teachers in today's society?

1. Teachers enjoy a high status

2. Teachers enjoy a moderate status

3. Teachers have no special status

4. Teachers have a lower status than they should 
34. What do regard as your best attribute in terms of being a teacher? Rank the following with 1 being the best and 8 (or 9) the worst.

Good command of the language

Good communication skills

Innovative in terms of teaching ideas

Strict disciplinarian

Empathy with students

Careful planner

Aware of latest trends in ESL teaching

Able to form good professional relationships with colleagues

Others (please specify)

35. Do you see teaching as a long-term career for you?

1. Yes, definitely

2. Probably

3. Not sure

4. Definitely not

36. In your first few years of teaching, who do you think will be most helpful to you?

1. Fellow first-year teachers

2. More experienced teachers

3. Panel chair

4. Principal

5. Senior teachers

6. Teacher-training supervisor

7. Others (Please specify) 
37. During your Practice Teaching module, who was most helpful to you?

1. Fellow first-year teachers

2. More experienced teachers

3. Panel chair

4. Principal

5. Senior teachers

6. Teacher-training supervisor

7. Others (Please specify)

38. Which of the following out-of-class activities do you think you will have to do as a first-year teacher? Circle all those which apply.

1. Be a form teacher

2. Be involved in English Club/Debating Club/Drama/other clubs

3. Train students for Speech Festivals and other performances

4. Be involved in extra-curricular activities

5. Be involved in religious activities

6. Counsel students

7. Meet parents

8. Attend meetings at school with principal/panel chair

9. Attend meetings at school with other teachers

10. Attend courses (e.g. at I.L.E.)

11. Mark papers

12. Prepare lessons

13. Prepare examinations

14. Administer examinations

15. Mark examinations 
39. Which of the following out-of-class activities do you think will take up the most time?

Being a form teacher

Being involved in English Club/Debating Club/Drama/other clubs

Training students for Speech Festivals and other performances

Being involved in extra-curricular activities

Being involved in religious activities

Counselling students

Meeting parents

Attending meetings at school with principal/panel chair

Attending meetings at school with other teachers

Attending courses (e.g. at I.L.E.)

Marking papers

Preparing lessons

Preparing examinations

Administering examinations

Marking examinations

40. In your future teaching, to what extent do you expect to be working closely with other teachers, e.g. sharing materials or discussing teaching ideas?

1. Very much

2. Some

3. A little

4. Not at all

41. In your future teaching, what do you think will help you most to continue to develop yourself as a teacher? Rank the following with 1 being the most helpful and 6 (or 7) the least.

Reading articles

Finding new materials

Designing new materials

Learning from other teachers

Getting feedback from panel chair

Attending conferences/seminars/workshops at the I.L.E. etc.

Others (Please specify) 
42. To what extent are you worried about your future as a teacher after the changeover of sovereignty of Hong Kong from British to Chinese rule?

1. To a large extent

2. To some extent

3. To little extent

4. To no extent

43. If you are worried about your future as a teacher after the changeover of sovereignty of Hong Kong from British to Chinese rule, what are your particular worries? (Please comment)

44. Do you think your professional relationships or responsibilities will change after the changeover of sovereignty of Hong Kong from British to Chinese rule?

1. Yes

2. Somewhat

3. No

If yes, in what ways? (Please comment) 


\section{Perceptions and Values}

45. Do you think that you have a definite philosophy of teaching?

1. Yes, very definite

2. Quite definite

3. Not very definite

4. Not definite at all

46. If you didn't answer "1" to question 45, which area(s) are you unsure about? Circle all those which apply.

1. Personal career goals and ambitions

2. Ideas on teaching content

3. Ideas on teaching methods

4. Ways to handle discipline

5. Professional relationships

6. Relationships with students

7. Professional responsibilities

8. Professional status

9. Working conditions

10. Salary

11. Others (please specify)

47. Please try to describe briefly your teaching philosophy. 
48. What do you think are the most important factors which affect what goes on in the classroom? Number the factors according to the following key:

Motivation of students

Language ability of students

Efficiency of English department

Competence of teacher

Backup of colleagues

Design of classroom

Class size

Location of school

Education Department

School policy

Examinations Authority

Businesses/employers

Government

Others (please specify)

$$
\begin{array}{ll}
1= & \text { Most important } \\
2= & \text { Very important } \\
3= & \text { Quite important }
\end{array}
$$

49. Which of the following skills do you consider the most important to teach? Number the skills according to the following key:
Reading
Writing
Speaking
$1=$ Very important
Listening
$2=$ Quite important
Grammar
Pronunciation 
50. Which of the following best sums up your feelings as regards the relationships a teacher should have with his/her students? Circle all those which apply.

1. The teacher should be a transmitter of knowledge.

2. The teacher should be a facilitator of learning.

3. The teacher should be in total control of all lessons at all times.

4. The teacher should allow students to decide the direction that their learning should take.

5. The teacher should try to become friends with the students.

6. The teacher should act like a parent.

7. The teacher should refrain from punishing students.

8. The teacher should be prepared to devote as much time as is necessary to helping his/her students.

51. Consider your strengths and weaknesses as a teacher and number the factors according to the following key:

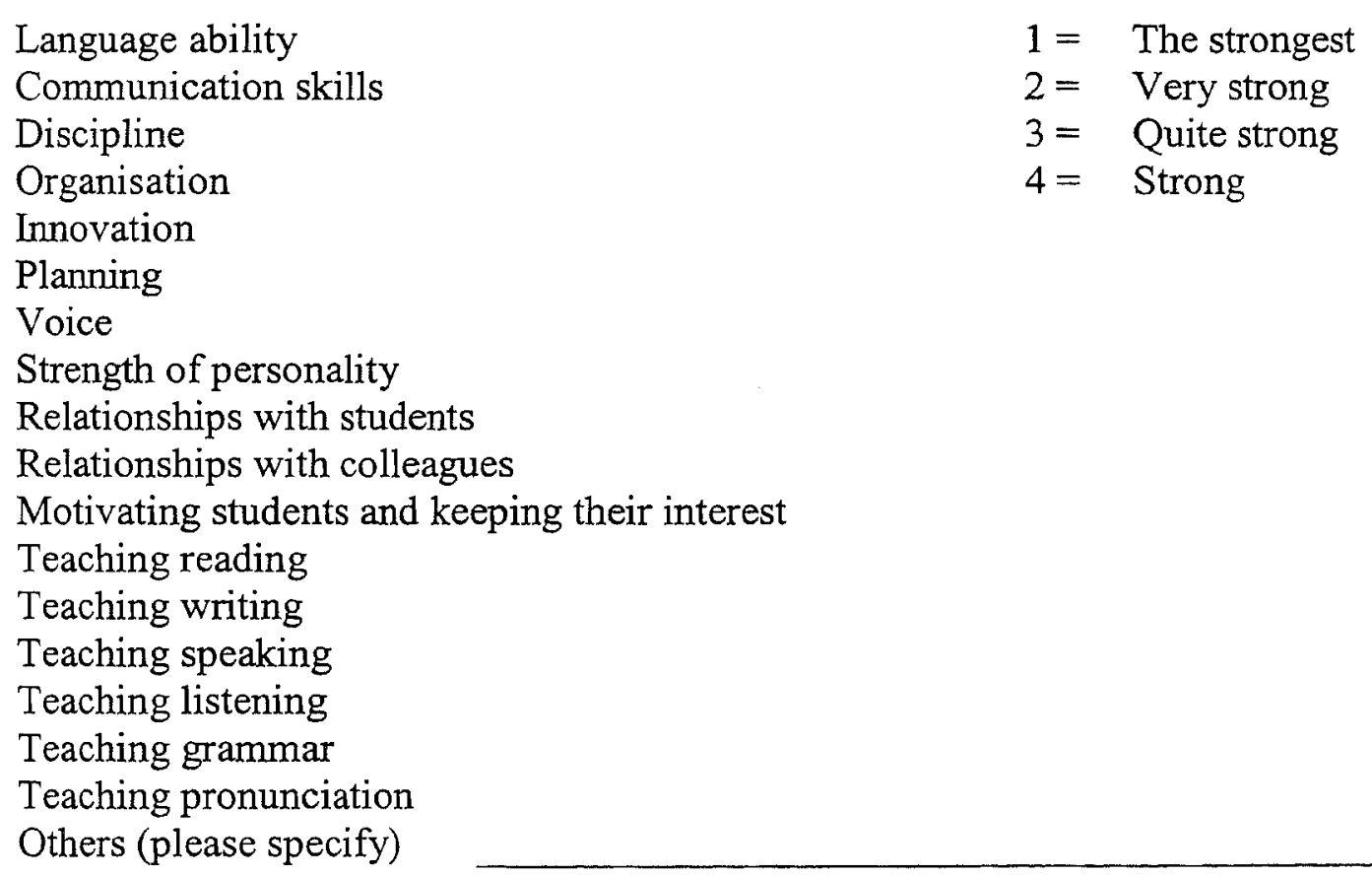

$$
\begin{array}{ll}
1= & \text { The strongest } \\
2= & \text { Very strong } \\
3= & \text { Quite strong } \\
4= & \text { Strong }
\end{array}
$$




\section{Personal Information}

52. Are you planning to teach English next year?
1. Yes
2. No
3. Not yet sure

53. What factors did you consider in making your decision about teaching (or not) next year? (Please comment)

54. If you do decide to teach, where and in what type of school would you like to teach?

55. If you don't go on to teach English, what do you think you might do instead?

Date:

Name:

You do not need to divulge your name if you do not wish to. However, at a later date I would like to interview a few people, particularly those who intend to teach next year. By adding your name, it makes it possible for me to contact you for this purpose.

Finally, I wish to offer my sincere appreciation and thanks for taking time out from your very busy schedule to help me in this project.

Best wishes for the future. 


\section{B.3 Transcriptions of Interviews with BATESL Graduates in 1997}

\section{Interviewee: $\quad$ Subject $\mathbf{N}(97)$ \\ Date: 23 July 1997}

Female, 24 years old.

Was unsure as to whether she would teach as she wished to explore other areas such as creative writing, journalism or voluntary work.

$\begin{array}{ll}\mathrm{AU} & \text { Researcher } \\ \mathrm{N}(97) & \text { Interviewee } \\ \ldots & \text { Missing words / Unfinished utterance } \\ \ldots & \text { Pause }\end{array}$

AU The first part of the questionnaire was Language Use and you said that the standard of your written and spoken English before you began the BATESL course and now is 'Quite good', 'Quite good', 'Quite good', 'Quite good'.

N(97) Yes, I believe so.

$\mathrm{AU} \quad$ So you don't think there has been any improvement during the course?

$N(97)$. A little bit. I think the major improvement is on the spoken and the listening in English, but written English, I can't say improvement or not because what we wrote in secondary school is quite different from what we wrote in university, so I think it's two kinds of writing. It's quite difficult for me to comment on whether I have improvement or not.

AU I suppose you can only tell if you've improved your writing or your speaking if you actually write or speak a lot outside the university, or outside of your work.

$\mathrm{N}(97) \quad$ What I can say is I'm more confident in both kinds of English. I write quite a lot outside school, but actually I don't have much chance to speak English outside school, so I think writing's okay, maybe a little bit better than before. Speaking, I think I'm more confident than before, I'm more concerned about my errors, I do quite a lot of self-corrections when I speak. So, maybe that's the improvement. 
AU You said that you always use English words or expressions when speaking Cantonese with friends. What kinds of English words or expressions would you use?

$\mathrm{N}(97) \quad$ Okay, when we talk about the academic aspect, we usually use some words like 'confidence', technical words or theories, something like that. But in social aspects, it's difficult to say because we use quite a lot of English, like ... for example, talking about films or music, we usually talk about, even though there is a Chinese name or Cantonese name, translated names, we use the English name to talk about the films, the director's name, something like that. Sometimes we may use some idioms, like 'practice makes perfect', when we talk.

AU Why do you think that is? Why use the English names when there are Cantonese translations?

$\mathrm{N}(97) \quad$ The major problem is the Cantonese translation is quite long and difficult to remember, and there are different translations sometimes, so probably we will use the English one. It's quite weird, the translation names sometimes.

AU And as for code-switching, you said 'Quite often'. So switching from Cantonese into English, rather than just a word or phrase but a bit of an extended speech.

$\mathrm{N}(97) \quad$ When I talk to some of my friends whose migrated to other countries, who have studied in other countries, sometimes we will code-switching. It's because they talk to me in this kind of mode, so I will respond to them.

AU Something I've noticed quite a lot this summer, perhaps because of the summer holidays but maybe because of the handover as well, that there's a lot of people who obviously live in Canada or America and they've come back to HK, and you can hear them speaking and they are code-switching a lot. Perhaps they live in Canada and they are meeting their old classmates and friends, so they are speaking in Cantonese.

N(97) Especially small children.

AU Yes, they are code-switching a lot. Okay, next we look at English in teaching. 'English should be the only language for all purposes'. You said, 'Quite disagree', 'It depends on the level of the students.'

$\mathrm{N}(97)$ Yes, that's my belief. Actually, after the practice teaching, or the internship, I found that though it is a Band 1 school, but their English proficiency was quite different. Some can, just like I taught in La Salle for my practice teaching, some can stand up and talk to me in English, they had no problems at all, but some even couldn't understand you, even you talk to them in 
simple English. I think if the students don't understand you in English, there's no point for you to still talk to them in English because education is to let them understand something. Of course if it's an English lesson, I think it's better for a teacher to use English as more as possible to expose them to English more, but for example, if you talk about some other subjects, or some other purposes, it might not be. The most important thing is to let the students understand the things, instead of to let them listen or speak English.

AU 'Lesson content can be presented in a mixed-code Cantonese-English', you said, 'Absolutely disagree'. So you believe that lesson content should all be in English.

N(97) Mm, or all in Cantonese, because I think that Cantonese will lower the students' language level, and it's quite difficult for Cantonese and Chinese mixed-code sometimes, they are two really different languages. There are technical problems when you are to make a mixed-code content and it will make students confused about these two kinds of languages, I think. So it's do no good to students' language level.

AU 'During your practice teaching, how much did you use only English in English lessons?'

N(97) Yes, I've told you I always ...

AU Because you were a good ...

N(97) Good school.

AU And how did the amount of pure English you used in English lessons change?' You said. 'It didn't change.' So you started off with only English and then continued with it. 'In what situations in an English lesson would or might you use Cantonese?' You said, 'To explain content', 'To establish discipline', 'Give instructions' and 'When students indicate they don't understand'. Any comment on that?

N(97) Not at all but I think for 'When students indicate that they don't understand', I think I will still try to explain to them in simple English, to demonstrate the meanings to them with some examples, something like that first, Cantonese will be the last resort for any explanations, I think.

AU 'To what extent do you see the use of English in schools being affected by the changeover in sovereignty?' You said, 'To little extent'.

$\mathrm{N}(97) \quad$ Yes, very little, because the world status of English is still very solid and HK is an international city, so I think it didn't change much. Maybe a little bit, because we can see that the status of Chinese is a little bit uprising, it's 
growing become more and more important. The two languages are both official languages now, so maybe a little bit affected, but not much.

AU So you think that because Chinese will be more emphasised, English perhaps will be less?

N(97) Yes, but just a little bit, I think.

AU Okay, and 'What, if any, changes do you expect there to be in the use of English in secondary schools as a result of the changeover?' You said, 'The importance of English is comparatively lower due to the rising importance of Chinese.' Yes, that's what have just said, isn't it?

N(97) Yes.

AU Okay, the next section is Instructional Planning and Decision-Making, 'It's impossible to have a successful lesson that hasn't been well-planned in advance.' You said you 'Quite agree' with that, 'Maybe it's possible for experienced teachers.'

N(97 But I think for amateur, it's no way a new teacher, a fresh teacher, first of all usually doesn't have confident, if he or she doesn't well-planned a lesson in advance and a student didn't understand a point when you explain to them over and over again and the lesson is running a bit out of time, a fresh teacher may not know how to deal with it. So, I think the plan is for this kind of incident or this kind of event. But probably an experienced teacher will not do.

AU So for experienced teachers, they perhaps don't need to plan so much in advance? I think that's what I've found, to a certain extent anyway. Once you have taught the same class or similar classes before, then you don't need to re-plan all the time. 'Teachers should write detailed lesson plans for every lesson they teach?' 'Absolutely disagree', 'It's just necessary for fresh teachers'.

N(97) Because I think if the lesson plans are too detailed, it will lower the flexibility of teaching, so it's not good because a teacher must be very flexible.

AU 'Teachers should be more responsive to students' needs than the set syllabus.' 'Quite agree', 'Teachers have to adjust the syllabus to match students' needs.' Do you think it's possible for teachers to do that?

N(97) I think it depends a lot on the panels or the principals, because I watch a TV programme about teachers who teach in Delia, a Band 5 school, and actually it doesn't follow the syllabus at all, because the level of the students are too low. For F1 students, the students may just know something from P4, 
something like that, so the progress very very slow. So the teachers just choose two or three points to teach them for the whole year. And the reporter asked her why, and she said the students only understand a little, better than none. I also believe that if the students' level is not up to the syllabus level, they will understand nothing. You just stand in front of them and talk and talk, and they understand nothing. It's no use. So I think teachers should try to adjust the syllabus, or should not just blindly follow the syllabus. They should think about the students' needs.

AU Very often, if it's an exam course then the syllabus is set and there's not much you can do, but if it's a lower school form, F1 to F3, then I think that teachers can pretty much set their own syllabus. They might have things that they are supposed to cover, but if the students are that weak and they are not going to be able to grasp very much, then you have to adjust the syllabus. 'When planning lessons, how detailed do you think the plans should be?' 'Quite detailed and quite flexible', so you've gone for the middle option there. 'During your practice teaching module, how often did you find you had to change your lesson plan?' 'Always'.

$\mathrm{N}(97) \quad$ Always.

AU Why was that?

$\mathrm{N}(97) \quad$ Actually, I'm quite aggressive. Usually I plan more than I can teach, and because the students are very responsive, and I had a lot of interactive activities with the students, so usually this kind of activities took much much more time than I planned, so I had to change a lot.

AU Well, I think it's probably one of the biggest worries for a teacher is when you plan a lesson what you plan will take less time than you actually planned it so you'll suddenly be stuck with 10 minutes left of the lesson and nothing planned to do. So you probably tend to plan more than you are likely to do. But again, that comes with experience that once you know your class you know how much they are likely to do, and with experience certain activities if they are going too quickly you can stretch them out. 'During your practice teaching module, on what basis did you plan your lessons? Rank the following.' You've said, 'Perceived needs of students' first, followed by 'Own ideas', 'Teacher's instructions', 'Syllabus', 'Course guidelines', 'Textbook teachers' instructions'. So you were pretty free to do what you wanted, were you, during your practice teaching?

$\mathrm{N}(97) \quad Y e s$, because my cooperating teacher's quite good, and she told me, just try out what you learned in university, don't mind anything. So I can be pretty free in my practice teaching.

AU It's quite short though, isn't it, the practice teaching? 
$\mathrm{N}(97) \quad$ Two weeks

AU Two weeks, yes. Perhaps the cooperating teacher thinks it's just two weeks so $\ldots$

$\mathrm{N}(97) \quad$ So it's okay.

AU At my school we had student teachers, but from the I of Ed, and they are there for ...

N(97) For a month.

AU Well, six weeks, something like that. So, we give them a syllabus to follow, pretty much the same syllabus that we follow, but we give them as much freedom as possible. Do you think it's long enough, the practice teaching?

$N(97) \quad I t$ 's too short because it's just like we have a taste of teaching, but we still don't know what to do. It's too short.

AU I know that you have observation and is it internship?

N(97) Internship, yes, but I know that some of my classmates had to teach for the whole two weeks of the internship. We are supposed to observe for the internship, but they still had to teach.

AU Is that they were told to teach, or they ...?

CC They were told to teach.

AU Three years ago we had two BATESL people at my school for internship, and they taught just one lesson. They said that they wanted to do that. They said that they wanted to observe for the first week and maybe help out, and then take a lesson in the second week.

N(97) That's what we are supposed to do.

AU To teach the whole lot is ...

N(97 Quite heavy, so actually, I don't think two weeks for practice teaching is enough. Not enough.

AU But I think the point is that the BATESL is not actually a teaching qualification. It's just a qualification in English, as much as anything, and then you are meant to do your Cert. Ed. on top to become a qualified teacher. 'During your practice teaching, what do you think were the factors that had the most influence on the decisions you made? Rank the following.' 
'Students' need', 'Regular teacher's input', 'Supervisor's advice', 'Fellow student teachers' advice'. How did you assess what the students' needs were?

N(97) Actually I will ask them, and they are very responsive, some of them even take initiative to tell me what they want to do or what the regular teacher usually does, something like that. We didn't need any very formal interview, we just chat. When I passed by their classroom in the recess, they will look for me, or I will look for them. It's not difficult for me to find out their needs.

AU And, 'To what extent do you think the changeover will have an effect on your lesson planning and decision-making?' 'To little extent'. Okay, Teaching Approach, 'Do you agree with the following statements, please indicate.' 'Examination requirements should determine what is taught in English lessons.' You 'Absolutely agree'.

N(97) I think it should be 'Quite agree' instead of 'Absolutely agree' when you ask me now, because I think the examination should reflect, and the English lesson they should reflect each other. What you had taught was going to be examined. We should leave a space for the students to use English, instead of just to test them what they are taught. Be more flexible for them to create something, be more creative. So I think the examination should reflect what is taught in English lessons, but we should still try to be a little bit tricky, to let the students create, to think instead of just recite or memorize something.

AU 'Students learn English better through interaction with other students than through direct instruction from the teacher.'

$\mathrm{N}(97) \quad$ Yes, because I think language is for communication, so interaction is very important.

$\mathrm{AU} \quad$ Is it possible, to get much interaction?

$\mathrm{N}(97$ It quite depends on the students' characters and their proficiency level, but different level can have different kind of interactions. You can have some basic ones for small children, or students who are mot so competent. So it's not so difficult for teachers to do so, because interaction not mean role play or discussions, there are different kinds of interaction.

AU 'A class should be strictly controlled by the teacher and all lesson content directed by the teacher.' 'Absolutely disagree'.

N(97) I think not strictly controlled. We should consider about the students, I think the students have a right to decide the content, because we should consider about their needs and their interests. 
AU I think the point there is that it seems to be what happens in most lessons in $\mathrm{HK}$ that teachers control the lesson strictly from the beginning to the end. They decide what is to be taught, and they direct the lesson completely, without any flexibility or the students having any say at all.

N(97) So when you talk about English to a lot of students, they just don't like it, they feel bored. That's why.

AU 'A teacher should be prepared to experiment with materials and teaching strategies from a variety of sources in his or her teaching.' This time you 'Absolutely agree'.

N(97) Does it mean try those materials beforehand, or the teaching strategies?

AU No, teachers should try different materials and different teaching strategies in their teaching. The word experiment I suppose means that perhaps they will take a few risks.

N(97 I agree with it because I think we can find out the best between those teaching strategies and materials that you have tried, it's a way for you to find out the most suitable strategies or the materials for the students.

AU And, 'In your practice teaching module did you find that students learn English better through interaction with other students or through direct instruction from the teacher?' You said there's No difference'. 'Since the duration of the practice teaching was too short. I could not observe students' actual progression.

N(97) Yes.

AU I suppose that's right, in two weeks you cannot really notice any change, can you?

N(97) No

AU Certainly in their progress. You might notice some change in their attitude. If you try different approaches, perhaps more interactive methods compared to less interactive methods.

N(97) When you talk about attitudes, obviously they like interactive method instead of being given direct instructions by the teacher. It's obvious.

AU It is these days, almost to the extent I've found now that when I go into a class, they sit there and wait for me to entertain them ... 
$N(97)$ I think it's better for the school, it seems you taught in Heep Yunn, so it's a girls' school, it's better, but when I taught in La Salle, those boys are very active, so they expect a lot of activities or a lot of active things for them to do.

AU That's true, there are girls in that school who don't like activities, they like simple, straightforward sit down reading things, grammar exercises, that kind of thing. They prefer that kind of thing, it's more like real learning. They feel that doing activities is like playing and they are not learning anything.

$\mathrm{N}(97) \quad$ They have a more solid feeling for writing, rather than English games, I think.

AU Okay, and 'During you practice teaching did you find that you controlled your lessons strictly and directed all lesson content?' 'Rarely'.

N(97) Actually, my decision depends on the students quite a lot, and depends on the school activities quite a lot, because during these two weeks, actually I think I taught for just 7 or 6 days because there were a lot of school activities. Suddenly they call them to ask them to go down to the playground to take photos. I don't know why. So it quite depends. I didn't design much, actually, I got quite a lot of constraints.

AU Yes, it happens all the time, you lose so many lessons for various activities. 'Did you experiment with materials and teaching strategies from a variety of sources?' 'Not much'. 'Would you describe your teaching style as learnercentered or teacher-centered?' You said, 'Mostly learner-centered'.

$\mathrm{N}(97) \quad$ Yes

$\mathrm{AU} \quad$ So as many learner-centered activities as possible. 'Which of the following factos do you think has the biggest influence on your approach to the teaching of English?' You've said, 'Teacher training'.

$\mathrm{N}(97) \quad$ Because my training give me the mode of teaching, because during this three years I have been encouraged to use the CLT, so gradually I believed in it quite a lot. So I tried to follow the CLT in my own teaching.

AU Next come things like, 'Personality of teacher', 'Motivation of students' and 'English proficiency of students'. Would you say these are more important than things like materials, teaching aids, classroom size, that kind of thing?

$\mathrm{N}(97) \quad$ Because I think those I ranked less important is quite easy to control sometimes, but for those I ranked important, it's quite difficult for me to control. So I think those are more important than the others. 
AU That's right, things like availability of teaching materials or teaching aids are important but there is nothing you can do to change them. And the last in this section, 'The changeover in sovereignty will affect my approach to teaching.' You have said ...

N(97) Not at all.

AU Not at all. Why do you think it won't affect your approach to teaching?

$N(97)$ I just won't change my approach. I don't think the ... I can't see the effect of the handover to my teaching approach, so I think it doesn't affect my teaching at all.

AU Okay, next section, Professional Relationships and Responsibilities, 'How confident are you in your ability to become a successful teacher?' You've put, 'Quite confident'.

$\mathrm{N}(97) \quad$ Because the most important thing is I am willing to devote my time and energy to the students, to be a teacher, I think in both extra-curricular activities and teaching academic things I can be a successful teacher.

AU And, 'How do you feel about the status of teachers?' 'Teachers enjoy a moderate status'. Do you think they should have a higher status?

$\mathrm{N}(97)$ Because society change, it's quite difficult for a teacher to have higher status and I think teachers are also human. I think they do something wrong, they may not be right all the time. Their own behaviour is quite controversial sometimes, so teachers may not enjoy a high status.

AU Do you think they don't have as high a status as doctors, or lawyers, or other professional people?

N(97) Yes, I think so. I think the status of those professionals is not as high as before. I think students still respect teachers. Actually, people still respect teachers but the way they respect teachers is different with the way in the past.

AU How is it different?

N(97) For example, in the past, when the parents see the teachers of their children they may be scared, or even bow to them. But nowadays parents are more educated so they won't do that, but they will still, when they have some problem with the children, they will ask the teachers to advise them. It's a kind of respect, I think. That's the difference. Parents will adjust, or justify the advice of the teachers. Maybe in the past, parents just blindly accept or follow the teachers' advice. That's the difference. 
AU 'What do regard as being your best attribute in terms of being a teacher.' You said, 'Innovative in terms of teaching ideas' and 'Empathy with students', 'Good command of the language', the lowest being, 'Able to form good professional relationships with colleagues'.

$\mathrm{N}(97) \quad$ Because I'm not very keen on this kind of things, establish relationships with the others. I would do it on purpose, so I think I rated it the least.

AU Don't you think it's important to have good professional relationships with colleagues?

N(97) I don't know.

AU Okay, 'Do you see teaching as a long-term career?' 'Definitely not'.

$\mathrm{N}(97)$ I've just told you I'm not looking for a teaching position. Actually, I like teaching, but I like to do something else also. It may not be a long-term career for me, for example, if I want to take some part-time course or save the money for my further studies, I will choose teaching since ... but I don't want to be a teacher for a long time. I'm afraid I will be stubborn if I be a teacher for a long time.

AU 'If you teach English in the future, who do you think will be most helpful to you?' 'More experienced teachers', and also, 'During practice teaching, who was most helpful?' Again, 'More experienced teachers'. Did you find them very helpful, during the practice teaching?

$\mathrm{N}(97) \quad Y e s$, because they can give me practical advice, practical strategies to solve some problem. I talked to my supervisor sometimes, but my supervisor was too theoretical sometimes and she helped me to solve the problem. So what I need is more practical things, I don't have the time to think, to solve the theory, so I think most experienced teachers helped me a lot, because these teachers understand more about the students.

AU Yes, because if they teach in that school, then they know the students, they know the situation you are dealing with. 'Which of the following out-of-class activities do you think you will be able to do as a teacher?' You said all except item 5, which is 'Be involved in religious activities'.

$\mathrm{N}(97)$ Because I'm an atheist, so I think I won't be invited to engage in this kind of activities.

AU A lot depends on the school. I don't know how it is in La Salle. La Salle is a Catholic school. My school is an Anglican school. They have religious activities, every morning assembly is a religious activity, which all the teachers have to go to. I guess you could class that as a religious activity. 
Once a month they have a prayer meeting at lunchtime and all the teachers go. I don't have to go because it is in Cantonese and I always manage to get out of it, but all the other teachers would go. And once a year at Easter we would have some kind of religious meeting and all of us had to go. Even I would have to go and sit there and not understand anything. So very often you find yourself having to do this because it's part of school policy.

$\mathrm{N}(97) \quad$ I interpreted this question in a different way. I misunderstood that I have to organise those religious activities. I think if you just say some assembly, I have to be involved in it.

AU Certainly all the others ... 'Be a form teacher' possibly, all these things you will be ... [tape ends] ... And 'Which of the following do you think will take up the most time?' The same list, 'Being a form teacher', 'Counseling students', 'Marking papers' and 'Preparing lessons'. Being a form teacher can take up a lot of time. I was a form teacher, I taught in Heep Yunn for six years and I was a form teacher every year.

N(97) English teachers are usually form teachers.

AU Yes, if you are the English teacher of the class then you see them for 7 lessons a week, so you get to know them very well anyway, but it can take up a lot of time. 'If you teach English in the future to what extent do you expect to be working with other teachers?' 'Some, to share , materials and teaching ideas.' Some, not very much?

N(97) Not very much, that's my style of working. I think I need help because I'm still fresh. I think teachers need help because it's easy to get frustrated sometimes, they may need others' encouragement or others' support or advice. I like to work alone, to prepare my teaching strategies, design my materials alone. I think some, but not very much.

AU 'What do you think will help you most to continue to develop as a teacher in your first year?' 'Learning from other teachers', which we've talked about. 'Reading articles', 'Designing new materials'. Do you think you would read many articles?

$\mathrm{N}(97)$ Because when I did the final-year project I found that some of the articles is quite useful for me to decide on teaching materials and to establish the teaching strategies, so I think reading articles is quite useful.

AU To what extent are you worried about your future as a teacher after the changeover in sovereignty?' 'To no extent', you said, 'I'm not worried at all, I think the prospect will be better.' Why do you think the prospect will be better? 
$\mathrm{N}(97) \quad$ Because as far as I know the Chinese govt. become more and more concerned about the importance of English, so they need quite a lot of professionals to teach English in mainland China, so I think the prospect's better.

AU So it could be they actually need more teachers?

$\mathrm{N}(97) \quad$ Yes

AU And, 'Do you think your professional relationships and responsibilities will change after the changeover?' Okay, and finally the last section is Perceptions and Values, 'Do you think you have a definite philosophy of teaching?' 'Quite definite'. You said your teaching philosophy is, 'Help students to look for their life direction and help students to discover their potential abilities.'

N(97) I think it's not difficult for a language teacher to do so because there's quite a lot of chance for you to read the literature with the students and literature can help students to search for their life directions and discover their potential abilities. These two things are the most important point of education, I think.

AU To discover their potential, good. Things that you might be unsure about, 'Personal career goals and ambitions'. Is that because you said that you don't really want to be a teacher?

N(97) Some people say, I don't want to be a teacher, they can be very definite, but I'm not this kind of person. I don't refuse to be a teacher but I know that it's not the thing that I want to do the most, so I'm quite confused.

AU And 'Ways to handle discipline'.

N(97) I need to think about classroom management because I think you do a lot with experience. You have to handle it in class; you can't just read of some ways from books to teach you how to handle the discipline. It doesn't work at all. So I think I have to learn while I teach.

AU A certain strength of character I think is the main thing. In some situations the students are able to sense that the teacher is unsure of what to do, and they can start to play on that. It takes a while for teachers to be able to recognize situations before they occur, so you learn how to deal with them, often through mistakes. I think I found in the overall results that these are the things that people are concerned about. 'What do you think are the most important factors which affect what goes on in the classroom?' You said, 'Education Department', 'School policy' and 'Examinations Authority' are most important. 
N(97) Yeah, because ... it's just like three things that you cannot change, you cannot fight against them. And motivation of students, if a student is really unmotivated, it is quite difficult for you to motivate them, and if they are not motivated, it's difficult to teach them. So I think these 4 things are the most crucial.

AU Certainly the ED, school policy, the EA, they set how the schools are run and everybody has to work within that system, so it's very important as you say. You said 'Language ability of students' is only 'Quite important', not 'Most Important'.

N(97) Because I think I can tailor-make some activities or something to match their language ability, or just by talking simple English, encourage them more, talk to them more, to encourage their language ability.

AU 'Which of the following skills do you consider the most important to teach?' All of them are important.

$\mathrm{N}(97) \quad$ Yes

$\mathrm{AU} \quad$ Which of the following best sums up your feelings as regards the roles or behaviours teachers should have in relation to his or her students?' 'The teacher should be a transmitter of knowledge', 'The teacher should be a facilitator of learning', 'The teacher should allow the students to determine the direction their learning should take' and 'The teacher should be prepared to devote as much time as is necessary to helping his or her students'. So things you haven't circled are, 'Teachers should be total control of all lessons at all times', which is something we had earlier. 'Teachers should try to become friends with the students', 'Teachers should act like a parent', 'Teachers should refrain from punishing students'. You don't agree that teachers should try to become friends with the students?

$\mathrm{N}(97) \quad$ I'm not sure is it possible to become friends. Actually the relationship between friends is quite different from the relationship between teachers and students. Sometimes if you are too friendly, or the students regard you as a friend, he or she may not behave properly in class, or some other students may be jealous of that student or they don't like that. So it's quite difficult to be friends with the students.

AU $\quad$ Yes, relations ...

N(97) You can keep good relationships, but not friends.

AU 'Consider your strengths and weaknesses ...', you've put 'Innovation' and 'Planning', 'Strength of personality', 'Motivating students'. Weakest, 
'Discipline', 'Voice'. For weakest, we can say less strong. So voice is not one of your strong points?

N(97) No, actually I can't project my voice all the time. They said I project wrongly, so after a long talk I will have a sore throat, so usually I can't project my voice.

AU Do you use a microphone?

N(97) Sometimes.

AU Sometimes I have to do too. My speaking voice is quite soft, but I taught you for a while, was my voice loud enough?

N(97) Okay.

AU 'Are you planning to teach English next year?' 'Not yet sure'. 'What factors did you consider when making your decision as to whether to teach or not?' 'Depends on if I can get other jobs.' So you are looking for other jobs as well?

N(97) Yes.

AU What kinds of other jobs are you looking for?

N(97) Do you know an organisation called the Warehouse Teenage Club?

AU $\quad$ Yes

$\mathrm{N}(97) \quad$ I like to organise some kinds of activities for these kinds of organisations. Or to be an editor or a creative writer because I like to write. This kind of job.

AU Better than teaching? Thank you very much.

N(97) You're welcome. 
Male, 23 years old.

Was keen to stay in education but did not know in what capacity. Planned to work as research assistant for a year before trying to find a teaching post.

$\begin{array}{ll}\text { AU } & \text { Researcher } \\ \mathrm{O}(97) & \text { Interviewee } \\ \ldots & \text { Missing words / Unfinished utterance } \\ \ldots & \text { Pause }\end{array}$

AU Okay, Language Use is the first section. You say your written English before you began the BATESL was 'Rather poor', both written English and spoken English were 'Rather poor', and now they are 'Quite good'.

$\mathrm{O}(97)$ I found there is quite a lot of improvement.

AU In what ways would you say they have improved?

$\mathrm{O}(97)$ Because in the syllabus of the cert or the A Level in HK I can just be trained in writing those descriptive or argumentative essays, so I think it is quite limited, so if there is other kinds of writing I need to write, I have no confidence at all in doing all those writing jobs. But after these few years in City U I was given quite a lot of chance to write, so I think there is quite a lot of improvement.

AU In speaking as well?

$O(97)$ Yes sure, especially in speaking, because I have to interact with those foreigners, so now I have no fear in talking with you, like I am now using English. But in those days in secondary school, I am scared if I have to use English. I can't express fully what I want to say. So I can see that there's very much improvement, especially in spoken English.

AU In confidence maybe. 'When speaking in Cantonese to friends, do you sometimes use English words or expressions (code-mixing)?' 'Quite often'.

$\mathrm{O}(97) \quad$ Yes

AU 'Code-switching' 'Rarely'.

$\mathrm{O}$ (97) Like most of the young people in HK, I think, if they are educated, should I use that word, I am not sure, we get adapted to using English words. Even 
you walk along the streets, you can listen some English words, so I think there's nothing special to say, like others.

$\mathrm{AU} \quad$ Is it because there is no Cantonese equivalent?

$\mathrm{O}(97)$ Sometimes I find this is very true, like 'presentation'. If I want to say like, I have to give a presentation this afternoon, I will use 'presentation' still, because I can hardly find any equivalent in Cantonese which can suit the translation of the English words. That sense I can't find.

AU And code-switching you say rarely.

$\mathrm{O}$ (97) No, code-switching is very rarely? Just some of the classmates, actually just one of them, they will switch to English to ask me questions or to talk with me, then simultaneously I will use English in response. I think it's a kind of politeness, I don't know. There are some other cases, like if Chinese lecturers will talk to me. When I talk to her first in Cantonese maybe she suddenly use English to respond, so I will use English then.

AU I think it is politeness. If someone speaks to you in English, even if you share another language, you will reply in English.

$\mathrm{O}(97) \quad$ It's interesting.

AU One thing I've noticed, which I was telling to Christine, is that I've noticed a lot of people, particularly young people, doing a lot of code-switching. It sounds like they are Chinese who are studying in Canada, they are studying in the States, they've come back, they are meeting their friends here and they are going out and talking Cantonese and switching to English for a few words or a few phrases and then back into Cantonese again. Or brother and sister, or the whole family.

$\mathrm{O}(97) \quad$ But it very much depends on how long they live in the foreign countries. It very much depends on their proficiency in English. Like me I won't use English very much because ... sometimes I can be frankly tell you that I can't express myself fully what I want to say in English. That's the limitation.

AU If you live in an English-speaking country then obviously you'll get better. 'In English lessons, English should be the only language used for all purposes.' You 'Quite disagree'.

$\mathrm{O}(97) \quad W h y$ 'all purposes'? Because I share the view with Angel Li, that sometimes the teacher can use Chinese, Cantonese to explain the abstract words, English words. It's so convenient, why don't you use it? In some cases, I mean. There should be some condition provided. 
AU In some schools though it is school policy that in English lessons you should use only English. You shouldn't use any Cantonese.

$\mathrm{O}(97) \quad$ It's silly. You shouldn't stick to that rule because some low proficiency students, you have to help them, in some convenient ways. In order to get rid of their fear. If you always use English, they are scared to listen to you, so finally you can't help them a lot.

AU And, 'In English lessons English content can be presented in mixed-code.' You said, 'Quite agree.'

$\mathrm{O}(97) \quad Y e s$, because I am taught in that way. It can be a success. There's quite a number of factors, I understand that there's many variables, but it can be carried out in this way. I should say.

AU You don't think there's anything wrong in teaching English and using mixedcode?

$\mathrm{O}(97)$ I understand that the chances for them to listen to a long English speech or some kind of discourse, maybe reduce the chance. That's the one drawback, but ...

AU That's okay, some people will just reject the whole idea of mixed-code.

$\mathrm{O}(97)$ It also depends on different personalities of the students. Sometimes they can't stand for all English, their interest may be reduced. But I don't believe we should use all English and code-mix in all cases should be banned.

AU 'During your practice teaching module, how much did you use only English in lessons?' You said, 'Usually.'

O(97) Because there was a low band school and their English standard was quite low. So I used Cantonese to offer those translations for them. I think it doesn't make sense to spend a long time using simple English to explain those English words. It just make the lesson longer, quite time-consuming.

AU 'During your practice teaching module, how much did the amount of English you used in English lessons change over the practice period?' You said, 'It increased.' So as time went on you were using more and more English.

$\mathrm{O}(97) \quad$ When I discovered that they can understand me, I used more English gradually. To see if they can comprehend the lessons if I use all English, that's my terminal goal. 
AU And, 'In what situations in an English lesson would or might you use Cantonese?' In all situations pretty much apart from 'To take roll-call', so as you said before, you have no objection to using Cantonese in English lessons.

O(97) Yes

AU 'To what extent do you see the use of English in secondary schools being affected by the changeover in sovereignty?' You put, 'To some extent.' The changes you expect there to be, 'The introduction to teaching Mandarin results in decrease in emphasis on English.'

O(97) Yes, there are three language here, I mean three spoken languages, people may put more emphasis on Mandarin than English. The use of English may be reduced to some extent. But it's still very important, the socio-economic factor in HK still dominates, so the parents may want their children to learn English well, still. That's what I believe. Generally, to a certain extent there's some effect.

AU So more emphasis on Mandarin will mean more emphasis on English. Next section is Instructional Planning and Decision-Making. 'It's impossible to have a successful lesson that hasn't been well-planned in advance.' You said you 'Absolutely agree' with this.

$\mathrm{O}(97) \quad$ Yes, I experienced it.

AU So you had lessons which you didn't plan in advance and which were not successful?

$\mathrm{O}(97)$ Extremely bad. Actually I found the students didn't realise that I hadn't planned it well. I will feel guilty, wasting their time.

AU I think it depends a lot on experience. 'Teachers should write a detailed lesson plan for every lesson they teach.' 'Absolutely agree, but it's impossible in a HK school context.'

$\mathrm{O}(97) \quad$ What do you think?

AU Yes, I would agree. 'Teachers should be more responsive to students' needs than the set syllabus.' Again, 'Absolutely agree, but do teachers have enough time to do so?'

O(97) Still the time constraint there.

AU So long as you follow the syllabus ... 
$\mathrm{O}(97)$ Yes, we are safe if we do so, no punishment can be exerted on us by the higher authority.

AU 'When planning lessons how detailed do you think the plans should be?' 'Loosely detailed and flexible'. Why would you say loosely detailed and flexible?

$\mathrm{O}(97)$ To me the time constraint is the main concern, so we can't write detailed lesson plans. So maybe loosely detailed, that will do, and flexibility is always very important. We should bare in mind that the lesson plan can be changed all the time, it depends on the situations you are in, especially in the classroom, there are so many students with different expectations.

AU 'During your practice teaching module, how often did you have to change your lesson plan?' You said, 'Always.'

$\mathrm{O}(97)$ I remember there are quite a number of times, so I find that the lesson plan can't be followed, actually. Say for example, I forgot to bring the books along with me, their homework book, so I need to ask the monitor to help me out to take the books down. The time, you can see what I've planned and the lesson plan, then everything should be put together. So can't follow the lesson plan so strictly. Some disciplinary problems I have to handle it, then it takes time as well.

AU There's always things happen and you have to change what you planned originally. Sometimes it works out okay, but discipline is something that comes into it, which you can't account for.

$\mathrm{O}(97) \quad$ So back to Q17? So if I planned a detailed lesson plan then that may not work because so many factors.

AU 'During your practice teaching module, on what basis did you plan your lessons?' 'Syllabus' ranked first followed by 'Perceived needs of students', 'BATESL course guidelines'. So you were given a syllabus by the cooperating teacher?

$\mathrm{O}(97) \quad$ Yes.

AU How did you perceive the needs of the students

O(97) According to my impressions, I mean the first two lessons when I interact with them, then I will have a judgement in my mind, how well their English is. Then I will adapt the lessons to their level.

AU You put 'Regular teacher's instructions' last, so the regular teacher, the cooperating teacher, didn't give you any instructions? 
$O(97)$ Actually not at all. He told me what I should do. That's just a set of syllabus, within the time limit I have to finish. And he said if I can't finish the area covered by the syllabus, he will help me. So actually no pressure.

AU Well it's only two weeks, isn't it?

$\mathrm{O}(97) \quad$ Three weeks.

AU So you don't have much time really?

$\mathrm{O}(97) \quad$ No

AU 'During your practice teaching module, what were the factors you think had the most influence on the decisions you made?' 'Students' needs' followed by 'Supervisor's advice'.

$\mathrm{O}$ (97) Students' needs I have mentioned before, and supervisor's advice, because the grade will be given by the supervisor, so being a student you will be very sensitive to a supervisor's advice. What they want to see and you give them what they want to see. Looking forward to getting a better grade, very practical.

AU Very practical, of course. 'To what extent do you think the changeover will have an effect on your lesson planning?' 'To no extent'.

$\mathrm{O}$ (97) I don't think there is any, it's my impression. I didn't take this into my consideration.

AU Next is Teaching Approach, 'Examination requirements should determine what is taught in English lessons.' 'Quite disagree'.

O(97) Sometimes I think teachers shouldn't only aim at teaching those examination skills, to help the students to get grade A in the examination. That's not the only aim for English lessons. Sometimes we may just inspire the students to think independently, maybe, to foster their creativity, but that's not the only point to consider.

AU 'Students learn English better through interaction with other students rather than through direct instruction from the teacher.'

$\mathrm{O}$ (97) That's what I've taught in BATESL course. Because we should give them chances to use their English instead of just told what to do, through direct instructions, but through interactions with each other, they can know how to use it. 
AU 'A class should be strictly controlled by the teacher and all lesson content directed by the teacher.' You said, 'Quite agree, it's because of the class size, you know.'

O(97) I think that may not be true, because there's another important factor, that is how well behaved those students are. If I taught an adult class in an evening school, could be a large class size, but it doesn't matter. I can let them do those activities by themselves. It may not be strictly controlled by me. But in HK schools, if you give them what they want to do, or let them control the classroom then it will be a mess.

AU Others have said who have, let's say, well behaved students that they would disagree with this, they say it's possible to let the students control the lesson to a certain extent. And 'The teacher should be prepared to experiment with materials and teaching strategies in his or her teaching.' For this one you 'Agree'.

$\mathrm{O}(97) \quad$ I think all those materials may not be suitable for your class, or the suggested teaching strategies in those teachers' textbooks, you may not use them in your class, your specific class, so we should always bear in mind that we shouldn't use those materials directly, just use them in your class, we should think about whether they are suitable or not, so we should experiment with those materials with the students first and you can confidently use them later in.

AU 'During your practice teaching module, did you find that your students learned English better through interaction with other students or through direct instruction from the teacher?' 'Through direct instruction, because discipline, they don't stick to English they will chat in Cantonese.' So you have to teach through direct instruction?

O(97) Reality.

AU Without any interaction with each other?

O(97) I've tried to get them into groups to ask English questions. Actually English questions are already written on the textbook, but they use Cantonese to ask each other. So, after you've explained it once in front of the class, to tell them that you have to use English, but they still use Cantonese. Only one or two pairs using English, but they talk to me using English, for sure. The others can listen to them, that maybe help, but then let them talk in Cantonese and they make a lot of noise.

AU 'During your practice teaching module, did you find that you controlled your lessons strictly and directed all lesson content?' You said 'Yes'.

$O(97) \quad$ Yes I'm very strict. 
AU 'Did you experiment with materials and teaching strategies from a variety of sources?' 'Somewhat'.

O(97) That means what I planned in the lesson, I experiment with them to see how they work in the classes, so somewhat.

AU 'Would you describe your learning style as learner-centered or teachercentered?' 'Mostly teacher-centered'.

$\mathrm{O}(97)$ I think I can't determine or describe right now because I haven't been a teacher for a very long time so it's difficult to say.

AU Certainly in you practice teaching ...

$\mathrm{O}(97) \quad$ Then I would say, teacher-centered.

AU 'Which of the following would you say has the most influence on your approach to the teaching of English?'

O(97) It's so difficult to rank, actually, there are so many items.

AU Difficult, yes. You put 'Class size', 'Classroom size' and 'Noise in classroom' as the first three grades. It's very much the physical constraints that seem to have influenced you.

O(97) Because when we talk about the approach, those physical factors may be quite influential. I think different levels of students I can still use different approach. I think those materials ... they are designed specifically for different approaches, that's my impression, and I think that may constrain me to which approach I should use, rather than the motivation and the proficiency. The same approach can be used to different levels of students.

AU Question 31, 'The changeover in sovereignty will affect my approach to teaching,' 'No'. The only way in which it might do I suppose is perhaps if the school changes, and there isn't any sign that the schools are going to change.

$\mathrm{O}(97)$ Within the three weeks we were in the schools, there's no special stimuli ... from EMI schools to CMI schools there may be some impact on their approach to teaching.

AU Now Professional Relationships and Responsibilities, 'How confident are you in your ability to become a successful teacher?' 'Unsure'.

$\mathrm{O}(97)$ Because I really don't know how I can get along with my colleagues and my students, and which school I will be in. I think those factors may disrupt my confidence in being a successful teacher. My English proficiency is also 
another factor. I don't speak very fluently. I have no experience in using simple English to explain those English terms or something like that. I think what I lack is the confidence, I mean the experience.

AU Well of course after just a little practice teaching, anybody would be unsure. 'How do you feel about the status of teachers in today's society?' 'Teachers have no special status'.

$\mathrm{O}$ (97) Compared to the old days. Back to 10 years ago, they are good, high status in HK society, but now people want to be doctors, engineers, accountants, businessmen. If they can gain a lot of money, those can have the high status in HK society. I don't know whether other people share my beliefs.

AU I think so. Question 4 is in some ways is a bit separate from that, you could say that teachers have no special status or a low status but do they also have a lower status than they should?

$\mathrm{O}$ (97) I would circle it as well. People may comment, you are only a teacher, you are just a teacher.

AU Its' not regarded as ...

$\mathrm{O}(97) \quad$ Prestigious

AU As the same level as doctors or lawyers.

$\mathrm{O}(97) \quad$ But we are professionals. Like teachers, social workers are also regarded as less high status.

AU And lower than they used to be. I don't know why.

O(97) Money oriented society.

AU Do you think there's a higher status of teachers in China than there is in HK?

$\mathrm{O}(97)$ I think in China the status may be higher for teachers, I'm not sure.

AU 'What do you regard as your best attribute in terms of being a teacher?' You put, 'Empathy with students', 'Innovative in terms of teaching ideas', 'Good communication skills'.

$\mathrm{O}(97)$ Because we are not just passing those knowledge to them, but to try to understand them, I think teachers always social workers to help them to ... to help them with all kinds of problems. So if I will be an English teacher, I won't just teach English. Other ethical ideas in my class, I don't know. Teach them to treat others better. 
AU And last on the list, 'Able to form good relationships with colleagues'.

O(97) It's still important but compared to others, it's less important.

AU 'Do you see teaching as a long-term career?' You said, 'Probably'.

$\mathrm{O}$ (97) They can run their business, they can set up a business, they can do other things, sure. I want to run the tutorial school maybe, to gain me a lot of money. But don't tell others, erase it!

AU 'If you teach English in the future, who do you think will be most helpful to you in your first year?' You've put, 'ED, reduce the class size in secondary schools.' [Tape ends] 'Who was most helpful to you in practice teaching?' You said, 'Students'. 'They showed understanding - cooperated when the supervisor came to observe.' 'Which of the following out-of-class activities do you think you'll have to do?' ...

O(97) That's usually the work.

AU And which of those, the same list, do you think will take up the most time? You put, 'Preparing lessons.'

$\mathrm{O}(97)$ If I'm a responsible teacher.

AU 'If you teach English in the future to what extent do you expect to be working with other teachers?' 'A little.' You wouldn't expect to work much with other teachers?

$\mathrm{O}(97)$ Why I circled \#3 is because when I was a student in secondary school, I can observe those teachers they rarely cooperated with each other, so that may affect my thinking.

AU Why do you think that is? Why don't teachers cooperate so much?

$\mathrm{O}(97) \quad$ They are not friends, I guess.

AU $\quad$... 'If you teach English in the future, what do you think will help you to develop most as a teacher in your first year?' You put, 'Designing new materials' and 'Finding new materials.' 'To what extent are you worried about your future after the changeover?' 'To no extent.' 'Do you think your professional relationships and responsibilities will change after the changeover?' 'No.' Finally, the last section, Perceptions and Values, 'Do you think you have a definite philosophy of teaching?' You said, 'Yes' 'Increase the chance of language use among students. Be conscious about the students' attitudes towards learning English. Give students a reason to learn English. Care about students including their personal lives, if they are willing to share 
with me.' Give students a reason to learn English, what kind of reason could you give them?

$\mathrm{O}$ (97) First of all they can equip themselves with these resources, I mean linguistic resources, speaking English, and they can obtain a better job. That's the first concern. Secondly, if they can read English they can explore themselves with different cultures, different kinds of knowledge by reading English magazines or different kinds of books in English. They can learn British or American or Australian culture by reading their literature. For example, when they are walking on the streets they encounter a foreigner, they are asking how to get to somewhere, they can respond to that. That's a kind of need, a reason to learn.

AU Okay, 'Important factors which affect what goes on in the classroom.' You put, 'Class size', 'School policy', 'Backup of colleagues' as most important.

$\mathrm{O}(97)$ Backup, I mean they won't say words behind me.

AU Backup actually means help.

$\mathrm{O}(97) \quad \mathrm{Oh}, \mathrm{I}$ see.

AU 'Which of the following skills do you consider most important to teach?' Why would you say grammar is most important?

$\mathrm{O}(97)$ Just because grammar is the means to express. We can't just take out grammar and teach them writing and speaking. If you don't know grammar, you don't know how to say. That's the means.

AU Would you say grammar or communication is the most important, or must you have grammar for communication?

$\mathrm{O}(97)$ If you use the grammar wrongly, you can't write or speak the meaning you want to express.

AU 'Which of the following best sums up the roles or behaviours a teacher should have in relation to his or her students?' ... Why do you say, 'The teacher should try to become friends with the students'?

$\mathrm{O}(97)$ Because if you respect them as a friend, then they will respect you, and when you teach them anything, they can accept it much more better.

AU 'The teacher should act like a parent'?

$\mathrm{O}(97)$ Sometimes, maybe. 
AU 'The teacher should refrain from punishing the students'?

O(97) I should circle it also.

AU Finally, '... your strengths and weaknesses as a teacher?' You put, 'Strength of personality', 'Relationships with students', 'Relationships with colleagues' and 'Teaching grammar' as the strongest. Before you said that you only expect to be working closely with other teachers 'A little'?

$\mathrm{O}(97)$ I mean if I can get along with them well, it will help me to live in that school, but not in the sense that they may help me in preparing the materials, sharing the materials, not that kind of academic, professional relationship.

AU Are you saying that relationships with colleagues is one of your strengths? You are good at making relationships?

O(97) I think so. 
Interviewee:

Date:

Female, 25 years old.

Planned to pursue a masters degree after graduation. She felt that she wanted to teach in a government school but that a masters would give her more scope than if she did a postgraduate diploma.

$\begin{array}{ll}\text { AU } & \text { Researcher } \\ \mathrm{P}(97) & \text { Interviewee } \\ \ldots & \text { Missing words / Unfinished utterance } \\ \ldots & \text { Pause }\end{array}$

AU Let's start of with Language Use, you said that your written English before you began the BATESL was 'Quite good', and it's still 'Quite good'.

$\mathrm{P}(97) \quad$ Yes

AU Your spoken English was 'Fair', but it's now 'Quite good'. So you think your written English has not improved any?

P(97) I think I haven't got enough training on the English proficiency and that's why I think I haven't got any improvement in written English, but as the exposure of written English has increased in these 3 years, that's why I think my spoken English is quite good now.

AU You don't think there's been enough attention paid to your English during the course?

$P(97) \quad Y e s$, especially for the proficiency, because we just focus on how to teach English and how to teach the students and that's why I think we haven't got enough attention on that aspect.

AU I thought about one third of the time was supposed to be given to your English proficiency.

P(97) You mean one third? Yes, I know, but only during Year 1, and after that we just focus on the teaching approaches and methods instead of the proficiency.

AU So they expect your English proficiency to have reached a suitable level after Year 1?

$\mathrm{P}(97) \quad$ After Year 1, yes. 
AU 'When speaking in Cantonese to friends who have Cantonese as their first language, do you use English words or expressions?' You said, 'Always.' What kinds of English words and expressions do you use?

$P(97) \quad L i k e$ 'sorry', 'tissue' and 'toilet' we always use English words because that's the norm in HK. Of course we don't use the whole sentence in English.

AU Just certain words. And code-switching you said, 'Quite often.'

P(97) Sometimes when I'm talking to my friends, especially the classmates, we sometimes use English. Just like joking. Then we'll switch to English.

AU I know people who do that, it's having fun with the language. One thing I have noticed this year, perhaps it's because of the summer holidays, or maybe because of the handover, but I've noticed a lot of what are probably overseas Chinese coming back to $\mathrm{HK}$, perhaps for a holiday, and you hear them speaking ....

$P(97) \quad$ English

AU Mixed-code, speaking Cantonese and then suddenly switching and saying something in English. Now English in teaching, 'In English lessons English should be the only language used for all purposes.' You said, 'Quite agree.' So in some situations you would use Cantonese?

$P(97) \quad Y e s$, for example, if you have to blame your students for doing something wrong, you can't use English because they won't understand you if you use English, and you have to code-switch to Cantonese again, or sometimes when give certain kinds of instructions to do an activity, you have to use English first then switch to Cantonese if they don't understand. In that kind of situation you have to use the mother tongue.

AU And 'In English lessons, lesson content can be presented in a mixed-code.' You 'Quite disagree' with this. So you would use just English to present the lessons, but you would use Cantonese to ....

P(97) It would be better if you could use English only, but just like I have said, in certain situations, you have to cope with the difficulty, you have to teach the syllabus, you have to follow the instructions of the panel chair, for example, so you have to use Cantonese sometimes. But I believe speaking, I think using English is good, rather than using mixed-code.

AU How about the school you were at for practice teaching?

$\mathrm{P}(97) \quad$ It's a Band 5 school so I have to use Cantonese quite often. 
AU It's quite important and it will affect quite a lot of the responses that you put on here. 'During your practice teaching, how much did you use only English in lessons?' You said, 'Usually.' And 'During your practice teaching, how much did the amount of English you use in lessons change? It actually increased. So you were using more English at the end of the period than you were at the beginning?

$\mathrm{P}(97) \quad$ At the beginning I had to use English explain everything, then I code-switch to Cantonese again and start from the beginning. It's like translation.

AU I see. The things that you would use Cantonese for, 'Discipline'. 'Friendly with students', 'Explaining vocabulary' and 'When they don't understand'. So most of the time you would use English, then Cantonese for more personal interaction?

$\mathrm{P}(97) \quad$ Yes.

AU 'To what extent do you expect the use of English within secondary schools to be affected by the changeover in sovereignty?' Which you didn't answer, you put, 'You mean the content area or English lessons?' That's a good question. Primarily I meant the English lessons.

P(97) For the English lessons, I don't think there will be much change. Maybe the English teacher have to be more focused on the English language instead of using Cantonese to explain the contents of the textbook. Now we have to follow the instructions of the govt., they said if the MOI in your school is Chinese, you have to use Chinese all the time for all the subjects, except English, and that's why I think for the English teacher they will try to focus more on English in English lessons.

AU And in other subjects? Perhaps in other content areas?

$\mathrm{P}(97) \quad$ I think they will use Cantonese.

AU It depends of course on the MOI in the school. As you know doubt know, the ED is going to insist that the majority of schools use English as the medium after 1997. And what changes you expect, it's probably as you just said actually. 'Those schools which have been streamed into the Chinese medium, they will have to use Cantonese to teach the content areas. In English lessons, I think both EMI and CMI schools can still use English to teach English.' So it's really only in the other content areas where if they change the medium, then we might see changes. Although perhaps in Chinese-medium schools, schools which go Chinese-medium from being English-medium before, perhaps even in English lessons, maybe they will teach totally English. Is that what you said before? They previously may have used some Cantonese in the 
lessons, but now that they feel the whole school is Chinese-medium, they want to keep the English lesson purely English.

$P(97) \quad$ Yes, that's what I mean.

AU And they get plenty of Chinese in the rest of the school. Next section is Instructional Planning and Decision-Making, 'It is impossible to have a successful lesson that hasn't been well-planned in advance.' Which you, 'Quite agree' with, and you said, 'It depends on the experience of the teacher.' How does it depend on the experience of the teacher?

$P(97) \quad I$ mean, if the teacher is very experienced then maybe she don't need to spend so much time on planning the lesson in advance and she can just teach based on the pace of the lesson and based on the ability of the students.

AU Of course, I agree with that, experience is very important. Even lessons which experienced teachers teach, which appear to be not planned, are planned in their head because they've taught that lesson many times before and they know what to expect. 'Teachers should write detailed lesson plans for every lesson they teach?' 'Absolutely disagree'.

$\mathrm{P}(97) \quad$ Yes, I think teachers have to plan the lesson in advance if they are not experienced, but as far as I know, many teachers won't plan for every lesson they teach. From my own experience, I've done a very detailed lesson plan because it's required by the department for that module we have to plan the whole lesson, but when I just go out to teach, I found it difficult to follow the lesson plan, because students are not the kind of students that you have expected, you have to change the whole lesson plan for sometimes.

AU Of course, it happens all the time that you plan lessons that you think will be great, but for some reason doesn't work. 'Teachers should be more responsive to student needs than the set syllabus.' You 'Quite disagree', 'Reality is reality. Teachers are quite often forced to follow and cover the syllabus to prepare students for the two public exams.' 'When planning lessons how detailed do you think the plan should be?' 'Loosely detailed and flexible'. Flexible so that you can change something if it doesn't work?

$\mathrm{P}(97) \quad$ Yes

AU 'During practice teaching how often did you find you had to change your lesson plan?' You said, 'Usually.' Why was that?

$\mathrm{P}(97) \quad$ Because I set quite a lot of activities and they don't know how to respond always because they used to be taught by a very experienced teacher, but she always use Cantonese and she always focus on the textbook page by page and then do all the comprehension exercises and go over all the grammatical 
points, but she won't do any activities. What we've learned here is designing some activities for your students and they can use English in the context, instead of just focus on the grammatical points, just learning the rules, and so I find it difficult to organise the activity and how to explain to the students, it's a great problem.

AU So there was some conflict between what you had been taught to do here and what the teacher wanted you to do?

$P(97) \quad Y e s$, maybe in higher banding schools you can do what you've learned here because their proficiency is high and you can use English as the MOI, and you can perform complicated tasks, but in lower banding schools you can't.

AU Is it just discipline that's the problem?

$\mathrm{P}(97) \quad$ For that school, discipline is not a problem because the students are just not good at English, they are not bad discipline.

AU 'During your practice teaching module on what basis did you plan your lessons?' You said, 'Syllabus' first, 'Regular teacher's instructions' and then 'BATESL course guidelines'. It's as we were saying, you were given a syllabus and tried to fit your own ideas into that. 'What were the factors that you think had the most influence on the decisions that you made?' Again, 'Regular teacher's input' first, followed by 'Supervisor's advice', and 'Fellow student teachers' and 'Students' needs'. And 'To what extent do you think the changeover of sovereignty of $\mathrm{HK}$ will have an effect on your lesson planning?' 'To no extent'. Well, we will see. Next section is Teaching Approach, 'Examination requirements should determine what is taught in English lessons.' 'Absolutely agree. Because students must sit for public exams'. Is that simply the reality as you see it? Do you think it is right? Do you see it as a good thing?

$\mathrm{P}(97) \quad$ It may not be a good thing because if you really want to learn a language, I think your interest and the motivation is the most important thing. But for $\mathrm{HK}$, the students have to sit for the public examination and the principal in schools will force the English teacher to follow the syllabus and how to train the students to be a good test-taker.

AU 'Students learn English better through interaction with other students rather than through direct instruction from the teacher.' Quite agree'. Is that something you believe?

$\mathrm{P}$ (97) Yes, I believe it's true, if you have to speak to your teacher, you speak under pressure, and you make more mistakes than usual, than with friends.

AU When students speak with fellow students, they don't feel that pressure. 
$\mathrm{P}(97) \quad$ Yes, they are more open and they will try to generate more ideas.

AU The class should be strictly controlled by the teacher and all lesson content directed by the teacher.' 'Quite agree'. Why do you quite agree with this?

$\mathrm{P}(97) \quad F o r$ some reason like if the lesson is not controlled by the teacher, then the students will feel loose and they don't know what to do. Based on my own experience when I was a F4 student, the teacher won't plan anything for us to do during the English lessons, and that's what I feel. I feel loose and I don't know what to do and I'm worried about my proficiency and I'm worried about my performance.

AU So you would like lessons that are not too strictly controlled but are controlled by the teacher?

$\mathrm{P}(97) \quad$ Yes.

AU 'A teacher should be prepared to experiment with materials and teaching strategies from a variety of sources.' You said you 'Absolutely agree'. 'Did you find that students learn English better through interaction with other students rather than through direct instruction from the teacher?' You said, 'Through interaction with other students.' 'I didn't know if they learned better but at least I found them motivated with interaction.' And 'During your practice teaching did you find that you controlled your lessons and directed all lesson content?' 'Sometimes'. Why only sometimes?

P(97) Actually for the first week I nearly taught nothing because they don't understand me and because I have to use English because it's required by the supervisor here. But I found doing nothing because they don't understand the instructions, and they also complained to their class teacher that I use English always, and so for the first week I taught nothing. So I have to adjust my instructions and I have to use Cantonese sometimes and that's why ....

AU 'During your practice teaching module, did you experiment with materials and teaching strategies?' 'Very much'. Although before you said that the cooperating teacher gave you a lot of guidelines, you were still able to experiment?

P(97) Yes, because I just squeeze what I have to do, required by the teacher there into a few lessons, and I used the other lessons to do my own things.

AU 'Would you describe your teaching style as learner-centered or teachercentered?' 'Mostly learner-centered'. And 'Which of the following factors has the most influence on your approach to the teaching of English?' You said, 'English proficiency of students' and 'Class size', followed by 'Exam requirements'. Class size you said is very high. 
$\mathrm{P}(97) \quad$ With a very large class you can't always use activities because you can't manage and you can't check every student at a time.

AU Question 31, 'The changeover in sovereignty will affect my approach to teaching. ' 'Disagree'. .... Personal Relationships and Responsibilities, ' How confident are you in your ability to become a successful teacher?' 'Quite confident'. 'How do you feel about the status of teachers?' 'Teachers enjoy a moderate status'.

P(97) Not very high.

$\mathrm{AU} \quad$ Why do you think that is?

P(97) People may appreciate a person who can make a lot of money. That's the reason. .... Especially the businessmen, they won't respect teachers because they don't think you are smart. Only a person who can make a lot of money is smart.

AU 'What do you regard as your best attribute in terms of being a teacher?' You said, 'Good command of the language', 'Empathy with students', 'Good communication skills'. And lowest, 'Able to form good professional relationships'. Why do you say that?

$\mathrm{P}(97)$ In a school there are many teachers and their age range from their twenties to the fifties. There may not be many fresh teachers in the school that you are teaching, and that's why I think it's quite difficult to form a good relationship with the other colleagues. And also they teach different subjects, and you can only communicate better with the English teacher, than other teachers. And if the English teachers in that school are in their fifties or in their forties, I think it's very difficult to communicate with the teachers.

AU Many teachers prefer to work by themselves.

$\mathrm{P}(97) \quad$ Maybe they are not that keen on teaching actually. For many English teachers in HK, they are not English major students when they were university students, and they may come from other disciplines, and as the salary of a teacher is really good in HK, they will join the education, but since they are not that keen on teaching, they will not share their ideas with other teachers and they just do what they have to do, follow the instructions by the ED or the principal and just do what they have to do and then nothing more.

AU 'Do you see teaching as a long-term career?' 'Not sure at the moment. Why are you not sure?

$P(97) \quad$ Because we are still young. Maybe it's affected by the intake of our course. As far as I know, many of our classmates in the BATESL course, put this 
course in a lower rank for the JUAS. When we were F7 students, we have to choose which course and which university you would like to enter, and many of us placed BATESL in a quite lower rank.

AU And most other people wanted to do what other kinds of courses?

P(97) I don't know, but not teaching. Communication and translation, but not teaching English. Maybe that's the reason.

AU 'If you teach English in the future, who do you think will be most helpful?' 'Fellow first-year teachers'. Rather than more experienced teachers?

$\mathrm{P}(97) \quad Y e s$, and your friends may understand you more because they are in the same situation as you, but more experienced teachers are not.

AU 'During your practice teaching, who was most helpful?' Your supervisor. Most people put more experienced teachers or panel chair.

P(97) Because I didn't meet the panel chair for the three weeks.

AU 'Which of the following out-of-class activities do you think you will have to do?' Pretty much all of them except ... and which of [tape ends] ... marking papers, marking anything. 'If you teach English to what extent do you expect to be working closely with other teachers?' 'Some'. 'If you teach English in the future, what do you think will help you most to develop?' 'Designing new materials', 'Finding new materials' and 'Learning from other teachers'. It looks like your development will be rather personal.

$\mathrm{P}(97) \quad$ Maybe at the moment, I don't know the real use of that kind of conference because the teachers in HK they give me the impression that they will only focus on their own and they won't attend any conferences if they are not forced to. They don't think it's workable to use the ideas in a conference. Or maybe they don't feel it's useful.

AU 'To what extent do you worry about your future as a teacher after the changeover?' 'To no extent'. Good, 'Do you think your professional relationships or responsibilities will change after the changeover?' 'No, as far as English teachers are concerned.' Do you think there will be any change in emphasis as regards English?

$\mathrm{P}(97) \quad$ You mean in HK or in schools?

AU In HK. Do you think English will become more important or less important?

$\mathrm{P}(97) \quad$ Less important. It will still be emphasised, but to a lesser extent. 
AU Why do you think that?

$\mathrm{P}(97) \quad$ Because we have our own language, we have Mandarin, we have Cantonese and English is a foreign language I would say, it's not the second language in $\mathrm{HK}$, and that's why I think there might be a change. It will become less important.

AU Or Putonghua will become more important?

$\mathrm{P}(97) \quad$ Yes.

AU And do you think that will affect English in schools?

$\mathrm{P}(97) \quad$ Actually we can see the effect now, the MOI in $70 \%$ of secondary schools has changed, and that's the effect it brings.

AU Perceptions and Values, 'Do you think you have a definite philosophy of teaching?' 'Not very definite', 'Teaching is not something which can be taught. I think you have to really teach a class to learn what is teaching and teaching can only be improved through teaching. 'What does that mean.

$\mathrm{P}(97)$ Even if somebody tell you, you can teach like this, you can teach like that, you may still fail, because your students are different from other students and you can't use others' philosophy and you can't use others' idea to teach your own students, and you have to for example, based on the proficiency of the students, based on the environment of the school or the policy made by the panel chair or the principal. All these things will affect your teaching. Even if you attend another course in teaching, you will make no change. You may still stay on the same situation.

AU Areas that you would be unsure about, 'Personal career goals and ambitions' and 'Ideas on teaching methods'. 'What do you think are the most important factors which affect what goes on in the classroom?' As most important, 'Competence of teacher' ....

$\mathrm{P}(97) \quad$ If the teacher is not confident, maybe he or she will have to use Cantonese all the time and he or she may make mistakes himself or herself, and it will affect what goes on in the classroom. Very often the performance of the students will be affected by the teacher. If the teacher is good, then the students are potentially good.

AU 'Which of the following skills do you consider the most important to teach?' 'Very important' 'Writing', 'Speaking', 'Grammar', 'Pronunciation', with 'Reading' and 'Listening' as 'Quite important'. Why would you say that reading and listening are not quite as important as the other four? 
P(97) For listening and reading, sometimes you can guess if you don't understand the vocabulary in the story, you can guess from the context, and also, if you don't understand what the speaker said, you can just guess while you are listening. But in writing you can't use that kind of strategy. You have to know everything before you write or before you speak. You have to know exactly the wordings or the grammar.

AU 'Which of the following statements best sums up your feelings as regards the role or behaviours a teacher should have in relation to his or her students?' ... Why should the teacher become friends with the students?

P(97) I think it will facilitate learning. If I don't understand the lecturer so good, I don't approach him or her so often, and I can't learn things from him or her so often.

AU Do you think its possible for teachers to become friends with students?

P(97) If your students are higher form students, you can.

AU 'Your strengths and weaknesses as a teacher?' None you put as the strongest, but as 'Very strong 'Teaching pronunciation', 'Teaching speaking', 'Voice'. All connected with speaking in some way, but none of them you feel as particularly strong.

P(97) Because I am not a native speaker of English.

AU 'Are you planning to teach English?' 'Yes', but now, no.

$\mathrm{P}(97) \quad$ Yes.

AU 'What are the factors which you considered when making your decision about whether to teach or not?' 'I'll see if I feel satisfied with my own teaching.'

$\mathrm{P}(97) \quad$ Originally I planned to start teaching, but I had applied for a place at CU and after I'd been accepted by the govt., I received a letter from CU and it said that they will offer me a place on the MA course and I want to finish the MA course first before I teach. Maybe after one year I'll still apply for the AEO and then I'll be a teacher.

AU You would rather do an MA than a Cert. Ed.?

P(97) If I do the Dip. Ed. or Cert. Ed., I have to teach for all my life and it wouldn't make any difference if you have the Cert. Ed. if you don't teach, they will just consider you as a Bachelor holder instead of a Masters student. 
Interviewee:

Subject Q(97)

Date:

4 August 1997

Male, 27 years old.

Had completed a higher diploma before enrolling on the BATESL course and was keen to teach after graduation, provided he could find a suitable position.

$\begin{array}{ll}\mathrm{AU} & \text { Researcher } \\ \mathrm{Q}(97) & \text { Interviewee } \\ \ldots & \text { Missing words / Unfinished utterance } \\ \text { - } & \text { Pause }\end{array}$

AU The first part is Language Use and you said that the standard of your written English was 'Quite good' before you began the BATESL course, and is now 'Very good', and your spoken English was 'Fair', and is now 'Quite good'. So you think that you've improved both written and spoken English?

Q(97) Yes, but first of all I would like to indicate my case so that you know something more about my background because my educational background is quite different with my classmates. I used to be a student who studied Public and Social Administration right here, so that I have already graduated and got my higher diploma in PSA. So, in my memory, my written English improve a lot during these three years because we have got so many opportunities to write to express our own opinions in English. Actually, during my secondary school, I didn't find any way to improve my English quite effectively or significantly, because at that time apart from schools English lessons we didn't have so many opportunities to practice English especially oral English or written English, but the situation improved a little bit during my F6 and F7. I think this is partially contribute by those course that I take at that time because I studied history at that moment. In HK A' Level examination we must answer in very very long essay, so that's why we have to write English very much, especially we have to write the essay at school, so that's why I think when I was promoted to tertiary education institutions I found that my written English is not bad, but for my oral English is rather poor. At least doing the real communications, I still find that I cannot communicate with a foreign native speaker quite easily, but after I promoted to my PSA course, unfortunately such tendency still preserved because most of my lecturers would like to use Chinese as their teaching medium at that time so I cannot practice my oral English. But we must write in English, so I still got some progress in written English. But after I take the BATESL course, the whole situation improved dramatically because I have to speak in English, I have to listen to English quite frequently, almost every day. So right now sometimes I even have my dreams in English. That's the situation improve a lot so since we have got a lot of opportunities to speak in English and practice our oral 
English so I found that after these three years I improve my oral English very much. For my written English, I still think I get some progress, so at the very beginning my standard is quite good, right now it's very good, at least above the average when compared with those tertiary institutions' students.

AU Good, 'When speaking Cantonese to friends who have Cantonese as their first language, do you use some English words or expressions?' You said, 'Frequently,' and code-switching, 'Rarely.'

Q(97) Yes

AU You actually use English words when you are speaking Cantonese, what kind of English words do you use? Or expressions?

$\mathrm{Q}$ (97) It's some kind of jargons about course, sometimes, and for most of the time we talk about our daily lives. Because most of my friends have already finished their university studies, or some of them at least have already established their professional status in their own careers, so sometimes we talk about some special terms since we found that it's very difficult for us to translate those words or terms in Cantonese especially we are not familiar with those translations from Taiwanese because it's very difficult. When we use those Windows applications to choose between English and Chinese produced by Taiwan companies, however, most of my friends choose English versions because we found that we are so familiar with English versions and we found that we are so familiar with the words and meanings and when we come across those words, we can know immediately what those word is. So we've no need to use the Chinese words to translate it or we don't like it so we just want to save our time and since we can understand each other or communicate with each other, so we use English words to speed up our conversations sometimes. But for the language usage, sometimes, I do not intend to speak in English to my friends if their first language is Cantonese, because of course I don't mind to speak to them in English, sometimes I even want to speak to them in English so that I can practice my oral English. Sometimes my friends refuse to do this because they just find that we are Chinese so we no need to speak in English. We have our own dignity sometimes. So they would not speak in Cantonese with me, so I do not always switch to English when I speak to my friends. But if their first language is not Chinese, I find that we have a common background to talk with different languages because in my family we talk in some Chinese dialects, Mandarin, of course I do not speak in Mandarin but I can understand Mandarin, Cantonese, because we are not Cantonese.

AU Fine ... 'In English lessons, English should be the only language used for all purposes.' With this you 'Quite agree'. You said, 'If some words are very difficult for students, Cantonese could be the quickest way to explain and then teachers can save time in the lessons.' 
Q(97) Yes, I think there's no reason to proceed if the students cannot understand the meaning of the English words, because that will prohibit them from learning or to get any progress. So, I just think that if they cannot understand the English words, of course the teacher should to explain them in some simpler way or try to simplify the meanings by other, English versions, however, if the students can the meanings then we can get the exact wordings in Cantonese so why don't you use Cantonese because it can save the time and help the students to understand the words quite easily. Of course I understand that for those elementary level students or intermediate level students, they need this kind of explanations but I don't recommend for those advanced level students because I think that sometimes, for those advanced level students they figure it out by themselves in English. They can try to give an explanation to the word meanings by themselves, they can use English to understand it, so we no need to use this but I recommend it for those lower level students.

AU Fine, and 'In English lessons lesson content can be presented in mixed-code Cantonese/English.' But you disagree with this, 'Absolutely disagree'.

Q(97) Yes, because I think that we need practice. I believe in practice and exposure theory because in HK we are limited to the exposure of English, even though when we switch on the television or switch to the English channel, the Chinese subtitles will annoy me sometimes. Sometimes we just look at those subtitles and forget about listening, forget about the English versions. I hate that. I just believe that if we do not allow our students to practice all the time, they will just forget it, or they can never proceed to another level. Somebody claim this as heavy duty routine for English lesson. I believe in this way because that's exactly my experience, how I improve my English during my learning in secondary school and also in the university. So I do not recommend those teachers who always speak in Cantonese or English, use both languages in the English lessons because it didn't help the students.

AU But from what you say in \#7, it's okay to ...

Q(97) To explain some words but not all the time because some words are very difficult for the students to understand. For instance, if you want to explain nationalism or industrialisation, or the difference between industrialisation and modernisation, if you want to explain these two words in English you may spend the whole lesson, that's meaningless, but if you just explain to them in Cantonese the exact wordings they can understand the difference between these two. So it save time, and sometimes we no need to explain all the words, the students are clever enough to understand some of these terms, so I just think that if we use Cantonese or Chinese for some words, it is all right. But if we always use this strategy on targets, I don't think it's acceptable. 
AU So long as the students don't become reliant on the teacher using Cantonese, they don't expect the teacher to use Cantonese every time they don't understand something.

Q(97) I discover during my practice teaching that most of my students came to rely on the teachers to explain all the difficult words to them, they seldom use the dictionaries right now, even though nowadays some electronic dictionaries is quite common and quite user-friendly in nature, they seldom use it, they just ask the teacher to explain to them. Even though I ask my students to go home and study and prepare for the lessons, look up in the dictionaries and find those difficult words, they never do that. So, if we always encourage the students by using mixed-code in English lessons, that's no use.

AU 'During practice teaching, how much did you use only English in lessons?' You said, 'Usually', and 'How much did the amount of pure English you used in lessons change?' It 'Didn't change at all'. You adopted a similar approach to what you said?

$\mathrm{Q}(97) \quad$ Yes but I made some concessions during my practice teaching because all my students are grade 5 students, so in other words that means I have to extend my proportion of using Cantonese in my lessons, I made some concessions, but I still insist for most of the time, I must give them English versions at first and try to explain to them in some simple English first and if they still can't understand it then I code-switch to Cantonese. That's my last resort, but I have to make concessions. For those Band 1 or 2 students I won't make such concessions indeed.

AU In fact in \#11, it says, 'In what situations in an English lesson would or might you use Cantonese?' Again, you said, 'To explain vocabulary' and 'To be friendly with students'.

Q(97) Sometimes I've found that the students may be hostile to the language teachers if they always speak to them in English or in a foreign language because they know that you are not native speakers you are just Cantonese speakers as well as themselves so they will think that you just want to establish you own status or authority so they will hate it, so I just want to be more friendly with my students. So at the first lessons I try to establish a warm atmosphere among the students by using some Cantonese for a brief introduction of myself.

AU 'To what extent do you see the use of English in secondary schools being affected by the changeover in sovereignty?' You said, 'To a large extent', and the changes you expect, 'The use of English in secondary schools will reduce to a significant level, for instance the strong guidance to the MOI may lead to a consequential loss of students' exposure towards English vocabulary.' 
Q(97) I think this question is two-fold. First of all, after the handover of HK, we must regard that some students will think that, oh, we are Chinese right now, so they have no need to think about the usage of English in their daily life, they have no need to use English. Forgive me, I must use the term, shortsighted, because they do not see that when they are promoted to tertiary education institutions, or they have to work in society, English is a must or a necessity. They don't realise this, but most of them tend to think about this. Change their attitude and change their incentive to learn English. They just think that we are Chinese ourselves so we do not need to learn English, and we have no need to use so much English in our lessons. That's the first influence. And the second influence is that the ED proclaims that all schools that do not achieve a certain level, that means the students' level is not so high enough to learn in English, they should use Chinese as the MOI in the classroom lesson. So according to these strong guidance for the MOI ... only about 100 secondary schools can use English as the teaching medium. So the vast opportunities of the students may lose the opportunities to learn in English in other subjects because they just regard, it's very interesting that they just regard English as a subject, so you just learn the subject, you just learn the grammar in the English lessons, but they don't realise that English is a live language. It's alive, in our daily lives. Whenever we talk something about our life with a foreigner, we need to use different vocab or to use English. We have to explain different terms such as ... some terms in physics, chemistry or biology. If we change all these subjects in Chinese, they won't be able to communicate with a foreigner, and they will face a very serious difficulties when they are promoted to the universities.

$\mathrm{AU}$ In fact the change in the MOI has come in now not officially anything to do with the changeover, it's something that they've been planning for a long time, but the fact that it's happening next year, plus the more cultural changes in the thinking of the Chinese people, may lead to, as you say, less exposure towards English. The next section is Instructional Planning and DecisionMaking, 'It's impossible to have a successful lesson that hasn't been wellplanned in advance.' You 'Quite agree' with this one.

$\mathrm{Q}$ (97) Of course I didn't mean that we should have a detailed lesson plan about every word that we spoke to our students, at least I should plan what we should talk in this schedule or in this time and that for next time we should talk something. We should collect two lessons together in accordance with some grammatical relationship or some grammatical linkage between the two lessons. We need to plan it otherwise the students may confuse.

AU And 'Teachers should write detailed lesson plans for every lesson they teach.' Which you 'Quite disagree with saying, 'It's impossible owing to the heavy workload, yet a lesson framework or a rough plan should be needed.' 'Teacher should be more responsive to students' needs than the set syllabus.' You 'Quite agree'. 'I agree but teachers may face pressure from schools, parents or 
students, if the teaching schedule is far behind the normal one which is based on the examination syllabus.' So basically, the examination syllabus is the allimportant one?

Q(97) Unfortunately, that's the first concern of the students and their parents. The only way to show that you are a successful student or a successful learner, you must pass through the public examination and get a good result such as credit or distinction. so you must teach the students in schedule according to the examination syllabus because the ED publish the syllabus and that's the guideline for what the students must learn. So you must teach them because all them will be tested during the public examinations and that's the only way. The teachers must face this kind of pressure, of course some teachers may feel that some students may be unable to cope with the examination syllabus because it's very difficult for them, especially those grade 5 students, so sometimes teachers may want to slow down a little bit and give some more explanations about some specific terms or some specific topics. However, it won't be easy for teachers to do this all the way because sometimes we need to meet a schedule so we must cut short our explanations sometimes, otherwise the students, parents or some other teachers may complain about your progress. That's the reasons we can't do so much things to help our students, even though the ED promote right now, what do they call, Curriculum Target Learning?

AU TOC, the Target Oriented Curriculum.

Q(97) Yes, the TOC. I don't think it's workable. Of course, it's original or fundamental principle is quite similar to that question, that teachers should be more responsive to students' progress, however, they don't realise that this also increases the workloads of secondary school teachers. We must have enough resources or manpower to cope with these changes, however, they never realise that. So they just ask you to use this principle, but we never able to do this because we only have two hands but not six hands or eight hands.

AU Right, and it's very often the examination which sets the needs. 'When planning lessons, how detailed do you think the planning should be?' 'Brief and very flexible'.

Q(97) Yes, from a case during my practice teaching I only write a brief, of course my module required us to write a very detailed lesson plans for grading, but in my opinion I just establish a very brief and flexible framework in my mind. I just let myself know that in this lesson I must teach some topics or teach some points in that lesson. If I cannot finish all of these I must give myself some allowance in future so that I can cope with these. I can change my plan quite easily so that's the reason why I oppose to write a very detail lesson plans because it just makes you become a very rigid person because every 
word every plan that I have made cannot be changed. So I do not agree with this.

AU Yes, and as you say in your practice teaching module, you usually had to change your lesson plan. Why was that?

$\mathrm{Q}$ (97) Two reasons, one reason is about the progress of my students. Sometimes I was quite disappointed with my students' progress. Of course that may be due to my poor teaching, but I just think that the students' level is very low so they cannot too accomplish with my progress, so I just have to change my plan and sometimes my cooperating teacher ask me to change my plan to do some other ways so I must do that. It's quite interesting that I discover the ED do not have a very thorough planning for those immigrant students because in my class during my practice teaching I found several new immigrant students. They told me that they had already studied F3 or F4 in China but when they come to HK they must study in F1 or F2 because their English is not good enough to cope with the learning in local schools. They have to learn English from the very beginning in F1, they cannot compare with other students, so when they talk to the ED they just say, you find a school by yourself and most of the time will be sent to Band 5 schools.

AU 'On what basis did you plan your lessons during your practice teaching?' You said first, 'Perceived needs of students' followed by 'Syllabus'. So you have to kind of balance the two?

Q(97) Yes.

AU And 'During your practice teaching module, what do you think were the factors that had the most influence on the decisions you made?' Again, 'Students' needs' followed by 'Other student teachers' advice'.

Q(97) And my 'Supervisor's advice' followed by 'Regular teacher's input'. Indeed I rank this because we have to deal with these problems, but actually I will only say that students' needs is the most significant reason and the most important one. The others are not that important but we have to deal with this.

AU 'To what extent do you think the changeover in sovereignty will have an effect on your lesson planning and decision-making?' 'To some extent', and you said, 'I may include more topics in my lessons so as to increase my students' exposure towards English.'

Q(97) Yes, that a compensation, because owing to the handover, the students' exposure to English may be limited in future, so I think I may need to include more topics. 
AU The next section is Teaching Approach, 'Examination requirements should determine what is taught in English lessons.' You 'Quite disagree', you say, 'I disagree with this statement, unfortunately this is a fact that we cannot resist certain situations in HK.' Okay, you disagree with it but there is nothing we can do about it?'

Q(97) Yes, I know that in the US sometimes we can modify our exam requirements or even though we can modify our students' progress according to their needs or their desires to become good learners and that's it. I think that's the best way because teaching is a two-way communication, if you want to feed your students with a huge amount of data, but they are unable to process it, that will be useless. So I just think that we should help our students according to their needs rather than examination requirements. Of course exam requirements will be some general standards perceived by the general public, that's the general guidelines for a successful teaching or learning progress, but I disagree with that because in HK we have already changed the nature of examinations, we just regard it as a means to single out those students who are unable to learn. We just want to think about those students, and let those students who are advanced in learning proceed to the next step. But we are not teaching our students how to learn, how to learn English through the learning process, how to improve their learning abilities, rather than memorize those data.

AU 'Students learn English better through interaction with other students rather than through direct instruction from a teacher.' You 'Quite agree', and then, 'During your practice teaching module, did you find that students learn English better through interaction with other students or through direct instruction from a teacher?' And then you find 'No difference between the two'.

Q(97) I want to clarify about this, I think that the students should learn much better if they can interact with somebody else because sometimes this kind of communication can stimulate their thinking process, and then they will become more easily in learning something new because they have partners and they can share with each other, they can share their opinions and those useful informations. This kind of process is very important because we can enhance the students' learning process but also their inter-personal communication ... however, in my practice teaching, they are grade 5 students and so in some small group work they are chatting all the time so there's no difference between the two because they are grade 5 students.

AU And, 'A class should be strictly controlled by the teacher and all lesson content directed by the teacher.' You 'Quite disagree', 'It depends upon the level of the students.' And then, 'During you practice teaching module, did you find that you controlled all your lessons strictly and directed all lesson content?' 'Always', 'Because of the low proficiency of the students.' 
Q(97) Because we are teaching English. Of course if the students are talking with each other I will tolerate some Cantonese, if they are really talking about the topics, but that's not the case in my class, most of the time they are chatting, chatting with each other about their idols, something like that. It's not directly about my lessons. If I do not control them quite frequently I will face serious discipline problems, so that's the reason why I always control my students during my lessons.

AU 'Teachers should be prepared to experiment with teaching material and strategies from a variety of sources.' 'Quite agree', 'Teachers need more support or input in terms of training.'

Q(97) I know that in HK so many publishers would like to provide some materials to teachers ... and some teachers they just like to adopt, directly from the publishers. They just adopt everything and they do not think that this is appropriate to our students or not. I just found that some materials are presented in a very complicated way and the students cannot deal with it ... [tape ends] ... should try to use different resources to teach the students, especially don't just use those materials provided by the publishers. I find that the materials are designed for those countries where English is the second language and the students have to use English in their daily life but in HK this isn't the case. Actually I'm quite happy with the BATESL course, in the last year we learned a lot about how to distinguish whether the materials are good or bad especially in listening, in reading, writing or speaking. It's very useful as we can apply in our teaching career.

AU And the next question asks, 'During your practice teaching module, did you experiment with teaching materials and strategies from a variety of sources?'

Q(97) Yes because we have been asked to classify some teaching materials and then write a report so that we can compare those teaching materials, so for instance, I have made some experiments about the listening materials, for instance, we compared the tempo, the delivery speed to our students. I discovered that some of the listening materials are not very good indeed.

AU 'Would you describe your teaching style as learner-centered or teachercentered?' 'Mostly teacher-centered'.

Q(97) Subconsciously I do not trust my students would behave themselves in the lessons so sometimes I tend to control them quite a lot. That's the reasons why I think my teaching approach is teacher-oriented in nature. However, I will try to change this as kind of learner-centered, at least I should establish this as two-way communication between teacher and learner. 
AU 'Which of the following factors has the most influence on your approach to the teaching of English?' You put 'English proficiency of students' as being the first ... why?

Q(97) I think that if I want to be, if my teaching approach is based upon learnercentered, it must be able to be, because we are language teachers, so they must be able to deal with the topics or the teaching materials, or they should be able to speak in English. Otherwise, it won't be able for us to establish a learner-centered approach because if they cannot deal with it, then the teacher will dominate the class. So English proficiency is very important for the students, because according to the ... level of students, we may adopt different levels. Of course, two-way communications between learner and teacher will be the best, however, if the student level are not able to cope with this kind of requirement, so we just change it to a teacher-dominated class.

AU And do you agree with the statement that, 'The changeover will affect my approach to teaching'? You 'Quite agree'. In what ways do you think it will affect your approach?

Q(97) I think it's about my ... teaching role in the classroom. If the changeover were not going to happen then I think my teaching role would not change so significantly, at least I can ask my students because you need to learn a lot about English because according to your examination requirements you need to learn in English and you need to work in a society in which English is the first or the second most important language. So, I no need to urge my students, so my teaching approach will be quite relaxed and I just ask my students and they can have a quite strong incentive to learn it. But since there is the changeover I must change my role, I must do a lot of work. In previous years the teacher may just adopt a passive role in teaching, they just think that my students they urge to learn it so I've no need to encourage you so you just learn it by yourself, we just provide some sorts of channels for you to learn English, that will be enough. Nowadays it's not the case, we need to encourage, we need to take an active role to encourage my students or our students to learn English.

AU Professional Responsibilities and Relationships, 'How confident are you in your ability to become a successful teacher?' You're 'Unsure'.

Q(97) Yes, I just think that my English would be all right, or above the average, but in my mind, language teachers should be a perfect language user, they should be able to master the language in every aspect. Then they should be qualified as a language teacher. Unfortunately, that's not the case in HK, most of the time, teachers who are not majoring in English, they are majoring in history or some other subjects, they will be required to teach English. That's not good for our students. And for my case, since I do not regard myself as a perfect language master, so I'm quite unsure about this. Of course I'm quite 
sure my students will like my teaching approach and they will like to learn English according to my encouragement, however, they need a teacher who will be able to help them to solve different kinds of language learning difficulties.

AU 'How do you feel about the status of teachers in today's society?' You said that, 'Teachers have no special status.'

$\mathrm{Q}$ (97) Yes, especially compared with other Asian countries like Taiwan, like Korea, like Singapore. In those countries they enjoy a higher social status. That means whenever the teachers talk something about their society and about their students the students will respect them, and they will be afraid, and I don't like to use the word afraid, but indeed in those countries the students are afraid of the teachers. So you will be able to establish a standard or establish a model for our students to learn the social values or something like that. But in HK you can't expect that the teachers enjoy such status.

Sometimes they just regard teachers as a kind of knowledge machine, we just provide some knowledge, that's it, so sometimes the teachers will feel they are not respected by students and their sense of achievement is very low.

AU 'What do you regard as being your best attribute in terms of being a teacher?' You said, 'Good command of the language', followed by 'Communication skills' and 'Innovative in terms of teaching ideas'.

Q(97) Yes, I mean good command of the language is compared with the normal standard of the university students, not exactly as I called before as a perfect language master.

AU And things such as 'Able to form good relationships with colleagues' and 'Strict disciplinarian' are ranked the lowest.

Q(97) I think that teaching is just a matter between the students and the teachers, but not the relationship with your colleagues. Of course sometimes we need to cooperate about our teaching progress, to let them know about this, but that's it. If you have a good professional relationship with colleagues, it doesn't mean that it will help you to improve your students' standard or help you to solve those problems that you face in the classroom. So I think that's not important, but it's very important for your promotion.

AU I think that in HK the professional relationships between colleagues are not as good as they should be.

Q(97) Yes that's quite true in HK, because according to my friend's comments, he's a teacher in a local secondary school, he told me that it's quite rare for teachers to have a deep affection between each other. Sometimes they have a 
relationship, but that kind of relationship is just based on competition between each other.

AU 'Do you see teaching as a long-term career?' You said you're 'Not sure'.

Q(97) Of course I can enjoy to teach my students if they can give me some positive rewards, not in money but in a sense of achievement or in a sense of friendship between us, that would be quite good. However, I cannot foresee that we can have such a good relationship in future, or at least it is not very easy for us to find it in local schools. So I don't want to be disappointed so I'm quite unsure this moment.

AU 'If you teach English in the future who do you think will be most helpful to you?' 'More experienced teachers', and 'During your practice teaching who was most helpful to you?' Again, 'More experienced teachers'.

Q(97) Because sometimes more experienced teachers can give you some direct information about the classes and about the level of students, and about the characters, so it save you a lot of time in dealing with the class. So you can smooth your lesson plans and also about your teaching ... quite easily, so sometimes their comments will be quite important. Especially some principals or some panel chairs may dislike some behaviors like they didn't like teachers who allow students to speak or to learn in groups. If they tell you about this you will never make such kinds of mistakes, or at least you can inform your panel chair about the reasons, so that may help to improve the relationships. And that also help to improve your teaching progress.

AU 'Which of the following out-of-class activities do you think you will have to do as a first year teacher?' Most of them except ...

Q(97) I think be a form teacher is very demanding, especially for a fresh graduate, or for those novice in teaching language, because we have to do a lot with lesson plans, language problems, but rather than students' emotional feelings or something like that.

AU The same list of activities again, but this time ' ... which do you think will take up the most time?' You've put, 'Attending meetings' both with the principal and panel chair, and 'Preparing lessons'.

$\mathrm{Q}$ (97) That's quite time-consuming. In the first few years of teaching career, I think we have to be familiar with the school environment so that we need to talk with other teachers and also with the principal and panel chair, and then since we haven't established our own teaching materials during the first few years so we need to spend a lot of time. After this first few years we just modify our teaching materials. 
AU 'If you teach English in the future, to what extent do you expect to be working closely with other teachers?' 'Some'.

$\mathrm{Q}(97) \quad$ Yes

AU 'If you teach English in the future, what do you think will help you most to develop in your first year?' You put, 'Designing and finding new materials' as being the things that would help you to develop most.

Q(97) I just think that of course we can learn from other teachers, from other experts, but that's their own experience, that may not be appropriate to you. So I just think that finding new materials and designing new materials will be more important than this.

AU And 'To what extent are you worried about your future as a teacher after the changeover in sovereignty?' 'To little extent', then you've added, 'The sociopolitical status of English may decrease as we're going to be a foreign language teacher.' So you are a little bit worried?

Q(97) Yes, because we need English so we need English teachers, and that's it, so I do not worry about my status because if I'm a qualified teacher, I can teach English. Of course I cannot compare with native speakers, or compare with those native speakers who teach English in HK. However, I have my advantages over them because I know Cantonese, I know the students' needs, so I do not worry about my status, or the threats by the ED's schemes about importing native speakers from other countries because we have our own advantages.

AU And 'Do you think your professional relationships or responsibilities will change after the changeover?' 'Yes', and you've said, 'My workload may increase and my status may decrease.'

Q(97) Status, I mean the govt. or even the school principals may pay more attention to the Chinese language teachers, rather than the English language teachers. They will provide more resources for these teachers because want to improve the Chinese standard of our students. However, I don't care about this because I know they must still provide some resources for those English language teachers, otherwise there will be no talents for the future.

AU Okay, and the last section is Perceptions and Values. 'Do you think that you have a definite philosophy of teaching?' You said, 'Quite definite', and your philosophy of teaching is, 'I'll pay attention to my students' needs rather than public examinations. I believe a learner-centered approach may enhance the learning/teaching progress.' Do you mean 'progress' here or 'process'?

Q(97) Process 
AU Okay, 'What do you think are the most important factors which affect what goes on in the classroom?' You ranked as most important, 'Motivation of students', 'Language ability of students', and 'Competence of teacher'.

Q(97) Yes, sometimes I've discovered that even though the competence of teachers may be very important as the students may have different questions, so you must have a high competence in English or in language so that you can solve their problems, and you must clear up their confusion otherwise they will just fossilize their mistakes and that will be no good.

$\mathrm{AU} \quad$ And things like 'Class size', 'Location', 'ED' are not so important?

$\mathrm{Q(97)} \mathrm{Of} \mathrm{course} \mathrm{class} \mathrm{size} \mathrm{may} \mathrm{help} \mathrm{us} \mathrm{to} \mathrm{reduce} \mathrm{the} \mathrm{work} \mathrm{load} \mathrm{but} \mathrm{if} \mathrm{the} \mathrm{students}$ have a strong motivation we can still manage to teach them in a good way.

AU 'Which of the following skills do you consider most important to teach?' All of them, very important?

Q(97) Yes, I just don't understand why some so-called experts proclaim that grammar is not so important. They tend to use some inductive, is that right? They ask the students to generate some grammar rules from the lessons, a deductive model? They proclaim there's no need to teach those grammar rules explicitly, we just allow the students to generalize. Unfortunately, some of the teachers, especially the English teachers complain about this because they find that the students are not able to generalize the grammar rules. Instead of this they fossilize the mistakes only to the confusion, so I think grammar lesson is a must, I must teach my students grammar, even though it's boring. Of course I will try to teach in an interesting way, but I must teach grammar to our students.

AU 'Which of the following best sums up your feelings as regards the roles or behaviors a teacher should have in relation to his or her students?'

Q(97) I think that I have already explained why I do not agree that teachers should be in total control of all lessons and I want to have further explanations of why I think students should not decide which direction their learning should take. Of course I always say that we should focus on their needs, but sometimes the students do not know what they want, and what they need. They do not possess that kind of knowledge or their maturity is not good enough to make such kind of decisions. And I think the teacher should be like a friend rather than a parent because they have parents at home, that their responsibility not our responsibility. So we just act as a friend and help them to cope with some of their problems.

AU And 'Consider your strengths and weaknesses as a teacher.' Your strengths, 'Communication skills' and 'Strength of personality' ... that pretty much 
replicates the previous responses. And finally, 'Are you planning to teach English next year?' You said, 'Not yet sure.' The factors affecting your decision are 'Social status of teachers', 'New teachers salary', 'Job satisfaction'.

$\mathrm{Q}$ (97) I marked job satisfaction as this is the most important factor that will affect my decision because we all know that we are paid the same salary as those other teachers. Of course salary is important, if I cannot find another job that is high paid as a teacher then I may change my mind. But I don't want to be a teacher whose motivation upon teaching is based upon salary. ... And I think that job satisfaction may not be so high as other jobs in HK because those students may not be cooperative enough. 


\section{Interviewee: $\quad$ Subject R(97) \\ Date: $\quad 6$ August 1997}

Female, 24 years old.

Was unsure as to whether she wanted to teach as she planned to take an MPhil degree after graduation.

$\begin{array}{ll}\text { AU } & \text { Researcher } \\ \text { R(97) } & \text { Interviewee } \\ \ldots & \text { Missing words / Unfinished utterance } \\ \text { - } & \text { Pause }\end{array}$

AU Language Use, you said that when you started the BATESL, both your written English and your spoken English were 'Quite good', and now they are 'Very good'.

R(97) Yes, I think so. I think I have made some improvement because of lots of practice here.

AU Do you think you've improved more here in three years than you did in secondary school?

$\mathrm{R}(97) \quad$ Yes, because it's more intensive here.

AU Would you say that you are stronger in spoken English or written English?

R(97) I guess I'm rather stronger in written English than spoken English because you don't need to face others when you write English and you can write whatever you like and you can erase whatever you like, but especially when I speak to a foreigner, I mean an English speaker, sometimes I've got a lot of slip of the tongue. I guess it's because of my level of confidence is too fluctuated. You will be threatened by their maybe speed. When I speak to Professor Richards, he speaks so fast, and when I speak to him I was threatened to a certain extent, but when I speak to Martha or Angel, I would speak better because they know your standard and they can take care of you in some way. And because of their status ... very frightening.

AU 'When speaking Cantonese to friends who have Cantonese as their first language, do you use some English words or expressions?' 'Quite often', which is code-mixing, and also switching between one language and the other, which is code-switching, you also said 'Quite often'.

R(97 Yes, I guess it's a question of habit nowadays. Maybe we are lazy, we won't think of the difficult terms used in Cantonese, we would use English to save 
time or because it is the shared knowledge in some way. We will prefer using English rather than Cantonese.

AU And code-switching, you sometimes switch into English?

R(97) Yes, sometimes if the words won't come into my mind, I mean L1, then English comes first, I will use English.

AU But for more than just a word or a phrase, you will actually switch into English?

$\mathrm{R}(97) \quad$ Yes, when we are speaking secrets, when we are speaking things that we won't let others know, then probably we will switch to English. This is especially frequent when I am speaking to my sisters, we don't want my mum to know what we are talking about so we will sometimes use English.

AU Now talking about English used in teaching, you said 'In English lessons English should be the only language used for all purposes.' You 'Quite disagree'.

R(97) Yes, in real situations there's some situations that you couldn't use English. If you want your students understand what you meant. This is true because I went to the real classroom and I saw what I just said and I have done some research on code-switching and in some cases it is necessary if you really want the students the understand what you are talking at that moment then you need to switch, especially when you explain vocabulary on abstract nouns and names of objects, you need to use some L1 to help them understand.

AU And as regards lesson content, you disagree that it can be presented in mixedcode. So you wouldn't present lesson content in mixed-code, you would only use Cantonese to explain things?

$\mathrm{R}(97)$ Say for example, in mathematics lesson, my mathematics teacher used mostly $\mathrm{L} 1$ in the lessons. I guess it's a question of habit. So I prefer using L1 in content lessons rather than English lessons. In English lessons we should use English.

AU 'During your practice teaching, how much did you use only English in English lessons?' 'Usually'.

$\mathrm{R}(97) \quad$ Yes

AU And ' ... how much did the amount of pure English you used change?' 'It increased'. So when you started off you were using more Cantonese at the beginning? 
R(97) And I started to increase to use English, because the students are used to what I taught and they can follow me so I started to increase using more English.

AU And ' ... in what situations would you use Cantonese?' 'To explain content' or 'To explain vocabulary'.

$R(97)$ Most probably I use Cantonese to explain vocabulary. Maybe out of the classroom I would use Cantonese, but I try not to do so in the classroom, to set up an example.

AU 'To what extent do you see the use of English in secondary schools being affected by the changeover?' You said, 'To little extent'. 'What changes do you expect?' 'The original status of L2 may be shared or taken over to a certain extent by Mandarin.'

$\mathrm{R}(97)$ I mean the importance of Mandarin is getting greater. It becomes more and more important, and maybe it shares more or less the same status as L2.

AU So you think as Mandarin becomes more and more important, English will become less important?

R(97) No, not less important, more or less the same status. Because when I was a primary student, I don't have lessons of Mandarin, but now my cousin who are primary school children, they have lessons of Mandarin. They know more Mandarin than me, though I have taken some courses in Mandarin.

AU Okay, the next section is Instructional Planning and Decision-making, 'It's impossible to have a successful lesson that hasn't been well-planned in advance.' You 'Quite agree'.

$\mathrm{R}$ (97) It is especially true for us, for inexperienced young teachers, because it gives us confidence when we have something in hand. If we don't have something in mind, something planned, when we go into the classroom, we would feel less confident. We need to have something planned in mind before we go into the classroom.

AU And 'Teachers should write detailed lesson plans for every lesson they teach.'

$\mathrm{R}(97) \quad$ For me I think it's true.

AU You 'Quite disagree'.

$\mathrm{R}(97)$ For inexperienced teachers, this is true, but for experienced teachers, I won't say it's not true but they may not have that necessary time to prepare a detailed lesson plan for each lesson. But I think if we prepare a detailed 
lesson plan we will have a better picture of the lessons ... And the definition of 'detailed' does vary, for me it's detailed, for you maybe it's not detailed.

AU 'Teachers should be more responsive to student needs than the set syllabus.' You 'Quite agree'.

$R$ (97) Yes, because they are your targets, they are the people who give you the most immediate response, so you need to take care of their needs.

AU 'When planning lessons, how detailed do you think the planning should be?' 'Quite detailed and quite flexible'.

R(97) You need to have that sort of detailed element because you may forget before teaching that lesson, and you need to have flexibility because they are so many unexpected classroom happenings and you need to have that sort of flexibility to change.

AU 'During your practice teaching module, how often did you have to change your lesson plans?' 'Sometimes'.

R(97) Only sometimes. The lesson went quite smoothly. I taught young students, F2, they were quite obedient. Frankly I prepared the lesson quite well and everything was in my hands.

AU So you didn't have to change much?

$\mathrm{R}(97) \quad$ Yes, little changes.

AU Then what might you have to change?

R(97) I remember there's a case I had to change, when the tape recorder was out-oforder. Then I couldn't play the tape. I had to read the thing.

AU 'During your practice teaching module, on what basis did you plan your lessons?' You said firstly your 'Own ideas', followed by 'Perceived needs of students' and 'Syllabus'.

$\mathrm{R}(97) \quad$ Yes

AU BATESL course guidelines actually last.

$\mathrm{R}(97)$ Because my tutor gave us so much flexibility, we could do on our own, and she gave us so little guidelines. But it's good; we could work on our own.

AU So you didn't have too much instruction from the cooperating teacher? 
$R$ (97) Because she told us that if she give us so many guidelines, it is not our practice teaching, it is her practice teaching. Then I need to work on my own. I have the set syllabus but she gave me so little guidelines, so much, not very helpful in fact. Most of the time they won't take care of you because they have other things to deal with, school administration things to deal with. So we need to count on our own.

AU And 'During your practice teaching, what the factors that had the most influence on the decisions you made?' You said, 'Supervisor's advice' first, followed by 'Regular teacher's input'.

R(97) My supervisor was [ ], in the middle of the internship she came and she talked to me and she gave me advice and that's why I chose it as \#1. And then I need to change, because of her advice, because it's good advice.

AU 'To what extent do you think the changeover in sovereignty will have an effect on your lesson planning and decision-making?' 'To no extent'.

$\mathrm{R}(97)$ I couldn't tell at that moment, I don't know. When you look at the, Basic Law? There's not much guidelines about education. I don't know if there are any big changes after 1997, so I couldn't tell.

AU Next section is Teaching Approach, 'Examination requirements should determine what is taught in English lessons.' You 'Quite disagree'.

R(97) Yes, to me learning English is not for examination, examination is just a process that I need to proceed to another stage of schooling, but in reality we need to do so, we need to have some examination requirements lessons. But to me if I could have that opportunity I won't teach examination requirements in English lessons. Maybe in some other time.

AU 'Students learn English better through interaction with other students rather than through direct instruction from a teacher.' You 'Quite disagree' with that.

R(97) I think students learn more interaction with the teacher than the students because traditionally teacher is the authority in the classroom, is the one who knows the most amount of knowledge, and he or she knows the most amount than students. We could have better interaction with teacher rather than students. Another thing is for teacher. If you have interaction with teacher, they could correct you when you have made mistakes. But when you have made mistakes, your peers couldn't correct you. That's not helpful then, that's not helpful interaction. It may be more relaxing interacting with peers, but academically, or if you want to get some educational benefit, you need to interact with teacher. 
AU 'Lessons should be strictly controlled by the teacher and all lesson content directed by the teacher.' You 'Quite disagree'.

$R(97)$ If you take care of student needs, then teacher cannot control all the lesson content, and a class should not be strictly controlled by the teacher because you are just teaching students, you are not having a parent-son relationship. Don't control them, if you want to have good classroom management. Maybe you can control them in a mild way. For me control is not a good word.

AU 'A teacher should be prepared to experiment with teaching materials and strategies from a variety of sources.' You 'Quite agree' with this.

$R(97)$ Yes, we need to be innovative, we need to try it out before we actually use them. We need to recycle them, so we need to do some experiment with the teaching materials and strategies and so on.

AU 'In your practice teaching module, did you find that students learn English better through interaction with other students or through direct instruction from the teacher?' This refers back to the other question, and you said, 'Through direct instruction'.

$R(97)$ Because from my observation they were not so familiar with the idea of discussion, group discussion and pair work, they were not so familiar with that idea, they just familiar with the traditional, that is whole-class approach, that is teacher-fronted pattern. They learned better from me, I guess.

AU And 'During your practice teaching module, did you find that you controlled all your lessons strictly and directed all lesson content?'

$\mathrm{R}(97) \quad$ Yes, because I was restricted by the set syllabus, I need to go through all before I left, so I need to direct all lesson content. So many restrictions.

AU And 'During your practice teaching module, did you experiment with materials and teaching strategies from a variety of sources?' You said, 'Very much'.

R(97) Yes, because I need to do some journal writing, and I need to tell [ ] what I experiment, what strategies I have used, and I need to evaluate them. So I need to do a lot of experiment in my lessons. And I guess it's helpful to do so, it's really helpful. And sometimes the strategy just comes at that moment. I haven't though of them before. Because of such and such a situation, I come up with such and such a strategy just at that moment, on the spot strategies.

AU 'Would you describe your teaching style as mostly learner-centered or teacher-centered?' 'Mostly teacher-centered'. 
R(97) Because I was teaching young learners, I had to show them some authority. But I have no opportunity to teach upper form students, which I really want to teach. If I had that opportunity I think my approach would change to more like learner-centered. You need to bring out this problem. It seems to me that this BATESL course is prepare you for teaching only junior form students ... we have not much coverage on how to teach senior form students, we need that.

AU 'Which of the following factors has the most influence on your approach to the teaching of English?' 'Class size', followed by 'English proficiency of students' and 'Motivation of students'.

$\mathrm{R}(97)$ Just drawing from my experience of practice teaching, I've got 45 in a class, I think class size is a problem. ... It is really terrible, 45 students in a class. When I'm talking to that side then another side start gossiping, and when I'm talking to individuals, the students at the back are talking quite loudly. I just couldn't control them. When I did marking, it was especially terrible. When I was marking compositions, 45 students and then I've got 2 classes, that means 90 copies.

AU The least important things are 'School policy', 'Noise in classroom' ...

R(97) I wouldn't expect there's absolute silence in the classroom, that's abnormal, so I rank it the lowest.

AU 'The changeover in sovereignty will affect my approach to teaching.' You 'Quite disagree'. I suppose similar to the last ...

$\mathrm{R}(97) \quad$ Yes

AU Professional Relationships and Responsibilities, 'How confident are you in your ability to become a successful teacher?' 'Very confident'.

R(97) Right, especially when I teach junior form students, because I have two years' experience of teaching them, during my internship in Year 1 and during my practice teaching in Year 3. I have confidence in teaching Form 2, only Form 2.

AU 'How do you feel about the status of teachers in today's society?' 'Teachers enjoy a moderate status'.

$\mathrm{R}(97)$ Yes, to me it is a moderate status, high status is lawyers, doctors.

AU Why do you think they have a higher status than teachers? 
$R(97) \quad B e c a u s e$ of social norms. Maybe because of their salary, I don't know. I just learn it from my schooling, that lawyers, doctors earn more.

AU They earn more so they have a higher status. 'What do you regard as being your best attribute as a teacher?' 'Good command of the language', 'Good communication skills', 'Innovative in terms of teaching ideas'.

$\mathrm{R}$ (97 Yes, we need to have good command of the language, especially because we are not native speaker, but we teach foreign language, that's why we need to have good command of language then native speakers.

AU The lowest being 'Able to form good professional relationships with colleagues' and 'Strict disciplinarian'.

$\mathrm{R}(97) \quad Y$ Yes again I'm only a teacher, I'm not a mother and I'm not a father. I just teach, I've not come to do discipline. And I don't like to control students.

AU Forming good relationships with colleagues?

R(97) [Tape ends] ... grows naturally. It just grows naturally; you don't need to pay much attention ... to think of ways to be a better colleague. I think I will have little problems in that respect.

AU I think so, you are a very friendly, open person.

R(97) Thanks.

AU 'Do you see teaching as a long-term career?' 'Not sure at the moment'.

R(97) Because I'm going to study M.Phil.

AU 'If you teach English in the future, who do you think will be most helpful to you in your first few years of teaching?' 'Panel chair'.

R(97) I mean the panel chair would be my second boss and I would have the most contact with him or her, besides principal.

AU And for your practice teaching module, you said that your 'Teacher-training supervisor' was most helpful.

R(97) She was, no I should say she is very helpful. She is always the one who speaks for us, and if we have any problems in teaching, classroom management, in school administration, she is the first one who speaks for us, very helpful. 
AU 'Which of the following out-of-class activities do you think you will have to do as a first-year teacher?' ... The same list, but this time which do you think will take up the most time? You put 'Counseling students'?

$R(97)$ Because the students nowadays are too mature, they have a very complicated mind and it really takes time to talk to them. If you want to be good counselor, then it should take up the most time.

AU More time than marking or preparing lessons?

R(97) I guess marking is just a question of habit, you can mark very fast, you can do it in an efficient way. But if you do some counseling, it really takes time and it depends on who you are talking to. Maybe some would take you more time, maybe some less, but I guess most probably, this kind of job will take up the most time, and you couldn't control. The marking, I suppose you could have some sort-of ways to control.

AU 'If you teach English in the future, to what extent do you expect to be working closely with other teachers?' You said, 'Very much', sharing ideas or teaching materials?

R(97) Yes, especially as we are inexperienced. We need to ask very often. If we want to learn within a short time, we need to ask. We need to work closely with my neighbours.

AU 'If you teach English in the future, what do you think will help you most to develop as a teacher?' You put 'Getting feedback from panel chair' and 'Learning from other teachers'.

R(97) Yes, because I am teaching English, I guess the most experienced English teacher may be the panel chair. So maybe his or her feedback would be the most helpful.

AU And, 'To what extent are you worried about your future as a teacher after the changeover?' 'To little extent'. What are your particular worries? You put, 'How Chinese see the role of English in HK, for example are they supportive to advance and improve the local language situation by giving enough funding to provide teaching aids and teaching materials to schools.'

$\mathrm{R}(97) \quad J u s t$ see how supportive they are. If they are supportive then students may become higher, but if they support more Mandarin in schools, we need to be careful.

AU 'Do you think your professional relationships or responsibilities will change after the changeover?' 'Somewhat'. 
$R(97) \quad I$ don't know. Maybe you ask me this question one year after the handover, two years after the handover, then I could have a better answer.

AU I might do that. Okay, Perceptions and Values, 'Do you think that you have a definite philosophy of teaching?' You said, 'Yes, very definite'. You put, 'To help students engage their whole-person learning, and to help students learn in a caring and sharing atmosphere, and help them to take responsibility and monitor their own learning.' The whole-person learning?

R(97) Not just teach them English, you have to help them learn from all perspectives. But we just start from teaching them English.

AU Help students learn in a caring and sharing atmosphere and help them take responsibility and monitor their own learning.

$\mathrm{R}(97)$ Because I couldn't be his or her teacher in his or her life. They need to monitor the learning. I just teach them the skills, I just teach them how, but they need to monitor themselves, they need to take care of their learning when they become grown up.

AU 'What do you think are the most important factors which affect what goes on in the classroom?' Most important are ... 'The most important skills to teach?' You put all of them 'Very important'.

R(97) Right

AU Some people might think that some are more important than others.

$\mathrm{R}(97)$ Because we have no criteria, or which aspects of them is the most important. We need to give them equal importance then.

AU 'Which of the following best sums up your feelings as regards the roles or relationships a teacher should have in relation to his or her students?' All of them except, 'A teacher should refrain from punishing students' and 'A teacher should remain in control of all lessons at all times'.

$\mathrm{R}(97) \quad$ Again I don't like to control students.

AU But you've also put that 'The teacher should act like a parent.' Whereas before you said that you are not a parent.

R(97) Yes, to a certain extent, when you are counseling them, maybe you need to be a parent. But when you are teaching, you couldn't be a parent. 
AU And 'Consider your strengths and weaknesses as a teacher.' 'Relationships with students' is ranked the strongest, along with 'Motivating your students and keeping their interest' and teaching of all the skills.

R(97) Right

AU And ones that are ranked not so strong are 'Discipline' and 'Voice'.

$\mathrm{R}(97)$ We could be cool to students in lessons but we don't want to do so. I could do something in discipline but I won't do so, I won't control students. So I would say I'm still strong in discipline but I don't control students in a very strict way. Everyone can be strong, but we just don't want to do so.

AU 'Are you planning to teach English next year?' 'Not yet sure'. 'What are the ' factors that affect your decision?' 'How to let students respect me.' 
Interviewee:

Date:

Female, 23 years old.

Planned to teach after graduation provided she could find a job.

$\begin{array}{ll}\text { AU } & \text { Researcher } \\ \text { S(97) } & \text { Interviewee } \\ \ldots & \text { Missing words / Unfinished utterance } \\ \text { - } & \text { Pause }\end{array}$

AU First of all Language Use, you said that your written English was 'Quite good' before you began the BATESL, and is still 'Quite good'. Your spoken English was 'Fair' and is now 'Quite good'. So you think that your spoken English has improved a bit, but your written English has not improved?

S(97) Because I think I have more chance to speak in English when I studied in this course, and compared with the secondary education, we seldom have chances to speak up in the class because most of the lessons are taught by the teachers and they seldom encouraged us to speak up. What I did in the lesson is just put down all the notes on the blackboard.

AU You don't think your written English improved any?

S(97) A little bit, I think I have learned more vocabulary items from this course, and I think I've learned more difficult words and the level of the words that I've learned is higher.

AU 'When speaking in Cantonese to friends who have Cantonese as their first language, do you sometimes use English words or expressions?' 'Quite often. Same with code-switching, again 'Quite often'. What sort of expressions would you use in English when you are speaking in Cantonese?

S(97) For example, I will say 'sorry' instead of [ ] in Cantonese. Some words like 'party', 'let's go to party', we won't say [ ] in Cantonese. So I think some words we are used to speak in English. So we still speak in English for some expressions. ... Some embarrassing words we will speak in English instead of Chinese because we will feel shy to speak in Cantonese.

AU Are there some words that you would use that most people wouldn't use?

S(97) Some technical words. I think most of the technical words we will use in this course, we will still speak in English with our classmates. We won't translate 
those technical words into Chinese, for example, the titles of the modules we will still speak in English.

AU How about code-switching? In what sort of situations would you do that?

$\mathrm{S}$ (97) In discussion. The discussion that we talk in this course, we will speak in English.

AU That's during the course, but outside when you are talking with friends?

S(97) Outside? I think it depends on the nationality of my friends. Maybe sometimes the names of the countries, I will speak in English. Like USA or Australia, because we always hear those kinds of words in English.

AU English in teaching, 'In English lessons, English should be the only language used for all purposes?' You 'Quite agree' with this. And 'In English lessons, lesson content can be presented in mixed-code Cantonese/English.' You 'Quite disagree' with that. So you would use only English within your English lessons?

S(97) I think I will, but if the students really don't understand my English, then improvement may use some Chinese for explaining some difficult vocabulary.

AU In fact, this question, \#11, and 'When students indicate that they don't understand.' You would also use Cantonese then?

S(97) But of course I will try my best to explain the difficult words in English first. I will use more examples to help them.

AU In fact during your practice teaching you said you always used only English in lessons. You didn't use any Cantonese at all?

S(97) I seldom used.

AU And 'How did the amount of pure English you used in lessons change?' 'It decreased'. So you were actually using more Cantonese?

S(97) I always use Cantonese when I have to handle the discipline problems because I have to handle the discipline problems first and if I still use English, they are out of control. So in that situation, I have to use Cantonese. Or the students will say, 'I don't know what you are talking about!' Then I can't do anything.

AU What sort of school did you teach at? 
S(97) It's a Band 5 school.

AU So to use only English is pretty good in a Band 5 school. 'To what extent do you see the use of English in secondary schools being affected by the changeover in sovereignty?' 'To some extent'. 'More subjects will be taught in Cantonese. The MOI will change to Cantonese eventually.' ... In your practice teaching, was your school officially English-medium?

$\mathrm{S}(97) \quad$ Yes, English-medium, it is supposed to be.

AU Did you find that most things were conducted in Cantonese?

S(97) I seldom observed other teacher's lessons, so I don't know what kind of medium the teachers use

AU And assemblies and notices, were they in English?

S(97) They were in Chinese.

AU So how can they call themselves English-medium?

S(97) The books that they use are in English.

AU Well I think most people would agree that for schools like that they should change to Chinese-medium officially. Instructional Planning and DecisionMaking next, 'It's impossible to have a successful lesson that hasn't been well-planned in advance.' You 'Quite agree'. Do you think that every lesson has to be well-planned?

S(97) Of course it is impossible to plan everything of the lesson in advance, but I think the teacher should think of the run-down of the lesson and prepare some materials for the lesson first.

AU Yes, and the next question, 'Teachers should write detailed lesson plans for every lesson they teach.' You 'Quite disagree' and say, 'A brief lesson plan is okay. 'But some lesson plan is needed?

S(97) Yes.

AU 'Teachers should be more responsive to student needs than the set syllabus.' 'Quite agree'. How much do you think you are able to deal with student needs in your teaching? In your practice teaching, were you able to?

S(97) Whenever I found that the students had problems, then I will stop my teaching, then I will re-teach that part first. Maybe I will change the method 
of teaching. If they really don't understand, then I have to give more examples, or I use another kind of teaching method.

AU 'In planning lessons, how detailed do you think the plans should be?" 'Quite detailed and quite flexible'. So you've gone for the middle option there. And 'During your practice teaching module, how often did you find you had to change your lesson plan?' 'Sometimes'. In what sort of situation would you have to change your lesson plan?

S(97) At first I don't know the standard of the students, so very often I will prepare more work for them, and they don't have enough time to finish that, so I always have to leave some work for the next day.

AU 'During your practice teaching module, on what basis did you plan your lessons?' Firstly, 'Syllabus', secondly, 'Regular teacher's instructions', third, 'Perceived needs of students'. So you were given a syllabus ...

S(97) What I have to teach.

AU Were you given much freedom in the way that you taught it?

S(97) I only have freedom to choose my teaching method and I have to teach certain items, certain language items. I was also asked to finish certain exercises.

AU 'During your practice teaching module, what were the factors which had the most influence on the decisions that you made?' 'Supervisor's advice' followed by 'Regular teacher's input'. So you say your supervisor was very influential?

S(97) Sometimes, because she always gave me some advice on the students' behaviour, and I had to take her advice in order to handle the situation, the bad situation.

AU Was it good advice?

S(97) Yes

AU 'To what extent do you think the changeover of sovereignty of HL will have an effect on your lesson-planning and decision-making?' 'To little extent'. 'Some meaning of difficult words may be explained in Cantonese.' What do you mean by that? You mean you may be able to use more Cantonese in the class?

S(97) I guess the school principal may allow us to use more Cantonese in our lessons. 
AU Because of the changeover?

S(97) Yes, especially for those Anglo-Chinese schools.

AU Teaching Approach, 'Examination requirements should determine what is taught in English lessons.' You 'Quite agree'. You think the examination requirements are the most important thing?

S(97) I think it's quite important in HK because the students are also quite examination-oriented. They may still think that learning English can help them to get a brighter future. They still take learning English as a subject.

AU 'Students learn English better through interaction with other students rather than through direct instruction from a teacher.' You 'Quite agree' and said, 'Students can have more practice when they do pair or group work.' And connected with this question, 'During your practice teaching module, did you find that students learn English better through interaction with other students or through direct instruction from a teacher?' Again you said, 'Through interaction with other students.' 'But this causes unexpected noises.' So although you found it was a better way of teaching, it created a lot of noise, right?

S(97) Yes, and it caused discipline problems, they are out of control, and I have to stop them, stop the activity.

AU Was it because they use it as an excuse to chat, or they get excited?

S(97) They get excited and they use that chance to chat with their classmates instead of practicing.

AU 'A class should be strictly controlled by the teacher and all lesson content directed by the teacher.' You 'Disagree' with this, 'Students can be guided to explicit the language items by themselves.' What do you mean by that?

S(97) I always use some activities to warm up the students at the very beginning of the lesson because I hope they can know what I am going to teach in this lesson. So I won't tell them, okay in this lesson I will teach you the present perfect tense. Instead of this I will use some activities first. I will let them play, I will let them talk, and then in order to get them to use this kind of tense.

AU And 'During your practice teaching module did you find that you controlled all your lessons strictly and directed all lesson content?' 'Sometimes', 'Because the students are very passive, they always waited for the lesson content provided by me.' So you found that your approach didn't always work? Because the students were very passive? ... 'Teachers should be 
prepared to experiment with teaching materials and strategies from a variety of sources.' 'Quite agree', then 'During your practice teaching did you experiment with teaching materials and strategies?' 'A little'. Only a little? Why was that?

S(97) Because I have to conduct many listening lessons and dictation lessons so I don't have enough lessons to do the practice in teaching grammar, so I don't have that kind of chance.

AU Why did you have to do so many listening lessons?

S(97) I just follow the syllabus of the school, and there must be two listening lessons in a week.

AU 'Would you describe your teaching style as learner-centered or teachercentered?' 'Mostly learner-centered'. And 'Which of the following factors has the most influence on your approach to the teaching of English?' You put first 'School, Principal's or ED policy', followed by 'Examination requirements' and 'Current trends in ESL teaching'. So you think school policy is the most influential, the most important?

S(97) Yes

AU 'The changeover will affect my approach to teaching.' You 'Quite disagree'. 'It won't affect too much in English lessons.'

S(97) And I think English is still an important language in HK nowadays. Instead I think maybe more English lessons will be taught in schools. They will increase the number of English lessons.

AU Why do you think that will happen?

S(97) Because in some schools, there will be a clear cut between English-medium and Chinese-medium, so for those Chinese-medium schools, they may have to increase more English lessons to compensate ... they may want to increase the chances for the students to speak up in English.

AU Professional Relationships and Responsibilities, 'How confident are you in your ability to become a successful teacher?' 'Quite confident'. ... 'How do you feel about the status of teachers in today's society?' 'Teachers have no special status'. Why do you think that is, and should they have a special status?

S(97) In the past the students would respect the teachers. They think that teachers are outstanding. But nowadays, when I taught in the practice teaching 
module, I don't think that the students respect me, they just take me as a friend.

AU Is that the normal thing now in HK? Maybe because you are still quite young?

$\mathrm{S}(97) \quad$ It is just a kind of career.

AU 'What do you regard as your best attribute in terms of being a teacher?' 'Good command of the language', 'Good communication skills'. 'Innovative in terms of teaching ideas'. So you don't think you would be very good at forming good professional relationships with colleagues? I just wonder why most people put this low on the list?

S(97) Maybe the other choices are more important than this one.

AU 'Do you see teaching as a long-term career?' 'Probably'. 'If you teach English in the future, who do you think will be most helpful to you during your first few years?' 'The panel chair'. I think you put first 'More experienced teachers' and then changed your mind.

S(97) I think both of them are also helpful.

AU And, 'During your practice teaching module, who was most helpful?' 'Teacher training supervisor'. 'Which of the following out-of-class activities do you think you will have to do as a first year teacher?' ... and of the same list which do you think will take up the most time? Mostly marking. 'If you teach English in the future, to what extent do you expect to be working closely with other teachers?' 'Some'. In your experience, do you find that teachers share ideas?

S(97) No, I think most teachers will share the work [tape ends] ... to prepare an examination paper, but they may not work together, they may work separately and combine different parts into a paper.

AU Teachers are very much individuals, they are quite happy to accept ideas, but whether they use them or not is another matter.

S(97) Some teachers may not like you to do so because it seems you think her work is not good enough.

AU 'If you teach English in the future, what do you think will help you most to develop as a teacher in your first year?' 'Designing new materials' you put first, 'Learning from other teachers'. And 'To what extent are you worried about your future as a teacher after the changeover of sovereignty?' 'To little 
extent'. 'Less English teachers will be employed. I'm afraid of being unemployed.' Do you think so?

S(97) I think less degree-holders will be employed as GM teachers.

AU 'Do you think your professional relationships and responsibilities will change after the changeover?' You said 'No'. Perceptions and Values, 'Do you think that you have a definite philosophy of teaching?' 'Quite definite', 'The objectives of a lesson should be quite clearly presented to a class. A variety of activities should be used to arouse students' interest and motivation to consider the learning pace of students.' To consider the learning pace of students, what do you mean by that?

S(97) That teachers should notice the understanding of the students. A teacher can't rush the lessons.

AU The previous question said that 'If you didn't answer 1 to this question, which areas are you unsure about?'

S(97) I think 'Working conditions'.

AU You mean the environment and that kind of thing?

S(97) The environment and the personality of my colleagues ... and the facilities of the school provided for me.

AU What do you think are the most important factors which affect what goes on in the classroom?' Most important 'Motivation of students', 'Language ability', 'Competence of teacher' ... 'Which of the following skills do you consider the most important to teach?' All of them. 'Which of the following best sums up your feelings as regards the roles or responsibilities a teacher should have in relation to his or her students?' You don't think the teacher should act like a parent?

S(97) No, the students will treat you like a friend.

AU The final question is 'Consider your strengths an weaknesses as a teacher.' Strongest being 'Language ability' and 'Communication skills' ... Similar to a previous question. ... So you think your language ability is quite strong?

S(97) No, I don't know why I put this item. I think I'm good at communicating with the colleagues and students.

AU Language ability is important, some schools look for near native like ability. 
S(97) But I think teaching skills is more important. Although the language ability of the teacher may be good, they may not know how to teach. They speak well; they write well, they don't have any teaching experience.

AU 'Are you planning to teach English next year?' 'Yes'. 'What factors did you consider?' 'Teaching environment and students' behaviour'. 
Interviewee:

Date:

Male, 25 years old.

Wanted to teach in a new school of he could as he felt that new schools would be less conservative and more open to innovation than older, more traditional schools.
AU Researcher
T(97) Interviewee
... Missing words / Unfinished utterance
Pause

AU Language Use to start with, you said that before you began the BATESL your written English was 'Fair' and now is still 'Fair'. Your spoken English was 'Rather poor' but is now 'Excellent'. So you think there has been not much improvement in your written English but a lot of improvement in your spoken English?

$\mathrm{T}$ (97) Yes, because the BATESL course provided me with internship, the practice teaching and the public speaking module, and also the various presentation and micro teaching and it provided many opportunities for me to practice speaking English. Much more opportunities.

AU More opportunities than to practice your writing?

$\mathrm{T}$ (97) Yes, much more than secondary school years because I remember in secondary school during F6 and F7, English lesson consisted just of listening and doing the written exercise, maybe the past paper for HKAL examination. So the most important point is that much more opportunities to polish and practice my speaking skills.

AU 'When speaking in Cantonese to friends who have Cantonese as their first language, do you sometimes use English words or expressions?' 'Quite often'. And also, do you switch from English and then back into Cantonese? Also 'Quite often'. In what sort of situations would you use English words or expressions?

$\mathrm{T}(97)$ In speaking Cantonese? Maybe this is a habit. Many Chinese people use the English word to substitute for the Chinese word, for example, 'interview'. In Chinese it is [ ] but nearly all the people say, 'interview' and seldom use the Chinese word because it is a trend for HK people to code-mix. It is a habit and because in HK English and Chinese are both maybe important, so they may use some English words when they are speaking in Chinese. 
AU And code-switching, when would you do that?

T(97) Maybe if I speak in Chinese, maybe the grammar is quite complicated in HK. There are some Chinese words are only used in HK, so if I speak to another person, I speak Chinese, maybe the grammar or the words they don't understand, so I speak English. Maybe the idea I present in Chinese is not very good, so I try to present it in English.

AU Now English in teaching, 'In English lessons, English should be the only language used for all purposes.' You 'Absolutely agree', you said that, 'More time to use the L2 prevent interlingual errors.' So you would use only English within English lessons?

T(97) Yes

AU And that will give them the maximum amount of exposure to the language?

$\mathrm{T}(97)$ Yes, the maximum amount of exposure is most important in an ESL classroom.

AU And 'In English lessons, lesson content can be presented in mixed-code.' 'Quite disagree', 'It will lead to interlingual errors.'

$\mathrm{T}(97) \quad$ I think it is because the student may misuse translation from Chinese to English. They may translate a whole Chinese sentence word by word into English, so it leads to some HK English, maybe Chinglish.

AU 'During your practice teaching, how much did you use only English in English lessons?' 'Always', and 'During your practice teaching, how much did the amount of English you used in English lessons change over the practice period?' 'It decreased'. So you said you used only English in English lessons, but that decreased during the practice time, was that because you were speaking less?

T(97) Yes, because the students didn't understand English very much, so if are presenting something wholly in English but the students they are fooling around, they don't understand. Maybe in their mind, English is just another planet.

AU So in that case you would use more Cantonese?

$\mathrm{T}(97) \quad$ Yes, I would use more Cantonese gradually.

AU And then in the next one, 'In what situations would or might you use Cantonese?' You actually said, 'No situation'. 
T(97) Because I use Cantonese to explain things as a last resort. If I speak English and the students don't understand, I use some gesture and some body movement or multimedia, maybe a tape recorder or the ... if they still don't understand. I may ask some bright student to explain. If still don't understand, I just use Chinese. I use Chinese as a last resort.

AU 'To what extent do you see the use of English being affected by the changeover in sovereignty?' You said, 'To a large extent' and then 'Official status of English decrease. The prestige of English will decrease. The use of Putonghua as a means or symbol of sovereignty. So you expect Putonghua to kind of take-over?

$T(97) \quad$ Yes

AU Okay, Instructional Planning and Decision-making, 'It's impossible to have a successful lesson which hasn't been well-planned in advance.' You didn't answer this one.

T(97) Maybe I'll answer it now ... This only apply to inexperienced teachers like us.

AU 'Especially for new teachers, they may not have the experience to predict students' responses and reactions.' You 'Absolutely disagree' that it is impossible to have a successful lesson that hasn't been well-planned in advance. In other words you agree that it is possible to have a successful language that hasn't been well-planned in advance. 'Teachers should write detailed lesson plans for every lesson they teach.' 'Quite disagree', again, 'Since teachers gain experience, lesson plan is less useful when the teachers familiarize the lesson.' So in other words, once a teacher has prepared and given a lesson, they have no need to re-plan the lesson.

T(97) Yes, I think so. I think these two statements only apply to new teachers or inexperienced teachers.

AU 'Teachers should be more responsive to student needs than the set syllabus.' 'Quite agree'. 'When planning lessons, how detailed do you think the plans should be?' 'Quite detailed and rigid'. So you are a believer in having a fixed lesson plan?

$T$ (97) Yes, for new teachers. In my experience it's like that, to create a detailed and rigid lesson plan.

AU 'During your practice teaching module, how often did you find you had to change your lesson plan?' 'Always'. Why was that? 
$\mathrm{T}(97)$ Because maybe I over-estimate the students' ability. Maybe too difficult to them, so I need to change much for the lesson plan and the pre-set teaching material.

AU Was that while the lesson as going on, you need to change?

$\mathrm{T}(97) \quad$ Yes, while the lesson was going on, I need to change the ... if I give the students 10 questions, I need them to answer easier questions only. Maybe three questions out of ten questions is too easy for them. They do just seven questions, which is easier.

AU 'During your practice teaching, on what basis did you plan your lessons?' 'BATESL course guidelines' followed by 'Regular teachers' instructions' and 'Textbook teacher's instructions'. How much freedom were you given by the school you were in to teach what you wanted to teach?

$\mathrm{T}(97) \quad$ Not much freedom because I have to finish the exam syllabus because the past teaching period is just before the examination, so the time was quite tight. So I had to finish what the teacher assigned me, the school teacher.

AU I see, it's probably not a good time to have the practice teaching.

$T$ (97) Yes, because there's no time to do additional games or activities. If the syllabus is not finished, they may have difficulty to face the English examination, which is just after the practice teaching period.

AU And 'During your practice teaching, what were the factors which had the most influence over the decisions you made?' 'Supervisor's advice', followed by 'Regular teacher's input' and 'Students' needs'. So again your supervisor was very influential?

$T$ (97) Yes, because she assessed me. If I don't follow her instructions, maybe she would reduce my marks. Generally speaking there's not much freedom during practice teaching, I have to obey the instructions of the cooperating teacher and the tutor of the university.

AU The next question was 'To what extent do you think the changeover will have an effect on your lesson planning and decision-making?' You said ' To little extent'. 'If the English syllabus is not changed, it will not affect my lesson planning.'

$\mathrm{T}(97)$ I chose \# 2 for this question for higher form students. I quite agree if the students who will sit for the public examination such as HKCEE or HKAL. But for junior form students, I chose \#3. 
AU Right, 'Examination requirements should determine what is taught ...' And 'Students learn English better through interaction with other students rather than through direct instruction from the teacher.' You 'absolutely agree' with this. 'Students learn things not only from teachers but also from classmates.' This ties in with Q26, 'During your practice teaching module, did you find that students learn English better through interaction with other students or through direct instruction from the teacher?' And you found that students did learn better through interaction with other students.'

T(97) Yes, for example, if the student exchange the composition each other, they may learn something about the writing style, grammar or even the vocabulary.

AU Did you have much opportunity to allow the students to learn through interaction?

$T(97) \quad$ Yes

AU A class should be strictly controlled by the teacher and all lesson content directed by the teacher.' You 'Absolutely disagree' with this saying that 'Students may feel bored.'

$\mathrm{T}(97) \quad$ Yes, and this may lead to some discipline problems.

AU And in fact 'During your practice teaching module, did you find that you controlled your lessons strictly and directed all lesson content?' You said, 'Always'. 'Because of class discipline problems.'

$T(97)$ Because in my teaching schools, at first I give them many games or activities, which are in small groups, and they are too happy and they create many noises. Maybe they like the activities too much and they play happily and forget the classroom discipline.

AU Yes it is quite difficult, especially with such large classes.

$T$ (97) Yes, to form a class into ten groups, each group with four students, it is difficult to manage. The class size is too big.

AU 'A teacher should be prepared to experiment with teaching materials and strategies from a variety of sources in his or her teaching.' You 'Absolutely agree' with this, which I guess is the philosophy of the BATESL course, and then Q28, 'During your practice teaching module did you experiment with teaching materials and strategies from a variety of sources?' You said, 'A little'. 
$T$ (97) Yes, because of the time constraints. After finish the syllabus for the first exam of F1 students.

AU 'Would you describe your teaching style as learner-centered or teachercentered?' 'Very teacher-centered'. 'Which of the following factors has the most influence on your approach to teaching?' First you put 'Noise in classroom', followed by 'Motivation of students' and 'School, principal or ED policy'.

T(97) Yes, because if the classroom is too noisy, the teacher in the next classroom may complain. Also, it may disturb the other students in other classes.

AU And the last question in this section, 'The changeover in sovereignty will affect my approach to teaching. 'Absolutely disagree'.

$\mathrm{T}(97)$ It's still the English lesson. It's important as HK is still the international city. The govt. hasn't told us that there's no English lesson, the nature of English should be demoted. They haven't said that.

AU The next section is Professional Relationships and Responsibilities, 'How confident are you in your ability to become a successful teacher?' 'Quite confident', and 'How do you feel about the status of teachers in todays' society?' 'Teachers have a lower status than they should.' Why do you think so?

$T(97) \quad B e c a u s e$ of the culture of the society. Because some people may think that the customer is always right. This theory may be applied to schools, that means the students are always right, they complain everything. The teaching method is boring, they punish the students etc. And the emergence of some private remedial classes outside the school. Such kind of teachers in private institute may like a superstar because they provide many tips, many internal tips, maybe some secret tips about the examination, and they can predict the questions of the public examinations. The students may think such kind of teachers is like a superstar, is superior, because the teacher in ordinary secondary school cannot do this. They are unable to predict the questions in public examinations.

AU So in fact some of these private tuition schools are actually gaining a higher reputation a than some secondary schools?

$T(97)$ Yes, higher than secondary schools, and this is not a good phenomenon.

AU 'What do you regard as being your best attribute in terms of being a teacher?' You said 'Good command of the language', 'Innovative in terms of teaching ideas', 'Strict disciplinarian'. So you think language is your best attribute? 
$\mathrm{T}(97) \quad$ Yes.

AU And lowest on the list is 'Able to form good professional relationships with colleagues'. So you think that is not so important?

$\mathrm{T}$ (97) Yes, in a school is different from in universities and colleges because in my opinion in universities or colleges is a little bit theoretical, but it is different in secondary school because they are all practical.

AU So it's difficult to form relationships? 'Do you see teaching as a long-term career?' 'Definitely'. And 'If you teach English in the future, who do you think will be most helpful to you?' 'More experienced teachers'. How does that link with the previous question about forming good relationships with colleagues? And 'During your practice teaching module, who was most helpful to you?' 'Panel chair'.

$T$ (97) That's because the panel chair has much more practical experience.

AU 'Which of the following out-of-class activities do you think you will have to do as a first year teacher?' ...

$\mathrm{T}(97)$ I think it depends on the culture of the schools. Some schools may pay more attention to the extra-curricular activities because in my secondary school, the principal is paying much more attention on activities, rather than regular classes.

AU And 'Of the same list, which of the following do you think will take up the most time?' 'Being a form teacher'.

$\mathrm{T}$ (97) Because a form teacher have to do many administration job of a class, such as the parents day, you meet 40 parents, and some of the parents are quite troublesome. If your class has many behaviour problem, you have many follow-up tasks.

AU 'If you teach English in the future, to what extent do you expect to be working closely with other teachers?' 'Very much'.

$\mathrm{T}(97) \quad$ Yes, teaching can be a team-work.

AU 'If you teach English in the future, what do you think will help you most to develop as a teacher in your first year?' You put 'Learning from other teachers', 'Getting feedback from panel chair' and 'Attending conferences'.

$\mathrm{T}(97)$ Getting feedback from the students is also useful, I doing it in practice teaching. 
AU Yes, and 'To what extent do you worry about your future as a teacher after the changeover?' 'To a large extent' 'Prestige of English will be increased, and more immigrants from Mainland China.'

$\mathrm{T}(97) \quad Y e s$, because most immigrants from Mainland China, they are unable to speak or write English, totally maybe illiterate in English. This creates some problems to the teachers.

$\mathrm{AU} \quad$ In that case, won't there be a need for more English teachers?

T(97) Yes, I really agree with you.

AU And 'Do you think your professional relationships and responsibilities will change after the changeover?' 'Somewhat'.

$\mathrm{T}(97) \quad Y e s$, because in the past, people may think that if you have English literacy, you have a high social status, but I think now this isn't totally valid.

AU Okay, Perceptions and Values next, 'Do you think you have a definite philosophy of teaching?' 'Not quite definite'. In fact you put, 'Studentcentered learning' as your teaching philosophy. And the previous question, any areas you are not sure about, you didn't circle any, so you are fairly confident about those areas.

$\mathrm{T}(97) \quad$ Yes.

AU Anything else you want to say about student-centered learning?

$\mathrm{T}(97)$ I think the students not only learn from teachers but from other classmates, and their motivation may be much higher to learn from other students, rather than teacher-student relationship. They may gain more confidence and they may not feel bored.

AU 'What do you think are the most important factors which affect what goes on in the classroom?' 'Motivation of students' being 'Most important' and 'Language ability of students' being 'Quite important'.

$\mathrm{T}(97) \quad$ Yes, the mot important point, the motivation of students and the language ability of students.

AU 'Which of the following skills do you consider the most important to teach?' You put all important.

$\mathrm{T}(97) \quad$ Yes, it is a balance, it creates a balance. 
AU 'Which of the following best sums up the roles and relationships a teacher should have with his or her students?' ...

T(97) Yes, I think teachers should try to become friends with the students because if the teacher have a god relationship with the students, it may facilitate the teaching process, and the students may be more motivated.

AU And finally, 'Consider your strengths and weaknesses as a teacher ...' Strongest being 'Language ability', 'Discipline' and 'Organisation'. So you think that the BATESL has been successful in bringing up your language ability to the required level?

$T$ (97) Because my English foundation is not very good, so I think if the student have got a $\mathrm{C}$ in the HKAL English, or HKCEE English, it is okay, but I only got a D. It is quite difficult to follow the classes, and lectures and tutorials. Because I think in BATESL, the students' English proficiency is not very good, at least one third of the class.

AU 'Do you plan to teach English next year?' 'Yes' 'If there's a vacancy I'll sure go to teaching.' 'If you decide to teach, where and in what type of school?' 'A newly established school.' Why have you said that?

$\mathrm{T}(97) \quad B e c a u s e$ in a newly-established school, most principals or panel chairs may not be so conservative, like some well-known secondary schools. They are more open and have much more room for me to explore the teaching approach.

AU And if you don't get a job by September, what will you do?

$\mathrm{T}$ (97) Maybe continue my study and teach part-time in a tuition school, then I can still gain some experience. 
Female, 24 years old.

Was hopeful of finding a teaching position in a Band Two or Three school near her home. Was working in a hotel at the time and would continue to do so if she could not find a teaching post.

$\begin{array}{ll}\text { AU } & \text { Researcher } \\ \text { U(97) } & \text { Interviewee } \\ \ldots & \text { Missing words / Unfinished utterance } \\ \text { - } & \text { Pause }\end{array}$

AU Language Use first of all, 'How would you describe the standard of your written English before you began the BATESL?' You said 'Fair', and '... the standard of your written English now?' Still 'Fair'. Spoken English was 'Very good' and is now still 'Very good'. So you think you haven't improved any?

U(97) Well because I don't have a similar standard to compare once before and once after my degree, so to be humble, I think I have to keep on my standard. Originally I want to say that my spoken English is excellent but I still find some grammatical mistakes when I'm speaking or when I'm not thinking seriously before I speak out, so I think 'Very good' is much better.

AU 'When speaking Cantonese to friends who have Cantonese as their first language, do you use some English words or expressions?' 'Quite often', and 'When speaking to friends ... do you switch into English and then back into Cantonese?' This time you said 'Frequently'. In what sort of situations would you use English words or expressions?

U(97) Actually it depends, if I found that my friends do not understand my English wordings, I will code-switch back into Cantonese immediately. However, sometimes I can't remember what the Cantonese meaning of that word, or I find that English is more appropriate, then I will keep on using English words.

AU And code-switching? In what sort of situations would you code-switch into English?

U(97) When I want to keep something secret, I will code-switch into English. Like when I talk to my little brother at home, I want to hidden something from my mum, I will talk to him in English. 
AU You are not the first person to give that reason. English in teaching, 'In English lessons, English should be the only language used for all purposes.' You 'Absolutely agree' with that. So you wouldn't use any Cantonese at all?

U(97) No, even when you are explaining vocabulary, you may use simpler words to substitute it. If you try to use Cantonese to explain the words, your students will force you to use Cantonese next time, and then you cannot keep on your English. Actually it's not good to them, so I say absolutely for all purpose.

AU I think that's true. You may be able to save time by telling them in Cantonese, but then they may begin to expect that and will not try to understand in English.

U(97) Maybe you can give more example. Maybe the students have a brief idea, they think it may like that, it may mean that, but they don't come from themselves, then you may give more examples so that they can confirm and you can confirm.

AU And 'In English lessons, lesson content can be presented in a mixed-code Cantonese-English.' You 'Quite disagree'.

$\mathrm{U}(97) \quad$ The reason is more or less the same as \#7.

AU 'During your practice teaching module, how much did you use only English in English lessons?' 'Always'.

U(97) Yes, actually no Cantonese at all because I'm quite lucky be allocated in a Band 1 school with the Band 1 students there, so they fully understand my English, and my cooperating teacher there asked me not to use Cantonese, because that's prohibited in the classroom. If they want to explain themselves, they have to use their language, in English, simple English, so that they can practice and they can know that even if they use simple English, they can express themselves. I think it's good to have that practice. I have been there when I was internship as well, they were F1 at that time. This year they were F2. That means I notice that they really improved their English, they don't feel embarrassed when they speak English. They are strong enough to stand in front of the class and have a presentation in English. So I believe that training is very important, since they were in F1, so I agree that use English is the best way. If you want to improve English, use English is the best way.

AU It might not be as easy in a Band 5 school, for example.

U(97) Then we have to use another way. First of all, at least up to my opinion, we may build up their interest. If they don't like English, you cannot talk to them, even a word or a letter. So build up their interest first. But how to build 
up their interest is another thing. For junior form students it's easier, you can use cartoons or pictures, it's easier. For senior students, they may find it's stupid, silly, and they concentrate on the syllabus for the public exam. Teachers may push them to make more practice on the examination, but that's what the HK examination is.

AU 'During your practice teaching, how much did the amount of pure English you use change?' 'It didn't change', so you used pure English all the time?

U(97) Yes

AU 'In what situations in an English lesson would or might you use Cantonese?' 'To explain vocabulary', 'To explain when students indicate that they don't understand'. That would be as a last resort?

U(97) Yes

AU And 'To what extent do you see the use of English in schools being affected by the changeover in sovereignty?' 'To some extent', and what changes you expect, 'Little English will be used, or it will not be praised as seriously as before.'

U(97) I mean when we were the British colony, when we use English, or when we have the ability to speak fluent English, the others will think you are welleducated. Although that may be one of the motivation for students to learn, if they want to stick to a higher social class, later, after the changeover, to me I think it will not be emphasised as before. Just like the legislative meeting, they use Cantonese. Of course the legislators can still use English, but that is not expected, and they are supposed not to use English anymore. And beforehand, English was the first version of law to follow, and afterward, we have to follow the Chinese version. So students may think that Chinese or Putonghua is more important later, and they have lost their interest, the motivation, the initiation to learn English. And I noticed from my job, some of my juniors are just F5 graduated and they ask me, why do I think English is so important, and I just tell the same thing, and they simply tell me, no, we are now 1997, we have to emphasise Chinese more. Then I will be stuck, I have nothing to say.

AU Especially in the hotel business, where you have to deal with foreign tourists

U(97) Because relatively, they don't have the ability to manage their English, they can manage Putonghua much better. It may be because they are the same language. But we still have foreign guests, so I ask them to improve more, but I can't get any improvement. 
AU Okay, Instructional Planning and Decision-Making, ' It's impossible to have a successful lesson that hasn't been well-planned in advance.' You 'Quite disagree'.

U(97) We should be flexible. Something you can't expected, though you have a very detailed lesson plan but there may be something you can't expected. Maybe one day there is a rainstorm warning and the students is going to leave as soon as possible, then how can you stop your lesson? How can you revise the lesson before they go? So we should have some flexibilities.

AU 'Teachers should write detailed lesson plans for every lesson they teach.' You 'Disagree'.

U(97) More or less the same. Teachers should write lesson plan, but not a very detailed one and we should allow flexibilities. If it is too detailed, you may lose yourself once when it cannot be followed.

AU And most teachers don't have time to write detailed lesson plans.

U(97) Maybe for the first year.

AU 'Teachers should be more responsive to student needs than the set syllabus.' You 'Absolutely agree'.

U(97) Yes

AU But is that possible?

U(97) For the junior form students, yes, it's possible. But for the senior form students, their needs is according to the syllabus. When you are responsible to their needs, actually you are responsible to the syllabus. Unlike the senior form students, the junior form students may want to have some interesting lessons so that they can pay attention, so I think to make them more like your lesson, you should be more responsible to their needs instead. But not for senior form students.

AU 'When planning lessons, how detailed do you think the plans should be?' 'Brief and very flexible'. And 'During your practice teaching module, how often did you find you had to change your lesson plan?' 'Usually'.

U(97) I can't say I did not make appropriate lesson plan, but as what I said before, the response is not expected as I planned so you have to change to fit them, so they still find my lesson attractive. So that's why I usually change my lesson plan. 
AU 'During your practice teaching module, on what basis did you plan your lessons?' 'Perceived needs of students' first, followed by 'Regular teacher's instructions' and then 'Syllabus'. Did you have a lot of freedom to teach what you wanted?

U(97) Freedom, yes, actually they are familiar with their teachers, and I follow her advice, and then I basically get the same response from the students. Especially later in the practice, when we become friendly with the others. So it's quite good. How can I get their needs? Because some of the students like to talk to me after class, so I know what they know, and I just ask them, do you find it interesting? Do you find it boring? Or, what kind of things do you want in my English lesson? Then they will simply tell you, I need this, or I need that. So I can change it according to what they want. And that's a very good school, when the students are not in the classroom, they talk to you in English. That's excellent.

AU 'During your practice teaching module, what were the factors which had the most influence on the decisions you made?' 'Student needs' followed by 'Regular teacher's input', 'Fellow student-teachers' advice'. 'To what extent do you feel the changeover of sovereignty will have an effect on your lessonplanning and decision-making?' 'To some extent'.

U(97) As I mentioned before, if the students do not find English as important as before, I may need to teach them some additional and practical English. In our generation we may spend some time on the literature, and may know how to appreciate the literature, but they may need to know some practical use, like how to write an application letter, or how to write a resume.

AU Next, Teaching Approach, 'Examination requirements should determine what is taught in English lessons.' You 'Quite disagree', 'Students need to learn much more than those required by the examination.'

U(97) That's it. The most difficult form is F4 and 5, because I think when they are promoted to F6, they may know how to manage their time. The teacher may spend some time on different English to them. But F4 and 5 students, they are so focused on the examination result, they don't have any spare time to what you said, even you tell them this is to equip yourself, so that you understand English better, they will simply tell you it is not included in the syllabus.

AU I taught mostly F6 and 7, and although you have to teach them the skills they need for the exam, you try to make it interesting for them, teach them about current affairs and the outside world.

U(97) Or maybe we can integrate the techniques into the world-wide information, like when you teach them how to make use of the scan and skim skills, we 
can give them an article, maybe talking about the ozone layer, talking about the flooding, something like that.

AU 'Students learn English better through interaction with other students rather than through direct instruction from a teacher.' You 'Quite agree'.

U(97) I prefer a controlled classroom, it's time-saving. It's more efficient.

AU Linked to that is 'During your practice teaching module, did you find that students learn English better through interaction with other students rather than through direct instruction?' And you said, No difference between the two.' Was that because you didn't really have much time to assess the ...

U(97) That's one of the reasons. And actually they are very initiative, and they are familiar with the grouping. When you ask them to group yourself, then they know in which situation is a pair, and which situation is a group of four. So, no difference to me.

AU And related to this idea of control, 'A class should be strictly controlled by the teacher and all lesson content directed by the teacher.' You 'Quite disagree' with this. 'Teacher-student interaction is recommended by my pupils.'

U(97) Because when you need to know the students' names, you need interaction. If no interaction, how can you know what their needs are? So if the class is strictly controlled by the teacher, it's a single way to teach. We now emphasise two ways, so I think interaction is important.

AU And connected with that one is, 'During your practice teaching, did you find that you controlled your lessons strictly and directed all lesson content?' 'Usually'. On the one hand you said that you like to control the lesson, but on the other you said that they need student-student interaction.

U(97) They have, they have student-student interaction. They have interaction, and when they are having the discussion I will walk around and notice what they are doing. So I wonder if this is teacher-student interaction. If it is, then I have. If it's not then I haven't.

AU What I meant by strictly controlled is the teacher-centered classroom where the teacher does all the talking and asks the students questions.

U(97) Then I may circle 'Sometimes'.

AU 'A teacher should be prepared to experiment with teaching materials and strategies from a variety of sources in his or her teaching. ' Absolutely agree'. Then, 'During your practice teaching module, did you experiment with teaching materials and strategies from a variety of sources.' 'Somewhat'. So 
you agree that teachers should, and you were able to to a certain extent during your practice teaching?

U(97) Yes, actually I got a lot of support from the cooperating teacher. When I need some materials, and I ask her, how can I got that, she gave me the advice in the case where I an make it myself, or she make it for me. So I got a lot of support from her. When I need some materials, I never worry about it's sources.

AU So you were given a certain part of the syllabus to cover?

U(97) No, I had total freedom.

AU 'Would you describe your teaching style as mostly learner-centered or teacher-centered?' 'Mostly teacher-centered'.

U(97) Actually it depends on the banding of school and my students. If it's like my students in the practice teaching school, I may give them more chance to be learner-centered because they can control themselves, they can help themselves or help the others. But if they are in like Band 4 or 5 schools, they need more help from teachers. So it depends, but to my own style, I really prefer teacher-centered. It will be more efficient, I still think that.

AU 'Which of the following factors do you think has the most influence on your approach to teaching?' 'English proficiency of students' followed by 'Class size' and then 'Noise in classroom'. The interesting thing is class size ...

U(97) It's horrible, when you've got a small size classroom and it's full of students, each with a table and a chair and you can imagine how horrible it is. You can stand there all around the room there are students, and school bags, no more. Just students, even 45 of them.

AU Actually lowest you put 'Personality of teacher' and 'Current trends in ESL teaching'. Why did you put 'Personality of teacher' so low?

U(97) Because you cannot change the personality. Personality cannot be changed easily, so I may say, I'd rather ignore it.

AU It was ranked quite high by most people.

U(97) Logically it should be.

AU And 'Noise in classroom' was ranked lowest but you ranked it so high. 
U(97) If they make so much noise, I don't think I can give them instruction. Then I don't think I can tell them what to do or even teach them. So noise is very important, and that's why I can give them instruction because they are quiet.

AU And do you agree with the following statement, 'The changeover in sovereignty will affect my approach to teaching.' You 'Quite disagree'. You don't think it will have any affect?

U(97) No, actually, some of the question and reason are the same. My approach my emphasise on the use of English, not the appreciation of English, even for senior form students.

AU Professional Relationships and Responsibilities, 'How confident are toy in your ability to become a successful teacher?' 'Quite confident'.

U(97) Yes, if I can be employed. Yes, I really believe that. At least if I can be a good teacher, if I can have a good inter-relation with my students, I can be very instructive and informative to my students. But I have no chance to explain to anyone of the principals.

AU 'How do you feel about the status of teachers in today's society?' 'Teachers have a lower status than they should'.

U(97) Yes, I think so, because when I was a student in senior form or junior form or even primary schools, I really respect my teacher very much. I think it's necessary to respect my teacher, but I can't find this respect from nowaday's students. They just think that teachers receive the salary from schools, and they paid for the school fee, and actually teachers are working for the salary and working for them. So teachers should be so good, only because of the salary, not because of the teacher.

AU 'What do you regard as being your best attribute in terms of being a teacher?' 'Good command of the language', 'Good communication skills', 'Empathy with students'. Last was 'Able to form good professional relationships with colleagues'. Why did you put this last?

U(97) Actually we should be cooperative with other teachers, we still have a chance to work together or to share the ideas, but as you know, personalities cannot be changed, and it's not easy to get along with the others. So if we cannot get along with the others, what should we do? We have to depend fully on ourselves. So I don't think it can be ranked in a higher priority, so I put it as the lowest.

AU 'Do you see teaching as a long-term career?' 'Probably'.

U(97) Yes, if I can be employed. 
AU 'If you teach English in the future, who do you think will be most helpful to you during your first few years?' 'Panel chair'.

U(97) Yes, maybe I can get immediate help from the experienced teacher, but if the panel chair doesn't understand you or doesn't accept your weakness, I think it will be quite harsh. And I believe that the panel chair should be the most experienced one, so he or she can give me the most help.

AU 'During your practice teaching module, who was most helpful to you?' 'More experienced teachers'.

U(97) Actually, I have one cooperating teacher and one panel chair, and my cooperating teacher is the more experienced teacher, and another one is the panel chair. She also gave me a lot of help. She has a lot to do, she taught F4, 5,6 and 7. She can give you a lot of ideas, but how to develop is up to me. So once when I get an idea, I ask my cooperating teacher and then she help me to integrate the ideas together, and help me how to present in the classroom. So most of the help is from her, from my cooperating teacher.

AU 'Which of the following out-of-class activities do you think you will have to do as a first-year teacher?' ... You didn't put 'Mark papers', by mark papers that was meant to be just marking generally.

U(97) Because when I'm taking [tape ends] ... when you mark books.

AU And ' ... of the same list which do you think will take up the most time?' You put 'Preparing lessons'. 'If you teach English in the future, to what extent do you expect to be working closely with other teachers?' 'Very much'.

U(97) If they are willing to share with you. Especially Cantonese, I can tell you Chinese is to a certain extent very selfish.

AU 'If you teach English in the future, what do you think will help you most to develop as a teacher in your first year?' You put 'Learning from other teachers' and getting feedback from the panel chair'.

U(97) Because they have more experience than me.

AU To what extent do you worry about your future as a teacher after the changeover of sovereignty?' 'To no extent'. 'Do you think your professional relationships or responsibilities will change after the changeover?' 'No'.

U(97) I still have to teach. I still have to teach English. I don't think the profession will be changed after the changeover of sovereignty. 
AU Perceptions and Values, 'Do you think that you have a definite philosophy of teaching?' 'Quite definite', and this is it, Not necessarily be the best teacher in the school, but I must be responsible to all my students.'

U(97) That's the basic criteria, I think.

AU 'Which areas are you unsure about?' You didn't circle any, so you are not unsure about anything?

U(97) Maybe I missed it, let's see, \#4, 6, 8, because I can't control them.

AU 'What do you think are the most important factors which affect what goes on in the classroom?' 'Most important' being 'ED/school policy', 'Exams Authority' and 'Govt.'.

U(97) They design the syllabus and the teachers follow the syllabus, so they are the most important factors.

AU 'Which of the following skills do you consider most important to teach?' 'Very important' 'Reading', 'Speaking', 'Listening' and 'Grammar'; 'Quite important' 'Writing' and 'Pronunciation'. Why would you say writing is a little less important than the others?

$\mathrm{U}(97)$ Writing is quite objective, I should say. Maybe my criteria to the writing is correct grammar, rich in content, and logical flow of the mind, that's enough. If they want to make it beautiful, that's okay. And for the pronunciation I notice that, of course if you can give a very good pronunciation, native-like speaker, that's great, but some of the pronunciation is very difficult for Chinese to make. I can't quote examples, but I notice that it did happen, it does exist, so pronunciation is a bit difficult to control. So I allow some flexibility there.

AU 'Which of the following best sums up your feelings as regards the roles or behaviours a teacher should have towards his or her students?' ... The only one you've missed out really, 'The teacher should be in total control of all lessons at all times'.

U(97) No, you can't totally control. Maybe you want to but you can't.

AU Teachers should act like a parent ...

U(97) It's not easy, maybe the teacher can have the ambitions to act like their parent, but once there is any legal responsibility, you can't say, oh and the student said, my teacher teach me in this way, teach me in that way, the parent just tell them, he or she is only a teacher to teach you knowledge, not really like a parent. 
AU And the teacher should refrain from punishing students?

U(97) Sometimes you have to punish them.

AU Finally, 'Consider your strengths and weaknesses as a teacher.' The 'Strongest', you put 'Teaching speaking', 'Teaching grammar', 'Teaching pronunciation'.

$\mathrm{U}(97) \quad$ Yes

AU And 'Very strong', 'Voice' and 'Teaching listening'.

U(97) Actually I believe this. I don't know why I have such idea. I just notice that my written ability is a bit different from my speaking ability.

AU The last page is about your plans. 'Are you planning to teach English next year?' 'Yes'. 'What factors did you consider, 'Stability of income and the attractiveness of holidays offered.' 'If you do decide to teach, in what kind of school would you like to teach?' 'Band Two/Three school near my home.' And 'If you don't, what might you do instead?' 'Join the hotel field.'

U(97) What I'm doing now. 


\section{B.4 Statistical Results of Questionnaire Surveys}

Kruskal-Wallace $H$ Test

Mann-Whitney $U$ Test

Chi-square Test

Spearman's rho Correlation

Significance
K-W

$\mathrm{M}-\mathrm{W}$

Chi-sq.

Corr.

Sig.

\section{Language Use}

Table 4.1: Self-perceived ability in written English

\begin{tabular}{|l|c|c|c|c|c|c|c|}
\hline & Excellent & $\begin{array}{c}\text { Very } \\
\text { Good } \\
2\end{array}$ & $\begin{array}{c}\text { Quite } \\
\text { Good } \\
3\end{array}$ & $\begin{array}{c}\text { Fair } \\
4\end{array}$ & $\begin{array}{c}\text { Rather } \\
\text { Poor } \\
5\end{array}$ & Mean & SD \\
\hline & & & & & & & \\
\hline $\mathbf{9 4 ( 3 )}$ & 0 & 14 & 28 & 6 & 0 & 2.83 & 0.63 \\
\hline $\mathbf{9 4 ( 1 )}$ & 0 & 4 & 18 & 16 & 2 & 3.40 & 0.73 \\
\hline $\mathbf{9 7 ( 3 )}$ & 0 & 8 & 16 & 6 & 0 & 2.93 & 0.69 \\
\hline & & & & & & & \\
\hline
\end{tabular}

\begin{tabular}{|c|c|c|c|c|c|c|}
\hline & Test & 3 groups & $\begin{array}{l}\text { Post- } \\
\text { hoc test }\end{array}$ & $\begin{array}{c}94(1) \\
v \\
97(3)\end{array}$ & $\begin{array}{c}94(3) \\
v \\
97(3) \\
\end{array}$ & $\begin{array}{c}94(1) \\
v \\
94(3) \\
\end{array}$ \\
\hline \multirow{2}{*}{$\begin{array}{l}\text { English writing } \\
\text { ability }\end{array}$} & $\mathrm{K}-\mathrm{W}$ & 13.65 & $M-W$ & 6.37 & 0.387 & 12.69 \\
\hline & Sig. & 0.001 & Sig. & 0.012 & 0.534 & 0.000 \\
\hline
\end{tabular}


Table 4.2: Self-perceived ability in spoken English

\begin{tabular}{|c|c|c|c|c|c|c|c|}
\hline & Excellent & $\begin{array}{c}\text { Very } \\
\text { Good } \\
2\end{array}$ & $\begin{array}{c}\text { Quite } \\
\text { Good } \\
3\end{array}$ & Fair & $\begin{array}{c}\text { Rather } \\
\text { Poor } \\
5\end{array}$ & Mean & SD \\
\hline & & & & & & & \\
\hline $\mathbf{9 4 ( 3 )}$ & 0 & 9 & 21 & 18 & 0 & 3.19 & 0.73 \\
\hline $\mathbf{9 4 ( 1 )}$ & 0 & 3 & 11 & 22 & 4 & 3.68 & 0.76 \\
\hline $\mathbf{9 7 ( 3 )}$ & 1 & 9 & 15 & 5 & 0 & 2.80 & 0.76 \\
\hline & & & & & & & \\
\hline
\end{tabular}

\begin{tabular}{|c|c|c|c|c|c|c|}
\hline & Test & 3 groups & $\begin{array}{l}\text { Post- } \\
\text { hoc test }\end{array}$ & $\begin{array}{c}94(1) \\
v \\
97(3) \\
\end{array}$ & $\begin{array}{c}94(3) \\
v \\
97(3)\end{array}$ & $\begin{array}{c}94(1) \\
v \\
94(3)\end{array}$ \\
\hline \multirow{2}{*}{$\begin{array}{l}\text { English speaking } \\
\text { ability }\end{array}$} & $\mathrm{K}-\mathrm{W}$ & 19.69 & $\mathrm{M}-\mathrm{W}$ & 17.73 & 4.501 & 8.301 \\
\hline & Sig. & 0.000 & Sig. & 0.000 & 0.034 & 0.004 \\
\hline
\end{tabular}

Table 4.3: Admitted use of code-mixing with Cantonese-speaking friends

\begin{tabular}{|c|c|c|c|c|c|c|c|}
\hline & $\begin{array}{c}\text { Always } \\
1 \\
\end{array}$ & $\begin{array}{c}\text { Frequently } \\
2 \\
\end{array}$ & $\begin{array}{c}\text { Quite } \\
\text { often } \\
3 \\
\end{array}$ & $\begin{array}{c}\text { Rarely } \\
4 \\
\end{array}$ & $\begin{array}{c}\text { Never } \\
5 \\
\end{array}$ & Mean & SD \\
\hline $94(3)$ & 12 & 17 & 15 & 3 & 1 & 2.25 & 0.98 \\
\hline $94(1)$ & 5 & 2 & 22 & 10 & 1 & 3.00 & 0.96 \\
\hline $97(3)$ & 6 & 6 & 16 & 2 & 0 & 2.47 & 0.90 \\
\hline
\end{tabular}

\begin{tabular}{|c|c|c|c|c|c|c|}
\hline & Test & 3 groups & $\begin{array}{l}\text { Post- } \\
\text { hoc test }\end{array}$ & $\begin{array}{c}94(1) \\
\mathbf{v} \\
97(3)\end{array}$ & $\begin{array}{c}94(3) \\
v \\
97(3) \\
\end{array}$ & $\begin{array}{c}94(1) \\
v \\
94(3)\end{array}$ \\
\hline \multirow{2}{*}{$\begin{array}{l}\text { Code-mixing with } \\
\text { friends }\end{array}$} & $\mathrm{K}-\mathrm{W}$ & 14.74 & M-W & 6.323 & 1.525 & 13.46 \\
\hline & Sig. & 0.001 & Sig. & 0.012 & 0.217 & 0.000 \\
\hline
\end{tabular}


Table 4.4: Admitted use of code-switching with Cantonese-speaking friends

\begin{tabular}{|c|c|c|c|c|c|c|c|}
\hline & $\begin{array}{c}\text { Always } \\
1\end{array}$ & $\begin{array}{c}\text { Frequently } \\
2\end{array}$ & $\begin{array}{l}\text { Quite } \\
\text { often } \\
3\end{array}$ & $\begin{array}{c}\text { Rarely } \\
4\end{array}$ & $\begin{array}{c}\text { Never } \\
5\end{array}$ & Mean & SD \\
\hline $94(3)$ & 3 & 2 & 18 & 23 & 2 & 3.37 & 0.89 \\
\hline $94(1)$ & 1 & 1 & 7 & 23 & 8 & 3.90 & 0.84 \\
\hline $97(3)$ & $\underline{0}$ & 3 & 9 & 14 & 4 & 3.63 & 0.85 \\
\hline
\end{tabular}

\begin{tabular}{|c|c|c|c|c|c|c|}
\hline & Test & 3 groups & $\begin{array}{l}\text { Post- } \\
\text { hoc test }\end{array}$ & $\begin{array}{c}94(1) \\
\mathbf{v} \\
97(3)\end{array}$ & $\begin{array}{c}94(3) \\
v \\
97(3)\end{array}$ & $\begin{array}{c}94(1) \\
\text { v } \\
94(3)\end{array}$ \\
\hline \multirow{2}{*}{$\begin{array}{l}\text { Code-switching } \\
\text { with friends }\end{array}$} & $\mathrm{K}-\mathrm{W}$ & 8.080 & $\mathrm{M}-\mathrm{W}$ & 2.230 & 0.983 & 8.262 \\
\hline & Sig. & 0.018 & Sig. & 0.135 & 0.322 & 0.004 \\
\hline
\end{tabular}

Table 4.5: Level of agreement on whether English should be the only language used in English lessons

\begin{tabular}{|l|c|c|c|c|c|c|}
\hline & $\begin{array}{c}\text { Absolutely } \\
\text { agree } \\
1\end{array}$ & $\begin{array}{c}\text { Quite } \\
\text { agree } \\
2\end{array}$ & $\begin{array}{c}\text { Quite } \\
\text { disagree } \\
3\end{array}$ & $\begin{array}{c}\text { Absolutely } \\
\text { disagree } \\
4\end{array}$ & Mean & SD \\
\hline & & & & & & \\
\hline $\mathbf{9 4 ( 3 )}$ & 14 & 26 & 7 & 1 & 1.89 & 0.72 \\
\hline $\mathbf{9 4 ( 1 )}$ & 13 & 23 & 4 & 0 & 1.78 & 0.62 \\
\hline $\mathbf{9 7 ( 3 )}$ & 5 & 11 & 10 & 4 & 2.43 & 0.93 \\
\hline & & & & & & \\
\hline
\end{tabular}

\begin{tabular}{|l|l|l|l|c|c|c|}
\hline & Test & 3 groups & $\begin{array}{l}\text { Post- } \\
\text { hoc test }\end{array}$ & $\begin{array}{c}\mathbf{9 4 ( 1 )} \\
\mathbf{v} \\
\mathbf{9 7 ( 3 )}\end{array}$ & $\begin{array}{c}\mathbf{9 4 ( 3 )} \\
\mathbf{v} \\
\mathbf{9 7 ( 3 )}\end{array}$ & $\begin{array}{c}\mathbf{9 4 ( 1 )} \\
\mathbf{v} \\
\mathbf{9 4 ( 3 )}\end{array}$ \\
\hline $\begin{array}{l}\text { English only in } \\
\text { English lessons }\end{array}$ & K-W & 10.85 & M-W & 9.587 & 6.823 & 0.483 \\
\cline { 2 - 7 } & Sig. & 0.004 & Sig. & 0.002 & 0.009 & 0.487 \\
\hline
\end{tabular}


Table 4.6: Level of agreement on whether lesson content can be presented in mixed-code in English lessons

\begin{tabular}{|l|c|c|c|c|c|c|}
\hline & $\begin{array}{c}\text { Absolutely } \\
\text { agree } \\
1\end{array}$ & $\begin{array}{c}\text { Quite } \\
\text { agree } \\
2\end{array}$ & $\begin{array}{c}\text { Quite } \\
\text { disagree } \\
3\end{array}$ & $\begin{array}{c}\text { Absolutely } \\
\text { disagree } \\
4\end{array}$ & Mean & SD \\
\hline & & & & & & \\
\hline $\mathbf{9 4 ( 3 )}$ & 1 & 15 & 25 & 7 & 2.79 & 0.71 \\
\hline $\mathbf{9 4 ( 1 )}$ & 2 & 6 & 25 & 7 & 2.93 & 0.73 \\
\hline $\mathbf{9 7 ( 3 )}$ & 2 & 7 & 13 & 8 & 2.90 & 0.88 \\
\hline & & & & & & \\
\hline
\end{tabular}

\begin{tabular}{|c|c|c|c|c|c|c|}
\hline & Test & 3 groups & $\begin{array}{l}\text { Post- } \\
\text { hoc test }\end{array}$ & $\begin{array}{c}94(1) \\
v \\
97(3)\end{array}$ & $\begin{array}{c}94(3) \\
v \\
97(3)\end{array}$ & $\begin{array}{c}94(1) \\
v \\
94(3)\end{array}$ \\
\hline \multirow{2}{*}{$\begin{array}{l}\text { Lesson content in } \\
\text { mixed-code }\end{array}$} & $\mathrm{K}-\mathrm{W}$ & 1.229 & (5) & & \multirow[b]{2}{*}{ 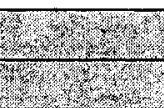 } & \\
\hline & Sig. & 0.541 & & 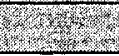 & & \\
\hline
\end{tabular}

Table 4.7: Level of use of English only in English lessons during practice teaching

\begin{tabular}{|l|c|c|c|c|c|c|}
\hline & $\begin{array}{c}\text { Always } \\
1\end{array}$ & $\begin{array}{c}\text { Usually } \\
2\end{array}$ & $\begin{array}{c}\text { Rarely } \\
3\end{array}$ & $\begin{array}{c}\text { Never } \\
4\end{array}$ & Mean & SD \\
\hline & & & & & & \\
\hline $\mathbf{9 4 ( 3 )}$ & 19 & 20.5 & 8.5 & 0 & 1.78 & 0.73 \\
\hline $\mathbf{9 4 ( 1 )}$ & & & & & & \\
\hline $\mathbf{9 7 ( 3 )}$ & 15 & 12 & 3 & 0 & 1.60 & 0.67 \\
\hline & & & & & & \\
\hline
\end{tabular}

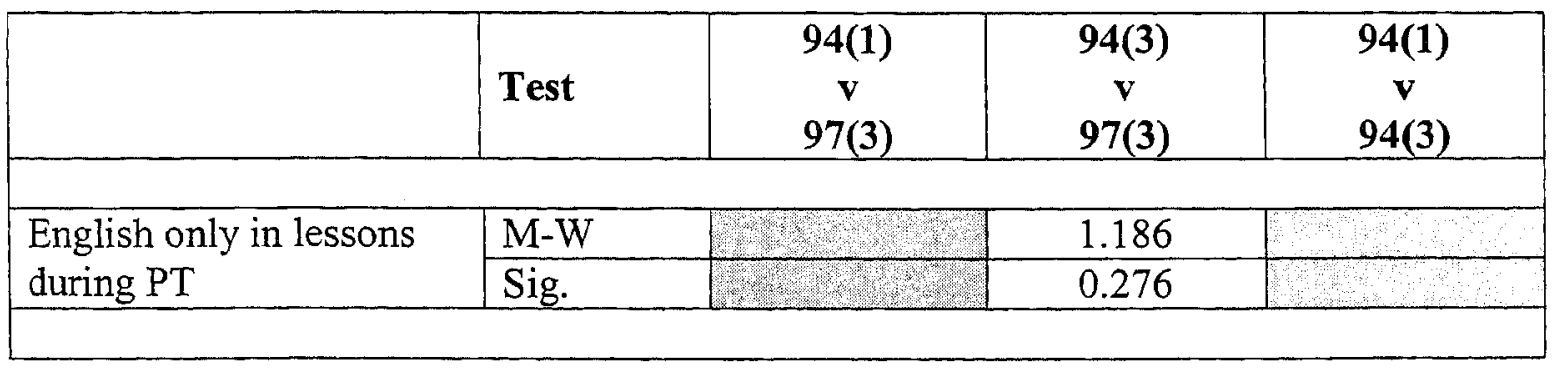


Table 4.8: Extent to which amount of pure English used in lessons during teaching practice changed during the period

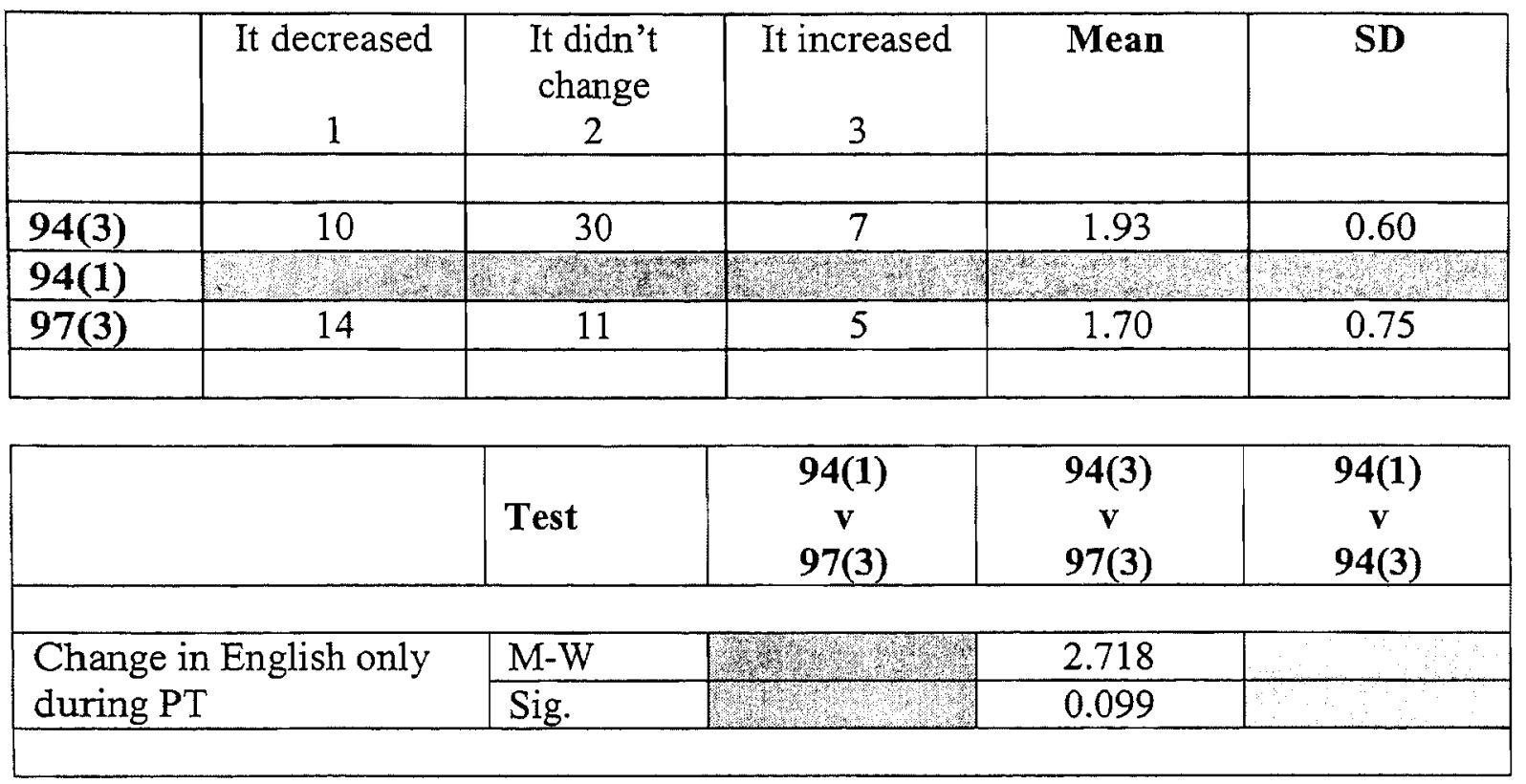


Table 4.9: Situations in which subjects would or might use Cantonese in English lessons

\begin{tabular}{|l|c|c|c|c|c|c|}
\hline \multirow{2}{*}{ Item } & \multicolumn{2}{|c|}{ 94(3) } & \multicolumn{2}{c|}{ 94(1) } & \multicolumn{2}{c|}{ 97(3) } \\
& Yes & No & Yes & No & Yes & No \\
\hline & & & & & & \\
\hline None & 3 & 45 & 5 & 35 & 2 & 28 \\
\hline To present lesson content & 7 & 41 & 2 & 38 & 3 & 27 \\
\hline To explain lesson content & 21 & 27 & 19 & 21 & 8 & 22 \\
\hline To establish discipline & 29 & 19 & 7 & 33 & 13 & 17 \\
\hline $\begin{array}{l}\text { To be friendly with } \\
\text { students }\end{array}$ & 22 & 26 & 16 & 24 & 11 & 19 \\
\hline & & & & & & \\
\hline
\end{tabular}

\begin{tabular}{|c|c|c|c|c|c|c|}
\hline & Test & 3 groups & $\begin{array}{l}\text { Post- } \\
\text { hoc test }\end{array}$ & $\begin{array}{c}94(1) \\
v \\
97(3) \\
\end{array}$ & $\begin{array}{c}94(3) \\
v \\
97(3) \\
\end{array}$ & $\begin{array}{l}94(1) \\
v \\
94(3) \\
\end{array}$ \\
\hline \multirow[t]{2}{*}{ None } & Chi-sq. & 1.268 & \multirow{2}{*}{\multicolumn{2}{|c|}{\begin{tabular}{l|l}
3 & \\
5 &
\end{tabular}}} & \multirow{2}{*}{\multicolumn{2}{|c|}{ 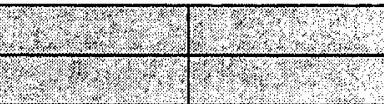 }} \\
\hline & Sig. & 0.530 & & & & \\
\hline \multirow{2}{*}{$\begin{array}{l}\text { To present lesson } \\
\text { content }\end{array}$} & Chi-sq. & 2.195 & \multirow{2}{*}{\multicolumn{4}{|c|}{ 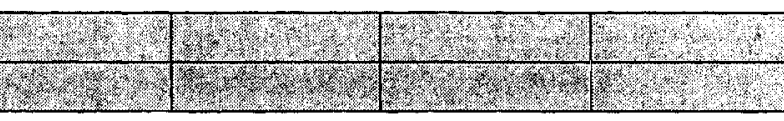 }} \\
\hline & Sig. & 0.334 & & & & \\
\hline \multirow{2}{*}{$\begin{array}{l}\text { To explain lesson } \\
\text { content }\end{array}$} & Chi-sq. & 3.400 & \multirow{2}{*}{ 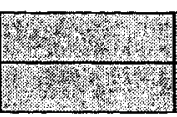 } & \multirow{2}{*}{ 3. } & \multirow{2}{*}{ The } & \multirow{2}{*}{ 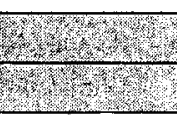 } \\
\hline & Sig. & 0.183 & & & & \\
\hline \multirow{2}{*}{$\begin{array}{l}\text { To establish } \\
\text { discipline }\end{array}$} & Chi-s & 16.60 & \multirow{2}{*}{\begin{tabular}{|l|} 
Chi-sq. \\
Sig. \\
\end{tabular}} & 4.411 & 1.535 & 14.90 \\
\hline & Sig. & 0.000 & & 0.036 & 0.215 & 0.000 \\
\hline \multirow{2}{*}{$\begin{array}{l}\text { To be friendly } \\
\text { with students }\end{array}$} & Chi-sq. & 0.697 & & 2 & & \\
\hline & Sig. & 0.706 & & & & \\
\hline
\end{tabular}




\section{Instructional Planning and Decision-Making}

Table 4.10: Level of agreement on it being impossible to have a successful lesson that hasn't been well planned in advance

\begin{tabular}{|l|c|c|c|c|c|c|}
\hline & $\begin{array}{c}\text { Absolutely } \\
\text { agree } \\
1\end{array}$ & $\begin{array}{c}\text { Quite } \\
\text { agree } \\
2\end{array}$ & $\begin{array}{c}\text { Quite } \\
\text { disagree } \\
3\end{array}$ & $\begin{array}{c}\text { Absolutely } \\
\text { disagree } \\
4\end{array}$ & Mean & SD \\
\hline & & & & & & \\
\hline $\mathbf{9 4 ( 3 )}$ & 15 & 24 & 5 & 4 & 1.96 & 0.87 \\
\hline $\mathbf{9 4 ( 1 )}$ & 13 & 23 & 4 & 0 & 1.78 & 0.62 \\
\hline $\mathbf{9 7 ( 3 )}$ & 11 & 14 & 5 & 0 & 1.80 & 0.71 \\
\hline & & & & & & \\
\hline
\end{tabular}

\begin{tabular}{|l|l|l|l|l|l|l|}
\hline & Test & 3 groups & $\begin{array}{l}\text { Post- } \\
\text { hoc test }\end{array}$ & $\begin{array}{c}\mathbf{9 4 ( 1 )} \\
\mathbf{v} \\
\mathbf{9 7 ( 3 )}\end{array}$ & $\begin{array}{c}\mathbf{9 4 ( 3 )} \\
\mathbf{v} \\
\mathbf{9 7 ( 3 )}\end{array}$ & $\begin{array}{c}\mathbf{9 4 ( 1 )} \\
\mathbf{v} \\
\mathbf{9 4 ( 3 )}\end{array}$ \\
\hline $\begin{array}{l}\text { Planning for } \\
\text { lesson success }\end{array}$ & K-W & 0.595 & & & & \\
\hline & Sig. & 0.743 & & & & \\
\hline
\end{tabular}

Table 4.11: Level of agreement on whether teachers should write detailed lesson plans for every lesson they teach

\begin{tabular}{|l|c|c|c|c|c|c|}
\hline & $\begin{array}{c}\text { Absolutely } \\
\text { agree } \\
1\end{array}$ & $\begin{array}{c}\text { Quite } \\
\text { agree } \\
2\end{array}$ & $\begin{array}{c}\text { Quite } \\
\text { disagree } \\
3\end{array}$ & $\begin{array}{c}\text { Absolutely } \\
\text { disagree } \\
4\end{array}$ & Mean & SD \\
\hline & & & & & & \\
\hline $\mathbf{9 4 ( 3 )}$ & 0 & 8 & 26 & 14 & 3.12 & 0.67 \\
\hline $\mathbf{9 4 ( 1 )}$ & 4 & 21 & 12 & 3 & 2.35 & 0.77 \\
\hline $\mathbf{9 7 ( 3 )}$ & 1 & 6 & 18 & 5 & 2.90 & 0.71 \\
\hline & & & & & & \\
\hline
\end{tabular}

\begin{tabular}{|c|c|c|c|c|c|c|}
\hline . & Test & 3 groups & $\begin{array}{l}\text { Post- } \\
\text { hoc test }\end{array}$ & $\begin{array}{c}94(1) \\
v \\
97(3)\end{array}$ & $\begin{array}{c}94(3) \\
v \\
97(3)\end{array}$ & $\begin{array}{c}94(1) \\
v \\
94(3) \\
\end{array}$ \\
\hline \multirow{2}{*}{$\begin{array}{l}\text { Detailed plans for } \\
\text { every lesson }\end{array}$} & $\overline{\mathrm{K}-\mathrm{W}}$ & 21.94 & $\mathrm{M}-\mathrm{W}$ & 9.370 & 1.683 & 20.11 \\
\hline & Sig. & 0.000 & Sig. & 0.002 & 0.195 & 0.000 \\
\hline
\end{tabular}


Table 4.12: Assessment of how detailed lesson plans need to be

\begin{tabular}{|l|c|c|c|c|c|c|c|}
\hline & $\begin{array}{c}\text { Very } \\
\text { detailed } \\
\text { and } \\
\text { precise } \\
1\end{array}$ & $\begin{array}{c}\text { Quite } \\
\text { detailed } \\
\text { and } \\
\text { precise } \\
2\end{array}$ & $\begin{array}{c}\text { Quite } \\
\text { detailed } \\
\text { and quite } \\
\text { flexible } \\
3\end{array}$ & $\begin{array}{c}\text { Loosely } \\
\text { detailed } \\
\text { and quite } \\
\text { flexible } \\
4\end{array}$ & $\begin{array}{c}\text { Brief and } \\
\text { very } \\
\text { flexible }\end{array}$ & Mean & SD \\
\hline & 0 & 5 & 22 & 13 & 8 & 3.50 & 0.90 \\
\hline $\mathbf{9 4 ( 3 )}$ & 0 & 4 & 28 & 4 & 4 & 3.20 & 0.76 \\
\hline $\mathbf{9 4 ( 1 )}$ & 0 & 2 & 14 & 8 & 6 & 3.60 & 0.89 \\
\hline $\mathbf{9 7 ( 3 )}$ & & & & & & & \\
\hline
\end{tabular}

\begin{tabular}{|c|c|c|c|c|c|c|}
\hline & Test & 3 groups & $\begin{array}{l}\text { Post- } \\
\text { hoc test }\end{array}$ & $\begin{array}{c}94(1) \\
v \\
97(3)\end{array}$ & $\begin{array}{c}94(3) \\
v \\
97(3)\end{array}$ & $\begin{array}{c}94(1) \\
v \\
94(3)\end{array}$ \\
\hline \multirow{2}{*}{$\begin{array}{l}\text { Extent of detail of } \\
\text { lesson plans }\end{array}$} & $\mathrm{K}-\mathrm{W}$ & 4.958 & 4 & PO & क & 3 \\
\hline & Sig. & 0.084 & & t & 4 & 3 \\
\hline
\end{tabular}

Table 4.13: Basis on which lessons were planned during practice teaching

\begin{tabular}{|c|c|c|c|c|c|c|c|c|c|}
\hline Item & anking & 1 & 2 & 3 & 4 & 5 & 6 & 7 & Mean \\
\hline \multirow[t]{3}{*}{ Syllabus } & $94(3)$ & 12 & 11 & 11 & 7 & 4 & 3 & 0 & 2.77 \\
\hline & 94(1) & 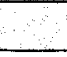 & - & & & & 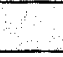 & & \\
\hline & $97(3)$ & 11 & 3 & 5 & 3 & 7 & 1 & 0 & 2.83 \\
\hline \multirow{3}{*}{$\begin{array}{l}\text { Regular teacher's } \\
\text { instructions }\end{array}$} & & 16 & & & & & & & 82 \\
\hline & $\frac{94(3)}{94(1)}$ & 16 & 8 & 8 & 4 & 8 & 4 & 0 & 2.83 \\
\hline & $97(3)$ & 9 & 9 & 5 & 2 & 3 & 2 & 0 & 2.57 \\
\hline \multirow{3}{*}{$\begin{array}{l}\text { BATESL course } \\
\text { guidelines }\end{array}$} & 94(3) & 1 & 5 & 7 & 12 & 10 & 11 & 1 & 4.32 \\
\hline & 94(1) & & & & & & & - & $\ldots$ \\
\hline & $97(3)$ & 5 & 4 & 8 & 2 & 5 & 6 & 0 & 3.53 \\
\hline \multirow{3}{*}{$\begin{array}{l}\text { Textbook Teachers } \\
\text { Instructions }\end{array}$} & $94(3)$ & 5 & 5 & 3 & 6 & 12 & 17 & 0 & 4.50 \\
\hline & $94(1)$ & & & 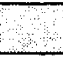 & & & & & \\
\hline & 97(3) & 0 & 3 & 2 & 9 & 3 & 13 & 0 & 4.70 \\
\hline
\end{tabular}




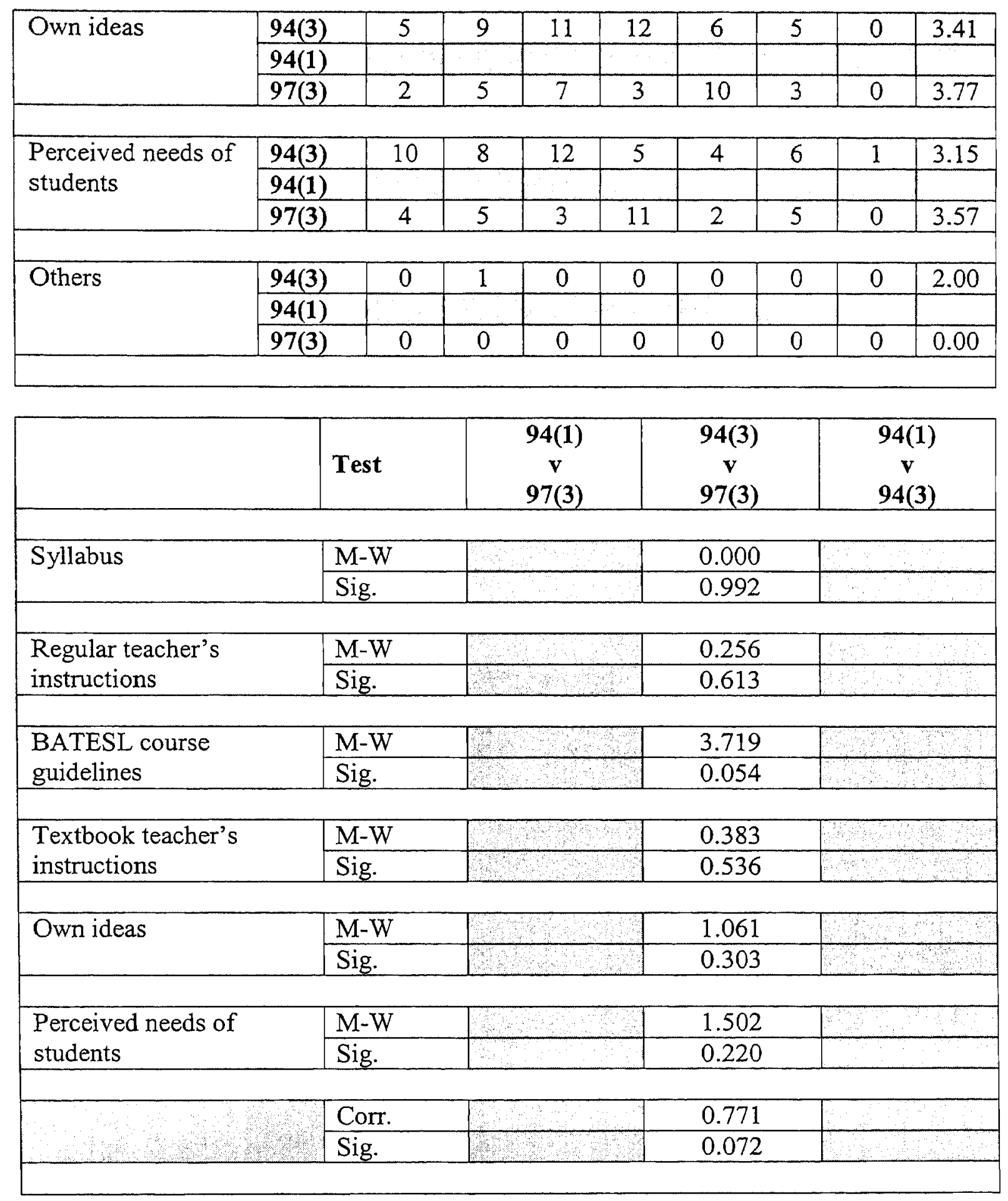


Table 4.14: Factors having most influence on decisions made during practice teaching

\begin{tabular}{|c|c|c|c|c|c|c|c|}
\hline Item & Ranking & 1 & 2 & 3 & 4 & 5 & Mean \\
\hline \multirow[t]{3}{*}{ Students' needs } & $94(3)$ & 14 & 15 & 12 & 7 & 0 & 2.25 \\
\hline & $94(1)$ & & 5 & 5 & 2 & 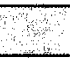 & \\
\hline & $97(3)$ & 7 & 8 & 7 & 8 & 0 & 2.53 \\
\hline \multirow{3}{*}{$\begin{array}{l}\text { Regular teacher's } \\
\text { input }\end{array}$} & 94(3) & 16 & 13 & 14 & 5 & 0 & 2.17 \\
\hline & 94(1) & & & & & & \\
\hline & $97(3)$ & 11 & 13 & 4 & 2 & 0 & 1.93 \\
\hline \multirow{3}{*}{ Supervisor's advice } & $94(3)$ & 9 & 13 & 12 & 13 & 0 & 2.62 \\
\hline & 94(1) & 8 & 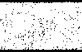 & & e & 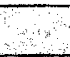 & \\
\hline & $97(3)$ & 10 & 7 & 11 & 2 & 0 & 2.17 \\
\hline \multirow{3}{*}{$\begin{array}{l}\text { Fellow student } \\
\text { teacher's advice }\end{array}$} & $94(3)$ & 7 & 10 & 9 & 21 & 0 & 2.94 \\
\hline & \begin{tabular}{|l}
$94(1)$ \\
\end{tabular} & 2 & 5 & Wh & 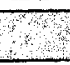 & . & \\
\hline & $97(3)$ & 2 & 3 & 8 & 17 & 0 & 3.33 \\
\hline \multirow{3}{*}{ Other factors } & $04(3)$ & 1 & 0 & 0 & 0 & 0 & 100 \\
\hline & $\frac{94(3)}{94(1)}$ & 1 & & 0 & 0 & & 1.00 \\
\hline & 97(3) & 0 & 0 & 0 & 0 & 0 & 0.00 \\
\hline & & & & & & & \\
\hline
\end{tabular}




\begin{tabular}{|c|c|c|c|c|}
\hline & Test & $\begin{array}{c}94(1) \\
v \\
97(3) \\
\end{array}$ & $\begin{array}{c}94(3) \\
v \\
97(3)\end{array}$ & $\begin{array}{c}94(1) \\
v \\
94(3) \\
\end{array}$ \\
\hline \multirow[t]{2}{*}{ Students' needs } & $\mathrm{M}-\mathrm{W}$ & 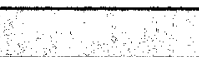 & 1.176 & \\
\hline & Sig. & & 0.278 & \\
\hline \multirow{2}{*}{ Regular teacher's input } & $\mathrm{M}-\mathrm{W}$ & VW & 1.268 & \\
\hline & Sig. & 2 & 0.260 & \\
\hline \multirow[t]{2}{*}{ Supervisor's advice } & M-W & - & 3.086 & \\
\hline & Sig. & 4 & 0.079 & \\
\hline \multirow{2}{*}{$\begin{array}{l}\text { Fellow student teacher's } \\
\text { advice }\end{array}$} & $\mathrm{M}-\mathrm{W}$ & की? & 1.889 & 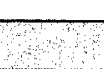 \\
\hline & Sig. & 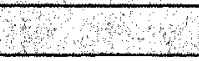 & 0.169 & \\
\hline & Corr. & me & 0.800 & \\
\hline & Sig. & & 0.200 & \\
\hline
\end{tabular}

Table 4.15: Level of agreement on whether teachers should be more responsive to student needs than the set syllabus

\begin{tabular}{|l|c|c|c|c|c|c|}
\hline & $\begin{array}{c}\text { Absolutely } \\
\text { agree } \\
1\end{array}$ & $\begin{array}{c}\text { Quite } \\
\text { agree } \\
2\end{array}$ & $\begin{array}{c}\text { Quite } \\
\text { disagree } \\
3\end{array}$ & $\begin{array}{c}\text { Absolutely } \\
\text { disagree } \\
4\end{array}$ & Mean & SD \\
\hline & & & & & & \\
\hline $\mathbf{9 4 ( 3 )}$ & 14 & 28 & 5 & 0 & $\mathbf{1 . 8 1}$ & $\mathbf{0 . 6 1}$ \\
\hline $\mathbf{9 4 ( 1 )}$ & 20 & 18 & 2 & 0 & $\mathbf{1 . 5 5}$ & $\mathbf{0 . 6 0}$ \\
\hline $\mathbf{9 7 ( 3 )}$ & 15 & 10 & 4 & 0 & $\mathbf{1 . 6 2}$ & $\mathbf{0 . 7 3}$ \\
\hline & & & & & & \\
\hline
\end{tabular}

\begin{tabular}{|l|l|l|l|c|c|c|}
\hline & Test & 3 groups & Post- & $\begin{array}{c}\mathbf{9 4 ( 1 )} \\
\mathbf{v} \\
\mathbf{9 7 ( 3 )}\end{array}$ & $\begin{array}{c}\mathbf{9 4 ( 3 )} \\
\mathbf{v} \\
\mathbf{9 7 ( 3 )}\end{array}$ & $\begin{array}{c}\mathbf{9 4 ( 1 )} \\
\mathbf{v} \\
\mathbf{9 4 ( 3 )}\end{array}$ \\
\hline
\end{tabular}


Teaching Approach

Table 4.16: Level of agreement on whether examination requirements should determine what is taught in English lessons

\begin{tabular}{|l|c|c|c|c|c|c|}
\hline & $\begin{array}{c}\text { Absolutely } \\
\text { agree } \\
1\end{array}$ & $\begin{array}{c}\text { Quite } \\
\text { agree } \\
2\end{array}$ & $\begin{array}{c}\text { Quite } \\
\text { disagree } \\
3\end{array}$ & $\begin{array}{c}\text { Absolutely } \\
\text { disagree } \\
4\end{array}$ & Mean & SD \\
\hline & & & & & & \\
\hline $\mathbf{9 4 ( 3 )}$ & 3 & 22.5 & 21.5 & 1 & 2.43 & 0.65 \\
\hline $\mathbf{9 4 ( 1 )}$ & 0 & 30 & 10 & 0 & 2.25 & 0.44 \\
\hline $\mathbf{9 7 ( 3 )}$ & 11 & 10.5 & 8.5 & 0 & 1.92 & 0.82 \\
\hline & & & & & & \\
\hline
\end{tabular}

\begin{tabular}{|c|c|c|c|c|c|c|}
\hline & Test & 3 groups & $\begin{array}{l}\text { Post- } \\
\text { hoc test }\end{array}$ & $\begin{array}{c}94(1) \\
v \\
97(3)\end{array}$ & $\begin{array}{c}94(3) \\
\text { v } \\
97(3)\end{array}$ & $\begin{array}{c}94(1) \\
v \\
94(3)\end{array}$ \\
\hline \multirow{2}{*}{$\begin{array}{l}\text { Exams determine } \\
\text { what is taught }\end{array}$} & $\mathrm{K}-\mathrm{W}$ & 9.087 & $\mathrm{M}-\mathrm{W}$ & 3.483 & 7.305 & 2.867 \\
\hline & Sig. & 0.011 & Sig. & 0.062 & 0.007 & 0.090 \\
\hline
\end{tabular}

Table 4.17: Level of agreement on whether students learn English better though interaction with other students rather than through direct instruction from a teacher

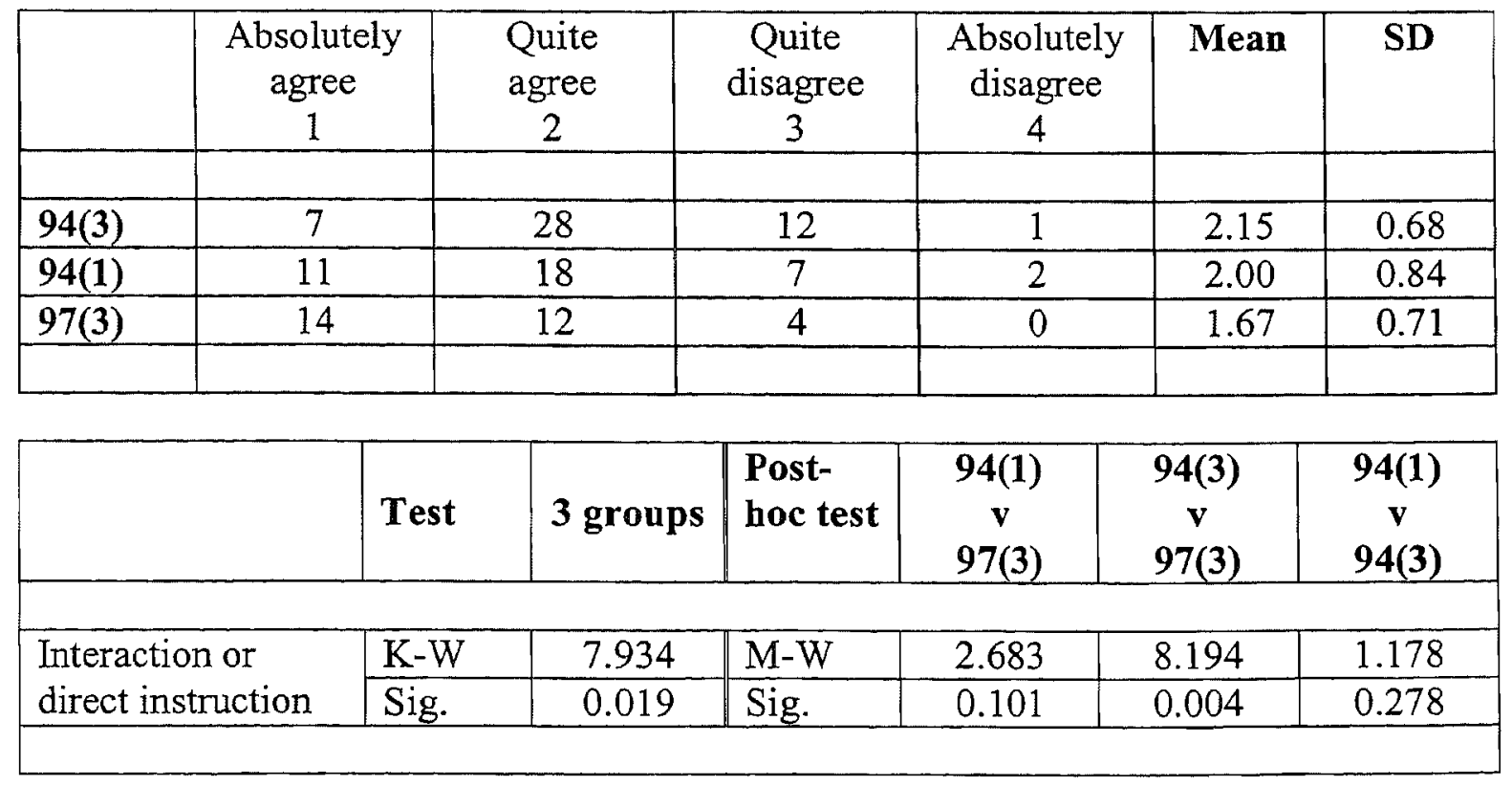


Table 4.18: Whether students learned English better through interaction with other students or through instruction from teacher during practice teaching

\begin{tabular}{|l|c|c|c|}
\hline & $\begin{array}{c}\text { Through interaction } \\
\text { with other students } \\
1\end{array}$ & $\begin{array}{c}\text { Through direct } \\
\text { instruction } \\
2\end{array}$ & $\begin{array}{c}\text { No difference between } \\
\text { the two } \\
3\end{array}$ \\
\hline & & & \\
\hline $\mathbf{9 4 ( 3 )}$ & 8 & 25 & 10 \\
\hline $\mathbf{9 4 ( 1 )}$ & & & 8 \\
\hline $\mathbf{9 7 ( 3 )}$ & 11 & 11 & \\
\hline & & & \\
\hline
\end{tabular}

\begin{tabular}{|c|c|c|c|c|}
\hline & Test & $\begin{array}{c}94(1) \\
\text { v } \\
97(3)\end{array}$ & $\begin{array}{c}94(3) \\
v \\
97(3)\end{array}$ & $\begin{array}{c}94(1) \\
\text { v } \\
94(3) \\
\end{array}$ \\
\hline \multirow{2}{*}{$\begin{array}{l}\text { Interaction or direct } \\
\text { instruction in } \mathrm{PT}\end{array}$} & Chi-sq. & & 3.951 & \\
\hline & Sig. & 8 & 0.139 & \\
\hline
\end{tabular}

Table 4.19: Level of agreement on whether a class should be strictly controlled by the teacher and all lesson content directed by the teacher

\begin{tabular}{|l|c|c|c|c|c|c|}
\hline & $\begin{array}{c}\text { Absolutely } \\
\text { agree } \\
1\end{array}$ & $\begin{array}{c}\text { Quite } \\
\text { agree } \\
2\end{array}$ & $\begin{array}{c}\text { Quite } \\
\text { disagree } \\
3\end{array}$ & $\begin{array}{c}\text { Absolutely } \\
\text { disagree } \\
4\end{array}$ & Mean & SD \\
\hline & & & & & & \\
\hline $\mathbf{9 4 ( 3 )}$ & 0 & 10 & 30 & 8 & 2.96 & 0.62 \\
\hline $\mathbf{9 4 ( 1 )}$ & 2 & 4 & 26 & 8 & 3.00 & 0.72 \\
\hline $\mathbf{9 7 ( 3 )}$ & 0 & 3 & 15 & 12 & 3.30 & 0.65 \\
\hline & & & & & & \\
\hline
\end{tabular}

\begin{tabular}{|l|l|l|l|l|l|l|}
\hline & Test & 3 groups & Post- & $\begin{array}{c}\mathbf{9 4 ( 1 )} \\
\mathbf{v} \\
\mathbf{9 7 ( 3 )}\end{array}$ & $\begin{array}{c}\mathbf{9 4 ( 3 )} \\
\mathbf{v} \\
\mathbf{9 7 ( 3 )}\end{array}$ & $\begin{array}{c}\mathbf{9 4 ( 1 )} \\
\mathbf{v} \\
\mathbf{9 4 ( 3 )}\end{array}$ \\
\hline
\end{tabular}


Table 4.20: Level of agreement on whether a teacher should be prepared to experiment with materials and teaching strategies

\begin{tabular}{|c|c|c|c|c|c|c|}
\hline & $\begin{array}{c}\text { Absolutely } \\
\text { agree } \\
1\end{array}$ & $\begin{array}{c}\text { Quite } \\
\text { agree } \\
2\end{array}$ & $\begin{array}{c}\text { Quite } \\
\text { disagree } \\
3\end{array}$ & $\begin{array}{c}\text { Absolutely } \\
\text { disagree } \\
4\end{array}$ & Mean & SD \\
\hline & & & & & & \\
\hline $\mathbf{9 4 ( 3 )}$ & 15 & 30 & 3 & 0 & 1.75 & 0.56 \\
\hline $\mathbf{9 4 ( 1 )}$ & 11 & 28 & 1 & 0 & 1.75 & 0.49 \\
\hline $\mathbf{9 7 ( 3 )}$ & 12 & 11 & 7 & 0 & 1.83 & 0.79 \\
\hline & & & & & & \\
\hline
\end{tabular}

\begin{tabular}{|c|c|c|c|c|c|c|}
\hline & Test & 3 groups & $\begin{array}{l}\text { Post- } \\
\text { hoc } \\
\text { test }\end{array}$ & $\begin{array}{c}94(1) \\
\mathbf{v} \\
97(3)\end{array}$ & $\begin{array}{c}94(3) \\
v \\
97(3) \\
\end{array}$ & $\begin{array}{c}94(1) \\
v \\
94(3)\end{array}$ \\
\hline \multirow{2}{*}{$\begin{array}{l}\text { Experiment with } \\
\text { materials/strategies }\end{array}$} & $\mathrm{K}-\mathrm{W}$ & 0.098 & ? & 9 & 5 & \\
\hline & Sig. & 0.952 & 1 & & & \\
\hline
\end{tabular}

Table 4.21: Description of teaching style

\begin{tabular}{|l|c|c|c|c|c|c|}
\hline & $\begin{array}{c}\text { Very leamer- } \\
\text { centered } \\
1\end{array}$ & $\begin{array}{c}\text { Mostly } \\
\text { learner- } \\
\text { centered } \\
2\end{array}$ & $\begin{array}{c}\text { Mostly } \\
\text { teacher } \\
\text { centered } \\
3\end{array}$ & $\begin{array}{c}\text { Very } \\
\text { teacher- } \\
\text { centered } \\
4\end{array}$ & Mean & SD \\
\hline & 1 & 20 & 26 & 1 & 2.56 & 0.58 \\
\hline $\mathbf{9 4 ( 3 )}$ & 2 & 22 & 15 & 1 & 2.38 & 0.63 \\
\hline $\mathbf{9 4 ( 1 )}$ & 0 & 15 & 14 & 1 & 2.53 & 0.57 \\
\hline $\mathbf{9 7 ( 3 )}$ & & & & & & \\
\hline & & & & & & \\
\hline
\end{tabular}

\begin{tabular}{|c|c|c|c|c|c|c|}
\hline & Test & 3 groups & $\begin{array}{l}\text { Post- } \\
\text { hoc test }\end{array}$ & $\begin{array}{c}94(1) \\
v \\
97(3)\end{array}$ & $\begin{array}{c}94(3) \\
v \\
97(3)\end{array}$ & $\begin{array}{c}94(1) \\
v \\
94(3)\end{array}$ \\
\hline \multirow[t]{2}{*}{ Teaching style } & $\mathrm{K}-\mathrm{W}$ & 2.397 & $\sqrt{25}$ & (m) & & 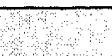 \\
\hline & Sig. & 0.302 & 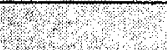 & & & \\
\hline
\end{tabular}


Table 4.22: Factors having most effect on teaching of English

\begin{tabular}{|c|c|c|c|c|c|c|c|c|c|c|c|c|c|c|c|}
\hline Item & king & 1 & 2 & 3 & 4 & 5 & 6 & 7 & 8 & 9 & 10 & 11 & 12 & 13 & $\bar{M}$ \\
\hline \multirow{3}{*}{ Class size } & $94(3)$ & 4 & 4 & 2 & 9 & 2 & 7 & 0 & 2 & 4 & 7 & 6 & 0 & 0 & 6.25 \\
\hline & $94(1)$ & 0 & 1 & 1 & 2 & 3 & 4 & 5 & 3 & 2 & 4 & 9 & 3 & 0 & 8.22 \\
\hline & $97(3)$ & 8 & 2 & 1 & 3 & 1 & 2 & 1 & 1 & 1 & 5 & 1 & 4 & 0 & 5.90 \\
\hline \multirow{3}{*}{ Classroom size } & $94(3)$ & 0 & 0 & 2 & 1 & 2 & 0 & 4 & 3 & 3 & 10 & 10 & 10 & 1 & \\
\hline & $94(1)$ & 0 & 1 & 0 & 0 & 0 & 1 & 0 & 2 & 0 & 5 & 10 & 19 & 0 & 1087 \\
\hline & $97(3)$ & 2 & 5 & 1 & 0 & 1 & 1 & 2 & 3 & 2 & 1 & 5 & 6 & 0 & 7.55 \\
\hline \multirow{3}{*}{$\begin{array}{l}\text { Noise in } \\
\text { classroom }\end{array}$} & $94(3)$ & 3 & 0 & 2 & 2 & 3 & 2 & 4 & 3 & 6 & 6 & 8 & 8 & 0 & 8.38 \\
\hline & $94(1)$ & 0 & 0 & 1 & 2 & 2 & 1 & 2 & 3 & 5 & 7 & 11 & 3 & 0 & 9.13 \\
\hline & $97(3)$ & 2 & 0 & 5 & 0 & 0 & 3 & 1 & 1 & 3 & 5 & 4 & 6 & 0 & 8.10 \\
\hline \multirow{3}{*}{ School policy } & 94(3) & 9 & 3 & 1 & 0 & 5 & 4 & 8 & 2 & 4 & 3 & 4 & 4 & 0 & \\
\hline & $94(1)$ & 9 & 1 & 2 & 2 & 5 & 3 & 4 & 2 & 3 & 4 & 2 & 1 & 0 & 6.00 \\
\hline & $97(3)$ & 1 & 4 & 4 & 1 & 3 & 1 & 2 & 1 & 2 & 5 & 4 & 1 & 0 & 6.62 \\
\hline \multirow{3}{*}{$\begin{array}{l}\text { Exam } \\
\text { requirements }\end{array}$} & $94(3)$ & 6 & 8 & 1 & 6 & 4 & 4 & 4 & 3 & 1 & 3 & 2 & 4 & 0 & 5.52 \\
\hline & 94(1) & 1 & 7 & 3 & 5 & 4 & 5 & 4 & 1 & 2 & 2 & 1 & 2 & 0 & 5.29 \\
\hline & $97(3)$ & 2 & 2 & 2 & 4 & 5 & 3 & 1 & 5 & 2 & 0 & 2 & 0 & 0 & 5.64 \\
\hline \multirow{3}{*}{$\begin{array}{l}\text { Current trends in } \\
\text { ESL teaching }\end{array}$} & $94(3)$ & 1 & 1 & 4 & 3 & 2 & 2 & 7 & 3 & 5 & 3 & 5 & 9 & 1 & 8.11 \\
\hline & 94(1) & 1 & 4 & 4 & 3 & 7 & 5 & 1 & 4 & 3 & 2 & 1 & 2 & 0 & 5.97 \\
\hline & $97(3)$ & 2 & 3 & 2 & 0 & 2 & 1 & 4 & 3 & 2 & 2 & 5 & 2 & 0 & 7.11 \\
\hline \multirow{3}{*}{$\begin{array}{l}\text { Personality of } \\
\text { teacher }\end{array}$} & 94(3) & 7 & 3 & 5 & 4 & 3 & 6 & 1 & 8 & 2 & 2 & 2 & 4 & 0 & 5.83 \\
\hline & $94(1)$ & 5 & 4 & 2 & 5 & 1 & 2 & 8 & 2 & 3 & 1 & 2 & 2 & 0 & 5.73 \\
\hline & $97(3)$ & 0 & 4 & 4 & 2 & 5 & 3 & 1 & 2 & 2 & 2 & 2 & 2 & 0 & 6.14 \\
\hline \multirow{3}{*}{$\begin{array}{l}\text { Motivation of } \\
\text { students }\end{array}$} & $94(3)$ & 8 & 11 & 11 & 3 & 5 & 0 & 5 & 3 & 1 & 0 & 0 & 0 & 0 & 3.57 \\
\hline & 94(1) & 10 & 4 & 8 & 8 & 0 & 3 & 0 & 4 & 1 & 0 & 0 & 1 & 0 & 3.87 \\
\hline & $97(3)$ & 1 & 5 & 5 & 7 & 3 & 1 & 2 & 0 & 4 & 0 & 0 & 2 & 0 & \begin{tabular}{|l}
4.97 \\
\end{tabular} \\
\hline \multirow{3}{*}{$\begin{array}{l}\text { English } \\
\text { proficiency of } \\
\text { students }\end{array}$} & $94(3)$ & 15 & 11 & 7 & 5 & 3 & 1 & 0 & 2 & 0 & 0 & 2 & 0 & 0 & 2.98 \\
\hline & 94(1) & 6 & 6 & 2 & 1 & 6 & 5 & 1 & 6 & 1 & 1 & 1 & 1 & 0 & 5.03 \\
\hline & $97(3)$ & 8 & 4 & 2 & 4 & 3 & 3 & 0 & 3 & 0 & 1 & 1 & 1 & 0 & \begin{tabular}{|l|l|}
4.27 \\
\end{tabular} \\
\hline \multirow{3}{*}{$\begin{array}{l}\text { Availability } \\
\text { quality of } \\
\text { teaching mats. }\end{array}$} & 94(3) & 2 & 1 & 6 & 4 & 5 & 8 & 8 & 5 & 6 & 1 & 0 & 0 & 0 & 5.89 \\
\hline & 94(1) & 0 & 5 & 3 & 3 & 3 & 3 & 3 & 4 & 9 & 3 & 0 & 1 & 0 & 6.43 \\
\hline & \begin{tabular}{|l}
$97(3)$ \\
\end{tabular} & 2 & 2 & 1 & 2 & 2 & 3 & 5 & 6 & 3 & 2 & 1 & 0 & 0 & 6.41 \\
\hline
\end{tabular}




\begin{tabular}{|c|c|c|c|c|c|c|c|c|c|c|c|c|c|c|c|}
\hline \multirow{3}{*}{$\begin{array}{l}\text { Availability/ } \\
\text { quality of } \\
\text { teaching aids }\end{array}$} & 94(3) & 0 & 0 & 1 & 4 & 6 & 6 & 6 & 6 & 6 & 6 & 4 & 1 & 0 & \\
\hline & $94(1)$ & 0 & 1 & 3 & 5 & 2 & 3 & 3 & 4 & 6 & 8 & 2 & 0 & 0 & \\
\hline & $97(3)$ & $\overline{0}$ & 0 & 1 & 0 & 4 & 2 & 7 & 1 & 5 & 5 & 3 & 1 & 0 & \\
\hline & & & & & & & & & & & & & & & \\
\hline \multirow[t]{3}{*}{ Teacher training } & 94(3) & 4 & 1 & 2 & 6 & 4 & 5 & 2 & 6 & 6 & 3 & 3 & 4 & 0 & 6.80 \\
\hline & $94(1)$ & 10 & 6 & 6 & 1 & 3 & 3 & 5 & 1 & 1 & 1 & 0 & 0 & 0 & 3.76 \\
\hline & $97(3)$ & 2 & 2 & 1 & 4 & 1 & 5 & 3 & 3 & 3 & 1 & 1 & 2 & 0 & 6.32 \\
\hline \multirow{3}{*}{ Other factors } & $94(3)$ & 1 & 0 & 1 & 0 & 0 & 0 & 0 & 0 & 0 & 0 & 0 & 0 & 0 & 2.00 \\
\hline & 94(1) & 0 & 0 & 0 & 0 & 0 & 0 & 0 & 0 & 0 & 0 & 0 & 0 & 0 & 0.00 \\
\hline & $97(3)$ & 0 & 0 & 0 & 0 & 0 & 0 & 0 & 0 & 0 & 0 & 0 & 0 & 0 & 0.00 \\
\hline
\end{tabular}

\begin{tabular}{|c|c|c|c|c|c|c|}
\hline & Test & 3 groups & $\begin{array}{l}\text { Post- } \\
\text { hoc test }\end{array}$ & $\begin{array}{c}94(1) \\
\mathbf{v} \\
97(3)\end{array}$ & $\begin{array}{c}94(3) \\
\mathbf{v} \\
97(3)\end{array}$ & $\begin{array}{c}94(1) \\
v \\
94(3)\end{array}$ \\
\hline \multirow[t]{2}{*}{ Class size } & $\mathrm{K}-\mathrm{W}$ & 9.073 & $\mathrm{M}-\mathrm{W}$ & 5.353 & 0.245 & 7.936 \\
\hline & \begin{tabular}{|l} 
Sig. \\
\end{tabular} & 0.011 & Sig. & 0.021 & 0.621 & 0.005 \\
\hline \multirow[t]{2}{*}{ Classroom size } & $\mathrm{K}-\mathrm{W}$ & 16.12 & $\mathrm{M}-\mathrm{W}$ & 13.09 & 3.687 & 8.369 \\
\hline & Sig. & 0.000 & Sig. & 0.000 & 0.055 & 0.004 \\
\hline \multirow[t]{2}{*}{ Noise in classroom } & $\mathrm{K}-\mathrm{W}$ & 0.641 & & & & \\
\hline & Sig. & 0.726 & 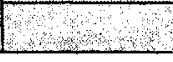 & + & 8 & \\
\hline \multirow{2}{*}{ School policy } & $\mathrm{K}-\mathrm{W}$ & 1.771 & 17. & $\sqrt{25}$ & 3 & \\
\hline & Sig. & 0.413 & 25 & & 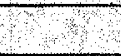 & \\
\hline \multirow{2}{*}{$\begin{array}{l}\text { Exam } \\
\text { requirements }\end{array}$} & $\mathrm{K}-\mathrm{W}$ & 0.221 & 20 & & & \\
\hline & \begin{tabular}{|l|} 
Sig. \\
\end{tabular} & 0.895 & 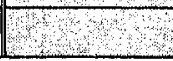 & & 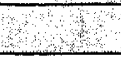 & \\
\hline \multirow{2}{*}{$\begin{array}{l}\text { Current trends in } \\
\text { ESL teaching }\end{array}$} & $\mathrm{K}-\mathrm{W}$ & 8.886 & M-W & 2.104 & 1.507 & 8.860 \\
\hline & Sig. & 0.012 & Sig. & 0.147 & 0.220 & 0.003 \\
\hline \multirow{2}{*}{$\begin{array}{l}\text { Personality of } \\
\text { teacher }\end{array}$} & $\mathrm{K}-\mathrm{W}$ & 0.288 & 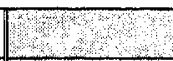 & & & \\
\hline & Sig. & 0.866 & 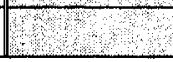 & & & \\
\hline \multirow{2}{*}{$\begin{array}{l}\text { Motivation of } \\
\text { students }\end{array}$} & $\mathrm{K}-\mathrm{W}$ & 5.714 & 5 & & & \\
\hline & Sig. & 0.057 & 5 & & 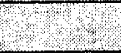 & - \\
\hline
\end{tabular}




\begin{tabular}{|c|c|c|c|c|c|c|}
\hline \multirow{2}{*}{$\begin{array}{l}\text { English } \\
\text { proficiency of Ss. }\end{array}$} & $\mathrm{K}-\mathrm{W}$ & 9.935 & $M-W$ & 1.313 & 3.010 & 9.700 \\
\hline & Sig. & 0.007 & Sig. & 0.252 & 0.083 & 0.002 \\
\hline \multirow{2}{*}{$\begin{array}{l}\text { Availty./quality of } \\
\text { teaching mats. }\end{array}$} & $\mathrm{K}-\mathrm{W}$ & 1.714 & 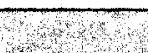 & $\sqrt{3}$ & The & 3 \\
\hline & Sig. & 0.425 & 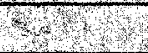 & $\sqrt{25}$ & 2 & $x_{2}$ \\
\hline \multirow{2}{*}{$\begin{array}{l}\text { Availty./quality of } \\
\text { teaching aids }\end{array}$} & $\overline{\mathrm{K}-\mathrm{W}}$ & 1.246 & 17 & 4 & 8 & 70 \\
\hline & Sig. & 0.536 & 7 & 70 & & 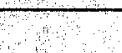 \\
\hline \multirow{2}{*}{ Teacher training } & $\mathrm{K}-\mathrm{W}$ & 18743 & $M_{W} W$ & 1034 & 0415 & 1657 \\
\hline & Sig. & 0.000 & Sig. & 0.001 & 0.519 & 0.000 \\
\hline \multirow{3}{*}{89} & & & & & & \\
\hline & 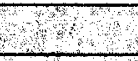 & 40 & Corr. & 0.637 & 0.836 & 0.886 \\
\hline & & & Sig. & 0.019 & 0.000 & 0.000 \\
\hline
\end{tabular}

\section{Professional Relationships and Responsibilities}

Table 4.23: Level of confidence in ability to become successful teacher

\begin{tabular}{|c|c|c|c|c|c|c|c|}
\hline & $\begin{array}{c}\text { Very } \\
\text { confident } \\
1\end{array}$ & $\begin{array}{c}\text { Quite } \\
\text { confident } \\
2\end{array}$ & $\begin{array}{c}\text { Unsure } \\
3\end{array}$ & $\begin{array}{c}\text { Not very } \\
\text { confident } \\
4\end{array}$ & $\begin{array}{c}\text { Not at all } \\
\text { confident } \\
5\end{array}$ & Mean & SD \\
\hline $94(3)$ & 1 & 24 & 18 & 5 & 0 & 2.56 & 0.71 \\
\hline $94(1)$ & 4 & 19 & 11 & 5 & 1 & 2.50 & 0.93 \\
\hline $97(3)$ & 2 & 16 & 11 & 1 & 0 & 2.37 & 0.67 \\
\hline
\end{tabular}

\begin{tabular}{|c|c|c|c|c|c|c|}
\hline & Test & 3 groups & $\begin{array}{l}\text { Post- } \\
\text { hoc test }\end{array}$ & $\begin{array}{c}94(1) \\
\mathbf{v} \\
97(3)\end{array}$ & $\begin{array}{c}94(3) \\
v \\
97(3) \\
\end{array}$ & $\begin{array}{c}94(1) \\
v \\
94(3)\end{array}$ \\
\hline \multirow{2}{*}{$\begin{array}{l}\text { Confidence in } \\
\text { ability as teacher }\end{array}$} & $\mathrm{K}-\mathrm{W}$ & 1.028 & 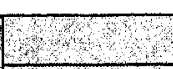 & 8 & I & 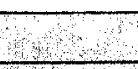 \\
\hline & Sig. & 0.598 & 28 & 28 & 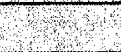 & 20 \\
\hline
\end{tabular}


Table 4.24: Opinions on status of teachers

\begin{tabular}{|c|c|c|c|c|c|c|}
\hline & $\begin{array}{c}\text { High status } \\
1 \\
\end{array}$ & $\begin{array}{c}\text { Moderate } \\
\text { status } \\
2 \\
\end{array}$ & $\begin{array}{c}\text { No special } \\
\text { status } \\
3 \\
\end{array}$ & $\begin{array}{c}\text { Status lower } \\
\text { than should } \\
\text { be } \\
4 \\
\end{array}$ & Mean & SD \\
\hline $94(3)$ & 1 & 15 & 17 & 15 & 2.96 & 0.85 \\
\hline $94(1)$ & 2 & 23 & 10 & 5 & 2.45 & 0.78 \\
\hline $97(3)$ & 3 & 13 & 8 & 6 & 2.57 & 0.93 \\
\hline
\end{tabular}

\begin{tabular}{|c|c|c|c|c|c|c|}
\hline & Test & 3 groups & $\begin{array}{l}\text { Post- } \\
\text { hoc test }\end{array}$ & $\begin{array}{c}94(1) \\
v \\
97(3)\end{array}$ & $\begin{array}{c}94(3) \\
v \\
97(3)\end{array}$ & $\begin{array}{c}94(1) \\
v \\
94(3)\end{array}$ \\
\hline \multirow[t]{2}{*}{ Status of teachers } & $\mathrm{K}-\mathrm{W}$ & 8.384 & $\mathrm{M}-\mathrm{W}$ & 0.316 & 3.343 & 7.978 \\
\hline & Sig. & 0.015 & Sig. & 0.574 & 0.068 & 0.005 \\
\hline
\end{tabular}

Table 4.25: Best attributes as teacher

\begin{tabular}{|c|c|c|c|c|c|c|c|c|c|c|c|}
\hline Item & anking & 1 & 2 & 3 & 4 & 5 & 6 & 7 & 8 & 9 & Mean \\
\hline \multirow{3}{*}{$\begin{array}{l}\text { Good command } \\
\text { of language }\end{array}$} & $94(3)$ & 18 & 6 & 5 & 3 & 6 & 4 & 2 & 0 & 2 & 3.11 \\
\hline & 94(1) & 14 & 6 & 9 & 3 & 1 & 3 & 2 & 1 & 0 & 2.82 \\
\hline & $97(3)$ & 15 & 6 & 5 & 0 & 1 & 2 & 1 & 0 & 0 & 2.20 \\
\hline \multirow{3}{*}{$\begin{array}{l}\text { Good } \\
\text { communication } \\
\text { skills }\end{array}$} & & & & & & & & & & & \\
\hline & \begin{tabular}{|l}
$94(3)$ \\
$94(1)$
\end{tabular} & $\frac{7}{10}$ & $\frac{14}{8}$ & $\frac{7}{5}$ & $\frac{8}{5}$ & $\frac{7}{3}$ & $\frac{3}{3}$ & $\frac{1}{2}$ & $\frac{1}{0}$ & $\frac{0}{0}$ & $\frac{3.25}{3.00}$ \\
\hline & $97(3)$ & 8 & 12 & 3 & 2 & 2 & 1 & $\frac{2}{1}$ & 0 & 0 & 2.48 \\
\hline & & & & & & & & & & & \\
\hline \multirow{3}{*}{$\begin{array}{l}\text { Innovative in } \\
\text { terms of teaching } \\
\text { ideas }\end{array}$} & $94(3)$ & 5 & 8 & 9 & 12 & 6 & 5 & 0 & 1 & 1 & 3.68 \\
\hline & $94(1)$ & 6 & 9 & 7 & 6 & 4 & 4 & 1 & 0 & 0 & 3.24 \\
\hline & $97(3)$ & 2 & 5 & 12 & 4 & 4 & 2 & 1 & 0 & 0 & 3.43 \\
\hline \multirow{3}{*}{$\begin{array}{l}\text { Strict } \\
\text { disciplinarian }\end{array}$} & $94(3)$ & 1 & 0 & 3 & 3 & 2 & 8 & 7 & 22 & 1 & 6.68 \\
\hline & $94(1)$ & 1 & 1 & 2 & 1 & 3 & 2 & 6 & 22 & 1 & 6.85 \\
\hline & $97(3)$ & 0 & 0 & 1 & 3 & 2 & 2 & 2 & 16 & 1 & 7.03 \\
\hline
\end{tabular}




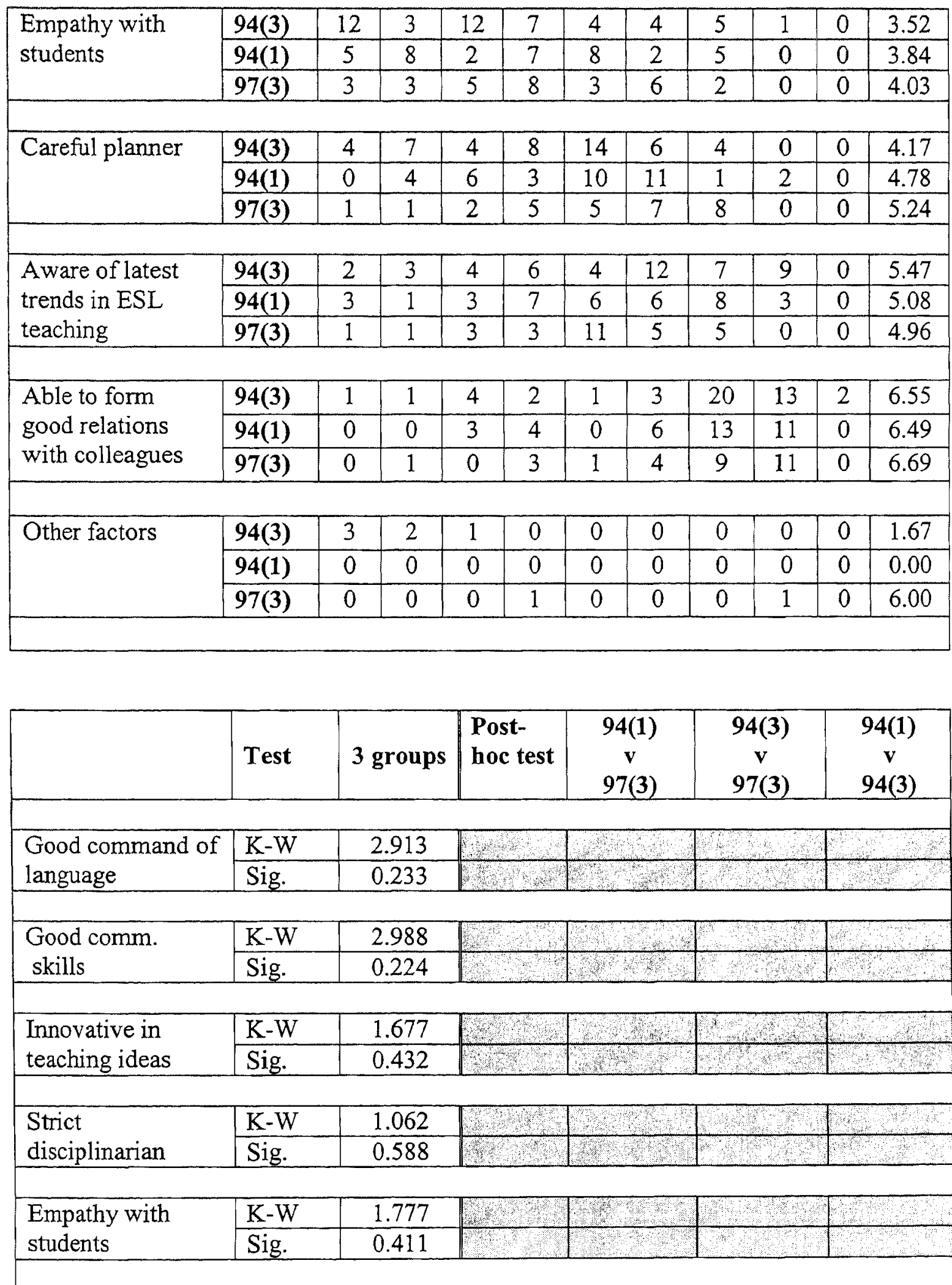




\begin{tabular}{|l|l|l|l|l|l|l|}
\hline Careful planner & $\mathrm{K}-\mathrm{W}$ & 7.515 & $\mathrm{M}-\mathrm{W}$ & 1.824 & 6.743 & 2.570 \\
\cline { 2 - 7 } & Sig. & 0.023 & Sig. & 0.177 & 0.009 & 0.109 \\
\hline \multirow{2}{|c|}{$\begin{array}{l}\text { Aware of latest } \\
\text { trends in TESL }\end{array}$} & $\mathrm{K}-\mathrm{W}$ & 2.201 & & & & \\
\hline & Sig. & 0.333 & & & & \\
\hline \multirow{2}{*}{$\begin{array}{l}\text { Good relations } \\
\text { with colleagues }\end{array}$} & $\mathrm{K}-\mathrm{W}$ & 0.470 & & & & \\
\cline { 2 - 7 } & Sig. & 0.791 & & & & \\
\hline & & & Corr. & 0.976 & 0.952 & 0.976 \\
\hline & & Sig. & 0.000 & 0.000 & 0.000 \\
\hline
\end{tabular}

Table 4.26: Views on teaching as a long-term career

\begin{tabular}{|c|c|c|c|c|c|c|}
\hline & $\begin{array}{c}\text { Yes, } \\
\text { definitely } \\
1\end{array}$ & $\begin{array}{c}\text { Probably } \\
2 \\
\end{array}$ & $\begin{array}{c}\text { Not sure } \\
3\end{array}$ & $\begin{array}{c}\text { Definitely } \\
\text { not } \\
4\end{array}$ & Mean & SD \\
\hline $94(3)$ & 4 & 10 & 23 & 11 & 2.85 & 0.87 \\
\hline $94(1)$ & 6 & 13 & 19 & 2 & 2.43 & 0.81 \\
\hline $97(3)$ & $\overline{6}$ & 10 & 12 & 2 & 2.33 & 0.88 \\
\hline
\end{tabular}

\begin{tabular}{|c|c|c|c|c|c|c|}
\hline & Test & 3 groups & $\begin{array}{l}\text { Post- } \\
\text { hoc test }\end{array}$ & $\begin{array}{c}94(1) \\
v \\
97(3)\end{array}$ & $\begin{array}{c}94(3) \\
v \\
97(3)\end{array}$ & $\begin{array}{c}94(1) \\
v \\
94(3)\end{array}$ \\
\hline \multirow{2}{*}{$\begin{array}{l}\text { Teaching as a } \\
\text { career }\end{array}$} & $\mathrm{K}-\mathrm{W}$ & 8.328 & $\mathrm{M}-\mathrm{W}$ & 0.221 & 6.134 & 5.527 \\
\hline & Sig. & 0.016 & Sig. & 0.638 & 0.013 & 0.019 \\
\hline
\end{tabular}


Table 4.27: People who would be most helpful in first years of teaching

\begin{tabular}{|l|c|c|c|c|c|c|}
\hline \multirow{2}{*}{ Item } & \multicolumn{2}{|c|}{$94(3)$} & \multicolumn{2}{c|}{ 94(1) } & \multicolumn{2}{c|}{ 97(3) } \\
& Yes & No & No & Yes & Yes & No \\
\hline & & & & & & \\
\hline Fellow first-year teachers & 5 & 43 & 8 & 32 & 7 & 23 \\
\hline More exp. teachers & 34 & 14 & 27 & 13 & 17 & 13 \\
\hline Panel chair & 4 & 44 & 1 & 39 & 6 & 24 \\
\hline Principal & 1 & 47 & 1 & 39 & 0 & 30 \\
\hline Senior teachers & 5 & 43 & 5 & 35 & 6 & 24 \\
\hline Teach-training supervisor & 6 & 42 & 4 & 36 & 4 & 26 \\
\hline & & & & & & \\
\hline
\end{tabular}

\begin{tabular}{|c|c|c|c|c|c|c|}
\hline & Test & 3 groups & $\begin{array}{l}\text { Post- } \\
\text { hoc test }\end{array}$ & $\begin{array}{c}94(1) \\
\mathbf{v} \\
97(3)\end{array}$ & $\begin{array}{c}94(3) \\
v \\
97(3) \\
\end{array}$ & $\begin{array}{c}94(1) \\
v \\
94(3)\end{array}$ \\
\hline \multirow{2}{*}{$\begin{array}{l}\text { Fellow first-year } \\
\text { teachers }\end{array}$} & Chi-sq. & 2.588 & & 3 & 7 & \\
\hline & Sig. & 0.274 & 2 & 7 & 2 & \\
\hline \multirow{3}{*}{$\begin{array}{l}\text { More exp. } \\
\text { teachers }\end{array}$} & & & & & & \\
\hline & Chi-sq. & 1.706 & 2 & (1) & 24 & 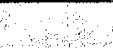 \\
\hline & Sig. & 0.426 & 3 & 5 & 5 & \\
\hline \multirow{2}{*}{ Panel chair } & Chi-sq. & 6304 & Chi-sa & 4051 & 1336 & 0511 \\
\hline & Sig. & 0.043 & Sig. & 0.044 & 0.250 & 0.475 \\
\hline \multirow{2}{*}{ Principal * } & & & & & & \\
\hline & & & 2 & & 5 & \\
\hline \multirow{2}{*}{ Senior teachers } & Chi-s & 1505 & & & & \\
\hline & Sig. & 0.471 & + & & & \\
\hline \multirow{2}{*}{$\begin{array}{l}\text { Teach-training } \\
\text { supervisor }\end{array}$} & $\mathrm{Chi}_{-} \mathrm{s}$ & 0213 & Chi-ga & 0003 & 0 & 0001 \\
\hline & Sig. & 0.899 & \begin{tabular}{|l} 
Sig. \\
\end{tabular} & 0.957 & 1.000 & 0.976 \\
\hline
\end{tabular}

* As at least one cell in $2 \times 3$ table had a value of zero, chi-square could not be considered reliable and so was not carried out. 
Table 4.28: Expectations of first-year teaching activities

\begin{tabular}{|l|c|c|c|c|c|c|}
\hline \multirow{2}{*}{ Item } & \multicolumn{2}{c|}{ 94(3) } & \multicolumn{2}{c|}{ 94(1) } & \multicolumn{2}{c|}{ 97(3) } \\
& Yes & No & Yes & No & Yes & No \\
\hline & & & & & & \\
\hline Be a form teacher & 35 & 13 & 14 & 26 & 23 & 7 \\
\hline Be involved in Eng. Club & 34 & 14 & 25 & 15 & 22 & 8 \\
\hline Train students for speech & 14 & 34 & 9 & 31 & 13 & 17 \\
\hline ECA & 37 & 11 & 31 & 9 & 24 & 6 \\
\hline Religious activities & 6 & 42 & 7 & 33 & 5 & 25 \\
\hline Counsel students & 24 & 24 & 26 & 14 & 18 & 12 \\
\hline Meet parents & 30 & 18 & 14 & 26 & 22 & 8 \\
\hline Meetings with superiors & 37 & 11 & 17 & 23 & 26 & 4 \\
\hline Meetings with colleagues & 36 & 12 & 27 & 13 & 28 & 2 \\
\hline Attend courses & 14 & 34 & 22 & 18 & 16 & 14 \\
\hline Mark papers & 41 & 7 & 26 & 14 & 28 & 2 \\
\hline Prepare lessons & 46 & 2 & 32 & 8 & 27 & 3 \\
\hline Prepare examinations & 41 & 7 & 24 & 16 & 26 & 4 \\
\hline Administer examinations & 35 & 13 & 11 & 29 & 21 & 9 \\
\hline Mark examinations & 43 & 5 & 29 & 11 & 28 & 2 \\
\hline & & & & & & \\
\hline
\end{tabular}

\begin{tabular}{|c|c|c|c|c|c|c|}
\hline & Test & 3 groups & $\begin{array}{l}\text { Post- } \\
\text { hoc test }\end{array}$ & $\begin{array}{c}94(1) \\
v \\
97(3)\end{array}$ & $\begin{array}{c}94(3) \\
v \\
97(3) \\
\end{array}$ & $\begin{array}{c}94(1) \\
v \\
94(3) \\
\end{array}$ \\
\hline \multirow{2}{*}{$\begin{array}{l}\text { Be a form } \\
\text { teacher }\end{array}$} & Chi-sq. & 17.32 & Chi-sq. & 10.33 & 0.011 & 11.22 \\
\hline & Sig. & 0.000 & Sig. & 0.001 & 0.918 & 0.001 \\
\hline \multirow{2}{*}{$\begin{array}{l}\text { Be involved in } \\
\text { English Club }\end{array}$} & Chi-sq. & 1.115 & Whe & & $\overline{3}$ & 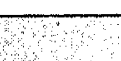 \\
\hline & Sig. & 0.573 & 5 & 5 & $2 \quad$ & \\
\hline \multirow{2}{*}{$\begin{array}{l}\text { Train students } \\
\text { for speech fest. }\end{array}$} & Chi-sq. & 3.578 & 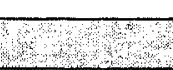 & & 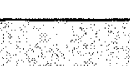 & \\
\hline & Sig. & 0.167 & & & & 2 \\
\hline \multirow{2}{*}{$\begin{array}{l}\text { Extra-curricular } \\
\text { activities }\end{array}$} & Chi-sq. & 0.099 & 2 & 25 & 50 & 4 \\
\hline & Sig. & 0.952 & 5 & 5 & 5 & 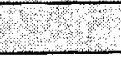 \\
\hline \multirow{2}{*}{$\begin{array}{l}\text { Religious } \\
\text { activities }\end{array}$} & Chi-sq. & 0.484 & 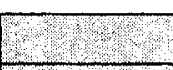 & & 7 & \\
\hline & Sig. & 0.785 & & & 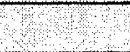 & \\
\hline
\end{tabular}




\begin{tabular}{|c|c|c|c|c|c|c|}
\hline \multirow[t]{2}{*}{ Counsel students } & Chi-sq. & 2.103 & 5 & 5 & DQ1 & \\
\hline & Sig. & 0.349 & & 5 & 5 & \\
\hline \multirow{2}{*}{ Meet parents } & Chi-sa. & 11.63 & Chi-sq. & 8.608 & 0.548 & 5.546 \\
\hline & Sig. & 0.003 & Sig. & 0.003 & 0.495 & 0.019 \\
\hline \multirow{2}{*}{$\begin{array}{l}\text { Meetings with } \\
\text { principal/panel }\end{array}$} & Chi-sq. & 18.51 & Chi-sq. & 12.31 & 0.562 & 9.596 \\
\hline & Sig. & 0.000 & Sig. & 0.000 & 0.454 & 0.002 \\
\hline \multirow{2}{*}{$\begin{array}{l}\text { Meetings with } \\
\text { colleagues }\end{array}$} & Chi-sq. & 6.689 & Chi-sq. & 5.347 & 3.060 & 0.291 \\
\hline & Sig. & 0.035 & Sig. & 0.021 & 0.060 & 0.590 \\
\hline \multirow{2}{*}{ Attend courses } & Chi-sa & 7309 & $\mathrm{Cl}_{-\mathrm{ca}}$ & 0000 & 3507 & 5002 \\
\hline & Sig. & 0.026 & Sig. & 1.000 & 0.058 & 0.025 \\
\hline & & & & & & \\
\hline \multirow[t]{2}{*}{ Mark papers } & Chi-sq. & 10.01 & Chi-sq. & 6.281 & 0.491 & 3.945 \\
\hline & Sig. & 0.007 & Sig. & 0.012 & 0.484 & 0.047 \\
\hline \multirow{2}{*}{ Prepare lessons } & Chi-sq. & 5.622 & & 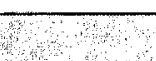 & 5 & 43 \\
\hline & Sig. & 0.060 & 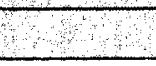 & 0 & 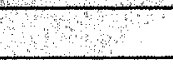 & 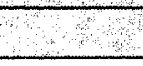 \\
\hline \multirow{2}{*}{$\begin{array}{l}\text { Prepare } \\
\text { examinations }\end{array}$} & Chi-sq. & 10.07 & Chi-sq. & 4.738 & 0.000 & 6.044 \\
\hline & Sig. & 0.007 & Sig. & 0.030 & 1.000 & 0.014 \\
\hline \multirow{2}{*}{$\begin{array}{l}\text { Administer } \\
\text { examinations }\end{array}$} & Chi-sq. & 21.20 & Chi-sq. & 10.824 & 0.000 & 16.26 \\
\hline & Sig. & 0.000 & Sig. & 0.001 & 0.984 & 0.000 \\
\hline \multirow{2}{*}{$\begin{array}{l}\text { Mark } \\
\text { examinations }\end{array}$} & Chi-sq. & 7.220 & Chi-sg. & 3.639 & 0.025 & 3.209 \\
\hline & Sig. & 0.027 & Sig. & 0.056 & 0.876 & 0.073 \\
\hline
\end{tabular}


Table 4.29: Expectations of which first-year teaching activities would take up the most time

\begin{tabular}{|l|c|c|c|c|c|c|}
\hline \multirow{2}{*}{ Item } & \multicolumn{2}{c|}{ 94(3) } & \multicolumn{2}{c|}{ 94(1) } & \multicolumn{2}{c|}{ 97(3) } \\
& Yes & No & Yes & No & Yes & No \\
\hline & & & & & & \\
\hline Be a form teacher & 16 & 32 & 17 & 23 & 13 & 17 \\
\hline Be involved in Eng. Club & 4 & 44 & 7 & 33 & 5 & 25 \\
\hline Train students for speech & 0 & 48 & 1 & 39 & 2 & 28 \\
\hline ECA & 8 & 40 & 8 & 32 & 6 & 24 \\
\hline Religious activities & 0 & 48 & 2 & 38 & 0 & 30 \\
\hline Counsel students & 8 & 40 & 13 & 27 & 7 & 23 \\
\hline Meet parents & 0 & 48 & 1 & 39 & 2 & 28 \\
\hline Meetings with superiors & 8 & 40 & 4 & 36 & 4 & 26 \\
\hline Meetings with colleagues & 7 & 41 & 2 & 38 & 4 & 26 \\
\hline Attend courses & 4 & 44 & 4 & 36 & 3 & 27 \\
\hline Mark papers & 26 & 22 & 6 & 34 & 19 & 11 \\
\hline Prepare lessons & 27 & 21 & 18 & 22 & 17 & 13 \\
\hline Prepare examinations & 11 & 37 & 10 & 30 & 14 & 16 \\
\hline Administer examinations & 3 & 45 & 0 & 40 & 3 & 27 \\
\hline Mark examinations & 15 & 33 & 9 & 31 & 13 & 17 \\
\hline & & & & & & \\
\hline
\end{tabular}

\begin{tabular}{|c|c|c|c|c|c|c|}
\hline & Test & 3 groups & $\begin{array}{l}\text { Post- } \\
\text { hoc test }\end{array}$ & $\begin{array}{c}94(1) \\
\mathbf{v} \\
97(3)\end{array}$ & $\begin{array}{c}94(3) \\
\mathbf{v} \\
97(3) \\
\end{array}$ & $\begin{array}{c}94(1) \\
v \\
94(3)\end{array}$ \\
\hline \multirow{2}{*}{$\begin{array}{l}\text { Be a form } \\
\text { teacher }\end{array}$} & Chi-sq. & 1.091 & 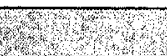 & $x_{2}$ & +8 & 19: \\
\hline & Sig. & 0.580 & 16 & & & ? \\
\hline \multirow{2}{*}{$\begin{array}{l}\text { Be involved in } \\
\text { English Club }\end{array}$} & Chi-sq. & 1.896 & 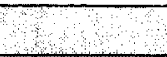 & & & \\
\hline & Sig. & 0.388 & & & & \\
\hline \multirow{2}{*}{$\begin{array}{l}\text { Train students } \\
\text { for speech fest* }\end{array}$} & Chi-sq. & & & 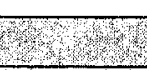 & 3 & $E$ \\
\hline & Sig. & & 2 & 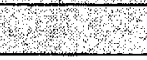 & 8 & \\
\hline \multirow{2}{*}{$\begin{array}{l}\text { Extra-curricular } \\
\text { activities }\end{array}$} & Chi-sq. & 0.209 & & $\sqrt{3}$ & 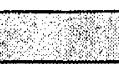 & 3 \\
\hline & Sig. & 0.901 & ? & $\sqrt{13}$ & 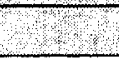 & \\
\hline \multirow{2}{*}{$\begin{array}{l}\text { Religious } \\
\text { activities * }\end{array}$} & Chi-sq. & 15. & & & & \\
\hline & Sig. & 5 & & & & \\
\hline
\end{tabular}




\begin{tabular}{|l|l|l|l|l|l|l|}
\hline \multirow{2}{*}{ Counsel students } & Chi-sq. & 3.026 & & & \\
\hline & Sig. & 0.220 & & & \\
\hline \multirow{5}{*}{ Meet parents * } & Chi-sq. & & & & \\
\hline & Sig. & & & & \\
\hline
\end{tabular}

* As at least one cell in $2 \times 3$ table had a value of zero, chi-square could not be considered reliable and so was not carried out. 
Table 4.30: Views on extent to which they might work closely with other teachers in the future

\begin{tabular}{|l|c|c|c|c|c|c|}
\hline & $\begin{array}{c}\text { Very much } \\
1\end{array}$ & $\begin{array}{c}\text { Some } \\
2\end{array}$ & $\begin{array}{c}\text { A little } \\
3\end{array}$ & $\begin{array}{c}\text { Not at all } \\
4\end{array}$ & Mean & SD \\
\hline & & & & & & \\
\hline $\mathbf{9 4 ( 3 )}$ & 9 & 33 & 6 & 0 & $\mathbf{1 . 9 4}$ & $\mathbf{0 . 5 6}$ \\
\hline $\mathbf{9 4 ( 1 )}$ & 25 & 14 & 1 & 0 & $\mathbf{1 . 4 0}$ & $\mathbf{0 . 5 4}$ \\
\hline $\mathbf{9 7 ( 3 )}$ & 16 & 13 & 1 & 0 & $\mathbf{1 . 5 0}$ & $\mathbf{0 . 5 7}$ \\
\hline & & & & & & \\
\hline
\end{tabular}

\begin{tabular}{|c|c|c|c|c|c|c|}
\hline & Test & 3 groups & $\begin{array}{l}\text { Post- } \\
\text { hoc test }\end{array}$ & $\begin{array}{c}94(1) \\
v \\
97(3)\end{array}$ & $\begin{array}{c}94(3) \\
v \\
97(3)\end{array}$ & $\begin{array}{c}94(1) \\
\text { v } \\
94(3)\end{array}$ \\
\hline \multirow{2}{*}{$\begin{array}{l}\text { Work with other } \\
\text { teachers }\end{array}$} & $\mathrm{K}-\mathrm{W}$ & 20.00 & $\mathrm{M}-\mathrm{W}$ & 0.585 & 10.21 & 17.65 \\
\hline & Sig. & 0.000 & Sig. & 0.444 & 0.001 & 0.000 \\
\hline
\end{tabular}


Table 4.31: What will be of most help in terms of development as a teacher in first year?

\begin{tabular}{|c|c|c|c|c|c|c|c|c|c|}
\hline Item & anking & 1 & 2 & 3 & 4 & 5 & 6 & 7 & Mean \\
\hline \multirow[t]{3}{*}{ Reading articles } & $94(3)$ & 3 & 5 & 4 & 8 & 14 & 10 & 1 & 4.31 \\
\hline & $94(1)$ & 3 & 1 & 4 & 6 & 7 & 17 & 0 & 4.68 \\
\hline & $97(3)$ & 7 & 1 & 4 & 7 & 3 & 7 & 0 & 3.65 \\
\hline \multirow{3}{*}{$\begin{array}{l}\text { Finding new } \\
\text { materials }\end{array}$} & $94(3)$ & 7 & 15 & 12 & 5 & 4 & 2 & 0 & 2.78 \\
\hline & $\begin{array}{l}94(1) \\
\end{array}$ & 2 & 11 & 5 & 8 & 10 & 1 & 0 & 3.43 \\
\hline & \begin{tabular}{|l|}
$97(3)$ \\
\end{tabular} & 5 & 6 & 8 & 6 & 4 & 0 & 0 & 2.93 \\
\hline \multirow{3}{*}{$\begin{array}{l}\text { Designing new } \\
\text { materials }\end{array}$} & $04(3)$ & 6 & & & 12 & & & 0 & \\
\hline & \begin{tabular}{|l|}
$4(1)$ \\
$94(1)$ \\
\end{tabular} & 4 & $\frac{0}{5}$ & $\frac{10}{7}$ & $\frac{10}{9}$ & 6 & 7 & 0 & 3.76 \\
\hline & \begin{tabular}{|l|}
$97(3)$ \\
\end{tabular} & 3 & 4 & 4 & 2 & 10 & 6 & 0 & 4.03 \\
\hline & & & & & & & & & \\
\hline \multirow{3}{*}{$\begin{array}{l}\text { Learning from } \\
\text { other teachers }\end{array}$} & 94(3) & 24 & 9 & 4 & 4 & 5 & 2 & 0 & 2.23 \\
\hline & $94(1)$ & 18 & 9 & 4 & 4 & 3 & 0 & 0 & 2.08 \\
\hline & $97(3)$ & 12 & 9 & 5 & 2 & 0 & 2 & 0 & 2.17 \\
\hline \multirow{3}{*}{$\begin{array}{l}\text { Getting feedback } \\
\text { from panel chair }\end{array}$} & $94(3)$ & 4 & 11 & 8 & 8 & 8 & 7 & 0 & 3.56 \\
\hline & \begin{tabular}{|l|}
$94(1)$ \\
\end{tabular} & 3 & 6 & 11 & 5 & 5 & 7 & 0 & 3.65 \\
\hline & $97(3)$ & 1 & 9 & 3 & 9 & 2 & 6 & 0 & 3.67 \\
\hline \multirow{3}{*}{$\begin{array}{l}\text { Attending } \\
\text { conferences etc. }\end{array}$} & $94(3)$ & 5 & 3 & 8 & 5 & 9 & 15 & 0 & 4.29 \\
\hline & \begin{tabular}{|l|l|}
$94(1)$ \\
\end{tabular} & 9 & 5 & 6 & 5 & 6 & 7 & 0 & 3.39 \\
\hline & $97(3)$ & 2 & 1 & 6 & 3 & 10 & 8 & 0 & 4.40 \\
\hline \multirow[t]{3}{*}{ Others } & 94(3) & 1 & 0 & 0 & 0 & 0 & 0 & 0 & 1.00 \\
\hline & \begin{tabular}{|l|}
$94(1)$ \\
\end{tabular} & 0 & 0 & 0 & 0 & 0 & 0 & 0 & 0.00 \\
\hline & $97(3)$ & 0 & 0 & 0 & 0 & 0 & 0 & 0 & 0.00 \\
\hline
\end{tabular}




\begin{tabular}{|c|c|c|c|c|c|c|}
\hline & Test & 3 groups & $\begin{array}{l}\text { Post- } \\
\text { hoc test }\end{array}$ & $\begin{array}{c}94(1) \\
v \\
97(3) \\
\end{array}$ & $\begin{array}{c}94(3) \\
v \\
97(3)\end{array}$ & $\begin{array}{c}94(1) \\
v \\
94(3) \\
\end{array}$ \\
\hline \multirow[t]{2}{*}{ Reading articles } & $\mathrm{K}-\mathrm{W}$ & 5.931 & (x) & 65 & & \\
\hline & Sig. & 0.052 & & $0 \quad$ & & \\
\hline \multirow{2}{*}{$\begin{array}{l}\text { Finding new } \\
\text { materials }\end{array}$} & $\mathrm{K}-\mathrm{W}$ & 4.747 & 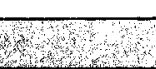 & c. & 1. & \\
\hline & Sig. & 0.093 & 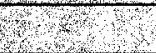 & $12 \times$ & & \\
\hline \multirow{2}{*}{$\begin{array}{l}\text { Designing new } \\
\text { materials }\end{array}$} & $\mathrm{K}-\mathrm{W}$ & 2.055 & 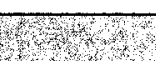 & $\sqrt{3 x}$ & 72 & $8 \times$ \\
\hline & Sig. & 0.358 & 12 & 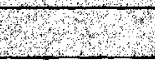 & 5 & \\
\hline \multirow{2}{*}{$\begin{array}{l}\text { Learning from } \\
\text { other teachers }\end{array}$} & $\mathrm{K}-\mathrm{W}$ & 0.148 & 6 & Wex & m & 28 \\
\hline & Sig. & 0.928 & (2) & 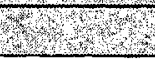 & & \\
\hline \multirow{2}{*}{$\begin{array}{l}\text { Getting feedback } \\
\text { from panel chair }\end{array}$} & $\mathrm{K}-\mathrm{W}$ & 0.092 & s? & try & 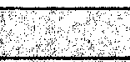 & \\
\hline & Sig. & 0.955 & 5 & 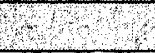 & & \\
\hline \multirow{2}{*}{$\begin{array}{l}\text { Attending } \\
\text { conferences etc. }\end{array}$} & $\mathrm{K}-\mathrm{W}$ & 6.105 & $\mathrm{M}-\mathrm{W}$ & 4.900 & 0.045 & 4.197 \\
\hline & Sig. & 0.047 & Sig. & 0.027 & 0.833 & 0.040 \\
\hline \multirow{2}{*}{ 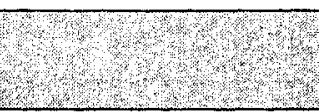 } & - & a & Corr. & 0.657 & 0.657 & 0.257 \\
\hline & 3. & 36. & Sig. & 0.156 & 0.156 & 0.623 \\
\hline
\end{tabular}




\section{Perceptions and Values}

Table 4.32: Opinions as to whether they have a definite philosophy of teaching

\begin{tabular}{|l|c|c|c|c|c|c|}
\hline & $\begin{array}{c}\text { Yes, very } \\
\text { definite } \\
1\end{array}$ & $\begin{array}{c}\text { Quite } \\
\text { definite } \\
2\end{array}$ & $\begin{array}{c}\text { Not very } \\
\text { definite } \\
3\end{array}$ & $\begin{array}{c}\text { Not definite } \\
\text { at all } \\
4\end{array}$ & Mean & SD \\
\hline $\mathbf{9 4 ( 3 )}$ & 4 & 21 & 20 & 3 & 2.46 & 0.74 \\
\hline $\mathbf{9 4 ( 1 )}$ & 3 & 18.5 & 17.5 & 1 & 2.41 & 0.67 \\
\hline $\mathbf{9 7 ( 3 )}$ & 9 & 14 & 6 & 1 & 1.97 & 0.81 \\
\hline & & & & & & \\
\hline
\end{tabular}

\begin{tabular}{|c|c|c|c|c|c|c|}
\hline & Test & 3 groups & $\begin{array}{l}\text { Post- } \\
\text { hoc test }\end{array}$ & $\begin{array}{c}94(1) \\
v \\
97(3)\end{array}$ & $\begin{array}{c}94(3) \\
v \\
97(3)\end{array}$ & $\begin{array}{c}94(1) \\
v \\
94(3)\end{array}$ \\
\hline \multirow{2}{*}{$\begin{array}{l}\text { Definite teaching } \\
\text { philosophy }\end{array}$} & $\mathrm{K}-\mathrm{W}$ & 8.596 & $M-W$ & 6.262 & 7.147 & 0.059 \\
\hline & Sig. & 0.014 & Sig. & 0.012 & 0.008 & 0.808 \\
\hline
\end{tabular}

Table 4.33: Factors that subjects felt unsure about

\begin{tabular}{|l|c|c|c|c|c|c|}
\hline \multirow{2}{*}{ Item } & \multicolumn{2}{c|}{ 94(3) } & \multicolumn{2}{c|}{ 94(1) } & \multicolumn{2}{c|}{ 97(3) } \\
& Yes & No & Yes & No & Yes & No \\
\hline & & & & & & \\
\hline Personal career goals & 21 & 27 & 12 & 28 & 16 & 14 \\
\hline Ideas on teaching content & 7 & 41 & 10 & 30 & 8 & 22 \\
\hline Ideas on teaching meths. & 9 & 39 & 11 & 29 & 6 & 24 \\
\hline Ways to handle discipline & 16 & 32 & 10 & 30 & 12 & 18 \\
\hline Professional relationships & 7 & 41 & 4 & 36 & 10 & 20 \\
\hline Relationships with stdnts. & 0 & 48 & 5 & 35 & 12 & 18 \\
\hline Professional respons. & 7 & 41 & 4 & 36 & 4 & 26 \\
\hline Professional status & 7 & 41 & 2 & 38 & 10 & 20 \\
\hline Working conditions & 8 & 40 & 6 & 34 & 6 & 24 \\
\hline Salary & 3 & 45 & 3 & 37 & 0 & 30 \\
\hline & & & & & & \\
\hline
\end{tabular}




\begin{tabular}{|c|c|c|c|c|c|c|}
\hline & Test & 3 groups & \begin{tabular}{|l|} 
Post- \\
hoc test
\end{tabular} & $\begin{array}{c}94(1) \\
\mathbf{v} \\
97(3)\end{array}$ & $\begin{array}{c}94(3) \\
v \\
97(3)\end{array}$ & $\begin{array}{c}94(1) \\
v \\
94(3)\end{array}$ \\
\hline \multirow{2}{*}{$\begin{array}{l}\text { Personal career } \\
\text { goals }\end{array}$} & Chi-sq. & 4.009 & & 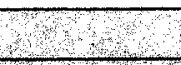 & & \\
\hline & Sig. & 0.135 & 2 & 28 & 2 & 0 \\
\hline \multirow[t]{2}{*}{ Ideas on content } & Chi-sq. & 2.141 & res? & & 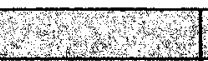 & 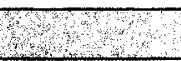 \\
\hline & Sig. & 0.343 & Fen & 25 & 20 & 2 \\
\hline \multirow[t]{2}{*}{ Ideas on methods } & Chi-sq. & 1.069 & $\sqrt{20}$ & & & $5 \%$ \\
\hline & Sig. & 0.586 & 2 & 8 & 4 & \\
\hline \multirow{2}{*}{$\begin{array}{l}\text { Ways to handle } \\
\text { discipline }\end{array}$} & Chi-sq. & 1.814 & Den & 3 & 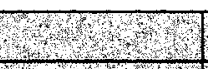 & 4 \\
\hline & Sig. & 0.404 & 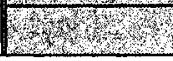 & min & (2) & ry \\
\hline \multirow{2}{*}{$\begin{array}{l}\text { Professional } \\
\text { relationships }\end{array}$} & Chi-sq. & 6.951 & Chi-sg. & 4.466 & 2.787 & 0.105 \\
\hline & Sig. & 0.031 & Sig. & 0.035 & 0.095 & 0.746 \\
\hline \multirow{2}{*}{$\begin{array}{l}\text { Relationships } \\
\text { with students * }\end{array}$} & & & S & & & \\
\hline & & 然 & & 8 & & \\
\hline \multirow{2}{*}{$\begin{array}{l}\text { Professional } \\
\text { responsibilities }\end{array}$} & Chi-sq. & 0.427 & & 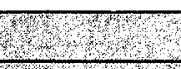 & 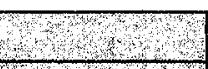 & \\
\hline & Sig. & 0.808 & 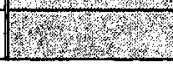 & (1) & (1) & 10 \\
\hline \multirow{2}{*}{$\begin{array}{l}\text { Professional } \\
\text { status }\end{array}$} & Chi-sq. & 10.32 & Chi-sq. & 7.797 & 2.787 & 1.263 \\
\hline & Sig. & 0.006 & Sig. & 0.005 & 0.095 & 0.261 \\
\hline \multirow{2}{*}{$\begin{array}{l}\text { Working } \\
\text { conditions }\end{array}$} & Chi-sq. & 0.309 & 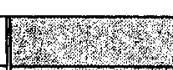 & 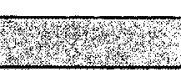 & (5) & \\
\hline & Sig. & 0.857 & 12 & $=2$ & 54 & \\
\hline \multirow[t]{2}{*}{ Salary* } & $+\quad$ & & & & & \\
\hline & & & & & & \\
\hline
\end{tabular}

* As at least one cell in the $2 \times 3$ table had a quantity of zero, chi-square could not be considered reliable and was not carried out. 
Table 4.34: Most important factors affecting what goes on in the classroom

\begin{tabular}{|c|c|c|c|c|c|}
\hline Item & anking & 1 & 2 & 3 & Mean \\
\hline \multirow{3}{*}{$\begin{array}{l}\text { Motivation of } \\
\text { students }\end{array}$} & $94(3)$ & 28 & 16 & 3 & 1.47 \\
\hline & $94(1)$ & 27 & 8 & 4 & 1.41 \\
\hline & $97(3)$ & 25 & 4 & 0 & 1.14 \\
\hline \multirow{3}{*}{$\begin{array}{l}\text { Language ability } \\
\text { of students }\end{array}$} & $94(3)$ & 25 & 13 & 7 & 160 \\
\hline & $94(1)$ & 8 & 12 & 16 & 2.22 \\
\hline & $97(3)$ & 17 & 8 & 3 & 1.50 \\
\hline \multirow{3}{*}{$\begin{array}{l}\text { Efficiency of } \\
\text { English } \\
\text { department }\end{array}$} & $94(3)$ & 5 & 14 & 19 & 2.37 \\
\hline & $94(1)$ & 6 & 13 & 14 & 2.24 \\
\hline & $97(3)$ & 8 & 4 & 13 & 2.20 \\
\hline \multirow{3}{*}{$\begin{array}{l}\text { Competence of } \\
\text { teacher }\end{array}$} & $94(3)$ & 23 & 12 & 8 & 1.65 \\
\hline & $94(1)$ & 10 & 14 & 13 & 2.08 \\
\hline & $97(3)$ & 21 & 4 & 2 & 1.30 \\
\hline \multirow{3}{*}{$\begin{array}{l}\text { Backup of } \\
\text { colleagues }\end{array}$} & $94(3)$ & 1 & 23 & $\overline{14}$ & 2.34 \\
\hline & $94(1)$ & 2 & 21 & 9 & 2.22 \\
\hline & $97(3)$ & 7 & 9 & 9 & 2.08 \\
\hline \multirow{3}{*}{$\begin{array}{l}\text { Design of } \\
\text { classroom }\end{array}$} & $94(3)$ & 2 & 6 & 30 & 274 \\
\hline & $94(1)$ & 1 & 4 & 28 & 2.82 \\
\hline & $97(3)$ & 3 & 4 & 16 & 2.56 \\
\hline \multirow{3}{*}{ Class size } & $94(3)$ & 7 & 16 & 16 & 2.23 \\
\hline & $94(1)$ & 5 & 12 & 16 & 2.33 \\
\hline & $97(3)$ & 12 & 7 & 5 & 1.71 \\
\hline \multirow{3}{*}{$\begin{array}{l}\text { Location of } \\
\text { school }\end{array}$} & $94(3)$ & 4 & 5 & 28 & 2.65 \\
\hline & $94(1)$ & 0 & 5 & 27 & 2.84 \\
\hline & $97(3)$ & 3 & 8 & 12 & 2.39 \\
\hline \multirow{3}{*}{$\begin{array}{l}\text { Education } \\
\text { Department }\end{array}$} & $94(3)$ & 1 & 10 & 25 & 2.67 \\
\hline & $94(1)$ & 5 & 18 & 9 & 2.13 \\
\hline & $97(3)$ & 13 & 6 & 4 & 1.61 \\
\hline
\end{tabular}




\begin{tabular}{|c|c|c|c|c|c|c|}
\hline \multirow[t]{3}{*}{ School policy } & $94(3)$ & 6 & \multicolumn{2}{|l|}{27} & 7 & 2.02 \\
\hline & $94(1)$ & 14 & \multicolumn{2}{|l|}{15} & 6 & 1.77 \\
\hline & $97(3)$ & 15 & \multicolumn{2}{|l|}{10} & 1 & 1.46 \\
\hline \multirow{3}{*}{$\begin{array}{l}\text { Examinations } \\
\text { Authority }\end{array}$} & $94(3)$ & 5 & \multicolumn{2}{|l|}{20} & 15 & 2.25 \\
\hline & 94(1) & 13 & \multicolumn{2}{|l|}{13} & 7 & 1.82 \\
\hline & $97(3)$ & 14 & \multicolumn{2}{|l|}{10} & 1 & 1.48 \\
\hline \multirow{3}{*}{$\begin{array}{l}\text { Businesses/ } \\
\text { employers }\end{array}$} & 94(3) & 0 & \multicolumn{2}{|l|}{8} & 30 & 2.79 \\
\hline & 94(1) & 0 & \multicolumn{2}{|l|}{12} & 20 & 2.63 \\
\hline & 97(3) & 3 & \multicolumn{2}{|l|}{5} & 16 & 2.54 \\
\hline \multirow{3}{*}{ Government } & 94(3) & 2 & \multicolumn{2}{|l|}{13} & 22 & 2.54 \\
\hline & 94(1) & 9 & \multicolumn{2}{|l|}{13} & 10 & 2.03 \\
\hline & 97(3) & 12 & \multicolumn{2}{|l|}{7} & 5 & 1.71 \\
\hline \multirow[t]{3}{*}{ Other factors } & $94(3)$ & 1 & \multicolumn{2}{|l|}{0} & 0 & 1.00 \\
\hline & 94(1) & 0 & \multicolumn{2}{|l|}{0} & 0 & 0.00 \\
\hline & $97(3)$ & 0 & \multicolumn{2}{|l|}{0} & 0 & 0.00 \\
\hline & Test & 3 groups & $\begin{array}{l}\text { Post- } \\
\text { hoc test }\end{array}$ & $\begin{array}{c}94(1) \\
v \\
97(3) \\
\end{array}$ & $\begin{array}{c}94(3) \\
v \\
97(3) \\
\end{array}$ & $\begin{array}{c}94(1) \\
v \\
94(3)\end{array}$ \\
\hline \multirow{2}{*}{$\begin{array}{l}\text { Motivation of } \\
\text { students }\end{array}$} & $\mathrm{K}-\mathrm{W}$ & 6.041 & \multirow{2}{*}{$\begin{array}{l}\text { M-W } \\
\text { Sig. }\end{array}$} & 3.039 & 6.255 & 0.493 \\
\hline & Sig. & 0.049 & & 0.081 & 0.012 & 0.483 \\
\hline \multirow{2}{*}{$\begin{array}{l}\text { Language ability } \\
\text { of students }\end{array}$} & $\mathrm{K}-\mathrm{W}$ & 16.01 & \multicolumn{2}{|l|}{$M-W$} & 0.276 & 11.31 \\
\hline & Sig. & 0.000 & Sig. & 0.001 & 0.599 & 0.001 \\
\hline \multirow{2}{*}{$\begin{array}{l}\text { Efficiency of } \\
\text { English dept. }\end{array}$} & $\mathrm{K}-\mathrm{W}$ & 0.579 & \multicolumn{2}{|l|}{ 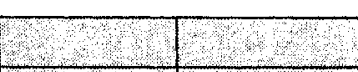 } & 3 & \\
\hline & Sig. & 0.748 & 2. & 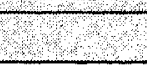 & & \\
\hline \multirow{2}{*}{$\begin{array}{l}\text { Competence of } \\
\text { teacher }\end{array}$} & $\mathrm{K}-\mathrm{W}$ & 16.23 & \multirow{2}{*}{\begin{tabular}{|l} 
M-W \\
Sig.
\end{tabular}} & 15.29 & 4.153 & 5.736 \\
\hline & Sig. & 0.000 & & 0.000 & 0.042 & 0.017 \\
\hline Backup of & $\mathrm{K}-\mathrm{W}$ & 1.840 & $=$ & 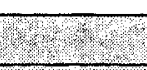 & 5 & (y) \\
\hline colleagues & Sig. & 0.398 & 20 & $2=$ & 1 & 5 \\
\hline
\end{tabular}




\begin{tabular}{|c|c|c|c|c|c|c|}
\hline \multirow{2}{*}{$\begin{array}{l}\text { Design of } \\
\text { classroom }\end{array}$} & $\mathrm{K}-\mathrm{W}$ & 2.145 & & & & \\
\hline & Sig. & 0.342 & $\sqrt{24}$ & 5 & 2 & \\
\hline \multirow{2}{*}{ Class size } & $\bar{K}-\mathrm{W}$ & 9195 & M-W & 7924 & 6223 & 0376 \\
\hline & Sig. & 0.010 & Sig. & 0.005 & 0.013 & 0.540 \\
\hline \multirow[t]{2}{*}{ Location of school } & $\overline{\mathrm{K}-\mathrm{W}}$ & 7.360 & $\overline{M-W}$ & 7.333 & 2.796 & 1.117 \\
\hline & Sig. & 0.025 & Sig. & 0.007 & 0.094 & 0.291 \\
\hline \multirow{2}{*}{$\begin{array}{l}\text { Hong Kong } \\
\text { Education Dept. }\end{array}$} & $\bar{K}-\mathrm{W}$ & 26.27 & $\overline{M-W}$ & 6.709 & 21.93 & 12.10 \\
\hline & Sig. & 0.000 & Sig. & 0.010 & 0.000 & 0.001 \\
\hline & & & & & & \\
\hline \multirow{2}{*}{ School policy } & $\mathrm{K}-\mathrm{W}$ & 12.05 & $\mathrm{M}-\mathrm{W}$ & 2.744 & 12.92 & 3.024 \\
\hline & Sig. & 0.002 & Sig. & 0.098 & 0.000 & 0.082 \\
\hline \multirow{2}{*}{$\begin{array}{l}\text { Hong Kong Exams } \\
\text { Authority }\end{array}$} & $\mathrm{K}-\mathrm{W}$ & 17.18 & $M-W$ & 2.780 & 16.83 & 5.966 \\
\hline & Sig. & 0.000 & Sig. & 0.095 & 0.000 & 0.015 \\
\hline \multirow{2}{*}{$\begin{array}{l}\text { Businesses or } \\
\text { employers }\end{array}$} & $\mathrm{K}-\mathrm{W}$ & 2.639 & & कख & & 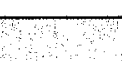 \\
\hline & Sig. & 0.267 & & & & \\
\hline \multirow{2}{*}{$\begin{array}{l}\text { Hong Kong } \\
\text { Government }\end{array}$} & $\mathrm{K}-\mathrm{W}$ & 16.58 & $\mathrm{M}-\mathrm{W}$ & 2.323 & 14.79 & 7.757 \\
\hline & Sig. & 0.000 & Sig. & 0.127 & 0.000 & 0.005 \\
\hline \multirow{2}{*}{ 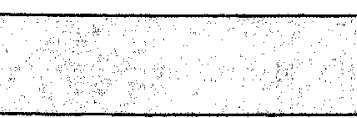 } & & 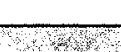 & C & 0637 & 0836 & 0886 \\
\hline & s. & 5n & Sig. & 0.019 & 0.000 & 0.000 \\
\hline
\end{tabular}


Table 4.35: Importance of language skills

\begin{tabular}{|c|c|c|c|c|c|}
\hline Item & anking & 1 & 2 & 3 & Mean \\
\hline \multirow{3}{*}{ Reading } & $94(3)$ & 27 & 16 & 3 & 1.48 \\
\hline & $94(1)$ & 19 & 10 & 3 & 1.64 \\
\hline & $97(3)$ & 21 & 4 & 3 & 1.36 \\
\hline \multirow{3}{*}{ Writing } & $94(3)$ & 32 & 11 & 3 & 1.34 \\
\hline & $94(1)$ & 19 & 14 & 3 & 1.97 \\
\hline & $97(3)$ & 21 & 6 & 1 & 1.29 \\
\hline \multirow{3}{*}{ Speaking } & $94(3)$ & 37 & 9 & 1 & 123 \\
\hline & \begin{tabular}{|l|}
$94(1)$ \\
\end{tabular} & 29 & 8 & 0 & 1.22 \\
\hline & $97(3)$ & 23 & 4 & 2 & 1.28 \\
\hline \multirow[t]{3}{*}{ Listening } & $94(3)$ & 29 & 12 & 3 & 1.41 \\
\hline & $94(1)$ & 21 & 12 & 4 & 1.50 \\
\hline & $97(3)$ & 17 & 7 & 2 & 1.42 \\
\hline \multirow{3}{*}{ Grammar } & $94(3)$ & 28 & 12 & 3 & 1.42 \\
\hline & $94(1)$ & 23 & 11 & 3 & 1.76 \\
\hline & $97(3)$ & 25 & 1 & 2 & 1.18 \\
\hline \multirow[t]{3}{*}{ Pronunciation } & $94(3)$ & 19 & 19 & 5 & 1.67 \\
\hline & $94(1)$ & 19 & 10 & 7 & 1.74 \\
\hline & $97(3)$ & 20 & 4 & 2 & 1.31 \\
\hline
\end{tabular}




\begin{tabular}{|c|c|c|c|c|c|c|}
\hline & Test & 3 groups & $\begin{array}{l}\text { Post- } \\
\text { hoc test }\end{array}$ & $\begin{array}{c}94(1) \\
v \\
97(3) \\
\end{array}$ & $\begin{array}{c}94(3) \\
v \\
97(3) \\
\end{array}$ & $\begin{array}{c}94(1) \\
v \\
94(3) \\
\end{array}$ \\
\hline \multirow[t]{2}{*}{ Reading } & $\mathrm{K}-\mathrm{W}$ & 1.544 & 40 & 95 & & Pro \\
\hline & Sig. & 0.460 & 5 & 2 & & \\
\hline \multirow{2}{*}{ Writing } & $\mathrm{K}-\mathrm{W}$ & 3.872 & 5 & 20 & 7 & 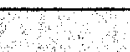 \\
\hline & Sig. & 0.144 & 2 & (2) & & 5 \\
\hline \multirow[t]{2}{*}{ Speaking } & $\mathrm{K}-\mathrm{W}$ & 0.003 & 25 & 25is? & 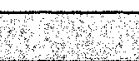 & 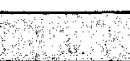 \\
\hline & Sig. & 0.998 & $2 \quad$ & मार & 2 & 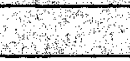 \\
\hline \multirow[t]{2}{*}{ Listening } & $\mathrm{K}-\mathrm{W}$ & 0.908 & 80 & 28 & 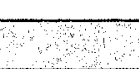 & \\
\hline & Sig. & 0.635 & & & & \\
\hline \multirow{2}{*}{ Grammar } & $\mathrm{K}-\mathrm{W}$ & 5.519 & & & & \\
\hline & Sig. & 0.063 & & 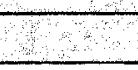 & & \\
\hline \multirow{2}{*}{ Pronunciation } & $\mathrm{K}-\mathrm{W}$ & 5.962 & क․ & & & \\
\hline & Sig. & 0.051 & & & & \\
\hline \multirow{3}{*}{ 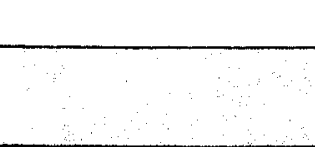 } & & & & & & \\
\hline & 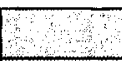 & & Corr. & -0.314 & 0.314 & 0.257 \\
\hline & & 5 & Sig. & 0.544 & 0.544 & 0.623 \\
\hline
\end{tabular}

Table 4.36: Feelings as regards relationships with students

\begin{tabular}{|l|c|c|c|c|c|c|}
\hline \multirow{2}{*}{ Item } & \multicolumn{2}{|c|}{ 94(3) } & \multicolumn{2}{c|}{ 94(1) } & \multicolumn{2}{c|}{ 97(3) } \\
& Yes & No & Yes & No & Yes & No \\
\hline & & & & & & \\
\hline Transmitter of knowledge & 30 & 18 & 26 & 14 & 26 & 4 \\
\hline Facilitator of learning & 40 & 8 & 25 & 15 & 29 & 1 \\
\hline Total control at all times & 0 & 48 & 2 & 38 & 2 & 28 \\
\hline Allow students to decide & 20 & 28 & 20 & 20 & 20 & 10 \\
\hline Become friends & 31 & 17 & 29 & 11 & 23 & 7 \\
\hline Act like a parent & 6 & 42 & 5 & 35 & 9 & 21 \\
\hline Refrain from punishing & 13 & 35 & 4 & 36 & 12 & 18 \\
\hline Devote as much time & 30 & 18 & 23 & 17 & 24 & 6 \\
\hline & & & & & & \\
\hline
\end{tabular}




\begin{tabular}{|c|c|c|c|c|c|c|}
\hline & Test & 3 groups & \begin{tabular}{|l|} 
Post- \\
hoc test
\end{tabular} & $\begin{array}{c}94(1) \\
v \\
97(3)\end{array}$ & $\begin{array}{c}94(3) \\
v \\
97(3)\end{array}$ & $\begin{array}{c}94(1) \\
v \\
94(3)\end{array}$ \\
\hline \multirow{2}{*}{$\begin{array}{l}\text { Transmitter of } \\
\text { knowledge }\end{array}$} & Chi-sq. & 5.661 & $x^{2}$ & Whot & 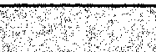 & 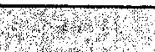 \\
\hline & Sig. & 0.059 & & 3. & & \\
\hline \multirow{2}{*}{$\begin{array}{l}\text { Facilitator of } \\
\text { learning }\end{array}$} & Chi-sq. & 13.02 & Chi-sq. & 9.494 & 2.042 & 3885 \\
\hline & Sig. & 0.001 & \begin{tabular}{|l} 
Sig. \\
\end{tabular} & 0.002 & 0.153 & 0.049 \\
\hline \multirow{2}{*}{$\begin{array}{l}\text { In total control at } \\
\text { all times * }\end{array}$} & 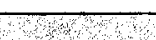 & 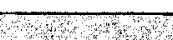 & & & & \\
\hline & & $\frac{5}{6 x}$ & $\sqrt{52}$ & 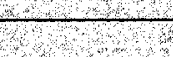 & 2 & 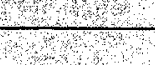 \\
\hline & & & & & & \\
\hline \multirow{2}{*}{$\begin{array}{l}\text { Allow students to } \\
\text { decide }\end{array}$} & Chi-sq. & 4.634 & 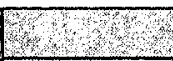 & 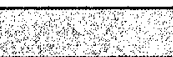 & 5 & 5 \\
\hline & Sig. & 0.099 & Why & 25 & 50 & 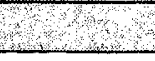 \\
\hline \multirow{2}{*}{$\begin{array}{l}\text { Become friends } \\
\text { with students }\end{array}$} & Chi-sg. & 1.427 & 2 & 4 & & $\sqrt{6 x}$ \\
\hline & Sig. & 0.490 & 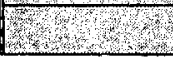 & & & \\
\hline \multirow{2}{*}{ Act like a parent } & Chi-sq. & 4.868 & SEl: & 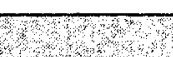 & & 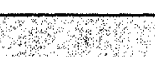 \\
\hline & Sig. & 0.088 & 1 & & & \\
\hline \multirow{2}{*}{$\begin{array}{l}\text { Refrain from } \\
\text { punishing Ss. }\end{array}$} & Chi-sq. & 8.598 & Chi-sq. & 7.131 & 0.883 & 3.063 \\
\hline & Sig. & 0.014 & \begin{tabular}{|l} 
Sig. \\
\end{tabular} & 0.008 & 0.347 & 0.080 \\
\hline \multirow{2}{*}{$\begin{array}{l}\text { Devote as much } \\
\text { time as nec. }\end{array}$} & Chi-sq. & 4.098 & & & & 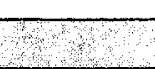 \\
\hline & Sig. & 0.129 & 18 & 20 & & \\
\hline
\end{tabular}

* As at least one cell in $2 \times 3$ table contained a value of zero, chi-square could not be considered reliable and so was not carried out. 
Table 4.37: Strengths and weaknesses as teachers

\begin{tabular}{|c|c|c|c|c|c|c|}
\hline Item & anking & 1 & 2 & 3 & 4 & Mean \\
\hline \multirow{3}{*}{ Language ability } & $94(3)$ & 1 & 20 & 17 & 4 & 2.57 \\
\hline & $94(1)$ & 5 & 10 & 16 & 5 & 2.58 \\
\hline & $97(3)$ & 4 & 8 & 3 & 15 & 2.97 \\
\hline \multirow{3}{*}{$\begin{array}{l}\text { Communication } \\
\text { skills }\end{array}$} & $94(3)$ & 6 & 17 & 12 & 8 & 2.51 \\
\hline & $94(1)$ & 3 & 16 & 12 & 6 & 2.57 \\
\hline & $97(3)$ & 4 & 8 & 3 & 14 & 2.93 \\
\hline \multirow{4}{*}{ Discipline } & & & & & & \\
\hline & $94(3)$ & 3 & 8 & 14 & 14 & 3.00 \\
\hline & $94(1)$ & 3 & 8 & 14 & 10 & 2.89 \\
\hline & $97(3)$ & 4 & 3 & 9 & 13 & 3.07 \\
\hline \multirow{3}{*}{ Organisation } & $94(3)$ & 7 & 13 & 19 & 6 & 2.53 \\
\hline & $94(1)$ & 2 & 14 & 12 & 6 & 2.65 \\
\hline & $97(3)$ & 4 & 9 & 10 & 7 & 2.67 \\
\hline \multirow[t]{3}{*}{ Innovation } & $94(3)$ & 8 & 16 & 14 & 7 & 2.44 \\
\hline & $94(1)$ & 4 & 16 & 9 & 5 & 2.44 \\
\hline & 97(3) & 7 & 10 & 5 & 5 & 2.30 \\
\hline \multirow{3}{*}{ Planning } & $94(3)$ & 7 & 17 & 13 & 5 & 248 \\
\hline & \begin{tabular}{|l|}
$94(1)$ \\
\end{tabular} & 5 & 16 & 9 & 5 & 2.40 \\
\hline & 97(3) & 8 & 10 & 6 & 5 & 2.28 \\
\hline \multirow[t]{3}{*}{ Voice } & 94(3) & 11 & 11 & 11 & 8 & 2.39 \\
\hline & 94(1) & 4 & 15 & 7 & 8 & 2.56 \\
\hline & $97(3)$ & 9 & 11 & 4 & 5 & 2.17 \\
\hline \multirow{3}{*}{$\begin{array}{l}\text { Strength of } \\
\text { personality }\end{array}$} & 94(3) & 8 & 17 & 13 & 6 & 2.39 \\
\hline & 94(1) & 5 & 20 & 10 & 2 & 2.24 \\
\hline & \begin{tabular}{|l|}
$97(3)$ \\
\end{tabular} & 12 & 7 & 6 & 4 & 2.07 \\
\hline & & & & & & \\
\hline \multirow{3}{*}{$\begin{array}{l}\text { Relationships } \\
\text { with students }\end{array}$} & 94(3) & 16 & 19 & 3 & 8 & 2.06 \\
\hline & $94(1)$ & 12 & 17 & 7 & 1 & 1.92 \\
\hline & $97(3)$ & 8 & 15 & 3 & 2 & 1.96 \\
\hline
\end{tabular}




\begin{tabular}{|c|c|c|c|c|c|c|}
\hline \multirow{3}{*}{$\begin{array}{l}\text { Relationships } \\
\text { with colleagues }\end{array}$} & 94(3) & 4 & 20 & 9 & 5 & 2.39 \\
\hline & 94(1) & 2 & 21 & 6 & 5 & 2.41 \\
\hline & $97(3)$ & 5 & 11 & 9 & 2 & 2.30 \\
\hline \multirow{3}{*}{$\begin{array}{l}\text { Motivating } \\
\text { students }\end{array}$} & 94(3) & 6 & 20 & 11 & 5 & 2.36 \\
\hline & $94(1)$ & 9 & 12 & 12 & 2 & 2.20 \\
\hline & $97(3)$ & 4 & 14 & 6 & 4 & 2.35 \\
\hline \multirow[t]{3}{*}{ Teaching reading } & 94(3) & 3 & 11 & 19 & 7 & 2.75 \\
\hline & $94(1)$ & 3 & 16 & 11 & 2 & 2.38 \\
\hline & $97(3)$ & 4 & 10 & 9 & 4 & 2.48 \\
\hline & & & & & & \\
\hline \multirow[t]{3}{*}{ Teaching writing } & 94(3) & 7 & 11 & 14 & 9 & 2.61 \\
\hline & 94(1) & 4 & 13 & 14 & 1 & 2.38 \\
\hline & $97(3)$ & 4 & 8 & 13 & 3 & 2.54 \\
\hline \multirow{3}{*}{$\begin{array}{l}\text { Teaching } \\
\text { speaking }\end{array}$} & $94(3)$ & 8 & 9 & 13 & 9 & 2.59 \\
\hline & 94(1) & 2 & 13 & 10 & 9 & 2.76 \\
\hline & $97(3)$ & 5 & 11 & 6 & 5 & 2.41 \\
\hline \multirow{3}{*}{$\begin{array}{l}\text { Teaching } \\
\text { listening }\end{array}$} & & & & & & \\
\hline & \begin{tabular}{|l}
$94(3)$ \\
$94(1)$
\end{tabular} & $\frac{3}{4}$ & $\frac{12}{15}$ & $\frac{16}{8}$ & $\frac{8}{6}$ & $\frac{2.74}{2.48}$ \\
\hline & $97(3)$ & 6 & 7 & 11 & 2 & 2.35 \\
\hline \multirow{3}{*}{$\begin{array}{l}\text { Teaching } \\
\text { grammar }\end{array}$} & $94(3)$ & 8 & 15 & 9 & 8 & 243 \\
\hline & \begin{tabular}{|l}
$94(1)$ \\
\end{tabular} & 3 & 14 & 14 & $\frac{0}{1}$ & $\frac{2.45}{2.41}$ \\
\hline & $97(3)$ & 4 & 12 & 9 & 3 & 2.39 \\
\hline \multirow{3}{*}{$\begin{array}{l}\text { Teaching } \\
\text { pronunciation }\end{array}$} & $94(3)$ & 3 & 13 & 11 & 12 & 282 \\
\hline & 94(1) & 2 & 15 & 10 & 4 & 2.52 \\
\hline & $97(3)$ & 4 & 10 & 10 & 3 & 2.44 \\
\hline \multirow[t]{3}{*}{ Other factors } & $94(3)$ & 0 & 0 & 0 & 0 & 0.00 \\
\hline & 94(1) & 0 & 0 & 0 & 0 & 0.00 \\
\hline & \begin{tabular}{|l|}
$97(3)$ \\
\end{tabular} & 0 & 1 & 0 & 0 & 2.00 \\
\hline
\end{tabular}




\begin{tabular}{|c|c|c|c|c|c|c|}
\hline & Test & 3 groups & $\begin{array}{l}\text { Post- } \\
\text { hoc test }\end{array}$ & $\begin{array}{c}94(1) \\
\text { v } \\
97(3) \\
\end{array}$ & $\begin{array}{c}94(3) \\
v \\
97(3) \\
\end{array}$ & $\begin{array}{c}94(1) \\
v \\
94(3)\end{array}$ \\
\hline \multirow[t]{2}{*}{ Language ability } & $\mathrm{K}-\mathrm{W}$ & 3.836 & 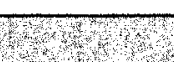 & 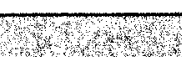 & Wres: & m5 \\
\hline & Sig. & 0.147 & 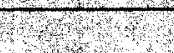 & $\sqrt{3}+6$ & 5 & 21 \\
\hline & & & & & & \\
\hline \multirow{2}{*}{$\begin{array}{l}\text { Communications } \\
\text { skills }\end{array}$} & $\mathrm{K}-\mathrm{W}$ & 3.176 & $2+2$ & 5 & $4 \quad$ & 7 \\
\hline & Sig. & 0.204 & 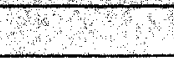 & 5 & 5 & \\
\hline \multirow{2}{*}{ Discipline } & V Y & 1057 & & & & \\
\hline & $\begin{array}{l}\text { K-W } \\
\text { Sig. }\end{array}$ & $\frac{1.051}{0.589}$ & & & & \\
\hline & & & & & & \\
\hline \multirow[t]{2}{*}{ Organisation } & $\mathrm{K}-\mathrm{W}$ & 0.344 & +6 & $\sqrt{3}$ & 46 & 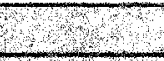 \\
\hline & Sig. & 0.842 & & 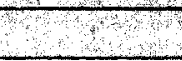 & & \\
\hline \multirow{2}{*}{ Innovation } & $\mathrm{K}-\mathrm{W}$ & 0.603 & & & & \\
\hline & Sig. & 0.740 & 5 & $x^{2}$ & 20 & 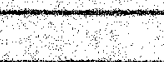 \\
\hline \multirow[t]{2}{*}{ Planning } & $\mathrm{K}-\mathrm{W}$ & 0.426 & 2 & & 2 & \\
\hline & Sig. & 0.808 & 0 & 28 & (2) & E \\
\hline \multirow{3}{*}{ Voice } & & & & & & \\
\hline & $\mathrm{K}-\mathrm{W}$ & 2.289 & 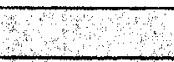 & (2) & 3 & 2 \\
\hline & Sig. & 0.318 & & & & \\
\hline \multirow{2}{*}{$\begin{array}{l}\text { Strength of } \\
\text { personality }\end{array}$} & $\mathrm{K}-\mathrm{W}$ & 2.364 & & & & \\
\hline & Sig. & 0.207 & & & & \\
\hline \multirow{2}{*}{$\begin{array}{l}\text { Relationships with } \\
\text { students }\end{array}$} & $\mathrm{K}-\mathrm{W}$ & 0.362 & एक & resta & & \\
\hline & Sig. & 0.835 & 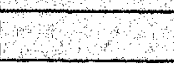 & & & \\
\hline \multirow{2}{*}{$\begin{array}{l}\begin{array}{l}\text { Relationships with } \\
\text { colleagues }\end{array} \\
\end{array}$} & $\mathrm{K}-\mathrm{W}$ & 0.124 & & & & \\
\hline & Sig. & 0.940 & Cos & 5 & 40 & 40 \\
\hline \multirow{2}{*}{\begin{tabular}{|l|}
$\begin{array}{l}\text { Motivating } \\
\text { students }\end{array}$ \\
\end{tabular}} & $\mathrm{K}-\mathrm{W}$ & 0.473 & $2 x$ & & & \\
\hline & Sig. & 0.789 & 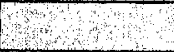 & +2 & 5 & \\
\hline \multirow[t]{2}{*}{ Teaching reading } & $\mathrm{K}-\mathrm{W}$ & 4.167 & 208 & 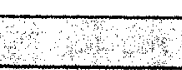 & & \\
\hline & Sig. & 0.124 & (2) & & & \\
\hline \multirow[t]{2}{*}{ Teaching writing } & $\mathrm{K}-\mathrm{W}$ & 1.311 & & & & \\
\hline & Sig. & 0.519 & & & & \\
\hline
\end{tabular}




\begin{tabular}{|c|c|c|c|c|c|c|}
\hline \multirow[t]{2}{*}{ Teaching speaking } & $\mathrm{K}-\mathrm{W}$ & 1.856 & 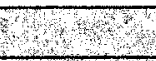 & 5 & P & 5 \\
\hline & Sig. & 0.395 & $2-12$ & 2 & 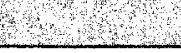 & \\
\hline \multirow{2}{*}{ Teaching listening } & $\mathrm{K}-\mathrm{W}$ & 2.965 & 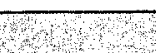 & Evise & $\sqrt{32}$ & 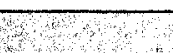 \\
\hline & Sig. & 0.227 & 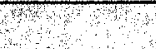 & & & \\
\hline \multirow{2}{*}{ Teaching grammar } & $V$ V & 0030 & & & & \\
\hline & Sig & 0.985 & & 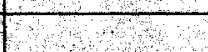 & $\square$ & $=$ \\
\hline & & & & & & \\
\hline \multirow{2}{*}{$\begin{array}{l}\text { Teaching } \\
\text { pronunciation }\end{array}$} & $\mathrm{K}-\mathrm{W}$ & 3.047 & 2 & 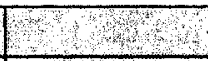 & + & \\
\hline & \begin{tabular}{|l} 
Sig. \\
\end{tabular} & 0.218 & Se & 8 & & 2 \\
\hline \multirow{2}{*}{20} & 18 & 20 & Corr. & 0.536 & 0.727 & 0.614 \\
\hline & 2 & 5. & Sig. & 0.026 & 0.001 & 0.009 \\
\hline
\end{tabular}

Effects of Changeover in Sovereignty

Table 4.38: 97(3) group's opinion as to the extent to which the use of English in secondary schools might be affected by the changeover in sovereignty

\begin{tabular}{|l|c|c|c|c|c|c|}
\hline & $\begin{array}{c}\text { To a large } \\
\text { extent } \\
1\end{array}$ & $\begin{array}{c}\text { To some } \\
\text { extent } \\
2\end{array}$ & $\begin{array}{c}\text { To little } \\
\text { extent } \\
3\end{array}$ & $\begin{array}{c}\text { To no } \\
\text { extent } \\
4\end{array}$ & Mean & SD \\
\hline & & & & & & \\
\hline $\mathbf{9 7 ( 3 )}$ & 8 & 14 & 7 & 1 & 2.03 & 0.79 \\
\hline & & & & & & \\
\hline
\end{tabular}

Table 4.39: 97(3) group's opinion as to the extent to which their lesson planning and decision-making might be affected by the changeover in sovereignty

\begin{tabular}{|l|c|c|c|c|c|c|}
\hline & $\begin{array}{c}\text { To a large } \\
\text { extent } \\
1\end{array}$ & $\begin{array}{c}\text { To some } \\
\text { extent } \\
2\end{array}$ & $\begin{array}{c}\text { To little } \\
\text { extent } \\
3\end{array}$ & $\begin{array}{c}\text { To no } \\
\text { extent } \\
4\end{array}$ & Mean & SD \\
\hline & & & & & & \\
\hline $\mathbf{9 7 ( 3 )}$ & 0 & 4 & 18 & 7 & 3.10 & 0.61 \\
\hline
\end{tabular}


Table 4.40: 97(3) group's level of agreement on whether the changeover in sovereignty will affect their approach to teaching

\begin{tabular}{|l|c|c|c|c|c|c|}
\hline & $\begin{array}{c}\text { Absolutely } \\
\text { agree } \\
1\end{array}$ & $\begin{array}{c}\text { Quite } \\
\text { agree } \\
2\end{array}$ & $\begin{array}{c}\text { Quite } \\
\text { disagree } \\
3\end{array}$ & $\begin{array}{c}\text { Absolutely } \\
\text { disagree } \\
4\end{array}$ & Mean & SD \\
\hline $\mathbf{9 7 ( 3 )}$ & 1 & 3 & 11 & 15 & 3.33 & 0.79 \\
\hline & & & & & & \\
\hline
\end{tabular}

Table 4.41: Extent to which the Yr. 3 (97) group are worried about their future as teachers after the changeover of sovereignty

\begin{tabular}{|l|c|c|c|c|c|c|}
\hline & $\begin{array}{c}\text { To a large } \\
\text { extent } \\
1\end{array}$ & $\begin{array}{c}\text { To some } \\
\text { extent } \\
2\end{array}$ & $\begin{array}{c}\text { To little } \\
\text { extent } \\
3\end{array}$ & $\begin{array}{c}\text { To no } \\
\text { extent } \\
4\end{array}$ & Mean & SD \\
\hline & & & & & & \\
\hline $\mathbf{9 7 ( 3 )}$ & 3 & 8 & 10 & 9 & 2.83 & 0.97 \\
\hline & & & & & & \\
\hline
\end{tabular}

Table 4.42: 97(3) group's opinions as to whether their professional relationships or responsibilities will change after the changeover of sovereignty

\begin{tabular}{|l|c|c|c|c|c|}
\hline & Yes & $\begin{array}{c}\text { Somewhat } \\
2\end{array}$ & $\begin{array}{c}\text { No } \\
3\end{array}$ & Mean & SD \\
\hline & & & & & \\
\hline $\mathbf{9 7 ( 3 )}$ & 1 & 11 & 18 & 2.57 & 0.56 \\
\hline & & & & & \\
\hline
\end{tabular}




\section{B.5 BATESL Students' Philosophies of Teaching}

The philosophies written by the respondents have been divided into three key areas relating to the focus on students, on teachers and on teaching. The raw figures refer to the number of philosophies categorised under each area and the percentages in respect of the total number of philosophies of each survey group are given in brackets.

\section{FOCUS ON STUDENTS}

\section{Cohort 1}

Student-centred approach $-24(60 \%)$

Sincerity to the student. Help them to have a good result in examination. Help the students to have a positively view towards life.

Of course I teach them and I want them to really know English, but I do want to be their friends and share their feeling not only towards learning in school but also towards their lives. I hate to force students to do anything.

Helpful and emphatic to the students especially the less-able students because they are the target group that needs help and support from us (humanized teacher).

\section{Cohort 2a}

Student-centred approach $-27(69 \%)$

I'll try my best to teach what I've learnt. Besides, try to be good relationships with students so I can solve their problems.

Teachers should try to communicate with students and let them think independently not just teach them throughout the lessons.

Teachers should have a close relationship with their students, lessons should not be boring and try to understand their students problems.

I teach my students and learn from them. I want to help my students under the constraints of the environment (e.g. within the school).

\section{Cohort 2b}

Student-centred approach $-9(39 \%)$

I'll pay attention to my students' need rather than the requirement of public examinations. I believe that a learner-centred approach may enhance the learning/teaching process.

The objectives of a lesson should be clearly presented to the class. A variety of activities should be used to arouse students' interest and motivation. To consider the learning pace of students.

Student-centred learning.

Student-centred approach to teaching is very important. 
Teachers should be prepared to help students always in every aspect, including academic performance, personality developments and their problems.

To create a pleasant environment in which students can have enjoyable lessons with me.

To teach what is required in the syllabus in a way that students can learn in a relaxed and interactive environment which enhances students' learning in English.

I want to pass what I have learnt in my life to my students.

To benefit my students instead of only finishing my job.

My teaching philosophy is rather simple. Whatever I do, I should think of my students' needs and lacks first, as they are the target students in setting my objectives in teaching.

Treat students as your friends and constantly discuss with them their needs and problems on their studies.
I will try my very best to transmit any knowledge to my student \& get their confidence.

Teaching in line with the needs of students and society.

Discipline first, teacher centred for second, then more student centred.

According to the student's needs, give help and advice. Tell the students all I know.

Keep close with the students; and show them I am concerned about them.

To be friendly to students, teaching contents is highly suitable to examination.

Be student-centred, flexible and interesting.

I think I am able to handle the problem of the students and I am cautious enough to teach them.

\section{I think good} communication with students is very important and I will lead my students to learn English in happy atmosphere and learn English from their daily lives rather than just from books.
Posses good language competence, realize students' needs and difficulties and be patient.

Increase the chance of language use among Ss.

Conscious about the Ss' attitudes to learning English. Give Ss a reason to learn English. Care about Ss including their personal life and if they are willing to share with me.

1. to help Ss engage the whole person learning, 2. help Ss learn in a sharing and caring atmosphere,

3. help them take responsibility and monitor their own learning.

Help Ss to look for their life direction.

Help Ss to discover their potential abilities.

Help Ss learn rather than teach Ss anything. 
I teach because I would like to help students to learn the language.

Always think about the students' feeling.

Build up good relationship with students, otherwise, the students will not listen to you. Students nowadays are rebellious.

My teaching should help the students to really use the language to communicate.

Try to have a balance among students' needs, abilities and examination requirements.

Teachers should guide the students to learn and not to control them. We should be very flexible and brave to try new materials and teaching ideas.

To help my students to develop interest in learning, and try my best to explore their horizon.

To teach students the content of the syllabus in an efficient and joyful way.

Students are our clients, friends and children whenever possible.
Communication between students and teachers is the most important. New and innovative ideas to teach English will be much more attractive to students nowadays.

Try to understand what's the difficulties and problems of students in their learning, help them solve problems properly.

Try my best to help teach the students to know not only how to use English properly, but how to be good people.

To have interaction with students, understand their needs and keep pace with the society of what they have to learn.

To raise the interest of student and give them encouragement all the time.

To inspirit [sic] student to eager to learning English.

Teaching is not only transmit knowledge but also help students to develop their own personality.

Arose students' interest to English. 
I strongly agree that the lessons should be studentcentred. The good relationship between students and teachers is very important. In fact, teachers can learn from the students. Also, I think teaching English should be creative and funny.

My teaching philosophy is to try my best to help students and motivate them to learn English as a second language.

A teacher should not only teach what he has learnt, but also care about the needs of students.

To motivate the students to learn and think.

Ss should be active

learners in all lessons.

Language training for specific needs of specific course participants.

I think my teaching philosophy depends very much on the school and students that I teach.
Having good relationship with students, giving knowledge to my students. And, cooperating with other teachers.

I want students to know more English and speak English fluently.

Student are eager to learn if they are interested in

Help the student not to be afraid of English and to master it easily.

Try to use an interesting approach to let students being interested in learning English; try to explain the different structure between Chinese and English Language. Try to build up a good relationship with students. 


\section{FOCUS ON TEACHERS}

\section{Cohort 1}

Responsibility of teachers

$-10(25 \%)$

A teacher should be responsible.

Well-prepared before the lesson.

Not too strict.

Be a good teacher and establish a certain reputation in the school.

To be responsible and sensible to students' needs.

Teachers should be responsible and hardworking. They should have an understanding of students' needs and interests.

Be a sensitive and sensible teacher.

Other than giving students knowledge of the subject, teachers should also teach students how to be better people.

Helping students to develop into a whole person.
Cohort 2a

Responsibility of teachers

$-6(15 \%)$

I realise that being a teacher is a social responsibility.

As a teacher he or she should be able to transfer the knowledge to the students as well as help the latter to establish good moral values.

Train the students to become more mature.

Help students to develop a positive value system. To help the weak. To try my very best to teach younger generations and help students to have correct value to life.

(1) Well-planned

(2) learning through activities

(3) Patience

(4) Get involved with my student

\section{Cohort 2b}

Responsibility of teachers $-7(30 \%)$

Be responsible.

Not necessarily be the best teacher in the school but I must be responsible to all my students.

Gain respect from students. Teach whatever I can, be responsible.

Teaching is not only a career, it's something that needs the teachers to be responsible for themselves and students since one regulates or supervise their teaching style. It aims to help students to develop their personal growth and also academic performance.

Gain respect from students.

Getting both relationship and respect from $S$ s.

I think as a teacher, I have to cultivate a sense of responsibility to students as well as to teach them the language. 
Share my philosophy and value of life with the students, help them grow healthily and share with them my knowledge.

My philosophy of teaching is to indoctrine [sic] students with enough knowledge which will prepare them for contributing to the society.

Teaching is a long-term commitment in which you use your knowledge of subject-matter as well as teaching and your heart to help students to develop themselves to find out their potential.
Teacher's competency $1(3 \%)$

Teaching method is very important. If a teacher does not know how to teach his/her student, the student will be affected directly.
Teacher's competency $2(9 \%)$

An English teacher should be very competent and proficient in English. $\mathrm{He} /$ she needs to reinforce himself/herself and keep going on training himself/herself even when he/she starts teaching.

Teachers should be competent in English. They should motivate students to participate in lessons and help them to build positive attitudes towards English.

Teacher's insecurity - 1 $(4 \%)$

I feel that students are more and more difficult to handle nowadays. My former English teachers always said that teaching secondary school students is an undesirable job. I worry that I might spend time on futile things. Even if I try my best, students won't appreciate my efforts. 


\section{FOCUS ON TEACHING}

\section{Cohort 1}

Definition of teaching - 4 $(10 \%)$

Emphasise more on communication. Grammar exercise a definite need. Encourage more private reading (Eng. magazine, movie, $T V$, radio etc.).

I think the communicative approach of learning is a good teaching philosophy.

A teacher should be neither too strict nor too lenient. He or she should be hard-working and hold the belief that teachers have a vital role to students' learning.

Training on accuracy is more important for lower form students. There should be a graded progress to the training in fluency for higher forms.

Teaching materials - 1

$(2 \%)$

Help myself to plan lessons and materials.
Cohort 2a

Definition of teaching - 1 (3\%)

I think teaching method is very important. Learning from doing is the best way to learn English.

Personal goal - $4(10 \%)$

Try my best to teacher.

Never forget about 'twoways communication'.

Keep a 'learning attitude'.

Must pay a great attention to what going on around me and be patient enough.

Being a teacher is my ambitious, it doesn't change forever

\section{Cohort 2b}

Definition of teaching - 3 $(13 \%)$

Teaching is not something that can be taught. I think you have to really teach a class in order to learn what is teaching and teaching can only be improved through teaching.

Teaching should be wellplanned, so teachers have to do a lot of work before and after lessons.

I think teaching is to plan activities for $S$ s to have a chance to learn the subject area, e.g., English. I believe that $S$ S can learn the language by doing activities which are carefully designed.

Teaching materials - 1 $(4 \%)$

S-oriented, authentic materials.

Personal goal - $1(2 \%)$

Keep trying. 


\section{APPENDIX C}

Appendix $\mathrm{C}$ consists of the following, which should be viewed in conjunction with Chapter 5 of the thesis:

C.1 Transcriptions of interviews with BATESL graduates in 1998

C.2 Main points to emerge during interviews with BATESL graduates in 1998

C.3 Lesson observation reports of 1997 and 1994 BATESL graduates

C.4 Transcriptions of interviews with BATESL course leaders in 1999

C.5 Main points to emerge from interviews with BATESL course leaders in 1999 


\section{C.1 Transcriptions of Interviews with BATESL Graduates in 1997}

Interviewees: Graduates N(97), O(97) and U(97)

Date:

3 August 1998

$\begin{array}{ll}\text { AU } & \text { Researcher } \\ \text { N(97) } & \text { Interviewee } \\ \text { O(97) } & \text { Interviewee } \\ \text { U(97) } & \text { Interviewee } \\ \ldots & \text { Missing words / Unfinished utterance } \\ - & \text { Pause }\end{array}$

AU OK, Language Use, you said that, not surprisingly, as a group this is, that your language had improved over the course of the BATESL, but what I found was that speaking had improved more than writing. At least that's what people said. Why do you think that is?

U(97) I think it depends on which kind of writing they're talking about. There were quite a lot of academic writings or just writing some short sentences to answer questions or ... but maybe we are talking about creative writing or something which is just not academic writing. And then there's more chances to talk during the course, rather than write, I think.

AU You had to use English all the time, when you were speaking?

U(97) With you!

N(97) And the lecturers.

$\mathrm{AU} \quad$ And amongst yourselves?

U(97) I don't think so; we still speak with the others in Cantonese.

N(97) Maybe just for fun, we will speak in English.

U(97) But it's rare, I can say.

AU Just when we met today, you two were speaking in English. Was that just because I was there?

$\mathrm{O}(97) \quad$ Yes. 
O(97) No, definitely not.

How about code mixing and code switching, which was the next one, leading onto this idea of when you're speaking to each other. As a whole, the group said that they code-mixed quite often and code-switched rarely. Do you find that you code-mix or code-switch as much now as you did then? When you are speaking now with people in Cantonese, do you still use a lot of English terms and expressions? Or has that sort of faded away now since you left the BATESL?

U(97) Well, I can say my code mixing has decreased. It may be that I can't do it in the classroom, so I get used to it, and the frequency lowered down. But for code-switching, it's always ... it depends on the circumstances. So I code-switch.

$\mathrm{O}(97)$ To me I - I've got virtually no chance to code-switch, but for the codemixing, I find that I'm trying to reduce the amount I code-mix, I don't know why, maybe because of the notion of language purity, I'm not sure. Because when I was in the BATESL course, I got a lot to discuss with my fellow classmates, I've got to discuss because most of the terms we usually don't say it in Cantonese. But after graduation, when I started working as a research assistant, most of my conversation is between my colleagues in the room and all of them are Cantonese-speaker. That's why I can say purely Cantonese in my daily life.

$\mathrm{N}(97) \quad$ I think for me, I do more code-mixing and code-switching now than what I did in university because in my workplace, some of them are $\mathrm{ABC}-$ American-born Chinese and their Cantonese is no good so we cannot communicate fully in Cantonese but they still want to talk in Cantonese. Even some Hong Kong people, it's very strange that they just like to talk to you in English, so now I did more code-switching. But I try to do less code-mixing, I don't like code- mixing, personally I think it's just like $[\mathrm{O}(97)]$ 's reason, language purity. But sometimes when I interview people I've got to get into their mode of conversation. I think I do more codemixing and code-switching now.

AU Certainly code-mixing has come in for some bad press. One of the things I have been doing is following the newspapers for the last year, education articles, some of them written by you as well, I'm collecting a database of newspaper articles and reports and so on. Some of them have been on code-mixing, not very many but one or two. This idea of code-mixing is one of the reasons why the government has brought in the change in the medium of instruction as they want to abandon and get rid of code-mixing in classrooms. You are either English-medium so you use only English or 
Chinese-medium and you use only Chinese. I like the quote, it's on page 6, Subject C said, 'Like, 'sorry', 'tissue' and 'toilet', we always use English words because that's the norm in Hong Kong. Of course we don't use a whole sentence in English.'

N(97) Actually I think we use these words is so common that even we don't regard it as code-mixing. Just like 'cut', cut something, we use this in English, rather than the Cantonese gai.

$\mathrm{O}(97) \quad$ Yes, I agree.

$\mathrm{N}(97) \quad$ It's so common that I don't regard it as code mixing.

AU I suppose there is a line between code-mixing as such and then if certain words or terms become code-mixed so often, eventually they become adopted into the language, don't they?

N(97) Yes.

AU And they become, sort of changed a little bit so the pronunciation will become changed a little bit and they become Chinese loan words. It's the same in any language.

Using English in the classroom, Table 4, the group were sort of mixed with regard to the use of English only in the classroom - about half disagreed and half agreed with using only English and mostly disagreed, just about, with using mixed-code. How do you feel about that now, about which languages should be used in the English classroom?

$\mathrm{O}(97) \quad$ I think teachers can use English only or code-mixing in classrooms, it depends on ...

U(97) School policy.

$\mathrm{O}(97) \quad$... the level of proficiency of your students, and sometimes, because I haven't been a full-time teacher, I'm sure that the school policy has got a very strong effect, some pressure exerted on teachers. Because there is no point in using English only if your students cannot comprehend what you are talking about. I think there's a kind of threshold level of your students' proficiency in order to benefit from the English-only classroom. That's what I mean.

U(97) Basically I have the same idea with $[\mathrm{O}(97)]$, but as I say, the school policy plays the most important role. If your principal tells you to use English, I don't think you can use Cantonese because suddenly he or she will walk around the corridor and check if you're really using English in your 
English lesson, or even sometimes in an EMI school to see if you are using English to teach other content area subjects. So even students don't have the ability to understand what you said, you still need to use English. But I think you may need to repeat what you said to make sure your students understand, if you're pushed to use English.

$\mathrm{O}(97) \quad$ How about if you use a few Cantonese words as a glossary to help them?

U(97) If you use the first time then your students will ask you to use the second time. And maybe you can pretend you don't understand Cantonese, what they say.

$\mathrm{O}(97) \quad$ Sure, it must be a very good school, isn't it?

U(97) For good school they know you are Cantonese.

AU It's interesting because the school I used to teach at, which was an English-medium school, but a lot of teachers taught in Cantonese - not English teacher, I don't know of any English teachers who teach in Cantonese. I heard of somebody teaching English Literature and using Chinese, this is A Level. Maybe now with the change in the medium of instruction, schools have to be clearly defined one way or the other...

N(97) Exactly.

AU $\quad \ldots$ then they will enforce it more strictly.

N(97) Exactly, of course I interviewed quite a number of principals from the EMI schools, they said they ... especially those that appealed to be EMI. So, they really got a lot of pressure from the government because there's sudden inspection from the educational services, something like that. And actually talking about code-mixing in the classroom, the ... changed now because EMI became the minority and the majority is CMI schools and a lot of teachers told me that they would code-mix in the way that they would use the mother tongue for most of the teaching and for some special terms because of course they have to think about the interface with higher education, and some technical terms they will use English. Today is like that, code mixing is like that; it's different from the past. Code mixing is using one or two Cantonese words, today is just opposite.

AU So using Cantonese and using one or two English words?

N(97) $\quad$ Yes

$\mathrm{O}(97) \quad$ You mean those CMI schools? 
N(97) The CMI schools, yeah.

AU Because that's one of the major issues that people have thought about is that in certain subjects, perhaps science subjects or economics, that some terms, maybe the translation doesn't exist, or is very difficult to find.

$\mathrm{O}(97) \quad$ And difficult to remember.

N(97) Yes, maybe for example the scientific terms got 12 English alphabets, but the Chinese translation has got 12 Chinese characters.

AU Table 8, the groups' opinion as to the extent to which the use of English in secondary schools might be affected by the changeover in sovereignty, to some extent. People thought things like - the prestige of English might become less and Putonghua would become more important. Less English would be used and mother tongue education, so this idea of the medium of instruction was being discussed at that time. Have you noticed any changes in English use since the changeover? I'm referring more to schools. [U(97)], you've been teaching a little this year?

U(97) Yes, but it seems no change in that school because it's classified as an EMI school, so the principal wants to maintain the prestige in that district. To me, and after my observation, there is no change, and it seems that the school wants to work even harder to impress the others, to tell the others that they are really capable to be an EMI school. So no change.

AU No change?

N(97) English use at school, or in society?

AU In society as well.

N(97) I think the importance of English doesn't change - at school, I mean doesn't overwhelmed by Putonghua, as the CDI, the Curriculum Development Institute, part of the Education Department, didn't do much things on Putonghua, so because of the mother-tongue education, pupils seem to pay more attention, even more attention to English, yeah, because they found that their exposure to English is decreasing. It seems that English is still very important at school. In society, I think, not much change because there are a lot of people rushing to learn Putonghua, but I think it's just an illusion. Just because of the handover they think that they should learn Putonghua, but when you ask them for their real reasons, they can't tell me, they say because it's 1997, but why? Why you have to learn just because of the 1997? I have to say that yeah, a lot of people are talking to me in Putonghua today than before, but I think English is important, is still rather important because just because of the 1997 there 
are more international trades with foreigners, more and more people come to Hong Kong, who speak English, so I think it's even more important now, English. The Mainland government's looking for a lot of English professionals too.

$\mathrm{O}(97)$ I think it very much depends on - whether individuals have to use Putonghua in their workplace. For example, some of my friends have got to speak Putonghua very often as they've got to go to Mainland China to deal with those people. But back to the question of the use of English in schools, I think that because of the MOI policy, most schools are forced to use Cantonese as the medium of instruction. It is obvious that there is a drastic change in the use of English in secondary schools, but I'm not sure if we can say that this is because of the handover of the sovereignty or should we say it's due to the government policy, the MOI policy, rather than the handover itself.

AU Yeas that's a big issue and these policies that have been changed, have been brought in. Another question was were they a result of the handover, or was the MOI a result of the handover, and most of the experts I have spoken to have said no, because it was already decided back in 1994 and even earlier that they were going to change the medium of instruction. But whether it proves to be a good time to do it, now, that people might accept it more after the handover, nobody has been able to say for sure. As you know there has been all kinds of controversy about it, parents have been crying and all kinds of things have happened about that. But personally I don't think there has been any change in the use of English yet, in the schools I've seen, because from September schools will have to change.

OK, Instructional Planning and Decision-Making was the next section. The general feeling just summarising these ideas was that the group felt that planning was important in lessons and still was important, but flexibility was more important - being able to change your planning as you went along. The fact there was not enough time in the school context to be able to write detailed lesson plans anyway, that the only reason you were doing it, that you had to write a detailed lesson plan for your supervisor in your practice teaching. I actually had one of the guys yesterday saying that when their supervisor came they prepared everything very well, the lesson plan and everything, but when the supervisor wasn't coming you would do what you like. We were discussing the idea of maybe it would be better if the supervisor didn't tell the teachers when they were coming, they could come at any time. That would be rather unfair in a way. It might make the student teachers prepare every lesson properly, just in case, but ...

U(97) Maybe for the first week, but not the following one because they will be very busy, they don't have time to prepare. 
U(97) Maybe it's a little bit short, it's better to be a bit longer ...

$\mathrm{O}(97) \quad$ For a month ...

U(97) Say one month.

AU Because it's what, two weeks, three weeks?

U(97) Three weeks.

N(97) Three weeks only.

AU Having gone through the questionnaires and talked to people I found that in some cases you have three weeks of practice teaching and some teachers did very little teaching because they lost a lot of days because of school functions and a lot of things and exams or tests were on so they couldn't do anything. It's very difficult, very difficult for the course tutors to be able to find schools which are willing to accept BATESL people. What thoughts do you have about your practice teaching, was it a good experience? Was it a realistic experience as regards teaching?

$\mathrm{O}$ (97) I think it's definitely not realistic because we were just responsible for teaching one class, English lessons; it's just one tenth? I don't know, one third of the usual teachers' duties. But it's still good to have a chance, for the students to be there - get to know what the students are really like in the real world. And try to prepare a good lesson for the supervisor to see. I'm sure it's very worthwhile.

At least you were in a class, in front of a class of students. That's the main thing. There are so many other things which you couldn't experience at the same time, like what it's like to go through the whole term and all the other things you have to do and the pressure builds and builds and builds.

N(97) As for me, it's totally unrealistic because I was quite playful at that time, I think. I wanted to prove some theoretical things or some methodology which has been all along rejected by those experienced English teachers, for example, drama or role-plays, they often rejected them. I just want to try to prove that all those methodologies could work in my practice teaching. So it's unrealistic but it's fun. I think the greatest reward is that the students told me that it's the greatest three weeks when you were with us. I think the students' encouragement, all this is the best reward, they still send me cards now. But I'm not a teacher now so I'm not sure if it ... 
O(97) Still works.

N(97) Still works, yeah. It's happy and it's fun. Actually I've tried to put what I've learned in reality, I think this experience is really good.

O(97) I've also tried other ideas that [BATESL Course Leader 1999] taught us, something like troubleshooting in listening practice. I just find it terrible because it just bores the students. Well what we refer to troubleshooting is sometimes we give the same ... pieces of recordings we play it several times to show the students what the sounds is really like.

N(97) So I think that's what we have to learn, we have to adjust the methodology depends on the students' level.

That's right, that's teaching.

$\mathrm{O}(97) \quad$ I'm sure practice teaching improves our confidence in real life situation. I'm sure that if I asked you to come into my classroom, I've got the confidence to speak in front of forty students.

AU It's interesting what you were saying there about your students saying it was the best three weeks. It happens that when you are a full-time teacher and you have student teachers come in and they take your class and the students love the student teacher because .... and there's a little bit of jealousy there because of course the full-time teacher has a lot of things to worry about, like the syllabus and making sure they cover everything and so often they are not able to do the things that the student teacher can do, as they can do what they like, they have no responsibilities, they can just do activities and have fun and after three weeks they go away again. In a way it's not a good situation to be in as some students can play around with the student teachers.

N(97) But I think if we can keep - on the right track - keep ... I mean don't get behind the syllabus or the schedules, why not try? And then I didn't fall behind the schedule. If I got any problems on my arrangements of works or the schedule I will inform my cooperating teacher to see how can we cooperate or how can we solve the problems together.

AU Table 16.

N(97) Page 21.

AU 'Extent to which their lesson planning might be affected by the changeover in sovereignty', To 'little' or 'no extent' most people said. How do you feel about that now? Has it had any effect? In terms of perhaps the content of lessons, do you think that would have changed any? Or will it change any 
in the coming school year? If schools are going to change from Englishmedium to Chinese- medium, can you see any change in the English class?

U(97) No.

AU Still be taught the same?

U(97) Yes, I think, with the same syllabus, I think.

AU Still with the same public examination?

U(97) Yes

AU Table 17, 'Should exam requirements determine what is taught in English lessons?' The group generally agreed with this. I like the comment, number 1 , 'Because students must sit for public exams. Students are examoriented.'

N(97) Students are exam oriented? Teachers are exam-oriented, I think, not students. It's not fair for the students.

U(97) No, I think students ...

$\mathrm{O}(97) \quad$ I think students ...

U(97) $\quad \ldots$ not teachers

N(97) Really?

$\mathrm{O}(97) \quad$ Yes.

U(97) Yes.

$\mathrm{N}(97) \quad$ I think both.

$\mathrm{O}(97) \quad$ No no no no, teachers are student-oriented, but students are examoriented.

U(97) Right.

N(97) Maybe principals are exam-oriented.

U(97) Society is exam-oriented, right?

AU It was interesting that the group felt that as a whole, exams should 
determine what is taught in English lessons. Later on there's support for student-student interaction, less control by the teacher and so on. So in terms of Teaching Approach, the content, the group thought examinations should determine what is taught, but you can still teach it in an interactive way, which is kind of odd, I find.

O(97) I don't know, maybe in lower forms, maybe you can teach in a more interactive models, but in upper forms, the Form 4 or above, less interactive. Maybe because the teacher just give them some mock papers to do, how can it be interactive? I'm not sure.

U(97) Well I agree with [O(97)], basically the trend is like that and for senior form students if you use some interactive approaches, they will say that you are wasting the time. So it's a norm, I can say it's a norm.

AU Students will say that?

U(97) Maybe even complain.

AU I wonder about the exams, will they change? Not the English exams, the other

exams, they must change.

N(97) Yes the Examinations Authority's doing a review with Hong Kong Baptist University to review the whole examination system in Hong Kong. I think it's for one or two years. They are talking about writing a more schoolbased assessment.

U(97) It's not good.

$\mathrm{O}(97) \quad$ I agree.

U(97) Just like TAS, right - Teachers ... Scheme - when I was in matriculation. I think the same as for [O(97)], right? It's usually for science subjects. Can you remember that?

$\mathrm{O}(97) \quad$ Oh yes, we ... but - when I was in upper secondary, two subjects has been involved in that scheme.

U(97) It must be chemistry and biology.

O(97) No not biology, chemistry and ...

U(97) Physics?

$\mathrm{O}(97) \quad$ No, only chemistry ... 
U(97) Are you sure?

$O(97) \quad$ Yes, I need to take the public examination first.

U(97) It has been changed already ...

$O(97) \quad J u s t$ two or three years ago they don't have group discussion or presentation because ...

U(97) They only have integrated reading and writing and so forth.

$\mathrm{O}(97) \quad Y$ es, and I just find that it's a very good change because the teachers are more aware of teaching students speaking skills, let them to speak up in front of the public, which is a good change because if the cert. level examination hasn't been changed like what they've done now, I just find that as a student I just find that I've got no chance to speak English because we are just given a lot of paper work to do, I mean paper examination, written test instead of having a chance to speak.

The new format they have, the role play and group discussion, when I was teaching in the secondary school, I found that the teachers who were teaching Form 3 were introducing that in Form 3, the idea of group discussion and role play, so it wouldn't be a big shock when they went into Form 4. And it was quite successful, the students quite enjoyed it. Although they were quite good students at the school I was at, I think it's definitely a better idea than the old system of interviewing.

Okay, let's move along, Table 24, teaching styles, almost exactly split down the middle between learner-centered and teacher-centered. Do you think that there's been any change? Perhaps if you haven't been teaching it's hard to judge, but imagine if you were and you were going to teach now, would you be more learner-centered or more teacher-centered?

U(97) Well, I think there can be many factors. If students or learners are active, then we can be learner-centered. If they are not then I don't think ... even we adopt the learner-centered activities or approach, they will not participate in that. So firstly it depends on students' attitudes and secondly it depends on teachers' style. Some of the teachers like to be teachercentered, to save up the time and to give students as much information as possible within a limited time, so at least we have two factors.

AU That's what came out in the comments, it very much depends on the school, on the students, the level of the students - if they are good, attentive students, you can be learner-centered, if they are not you have to exert more of a control over them which necessitates you having to be more teacher-centered. 
$O(97) \quad$ I don't know because I can think of an example, say for example a teacher tried to initiate a discussion among the students but I guess it's regarded as a learner-centered activity, but the students just don't like that kind of a discussion. Then the teachers switch to the teacher-centered approach. But if you say that this is a typical case to show that it depends on your students, but I don't think it must be depends on your students because maybe you just can't initiate a very interesting topic or sometimes it depends on the teacher themselves rather than we just push all the responsibility on the students' shoulder. It must be the problem of the students.

AU The factors that the group felt had the most affect on their teaching Table 25 - English proficiency and motivation of students came out top as they had done in 1994. Whether the changeover will affect the approach to teaching - Table 26 - most people said no, again, as we discussed earlier, there's probably been no effect, or it's been hard to judge any effect so far. The main effect is going to be the change in the medium of instruction, which won't happen until next year. Professional

Relationships and Responsibilities, level of confidence felt by the group in their ability to become a successful teacher - the mean was 2.37 somewhere between 'Unsure' and 'Quite confident'. How would you feel now? [U(97)], do you feel more confident?

U(97) Yes I am - I have been to a range of schools — and they are Band 3 and Band 1 students and even they are in different bandings I can communicate with them and I can give them different level of knowledge and based on their proficiency.

AU You said before you feel quite confident.

$\mathrm{O}(97) \quad$ I'm still confident.

AU You've not had any opportunity to do any teaching, I suppose?

$\mathrm{O}(97)$. No, not for the last year

AU I did, I taught you for a little while. Mind you I was a little different as I had been a teacher. They allowed me to take a couple of sessions a week, which was good, it kept me in practice. But there's nothing like teaching regularly to increase your confidence. I must say I find it strange that even though I've been teaching for over ten years now that after a long gap, even just a few months over the summer, then it's time to teach again, a new class and you still feel nervous. [Tape ends]

Subject B said, 'Because I really don't know how I'll be able to get along with my colleagues and my students and which school I'll be in.' This is 
you, isn't it? I forgot about that. 'I don't speak very fluently and I have no experience in using simple English and those specific terms. What I lack is the confidence, I mean the experience.' So you will teach? Sometime in the future you will teach?

O(97) Yes, sometime.

AU You'd prefer to teach at a tertiary level or a secondary level?

O(97) No no no, I prefer teaching in a secondary school. That's what I think. I just got no response from the schools.

N(97) So you are on the top of the waiting list?

$\mathrm{O}(97) \quad$ Yes

AU Status of teachers ... pretty much in the middle of 'No special status' and 'Moderate status'. What do you think about the status of teachers now? Do you think it has changed any in the last year?

$\mathrm{O}$ (97) Because of the decline in the Hong Kong economy, the status become more and more better because the income and the stability.

AU But is it the job that has become a higher status or teachers? It's now become more of a desirable job ...

$\mathrm{O}(97) \quad$ But the status is still the same.

AU There's quite a lot in the newspapers about people complaining not about the status of teachers but about the quality of teachers.

N(97) Exactly, I think ...

$\mathrm{O}(97) \quad$ They've agreed to set up some sort of examination for the teachers? For English teachers, to test their ...

U(97) Not only English teachers, all the teachers in both primary and secondary schools.

$\mathrm{O}(97) \quad$ They want to professionalise the job of teaching by asking the teachers to get the Cert. Ed. or the Bachelor of Education. I was told the other day that by 2003 every English teacher should have a Cert. Ed. or Dip. Ed.

$\mathrm{N}(97) \quad$ But I think to a certain extent the status or quality is not so closely related, that means I think the status is lowering because today there are more and more people can be teachers and there are a lot of opportunities for you to 
further your studies and become a teacher especially now the economy is so difficult that people are rushing to become a teacher. So, it seems that it's no more a difficult job, to be a teacher, no more difficult for you to be a teacher, so the status is lowering. Another reason is that the parents are more and more educated. In the past they were less educated and they absolutely and totally depends and believe in the teachers, but today, a lot of parents are challenging the teachers. No matter they are reasonable or not, they try to challenge the teachers, and that's the reason why I think the status of teachers is lowering.

Yes, I'd agree. Always when the economy goes down, teaching becomes a more attractive job. You find that when the economy picks up again then people will not want to be teachers any more, they want to go into business. It's a safe job, as you say, the salary is pretty good, the holidays are good, there's security. I think what the Chief Executive has said is that they want all teachers to be university graduates so the idea of a C.M.I. [C.M.] eventually will be phased out. You'll not be able to teach just with a teaching certificate. You'll have to be a university graduate. Then hopefully eventually, like it is in the UK, you'll have to be a university graduate and have a certificate of education, or have a B.Ed. So, be a graduate and a trained teacher before you can actually teach. And then, in England you have to have both, then your first year in teaching is a probationary year. Only if you are considered satisfactory after that first year can you call yourself a qualified teacher.

$\mathrm{N}(97) \quad$ Probation for one year.

AU Yes, whereas in Hong Kong, in the past, people could do two years Cert. Ed. then they could teach. It doesn't necessarily mean that someone who gets a bachelor's degree and a cert. ed. is going to be a better teacher than someone who hasn't got that qualification. Some people are just naturally good anyway. The idea is to raise the professionalism and naturally that should raise the status as well. If it becomes harder to be a teacher, the status should become higher. The status of lawyers and doctors is high because they have to train for five or six years at great expense to the government before they are qualified.

The idea of whether people saw teaching as a long-term career didn't really change after three years of the BATESL. I don't know if that would please the course leaders or not.

Some of the comments are quite interesting. Subject A found that, 'Reading articles is quite useful because when I did the final-year project I found that some of the articles is quite useful for me.'

'The demand for English teachers will diminish.' Do you see the demand 
for English teachers diminishing?

$N(97) \quad$ No, of course not.

$\mathrm{U}(97) \quad$ Why not?

N(97) Why yes?

U(97) Because we will have more expatriate teachers and more local teachers, so the demand will decrease. You may say that we will have new schools, but at the same time...

N(97) As far as I am aware those CMI schools will get four more English teachers ...

O(97) Excuse me, they can choose the salary which is paid for additional GM, they can use that for resources, for buying machines or buying facilities, setting up self-access centres, something like that. I mean, most principals don't use the amount of money for employing additional teachers, instead they just use it as ...

N(97) What I mean is the NET teacher, the native teacher is an extra one, so the basic numbers of English teachers ...

U(97) Will decrease.

N(97) ... didn't change.

U(97) No, first of all they will have a decreasing number of classes, say we will have each level from Form 1 to Form 3 in my mother school we will have five classes each, the same as Form 4 and Form 5. In most schools they may have six classes or even eight classes each in Form 1 to Form 3, then principal want to lower the gap of the class number, that will cut down the number of class in Form 1 to Form 3. Then the demand for English teachers will decrease.

Can you explain that to me again? Why will the demand for English teachers decrease?

U(97) Because the number of classes in junior form will decrease in some of the schools, especially those CMI ones.

AU How can the classes decrease?

$U(97)$ Because there are less students in that level, so the principal combined them together. Say they may have thirty-five in six classes, so they can 
gather together as forty in five classes.

$\mathrm{O}(97) \quad$ More students in one class and less number of classes?

U(97) Yes, you get that?

AU Yes, increase the class size.

$\mathrm{U}(97) \quad Y$ ou may say that but maybe the demand for the position decrease. When the population is steady ...

N(97) That means there are less and less, there are fewer and fewer children?

$\mathrm{O}(97) \quad$ Low birth rate.

U(97) Yes.

AU But the population is increasing; it's going to increase to about eight million in the next twenty years.

N(97) As far as I know they are planning to narrow down the class size, so theoretically if they narrow down the class size there will be more classes.

U(97) No.

$N(97) \quad$ Because of the lower birth rate.

$\mathrm{U}(97)$ If they lower down the classes gap, then the number of classes will be decreased.

N(97) Then more children, smaller class size.

U(97) No more children and larger class size.

$\mathrm{O}(97) \quad$ But long-term, what's the policy that will be put forward by the government, I mean I was told that the class size will be reduced, but is it the case ... as you know?

U(97) In different districts.

U(97) In different districts, that means I guess more or less the same, I mean number of teachers required. I think it's really a threat, those native teachers of English those expatriate teachers, it's a threat ... to me.

AU I don't think it can be seen as a threat because they are just employing two in each school at the most, they are hoping to, but they've found that they 
just can't find enough teachers, native English teachers.

N(97) I think we have to see them, to wait for the feedback, maybe the principals don't like the native teachers.

O(97) The point is the government doesn't want to listen to our voices.

U(97) Our voices, what do you mean by our voices?

$\mathrm{O}$ (97) Because the policy reflects what is in the Chief Executive's mind. It represents that they think that the native speakers of English means better English input for those learners. That's the point, that's the threat, instead of the teachers themselves.

AU Instead of raising the standard of local teachers?

N(97) Exactly.

AU I think one reason it's been done is to make parents happy. If suddenly their children are going to have to go to a Chinese-medium school, they said, 'Okay, but we are not going to put any less emphasis on their English, we are going to employ native-speaking teachers to help with their English, don't worry about that.'

N(97) I think it's a stupid prejudice or perception that a native speaker is better to teach English in Hong Kong, it's a conventional thought, but after professional training I think it may not be true. I think the local teachers; Hong Kong people can be very very great as a teacher as well. To a certain extent, the communication between the local teachers and the students, the local students is better than the native-speaking teachers and the local students. I think we shouldn't blindly think that the NET scheme can polish up the English of the students or can polish up English education in Hong Kong.

AU Sure, but if you look at it in comparison with other countries, perhaps, in foreign language learning, if we regard English as a foreign language in Hong Kong, which basically it is I think, if you learn a foreign language in other countries, like in England if you learn French, then we would have a French Assistant. And he or she, and it was usually a she, would be a natural French person and would come in and would help, mainly for speaking practice, and so students would get their accent right and so on. I think from that point of view, native speakers have their place, but I'd agree that they can't just bring in the native English teachers and give them the best classes and let the local teachers do the best they can. It doesn't work like that and it's not good for the morale of local teachers. The idea, as we said before, is to make sure the local teachers are better 
trained.

Okay, [Perceptions and Values] philosophy of teaching, it's on page 44, 45 , the philosophies of teaching, which I grouped here. Subject B's philosophy of teaching focused on student needs, 'Increase the chance of language use among students, conscious about the students' attitudes towards learning English. Give students a reason for learning English. Care about students including their personal life. If they're willing to share with me.' What I found is that most of the students expressed student needs in their teaching philosophies, a desire to care for the students and look after their needs. And responsibility was another one that came out. I divided them up into areas and responsibility came out quite high, as does competency and even teachers' insecurity. And then student-centered approach was very high as well. If I asked you now, what is your philosophy of teaching, what would you say?

U(97) Practice makes perfect, to both teachers and students. If you teach more, you know how to teach better, especially when you have to deal with different students in the same class. And when you need to keep them to pay full attention in your class, that really needs experience.

$\mathrm{N}(97) \quad$ I think as for me, my philosophy has never changed, that is I want to English, I mean I want to use literature, writings to help students to find out their own directions, their visions of life. I think that's the most important. To help them to grow up ... not help them to grow up but to be with them.

$\mathrm{O}(97) \quad$ How about using Chinese to do this?

N(97) That's okay, the most important thing is try to fully utilize your subject, I mean for example you're teaching EPA or anything, that's not the point, the point is walk along with your students at the most crucial stage, when they're growing up.

$\mathrm{O}(97) \quad$ To me, I think my philosophy remains unchanged because I still think that the reasons why students have to learn English is still playing a very important role to them, I mean as teachers we should try to think from the position of those students. Why I should sit here and listen to you, $[O(97)]$ you teach me English, why I have to learn English. And try to understand them, the reasons to learn and it may help the teachers decide what to teach, and how the students can learn better, provide a better environment for the students to learn. I guess - it's student-centeredness.

AU Listen to the students, listen to the students. That came out so strongly and didn't really change very much, everybody was student-centered at the beginning of the BATESL and was still student-centered at the end. 
Page 50, teaching skills, in 1994, the group felt that speaking and listening were the most important, but now the group felt that grammar was the most important to teach. Why do you think that is?

O(97) Vocabulary.

N(97) Maybe it is the norm of the local education? I think maybe they change their mind first, they are a teacher now and they found that in the local curriculum, no matter which level, a lot of emphasis placed on grammar. That's why they think that grammar is the most important. As for myself, I still think that speaking and listening is the most important, especially for the real-world communication.

AU Speaking and writing, the production skills came out highest, and then listening was quite low down this time. I was thinking that perhaps because at the end of the course you'd all been teachers and you'd been teaching, at least for a little while, and you realise that when you're a teacher, and you see their composition books, and you've got to mark those books and you've got a big pile like this and you've got to mark the grammar, you realise how important grammar is. For the students perhaps in real-life communication in speaking and listening, maybe even pronunciation are quite important, but how often do secondary school students actually need to communicate in English, so grammar becomes fundamental for them. Would you agree?

U(97) Yes, sure.

$\mathrm{O}(97) \quad$ And also the examination test the students' grammar knowledge, rather than those four skills. Students need those four skills but usually when they got into the practice in the mock papers they can learn it from trial and error. But grammar is the fundamental thing for all the papers in the public examination.

AU Okay, relationships a teacher should have with his or her students 'Teachers should try to become friends with the students' was ranked sort of about the middle. It was actually ranked higher in the early one, in fact in 1994 that was ranked first, 'Teachers should try to become friends with the students.'

N(97) We were still students at that time ... lecturers to be our friends. After the practice teaching you find it's very difficult to be friends, I mean the line is very unclear. If you are too friendly with the students, the class may be totally out of control but if you are not friendly with the students ...

U(97) They will find your lesson boring - maybe they find you boring. 
N(97) Today's students are always looking for love and concern of the teachers, if you are not friendly with them they find that, 'Oh you are not care with me, you don't love me,' something like that. Then they may jump down from the ... it's very difficult to adjust the position of teacher, but what I'm sure is that you can't be too friendly with the students.

$\mathrm{O}(97) \quad$ It's interesting because a friend of mine who has been teaching in a secondary school, he got a quite good relationship with the students, but maybe because of the good relationship, some other colleagues feel jealous about that and ...

U(97) It's usual ...

$\mathrm{O}(97) \quad \ldots$ and say some bad things behind his back, especially ...

N(97) So narrow-minded ...

$\mathrm{O}(97) \quad \ldots$ say to the principal, and then he can't get promoted.

N(97) I think the most important thing is to let your students respect you because sometimes if you find you are friends with them they may not show you respect. It's no good, I think.

U(97) Maybe students have to respect you at least in the classroom and out of the classroom they can be very friendly with you. It seems that this problem can be solved but it's hard to control. You can't tell them, Now you have to respect me,' even if you give them an explanation, they may not be able to follow.

N(97) Yeah, exactly.

AU I notice your comments here, Subject A was not so sure, Subject $G$ was in agreement, 'Teachers should try to become friends with the students.'

Subject A was not so sure, 'I'm not sure it is possible to become friends. If you are too friendly the students may not behave properly in class.' This is basically what you've just been saying, isn't it? Some other teachers may be jealous. Yes, I found that, especially teaching in girls' school ... not from my point of view, from other teachers. I noticed that some of the younger female teachers would try to become friends with the students, and act like the students - laughing and giggling like schoolgirls and trying to become friends with the students by behaving on the same level. I don't know what they were like in the class, maybe they were totally professional, but outside of class ... I didn't like that.

Okay, I've asked you about your practice teaching, how do you feel about the BATESL now? 
U(97) We need promotion for the BATESL.

AU The course needs to be promoted?

U(97) Yes, to school principals, at least, to tell them what the graduates know and what they are expected to teach - the course needs to be promoted.

AU Right, I think that you may have mentioned this to me, or somebody did last year as well, that people were finding it hard to get a job because headmasters and principals were not ... didn't know what the BATESL was. They knew what Cert. Ed. was from the Institute of Education and they knew what a degree was from Hong Kong $U$ or something, but the BATESL from City U?

U(97) Maybe you will get the first question is, 'Tell me what did you leam from your course?' This can be your first question.

What would you say? I learned how to be an English teacher?

U(97) I focus on internship and practice teaching and the teaching skill we learnt. This, I think.

AU I think that's a good point. They should promote the BATESL as producing the cream of the English teachers - specialist English teachers for Hong Kong secondary schools, that's the whole idea of the course. It's a crime that the graduates from the course can't find jobs as English teachers. It seems to me crazy. How do you feel about the BATESL now?

N(97) I'm not sure. I don't have such practical comments on this; I've just got some personal feelings. I think campus life's always great and if you ask me to look back for the three years I can just remember three things: India tour, Singapore tour, penguin boy [?]. All these are enough for my life, the drama, the tours that we choose, the friendships, the friendships with lecturers even. So that's it!

O(97) I like the BATESL course still.

AU You like it so much that you are still there! You don't want to leave.

$\mathrm{O}(97) \quad$ I stay there because three months before my graduation I was offered this position. That's why I'm still there. I think although sometimes I found some of the modules are not that practical, but generally I think ... it's really able to equip teacher trainees to be a good teacher. That's my general opinion.

AU It would be interesting for the department to survey the graduates to see 
just how many of them are actually teaching. Do they do that; do they keep in touch with you?

$\mathrm{N}(97) / \mathrm{O}(97) \quad$ Not from time to time.

AU But if they did it say one year later, five years later and so on ...

N(97) Actually we talked once ...

U(97) Only.

$\mathrm{N}(97) \quad \ldots$ after graduation like we are doing now to try to do some survey or improve the course and they publish a newsletter and through that we can keep in touch through the newsletter. I think they are doing quite a number of things in trying to promote the course and trying to increase the status of the course and trying to help graduates to have a better future, better prospects and so on. I really appreciate they effort, although I don't know what they are doing

AU $\quad$ Right ...

N(97) Actually I want to ask you a stupid question, what exactly is your thesis talking?

AU My thesis is talking about learning to teach English in Hong Kong and the effect of the changeover and how all of this has affected teaching in Hong Kong and teacher education in Hong Kong, so I've been able to look at you as my subjects and how your views have changed since 1994, and the comparison between you and another group which graduated in 1994, plus the survey of what's been in the newspapers and interviews with lots of different people all combined into a general survey of how English teaching is now. 


\section{Interviewees: $\quad$ Graduates $Q(97)$ and $T(97)$ \\ Date:

$\begin{array}{ll}\text { AU } & \text { Researcher } \\ \text { Q(97) } & \text { Interviewee } \\ \text { T(97) } & \text { Interviewee } \\ \ldots & \text { Missing words / Unfinished utterance } \\ \text { - } & \text { Pause }\end{array}$

AU Although you are not actually teaching at the moment, have you done any teaching at all this year?

$\mathrm{T}$ (97) Something maybe related to teaching. I'm working as a part-time marker in an education company, mainly marking some business-related assignments.

AU I see. What sorts of students are doing this?

$\mathrm{T}$ (97) They are mostly mature students working. They attend some course on business, mainly on diploma level. The course cover something about banking, finance, accounting and management.

AU Right. So you've been marking their assignments?

T(97) Yeah.

AU How've you found that?

$T$ (97) Some of the students, maybe the presentations skill is quite weak, but some of them are very good.

AU A big variation.

$T(97) \quad$ Yes, a big variation.

AU $\quad[Q(97)]$, have you been teaching?

Q(97) Yes, I used to be a supply teacher for the Education Department for about two months. So I teach both primary schools and secondary schools. It's quite a nice experience, but unfortunately it forced me to give up my willingness to be a secondary school teacher.

AU Really? Why was that? 
Q(97) Because, actually I was disappointed with the standard of the students even though I was sent to a Band 2 secondary school, it's supposed that the students are able to learn in English, even though not all of them were graded at that level. But however, even the best class in the form, I still found that they are not up to the level to be taught in English. It's terrible because if you mark their composition, it's a kind of torture, you know that - you cannot find a single sentence which is complete and perfect in grammar, so, from the very beginning until the very end you have to use your red pen to mark every detail.

AU Yes, I found that even though I was marking Band 1 students it was often the case.

Q(97) It's exhausting.

$T$ (97) I think so, even such kind of mistakes makes it difficult to understand what they write, their ideas, and this is a major problem of my experience.

AU It's something that comes up later so we'll leave it for now. Let's start of with Language Use, which was the first section. From what the group said in the questionnaires, basically, not surprisingly, most of the group said that their English proficiency had improved from the beginning of the BATESL to the end of it. Although what I noticed was that people said that their spoken English had improved more than their written English.

T(97) I think so.

AU Why do you think that is?

T(97) Maybe about the improvement in the spoken English, maybe in that environment, in the BATESL, it forced you to use more English, you have no chance to use Chinese to speak with lecturers. And it's the major reason I think.

AU The lecturers insisted on you using English?

$T$ (97) Maybe the environment forced you to use English. We had no choice.

AU How about writing? Did you do much writing?

Q(97) Not so much in my mind, however, since we have improved a lot during the course from written skills, speaking skills, something like that. I think we learned something about how to improve the writing style, some techniques, some skills to improve our writing style. For instance, maybe before the BATESL course our written standard is up to B or C level, but 
after the BATESL course, it may be up to B or A. Not just because we improved a lot, but because we have learned some skills and techniques to improve our writing style. We know something should be written in a certain style or a certain format, so we know how to make use of the language to use in the correct positions and at the correct time, so it's a kind of improvement.

AU How do you feel about your language proficiency now, one year later? Do you think in the meantime it has improved, or has it got worse? What would you say?

Q(97) Downgrade a little bit because during the time when I was still a student in the BATESL course, I have a lot of exposure to different kinds of English, like Australian English, American English and so on and then I was able to pick up the language quite easily and I was able to use the language conveniently. However, after my graduation, I discovered that my exposure towards English decrease a lot, to a significant level, so I was enforced to use Cantonese most of the time. So I realised that after I cannot make use of English in my daily life, I realised that my proficiency downgrade a little bit, not so significant but I realise that

AU Would you have the same feeling?

$\mathrm{T}(97) \quad$ The same feeling because due to my job nature there's less opportunity to use English and I have to write Chinese, read Chinese much more, comparing to the BATESL course. And the main reason is that I can choose to use Chinese or English because in terms of communicate with other people in the office, it's much more convenient to use Chinese. Maybe some of the people in the office, the English standard is not good, so it is much more convenient and better to use Chinese as a communication language.

AU Does that worry you at all, the fact that your English is not going to get better?

Q(97) Yes, I agree that ... I worry about this for several months time so actually I plan to employ an English teacher, private English teacher to help me to improve my oral English. Because right now my job duty is working as a kind of managerial staff in the Hong Kong government, it's quite important that I must communicate with those different kinds of people including those foreigners and foreign staff in various departments so I must be able to communicate with them effectively so I must improve my English. And I'm quite lucky that as the managerial staff in the government we can receive different kinds of training including language. So we can ask our department or even the government to sponsor our studies in language. 
I was going to ask that actually, if your department provided any help.

Q(97) Yes, they provide training. You can attend some course or you can employ some private teachers. Of course they must be a qualified teacher from their list.

AU How about you [T(97)]? Have you thought about doing anything positive about maintaining your English standard?

T(97) Yes, maybe I have to use Chinese in the office but outside of the office I use English as much as possible. Maybe I haven't planned to employ a private tutor but I use a self-study approach. I try to read more English books and try to communicate with some friends using English as a mother tongue. In summer maybe expose more in English as possible outside the office.

AU Why in summer?

$\mathrm{T}(97) \quad$ In summer?

$\mathrm{Q}(97) \quad$ Why in summer, and not in other seasons?

$\mathrm{T}(97) \quad$ In summer?

AU $\quad$ You said ...

Q(97) You try to have more opportunities to improve your English in summer, so why you think so?

$\mathrm{T}(97) \quad$ Not in summer, maybe you misunderstand it.

AU Moving on, you said that when speaking English with friends, looking at the idea of code-mixing and code-switching, the group admitted to codemixing a lot more than code-switching - do you still find that you codemix when speaking in Cantonese as much as you did before?

Q(97) Yes - right now I realise that I even added in - not just code-mixing it seems that I substitute English for even a whole sentence in Cantonese because sometimes when I communicate with my

friends sometimes I realise that it's much better for me to speak or to express myself in English so sometimes I just substitute English into my sentence so for instance after a few words I may speak some sentence of English. It's a kind of ... it's a tendency that I increase to use that kind of sentence structure.

AU So actually code-switching rather than code-mixing? 
Yeah, it include transfer from code-mixing to code-switching - maybe.

AU How about you Clifford, do you find that you're still code-mixing when you're speaking?

$T(97) \quad$ Still a little code-mixing, but the difference is not so obvious.

AU Code-switching at all?

T(97) Code-switching still the same thing, not much change. Maybe I don't like code-mixing and code-switching. This is my habit of ...

AU You'd rather just speak English, or speak Cantonese?

$\mathrm{T}(97) \quad$ Yes, I prefer this.

AU It's just that what I've found is that the university environment, students in Hong Kong ... it's just a natural way for students to speak. Perhaps out of that environment for a while, you may find that that falls off, but it hasn't?

Q(97) Yeah, because it may be due to working environment and also our circle of friendships because most of our friends, our colleagues have already received their education in university so they will be able to understand most of the English terms. And also they prefer to use that kind of terms because sometimes it's really difficult to explain some terms in Chinese so if you speak the English word they will be able to understand the meaning directly.

Right, I was thinking that, if your friends are university graduates then that language pattern that was developed within university will be carried on and you find that most educated people in Hong Kong, Chinese people do speak using a lot of mixed-code. As regards the use of English in teaching, what I found was the group's views on using just English or using mixedcode were not very clear and there was generally a mixture between those agreeing that you should only use English and people disagreeing saying it's okay to use Chinese. How do you feel about that now, how do you feel about the use of English and Chinese within teaching?

$\mathrm{T}(97) \quad$ I prefer using a mixed-code within teaching in the classroom because as in Hong Kong the students' English standard is not so good. If you try to use English for all purpose at all time they may not understand what you say and they may bored and other problems may arise such as discipline problems. And if you can use some Chinese, maybe explaining vocabulary so the students may understand what you say, if you speak some Chinese, so they have some feeling of achievement since they understand what you say and they learn something and create a good learning atmosphere. Use 
English only, the students may feel bored and it doesn't create a good learning atmosphere.

$\mathrm{AU} \quad$ That came out pretty well in the results as well, later on in the section where it said for what purposes would you use English it was mainly when students indicate that they don't understand or to explain vocabulary. Would you agree with that, $[\mathrm{Q}(97)]$ ?

Q(97) I think that I have changed a little bit but not so much. In my mind I remember that years ago I talked to you that I insist to use pure English in the classroom. However, I can tolerate code-switching but not codemixing. But after several months of teaching experience I prefer codemixing rather than code-switching. But still that I insist on using pure English in the classroom, however, sometimes for the purpose of efficiency or teaching purpose, we can use code-mixing rather than codeswitching. I realise that code-switching is time consumption in the real classroom environment, sometimes we have to teach a lot but we do not have so much time so if we always explain something in English one time and then use Chinese to explain a second time it's time consumption and I don't think that such kind of time-consuming activities would be a good method because I realise that sometimes you just use Chinese to explain some words will be enough. So, after some months of teaching in secondary schools and in primary schools I realise that code-mixing is more effective that code-switching.

AU So simply delivering the lesson in English and occasionally just a quick explanation in Cantonese just the translation of a word or ... keeping the amount of Cantonese that you are using to a minimum.

Q(97) Yeah.

AU Page eleven - the effect of the changeover in sovereignty on the use of English in secondary schools - the mean there was around 2,

people thought 'to some extent' - actually quite a few thought 'to a large extent'. Have you noticed any change in the use of English in schools, although I know you haven't been in schools very much?

T(97) I'm not clear about this.

$\mathrm{AU} \quad$ Even your opinion as a member of the public?

Q(97) I think in the school environment it change a lot because most of the schools have been enforced to use Cantonese as the teaching medium, except in the lessons of English, so it change a lot, but not to some extent to most of the schools. But to some of ... however it change the teaching 
pattern in the English lesson because they have to use different kinds of activities and even those exercise to reinforce the learning of the students in lessons, so it change the teaching pattern in the English lesson and also for the whole environment it change a lot because only about one third of the secondary schools was still allowed to teach in English, about two thirds of them were enforced to teach in Chinese. So for the vast majority of the schools the environment has been changed and they must use Chinese.

AU But that's not actually going to take place until next September, the change in the medium of instruction.

Q(97) But they have already to plan and to change the lesson pattern.

AU How do you think that is going to affect schools? Is there going to be any great change in the majority of schools as they are now going to be officially Chinese medium?

Q(97) Yes, for the grades of students, that's the first impact. The second impact is for the students, their exposure towards different kinds of English vocabulary will be limited and also they have to face a lot of exercise and also some kinds of extra activities so as to reinforce their learning.

AU How do you feel about the medium of instruction issue? Have you been following it in the newspapers?

$\mathrm{T}$ (97) Yes, yes - maybe there is some change - maybe the students use more Chinese after 1997 because ... I'm not very clear about it since I haven't teach in school after graduation.

AU Thinking about the school you did your practice teaching in, was that an English-medium school?

$\mathrm{T}$ (97) Now it is a Chinese-medium school, after 1997 but when I was ... during the practice teaching, some of the subjects in that school were taught in Chinese, some subjects are taught in English.

AU Did it depend on the subject or depend on the teacher?

T(97) Depend on the subject. Some subjects such as history or integrated science they teach in English, it is more convenient, but geography was teach in Chinese. But after 1997 that school was officially a Chinese medium of instruction school.

AU So presumably all those subjects will be taught in Chinese? 
AU Do you think that will be a problem for the school or the teachers?

$\mathrm{T}(97) \quad$ It's a problem for the school but I don't think it's a problem for the teachers or students because from my experience, my secondary school, my mother school, I remember in Form 6 and Form 7, all the subjects are teach in English. But after one year later, the Form 6 and Form 7 students, the medium of instruction was changed into Chinese and the passing rate is much higher. And even more students has got $\mathbf{A}$ in other subjects, except English. This is a fact. I think it is better, to use Chinese to learn other subjects.

I've found that the whole idea of the medium of instruction has been a major news story. Another part of what I've been doing is following the issues that have been reported in the newspapers, the English newspapers to do with English and English teaching.

Q(97) But I think the government should not push secondary schools to adapt to this policy so quickly because it certainly increase the burden of those schools and the teachers because for the schools part they have to find those teaching materials to support the teachers and also the lessons and they suddenly realise that in the market they cannot find enough materials, especially written in Chinese to teach the relevant subjects and that's the first problem. And the second problem is that the teachers have to prepare the notes, to rewrite their notes once again, to teach the students, and the most significant problem is that several terms like the names in history and also the terms in mathematics, sometimes we may have different translations in China or in Taiwan or in Hong Kong. So, we do not have a unified system for that kind of terms and also for some names for specific persons, so if the teachers have to teach that kind of terns in Chinese, they may face the problems that which terms or which names they should use or because they have to translate it in Cantonese. However, the students have to face the public examinations, so which is the standard? And that's the problem, so a lot of teachers complained that they have to rewrite their notes and they have to face that kind of problem so their pressure and also their workload increase a lot.

AU That has been something that has come up as well, the fact that it will be quite difficult for a lot of teachers to suddenly change from teaching in English to teaching in Chinese because they were taught in English when they were students, they learned how to teach in English when they did their PCEd or whatever they did, so to suddenly change requires quite a lot, plus a change in textbooks and everything. There are a lot of issues. I tend to think it is going to be like the new airport, in that in the beginning there will be lots of problems, but after a while they'll eventually be okay 
but ...

Q(97) Yeah, I think in the long term it may be good to some of the students who are classified as those students who should not use English as the medium of instruction because in the long run if they can learn in mother tongue that means they can increase their interest towards different kinds of subjects, so in the long term it's good to that kind of students. But the policy cannot ensure a smooth transformations from the true process.

Okay, Planning and Decision-Making was the next section - generally the group felt that lessons should be well-planned but that it was not possible to plan every lesson in detail. Page 17, that's Table 11, 'Should teachers be more responsive to student needs than the set syllabus?' The group generally agreed that student needs were most important. Do you still agree with that?

T(97) I'm not quite agree with that because the resource is limited so the teacher cannot be too responsive to every student, since there are forty students in a class. It is impossible. Even if it is possible, the teachers maybe spend too much time to the students, and it is not balanced. From a commercial point of view, I don't agree with this statement because I think the students should fit the syllabus by adjusting themselves — by adjusting themselves.

AU It is tricky isn't it? The idea of the syllabus is that it's been designed - by experts based on what they think the students do need, based on a lot of factors which students themselves don't realise as they are too young.

T(97) Students should put some effort on fitting the syllabus, yes.

And I think that it is - idealistic in a way that when you read that you think, students' needs, that's what you have to deal with and we have to be student-oriented and so forth - slightly less agreement in Year 3 than in Year 1, so in Year 1, everyone agreed a bit more with that statement than they did in Year 3.

Q(97) I think in the realistic situations, most of the school teachers would try to do so for those experienced teachers would be able to do so because they are mature enough and they have enough experience to identify the different kinds of students. And even those for that kind of teachers, they will use a selective method, that means they will only select those outstanding students and also the worst students in the class, and then they give them some sort of extra practice or guidance so as to help them. For the outstanding students of course they hope they can get a distinction in the public examination so they have to give them some extra guidance and practice for them. For those worse students, they hope that they wouldn't 
fail in the public examinations and so they need some kind of specific guidance or extra practice, so I think for those experienced teachers they will try to single out these kind of students, rather than the general grade students.

And often, as you said, [T(97)], there's so many students in the class that you can't get to know what each of their needs are. You know who the weaker ones are and you try to help those and the best ones, it's those in the middle that are the ones that usually get neglected - most of them can be okay and they can carry on but some may veer from the middle down to the bottom if they find that their needs are not being catered for. Let's see what else is interesting - Table 16, Page 21, the extent to which your lesson planning and decision-making might be affected by the changeover in sovereignty. 'To little extent' - people thought it wouldn't really change. [Q(97)], you actually said, 'Yes, that's a compensation because owing to the handover the students' exposure to English may be limited in future so I think I may need to include more topics.'

Q(97) Yeah, I think so, and I realise that during my teaching experience when I was working as a supply teacher in secondary school, I realise that some of the English teachers have already started to do something to improve their students' exposure because they realise that the secondary schools that use English as the teaching medium, they must do something more to improve their students' English, so they plan a lot of activities for the English Club, for the ... students' activities and also they encourage the students to do something more so as to improve their English. So I realise that and I still insist that statement.

AU That's another thing that's come up a lot, that people will see the schools that are going to become Chinese medium in order to compensate perhaps for their reduction in the amount of English they are exposed to maybe to plan possibly more extra-curricular activities, English activities. And the next table was whether exam requirements should determine what is taught in English lessons, which is now in the Teaching Approach section. The mean here was 1.93 which is actually 'Agree', so in general the group agreed that examination requirements should determine what is taught in English lessons - which is not unusually, but what we found from other questions was that the group were in favour of a student-centered approach and activities and experimentation and catering for students' needs, but on this question they've said that exam requirements should determine what is taught. Would you still agree with that?

T(97) I still agree with that because the students have to sit for examination because it is more clear to determine the level of the students, after the examination. After the examination the student has received a grade, so I think examination cannot be ... maybe ... it cannot be avoided, so the 
syllabus should follow the examination. And in a commercial society such as in Hong Kong, the grade is very important, the examination grade, it is very important, even in finding a school or in finding jobs so that all students should be examination-oriented, and due to this, the syllabus should follow the examination.

Right, the conclusion I came to was that the group agreed that ... [tape ends] ... anything you want it to be, as long as it's effective. Your comment on this one, [Q(97)], was 'I think that we should help our students according to their needs rather than exam requirements.' You disagreed on this one.

Which page?

Twenty-three.

Twenty-three - I think it's a correct direction but it's not possible, that means it's impossible to do so in the realistic situation after seven months of teaching. I realise that the school principal from last time will enforce those language teachers to teach according to the examination syllabus and also require the teachers to help the students to pass the examinations as much as possible, because it directly affects the reputation of the schools. So, for most of the time, the school master or principal or even the panel will enforce you to teach according to the public examination syllabus, so it's impossible to ignore their requirements. I think I may change my mind a little bit so that both is important. Both is important right now. Because it's impossible, so I may compromise. We say that we have to teach in accordance with the examination syllabus, however, we should try our very best to help our students and let them know how to learn and how to proceed to the next level. I may change my opinion a little bit.

The group generally agreed that students learn better through interaction with other students rather than through direct instruction from a teacher. Do you still agree with this?

Q(97) Yes.

$\mathrm{T}(97) \quad$ Yes.

Later on we had a question, in Table 21, whether there was any difference during practice teaching between students learning through interaction with other students or through direct instruction, it actually came out about equal. Eleven thought through interaction, eleven through direct instruction and eight thought no difference, with many people saying that the practice teaching was too short to assess whether either method was 
any better than the other. One year on, or more than one year on, how do you feel about your practice teaching now? Do you think it was successful or not successful or too short?

$\mathrm{T}$ (97) It was a little bit short because in Education Institution, the practice teaching is maybe eight weeks long, much more longer than the BATESL, but this phenomenon maybe only happened in my group that my tutor tell me she may come in Thursday and I just prepare to do better in Thursday. In other days I just fooling around and doing others and I think it is not a good idea to let the student know when the tutors come.

AU As far as I remember I think that is how it happened when I was training to be a teacher as well. I think that tutors or supervisors feel that it is putting too much pressure on young teachers to say, 'Well I will come at any time so you have got to be on your toes.' They realise that student teachers are under a lot of pressure and so they'll give plenty of warning of when they are coming. Even here, working in a university, you get assessed and a senior lecturer will come and watch you teach. How about you $[\mathrm{Q}(97)]$, how do you feel about your practice teaching?

Q(97) It's a very nice experience, I received positive feedback from my students and I really think that they were interested in my teaching and they had increased their interest towards English and they would try to learn more. If I could have been their English teacher I think at least their attitude towards English may have been changed. I think especially for those lower form students, the students attitudes towards the language is very important. If you have a positive attitude towards the class and also towards the subject then the students may realise that and they will be encouraged to take part in the lessons. However, if you just ignore their needs or sometimes you just think that it is a routine work, it's just a normal lesson then the students will also realise that and they will just ignore you, so a kind of communication between teachers and students is very important. But I do find some problems during my practice teaching because I realise that in most of the classrooms in Hong Kong, teachers, especially those experienced teachers, know how to simplify their language; they use some simplified words or patterns to teach the students. And our students are very familiar to this kind of patterns, so when I used to be a practice teacher in the class, I realised that I faced the problem because I do not know how to simplify the language because I always insist that the students should face the realistic situation so we shouldn't use any simplified language to them because they have to adapt to the realistic situation and environment, so I suddenly realised if I speak in the normal speed, something like two hundred and ten syllables per minute, it's not the normal way. The normal way would be up to two hundred and thirty syllables per minute. Even though I speak something like two hundred and ten syllables per minute, the students still have difficulties to 
understand what I am speaking. So I have to slow my speech and then I try to simplify my language, but I have difficulties in that way because I do not know what kind of simplified language I should use. For instance, sometimes I ask my students to do something, they may not be able to understand my meaning quite exactly so I have to re-explain to them or sometimes to use Cantonese. And that's my problem and I do not know whether some of my classmates or the new teachers will face that kind of difficulties or not.

Oh I'm sure every teacher will find that. You have to find the right level of English that your class will understand. You go through different techniques in which you lower down your level of English, with experience you can find which level your students can understand.

Q(97) And for those lower form students, I was really scared by their - level towards English. I asked them to write the 26 alphabets, in order, that's very simple, right? But it seems that some of them cannot do so. It seems that we have some problems with the primary schools teaching, because my nephew right now, he's studying in the primary school. I realise that for most of his homework, just try to enforce him to memorise those rules and those data, and that's all, but they have never given the reasons to the students, why they have to memorise them, so I asked my nephew to try to memorise those alphabets in order because it's very useful when you have to use a dictionary and learn how to spell some words, it's quite important. However, he just told me that, 'My teachers, they never taught me like this.' And I realise that even though we have to memorise some of the vocabulary, it's not in alphabetical order, so I no need to learn it, and that's the reason. So, I realise that - even for the primary schools students right now, they do not have that sense to learn English from the foundation. It seems that the teachers just want to enforce the students to learn those rules and also the set syllabus, and so on. They have never tried to give the reasons to the students that you have to learn in that way because of some reasons. They have never taught the students like that, so the students were just asked to memorise, day to day, month to month. And finally the result is not that significant because I realise that pattern in the lower forms in the secondary schools. It's terrible.

$\mathrm{T}(97) \quad$ But I think this phenomenon exists not only in secondary schools but also in adults. When I working in the companies, I received some fax from other companies. The writers' English standard is still at a terrible level. And I think the reason is that the education system has some problems, maybe in flexibility is the main problem.

I guess the Chinese approach to learning has always been through memorisation, that's how you learn to read and write Chinese, you have to learn to recognise and to write all the characters. In other subjects it's 
always traditionally been the way, of memorisation. There are new initiatives like the TOC being brought in, which hopefully will change this. And there is a lot of resistance to this because it's a comfortable, easy way to teach - this is it, you memorise it, you are rather right or wrong.

Q(97) It increase the burden of those teachers even those right now, some of the schools try to split the class into two large groups and both groups have their own English teachers. However, it's very difficult for those teachers to adapt to that kind of environment because in the realistic situations, English teachers have to teach both upper forms and lower forms, most of the time they have to teach three or four classes in the school, so it's really impossible for them to teach according to the TOC. I know that there's a team in the education department to try to develop the teaching materials for the TOC and also the curriculum for the TOC. However, it seems that those teachers in the front line cannot receive so much help.

Well it will have its problems, I guess, but hopefully they'll be sorted out as it goes along. And the problem often is, particularly now that we have had a big political change, is that requirements can change and the emphasis that might have been put on an initiative in the past can suddenly change and that's what we find with the Education Department, they come up with new ideas and suddenly everything is going to change, like it might be, continuous assessment has been mentioned, four year university degrees has been mentioned, and these are major changes, which teachers don't like. Teachers are saying, 'Oh God, they're always changing things, why don't they just leave it alone?' I guess you can't keep everybody happy. Right, Teaching Style, Table 24, it was pretty much in the middle, the group felt that they were between learner-centered and teachercentered. Do you think your teaching style will have changed in the last year?

Q(97) I think Subject H's opinion is quite close to the realistic situation, so ...

AU It depends on the banding of the students?

Q(97) Yeah, it really depends on the banding of students, and really, teachercentered approach is really efficient in the classroom practice. It's real, but I still prefer learner-centered because it help the students overcome some obstacles in learning, because sometimes the students were afraid of the teacher's authority and so on and they were scared of expressing themselves in front of the whole class. But is we try to adopt a learnercentered approach, it may help the students to overcome their own difficulties. For instance, in a small group discussion sometimes the students will be able to express themselves much better than in front of the whole class, especially in front of the teacher. I still prefer learnercentered but Subject H's opinion is quite close to the realistic situation. 
$T$ (97) I think about the learner-centered and the teacher-centered, just depending on the students. In my experience, higher banding students can be more learner-centered, and on the other side of the coin, lower band students should be more teacher-centered. Just depends on the students. I think there's no absolute answer about which one is the best.

AU Do you feel that the changeover will affect your approach to teaching? Table 26, most people disagreed with this, they didn't think that the changeover would have any affect on their teaching approach.

Q(97) I still insist on my own opinions stated before. I still think so.

$\mathrm{T}$ (97) I think there's no obvious change, in terms of the English lessons.

AU 'We need to encourage, we need to take any active role to encourage students and teachers to learn.' Most people felt, like Subject H, that English would be important and that therefore it was still important to teach it and that teaching approach would not change. Do you think it will change in schools which are changing from English medium to Chinese medium?

$\mathrm{Q}(97) \quad$ Can you explain your question, sorry?

AU Yeah, do you think the teaching approach will change in English lessons if a school changes from being a Chinese-medium school to ... from being an English-medium school to being a Chinese-medium school? So in a Chinese-medium school the English lesson might be the only time they have any English input. Does the approach to teaching English need to change?

T(97) Generally, the teaching approach may not change much because the different subjects are independent, the teaching method or something, but I think the learning atmosphere may be a little bit less than in the past. Because after the changeover in sovereignty, the status of Chinese is more important as a symbol of sovereignty. So the students may not want to learn English so actively. Maybe some changes on the attitude of the students. I don't think there's some obvious change on teaching methods. Any thoughts, $[\mathrm{Q}(97)]$ ?

$\mathrm{Q(97)} \mathrm{I} \mathrm{reserve} \mathrm{a} \mathrm{bit} \mathrm{of} \mathrm{different} \mathrm{opinions} \mathrm{because} \mathrm{I} \mathrm{know} \mathrm{that} \mathrm{the} \mathrm{first} \mathrm{impact}$ that a school change from an English medium to a Chinese medium is that they receive those students with lower grade in performance. That's the first impact, so in order to improve or to compensate for that kind of loss, the school must establish their reputations through their teaching and also through the teaching of English. So I think that those schools may be even 
more eager to teach English in a more effective way, they may be try to even organise more activities and more practice for their students. I think the teaching style or teaching approach may not change as you first state, however, the level of exposure may be compensate by other kind of activities.

AU Okay, Professional Relationships and Responsibilities, your confidence in your ability to become a successful teacher: most of the group were between 'Quite confident' and 'Unsure'. How do you feel now? Neither of you is teaching now, [Q(97)], you said that you don't want to become a teacher after your experience as a supply teacher?

Q(97) I mean, actually up to right now, if I was forced to teach in accordance with the current organising systems or patterns, I would give up to become a teacher. Because right now the language teachers have to teach up to four classes in the secondary schools. It's impossible for the teachers to do so many things to help the students to improve their language proficiency. In my mind, one or two classes would be the maximum; three or four classes is incredible, we must be a super man or super women because we have to mark their assignments and give them comments, and for every class we have up to forty students. So, for two classes we have to face eighty students. It's really exhausted because you have to spend a lot of time to single out the problems and then try to give them some useful comments. So I think it's - really a kind of torture.

AU [T(97)], do you think you might go back into teaching?

T(97) If I have the opportunity I think I may want to become a teacher again, but I think the competition is too keen. Maybe a few years later.

AU Is it very difficult to get a job in teaching now?

$\mathrm{T}(97) \quad$ Yes.

AU Why do you think that is?

Q(97) I think in recent years according to the economic situation, most of the graduates from the universities were enforced to take part into the schools because they could not find other jobs, except in secondary schools or primary schools. And that's one of the reasons why we have to face keen competitions. And the second reason is that right now most of the schools' principals or masters or panels they would like to employ those students who have really majored in their own subjects, so they have to teach the subjects that they have really majored in. So, I think right now we have to face keen competitions and also face the increasing requirements form the school side. Nevertheless, I think it's not difficult for my classmates to 
find a job because we are qualified as professionals who are able to teach English as a second language. Indeed, up to right now I still receive some positive feedback from my classmates and from some schools that they would like to employ our graduates because we have already established quite a good reputations in previous years and also we are qualified to do so because we are graduates who are majored in teaching English as a second language, so they would like to employ us.

So, I think we may still face keen competitions but our situation is much better than the others because right now the government try to employ more language talents to teach the language class, even though some of them would like to employ native speakers as English teachers, however we still have a strong points in competition with those native speakers because we are local students and we are local citizens we notice about the needs of the students and we know about their problems in their daily lives, so we can try to adapt our teaching approach and also adapt our own teaching style to suit to the needs of the students. So that's our strong points when compared with those native speakers. And also we have qualifications and so I don't really think it is difficult for us but the thing is that we may face competition.

Okay - how do you feel about the status of teachers in society? A year ago the group were in the middle somewhere - 'No special status' or moderate status. Do you think the status of teachers is pretty much the same as it was a year ago or is it higher now because it's more difficult to find a job?

Q(97) No, I don't think the difficulties to find a job will increase the teachers' status, because they just find a kind of outlet, a temporary outlet for those graduates. After the economic situation improve they may still quit the career and try to find another job. So I don't think that may help to improve our special status. However, I think the government policy, the governmental policy in language may increase the status of those language teachers because right now they insist that some of the language teachers may have to pass a means test so that they may be qualified as a secondary school teachers to teach in the secondary schools and also they can ... they may receive extra salaries for their jobs if they can pass the means test. So that may help to increase the status of the language teachers but not for the overall teachers.

AU How do you feel about the status of teachers, [T(97)]? Do you think teachers have a ... or do you think it's changed in any way?

$\mathrm{T}(97) \quad$ I don't think that the status of the teachers has changed.

AU There's been a ... following the newspapers, because of this issue of the 
medium of instruction and so on and the one that you mentioned, the testing of teachers, they call it benchmarking, that the status of teachers and the proficiency of teachers has been increased quite a lot. And with generally the public agreeing that language teachers should be tested, if they have got a certain proficiency of language they shouldn't teach language. Whereas at the same time, the teachers disagree with it, the Professional Teachers Union disagrees saying, 'We've already qualified and so why should we have to do another test?'

Q(97) Yeah, I think that if those teachers who are majoring in teaching English and also are majoring in education, should be able to escape from the means test, they should be allowed to do so because they have already achieved their academic status and recognised by the university. So I think if we cannot trust the university, what can we do? So I just think that we may ask the teachers to pass the means test, however, I don't think that it should only be used for the language teachers, but for the overall teachers. So, it's not a kind of discriminations, just help us to single out the overall figures, whether we have to do some improvements or not, and that will be useful. However, those students who are majoring in English or language should be avoid from that kind of means test. I think they have proved their abilities in the universities and have been recognised by the honour degrees and so there's no need for them to face another means test. It's meaningless. But for those teachers who are not majoring in language but they want to teach in English, that may be useful.

AU That has been call for that, not only language teachers, but all teachers to be tested for their language ability.

Teaching Approaches ... Philosophy of teaching - Table 39 - at that time, most people had a definite or quite definite philosophy of teaching, and those are listed on the following pages into different areas, those who focused on teachers and those who focused on students - or on teaching. I found a great deal of them were student-oriented, student-centered.

People said how they wanted to do all they could to help their students students' needs - student-centered learning - 'Help students to look for their life direction.' And the other major feature to come out was this idea of responsibility, being responsible. If I asked you now, what would your philosophy of teaching be? If I asked you now, as a non-teacher?

T(97) Philosophy of teaching - quite difficult to answer - to teach students to help them to achieve a balance of life maybe - not only in English, not only in the academic field, but also in the other fields, such as character, or others.

AU So, a student-centered approach? You base your philosophy around [Tape ends]. 
Q(97) I still focus on the student-centered approach because education is not just to teach the students something but we should be aware of their needs and help them to achieve some kinds of achievements in their lives so they can learn and pick up the skills and techniques how to learn, and it will be useful for their life. So I will still focus on the student-centered approach. However, in addition to this right now I would add in some kind of social exposure in my teaching approach because I realise that most of the graduates are .. even those for the university students, their social experience is not that rich enough to face the requirement of the society. Some of the employers realise that ... I think most of the employers still think that now are graduates are not mature enough to meet their requirements. Today I opened the newspaper and I read some interviews with those graduates and I realise that some of them are still kids as some of them still think that they are graduates form the university and so they should be promoted to the managerial level, but rather than the fundamental level, or something like that.

And I think that according to my working experience its totally different with their perceptions. I realise that sometimes we have to work fairly hard even though we have to work from the very beginning level, from the fundamental level. If you work hard, and you posses a positive attitude and you are able to maintain a very good social relationship with you colleagues you will be able to help yourself to be promoted to the upper level, and also it will be able to help you to pick up the job quite easily. And I realise that most of the students and for those F5 graduates and for those university students, they were not sophisticated enough to do so. So right now if I were a teacher in the secondary schools, I would try to expose my students to the social requirements and let them know about the realities. Let them know that they should establish their own attitudes and their positive attitudes towards working and towards learning, otherwise they won't be successful.

AU Things that the group felt unsure about - personal career goals and ambitions was the biggest one - so as far as your career goals are concerned now, you're still unsure? Are you still unsure perhaps about your career goals?

$\mathrm{T}(97) \quad$ Sure.

AU You're sure?

$\mathrm{T}(97) \quad$ Yes.

AU So what do you hope to achieve?

$\mathrm{T}(97) \quad$ Maybe continue, maybe go back to college after a few years. Then I will 
teach again.

Teach at secondary or a higher level?

$\mathrm{T}(97) \quad$ Secondary school. This is my goal.

Q(97) And I realise that even though I was trained to teach English in a secondary school class, my language background allowed me to do some other jobs, and even though they may not be related to the teaching field, I can still make use of my language ability to help to improve my job performance and so on. So I find that my goal is quite clear that I have to improve my language abilities in future. And of course I'm going to receive some trainings and I'm going to study my master degree in language. And so I can improve my language level. I hope that I can make use of what I have learned in the university - to - to help to improve my job performance.

AU So you plan to do a masters degree?

Q(97) Yes, actually I've been accepted by the university as a masters degree student. I'm going to study the master's degree in linguistics.

Here in Hong Kong?

Q(97) Yes.

AU When will that start?

Q(97) September.

AU Oh this September?

Q(97) Yes, in part-time mode.

AU Okay, as far as teaching skills are concerned, Table 42, I found it interesting that the group had rated grammar as the most important.

Q(97) Table 41.

AU Oh yes, Table 41. The group had rated grammar as the most important to teach, and then speaking and writing. So in fact, in Year 3, the group felt that teaching grammar was the most important, followed by speaking and writing, whereas in Year 1, speaking was first and listening was second and grammar was actually fifth. It was quite an interesting change, I thought. Why would you think that suddenly more people felt that grammar was more important? 
$\mathrm{Q}$ (97) Because in realistic situations we have to make more use of the language in the commercial sector, and also in the teaching areas we have to make use of the language. But if we are not able to produce the language, for instance to write a letter in a perfect style without any grammatical mistakes, it will be disastrous because sometimes we have to communicate with foreigners and our customers will be foreigners.

What do you think about it?

$\mathrm{T}(97) \quad$ The other skill is more practical. Grammar is a little bit more theoretical, I think. Because speaking, listening, reading and writing is a very practical skills. So in the later stage I think the speaking, listening and reading skills is more important.

So we have to communicate with them and sometimes paperwork is a must in our job-related duties and so if we can ... if we do not posses a good foundation in grammar, it will be quite dangerous. Because sometimes we may write a letter and it is full of grammatical mistakes. If you were the customers, I don't think you will be happy about this. So even in the government right now - actually, in previous years after two months of teaching in local secondary schools, I've been employed by the Education Department to help them to finish a project. So I realise that even in the Hong Kong Government, most of the employees and the staff are eager to learn grammar because they have to do a lot of paperwork and they have to write in English and so they have to learn grammar. And so grammar is very important. It's not less important than any other things.

AU I wondered about this and I thought perhaps it had something to do with the teaching practice. When you become a teacher and you realise, as you said before, that you have to mark students' work and you realise how many mistakes they make and you have to correct every mistake, then you realise how important grammar is. That brings it into more of a focus and you become more aware of the grammar. Perhaps everybody when they were beginning the BATESL did not think about that too much. Speaking and listening are most important because they are the communicative skills. It goes against a lot of ideas that grammar is not important, but in fact as a school teacher you soon realise that it is.

That's covered the 1997 survey - thinking about the BATESL course now, one year on, how do you feel about it? Are you glad you did it?

Q(97) First of all, I realise that I learnt a lot, Actually, much more than my expectation. I improved my language abilities during these three years. Secondly, I found that I changed my attitude towards learning and also my attitudes towards social relationships, because when I was still a student who was studying in the course of Social Administration, I found that at that time I just think that social relationship is just a kind of social 
activities and that's all, not more than that. But during the BATESL course I realise that it's not just a kind of social activities, actually sometimes human relationships is a friendship and also our attitudes towards different kinds of things may change the result, so during the BATESL course I learned to a rather positive attitude towards learning and also towards the social relationships, and I realise that I met a lot of good friends and I improved myself. At least I have enough confidence to organise various activities by my own. And also I think that course helped me to overcome my fears towards English, yes, because before I was a student in the BATESL course, I realised that I was afraid of speaking English because I'm afraid to make mistakes and so on and so forth, but after three years of study in the BATESL course, I overcome that kind of difficulties and then right now, even though my oral proficiency may not be so good, but at least I will be able to communicate with people in English quite effectively. So, I think I learnt a lot from the BATESL course, and also of course, I have earned a special status in teaching. Even though I'm not going to be a teacher in my future career, I still think that's useful because it's still a kind of special status, especially in other countries like United States or United Kingdom. It's a kind of special status, so why not? It's a good course.

$\mathrm{T}(97)$ First of all, after attending the BATESL course, I've learned some new ways and different ways of thinking. And secondly, it improve my English standard, especially in oral English. And thirdly, I learned various research skills during the BATESL course. The most happy one is that I like the BATESL course because during the primary and secondary school English was my favourite subject. Generally it is an excellent course.

AU Right - any thoughts you have about education in Hong Kong now?

$\mathrm{T}(97) \quad$ About the education system in Hong Kong?

AU Yes, I know we said earlier that the education system relies too much on rote learning and memorization. Any changes you can foresee in the future?

$\mathrm{Q}$ (97) I think we may establish a special status for those language teachers in the coming years. I do not know how about ten years later, but for the coming years - two to three years, it seems that for those language teachers, especially those who can pass the means test or those who have already earned a special status or qualifications, in teaching areas, they will be able to establish a special status in the education system because they are viewed as a kind of special talent to the education system and ... but for the other subjects there's not so much change except the medium of instruction. I think for the vast majority the overall situation may not change so much except the medium of instruction and the language 
teachers' status.

Any thoughts?

T(97) I think the Government, the Education Department to divide students that some students can go to the English secondary school or Chinese, or some other students go to the Chinese secondary school is not a good method. And in my opinion I think all secondary schools are using Chinese as a medium of instruction is better because it may cause some parents to force their students, force their sons and daughters to go to an English secondary school, even though they are not suitable to learn in a English secondary school. And also, it is also not possible to turn all secondary schools into using English as a medium of instruction because now Hong Kong is no longer a colony of UK, so I think the best idea is to turn all secondary schools are Chinese secondary schools.

AU So how do you feel about English in Hong Kong, about the status of English?

Q(97) It's still important ...

T(97) It's still important, yes, I agree with this.

Q(97) Because most of the employers still think that English is the first language in the commercial sector, but not Chinese. We have to do business with other parts of the world and English is the common language. So English is still the first language. In the government, the official status of course has been replaced by Chinese. However, English is still the official language, it's still the official language. Most of the documents are written in Chinese and also English, so we still have to use English. In the teaching areas I think English enjoy a kind of ... a very special status. Right now they regard those who are able to learn or teach in English will be - those who have strong abilities in that area. So, it's a kind of special social perception and status.

Any thoughts on the status of English after 1997 ?

T(97) I think after 1997 English is still important in commercial sector, but I think in the political sector, in terms of political, the status of English is gradually decrease. And that's all.

Q(97) But I think English is still useful in several areas. We cannot replace English with Chinese, especially in the law section, we cannot replace it. Actually, it's almost impossible as we have to replace some of the terms, and it's really impossible to do so. So I think in several area, English may preserve its influence, to a large extent, and concerning the education 
system, I ... the Education Department may change the system in about 4 to 5 years time because they need figures to support that all schools should be taught in Chinese but not in English. Right now they allow one third of the secondary schools to use English because of the pressure from the general public, so they need figures to support their claims. So after 4 to 5 years, those students who will use Chinese as the teaching medium will face the public examinations. Then they will get the figures and they can compare the figures with those English medium schools, so if the figure is positive or in favour of those Chinese-medium schools, then the Education Department may announce that they may change the system and they may enforce that all of the schools may use Chinese as the medium of instruction. Right now they just want to find some figures so they allow some time to do so.

That's an interesting idea, that eventually they will all be Chinese-medium schools.

Q(97) Except the language subjects. Actually, from the very beginning they hope to do so, but based on strong pressure from the general public who are opposed to that kind of opinion, so thy allow some schools to preserve English as their medium of instruction. However, it seems that they will compare the figures with the public examinations in the future, so if they find that it's in favour of those Chinese-medium schools, they may enforce those English-medium schools to give up their attitudes towards using English as the medium of instruction.

AU Interesting, although Hong Kong being a free market, of course, what would happen is what is happening now, in that international schools become more and more popular and those parents who can afford it are now sending their children to international schools because they will get an education in English if they don't qualify to go to any of the local English-medium schools. 


\section{C.2 Main Points to Emerge During Interviews with BATESL Graduates in 1998}

\section{Language Use}

$\underline{N(97)}$

(Journalist)

$\underline{\mathrm{O}(97)}$

(Teacher)

$\underline{Q(97)}$

(Office

worker)
She had increased both code-mixing and switching due to her workplace environment (a high number of overseas-born Chinese).

Code-mixing is such a common feature of Hong Kong Cantonesespeaking people that it is not even considered as code-mixing.

As a result of the change to CMI, code-mixing has changed from predominantly English with some Cantonese to predominantly Cantonese with some English.

Students are paying more attention to English because of fears of loss of exposure to the language with change to CMI.

In society, English is as important if not more so than ever due to increased trade links with international community.

He was reluctant to code-mix for reasons of language purity.

Language choice in the classroom depends on school policy and proficiency of students.

There has been and will be a reduction in use of English in schools, which will be due to MOI policy and not the handover.

The writing skills of the group as a whole had improved during the BATESL course as a result of exposure to different genres of (mainly) academic writing.

His English proficiency had declined slightly since leaving the course as a result of reduced exposure and chance for practice. He planned to make efforts to improve his English standard.

He had increased use of code-switching with friends since graduation and felt that the university-educated in Hong Kong make a great deal of use of mixed-code.

He had become more tolerant of the use of code-mixing in the classroom but felt that code-switching takes up too much time. 
The proposed change in MOI to Chinese would result in raised student interest and improved performance in content areas but also in a requirement for extra English lessons or activities. Also, schools would find it difficult to find sufficient teaching materials designed for $\mathrm{CMI}$ and to get accurate, definitive translations of English terms into Chinese.

$\underline{T(97)}$

His English proficiency had declined because of lack of opportunity (P/t marker) to use English in work situations (he was not teaching). He planned to make efforts to improve his English standard.

Little or no obvious change in his code-mixing or code switching since graduation. Did not like to code-mix or switch with friends or colleagues.

He preferred to use mixed-code in teaching to aid students' understanding and motivation.

The change in MOI would result in improved performance in content areas but requirement for extra English lessons or activities.

U(97) Language use is monitored by school management, and so teachers (Hotelier) have no choice but to use the language designated by the school.

Instructional Planning and Decision-Making

N(97) Practice teaching was too short.

(Journalist)

Used practice teaching as opportunity to use innovative practices.

Sought advice of cooperating teacher.

Teachers are more exam-oriented than students.

O(97) Practice teaching was too short but still worthwhile as it increased his (Teacher) confidence.

Teachers are student-oriented but students are exam-oriented.

Q(97)

(Office worker)
Experienced teachers will give personal attention to both outstanding students and very weak ones but not those in-between. 
Evidence of schools putting more resources into English learning in $\mathrm{CMI}$ schools in the form of more English Club activities.

$T(97)$

$(\mathrm{P} / \mathrm{t}$ marker $)$

Teachers cannot be responsive to student needs due to large class sizes.

$\underline{\mathrm{U}(97)}$

Practice teaching was too short.

(Hotelier)

Students are more exam-oriented than teachers.

Society is exam-oriented.

\section{Teaching Approach}

$\underline{O(97)}$

(Teacher)

$\mathrm{Q}(97)$

(Office worker)

$\mathrm{T}(97)$

(P/t marker)
Interactive teaching is possible in lower forms but upper forms require exam practice only.

Support for increased focus on spoken interaction in public examinations.

Teaching style depends only on the teacher.

Had previously believed in student needs deciding teaching approach but was now split on student needs vs. examination requirements.

Was able to adopt student-centred approach during practice teaching but found he was unable to simplify his language in class such that the students could understand.

Criticised the language proficiency of students and put partial blame on rote learning methods in primary schools.

Criticised TOC.

Still prefers learner-centered approach but within constraints.

Still believed that syllabus should be examination-led.

Believed that teaching practice was too short and the supervisor only got to see a small proportion of what they did. 
Commented on low English proficiency in the workplace.

Approach depends on the ability of students.

$\underline{\mathrm{U}(97)}$

Students in upper forms will complain if the teacher tries interactive

(Hotelier) activities that are not exam-focused.

Teaching style depends on students' attitudes and on teacher's approach

\section{Professional Relationships and Responsibilities}

N(97) The status of teachers is falling due to increased numbers of suitably (Journalist) qualified and/or dedicated people entering the profession and increasingly proactive parents challenging teachers' authority.

Local teachers are better able to communicate with students than expatriate teachers.

$\mathrm{O}(97) \quad$ Would like to teach in secondary school but finding it difficult to get (Teacher) a position.

Status of teachers has improved because of economic downturn.

The government is attempting to raise professionalism of teachers.

Extra resources for CMI schools can be spent on more teachers or equipment/facilities.

Local teachers threatened by NETs as government prefers to hire NETs rather than improve local teachers.

$\mathrm{Q(97)}$

(Office worker)

Would not wish to teach at the moment because of the excessive workload faced by teachers.

Feels that status of teachers will be increased as a result of language Benchmarking. 
$\underline{T(97)}$

(P/t marker)

$\underline{\mathrm{U}(97)}$

(Hotelier)
Would like to teach but experiencing difficulty in finding a job due to economic downtum.

Believes the BATESL has a good reputation amongst schools

Believes local teachers have advantages over NETs in that they understand the needs of the students better.

Is confident in her own ability to teach.

There will be more English teachers, both local and NETs.

Falling rolls will result in fewer classes.

NETs seen as a threat to local teachers.

\section{Perceptions and Values}

$\underline{N(97)}$

(Journalist)

The teacher should be a guide to students to help them develop.

Speaking and listening are the most important skills for real-world communication.

Changed emphasis in teachers relationships with students: no longer believes that the teacher can be friends with the students.

$\underline{O(97)}$

(Teacher)

The teacher should try to understand the students and their needs.

The examination-driven curriculum places emphasis on grammar.

Some teachers become jealous of other teachers' good relationships with students.

$Q(97)$

It is important to teach students to be socially responsible.

(Office

worker)

Not sure about teaching in the future but hopes to continue further study and improve language skills.

Importance of correct grammar in writing in the workplace. 
Is determined to teach in secondary school in the future.

$(\mathrm{P} / \mathrm{t}$ marker $)$

Practical language skills such as speaking, listening and reading are more important than grammar.

U(97) Experience is very important.

(Hotelier)

Teacher-student relationships can be different outside of class to in class.

Post-graduation Reflections

$\underline{N(97)}$

Most valuable aspect of BATESL was campus life and relationships.

(Journalist)

$\underline{O(97)}$

BATESL was useful preparation for being a teacher.

(Teacher)

$Q(97)$

(Office

worker)

Through the BATESL course he had improved his language skills, gained a more positive attitude towards learning, and increased his confidence in social relationships.

Language teachers will achieve a special status in the future as a result of their real value becoming more recognised.

Status of English will remain high.

The government will wait until students in CMI schools reach external examination level so as to compare results with EMI before deciding on whether to implement CMI in all schools.

T(97) Through the BATESL course he had improved his language skills, (P/t marker) learned new ways of thinking and gained research skills.

All secondary schools should become CMI.

English will remain important in the commercial sector but will become less important in the political sector.

$\underline{\mathrm{U}(97)}$

(Hotelier)

The BATESL course needs better promotion.

Most valuable aspect of BATESL was teaching skills learned during 
practice.

Appreciative of efforts by BATESL to maintain contact with graduates. 


\title{
C.3 Lesson Observation Reports of 1997 and 1994 BATESL Graduates
}

\author{
Teacher: \\ Observer: \\ Graduate $O(97)$ \\ School: \\ Alan Urmston \\ Self-financing private school for post-secondary students in \\ Kowloon
}

\section{Background}

The teacher graduated from the BATESL course in 1997. After graduation he worked in the English Department at the City University as a research assistant for one year. He had been working in the school since September 1998.

The school is privately run and offers diploma and A Level courses mainly for students who wish to improve on the HKCEE or A Level grades that they gained in secondary school. They can study vocational courses such as business, hotel management and design, as well as core A Level Use of English and Chinese Language and Culture.

\section{Observation 1}

Date: 8th June 1999

Class: Use of English Year 1

Topic/Skills: Oral group work and presentation practice based on plan for summer holidays

The class consisted of about 40 students, mostly girls and took place in the multi-media language lab (MMLL). They have 3 double lessons of English each week, in two of which they will use the computers. The students were due to begin their end-of-year exams in two days time. The $T$ used the fact that it was the end of the term to get them to think about ways in which they could work on improving their English over the summer and use this as the basis for speaking practice.

\section{Lesson Record}

10:39 Ss enter room while T writes on WB:

My Summer Plan

$\mathrm{T}$ interacts with individual Ss in $\mathrm{C}$, then speaks to whole class. 
$T$ introduces $\mathrm{O}$ (in $\mathrm{E}$ ).

T says that as it is the second last lesson he would like to have a review of the course and look forward to the summer holiday. He says that he wants to ask them a few questions. Then writes:

(1) The most unforgettable learning experience.

T explains that this can be in any subject and can be good or bad.

(2) Words/Expressions $\rightarrow$

T gives Ss one minute to talk about (1) with their partner, then they will speak in front of the class.

10:46 A S says that 'Learning Accounts' was unforgettable. When the T asked why, she said, 'Because it was difficult.' So $T$ writes on right side of WB:

\section{(1) Difficult hard}

$T$ chooses another $S$ to give their experience. The $S$ mentions a subject called 'Social Ethics' because it is fun. $T$ writes:

(2) a lot of fun

Another S says 'UNO', then explains that it was because if they lost they had to give a presentation. $T$ writes:

(3) excitement

10:50 The T now asks the Ss to give him words and expressions that they have learned. A S says 'However' and explains that when reading a newspaper the uses of 'however' are different to those she is used to. $\mathrm{T}$ writes:

$$
\text { However } \rightarrow \text { a surprise! (4) useful }
$$

Another S says 'It seems that'. The T asks why this expression is unforgettable and the $S$ replies that it is useful. $T$ writes:

\section{(3) Reflections upon my experience.}

$\mathrm{T}$ tells the class that he has shared his experience of teaching with them as it is his first job. They have been part of his experience.

Next, the T tells the class to think of the four things, 'Difficult', 'Excitement', 'A 
lot of fun' and 'Useful' and relate them to a plan for the summer, linking the ideas of 'Unforgettable experience', Words / Expressions' and "Reflections'.

10:57 T tells the Ss to discuss in groups of 4 a summer plan which they will then present to the rest of the class. He tells them to write down their ideas on paper.

T writes:

$$
\begin{aligned}
& \text { imaginative } \\
& \text { creative } \\
& \text { not necessary } \\
& \text { to carry it out }
\end{aligned}
$$

What to do this summer:

T says that there may be things that they will definitely do $(\bullet)$ and some that they might do (*).

The Ss are working in groups discussing while the $\mathrm{T}$ walks around talking to groups and answering their questions.

11:05 T tells Ss that they have 4 more minutes. Ss are speaking together in a mixture of $\mathrm{E}$ and $\mathrm{C}$.

11:10 T tells Ss to write down their schedule in terms of how often they will do things.

Next, T says that it is time to choose people to tell the class their plan. He chooses a group and one S stands up and says, 'Practice English by playing the Internet.' The $\mathrm{T}$ tells the $\mathrm{S}$ to give them more details, so the $\mathrm{S}$ says a little more. $T$ writes:

\section{- Play the Internet (Communicate with others)}

T chooses another $S$ who talks about what he will do. $T$ has to ask him to repeat and then explain what he will do. The S says he will have to do 100 questions for another subject. $T$ asks the $S$ if he will do anything else.

11:15 The T asks another $\mathrm{S}$, who says that she will do 'Section $\mathrm{C}$ and Section $\mathrm{E}$ everyday'. The $\mathrm{T}$ jokes with her that she should write it down and sign it. $\mathrm{T}$ 
then writes:

$$
\begin{aligned}
& 100 \text { Qs } \\
& \text { Section } C+E \text { every day }
\end{aligned}
$$

$\mathrm{T}$ jokes about taking 'Vitamin $\mathrm{C}$ and $\mathrm{E}$ ' every day.

T chooses another $\mathrm{S}$ who says, 'Watching English TV programmes,' and then 'Discovery Channel' and 'twice a week'. T writes:

\section{Discovery Channel twice a week}

11:19 Another S says, 'Listen to BBC news' and T writes it on the WB. He then asks if it meets the four requirements and the S says that it is 'Difficult' and 'Useful' but not 'Fun' or 'Exciting'.

Another S suggests watching 'Sesame Street' and T writes it on the WB.

11:22 T says that they have quite a number of suggestions, then writes:

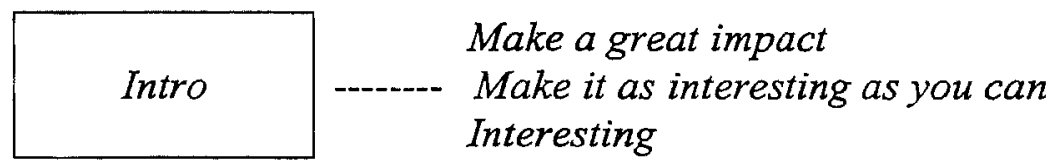

\section{Speech $\leftarrow$ Organise}

\section{Concl.}

$\mathrm{T}$ tells class to think about how to prepare a 2-minute speech on the points raised and then present it to the class. He gives them 10 minutes to prepare.

11:26 T explains what they have to do in $\mathrm{C}$ for clarification. They have to prepare in groups then choose one $\mathrm{S}$ to speak for the group. They will then have an award 
for 'best speaker'.

$T$ interacts with Ss in both $\mathrm{E}$ and $\mathrm{C}$. Some Ss need encouragement to prepare. $\mathrm{T}$ then sits at the front desk while Ss work in groups. Ss are speaking mainly $\mathrm{C}$ with some E.

11:34 T tells them they have 5 minutes left. $T$ interacts with $S$ s in $C$, going round the different groups.

11:40 T tells Ss that they seem to be doing nothing to prepare, that they think that someone else will do it. He tells them to give individual presentations to their group members and then choose the 'worst one' or the 'least best one' to give their presentation to the class. He remarks that some of them do not have paper in front of them so they must be doing it in their heads.

$\mathrm{T}$ tells Ss to give their presentations in their groups. Some are doing it while others are busy preparing.

$\mathrm{T}$ interacts with $\mathrm{Ss}$ in $\mathrm{C}$.

$\mathrm{T}$ then listens to a $\mathrm{S}$ speak in $\mathrm{E}$ then tells him to put in more examples to make it more 'interesting' or 'colourful'.

11:49 T speaks to class in C. It seems that he is encouraging Ss to keep them on task. T writes:

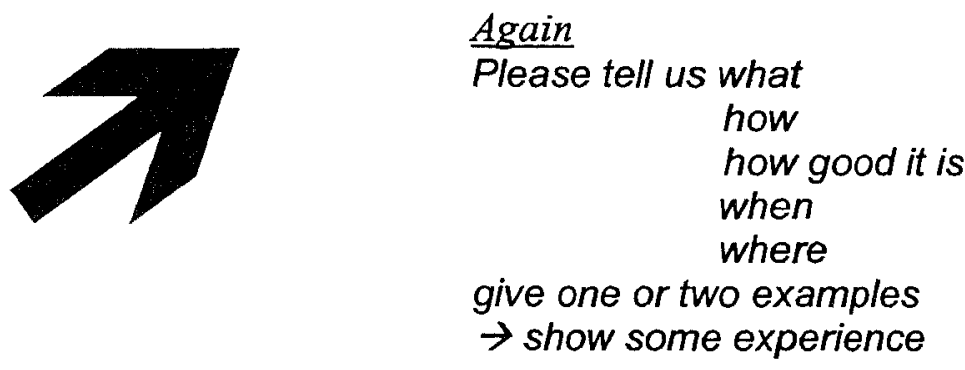

11:54 T chooses a group and tells them to send one member out to give their speech. A S comes out and class applauds. S speaks for about 30 seconds and then runs out of things to say.

Another $\mathrm{S}$ comes out and speaks quite well for about 1 minute.

A third $\mathrm{S}$ comes out and gives a good speech for about $1 \frac{1}{2}$ minutes.

12:00 T remarks that they can make their speeches more colourful by using examples and experiences.

$T$ then chooses another group but none of them are willing to come out and speak so he chooses another group and encourages a $S$ to come out. The $S$ 
gives a short speech. She uses examples as advised by the T. She speaks for about 1 minute.

T talks about 'Sesame Street' to show Ss how to illustrate their speeches.

12:05 T tells class to hand in their summer plans and he will make copies of them so that he will be able to tell whether they have fulfilled them or not.

$\mathrm{T}$ then leaves the room.

T returns. Apparently another $\mathrm{T}$ was going to come into the class but has already left for lunch.

12:09 T speaks to class in $\mathrm{C}$, with some interaction with individual Ss. $\mathrm{T}$ then writes:

$$
\begin{aligned}
& \text { get out get in } \\
& \text { It seems that! } \\
& \text { vigorous } \rightarrow \text { Reaction } \\
& \text { Language }
\end{aligned}
$$

T seems to be talking about colloquial expressions or vocabulary, then about presentations and vocabulary from another class.

12:17 T speaks to individual Ss in C. It seems that he has 10 minutes to fill.

12:19 Ss pack up their things and $T$ tells them they can go.

\section{Post-Observation Remarks}

The $\mathrm{T}$ felt that the Ss were not in the mood for working as they were winding down for the exams which would start in two days time. The T said that the Ss don't like planning.

\section{Observation 2}

Date: 8th June 1999

Class: Business English Year 1

Topic/Skills: Job Applications - interviews, CVs, letters of application

The class were studying for a Diploma in Business Studies. Most of them had failed HKCEE English. The class consisted of about 35 students, almost all girls. In the 
previous lesson, they had taken part in mock job interviews and had submitted CVs and application letters. The teacher was giving feedback on these.

1:17 T calls class to order, speaking in C. He announces the names of 'candidates' and Ss applaud each name [these were the students who would be shortlisted for 'the job' based on their performance in interview and CV/letter].

1:21 T holds up some papers and 2Ss come out to distribute them. They appear to be CVs and application letters.

$T$ leaves the room. Ss are getting their work back and reading through it and discussing it.

T returns and calls a $\mathrm{S}$ to come out. T says something to the $\mathrm{S}$ and he (the $\mathrm{S}$ ) leaves the room.

$\mathrm{T}$ asks for work from Ss who were absent.

1:28 T speaks about application letter. He talks through the structure (in C) of a letter and writes:

Address

$\left.\right|_{\rightarrow} ^{\text {Dear Sir/Madam }}$

T uses a visualiser to show a model of an application letter. Using a laser pointer he goes through the address, salutation, heading and so on (all in $\mathrm{C}$ with the occasional $\mathrm{E}$ term thrown in).

1:36 T then puts a CV on the screen. He talks through the different sections of the $\mathrm{CV}$, explaining and pointing some things out. Then writes:

\section{Extra-curricular activities}

and explains what it means. He then continues through the CV. 
1:45 T switches to $E$ and asks a $S$ what should be put under 'Referees'. $S$ answers (in $\mathrm{E}$ ) and $\mathrm{T}$ re-confirms it. $\mathrm{T}$ then switches back into $\mathrm{C}$.

$\mathrm{T}$ asks if they have any questions. He switches off the visualiser and says 'Interview'. He then demonstrates bad posture and the Ss laugh.

1:51 T explains extra-curricular activities in terms of how an interviewer might ask, 'Do you have any extra-curricular activities?'

$T$ tells the class about the interview he had with one $S$ and asks the $S$ to relate it (in E). The $\mathrm{S}$ calls for another $\mathrm{S}$ to answer. This $\mathrm{S}$ speaks in C.T then gives an example, 'I went camping with my friends.' 'I found it quite difficult to work with friends.'

A S speaks in $\mathrm{C}$ and $\mathrm{T}$ responds in $\mathrm{C}$.

T gives sample expression, ' It would be fine with me.'

1:58 Ss put their things away.

$\mathrm{T}$ talks about $\mathrm{CV}$ and asks if they have any problems.

2:00 T tells the class that in the next lesson they will do model business letter.

Ss leave the classroom. Some stay behind to talk to the T.

\section{Post-Observation Remarks}

In the next lesson the best Ss will demonstrate their interviews in front of the class.

1.. Language use - Sometimes he will conduct the whole lesson in $\mathrm{E}$, sometimes all in C. He will introduce new topic in $\mathrm{E}$ as they tend to pay attention to something new. For revision or review, he will use $\mathrm{C}$. So long as they have the chance to use $\mathrm{E}$, both spoken and written, the use of $\mathrm{C}$ is felt to be $\mathrm{OK}$. The $\mathrm{T}$ will use $\mathrm{C}$ for giving instructions, explaining vocabulary and grammatical structures. If the Ss are not on task then he will repeat the instructions in $\mathrm{C}$ as they may not listen if he uses $\mathrm{E}$. As regards language use in the school, the principal encourages Ts to use $\mathrm{E}$ but there is no formal policy. Most Ts use C.

2. Materials development - The $\mathrm{T}$ usually uses his own materials but sometimes consults textbooks. Ss books are exam-practice books. He said that he doesn't have the time to prepare his own materials but plans to spend some part of the summer doing so. 
the time to prepare his own materials but plans to spend some part of the summer doing so.

3. Teaching strategies - $T$ uses interactive activities, mainly groupwork, for speaking and listening and to a certain extent, writing, but not for Paper $\mathrm{C}$ (reading, usage) or Paper E (practical skills). The content of instruction is described as examinationoriented as the reason the Ss attend the school is to improve their exam grades, or as T described, 'To make them function in the examination.' They have the flexibility to teach what they want, so long as there are no complaints from the Ss.

4. Roles and responsibilities - The $\mathrm{T}$ is an advisor for the SU. He is also mc of the Ss fashion show and has had to escort visitors from the UK to Macau.

5. Relationships with Ss - Described as 'OK'. He spends time with Ss outside of class and they will come to him to ask questions.

6. Relationships with colleagues - Described as 'Very good'. He shares ideas with one colleague as they rarely have official meetings. They tend to work by themselves.

7. Status - He feels that what he is doing is valuable and that it is what he wants to do. He plans to teach in this school for another year and then look for another institution, perhaps a TI. He finds the work enjoyable but hard. He feels like he is an actor, trying to come up to the expectations of the Ss is a pressure. Sometimes he feels frustrated about Ss' attitudes if they are impolite or not interested and don't see the importance of being proficient in $\mathrm{E}$.

8. Effects of benchmarking - He feels confident in his ability to talk about teaching.

9. Effects of Ss from Mainland - He sees it as a challenge as there will be more jobs.

\section{Observer's Remarks}

In both lessons the teacher was in control throughout. He maintained a brisk pace and was always ready to encourage students to keep on task. In the first lesson observed, he used mostly English for presenting lesson content and a mixture of Cantonese and English for explanation, clarification, and interaction with students on a one-to-one and whole-class basis. As the lesson progressed, he used more and more Cantonese for all purposes, including presenting and explaining content. The teacher used the students' own experiences as a stimulus to get them to engage in discussion. He encouraged the students to speak and used group activities as a way to foster communication between students, although the students seemed to use predominantly Cantonese to interact with each other. His use of the whiteboard to consolidate students' responses was effective. The teacher seemed to have good relationships with the students and they responded well to his teaching. He was able to make them laugh and keep the atmosphere friendly but businesslike. 
The second lesson was more teacher-directed and instructional as the teacher gave feedback on the students' assignments. Mostly Cantonese was used for explanation and for interaction with students, who also used mostly Cantonese. 
Teacher:

Observer:

School:
Graduate U(97)

Alan Urmston

Band 4/5 Government-aided co-ed secondary school in New Territories new town

\section{Background}

The $\mathrm{T}$ graduated from the BATESL in 1997 and was in her first year in the school. She had wanted to teach but had spent the first year after graduation working in another field. She would not be staying on at the school after her contract expired at the end of the current school year.

\section{Observation 1}

Date: 31 May 1999

Class: Form 2 English class

Topic/Skills: Preparation for writing composition

The class was a full Form 2 class of about 40 students. In each cycle the class had 9 English lessons plus 1 from the NET, which would be used for speaking. In this lesson, the teacher prepared the students to write a composition about pandas.

\section{Lesson Record}

9:39 T tells class to greet $\mathrm{O}$.

A S gives out the composition books while the $\mathrm{T}$ gives out the w/s. T interacts with Ss in C. One S comes out to talk to T.

$\mathrm{T}$ writes on $\mathrm{BB}$ :

$$
\text { Reporting about pandas May 31,99 }
$$

$\mathrm{T}$ talks to class in $\mathrm{C}$ with some $\mathrm{E}$ terms interspersed such as 'Suggested plan', 'Country where they are found' and 'Plan'. Then she writes:

\section{$\underline{\text { Plan }}$}

Para $1+$ Para 3

Para 2

Para 4

Para 5

A S calls out and Ss laugh. 
$\mathrm{T}$ talks through the questions on the $\mathrm{w} / \mathrm{s}$, reading parts out and explaining in $\mathrm{C}$.

Some Ss calling out in C, class is generally quiet.

9:53 T writes:
1) In which country are they found?
China their food is there.

$\mathrm{T}$ asks questions and Ss answer. $\mathrm{T}$ writes:

They are found in China.

Because their food is there.

2) Do they have a home?

No ........ pandas always move about looking for food.

They do not have a home.

(As) Because they always move about to look for food.

As she writes, the $T$ explains and Ss copy from BB onto their w/s.

9:58 T writes:

3) They live alone because they need a large area to collect food.

$T$ interacts with Ss, many are laughing. $T$ writes:

They live in groups in late spring or early summer during their very brief mating season.

Some Ss are not sure about some of the words written. The T spells them out and explains them (in C).

A S cleans the BB. Some Ss put up their hands. A S goes out to get chalk.

10:05 T says she will give them information to help them to write their compo (in C). Then writes:

4) They usually eat bamboo trees, mainly stems and leaves.

Therefore, they live in bamboo forest.

They eat up to 14 hours a day. 
5) They are black and white in colour.

6) Female pandas weigh $80 \mathrm{~kg}$ while male pandas weigh $100 \mathrm{~kg}$.

7) There are less than 100 pandas in the world.

8) The number is decreasing because many people hunt them and there are fewer areas of bamboo.

Some Ss call out and $\mathrm{T}$ answers (in C).

8) Baby pandas die easily.

When they are born, they only weigh $80-140 \mathrm{~g}$.

The Chinese Governent provides

New born babies have little fur.

The Chinese Government provides

large area for pandas to live as one of the ways to help them.

10:10 Bell rings. T carries on writing:

$\rightarrow$ Panda is one of the endangered species.

They are found in China because they can find food there.

10:12 T gives Ss instructions as regards writing of compo.

T tells Ss to stand up and say good-bye to $O$.

\section{Post-Observation Remarks}

The $\mathrm{T}$ remarked that some of the Ss are very weak and will simply copy the sentences she wrote on the $\mathrm{BB}$ and produce them as a composition, while others can produce a logical essay, rearranging the sentences. She would give the Ss a range of marks form $\mathrm{B}+$ to $\mathrm{E}-$.

This was the first time the $\mathrm{T}$ had tried this method of composition preparation. She had found information on pandas from the internet and summarized it to answer the questions on the w/s. The students would do 4 compositions each term and required a 
lot of information support. They would write letters, extract information from texts and so on.

Extra provision for E within the school was provided by an English Week and English Corner.

\section{Observer's Remarks}

The $\mathrm{T}$ used approximately $90 \%$ Cantonese during the lesson, which she said was necessary in order for the students to be able to understand what she was saying. She had begun the year by using English, but the class had objected to this and so the T now uses Cantonese for all purposes and intersperses it with key phrases in English. The Ss are lively and tend to make a lot of noise, but the $\mathrm{T}$ seems to have a good relationship with them and is able to keep them on task by interacting with them, rather than using a more authoritative approach.

\section{Observation 2}

Date: 31 May 1999

Class: Form 3 English class

Topic/Skills: Oral examination based on dialogues

In this class, the $\mathrm{T}$ administered an oral examination to a class of 30 Form 3 students. The $T$ sat at the T's desk and the Ss came out in pairs to read a short dialogue that they had prepared in advance. They could choose whichever dialogue they wanted to do from a choice of 4 . The $\mathrm{O}$ sat next to the $\mathrm{T}$ at the front desk.

\section{Lesson Record}

10:35 $\quad T$ greets class and class greet $\mathrm{O}$ and $\mathrm{T}$.

T speaks to class and a $\mathrm{S}$ brings out two chairs.

A S comes out and T speaks to her, then she leaves the classroom. The Ss are reading their w/s. A S comes out and sits at the T's desk. Two other Ss come out and ask the $\mathrm{T}$ something from a textbook. Two Ss sitting at the front ask the $T$ about the $w / s$.

10:43 A S sits at the T's desk and T speaks to him (in C).

The $S$ who left the room returns with copies of $w / s$.

T tells class to be quiet. 
Another S comes out to join the one sitting at the T's desk. The two then read the dialogue from the $\mathrm{w} / \mathrm{s}$. The $\mathrm{T}$ stops them to tell the class to make less noise.

The Ss resume reading the dialogue.

The $\mathrm{T}$ then tells a $\mathrm{S}$ to stand at the $\mathrm{BB}$ to write down the names of any $\mathrm{Ss}$ who misbehave.

10:49 Two more Ss come out the T's desk to read dialogue. Ss' reading is weak. They try to read it but sometimes they laugh. The $T$ tells them to read it again after giving them some help with some of the words and phrases.

10:54 Two more Ss come out to T's desk to read. T listens then tells them to read it again.

Class is quiet.

10:58 Two more Ss come out to read. The T gives them the opportunity to read it again but they don't want to.

Two more Ss come out to read. They have difficulty with some words but once they recognise the word they can say it a second time. Their pronunciation is not bad but stress and intonation are poor.

11:04 Two more Ss come out to read. They do it very well and don't choose to read it again.

11:06 Two more Ss come out. Their reading is quite weak. They read it again and the $\mathrm{T}$ gives them some praise.

11:10 The next pair of Ss come out. They read quite well and have obviously been practicing, although they do make mistakes. They decide to read it again.

11:13 The next pair come out. They read it twice.

11:16 Two Ss come out who haven't been practicing. The second is very poor and cannot read the words. He uses some $\mathrm{C}$ and the other $\mathrm{S}$ laughs. Later the second $\mathrm{S}$ shows that he can read the words but is not taking it seriously. They don't read it again.

11:20 The next pair are quite good and decide to read it twice.

11:23 The next pair read. The $T$ interrupts them, perhaps to tell them to read with more expression in their voices. They then read it better. 
11:26 The next pair read quite well and read it twice.

11:30 One $\mathrm{S}$ comes out by himself. The $\mathrm{T}$ takes the second role in the dialogue.

11:32 The $\mathrm{S}$ who was acting as class monitor comes out to read and the $\mathrm{T}$ takes the second role. The $T$ reads through the whole conversation first to help him.

11:35 T speaks to class, giving general feedback.

Bell rings.

Ss say good-bye to $\mathrm{O}$ and $\mathrm{T}$.

\section{Post-Observation Remarks}

1. Use of Cantonese - $\mathrm{C}$ is used for all purposes except for oral exams and attainment tests. The $\mathrm{T}$ did not do oral work with her Ss (this was done by the NET), only grammar, so $\mathrm{C}$ is used almost exclusively since the school is CMI and has been since the $\mathrm{T}$ started working there.

2. Use of materials - Ts have to follow the scheme of work and use the textbook. There were no teacher-designed activities.

3. Teaching strategies - Interactive strategies used with F1 class but not with F2 due to lack of S motivation.

4. Content of instruction - Mainly grammar-based, marks-oriented.

5. Roles and Responsibilities - Housemaster, Extra-Curricular Activities Committee member, Careers Committee member.

6. Relationships with Ss - Depends on class and Ss' attitude and level of appreciation. She felt closest to her former F1 Ss and felt that Ss tend to cling to their F1 class teacher.

7. Relationships with colleagues - Good, she was able to go to Panel for advice.

8. Status of self and profession - The $T$ feels that she is wasting her time as she really wants to teach the Ss and be a good T but many Ss are only interested in what is on the syllabus. She feels that she might be better teaching higher forms. She also feels that the workload is too heavy for most Ts.

9. Effects of benchmarking - Some older Ts are concerned about it. The T feels that older Ts are often reluctant to take on new skills and new technology. 
10. Effects of Ss from Mainland - The Ss from the Mainland tend to be more motivated and diligent and their grounding in $E$ tends to be better than local Ss. Although some can be quite poor, others are very good.

11. Use of methods learned in BATESL - $T$ has to use totally a grammar-based approach, fully dominated by the $T$ and conducted in Cantonese. The communicative approaches encouraged but Ss are unable to adopt it because of poor English language skills. They are particularly weak in spoken English and are unwilling to use it.

12. Adoption of innovations in teaching - $T$ admits to making use of internet to get information and use of Powerpoint in teaching. She found that the Ss were interested in this as they like hi-tech. The $T$ would also make use of materials from seminars she had attended. She felt that she was more open to innovations than some colleagues, not necessarily because of the BATESL, but because some Ts were fossilized in their old methods. She feels that she will become more open to new innovations as she becomes more experienced as she is eager to adopt new technologies in her teaching. As an innovator, it depends on the support she gets from ED and the school. She sees herself as being central to her colleagues in this aspect as she is more willing to learn new things.

13. Changes to beliefs, assumptions and knowledge - The $T$ felt that her beliefs had changed in that she used to believe that if she were close to her students she could communicate with them easily and then they would pay more attention to her lessons. She now feels that this is only applicable to senior form students. Her assumption, that learning attitude is positively correlated to academic achievement has been proved to be correct from her observations.

\section{Observer's Remarks}

The $\mathrm{T}$ appears to be dissatisfied with her teaching situation. She feels frustrated that she is unable to use the methods and approaches that she learned during the BATESL course due to students' lack of motivation and the restrictions placed upon teachers by the school. She places a great deal of importance on being able to communicate with her students, and this is a feature of her lessons, but she expressed the view that she can only really communicate and motivate senior form students. She sees herself as open to innovations and as an innovator herself, but again, the constraints of heavy workload, having to stick to the syllabus and the reluctance of other teachers to adopt new ideas mean that she is frustrated. Consequently, she will be looking for a new job after her present contract ends in August. 
Teacher:

Observer:

School:
Graduate W(97)

Alan Urmston

Band 3/4 Government-aided co-ed secondary school in Kowloon new town

\section{Background}

The school is new, only having been open for three years. At the time of the observation, there were Form 1 and 2 classes and Form 4 and 5. The school was described by the teacher as a Band 3 or 4 Chinese - medium (CMI) secondary school in a new town in Kowloon.

In addition to their English class, the students are required to attend an English Enhancement Scheme that takes place over 3 or 4 mornings during school holidays. In this scheme they take part in activities and use only English. This scheme was thought to be an Education Department initiative for CMI schools and for which the school received funding.

The teacher worked from a syllabus that was prepared at the beginning of the year by the Form Coordinator. However, teachers were free to teach in whatever manner they wished. The teacher said that the students were taught all the four language skills but there was a heavy bias towards the teaching of grammar.

In terms of materials, they worked from the textbook for $30-40 \%$ of the time as well as using a class reader and listening comprehension book. There was also an intensive reading scheme which the students did for 2 lessons per month. All other materials were produced by the teachers themselves. One teacher would prepare materials and they would be shared amongst the other teachers.

The teacher had been teaching at the school as a supply teacher from November 1998. She said that she had been employed as a replacement for a NET (Native English Teacher) who had not taken up employment at the school. She had spent one year in the UK studying for an MBA after graduating from the BATESL course in 1997. She intended to remain at the school on a permanent contract from September 1999.

When asked what she thought she had gained from the BATESL that had helped her in her teaching, she said that the course, particularly the practice teaching and micro teaching had given her the confidence to speak in front of a class and the ability to present a lesson. However, she found that she could not adopt the strategies learned on the course, such as communicative approach, due to the low ability level of the students. She felt that she would like to be more interactive (in English) with the students but their lack of understanding meant that she had to use Cantonese in order to get her message across and to save time in order to cover the syllabus. 


\section{Observation 1}

Date: 17 May 1999

Class: Form 1 English

Topic/Skills: Asking and answering 'wh' questions.

The class observed was a 'split-class' group of $14 \mathrm{~F} 1$ students. It was the policy of the school to split F1 classes for English for all but one class of the ten in the teaching cycle. The teacher described the students as being of quite high ability but weak in English.

\section{Lesson Record}

9:48 T brings Ss into class and Ss say 'Good morning' to $O$ and $T$.

$\mathrm{T}$ tells class that they do not need their reader today.

$\mathrm{T}$ asks Ss if they remember how to ask questions, then repeats in $\mathrm{C}$.

$\mathrm{S}$ gives question form 'What is your name?' and $\mathrm{T}$ tells her to write it on $\mathrm{BB}$.

$T$ then elicits other question forms from Ss and tells them to write them on $\mathrm{BB}$.

9:51 T asks Ss where they were before, repeating it in $\mathrm{C}$ for clarification. Ss call out answers.

The $\mathrm{T}$ explains in $\mathrm{C}$ and then $\mathrm{Ss}$ give question form in $\mathrm{E}$. $\mathrm{T}$ tells some $\mathrm{Ss}$ to write question forms on $\mathrm{BB}$.

$T$ asks the class how to spell 'favourite' and the Ss call out the spelling as $T$ writes it on BB.

9:53 Ss write question forms on $\mathrm{BB}$ while $\mathrm{T}$ gives encouragement and asks individual Ss for other question forms.

Ss do not have any books or $\mathrm{w} / \mathrm{s}$ in front of them.

$\mathrm{T}$ checks question forms on $\mathrm{BB}$.

$\mathrm{T}$ then asks about tense forms (in C) and Ss give question form 'What are you going to do after this lesson?'

9:57 T gives out w/s of question forms that Ss had written in previous lesson then 
tells Ss to read the questions out loud in unison. As they are doing this, T gives out a questionnaire and tells Ss to repeat one or two questions.

10:00 T brings Ss attention to the questionnaire and asks them if they know what a questionnaire is. She then explains in C.

T tells a $S$ to read out the instructions on the questionnaire. $T$ has to help the $S$ with some words, such as 'partner'. T then asks the Ss if they know what 'partner' means, then asks some Ss to name their partner.

T explains that Ss must work in pairs to ask each other questions and complete the questionnaire. She then repeats her instructions in C.

10:03 As Ss ask each other questions, $T$ walks around monitoring them. Ss ask for spellings and $\mathrm{T}$ writes on $\mathrm{BB}$ :

\author{
Government \\ Po Leung Kuk \\ King Lam Estate \\ Po Lam \\ Hau Tak
}

10:07 T writes on $\mathrm{BB}$ :

$$
\text { purple }
$$

T tells Ss to try to speak in English.

$\mathrm{T}$ writes spaghetti on $\mathrm{BB}$ then erases it and tells $\mathrm{S}$ that it is in their book.

$\mathrm{T}$ tells two Ss at back of class to ask and answer questions.

T writes pink on $\mathrm{BB}$.

10:11 T tells class to stop talking and writing and to listen to $2 \mathrm{Ss}$ who will demonstrate. The Ss stand up at their desks and one asks the other questions to elicit the information needed to complete the questionnaire. The $T$ guides them through it by telling them the next question to answer and giving help and encouragement where needed.

$\mathrm{T}$ then stops the Ss who are demonstrating and tells 2 more $\mathrm{Ss}$ to continue through the questions.

The $\mathrm{T}$ interacts with one of the demonstrating Ss (in $\mathrm{E}$ and $\mathrm{C}$ ) as regards the question on hobbies. 
10:15 Bell rings

$\mathrm{T}$ talks to Ss in $\mathrm{C}$ about their reader (what to bring to the next class), and tells them to hand in their $w / s$. $T$ then tells them to bring their reader to the next lesson.

$\mathrm{T}$ tells Ss that their $\mathrm{h} / \mathrm{w}$ is to write corrections on their previous work. She then calls out some names and gives instructions (in $\mathrm{C}$ ).

T writes on $\mathrm{BB}$ :

\section{Writing Corrections}

T tells Ss to stand up. When they are quiet, she tells them to say goodbye to O and herself.

Ss leave the class. One Ss stays behind and T says something to him (in C), then he leaves.

\section{Post-Observation Remarks}

The $T$ said that she found that the Ss did not use English enough and that they just told each other the answers, unless she stood near to them. However, she was pleased that they had learned the question forms and could say them without having the worksheet. Although she had found that they could not consolidate what they had learned. She also found that they were very weak in spelling.

The $T$ said that she got the $S$ s to write on the BB because they liked doing it and it stopped them from falling asleep.

In the next lesson the class would finish the question and answer activity and then they would carry out a survey of the findings.

\section{Observer's Remarks}

I thought that the lesson worked quite well in that the students were able to practice all four of the language skills in order to perform the main task of the lesson. They had to read the questions, speak to each other and listen to get the answers and then write them down. Although the teacher expressed the view that she had been unable to use a communicative approach in her teaching due to the level of the students, I think this lesson proved that it is possible to be communicative with fairly low proficiency students. That the students found the lesson content interesting was quite obvious. They 
participated actively and seemed to enjoy speaking to each other and finding things out about each other.

Having the students write the questions on the board at the start of the lesson was a nice way of 'warming them up' and getting them actively involved and they did seem to enjoy doing it. It was also a good idea not to give them back their worksheets at this stage as it made them think about the topic rather than simply read from a sheet.

The questionnaire activity seemed to work well with most of the students getting into their role. There was a problem of the students slipping into Cantonese whenever the teacher was not standing next to them and I think this is inevitable as they were communicating with each other and their natural inclination is to use their L1. The fact that the teacher uses Cantonese for explanation is likely to lead to the students using it also. The fact that the school is CMI means that the students expect to be given instructions and explanations in Cantonese. However, they should realise that in practice situations like this one then English should be the only language used.

In general the lesson seemed to accomplish its aims of getting the students to ask each other questions in a grammatically correct format. The teacher was firm and the students responded well to her. She used approximately 60\% English and 40\% Cantonese, using the latter for explanation, giving instructions and some lesson content.

\section{Observation 2}

Date: 20 May 1999

Class: Form 1 English

Topic/Skills: Identifying parts of the body, pronunciation, physical response to instructions, written follow-up and consolidation.

The class observed in this lesson was a complete class of about 35 students, some of them the same as those observed in the previous observation. The teacher explained that the class had a problem of interest in the subject and amongst some an unwillingness to participate in class activities. The teacher also described them as being frequently noisy.

In this lesson, the teacher would be working from the textbook on identifying parts of the body. They had already done some preliminary work on this in the previous lesson.

\section{Lesson Record}

10:36 T tells Ss to turn to p.33 then explains in C. T then tells Ss to remember the words that they had learned yesterday.

Whilst Ss are reading their books, the $\mathrm{T}$ draws pictures of body on BB (head \& 
torso, arm \& hand, foot)

The T tells the Ss to close their books and write down the parts they know and tell her where they are. Then she tells some Ss to go to the $\mathrm{BB}$ and label the drawings.

The $T$ speaks in $C$ and Ss put up their hands. The $T$ then calls on some to go out to BB.

'Where is the chin?' T says then repeats in $\mathrm{C}$.

The Ss are laughing and seem to be enjoying the activity.

10:43 T uses $\mathrm{C}$ to ask for more parts of the body and Ss go out to label drawings on $\mathrm{BB}$.

T uses mixed-code and code switching to both give instructions, ask questions and give explanations.

10:45 T tells Ss to repeat after her as she reads through the different parts of the body. She stops to explain 'beard' then concentrates on pronunciation of 'moustache' and 'thumb' and gets them to repeat them several times.

$\mathrm{T}$ then tells $\mathrm{Ss}$ to read the words aloud as she points to them on $\mathrm{Bb}$, occasionally stopping to get them to repeat certain words.

10:47 T tells the Ss that they are going to play a game. She gives an explanation of the game in a mixture of $\mathrm{C}$ and $\mathrm{E}$.

T gives instruction and Ss follow: 'Raise your left hand'

'Touch your chin'

'Touch your neck'

'Raise your both hands'

'Stand up'

'Touch your chin with your left hand'

'Point to your lips'

'Put your right hand on your left

shoulder'

'Touch your chin with your thumb'

'Sit down'

10:52 T tells Ss to write down a sentence or command like she had told them. Then she explains in $\mathrm{C}$ and gives examples in $\mathrm{E}$.

$\mathrm{T}$ writes examples on $\mathrm{BB}$ :

Put your left hand on your right shoulder. 
Point to your lips.

Raise your right hand.

10:54 T tells 2 Ss to stand up and tell each other their commands. The Ss are noisy and excited.

T tells 2 more Ss to stand and give command. T has to repeat the command as first $\mathrm{S}$ cannot hear.

T tells 2 more. She tells the first $S$ to speak louder as class is noisy. The $S$ seems not sure of what to say. T has to repeat so that the class can hear. She then tells them to reverse their roles and speak louder. The $\mathrm{S}$ does the action.

10:58 T tells Ss she has a word puzzle for them and gives it out.

$T$ explains in $C$ then in $E$ to do the puzzle without looking at their books. Ss do puzzle. T reminds them not to look at their books or at BB.

A S asks about \#5 in C. T tells them, in $\mathrm{E}$, it is a finger, "but what kind of finger?'

T tells class to raise their hands if they want to ask a question.

11:02 T speaks in $\mathrm{C}$ in response to a $\mathrm{S}$ 's question.

$\mathrm{T}$ explains \#2 in $\mathrm{E}$.

Ss ask in $\mathrm{C}$ about different clues and $\mathrm{T}$ responds in a mixture of $\mathrm{C}$ and $\mathrm{E}$, giving them more clues. There is some interaction between $\mathrm{Ss}$ and $\mathrm{T}$.

11:04 T calls on a $S$ to write the answers to the puzzle on the BB. S erases what is on BB. Some Ss raise their hands to answer and another S asks (in C) to be given the chance to answer. First $\mathrm{S}$ writes answers on $\mathrm{BB}$.

11:06 Bell rings.

T says 'Let's check the answers' and then turns to BB and writes answers from \#11 onwards to add to those $\mathrm{S}$ had written.

$\mathrm{T}$ tells Ss to read out the answers one by one after her, getting them to repeat 'chest', 'chin', 'shoulder' and 'wrist'.

$\mathrm{T}$ tells class to do the exercises on p. $35 \& 36$, she then writes it on the BB. She also tells them to keep their $\mathrm{w} / \mathrm{s}$ and bring it to lesson tomorrow. 


\section{Post-Observation Remarks}

1. Use of Cantonese - The $T$ said that she uses $C$ to give instructions, for classroom control and to tell the Ss why she is telling them to do something. She will not give them a translation of anything that they find difficult, instead she will give them clues to try to get them to think for themselves. In addition, she will never write $\mathrm{C}$ on the $\mathrm{BB}$, although she said that some $\mathrm{E}$ teachers do, and she tells her Ss not to write $C$ in their books. This was due to her own experience as a learner, when having to avoid the use of $\mathrm{C}$ helped to push her to learn $\mathrm{E}$.

2. Use of materials - The materials used were a mixture of published materials (core textbook, listening book, reader) and teacher-produced materials. Each teacher in the year group was given responsibility for developing materials for different topics and then other $\mathrm{Ts}$ would share them. The $\mathrm{T}$ said that they were given a lot of flexibility as regards how they teach.

3. Roles \& responsibilities - The $T$ was in her first year at the school but was a member of the Extra-curricular Activities Committee which organised English activities for the Ss during holidays.

4. Relationships - She said that her relationships with the Ss were good with the lower forms, especially the girls, but not with the upper forms (F4). With colleagues, relationships were very good and she could share both ideas and problems.

5. Status of the profession - She felt that some Ts were not committed to teaching as a career and were always complaining about class size, workload, materials support and insufficient support and equipment for IT.

6. Extent of use of BATESL methods \& practices - The BATESL basically prepared them for teaching and the techniques learned in the course she was able to use for 10 - $20 \%$ of the time. She felt that the course would be improved by having videos of real lessons so that the BATESL students could see get an impression of the behaviour of students in real classroom as it was often hard for them to visualise the real situation.

7. Evidence of changes in self - The $T$ felt that that she needed more patience and experience in order to get more satisfaction from the job. She did feel that she was more objective than she had been in the past in that she was better able to step back and judge what the Ss needed. She felt that there had been no real changes in her beliefs as teaching had been what she had expected. She was still learning to shorten the distance between theory and practice and what she should and shouldn't do. 


\section{Observer's Remarks}

The $T$ used a visual and physical approach to teach the Ss how to identify and pronounce the different parts of the body. As in the previous lesson, the Ss enjoyed coming out to the $\mathrm{BB}$ and labeling the parts. This was evidenced by the number of Ss who were keen to come out. In addition, they enjoyed the physical actions of touching their body parts as the teacher gave them instructions. The crossword puzzle was a nice way of consolidating what they had learned during the lesson.

It was important to spend some time on the pronunciation of certain words such as 'moustache', 'chin', 'chest', 'wrist' and 'shoulder'. These contain the consonant sounds that many Chinese students find difficult.

I would suggest that a way of continuing and developing this topic might be to get the students to bring in pictures and to go on to describe the features of them, using both the vocabulary learned as well as descriptive adjectives. 
Teacher:

Observer:

School:
Graduate G(94)

Alan Urmston

Band 2/3 Government-aided co-ed secondary school on Hong Kong Island

\section{Background}

The teacher graduated from the BATESL course in 1994 and was a member of the First-year Teacher Project which followed five of the graduates in their first year in the classroom. He had been teaching in the same school since graduation. During that time he had also completed his Certificate of Education and was in the second year of the MATESL course at City University.

\section{Observation 1}

Date: 26 May 1999

Class: Form 4 English, remedial group.

Topic/Skills: Understanding requirements and format of oral exam; practice in exam skills

The class was a small F4 group of about 15 students described by the teacher as a 'remedial group'. As the end-of-year examinations were coming up, the teaching was mainly focused on that. The $T$ described the class as lacking in motivation more than ability. In the class to be observed, the teacher was preparing the students for an oral exam that they would have the following week.

\section{Lesson Record}

3:00 T arrives then class arrives and waits outside until $\mathrm{T}$ tells them to enter. Ss greet $\mathrm{T}$ and $\mathrm{O}$.

T explains to Ss that they need to prepare for their forthcoming oral exam so he gives out vocabulary list. Then T gives some instructions about next day's lesson.

3:04 T tells Ss that they will have their oral exam next week so they are going to practice. He gives out two worksheets and tells them that they will do both if they have time.

T explains situation to Ss by referring to possible experience they may have had. He tells them that they have five hints and reads them out. The T then tells the Ss that they have 3-5 minutes to form questions based on the hints given. 
3:08 Ss working in pairs as $T$ circulates to check what they are doing.

T says, 'Sorry to interrupt you' and tells them to think of another question for \#6 and to write the question down on their paper.

3:09 T writes on BB:

1.

2.

3
4.

5.

6.

3:10 T tells Ss that they have one more minute and reminds them to think of a question for $\# 6$.

3:11 T tells class that time is up, then chooses a $\mathrm{S}$ to go out to $\mathrm{BB}$ and write a question for \#1. S writes:

\section{What thing did you lose?}

$T$ chooses another $S$ to write a question for $\# 2$ :

\section{Where did you lost it?}

Another S writes 3\#:

\section{When did you lost it?}

Another S writes \#4:

\section{Can you descript that thing?}

Another S writes \#5:

\section{How much did it cost?}

$T$ tells Ss not to write down the questions as they may not be correct. Then another $\mathrm{S}$ is chosen to write \#6:

\section{What do you want me to do?}

Then the $\mathrm{T}$ goes through the questions and asks the Ss if they are correct, using a $\mathrm{C}$ phrase and asking them to correct any mistakes. The $\mathrm{T}$ then corrects the mistakes in the questions. 
3:16 The T discusses \#6 and tells the class that it may not be acceptable even though it is grammatically correct.

The $T$ goes back to the situation and asks the Ss where they can find the information they need and the Ss answer, 'On the paper.'

$\mathrm{T}$ chooses a $\mathrm{S}$ to stand up and begin the role play by introducing himself. Then he demonstrates how to introduce themselves.

$\mathrm{T}$ chooses another $\mathrm{S}$ to demonstrate. The $\mathrm{S}$ stands up and demonstrates introducing himself.

3:21 The T tells the Ss to write down his responses to the questions so as the Ss read out the questions, he answers them and the Ss write down the T's responses.

3:22 A S asks the $T$ to repeat a response but the $T$ answers that in the exam responses won't be repeated. But he does repeat it anyway. Another S asks \#5 and the $\mathrm{T}$ answers, followed by \#6. A $\mathrm{S}$ then asks the question, 'How can I contact you?' The $T$ praises the $S$ for a good question then gives a phone number as answer and the class write it down.

3:25 Next the $\mathrm{T}$ says that he has changed his role and he is now the boss, so he asks the Ss questions on what he said before. He chooses Ss to answer the questions which are not in the same order as in the first part.

The $T$ tells the class that they will not have time to do the second sheet so they can do it themselves for practice.

3:28 T tells Ss to pass their exercise books to the front while one $S$ passes out other exercise books. Another S cleans the BB. T reminds class about dictation on Friday.

T asks a $\mathrm{S}$ why he has not handed in his book and $\mathrm{S}$ gives an excuse.

$\mathrm{T}$ asks if they have any questions about the exam.

3:30 Bell rings and $\mathrm{T}$ dismisses class.

\section{Post-Observation Remarks}

The T had to carry out some duties and so there was no post-lesson discussion 


\section{Observer's Remarks}

The lesson was nicely paced and quite a lot was done. The Ss were involved in what was going on and seemed to acquire the content such that the lesson seemed to fulfill its objectives. The Ss were able to formulate questions and understand how the exam worked. They enjoyed writing questions on the $\mathrm{BB}$ and correcting them.

The lesson was T-centered and the Ss had little chance to do anything interactive or communicative, although they did work in pairs to formulate the questions. Perhaps due to a lack of time they did not get the chance to actually practice the full role play by themselves.

\section{Observation 2}

Date: 28 May 1999

Class: Form 4 English, remedial group.

Topic/Skills: Vocabulary dictation test \& grammar revision.

The class was the same one as in the previous observation. In this class they would again be preparing for the upcoming examinations. They would first have a dictation test of twenty words they had learned previously. Then they would do some revision work from their textbook on the present perfect continuous tense.

\section{Lesson Record}

3:00 T greets class.

$\mathrm{T}$ talks about coming examinations then tells class to get out their dictation books and to put their other books away.

Some Ss who may have been absent in previous lesson did not know that there would be a dictation. $\mathrm{T}$ tells them to do their best.

3:03 T reads out the words and repeats each one once:

$\begin{array}{ll}\text { '\#1 } & \text { essential } \\ \# 2 & \text { prospective } \\ \# 3 & \text { impression } \\ \# 4 & \text { process } \\ \# 5 & \text { presentable } \\ \# 6 & \text { makeup } \\ \# 7 & \text { candidates } \\ \# 8 & \text { panel interview } \\ \# 9 & \text { techniques }\end{array}$




$\begin{array}{ll}\# 10 & \text { lasting } \\ \# 11 & \text { occupation } \\ \# 12 & \text { confident } \\ \# 13 & \text { keenness } \\ \# 14 & \text { boastful } \\ \# 15 & \text { applicants } \\ \# 16 & \text { beaming } \\ \# 17 & \text { lap } \\ \# 18 & \text { eye-contact } \\ \# 19 & \text { slump } \\ \# 20 & \text { punctual ' }\end{array}$

T says, 'Pay attention,' then repeats the whole list a third time.

Then the T went through the words one more time quickly and gave the Ss one minute to tidy up.

$\mathrm{T}$ gives Ss instructions for dictation exam and for next lesson.

3:08 T tells Ss to pass their books to the front.

3:09 T asks the Ss if they are tired and they answer that they are. He tells them that will be able to go home after 20 minutes. Then he tells them to turn to page 116 for a revision of the present perfect tense in the passive voice.

T writes on $\mathrm{BB}$ :

The company has received many applications for this job.

He asks one or two Ss why they haven't brought their books. One S answers in $\mathrm{C}$ and $\mathrm{T}$ replies that he doesn't understand $\mathrm{C}$ so the $\mathrm{S}$ repeats in $\mathrm{E}$.

$\mathrm{T}$ asks what tense is used in the sentence on the $\mathrm{BB}$. Ss call out wrong answers and $\mathrm{T}$ shows mock incredulity. Then he writes in a corner of the BB:

I eat an apple.

Then he talks the class through the transformation of above sentence to the passive:

An apple is eaten by me.

Then he asks for a transformation of sentence on BB:

Many applications .... 
Then asks for word that must be added for present perfect in passive and writes:

$$
\begin{aligned}
& \text { has/have + been } \\
& + \text { past participle }
\end{aligned}
$$

Then goes back to original sentence:

Many applications have been received for the job.

3:16 T refers Ss to book and example, 'The employer has interviewed ten people for the job.' $T$ talks the Ss through the transformation.

$T$ then reads through 'Practice' instructions and explains to Ss what they must do. Then he talks through the examples in 'Part $\mathrm{A}$ ' and tells them to finish the exercise in pairs. He gives them 5 minutes to do it.

3:21 Ss work in pairs on exercise. T walks around monitoring what they are doing.

3:26 T tells a $S$ to read the first sentence. Then he asks another $S$ if he agrees and tells him to read it out as well. $T$ has to tell the $S$ to speak louder.

Next the $T$ tells another $S$ to read the next sentence and so on through the rest of the passage, occasionally asking for confirmation or correction if they get it wrong.

3:29 The T says that they don't have time to do the next exercise. It is a speaking activity but tells them to do it as a written one at home.

3:30 Bell rings. Ss stand up and say good-bye to $\mathrm{O}$ and $\mathrm{T}$. $\mathrm{T}$ reminds class to prepare for their dictation.

\section{Post-Observation Remarks}

1. Use of Cantonese/Chinese - The $T$ uses only $E$ in the classroom. Outside of the classroom he will use $\mathrm{C}$ to interact with lower form and/or Ss whose $\mathrm{E}$ is weak.

2. Medium of instruction - The school is EMI and so has not changed. If anything, the $T$ has noticed more emphasis on $E$ in terms of activities, notices etc., as if there has been a renewal of commitment to being EMI.

3. Use/development of teaching materials - The $\mathrm{T}$ uses only published materials as he has no time to develop his own. There is no official school policy on materials development but some Ts will do some on an ad-hoc basis. 
4. Content/mode of instruction - The $T$ said that he will use interactive activities for between 60 and $70 \%$ of the time with less motivated Ss to try to maintain their attention. He felt that his teaching had become less communicative since graduation from the BATESL course and more grammar-based and exam-oriented. Ss and parents expected uniformity and to teach what Ss needed to succeed in exams.

5. Roles and responsibilities - The $T$ had been promoted to Junior Panel Head with responsibility for F1-3; LAC Coordinator, responsible for F1 bridging programme; EMI coordinator, responsible for dealing with HKIEd. as regards EMI, but has not been able to participate as yet; and Careers Master, which takes up approx. $40 \%$ of working time.

6. Relationships - Good relationships with Ss that he teaches but some disagreements with $\mathrm{F} 6$ class as form teacher. In the past he was very strict but has since learned to lower his expectations and be more accepting of Ss' weaknesses. Very good relationships with colleagues, although there may sometimes be differences of opinion. In the past he was able to take sides in a dispute, but increased responsibility means that he must now remain neutral.

7. Status - Less experienced colleagues may seek his advice and other colleagues due to his position of authority. He feels that the status of Ts is not high enough but that the govt. is taking measures to raise it.

8. Effects of benchmarking - Not worried personally, he feels that if Ts are qualified so why have another test? It is not fair on the Ts.

9. Effects of Ss from Mainland - They do get some Ss from China but mainly in the higher forms.

10. Use of methods/approaches from BATESL - Not much now but some things such as lesson format - 'ppp' \& use of 'warmers' etc. Main problem is lack of time to prepare, but will use some of the things he learned when he can. School policy is also against anything that strays away from the syllabus.

11. Use of innovations in teaching - From time to time the $T$ will make use of aids such as language learning websites so as to try to keep his teaching fresh and interesting for the Ss. He will also advise that the textbook is changed as often as possible. He feels that graduates from the BATESL or other, similar courses are more open to innovations than other teachers who did not do such courses. He describes such teachers as being closed to new ideas. He feels also that as he becomes more experienced, he also becomes more open to innovations. This is an effect of advancements in technology that can be used in teaching.

12. Teacher as innovator - He feels that he does take a central role in his group of colleagues by making suggestions and having other Ts work with him. As he is still studying for his MA, he finds that colleagues will come to him for ideas. As far as 
developing the curriculum is concerned, the Ts will work together, although there had been no real changes in lower form or exam curricula. He feels that the curriculum is changing because of the changing needs of Ss, it is becoming more skills-based.

13. Changes in BAK - He feels that he has become more flexible and that what was learned in the BATESL was not practical. He will put more effort into teaching Ss who are motivated and will do only the bare minimum for those who are not. He has learned how to allocate his time and energy to the best effect.

\section{Observer's Remarks}

The $\mathrm{T}$ seems more at ease with himself and his teaching compared with five years ago. This is understandable. He is also very accomplished and confident in the classroom. It would seem that he is now starting to settle down into the role of teacher. After he finishes his MA then he will be able to devote more time to his teaching and perhaps employ some of the methods and practices learned in the BATESL and incorporate more innovations in his teaching. He still seems to believe that what he learned in the BATESL is essentially worthwhile (more so than the P.C.Ed.) and that it has given him a solid background from which to develop his career. 
Teacher:

Observer:

School:
Graduate H(94)

Alan Urmston

Band 3/4 Government-aided co-ed secondary school in New Territories new town

\section{Background}

The teacher graduated from the BATESL course in 1994 and was a member of the First-year Teacher Project which followed five of the graduates in their first year in the classroom. The present year was her third in the school; she had been teaching there since it opened.

The school, being newly-built is well-appointed, having OHP and TV/video in each classroom. In addition, due to funds gained through the Government's Quality Education Fund (QEF), the school has a fully-equipped multi-media language lab (MMLL) with space for up to 40 students and a full-time technician. The $T$ said that the MMLL was generally used for S self-access work (as it saved on marking) and writing. The $T$ made use of the MMLL once or twice per month with the class and would tell them to use it for self-access during lunch times or after school.

\section{Observation 1}

Date: 1 June 1999

Class: Form 1 English class

Topic/Skills: Use of 'too' and 'enough' with adjectives

The lesson to be observed took place in the MMLL with a high-ability Form 1 group of 20 Ss. These Ss would have 7 lessons of E per cycle plus 2 with the NET. The T would focus on grammar and writing, leaving speaking and listening to the NET. The materials used in the class were based on the Ss' textbook, using Powerpoint to present the language items and Tense Buster for practice.

\section{$\underline{\text { Lesson Record }}$}

12:02 T brings Ss into room one group at a time [each group consisting of $4 \mathrm{Ss}$ ] and tells them at which computer terminal they should sit. the Ss are a little excited. The $\mathrm{T}$ tells them not to touch their computers.

Ss greet $\mathrm{O}$ and $\mathrm{T}$.

$\mathrm{T}$ tells a $\mathrm{S}$ to give her the scores for the different groups. The $\mathrm{S}$ replies in $\mathrm{C}$ and the $T$ writes up the scores for each group on the whiteboard. 
T says that 2 Ss need to come to the MMLL 2 times that week, which she repeats in $\mathrm{C}$.

12:05 T gives out prizes for the groups. She describes the prizes and tells the Ss from each group to stand up. She then tells one $S$ to give out the prizes to the others in their group.

$\mathrm{T}$ asks another group what prizes they want.

12:08 The $\mathrm{T}$ uses the big screen at the front of the room to give a Powerpoint presentation of the uses of 'too' and 'enough'. As she touches the screen the frame changes. The $T$ asks a $S$ for the missing adjective then when the $S$ answers she checks the answer by touching the screen and the missing word appears in the sentence. If the $S$ answers correctly the $T$ gives the $S$ 's group 10 points. The $\mathrm{T}$ then tells them that they need to learn this, repeating in $\mathrm{C}$.

T explains the use of 'too ' as alternative to 'not enough'.

She then gives examples and calls on different groups to answer, offering prizes for correct answers.

12:13 T changes her screen to the practice exercises. She tells the Ss to 'Press 1' and then explains what she means in C. She explains how to use the programme to choose the correct answer to the questions in a cloze exercise. They must choose either 'too' or 'enough'. T puts a question up on the screen and Ss choose either 'too' or 'enough' by pressing ' 1 ' or ' 2 '.

T chooses another question and explains what 'shy' means.

$T$ calls up a frame on the screen which shows the individual scores of each $S$ in terms of their terminal number and makes some comments.

12:19 T goes on to the next question and Ss choose the answer. She then again checks the running scores of the class and allocates scores to each group. The Ss seem to be enjoying the competitive element.

12:23 T tells Ss to do an exercise from Clarity. They need to type their name and then do the revision exercise from Tense Buster. The T has put some of the vocabulary from the exercise into a box and she goes through them asking for their meanings. The Ss put up their hands and offer translations of the words. The $T$ needs to give some clues (in E) to help them to get the correct meaning. When they get the correct meaning, the $\mathrm{T}$ gives their group some points.

12:27 T tells the class to do the exercises themselves before they leave the classroom. She walks around and helps some Ss to find the exercise, using the big screen to show them. She scolds one $S$ for being too slow in finding the exercise. 
12:30 Bell rings.

Ss continue working. The $T$ gives them 10 seconds and counts them down before telling them to look at the big screen. She explains what they have to do, with accompanying explanations in $\mathrm{C}$. She also gives them a look at the answers.

$\mathrm{T}$ tells the Ss to come back to the MMLL themselves to finish all the exercises and then print the report and hand it in to her before Thursday (two days later). She goes over this in $\mathrm{C}$. Then she explains how to print.

12:35 T tells the class to stand up and tell the group with the most points to collect their prizes.

$\mathrm{T}$ dismisses class.

\section{Post-Observation Remarks}

The $\mathrm{T}$ felt that the lesson had been badly timed resulting in the Ss having to rush the exercise at the end. This was because she had to go to collect the Ss from their previous lesson, which usually ran late. In addition, she felt that the prize-giving had taken longer than expected. As a result, she had skipped two exercises that she had originally planned for the Ss to do and gone straight on to the more difficult revision exercise. She felt that they would be able to do this exercise without necessarily doing the first two, although she had told them to do these themselves anyway.

1. Use of Cantonese - The $\mathrm{T}$ said that she used $\mathrm{C}$ more with her $\mathrm{F} 4$ class $(50-80 \%)$ than with her $\mathrm{F} 1$ class (10-20\%). She used $\mathrm{C}$ to explain grammar, to explain difficult words, for discipline. She often used $\mathrm{C}$ to tell jokes and experiences to show her Ss that $\mathrm{E}$ is important. The school was CMI for F1-F3 and had been since it opened. F4 was EMI as all the Ss had come from other schools, some EMI and some CMI. The $T$ felt that $E$ was given a lot of emphasis within the school. There were 2 NETs, a lower $T: S$ ratio for $E$ (1:20 for lower forms and 1:30 for upper) than most other schools, and extra Ts to teach the best Ss.

2. Use of materials - The $\mathrm{T}$ estimated that at the beginning of the term, maybe $70 \%$ of the materials used were published and the rest were adapted. Later this would change to about 50-50. The materials would be supplemented with games and activities designed by the $\mathrm{T}$. There was no $\mathrm{T}$-in-charge for materials in $\mathrm{F} 1$, but there was for F2 and F3. She had taken on the role of preparing materials for F1 Ss.

3. Teaching strategies - The T makes a lot of use of group work including competition between the groups and group discussion. The $\mathrm{T}$ tries to make her lessons interactive 
and communicative but sometimes there is not the time to plan specific activities. She likes them to work in groups.

4. Content of instruction - Grammar and writing with a little reading, mainly examoriented.

5. Roles and responsibilities - She was a member of the MMLL Team, responsible for developing materials for use in the MMLL and for helping other Ts to get the most out of the facility. She was Resource Manager (for English resources), F5 Coordinator, Academic Section (to overlook academic processes of the school), PTA (to hold meetings, design notice boards etc.), ECA and form teacher.

6. Relationships with students - She thinks that $80 \%$ of the Ss think that she is strict. Outside class she is friendly with Ss. Although she tends to spend more time with the upper forms, she is more friendly with the lower forms.

7. Relationships with colleagues - Good to above average relationships with most Ss but not with all. She feels that she is rather outspoken which might lead to some Ss not liking her. Perhaps the computer teachers are jealous of her use of the MMLL, or maybe Chinese teachers are jealous of the special status of English.

8. Status of self and profession - She is happy to be a $T$, but not happy with the status of Ts. She feels that people should know more about what it is like to be a $T$ then they might understand and the status of the profession might rise. She feels that teaching $\mathrm{E}$ is more dynamic than other subjects.

9. Effects of benchmarking - It will mean more work as Ts will need to prepare for it. She feels that it is unfair to test $T s$ as they are qualified already and the benchmarking cannot reveal the whole picture of what it takes to be an E T.

10. Effect of Ss from Mainland - There are a few Ss in the school from the Mainland. They are very good but not in $\mathrm{E}$, and they are eager to learn.

11. Use of methods/approaches from BATESL - She uses more with the lower forms as she has more time and the help of colleagues, but with the senior forms she uses a more grammatical approach. She is still aware of the approaches learned in the course and feels that they make up the basis of her knowledge of teaching. She feels that she is not using so much because of lack of time and energy, but she feels that her present school is not as restrictive as her previous school.

12. BATESL as preparation for teaching - The $T$ felt that the course did not prepare $T$ s adequately enough for the Hong Kong teaching context in terms of Ss' behaviour, ability and motivation, as well as the roles and responsibilities they need to take on and the benefits of using IT in teaching. 
13. Use of innovations in teaching - She feels that she is using innovations, such as games, group work and process writing on the computer. She is the only $T$ who uses groups in her classes. It could be because most of her colleagues are not as experienced as she is and so are not as confident in using innovations. As she becomes more experienced, she feels that she becomes more open to innovations and learns how to adapt to change. She feels that she is central to her group of colleagues, especially in the development of materials. She feels that she is now an experienced $\mathrm{T}$.

14. Curricular evolution - Through staff development, innovation is being fed into the curriculum. An example is the use of MM. If something is found to be good then it is shared and included in the curriculum.

15. Changes in beliefs - Her beliefs haven't changed, she still believes in discipline before teaching, being prepared (in terms of materials, ideas and knowledge) and being talkative at the right time. In addition, she now believes that $\mathrm{E}$ should be creative and fun.

\section{Observer's Remarks}

The $T$ has an air of authority and confidence and clearly sees herself as one of the more experienced and senior members of the teaching staff. She seems to have developed her own teaching style and philosophy and is happy with it. Although she is not happy with the status and situation of teachers in general in Hong Kong, she seems satisfied in her own situation. She is adopting innovations in her teaching, within the constraints of the system, and sees herself as an innovator and participant in curricular evolution. 


\title{
C.4 Transcriptions of Interviews with BATESL Course Leaders in 1999
}

\author{
Interviewee: Course Leader (1994) of BATESL, Department of English, \\ City University of Hong Kong \\ Date: \\ 5 October 1999
}

$\begin{array}{ll}\text { AU } & \text { Researcher } \\ \text { CL(94) } & \text { Interviewee } \\ \ldots & \text { Missing words / Unfinished utterance } \\ \text {. } & \text { Pause }\end{array}$

AU How about the BATESL, they're the focus of my work.

CL(94) Well I'm not so intimately involved with them, I'm mainly MATESL now but I don't teach them. I haven't seen a significant change, no. I still think proficiency is a problem for a significant minority of them. I think this year the entry results were a little bit better than in the past but I don't think it's significant. I really don't think we should be taking people below $\mathrm{C}$, but we do.

AU He said you'd been taking in more mature students.

CL(94) Yes, that's a good trend.

AU Anything in their approach, like their desire to teach, or their ideals.

CL(94) I don't know them that well, but those that I've taught my impression is that more of them want to go into teaching than I thought, whether that's just an impression from the year I taught last year. The real trouble with the BATESL is not being able to have a fourth year to run straight through and then they have to compete for places at other institutions and then sometimes they get turned down and if they do get in a lot of them don't get credit for what they've done and they're doing a lot of stuff which is Mickey Mouse to a lot of stuff they've done here and that's a problem that has to be solved.

AU They have told me that they find it difficult to find a job because very often the school principals don't know about them, the BATESL. If they're not a Hong Kong $U$ or $C U$ graduate or I.Ed., they tend to be lower down the frame.

CL(94) It's a shame that's still true, but we're building up. I've been very impressed, I mean I haven't seen a great number but I've seen a number of our students 
in schools and my impression is that they have a very very hard first year, first two years, but after that, those that survive seem to be doing quite well. And I don't know there's any way you could help them over that. Maybe we should have more outreach after the course, but how we would build that in with time and resources, but I do feel we need to give them more support in their first year of teaching.

AU With the group that we worked with, with [Co-Investigators of First-year Teacher Project], they got that as part of our project, and [G(94)] and [other graduate] and [H(94)], who I've seen, are doing really well.

CL(94) Oh [other graduate], because I was worried about [him].

AU He's still in the same school, he seems to be happy with what he's doing.

CL(94) $[G(94)]$ I've seen, of course, because he came back to do the MA.

AU Yes, he's now assistant panel chair, and [H(94)] I saw.

CL(94) $\quad[\mathrm{H}(94)]$ I wouldn't have thought should would stick at it.

AU Neither would I, but she has. She said the first year was really tough and so she moved to a new school, which is often a good idea.

$\mathrm{CL}$ (94) I'd like to see the results. It seems to me that it's very hard to know how much of what they get here sticks after the trauma of that first year. If the students did their first year and then come here, that would be the ideal. I still can't see why we can't run it part-time but there still seems to be resistance to running the BATESL part-time.

AU I think generally speaking what they told me was that once they'd got through that first couple of years and then they started falling back on the stuff they'd learned here, and started bringing it in as they'd gained in confidence and established themselves in the school.

$\mathrm{CL}$ (94) I do think we need to do more, whatever you want to call it, teaching English in large classes and large ill-disciplined classes. I know they get a certain amount of that, more than they did from ... particularly. I think we were a bit unrealistic in the beginning in not quite having enough of that.

AU What would be nice and I was speaking to [CL(99)] about this is to have a detailed knowledge of where the graduates go, whether they stay in teaching.

CL(94) Do we have that? 
AU $\quad[C L(99)]$ 's tried it but he said it's very difficult keeping track of them.

CL(94) It's interesting to see a trickle coming through to the MA, I think they think it' $d$ be easier coming here after being here. If it were me I'd want to go somewhere else, to get some fresh ideas, but it's nice to see.

AU Yes, as you say, maybe they just like the place and find it easier.

CL(94) What surprises me is the way we keep up the MATESL numbers, I mean we've got good students. As you know it's up to the whim of the principals as to whether they get any credit for it or any time off, very few get any time off for it, they don't even get time off for extra-curricular activities. And they get very little credit for getting it, but we still get them coming through and I don't think they all do it because they hope to escape into tertiary because they know it's not possible.

AU They get credit for it if they don't have any other English teaching qualification.

CL(94) I don't know, if they haven't got post-graduate diploma they haven't got qualified teacher status, MA or no MA. Our MA doesn't give them QTS. A sensible principal will take it into account when promoting, but principals aren't exactly sensible. Promotion doesn't necessarily go on qualifications and ability as you know, seniority, friendship.

AU If the teachers are graduating from the MATESL and going into schools, you are getting a more and more expert workforce.

CL(94) The thing about the teachers here is the real or imagined feeling of powerlessness. They don't believe that they can change things. They believe that they are at the whim of the principal and the Government, Education Department, and to some extent they are but sometimes it seems to me to be an excuse.

AU Yes, after all, they are the master in their own classroom. In most schools in my experience teachers can teach in the way they want.

CL(94) Still the biggest impression when I go out is just the sheer variety of schools. You go into one class and there's a riot going on, a real full-scale riot, you go into another one and they're all lining up outside the door and saying, good morning. There's an incredible difference between the schools and even maybe within schools and I don't know whether it's down to class or simply banding, the area. Like in Britain it's nice to have a lot of variety in schools, a lot of de-centralisation, but here there's a little bit more standardisation in the curriculum so there is this distinction in the streaming and the language, and also the principal didn't have quite so much power. 
They're a bit too autonomous some of the schools, that's my feeling.

AU And the school authorities have a lot of power too, most of them being Christian.

CL(94) You certainly don't want them being run by the Education Department in a more hands-on way, but you'd hope that the professional association ... 
Interviewee:

Date:

$\mathrm{AU}$

CL(99)

...

-

AU

CL(99)

AU

CL(99) Oh, that's things that they were unsure about, those who did not have a definite philosophy of teaching had to say what things they were unsure about

CL(99) I remember around $97 \ldots$ who were the people in that class, the trouble is they begin to flow ...

AU I know, the ones I've spoken to are B (97), I (97), H (97), A (97), they were the group that I'm following and I've observed some of their classes.

CL(99) You've observed them teaching? How were they doing?

AU Pretty well, B (97)'s teaching in a private school, it's one of these postsecondary pre-vocational schools. He seems to be doing okay. 
CL(99) Yeah, I should imagine he'll do well in most places. H (97) was one of those ... averaging on the weaker side, how is she getting on?

AU She's determined, very determined, she hasn't taught straight away after graduation, like a lot of them she found it hard to get a job. What they said was that a lot of school principals didn't recognise the BATESL. Institute of Education, Hong Kong U, they were familiar with, but the BATESL, it didn't have the kudos, I think. They weren't sure about it. $H$ (97) hung out and then she got a job in a school in one of the new towns. She's done a year, she only did a year, she wasn't that happy with the school. She tried her best. She was teaching mostly through Chinese, Cantonese in the lessons. The students were very weak. But she still seemed to have that spark in her.

CL(99) Well just last year we sent out about five of these things, between questionnaires, publicity, but I think most of them either get binned or just passed on to the careers master without looking at them even though you try addressing things to head teachers, you know, I've got this project at the moment where I bought in staff, one of these tertiary-secondary interview projects and I've been able to buy in part-time staff to buy out members of BATESL teams to get them out into schools so they have more contact with schools, and I use the letter to that as there's publicity explaining about the course. But the irony is that things happen like in the TESOL Quarterly, they had their special edition on second language teacher education, their best practice section where they selected articles which they regarded as examples from around the world of best practice, there was an article by John Flowerdew on the BATESL. ${ }^{1}$

AU In fact nearly all of the articles were in some way connected with the English Department at City U. City U is recognised world-wide within the field, but locally it seems to' be a battle.

CL(99) Well we made a special effort last year but I despair, because a cycle's going round face to face with people, you invite them when we have our English teaching practice do's, they're busy people, they don't want to come to it. I've put out about five different motions, publicity, we've had open days, could you pass these onto your career master, then ...

AU I think it's just that the market is so much tougher now. There are so many experienced teachers, who have perhaps gone out of teaching and have come back to it because of the state of the economy and so on and some who had emigrated, and so for the fresh graduates, it's difficult anyway, especially if they're not from the I.Ed. Have you noticed any trends over the years in the

\footnotetext{
${ }^{1}$ Flowerdew, J. (1998). Language learning experience in L2 teacher education. TESOL Quarterly, 32(3), pp. 529-535.
} 
BATESL? Is it a case of some years they are good, some years they are not so good?

CL(99) That's exactly it. When we kicked off we had three years of going up and up on our students' academic ability measured by the points in their JUPAS and the English proficiency as regards how many of our intake, what proportion of them get a grade $\mathrm{C}$ or above in Use of English. So the first few years started off like that and then it's a D and people start shouting and screaming at the state of the course, but what I've seen is that we have to, in terms of programme structure, get one that is flexibly geared so that when we do have a dip in the intake, we can give them much more proficiency, we can have a whole range of fallback measures, have a certain benchmark at the end of Year 1 and if they don't reach it have a couple of fallback options they can do among themselves and if they still haven't reached it, boot them out at the end of Year $2 \ldots$ otherwise you just have a nice smooth course for what you expect to be your average or good intake. When we kicked off, despite the fact that we happened to be building a unified degree programme with your intake that you expect, here we were routinely pretty good but we just have this frustration. One of our best years ever in terms of English proficiency was one of our worst years ever in terms of how many students put us up in their category A choice ... so you can either get a year that has got intellectually extremely more acute, proficiency much better than any we've previously had, motivation, but some quirk of the JUPAS system has put us in with a load of people that had us down for the most part between choices eight and twelve. There's a lot of fluctuation there, it's not just a case of proficiency or academic ability. The last two years we've had hiccups again and fingers crossed we're going to go even further because what I've done as programme leader is expanded the number of non-JUPAS people that we are getting. We're getting these experienced people with good qualifications and good Use of English ...

AU Or people who have the teaching certificate but they don't have a degree.

CL(99) We don't get so many of those.

$\mathrm{AU} \quad$ Or who just want a break, they just want to spend three years out of work.

CL(99) We generally get about one every year.

AU It's a lot of money, isn't it, if you're not working?

CL(99) So what would you say is the most striking or salient thing that you've found with the BATESL graduates?

AU I suppose the overall feeling is this sort of, not cynicism as such, but this sort 
of falling off in ideals from when they first started compared to when they come to graduate. Do you have numbers of those who actually teach and how long they actually stay in teaching.

CL(99) We don't have how long they stay in teaching but we have the numbers who go into teaching, it's consistently between 70 to 80 per cent and they've generally been 75 to 80 per cent. But I'm surprised to hear that you find that there's a dropping off in idealism because I get the admissions things where I see what choice they put us, first, second, third, fourth, fifth, twentieth, well I see the bands, A, B, C, D or E and consistently 50 per cent have had us in ... Grade $\mathrm{C}$ or below, so we've had a kind of minority putting us in their first three choices.

AU The teaching is not their preferred career, at least at the beginning, they're looking at something that's going to give them a degree and improve their English and give them a chance to work in a field where English is required.

CL(99) Yeah, so we've always been gratified that so many of them have gone into teaching, but I did want to use those figures initially as a sort of earlywarning device to see which students were likely to be less motivated and act accordingly and give them support accordingly. But what I found was that there was no very strong relationship between the ways that they interact and perform and their choice. I think if they put us up in Band A, their first three choices, then, yeah they were pretty keen, but the ones who have us down at twelfth, thirteenth choice out of twenty, some of them will be treading the water a little bit ... but there were others who were ... one year I did the thing of trying to guess what their choice was and there were some that I was putting up in the top five choices and they were round about fourteenth, so ...

AU I think there are so many different factors involved, whether they have a good experience in their practice teaching, who they make friends with, perhaps if they're on the doubtful side but they have two or three friends who are really into teaching and are enthusiastic, it can rub off on them. A lot of different factors and I think also a general feeling is, and I'm sure you've had this as well, they think their practice teaching is too short, they want more experience.

CL(99) Well, we're giving them a ...

AU It's always theory against practice. Theory's okay but it doesn't really prepare you for when you're stood in front of that class on a Monday morning.

CL(99) Well really for more practice we need the extra year and then we could make the BATESL a B.Ed. course. But with the changeover, we've made the 
practice teaching five weeks, but it's still a small amount.

AU It's still short but ...

CL(99) But it's better than three weeks. But the cost is that they've got to lose the two weeks they get in the second year ... so we run it into a five-week stretch. But what they do do is a classroom observation, which is going to be based on videos, mainly, but we've worked it so that they go in one day a week into schools for observation. As programme leader nobody has ever come to me and said, we need to cut this, everybody has always said, we need to expand this, we need to expand this ... and at the end of the day it's a university degree education and that's what these students care about. There's a certain point where you have to say, look I know that about 50 to 60 per cent of these students are so immature or they've got pressures on them or things like that, it's time to retract, they'll go off and get a second job ... but there's 30,40 per cent, you've got to give them a chance because there's so much that we do on these courses that's so that these poor proficiency students, weakly motivated students and to make sure that they can't do wrong, to boost them up, right? And so the best thing you can do for the good students ... is to let them shine in their own way. I'm against overloading what is already an overloaded timetable, where you've got eighteen contact hours a week, where does the real university learning take place? Where do they start learning things for themselves, and I'm very glad that we introduced this reading week, although I know there's some students who just do some private lessons to get themselves some extra money, there are other students who are very good, that reading week for them it's fabulous, they can actually get these ideas that have interested them and they can deepen their knowledge of them, expand it and the funny thing is that in terms of staff input and time and effort, what it requires is getting the staff to lay off. A lot of staff still have the grammar, secondary school mentality, directing ...

AU The more exposure they get the better.

CL(99) This year's graduates have given me some of the greatest pleasure because it was our worst year in terms of intake and there were lots of kids there who were ... so that we can take them and we can actually allow them into a classroom, we've done virtually a miracle. We had to terminate four people, but I think it's the proof of the programme that this pretty mediocre intake, the ones who came in were pretty shaky, we got up to a good, set level, they could go into a classroom, they've got the skill, they've got the knowledge, the teaching skills to not create any disasters, not harm anybody and sufficient knowledge to be able to make sense of that experience that they are getting, rapidly assimilate it and out the various bits together and turn into fairly solid teachers. With the good students, you can't do much damage to them and they go out into the schools and do a good job but getting in 
some of these ... people at the beginning and seeing how we manage to build up to a respectable level, they are never going to be the bright shining stars that ... but you get an intake like that and you know what Hong Kong's like, everybody says education's really important and English is really important and education is very Confucian-ethic and when it comes to what they want their kids to do it's be a lawyer, be a businessman. An English teacher? Why don't you try something else? Students come in the first couple of weeks of the academic year and there was some girl from the Law Department crying, her dad had forced her into law and it was exactly as she'd expected it to be. The people were absolutely horrible and the subject was terrible and was there no way she could get in. It's one of those great lip-service things, isn't it? I would definitely say that the general quality of teaching is improving slightly each year. The general quality of English language usage in terms of the proportion of people able to use good English, I'm not talking about your chief executive officer or your financial wheeler and dealer types, but people who can knock a bit of English together because they need to use it in their job.

AU I've talked to some of the older graduates and what they've said because they graduated in $1994 \ldots$

CL(99) That was our first intake?

AU That was the first intake. Now they're able to reflect, they've been teaching five years now, now they're able to reflect on what they're doing, they're able to think about the things they learned in the BATESL and going back and having a look at and re-reading some of the stuff they did then. Perhaps they've got through the first couple of years where it's just madness and they want to just keep their head above water. Now they're experienced teachers and they're able to see how everything that they were taught then can be used. They seem very happy with what they're doing and satisfied and it's nice to see. I don't know what percentage of them that is.

CL(99) I'm going to mail out a questionnaire actually, to all our previous graduates, getting their comments, things that could be improved, changed. If you had any specific points that you would like put in, well I've got to do the ... in the next two weeks. Would you be able to ... I'd be very happy to have you put in plus there'd be somebody to collate the results.

AU Oh yes, that would be great. Are you still able to contact these people? Some of the addresses might be out of date.

CL(99) Well that's the only thing, we don't have an up-to-date list but we can send ... and it'd be for every cohort that's graduated. 
very quickly and the rest ... as you said before, you'll get some who will look at it and say, oh, I can't be bothered, but if it's something very quick they can do, five minutes, they'll do it. Say have a part one that they can do quickly and a part two.

CL(99) Well I was going to do a part one, end of story.

AU Just the basic information would be very nice to get, whether they went into teaching upon graduation or whether they did something else and then went into teaching, or never went into teaching, or if they went into teaching are they still in teaching? Particularly the two groups that I focused on, 94 and 97.

CL(99) I sent out my four hundred and four questionnaires to schools, just a quick, dirty questionnaire along the lines of, we've got these BATESL ... it was another thing that I was doing last year, it was partly to get information and partly to raise the profile. I wrote to the principals and the BATESL

graduates have been teaching for a few years and we'd like to have a bit of feedback on them and basically it was, have you had any of our BATESL graduates, yes or no and if so how would you rate them? And a couple of different characteristics. And again we asked, coming in as new English teachers, have they had new English teachers from other institutes, I.Ed or $\mathrm{HKU}$, and if so how would they rate them? So we could compare ourselves. I think we only got about 10 per cent and it was something that took, it was a three-minuter, it literally was a one page ...

AU Yes, it is disappointing. Even if they're not able to do it themselves, they can pass it on, give it to the panel head or somebody.

CL(99) That's what we asked them to do ... and it would have been nice just to get an impression of just how many different schools our graduates have gone into. I think we ended up getting about forty replies.

AU I don't know if there's any way of doing these things through the Education Department. If they've got ED on them perhaps they're more likely to do it, they think it's official.

CL(99) We gave them reply paid envelopes.

AU I think it's only people who've done higher education and that have done research themselves are likely to do it.

CL(99) I can understand it when they don't have much time. 


\section{C.6 Main Points to Emerge from Interviews with BATESL Course Leaders}

The main points to emerge from the interviews are listed under appropriate headings. Direct quotations from the interviews are shown in italics. The two course leaders are referred to as CL(99) (Course Leader 1999) and CL(94) (Course Leader 1994).

\section{Language proficiency}

CL(94) English proficiency is a problem for BATESL students.

The course should not admit students with lower than Grade C in Use of English.

CL(99) The language standard of the BATESL intake has fluctuated over the years.

Fallback measures are needed to support BATESL students with low proficiency.

The standard of English amongst the general public seems to be improving as more people need to use English for their work and are acquiring what they need.

\section{Changes in intake}

CL(99) As a result of expansions in the intake criteria, more experienced teachers with higher proficiency are entering the BATESL.

The 1999 graduates were the weakest to date in terms of qualifications and proficiency but they were able to graduate with the skills needed to teach successfully in schools.

Number of graduates becoming teachers

CL(94) It seemed that more graduates wanted to go into teaching than had previously.

CL(99) Consistently $75-80$ per cent of BATESL graduates go into teaching.

$$
\mathrm{C}-104
$$




\section{Post-graduation}

CL(94) BATESL graduates often have difficulty getting into post-graduate certificate and diploma courses in education.

BATESL graduates often find that they have already done much of the content of post-graduate certificate and diploma courses in education.

More support for graduates after graduation

CL(94) BATESL graduates teaching in schools tend to have a very difficult first year or two but then go on to do quite well.

There should be more support for the BATESL graduates teaching in schools from the course organisers.

\section{Need for changes to course}

CL(94) The BATESL course should be run part-time to enable serving teachers to enrol.

The BATESL should have a fourth year so that graduates are able to complete their training in education.

CL(99) An extra year could give the BATESL students more practice and lead to the course becoming a B.Ed.

\section{Lack of recognition of course}

CL(99) Promoting the BATESL course is very difficult as teachers and principals tend not to read publicity material or attend open days.

\section{Practice teaching}

CL(94) BATESL students need to be given more practice in teaching large and undisciplined classes so as to prepare for the real world. 
CL(99) Practice teaching has been extended from three weeks to five with more observation using videos.

Teachers as change agents

CL(94) Teachers in Hong Kong feel powerless to enact change, although sometimes this may be an excuse for not trying.

Wide variety of teaching situations

CL(94) There is a great deal of variety between schools and within schools especially in terms of discipline and student motivation, which could be a result of banding or social class.

\section{Autonomy of schools and school authorities}

CL(94) In Hong Kong, schools have too much autonomy and the school authorities, which are usually religious organisations, have a great deal of power also.

\section{Low status of English teaching}

CL(99) There is a contradiction within people in Hong Kong who believe that education is important but prefer their children to go into law or business rather than teaching. 